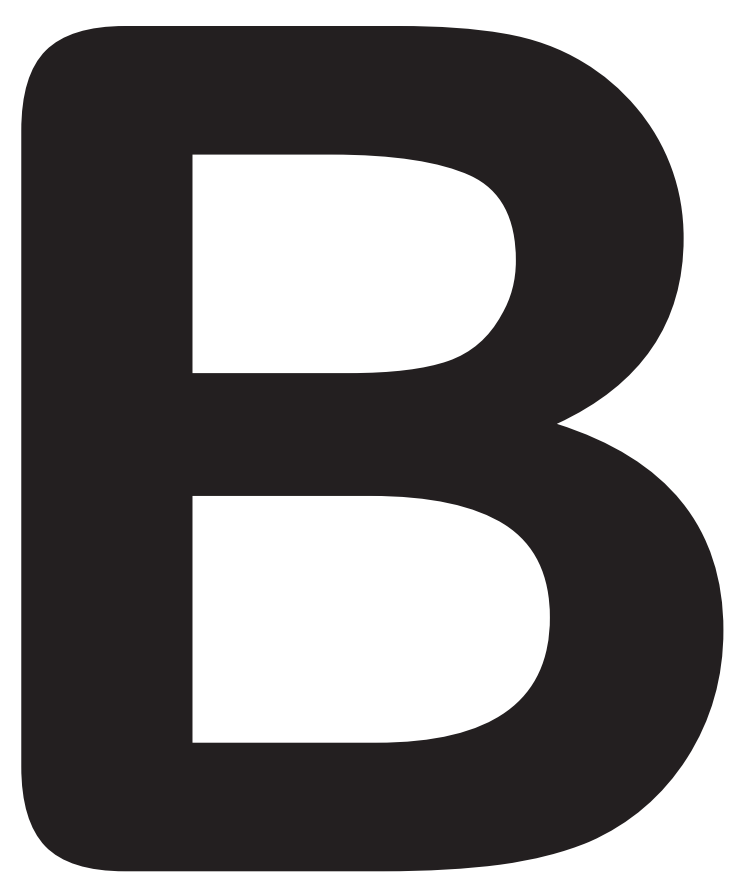

\title{
Scientific Sessions (SS)
}

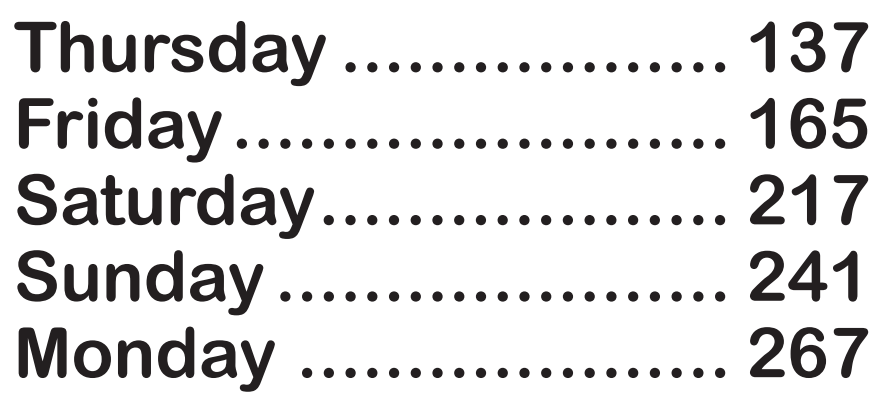




\section{Thursday, March 3}




\section{Abdominal Viscera}

\section{SS 101a}

\section{Hepatocellular carcinoma: screening, diagnosis and management}

\section{Moderators:}

L. Grazioli; Brescia/IT

B. Guiu; Dijon/FR

\section{B-001 14:00}

Hepatocellular carcinoma nodule conspicuity during arterial phase: comparison of contrast-enhanced 64-row multidetector CT with bolus tracking and MR imaging with bolus chase

E. Quaia, L. Giarraputo, A. Lorusso, B. Cabibbo, M.A. Cova; Trieste/IT (quaia@units.it)

Purpose: To compare the HCC nodule conspicuity during the arterial phase between contrast-enhanced 64-row multidetector CT with bolus tracking and MR imaging with bolus chase.

Methods and Materials: Thirty-one biopsy-proven HCCs (diameter, 1-3 cm) in 18 consecutive cirrhotic patients ( 12 males and 6 females; age: $50 \pm 13$ years) scanned by multiphase contrast-enhanced 64-row multidetector CT and, after 7-21 days, by MR imaging were retrospectively analysed. CT started 18 seconds after $140 \mathrm{HU}$ threshold was reached in the abdominal aorta after intravenous contrast injection (370-400 mg I/mL, $4 \mathrm{~mL} / \mathrm{sec}$ at $2 \mathrm{~mL} / \mathrm{kg}$ followed by $50 \mathrm{~mL}$ of saline flush). MR imaging was performed by 3D T1-weighted THRIVE breathold sequence (TR/ $\mathrm{TE}=3 / 1.57 \mathrm{msecs})$ before and after Gd-BOPTA injection $(0.1 \mathrm{mmol} / \mathrm{kg} ; 2 \mathrm{~mL} / \mathrm{sec})$ with contrast injection starting after contrast visualisation in abdominal aorta at MR fluoroscopy. Nodule conspicuity at arterial phase was calculated off-site by quantifying $\mathrm{HCC}$ and liver attenuation (CT) or intensity (MR) by manually drawn ROls on a dedicated workstation. The contrast-to-noise ratio (CNR) on CT [(Attenuation - Attenuation $\left.{ }_{\text {liver }}\right) /\left(S D\right.$ background)x100], and MR imaging [[(Intensity ${ }_{\text {nodule }}$ - Intensity $_{\text {. }}$ )/(SD background)x100] were calculated and compared using Wilcoxon test Results: In 20 HCCs concordant hypervascular profile was shown between CT and MR during the arterial phase, while discordant vascularity profile was identified in 11 nodules appearing isovascular on CT and hypervascular on MR. MR imaging revealed a higher $(P<0.05)$ HCC conspicuity than CT (median CNR, $164 \pm 145$ vs $89 \pm 45$, MR vs CT) during arterial phase.

Conclusion: Bolus-chase MR imaging produces higher HCC nodule conspicuity during arterial phase than $\mathrm{CT}$.

\section{B-002 14:09}

Differential diagnosis among indeterminate lesions in patients undergoing surgery for hepatocellular carcinoma using combination of Gd-EOB-DTPAMRI and contrast-enhanced intraoperative ultrasound using Sonazoid

J. Arita, M. Takahashi, S. Hata, J. Shindoh, T. Aoki, Y. Beck, Y. Sugawara,

K. Hasegawa, N. Kokudo; Tokyo/JP (jarita-tky@umin.ac.jp)

Purpose: Diagnostic roles of hepatobiliary phase of Gd-EOB-DTPA-MRI (HB-EOB) and Kupffer phase of contrast-enhanced intraoperative ultrasound using Sonazoid (Kup-CEIOUS) for differentiating hepatocellular carcinoma (HCC) among lesions showing atypical pattern were assessed.

Methods and Materials: Consecutive 79 patients who received hepatic resection for HCC underwent both preoperative HB-EOB and intraoperative Kup-CEIOUS Images of $\mathrm{HB}-\mathrm{EOB}$ were obtained 20 minutes after intravenous injection of $\mathrm{Gd}$ EOB-DTPA. Sonazoid was injected following fundamental intraoperative ultrasound and whole liver was screened 15 minutes after injection (Kup-CEIOUS). Resected lesions were diagnosed through histological examination; and unresected lesions through postoperative follow-up using dynamic CT.

Results: A total of 204 lesions were detected including 93 lesions showing typical pattern in preoperative dynamic CT, which were all diagnosed as HCC. Of 111 lesions showing atypical pattern, 21 were hypoechoic during Kup-CEIOUS and all but one of them were diagnosed as HCC. Among 90 lesions that were not hypoechoic during Kup-CEIOUS, 45 each were hypointense and not hypointense in HB-EOB, respectively. Six of 45 lesions belonging to the former group were resected and 4 of them $(66 \%)$ were histologically diagnosed as HCC, whereas only 8 of 39 unresected lesions $(21 \%)$ were diagnosed as $\mathrm{HCC}$ through postoperative follow-up. None of the remaining 45 lesions that were neither hypointense in HB-EOB nor hypoechoic during Kup-CEIOUS was diagnosed as HCC.
Conclusion: Hypoechoic lesions during Kup-CEIOUS should be resected. Otherwise, a lesion should not be resected if it is not hypointense in HB-EOB. Twenty percent of the other group are estimated to be HCC.

\section{B-004 14:18}

Imaging features of early hepatocellular carcinoma defined by new histologic criteria on quadruple-phase CT and gadoxetic acid-enhanced MR imaging

H. Rhee, M.-J. Kim, M.-S. Park, J.-Y. Choi; Seoul/KR (hjinrhee@gmail.com)

Purpose: To compare the imaging features of early hepatocellular carcinoma (HCC) defined by new histologic criteria between quadruple-phase CT and gadoxetic acid-enhanced MRI.

Methods and Materials: We analysed imaging findings of pathologically proven 33 early HCCs (mean diameter $14.6 \mathrm{~mm}$ ) in 27 patients who underwent quadruplephase CT and gadoxetic acid-enhanced MRI at 3.0 Tesla unit before surgery. Two radiologists retrospectively reviewed $\mathrm{CT}$ and $\mathrm{MRI}$ in separate sessions, then recorded the attenuation or signal intensity in precontrast and each of dynamic phase images. On review of MR images, signal drop in in-and-out of phase images and signal intensity on hepatobiliary phase were also recorded.

Results: 25 of 33 lesions (75\%) were identified on at least one phase of dynamic CT. Four lesions (12\%) showed arterial enhancement; however, none of these lesions showed washout in late phase. On gadoxetic acid-enhanced MRI, 31 of 33 lesions (93\%) were identified. Nine lesions (27\%) demonstrated arterial enhancement and 6 of 9 lesions showed washout. On dual-echo images, 8 lesions showed signal drop on opposed phase images, and more than $10 \%$ of fatty change was reported in all of these lesions. 29 lesions $(89 \%)$ are hypointense on hepatobiliary phase images. Typical enhancement pattern of HCC (arterial enhancement and late washout) was identified in none of early HCC on CT, and $6(18 \%)$ lesions on gadoxetic acid-enhanced MRI.

Conclusion: Imaging features of early HCC are often atypical; however, typical enhancement pattern may be more commonly seen on gadoxetic acid-enhanced MRI.

\section{B-005 14:27}

Detection of hepatocellular carcinoma in liver transplant candidates: intraindividual comparison of gadobenate dimeglumine-enhanced MR imaging and multiphasic 64-slice CT

M. Di Martino, G. De Filippis, D. Geiger, R. Di Miscio, M. Mennini, C. Catalano,

R. Passariello; Rome/IT (dimase81_6@hotmail.com)

Purpose: To intraindividually compare gadobenate dimeglumine (MultiHance ${ }^{\circledR}$, Bracco)-enhanced MRI and 64-slice CT for detection of HCC in patients with cirrhosis.

Methods and Materials: Fifty-five consecutive patients underwent MRI at $1.5 \mathrm{~T}$ and 64-slice CT at a mean interval of 14 days. All patients underwent transplantation within 60 days. MR acquisitions comprised unenhanced breath-hold T2W images and volumetric 3D gadobenate dimeglumine-enhanced $(0.1 \mathrm{mmol} / \mathrm{kg}$ bodyweight) $\mathrm{T} 1 \mathrm{~W}$ GRE images acquired at 25s, 60s, 180s (dynamic phase) and 90 min (hepatobiliary phase). A triple-phase CT-protocol was utilised with scans initiated at $18 \mathrm{~s}, 60 \mathrm{~s}$ and 180 s after a trigger threshold of $150 \mathrm{HU}$ above baseline was achieved in the aorta. Image analysis was independently performed by three observers in two sessions separated by 4 weeks. Findings were compared directly with explanted liver pathologic results. Diagnostic accuracy was evaluated using the JAFROC method. Sensitivity and specificity with corresponding $95 \%$ confidence intervals were determined Results: A total of $76 \mathrm{HCC}$ nodules were present in explanted livers. On a lesion-bylesion basis, the mean sensitivity gadobenate dimeglumine-enhanced MRI for the detection of lesions was significantly higher than that of CT (76\% vs $62 \%$; $<<.05)$. Likewise a slightly higher specificity was also noted (84\% vs $82 \%$ ) although the difference was not significant. Undetected lesions were typically small HCC nodules $(<5 \mathrm{~mm})$. The mean area under the JAFROC curve for gadobenate dimeglumineenhanced MRI $(0.81)$ was significantly higher than that of CT $(0.72)(P<.05)$.

Conclusion: Gadobenate dimeglumine-enhanced MRI is significantly more sensitive than 64-slice CT for the detection and diagnosis of HCC in patients with cirrhosis prior to liver transplantation.

B-007 14:36

Comparison of 64-slice CT and 3-T-MRI with DWI for the detection of hepatocellular carcinoma

B. Maiwald, D. Lobsien, T. Kahn, P. Stumpp;

Leipzig/DE (Bettina.Maiwald@medizin.uni-leipzig.de)

Purpose: We compared contrast-enhanced 64-slice CT with 3-Tesla MRI using GdEOB-DTPA and diffusion-weighted imaging in diagnosing hepatocellular carcinoma 
Methods and Materials: 43 patients (median age 61.4 years; 36 males, 7 females) with suspected or proven HCC underwent a 3-phase-liver-CT with lopromide. An additional 3-Tesla-MRI-examination with Gd-EOB-DTPA was performed within the next two days. The anonymised images were evaluated by a radiologist with respect to number and size of liver lesions. Detectability of lesions and image quality were evaluated with a 5-point Likert-scale. Results were compared with the gold standard of histological findings after tumour resection or biopsy or with follow-up-examinations. Sensitivity, specificity, positive and negative predictive values (PPV and NPV) were evaluated for both methods.

Results: MRI detected more liver lesions per patient (3.9 versus 3.4 in CT). Size of lesions was greater in MRI with an average greatest diameter of $22.8 \mathrm{~mm}$ in CT and $28.1 \mathrm{~mm}$ in MRI. Detectability of lesions was superior for CT (2.3 vs. 2.8 for MRI). Image quality was rated similar for CT and MRI (2.2 vs. 2.3). Sensitivity, specificity, PPV and NPV were evaluated superior for MRI (sensitivity $92 \%$, specificity $77.8 \%$, PPV $85.2 \%$ and NPV $87.5 \%$ vs. sensitivity $88 \%$, specificity $66.7 \%$, PPV $78.6 \%$ and NPV $80 \%$ for CT).

Conclusion: Although 64-slice CT-scans of the liver give a better detectability of liver lesions compared to 3 T-MRI, less lesions per patient were detected. Gd-EOBDTPA-enhanced MRI showed higher sensitivity, specificity, positive and negative predictive values in detection of $\mathrm{HCC}$.

\section{B-008 14:45}

Value of MDCT angiography in the evaluation of the ectopic blood supply of hepatocelular carcinoma

B. Song, G. Chen, Z. Li; Chengdu/CN (cjr.songbin@vip.163.com)

Purpose: To investigate the value of multidetector-row spiral CT angiography in assessing the ectopic blood supply of hepatocellular carcinoma (HCC) and addressing the underlying morphological and pathological factors.

Methods and Materials: 306 patients with a total of $373 \mathrm{HCC}$ lesions were prospectively included in the study. Standardised MDCT multi-phasic acquisition was performed for all patients. Thin-slice source images were reconstructed using techniques of multi-planar reconstruction, curved planar reconstruction, maximum intensity projection and volume rendering to display the hepatic artery and arterial vasculature in the upper abdomen. The angiography images were interpreted by three experienced abdominal radiologists. Factors such as the size, location, pseudocapsule of $\mathrm{HCC}$ lesions and previous history of transarterial chemoembolisation (TACE) therapy were analysed with the incidence of the ectopic blood supply. Results: Ectopic blood supply was found in $115 \mathrm{HCC}$ lesions (30.8\%). Diverse source of ectopic arteries participating in HCC blood supply were shown, including internal mammary artery, intercostals artery, inferior phrenic artery, left and right gastric artery, cystic artery, epiploic branch of gastroduodenal artery, superior mesenteric artery, right renal artery, right adrenal artery, right lumbar artery and abdominal wall artery. Statistic analysis showed that the tumour size, the location and the status of pseudocapsule of $\mathrm{HCC}$ lesion were significantly correlated with the presence and the source of ectopic arteries, while the history of TACE therapy was slightly correlated. Conclusion: MDCT angiography is able to provide comprehensive evaluation of the ectopic blood supply of $\mathrm{HCC}$ and the related risk factors.

\section{B-009 14:54}

Intra- and inter-observer agreement of perfusion CT (pCT) measurements in hepatocellular carcinoma (HCC): experience in 103 exams

G. Petralia ${ }^{1}$, S. Viotti ${ }^{1}$, R. Montefrancesco, ${ }^{2}$, S. Raimondi ${ }^{1}$, L. Bonello ${ }^{1}$,

P. Summers ${ }^{1}$, M. Bellomi'; ${ }^{1}$ Milan/IT, ${ }^{2}$ Lecce/IT (giuseppe.petralia@ieo.it)

Purpose: To assess intra- and interobserver agreement of perfusion CT ( $\mathrm{pCT}$ ) measurements in patients with hepatocellular carcinoma (HCC).

Methods and Materials: After approval by our Institutional Ethics Committee, $103 \mathrm{pCT}$ exams performed in patients with $\mathrm{HCC}$ were analysed using a dedicated software package (perfusion CT 3 ), which provided the following measurements: blood flow (BF), blood volume (BV), mean transit time (MTT) and permeability surface area product (PS). The results from prospective $\mathrm{pCT}$ analyses by two readers were analysed for inter-observer agreement. One reader repeated his analysis for intra-observer agreement assessment. Intra- and inter-observer agreement was assessed using the Bland-Altman approach.

Results: The geometric mean of the ratios ( $95 \%$ limits of agreement) for intra-observer agreement was 1.00 (0.55-1.83) for BF, 0.99 (0.69-1.41) for BV, 0.99 (0.70-1.40) for MTT, and $1.10(0.47-2.60)$ for PS, with a higher interclass correlation coefficient (ICC) for BF and BV (0.94 and 0.98, respectively) than for MTT and PS (0.90 and 0.90 , respectively). The geometric mean of the ratios ( $95 \%$ limits of agreement) for inter-observer agreement was $0.92(0.50-1.66)$ for $\mathrm{BF}, 0.90(0.45-1.80)$ for $\mathrm{BV}, 1.12$ (0.62-2.03) for MTT, and 0.79 (0.16-3.80) for PS, with a higher ICC for BF and BV ( 0.94 and 0.98 , respectively) than for MTT and PS ( 0.68 and 0.71 respectively).
Conclusion: The higher intra- and inter-observer agreements suggest that BF and BV measurements are likely to be more suitable than MTT and PS for possible clinical use. The wide limits of agreement may however limit their utility.

\section{B-010 15:03}

Clinical utility of non-enhanced CT spectral imaging for differentiating hepatocellular carcinoma from hepatic haemangioma: initial experience

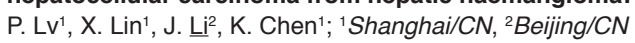

(Jianying.Li@med.ge.com)

Purpose: To investigate the utility of CT spectral imaging with single tube, fast $80 \mathrm{kVp}$ and $140 \mathrm{kVp}$ switching scan mode during non-enhanced liver scans in differentiating hepatocellular carcinoma $(\mathrm{HCC})$ from hepatic haemangioma $(\mathrm{HH})$. Methods and Materials: This prospective study received IRB approval and patients' informed consent. We analysed 53 patients ( 35 men) with 56 lesions (18 $\mathrm{HH}, 13 \mathrm{HCC}$ with cirrhosis and 25 with $\mathrm{HCC}$ but without cirrhosis) scanned on a Discovery CT750HD with spectral imaging scan mode during the non-enhanced phase. The values of effective iodine content $(\mathrm{elC})$ and Effective- $Z$ were obtained from the iodine-based material decomposition images. The slope $(k)$ of CT number changes as function of photon energy for the lesions was calculated as the CT values at two energy levels $(50 \mathrm{keV}$ and $130 \mathrm{keV})$ divided by the energy difference (80keV) from the spectral $\mathrm{HU}$ curve generated from a set of single energy images. Two-sample t test was performed for these quantitative parameters between $\mathrm{HH}$ and HCC with and without cirrhosis.

Results: The values of elC, $\mathrm{k}$ and Effective- $\mathrm{Z}$ in $\mathrm{HH}$ patients $(0.98 \pm 0.62 .0 .59 \pm 0.37$ and $8.14 \pm 0.40$ ) were significantly higher than those in HCC patients with cirrhosis $(0.58 \pm 0.39 .0 .37 \pm 0.23$ and $7.90 \pm 0.25)(P<0.05)$ and HCC without cirrhosis $(0.62 \pm 0.38 .0 .34 \pm 0.23$ and $7.93 \pm 0.24)(P<0.05)$. The sensitivity and specificity of differentiating hepatocellular carcinoma from hepatic haemangioma by using these parameters were $61 \%$ and $82 \%$, respectively.

Conclusion: CT spectral imaging for non-enhanced liver scans maybe useful for differentiating small haemangiomas and hepatocellular carcinomas.

14:00 - 15:30 Room C

GI Tract

\section{SS 101b}

\section{Imaging of the oesophagus and stomach}

Moderators

S.A. Jackson; Plymouth/UK

A.M. Riddell; Sutton/UK

\section{B-011 14:00}

Tumour angiogenesis and first-pass perfusion using a low-dose method at multidetector row CT in oesophageal squamous cell carcinoma: radiologic-pathologic correlation

Z.G. Yang, T.W. Chen; Chengdu/CN

Purpose: To investigate correlations between first-pass perfusion CT parameters using a low-dose method technique and immunohistochemical markers of angiogenesis within esophageal squamous cell carcinomas.

Methods and Materials: Thirty-two patients with oesophageal squamous cell carcinomas underwent first-pass perfusion scan with 64-section multidetector row $\mathrm{CT}$ at $50 \mathrm{mAs}$. Perfusion parameters including perfusion (PF, in $\mathrm{ml} / \mathrm{min} / \mathrm{ml}$ ), peak enhanced density (PED, in HU), time to peak (TTP, in second) and blood volume $(\mathrm{BV}$, in $\mathrm{ml} / 100 \mathrm{~g}$ ) were measured. Post operative tumour specimens were assessed for microvessel density (MVD) and vascular endothelial cell growth factor (VEGF) expression. Correlation tests were performed to determine associations between perfusion parameters and MVD, or VEGF expression. Threshold of perfusion parameter was obtained to discriminate VEGF-positivity from VEGF-negativity in this tumour. Results: Mean PF, PED, TTP and BV were $38.47 \pm 30.26 \mathrm{ml} / \mathrm{min} / \mathrm{ml}, 24.68 \pm 9.65 \mathrm{HU}$, $28.35 \pm 9.03 \mathrm{~s}$, and $11.82 \pm 6.06 \mathrm{ml} / 100 \mathrm{~g}$, respectively. PED and BV were correlated with MVD ( $r=0.436$ and $r=0.648$, respectively; all $p<0.05)$, whereas PF and TTP were not correlated with MVD $(r=0.151$ and $r=0.287$, respectively; all $p>0.05)$. In VEGF-positive group, PED and BV were correlated with VEGF expression ( $r=$ 0.576 and $r=0.765$, respectively; all $p<0.05)$, but PF and TTP were not correlated with the expression $(r=0.361$ and $r=0.239$, respectively; all $p>0.05)$. A threshold of BV $(10.23 \mathrm{ml} / 100 \mathrm{~g})$ achieved a sensitivity of $94.4 \%$, and specificity of $92.9 \%$ for discrimination of VEGF-positivity from VEGF-negativity.

Conclusion: The BV value of first-pass perfusion CT by using this low-dose method could reflect the underlying angiogenesis, and can be an indicator for evaluating this angiogenesis within oesophageal tumours. 


\section{B-012 14:09}

Relationship between presenting gastric symptoms and prediction of patterns of gastric emptying

L.I. Sonoda, M.Y.Z. Halim, S.J. Middleton, K.K. Balan; Cambridge/UK (luke@sonoda.co.uk)

Purpose: In patients presenting with upper gastrointestinal (GI) symptoms, clinical impression of rapid or delayed gastric emptying (GE) may be misleading. This study was undertaken to determine the frequency of rapid/delayed GE compared with clinical presentation in patients referred for GE scintigraphy.

Methods and Materials: A retrospective review of 642 consecutive GE studies was performed. Each patient received a standard solid meal containing two large eggs labelled with $12 \mathrm{MBq}$ of 99mTc-tin colloid and a glass of water. Static imaging in upright position (anterior and posterior 60 second) every 15 minutes was performed for $150 \mathrm{~min}$. using a large field of view gamma camera fitted with a low-energy collimator and a computer system. GE was classified as normal, rapid and delayed based on half-emptying time and percentage retained at $150 \mathrm{~min}$. Results: $70(11 \%)$ patients with suspected rapid and $572(89 \%)$ with suspected delayed GE were studied. Normal, rapid and delayed GE, respectively, were observed in $11(16 \%), 54(77 \%)$ and $5(7 \%)$ patients with the clinical suspicion of rapid GE and $178(31 \%), 215(37 \%)$ and $178(31 \%)$ patients with suspected delay in GE. One patient with suspected delay had a biphasic GE pattern. Positive Predictive Value (PPV) of clinical suspicion for rapid GE was $77 \%$ while PPV of clinical suspicion for delayed GE was $31 \%$

Conclusion: GE scintigraphy is very useful in the evaluation of patients with various GI symptoms, especially those with symptoms of a delayed GE, where clinica impression is often misleading.

\section{B-013 14:18}

CT texture analysis in patients with oesophageal cancer: association with tumour metabolism and stage

B. Ganeshan, K. Skogen, I. Pressney, D. Coutroubis, K. Miles; Brighton/UK (b.ganeshan@sussex.ac.uk)

Purpose: To assess the potential for texture analysis of CT images in oesophageal cancer to predict tumour metabolism and tumour stage.

Methods and Materials: The study comprised 29 patients with oesophageal cancer of whom 8 were excluded due to oesophageal stents $(n=6)$ or prior chemotherapy and radiotherapy $(n=2)$. Non-contrast enhanced CT images of the primary oesophageal lesion obtained from PET-CT examinations underwent texture analysis in 21 patients. Texture analysis was performed using TexRAD, a software algorithm that selectively filters and extracts textures at different anatomical scales between filter value $=0.5$ (fine detail) and filter value $=2.5$ (coarse features) with quantification as mean grey-level intensity (MGI), entropy and uniformity. Texture parameters were compared to average tumour FDG uptake (standardized uptake value: SUVavg) and tumour stage as determined by endoscopic ultrasound (nodal involvement) and PET (distant metastases). Results: Coarse texture features correlated with SUVavg (Entropy: $r=0.75, p=0.0001$; Uniformity: $r=-0.75, p=0.00008)$. Medium texture also correlates with SUVavg (Entropy: $r=0.54, p=0.01$; Uniformity: $r=-0.54, p=0.01$ ). Medium texture (MGI > 1.66) identified patients above stage II (sensitivity $=85 \%$, specificity $=75 \%$, accuracy $=81 \%, p<0.02$ ). Conclusion: $\mathrm{CT}$ texture analysis has the potential to identify oesophageal cancers with adverse biological features.

\section{B-014 14:27}

Preoperative locoregional ( $\mathrm{T}$ and $\mathrm{N}$ ) staging of gastro-oesophageal cancer: magnetic resonance imaging (MRI) including diffusion weighted sequences versus endoscopic ultrasound (EUS)

M. Cellina, F. De Cobelli, F. Giganti, C. Losio, E. Orsenigo, S. Kusamura,

P. Arcidiacono, C. Staudacher, A. Del Maschio; Milan/IT (cellina.michaela@hsr.it)

Purpose: The aim of this study was to determine the accuracy of Magnetic Resonance (MR), including diffusion-weighted-imaging (DWI), in the preoperative locoregional staging $(T, N)$, of gastro-oesophageal neoplasms, compared with Endoscopic ultrasound (EUS), currently the most widely used technique.

Methods and Materials: From November 2009 to June 2010, 22 patients affected by gastro-oesophageal cancer (6 gastro-oesophageal junction, 2 oesophageal and 14 gastric cancer) were evaluated by an experienced radiologist with a 1.5-T system, using a five-elements-cardiac-coil. The MR-protocol included DWI performed at b value of 0 and $600 \mathrm{~s} / \mathrm{mm}^{2}$. Apparent diffusion coefficients (ADC) of the primary tumour and lymphonodes were calculated. 21 out of 22 patients underwent EUS performed by three experienced gastroenterologists. We classified T-status in stages T1-T2 (organ-confined neoplasia) as negative (T-) and T3-T4 (infiltrating neoplasia) as positive $(\mathrm{T}+)$ and lymphonode stage as negative $(\mathrm{N}-)$ or positive
$(\mathrm{N}+)$. The results of MR and EUS were compared with histopathological findings. Sensitivity, specificity, positive predictive value (PPV), negative predicted value (NPV) and accuracy were calculated for both $\mathrm{T}$ and $\mathrm{N}$ stages.

Results: Sensitivity, specificity, PPV, NPV and accuracy calculated for T stage were as follows for MR: $55.5 \%, 76.9 \%, 62.5 \%, 71.4 \%$ and $68 \%$; while for EUS: $57 \%, 76.9 \%, 57 \%, 76.9 \%$ and $70 \%$; for $\mathrm{N}$ stage, for MR: $80 \%, 75 \%, 72 \%, 81 \%, 77 \%$; for EUS: $62.5 \%, 66.6 \%, 55.5 \%, 72.7 \%, 65 \%$. Mean ADC of primary tumours was $1.32 \times 10^{-3} \mathrm{~mm}^{2} / \mathrm{s} \pm 0.42$; of lymphonode was $1.42 \times 10^{-3} \mathrm{~mm}^{2} / \mathrm{s} \pm 0.35$

Conclusion: In staging gastro-oesophageal cancer, MR and EUS are comparable for T evaluation; MR seems to be superior than EUS in the evaluation of $\mathrm{N}$-stage due to the added value of diffusion-weighted imaging.

\section{B-015 14:36}

Diagnostic performance of multidetector-row computed tomography in the staging of stomach cancer: a comparative analysis with histopathology according to the $7^{\text {th }}$ edition of the American Joint Committee on Cancer (AJCC) cancer staging manual

S. Moon, I. Joo, J. Lee, S. Kim, J. Han, B. Choi; Seoul/KR (radmsk@hotmail.com)

Purpose: To evaluate the MDCT performance for the preoperative staging of stomach cancer using the $7^{\text {th }}$ edition of the AJCC staging system and histopathologic results as the reference.

Methods and Materials: For establishing the new CT criteria for T2 and T3 according to the new staging system, two radiologists reviewed MDCT images of 50 patients with surgically confirmed T2 and T3 cancers. With the new CT criteria, two radiologists, blinded to endoscopic and histopathologic results, independently reviewed the preoperative MDCT images of additional 114 patients with surgically confirmed stomach cancers, and determined their TNM staging. The sensitivity, specificity, and accuracy of the TNM staging were calculated.

Results: New CT criteria differed between T2 and T3 by the presence of fullthickness tumour involvement and perigastric fat infiltration $(p<0.0001)$. The overal accuracy of T staging was $72.8 \%$ (83/114) for reviewer 1 and $80.7 \%(92 / 114)$ for reviewer 2, while the overall accuracy of $\mathrm{N}$ staging was $69.3 \%(79 / 114)$ for reviewer 1 and $67.5 \%$ (77/114) for reviewer 2. In differentiating $T 1$ from advanced cancer $(\geq T 2)$, CT showed excellent performance with 88.2\% (60/68) sensitivity for reviewer 1 and $91.2 \%(62 / 68)$ for reviewer 2. However, CT showed low performance for differentiating T2 from T3 with sensitivity and specificity of $33.3 \%(4 / 12)$ and $50.0 \%(9 / 18)$ for reviewer 1 , and $16.7 \%(2 / 12)$ and $66.7 \%(12 / 18)$ for reviewer 2 , respectively. Conclusion: MDCT showed good overall performance in the preoperative T staging of stomach cancers according to the $7^{\text {th }}$ edition of the AJCC staging system, although it was limited in differentiating the T2 and T3. Meanwhile, MDCT showed similar performance for $\mathrm{N}$ staging, as stated in the previous staging system.

\section{B-016 14:45}

Support vector machine for diagnosis of lymph node metastasis in gastric cancer with multidetector computed tomography

Z.-L. Wang, X.-P. Zhang, L. Tang, Y.-S. Sun, K. Cao, Y. Cui; Beijing/CN (atjbjcui@hotmail.com)

Purpose: To evaluate lymph node metastasis (LNM) of gastric cancer with the support vector machine (SVM) model, which is based on the tumour and lymph node information on MDCT.

Methods and Materials: The institutional review board approved this retrospective study. 175 patients ( 125 men, 50 women, mean age 59 years, range 30-85 years) with gastric cancer who underwent MDCT before surgery were included. The tumour maximum diameter, serosal invasion, Borrmann classification, number of all visible lymph nodes, maximum lymph node short diameter and lymph nodes station were measured and evaluated by two radiologists manually on CT images. A SVM model was built with these 6 indicators above as input index. The output index was that LNM of the patient was positive or negative on pathology. A standard machine-learning technique called $\mathrm{k}$-fold cross-validation was used to train and test SVM model. We evaluated the SVM model with the receiver operating characteristic (ROC) curve. And another radiologist classified the LNM of patients using maximum lymph node shor diameter as criterion. We compared SVM model with the radiologist by ROC curve. Results: There were 134 LNM positive cases and 41 negative. The means of the sensitivity, specificity and AUC of SVM model were $88.5 \%, 78.5 \%$ and 0.876 , respectively. While the diagnostic power of the radiologist classifying LNM by maximum lymph node size was $63.4 \%, 75.6 \%$ and 0.757 .

Conclusion: SVM model can help diagnose the lymph node metastasis of gastric cancer preoperatively. It is better than the traditional lymph node size criterion. 


\section{B-017 14:54 \\ Review of the radiological findings and study of the incidence of internal herniations after laparoscopic Roux-en-Y gastric bypass surgery \\ P. Seynaeve', C. Lecluyse ${ }^{1}$, K. Carels ${ }^{1}$, L. Cardoen'1, S. Traen ${ }^{1}$, B. Deylgat ${ }^{2}$, F. Van Rooy', D. Devriendt', F. Vansteenkiste ${ }^{1} ;{ }^{1}$ Kortrijk/BE, ${ }^{2}$ Leuven/BE (clarisse.lecluyse@azgroeninge.be)}

Purpose: To comprehensively describe the radiological findings and to study the incidence of the different types of internal herniation after laparoscopic Roux-en- $Y$ gastric bypass surgery (RYGBP).

Methods and Materials: A series of 726 laparoscopic RYGBP were retrospectively reviewed late December 2009. 51 internal herniations who required surgica treatment were studied. CT examinations were performed with a 64 slice helical scanner (GE Milwaukee) with IV contrast, no oral contrast was given due to the emergency room setting of these examinations. The $\mathrm{CT}$ images were reviewed by 2 independent radiologist readers (CL,PS) and 1 non involved abdominal surgeon (FV) and correlated with the postoperative findings.

Results: 51 internal herniations were detected by CT in a series of 726 RYGBP patients in a time period of 9 months which correlates with an incidence of $7 \%$. This is slightly higher than the incidence reported in the literature of $3.1-4.5 \%$. Of these herniations only $4(7.8 \%)$ involved the Petersen hernia space, the remainder involved the jejunojejunostomy defect $(92.2 \%)$. No herniations through the transverse mesocolon were detected.

Conclusion: The incidence of internal herniation after laparoscopic RYGBP surgery in our series was $7.8 \%$. The main location for internal herniation was the jejunojejunostomy defect, the Petersen hernia space was the second most affected location No herniations through the transverse mesocolon were detected. CT is the modality of choice to evaluate the different internal herniations. A $100 \%$ detection rate for the different types of internal herniation was found using contrast-enhanced helical CT.

\section{B-018 15:03}

Time-resolved MRI reveals motility changes after laparoscopic sleeve gastrectomy

T. Baumann, A.-O. Schäfer, W.K. Karcz, M. Langer;

Freiburg/DE (tobias.baumann@uniklinik-freiburg.de)

Purpose: Laparoscopic sleeve gastrectomy (LSG) is a frequently performed bariatric procedure. It has been assumed from nuclear-medicine studies that stomach motility is altered after LSG, but direct visualisation has not been available. It was the purpose of this study to evaluate gastric peristalsis before and after LSG by means of time-resolved magnetic resonance imaging (MRI).

Methods and Materials: In this prospective study five female patients with a mean BMI of $51.6 \mathrm{~kg} / \mathrm{m}^{2}$ underwent MRI before surgery and six days and six months after LSG. Cine steady-state free precession sequences were used to analyse the gastric motility after ingestion of $500 \mathrm{ml}$ water with a temporal resolution of 0.86 $\mathrm{s}$. Gastric volume was measured on axial image stacks acquired every 5 minutes after fluid ingestion. Emptying half-times were calculated.

Results: Mean excess body-mass-index loss was $60.6 \%$ after six months. Dynamic analysis showed that antral propulsive peristalsis was preserved immediately after surgery and during follow-up, but fold speed increased significantly from $2.7 \mathrm{~mm} / \mathrm{s}$ before LSG to $4.4 \mathrm{~mm} / \mathrm{s}$ after six months. The sleeve itself remained without recognisable peristalsis in three patients and showed only uncoordinated motion in two patients. Consequently the fluid transport through the sleeve was markedly delayed whereas the antrum showed accelerated propulsion with emptying half-time decreasing from 16.5 minutes preoperatively to 7.9 minutes six months after surgery. Conclusion: Dynamic MRI could for the first time provide a detailed analysis of gastric motility after LSG. The sleeve stomach represents an immobile tube, whereas the preserved antrum shows accelerated folds and emptying after six months.

\section{B-019 15:12}

Patterns of recurrence after resection of gastrointestinal stromal tumors (GIST) A.A.O. Plumb, R. Kochhar, M.B. Taylor, M. Leahy; Manchester/UK (aaoplumb@doctors.net.uk)

Purpose: To determine the timecourse, anatomical pattern and imaging appearances of recurrent gastrointestinal stromal tumours (GIST) after surgical resection, and to determine factors associated with higher recurrence rates.

Methods and Materials: Cases were identified from the hospital GIST database. Clinical features, surgical details, histopathologic results and follow-up imaging were reviewed. Where recurrences were found, locations of recurrence and the conspicuity of liver lesions on arterial versus portal phase imaging were recorded. Results: 91 cases with complete excision were identified, of which 66 were gastric The majority presented with bleeding (35\%) or abdominal pain $(20 \%) .28$ cases were low-risk, 22 intermediate and 41 high-risk. There were 19 relapses, all in the highrisk group. $50 \%$ of relapses occurred within 1 year and $85 \%$ within 3 years. When compared to high-risk tumours without relapse, the relapsed group was more likely to be non-gastric $(p<0.05)$, a large tumour (mean $14 \mathrm{~cm}$ versus $10 \mathrm{~cm}, p<0.05$ or a high mitotic rate (mean 40 per 50 high-power field versus $7, p<0.05$ ). Commonest sites for recurrence were the liver and mesentery. Some liver lesions were more conspicuous in the arterial phase and others better seen in the portal phase. Conclusion: Recurrence after complete excision of GIST is commonest in the liver and mesentery. The majority of recurrences occur early ( $<1$ year) but late recurrences do occur. Dual-phase imaging of the liver is recommended as conspicuity is variable. Relapse is more likely with larger, more mitotically active tumours even within the high-risk category, suggesting a "very high risk" subcategory exists.

\section{B-020 15:21}

Two different ways for screening of cirrhosis-related oesophageal varices: Doppler haemodynamic indices of portal system versus "Platelet count/ splenic diameter" ratio

A. Hekmatnia, R. Barikbin, P. Adibi, N. Omidifar, M. Farghadani, B. Tavakoli,

S. Etezazian, A. Ghazavi, F. Hekmatnia; Esfahan/IR (hekmatnia@med.mui.ac.ir)

Purpose: Comparing accuracy of Doppler sonography versus PC/SD ratio for the screening of oesophageal varices (EV) in cirrhotic patients to find a less expensive, safer and more available way than upper gastrointestinal endoscopy (UGIE) for those patients needs prophylactic actions of EV bleeding to lessen Socio-medical burden of increasing UGIE.

Methods and Materials: During two years patients with cirrhosis referred to Al -Zahra hospital enrolled. Clinical examination, haematology and liver function tests, Grey-scale ultrasonography and Doppler sonography of hepato-portal system were done. Degree of oesophageal varices was assessed at UGIE; Paquet's grades 0 - III were classified as group A (0-I; No or Mild EV) and B (II-III; moderate to severe EV). Portal haemodynamic indices (liver vascular, portal hypertension and congestive index) and the relationship of the presence and degree of EVs with $\mathrm{PC}$ SD ratio and Doppler results were evaluated.

Results: 50 consecutive cirrhotic patients $(52.1 \pm 16.2$ year; 41 males and 9 females) were enrolled: $38 \%$ patients in group A while $62 \%$ in group $\mathrm{B}$. $\mathrm{PC} / \mathrm{SD}$ ratio significantly predicted high-risk EVs. It was significantly $(p<0.0001)$ different between groups. Porta hypertension index had statistically significant correlation with degree of $E V s(p<0.029)$ Doppler ROC area under the curve was not significant, but was close (AUC=0.64). Conclusion: $\mathrm{PC} / \mathrm{SD}$ ratio and Doppler results are independently associated with the presence of EV and can predict its severity. PC/SD ratio can be more helpful for primary screening of EVs because it is more sensitive and accurate and cheaper than Doppler.

14:00 - 15:30 Room D1

Chest

\section{SS 104}

\section{Lung cancer imaging}

Moderators:

A. Devaraj; Cambridge/UK

J.D. Dodd; Dublin/IE

\section{B-021 14:00}

Feasibility of CT-perfusion integrated in routine whole body ce-FDG-PET/ CT study in lung cancer: relationship of perfusion measurements and glucose metabolism parameters

C. $\underline{\text { Capraro }}^{1}$, D. Ippolito ${ }^{1}$, L. Guerra ${ }^{1}$, G. Di Pisa ${ }^{2}$, C. Messa' 1 S. Sironi'; ${ }^{1}$ Milan/IT, ${ }^{2}$ Monza/lT (cristinacapraro@hotmail.it)

Purpose:To assess the feasibility of integrated CT-perfusion (CT-p) into a routine full diagnostic clinical protocol of whole-body FDG-PET/CT in patients with lung cancer in order to evaluate the relationship between perfusion measurements and glucose metabolism.

Methods and Materials: Forty-three consecutive patients with known or suspected lung cancer who were referred for FDG-PET/CT staging were prospectively enrolled in our study. After acquisition of a low-dose PET/CT, a split injection ce-CT protocol including a 30 sec CT-perfusion scan of the lung lesion (dynamic acquisition of 4 dynamic slice/scan; 30 scans; i.v. administration of $50 \mathrm{ml}$ of iodinated contrast; $5 \mathrm{ml} / \mathrm{sec}$ )and diagnostic whole body ce-CT (i.v. administration of $80 \mathrm{ml}$ of contrast; $3.5 \mathrm{ml} / \mathrm{sec}$; $60 \mathrm{sec}$ delay) were performed. CT perfusion data were used to calculate a range of parameters of tumour vascularity (BF, BV, MTTT and PS). 
Results: The perfusion images were analysed using a commercially available software (Perfusion 3.0; Advantage; GE) which generated a quantitative map of arterial perfusion. Regression analysis was used to analyse the statistical relation in terms of size, location, tumour histotype, for SUVmax (standardised uptake value) and for perfusion parameters. In our series, a trend for perfusion parameters MTT and PS was found being higher in small tumours; particularly, a significant difference was achieved in relation to the size $(p=0.03)$ between tumour $>3 \mathrm{~cm}$ and tumour $<3 \mathrm{~cm}$ (mean value MTT: 9.2 vs $4.7 \mathrm{sec}$; $\mathrm{m} . \mathrm{v}$. PS $14.9 \mathrm{vs} 4.8 \mathrm{ml} / \mathrm{min} / 100 \mathrm{~g}$ ). Interestingly, highest correlations (linear regression) were detected between $\mathrm{BV}, \mathrm{BF}$ and SUVmax for all size of the tumour. Conclusion: The integrated CT-perfusion protocol into a ce-PET/CT examination appears to be a technically feasible tool providing functional information about tumour vasculature and tumour pathophisiology related to metabolism and angiogenesis, thus helping the therapeutic approach.

\section{B-022 14:09}

Diffusion-weighted MR imaging in comparison with integrated $\left[{ }^{18} \mathrm{~F}\right]-\mathrm{FDG}$ $\mathrm{PET} / \mathrm{CT}$ for $\mathrm{N}$-staging in patients with lung cancer

S. Pauls ${ }^{1}$, S.A. Schmidt' ${ }^{2}$ M.S. Juchems ${ }^{2}$, O. Klass², M. Luster ${ }^{2}$, S.N. Reske ${ }^{2}$,

H.-J. Brambs ${ }^{2}$, S. Feuerlein ${ }^{2} ;{ }^{1}$ Lübeck/DE, ${ }^{2}$ Ulm/DE (s.pauls@sana-luebeck.de)

Purpose: To determine the diagnostic accuracy of diffusion-weighted imaging (DWI) for staging of thoracic lymph nodes in patients with lung cancer, and to compare these results with conventional MRI and PET/CT.

Methods and Materials: 20 patients with lung cancer were included in this study. In all patients FDG-PET/CT was routinely performed to stage lung cancer. Additionally, MRI (1.5 T) was performed (non-contrast T1w, T1w post contrast, T2w, DWI sequences: b-values $\left.0,400,800 \mathrm{~s} / \mathrm{mm}^{2}\right)$. With PET/CT there were five patients with N0, three patients with $\mathrm{N} 1$, five patients with $\mathrm{N} 2$ and seven patients with N3. Image analysis was performed by two radiologists (R1 and R2). First the T1 post contrast sequence was analysed. In a second session, the DWI sequence $(b=800)$ was analysed.

Results: MR imaging with or without DWI agreed with the results of the PET/ CT regarding the $\mathrm{N}$ stage in $80 \%$ of the patients - $15 \%$ were understaged and $5 \%$ overstaged. Interobserver agreement was $r=0.98$ for DWI and $r=1.0$ for MRI. Compared with PET/CT MRI overstaged one and understaged four patients, while DWI overstaged one and understaged three patients: correlation coefficients of 0.814 (R1 and R2) for MRI and 0.815 (R1) and 0.804 (R2) for DWI. ADC values were not significant different regarding the localization of lymph node metastases. Conclusion: DWI does not show a clear advantage over conventional MR protocols in the $\mathrm{N}$ staging of lung cancer. MRI with or without DWI shows a moderate correlation with PET/CT with a tendency for understaging

\section{B-023 14:18}

\section{Correlation of iodine-related attenuation in dual energy CT with} standardised uptake value of FDG-PET-CT in lung cancer G. Schmid-Bindert, T. Henzler, C. Tianging, P. Apfaltrer, C. Manegold, S.O. Schönberg, C. Fink; Mannheim/DE (christian.fink@umm.de)

Purpose: To investigate whether iodine-related attenuation (IRA) measured with contrast-enhanced dual energy CT (DECT) correlates with maximum standardised uptake value (SUVmax) of FDG-PET-CT in patients with lung cancer.

Methods and Materials: In a retrospective analysis 37 patients (mean age 69 years) with histologically proven lung cancer (29 NSCLC, 8 SCLC) who underwent DECT as well as FDG-PET were identified from the RIS. The mean interval between both exams was 39 days; in 17 patients the interval was 20 days or less. Contrast-enhanced DECT was performed on a first-generation dual source CT (Definition, Siemens) using a slice collimation of $1.2 \mathrm{~mm}, 80$ and $140 \mathrm{kV}$ tube voltages, and a scan delay $35 \mathrm{~s}$. Using a commercial postprocessing algorithm (virtual non contrast - VNC) the mean and maximum IRA of FDG-positive primary tumours $(n=32)$ and lymph nodes $(n=71)$ were determined and correlated to the SUVmax of FDG-PET-CT exams (Biograph mCT, Siemens).

Results: In the entire cohort there was no meaningful correlation between the mean and maximum IRA and SUVmax, if all lesions were considered. However, for primary tumours a weak correlation between maximum IRA and SUVmax was observed $(r=0.51, p=0.003)$. If only patients were considered who received DECT and FDG-PET-CT within an interval of 20 days or less there was a strong correlation between maximum IRA and SUVmax of primary tumours $(r=0.77, p=0.002)$. Conclusion: IRA of DECT correlates with SUVmax of FDG-PET-CT in primary tumours and might be valuable as a functional surrogate parameter for response assessment in lung cancer.
B-024 14:27

Diagnostic accuracy of FDG PET/CT and CT for lymph node metastasis of T1 non-small cell lung cancer manifesting as subsolid nodules C. Park, S. Lee, J. Paeng, J. Goo, H. Lee, J. Kim; Seoul/KR (cmpark@radiol.snu.ac.kr)

Purpose: To retrospectively evaluate the diagnostic accuracy of FDG-PET/CT and CT examinations for nodal staging of T1 non-small cell lung cancers (NSCLC) manifesting as subsolid nodules, and to compare them with solid T1 NSCLCs. Methods and Materials: From January 2005 to February 2010, among 5873 patients diagnosed with NSCLC, 347 patients $(\mathrm{M}: \mathrm{F}=200: 147$, mean age $61.9 \pm 10.4$ years) who had a pathologically proven T1 NSCLC with lymph node staging and available preoperative FDG-PET/CT images were included in this study. From these 347 patients, 112 patients ( $M: F=47: 65$ ) were categorised into subsolid NSCLC group and the remaining 235 patients ( $M: F=153: 82$ ) into solid NSCLC group. Sensitivity, specificity, and accuracy in nodal staging were compared on patients' basis between FDG PET/CT and CT examinations, and between the subsolid and solid NSCLC groups. Results: Nodal metastases were found in 7 patients $(6.3 \%)$ in subsolid NSCLCs group and $63(26.8 \%)$ patients with solid NSCLCs $(P<0.05)$. In the subsolid NSCLC group, sensitivity, specificity, and accuracy of FDG-PET/CT were $14.3 \%$, $87.6 \%$, and $83.0 \%$, respectively, and those of CT were $14.3 \%, 95.2 \%$, and $90.2 \%$, respectively. There were no significant differences in diagnostic accuracy in the subsolid NSCLC group, between FDG-PET/CT and CT (P>0.05). In the solid NSCLC group, sensitivity, specificity, and accuracy of FDG-PET/CT were $25.4 \%$, $82.0 \%$, and $66.8 \%$; those of CT were $23.8 \%, 88.4 \%$, and $71.1 \%$, respectively. Conclusion: The sensitivities of PET/CT and CT for nodal metastasis is very low in subsolid NSCLC, and FDG-PET/CT does not provide additional diagnostic accuracy for nodal staging in subsolid T1 NSCLC.

\section{B-025 14:36}

Lung cancer screening: sensitivity of ultra-low-dose versus low-dose MDCT in pulmonary nodule detection

C. Rampinelli, L. Funicelli, S. Raimondi, S. Meroni, D. Origgi, M. Bellomi; Milan/IT

Purpose: To compare the sensitivity of ultra-low-dose Multi-Detector-ComputedTomography (MDCT) with low-dose MDCT in pulmonary nodule detection.

Methods and Materials: This prospective study was approved by the institutional Ethics Committee and all participants signed an informed consent. A 16-detector row CT (1.25 mm section-thickness) with low-dose (LD-MDCT, $30 \mathrm{~mA}$ ) and ultra-low-dose (ULD-MDCT, $10 \mathrm{~mA}$ ) tube currents was performed subsequently, on different breath-holds, in 109 subjects. Body Mass Index (BMI) was reported for each subjects. Two radiologists independently reported all nodules (location, size, attenuation) for each technique and a consensus was obtained in a second session. McNemar's test was used to assess differences in pulmonary nodule detection with the two techniques. Sensitivity of each technique was calculated with $95 \%$ confidence intervals $(\mathrm{Cl})$. P-values $<0.05$ were considered significant. Results: The sensitivity for all nodules $(n=790)$ was $74 \%(95 \% \mathrm{Cl}, 71 \%-77 \%)$ and $72 \%(95 \% \mathrm{Cl}, 69 \%-74 \%)$ for LD-MDCT and ULD-MDCT, respectively (McNemar's test p-value: 0.46$)$, while the sensitivity for nodules $>4 \mathrm{~mm}$ was $80 \%(95 \% \mathrm{Cl}, 76 \%$ $84 \%$ ) and $78 \%$ (95\% Cl, 74\%-82\%), respectively. We found a similar agreement for subject with $\mathrm{BMK}<25$ and $\mathrm{BMl} \geq 25$ between the two techniques.

Conclusion: A dose reduction to $66 \%$ of the corresponding dose at low-dose chest CT was shown to cause no significant difference in pulmonary nodule detection rate with thin-section MDCT.

\section{B-026 14:45}

Influence of body region adapted reference tube current modulation on radiation dose for oncologic staging examinations of the thorax and abdomen with computed tomography

G. Pache, J. Buerk, S. Bulla, M. Langer, P. Blanke;

Freiburg/DE (gregor.pache@uniklinik-freiburg.de)

Purpose: To evaluate the influence of a body region adapted switch of the reference tube current (ref. mAs) for automated exposure control (AEC) in combined computed tomography (CT) staging examinations of the thorax and abdomen as compared with either fixed ref.mAs or two subsequent examinations with matched ref.mAs. Methods and Materials: In this institutional review board approved prospective study, 315 patients, referred for CT examinations of the thorax and abdomen (Siemens Definition, Forchheim, Germany) were randomised to three groups: A: subsequent thorax (120 ref.mAs) and abdomen (240 ref.mAs) scan, B: combined scan (240 ref.mAs) or C: combined scan with adaptive switch of ref.mAs (thorax 120 ref.mAs/thorax 240 ref.mAs) at the height of the diaphragm. Scan length, 
objective image quality were assessed. Radiation dose estimates were calculated from the dose length product.

Results: There were no significant differences concerning patient age and BMI ([kg/ $\mathrm{m}^{2}$ ]: $\mathrm{A}: 25.4 \pm 4.0$; $\left.\mathrm{B}: 25.4 \pm 4.8 ; \mathrm{C}: 25.2 \pm 3.6\right)$. Mean total scan length was comparable for all groups ([cm]: A: $63.8 \pm 3.6$; B: $63.2 \pm 3.3 .3 \mathrm{~cm} ; \mathrm{C}: 63.4 \pm 3.9)$, not including redundant coverage [group $A(12.2 \pm 2.3 \mathrm{~cm})$ ]. Image noise at the height of the pulmonary artery bifurcation and renal arteries was comparable for all groups, whereas group B showed significant less image noise at the height of the shoulder girdle. Group C exhibited a significant dose reduction of $16.3 \%$ and $13.5 \%$ as compared to groups $A$ and $B$ (estimated effective dose $(m S v)$ : $A: 15.3 \pm 4.1$; B: $14.8 \pm 4.9 ; \mathrm{C}: 12.8 \pm 3.2, \mathrm{p}<0.001)$

Conclusion: Body region adapted reference tube current modulation allows for significant dose reduction for oncologic CT staging examinations of the thorax and abdomen. As compared to subsequent scans redundant imaging is avoided.

\section{B-027 14:54}

(EPOS)

\section{Review of urgent lung cancer referrals recommended by radiologists} reporting chest $x$-ray

S. Chellamuthu, M. Bansal, S. Matthews; Sheffield/UK (csanthi@gmail.com)

Purpose: A policy was introduced in Sheffield Teaching Hospitals in 2003 by the radiologists to refer all the patients with definite mass lesion, lobar collapse and unresolved pneumonia to the lung cancer clinic. The aim of this study was to determine the percentage of malignancy, whether the referrals were appropriate, assess waiting times on chest clinic and subsequent CTs and identify any weaknesses in the direct referral process.

Methods and Materials: Retrospective review of all the CXR referrals in the year 2008. The chest x-ray pattern and appearance, source of referrals, waiting times, referral to previous imaging, the appropriateness of referral and the percentage of malignancy were all recorded.

Results: Chest x-rays of 163 patients referred as per protocol were reviewed, of which $52 \%$ of referrals were from GP and $48 \%$ from hospital practitioners. 133 had mass lesion/nodules, 13 atelectasis, 12 consolidation, 4 pleural effusion and 1 mediastinal widening. Malignancy was detected in $53 \%$ of the total referrals, $75 / 163$ mass lesions, $6 / 6$ atelectasis, $1 / 7$ consolidation, $1 / 4$ pleural effusion and $13 / 163$ had no final diagnosis. $87 \%$ of the patients were seen in the clinic within a month and $83 \%$ had CT within the 20 days of CXR and $92 \%$ had CT prior to the clinic appointment. $86 \%$ of the referrals were appropriate

Conclusion: Mass lesion and lobar atelectasis were associated with a high rate of malignancy. This policy increased the number of referrals to the cancer clinic and proved to be an efficient service in identifying and treating the malignancy early.

\section{B-028 15:03}

Relationship between lung cancer and pulmonary emphysema

N. Sverzellati' ${ }^{1}$, G. Randi², E. Calabrò ${ }^{2}$, M. Silva ${ }^{1}$, J.-M. Kunhigk ${ }^{3}$, A. Marchianò2, M. Zompatori ${ }^{4}$, U. Pastorino $2 ;{ }^{1}$ Parma/IT, ${ }^{2}$ Milan/IT, ${ }^{3}$ Bremen/DE, ${ }^{4}$ Bologna/IT (nicolasve@tiscali.it)

Purpose: To determine the relationship between lung cancer and emphysema by using high-precision 3D image analysis tools on volumetric thin-section CT data. Methods and Materials: Low-dose thin-section CT emphysema features of subjects with either symptomatic or screening-trial detected lung cancer $(n=124)$ were compared with those of 987 controls recruited by the same lung cancer screening trial. All CT scans were obtained according to the same protocol and analysed with a prototypical emphysema detection software (MeVis research), which provides a region-based assessment of the following parameters: lung volumes, mean lung density, emphysema index and four classes of emphysema clusters with different volumes. Multiple regression models were used to assess emphysema features as potential risk factor for lung cancer, after allowance for gender, age, body mass index $(\mathrm{BMI})$, smoking history, forced expiratory volume in one second $\left(\mathrm{FEV}_{\mathrm{f}}\right)$, forced vital capacity (FVC), and FEV,/FVC ratio.

Results: We found no compelling evidence to suggest that any emphysema feature was associated with lung cancer. No differences in terms of emphysema phenotype were observed between subjects with screening-detected lung cancer and those with cancer detected outside the screening trial. The likelihood of lung cancer was significantly higher amongst those with airflow obstruction on spirometry (odds ratio $=0.91,95 \%$ confidence interval $[\mathrm{Cl}]$ 0.88-0.93).

Conclusion: These results show an increased risk of lung cancer associated with airway obstruction. However, emphysema per se is not associated with an increased risk of lung cancer.

\section{B-029 15:12}

Course and variation of the intercostal artery: CT angiographic study and clinical relevance

E.J. Helm, N. Rahman, F.V. Gleeson; Oxford/UK (emmajhelm@hotmail.com)

Purpose: The intercostal artery (ICA) is vulnerable to injury during pleural intervention. The purpose of this study was to describe the course of the ICA in order to define the length and position of unprotected artery.

Methods and Materials: CT pulmonary angiograms demonstrating the ICA, performed for suspected PE, were analysed. Coronal MIP images were created to show the relationship of the ICAs to the ribs and custom-designed software was used to calculate the distance from each artery to the edge of the rib above and below. Analysis included the position and extent of unprotected artery and the variation in position with age (SD and coefficient of variation).

Results: 298 arteries from 47 patients were analysed (median age 60; IQR 47 to 69). The percentage of arteries that was exposed decreased progressively with increasing distance from the spinous process. Immediately adjacent to the transverse process $83.4 \%$ of arteries were not protected by the intercostal groove (ICG) at sites of potential pleural intervention. However, $7 \mathrm{~cm}$ lateral to the spinous process $99.0 \%$ of ICAs were protected by the ICG. Variability of arterial position was positively correlated with both age and rib space number $(p=0.009$ and $p=<0.001)$. Conclusion: The intercostal artery is not protected by the intercostal groove posteriorly, making it vulnerable to damage during pleural intervention. Performing pleural intervention at least $7 \mathrm{~cm}$ lateral to the spinous process significantly reduces the risk of ICA injury.

\section{B-030 15:21}

Whole tumour perfusion of peripheral lung carcinoma: evaluation with first-pass low-dose $\mathrm{CT}$ perfusion imaging

Z.G. Yang, Y. Li; Chengdu/CN

Purpose: To prospectively assess the feasibility of a whole-tumour perfusion technique using low-dose 64-detector row MDCT and to analyse the variation of CT perfusion parameters of peripheral lung carcinoma.

Methods and Materials: Ninety-seven pathologically confirmed primary peripheral lung carcinoma were performed with low-dose whole-tumour perfusion CT, which was subdivided into subgroups including pathological types, sizes or the status of lymph node metastasis. Dynamic acquisitions encompassing the whole tumour were adopted to quantify perfusion of the whole-tumour during first-pass of contrast medium with a 64-detector MDCT. A 40-mAs tube current product was used to reduce the radiation exposure. Perfusion, peak enhancement (PEI), time to peak (TTP) and blood volume (BV) were measured and statistically compared among subgroups. Results: Mean values for low-dose perfusion, PEI, TTP and BV of the 97 lung carcinomas were $61.2 \mathrm{ml} / \mathrm{min} / \mathrm{ml} \pm 47.5$ (range, 6.7-238.7), $58.9 \mathrm{HU} \pm 46.9$ (range, 13.5-235.2), 33.7 $\mathrm{sec} \pm 9.8$ (range, 11.5-60) and $31.6 \mathrm{ml} / 100 \mathrm{~g} \pm 25.1$ (range, 3.4120.3 ), respectively. No statistical differences were found in all perfusion parameters among histological types ( $p>0.05$ ), whereas considerable differences with higher perfusion, $\mathrm{PEI}$ and $\mathrm{BV}$ were noted in tumours with $\mathrm{T} 1$ stage than in those with $\mathrm{T} 2$ stage and in those with T4 stage $(p<0.05)$, and perfusion of the tumours with distant metastasis manifested significantly higher than those of the tumours without distant metastasis $(p<0.05)$, No statistically significant differences were found between nodal metastasis positive and negative groups $(p>0.05)$.

Conclusion: Low-dose perfusion imaging could provide a feasible method for assessment of whole-tumour perfusion of peripheral lung carcinomas, and perfusion parameters were associated with tumour size and distant metastasis. 
$14: 00-15: 30$

Room D2

\section{Interventional Radiology}

\section{SS 109}

\section{Interventional oncology}

\section{Moderators:}

L. Crocetti; Pisa/IT

S.K. Venkatesh; Singapore/SG

\section{B-031 14:00}

Efficacy of microwave ablation therapy of pulmonary metastases: tumour control and survival

T.J. Vogl, S. Zangos, M.G. Mack, N.N. Naguib, N.E. Nour-Eldin;

Frankfurt a. Main/DE (t.vogl@em.uni-frankfurt.de)

Purpose: To prospectively evaluate effectiveness, follow-up imaging and safety of microwave ablation in patients with pulmonary metastases.

Methods and Materials: This prospective study was approved by the institutional ethical committee board; informed consent was obtained. The study included 180 ablation sessions for lung tumours in 120 patients ( 47 males/73 females; mean 58.9 years, SD:12.1) between 2007 and 2010. Pulmonary metastases originated from colorectal cancer $(n=70)$, breast cancer $(n=30)$, hepatocellular carcinoma $(n=15)$ and renal cell cancer $(n=5)$. Exclusion criteria for ablation were lesions $>5 \mathrm{~cm}$ in diameter or $>5$ in number and coagulopathy. Percutaneous microwave ablation was performed under CT-fluoroscopy guidance. Tumour criteria, response and post ablation complications were assessed. Mixed linear modeling and logistic regression were performed.

Results: Preablation tumour volume was $2.1 \mathrm{~cm}^{3}$ (range, 0.32- $5.4 \mathrm{~cm}^{3}, \mathrm{SD}: 1.59$ ), mean largest axial diameter was $1.9 \mathrm{~cm}(0.6-5 \mathrm{~cm}$, SD:1.2). Kaplan-Meier analysis yielded a 1-year survival of $85.5 \%$. At follow-up (6-24 months, mean:9.2), tumour control response was more favourable for lesions $<3 \mathrm{~cm}$ in diameter (tumour recurrence in $11.4 \%(8 / 70)$ ) versus tumours $>3 \mathrm{~cm}$ (tumour recurrence in $32 \%$ $(16 / 50)(\mathrm{P}=0.01)$. No procedure-related mortality within 3 months post ablation was documented. Histopathology was statistically nonsignificant for end results. Immediate complications included pneumothorax (11.1\%), haemoptysis (9\%) and pulmonary haemorrhage (5\%).

Conclusion: Microwave ablation is a minimal-invasive procedure and may be safely applied to pulmonary metastases. Tumour control response is favourable for lesions up to $3 \mathrm{~cm}$ in diameter with increased incidence of recurrence for larger tumours. Histopathology of metastases is statistically nonsignificant for results.

\section{B-032 14:09}

Role of pulmonary transarterial chemoembolisation followed by microwave ablation therapy in the treatment of pulmonary metastases N.-E.A. Nour-Eldin, N.N. Naguib, A. Ola, T. Gruber-Rouh, T. Lehnert, T.J. Vogl; Frankfurt a. Main/DE (nour410@hotmail.com)

Purpose: To prospectively evaluate the effectiveness of combined use of transpulmonary artery chemoembolisation (TPCE) followed by microwave ablation (MWA) on tumour control and patients survival.

Methods and Materials: This prospective study included 80 patients ( 38 males, $42 \mathrm{fe}$ males; mean age 56.9 years). Pulmonary metastases were originating from: colorectal carcinoma ( $n: 50)$, breast ca $(n: 15)$, hepatocellular ca ( $n: 15)$. From 2-4 sessions of TPCE were performed with 4 weeks interval before MWA. The pulmonary artery segment supplying the tumours was selectively catheterised through transfemoral vein approach. The chemotherapeutic agent mitomycin $C\left(10 \mathrm{mg} / \mathrm{m}^{2}\right)$ was injected followed by $5-10 \mathrm{ml}$ lipiodol and starch particles (Embocept). MWA was performed under CT-fluoroscopy guidance. Tumour criteria, response and post-ablation complications were assessed. Follow-up accomplished using CE-CT and MRI.

Results: At follow-up (6-24 months, mean: 9.2months), 20\% (16 of 80 ) of patients had residual or progressive disease at the ablation site, predicted by using index size of larger than $3 \mathrm{~cm}(\mathrm{P}=0.01)$. According to RECIST criteria: complete response was achieved in 55 patients $(68.8 \%)$, stable disease in 9 patients $(11.3 \%)$ and tumour progression in $20 \%$. Mean time to tumour progression was 8.7 months. KaplanMeier analysis yielded an actuarial survival of $90 \%$ and $80 \%$ at 1 and 2 years, respectively. Histopathology was statistically non-significant factor for end results of treatment $(\mathrm{P}=0.07)$. All patients tolerated the repeated TPCE treatments without major adverse effects. Microwave-associated complications were: pneumothorax $(8 \%)$, haemoptysis $(6 \%)$ and pulmonary haemorrhage $(8 \%)$. No procedure-related mortality within 3 months post-treatment.

Conclusion: TPCE might be combined with ablation in the management of pulmonary metastases to induce synergetic effect in order to potentiate tumor control and improve survival.

\section{B-033 14:18}

MRI chest parameters used in the assessment of tumour response postmicrowave ablation of pulmonary neoplasms

N.-E.A. Nour-Eldin, N.N. Naguib, A. Ola, T. Gruber-Rouh, T. Lehnert, T.J. Vogl; Frankfurt a. Main/DE (nour410@hotmail.com)

Purpose: To determine the value contrast enhanced (CE-MRI) follow-up in the assessment of tumour response of microwave (MW) ablated pulmonary metastases by correlating the results with CE-CT.

Methods and Materials: This prospective study included 130 ablation sessions for pulmonary metastases in 80 patients. CE-MRI chest scanning was performed 1 week before the ablation and at 24 hours, 3, 6, 9 and 12 months post-ablation. Thin section CT volumetric measurement of the lesions was performed at the same time periods as a second parameter for comparison. The lesion MRI enhancement intensity in each study was estimated, and the ratio to the paraspinal muscle enhancement intensity at the same level was measured (lesion muscle signal (LMS) ratio). The correlations between post-ablation follow-up CT volume of tumours and CE-MRI LMS ratio at the follow-up periods were assessed.

Results: The preablation tumour volumes range: $0.26-5.29 \mathrm{~cm}$ (mean: $1.75 \mathrm{~cm}^{3}$, SD: 1.59). LMS ratio $<1$ was associated with post-ablation reduction of tumour volume (denoting scaring), while LMS ratio $>1$ were noted in preablation due to high contrast enhancement of the tumour, in $24 \mathrm{~h}$ post-ablation due to the inflammatory response associated with the thermal ablation and due to tumour residue or progress. Weak correlation was detected between the LMS-ratios and CT-volumetric changes in $24 \mathrm{~h}$ post-ablation.Strong correlation between the LMS ratios was estimated between the follow-up periods of 3 months (Spearman $R$ : 0.62, $\mathrm{p}=0.0021$ ), 6 months (Spearman R: 0.66, $\mathrm{p}=0.001$ ), 9 months (Spearman $\mathrm{R}$ $0.61, p<0.001$ ) and 12 months (Spearman R: 0.7, p<0.00001).

Conclusion: CE-MRI follow-up of the MW ablated lung tumours can be used effectively to assess the tumour response to ablation using LMS ratio as a parameter of assessment.

\section{B-034 14:27}

Regional chemoperfusion (TACP) and transpulmonary chemoembolisation (TPCE) in thoracic lesions: online therapy control with $\mathrm{C}$-arm computed tomography

T.J. Vogl, N.N. Naguib, N.E. Nour-Eldin, T. Lehnert, E.C. Mbalisike;

Frankfurt a. Main/DE (t.vogl@em.uni-frankfurt.de)

Purpose: To evaluate the role of $\mathrm{C}$-arm CT for online fluoroscopy in regional transarterial chemoperfusion (TACP) and transpulmonary chemoembolisation (TPCE) of primary and secondary malignant thoracic lesions.

Methods and Materials: From 2008 to 2009, 31 patients (20 males/11 females, average age: 61.7 years, range 22-84) with 53 thoracic malignant lesions from different origin (primary or secondary pulmonary lesions $(n=37)$, pleural mesothelioma $(n=16)$ ) were treated with TACP or TPCE using flat-detector CT (FD-CT). A C-arm CT of the latest generation was used to localise the lesions before local chemotherapy (Artis Zeego, Siemens, Forchheim). For TACP, a $220^{\circ}$ rotation and a volume of $150 \mathrm{ml}$ (ratio of 1:2 contrast/normal saline), delay $2 \mathrm{sec}$, flow $12 \mathrm{ml} /$ sec was used. For TPCE, a volume of $75 \mathrm{ml}$ (ratio of 1:2 contrast/normal saline) delay $2 \mathrm{sec}$, flow $3 \mathrm{ml} / \mathrm{sec}$ was used.

Results: TPCE C-arm CT allowed the evaluation of the degree of perfusion of the tumour, and the geographic areas of enhancement correlated with the postinterventional lipiodol uptake in the MSCT. In the TACP the involved intercostal arteries were visualised, and in $30 \%$ of interventions the catheter had to be repositioned for the following intervention.

Conclusion: C-arm CT provides additional information on the vascular characteristics and perfusion of lung lesions resulting in a change of interventional strategy in a relevant number of patients.

\section{B-035 14:36}

Transarterial thoracic chemoperfusion in the treatment of malignant pleural mesothelioma: assessment using C-arm CT and CT E.C. Mbalisike, J. Gurung, A. Azizi, P. Farshid, A. Davishi, T.J. Vogl; Frankfurt a. Main/DE (embalisike@yahoo.com)

Purpose: To evaluate the effect of regional transarterial thoracic chemoperfusion (TACP) in the palliative treatment of recurrent malignant pleural mesothelioma (MPM). Methods and Materials: 23 patients (4 females and 19 males) age range 26-85 years (mean age 64.3, SD 10.7) were assessed. 71 sessions (mean session of 3.1 / patient) were done. C-arm CT was used to confirm catheter position and diameter of intercostals arteries. Response of MPM was assessed using MSCT. Mesothelioma volume (MV) and the aerated lung volume (ALV) were evaluated and a relationship between both attempted. Statistical tests used were Hahn's regression prognosis 
interval, Spearmann-Rang correlation coefficient and interclass correlation (ICR). Results: Using the C-arm CT, catheter position was altered in 3 patients. A mean intercostals artery diameter of 3.829 was obtained for the patients having MPM and a mean of 2.533 for patients without. Changes of MV/ALV showed a confidence interval of 0.95 for increasing regression coefficient (-4.353 and 6.143) at the last cycle. A mean of $13.10 \% \mathrm{MV}$ changes was found in $65 \%$ of patients that responded. Correlation between ALV and MV yielded rho $=0.916, P=0.002$ using Spearmann-Rang correlation coefficient. Correlating changes of MV/ALV and patient symptom yielded, for chest pain $\mathrm{P}=0.007$, ICR (rho) $=0.95$, repeatability $=0.858$. For dyspnoea rho $=0.95$, repeatability $=0.761$, and for weight loss rho $=0.95$, repeatability $=0.688$. Conclusion: TACP showed promising results when used to treat patients with MPM. This result was shown in the $65 \%$ of patients that responded to treatment.

\section{B-036 14:45}

Transpulmonary chemoembolisation (TPCE) in nonresectable lung metastases: tumour control and survival

T.J. Vogl, R. Hammerstingl, S. Lindemayr

Frankfurt a. Main/DE (t.vogl@em.uni-frankfurt.de)

Purpose: To evaluate tumour response with volumetric assessment for tumour sizes after treatment of nonresectable lung metastases with transpulmonary chemoembolisation (TPCE) in palliative intention.

Methods and Materials: Between 2001 and 2009, 194 patients (95 females, 99 males; mean: 57.4 years) suffering from 3,112 unresectable lung metastases were treated with 1 to 12 TPCEs (mean: 3.6 sessions/patient). They featured a mean of 16.0 metastases of different origins: colorectal carcinoma $(n=74)$, breast cancer $(n=27)$, renal cellular carcinoma $(n=14)$, sarcoma $(n=14)$, melanoma $(n=6)$ and others $(n=59)$. Tumour-feeding arteries were selectively probed after puncturing the femoral vein, and 5-10 ml lipiodol, 5-10 mg mitomycin and microspheres (Spherex) were administered during balloon protection. At 4-week intervals, diagnosis and follow-up were accomplished by unenhanced and contrast-enhanced CT.

Results: All patients tolerated the repeated treatments without adverse effects. No complications or influences on labaratory parameters were observed. In $22.6 \%$ $(n=44)$ moderate to high lipiodol uptake was found while $77.3 \%(n=150)$ of the treated tumours showed a low storage. In $22.1 \%(n=43)$ of embolised metastases, tumour volume was resolved to $21.5 \mathrm{ml}$ on average. In $26.3 \%(n=51)$ tumourvolume remained unchanged and in $51.5 \%(n=100)$ volume increased to $56.3 \mathrm{~m}$ on average. According to the RECIST criteria, "partial response" was recorded in 43 cases, "stable disease" in 51 cases and "progressive disease" in 100 cases. Mean survival time was 1,047 days (Kaplan-Meyer analysis).

Conclusion: According to these findings, TPCE might be a well-tolerated procedure for the palliative treatment of nonresectable lung metastases.

\section{B-037 14:54}

\section{Long-term results after laser ablation of pulmonary metastases}

B. Mensel, K. Bock, M. Mohr, N. Hosten, C. Rosenberg; Greifswald/DE

Purpose: Surgical resection is the only known curative therapy of pulmonary metastasis. Thermal ablative techniques, such as laser ablation, are established palliative modalities in multimodal cancer therapy. This study demonstrates longterm outcome after CT-guided laser ablation of pulmonary metastases.

Methods and Materials: 135 procedures of laser ablation have been performed in 64 inoperable patients to treat 108 pulmonary metastases of different primary entities. Long-term survival was correlated with initial technical success, primary entities, size and count of treated metastases.

Results: Primary effectiveness rate was $78 \%$. Benifit of tumour size and count to initial technical success was statistically significant $(p=0.0004)$ when being limited to 2 metastases per patient and size of maximum $2.1 \mathrm{~cm}$. Overall median survival was 23.1 months (Cl 95\%, 11.8-34.5 months) as compared with 32.4 months ( $\mathrm{Cl}$ 95\%, 17.5-47.3 months) in group with technical complete ablation. 1-, 3- and 5-year survival rates were $81 \%, 44 \%$ and $27 \%$ in this group. Local progression rate after complete ablation was $19.4 \%$. Survival $(p=0.008)$ and abscence of local progression $(p=0.039)$ significantly benifited from initial technical success. Patients suffering from metastases of CRC or squamous ENT tumours superiorily benifited from laser ablation according to long-term survival (1-year-survival $80 \%$ and $100 \%$, respectively). Mortality rate was $0 \%$. Conclusion: Percutaneous laser ablation is an established modality in multimodal cancer therapy. Consecutive improvement of patient survival is evident according to results of this study. Tumour size (below 2.1) and count (below 2) as well as initial technical success, comprising complete treatment of tumour burden, are positive prognostic factors.

\section{B-038 15:03}

Distant infusion of saline may enlarge coagulation volume during radiofrequency ablation of liver tissue using cool-tip electrodes without impairing their predictability

P. Tobajas Morlana ${ }^{1}$, F. Burdío², E. Berjano ${ }^{3}$, R. Quesada ${ }^{2} ;{ }^{1}$ Zaragoza/ES, $^{2}$

${ }^{2}$ Barcelona/ES, ${ }^{3}$ Valencia/ES (PILARTOBAJAS@YAHOO.ES)

Purpose: We hypothetised that a low-flow continous perfusion $(0.1 \mathrm{~mL} / \mathrm{min})$ of saline at 2-mm distance from a cool-tip does not reduce the predictability of the lesion shape and may enlarge the achieved coagulation volume in a safe fashion. Methods and Materials: Our experimental protocol was approved from the local ethical committee. Forty-eight ablations were performed on 12 adult pigs: group 1, n $=24$ (cool-tip with 2-mm distant perfusion of saline); group 2, $\mathrm{n}=24$ (simple cool-tip). Coagulation volume, long (LCD) and short (SCD) coagulation diameters, surface area-to-volume ratio $\left(\mathrm{SR}\left(\mathrm{cm}^{-1}\right)\right)$ and sphericity $(\mathrm{S})$ were assessed macrocopically, sonographically and by MRI with and without digital reconstruction. Coefficient of variability $(\mathrm{CV})$ was calculated for each measure and compared among groups. Results: Both SCD and V were significantly greater with slightly higher $\mathrm{CV}$ in group $1 ;\left(2.46 \pm 0.606 \mathrm{~cm}\right.$ and $19.54 \pm 11.39 \mathrm{~cm}^{3} ; 1.86 \pm 0.55 \mathrm{~cm}$ and $9.21 \pm 5.74 \mathrm{~cm}^{3} ;$ for groups 1 and 2, respectively, $\mathrm{p}<0.05$, to macroscopic study and $2.41 \pm 0.58 \mathrm{~cm}$ and $15.03 \pm 9.21 \mathrm{~cm}^{3} ; 1.8 \pm 0.52 \mathrm{~cm}$ and $7.74 \pm 5.03 \mathrm{~cm}^{3}$, respectively, to MRI). No significant differences $(p<0.05)$ were observed between LCD and S. SR showed significant lower ratios in group 1, measured macroscopically and by MRI with digital reconstruction $(3.15 \pm 0.65$ and $2.69 \pm 0.57 ; 3.86 \pm 0.82$ and $3.46 \pm 1.12$ for groups 1 and 2 , respectively, $p<0.05$ ).

Conclusion: Distant infusion of saline enlarges coagulation volume during radiofrequency ablation of liver tissue using cool-tip electrodes without impairing their predictability.

B-040 15:12

Clinical application of virtual navigator and GPS in liver tumour ablation X. Xie; Guangzhou/CN (xxy1992@21cn.com)

Purpose: To evaluate the clinical value of real-time virtual navigator system and GPS as the new ultrasound guidance tools for percutaneous ablation therapy of malignant liver lesions.

Methods and Materials: Real-time virtual navigator-guided percutaneous ablations were applied in 76 patients with 125 malignant liver lesions and GPS-guided percutaneous ablations were applied in 20 patients with 23 lesions. For navigator group, $64.8 \%(81 / 125)$ lesions were undetectable on conventional ultrasound (US). The consuming time of image fusion was recorded and the local treatment response was observed. The influence of GPS to clinical outcome was evaluated on a 4-point scale: substantial useful, increased confidence, added some information, no change.

Results: For navigator group, the average fusion time of the ultrasound and CT image was $13.2 \pm 12.8 \mathrm{~min}$ ( $5 \mathrm{~min} \sim 55 \mathrm{~min}$ ). Complete ablation rate was $86.4 \%$ (38/44) in detected lesions by ultrasound and $91.6 \%(74 / 81)$ in undetected lesions by ultrasound. For GPS group, the influence of GPS to clinical outcome is substantial useful in $11(55.0 \%)$ cases, increased confidence in $5(25.0 \%)$ cases, added some information in $2(10.0 \%)$ cases, no change in $2(10.0 \%)$ cases. No treatment associated complications were found.

Conclusion: Ablation assisted by real-time virtual navigator or GPS is safe and efficacious, especially for undetectable lesion by conventional ultrasound or multipuncture ablation lesions. 
$14: 00-15: 30$

Vascular

\section{SS 115}

\section{Aorta}

Moderators:

E. Bruguiere; Paris/FR

T. Pfammatter; Zurich/CH

\section{B-041 14:00}

Subtle dissection of the ascending aorta: assessment with ECG-gated MD-CT S. Schotten, L. Conzelmann, K.-F. Kreitner, C. Düber, C.-F. Vahl, K. Oberholzer; Mainz/DE (sebastian.schotten@gmx.de)

Purpose: To analyse the value of ECG-gated MD-CT for diagnosis of subtle or discrete dissection of the ascending aorta, defined as an intimal tear without an intimomedial flap or evident intramural haematoma and to correlate these results with intraoperative findings and histopathology.

Methods and Materials: 32 patients with suspected Stanford type A dissection preoperatively underwent contrast-enhanced ECG-gated 64- $(n=30)$ or 256-slice $(n=2)$ MD-CT (Brilliance $64 \AA$, iCT 256®, Philips Medical systems). Two readers in conference retrospectively analysed the image material with focus on the detection of intramural haematomas, pericardial and pleural effusions, mediastina haemorrhage, and intimal flaps or tears. Imaging findings could be correlated with intraoperative findings as well as with histopathology.

Results: Histopathology revealed a dissection of the ascending aorta with an intimomedial flap in $26 / 32(81 \%)$ patients, an intramural haematoma in $2(6 \%)$, and a limited intimal tear in the ascdending aorta in $4 / 32(13 \%)$ patients, respectively. ECG-gated MD-CT depicted all type A dissections and the intramural haematomas. However, only 2/4 patients with subtle intimal tears were delineated by MD-CT. Conclusion: Subtle aortic dissections are a challenging entity among pathologies of the ascending aorta presenting clinically as an acute aortic syndrome. ECG-gating limits motion artefacts of the aortic root and ascending aorta, and therefore improves identification of discrete intimal injuries. Nevertheless, even with ECG-gating, not al subtle aortic dissections could be identified in the present study. Therefore, we suggest that additional imaging modalities should be considered in clinically doubtful cases.

\section{B-042 14:09}

Aortic elasticity assessment: a prospective study by using

electrocardiographically gated dual-source $\mathrm{CT}$

N. Lii', C. Liu'1 , J. Chen²; 'Jinan/CN, ${ }^{2}$ Beijing/CN (lining_820313@126.com)

Purpose: To provide a new insight into elastic properties of the thoracic aorta and evaluate its age relevance in patients without aortic disease by using ECG-gated dual-source CT (DSCT).

Methods and Materials: 56 subjects with confirmed diagnosis of no cardiovascular disease, selected from 2,700 people were divided into three groups according to their age $(A<40, B 40-50, C>50)$. Original data were reconstructed in $5 \%$ steps throughout the RR interval. The maximum and minimum diameter phase of the ascending aorta $(\mathrm{AA})$, the elastic parameters such as relative diameter change $(\% \mathrm{Ao})$, aortic distensibility $(A \circ D)$, compliance $(A \circ C)$ and stiffness index (AoSI) were calculated on the plane which is located $20 \mathrm{~mm}$ or $25 \mathrm{~mm}$ above the sinotubular junction. The above parameters were also calculated on the same plane of the descending aorta (DA). Results: All data of the patients were successfully evaluated. There was an elasticity difference between AA and DA.The $95 \%$ confidence intervals $(\mathrm{Cl})$ of the maximum and minimum diameter phases of AA were at [22.68\%, 25.35\%] and $[92.4 \%, 94.6 \%]$ of RR interval, which were $[24.35 \%, 26.9 \%]$ and $[95.38 \%, 97.83 \%]$ for DA. There was no significant correlation between the phases and the average heart rate $(r<0.3)$. An age-dependent decrease of aortic elasticity was determined in all groups, which was more obvious in group $A$.

Conclusion: As a one-stop shop method, retrospective ECG-gated DSCT can provide valuable functional information of the aorta at the same time to show the structure changes, which would be helpful in cardiovascular disease treatment planning and therapy control.

\section{B-043 14:18} Haemodynamic imaging of the thoracic aorta by computational fluid dynamics (CFD): status quo of the project at two years from the presentation of the method (ECR 2008)

M. Midulla ${ }^{1}$, R. Moreno ${ }^{2}$, M. Chau ${ }^{2}$, A. Negre-Salvayre ${ }^{2}$, I. Berry², J.-P. Pruvo', H. Rousseau'; ; ${ }^{2}$ ille/FR, ${ }^{2}$ Toulouse/FR (marcomidu@gmail.com)

Purpose: A project dedicated to the functional analysis of the thoracic aorta has been developed over the last two years. After the in vitro phase, the method, presented at the ECR 2008, has been developed in its clinical applications.

Methods and Materials: 40 subjects were studied: 20 volunteers ( 12 male; mean age 20.4) and 20 patients (14 male; mean age 62.2) with different thoracic aortic diseases treated by thoracic endovascular aortic repair. The CFD imaging was reviewed by two vascular radiologists and compared to the native MRI. Quantitative measures of the haemodynamic parameters (velocity, wall shear stress, compliance) were performed at different standardised levels (aortic arch, isthmic region, descending aorta). Results: Blood flow patterns were characterised in both patients and volunteers groups. Velocity (peak values, $\mathrm{m} / \mathrm{sec}: 2.10 \pm 0.3$ ) and WSS (systolic, Pa: $0.3 \pm 0.2$ vs $0.4 \pm 0.2$ ) values were extracted. Focal alterations of WSS were founded in 8 subjects (40\%) of the TEVAR group and were directly correlated with morphologic findings on MRI related to the stent graft's anatomy. In 2 cases the CFD findings allowed to preview the clinical evolutions of the pathology as confirmed in the scheduled follow-up. An increased aortic compliance was found in the TEVAR group compared to the volunteers (diameter compliance, $\mathrm{Cd}=2 \Delta \mathrm{d} / \Delta \mathrm{P}$ and distensibility coefficient, $\mathrm{DC}=\mathrm{Cd} / \mathrm{d})$.

Conclusion: The CFD imaging provides a feasible and reliable method for the functional study of the thoracic aorta. It should be a useful tool for a better comprehension of the physiologic consequences of the endovascular treatments by stent graft implantation.

B-044 14:27

Computational simulation of 4D blood flow dynamics of the thoracoabdominal aorta: prediction of long-term changes in aneurysm morphology

T. Ueda ${ }^{1}$, H. Suito 2 , G.D. Rubin ${ }^{3}$, D. Sze ${ }^{4} ;{ }^{1}$ Chiba/JP, ${ }^{2}$ Okayama/JP,

${ }^{3}$ Durham, NC/US, ${ }^{4}$ Stanford, CA/US (takuedarad@gmail.com)

Purpose: To simulate blood flow dynamics of the thoracic and abdominal aorta and assess wall stresses with the intent of predicting long-term morphological changes of aneurysms.

Methods and Materials: Thoraco-abdominal CT scans from 10 patients with aortic aneurysms that demonstrated varying degrees of torsion were evaluated. The computational dynamics of aortic blood flow were derived using Navier-Stokes equations for an incompressible viscous fluid with blood assumed to be a nonNewtonian fluid. Blood flow dynamics were simulated based on a finite-difference approximation with a fictitious domain method by using flow dynamic data at the aortic root as the boundary condition. To determine how flow dynamics affect aortic morphology, we computed force-field parameters including averaged shear stress, expansion force and bending force on the aortic wall. Cardiac-phase-dependent 4D flow fields were computed and visually assessed.

Results: All 10 thoraco-abdominal aorta were simulated fully. The shear stresses, expansion forces, and bending forces were visualised. Strong curvature was insufficient to generate large shearing stress on the aortic wall. Aortic tortuosity (elongation) causes swirling flow and enhanced the shearing stresses on the wall. Conclusion: Computational simulation clarified the relationship between morphology and haemodynamics of the aorta, emphasising significance of swirling flow due to aortic torsion which causes large shearing stresses on the aortic wall. These stresses may result in long-term morphological changes in the aortic wall.

\section{B-045 14:36}

In vivo and in vitro validation of aortic flow quantification by time-resolved three-dimensional velocity-encoded MRI

F. Rengier ${ }^{1}$, M. Delles ${ }^{2}$, M. Müller-Eschner ${ }^{1}$, Y. Hoo', R. Unterhinninghofen²,

S. Ley ${ }^{1}$, R. Dillmann ${ }^{2}$, H.-U. Kauczor ${ }^{1}$, H. von Tengg-Kobligk ${ }^{1} ;{ }^{1}$ Heidelberg/DE, ${ }^{2}$ Karlsruhe/DE

Purpose: The potential of three-dimensional velocity-encoded cine MRI (3D VEC $\mathrm{MRI}$ ) lies in the possibility of three-dimensional flow quantification which may be used for risk stratification in the future. The purpose of this study was to validate aortic flow quantification by 3D VEC MRI in vitro and in vivo.

Methods and Materials: 3D VEC MRI data sets were acquired at $1.5 \mathrm{~T}$ in an elastic tube phantom with constant and pulsatile aortic flow as well as in 11 healthy volunteers ( $23 \pm 2$ years, 7 men). Two-dimensional through-plane VEC MRI at six equidistant levels in vitro and three locations in vivo (ascending aorta/aortic arch/ 
descending aorta) was used as gold standard. The percental difference of the 3D VEC MRI measurement to the gold standard was defined as parameter for accuracy. Results: In vitro, 3D VEC MRI significantly overestimated average area, and significantly underestimated average aortic velocity and flow with accuracy values for area, velocity and flow of $13.6 \%(p<0.001),-18.1 \%(p<0.001)$ and $-7.4 \%$ $(p<0.001)$. Accuracy was significantly higher in the iso-centre compared to offcentre $(p=0.001)$. In vivo, 3D VEC MRI underestimated average velocity (all locations $p<0.001)$ and overestimated average area $(p=n . s . /<0.001 /<0.001)$. Average flow was significantly underestimated in the ascending aorta $(p=0.035)$, but tended to be overestimated in the aortic arch and descending aorta.

Conclusion: 3D VEC MRI tends to overestimate average aortic area and to underestimate average aortic velocity, resulting in significant underestimation of average flow in the ascending aorta. Flow quantification by 3D VEC MRI must be interpreted with care regarding current limitations.

\section{B-046 14:45}

Quantitative measurements of haemodynamics of pulmonary and systemic circulation in healthy volunteers with phase-contrast MR imaging on 3.0 tesla system

R.P. Wang ${ }^{1}$, C.H. Liang ${ }^{2}$, M.P. Huang ${ }^{2} ;{ }^{1}$ Guiyang/CN, ${ }^{2}$ Guangzhou/CN (wangrongpin@126.com)

Purpose: To measure the haemodynamic information of great vessels of pulmonary and systemic circulation using phase-contrast imaging sequence on 3.0 tesla MR system (PC-MRI), and to validate the accuracy of the results from PC-MRI sequence in vivo simultaneously.

Methods and Materials: Fifteen healthy volunteers were performed on GE 3.0 tesla MR scanner using PC-MRI sequence to evaluate the blood flow status in main pulmonary artery (MPA), right pulmonary artery (RPA), left pulmonary artery (LPA), ascending aorta (AA), superior vena cava (SVC) and inferior vena cava (IVC). The average flow and regurgitation fraction of each vessel were calculated for each volunteer during one cardiac cycle. Paired-samples t-test was employed to assess the statistical significant difference. Results: No significant difference was found in the average flow and regurgitation fraction between MPA and AA. The average flow of RPA was significantly higher than that of LPA $(t=3.092, P=0.004)$, while the regurgitation fraction of RPA was significantly lower than that of LPA $(t=-5.502, P=0.001)$. The average flow of SVC was significantly lower than that of IVC $(t=-6.866, P<0.001)$, while the regurgitation fraction of IVC was significantly higher than that of SVC $(t=4.250, P<0.001)$. We obtained a high correlation ( $r=0.939 \sim 0.991)$ among average flow of MPA, AA and vena cava, and the ratio of the three were 1:1.009:0.974.

Conclusion: The average flow of great vessels can be accurately measured with 3.0 T PC-MRI sequence. The flow volume and regurgitation fraction of great vessels in healthy volunteers may be used as a reference for cardiopath.

\section{B-047 14:54}

Follow-up with CT imaging after endovascular abdominal aneurysm repair: how much is the dose?

R.M. Trimboli' ${ }^{1}$, G. Mauri ${ }^{1}$, G. Tosi ${ }^{2}$, G. Brambilla ${ }^{2}$, G. Di Leo ${ }^{3}$, G. Cornalba',

F. Sardanelli ${ }^{3} ;{ }^{1}$ Milan $/ I T,{ }^{2}$ Rozzano/IT, ${ }^{3}$ San Donato Milanese/IT

Purpose: To estimate the patient's cumulative radiation dose for a routine CT scan in following up patients treated with endovascular prosthesis for abdominal aneurysm. Methods and Materials: Between December 1998 and September 2008,122 patients (mean age $74 \pm 7$ years) have been treated with endovascular repair of abdominal aortic aneurysm. They were followed up with contrast-enhanced 16-row or 64-row multidetector CT. For each patient, we counted the number of CT scans during follow-up. Exposure data were collected for an independent sample of 10 patients and the corresponding estimated effective dose per CT scan was applied for the first large series. In such a way we calculated the patient's cumulative effective dose in the follow-up period. Effective dose of each CT scan was calculated multiplying the dose-length product by 0.016 that is mean of conversion factors for thorax and abdomen.

Results: The number of CT scans ranged from 1 to 15 with a median of 3 CT examinations. Tube voltage was $120 \mathrm{kVp}$ and mean \pm standard deviation tube current was $250 \pm 77 \mathrm{mAs}$ (range 135-400 mAs). The mean \pm standard duration of follow-up was $21 \pm 19$ months. The mean effective dose per CT scan was $30 \pm 12 \mathrm{mSv}$ (range 11-45 mSv), and the median patient cumulative effective dose was $88 \mathrm{mSv}$ (range 29-440 mSv).

Conclusion: Following up patients treated with endovascular repair of abdominal aortic aneurysm using routine CT determines a very high cumulative effective radiation doses.
B-048 15:03

Aortic neck dilation after endovascular abdominal aortic aneurysm repair: should be predicted?

R. Dattesi, R. lezzi, M. Santoro, F. Pirro, M. Nestola, F. Snider, L. Bonomo; Rome/IT (robertadattesi@gmail.com)

Purpose: To evaluate whether dynamic CT can provide functional vessel information predicting the outcomes of aortic neck in patients undergoing EVAR.

Methods and Materials: We prospectively enrolled 20 patients with AAA and 20 patients without AAA. All patients underwent 64-slice ECG-gated abdominal CTA examinations. Axial pulsatility measurements were manually taken by three readers in both systolic and diastolic phase at 3 levels: $2-\mathrm{cm}$ above the highest renal artery, at the level of renal arteries, $1-\mathrm{cm}$ below the lowest renal artery. Systolic and diastolic blood pressures were measured in the brachial artery in order to calculate the arterial wall distensibility expressed as pressure strain elastic modulus (Ep). Cross-sectional area change, wall distensibility, and Ep value were determined and statistically compared. Results: A significant aortic pulsatility during the cardiac cycle was obtained in both groups. When comparing the two groups, no significant differences were found in terms of Ep values in the suprarenal and juxtarenal level, whereas in AAA group a significant higher value was obtained in the infrarenal level. A subgroup of patients with AAA (45\%) was identified with a significantly higher Ep value obtained in infrarenal level. An higher pressure strain elastic modulus is related to a lower distensibility and a lower compliance and potentially to an higher aortic wall degeneration.

Conclusion: Dynamic CT imaging could provide insight into the pathophysiology of abdominal aorta allowing the identification of patients with higher infrarenal distensibility, potentially predicting a post-EVAR proximal neck dilation in the follow-up, and consequently changing the stent-graft selection.

\section{B-049 15:12}

Whole-body MRA in Takayasu arteritis: systemic assessment of arterial stenosis and related mural involvement

C. Zini, A. Napoli, G. Cartocci, L. Molisso, L. Di Mare, F. Zaccagna, P. Di Paolo,

C. Catalano, R. Passariello; Rome/IT (zini.chiara@gmail.com)

Purpose: To assess vessel involvement and activity of Takayasu arteritis (TA) with whole-body MRA (WB-MRA).

Methods and Materials: 23 patients with diagnosed TA underwent WB-MRA with gadobenate dimeglumine (MultiHance). According to the Birmingham vasculitis activity score (BVAS), 16 (70\%) patients were classified as having active/persistent disease (AD) and $7(30 \%)$ patients as having remissive disease (RD). The arterial system was divided into 32 segments and 5 regions. Image quality, wall thickness (WT) and wall signal intensity (SI) were evaluated by expert readers who were blinded to all clinical data. Quality analysis was performed using Wilcoxon's rank test; statistically significant differences in SI values of $A D$ and RD were tested using an unpaired t-test on a per region basis.

Results: A total of 736 arterial segments with good to excellent image quality were assessed. In 12 patients WBMRA depicted vessel involvement that could not be assessed by mono-station MRA; 5 extra-vascular findings were identified at MRA. The vessel wall thickness of the AD group $(4.2 \pm 1.9 \mathrm{~mm})$ was thicker than that of the RD group $(2.9 \pm 2.4 \mathrm{~mm} ; \mathrm{p}<0.05)$. The post-contrast signal intensity ratio was significantly different between the two groups (active/persistent vs remissive, $1.8 \pm 0.6$ vs $1.1 \pm 0.5 ; p<0.05$ ). Per region analysis revealed higher wall intensity in the carotid and subclavian regions $(p<0.05)$ in both groups.

Conclusion: WB-MRA allows a comprehensive assessment of vessel involvement. MRA could be useful modality for TA activity evaluation, which is mandatory for therapy management.

\section{B-050 15:21}

MS-CT vs contrast-enhanced ultrasound in detection of endoleak following endovascular aneurysm repair

D.-A. Clevert, W. Sommer, S. Weckbach, M.F. Reiser;

Munich/DE (Dirk.Clevert@med.uni-muenchen.de)

Purpose: The purpose of this study was to compare colour-duplex-ultrasound (CDU), contrast-enhanced ultrasound (CEUS) and multislice-computed-tomography (MS-CT) angiography in the routine follow-up of patients following endovascular repair (EVAR) of abdominal aortic aneurysm (AAA).

Methods and Materials: 129 consecutive patients with AAA underwent EVAR and were imaged with CDU, CEUS and MS-CT angiography.CEUS was examined using 0.9-1.2 $\mathrm{ml}$ intravenous injection of SonoVue (Bracco, Italy) and using a multifrequency transducer 2-4 MHz (Siemens, Sequoia, Acuson or S 2000). Each imaging modality was evaluated for the detection of endoleaks. 
Results: CTA was used as gold standard in determining the presence of endoleaks. CDU was true positive for endoleaks in $17 / 129$ patients (13.2\%) and false positive for endoleaks in $6 / 129$ patients $(4.6 \%)$. The sensitivity of CDU was therefore $32.3 \%$ and its specificity $92.8 \%$; the positive and negative predictive values were 0.71 and 0.72 , respectively. CEUS was true positive for the detection of endoleaks in 45/129 patients (34.9\%) and false positive in $6 / 129$ patients $(4.6 \%)$. The sensitivity of CEUS was therefore $100 \%$ and its specificity $93 \%$; the positive and negative predictive values were 0.88 and 1 . In the follow-up the six false positive endoleaks in CEUS were confirmed in three cases as true positive endoleaks by CEUS and MS-CT. Conclusion: CEUS seemed to be equal in demonstrating endoleaks after EVAR in comparison to MS-CT angiography and may be considered as a primary surveillance modality whereas duplex ultrasound scanning alone is not as sensitive as CEUS and MS-CT angiography in detection of endoleaks.

14:00 - 15:30

Room E2

\section{Musculoskeletal}

\section{SS 110}

\section{Tumours, bone marrow and whole body}

Moderators:

M. Adriaensen; Heerlen/NL

J.F.M. Meaney; Dublin/IE

\section{B-051 14:00}

\section{MRI-based assessment of therapy monitoring in neoadjuvant} chemotherapy of soft tissue sarcoma

L. Umutlu, E. Stylianou, S. Bauer, F. Grabellus, G. Taeger, T.C. Lauenstein;

Essen/DE (Lale.Umutlu@uk-essen.de)

Purpose: The use of tumour necrosis factor in isolated limb perfusion (ILP) has shown to be a promising treatment of locally advanced limb soft-tissue sarcomas (STS). However, the assessment of therapy response is an important issue of neoadjuvant cancer treatment. The aim of this study was to evaluate unidimensional (RECIST), 2D and 3D assessment of morphologic cancer response.

Methods and Materials: 38 patients with histologically proven soft-tissue sarcoma were examined (a) prior and (b) 4 weeks after ILP on a 1.5 Tesla scanner (Avanto, Siemens Healthcare). The imaging protocol included contrast-enhanced T1w-3D spoiled gradient-echo-sequences (Gadovist ${ }^{\circledR}$, Bayer Schering Pharma). All malignant lesions were ssessed in the baseline and follow-up examination according to RECIST (unidimensional), 2D and 3D measurements. Results were correlated to pathologic response evaluation. The morphologic changes were compared by Wilcoxon signed rank test.

Results: According to histopathology 30 patients were determined as responders and 8 patients as non-responders. Statistical analysis of responders revealed a tumour size decrease under therapy in all three measurements (RECIST $-5.7 \%$; $2 \mathrm{D}$ $-14.5 \%$; 3D $-31 \%$ ). However, only 2D measurements proved statistical significance $(p=0.046)$ comparing responders and non-responders. 2 out of 8 non-responders showed tumour progress under therapy, leaving 6 non-responders with a moderate, but non-statistically significant tumour size decrease.

Conclusion: RECIST and 3D volumetric assessment of tumour size of STS under ILP does not provide sufficient evaluation of therapy response, leaving only bidimensional assessment as a reliable marker. Nevertheless, further response evaluation criteria including functional parameters should be considered in the future.

\section{B-052 14:09}

Whole-body MR-imaging allows to differentiate indolent from aggressive systemic mastocytosis

H.J. Michaely, G. Metzgeroth, S. Haneder, P. Riffel, S.O. Schönberg, A. Reiter; Mannheim/DE (henrik.michaely@umm.de)

Purpose: To evaluate if whole-body MR-imaging allows differentiating between aggressive systemic mastocytosis (ASM) and indolent systemic mastocytosis (ISM). Methods and Materials: In this IRB-approved study, 30 consecutive patients (18 F $12 \mathrm{M}$ ) with clinically proven mastocytosis underwent a whole-body (wb) MR-exam at $1.5 \mathrm{~T}$ (Siemens Avanto) including coronal wb-T1w-TSE imaging, coronal wbSTIR-imaging and dynamic contrast-enhanced T1w-VIBE imaging of the abdomen. Image evaluation included the pattern and extent of pathologic bone marrow signal in the spine and the extremities, the presence of ascites, pathologic lymph nodes, hepatosplenomegaly and focal organ lesions. The clinical diagnosis of ASM and ISM was based on the current WHO-criteria.
Results: 17 patients were diagnosed with ASM, 13 with ISM. 15/17 ASM patients revealed a pathologic bone marrow pattern (sclerosis in 10/17, tumour cell infiltration in $5 / 17$ patients) extending into the extremities compared to only 1 patient in the ISM group (sclerotic bone marrow). In ASM pathologic lymph nodes, hepatosplenomegaly and ascites were present in 9/17, 16/17 and 13/17 patients while in the ISM group these findings were seen in $1 / 13,2 / 13$ and $0 / 13$ patients. Focal organ lesions were exclusively found in 4/17 ASM patients.

Conclusion: Wb-MRI is able to differentiate between ASM and ISM based on the specific pattern of bone marrow changes in combination with the presence of pathologic lymph nodes, focal organ lesions and ascites.

\section{B-053 14:18}

Diagnostic value of whole-body MRI in patients with sickle cell disease and its impact on patient management

S. Khedr, A. Gaballa; Jiddah/SA

Purpose: The aim of work was to assess the role of whole-body MRI in patients with sickle cell anemia.

Methods and Materials: 27 patients with known sickle cell anaemia were included in this study, complaining of acute painful vaso-occlusive crisis. Most of the patients complained of bony pain and swellings. Few of them complained of old stroke and chest pain. The whole-body (W.B) MRI studies were performed. Three coronal (T1, T2, and STIR) sequences were performed for whole-body MR imaging. In selected cases, dedicated examination of certain body parts were performed.

Results: Bone infarcts were the most common presentation, modularly and vertebral bone infarcts were found in all patients. Bilateral proximal femoral head epiphysis avascular necrosis wasfound in 15 patients. Osteomylitis was diagnosed in 6 patients, and in 2 of them the diagnosis was confirmed by bone scan due to difficulty in differentiating between bone infarct and osteomylitis. 2 cases with septic arthritis and 2 cases with myonecrosis of the muscle were also found. 5 patients with old brain infarcts and 2 patients with pneumonia were seen as well.

Conclusion: Whole-body MRI can help identifying multisystem affection in sickle cell disease in a single session. MRI is a useful imaging tool in distinguishing acute osteomylitis and bone infarct. Knowledge of the range of imaging findings is crucial in order to accurately depict the complication and initiate appropriate therapy.

\section{B-054 14:27}

(EPOS)

Whole-body MRI with diffusion sequences as a biomarker of MGUS and myeloma

E. Brillet, T. Josseaume, O. Decaux, T. Lamy de la Chapelle, R. Duvauferrier; Rennes/FR(e.brillet@gmail.com)

Purpose: Our work aimed to determine if the classification of Durie and Salmon plus (DSP) based on diffusion whole body MRI can be used as a biomarker for diagnosis, and follow-up of patients with MGUS or myeloma.

Methods and Materials: We followed between November 2008 to April 2010 a cohort of 63 initially non-treated patients (21 MGUS and 42 myeloma). 25 patients also had 2 diffusion whole body MRI (7 relapsing MGUS and 18 de novo myelomas). The protocol of diffusion whole-body MR included a sagittal T1 sequence of the spine, and coronal STIR and axial diffusion sequences of the whole body. The classification of DSP was compared with the classification of Salmon and Durie and the pasmacytosis. Within the follow-up period the evolution of DSP without and then with diffusion sequences was compared with the clinical and the monoclonal peak outcomes.

Results: We found a correlation between DSP and DS $(p=0.001)$ and the medullary plasmacytosis $(p=0.0075)$. There is a correlation between the deterioration of DSP and the deterioration of the clinioco-biological status. The correlation seems more important if the DSP was calculated with the diffusion compared to the DSP calculated without taking the diffusion into consideration $(p=0.0123$ vs $p=0.0377$ ) Conclusion: The DSP classification including diffusion sequences is a good reflection of the tumour mass and its evolution can be used in the follow-up of patients.

\section{B-055 14:36}

Whole-body low-dose computed tomography (WBLDCT) in staging of patients with multiple myeloma

V. Besostri, D. Ippolito, M. Crivellaro, E. Pogliani, A. Di Lelio, S. Sironi; Milan/IT (valeriabesostri@gmail.com)

Purpose: To assess the role of whole-body low-dose computed tomography (WBLDCT) in the diagnosis and staging of patients with suspicion of multiple myeloma (MM).

Methods and Materials: A total of 99 patients, with early multiple myeloma, underwent unenhanced whole-body low-dose CT protocol study, performed on 16-slice scanner (Philips, Brilliance, 16P): tube voltage $120 \mathrm{KV}$; tube current time product 
$40 \mathrm{mAs}$; collimation $16 \times 0.75$. All the patients were clinically staged according to Durie and Salomon System. Diagnosis of osteolytic lesions was performed on the basis of axial and multiplanar reformatted images, whereas the assessment of spinal misalignment and fracture was done using multiplanar reformatted images Results: In all 99 patients, image resolution was diagnostic, enabling correct classification of multiple myeloma patients. WBLDCT showed a total of 216 pathologic bone findings in 64 patients. CT scanning resulted in complete evaluation of the bone lesions in these areas of the skeleton: skull (30), humerus (9), femur (16), ribs (3), scapulae (10), pelvis (30), clavicle (10), sternum (6), cervical (21), dorsal (40), lombar (31) and sacral rachis (10). In 30 patients the CT detection of bone involvement was the only criterion for the treatment. Furthermore, in 16 patients WBLDCT scanning demonstrated pulmonary or pleural lesions ( 9 cases due to infective source and 6 cases due to multiple myeloma localisations) and 1 case of renal neoplasia as a related pathology. The overall dose delivered to each patients was $3.2 \mathrm{mSv}$.

Conclusion: Whole-body low-dose CT investigational protocol is a reliable imaging-based method for the direct management of patients with multiple myeloma. WBLDCT has a superior reproducibility and it is faster than conventional radiography, being, furthermore, able to demonstrate extraosseous findings.

\section{B-056 14:45}

Role of diffusion-weighted whole body MR imaging with background body signal suppression for disease detection and staging in patients with multiple myeloma: preliminary results

P.A. Bonaffini, D. Ippolito, I. Macchi, F. Rossini, A. Di Lelio, S. Sironi; Monza/IT (pa.bonaffini@gmail.com)

Purpose: To assess the overall diagnostic capability of whole-body MRI, with diffusion-weighted imaging with background body signal suppression (DWIBS) in combination with STIR T2 sequences, for detection of bone marrow lesions in patients with multiple myeloma (MM).

Methods and Materials: A total of 27 patients with MM underwent 1.5 T (Achieva Philips) WB-MRI study from the skull vertex to the feet, with a stepping table technique.The protocol consists of coronal short tau inversion recovery (STIR) T2, T1 TSE and DWIBS sequences, in order to characterise bone involvement in terms of pattern, size and distribution. DWIBS images were compared with standard T1 and STIR images in five districts (skull; spine; sternum and ribs; pelvis; upper and lower limbs), in patients with focal (15), diffuse (6) or combined (6) disease. All patients were clinically staged according to Durie and Salomon System.

Results: 153 focal bone lesions were detected. 151/153 lesions (98\%) were correctly identified with T1 and T2 sequences, while $138 / 153$ lesions $(90 \%)$ with DWIBS alone. In patients with diffuse disease DWIBS showed an homogenous increased bone intensity signal, with a greater sensitivity in demonstrating the widespread involvement of bone marrow than T2 sequences, due to inhomogeneous or partially increased of intensity signal. Our analysis showed that a more accurate result were achieved with T2 and T1 images combined with DWIBS images than T2 and T1 images alone, particularly in widespread form. Furthermore, in 3 patients with focal lesion in T2, DWIBS showed a diffuse involvement, respectively, of sternum and of both proximal femur.

Conclusion: Whole body staging with DWIBS sequences in patients with MM appears to be technically feasible and a promising tool in detection and characterisation of bone marrow lesions in alternative to multimodality approaches.

\section{B-057 14:54}

Whole body MRI for staging of patients with multiple myeloma in complete or partial remission after stem cell transplantation

C. Weber, H. Hentschel, N. Kröger, G. Adam, P. Bannas;

Hamburg/DE (C.Weber@amalie.de)

Purpose: To determine the diagnostic value of whole body MRI (WBMRI) in staging of patients with multiple myeloma (MM) after stem cell transplantation (SCTX) in complete or partial remission (CR, PR) in comparison to MRI of the spine alone. Methods and Materials: 40 patients with MM were examined twice on a $1.5 \mathrm{~T}$ scanner with a WBMRI protocol using fat-suppressed STIR, T1-FEE, and contrastenhanced fat-suppressed T1 sequences. Mean time between baseline and follow-up WBMRI was 1.3y (0.2-2.8y). WBMRI findings of involvement of the skeletal system and extraosseous relapse were analysed between the groups and correlated to an isolated analysis of axial skeleton MRI. Two blinded readers independently evaluated baseline and follow-up WBMRI.

Results: At baseline WBMRI 20 patients were in $\mathrm{CR}$ and 20 patients in PR. Baseline and follow-up WBMRI of 9 of all 40 patients (23\%) revealed no pathological findings (CR group: 6/20 (30\%); PR group 3/20 (15\%)). Follow-up WBMRI revealed regredient findings of bone involvement in 5 of 40 patients (13\%) (CR group: 1/20 (5\%),
PR group 4/20 (20\%)). 12 of 40 patients (30\%) had no change of bone involvement (CR group: $8 / 20$ (40\%), PR 4/20 (20\%)). 14 of 40 patients (35\%) had progressive bone involvement. Progredient findings were located in the axial skeleton in 1/14 patients $(7 \%)$, in $9 / 14$ patients $(64 \%)$ both in the axial and extra-axial skeleton and in $4 / 14$ patients $(28 \%)$ outside the axial skeleton.

Conclusion: WBMRI-based staging after SCTX in patients with MM is a significant prognostic tool and recommended for post-therapeutic monitoring.

\section{B-058 15.03}

F-18-FDG PET/CT versus whole-body MRI in the evaluation of multiple myeloma after stem cell transplantation

C. Weber, S. Klutmann, T. Derlin, N. Kröger, G. Adam, K. Peldschus; Hamburg/DE (C.Weber@amalie.de)

Purpose: To compare the relative contribution of F-18-FDG-PET/CT and WBMRI in the evaluation of MM patients after SCTX.

Methods and Materials: Thirty patients ( 11 women, 19 men; age, $55.3 \pm 9.9$ years remission status, 22 complete remission, 8 partial remission) during follow-up after SCTX (median follow-up 28 months) were retrospectively studied. All patients underwent whole body F-18-FDG-PET/CT and WBMRI within 1 month of each other. Bone involvement observed by WBMRI was characterised in terms of pattern, size and contrast enhancement, bone involvment observed by F-18-FDG-PET/CT was characterised in terms of pattern, size and tracer accumulation in 10 regions of the skeleton. Extraosseous lesions were likewise classified. Imaging findings, clinical remission status, and immunological and serum markers were compared. Results: F-18-FDG-PET/CT detected a total of 10 lesions in 6 patients. WBMRI detected a total of 97 lesions in 20 patients. On a per patient basis, F-18-FDG-PET had a sensitivity of $50 \%$, a specificity of $91 \%$, positive predictive value of $67 \%$, and a negative predictive value of $83 \%$. WBMRI had a sensitivity of $88 \%$, a specificity of $41 \%$, a positive predictive value of $35 \%$, and a negative predictive value of $90 \%$. Comparison of F-18-FDG-PET and WBMRI findings revealed concordant results in only $5(4.7 \%)$ of the 102 lesions.

Conclusion: In treated patients, the positive predictive value of MRI is disappointingly low. Therefore, F-18-FDG-PET/CT, despite its limited capacity in detecting lesions in the post-treatment setting, might be more suitable than MRI.

\section{B-059 15:12}

Evaluation on Murin model of osteosarcoma tumour hypoxia by MRI BOLD and correclation with diffusion MRI and F-MISO TEP-CT: preliminary study B. Dallaudiere ${ }^{1}$, V. Hummel ${ }^{1}$, J. Monteil' ${ }^{1}$, E. Schouman-Clayes ${ }^{2}$, P. Preux ${ }^{1}$,

A. Maubon ${ }^{1} ;{ }^{1}$ Limoges/FR, ${ }^{2}$ Paris/FR (bendallau64@hotmail.fr)

Purpose: The concentration of oxygen in tumours has therapeutic and prognostic implications in many solid tumours. BOLD MRI has been proposed to try to nonin vasively monitor the level of tissue oxygenation. We are interested in osteosarcoma hypoxic because they are the most frequent primary malignant bone tumours. The main purpose of our study was to assess the level of oxygenation in rodent model of osteosarcoma by BOLD MRI and to correlate the value of ADC maps and PET-CT binding to F-Miso.

Methods and Materials: We quantified BOLD MRI at 3 Tesla in ambient air and in 8 litres of oxygen after impregnating 2 and 3 minutes of the signal osteosarcomas transplanted in eight male rats on days 10,17 and 24 post transplant. We compared these results with hypoxic anatomopathologic study (HIF-1), ADC map and value of the ratio of SUV max between diseased tissue and healthy PET-CT F-Miso, with a significance level of 0.05

Results: The results showed significant differences $(p<0.005)$ of signal drop of the tumour under $\mathrm{O}^{2}$ to 2 and 3 minutes compared with study air, no impact from the time of impregnation of oxygenation or the tumour volume. There is no correlation between the fall of the BOLD signal and the coefficient ADC. We denote a significant correlation $(p<0.05)$ between the MRI signal intensity in BOLD and PET CT. Conclusion: BOLD imaging is a promising non-irradiating tool for non-invasive functional imaging of osteosarcoma, but new developments are necessary to allow its use in clinical practice.

\section{B-060 15:21}

The application of diffusion tensor imaging in tumour of bone and soft tissue

D.M. Deng ${ }^{1}$, Q.F. Meng 2 ; ${ }^{1}$ Nangning/CN, ${ }^{2}$ Guangzhou/CN (ddemao@163.com)

Purpose: To explore the value of DTI in diagnosis of bone and soft tissue tumours. Methods and Materials: Routine scanning of MR and DTI was performed prospectively on 42 cases with bone and soft tissue tumours betiding at calf, thigh or haunch. We analysed the statistical difference of DTI measurements between benign and malignant tumours' parenchyma, and between two groups' parenchyma 
from different tissues. We did peritumoural myofiber tracking to 26 cases suspected of invasion into adjacent muscles in routine MR.

Results: The value of $\lambda_{1}, \lambda_{2}, \lambda_{3}$ and ADC of malignant parenchyma was lower than that of benign ones, the FA values of parenchyma in malignant and benign tumours had no difference. The value of $\lambda_{1}, \lambda_{2}, \lambda_{3}$ and ADC of parenchyma in tumours from fibre and muscle tissues was lower than those from other tissues, with much overlap between the 2 groups; and the FA value was higher than that of the latter ones, with little overlap. Of the 15 tumours with peritumoural muscles invasion, 8 cases were diagnosed correctly by the technology; of the 11 tumours with peritumoural muscle compression, 6 cases were diagnosed correctly.

Conclusion: DTI has important application value in diagnosis of bone and soft tissue tumours. The value of $\lambda_{1}, \lambda_{2}, \lambda_{3}$ and ADC of benign parenchyma is much higher than that of malignant ones, with no difference in the FA value of the two groups. The FA value may provide useful information to judge the tumour source (fibre or muscles). The fibre trace is valuable to demonstrate the relationship between tumour and peritumoural muscle.

\section{4:00 - 15:30}

Room F1

\section{Genitourinary}

\section{SS 107 \\ Gynaecologic masses}

Moderators:

C.D. Alt; Heidelberg/DE

K. Kinkel; Chêne-Bougeries/CH

\section{B-061 14:00}

Endometrial cancer: correlation of apparent diffusion coefficient with tumour grading, depth of myometrial invasion and lymph node metastases G. Rechichi, S. Galimberti, M. Signorelli, C. Talei Franzesi, P. Perego, M.G. Valsecchi, S. Sironi; Monza/IT (gilda.rechichi@gmail.com)

Purpose: To investigate (a) whether ADC values of endometrial cancer differ from those of normal endometrium and myometrium, and (b) whether they vary according to histologic tumour grade, depth of myometrial invasion, and lymph node metastases.

Methods and Materials: Seventy patients with histologically proven endometrial cancer and 36 with normal endometrium (control group) were enrolled in this prospective study. T2-weighted, dynamic T1-weighted and diffusion weighted (DW) images with b-values of 0 and $1000 \mathrm{~s} / \mathrm{mm}^{2}$ were obtained in all patients. $A D C$ values of endometrial cancer, normal endometrium and myometrium were recorded. Tumour grade, depth of myometrial invasion, and lymph node status were assessed at postoperative histopathological analysis.

Results: The mean (+SD) ADC value $\left(10-3 \mathrm{~mm}^{2} / \mathrm{s}\right)$ of endometrial cancer $(0.77+$ 0.12 ) was significantly lower than that of normal endometrium $(1.31+0.11$, $p$-value $<0.0001)$ and normal myometrium $(1.52+0.21$, p-value $<0.0001)$, with no overlap between the two former distributions. There was no significant difference between ADC values of endometrial cancer tussue in: (a) patients with tumour grade $1(0.79$ $+0.08, n=14)$, grade $2(0.76+0.14, n=40)$, or grade $3(0.75+0.12, n=16),(p-$ value $=0.67) ;(b)$ patients with deep $(0.77+0.13, n=18)$ and superficial myometria invasion $(0.76+0.12, n=52)$, $(p$-value $=0.87)$; $(c)$ patients with $(0.78+0.10, n$ $=6)$ and without lymph node matastases $(0.75+0.14, n=39)$, $(p$-value $=0.64)$. Conclusion: ADC values allow differentiating normal endometrium from endometrial carcinoma; however, they do not correlate with histologic tumour grade, depth of myometrial invasion and presence of lymph node metastases.

\section{B-062 14:09}

Diffusion-weighted MR imaging with background body signal suppression (DWIBS) for the evaluation of endometrial cancer

J. Seo, C. Kim, B. Park, H. Kim; Seoul/KR (hyunsu83.kim@samsung.com)

Purpose: To prospectively assess the feasibility of diffusion-weighted MR imaging with background body signal suppression (DWIBS) in endometrial cancer, to evaluate the depth of myometrial invasion by combined T2-weighted imaging (T2WI) and DWIBS, and to investigate the difference of tumour ADC value according to tumour grade.

Methods and Materials: 52 consecutive patients with biopsy-proven endometria cancer were examined by 3 T MR scanner. MR sequences of all patients treated with surgery included T2WI, DWIBS and dynamic contrast-enhanced T1-weighted imaging (DCEI). ADC was calculated in the tumours and normal myometrium. Depth of myometrial invasion (revised 2009 FIGO staging) was assessed by two radiologists in consensus for each MR sequence, on a 5-point scale. Mean ADC of the tumour was analysed according to the grade.

Results: Among 52 patients, 49 had endometrioid adenocarcinoma and the remaining 3 serous adenocarcinoma. Mean ADC of the tumours and normal myometrium was 0.88 and $1.46 \times 10^{-3} / \mathrm{mm}^{2} / \mathrm{s}$, respectively $(P<0.001)$. At ROC analysis for assessing myometrial invasion, Az value of T2WI, combined T2WI and DWIBS and DCEI was $0.848,0.893$ and 0.901 , respectively $(P>0.05)$. In endometrioid adenocarcinoma, a significant difference of mean ADC between grade $1(0.96)$ and $2(0.75)$ or $3\left(0.74 \times 10^{-3} / \mathrm{mm}^{2} / \mathrm{s}\right)$ was found $(\mathrm{P}<0.01)$.

Conclusion: DWIBS at $3 \mathrm{~T}$ was useful to differentiate of endometrial cancer and normal myometrium. Combined T2WI and DWIBS could predict the depth of myometrial invasion accurately. Moreover, the mean ADC between grade 1 and 2 or 3 was signficantly different.

\section{B-063 14:18}

Accuracy of magnetic resonance imaging in predicting residual disease in local advanced cervical carcinoma (FIGO > IIB) after chemoradiation therapy: correlation with histopathologic results

M. Miccò, B. Gui, A. Valentini, G. D'agostino, D. Smaniotto, L. Bonomo; Rome/IT (miccomaura@yahoo.it)

Purpose: To evaluate diagnostic accuracy of magnetic resonance imaging (MRI) in predicting response to neoadjuvant chemoradiotherapy in patients with local advanced cervical carcinoma. MRI was performed before and after therapy: imaging findings were correlated with post-surgical histopathology

Methods and Materials: Between 2003 and 2009 MRI was performed in 41 patients (mean age 48) who fulfiled following criteria:1) histologic proven cervix cancer (FIGO > IIB); 2) external pelvic radiation (3960 pelvis + 1080 lymph nodes = $5040 \mathrm{cGy}$ ) and concomitant chemotherapy; 3) MRI studies obtained before initiation and after 4 weeks at conclusion of treatment; 4) radical hysterectomy at least 4 weeks after MRI. Post-treatment MRI findings (tumour volume, relative signal intensity of the lesion on T2 weighted images) and post-surgical histological results were compared. Statistical analysis (logistic regression) was performed by Medcalc software.

Results: At histology no macroscopic residual disease was found in $38 / 41$; MRI correctly identified no residual disease in $27 / 38$ with sensibility, specificity, accuracy, NPV and PPV of $97 \%, 71 \%, 84,96 \%$ and $77 \%$, respectively. In this group reduction of relative signal intensity of cervical region was identified (pre-treatment mean value 3 vs 2 post-treatment value). 28/38 patients presented inflammatory local process at histology. MRI correctly identified post-irradiation changes in 17/28 as hyperintense lesion with elevated RSI of cervical region (OR 5.8; 95\% Cl 1.2-27; $p=0.004$ ) with a cut-off value $>1.8$ (sensibility $89 \%$,specificity $63 \%$ ).

Conclusion: This study demonstrated high diagnostic accuracy of MRI in predicting local response to therapy and in identifying post-irradiation/inflammatory changes evaluating the relative signal intensity value of cervical region on T2 weighted images.

\section{B-064 14:27}

Locally advanced cervical cancer: MR imaging in prediction of response after preoperative chemoradiation therapy

G. Restaino ${ }^{1}$, M. Occhionero $^{1}$, M. Missere ${ }^{1}$, G. Macchia ${ }^{1}$, G. Ferrandina ${ }^{1}$,

F.M. Danza ${ }^{2}$, G. Sallustio ${ }^{1} ;{ }^{1}$ Campobasso/IT, ${ }^{2}$ Rome/IT (gennares@hotmail.com)

Purpose: To assess the accuracy of MRI in evaluating the LACC response to concurrent preoperative chemoradiation therapy, using qualitative and dimensional and volumetric quantitative criteria.

Methods and Materials: $1.5 \mathrm{~T}$ pelvic MRI pre- and post-chemoradiation therapy in 55 patients with LACC (53 y/o; 30-79) were retrospectevely qualitatively evaluated by two radiologists with 10 and 7 years of practice in gynaecologic MRI for presence or absence of residual disease. The quantitative analysis included: tumour diameters, tumour volumes calculated with ellypsoid method and with 3-D volumetry according Simpson's modified formula before and after treatment, and fractional regression rate. The MRI findings were correlated to histopathologic findings with Wilcoxon Signed-Rank test and with Mann-Whitney test.

Results: qualitative evaluation showed sensitivity, specificity, accuracy, positive predictive value, negative predictive value, LR+, and LR-: $93 \%, 53 \%, 63.6 \%, 42 \%$, $96 \%, 1.965$, and 0.127 . Quantitative analysis showed that the diameter of residual disease measured by MRI was on average twice the true diameter measured at histology (18 $\mathrm{mm}$ versus $9 \mathrm{~mm}$ ). No quantitative dimensional criterion showed significant differences between true positive and false positive cases.

Conclusion: Qualitative MRI has very high sensitivity and negative predictive value but low specificity and positive predictive value for predicting presence of residual disease after chemoradiation treatment in LACC. Evaluation of quantitative dimensional parameters did not improve the accuracy of the technique and was of no help in differential diagnosis between true and false positives qualitative evaluations. 


\section{B-065 14:36}

Detection of metastatic lymph nodes in patients with ovarian cancer by $\mathrm{CT}$, MRI, and PET or PET/CT: a systematic review and meta-analysis

Y. Yuan, S. Liu; Shanghai/CN (imyy-mn@163.com)

Purpose: Recognising the location of metastatic nodes is important for selecting treatment and predicting the possibility of optimal resection. The purpose of this study was to systematically review the diagnostic accuracy of CT, MRI, and PET or PET/CT for detection of metastatic lymph nodes in patients with ovarian cancer. Methods and Materials: Relevant studies were identified with MEDLINE and EMBASE from January 1990 to July 2010. We estimated the weighted summary sensitivities, specificities, OR (odds ratio), and summary receiver operating characteristic (sROC) curves of each imaging technique and conducted pair-wise comparisons using the two-sample Z-test. Meta-regression, subgroup analysis, and funnel plots were also performed to explain the between-study heterogeneity. Results: Eighteen eligible studies (CT 10, MR 5, PET or PET/CT 14) were included, with a total of 882 patients. PET or PET/CT was a more accurate modality (sensitivity $70.0 \%$, specificity $97.1 \%$ ). No significant difference was detected between CT (sensitivity $42.6 \%$, specificity $95.0 \%$ ) and MR imaging (sensitivity $54.7 \%$, specificity $88.3 \%$ ). Meta-regression analyses showed that sample size, year of publication, mean age, percentage of serous cancer, percentage of advanced ovarian cancer, and prevalence of lymph node metastasis had no significant influence on the between-study heterogeneity. Subgroup analyses revealed no statistical difference as well. Funnel plots with marked asymmetry suggested a publication bias. Conclusion: This meta-analysis showed that FDG-PET or FDG-PET/CT performs better than contrast enhanced CT and MR imaging in the detection of lymph node metastasis in patients with ovarian cancer.

\section{B-066 14:45}

Signal of uterine fibroids on T2W MR images as a predictive value of tumour volume reduction after intraarterial embolisation

T. Belsan, N. Profantova, M. Mara; Prague/CZ (tomas.belsan@uvn.cz)

Purpose: Uterine fibroids have different signal characteristics on magnetic resonance imaging (MRI). The aim of this study is to prove weather there is any significant difference of tumour volume reduction after uterine artery embolisation (UAE) in fibroids with different signal intensity on T2 weighted (T2W) images.

Methods and Materials: Study involves 69 females with uterine fibroids, 70 uterine fibroids were assessed. All patients underwent initial MRI and follow-up MR 6 months after UAE. In patients with multiple fibroids, only the significantly larges was included. Fibroids were measured in three orthogonal diameters, tumour volume was calculated. Accordingly the initial MRI fibroids were divided into two groups by their signal in T2W. Group 1: fibroids of markedly low signal (isosignal to sceletal muscle). Group 2: fibroids of mixed singal in T2W (higher to sceletal muscle). Tumour volume regress was calculated in percentage.

Results: Group 1 - 22 fibroids with markedly low signal in T2W - average tumour volume regress was $39.7 \%$ (range 8-75\%), only 3 tumours regressed more than $50 \%$. Group 2 - 48 fibroids with mixed signal in T2W - average tumour volume regress was $69.3 \%$ (range $30-100 \%$ ).

Conclusion: After $\mathrm{UAE}$, there is significant difference of volume reduction of fibroids with markedly low signal and of fibroids with mixed signal on T2W images. By this, MRI has predictive value for uterine fibroid volume regress based on signal intensity of tumours on $\mathrm{T} 2 \mathrm{~W}$ images.

\section{B-067 14:54}

Prediction of the predominant side of arterial supply to the uterine leiomyomas using uterine artery Doppler before uterine artery embolisation

N.N.N. Naguib, N.-E.A. Nour-Eldin, T. Lehnert, T. Gruber-Rouh, T.J. Vogl; Frankfurta.Main/DE (nagynnn@yahoo.com)

Purpose: To test if uterine artery (UA) Doppler performed before uterine artery embolisation (UAE) can predict the predominant side of supply to the uterine leiomyomas.

Methods and Materials: The study was prospectively performed on 38 females (mean age: $45.3 \pm 4.77$ years). The UA peak systolic velocity (PSV), end diastolic velocity (EDV) and resistive index (RI) were measured in both sides. The side with lower RI or with higher PSV or EDV was suggested as the predominant side of supply (for each patient 3 suggestions were provided). Measurements were performed trans-abdominally before UAE. After UAE, the predominant side was determined based on the tumour blush following contrast-medium injection in each side and based on the UA diameter measured $2 \mathrm{~cm}$ distal to its origin.

Results: The suggested side of predominant supply based on the RI was the right in 18 patients $(47 \%)$ and the left in $20(53 \%)$, based on the PSV and EDV measurements it was the right in $18(47 \%)$ and $16(42 \%)$ and the left in $20(53 \%)$ and $22(58 \%)$ patients, respectively. After UAE, the predominant side was the right in $19(50 \%)$ and the left in $19(50 \%)$. The mean left UA diameter was $3.58 \pm 0.67 \mathrm{~mm}$ (range 2.3-5.1 $\mathrm{mm}$ ) and the right was $3.55 \pm 0.88 \mathrm{~mm}$ (range: $2.2-5.8 \mathrm{~mm}$ ). The RI correctly identified the predominant side in $31(81.6 \%)$ patients (sensitivity=0.82), while the PSV and EDV correctly identified the predominant side in $15(39.5 \%)$ and 21 $(55.3 \%)$ patients, respectively, showing a sensitivity of 0.39 and 0.55 , respectively. Conclusion: Pre-interventional UA Doppler can predict the predominant side of arterial supply to the leiomyomas with the RI showing the highest sensitivity ( $\mathrm{S}=0.82$ ).

\section{B-068 15.03}

Uterine myomas treated by uterine artery embolisation, angioMRI at $48 \mathrm{~h}$ is predictive of evolution at 6 months

B. Layré, J. Manelfe, B. Vedel, P. Bouillet, Y. Aubard, A.J.M. Maubon; Limoges/FR (antoine.maubon@unilim.fr)

Purpose: Compare uterine myomas vascularisation by angioMR at $48 \mathrm{~h}$ and 6 month after UAE with microparticles, correlation with myoma shrinkage and clinical evolution.

Methods and Materials: Patients with symptomatic uterine myomas, treated with bilateral UAE using 500-700 $\mu \mathrm{m}$ trisacryl gel particles. Pelvic MRI with T2 and dynamic 3D T1w with IV gadolinium injection, performed at $48 \mathrm{~h}$ and 6 months afte UAE. Morphologic, volumetric with dedicated 3D software, and functional analysis of myomas. Evaluation of residual myoma perfusion at $48 \mathrm{~h}$ and 6 months. Correlation with clinical evolution

Results: Eighty seven patients were included, mean age 40.9 years (26-52). Mean myoma volume by uterus $222 \mathrm{~cm}^{3}\left(12-1022 \mathrm{~cm}^{3}\right)$ at $48 \mathrm{~h}, 78 \mathrm{~cm}^{3}\left(0-522 \mathrm{~cm}^{3}\right)$ at 6 months, mean myoma volume reduction $68.1 \%(17-100 \%)$. Totally devascularised myomas show a high signal in $\mathrm{T} 1$ and $\mathrm{T} 2$ at $48 \mathrm{~h}$, a high signal $\mathrm{T} 1$ and low signal $\mathrm{T} 2$ at 6 months. A significant persistent enhancement was found in 19 myomas in 8 patients at 6 months (mean myoma volume by uterus $89 \mathrm{~cm}^{3}$ ), mean myoma residual vascularisation: $55 / 89 \mathrm{~cm}^{3}$. It was present in the same 19 myomas in the same 8 patients on the $48 \mathrm{~h} \mathrm{MRI}$ (mean myoma volume by uterus $171 \mathrm{~cm}^{3}$ ), mean residual vascularisation $20 / 171 \mathrm{~cm}^{3}$. Clinical outcome was favourable in $86 / 87$ cases at 6 months. No correlation was found between a persistent vascularisation at 6 months and unfavourable clinical outcome.

Conclusion: MRI with GD injection at $48 \mathrm{~h}$ is predictive of myoma vasularisation evolution at 6 months after UAE. Clinical outcome at 6 months was not correlated with persistent vascularisation.

\section{B-069 15:12}

Real-time sonoelastography performed in addition to conventional ultrasound: improved early detection of ectopic pregnancy V. Gazhonova, S. Churkina, A. Zubarev; Moscow/RU

Purpose: To evaluate the possibilities of real-time sonoelastography in early detection of ectopic pregnancy.

Methods and Materials: Endovaginal ultrasound with elastography was performed in 56 women (19-38 y.o). with a positive pregnancy test and with suspected complications of early pregnancy. Urinary and serum $\beta$-hCG levels were measured on the day of the patient's hospitalisation (Second International Units). Sonoelastography was performed with the HI VISION 900 and Preirus (Hitachi Medical Corporation) with an endocavity transducer, 8-4 MHz frequency (EUP - V53 W, Hitachi). The elastographic images were assessed by 2 radiologists using a 4-point grading score for the presence or absence of ectopic pregnancy. Inter-observer agreement and diagnostic confidence levels were calculated. We assessed the accuracy of sonoelastography for the detection of ectopic pregnancy by comparing the findings of sonoelastography with surgical results.

Results: 25 women were proven to have an ectopic pregnancy. All 25 were ac curately detected by endovaginal ultrasound with elastography. The "blue eye" sonoelastographic sign was seen in every case of extrauterine pregnancy and had an $80 \%$ diagnostic confidence of ectopic pregnancy in women with $\beta$-hCG levels lower than $1000 \mathrm{mlU} / \mathrm{ml}$, and a $100 \%$ diagnostic confidence when levels were above the discrimination point of $1000 \mathrm{mlU} / \mathrm{ml}$. Inter-observer agreement revealed Kappa estimates ranging between 0.86 and 0.93 , indicating almost perfect conformity in the assessment of pathological changes between reader 1 and reader 2 . Conclusion: The "blue eye" sign can be used for the detection of extrauterine pregnancy in doubtful cases of serum $\beta$-hSG levels lower than $1000 \mathrm{mIU} / \mathrm{ml}$. 


\section{B-070 15:21}

The value of endovaginal sonoelastography (EVSE) in emergency gynaecological pathology

S. Churkina, A. Fedorova, V. Gazhonova; Moscow/RU (14churkina@mail.ru)

Purpose: To evaluate the usefulness of EVSE in the diagnosis of emergency gynecological pathology.

Methods and Materials: We study 140 women with acute lower abdomen pain and menorrhagia. All pts underwent conventional US and endovaginal sonoelastography on HI VISION 900, HI VISION Preirus (Hitachi Medical Corporation). We used modified Tsukuba sonoelastography classification for evaluation of the EVSE data. US results were compared with surgical and hystomorphological data. Results: The "blue eye" sign was characteristic for ectopic pregnancy in $100 \%$ o cases. The "reverse cystic type" showed by EVSE in 100\% of cases with haemorrhagic continence. EVSE increased the diagnostic confidence of US in the cases of ectopic pregnancy in $38 \%$ of cases, ovarian apoplexy - in 7\%, in $9 \%$ with missed abortion, in $21 \%$ with tuboovarian pathology, in $13 \%$ with fibroid and ovarian torsion, in $20 \%$ with pyosalpinx, in $18 \%$ with hydrosalpinx, and in $21 \%$ with haematometra. The overall sensitivity of US in the diagnosis of emergency gynaecological pathology increased from $77 \%$ up to $95 \%$ with EVSE.

Conclusion: EVSE increased the diagnostic confidence of US in emergency gynaecological pathology, especially in the cases with ectopic pregnancy and haemorrhage.

\begin{tabular}{ll}
\hline 14:00 - 15:30 Room F2 \\
\hline
\end{tabular}

\section{Breast}

\section{SS 102}

\section{Ultrasonography: new developments}

Moderators:

G.I. Kirova; Sofia/BG

F. Pediconi; Rome/IT

\section{B-071 14:00}

Preoperative ultrasound-guided biopsy of sentinel lymph nodes using microbubbles and contrast-enhanced ultrasonography in patients with breast cancer

A.R. Sever ${ }^{1}$, P. Mills ${ }^{1}$, J. Weeks ${ }^{1}$, K. Cox ${ }^{1}$, D. Fish' ${ }^{1}$, H. Devalia', S. Jones ${ }^{1}$, P. Jones ${ }^{1}$, W. Mali ${ }^{2} ;{ }^{1}$ Maidstone/UK, ${ }^{2}$ Utrecht/NL (ali.sever@nhs.net)

Purpose: To assess the feasibility of US-guided needle biopsy of the sentinel lymph node (SLN) identified following intradermal injection of microbubbles.

Methods and Materials: In this prospective study, 99 patients with proven invasive breast cancer, with no clinically or sonographically abnormal axillary lymph nodes, received an intradermal microbubble injection at the areola margin. When an enhancing axillary lymph node was visualized, percutaneous US-guided fine needle aspiration (FNA) or 16-G core biopsy was performed. Depending on the biopsy results patients had conventional SLN biopsy or axillary lymph node dissection (ALND).

Results: SLN was successfully identified in 97 of the 99 cases (98\%). Eighteen of these 97 cases (19\%) were lymph node positive. Eleven of the $18(61 \%)$ patients had a positive US-guided biopsy and therefore were treated with an immediate ALND. The remaining 86 patients had conventional SLN biopsy with blue dye and isotope injection. In seven cases a positive lymph node was identified, and patients underwent a completion ALND. Three of the 97 biopsies were reported as inadequate. In 96\% (90/94) of patients undergoing conventional SLN biopsy, the lymph node showed histological evidence of a previous needle biopsy, including the seven false negative cases. Of these seven cases there were three micrometastasis and one isolated tumor cells (ITC).

Conclusion: SLNs can be identified and biopsied using preoperative contrastenhanced US, and this reduces the need for a second operation in more than $50 \%$ of the cases. However if the needle biopsy is negative, conventional operative SLN biopsy is still indicated.

\section{B-072 14:09}

Hyperechoic nodules of the breast: are they always benign?

A. Linda, M. Lorenzon, V. Londero, A. Dal Col, C. Zuiani, M. Bazzocchi; Udine/IT (annalinda33@gmail.com)

Purpose: To evaluate the frequency of hyperechoic breast cancers in a large ultrasound (US)-guided core-needle-biopsy (CNB) series and to identify sonographic features able to predict the presence of malignancy in case of hyperechoic lesions.
Methods and Materials: The records of 4511 consecutive US-guided breast CNB (2347 benign, 1849 malignant and 315 high-risk) performed between 2000 and 2009 were retrospectively reviewed. Twenty-five (5.5\%) lesions were classified as hyperechoic. For each lesion, images were reviewed by two experienced breas radiologists, and sonographic features were recorded according to the BI-RADS lexicon; in addition, the presence hypoechoic areas within the lesion was noted. The pathologic results were reviewed and the frequency of hyperechoic cancers among all malignancies was calculated. Differences of sonographic features between benign and malignant hyperechoic nodules were assessed using the Fisher's exact test.

Results: Nine (36\%) of 25 hyperechoic lesions proved to be malignant (4 invasive ductal carcinomas not-otherwise-specified,2 invasive lobular carcinoma, 1 mucinous carcinoma, 1 neuroendocrine carcinoma and 1 intraductal papillary carcinoma), corresponding to $0.4 \%(9 / 1849)$ of all malignancies. The remaining 16 lesions were benign. Malignant hyperechoic lesions presented lobular/irregular shape ( $9 / 9$ versus $14 / 16, p=0.520$ ), not parallel orientation ( $6 / 9$ versus $1 / 16, p=0.003$ ), noncircumscribed margins ( $9 / 9$ versus $7 / 16, p=0.008)$, posterior acoustic shadowing (6/9 versus $5 / 16, p=0.115)$, vascularization $(6 / 9$ versus $7 / 16, p=0.410)$ and hypoechoic areas $(7 / 9$ versus $7 / 16, p=0.208)$ more frequently than benign hyperechoic lesions, although a significant difference was found only for lesion orientation and margins Conclusion: Hyperechoic malignancies do occur. Sonographic features commonly used to identify a breast malignancy on US can be helpful to differentiate malignant from benign hyperechoic nodules.

\section{B-073 14:18}

Preliminary results of a prototype automated breast ultrasound system combining transmission and reflection 3D imaging

U. Saueressig', V. Ivanovas ${ }^{1}$, M. Windfuhr-Blum ${ }^{1}$, P. Pelegretti ${ }^{2}$, E.C. Kotter ${ }^{1}$, M. Langer'; ' $F$ Freiburg/DE, ${ }^{2}$ Genoa/lT (ulrich.saueressig@uniklinik-freiburg.de)

Purpose: Evaluation of the viability of an automated breast ultrasound system in breast imaging. The results of the automated warm bath ultrasonography (WBU), combining transmission and reflection images, were compared with findings from routine mammography and handheld ultrasound.

Methods and Materials: 60 patients, undergoing routine examination of the breast with mammography and handheld ultrasound, were examined with the WBU prototype (Techniscan Medical Systems, Salt Lake City, Utah, USA). In cases of suspected malignancy, vacuum core biopsy was conducted. After routine examinations, 12 patients were diagnosed with histologically confirmed breast cancer. 13 patients showed cysts, 12 fibroadenomas and 23 cases no significant findings. The WBU system acquires a 3D set of transmission and reflection images simultaneously. They contain information on ultrasound reflection, speed and attenuation of sound. Results: 10 out of 12 carcinomas could be detected in the WBU examination. In 2 cases, carcinoma close to chest wall lay outside the field of view. Fibroadenomas and cysts were depicted reliably. Lesion characteristics differ markedly from handheld ultrasound. Lesion characterisation may be improved by speed of sound and attenuation imaging.

Conclusion: The WBU technique offers a new way of examing the breast, combining features of reflection with transmission imaging. This may lead to improved lesion characterisation. As an automated examination, objectivity and reproducibility are key features. The difference of image characteristics compared with conventional ultrasound implies a learning curve.

\section{B-074 14:27}

First experience of an automated breast volume scanner for the identification and diagnosis of breast cancer

D.-A. Clevert, N. Wöhrle, K. Hellerhof, M.F. Reiser

Munich/DE (Dirk.Clevert@med.uni-muenchen.de)

Purpose: To evaluate the performance of an automated breast volume scanne (ABVS) versus conventional hand-held ultrasonography (HH-US) by comparing lesion visibility.

Methods and Materials: Scanning breast ultrasound was performed on 57 breasts in 29 patients in addition to mammography or MRI. Using automated scanning parameters, three to five views were taken of each breast by trained radiologists, using the Siemens ACUSON S2000 ${ }^{\mathrm{TM}}$ ABVS. Imaging of the lesions using the ABVS was performed with a 14L5BV transducer and compared with visibility of these same lesions using $\mathrm{HH}$-US with a $13 \mathrm{MHz}$ transducer. All lesions were classified according to BI-RADS assessment.

Results: A total of 29 patients were succesfully examined. Median patient age was 54 years (range 13-80). Median ABVS scanning time was for each breast 8.1 minutes (range 3-15), interpretation time was 7.3 minutes (range 3-18). In 17 patients HH-US depicted 25 lesions which were confirmed by the ABVS. There 
were 7 carcinomas and 18 benign lesions (4 fibroadenomas, 1 peripheral papillomas, 8 cystic lesions, 5 post surgical scars). No benign lesion was classified as BI-RADS 5 with ABVS or HH-US. All breast cancers were found with the ABVS and correctly classified as BI-RADS 4 or 5 . There was good agreement regarding BI-RADS classification of HH-US and ABVS. The size of all lesions ranged from 5 to $35 \mathrm{~mm}$ (median $17 \mathrm{~mm}$ ).

Conclusion: These preliminary results suggest that images made with the ABVS allows detection of solid and cystic lesions and their BI-RADS classification with a high reliability in comparison to $\mathrm{HH}-U S$.

\section{B-075 14:36}

Automated breast volume scanning: advantages, drawbacks and preliminary results

M.D.F. de Jong, I.J.M. Dubelaar, T.A. Fassaert, M.J.C.M. Rutten;

ss Hertogenbosch/NL (mdjfdejong@hotmail.com)

Purpose: Automated Breast Volume Scanning (ABVS) is a promising new technique for the evaluation of breast tissue. With ABVS 3D-images are available for review and post-processing. We describe the advantages, drawbacks and applications of ABVS and illustrate our primary clinical results in 3D breast ultrasound (US). Methods and Materials: Forty consecutive patients were scanned with a 3D-US scanner (Acuson S2000, Siemens, Erlangen) using a 5-14 MHz broadband transducer. Each breast was evaluated with 3 to 5 scans, depending on its cup size. Examination took 10 to 15 minutes per patient. ABVS findings were correlated with mammography, handheld 2D US and magnetic resonance imaging (MRI) findings. All patients filled up a questionnaire about their experience, including a VAS score. Results: In all patients scanning was technically successful, breast tissue was visible from skin to thoracic wall. In all patients with cysts and solid tumours at MRI, ABVS were able to depict them. All patients stated ABVS-scanning equally or more bearable in contrast to MR-scanning and mammography. Experiences compared with handheld 2D-US were similar. In most patients axillary lymfnode evaluation was not possible.

Conclusion: Due to the imaging reconstruction possibilities and high-resolution ABVS can be an additional technique in evaluating breast tissue. Almost all of the breast tissue is scanned and available for postprocessing and evaluation in PACS or a workstation. Patients experience less discomfort in comparison with MRI and mammography. Axillary lymfnodes should be evaluated by handheld US.

\section{B-077 14:45}

Shear-wave sonoelastography features of benign and malignant breast lesions

G. Ivanac, A. Hrkac-Pustahija, R. Huzjan-Korunic, I. Cikara, B. Brkljacic;

Zagreb/HR (gordana.augustan@zg.htnet.hr)

Purpose: Shear-wave sonoelastography (SWE) generates, captures and quantifies velocity of the shear wave and enables quantitative analysis of breast lesion elasticity by producing real-time quantitative colour-coded map of local tissue elasticity. The aim of this study was to determine and compare qualitative and quantitative features of benign and malignant breast lesions using SWE.

Methods and Materials: Aixplorer scanner (Supersonic Imagine) with high-frequency transducer was used. B-mode and SWE of breast lesions was performed in 174 women. Colour scale indicated tissue elasticity in kilopascals $(\mathrm{kPa})$, soft lesions being blue and hard lesions red. Maximum, minimum and mean kPA E-values were recorded in each lesion and E-ratios calculated between the lesion and normal parenchyma. In malignant lesions SWE area of bright colour was measured and compared with tumour area measured by B-mode. 82 lesions were malignant, as confirmed by core-biopsy, and 106 benign.

Results: Mean E-values for malignant lesions were 181.0 kPa (range 61.1-300), and for benign lesions $40.9 \mathrm{kPa}$ (range 10.7-157 kPa) $(p<0.01)$. Mean E-ratios between lesion and normal parenchyma was 8.39 (range 2.0-78.5) for malignant lesions, and 1.25 (range $0.6-2.8)$ for benign lesions $(p<0.01)$. Mean area of malignant lesions measured by SWE was $3.81 \mathrm{~cm} 2$ (range 1.48-8.59 $\mathrm{cm} 2$ ), and by B-mode $1.6 \mathrm{~cm} 2$ (range $0.35-4.4 \mathrm{~cm} 2)(p<0.01)$

Conclusion: Malignant lesions have significantly higher elasticity and E-ratios to normal parenchyma than benign lesions. Area of malignant lesions measured by SWE is significantly larger compared with B-mode measurement. SWE could be complimentary to B-mode in evaluation of sonographic features of breast lesions.

\author{
B-078 14:54 \\ Significant differentiation of focal breast lesions: raw data-based \\ calculation of strain ratio \\ M. Grigoryev, A. Thomas, T. Slowinski, S. Filimonow, T. Fischer; \\ Berlin/DE (maria.grigoryev@charite.de)
}

Purpose: Initial data suggest that elastography can improve the specificity of ultrasound in differentiating benign and malignant breast lesions. We compared elastography, B-mode ultrasound and mammography to determine whether raw data calculation of strain ratios (SRs) can further improve the differentiation of focal breast lesions.

Methods and Materials: 201 women with histologically proven focal breast lesions ( 85 benign, 116 malignant) were included at two German breast centers. Patients underwent standardised ultrasound procedure using high-end ultrasound system (Aplio XG, Toshiba, Japan) with a 9-MHz broadband linear transducer. Two experienced readers analysed B-mode scans and mammograms using the BI-RADS criteria; elastograms were analysed using the Tsukuba score. SRs were calculated from a tumour-adjusted ROI; comparable ROI was placed in the lateral fatty tissue. Sensitivity, specificity, and cut-off values were calculated for SRs (ROC analysis). Results: Median age was 53 years [range 19-80]. Lesion diameter was $17.4 \pm 9.8 \mathrm{~mm}$ (mean \pm SD). Sensitivity and specificity were $85 \% / 60 \%$ for B-mode scanning, $85 \% / 68 \%$ for elastography, $78 \% / 62 \%$ for mammography, and $95 \% / 74 \%$ for SRs. SR cut-off value of 2.27 (AUC 0.907) allowed significant differentiation $(p<0.001)$ of malignant and benign lesions. The quantitative $S R$ calculation was superior to subjective interpretation of B-mode scans and sonoelastograms with a positive predictive value of $83 \%$ versus $78 \%$ and $74 \%$, respectively, and was equal to that of mammograms.

Conclusion: Calculation of strain ratios contributes to the standardisation of sonoelastography with high sensitivity and allows significant differentiation of benign and malignant breast lesions with a higher specificity compared to B-mode, subjective evaluation of elastography and mammography.

\section{B-079 15:03}

Qualitative and semi-quantitative evaluations of solid breast lesions by sonoelastography

H. Yerli, T. Yilmaz, T. Kaskati, H. Gulay; Izmir/TR (hasanyerli@yahoo.com)

Purpose: To determine whether the use of qualitative elasticity scoring method together with semi-quantitative strain index method by sonoelastography (SE) is useful to differentiate between benign and malignant breast masses.

Methods and Materials: Some 78 lesions in 71 consecutive patients with solid breast masses ( 62 benign, 16 malignant) were prospectively included in this study. For each lesion, B-mode US and SE images were obtained. After elasticity scores had been determined with 5-point scoring method, strain indexes of the lesions were calculated using the same-level and normal-appearing breast region as an internal reference by means of the method of strain ratio measurement. The findings were compared with histopathology. Considering the receiver operating curves, the diagnostic performances for the elasticity scoring and the strain index methods were determined.

Results: The mean scores on SE were $2.69 \pm 0.59$ for benign lesions and $3.75 \pm 0.68$ for malignant lesions. The mean stiffness index values were $2.03 \pm 2.67$ for benign lesions and $5.97 \pm 4.45$ for malignant lesions. The area under the curve value was 0.864 for 5 -point scoring method and 0.840 for strain index method $(P$ $=706$ ). Sensitivity and specificity for 5 -point scoring method were $80 \%$ and $95 \%$, respectively; $87.5 \%$ and $72.6 \%$ for B-mode US; and $80 \%$ and $93 \%$ for strain index method when a cutoff point of 3.52 was used. A semi-quantitative evaluation using the strain index method did not contribute to qualitative evaluation by scoring. Conclusion: After 5-point scoring by SE, additional measurement of the strain index is not mandatory to differentiate between benign and malignant breast masses.

\section{B-080 15:12}

Ultrasound elastography as an adjunct to conventional ultrasound, comparing sensitivity in the pre operative assessment of axillary lymph nodes

K.A. Taylor, S. O'Keeffe, M.G. Wallis, R. Sinnatamby, G.M. Treece;

Cambridge/UK (kathryn.taylor@addenbrookes.nhs.uk)

Purpose: The imaging modality of choice for preoperative assessment of axillary metastase in suspected breast cancer is currently conventional ultrasound $(\mathrm{CU}) \pm$ needle biopsy. Despite preventing unnecessary surgical sentinel lymph node biopsy, it has recognised limitations notably a significant false negative rate. Ultrasound elastography (UE) is introduced as an adjunct to CU to compare sensitivity in the axilla for the first time. 
Methods and Materials: A pilot cohort of 50 women with sonographically suspicious (BI-RADS 4 or 5) solid breast lesions underwent routine $\mathrm{CU}$ of ipsilateral axillary lymph nodes (ALNs), followed by quasi-static UE. Elastograms created using non-commercial in house software were displayed in greyscale (white/ soft, black/ stiff) alongside simultaneous CU images. Elastograms were scored 1-4 (normal-malignant) in terms of apparent stiffness and compared with visual $\mathrm{CU}$ findings and reference standard of surgical histology.

Results: 21 (42\%) patients had metastatic ALNs and 29 (58\%) patients had normal ALNs at final surgical histology. 23 were morphologically abnormal on CU, of which 19 were suspicious/malignant on UE (score 3/4) and 16 malignant at surgical histology. Adding UE to CU reduces the false positive rate from $44 \%$ to $19 \% .27$ were morphologically normal on CU of which 5 were malignant at surgical histology. Importantly all 5 were suspicious/malignant (score 3/4) on UE. Adding UE to CU raises sensitivity by $24 \%$.

Conclusion: UE shows promise at differentiating benign from malignant ALNs and as an adjunct to CU potentially increases sensitivity in identifying abnormal ALNs for biopsy, improving preoperative diagnosis.

\begin{tabular}{ll}
\hline $14: 00-15: 30$ & Room G/H \\
\hline
\end{tabular}

\section{Head and Neck}

\section{SS 108}

\section{Ear, eye and paranasal sinuses}

Moderators:

N. Dfouni; Geneva/CH

K. Zelenak; Martin/SK

\section{B-081 14:00}

Vitreoretinal disorders: preliminary evaluation at CEUS

M. Bertolotto ${ }^{1}$, G. Serafini ${ }^{2}$, L.M. Sconfienza ${ }^{2}$, M. Cavallaro ${ }^{1}$, F. Lacelli²,

M.A. Cova ${ }^{1} ;{ }^{1}$ Trieste/IT, ${ }^{2}$ Pietra Ligure/IT (bertolot@univ.trieste.it)

Purpose: To investigate whether CEUS may be helpful in adjunct to grey-scale and colour Doppler US for evaluation of vitreoretinal disorders.

Methods and Materials: US was performed on 36 consecutive patients (19 men, 17 women) with vitreous haemorrhage and suspicious retinal detachment (RD), choroidal detachment (CD), and posterior vitreous detachment (VD) mimicking RD. After preliminary grey-scale and colour Doppler interrogation, CEUS was performed using low acoustic power, contrast specific modes. A2.4-4.8 mL bolus of SonoVue was injected, followed by saline flush. All examinations were digitally recorded for retrospective analysis. Confirmation of US and CEUS findings was obtained at surgery $(n=12)$ or with clinical evaluation during the follow-up.

Results: There were $24 \mathrm{VD}, 19 \mathrm{RD}, 7 \mathrm{CD}$. In 14 patients different vitreoretinal disorders were associated. VD were not vascularised at CEUS, RD and CD were vascularised in all cases. In RD the vascularised choroid was identified outside the detached retina, while in CD no external vascularised structures were recognised within the globe. In 22 patients differentiation between VD, RD, and CD improved using CEUS. Vascularisation was evident at CEUS in 5 RD/CD not vascularised at colour Doppler interrogation. In 3 cases echogenic VD adherent to the optic disk was correctly characterised at CEUS. In 3 patient RD within organised haemorrhage was identified at CEUS only. In 10 cases, the extent of RD and CD was better assessed at CEUS.

Conclusion: CEUS may be used in equivocal cases to improve characterisation of vitreoretinal disorders.

\section{B-082 14:09}

\section{Orbital Doppler in optic neuritis}

M. Karami Nogourani; Esfahan/IR (m_karami@med.mui.ac.ir)

Purpose: To evaluate orbital blood flow velocities and optic nerve diameter with Doppler sonography in patients with acute unilateral optic neuritis (ON).

Methods and Materials: Orbital Doppler and grey-scale sonography was performed in 46 eyes of 23 patients aged 19-47 years with acute unilateral ON. ON was diagnosed by an ophthalmologist on the basis of clinical presentation, presence of decreased visual acuity and assessment of visual evoked potentials. The peak systolic (PSV) and end-diastolic (EDV) blood flow velocities and resistance (RI) and pulsatile $(\mathrm{PI})$ indices of the ophthalmic $(\mathrm{OA})$, central retinal (CRA) and posterior ciliary (PCAs) arteries and optic nerve diameter were measured in both eyes. We compared results from affected and unaffected eyes using the paired t-test. The area under the receiver-operating characteristic $(\mathrm{ROC})$ curves was used to assess diagnosis of $\mathrm{ON}$ on the basis of measured blood flow parameters of the OA, CRA and PCAs and optic nerve diameter.
Results: Optic nerve diameter in eyes with $\mathrm{ON}$ was significantly higher than that of the control eyes $(P<0.001)$. The mean $(S D)$ optic nerve diameter was $4.1(0.8)$ $\mathrm{mm}$ in affected eyes and $3.0(0.4) \mathrm{mm}$ in unaffected eyes (P0.05). The mean Rl in the PCAs $(\mathrm{P}<0.05)$ was slightly lower in the eyes with $\mathrm{ON}$ than in the contralatera eyes. The area under the ROC curves was 0.928 for optic nerve diameter. Conclusion: Optic nerve diameter was related to ON; RI of PCAs was slightly related to $\mathrm{ON}$, but other orbital blood flow parameters were not.

\section{B-084 14:18}

Role of ocular Doppler ultrasonography in primary open-angle glaucoma A. Alconchel, L. Pablo, J.J. Alconchel, P. Seral, J. Remírez; Zaragoza/ES (aralodia@hotmail.com)

Purpose: Primary open-angle glaucoma is a prevalent chronic disease traditionally related with increased intraocular pressure. Studies have proved that an altered perfusion of the optic nerve head is also an important patogenic factor. Ocular colour and pulsed Doppler US permits to evaluate the optic nerve microcirculation and detect haemodynamic differences between patients having progressive versus stable glaucoma.

Methods and Materials: 59 patients diagnosed of open-angle glaucoma who met the inclusion/exclusion criteria were divided into two groups according to the presence of stable versus progressive disease assessed by HRT scans. Colour and pulsed Doppler imaging of ophtalmic artery, central retinal artery and vein, and posterior ciliar arteries measurements (peak systolic velocity, end-diastolic velocity, mean velocity, and resistivity and pulsativity indices). One eye randomly chosen of each patient was taken into account in the statystical analysis.

Results: During an average follow-up period of 36 months, 22 of the 59 patients $(37.3 \%)$ showed progression of glaucomatose retinal damage, and 37 patients $(62.7 \%)$ were stable. CDI measurements were compared using the Mann-Whitney $U$ test, and showed statistically significant differences $(P$-value $>0.05)$ between both groups, with decreased $\mathrm{RI}$ and $\mathrm{PI}$ in the OA, and PSV and MV in CRA in patients with glaucoma progession. No differences in flow velocities were found for the rest of the retrobulbar vasculature.

Conclusion: Changes of ocular perfusion in patients with glaucoma have been associated with progression of the desease. Colour Doppler US imaging is a promising method for detecting haemodynamic defects that might imply changes in its treatement.

\section{B-085 14:27}

Detection of intraorbital foreign bodies using MDCT

C. Friedrich, P. Hoffstetter, A.G. Schreyer;

Regensburg/DE (chris.friedrich@me.com)

Purpose: Judging the possibilities of detection of orbital foreign bodies in multidetector CT (MDCT) with a focus on glass slivers.

Methods and Materials: Systematic measuring of Hounsfield units (HU) of 20 different materials, containing 16 different types of glass. The measurements were performed using an orbita phantom scanned in a 16 slice MDCT. Using the resulting density values, the smallest detectable volume was calculated. We then produced slivers of 5 different glass types in the sub-millimetre range and calculated their volume. Those microslivers underwent another CT scan using the same protocol as mentioned above to discern the limit of detection for microslivers made of different materials.

Results: Glass has comparatively high density values of at least $2000 \mathrm{HU}$. The density of glasses with strong refraction is significantly higher and reaches up to $12400 \mathrm{HU}$. We calculated a minimum detectable volume of $0.07 \mathrm{~mm}^{3}$ for glass with a density of $2000 \mathrm{HU}$. Only glass slivers with a density higher than $8300 \mathrm{HU}$ were experimentally detectable in the sub-millimetre range up to a volume as small as $0.01 \mathrm{~mm}^{3}$.

Conclusion: Due to its high density of at least $2000 \mathrm{HU}$, glass is usually easily recognisable as an orbital foreign body. The detection threshold depends on the object's density and size and can be as low as $0.01 \mathrm{~mm}^{3}$ for glass with high density. Detection of glass as an orbital foreign body seems to be secure for slivers measuring at least $0.2 \mathrm{~mm}^{3}$ for all types of glass.

\section{B-086 14:36}

Diffusion MR in detection of postoperative recurrent cholesteatoma

C. Fernández Martín, R. Martin Boizas, J.M. Sánchez Reyes,

B. Lannegrand Menéndez, J. Esteban Sanchez; Madrid/ES

Purpose: To accomplish a diffusion MRI prospective study of the utility for detecting recurrent cholesteatoma after radical surgery in patients between February 2009/September 2010. 
Methods and Materials: 40 patients were included in our study after a radical mastoidectomy intervention ussing the following protocol for the MRI exam: sagittal 3DT2, axial T1WI fast SE, diffusion (b0, b300, b600 seg/mm), postgadolinium axial T1WI fast SE, delayed postgadolinium coronal GET1 WI. Hyperintensity on b600 values was interpreted as recurrent cholesteatoma. TI report was done on two different moments by an experienced neuroradiologist. It was classificed as: CC (clearly cholesteatoma), DC (doubful cholesteatoma), CF (clearly fibrosis), DF (doubtful fibrosis). Results were compared with postsurgery reports.

Results: Recurrent cholesteatoma was properly identified in 8 patients with PPV $100 \%$. 6 of 7 patients were confirmed at surgery as fibrosis with only 1 false negative NPV $80 \%$. Sensitivity and specificity were $89 \%$ and $100 \%$, respectively. Conclusion: Results for specificity and PPV in our sample (100\%) support the utility of IPD for detection of recurrent cholesteatoma. Our study shows the necessity of using 3DT2 sequences together with diffusion MRI to proper anatomical localization. Previous CT is also essential to determinate bony structures and compare with 3DT2 images.

\section{B-087 14:45}

Detection of residual and recurrent cholesteatoma with non-epi diffusion weighted imaging: the Dutch experience

M.H.G. Dremmen, P.A.M. Hofman, J.R. Hof, R.J. Stokroos, A.A. Postma;

Maastricht/NL (marjolein.dremmen@gmail.com)

Purpose: The accuracy of MRI for detection of residual or recurrent cholesteatoma in our hospital was retrospectively investigated.

Methods and Materials: Between 2005 and 2010, 56 non-EPI DW MR imaging studies were performed in 51 patients previously operated for cholesteatoma. T1-, T2- and postgadolinium T1-weighted images were followed by non-EPI DWI in the transverse and coronal plane. The imaging findings were correlated with clinical and intraoperative findings. Twenty-six cases underwent second-look surgery, 8 cases with clinically confirmed suspicion of residual cholesteatoma are under close clinical follow-up. Twenty-two cases without evidence of recurrent cholesteatoma are under follow-up (follow-up ranging from 8 to 38 months)

Results: Non-EPI DW MRI sequences showed restricted diffusion in 36 cases. Second-look surgery confirmed cholesteatoma in 24/26 cases; in 1/24 cases an empyema was diagnosed and in one case no cholesteatoma was found at surgery. In 2/36 cases restricted diffusion was accompanied by hyperintense signal on the T1-weighted images which was consistent with transplanted fat; without conventional imaging restricted diffusion in this two cases would be misinterpreted as cholesteatoma. The positive predictive value for detection of cholesteatoma with the full imaging protocol was $94 \%$ (34/36); whereas with non-EPI DWI as the only sequence this would drop to $88 \%(32 / 36)$.

Conclusion: Residual or recurrent cholesteatomas after primary cholesteatoma surgery can be reliable detected by restricted diffusion on non-EPI DW MR imaging. DWI imaging without conventional sequences will increase the number of falsepositive findings in our patient setting, because transplanted fat within the cavity may show restricted diffusion.

\section{B-088 14:54}

Reducing the radiation dose for low-dose CT of the paranasal sinuses using iterative reconstruction: feasibility and image quality

S. Bulla, F. Hassepass, P. Blanke, T. Kraus, M. Langer, G. Pache;

Freiburg im Breisgau/DE (stefan.bulla@uniklinik-freiburg.de)

Purpose: To retrospectively evaluate image quality of dose-reduced CT of the paranasal-sinus using the iterative reconstruction (IR) technique.

Methods and Materials: In this retrospective study 80 patients (mean age: $46.9 \pm 18$ years) underwent low-dose CT of the paranasal-sinus (Siemens Definition, Forchheim, Germany), with either standard settings (A: $120 \mathrm{kV}, 60 \mathrm{mAs}$ ) reconstructed with conventional filtered back projection (FBP) $(n=20)$ or with tube current-time product lowering of $20 \%, 40 \%$ and $60 \%$ (B: $48 \mathrm{mAs}, \mathrm{C}: 36 \mathrm{mAs}$ and D: $24 \mathrm{mAs}$ ) using iterative reconstruction ( $\mathrm{n}=20$ each). Subjective image quality was independently assessed by three blinded observers using a semiquantitative fivepoint grading scale ( $1=$ poor, $5=$ excellent). Effective radiation dose was calculated from the dose-length product. Mann-Whitney-U-test was used for statistical analysis. Results: Mean effective doses were $0.28 \pm 0.03 \mathrm{mSv}(\mathrm{A}), 0.23 \pm 0.02 \mathrm{mSv}(\mathrm{B})$, $0.17 \pm 0.02 \mathrm{mSv}(\mathrm{C})$ and $0.11 \pm 0.01 \mathrm{mSv}(\mathrm{D})$ resulting in a maximum dose reduction of $61 \%$ with IR as compared to the standard low-dose CT. Best image quality was observed at $48 \mathrm{mAs}(4.8 ; \mathrm{p}<0.05)$, whereas standard low-dose CT and maximum dose reduction $(D)$ provided the same subjective image quality $(4.3 ; p$ : n.s). Interobserver agreement was excellent ( $\mathrm{k}$ values 0.77-0.93).

Conclusion: As compared with filtered back projection, application of iterative reconstruction allows for significant dose reduction of up to $61 \%$ for paranasal-sinus low-dose CT without loss in diagnostic image quality.

\author{
B-089 \\ 15:03 \\ Comparison of CT, PET and PET-CT in detection of paranasal sinus \\ tumours \\ S. Shaikh; Hyderabad/IN
}

Purpose: CT has been the main imaging modality to detect primary tumours in paranasal sinus tumours. We compared the sensitivity of FDG-PET, CT and their combination PET-CT to detect primary tumours in Paranasal sinuses.

Methods and Materials: Retrospective data review of 22 patients with clinically suspected paranasal sinus tumours who underwent whole-body contrast-enhanced CT, FDG-PET or both. We reviewed the imaging reports for any findings suspicious for malignancy. We then noted if these findings correlated with the actual location of any primary tumour (confirmed by pathology) and calculated the sensitivity, specificity and accuracy for these two imaging modalities separately and in combination. Results: Of 22 patients (ages 29-68, $F=14, M=8$ ), 10 had extrasinus extension, 4 had bone destruction, 3 had nasal extension, 2 had local disease, 2 had intracranial extension and 1 had CSF rhinorrhea. 20 underwent CT, which correctly identified $9 / 11$ with tumours (sens $=81 \%), 9 / 9(\mathrm{spec}=100 \%)$ without tumours, with accuracy of 18/20 (90\%). 18 underwent FDG-PET, which correctly identified 9/9 with tumours (sens=100\%), 8/9 without tumours (spec=89\%), with accuracy of $17 / 18(94 \%)$. Sensitivity, specificity and accuracy for diagnostic CT, FDG-PET and combined PET-CT were 100\% (12/12), 90\% (9/10), and 95\% (21/22), respectively.

Conclusion: FDG-PET is more sensitive than CT in detection of primary Paranasal sinus tumors, whereas $\mathrm{CT}$ is more specific. Interestingly, the diagnostic performance of PET-CT combined was only slightly greater than that of FDG-PET alone.

\section{B-090 15:12}

Cervicofacial tissue infarction in patients with invasive fungal sinusitis: its prevalence and characteristic MR imaging findings

J. Seo, H.-J. Kim, E. Kim, H. Lee, S. Kim; Seoul/KR (jungmin1130@gmail.com)

Purpose: Although tissue infarction is a well-known histologic feature of invasive fungal sinusitis, it has rarely been emphasised in radiologic literature in the head and neck. The purpose of this study was to investigate the prevalence and the characteristic MRI findings of cervicofacial tissue infarction (CFTI) in invasive fungal sinusitis.

Methods and Materials: We retrospectively reviewed MR images in 33 patients with histologically proved invasive fungal sinusitis. CFTI was defined areas of necrosis. We divided CFTIs into those in the nasal cavity, and those extending to the extranasal areas. Attention was paid on the characteristics of CFTI on contrast-enhanced T1WI (CET1WI). Preservation of intervening fat on precontrast T1WI in cases of extrasinonasal CFTI and the presence of bone change on CT were recorded. Results: CFTI was found in 17 of 33 patients $(52 \%)$. All of 17 patients had sinonasal CFTI, in whom 13 patients also had extranasal CFTI. 9 patients were infested with Mucor, and 6 with Aspergillus. 11 patients were immunocompromised and 6 patients had diabetes. On CET1WI, all lesions were characteristically ill-defined nonenhancing lesions. In cases of extrasinonasal CFTI, there was no respect for the intervening tissue planes, extending to the adjacent soft tissue, orbit, bony structure, or intracranial cavity. In 12 of 13 lesions with extrasinonasal CFTI, intervening fat was preserved on T1WI. 16 lesions with CFTI demonstrated no erosive or destructive bone changes on CT.

Conclusion: On CET1WI, CFTls were demonstrated in more than half of the patients with invasive fungal sinusitis. They may be a pathognomonic sign on MRI caused by this fulminant infection. 


\section{Cardiac}

\section{SS 103}

\section{MR imaging of coronary artery disease}

\section{Moderators:}

M. Gardarsdottir; Reykjavík/IS

A. Stadler; Vienna/AT

\section{B-091 14:00}

An intra-patient comparison of 3 Tesla and 1.5 Tesla in CMR for detection of coronary artery disease

P. Bernhardt, R. Gradinger, T. Walcher, J. Spieß, D. Walcher, W. Rottbauer; UIm/DE (peter.bernhardt@uniklinik-ulm.de)

Purpose: Cardiac magnetic resonance (CMR) imaging including adenosine perfusion and late Gd-DOTA enhancement (LGE) at 1.5-T has been established for non-invasive detection of significant coronary artery disease (CAD). However, little is known about the potential advantages of 3.0-T. Our objective was to compare a compiled clinical routine CMR protocol performed at both 1.5-T and 3.0-T in patients with suspected CAD undergoing coronary $x$-ray angiography.

Methods and Materials: 52 evaluable patients $(62.3 \pm 10.2$ years) with suspected CAD were scanned at both 1.5-T and 3.0-T including functional imaging, adenosine stress and rest perfusion and LGE imaging. CMR images were analysed by two blinded readers in consensus. A significant CAD was diagnosed by quantitative analysis on conventional $x$-ray angiography in vessels with a diameter of $>2 \mathrm{~mm}$ (using two thresholds: $>50 \%$ and $>70 \%$ in coronary stenosis).

Results: Diagnostic accuracy of the combined analysis of perfusion and LGE imaging yielded better values at 1.5-T and 3.0-T than the analysis of perfusion images alone. Specificity and sensitivity at 3.0-T were superior to $1.5-\mathrm{T}$ in detecting coronary stenoses $50 \%$ ( $90 \%$ vs. $75 \%$ and $84.4 \%$ vs. $75 \%$ ) and $>70 \%$ ( $88 \%$ vs. $80 \%$ and $96.3 \%$ vs. $88.9 \%$ ). Diagnostic accuracy was higher at $3.0-\mathrm{T}$ in comparison to 1.5-T for detection of left anterior descending and left circumflex stenosis, but not for right coronary artery stenosis.

Conclusion: CMR imaging with Gd-DOTA at 3.0-T in a routine clinical setting is superior to 1.5-T in detection of significant CAD. 3.0-T might become the preferred CMR field strength for evaluation of CAD in clinical practice.

\section{B-092 14:09}

Quantitative assessment of myocardial strain with DENSE MRI in patients with coronary artery disease

H. Miyagi ${ }^{1}$, K. Kitagawa' ${ }^{1}$ S. Kato ${ }^{1}$, S. Takase ${ }^{1}$, Y. Yoon ${ }^{1}$

A. Sigfridsson ${ }^{2}$, M. Nagata 1 , H. Sakuma $1 ;{ }^{1}$ Tsu/JP, ${ }^{2}$ Linkoping/SE

(h-miyagi@clin.medic.mie-u.ac.jp)

Purpose: The purpose of this study was to determine if strain assessment with DENSE MRI (displacement encoding with stimulated echoes) can provide more accurate detection of altered systolic function as compared with cine MRI in patients with coronary artery disease (CAD).

Methods and Materials: Twenty-one patients with suspected CAD (age $63 \pm 12$ years) were studied with a $1.5 \mathrm{~T}$ MR imager and 32 channel coils. Multi-phase DENSE MRI was acquired on short axis planes to quantify radial (e1) and circumferential (e2) strains. Cine MRI and late gadolinium enhancement (LGE) MRI were also obtained. Percent systolic wall thickening (\%SWT) on cine MRI, e1 and e2 were determined using the AHA 16-segment model.

Results: DENSE MRI was successfully obtained in all subjects in $17 \pm 3 \mathrm{sec}$ per slice location. The mean image analysis time was $24 \pm 2 \mathrm{sec}$. LGE was observed in $79(24 \%)$ of 336 segments, including 56 segments with subendocardial infarction. On visual analysis, reduced systolic contraction in the area of infarction was detected in $58(73 \%)$ of 79 segments by cine MRI, $67(85 \%)$ of 79 segments by e1, and $71(90 \%)$ of 79 segments by e2. The area under ROC curve for predicting infarct segment was $0.75[95 \% \mathrm{Cl} 0.65-0.85]$ by $\%$ SWT, 0.85 [0.76-0.93] by e1 and $0.90[0.83-0.96]$ by e2.

Conclusion: Breath-hold DENSE MRI allows for quantification of myocardial strain in patients with CAD without manual interaction. Circumferential myocardial strain is the most sensitive index for detecting abnormal systolic contractile function in segments with LGE.

\section{B-093 14:18}

Cardiac diffusion-weighted MR imaging in recent, subacute and chronic myocardial infarction: a pilot study

J.-P. Laissy', V. Gaxotte1, I. Klein'1, A. BenDriss', L. Feldman'1, A. Ribet ${ }^{2}$, E. Schouman-Claeys ${ }^{1}$, G. Steg 1 , J.-M. Serfaty ${ }^{1} ;{ }^{1}$ Paris/FR, ${ }^{2}$ Velizy/FR (jean-pierre.laissy@bch.ap-hop-paris.fr)

Purpose: Diffusion-weighted imaging (DWI) has recently been implemented in order to depict infarct-related myocardial oedema and/or necrosis as a marker of acute but not chronic myocardial injury in patients with myocardial infarction (MI). We investigated the clinical utility of DWI to detect recent MI and to differentiate it from subacute and chronic MI, with delayed enhancement MR sequences (DE) as reference.

Methods and Materials: Seventy-four MI patients were studied in 3 groups. Group A included 34 recent (i.e. $<8$ days) MI patients; group B, 22 patients with subacute (i.e. 9-90 days) Ml; group C, 18 patients with chronic ( $>90$ days) Ml; a fourth group (group D) included 24 control patients with no suspicion of $\mathrm{MI}$ and normal DE imaging. DWI (b, 250 to $500 \mathrm{sec} / \mathrm{m}^{2}$ ) and DE images were examined visually by 2 blinded observers for the presence or absence of hyperintense areas in corresponding segments.

Results: The overall number of segments affected at DE was 272 (110 in recent, 94 in subacute and 68 in chronic MIs). Qualitative assessment of DWI compared to DE images per-patient yielded a sensitivity of $97 \%$ and a specificity of $61 \%$ to differentiate recent from chronic lesions, and a sensitivity of $97 \%$ and a specificity of $14 \%$ to diagnose recent from subacute MI. The relative apparent diffusion coefficient (ADC) or ADC ratio was significantly different between groups.

Conclusion: DWI is a sensitive technique to diagnose recent MI. An imaging approach combining DWI and DE MR sequences accurately differentiates recent from chronic Mls.

\section{B-094 14:27}

Edema assessment in acute myocardial infarction: low b-value DWI versus triple IR FSE

V. Di Molfetta, L. Natale, A. Meduri, R. Marano, I. Porto, L. Bonomo; Rome/IT (ivdimolfetta@gmail.com)

Purpose: Oedema assessment is crucial in the diagnosis and management of infarcted patients. Aim of the study was the evaluation of the feasibility of diffusion weighted imaging in oedema recognition.

Methods and Materials: We studied 15 pts with acute myocardial infarction (group 1), 6 pts with chronic myocardial infarction (group 2) and 6 pts referred for arrhythmias (group 3). A conventional cardiac protocol based on T2 triple IR-FSE, cine-SSFP, rest-FGRET and IR-FGRE sequences was employed. Furthermore, an ECG-gated, breath held DWI sequence with $b=100$ was acquired in short axis. Results: First, presence or absence of oedema was evaluated. Second, SNR was measured in acute infarcted area, in scar area and in remote regions. Pts in groups 2 and 3 showed no areas of oedema both on T2 triple IR-FSE and DWI sequences. In all pts of group 1, T2 triple IR-FSE showed areas of increased SI concordant with delayed enhancement (DE) areas, while DWI was negative in 1 pts. Furtherly, DWI showed increased $\mathrm{SI}$ areas in lateral wall in 2 pts, one negative and one positive at DE, the last negative on T2 triple IR-FSE.

CNR measurements in acute infarcted areas was significantly lower in T2 triple IR-FSE, compared to $b=100 \mathrm{DWI}$ (mean 24.1 vs mean 67.8 ).

Conclusion: Low b value DWI is feasible for oedema assessment and represents an alternative method to T2 triple IR-FSE in non-cohoperative pts due to shorter apnea; furthermore, CNR is higher. However, false positive can occur in lateral wall due to susceptibility.

\section{B-095 14:36}

T2* measurement in acute myocardial infarction: insight haemorrhage and microvascular infarction

L. Natale ${ }^{1}$, A. Meduri ${ }^{1}$, A. Bernardini ${ }^{2}$, C. Napolitano ${ }^{1}$, R. Marano ${ }^{1}$, L. Bonomo ${ }^{1}$;

${ }^{1}$ Rome/IT, ${ }^{2}$ Teramo/IT (Inatale@rm.unicatt.it)

Purpose: Microvascolar obstruction and haemorrhage represent negative prognostic factors in acute myocardial infarction. We preliminarily evaluated T2* measurement to assess their presence.

Methods and Materials: 35 consecutive patients with first acute myocardial infarction treated by primary $\mathrm{PCl}$ were studied employing a conventional cardiac MR protocol (T2 triple IR-FSE, cine-SSFP, rest FGRET and 2D IR-FGRE); further an ECG-gated T2* multiecho FGRE sequence at the level of the infarcted area was acquired. Echo times ranged from $1.8 \mathrm{~ms}$ to $20 \mathrm{~ms}$. T2* was calculated using the Reportcard 4.0 software (GE Medical Systems). 
Results: Using a cut-off value of $20 \mathrm{~ms}$, obtained from another study in normal subjects, T2* values were lower (ranging betwen 12 and $18 \mathrm{~ms}$ ) in 9 patients. In these patients, T2 triple IR-FSE images were postive for haemorrhage in 5, while FGRET and or IR-FGRE were positive for microvascular obstruction in 7. All patients with haemorrhage showed also microvascular obstruction.

Conclusion: $\mathrm{T}^{*}$ multiecho imaging is feasible for $\mathrm{T}^{*}$ measurements in patients with acute myocardial infarction. Furthermore, haemorrhage and severe microvascular obstruction represent the same type of complication as all patients with T2 triple IR FSE positive for haemorrhage showed microvascular obstrution, and $\mathrm{T}^{*}$ imaging was positive in the other patients with microvascular obstruction. The discrepancy is due to the superior sensitivity of $\mathrm{T}^{*}$ compared with $\mathrm{T} 2$ imaging.

\section{B-096 14:45}

Magnetisation transfer steady state free precession imaging of myocardial injury

L.M. Winter, M. Zellweger, O. Weber, O. Bieri, J. Bremerich; Basle/CH (winterl@uhbs.ch)

Purpose: To identify and characterise myocardial injury with magnetisation transfer (MT) balanced steady state free precession (SSFP) magnetic resonance (MR) imaging.

Methods and Materials: Twenty four patients with suspected myocardial injury such as infarct $(n=13)$, myocarditis $(n=6)$, or other $(n=5)$ were subjected to cardiac MR (1.5 Tesla with phased array body coil) comprising precontrast SSFP, magnetisation transfer SSFP (MT-SSFP) and delayed contrast enhanced $(0.1 \mathrm{mmol} /$ $\mathrm{kg} \mathrm{Gd}$-chelate) inversion recovery fast low angle shot (LE) images. Signal intensities (SI) were measured in blood, skeletal muscle, normal and injured myocardium. MT ratio was calculated as $\left(\mathrm{SI}_{\mathrm{SSFP}}\right.$ - SIMT-SSFP)/SISSFP \pm standard error of the mean. Results: MT ratios in blood, skeletal muscle, normal and injured myocardium were $0.03 \pm 0.01,0.3 \pm 0.01,0.31 \pm 0.03,0.21 \pm 0.06$, respectively. Moreover, injury that was visible on LE images was identified on MT images in 13 patients with a trend to lower MT ratios in subacute injury and higher MT ratios in chronic injury. Conclusion: Different MT ratios can be measured in blood, normal and injured myocardium with MT magnetic resonance imaging. Moreover, MT imaging may enable further characterisation of myocardial injury with respect to age and nature of the lesion.

\section{B-097 14:54}

Myocardial $\mathrm{T}_{1}$-mapping in chronic myocardial infarction: unenhanced and contrast-enhanced MR imaging

K.U. Bauner ${ }^{1}$, A. Biffar ${ }^{1}$, D. Theisen ${ }^{1}$, A. Greiser ${ }^{2}$, M.F. Reiser ${ }^{1}$, B. Wintersperger ${ }^{1}$, ${ }^{1}$ Munich/DE, ${ }^{2}$ Erlangen/DE (Kerstin.Bauner@med.uni-muenchen.de)

Purpose: To differentiate normal from infarcted myocardium by means of $T_{1}$-maps in unenhanced and contrast-enhanced scans.

Methods and Materials: 18 patients with myocardial infarction were examined at 1.5 T (Magnetom Avanto, Siemens Healthcare). A modified Look-Locker-InversionRecovery (MOLLI) sequence (TR/TE $200.7 / 1.03 \mathrm{msec}$; TI 100-4000 msec; flip $\left.35^{\circ}\right)$ was performed pre- and $10 \mathrm{~min}$ post-contrast $(0.15 \mathrm{mmol} / \mathrm{kg}$ Gadobutrol, Bayer-Schering-Pharma) at three short axis positions. $\mathrm{T}_{1}$-value signal intensities of myocardial and infracted tissue were measured at 11 time points with two blocks of 3 and a third block 5 consecutive image acquisitions. Within each block $\mathrm{Tl}$ increased by steps of $80 \mathrm{msec}$. $\mathrm{T}_{1}$-maps were created and areas of normal and infarcted myocardial tissue were identified according to the 3-standard-deviation method. The analyses of $T_{1}$-values were performed for normal myocardium (MYO), infarcted myocardium (CMI) and the left ventricular cavity (LVC). In addition $\mathrm{T}_{1}$ ratios of MYO/LVC and CMI/LVC were calculated. Student's t-test was used for statistical analysis.

Results: The comparison of $\mathrm{T}_{1}$-values of $\mathrm{MYO}$ and $\mathrm{CMI}$ revealed significant differences in pre-contrast scans $(1024 \pm 38 \mathrm{vs} .1200 \pm 57 \mathrm{msec} ; \mathrm{p}<0.001)$, as well as CMI T -values in comparison with LVC $(1200 \pm 57$ vs. $1502 \pm 70$ msec; $p<0.001)$ The calculated ratios of MYO/LVC and CMI/LVC were also significantly different $(0.68 \pm 0.04$ vs. $0.79 \pm 0.05 ; p<0.001)$ in pre-contrast scans. In post-contrast evaluations differences of $\mathrm{T}$-values in MYO and CMI were equally high $(365 \pm 46$ vs. $231 \pm 52$ msec; $\mathrm{p}<0.001)$ resulting in significantly different $\mathrm{T}_{1}$-ratios of $\mathrm{MYO} /$ $\operatorname{LVC}(1.5 \pm 0.19)$ and CMI/LVC $(1.0 \pm 0.11 ; p<0.001)$

Conclusion: MR-measurements of $T_{1}$-values with the LVC as reference allow for differentiation of infarcted areas from normal myocardium in enhanced and unenhanced imaging.

\author{
B-098 15:03 \\ Comparison of free-breathing 3D and conventional 2D late gadolinium \\ enhancement cardiac MR for the evaluation of left ventricular infarction

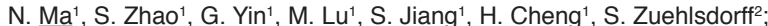 \\ ${ }^{1}$ Beijing/CN, ${ }^{2}$ Chicago, IL/US
}

Purpose: To compare a new free-breathing 3D late gadolinium enhancement (LGE) cardiovascular magnetic resonance (CMR) with conventional 2D LGE in patients with prior myocardial infarction.

Methods and Materials: Patients with prior myocardial infarction were studied using a segmented 2D phase-sensitive inversion-recovery (PSIR) turbo FLASH sequence followed by a navigator-gated 3D PSIR turbo FLASH sequence on a 1.5 T MR scanner for myocardial late enhancement after the administration of gadopentetate dimeglumine. All images were evaluated by two independent cardiovascular radiologists. Overall image quality was graded. The hyperenhanced myocardial scars were compared quantitatively in volume and scored qualitatively on the basis of size (area) and location.

Results: 23 patients were successfully scanned using both approaches and analysed qualitatively and quantitatively. There was a high correlation between the hyperenhanced lesions volumes determined by 2D PSIR and 3D PSIR $\left(R^{2}=\right.$ 0.86 ). The average hyperenhancement lesion score (maximum possible score 68 ) between both readers based on scar area per patient was 31.85 (range 8.548) for 3D PSIR and 26.91 (range 8.5-44.5) for $2 D$ PSIR $(p<0.01)$. The average hyperenhancement lesion score based on scar location (maximum possible score 34) per patient was 19.46 (range 3.5-28.5) for 3D PSIR and 16.89 (range 4.5-26) for 2D PSIR $(p<0.01)$. The mean scar volume over all cases was $31.41 \mathrm{ml}$ (range 8.6-63.8) for 3D PSIR and $23.42 \mathrm{ml}$ (range 7.4-44.6) for 2D PSIR ( $<<0.01$ ). Conclusion: Free-breathing 3D PSIR turbo FLASH provides a promising approach for accurate scar quantification and detection of left ventricular myocardial scar particularly for patients who cannot perform repeated breath-holding.

\section{B-099 15:12}

Role of oedema, microvascular obstruction and infarct size in left ventricular remodelling after acute myocardial infarction: MRI evaluation F. Ferrara ${ }^{1}$, L. Natale ${ }^{1}$, A. Meduri' ${ }^{1}$ A. Bernardini' ${ }^{2}$, A. Lombardo'1, L. Bonomo' ${ }^{1}$ Rome/IT, ${ }^{2}$ Teramo/IT (ferdinandoferrara@live.it)

Purpose: To evaluate the role of oedema, microvascular obstruction and infarct size in left ventricular remodelling after acute myocardial infarction (AMI).

Methods and Materials: 32 consecutive pts with first AMI treated by $\mathrm{PCl}$ underwent MRI to assess end-diastolic and end-systolic volumes, within 7 days and 6 months after infarction. MRI protocol consisted of triple IR-FSE sequence for oedema assessment, cine-SSFP for regional and global systolic function assessment, FGRET for first pass evaluation and IR-FGRE for infarct size assessment. Left ventricular remodelling was defined as $20 \%$ increase of end-systolic volume. A 17-segment model was used for semiquantitative grading system of microvascular obstruction and infarct size, based on transmurality.

Results: 14 pts showed remodelling at follow-up. Mean EDV increased from $106 \pm 30 \mathrm{ml}$ to $153 \pm 36 \mathrm{ml}$, while ESV increased from $60 \pm 17 \mathrm{ml}$ to $91 \pm 23 \mathrm{ml}$. Microvascuar obstructon at first-pass was found in 26 pts (81\%). Oedema, microvascular obstruction and infarct size scores were, respectively, $4.0 \pm 1.6,2.4 \pm 1.1$ and $3.3 \pm 1.6$ in pts with remodelling and $2.9 \pm 2.2(p=N S), 1.4 \pm 0.9(p=0.04)$ and $2.6 \pm 1.7(p=N S)$ in pts without remodelling. Furthermore, pts with remodelling showed a higher number of segments with transmurality $>75 \%$ of perfusion defects (=microvascular obstruction) $(p=0.008)$ and delayed enhancement $(p=0.043)$. Conclusion: Transmural extension $>75 \%$ of microvascular obstruction and infarct size affects significantly left ventricular remodelling. Microvascular obstruction resulted as the strongest predictor.

\section{B-100 $15 \cdot 21$}

3D PSIR gradient echo for imaging myocardial infarction

A.M. Huber, K. Herrmann, M. Settles, M. Betz, A. Beer, E. Rummeny;

Munich/DE (armin.huber@roe.med.tu-muenchen.de)

Purpose: The aim of the study was to assess the diagnostic accuracy in imaging viability of the myocardium with a 3D phase-sensitive gradient echo sequence.

Methods and Materials: 19 patients with myocardial infarction were examined at a 1.5 Tesla MR System (Achieva, Philips) 10 minutes after application of contrast material with a 3D phase-sensitive inversion recovery (PSIR) multislice technique (IR gradient echo, TR/TE/bandwidth: $5.0 \mathrm{msec}, 2.4 \mathrm{msec}$, voxel size $1.25 \times 1.25 \mathrm{x}$ $4.0 \mathrm{~mm}^{3}$ ) that allows to image the entire short axis during three breath-holds, and with a segmented 2D single slice technique (IR gradient echo, TR/TE/bandwidth $12.0 \mathrm{msec}, 4.4 \mathrm{msec}, 140 \mathrm{~Hz} / \mathrm{Px}, 1.8 \times 2.3 \times 8 \mathrm{~mm}^{3}$ ), which requires one breath- 
hold per slice (reference technique). The PSIR technique was used with a standard inversion time of $200 \mathrm{msec}$. The reference sequence was used after individual adaptation of the inversion time. The areas of hyperintense infarctions on selected slices and the entire volumes were compared for both sequence techniques. Results: The 3D PSIR gradient echo sequence provided sufficient image quality for evaluation in all patients without individual adaptation of the inversion time. The assessment of the area of infarction within one slice $(r=0.96, p<0.002)$ and the volume of the entire infarction is possible with excellent correlation of both techniques $(r=0.95, p<0.003)$

Conclusion: The 3D PSIR GE sequence allows stable imaging of myocardial infarction during a shorter acquisition time without individual adaptation of inversion time. Compared to the reference technique the infarct areas and volumes were determined accurately.

Room L/M

\section{Neuro}

\section{SS 111}

\section{Brain tumours: grading, treatment and follow-up}

Moderators:

D. Goldsher; Haifa/lL

A. Levinsson; Malmö/SE

\section{B-101 14:00}

MR perfusion and diffusion-weighted imaging in the follow-up of recurrent glioblastoma treated with dendritic cell immunotherapy: a pilot study S. Van Cauter ${ }^{1}$, M. Vrabec ${ }^{2}$, U. Himmelreich', S. Van Gool ${ }^{1}$, S. Sunaert ${ }^{1}$, S. De Vleeschouwer ${ }^{1}$, D. Suput ${ }^{2}$, P. Demaerel ${ }^{1} ;{ }^{1}$ Leuven/BE, ${ }^{2}$ Ljubljana/SI (sofie.vancauter@uz.kuleuven.be)

Purpose: To determine the value of MR-PWI and MR-DWI to differentiate immunotherapy-induced inflammatory response from recurrent glioblastoma tumour. Both present as contrast-enhancing lesions on conventional MRI.

Methods and Materials: Patients with recurrent glioblastoma, who could obtain a (near)-total resection were treated with dendritic cell immunotherapy according to the HGG-immuno-2003 trial. A retrospective analysis of $32 \mathrm{MRI}$ examinations (mean follow-up: 21 months) in 8 patients, was performed. For statistical analysis, the 32 examinations were divided into three groups: 0 -obtained in patients that remained stable during the follow-up period, 1a-obtained in progressive patients at time points before definite progression, $1 \mathrm{~b}$-obtained in patients at or after progression. Results: Maximum lesional rCBV ratios were highest in group $1 \mathrm{~b}$ (Studenttest: $9.25 \pm 2.68, p<0.001)$ and were higher in group 1a $(4.87 \pm 1.61, p<0.001$, group 1a), compared to group $0(1.22 \pm 0.47)$. Minimum ADCs in the contrastenhancing regions were lower in group $1 \mathrm{a}\left(0.62 \pm 0.06 \times 10^{-3} \mathrm{~mm}^{2} / \mathrm{s}\right)$ than in group 0 , $\left(1.03 \pm 0.43 \times 10^{-3} \mathrm{~mm}^{2} / \mathrm{s}, \mathrm{p}=0.01\right)$ and higher in group $1 \mathrm{~b}(0.76 \pm 0.08)$ compared with $1 \mathrm{a}(\mathrm{p}=0.02)$. Minimum ADCs in the FLAIR-hyperintense region were lower in group 1a $(0.62 \pm 0.06 \mathrm{~s}, \mathrm{p}=0.02)$ compared with group $0(0.76 \pm 0.16)$, but not significanty different in group $1 \mathrm{~b}(0.68 \pm 0.07)$ from groups 0 and $1 \mathrm{a}(\mathrm{p}=0.33, p=0.10)$. The mean ADCs of the FLAIR-hyperintense region and mean ADCs of the contrastenhancing lesion were not significantly different.

Conclusion: Maximum lesional rCBV ratios and minimum ADC values in the contrast-enhancing area are potential radiological markers to differentiate between immunotherapy-induced inflammatory response and recurrent tumour growth in glioblastoma patients treated with immunotherapy.

\section{B-102 14:09}

Application of whole brain VPCT in the preoperative assessment of intraaxial brain tumours

A. $\underline{X y d a}^{1}$, E. Klotz ${ }^{2}$, U. Haberland ${ }^{2}$, C. Bock ${ }^{1}$, G. Erb' ${ }^{3}$, R. Schramm ${ }^{1}$,

M. Knauth', P. Schramm $1 ;{ }^{1}$ Göttingen/DE, ${ }^{2}$ Forchheim/DE,

${ }^{3}$ Konstanz/DE (iroxida@yahoo.com)

Purpose: Evaluation of the role of VPCT in the preoperative differentiation of intra-axial brain tumours.

Methods and Materials: Whole brain VPCT data from 59 consecutive patients with intra-axial tumours were analysed. Two readers independently evaluated VPCT data. Volumes of interest (VOIs) were drawn around the entire tumour on maximum intensity projection (MIP) volumes, and then mapped automatically onto the cerebral blood volume (CBV), flow (CBF) and permeability ( $\left.\mathrm{K}^{\text {trans }}\right)$ maps. As control, a second VOI was placed in the contralateral cortex. The perfusion parameters were quantified and compared to those of the control hemisphere. Their diagnostic power was analysed by ROC curve analysis.
Results: Histopathologic diagnosis revealed 29 high grade gliomas WHO III/IV, 17 low grade WHO II and 6 lymphomas. Four patients had metastases and 3 tumour like lesions. Mean values of $\mathrm{K}^{\text {trans }}, \mathrm{CBV}, \mathrm{CBF}$ in high grade gliomas were significantly higher compared to normal parenchyma and to low grade $(p<0.01)$. High grade gliomas were differentiated from lymphomas based on the significantly increased CBV and CBF values ( $p<0.0001$ and $p=0.0004$, respectively). Lymphomas were differentiated from low grade through the significant elevation of $K^{\text {trans }}(p=0.0029)$. $K^{\text {trans }}$ demonstrated the highest sensitivity $(97 \%)$ and specificity $(100 \%)$ for the comparison between low and high grade as well as between low grade and lymphomas (100\%, 100\%, respectively). CBV showed the highest sensitivity and specificity for the differentiation of high grade from lymphomas ( $93 \%$ and $83 \%$, respectively). Conclusion: Perfusion values correlate well with histopathological grade facilitating the differentiation among lymphomas, low and high grade gliomas.

\section{B-103 14:18}

Glioma grading: the role of combined perfusion MR imaging and singlevoxel MR spectroscopy compared to conventional MR imaging

G. Sparacia, P. Purpura, E. Di Giovanna, C. Gagliardo, M. Midiri; Palermo/IT (sparacia@unipa.it)

Purpose: To assess the contribution of combined perfusion MRI and single-voxe MRS in grading primary gliomas compared with conventional MRI.

Methods and Materials: 32 patients with primary cerebral glioma underwent conventional MRI, dynamic contrast-enhanced T2* w perfusion MRI and single-voxel proton MRS. Gliomas were graded as low or high based on conventional MRI. The rCBV measurements were obtained from regions of maximum perfusion normalised between tumour and healthy tissue. Metabolite ratios ([Cho]/[Cr], [Cho]/[NAA], [NAA] $[\mathrm{Cr}])$ were measured with TE: $34 \mathrm{~ms}$. Tumour grade determined with the three methods was then compared with that from histopathologic grading. Logistic regression and ROC analyses were performed to determine which parameters best increased diagnostic accuracy (sensitivity, specificity, positive and negative predictive values). Results: Statistically significant differences were found for rCBV tumour/normal tissue ratio, and NAA/Cr ratio in tumour and $\mathrm{Cho} / \mathrm{Cr}$ ratio in tumour between lowand high-grade tumours. The best performing single parameter for glioma grading was normalised $\mathrm{rCBV}$ value. Combined $\mathrm{rCBV}$ tumour/normal tissue ratio and NAA/Cr tumour ratio increased overall accuracy in glioma grading. ROC curves demonstrated a rCBV tumour/normal tissue ratio of $>1.16$ and NAA/Cr tumour ratio of $<0.44$ has the higher probability for a neoplasm to be a high-grade glioma. Conclusion: The rCBV measurements and metabolite ratios both individually and in combination can increase the accuracy when compared with conventional MRI alone in determining glioma grade. The best performing parameter was found to be the rCBV measurements. Threshold values can provide a means for guiding treatment and predicting postoperative patient outcome.

\section{B-104 14:27}

The characterisation of anaplastic transformed WHO grade II gliomas by means of advanced magnetic resonance imaging

G. Stasik-Pres, A. Hebda, B. Bobek-Billewicz; Gliwice/PL (gabastasik@poczta.onet.pl)

Purpose: To characterise diffusion-weighted imaging (DWI), diffusion tensor imaging (DTI), perfusion weighted imaging (PWI) and proton magnetic resonance spectroscopy (1HMRS) parameters suggesting anaplastic transformation of WHO gradell gliomas.

Methods and Materials: Analysed group consisted of 47 (age $42 \pm 12$ years) consequtive patients diagnosed in Maria Sklodowska - Curie Memorial Cancer Center and Institute of Oncology, Gliwice Branch, between 2006-2009. 39/47 (mean follow-up 16.4 months,range 2-37) WHO gradell gliomas did not transform to higher grade -nontransformers group. 8/47 (mean follow-up 15 months, range 6-24) WHO gradell gliomas transformed to higher grade (Astrocytoma Anaplasticum WHOIII or Glioblastoma Multiforme WHOIV), which was histopathologically confirmed -transformers group. Selected parameters were analysed: $\mathrm{rCBV}_{\max } \mathrm{rCBF}_{\max }$ normalised to normal-appearing white matter, $\mathrm{ADC}_{\text {min }}, \mathrm{Fa}_{\min },(\mathrm{Cho} / \mathrm{Cr})_{\max },(\mathrm{Cho} / \mathrm{NAA})_{\max }$ ratio. Results: Higher normalised rCBF $2.93 \pm 1.28$ vs. $1.85 \pm 0.62 ; p<0.05)$ and trend towards higher normalised $\mathrm{rCBV}_{\max }(2.57 \pm 0.99$ vs. $1.84 \pm 0.55 ; \mathrm{p}<0.08)$ were in transformers group in comparison with nontransformers group. Transformers group characterised higher (Cho/NAA) ratio (3.69.2.90-4.83 vs. 1.90.0.87-3.48; $p<0.001$ ), whereas $(\mathrm{Cho} / \mathrm{Cr})_{\max }$ ratio (1.69.0.94-3.78 vs. 1.96.1.18-3.38; $\left.\mathrm{p}=\mathrm{NS}\right)$ was not different in comparsion with nontransformers group. No statistically significant difference between analysed groups was stated in terms of: $\mathrm{ADC}_{\min }\left(0.882 \pm 0.268^{*} 10^{-3} \mathrm{~mm}^{2} / \mathrm{s}_{\text {_ }}\right.$ transformers vs. $1.07 \pm 0.293^{*} 10^{-3} \mathrm{~mm}^{2} / \mathrm{s} \_$nontransformers; $\left.\mathrm{p}=\mathrm{NS}\right), \mathrm{Fa}_{\min }(0.122 .0 .093$ 0.145 transformers vs. $0.126 .0 .061-0.332$ nontransformers; $p=N S$ ). 
Conclusion: Among advanced MR neuroimaging methods significant predictive factors which suggest anaplastic transformation of WHO II gliomas might be normalised $\mathrm{rCBF}_{\max ,}, \mathrm{rCBV}_{\max }$ and $(\mathrm{Cho} / \mathrm{NAA})_{\max }$ ratio.

\section{B-105 14:36}

Pseudoprogression in glioblastoma multiforme after radiation and temozolomide: a retrospective population-based study of 74 patients J. Cohnen, H. Bock, P. Schramm, M. Knauth;

Göttingen/DE (joseph.cohnen@med.uni-goettingen.de)

Purpose: Pseudoprogression (PSPD) following radiation and chemotherapy in patients with glioblastoma multiforme is difficult to distinguish from progressive disease. The aim of our study was to identify early signs of PsPD in brain MRI after standard therapy including surgery, radiation and temozolomide.

Methods and Materials: Out of 74 patients with standard therapy, we retrospectively identified 47 with early progression in MRI within the first 6 months. Patients were divided in groups of "real" early progression versus pseudoprogression. We analysed DWI-ADC, also T2*-DSC-PWI changes in both groups.

Results: Preceding studies have shown that median survival in patients with GBM was better in patients with PSPD than with PD. We identified 18 patients with median survival more than 12 months and early signs of progression in MRI within the first 6 months after therapy. In patients with PSPD, no signs of hypervascularisation were found (T2*-DSC-PWI), whereas in patients with early progressive disease restricted diffusion and hypervascularisation were found.

Conclusion: Pseudoprogression is not an uncommon phenomenon after standard therapy of GBM. The amount of $30 \%$ given in the literature can be reaffirmed in our study. To our knowledge, this is the largest study population of patients with MRI signs of early progression. Our study strengthens the role of PWI and DWI as a diagnostic tool to identify PSPD, especially, since no definite examination procedures have been identified so far for reliably detecting PSPD and separating it from progressive disease.

\section{B-106 14:45}

Comparison of MRS, MR perfusion, and PET scan to formulate an appropriate protocol for evaluation of suspected recurrent gliomas G.S. Gujral, R. Deshpande, N.S. Saini; Delhi/IN (girigujral@gmail.com)

Purpose: Comparison of MRS, MR perfusion, and PET scan to formulate an appropriate protocol for evaluation of suspected recurrent gliomas.

Methods and Materials: 30 patients with total resection of their brain tumours were followed up with MR spectroscopy, dynamic susceptibility contrast perfusion scan and FDG PET. Recurrence detected on imaging was confirmed by histopathology, the gold standard.

Results: 14 of the 30 patients showed evidence of recurrence which was confirmed by histopathology in 12 patients. In one patient histopathology could not be done as the lesion was very small and deep seated and in the other a cerebellar lesion, histopathology would have resulted in deterioration of the patient's condition. However, all three imaging modalities showed evidence of recurrence in these two cases. Using a Cho/Cr cut-off of 2.4 , MRS showed a sensitivity of $83 \%$ and a specificity of $100 \%$ to detect recurrence. Using a cut-off value for rCBV of 2.6, perfusion MRI showed a sensitivity of $91.7 \%$ and a specificity of $100 \%$. PET exhibited a sensitivity of $83.3 \%$ and a specificity of $50 \%$.

Conclusion: All modalities detected recurrence but perfusion was the most sensitive as it detects early microvascular changes and increased blood flow within recurrent tumour. Advanced MR techniques such as perfusion are very robust in picking up recurrent tumour and should form an integral part of any protocol to detect recurrent tumours.

\section{B-107 14:54}

MGMT promoter methylation in glioblastoma can be predicted using diffusion weighted imaging

A. Hiwatashi, T. Yoshiura, K. Yamashita, H. Kamano, H. Honda; Fukuoka/JP

Purpose: Methylation of the O6-methylguanine-DNA methyltransferase (MGMT) promoter is related to the treatment effect of temozolomide, which is the only chemotherapeutic drug that prolongs survival in glioblastoma. The purpose of this retrospective study was to evaluate the value of magnetic resonance (MR) imaging including the apparent diffusion coefficient (ADC) on 3 T MR scanner to predict the status of MGMT promoter methylation.

Methods and Materials: This study included 27 patients (15 females and 12 males) with glioblastomas. All patients obtained unenhanced T2-weighted and fluid attenuated inversion recovery images, contrast enhanced T1-weighted images and diffusion-weighted images on $3 \mathrm{~T}$ MR scanner before surgery. Minimum $A D C$ ratio (mADCR) was calculated by the minimum ADC in tumour divided by that in the normal appearing white matter. Tumour volume on unenhanced and enhanced images was also calculated. Statistical analysis was performed with Mann-Whitney U test.

Results: Eight out of 27 patients showed methylation of the MGMT promoter. Glioblastomas with MGMT promoter methylation showed higher $\operatorname{mADCR}(1.19 \pm 0.19)$ than those without $(0.99 \pm 0.22)(P<0.05)$. There was no statistically significant difference in tumour volume on both unenhanced and enhanced images $(P>0.05)$. Conclusion: Higher mADC in glioblastoma is related to the status of MGMT promoter methylation and potentially predicts better prognosis.

B-108 15:03

T2*-weighted dynamic susceptibility-weighted contrast-enhanced magnetic resonance imaging to distinguish recurrence from pseudoprogression in patients affected by glioblastoma multiforme treated with radiotherapy in association with temozolamide: a preliminary study S. Pugliese, A. Romano, G. Trasimeni, G. Minniti, A. Bozzao; Rome/IT (silviapugliese@gmail.com)

Purpose: In patient affected by GBM the treatment scheduled is based on surgery followed by radiation therapy (RT) associated with temozolamide chemotherapy. Temozolamide can be responsible for early radiation necrosis also indicated as pseudo-progression (PP). Being able to distinguish this entity from recurrence (RGBM) is crucial for the following treatment. Our purpose was to evaluate the reliability of $\mathrm{T}^{*}$-weighted dynamic susceptibility-weighted contrast-enhanced (DSC) MR imaging to differentiate PP from RGBM.

Methods and Materials: Fifty patients affected by GBM were prospectively followed-up (every 2 months) by DSC-MR after surgery and RT associated with temozolamide. In twelve of them MR imaging revealed a new area of contrast enhancement inside the radiation field within 4 months. The diagnosis of PP or ERT was established subsequently with clinico-radiologic follow-up or surgical resection. The MR evaluation was performed drawing multiple ROIs ( $4 \mathrm{~mm}^{2}$ each) inside the contrast-enhancing lesion then transferred on the CBV maps avoiding cortical vessels. Three relative cerebral haemodynamic parameters were evaluated: relative cerebral volume $(\mathrm{rCBV})$, relative peak height $(\mathrm{rPH})$ and relative percentage of signal recovery (rPSR). Welch t tests were used to compare measurements between groups.

Results: Although not reaching statistical significance, mean rCBV (4.7 vs 4.2, P $=0.6$ ), maximum $\mathrm{rCBV}(5.1$ vs $4.4, \mathrm{P}=0.7)$ and $\mathrm{rPH}(0.96$ vs $0.84, \mathrm{P}=0.2)$ were higher in RGBM than in PP while rPSR values were lower (1 vs 1.5, P = 0.4). Conclusion: These findings are in agreement with the few reported in literature and support the potential role of DSC perfusion MR imaging to differentiate RGBM from PP.

\section{B-110 15:12}

Treatment monitoring in gliomas: comparison of dynamic contrastenhanced and spectroscopic MR imaging techniques for identifying treatment failure

M.-A. Weber ${ }^{1}$, J. Vöglein' ${ }^{1}$, J. Tüttenberg' ${ }^{2}$ M. Weimer ${ }^{1}$,

L. Gerigk ${ }^{1}$, H.-U. Kauczor ${ }^{1}$, M. Essig ${ }^{1} ;{ }^{1}$ Heidelberg/DE,

${ }^{2}$ Mannheim/DE (MarcAndre.Weber@med.uni-heidelberg.de)

Purpose: To evaluate whether dynamic susceptibility-weighted contrast-enhanced (DSC), dynamic contrast-enhanced (DCE) and proton spectroscopic imaging $\left({ }^{1} \mathrm{H}-\mathrm{MRSI}\right)$ can identify progression and predict treatment failure during follow-up earlier than morphologic MRI signs.

Methods and Materials: Thirty-seven patients with gliomas (21 women, 16 men; 48 years \pm 14 ) were assessed prospectively by ${ }^{1} \mathrm{H}-\mathrm{MRSI}$, DCE and DSC perfusion $\mathrm{MRI}$, each after a single dose of gadobenate dimeglumine during follow-up. Tumour progression (PD) was defined according to RECIST. DSC, DCE, and MRSI image analysis comprised a detailed semiquantitative region of interest $(\mathrm{ROI})$ analysis of the different parameters. Wilcoxon signed rank and rank sum test and Cox regression were used for statistical analysis.

Results: Twenty patients showed PD (54\%). In PD, significant positive differences between log2-transformed $\mathrm{ROI}$ ratios at the last measurement in comparison with baseline could be detected both for DSC tumour blood flow $(p<0.006)$ and volume $(p<0.001)$ and for maximum choline within tumour tissue $(p=0.0029)$ and choline/ creatine $(p=0.032)$, but not choline $/ \mathrm{N}$-acetyl-aspartate $(p=0.37)$. In contrast, these parameters were not significantly higher at last measurement in stable disease. Also, the differences between last value and baseline were significantly different between PD and stable disease for tumour blood flow $(p<0.004)$, volume $(p<0.002)$ and for maximum tumour choline $(p=0.0011)$. The time-dependent difference to baseline of DSC tumour blood flow normalized on grey matter was the best prognostic parameter for PD at Cox analysis (hazard ratio 2.67, $\mathrm{p}=0.01$ ).

Conclusion: DSC perfusion imaging can identify progression and predict treatment failure during follow-up of gliomas with the best diagnostic performance. 
14:00 - 15:30

Room N/O

\section{Molecular Imaging}

\section{SS 106}

\section{PET CT as a tool for molecular imaging}

\section{Moderators:}

A. Hauser; Bruderholz/CH

M. Schwaiger; Munich/DE

\section{B-111 14:00}

Focal thyroid incidentaloma detected by FDG-PET/CT; correlation to pathological and clinical follow-up

A. Sundin, F. Arnberg, J. Zedenius, I.-L. Nilsson; Stockholm/SE

(anders.sundin@ki.se)

Purpose: [18 F]Fluoro-deoxy-glucose positron emission tomography and concomitant CT (PET/CT) is established for especially oncological imaging, and its increasing use yields more incidental image findings. Focal thyroid uptake of FDG is associated with a significant risk of thyroid malignancy but the reports of prevalence and significance are contradictory. In the present study, we assessed the Stockholm city regions prevalence of focal thyroid incidentalomas and their nature by means of pathological and clinical correlation.

Methods and Materials: During 2006 and 2009, 3,637 patients in the Stockholm city region were examined by FDG-PET/CT. The anatomical position of focally increased thyroid FDG accumulations was registered and their standardised uptake value (SUV) was measured. Data on diagnostic and therapeutic follow-up were retrieved from the medical files.

Results: $62(1.7 \%)$ had increased thyroid FDG uptake which was focal in 35 (0.96\%; 10 men and 25 women). Ultrasound and fine needle cytology was performed in $23 / 35(66 \%)$ of the cases and $13 / 23(61 \%)$ were malignant. Surgery was performed in 10 cases. Benign cytology was found in 9 patients (thyroiditis, colloid or hyperplastic nodules). The tumour size ranged from 8 to $40 \mathrm{~mm}$. The maximum tumour SUV/normal thyroid SUV-ratio was significantly higher for malignant than benign nodules $(8.9 \pm 10.1$ vs. $3.5 \pm 1.1 ; p<0.05)$.

Conclusion: Incidental findings of focal thyroid FDG uptake are associated with a significant risk of a thyroid cancer. If a thyroid carcinoma may change the patient's treatment plan or prognosis, a diagnostic work up with ultrasound and fine needle cytology is recommended.

\section{B-112 14:09}

PET/CT imaging of small objects with the clinically implemented 40-detector biograph $\mathrm{mCT}$

H. Kayed, M. Sadick, S.O. Schönberg; Mannheim/DE (hany.kayed@umm.de)

Purpose: Small animal imaging with clinically implemented PET/CT (CPET/CT) scanners is inferior compared with animal PET/CT scanners. In this experiment we quantitatively analysed the applicability of the recently introduced Biograph $\mathrm{mCT}$ in imaging small objects.

Methods and Materials: A single-spot phantom was constructed simulating a small mouse of $30-\mathrm{g}$ body-weight bearing an ${ }^{18} \mathrm{~F}$-FDG PET positive tumor. The phantom was subjected to scanning with the Biograph $\mathrm{mCT}$ under clinical settings. The dataset was reconstructed and quantified using the TrueX algorithm and the TrueD software tool, respectively. Analysis of the SUV (standard uptake value) was performed using different doses $(1,10$ and $20 \mathrm{MBq})$ of ${ }^{18} \mathrm{~F}-\mathrm{FDG}$ in different Gaussian postfilter reconstructions at 1 and $3 \mathrm{~mm}$.

Results: CT images showed phantom dimensions close to the manufactured dimensions in comparison with PET images. The 1-mm filter showed augmented SUVs compared with $3 \mathrm{~mm}$ filter irrespective of the dose. Low doses, such as $1 \mathrm{MBq}$, showed augmented SUVs compared with high doses $(20 \mathrm{MBq})$. The average SUV showed a linear relationship and consistent data compared with the maximum SUV. Conclusion: Clinically implemented Biograph $\mathrm{mCT}$ with the TrueX reconstruction algorithms and TrueD quantification software are feasible for imaging small animals bearing very small tumours. These results show that application of low doses, determination of the volume of interest (VOI) in the reconstructed CT images, fused with the reconstructed PET images in $1 \mathrm{~mm}$ filter as well as measuring the SUV might be the optimal settings for evaluating small lesions.

\section{B-113 14:18}

Multimodality imaging for assessment of early tumour response to antiangiogenic therapy with anticalins in a preclinical sarcoma model with MRI and FDG-PET

R. Meier', R. Braren ${ }^{1}$, A. Steingötter ${ }^{2}$, Y. Kosanke ${ }^{1}$, H. Gille ${ }^{3}$,

E.J. Rummeny', M. Schwaiger ${ }^{1}$, A.J. Beer' ${ }^{1}{ }^{1}$ Munich/DE, ${ }^{2}$ Zurich/CH,

${ }^{3}$ Freising/DE (reinhardt.meier@gmail.com)

Purpose: The purpose was to compare the performance of DWI and FDG-PET for monitoring early response to a novel antiangiogenic therapy directed to VEGF with the anticalin PRS-050.

Methods and Materials: Anticalins are a novel class of targeted protein therapeutics based on the human lipocalin protein scaffold. The PEGylated anticalin angiocal (PRS050-PEG40) is directed against VEGF-A. Thirty-one mice were implanted subcutaneously A673 rhabdomyosarcoma xenografts and underwent DWI-MR (1.5 T Achieva, Philips, Netherlands) and FDG-PET (Siemens Inveon, Germany) imaging before and 2 days after i.p. injection of angiocal $(n=13)$, avastin $(n=6)$ or PBS $(n=12)$. Tumour size was measured manually with a caliper. Differences between therapeutic and control groups were tested for significance with analysis of variance $(p<0.05)$.

Results: Tumour size was not significantly different in the therapeutic compared to the control group $(p=0.977)$ on day 2 after therapy onset. The FDG-PET signal decreased significantly in the therapeutic group (anticalin: $-17.6 \pm 6 \%$, avastin: $-26.8 \pm 8 \%$ ); controversially there was a slight increase in FDG signal in the contro group $(2.1 \pm 1 \%)(p<0.05)$. The therapeutic group showed a significant increase in ADC value (anticalin: $9.7 \pm 3 \%$, avastin: $7.0 \pm 3 \%$ ), while the control group showed a decrease $(-8.8 \pm 2 \%)(p<0.05)$.

Conclusion: Although tumour size was not substantially influenced by angiocal therapy on day 2 after therapy onset, both FDG-PET signal and ADC value showed significant changes in the therapy versus control group as early as two days after treatment. Therefore, both modalities are promising for monitoring early response of antiangiogenic therapy.

\section{B-114 14:27}

Could tumoral lesion depiction with morphological imaging techniques (CT and MRI) be improved in contrast to FDG-PET-CT? A retrospective analysis M. Alemany, M. Bertomeu, C. Trampal; Barcelona/ES (malemany@crccorp.es)

Purpose: To analyse why tumoral lesions detected by FDG-PET/CT in oncological patients were not reported in previously performed CT or MRI.

Methods and Materials: We retrospectively analysed 154 consecutive oncologic patients who were examined with CT/MRI and with FDG-PET/CT for the following reasons: staging, restaging, suspicion of recurrence (either because of an increase in tumour markers or because of morphological imaging techniques reported as negative or not conclusive), and evaluation of therapy response. Image examinations were performed according to standard protocols.

Results: FDG-PET/CT detected tumoral lesions that had not been reported by CT or MRI in $31 / 154$ patients $(20.13 \%)$. When CT or MRI images were analysed retrospectively we found that 3 lesions $(9.6 \%)$ were invisible to morphological techniques, 12 lesions $(38.46 \%)$ were visible but not reported as malignant, 2 lesions $(7.7 \%)$ were not visualised because they were not included in the field of CT or MRI examination or were not identified due to an inadequate window level, 12 lesions $(38.46 \%)$ were not reported but detected retrospectively compared with PET findings and, finally, CT/MRI were unable to differentiate between fibrosis/ postradiation changes and viable tumour in 2 patients $(7.7 \%)$.

Conclusion: FDG-PET/CT is superior to morphological imaging techniques in detecting tumoral lesions because it detects metabolic changes which precede morphological ones. In addition, FDG-PET is able to diagnose viable tumour inside morphological changes due to fibrosis/postradiation. However, the results of CT and MRI could be improved by changing imaging protocols and the methodology of image analysis.

\section{B-115 14:36}

The clinical utility of ${ }^{18} \mathrm{~F}-\mathrm{FDG}$ PET in the differentiation of malignant and benign bone lesions and correlation with morphological changes on $\mathrm{CT}$ in PET-CT studies

M.N. Cabrera, M. García, L. Ortiz, L. Lapeña, A. Ortega, C. Rodríguez, J.L. Carreras, M.L. Vega; Madrid/ES

Purpose: To assess concordance/discordance of skeletal findings on PET and CT components in PET-CT studies.

Methods and Materials: Studies performed from 12/2008 to 12/2009 were revised, selecting those with bone lesions in PET, CT or in both techniques. The gold standard was clinical follow-up (minimum 6 months), correlation with other imaging techniques and/or biopsy (23 cases). Lesions were classified into the following 
groups: 1) concordant: 1.1) suggestive of malignancy in both components, 1.2) suggestive of benign lesions; 2) discordant: 2.1) suggestive of malignancy on CT without FDG uptake, 2.2) suggestive of malignancy in PET without alterations in $C T, 2.3)$ suggestive of benign lesions on CT with FDG uptake.

Results: Of 1320 PET-CT studies, we found bone lesions in 243 patients. We review data from 124 patients, six of them were lost to clinical follow-up. They were 41 men, 77 women, mean age 61.4 (range 7-84). 73 were concordant, 70 suggesting malignancy, with 4 false positive (FP) results, and 3 suggestive of benign lesions (true negatives). We found 45 cases of disagreement: In 4 patients, CT identified blastic malignant lesions (subgroup 2.1), in 25, PET revealed the presence of malignant lesions (subgroup 2.2), 23 true positives (TP) and 2 FP. Benign lesions were noted on CT with FDG uptake of variable degree in 16 (subgroup 2.3), being CT very useful for diagnosis.

Conclusion: In PET-CT, PET reduces FN of CT and CT reduces FP results of PET. The combined analysis is essential for a correct caracterisation of bone lesions.

\section{B-116 14:45}

\section{Additional value of a conjugated interpretation of FDG PET/CT and} contrast enhanced CT when staging malignant lymphoma

F. Ricard, P.J. Valette, C. Tychyj-Pinel, C. Houzard, F. Chateau, A.F. Manichon,

I. Orliaguet, F. Giammarile, F. Rety; Pierre-Bénite/FR (fabienricard@aliceadsl.fr)

Purpose: To introduce the additional benefit for both FDG PET and CT readers of a conjugated interpretation of FDG PET-CT and enhanced CT when staging malignant lymphoma.

Methods and Materials: FDG PET/CT and enhanced CT done at initial staging were retrospectively reviewed in 47 patients with malignant lymphoma ( 24 Hodgkin disease, 23 B cell lymphoma) with separate interpretation of FDG PET/CT (2 nuclear physicians) and enhanced CT ( 2 radiologists), then with conjugated analysis of both modality ( 1 nuclear physician and 1 radiologist specialiSed in both FDG PET and CT interpretation in lymphoma). Intraclass correlation and weighted kappa ( $\mathrm{kW}$ ) were used to figure interobserver variability and agreement to a clinical standard of reference. Results: Considering Ann Arbor stage, interobserver agreement was excellent for each interpretation modality, and was perfect in conjugated analysis ( $\kappa \mathrm{W}=1)$ with almost perfect correlation to the referent stage $(\kappa w=0.98)$. FDG PET-CT and enhanced CT were complementary getting better interobserver agreement with conjugated analysis of nodal sites $(0.79-0.83=0.86)$ and extranodal sites $(0.84$ $0.91=>0.95$ ). FDG PET improved morphologic analysis for involved small-sized lymph nodes mainly in cervical $(0.78->0.88)$, supraclavicular, hilar $(0.71->0.74)$ and pelvic (0.63->0.97) areas. Enhanced CT improved functional analysis mainly for spleen involvement ( $\mathrm{kW}=1.00$ in conjugated analysis) and reported frequent incidental findings (8/47 patients with major clinical impact).

Conclusion: Metabolic and morphological information are complementary for staging malignant lymphoma. Conjugated interpretation of enhanced CT and FDG PET-CT offer more reliable results than separate analysis of both modality.

\section{B-117 14:54}

Comparison between PET/CT and PET and CT in the initial staging of patients with Hodgkin's lymphoma

V. Marchesi, F. Fiocchi, G. Ligabue, E. Liardo, M. Cucca, B. Bagni, P. Torricelli; Modena/IT (vanessa.2610@hotmail.it)

Purpose: Compare retrospectively the concordance of combined PET/CT versus PET and CT in initial staging of patients with Hodgink's lymphoma $(\mathrm{HL})$.

Methods and Materials: Forty patients with $\mathrm{HL}$ (mean age $48.8 \pm 16.9 \mathrm{y} / \mathrm{o}$; $42.5 \%$ male) underwent combined PET/CT (first group) and 47 patients (mean age $43 \pm 15.6$ y/o; $46.8 \%$ male) underwent separately PET and CT within 4 days (second group) for initial staging of HL. PET/CT, PET and CT images were interpreted independently by one radiologist and one nuclear physician. Discordant $\mathrm{PET} / \mathrm{CT}$ results of the first group were read in consensus. Lymph-node regions were classified on the basis of Kaplan-Rosenberg plus extra-nodal site (14 regions/ patient). Patient stage was based on Ann-Arbor classification. Clinical stage was considered gold standard.

Results: Regarding first group, among 560 regions PET and CT results were concordant in $524(93.6 \%)$ and discordant in 36 regions $(6.4 \%)$; consensus reading confirmed positive all 36 regions (131 positive; 429 negative regions). Regarding second group, among 658 regions PET and CT results were concordant in 589 $(89.5 \%)$ and discordant in 69 regions (10.5\%). Among concordant sites both gave 112 positive and 477 negative regions. In first group, PET/CT stage was concordant to clinical stage in $92.5 \%$ of cases; PET/CT understaged $7.5 \%$ of patients. In second group, PET stage and CT stage were, respectively, concordant to clinical stage in $87.2 \%$ and $74.5 \%$ of cases. PET overstaged $6.4 \%$, understaged $6.4 \%$ of patients; CT overstaged $10.6 \%$, understaged $14.9 \%$ of patients.

Conclusion: PET/CT provides more exhaustive information compared to separate $\mathrm{PET}$ and $\mathrm{CT}$ for initial staging of $\mathrm{HL}$.

\section{B-118 15:03}

Evaluation of functional parameters in monitoring breast cancer treatment: 18 F-FDG-PET/CT mammography vs breast MRI

L. Umutlu, T.A. Heusner, A. Koeninger, F. Otterbach, R. Kimmig, A. Stahl,

G. Antoch, T.C. Lauenstein; Essen/DE (Lale.Umutlu@uk-essen.de)

Purpose: Aim of this study was the evaluation of functional parameters of 18-FFDG-PET/CT mammography (SUV) with CAD-based analysis of breast MRI as monitoring tools of breast cancer under neoadjuvant chemotherapy (NCTX).

Methods and Materials: 18 patients with breast cancer underwent both imaging modalities (a) prior and (b) after the third cycle of NCTX. Breast MRI was performed on a 1.5 Tesla scanner (Magnetom Espree, Siemens Healthcare). For dynamic imaging six dynamic T1-weighted gradient-echo sequences were collected (Gadovist, Bayer Schering Pharma). Contrast kinetics and pharmacokinetics were analysed quantitatively using a computer-aided detection system (CAD, iCAD) 18-F-FDG-PET/CT mammography examinations were performed within a time period of 48 hours on a Biograph PET/CT system (Siemens Molecular Imaging) and standardised uptake values (SUV) were assessed. Results were correlated to pathologic response evaluation. Functional changes were compared by Wilcoxon signed rank test.

Results: According to histopathology all patients were pathologic responders. The SUV values showed a statistical significant under NCTX $(p=0.018)$. CAD-based analysis of breast MRI revealed a significant reduction of fast postinitial washout patterns $(p=0.07)$ and a significant increase of tumour tissue fibrotisation $(p=$ 0.08 ). Pharmacokinetic parameters, by means of permeability and extracellular volume fraction, also revealed significant decrease.

Conclusion: Both imaging modalities enable a valid evaluation of functional therapy response of malignant lesions under NCTX. Due to availability reasons, the omission of radiation and its superior (preoperative) assessment of residual tumour extent breast MRI is recommended for therapy response evaluation in clinical routine.

\section{B-119 15:12}

Value of iodinated contrast administration in computed tomography exam for staging and restaging Hodgkin disease: comparison with 18 f-FDG PET/CT

C. Capraro, D. Ippolito, L. Guerra, L. Monguzzi, C. Messa, S. Sironi; Milan/IT (letizia.monguzzi@gmail.com)

Purpose: To assess the diagnostic value of administration of iodinated contrast in coregistered 18-fluorodeoxyglucose-positron-emission-tomographic (FDG/PET) with unenhanced low dose CT (IdCT) and contrast-enhanced CT (ceCT) for the staging and restaging of disease in patients with Hodgkin disease $(\mathrm{HL})$.

Methods and Materials: A total of 30 patients with histologically proven $\mathrm{HL}$ underwent a PET/IdCT+ceCT studies, performed in a single step procedure. Eighteen studies were performed for staging and 28 for the evaluation of residual disease after first line of chemotherapy (restaging). The two different datasets of images were evaluated by two expert readers blinded about the clinical data. Finally, the stage of disease was then defined according to PET/IdCT and PET/ceCT following the Ann Arbor classification. For restaging studies, images were interpreted considering the presence of residual mass with or without residual activity according to the International Harmonization Project.

Results: Of 630 sites considered as potentially involved by the disease, PET/ldCT and $\mathrm{PET} / \mathrm{ceCT}$ were concordant in 622/630 (98.7\%) in staging studies and in 28/28 (100\%)of the restaging studies. PET/IdCT and PET/ceCT were concordant with standard reference in $16 / 18(88.9 \%)$ and in $17 / 18$ (94.4\%) patients, respectively, in staging studies. In comparison between restaging PET/IdCT and PET/ceCT results and standard reference both examinations were concordant with standard reference in $26 / 28$ (92.8\%)studies.

Conclusion: In our study the addition of ceCT to PET/IdCT did not show significant added diagnostic value both in staging and, in particular, in restaging studies. If these results will be confirmed in a larger patients population, PET/IdCT could be considered the standard imaging technique for staging and restaging $\mathrm{HL}$, sparing addicted radiation burden and iodinate contrast administration to the patient.

\section{B-120 15:21}

Investigation of dose minimisation protocol for 18 F-FDG PET-CT in the management of lymphoma post-chemotherapy follow-up

L.I. Sonoda, B. Sanghera, T. Mills, W. Wong; London/UK (luke@sonoda.co.uk)

Purpose: ${ }^{18}$ F-FDG-PETCT plays an important role in the management of post-chemotherapy follow-up in lymphoma patients. Some centres perform prechemotherapy baseline CT and first post-chemotherapy PETCT from skull base to pubic symphysis. With a concern of radiation burden, especially in young patients, 
this study aimed to assess if PETCT acquisition area could be minimised, and how much radiation dose could be reduced.

Methods and Materials: Retrospective data analysis of 100 consecutive lymphoma patients ( 55 male, mean 41.7 years-old, Hodgkin's lymphoma 50, non-Hodgkin's lymphoma 50) was performed to record sites of disease on pre-chemotherapy CT and post-chemotherapy PETCT. The potential reduction in radiation dose and timesaving achieved with PETCT scans limited to sites of known disease identified on pre-chemotherapy CT was calculated.

Results: No FDG-uptake was seen in 72 of the 100 subjects. FDG-uptake at known disease sites was seen in 24 cases. Of the remaining 4 cases one had clinically significant pathology, a rectal adenocarcinoma. PETCT scans did not reveal any unexpected sites of lymphoma. Limiting PETCT to sites of known disease would have resulted in a mean radiation dose saving of $4 \mathrm{mSv}(27.3 \%)$, with a mean timesaving of 16 minutes per PETCT.

Conclusion: Our study suggests young lymphoma patients may benefit from reduced radiation dose / scan time by limiting PETCT to sites of known disease with low risk of missing significant pathology. However in older patients, with increased incidence of asymptomatic synchronous malignancies, we recommend wholebody PETCT is advisable unless pre-chemotherapy PETCT has been performed.

\section{4:00 - 15:30}

Room Q

\section{Computer Applications}

\section{SS 105}

\section{CAD, image analysis}

Moderators

P. Badura; Gliwice/PL

P. Mildenberger; Mainz/DE

\section{B-121 14:00}

Computer-aided detection systems in CT colonography - mitigating reviewers experiences in emerging radiology groups for cost-effective practices: a case study on the NCIA ACRIN 6664 datasets F.A.R. Chandelier ${ }^{1}$, L.A. Stein ${ }^{2} ;{ }^{1}$ Granby, QC/CA, ${ }^{2}$ Montreal, QC/CA (lawrence.stein@muhc.mcgill.ca)

Purpose: The aim of the present study is to assess the performance of a new Computer-Aided Detection (CAD) system for lesions $>9 \mathrm{~mm}$ on tagged patien preparations, while preserving a cost-effective practice amongst inexperienced and experienced reviewers.

Methods and Materials: CT colonography (CTC) is defined as a legitimate colorectal cancer screening method, as suggested by systematic meta-analysis concluding "there is convincing diagnostic evidence for the detection of lesions $>9 \mathrm{~mm}$ and strong diagnostic evidence for lesions $6-9 \mathrm{~mm}$ " [Digestion:80;2009]. Such patientfriendly screening protocol increases the demand for CTC readings, shifting CTC practices to outside specialised academic centres. As more inexperienced readers are involved, proper training and tools should be provided to maintain quality standards defined as $>83 \%$ detection for lesions $>9 \mathrm{~mm}$. The stand-alone performance of CADENS Imaging CAD Colon was investigated in an asymptomatic population representative of the variability in existing clinical settings, compared to optically reported lesions $>9 \mathrm{~mm}$. The ACRIN6664 database provided by the USA Nationa Cancer Institute represented the population of interest. This CAD will be involved in an academic protocol (McGill University, Quebec, Canada) to prospectively investigate its overall contribution to both experienced and inexperienced readers. Results: The CAD featured a detection rate similar to experienced radiologists, with a per-lesion sensitivity of $82 \%$ and a CAD average false-positive of 5/patient, delivering the cost-effective $83 \%$ per-patient detection-threshold for lesions $>9 \mathrm{~mm}$ Conclusion: CAD for CTC can significantly contribute in the emergence of costeffective CTC practices depicting variable reviewers' expertise. It is a unique opportunity to answer the immediate and ever growing demand for CTC readings.

\section{B-123 14:09}

CT colonography on the iPhone ${ }^{\circledR}$ : evaluation of performance for $2 \mathrm{D}$ image reading

L. Faggioni, E. Neri, P. Vagli, E. Picano, C. Bartolozzi; Pisa/IT

(lfaggioni@sirm.org)

Purpose: To evaluate the effectiveness of the iPhone ${ }^{\circledR}$ as a mobile device for $2 \mathrm{D}$ reading of CT Colonography (CTC) datasets.

Methods and Materials: We retrospectively reviewed 67 CTC examinations performed in a colorectal cancer screening setting. CTC images had been acquired in the supine and prone position using a low radiation dose and a faecal tagging protocol based on oral administration of iodinated contrast material. All datasets were wirelessly imported in DICOM format on a iPhone 4® 32GB (Apple Inc) running the OsiriX iPhone app version 1.1.3 (www.osirix-viewer.com) from a Macintosh desktop computer (iMac® ${ }^{\circledR} .06 \mathrm{GHz}$ ) connected to our hospital PACS and running OsiriX 3.7.1. Two experienced raters read CTC datasets independently on the $\mathrm{iMac}{ }^{\circledR}$ and on the $\mathrm{iPhone}{ }^{\circledR}$. Lesions included 25 polyps sized less than $6 \mathrm{~mm}(37.3 \%), 29$ between 6 and $9 \mathrm{~mm}(43.3 \%), 10$ between $10 \mathrm{~mm}$ and $30 \mathrm{~mm}$ $(14.9 \%)$, and 3 colonic masses $(4.5 \%)$. Lesion detection rate and time needed to read each entire CTC study were recorded.

Results: All lesions diagnosed on the iMac ${ }^{\circledR}$ were also detected on the iPhone $\AA$. However, the time required to complete reading of CTC datasets was significantly longer using the iPhone ${ }^{\circledR}\left(13.31 \pm 6.29\right.$ minutes) than with the $\mathrm{iMac}{ }^{\circledR}(5.51 \pm 2.45 \mathrm{~min}-$ utes; $p<0.01$ ), predominantly due to the need to repeatedly pan and zoom over the various colonic segments on the small iPhone ${ }^{\circledR}$ screen

Conclusion: The iPhone ${ }^{\circledR}$ can reliably be used for preliminary $2 \mathrm{D}$ reading of CTC datasets. However, it requires a substantially longer time for complete image analysis compared with a desktop workstation.

\section{B-124 14:18}

Automatic segmentation of the pancreas in contrast-enhanced CT data M. Erdt ${ }^{1}$, K. Drechsler ${ }^{1}$, M. Hammon ${ }^{2}$, M. Uder ${ }^{2}$, A. Cavallaro $2 ; 1{ }^{2}$ Darmstadt/DE, ${ }^{2}$ Erlangen/DE (marius.erdt@igd.fhg.de)

Purpose: Pancreas segmentation in CT images is desirable for computer-aided diagnostics and operation planning, but so far considered unfeasible to automate. A method for fully automatic pancreas segmentation from contrast-enhanced CT data is introduced.

Methods and Materials: Common portal phase CT scans of 40 patients were acquired. Liver, spleen and supporting vessels were automatically segmented. A subset of the data was used to build a pancreas tissue classifier using boosted Fourier and wavelet features. The built classifier was used for adapting a statistical geometric model in order to automatically segment the pancreas in the remaining images. Manual volume segmentation of the pancreas is taken as the gold standard. Threefold cross-validation was used with disjunct training and test folds. Results: Correlation with gold standard delineation was performed. Averaging resulted in $12 \%$ volume difference, $2.4 \mathrm{~mm}$ average surface distance, $4.5 \mathrm{~mm}$ RMS error, $23.4 \mathrm{~mm}$ max surface distance and $76 \%$ positive predictive value.

Conclusion: Machine learning approaches combined with statistical shape modelguided segmentation can be used to segment the pancreas in contrast-enhanced CT data. Since this method is fully automatic and provides a sufficient accuracy, it enables new applications in computer-aided diagnosis and operation planning which were infeasible so far due to manual interaction needs. It is also a key factor towards automated full-body segmentation in CT.

\section{B-125 14:27}

Verification of feasibility and clinical role of computer-assisted analysis of functional parameters of the lung obtained from CE-MS-CT

A. Malich, S. Mikulik, D. Hentrich; Nordhausen/DE (sylvia.mikulik@shk-ndh.de)

Purpose: This study aimed to analyse clinical role of CAD-based determination lung functional parameters of the lung obtained from MS-CT compared with whole body plethysmographic data.

Methods and Materials: 88 patients were randomly selected who underwent MS $\mathrm{CT}$ and whole-body plethysmography within $<3$ days. Body plethysmography was performed using master-screen-body (Jaeger, Germany, version V4.67). CT was performed using 128-row CT (0.5 mm slice thickness, Siemens Definition AS+) All data were analysed using CAD (MeVis Pulmo3D,Fraunhofer MeviS, Germany). Vital capacity (VC), FEV1, intrathoracal gas volume (ITGV) were matched with CAD-based lung volume analysis (vol), mean low density (MLD), low- and high attenuation values (LAV/HAV), lung weight (w), P15, PK (maximum of histogramm) and FWHM (full width half maximum).

Results: CAD-analysis was available at all cases. Analysis required 1 minute acquisition time. Correlation coefficients of VC versus Vol, MLD, LAV, HAV, weight, FWHM were 0.55 (<.01); -0.30 (<.05), -0.29 (<.05); 0.01 (n.s)., 0.55 (<.01), -0.29 $(<.05)$. Correlation coefficients of FEV1 versus Vol, MLD, LAV, HAV and weight were. 13 (n.s)...02 (n.s)., -.25 (p<.05), -.04 (n.s). and -.37 (p<.01), respectively. Correlation coefficients of ITGV versus Vol, MLD, LAV, HAV, FWHM, P15, PK were.77 (<.001), -.60 (<0.001), -.50 (<.01), -.52 (<.01), -.21 (n.s)., -.59 (p<.001) $-.55(p<0.01)$. Degree of emphysema and fibrosis can be obtained from CT-data semiautomatically. 
Conclusion: CAD-analysis of volume, mean low density and lung weight calculation using MS-CT allow a reliable analysis of functional lung parameters with highly significant correlation to plethysmographic values and thus offer the opportunity to screen lung function at all patients undergoing MS-CT due to several reasons and perspectively to quantify degree of emphysema and fibrosis.

\section{B-127 14:36}

Separation of cortical and cancellous bone by 3D-texture-based analysis: a novel approach of threshold-independent calculation of cortical thickness A. Valentinitsch ${ }^{1}$, J. Patsch ${ }^{1}$, J. Deutschmann ${ }^{1}$, L. Fischer ${ }^{1}$,

G. Langs ${ }^{2}$, F. Kainberger ${ }^{1} ;{ }^{1}$ Vienna/AT, ${ }^{2}$ Cambridge, MA/US

(alexander.valentinitsch@meduniwien.ac.at)

Purpose: To propose a fully automated threshold-independent method to segment cortical and cancellous bone in high-resolution peripheral quantitative computed tomography (HR-pQCT) images based on texture features

Methods and Materials: We scanned 14 structure phantoms of radius sections (7 males and 7 females, isotropic resolution $41 \mu \mathrm{m}$ and $82 \mu \mathrm{m}$ ) in a HR-pQCT. Expert segmentations on $41 \mu \mathrm{m}$ data registered to $81 \mu \mathrm{m}$ data served as grunt truth (GT). The method learns and selects texture features from a set of annotated training images. It learns a classifier that segments the bone segments with high accuracy. We evaluated segmentation error and compared the cortical thickness (Ct.Th) measurement with a state-of-the-art method. To test the agreement of Ct. Th between GT and other segmentation method, a two-tailed Student's t-test was then used to check whether linear regression slopes and intercepts were significantly different from the equality line.

Results: The mean Dice similarity coefficient of GT and automatic segmentation is $0.904( \pm 0.045)$. There is no significant bias of the two tailed Student's t-test between measurement and GT with slope $=0.984(p=0.85)$ and intercept $=0.066(p$ $=0.32$ ) for our method, while both deviated significantly from GT for the standard method (slope $=1.377: p<0.01$, intercept $=-0.319: p<0.01$ ). The mean absolute measurement error for the proposed method is $0.07 \mathrm{~mm}$

Conclusion: Experiments show that the proposed method measures the cortical thickness accurately. It outperforms a state of the art method in terms of bias and accuracy. This results in reliable thickness measurements, and provides an accurate basis for the study of the transitionary zone between cortical and cancellous bone.

\section{B-128 14:45}

The diagnostic value of direction information from diffusion tensor imaging of the prostate

J.C. Apitzsch, F. Schoth, F. Goerg, N.A. Kraemer, C. Plumhans,

M. Weibrecht, D. Pfister, T. Braunschweig, C.K. Kuhl;

Aachen/DE (apitzsch@rad.rwth-aachen.de)

Purpose: Diffusion weighted imaging of the prostate is a common method of evaluating prostatic carcinoma: We assessed the diagnostic value of direction information resulting from diffusion tensor imaging of the prostate.

Methods and Materials: MRI of the prostate was performed in 18 patients (range 54-74 years, mean age 67 years) at 3 Tesla (Archiva, Philips Medical Systems, Best, the Netherlands) combining an endorectal coil with a six channel surface coil. In additiona to multiplanar T2/TSE, 3D-Spectroscopy, DCE a DTI-sequence (Single-Shot SE-EPI, FOV $110 \times 100 \times 60 \mathrm{~mm}$, Vox $1.7 \times 1.7 \times 3 \mathrm{~mm}$, TE $64 \mathrm{~ms}$, TR $3713 \mathrm{~ms}$, B 1000) was performed for 32 directions. The results were compared with the histology either retrieved from prostatectomy or from prostate biopsy which had been performed in 10 of the patients. Further, the diagnostic value was compared to that of examining the pictures without DTI information.

Results: The mean fractional anisotropy in the peripheral zone was $0.24 \pm 0.08$. A disturbance of the tangential orientation of the main diffusion direction in the peripheral zone was chosen as an indicator of carcinoma. The sensitivity/specificity of the reading of the direction information alone (superimposed to T1 weighted images) was $80 \% / 75 \%$ of this cohort.

Conclusion: DTI of the prostate provides information about prostatic microarchitecture in the peripheral zone and may help along with other criteria to increase the diagnostic accuracy in the diagnosis of prostate cancer. Clinical relevence/ application: The analysis of direction information from DTI images may improve noninvasive assessment of prostate carcinoma and provide useful information before prostate biopsy and/or operation.

\section{B-129 14:54}

Feasibility of texture-based classification and grading for initial diagnosis and therapy monitoring of oesophageal cancer at arterial-phase CT: preliminary results

R. Schernthaner, M.E. Mayerhöfer, W. Matzek, S. Baroud, N. Bastati-Huber, D. Muin, A. Ba-Ssalamah; Vienna/AT (ruediger.schernthaner@meduniwien.ac.at)

Purpose: To determine the feasibility of arterial CT texture analysis for the classification and grading of oesopghageal cancer before and after neoadjuvant chemotherapy.

Methods and Materials: 57 patients with oesophageal cancer that underwent CT (different scanners/protocols/slice thickness) before and after neoadjuvant chemotherapy were retrospectively included in the study. 16 patients were excluded due to too small tumour size $(n=3)$, missing arterial scan $(n=9)$ or missing initial histology $(n=4)$, leaving 41 patients for further analysis ( 23 adenocarcinoma, 18 squamous-cell carcinoma; both groups with pathohistological grade 2 or 3 ). Texture features derived from the run-length and co-occurrence matrix, gradient, histogram, autoregressive model and wavelet transform were calculated. An optimised subset for the linear discriminant analysis was selected using Fisher coefficients.

Results: Looking at the baseline examination of all patients (different scanners), the misclassification rate was $51.4 \%$. Selecting only the baseline examinations performed on a 64-row CT scanner with a fixed scan protocol and a slice thickness of $0.75 \mathrm{~mm}(n=18)$, no lesion was misclassified. Looking at the follow-ups after chemotherapy performed on the same 64-row CT scanner with the same protocol, 11 patients showed stable disease after neoadjuvant chemotherapy; only one of these was misclassified as progressive disease $(9.1 \%)$. 3 cancers were upgraded at restaging, 4 were downgraded; of these 7 patients, only one patient with complete remission was misclassified as grade 2 squamous-cell carcinoma.

Conclusion: CT-texture-based classification and grading of oesophageal cancer seems feasible for initial diagnosis and therapy monitoring with a fixed protocol on the same CT scanner.

\section{B-130 15:03}

Non-invasive low-cost system for symptomatic versus asymptomatic plaque classification in carotid ultrasound

L. Saba ${ }^{1}$, R.U. Acharya ${ }^{2}$, F. Molinari ${ }^{3}$, A. Nicolaides ${ }^{4}$, J.S. Suri ${ }^{5}{ }^{1}$ Cagliari/IT, ${ }^{2}$ Singapore/SG, Turin/IT, ${ }^{4}$ London/UK, ${ }^{5}$ Denver, CO/US (lucasaba@tiscali.it)

Purpose: Low-cost non-invasive computer-aided diagnosis system was developed to automatically classify symptomatic and asymptomatic classes.

Methods and Materials: The classification is based on (i) Discrete Wavelet Transform (DWT), (ii) wavelet Package (WP) and combination of DWT and WP. The features selected were detailed and approximate coefficients for DWT method; energy, average horizontal and vertical WP coefficients for WP method; and a combination of these five (two of DWT and three of WP) features. These extracted features were fed to Variable-Increment (VIM) Perceptron functionality for automated decision making. We have also proposed a plaque malignancy index (PMI), a number to identify the symptomatic and asymptomatic carotid plaque class. This PMI can be used as an adjunct tool by the physicians during their screening to cross check their diagnosis.

274 carotid plaque ultrasound images (137 asymptomatic plaques and 137 symptomatic plaques) associated with retinal or hemispheric symptoms (33 stroke, 60 TIA, and $44 \mathrm{AF}$ ) were used for this work.

Results: Results show that combination of DWT with WP showed the best results. To be specific, the classification accuracy, sensitivity and specificity of the DWTbased system was $89.1 \%, 90.7 \%, 84.4 \%$, respectively, and $89.7 \%, 89.6 \%, 90.7 \%$, respectively, for wavelet package based system, and the combination of DWT and WP achieved $90.3 \%, 90.2 \%, 90.6 \%$, respectively. PMI clearly showed the separation of symptomatic and asymptomatic plaques.

Conclusion: Results of this study showed that combination of DWT- and WP based features yield accuracy better than $90 \%$ with ability to classify symptomatic versus asymptomatic plaques. 
Friday, March 4 
$10: 30-12: 00$

Room C

\section{Abdominal Viscera}

\section{SS 401a}

\section{Imaging the pancreas: challenges and controversies}

Moderators:

R.F. Dondelinger; Liège/BE

A.-S. Rangheard; Le Kremlin-Bicêtre/FR

\section{B-131 10:30}

Evaluation of pancreatic ductal variants using secretin-enhanced magnetic resonance cholangiopancreaticography

P. Thamm, J.-P. Kühn, R. Bühlow, K. Hegenscheid, H. Völzke, M. Lerch,

N. Hosten, R. Puls; Greifswald/DE (patrick.thamm@uni-greifswald.de)

Purpose: To investigate the prevalence of pancreatic duct variants in an ongoing epidemiological study using noninvasive secretin-enhanced magnetic resonance cholangiopancreaticography (SMRCP).

Methods and Materials: A total of 360 volunteers with a mean age of $57( \pm 13)$ years, 194 women and 166 men, and a mean body mass index of $27.8( \pm 4.3)$ $\mathrm{kg} / \mathrm{m}^{2}$ underwent prospective sMRCP. Navigator-triggered T2-weighted 3D turbo-spin-echo SMRCP with reconstruction of 3D-MIP was performed statically over 10 minutes after slow bolus injection of $1 \mathrm{U} / \mathrm{kg}$ secretin at $1.5 \mathrm{~T}$ (Magnetom Avanto, Siemens). Two readers evaluated the datasets for anatomic variants and pathologies of the pancreatic duct system. In cases of disagreement, a consensus reading was performed. Pancreatic duct variants were classified using a modified version of the guidelines of the Indiana University School of Medicine Office of Visual Media (IUSM 2005).

Results: All SMRCP examinations were of diagnostic quality. A nonvarian pancreatic duct was observed in only $61 / 360$ cases (17\%). Anatomic pancreatic duct variants were seen in $299 / 360$ cases (83\%), among them $67.5 \%$ with no accessory duct or an accessory duct not patent at the minor papilla; $7.5 \%$ with an complete or incomplete pancreas divisum. Clinical and morphologic signs of chronic pancreatitis were noted in 8 subjects $(2.2 \%)$, none of which showed some form of pancreas divisum.

Conclusion: Noninvasive secretin-enhanced MRCP allows screening for pancreas duct variant. Anatomic variants of the pancreatic duct system are more frequently seen in MRCP than described in anatomical data.

\section{B-132 10:39}

Role of diffusion weighted imaging (DWI) in differentiating inflammatory from neoplastic pancreatic masses

R. Sharma, S. Mahalingam, S. Gamanagatti, P. Garg, D.N. Srivastava;

New Delhi/IN (raju152@yahoo.com)

Purpose: To evaluate the role of diffusion weighted imaging (DWI) in differentiating inflammatory from neoplastic pancreatic masses.

Methods and Materials: Forty-six patients with a suspicion of a pancreatic neoplasm on ultrasound underwent dynamic contrast-enhanced MR (CEMR) with DWI at b-values of 0,500 and 1000 . The degree of restriction was assessed on a visual scale and neoplasms were identified on the basis of strong restriction. The CEMR images were reviewed first and lesions were classified into inflammatory and neoplastic, by consensus of three radiologists, blinded to the final outcome, followed by a review of DWI images along with CEMR. The gold standard for diagnosis was histopathology $(n=30)$ or clinical follow-up for 6 months $(n=16)$. Based on the gold standard, there were 31 neoplasms and 15 inflammatory masses (focal pancreatitis). The ADC values for proven neoplasms $(n=31)$ and normal pancreas were measured.

Results: CEMR had a sensitivity of $96.8 \%$ and specificity of $85.7 \%$ in differentiating inflammatory from neoplastic masses. The addition of DWI raised the sensitivity to $100 \%$ and specificity to $92.8 \%$. The accuracy of CEMR alone was $93.4 \%$ and the addition of DWI increased it to $97.8 \%$. The mean ADC of pancreatic tumours was $1.32 \pm 0.33 \times 10^{-3} \mathrm{~mm}^{2} / \mathrm{sec}$, while the mean ADC of normal pancreas was higher at $1.72 \pm 0.24 \times 10^{-3} \mathrm{~mm}^{2} / \mathrm{sec}$. The mean normalised ADC (ADC ratio of neoplastic mass to normal pancreatic tissue) was $0.77 \pm 0.13$

Conclusion: Addition of DWI improves the diagnostic accuracy of CEMR in differentiating inflammatory from neoplastic masses.

\section{B-133 10:48}

Apparent diffusion coefficient as an imaging biomarker to predict the survival in patients with unresectable pancreatic cancer

H. Nishiofuku, N. Marugami, T. Tanaka, H. Anai, S. Sueyoshi, M. Sho, Y. Nakajima, K. Kichikawa; Kashihara/JP

Purpose: The purpose of this study is to investigate whether the apparent diffusion coefficient (ADC) on diffusion-weighted MR images at $1.5 \mathrm{~T}$ can predict the survival in patients with unresectable pancreatic cancer.

Methods and Materials: The institutional review board approved this study. The subjects were 31 patients (20 males, 11 females; mean age 65.7 years; range 53-79 years) with histologically confirmed unresectable pancreatic cancer who were treated with first-line chemotherapy (gemcitabine alone 17, TS-1 alone 7 , gemcitabine plus TS-1 7) between July 2007 and December 2009. The minimum $A D C$ ( $m i n A D C$ ) value of each primary tumour was determined by placing 3 regions of interest on ADC maps at pretreatment and 4 weeks after initiation of treatment, and the relative change of minADC value (\%ADC) was determined. The effect of \%ADC on overall survival (OS) was modelled by Cox proportional hazards regression. Results: Median time-to-progression was 4.0 months and median OS was 11.7 months. Patients with an increase of $>0 \%$ of the baseline minADC after 4 weeks of treatment $(n=19)$ had a significantly better median survival than patients with a decrease of $<=0 \%(n=12)(18.9$ vs 5.6 months; $P=0.001)$. The \%ADC was the strongest independent predictor of $O S$ in multivariate analysis $(P=0.009)$ Conclusion: The relative change of ADC at 4 weeks from the initiation of treatment may serve as a useful imaging biomarker in unresectable pancreatic cancer.

\section{B-134 10:57}

Endocrine tumours of the pancreas: discordant patterns of enhancement at contrast-enhanced CT, contrast-enhanced MRI and CEUS

G. Foti, N. Faccioli, R. Manfredi, R. Pozzi Mucelli; Verona/IT (gfoti81@yahoo.it)

Purpose: To compare contrast-enhanced computed tomography (CECT) contrast-enhanced magnetic resonance imaging (MRI) and contrast-enhanced ultra-sonography (CEUS) enhancement patterns of functioning (FPET) and nonfunctioning pancreatic endocrine tumours (NPET)

Methods and Materials: In a 6-year-period, 125 consecutive patients preoperatively studied with at least two contrast enhanced exams (98 NPETs, 27 FPETs) were included in our retrospective study. A total of $94 \mathrm{CT}, 83 \mathrm{MRI}, 69 \mathrm{CEUS}$ studies were performed; all CT and MRI exams were performed before and after i.v. administration of contrast material, including arterial pancreatic ( $45 \mathrm{sec}$ ), portal venous ( $80 \mathrm{sec}$ ) and late venous phase $(120 \mathrm{sec})$; CEUS was performed with continuous observation of lesion enhancement from unenhanced (B-mode) to late venous phase. Student's $t$ test was used to compare enhancement patterns (hyper-iso-hypovascularity in comparison with spared pancreas, homo-inhomogenous enhancement, peak enhancement phase). A value of $p<0.05$ was considered significant.

Results: Lesion hypervascularity was depicted in 76/94 (80.8\%) CT, 70/83 (84.3\%) MRI and 54/69 (78.2\%) CEUS exams, without significant difference between NPET and FPET $(p>0.05)$. A non-significant difference was determined as cocers enhancement patterns (hypervascularity and homogeneity) between CT and MRI ( $p$ $=0.1)$, CT and CEUS $(p=0.1)$, MRI and CEUS $(p>0.05)$. Conversely, there was a significant difference regarding the peak enhancement phase between $C T$ and MRI with respect to CEUS ( $p=0.03$ ).

Conclusion: In case of doubtful findings, an additional enhanced study may be useful to confirm hypervascularity, representing the key for characterising NPET and for identifying FPET.

\section{B-135 $11: 06$}

Preliminary results on the influence of combined chemoradiation on MRdiffusion-weighted imaging parameters in primary inoperable pancreatic carcinomas

K.M. Grünberg, D. Simon, M. Münter, A. Lemke, M. Ganten, M. Klauß,

S. Delorme, H.-.P. Schlemmer, B. Stieltjes; Heidelberg/DE

Purpose: To evaluate changes of quantitative parameters obtained from intravoxe incoherent motion diffusion-weighted imaging (IVIM-DWI) in patients with primary inoperable pancreatic carcinoma during a course of combined chemoradiation (CR). Methods and Materials: IVIM-DWI echo-planar imaging in expiratory-breath hold at $1.5 \mathrm{~T}$ was performed in 19 patients. We used eleven $\mathrm{b}$ values $\left(0-800 \mathrm{~s} / \mathrm{mm}^{2}\right)$. Mean tumour apparent diffusion coefficient (ADC), the true diffusion constant (D) and the perfusion fraction $(f)$ values were calculated to three time points during the course of therapy: before, during and after the end of radiation. Ca-19.9 determination in two time points (before and four weeks after the end of radiation) enabled a correlation of the diffusion parameters with the clinical course. Only 13 patients with tumour regression were analysed. 
Results: $f$ is at the end of therapy higher than before and during therapy $(0.0911$, $0.0681,0.0686$, respectively) but without significance. Mean ADC and D values are significantly higher at the end of therapy $(0.0015)$ compared to onset of therapy $(0.0012, p<0.0001,0.0002$, respectively) and during therapy $(0.0013$ and 0.0014 , respectively, $\mathrm{p}<0.05$ ).

Conclusion: These results show that tumour $A D C$ and $D$ values increase significantly during the course of combined $\mathrm{CR}$ in patients with partial remission. There is an increase of $f$ value during course but without significance, and therefore without effect of perfusion on ADC. In the course, ADC proved to be a sufficient parameter. Further studies with higher population will be necessary to prove the possible value of noninvasive DWI on predicting therapy outcome, e.g. resection probability or progression-free survival time.

\section{B-136 11:15}

Pancreatic ductal adenocarcinoma versus mass-forming pancreatitis: usefulness of combined diffusion and perfusion MR imaging

P. Boraschi, F. Donati, S. Salemi, R. Gigoni, C. Bartolozzi, F. Falaschi; Pisa/IT (p.boraschi@do.med.unipi.it)

Purpose: To evaluate the usefulness of combined diffusion and perfusion MR imaging in the differential diagnosis between pancreatic adenocarcinoma and mass-forming pancreatitis.

Methods and Materials: Twenty patients without pancreatic disease and thirty-five patients with pathologically confirmed pancreatic lesions (ductal adenocarcinoma, $n=$ 20; focal chronic pancreatitis, $n=12$; autoimmune pancreatitis, $n=3$ ) underwent MRI at 1.5 T-device. Diffusion-weighted MRI was performed using a free-breathing SS-EPI sequence (TR/TE, 3000-4500 ms/64-80 ms; thk, $5.0 \mathrm{~mm} / \mathrm{gap}, 1.0 \mathrm{~mm}$; 25-30 seconds) with b-gradient-factor value of $500 \mathrm{sec} / \mathrm{mm}^{2}$. Dynamic contrast-enhanced MR perfusion consisted of a 3D free-breathing LAVA sequence (TR/TE, $2.28 \mathrm{~ms} / 1.05 \mathrm{~ms}$; thk, $10.0 \mathrm{~mm} / \mathrm{sp},-0.0 \mathrm{~mm}$; 1 second) repeated up to 5 minutes after injection of $7 \mathrm{~mL}$ Gd-BOPTA (MultiHance, Bracco) and $20 \mathrm{~mL}$ saline flush at flow rate of $4 \mathrm{~mL} / \mathrm{sec}$. All MR diffusion and perfusion images were processed by two radiologists in conference that calculated mean ADC value for both normal pancreas and each focal lesion and classified four time-signal-intensity curve (TSIC) shapes: type 1 (quick enhancement and quick decay followed by slowly decaying); type 2 (slow enhancement followed by slow constant enhancement); type 3 (fast enhancement followed by signal plateau); type 4 (fast enhancement followed by slowly decaying plateau).

Results: All 20 patients with normal pancreas presented mean ADC values of $1.73 \pm 0.027 \mathrm{~mm}^{2} / \mathrm{s}$ and a TSIC-type 1 . In all 20 ductal adenocarcinomas mean ADC values were $1.44 \pm 0.004 \mathrm{~mm}^{2} / \mathrm{s}$ and a TSIC-type 2 was observed. In 12 patients with focal chronic pancreatitis and in 14 post-obstructive chronic pancreatites mean ADC values were $2.14 \pm 0.005 \mathrm{~mm}^{2} / \mathrm{s}$ and a TSIC-type 3 was recognised, whereas for autoimmune pancreatitis ADC values were $1.20 \pm 0.004 \mathrm{~mm}^{2} / \mathrm{s}$ and a TSIC-type 4 was identified. Conclusion: Combined diffusion and perfusion MR findings can improve the differential diagnosis between pancreatic adenocarcinoma and mass-forming pancreatitis.

\section{B-137 11:24}

Small cystic lesions of the pancreas: incidence and size changes in a large series population

G. Morana 1 , M. Fuser ${ }^{2}$, C. Cugini ${ }^{1}$, L. Cancian 1 , F. Ferro ${ }^{3} ;{ }^{1}$ Treviso/IT, ${ }^{2}$ Padua/IT, ${ }^{3}$ Perugia/lT (gmorana@ulss.tv.it)

Purpose: To evaluate the incidence and size changes of small pancreatic cysts in a large series population.

Methods and Materials: We reviewed the reports of all MR of the abdomen in the period March 2005-March 2010

Results: We evaluated 4463 patients: 394 (8.8\%) patients have been selected with at least one cystic lesion of the pancreas $<4 \mathrm{~cm}$. 174 were male $(44.2 \%), 220$ female $(55.8 \%) .140$ patients had a single lesion. Medium diameter of the lesion at diagnosis was $15 \mathrm{~mm}$ : 54 patients less than $10 \mathrm{~mm}, 57$ between 10 and $20 \mathrm{~mm}, 21$ between 20 and $30 \mathrm{~mm}$ and 8 more than $30 \mathrm{~mm}$. 86 patients were followed up at $6,12,24$ or 36 months, 254 patients had a multifocal lesion. Medium diameter of the lesion at diagnosis was $16 \mathrm{~mm}$ : 95 patients less than $10 \mathrm{~mm}, 106$ between 10 and $20 \mathrm{~mm}$, 31 between 20 and $30 \mathrm{~mm}$ and 22 more than $30 \mathrm{~mm}$. 173 patients were followed up at $6,12,24$ or 36 months. No differences in age and sex was evident between monofocal and multifocal lesions. The incidence of multifocal is statistically different from single lesion. The lesions do not show significant increase of the diameter in the follow-up up to 36 months, either in the single lesion or in the multifocal.

Conclusion: Small cystic lesion of the pancreas is not infrequent in daily practice. Follow-up is a safe procedure as there is no significant variation in size of the lesion.

\section{B-138 11:33}

Gadoxetic acid-enhanced magnetic resonance imaging for evaluation of pancreatic adenocarcinoma and liver metastasis

H. Morisaka, U. Motosugi, T. Ichikawa, H. Sou, T. Araki; Chuo/JP (morisakahiroyuki@hotmail.co.jp)

Purpose: To compare the diagnostic abilities of gadoxetic acid (Gd-EOB-DTPA)enhanced magnetic resonance imaging (EOB-MRI) with that of contrast-enhanced multidetector-row computed tomography (CECT) for pancreatic adenocarcinoma and liver metastasis

Methods and Materials: This study included 39 patients with pancreatic adenocarcinoma (23 men and 16 women), and 40 control individuals without pancreatic lesions ( 20 men and 20 women). A total of 48 liver metastases were detected in 13 of the 39 patients with pancreatic adenocarcinoma. Two radiologists independently reviewed EOB-MRI and CECT results and rated the level of confidence regarding the presence of pancreatic adenocarcinoma and of a liver metastasis in the entire liver by using a 5-point scale. To compare the diagnostic abilities of the 2 modalities for pancreatic adenocarcinoma, a receiver operating characteristic $(\mathrm{ROC})$ analysis was performed. The sensitivities, specificities and accuracies of the 2 modalities were also calculated. For the diagnosis of liver metastasis, the sensitivity, specificity and accuracy on a patient-by-patient basis and the sensitivity and positive predictive value (PPV) on a lesion-by-lesion basis were calculated.

Results: EOB-MRI did not significantly differ from CECT with regard to the sensitivity, specificity, accuracy and Az value for the detection of pancreatic adenocarcinoma. For the detection of liver metastasiss, the 2 modalities differed with regard to only the sensitivity that was calculated on lesion-by-lesion basis (EOB-MRI vs CECT: $90 \%$ vs $71 \%$ )

Conclusion: The diagnostic ability of EOB-MRI for pancreatic adenocarcinoma was equivalent to that of CECT. The sensitivity of EOB-MRI might be higher than that of CECT for the detection of liver metastasis.

10:30 - 12:00 Room D1

Chest

\section{SS 404}

\section{Pulmonary nodules: detection and characterisation}

Moderators

E. Rizzo; Genolier/CH

M. Scaglione; Castell Volturno/IT

B-141 10:30

Binary logistic regression analysis of multi-detector CT features of

pulmonary benign and malignant focal ground-glass opacity

L. Fan, S.-Y. Liu, X.-S. Xiao; Shanghai/CN (fanli0930@163.com)

Purpose: To retrospectively evaluate multi-detector computed tomography features of pulmonary focal ground-glass opacity (fGGO) in order to reveal the different features between benign and malignant fGGO and conduce to diagnosis and differential diagnosis.

Methods and Materials: Demographic data, lesion location, size, attenuation value and MDCT features (shape, margin, interface, internal characteristics, and adjacent structure) in a set of 82 fGGO were analysed. Difference between benign and malignant fGGO was analysed using chi-square test and Fisher exact test or Mann-Whitney $U$ test. A two-sided value of $p<0.05$ was used as the criterion to indicate a significant difference. Binary logistic regression analysis was performed to draw a regression equation.

Results: The set of fGGO consists of 21 benign and 61 malignant lesions. No statistical differences were found between benign and malignant fGGO in terms of demographic data, location, size and attenuation value. The frequency of lobulation $(p=0.000)$, spiculation $(p=0.008)$, spine-like process $(p=0.004)$, well-defined but coarse interface $(p=0.000)$, other air-containing space $(p=0.000)$, pleural indentation $(p=0.000)$ and vascular convergence $(p=0.006)$ was significantly higher in malignant fGGO than in benign fGGO. By binary logistic regression analysis, lobulation, interface and pleural indentation were important indicators for malignant diagnosis, and the corresponding odds ratio were 8.122, 3.139 and 9.076; moreover, well-defined but coarse interface was the most important among interfaces. With the three indicators for malignant diagnosis, sensitivity, specificity and accuracy were $93.4,66.7$ and $86.6 \%$, respectively.

Conclusion: An fGGO with lobulation, well-defined but coarse interface, and pleural indentation is more likely to be malignant. 


\section{B-142 10:39}

The volumetric measurement accuracy for ground-glass opacity nodules with low-dose high-definition CT: a chest phantom study

E. Ning, W. He, Y. Xu, D. Ma, J. Li; Beijing/CN (jianying.li@med.ge.com)

Purpose: To study the volumetric measurement accuracy of a low-dose CT for ground-glass opacity (GGO) nodules on a high-definition Discovery CT750 HD (HDCT) using a chest phantom.

Methods and Materials: An anthropomorphic chest phantom of $300 \mathrm{~mm}$ width containing a chest wall, heart, mediastinum and 16 artificial ground-glass opacity nodules with known volumes $\left(\sim 900 \mathrm{~mm}^{3}\right)$ was scanned on a HDCT with the following protocol: $100 \mathrm{kVp}$, automatic tube current modulation for noise index of 30 , $1.25 \mathrm{~mm}$ collimation. Images were reconstructed with slice thickness of $0.625 \mathrm{~mm}$ and BONE algorithm with $30 \% \mathrm{ASiR}$ (Adaptive Statistical Iterative Reconstruction). Nodule volumes were measured using a commercial volumetric software package. The GGO nodule volumes measured from the low-dose HDCT images were compared with the reference-standard volumes from the phantom. Effective dose was calculated on the basis of dose length product and volume $\mathrm{CT}$ dose index.

Results: All artificial GGO nodules were successfully identified and measured. The radiation dose for the whole-chest scan was $0.94 \mathrm{mSv}$. A good agreement was observed between the measured GGO nodule volumes and the reference-standard volumes from the phantom with average error of $-5 \%$ (0 to $-17 \%$ ).

Conclusion: High-resolution and low-dose GGO nodule detection and accurate volume measurement can be achieved on HDCT with Gemstone detector and ASIR algorithm.

\section{B-143 10:48}

\section{First-pass perfusion CT on 320-detector row CT: comparison of} differentiation capability of malignant from benign pulmonary nodules with first-pass dynamic MRI and PET/CT

Y. Ohno ${ }^{1}$, M. Nishio', Y. Onishi', H. Koyama', K. Matsumoto ${ }^{2}$, T. Yoshikawa ${ }^{1}$, S. Matsumoto', D. Takenaka', K. Sugimura'; ${ }^{1}$ Kobe/JP, ${ }^{2}$ Kofu/JP (yosirad@kobe-u.ac.jp)

Purpose: To compare diagnostic capabilities of pulmonary nodules among first-pass perfusion CT on 320-detector row CT, first-pass dynamic MRI and FDG-PET/CT. Methods and Materials: 25 consecutive patients with 61 nodules underwent chest perfusion $\mathrm{CT}$, dynamic MRI, PET/CT and microbacterial and/or pathological examinations. 61 nodules were classified into two groups based on the final diagnoses: malignant $(n=44)$ and benign nodules $(n=17)$. On perfusion CT, blood flows were calculated by single- $\left(\mathrm{PF}_{\mathrm{S}}\right)$ and dual-input maximum-slope $\left(\mathrm{PF}_{\mathrm{D}}\right)$ and patlak-plot $\left(\mathrm{PF}_{\mathrm{P}}\right)$ methods. Blood volume was also determined by patlak-plot method $\left(\mathrm{BV}_{\mathrm{P}}\right)$. On dynamic MRI, maximum enhancement ratio (MER) and maximum slope of enhancement ratio (MSER) were calculated. On PET/CT, maximum value of SUV (SUV ) was determined. Then, all indexes were evaluated by ROI measurements, and compared between two groups by Student's t-test. To compare diagnostic capability among all indexes, ROC analyses were performed. Finally, sensitivity, specificity and accuracy were compared with each other by using McNemar's test. Results: $\mathrm{PF}_{\mathrm{S}}, \mathrm{PF}_{\mathrm{D}}, \mathrm{MER}, \mathrm{MRER}$ and $\mathrm{SUV}_{\text {max }}$ were significantly different between two groups $(p<0.05)$. Area under the curves of PFS $(A z=0.85)$ was significantly larger than that of $\mathrm{PF}_{\mathrm{D}}(\mathrm{Az}=0.73, \mathrm{p}<0.05)$ and $\mathrm{SUV}_{\text {max }}(\mathrm{Az}=0.70, \mathrm{p}<0.05)$. When feasible threshold values were adopted, accuracies of $\mathrm{PF}_{\mathrm{S}}(85.2 \%)$ and MRER $(85.2 \%)$ were significantly higher than that of SUV Sax $_{\text {max }}(73.8 \%)$. In addition, specificity of $\mathrm{PF}_{\mathrm{s}}(70.6 \%)$ was significantly higher than that of $\mathrm{PF}_{\mathrm{D}}(29.0 \%, \mathrm{p}<0.05)$. Conclusion: First-pass perfusion CT and dynamic MRI were more accurate methods for differentiation of malignant from benign nodules as compared with PET/CT.

\section{B-144 10:57}

Dual energy computertomography in the evaluation of pulmonary nodules J.-C. Altenbernd, T. Heusner, S. Ladd, A. Ringelstein, M. Forsting, G. Antoch; Essen/DE (jens.altenbernd@gmx.de)

Purpose: To determine the clinical utility of dual energy computed tomography (DECT) for evaluation of pulmonary nodules.

Methods and Materials: This study was approved by the institutional review board, and informed consent was obtained. CT scans were obtained with arterial delay and 3 minutes after contrast material injection in 25 patients ( 14 men, 11 women; mean age 59 years \pm 11 ). 18 patients had pulmonary metastases, 7 had NSCLC. 65 pulmonary nodules were assessed with dual energy CT in these 25 patients. All image sets included virtual non-enhanced images, $80 \mathrm{kV}, 140 \mathrm{kV}$, and averaged images. CT attenuation (HU) of pulmonary nodules, muscle, aorta, and air in between image sets were compared and the iodine uptake in pulmonary nodules, the signal-to-noise ratio (SNR) and the contrast-to-noise ratio (CNR) were calculated.
Results: The mean iodine uptake (overlay) of pulmonary nodules in the arterial scan was $53 \mathrm{HU} \pm 32$ and in the delayed scan $57 \mathrm{HU} \pm 35(\mathrm{p}=0.37)$. The mean CT attenuation of pulmonary nodules on virtual non-enhanced images was $9 \pm 45$ when virtual images were based on the arterial scan, and $10 \pm 46$ when based on the delayed scan $(p=0.43)$. Aortic virtual non-enhanced CT attenuation was $56 \mathrm{HU} \pm 17$ when based on the arterial scan, and $49 \mathrm{HU} \pm 13$ when basing virtual non-enhanced images on the delayed scan $(p=0.001)$.

Conclusion: Dual energy CT allows reconstruction of virtual non-enhanced scans from both, arterial and delayed CT images, with comparable accuracy in pulmonary nodules.

B-145 11:06

(also

Impacts of ASiR reconstruction on volumetric measurement with low-dose HDCT: phantom study

L. Zhang', Q. Wang', T. Yu', Y. Ying', Z. Liu', Y. Dai', H. Cao²; ${ }^{1}$ Tianjin/CN,

${ }^{2}$ Beijing/CN (miracle_99.student@sina.com)

Purpose: To prospectively evaluate the impacts of low radiation dose adaptive statistical iterative reconstruction (ASiR) on automated volumetric measurements of pulmonary nodules with different diameters and density in a phantom using High-Definition CT (HDCT)

Methods and Materials: A total of 16 pulmonary nodules with 4 diameter categories $(2.5 \mathrm{~mm}, 5.0 \mathrm{~mm}, 10.0 \mathrm{~mm}, 20.0 \mathrm{~mm})$, and 5 attenuation categories $(-100 \mathrm{HU}$ $-60 \mathrm{HU}, 0 \mathrm{HU}, 60 \mathrm{HU}, 100 \mathrm{HU}$ ) were placed in a lung phantom. The phantom was kept beneath solution with attenuation of $50 \mathrm{HU}$, and was scanned by HDCT under different $\mathrm{mA}(25 \mathrm{~mA}, 50 \mathrm{~mA}, 100 \mathrm{~mA}, 200 \mathrm{~mA})$, using scan mode of HD. 5 levels $(10 \%, 30 \%, 50 \%, 70 \%, 100 \%)$ of slice ASiR were performed according to each scan. Nodule theoretical volumes were calculated using the formula $V=(4 / 3)$ $\pi r^{3}$. Automated nodule volume measurements were performed using Lung VCAR Single Protocol (GE, AW 4.4 workstation). Absolute percentage error (APE) was calculated to estimate the accuracy of volumetric measurement.

Results: $1.30 \%$ ASiR reconstruction yielded the minimal error, there were statistically significant differences in comparison with the other 4 level ASiR reconstruction $(p<0.05)$. 2. There were no statistically significant differences in APE among 4 different scanning tube current $(p<0.05)$, with the $200 \mathrm{~mA}$ tube current yielding the minimal error.

Conclusion: $30 \%$ ASiR reconstruction following HD scan suits the most for nodule volumetric measurement, it can maximally reduce in $\mathrm{x}$-ray dose while preventing structure details, and then yield satisfactory accuracy for pulmonary nodule volumetric measurement.

\section{B-146 11:15}

Volumetry of artificial lung nodules on 64-slice computed tomography (CT): precision of automatic vs semiautomatic method

L. Calandriello, A. Larici, A. del Ciello, A. Caulo, S. Santoro, M. Ciresa,

M. Amato, L. Bonomo; Rome/lT (Icalan@hotmail.it)

Purpose: To assess the precision of volumetry of artificial solid (S) and non-solid (NS) lung nodules using a tridimensional (3D) software equipped with full automatic and semiautomatic methods. To assess the reproducibility of semiautomatic volumetry.

Methods and Materials: A chest phantom containing 28 artificial nodules (14 S, 14 NS) of known diameter and volume underwent 64-slice CT scan (slice thickness/ interval $0.625 \mathrm{~mm}$, lung kernel, $100 \mathrm{mAs}$, pitch 1.375). Nodules diameter ranged between 2.9 and $25.3 \mathrm{~mm}$ for $\mathrm{S}$ and 3.1 and $11.1 \mathrm{~mm}$ for NS. Two chest radiologists calculated the volume of each nodule independently in two reading sessions, 30 days apart, using a 3D software equipped with full automatic and semiautomatic methods (Median LMS, Median Technologies). To assess the precision, standard deviation (SD) of the mean differences between the real and estimated volumes was calculated for each nodule with both methods. Inter- and intra-observer variabilities of semiautomatic volume measurements were also assessed by SD calculation. Results: Full automatic method of volume assessment has demonstrated higher variability for NS nodules $(S D= \pm 62.5)$ than for $S$ nodules $(S D= \pm 5.9)$. Semiautomatic method has shown higher reproducibility in volume assessment for both $S(S D= \pm 4)$ and NS $(S D= \pm 7.6)$ nodules. A very low inter- and intra-observer variabilities ( $S D= \pm 0.2 ; S D= \pm 0.9$ ) was reported for semiautomatic measurement. Conclusion: Semiautomatic volumetry of artificial lung nodules has proved to be more precise than the full automatic one, particularly for NS nodules. Semiautomatic volumetry has also demonstrated a very high inter- and intra-observer reproducibility in assessing nodule volume. 


\author{
B-147 11:24 \\ Accuracy and reproducibility of pulmonary nodule at dual-source CT: \\ volumetric measurements with comparison of manual, RECIST and lung \\ care software methods: a phantom study \\ J. Paul, R.W. Bauer, T.J. Vogl, W. Maentele; \\ Frankfurt a.Main/DE (ralfwbauer@aol.com)
}

Purpose: To evaluate the effect of slice thickness on the detection of pulmonary nodule dimension and prove the efficiency of lung care software for the calculation of nodule volume and also to evaluate the diagnostic accuracy of pulmonary nodule at various locations clinically using dual-source CT.

Methods and Materials: A DSCT scanner, an anthropomorphic phantom with two sets of tissue equivalent $(30 \mathrm{HU}$ ) round nodules (diameters- $2.3,4$ and $5 \mathrm{~mm}$ ) we produced images of thorax with pulmonary nodules. Four different protocols including dual energy and flash mode and four reconstruction methods with two different slice thicknesses were used for the scanning. We used Lin's concordance correlation coefficient statistical analysis with SPSS software as an appropriate statistical procedure.

Results: Using manual ruler and RECIST method, the per cent deviation of $\pm 13.06 \%$ and $\pm 11.08 \%$ were noted for $2.0 \mathrm{~mm}$ nodule measured in $5.0 \mathrm{~mm}$ slices, and for $1.0 \mathrm{~mm}$ slices $\pm 3.6 \%$ and $\pm 2.71 \%$, respectively. Further, volume determination of $2 \mathrm{~mm}$ nodule by lung care software showed the advantage of $3.57 \%, 3.43 \%, 0.49 \%$ and $0.57 \%$ compared with RECIST method for Th. $5.0 \mathrm{~B} 31 \mathrm{f}$ Th.5.0B80f, Th.1.0B31f, and Th.1.0B70f, respectively. The different positions of $5.0 \mathrm{~mm}$ diameter nodule measured by manual ruler shows the mean per cent difference of $2.77 \%, 4.1 \%, 1.79 \%$ and $4.45 \%$ for different reconstructions.

Conclusion: Image slice thickness greatly affects the determination of pulmonary nodule dimension and thinner slice will dramatically improve accuracy. Lung care software showed highest accuracy for the calculation of volumetry compared with other methods. Further, the location of pulmonary nodules, whether it was medial or lateral did not affect its diagnostic accuracy.

\section{B-148 11:33 \\ Prospectively respiratory-triggered high-pitch spiral acquisition for free-breathing dual source CT of the lung: qualitative and quantitative comparison in an ex vivo system \\ J. Dinkel' ${ }^{1}$, C. Hintze', T. Allmendinger ${ }^{2}$, A. Sulzmann ${ }^{3}$, C. Thierfelder ${ }^{2}$, \\ J. Biederer ${ }^{4}$, H.-P. Schlemmer'; ${ }^{1}$ Heidelberg/DE, ${ }^{2}$ Forchheim/DE, \\ ${ }^{3}$ Heiligkreuzsteinach/DE, ${ }^{4} \mathrm{Kiel} / \mathrm{DE}$}

Purpose: Computed tomography (CT) of the lung can be affected by respiratory artefacts especially in infants and incompliant patients. The objective was to analyse image quality of prospective respiratory-gated high-pitch (HP) CT in a ventilated ex vivo system.

Methods and Materials: Four explanted porcine lungs were inflated inside a chest phantom (artiCHEST, PROdesign, Germany) and prepared with 20 artificial nodules simulating metastases (mean diameter: $11.3 \mathrm{~mm} \pm 6$ ). The lungs were ventilated by a computer-assisted diaphragm and scanned with a dual source CT scanner (Definition Flash, Siemens, Germany). Prospective gated HP-CT (pitch=3.4, scan time $0.7 \mathrm{~s}$ ) and conventional breath-hold CT scan were acquired in end inspiration phase. Non-gated HP-CT and conventional CT were also acquired. Semiautomatic volumetry of the nodules was performed (Oncology, Siemens, Germany).

Results: The experiment provided a realistic respiration. The slow time resolution of conventional non-gated CT led to partial projection artefacts and inaccuracy of nodule delineation (mean nodule volumes: $772 \mathrm{~mm} 3$ ). All artefacts and nodule inaccuray predominated close to the diaphragm compared with the upper/middle parts of the lung $(p<0.05)$. With an inplane temporal resolution of $75 \mathrm{~ms}$, the accuracy of prospective HP-CT was particularly improved. The mean volumes from the gated HP-CT, non-gated HP-CT and breathold CT scans were not significantly different $(682,691$ and $672 \mathrm{~mm} 3$, respectively).

Conclusion: Prospective HP-CT revealed excellent quality without relevant motion artefacts. Prospective HP-CT is promising and has the potential to increase significantly the quality of lung imaging in incompliant patient. HP-CT without gating is satisfactory for nodule detection and quantification.

\section{B-149 11:42}

CT-guided marking of pulmonary nodules with a special lung marking wire before video-assisted thoracoscopic surgery: review of 184 cases

P. Stumpp, M.-K. Schulze, U. Eichfeld, T. Kahn;

Leipzig/DE (Patrick.Stumpp@medizin.uni-leipzig.de)

Purpose: Minimally-invasive techniques such as video-assisted thoracoscopic surgery (VATS) nowadays are the method of choice for resection of small pulmonary nodules in the periphery of the lungs. For a quick and save intraoperative identi- fication of the lung nodule a preoperative marking is necessary and reasonable. We report about 184 markings with a special lung marking wire, which is placed around the pulmonary nodule preoperatively using CT-guidance.

Methods and Materials: In 184 patients $(97 \mathrm{~m}, 87 \mathrm{f}$, mean age: $58.1+/-13.7$ years) with suspicious pulmonary nodules planned to undergo resection with VATS a special lung marking wire was placed preoperatively under CT-guidance. We evaluated technical success, safety, necessity of conversion to thoracotomy and histology in all patients.

Results: Marking procedure was successful in 181 cases (98.4\%). There were no major complications, minor adverse events such as pneumothorax (99 cases $=$ $53.3 \%$; maximum size $10.6+/-10.9 \mathrm{~mm}$ ) or a perifocal bleeding ( 56 cases $=30.4 \%$ ) did not necessitate a preoperative therapy. Complete resection of the marked pulmonary nodule was successful in $98.4 \%$ of patients. Conversion to thoracotomy was necessary in 29 patients $(15.9 \%)$ due to bleedings, adhesions, malignancy or wire dislocation. Histology revealed a benign nodule in 96 cases (52.5\%) and a malignant lesion in 87 cases $(47.5 \%)$ out of which only 21 nodules $(11.5 \%)$ turned out to present a primary pulmonary carcinoma.

Conclusion: CT-guided marking of pulmonary nodules using a special marking wire followed by thoracoscopic resection is an efficient and safe method for diagnosing suspicious nodules in the periphery of the lung.

\section{B-150 11:51}

CT-guided chest core biopsy: can we perform it in outpatients? A. Zanello, C. Monti, E. Passera, G. Chiesa, F. Gentinetta, E. Angeli; Bergamo/IT (alessandro.zanello@gavazzeni.it)

Purpose: To evaluate safety and efficacy of CT-guided chest cutting-needle biopsy. Methods and Materials: Between April 2007 and August 2010, 106 CT guided needle biopsies were attempted in 104 out-patients with chest lesion. A $18 \mathrm{G}$ co-axia system and a 64-slice multi-slice CT were used. Technical success, histological diagnosis and complications were retrospectively evaluated.

Results: Specimens were obtained in all cases, with no technical problem. The sampling was diagnostic in 100 out of the 106 biopsies (94.3\%). The results indicated chest cancer in 88 patients $(83.5 \%), 3$ biopsies indicated metastatic disease $(2.8 \%), 6$ excluded the presence of malignancy $(5.6 \%), 2$ were granulomatous disease $(1.8 \%)$ and 1 was a solitary fibrous tumour of the pleura $(0.9 \%)$. Complications included pneumothorax (36.7\%), isolate parenchymal bleeding ( $8.4 \%)$, parenchymal bleeding associated to pneumothorax $(4.7 \%)$ and haemoptysis $(3.7 \%)$. Mild or no significant complications occurred in 75 cases $(72 \%)$. Only in 3 cases $(2.8 \%)$ a precautional night in hospital was necessary, in 1 case because of bronchial hemorrhage with desaturation, and in 2 drained pneumothorax. All the remaining patients left the radiological department after 2 to 4 hours observation. Conclusion: Micro-histological CT guided needle biopsy is a safe and effective procedure. Multislice-CT guidance allows the choice of the appropriate path. The procedure can be performed in out-patients avoiding unnecessary hospitalization.

$10: 30-12: 00$

Room D2

Interventional Radiology

\section{SS 409}

\section{Chemoembolisation of liver tumours}

Moderators:

N. Fotiadis; London/UK

J. Tacke; Passau/DE

\section{B-151 10:30}

A prospective randomised controlled trial of adjuvant trans-arterial intrahepatic ${ }^{131}$-lipiodol following curative resection of hepatocellular carcinoma: technical considerations and results

B.S. Tan, A.Y.F. Chung, L.L.P.J. Ooi, D. Machin, C.H. Thng, R. Lo, K.H. Tay, A.S.W. Goh, K.C. Soo; Singapore/SG (tan.bien.soo@sgh.com.sg)

Purpose: To evaluate whether adjuvant trans-arterial injection of ${ }^{131}$-lipiodol is effective against recurrence of surgically resected hepatocellular carcinoma (HCC). Methods and Materials: This multi-centre prospective randomised controlled tria enrolled 103 patients from June 2001 through March 2007 who were 4-6 weeks postoperative for curative resection of $\mathrm{HCC}$ with complete recovery (52 randomised to adjuvant ${ }^{131}$-lipiodol, 51 to control). In the patients randomised to the adjuvant group, ${ }^{131} 1$-lipiodol was administered trans-arterially following selective hepatic arteriography. Patients were followed-up every 3 months for 1 year, then every 6 months thereafter. Recurrence-free survival (RFS) was the primary endpoint and overall survival (OS) secondary, both evaluated using the Kaplan-Meier technique. 
Results: The ${ }^{131}$-lipiodol group showed improved RFS (HR $=0.75$ [95\% Cl 0.45 to $1 \cdot 25, \mathrm{p}=0.27])$ and, to a lesser degree, OS $(\mathrm{HR}=0.90[95 \% \mathrm{Cl} 0.51$ to $1.58, \mathrm{p}=$ $0 \cdot 72])$ compared to control, though neither reached statistical significance and the improvements were less than those seen in a previous similar trial. The combined results showed a non-significant improvement of RFS for the ${ }^{131}$ |-lipiodol group (HR $=0.67[95 \% \mathrm{Cl} 0.44$ to $1.03, \mathrm{p}=0.07])$, with a less striking trend in OS $(\mathrm{HR}=0.69$ [95\% $\mathrm{Cl} 0.44$ to $1 \cdot 11, \mathrm{p}=0 \cdot 13])$. This trial's data also showed a non-significant improved RFS, though not improved OS, in patients without vascular invasion. There were 2 serious adverse events reported, one patient with hypothyroidism caused by ${ }^{131}$-lipiodol and one hepatic artery dissection during treatment.

Conclusion: Our results do not support the use of adjuvant ${ }^{131}$-lipiodol as a standard postoperative treatment for patients who have surgical resection of an HCC.

\section{B-152 10:39}

Multi-centre phase II study of SIR-spheres plus Sorafenib in patients with non-resectable hepatocellular carcinoma. The Asia-Pacific HCC trials group protocol 05 (AHCC05)

M.C. Burgmans, R.H.G. Lo, K.H. Tay, B.S. Tan, F.G. Irani, L.S. Khoo,

A.S.W. Goh, K.C. Soo, P.K.H. Chow; Singapore/SG (mburgmans@hotmail.com)

Purpose: Both loco-regional therapy with selective internal radiation therapy (SIRT) and systemic treatment with Sorafenib have shown to increase survival in patients with inoperable HCC. Combined treatment with SIRT and sorafenib may offer additional survival benefit.

Methods and Materials: Prospective multicentre study of combination sequential SIRT-sorafenib therapy. Patients with inoperable HCC (Barcelona Clinic Liver Criteria (BCLC) stages $B$ and C) were eligible. Enrolled patients were administered SIR-spheres (maximum dose $3 \mathrm{GBq}$ ) and subsequently administered sorafenib therapy (400 mg b.i.d). Monthly clinical and biochemical follow-up as well as 3-montly CT was performed. Using an A'Hen's single stage design, we assumed target tumour response rate of $30 \%$ and no further interest response rate of $10 \%$ with type lerror of 0.05 and power of 0.85 . Results: Between June 2008 and June 2009, 35 patients were enrolled. Median follow-up was 47 weeks (range 10-94 weeks). Overall tumour response was 35.5\% with $12 \%$ complete regression, $23.5 \%$ partial regression, $44 \%$ stable disease and $21 \%$ progressive disease. Disease control rate in BCLC B and C patients was $100 \%$ and $68 \%$, respectively. Median overall survival (OS) was 47 weeks ( $95 \% \mathrm{Cl} 30$ to 70 weeks). OS for BCLC B and BCLC C patients was 73 and 35 weeks, respectively. Median Time to Disease Progression (TTP) was 39 weeks (95\% Cl 27-73 weeks). TTP has not been reached for BCLC B patients and was 35 weeks for BCLC C patients. Conclusion: The SIRT-sorafenib combination therapy demonstrated good efficacy in advanced HCC especially in BCLC B patients and exceeded expected response rate.

\section{B-153 10:48}

Tumour response to transarterial chemoembolisation in hepatocellular carcinoma: are the amended RECIST criteria reliable?

I. Bargellini, A. Cicorelli, V. Battaglia, B. Parentini, P. Carrai, R. Cioni, F. Filipponi, C. Bartolozzi; Pisa/IT (irenebargellini@hotmail.com)

Purpose: To retrospectively analyse the tumour response to transarterial chemoembolisation (TACE) in a series of consecutive HCC transplanted patients and evaluate the agreement between amended RECIST criteria and percentage of tumour necrosis, assessed at pathology.

Methods and Materials: From January 1996 to December 2009, 384 cirrhotic patients $(\mathrm{M} / \mathrm{F}=336 / 48$, mean age $55 \pm 7$ years) with $\mathrm{HCC}$ underwent liver transplantation (LT). Among them, $200(52 \%)$ patients (mean number of nodules $2.2 \pm 1.6$, mean diameter $31.2 \pm 15 \mathrm{~mm}$ ) were treated by TACE prior to LT. The CT examinations performed one month after TACE were retrospectively reviewed by two experienced radiologists in consensus, to assess tumour response to TACE according to the amended RECIST criteria. After LT, percentage of tumour necrosis was pathologically assessed on the explanted liver.

Results: CT follow-up at 1 month was available in 165/200 (82.5\%) patients; in the remaining cases, LT was performed within the first month after TACE. According to the amended RECIST criteria, the objective response rate was 87.3\% (144/165), with 79 cases $(47.9 \%)$ of complete response. On the explanted livers, mean percentage of tumour necrosis was $65 \pm 38 \%$ (range $0-100 \%$ ), with $52 \%$ of patients with necrosis $\geq 90 \%$. A significant correspondence $(p<.0001)$ was found between amended RECIST criteria and percentage of tumour necrosis; mean rate of necrosis was $83 \%$ in patients with complete response, $75 \%$ in patients with partial response and $23 \%$ in subjects with stable disease. Conclusion: TACE is effective, inducing total /subtotal necrosis in over $50 \%$ of the patients. The amended RECIST criteria assessed by triphasic CT are reliable.
B-154 10:57

Treatment of hepatocellular carcinoma: prospective study of Doxorubicin-eluting bead embolisation compared with conventional chemoembolisation

M. Ferrer-Puchol, C. la Parra, E. Esteban-Hernández, M. Vaño, A. Vera, M. Forment, J. Cano, O. Cosín; Alzira/ES (eesteban@hospital-ribera.com)

Purpose: This study assesses the comparison between using conventional transarterial chemoembolisation (TACE) and chemoembolisation with drug eluting-beads (DEB-TACE) in patients with hepatocellular carcinoma (HCC).

Methods and Materials: We present 72 patients diagnosed with HCC. The study included 25 consecutive patients treated with doxorubicin TACE (Group A) and 47 consecutive patients treated with doxorubicin DEB-TACE (Group B) recruited from January 2000 to December 2009. All patients were in compensated Child-Pugh cirrhosis. Response was studied at 6 months with Computed Tomography (CT) or Magnetic Resonance (MR), according to RECIST criteria. Comparisons among two groups of treatment were made by ANOVA, Chi-square, T-test and Kaplan-Meier Estimator with a Log-Rank test.

Results: The age, size, number of tumours and liver reserve of patients in two groups were statistically similar. Number of sessions for patient was Group A mean $1.32 \pm 0.67$ times, Group B mean $2.13 \pm 0.95$ times. Mean dose of doxorubicine for patient was $50.60 \pm 29.95 \mathrm{mg}$ in Group A and $231.91 \pm 110.2 \mathrm{mg}$ in Group B. Complete response (total necrosis) of tumour was observed in $5.6 \%$ in Group A and $13.9 \%$ in Group B. According to RECIST criteria no significant differences were found. DEB-TACE was better tolerated with fewer immediate complications $(p=0.001)$. No difference in survival was found. Group A: mean 686.24, median 709 days, and Group B: mean 765.32, median 672 days.

Conclusion: In patients with unresectable HCC, transcatheter therapy with Doxorubicin-eluting beads is safe and better tolerated despite the higher mean total dose administered.

\section{B-155 11:06}

Transarterial chemoembolisation of unresectable hepatocellular carcinoma (HCC): drug-eluting-bead embolisation (DEB-TACE) vs conventional TACE with cisplatin/lipiodol (cTACE)

P. Wiggermann ${ }^{1}$, C. Brosche ${ }^{2}$, T. Brauer ${ }^{2}$, D. Sieron ${ }^{2}$, C. Stroszczynski ${ }^{1}$; ${ }^{1}$ Regensburg/DE, ${ }^{2}$ Dresden/DE (philippwiggermann@googlemail.com)

Purpose: Outcome evaluation of DEB-TACE compared to cTACE in patients with unresectable HCC.

Methods and Materials: Forty-four consecutive patients with an unresectable $\mathrm{HCC}(\mathrm{BCLC} A / \mathrm{B})$ were analysed. All patients were either treated with one or more procedures of CTACE $(n=22)$ or with one or more procedures of DEB-TACE $(n=$ 22). A total of 89 TACE procedures were performed. Two radiologists evaluated tumour response rates in accordance with the European Association for the Study of the Liver (EASL) response criteria by consensus reading.

Results: Objective response (OR, complete response + partial response) for DEB-TACE was $22.7 \%$; a further $68.2 \%$ was stable disease (SD). The respective response rates for the CTACE were OR 22.7 and SD 31.8\%. Mean survival after DEB-TACE was significantly prolonged with $651 \pm 76$ days vs. $414 \pm 43$ days for cTACE $(p=0.01)$.

Conclusion: This preliminary study indicates that DEB-TACE may have a higher efficacy than cTACE

\section{B-156 11:15}

Intra-procedural C-arm dual-phase cone-beam CT imaging to predict response of hepatocellular carcinoma during drug-eluting bead transcatheter arterial chemoembolisation

R. Loffroy' ${ }^{1}$, M.D. Lin², P. Rao ${ }^{2}$, N. Bhagat ${ }^{2}$, E. Liapi ${ }^{2}$, N. Noordhoek ${ }^{3}$, A. Radaelli ${ }^{3}$, J. Blijd ${ }^{3}$, J.F. Geschwind ${ }^{2} ;{ }^{1}$ Dijon/FR, ${ }^{2}$ Baltimore, MD/US, ${ }^{3}$ Best/NL (romaric.loffroy@gmail.com)

Purpose: To investigate whether intra-procedural dual-phase C-arm cone-beam CT (DPCBCT) can predict hepatocellular carcinoma $(\mathrm{HCC})$ response treated using drug-eluting bead transarterial chemoembolisation (DEB-TACE) as compared to contrast-enhanced MRI, imaging gold-standard. Both modalities capture early- and delayed-phase tumour enhancement.

Methods and Materials: Forty-seven lesions in 27 patients (15 males, age $61.9 \pm 10.7$ years) with unresectable HCC treated with DEB-TACE were retrospectively analysed. MRI was performed 1 month before and after DEB-TACE. Intra-procedural DPCBCT imaging was done immediately before and after DEBTACE. Pre- and post-procedural DPCBCT tumour enhancement (TE) at early- and delayed-phases was assessed by two experienced radiologists blind to MRI findings. Tumour response (TR) was measured according to European Association 
for the Study of the Liver criteria (EASL). The change in TE compared between modalities.

Results: A favourable (complete or partial) EASL TR was achieved in $74.5 \%$ and $76.6 \%$ of lesions at 1 month post DEB-TACE on MR early- and delayed-phases, respectively. Paired T-tests comparing pre- and post-TACE TE showed statistically significant average reduction in TE for both modalities by phase and lesion $(p<0.01)$. MRI TE decrease post-TACE linearly correlated with DPCBCT, Pearson correlation coefficient was excellent (0.80). A statistically significant relationship between post-TACE DPCBCT TE and favourable MRI TR was found: every 1 unit TE increment in DPCBCT resulted in decrease of TR by $5 \%(95 \% \mathrm{Cl}$; range $0.91-0.99 ; \mathrm{p}=0.027)$ and $4 \%(95 \% \mathrm{Cl}$; range 0.93-1.00; $p=0.034$ ), for early and delayed-phases, respectively, on MRI

Conclusion: HCC 1-month post-DEB-TACE response as seen in MRI can be predicted by DPCBCT during the procedure.

\section{B-157 11:24}

Relationship of apparent diffusion coefficient to survival for patients with unresectable primary hepatocellular carcinoma after chemoembolisation X.-D. $\underline{Y e}^{1}$, Z. Yuan ${ }^{2}$, S. Dong ${ }^{1}$, X.-S. Xiao ${ }^{1} ;{ }^{1}$ Shanghai/CN, ${ }^{2}$ Nanjing/CN (yuanyxd@163.com)

Purpose: To evaluate the prognostic value of apparent diffusion coefficient (ADC) values from MR diffusion-weighted imaging of unresctable hepatocellular carcinoma after chemoembolisation.

Methods and Materials: our study was proved by our institute and informed consent was obtained from all patients before commencement of the study. Twenty-three patients with unresctable hepatocellular carcinoma were scanned immediately before and after chemoembolisation within $24 \mathrm{~h}$ using conventional anatomical MR imaging and diffusion-weighted imaging, from which ADC values in the lesions were measured. The changes in ADC values after chemoembolisation were calculated. The relationship between the lesion ADC and the survival time was analysed by correlation analysis. The overall cumulative survival was analysed by the KaplanMeier method, and survival curves were compared by the log-rank test. Results: The mean overall survival period was $(25.0 \pm 8.7)$ months. The pre-chemoembolisation lesion ADC value was $(1.36 \pm 0.249) \times 10^{-3} \mathrm{~mm}^{2} / \mathrm{s}$; the change in ADC values post-chemoembolisation was $(0.377 \pm 0.332) \times 10^{-3} \mathrm{~mm}^{2} / \mathrm{s}$. There were significant linear regression relation between the survival time and pre-chemoembolisation lesion ADC values $(r=-0.698, P<0.001)$ or the changes in ADC value post-chemoembolisation $(r=0.702, P<0.001)$. And Log-rank test showed that pre-chemoembolisation ADC values $\left(\chi^{2}=7.339, P=0.007\right)$ or the changes in ADC value post-chemoembolisation $\left(\chi^{2}=9.820, P=0.002\right)$ significantly influenced the overall cumulative survival. Conclusion: Pre-treatment ADC values as well as changes in ADC values after treatment may provide useful information for predicting survival for patients with unresctable hepatocellular carcinoma.

\section{B-158 11:33}

Tumour response in liver metastases from the most common primary sites after repetitive transarterial chemoembolisation (TACE): a competitive evaluation

P. Farshid, A. Davishi Nakhl Ebrahimi, A. Azizi, N. Naguib, E. Mbalisike,

T.J. Vogl; Frankfurt a. Main/DE (parvizfarshid@gmail.com)

Purpose: Competitive evaluation of results in the palliative treatment of patients with liver metastases from the most common primary sites using transarterial chemoembolisation (TACE)

Methods and Materials: TACE was repeatedly performed in 105 patients with liver metastases from colon (31), breast (21), the uveal malignant melanoma (13), pancreas (31) and stomach (9). Radiologists retrospectively evaluated tumour response by MRI. The response evaluation criteria in solid tumours (RECIST) and specific growth rate (SGR) were used to assess tumour response. Survival parameters were calculated by Kaplan-Meier method.

Results: Tumour response after TACE [mean, 4.18 sessions per patient (range: 2-12)] following RECIST and SGR evaluation showed no statistically significant difference between all groups [7.1015, $(p=0.5257)$ and 4.5121, $(p=0.3411)]$. Mean of SGR (\% per day), for liver metastases from colon, breast, uveal malignant melanoma, pancreas and stomach was $-0.241,-0.208,-0.12,-0.099$, and -0.074 , respectively. Mean survival time and survival rate ( 1 and 3 years) from first TACE were for colon, 27, 89.5\%, 51\%; breast, 25.9, 88\%, 49\%; malignant melanoma, $20.83,82.5 \%$, $47.5 \%$; pancreas, $16.72,62 \%$, 33\%, and stomach $13,55 \%$, $28 \%$. Median TTP and progression-free survival rate for 1 year were for liver metastases from colon 4.1, 89\%; breast 3.5, 91\%; uveal melanoma 4.3, 88\%; Pancreas 2.91, $90 \%$, and stomach $3.38,53 \%$.

Conclusion: Despite lack of statistically significant difference in local tumour response after TACE using size- and volume-based evaluation, survival indexes were better in colon, breast, uveal melanoma, pancreas and stomach liver metastasis, respectively.

\section{B-159 11:42}

Differences in local tumour control of liver metastases from colorectal cancer between the treated liver lobes: treatment with transarterial chemoembolisation (TACE)

T.J. Vogl, A. Salega, S. Zangos, N.N. Naguib;

Frankfurta. Main/DE (t.vogl@em.uni-frankfurt.de)

Purpose: To evaluate differences in treatment response of palliative and symptomatic repeated transarterial chemoembolisation (TACE) of metastases from colorectal cancer between left and right liver lobes.

Methods and Materials: Between January 2009 and March 2010, repeated TACE was performed in 28 patients (mean:54.61 years, range:27-79) with unresectable metastases of colorectal cancer in both liver lobes. Local chemotherapy protocols consisted of Mitomycin/Irinotecan/Cisplatin ( $n=19)$, Mitomycin/Gemzar/Cisplatin $(n=5)$, Oxaliplatin $(n=1)$, and 3-bromopyruvate (3BP) $(n=1)$ (147 sessions mean:5.3 sessions/patient). The overall response was assessed by separately measuring the volume in unenhanced MRI for target lesions in each liver lobe. The absolute change in tumour size was classified as progressive disease (PD), stable disease (SD) and partial response (PR).

Results: Mean volume change in 147 treatments was a tumour growth of $5.18 \%$, matching the criteria for stable disease. The mean response on metastases of the right liver lobe was a reduction in size by $7.59 \%$ versus an increase of $18.01 \%$ in the left lobe lesions. According to the volume change in the left liver lobe 14 patients were classified as SD, 9 as PD and 5 as PR. The right volume changes were classified as follows: 15 SD, 5 PD and 8 PR.

Conclusion: Repeated TACE is a relevant palliative treatment option in liver metastases of colorectal cancer. However, the different vascularisation of the left liver lobe causes a more difficult access to the targeted tissues. Hence the used agents can be applied more effectively to the right liver lobe resulting in a better tumour control.

\section{B-160 11:51}

90Yttrium-radioembolisation of chemotherapy-refractory colorectal cancer liver metastases

P.M. Paprottka, R.T. Hoffmann, F. Räßler, C. Trumm, G.P. Schmidt, M.F. Reiser, T.F. Jakobs; Munich/DE (philipp.paprottka@med.uni-muenchen.de)

Purpose: To evaluate safety and survival outcomes of radioembolisation with 90 Yttrium-microspheres in patients with colorectal cancer liver metastases in whom currently available therapies had failed.

Methods and Materials: One hundred patients with colorectal cancer liver metastases in whom chemotherapy had failed, underwent single-session, whole-liver $90 \mathrm{Y}$ radioembolisation. Response and toxicity were assessed by computed tomography/ magnetic resonance imaging utilising the response evaluation criteria in solid tumours (RECIST) and the National Cancer Institute's Common Terminology Criteria for Adverse Events (CTCAE v3.0). Furthermore, tumour-marker levels, laboratory/ clinical toxicities and survival were evaluated.

Results: The median activity delivered was $1.87 \mathrm{GBq}$. Imaging follow-up at 3 months demonstrated partial response, stable disease and progressive disease in $20.8 \%, 75 \%$ and $4.2 \%$, respectively. Median overall survival was 11.2 months with improved survival for patients with a decrease in carcinoembryonic antigen level (18.7 vs 5.2 months) and imaging response ( 28.7 vs 4.4 months; $p<0.001$ ) Except for one instance of treatment-associated cholecystitis (grade 4 toxicity) there were no acute or delayed toxicities according to the CTCAE criteria higher than grade 2. No radiation-induced liver disease was noted.

Conclusion: Single-session, whole-liver radioembolisation with 90Yttrium-microspheres is a safe and effective treatment option for patients in whom currently available therapies had failed. Anti-tumoural effect is supported by good local tumour control, decrease in tumour marker levels and significant improved survival for patients with imaging response. Further investigation is warranted to determine the optimal use of this emerging therapeutic modality. 


\section{GI Tract}

\section{SS 401b}

\section{MRI of rectal cancer}

\section{Moderators:}

M. Bellomi; Milan/IT

C. Hoeffel; Reims/FR

\section{B-161 10:30}

Can restaging-MRI identify changes in nodal distribution that may explain a better selection of the yNO rectal cancer patients?

M. Maas, D.M.J. Lambregts, M. Berkhof, G.L. Beets, R.G.H. Beets-Tan; Maastricht/NL (moniquemaas@live.nl)

Purpose: Recent publications show that MRI in rectal cancer is more accurate in selecting yNO-patients after chemoradiation (CRT) than in selecting the cNO-patients at primary-staging. This improved accuracy facilitates selection of patients for local excision after CRT. We aimed to evaluate whether changes in nodal distribution and size after CRT can explain this phenomenon.

Methods and Materials: We included 46 patients with locally advanced rectal cancer (LARC) who underwent preoperative CRT (group A) and 26 patients with non-LARC who underwent immediate surgery with/without preoperative 5x5Gy (group B). Group A underwent MRI before and 6-8 weeks after CRT and group $B$ underwent pretreatment-MRI only. Nodes were analysed on 3DT1W-GRE with $1 \mathrm{~mm}^{3}$ isotropic voxels. For each node, short axis diameter was measured. For group A, nodes were measured and matched on pre- and post-CRT MRI. Results:In group A, 882 nodes were identified on pre-CRT-MRI, of which 380 (43\%) disappeared after CRT. Mean number of nodes/patient decreased from $21( \pm 9)$ pre-CRT to $13( \pm 7)$ post-CRT $(p<0.0001)$. Mean nodal size $(\mathrm{mm})$ decreased from $3.8( \pm 2)$ pre-CRT to $2.6( \pm 1.8)$ post-CRT $(p<0.0001)$. Mean size on pre-CRT-MRI of the 380 nodes that disappeared after CRT was smaller than that of the nodes that were still visible after CRT: $2.9( \pm 1.4)$ vs. $3.8( \pm 2.0) \mathrm{mm}(p<0.0001)$. In group B 558 mesorectal nodes were visualised (mean $22( \pm 11)$ nodes/patient). Conclusion:Pretreatment number of nodes does not differ between LARC and non-LARC. After CRT, mean number and size of nodes decrease significantly. Most small nodes disappear after CRT. These findings may contribute to the fact that size-criteria work better for restaging-MRI after CRT.

\section{B-162 10:39}

The added value of diffusion weighted imaging in combination with T2 weighted imaging for the evaluation of rectal cancer response to neoadjuvant treatments

L. Monguzzi', D. Ippolito', C. Capraro', M. Colombo', C. Talei Franzesi', F. Invernizzi', E. De Ponti', S. Sironi'; ' ${ }^{M}$ Monza/IT, ${ }^{2}$ Desio/lT (letizia.monguzzi@gmail.com)

Purpose: To assess the added value of DWI in combination with T2-weighted imaging in determining of tumour response to CRT, as compared to T2WI alone, correlated with tumour histopatologic downstaging as reference standard. Methods and Materials: A total of 29 patients with LARC, who underwent CRT and subsequent surgery, underwent pre- and post-CRT MRI examination (1.5 T scanner, Achieva, Philips) with following protocol: multiplanar T2 and T1 TSE images and in addition DWI sequences (b factor: 0 and $1000 \mathrm{~mm}^{2} / \mathrm{sec}$ ). Two readers in consensus, blinded to the results, evaluated T2WI without and with DWI to detect the presence of complete response by a four-point scale as follows (1: definitely absent; 2 : probably absent; 3 : probably present; 4 : definitely present). Mean value of ADC of pre- and post-CRT DWI were compared between responders and non-responders patients and correlated with histopatological response classified as tumour regression grade (Mandard's criteria; TRG1 = complete regression, TRG5 = no regression).

Results: All of the patients underwent total mesorectal excision and Mandard's criteria were calculated (responders: TRG1-2; non-responders: TRG3-5). Twenty tumours $(75 \%)$ showed complete (TRG1) or subtotal regression (TRG2) and were classified as responders; nine tumours $(25 \%)$ were classified as non-responders (TRG3-5). Mean ADC value in MR1 examination was $0.86 \pm 0.22 \times 10-3 \mathrm{~mm}^{2 /}$ $\mathrm{sec}$, whereas mean values after CRT was $1.43 \pm 0.25 \times 10^{-3} \mathrm{~mm}^{2} / \mathrm{sec}$. On T2W images both readers correctly identified 12 of 18 patients with complete response (specificity $65 \%$ ) while after addition of DWI the readers correctly identified 170 f the complete responders (spec 95\%) patients. The analysis showed that each of the two readers achieved more accurate results with T2 imaging combined with DW images than T2W images alone.
Conclusion: DW-MR imaging is a non-invasive imaging approach and a sensitive technique that allows the identification of spatially distinct regional responses to therapy within tumour tissues.

\section{B-163 10:48}

Diagnostic value of MRI, diffusion-weighted MRI and fusion images for the detection of locally recurrent rectal cancer

D.M.J. Lambregts, M. Maas, V.C. Cappendijk, G.L. Beets, R.G.H. Beets-Tan; Maastricht/NL (d.lambregts@mumc.nl)

Purpose: To assess the value of [1] diffusion-weighted MRI (DWI) and [2] T2W+DWI fusion images, in addition to standard T2W-MRI for the detection of a local recurrence after rectal cancer surgery.

Methods and Materials: 42 patients with a clinical suspicion of recurrence (increased CEA, clinical symptoms and/or abnormal CT-findings) underwent MRI (1.5 T), consisting of T2-weighted and DWI (b0.500.1000) sequences. T2W+DWI fusion-images were generated on an offline workstation. Two independent readers scored the likelihood of recurrence [1] on T2W-MRI, [2] after addition of DWI and [3] on T2W+DWI fusion images. The reference standard consisted of histology $(n=20), P E T$ and/or endoscopy + clinical follow-up.

Results: $19 / 42$ patients had a local recurrence. On T2W-MRI, reader 1 achieved an area under the ROC-curve (AUC) of 0.99 , sensitivity $100 \%$ and specificity $83 \%$. For reader 2 these figures were $0.87,84 \%$ and $74 \%$. After addition of DWI, reader 1 achieved an AUC of 0.98 , sensitivity $100 \%$ and specificity $91 \%$. Compared with T2W-MRI, the difference in AUC was not significant $(p=0.78)$. For reader 2 these figures were $0.91,89 \%$ and $83 \%(p=0.09)$. Fusion images did not improve accuracy compared with T2W-MRI+DWI, but were helpful for a better anatomical correlation. Interobserver-agreement was $\mathrm{k} 0.69$ for T2W-MRI, 0.82 for T2W-MRI+DWI and 0.84 for the fusion images.

Conclusion: MRI has a high accuracy for the detection of locally recurrent rectal cancer. Addition of DWI does not significantly improve its performance. However, with DWI specificity and interobserver-agreement increase. Fusion images do not further improve accuracy.

\section{B-164 10:57}

Diffusion-weighted magnetic resonance imaging combined T2-weighted imaging evaluation in the pre-operative staging of primary rectal carcinoma R. Rong, X.Y. Wang, X.X. Jiang, X.W. Sun; Beijing/CN (thornapplezixi@gmail.com)

Purpose: To investigate the added value of diffusion-weighted (DW) MR imaging in the evaluation of pre-operative assessment of T staging in patients with primary rectal carcinoma.

Methods and Materials: 27 patients have been selected in the study: 18 males and 9 females (mean age $60.7 \pm 13.9$ years; range $27-83$ years). Retrospectively, one radiologist scored T2-weighted MR images and, in a second reading, T2-weighted images combined with DW MR images for tumour penetration through rectal wall and extension into mesorectal fascia. Spearman correlation was used to evaluate the pre-operative staging consistency of T2-weighted and combined T2-weighted plus DW MR images according to histopathological results. ROC curve was drawn to evaluate the diagnostic efficacy of the two.

Results: For T staging of rectal cancer, addition of DW imaging correctly staged patients with rectal cancer more often than T2-weighted imaging (Spearman correlation: 0.695 vs. $0.456, P<0.05$ ). Addition of DW MR imaging was superior to T2-weighted imaging in the differentiation between T11T2 tumour (positive group) and T3IT4 tumour (negative group) (sensitivity: 0.625 vs. 0.25 ; spe: 0.947 vs. 0.895 PPV: 0.833 vs. 0.5 ; NPV: 0.857 vs. 0.739 ; Acc: 0.852 vs. 0.704 ). The dignostic efficacy of addition of DW MR imaging was superior to conventional T2-weighted MR imaging in the preoperative T staging (the areas under the ROC curve of the two are, respectively, 0.826, $P=0.009$ and $0.651, P=0.222$ ).

Conclusion: Adding DW MR imaging to conventional T2-weighted MR imaging yields better diagnostic efficacy than conventional T2-weighted MR imaging alone in the evaluation of border-line T3 tumours.

\section{B-165 11:06}

Value of DWI for treatment monitoring after chemoradiation in rectal cancer: can ADC be used as a parameter for response?

D.M.J. Lambregts, M. Maas, F.C.H. Bakers, V.C. Cappendijk, G.L. Beets, R.G.H. Beets-Tan; Maastricht/NL (d.lambregts@mumc.nl)

Purpose: To evaluate the value of $A D C$ as a parameter for monitoring response to preoperative chemoradiation (CRT) in rectal cancer.

Methods and Materials: 30 locally advanced rectal cancer patients underwent neoadjuvant CRT followed by a 6-8 week time interval and subsequent surgery. 
All patients underwent MRI, including DWI (b-values 0.500.1000) before and 6-8 weeks after CRT. Mean ADC of the primary tumour was measured at both timepoints. When after CRT no clear tumour or scar tissue could be identified, ADC of the primary tumour bed was measured. Histology was the standard reference. The relative change $(\Delta)$ in ADC (\%) after treatment was compared for good and poor-responding tumours. A good response was defined as a histological tumour regression grade (TRG, Mandard) of 0-2 and a poor response as TRG3-4.

Results: At histologic evaluation 17 patients had a TRG0-2 and 12 patients a TRG3-4. After chemoradiation, a significant increase in ADC was observed in all patients: mean pre-treatment ADC was $1.11 \pm 0.22$ versus $1.47 \pm 0.25^{*} 10^{-3} \mathrm{~mm}^{2} / \mathrm{s}$ after treatment $(p<0.001)$. The relative increase in ADC $(\%)$ was larger in the good responders (43\% ADC-increase) than in the poor-responders $(31 \%)$ but this difference was not statistically significant $(p=0.2)$.

Conclusion: $\triangle A D C$ tends to be higher for the good responders as compared with the poor responders, although the difference was not significant. This could partly be attributed to the small number of patients. Larger studies will show whether relative changes in ADC will be useful to discriminate between good- and poorresponding tumours.

\section{B-166 $11: 15$}

\section{Spectral fat saturation inversion recovery MR sequence after gadolinium} injection to differentiate fibrotic and neoplastic strands in the mesorectal fat in patients with restaging of rectal carcinoma after neoadjuvant chemoand radiation therapy

E. Quaia, V. Ulcigrai, C. Federica, M. Cavallaro, M.A. Cova; Trieste/IT (quaia@units.it)

Purpose: To retrospectively assess the capabilities of contrast-enhanced spectral fat saturation inversion recovery (SPIR) MR sequence in the differentiation between fibrotic and neoplastic strands within the mesorectal fat in the restaging of patients with locally advanced rectal carcinoma after neoadjuvant radiochemotherapy.

Methods and Materials: Forty consecutive patients (27 males, 13 females; mean age $\pm S D, 65.1 \pm 8.5$ years) with locally advanced rectal carcinoma underwent preoperative neoadjuvant radio-chemotherapy followed by surgery. MR imaging was performed 15-25 days after completion of neoadjuvant therapy by T2-weighted fast spin-echo (TR/TE, 2500/100 msec) and T1-weighted SPIR sequence (TR/ TE, $570 / 50 \mathrm{msec})$ after Gd HP-DO3 A injection $(0.1 \mathrm{mmoL} / \mathrm{kg})$. Two experienced radiologists $(5,10$ years) visually analysed in consensus the MR images obtained before and after neoadjuvant therapy and evaluated the evidence of enhancing nodules or strands. Logistic regressions were conducted to test the significance of MR image findings with histologic analysis.

Results: After neoadjuvant treatment, the disease was limited to the wall $(n=18$ patients), or revealed mesorectal fat infiltration $(n=22)$ at histology. In 20 patients enhancing strands in the mesorectal fat were identified, and reticular enhancing strands reaching the mesorectal fascia presented the highest correlation with mesorectal fat tumoural infiltration (OR $28.52,95 \% \mathrm{Cls}$ : 1.34-604.1), while mesorectal enhancing nodules (OR.295, 95\%Cls:.024-3.58) and separate enhacing strands reaching or not the mesorectal fascia (OR.07 or.12, 95\%Cls:.007-.65 or.012-1.25) revealed the lowest correlation.

Conclusion: The fibrotic or tumoural nature of the enhancing strands within the mesorectal fat evident after neoadjuvant radiochemotherapy may be characterized by contrast-enhanced SPIR MR sequence.

\section{B-167 11:24}

Selection of patients with rectal cancer for local excision with lymph-nodespecific contrast-enhanced MRI

M. Maas ${ }^{1}$, D.M.J. Lambregts ${ }^{1}$, J.W.A. Leijtens ${ }^{2}$, S.M.E. Engelen ${ }^{1}$,

R.M. van Dam ${ }^{1}$, G.L. Beets ${ }^{1}$, R.G.H. Beets-Tan ${ }^{1} ;{ }^{1}$ Maastricht/NL, ${ }^{2}$ Roermond/NL (moniquemaas@live.nl)

Purpose: Patients with T1-2 rectal cancer have $5-20 \%$ risk for nodal metastases, which cannot be accurately evaluated with standard imaging. To minimise the risk for local recurrence (LR), total mesorectal excision (TME) is performed in these patients. Accurate prediction of $\mathrm{NO}$-status could make local excision (LE) feasible. The aim of this study is to evaluate whether contrast-enhanced (CE)-MRI is useful in selecting patients with NO-status for LE.

Methods and Materials: 38 patients who underwent LE for rectal cancer were included and underwent T2W\&T1W-CE-MRI with ultrasmall superparamagnetic particles of iron-oxide (USPIO; $n=26$ ) or gadofosveset $(n=12)$ to evaluate $N$-stage and residual tumour. A radiologist experienced in rectal $\mathrm{MRI}$ and the CE-images evaluated the images. In case of NO-status, patients underwent follow-up with regular visits, endoscopy and/or MRI as part of a prospective study. Patients with $\mathrm{N}+$ status underwent TME ( \pm neoadjuvant therapy).
Results: CE-MRI indicated N0-status in 35/38 patients, who underwent follow-up. 3 patients had suspected $\mathrm{N}+$ status, of whom 2 had surgery, which confirmed the $\mathrm{N}$ +status in one patient, but refuted $\mathrm{N}+$ status in the other (false-positive). The third patient underwent chemoradiation which sterilised the nodes. These 3 patients are disease-free and alive. Median follow-up for all patients is 31 months (0-65). Three luminal and one nodal LR were found in the follow-up group. All recurrences were curatively treated. 2 -year disease-free survival is $94 \%$.

Conclusion: CE-MRI with lymph-node-specific contrast-agents is useful for selecting N0-patients for LE. The low LR-rate and resectability of the local recurrences suggest that CE-MRI can play a role in safely selecting T1-2N0 tumours for LE, leading to lower treatment-related morbidity.

\section{B-168 11:33}

Usefulness of dynamic contrast-enhanced magnetic resonance imaging for evaluation of therapeutic response to neoadjuvant chemoradiation therapy in locally advanced rectal cancer

S. Kim, J. Lee, J. Han, B. Choi; Seoul/KR (radiresi@gmail.com)

Purpose: To evaluate the efficacy of dynamic contrast-enhanced magnetic resonance imaging (DCE-MRI) for assessing the therapeutic response to neoadjuvant chemoradiation therapy (CRT) for locally advanced rectal cancer.

Methods and Materials: Between June 2009 and August 2010, 50 patients (32 males, 18 females; mean age, 64 years; range, $40-82$ years) with locally advanced rectal cancer ( $\geq$ T3 or lymph node positive) who underwent CRT and subsequent surgery, were included in this study. All patients underwent pre- and post-CRT, 1.5-T DCE-MRI. A radiologist blinded to the final pathology drew a region of interest along the border of the tumour on the pre-and post-CRT MRI. Perfusion parameters, i.e. the volume transfer constant $\left(\mathrm{K}^{\text {trans }}\right)$, rate constant $\left(\mathrm{K}_{\mathrm{ep}}\right)$, and volume of extracellular extravascular space $(v)$ were calculated using the general kinetic model for the two compartments. The mean values of the perfusion parameters were compared not only between the T-downstaged group and the non-downstaged group, but also before and after CRT in each group. The histopathology results served as the reference standard.

Results: The mean $\mathrm{K}^{\text {trans }}\left(\mathrm{min}^{-1}\right)$ significantly decreased after CRT from $1.24 \pm 0.53$ to $0.76 \pm 0.45$ in the T-downstaged group $(n=24)(P=0.0007)$, and it also tended to decrease from $1.02 \pm 0.53$ to $0.87 \pm 0.48$ in the non-downstaged group ( $n=$ 26) $(P=0.2358)$. None of the other parameters showed significant differences. Conclusion: A significant decrease in the mean $\mathrm{K}^{\text {trans }}$ was a useful indicator fo differentiating favourable responders from poor responders to CRT for locally advanced rectal cancer.

\section{B-169 11:42}

Gadofosveset-enhanced MRI for nodal staging in rectal cancer: predictive criteria

D.M.J. Lambregts, M. Maas, I.J.G. Rutten, W. Backes, R.G. Riedl,

F.C.H. Bakers, V.C. Cappendijk, G.L. Beets, R.G.H. Beets-Tan; Maastricht/NL (d.lambregts@mumc.nl)

Purpose: Recently, we showed that MRI using a new lymph-node contrast (gadofosveset) has high accuracy for nodal staging in rectal cancer (results in press) The Aim of the current study was to determine the most accurate imaging criteria for nodal evaluation using gadofosveset-enhanced MRI.

Methods and Materials: 33 patients underwent 3D T1-weighted MRI before and after administration of gadofosveset-contrast. For each visible lymph node the signal intensity $(\mathrm{SI})$ was measured by placing an $\mathrm{ROI}$ on both the pre- and post-contrast MRI. Furthermore, the presence of a nodal relief effect was scored. Finally, nodes were subjectively scored (using a 5-point confidence score) by an MR-rectum expert, based on visual evaluation of SI and nodal relief. Nodes were drawn on an anatomical template for lesion-by-lesion histologic validation.

Results: 289 nodes (55 malignant) were analysed. On pre-contrast MRI, the SI did not differ between benign and malignant nodes $(p=0.12)$. On gadofosvesetMRI, SI was significantly higher in the benign nodes $(p<0.0001)$, which resulted in an area under the ROC-curve (AUC) of 0.74 for prediction of metastatic nodes. The nodal relief rendered an AUC of 0.85 . The combination of SI and nodal relief rendered an AUC of 0.88 . When analysed visually, AUC for discrimination of metastatic nodes was 0.92

Conclusion: Only benign nodes show uptake of gadofosveset-contrast. The most optimal criteria for prediction of metastatic nodes are the nodal signal intensity on gadofosveset-MRI and the presence of a nodal relief. When analysed visually, these criteria provide high accuracy for discrimination of metastatic nodes. 


\section{B-170 11:51}

Rectal adenocarcinoma: intratumoural oxygenation level assessed with BOLD MRI and correlates with prognostic factors - initial experience Y. Liu, R. Xu; Shenyang/CN (liulio016@yahoo.com.cn)

Purpose: To establish the relationships between Intratumoral oxygenation level and immunohistologic markers of angiogenesis and metastasis in colorectal cancer. Methods and Materials: This study was approved by the local ethics committee, and written informed consent was obtained from all patients. BOLD MR imaging was performed in 51 patients with rectal adenocarcinoma to estimate $\mathrm{R}^{*}$ in tumour parenchyma, which is inversely related to tissue $\mathrm{Po}_{2}$. After surgery, resected specimens were sectioned and stained immunohistochemically to identify CD34 for quantification of microvessel density (MVD), and to identify matrix metalloproteinase-2 (MMP-2) for estimating of metastasis. The $\mathrm{R}^{*}$ value in tumour parenchyma was correlated with MVD and MMP-2 expression by using Pearson or Spearman rank correlation analysis, with significance assigned at the $5 \%$ level.

Results: R2* value in tumour parenchyma is $26.6 / \mathrm{sec} \pm 3.89$; it was correlated positively with MVD $(r=0.333, P=0.017)$. However, there were no significan associations between $\mathrm{R}^{*}$ values in tumour parenchyma and MMP-2 score (rho $<\mathrm{Or}=0.158, \mathrm{P}>\mathrm{Or}=0.27$ ).

Conclusion: BOLD MRI is feasible in patients with colorectal tumours. R2* values in rectal adenocarcinoma correlate positively with immunohistochemical marker of tumor angiogenesis; it may reflect the microvascularity of rectal tumors.

\section{$10: 30-12: 00$} Room F1

\section{Musculoskeletal}

\section{SS 410}

\section{Cartilage and bone imaging}

\section{Moderators:}

G. Andreisek; Zurich/CH

A. Blum; Nancy/FR

\section{B-171 10:30}

MRI-detected prevalent cartilage damage and cartilage loss over time predict incident bone marrow oedema-like lesions at the tibiofemoral compartments in persons with or at risk for knee osteoarthritis: the multicenter osteoarthritis (MOST) study

M.D. Crema' ${ }^{1}$, F.W. Roemer', K. Wang ${ }^{1}$, D.T. Felson ${ }^{1}$, M.D. Marra ${ }^{1}$, M.C. Nevitt ${ }^{2}$ J. Torner ${ }^{3}$, C.E. Lewis ${ }^{4}$, A. Guermazi'; 'B Boston, MA/US, ${ }^{2}$ San Francisco, CA/US, ${ }^{3}$ lowa City, IA/US, ${ }^{4}$ Birmingham, AL/US (michelcrema@gmail.com)

Purpose: Bone marrow oedema-like lesions (BMLs) are predictors of cartilage loss in knee osteoarthritis (OA). Inversely, loss of cartilage may remove a tissue protecting underlying bone, increasing loading and possibly damage to bone. We assessed the association of prevalent cartilage damage and cartilage loss over time with incident BMLs in the same subregion of the knee.

Methods and Materials: MOST is a longitudinal study of persons who have or are at risk for knee OA. Subjects with available baseline (BL) and 30 months follow-up (FU) 1.0 T MRIs were included. MRIs were read using the WORMS system. Tibiofemoral compartments were divided into 10 subregions. Cartilage morphology was scored from 0 to 6 . BMLs were scored from 0 to 3 . BL cartilage damage was defined as grade $\geq 2$. Cartilage loss had two groups: incident (grades 0 at BL and $\geq 2$ at $\mathrm{FU}$ ) and progression (BL cartilage damage and increased grade at FU). Incident BMLs were defined as grades 0 at $B L$ and $\geq 1$ at FU. Logistic regression was applied, adjusting for potential confounders.

Results: 1284 subjects were included. Medially, BL cartilage damage, incident cartilage loss and progression of cartilage loss were associated with incident BMLs, with odds ratios (ORs) of $3.5[95 \% \mathrm{Cl} 2.6,4.6], 7.1[95 \% \mathrm{Cl} 4.8,10.5]$, and $7.0[95 \% \mathrm{Cl} 4.7,10.4]$, respectively. Laterally, ORs were $3.4[95 \% \mathrm{Cl} 2.2,5.3], 4.3$ $[95 \% \mathrm{Cl} 2.3,8.3]$, and $8.9[95 \% \mathrm{Cl} 4.8,16.4]$ respectively.

Conclusion: Prevalent cartilage damage and cartilage loss strongly predict incident BMLs, supporting the close interrelation of the osteochondral unit in the progression of knee OA.

\section{B-172 10:39}

MRI-based cartilage T2 measurements and focal knee lesions correlate with BMI: 36-month follow-up data from the osteoarthritis initiative

T. Baum, G. B. Joseph, J. Carballido-Gamio, L. Nardo, H. Alizai, M.C. Nevitt, J. Lynch, C.E. McCulloch, T.M. Link; San Francisco, CA/US (thbaum@gmx.de)

Purpose: To compare 3 T MRI-based knee cartilage T2 measurements and focal knee lesions of normal, overweight and obese individuals over a 36 months period. Methods and Materials: 231 asymptomatic individuals (128 males, 103 females) aged 45-55 years with risk factors for knee osteoarthritis $(\mathrm{OA})$ and no radiographic $\mathrm{OA}$ were randomly selected from the Osteoarthritis Initiative (OAI) incidence cohort. 78 individuals had normal weight, 84 individuals were overweight and 69 individuals obese. All individuals underwent $3 \mathrm{~T}$ MRI of the right knee at baseline and 36-month follow-up. Focal knee lesions were assessed using the whole organ MRI score (WORMS). T2 measurements (mean T2, texture and laminar analysis) were performed in five compartments (patella, medial/lateral femur/tibia). Groups were compared using multivariate regression models adjusted for gender, age, $\mathrm{KL}$-score and OA risk factors other than overweight/obesity.

Results: Compared to normal individuals, overweight and obese individuals had more and more severe meniscal and cartilage lesions at baseline and after 36 months $(p<0.05)$. Baseline prevalence of meniscal (cartilage) lesions was $50 \%$ vs. $68 \%$ vs. $73 \%$ ( $71 \%$ vs. $80 \%$ vs. $91 \%$ ). Mean T2 values and all texture parameters were significantly different in all compartments for the three groups at baseline $(p<0.05)$. Obese individuals showed the highest mean T2 values and the most heterogeneous cartilage. In contrast to texture analysis parameters, mean T2 values were no longer significantly different between the groups after 36 months. Conclusion: Overweight and obesity are associated with more and more severe knee lesions and advanced cartilage degeneration according to T2 measurements.

\section{B-173 10:48}

New isotropic 3D PD-SPACE MR imaging in the comparison to isotropic 3D True-FISP and standard 2D MRI to depict the 3D MOCART score

G.H. Welsch', L. Zak', A. Mauerer ${ }^{2}$, T.C. Mamisch ${ }^{3}$, S. Marlovits ${ }^{1}$,

D. Paul' ${ }^{2}$, L. Lauer ${ }^{2}$, S. Trattnig $1{ }^{1}$ Vienna/AT, ${ }^{2}$ Erlangen/DE, ${ }^{3}$ Berne/CH (welsch@bwh.harvard.edu)

Purpose: To use the capabilities of the new isovoxel sequences and their 3Dmultiplanar-reconstruction (MPR), an new isotropic 3D proton-density, turbo-spinecho (PD-SPACE) sequence was compared to an isotropic 3D true-fast-imaging with steady-state-precession (True-FISP) sequence and to a set of 2D standard MR sequences in their depiction of the 3D-magnetic resonance observation of cartilage repair tissue (MOCART) score.

Methods and Materials: Sixty consecutive MR scans on 37 patients $(32.8 \pm 7.9$ years) during standard follow-up intervals after matrix-associated autologouschondrocyte-transplantation (MACT) of the knee at 3 Tesla were prospectively included. The new 3D-MOCART score with eleven variables was assessed using the standard $2 \mathrm{D}$ sequences ( $\sim 15$ minutes) and the MPR of each isotropic sequence (3D-PD-SPACE $\sim 7$ minutes; 3D-True-FISP $\sim 6$ minutes).

Results: The correlation between the 3D-MOCART scoring performed by the different sequences was highly significant for the variables 1) defect fill, 2)cartilage interface, 3) bone interface, 4) surface, 7) subchondral lamina, 8) chondra osteophyte, and 11) effusion (Pearson-coefficients 0.514 to $0.865(p<0.001)$ ). The variables 5) structure, 6) signal intensity, 9) bone marrow oedema, and 10) subchondral bone showed lower correlations with best results with respect to the 3D-PD-SPACE sequence $(0.307$ to $0.633(p=0.016$ to $p<0.001)$ ). Grading of subjective quality revealed good results for all sequences $(p \geq 0.05)$. Artefacts were most often visible on the 3D-True-FISP sequence $(p<0.05)$.

Conclusion: Different isotropic sequences can be used for the $3 \mathrm{D}$ evaluation of cartilage repair tissue with the benefits of isotropic 3D-MRI, MPR and a significantly reduced scan time.The 3D-PD-SPACE sequence reveals best results due to a better performance in the subchondral bone and because of the suppression of susceptibility artefacts produced by implantation and previous surgeries.

\section{B-174 10:57}

Factors associated with prevalent meniscal extrusion in persons with or at risk for knee osteoarthritis: the multicenter osteoarthritis (MOST) study M.D. Crema $^{1}$, F.W. Roemer ${ }^{1}$, M. Englund ${ }^{1}$, K. Wang ${ }^{1}$, D.T. Felson ${ }^{1}$,

M.C. Nevitt ${ }^{2}$, M.D. Marra' ${ }^{1}$ C.E. Lewis ${ }^{3}$, A. Guermazi ${ }^{1} ;{ }^{1}$ Boston, MA/US, ${ }^{2}$ San Francisco, CA/US, ${ }^{3}$ Birmingham, AL/US (michelcrema@gmail.com)

Purpose: Meniscal extrusion is a risk factor for knee osteoarthritis (OA). Understanding risk factors for meniscal extrusion is important in order to develop targeted treatment. We aimed to assess the cross-sectional associations of meniscal tears, knee malalignment, cartilage damage, and body mass index (BMI) with meniscal extrusion 
Methods and Materials: MOST is a study of individuals who have or are at risk for knee OA. Subjects with available baseline $(\mathrm{BL})$ knee radiographs and 1.0 T MRIs were included. MRIs were read using the WORMS system. Meniscal tears were graded from 0 to 4 , where grades $\geq 1$ exhibit tears. Meniscal extrusion was graded from 0 to 2 , where grades $\geq 1$ exhibit extrusion. Tibiofemoral cartilage morphology was scored from 0 to 6 , where grades $\geq 2$ exhibit cartilaginous defects. Associations were assessed using logistic regression. Multi-adjustments were performed when testing each predictor.

Results: 1527 subjects were included. Medially, meniscal tears, varus malalignment, and cartilage damage were significantly associated with meniscal extrusion, with odds ratios (OR) of $6.5[95 \% \mathrm{Cl} 5.1,8.2], 1.9[95 \% \mathrm{Cl} 1.5,2.3]$, and $1.4[95 \% \mathrm{Cl}$ $1.1,1.7]$, respectively. Laterally, meniscal tears, valgus malalignment, and cartilage damage were significantly associated with meniscal extrusion, with ORs of 10.5 [95\% $\mathrm{Cl} 7.3,15.2], 2.0[95 \% \mathrm{Cl} 1.4,3.0]$, and $2.2[95 \% \mathrm{Cl} 1.5,3.2]$, respectively. No association was found for BMI.

Conclusion: Not only meniscal tears are associated with meniscal extrusion but also other factors such as knee malalignment and cartilage damage. Meniscal extrusion is a result of the complex interrelation of the different joint tissues involved in the OA process.

\section{B-175 11:06}

Clinical application of CT-arthrography as a measure for cartilage quality, validated by in-vitro contrast-enhanced $\mu \mathrm{CT}$

J. van Tiel, M. Siebelt, J.H. Waarsing, M. van Straten, G.P. Krestin,

G.J. Kleinrensink, J.A.N. Verhaar, H. Weinans, E.H.G. Oei; Rotterdam/NL

(j.vantiel@erasmusmc.nl)

Purpose: Imaging techniques, e.g. delayed Gadolinium-enhanced MRI of cartilage (dGEMRIC), that accurately and quantitatively measure cartilage quality are increasingly important in osteoarthritis research. X-ray attenuation values in ex-vivo contrast-enhanced $\mu \mathrm{CT}$ have been used as a measure of glycosaminoglycans (GAG) distribution in the cartilage, and hence cartilage quality. The purpose of this study was to determine whether differences in GAG distribution can also be detected using a clinical application of CT-arthrography (CTa).

Methods and Materials: On six human cadaveric knee joints a CTa was performed with a second-generation dual-source multidetector spiral CT scanner (Siemens, Germany) after intra-articular injection with a negatively charged contrast agent (Hexabrix 320, Guerbet). After CTa, the knees were dissected into five parts: both medial and lateral femoral condyles and tibial plateaus and the patella. Subsequently, all parts were scanned using ex-vivo contrast-enhanced uCT (Skyscan, Belgium) as a reference standard. X-ray attenuation values from both techniques were correlated between seven anatomically matching regions of interest (ROIs) in the cartilage using a Pearson's correlation coefficient.

Results: X-ray attenuation of the different cartilage ROIs in the clinical CTa scans ranged from 144 - 236 Hounsfield Units. Comparison with the ex-vivo contrastenhanced $\mu C T$ resulted in a correlation coefficient of $r=0.80$ and a $R 2=0.65$ (95\% Cl 0.58-0.92, $\mathrm{p}<0.0001$ )

Conclusion: The excellent correlation between clinical CTa and ex-vivo $\mu C T$ shows that clinical CTa can be used to discriminate between GAG distributions within different cartilage regions and can thus be a cheap alternative for imaging techniques such as (dGE)MRI (C).

\section{B-176 11:15}

In vivo diffusion tensor imaging of the articular cartilage at $7 \mathrm{~T}$ : a feasibility study

J.G. Raya ${ }^{1}$, A. Horng², O. Dietrich², L.S. Beltran'1, S. Krasnokutsky', M.F. Reiser ${ }^{2}$, M. Recht ${ }^{1}$, C. Glaser ${ }^{1} ;{ }^{1}$ New York, NY/US, ${ }^{2}$ Munich/DE (Jose.Raya@nyumc.org)

Purpose: To demonstrate the feasibility of in vivo diffusion tensor imaging (DTI) of articular cartilage with a line scan diffusion imaging (LSDI) sequence at 7 T using a dedicated knee coil.

Methods and Materials: The right knee of 15 healthy volunteers $((29 \pm 3)$ y) was two time scanned with knee reposition between the scans on a whole body $7 \mathrm{~T}$ scanner using a birdcage transmit, 28-channel receive knee coil with a fat-saturated LSDI sequence $(T E / T R / T R e f f=45 / 180 / 2880 \mathrm{~ms}$, matrix $=256 \times 128$, b-values = $10,450 \mathrm{~mm}^{2} / \mathrm{s}, 6$ directions, FOV $=154 \times 77 \mathrm{~mm}^{2}$, thickness $=2 \mathrm{~mm}$, acquisition time $=2: 47 \mathrm{~min} /$ slice). Five axial slices over the patellar cartilage were acquired with a $3 \mathrm{~mm}$ gap to cover the complete patellar cartilage. ADC and FA maps and their mean values for cartilage, synovial fluid and muscle were calculated. SNR was calculated in the b0 image. Reproducibility was assessed by root-mean-square of coefficient of variation.
Results: SNR was $(20.9 \pm 4.6)$ in cartilage, $(18.0 \pm 2.4)$ in muscle and $(52.7 \pm 7.0)$ in synovium. Mean ADC and FA values were, respectively $(1.03 \pm 0.06) \times 10^{-3} \mathrm{~mm}^{2} / \mathrm{s}$, $(0.44 \pm 0.05)$ for cartilage, $(1.18 \pm 0.09) \times 10^{-3} \mathrm{~mm}^{2} / \mathrm{s},(0.34 \pm 0.04)$ for muscle and $(2.59 \pm 0.08) \times 10^{-3} \mathrm{~mm}^{2} / \mathrm{s} /(0.083 \pm 0.005)$ for synovium. ADC decreased from the articular surface $(1.52 \pm 0.23) \times 10^{-3} \mathrm{~mm}^{2} / \mathrm{s}$ to the bone-cartilage interface $(0.83 \pm 0.19) \times 10^{-3} \mathrm{~mm}^{2} / \mathrm{s}$. FA increased form the surface $(0.23 \pm 0.17)$ to the deep radial zone of the cartilage $(0.73 \pm 0.32)$. Mean reproducibility of ADC and FA were $6.5 \% / 8.4 \%$ in cartilage, $7.3 \% / 9.8 \%$ in muscle and $2.5 \% / 5.3 \%$ in synovium. Conclusion: For a first time in vivo acquisition of cartilage DTI data become feasible. In vivo ADC and FA showed a good reproducibility and are consistent with published ex vivo results.

\section{B-177 11:24}

Cartilage quality assessment using gagCEST and sodium MRI at 7 Tesla

B. Schmitt ${ }^{1}$, S. Zbyn², D. Stelzeneder ${ }^{2}$, V. Jellus ${ }^{3}$, D. Paul ${ }^{4}$,

P. Bachert 1 , S. Trattnig ${ }^{2} ;{ }^{1}$ Heidelberg/DE, ${ }^{2}$ Vienna/AT, ${ }^{3}$ Erlangen/DE,

${ }^{4}$ Freiburg/DE (b.schmitt@Dkfz-Heidelberg.de)

Purpose: gagCEST and 23Na MRI provide a contrast sensitive to glycosaminoglycan (GAG) content of articular cartilage. The aim of our study was to compare a newly developed gagCEST imaging method with $23 \mathrm{Na}$ imaging in patients after cartilage repair surgeries (matrix-associated autologous chondrocyte implantation and microfracture).

Methods and Materials: One female (age: 67.3 years), and 11 male (mean age: $29.3 \pm 6.04$ years) patients were examined using a $7-T$ whole-body system with approval of the local ethics committee after written informed consent was obtained. A modified 3D-GRE sequence and a 28-channel knee coil were used for gagCEST imaging. $23 \mathrm{Na}$ imaging was performed with a circularly polarized knee coil using a modified GRE sequence. Statistical analysis of differences and Pearson correlation were applied.

Results: The average asymmetry in gagCEST z-spectra summed over all offsets from 0 to $1.3 \mathrm{ppm}$ was $8.01 \pm 0.2 \%$ (mean \pm SEM) in native cartilage and $4.6 \pm 0.2 \%$ in repair tissue. A strong correlation $(R=0.885, p=0.0001)$ was found between ratios of native cartilage to repair tissue obtained with gagCEST, and sodium imaging. The mean dimensionless ratios between native cartilage and repair tissue were $1.39 \pm 0.3$ (range: 1.05 - 1.9) for gagCEST, and $1.34 \pm 0.21$ (range: $1.1-1.78$ ) for sodium MRI (mean \pm SD).

Conclusion: The high correlation between the new gagCEST method and $23 \mathrm{Na}$ imaging results demonstrates the GAG specificity and reliability of the technique. Both techniques suggest that cartilage repair tissue has lower GAG content than native tissue.

\section{B-178 11:33}

7T 23 Na (Sodium) imaging after knee matrix-associated autologous chondrocyte transplantation: preliminary results

S. Trattnig ${ }^{1}$, G.H. Welsch ${ }^{2}$, V. Juras ${ }^{1}$, M.E. Mayerhoefer ${ }^{1}$, D. Stelzeneder ${ }^{1}$, T.C. Mamisch ${ }^{3}$, O. Bieri ${ }^{4}$, K. Scheffler ${ }^{4}$, S. Zbyn ${ }^{1} ;{ }^{1}$ Vienna/AT, ${ }^{2}$ Erlangen/DE, ${ }^{3}$ Berne/CH, ${ }^{4}$ Basle/CH (siegfried.trattnig@meduniwien.ac.at)

Purpose: To evaluate the feasibility of sodium imaging at $7 \mathrm{~T}$ indicative of glycosaminoglycans in repair and native cartilage after matrix-associated autologous chondrocyte transplantation (MACT) and compare results with delayed gadoliniumenhanced MRI of cartilage (dGEMRIC) at $3 \mathrm{~T}$.

Methods and Materials: Six females and six males with mean age $32.8 \pm 8.2$ [standard deviation] and $32.3 \pm 12.7$ years were included, with mean follow-up $56 \pm 28$ months after MACT. At $3 \mathrm{~T}$, variable 3D-GRE dual flip-angle technique was used for T1-mapping before and after contrast agent administration. At 7T, sodium MR of all patients using 3D-GRE sequence was performed with mean delay $70.5 \pm 80.1$ days. $23 \mathrm{Na}$-only transmit/receive knee coil was used. Statistical analysis of variance and Pearson correlation were applied.

Results: Mean signal-to-noise ratio (SNR) in native cartilage was 24 and in transplants $16(p<0.001)$. Mean sodium intensities normalised by reference sample were $174 \pm 53$ for repair tissue within cartilage transplant and $267 \pm 42$ for healthy cartilage $(p=0.002)$. Post-contrast T1 values were $510 \pm 195 \mathrm{~ms}$ for repair tissue and $756 \pm 188 \mathrm{~ms}$ for healthy cartilage $(p=0.005)$. Mean score of magnetic resonance observation of cartilage repair tissue (MOCART) was $75 \pm 14$. Association between post-contrast T1 and normalised sodium values show high Person correlation coefficient $(R)$ of $0.706(p=0.001)$. High correlation $R=0.836(p=0.001)$ was found between ratios of normalised sodium values and ratios of T1 post-contrast values. Conclusion: Using modified 3D-GRE sequence at 7T, sufficiently high SNR in sodium images was achieved, allowing for the differentiation of repair tissue and native cartilage after MACT. Strong correlation was found between sodium imaging and dGEMRIC in patients after MACT. 


\section{B-179 $11: 42$}

Comparison of high-resolution peripheral quantitative $\mathrm{CT}$ and micro-CT for the assessment of bone microstructure

J.H. Deutschmann, J. Patsch, A. Valentinitsch, H. Resch, M. Mousavi, G. Weber, C. Schueller-Weidekamm, F. Kainberger; Vienna/AT (deutschmann_julia@yahoo.de)

Purpose: To compare the performance of three high-end modalities for the ex-vivo assessment of bone microstructure.

Methods and Materials: 3 bone samples from femoral heads of male patients with osteoporotic hip fractures $(n=1)$ and osteoarthritis of the hip $(n=2)$ were investigated with a HR-pQCT scanner (Scanco XtremeCT) at a resolution of 41 um. Moreover, the cubes were scanned with two other dedicated high-resolution CT-scanners (Scanco $\mu$ CT 40 and Viscom X8060 NDT) at a resolution of $20 \mu \mathrm{m}$, within the Research Network Osteology (RNO) Vienna which is an interdisciplinary platform for osteologic research focusing on qualitative and quantitative bone assessment in anatomical, anthropological and clinical bone samples. Parameters of bone microstructure were compared by mean absolute differences (\%) of the measurements

Results: The comparison of HR-pQCT and Scanco $\mu \mathrm{CT} 40$ showed an overestimation of trabecular bone volume $(+15 \%)$ and trabecular thickness $(+33.1 \%)$ by the HR-pQCT. Trabecular number was underestimated by the HR-pQCT scanner $(-11.3 \%)$. The Viscom X8060 NDT $\mu$ CT provides high image quality for 3D reconstructions, but still lacks a standardised evaluation software for quantitative microstructure analysis.

Conclusion: HR-pQCT and micro-CT provide divergent results in ex-vivo human bone morphometry, which are most likely to be due to different resolutions and partial volume effects. This should be kept in mind when studies on bone microarchitecture are compared or cited. The Viscom system provides images of high quality, but still lacks standardised quantification options.

\section{B-180 11:51}

Prediction of bone strength by $\mu \mathrm{CT}$ and MDCT-based finite-elementmodels: how much spatial resolution do we need?

J.S. Bauer', I. Sidorenko', D. Mueller', A.S. Issever ${ }^{3}$, S. Majumdar ${ }^{4}$, F. Eckstein ${ }^{5}$ E.J. Rummeny', T.M. Link', C.W. Räth2; ${ }^{1}$ Munich/DE, ${ }^{2}$ Garching/DE, ${ }^{3}$ Berlin/DE, ${ }^{4}$ San Francisco, CA/US, ${ }^{5}$ Salzburg/AT

Purpose: Finite element models (FEM) stand in the forefront in predicting bone strength and fracture risk. FEM have successfully been calculated with different spatial resolutions for bone specimens and whole bones. Usually, the highest resolution available was used for these calculations; however limitations exist regarding available calculation time and memory. Thus, the purpose of this study was to compare FEM based on datasets with decreasing spatial resolution regarding their ability to predict biomechanically tested maximum compressive strength (MCS).

Methods and Materials: 20 cylindrical trabecular bone specimens (diameter $12 \mathrm{~mm}$, length $15-20 \mathrm{~mm}$ ) were harvested from formalin-fixed human thoracic spines. All specimens were examined by Micro-CT (central core of specimen with a diameter of $8 \mathrm{~mm}$, isotropic resolution $26 \mu \mathrm{m}$ ) and whole body MDCT (whole specimen, resolution $200 \times 200 \times 500 \mu \mathrm{m} 3$ ). Resolution of the Micro-CT datasets was degraded stepwise to 200 um isotropic and FEM were calculated for all original and degraded datasets. Bone strength (MCS) was determined in a biomechanical test. Results: Biomechanically determined MCS showed high correlations with all FEM, up to $r=0.75$ (8-mm-diameter $u C T$-datasets) and up to $r=0.83$ (12-mm-diamete MDCT-datasets). Degrading of spatial resolution did not significantly change correlation in MDCT and $\mu C T$-based FEM. By only modelling the central MDCT-dataset (8 mm-diameter), like in case of $\mu \mathrm{CT}$, correlation decreased to $r=0.65$.

Conclusion: Micro-CT- and MDCT based finite element models could equally predict MCS. While degrading spatial resolution showed no decrease in correlations, it is mandatory to include the whole bone in the FEM, as local variations in bone structure have a great impact on bone strength
$10: 30-12: 00$

Room F2

Breast

\section{SS 402}

Mammography: from screening to digital technology

Moderators:

F. Diekmann; Berlin/DE

G. Forrai; Budapest/HU

\section{B-181 10:30}

Increased detection of clinically relevant cancers using FFDM in breast cancer service screening: a multi-center study

A.M.J. Bluekens ${ }^{1}$, N. Karssemeijer², M.J.M. Broeders², G.J. den Heeten ${ }^{2}$. 1Tilburg/NL, ${ }^{2}$ Nijmegen/NL (n.bluekens@elisabeth.nl)

Purpose: To study the performance of full-field digital mammography (FFDM) in breast cancer service screening compared with screen-film mammography (SFM). Methods and Materials: Preceding the nation-wide transition to FFDM in the Dutch screening programme, the feasibility of FFDM was studied in 3 screening regions. Qualified screening radiologists were involved in double reading of both FFDM and SFM images, with differences of opinion resolved in consensus. Screening outcomes obtained with both modalities were compared using the Chi-square test. Furthermore, radiological features of referrals and pathological characteristics of detected cancers were assessed.

Results: Nearly 1.2 million screening mammograms were performed $(152,515$ digital and 1,045,978 conventional) between 2003 and 2007. Subsequently, 18896 women were referred (SFM 1.5\%; FFDM 2.4\%). Preliminary results show that referral on the basis of microcalcifications increased vastly with FFDM. Breast cancer was diagnosed in 6410 women with a detection rate of $6.2 \%$ with FFDM and $5.2 \%$ 。 with SFM $(p<.00)$. Significantly more ductal carcinoma in situ (DCIS) $(p<.00)$ and invasive carcinoma $(p=.01)$ were found with FFDM. The increase in DCIS detection constituted a near doubling of the detection of high grade DCIS $(p<.00)$

Conclusion: With FFDM in population-based breast cancer screening detection performance is significantly better than with SFM. While referral increased, detection improved disproportionately with FFDM. Since this improvement is largely due to detection of clinically relevant cancers, such as high grade DCIS and invasive carcinoma, FFDM might be able to achieve a higher mortality reduction than reported for SFM in service screening.

\section{B-182 10:39}

Grade, size and surgical treatment of ductal carcinoma in situ detected by digital mammography screening

C. Biesheuvel 1 , S. Weigel ${ }^{1}$, T. Decker ${ }^{2}$, W. Heindel ${ }^{1} ;{ }^{1}$ Münster/DE,

${ }^{2}$ Neubrandenburg/DE (biesheuvel@referenzzentrum-ms.de)

Purpose: Mammography screening leads to detection of ductal carcinoma in situ (DCIS), typically $20 \%$ of all detected malignancies. We aimed to examine grade, size and type of surgical treatment of DCIS, detected in the prevalence phase of digital mammography screening.

Methods and Materials: 3166 breast cancers were detected at the first screening round (2005-2007) of the German breast cancer screening program in NordrheinWestfalia of which $611(19.3 \%)$ were DCIS. We evaluated 551 cases of DCIS detected by digital screening where post-operative histological grade, size and type of surgery were available. The Chi square test was applied to compare subgroups Results: $39.0 \%(215 / 551)$ of DCIS cases were of grade $3,41.6 \%(229 / 551)$ and $19.4 \%(107 / 551)$ were of grade 2 and 1, respectively. $25.6 \%(141 / 551)$ of the lesions were $<10 \mathrm{~mm}, 30.7 \%$ (169/551) were $10-19 \mathrm{~mm}, 16.9 \%$ (93/551) were 20-29 mm, 8.7\% (48/551) were 30-39 mm and $18.1 \%$ (100/551) were $\geq 40 \mathrm{~mm}$ Lesion size did not differ among the grading subgroups $(p=0.73)$. Breast conserving therapy (BCT) was performed in $81.7 \%$ of all DCIS patients. Whereas the BCT rates differed significantly between cases with lesion size $<40 \mathrm{~mm}$ and $\geq 40 \mathrm{~mm}$ with $89.6 \%(404 / 451)$ versus $46.0 \%(46 / 100)(p<0.001)$, grade did not influence type of surgery $(p=0.36)$.

Conclusion: The proportion of non-high grade DCIS detected by digital mammography screening was larger than reported for analogue screening $(61.0 \%$ vs approximately $40 \%$ ), whereas the BCT rate was relatively high ( $81.7 \%$ vs. approximately $70 \%$ in analogue screening). Lesion size, rather than grade, determined whether BCT could be applied. 


\section{B-183 10:48}

Second breast cancer size and survival following treatment for primary G.S. MacLennan ${ }^{1}$, G. Lawrence ${ }^{2}$, C. Boachie', S.D. Heys' ${ }^{1}$, F.J. Gilbert ${ }^{1}$; ${ }^{1}$ Aberdeen/UK, ${ }^{2}$ Birmingham/UK (f.j.gilbert@abdn.ac.uk)

Purpose: Currently there is uncertainty surrounding the role of surveillance mammography following primary breast cancer treatment. If in women with ipsilatera breast tumour recurrence (IBTR) or metachronous contralateral breast cancer (MCBC) smaller subsequent cancers are associated with improved survival then one could infer potential benefit from surveillance mammography.

Methods and Materials: Survival in women with second cancers diagnosed between $1^{\text {st }}$ Jan 1990 and $31^{\text {st }}$ Jan 2007 from the West Midlands Cancer Intelligence Unit Breast Cancer Registry were analysed using Cox regression models. Outcomes were time from diagnosis of second cancer to all cause death and to breast cancer death. Estimates are hazard ratios (HR) and $95 \%$ confidence intervals. Risk factors included were prognostic factors of the primary tumour, size of the second tumour and time to second tumour.

Results: There were 613/1174 all cause deaths after IBTR. Poorer prognosis was associated with second cancers $>2 \mathrm{~cm}$ compared with $<1 \mathrm{~cm}$, HR $1.75(1.29$, 2.37), and second cancers within 60 months HR $1.66(1.35,2.04)$. After MCBC there were 358/975 all cause deaths and risk factors were similar; HR 2.14 (1.49, $3.06)$ for size and HR $1.29(1.01,1.63)$ within 60 months.

Conclusion: In women with second cancers those with tumour size $>2 \mathrm{~cm}$ in diameter have a significantly greater risk of death. Also, time to the second cancer is important so frequency of surveillance mammography should be considered for maximum benefit. Caution is required interpreting these results as lead time bias from mode of detection may be a factor.

\section{B-184 10:57}

Density is in the eye of the beholder: visual versus semi-automated assessment of breast density on standard mammograms

M. Lobbes, J. Cleutjens, V. Lima Passos, C. Frotscher, C. Boetes; Maastricht/NL (marclobbes@planet.nl)

Purpose: BI-RADS guidelines advise assessment of breast density as part of the mammographic evaluation. In general, visual inspection is used to assess breast density in everyday clinical practice. Comparison of visual versus semi-automated analysis of breast density was never evaluated.

Methods and Materials: Breast density was assessed by an experienced and an inexperienced reader in 200 mammograms. Breast density was also assessed by dedicated software using a thresholding technique. Breast density was scored according to the BI-RADS classification system. The correlation between breast density classification of both readers, as well as the correlation between their assessment versus the semi-automated analysis, was expressed as the weighted $\kappa$ value. Results: Breast density measurements of the left and right breast and in both projections were excellent and highly significant (intraclass correlation coefficients (ICC) of $>0.9, p<0.0001)$. There was frequent disagreement between the BI-RADS categorisation of the experienced and unexperienced reader ( $42 \%$ of the cases). The experienced reader correctly classified the BI-RADS classification in $58.5 \%$ of the cases. The classification was overestimated in $35.5 \%$ of the cases, and underestimated in $6.0 \%$ of the cases. The correlation between the classification of both readers versus the semi-automated analysis was considered only fair with weighted $\mathrm{\kappa}$ values of 0.367 (experienced reader) and 0.232 (unexperienced reader). Conclusion: Visual assessment of breast density on mammograms is inaccurate and observer-dependent. Semi-automated analysis of breast density can aid in the improvement of inter-observer variation of breast density classification on mammograms.

\section{B-185 11:06}

HighReX: a multi-reader, multi-centre observer study comparing diagnostic accuracy of photon counting tomosynthesis and full field digital mammography

M.G. Wallis ${ }^{1}$, E. Moa ${ }^{2}$, F. Zanca ${ }^{3}$, K. Leifland ${ }^{4}$, M. Danielsson $2 ;{ }^{1}$ Cambridge/UK, ${ }^{2}$ Solna/SE, ${ }^{3}$ Leuven/BE, ${ }^{4}$ Stockholm/SE (matthew.wallis@addenbrookes.nhs.uk)

Purpose: To compare, in an observer study, detection accuracy of full field digital mammography (FFDM) with that of two-view and single view (MLO) photon-counting tomosynthesis (PhCT).

Methods and Materials: Sequential cases were collected from screening assessment and referral clinics in two European centres. In addition to standard management (Sectra MicroDose Mammography D40, GE Senograph DS and Essential) all women with breast composition BIRADS 2 or more underwent an examination on a prototype PhCT system, a modified Sectra MicroDose Mammography (L30), tomo angle 11 degrees. Average glandular dose 0.7 mGy (range 0.3-1.4) per PhCT exposure. After training, ten accredited film readers read a set of 130 examinations, 64 with verified abnormalities and 66 with no findings, using two-view FFDM and two-view PhCT over 2 sessions. A further ten readers reviewed the same cases using two-view FFDM but single view (MLO) PhCT. The multireader-multicase (MRMC) ROC methodology was applied and the PROPROC fitted area under the ROC curve was used Figure of Merit. Results: No statistically significant difference was reached between detection accuracy with either FFDM and 2 views PhCT (readers' average $\mathrm{AUC}_{\mathrm{FFDM}}=0.773$, $\mathrm{AUC}_{\text {PhCT }}=0.810, \mathrm{p}$-value $=0.22$ ), or FFDM and single MLO PhCT (readers' average $A U C_{F F D M}=0.708, A_{U C} C_{\text {PhCT }}=0.680, p$-value $=0.32$ ). Average reading times were 125 $\mathrm{s}$ for two-view PhCT, $97 \mathrm{~s}$ for the single MLO PhCT, and $71 \mathrm{~s}$ for two-view FFDM Conclusion: No differences were found in detection accuracy either between dualview PhCT and dual-view FFDM or between single-view PhCT and dual-view FFDM.

\section{B-186 11:15}

Digital breast tomosynthesis versus digital mammography in a clinical setting: side-by-side feature analysis of cancer conspicuity P. Skaane, R. Gullien, H. Bjorndal, U. Ekseth, U. Haakenaasen, G. Jahr; Oslo/NO (per.skaane@ulleval.no)

Purpose: Compare cancer conspicuity of digital mammography and tomosynthesis in a clinical setting.

Methods and Materials: 129 women underwent mammography (2D) and digital breast tomosynthesis (DBT). Indication included lump in $30(23 \%)$, abnormal mammographic findings $54(42 \%)$, and surveillance $45(35 \%)$ women. $20(16 \%)$ had a benign biopsy, $25(19 \%)$ a cancer, and $84(65 \%)$ no biopsy. BI-RADS density was 1 in $10(8 \%), 2$ in $70(54 \%), 3$ in $43(33 \%)$ and 4 in $6(5 \%)$ women. 2 D exams were interpreted on-site and DBT retrospectively after women left the department. Sideby-side feature analysis of cancer conspicuity was performed by 3 external breast radiologists trained with cases not included in the study. The readers interpreted bilateral $2 \mathrm{D}$ and $\mathrm{DBT}$ of 50 women without information about prior imaging, indication, pathology, or cancer mix in the test set. The test set included 23 cancers. Results: DBT resulted in 2 false positive recalls and 2 additional cancers. Feature analysis of cancer conspicuity for the 3 readers (2D higher, equal, and DBT higher score): Reader A 1.14,8; reader B 1.13, 9; reader C 3.5, 15, respectively. Conspicuity was higher for $2 \mathrm{D}$ in 5 , equal in 32 and higher for DBT in 32 interpretations. Scores for 14 cancers presented as spiculated mass or distortion:1.10 and 31 respectively. For $5 \mathrm{DCIS}$, scores were 3,12 and 0 , respectively. The mean cancer scores was 3.42 for $2 \mathrm{D}$ and 3.98 for DBT

Conclusion: Tomosynthesis shows higher conspicuity for malignancy than mammography, especially for spiculated masses and distortions.

\section{B-187 11:24}

Role of mammography cranio-caudal view in adjunction to digital breast tomosynthesis: clinical performance study

G. Gennaro', E. Bezzon', E. Baldan', R. Chersevani², C. di Maggio',

M. La Grassa ${ }^{3}$, L. Pescarini', I. Polico', A. Proietti'; ${ }^{1}$ Padua/IT, ${ }^{2}$ Gorizia/lT, ${ }^{3}$ Aviano/IT (gisella.gennaro@ioveneto.it)

Purpose: To evaluate the potential added value of mammography cranio-cauda (CC) view in adjunction to breast tomosynthesis (DBT) compared to standard twoviews digital mammography (FFDM).

Methods and Materials: The study included 250 consenting women with breast lesions classified as suspicious at mammography and/or ultrasound. They underwent bilatera digital mammography (CC, MLO) and tomosynthesis (MLO). Six readers reviewed the images per-breast in individual reading sessions, each including both FFDM and DBT images; after DBT evaluation, mammography CC-view of the same breast was added and evaluation refined using both modalities. Clinical assessments on 469 breasts from the six readers were compared with the truth and multiple-reader-multiple-case (MRMC) receiver operating characteristics $(\mathrm{ROC})$ analysis performed. Clinical performance of mammography versus tomosynthesis and tomosynthesis plus mammography CC-view were compared using sensitivity, specificity, and area under the ROC curve (AUC). Results: Mean sensitivity was the same with FFDM and DBT (72.8\% vs. $73.0 \%)$ while increased significantly adding FFDM $(C C)$ to DBT $(76.2 \%, p=0.004)$, leading to 2.1 extra-cancers detected, on average. Mean specificity was significantly higher with DBT than FFDM $(86.9 \%$ vs. $83.1 \%, p=0.025)$, but the adjunction of FFDM (CC) to DBT reduced specificity, even not significantly. AUCs averaged across the six readers gave 0.831 (FFDM), 0.844 (DBT), and 0.852 (DBT+FFDM (CC)), respectively, but no difference between modality pairs was significant

Conclusion: Adjunction of mammography CC-view to tomosynthesis MLO increased mean DBT sensitivity, with no significant effect on specificity. The overal increase in clinical performance (AUC) due to the combination of DBT and FFDM was not statistically significant. 


\author{
B-188 $11: 33$ \\ A novel CAD application designed to presort cases for maximising the \\ prevalence of breast cancer in a prioritised subset \\ R.B. Lederman, I. Leichter, P. Zagdanski, J. Stoeckel; Jerusalem/IL \\ (isaac.leichter@siemens.com)
}

Purpose: To determine the optimal operating point of a CAD algorithm, run in the background, to increase breast cancer prevalence in a subset of cases, thereby heightening reader awareness of subtle malignant findings

Methods and Materials: Radiologists are reluctant to accept CAD prompts, since almost all are false. The reader's awareness could, however, be heightened in a prioritised subset knowing that the prevalence of breast cancer is increased. 15892 mammograms (280 malignant) were run with a prototype CAD algorithm (Siemens) at several operating points, displaying findings with varying certainty of malignancy, normalised from $0-100$. Since cancers are usually visible in both views, while false marks tend to occur randomly, both normal and malignant cases were analysed regarding the percentage of cases with matching CAD marks of the same type (i.e. masses or clusters) in both views, for each operating point. The optimal operating point, yielding the highest prevalence of cancer in the prioritised subgroup, was explored.

Results: At an operating point corresponding to a certainty level of 80 , only $10.2 \%$ (1595 cases) of the normal cases had, coincidentally, matching CAD marks, while in the malignant cases, $65.0 \%$ (182) had matching CAD marks. Thus, by presorting, the prevalence of breast cancer in the prioritised subgroup was increased nearly 6 times, from $1.76 \%$ (280 of 15892 ) to $10.24 \%$ (182 of 1777).

Conclusion: With an optimised operating point, presorting by matching CAD marks resulted in a prioritised subgroup with a substantially increased cancer prevalence that could heighten reader awareness, thereby avoiding oversights.

\section{B-189 11:42}

Could "extreme-CAD" in screening mammography be used to cherry-pick cases for computer-only reading?

R.B. Lederman, I. Leichter, E. Ratner, J. Stoeckel; Jerusalem/L (izhak@hadassah.org.il)

Purpose: To evaluate an innovative CAD application for automated cherry-picking of definitely normal cases and obvious cancers for computer-only reading.

Methods and Materials: A CAD algorithm assigns each suspicious finding a certainty level of malignancy, normalised from 0-100. Only candidates above a selected certainty are displayed. When operated at low certainty (many false CAD marks but extremely high sensitivity), almost all cases with no marks should be normal and bypass review by a radiologist. Operating at high certainty (almost no false marks but lower sensitivity) and searching for cases with matching CAD marks of the same type in both views, should identify obvious cancers for automatic recall. When run at high and low certainties, the algorithm functions as "extreme-CAD", enabling automatic cherry-picking of both definitely normal cases and obvious cancers. 15892 mammograms (280 malignant) were run at various certainty levels with a prototype CAD algorithm (Siemens) to investigate the feasibility of developing "extreme-CAD".

Results: Operating at " 10 " certainty, yielded 1411 normal cases and 1 malignant case with no CAD marks, allowing "Extreme-CAD" to automatically bypass review of $9 \%$ of the normal cases with only $0.36 \%$ missed cancers. When subsequently operated at "100" certainty, $17.1 \%$ of the malignant cases had matching CAD marks allowing automatic recall with only $0.06 \%$ recall of normal cases.

Conclusion: "Extreme-CAD" could be used to cherry-pick for computer-only reading $9.3 \%$ of the cases, including definitely normal cases and obvious cancers, with a negligible number of missed cancers. The remaining cases are interpreted by a radiologist.

\section{B-190 11:51}

Does the application of CAD prior to planned stereotactic biopsy prevent unneccesary biopsies of B2 lesions? An analysis of screening-selected cases

A. Malich, A. Kott, M. Klima, C. Roth;

Nordhausen/DE (ansgar.malich@shk-ndh.de)

Purpose: Despite independent double reading and preinterventional consensus conference, the number of lesions recommended for stereotactic biopsy due to suspicious microcalcification-clusters finally proven as B2 is still high. This study aimed to verify whether preinterventional application of CAD allows a reduction of unnecessary biopsies.

Methods and Materials: All screening-selected stereotactic biopsies (9G) performed at our hospital in the previous year were retrospectively analysed by $C A D$
(R2,Hologic). Placed ROls at all 3 sensitivity levels were matched to histopathology by two radiologists in consensus. PPV, NPV, sensitivity, specificity, FP-rate of CAD were analysed at those microcalcification-clusters being scored as BI-RADs 4 in blinded double reading and consensus conference under the conditions of the German screening program.

Results: 59/114 lesions were proven B2, 2/114 B3a, 14/114 B3b, 4/114 B4, 33/114 B5a and 2/114 B5b. Sensitivity of histologically proven B3b/4/5 lesions was (at all three different sensitivity levels) $84.9 \% / 88.7 \% / 88.7 \%$. Taking into account B4/5 only, sensitivity is $87.2 \% / 89.7 \% / 89.7 \%$. The number of correctly nondetected B2/B3a-lesions caused a specificity of $47.5 \% / 47.5 \% / 40.0 \%$,resp. NPV was $78.4 \% / 78.1 \% / 77.4 \%$; PPV 58.4\%/57.3\%/56.6\%. FP-rate of MC-markers was $0.10 ; 0.18 ; 0.20 /$ image, resp. FP-rate of mass-markers was $0.30 / 0.41 / 0.50$. Including CAD-marks on histologically proven $\mathrm{B} 2$ lesions as FP, FP-values for MC-markers were $0.40 / 0.48 / 0.55$ and for mass-markers $0.32 / 0.42 / 0.53$.

Conclusion: Preinterventional application of $\mathrm{CAD}$ reduces unnecessary biopsies of benign lesions which were selected for biopsy by double reading and consensus conference in a screening population. CAD-application in such a highly selected group causes a slight decrease of sensitivity (approx. 10\%), thus yielding a significantly higher overall accuracy.

10:30 - 12:00 Room G/H

\section{Head and Neck}

\section{SS 408}

\section{Challenges in head and neck cancer}

Moderators:

M.I. Furmanek; Warsaw/PL

H.C. Thoeny; Berne/CH

\section{B-191 10:30}

Tumour thickness in oral cancer using an intra-oral ultrasound probe, with emphasis on the radiologic technique

H.J. Teertstra, W.L. Lodder, I.B. Tan, F.A. Pameijer, L.E. Smeele,

M.-L.F. van Velthuysen, M.W.M. vd Brekel; Amsterdam/NL (h.teertstra@nki.nl)

Purpose: To investigate tumour-thickness measurement with intraoral ultrasound. Clinical results were recently published (Eur Radiol. 2010 Aug 3, W.L.Lodder et al); the current presentation will focus on the radiologic technique.

Methods and Materials: A retrospective data analysis was undertaken for a total of 65 patients with a T1-2 oral cavity cancer, who were seen at a tertiary referral centre between 2004 and 2010. The correspondence between tumour thickness measured by ultrasonography and histopathology was assessed by Pearson's correlation coefficient, and also between tumour thickness and the development of neck metastasis. Results: In 11 cases, intra-oral measurement was not optimal due to limited mouth opening $(n=2)$ or impossibility to depict the lesion $(n=9)$. Tumour thickness measured by US correlated well with histopathology $(n=23, R=0.93)$. Tumour thickness of $\leq 7 \mathrm{~mm}$ carries a risk of lymph node metastasis of $12 \%$, whereas in tumours exceeding $7 \mathrm{~mm}$ this risk is $57 \%(p=0.001)$. Twenty-five per cent developed neck metastasis and $19 \%$ had local recurrence.

Conclusion: Tumour thickness is an important predictive marker for lymph node metastases. As such, it can help in decision-making with regard to management of the primary tumour and neck. Intraoral ultrasound is feasible to measure tumor thickness, especially in tongue tumours. Based upon our findings, a wait-and-see policy is only warranted for superficial lesions with tumour thickness of less than $7 \mathrm{~mm}$, but only if regular follow-up using US-guided aspiration of the neck is ensured.

\section{B-192 10:39}

Endocavitary sonography in the study of oral cavity tumours

M. Morella, M. Moschetta, S. Capodiferro, E. Maiorano, G. Favia, G. Angelelli; Bari/lT (marco.moschetta@gmail.com)

Purpose: This study aims to evaluate the role of endoral sonography in the preoperative management of oral cavity tumours using high frequency transducers. Methods and Materials: Five sane volunteers and nine patients affected by oral cavity tumours (minor salivary glands in 2 cases and carcinomas in the remaining 7) underwent endoral sonography examination using intraoperative $13 \mathrm{MHz}$ transducers. Sane volunteers ultrasound images were compared with anatomical images, the latest considered as the reference standard. Ultrasound images obtained by pathologic patients were compared with postoperative histological findings. Particularly, tumour sizes and depth of infiltration within the histological stratification were evaluated. 
Results: In sane volunteers, single anatomical layers were differentiated in all cases: cheek mucosal, submucosal and muscular layers; tongue mucosal and muscular layers; hard palate mucosa and periosteum. In neoplastic patients, tumour sizes were correctly evaluated in all cases, while depth of infiltration was correctly determined in 8 out of 9 cases, with sensitivity and diagnostic accuracy values of $89 \%$.

Conclusion: Sonography examination seems to be a promising tool for the study of oral cavity tumours because it allows to demonstrate the tumour depth of infiltration.

\section{B-193 10:48}

Dual energy CT of head and neck cancer: average weighting of low and high voltage raw data improves lesion delineation and image quality J.M. Kerl, A. Tawfik, R.W. Bauer, T.J. Vogl, M.G. Mack;

Frankfurt a. Main/DE (matthias.kerl@kgu.de)

Purpose: To compare between five dual energy image data sets pure $80 \mathrm{kv}, 140$ $\mathrm{kv}$ and average images with three different weighting factors (WF) for the detectability of head and neck squamous cell carcinoma (HNSCC).

Methods and Materials: 35 patients with HNSCC who underwent a contrast-enhanced dual-source scan in dual energy mode (Siemens Flash) included in this study. Images were reconstructed as pure $80 \mathrm{kv}, 140 \mathrm{kv}$, and average weighted image data sets in 3 different WF $(0.3,0.6,0.8)$ incorporating $(30 \%, 60 \%, 80 \%$ from the $80 \mathrm{kv}$ tube, respectively). Vascular enhancement, objective noise, lesion and muscle attenuation and CNR were recorded in the 5 image data sets. Three radiologists in consensus reviewed all images and rated the image quality and lesion detectability on a 5-point scale. Results: Tumour enhancement and muscle attenuation increased with decreasing tube voltage from $140 \mathrm{kVp}$ through $80 \mathrm{kVp}$, but the total increase in muscle attenuation from $140 \mathrm{kVp}$ through $80 \mathrm{kVp}(21 \mathrm{HU}, 36 \%)$ was small compared to that of the tumour $(74 \mathrm{HU}, 97 \%)$, resulting in an increase in tumour-muscle difference. CNR was significantly higher at WF 0.6 than at 0.3 , and 0.3 than at $140 \mathrm{kV}(\mathrm{p}<0.0001)$. In addition, WF 0.6 showed the best image quality, followed by $0.8,0.3$ and $80 \mathrm{kV}$ dataset. Conclusion: Mixing the dual energy raw data from the $80 \mathrm{kv}$ and $140 \mathrm{kv}$ tubes in WF 0.6 results in a high CNR and the best subjective overall image quality and lesion detectability.

\section{B-194 10:57}

Clinical impact of FDG PET-CT on management decisions in patients with head and neck tumours

P. Bhatnagar ${ }^{1}$, J. Martin ${ }^{2}$, A. Goldstone ${ }^{1}$, F.U. Chowdhury ${ }^{1}$, K. Dyker ${ }^{1}$, M. Sen ${ }^{1}$

A.F. Scarsbrook'; ' Leeds/UK, ${ }^{2}$ Bradford/UK (drpriyabhatnagar@gmail.com)

Purpose: To review the impact of FDG PET-CT in the management pathway of patients with head and neck tumours.

Methods and Materials: Consecutive patients with primary head and neck tumours undergoing FDG PET-CT between April 2007 and December 2009 were retrospectively analysed. Scan indication, findings on PET-CT compared to conventional imaging and impact on subsequent patient management were evaluated. Clinical impact was divided into: major: detection of occult disease or characterisation of indeterminate lesion (s) on conventional imaging; minor: confirmation of suspected metastases seen on conventional imaging; no impact.

Results: 77 patients underwent 92 PET-CT scans during the study period (mean age 57 , range 14 to 83 years) including 38 patients with oro-pharyngeal carcinoma, 5 with naso-pharyngeal carcinoma, 25 with squamous carcinoma in cervical nodes but unknown primary and 9 with other primary sites in the head and neck. 56 scans were performed for staging, 36 scans were for restaging or assessment of suspected disease recurrence. In 31 cases (33\%) PET-CT had a major impact on subsequent patient management. 6 patients had unsuspected nodal involvement, 5 patients had occult primary tumours and 2 had unsuspected metastatic disease. In a further 18 patients, PET-CT clarified indeterminate lesions as either malignant (11 patients) or benign (7 patients). PET-CT had a minor impact on 41 patients (46\%) and no impact on 20 cases $(21 \%)$.

Conclusion: FDG PET-CT has a valuable role in the management of patients with head and neck tumours by improving staging accuracy and helping characterise indeterminate lesions.

\section{B-195 11:06}

Diagnostic significance of magnetic resonance imaging in preoperative evaluation of patients with laryngeal tumours

B. Banko, J. Milovanovic, M. Kratovac-Dunjic, G. Lilic, R. Milenkovic,

T. Stosic-Opincal, R. Maksimovic; Belgrade/RS (bojanbanko@yahoo.com)

Purpose: To evaluate diagnostic accuracy of pretreatment surgical magnetic resonance images (MRI) in assessment of patients with laryngeal tumours.

Methods and Materials: The prospective study included 34 patients (mean age $62.2 \pm 5.1$ years) with suspected tumours of the larynx who underwent laryngeal endoscopy, followed by MRI. The MRI images were evaluated for invasion of paraglottic, preepiglotic space, cartilage invasion, extension to extralaryngeal tissue and lymph nodes, and were compared with surgical findings.

Results: In 15 patients (44\%) the tumour was glottic, in 10 patients (29\%) supraglottic and in $9(15 \%)$ patients transglottic. Ten patients $(29 \%)$ had subglottic extension. No statististical difference was seen in number of patients with positive MRI findings in comparisson with surgery for extension to subglottic subside (29\% vs $38 \%$ ), growth to the anterior commisure ( $48 \%$ vs $48 \%$ ), preepiglottic space (33\% vs $29 \%$ ) and cartilage infiltration (19\% vs 14\%), respectively. However, infiltration of the paraglottic spaces was observed more frequently on MRI ( $81 \%$ vs $52 \%), p<0.05$. Twelve tumor specimens were classified as T2 (36\%), 18 as T3 (53\%) and 4 as T4 (11\%). According to our MR findings, 4 (12\%) patients had T2, 26 (76\%) had T3 and 4 (12\%) patients had T4 staging. The MRI staging was correctly classified in $77 \%$ patients and the overall correlation was statistically significant $(\rho=0.59,<0.05)$. Fifteen patients had metastases in regional lymph nodes and only two were detected on MRI.

Conclusion: MRI has been shown to be a reliable method for presurgical assessment of patients with tumour of the larynx.

\section{B-196 11:15}

MRI with surface microcoils in the follow-up of laser resections for glottic tumours: initial experience

D. Farina, P. Rizzardi, M. Ravanelli, R. Maroldi; Brescia/IT (jam.81@virgilio.it)

Purpose: To assess the effectiveness of MRI with surface micro-coils in the follow-up of glottic tumours treated by endoscopic laser surgery.

Methods and Materials: Nineteen patients treated for primary glottic tumour (5 T1, 9 T2, 5 T3) by endoscopic laser resection underwent MRI with surface coils (two $4 \mathrm{~cm}$ loop coils). TSE T2 sequences were applied on axial and coronal planes Signal/noise (SNR) was calculated for lateral crico-arythenoid muscle (LCAM), scars and recurrences. Contrast/noise (CNR) between LCAM and scar and between LCAM and recurrence was also calculated.

Results: Submucosal recurrences were proven in $9 / 19$ patients, $3 / 9$ cases completely negative on endoscopy. MRI detected recurrence in $8 / 9$ cases. In one case imaging findings were considered doubtful and the patient was addressed to biopsy. No false negative and false positive were seen. Mean SNR of submucosal recurrences was significantly higer than those of LCAMs and scars (10.23; 5.39 and 3.77, respectively, on axia TSE-T2 images; 22.33; 7.69 and 3.26, respectively, on coronal TSE-T2 images). Mean CNR between LCAMs and scars was significantly higer than CNR calculated between LCAMs and recurrences ( 2.43 vs -4.32 on axial images; 2.37 vs -8.26 on coronal images). Conclusion: MRI with surface microcoils is effective in the detection of submucosal recurrences after laser larynx surgery even if endoscopy is completely negative. TSE-T2 sequences enable differential diagnosis between scar tissue and recurrent tumours on both axial and coronal planes.

\section{B-198 11:24}

Evaluation of role of ultrasonography and Doppler in assessment of cervical lymphadenopthy

H.V. Vadvala, N. Patel, A. Arya, S. Shah, H. Rajpura; Ahmedabad/IN (harshna_21984@yahoo.co.in)

Purpose: To determine the most reliable ultrasonographic parameters for differentiation of benign and malignant cervical lymph nodes with emphasis on intranodal vascularity and spectral indices.

Methods and Materials: A prospective study was conducted on 94 patients using high-resolution $7.5 \mathrm{MHz}$ linear transducer. Size, L/T Ratio, echogenic hilum, noda cortex, nodal margin, necrosis and calcification were evaluated on grey-scale ultrasound. On colour Doppler, type of intranodal vascularity was characterised as central, peripheral, mixed or abnormal flow such as displacement, aberrant vessel and focal absence of perfusion. $\mathrm{RI}$ and $\mathrm{PI}$ values $<0.6$ and $<1.2$, and $>0.8$ and $>1.5$ were considered as specific for benign and malignant lymph nodes, respectively. Diagnosis on each mode was categorised and histopathologically correlated. Results: $\mathrm{L} / \mathrm{T}$ ratio of $<2$, partial or complete absence of hilum with eccentric cortex and heterogeneous echotexture showed a sensitivity of $71.4 \%, 68.3 \%$ and $65.3 \%$, respectively, for malignant nodes. Grey scale, colour Doppler, spectral Doppler and combined grey scale and Doppler showed sensitivity and specificity of $(86.2 \& 74.6 \%)$, (82.1\&90\%), (82.2\&94.2\%) and (93\&88.3\%), respectively, for diagnosis of malignant nodes. Sensitivity and specificity for cut off values of R $>0.676$ and $\mathrm{PI}>1.2$ for malignant nodes were $81.5 \%$ and $88.75 \%$, respectively. Conclusion: The $\mathrm{L} / \mathrm{T}$ ratio, hilum and echogenecity were the critical indicators for diagnosing malignant nodes on grey-scale sonography. The distribution of intranodal vascularity was found more accurate than spectral indices in differentiating cervical lymphadenopathy. Addition of colour Doppler to conventional ultrasonography improved the diagnostic accuracy for characterisation of lymph nodes. 


\section{B-199 11:33}

Optimisation of reconstruction algorithms in Truepoint HD PET/CT for improved diagnostic accuracy of lymph node metastases in head and neck squamous cell carcinoma (HNSCC)

M. Sadick', H. Kayed ${ }^{1}$, R. Pinol', S. Frey ${ }^{1}$, H. Sadick', C. Fink',

J. Brade', S.O. Schönberg', V. He ${ }^{2} ;{ }^{1}$ Mannheim/DE, ${ }^{2}$ Wuhan/CN

(maliha.sadick@medma.uni-heidelberg.de)

Purpose: Reconstruction parameters (RP) influence image quality and diagnostic accuracy in lymph node (LN) metastases detection in HNSCC. Optimised RP was evaluated using histopathological verification as gold standard.

Methods and Materials: RP consisted of fixed 3 iterations and 24 subsets applying increasing Gaussian filter sizes of 1, 3 and $6 \mathrm{~mm}$ FWHM with decreasing spatial and contrast resolution and noise. 28 patients ( 7 female, 21 male; mean age 61 years) underwent PET/CT (Siemens Biograph TruePoint ${ }^{T M}$ ) for initial staging of HNSCC. Data was assessed independently by two readers for pathological $L N$ according to the TNM classification in HNSCC. Diagnostic accuracy for both readers was evaluated and histology was correlated with the change in the standard uptake value (SUVmax) according to the three filter sizes.

Results: In total, 23 benign and 41 malignant LN were confirmed histologically The SUV mean values were significantly different for all filters (t-test $p<0.0001$ ) $6 \mathrm{~mm}$ benign $2.8 /$ malignant $4.4 ; 3 \mathrm{~mm}$ benign $3.1 /$ malignant $5.2 ; 1 \mathrm{~mm}$ benign $3.1 /$ malignant 5.3. Filter one proved most sensitive for SUV differentiation. Sensitivity for malignant LN detection increased with decreasing filter size: $6 \mathrm{~mm} \mathrm{88 \% /50 \%}$ sensitivity/specificity (Chi Square p < 0.0021), $3 \mathrm{~mm} 91 \% / 27 \%$ sensitivity/specificity (Chi Square p < 0.05), $1 \mathrm{~mm} 92 \% / 26 \%$ sensitivity/specificity (Chi Square $p<0.05$ ). Conclusion: Reconstruction parameters adapted to low photon attenuation areas like the head and neck region improve diagnostic accuracy of LN metastases detection in HNSCC and contribute to precise surgical resection.

\section{B-200 11:42}

Grey and power Doppler study of biopsy proven Kikuchi disease: analysis of 175 cases

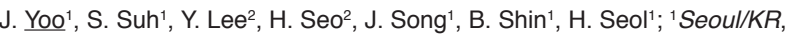

2Ansan/KR (jewel8@hanmail.net)

Purpose: Kikuchi disease (KD) is a benign, self-limiting cause of cervical lymphadenopathy. The purpose of this study is to characterise the ultrasound (US) features of KD that can contribute in differentiating KD with other diseases causing cervical lymphadenopathy.

Methods and Materials: US findings of 175 patients with biopsy proven KD were retrospectively reviewed. The mean age of the patients was 27.3 years and the male-to-female ratio was 39:136. Pathologic findings were classified into proliferative $(n=57)$, necrotising $(n=109)$, and xanthomatous $(n=9)$ types. US findings were evaluated according to grey and power Doppler study. On grey scale, lymph nodes (LNs) were assessed by size, shape (shortest axis/longest axis), location presence of conglomeration, gross necrosis, echogenic nodal hilum, and increased perinodal echogenicity. On power Doppler, vascular pattern was assessed.

Results: The mean maximum diameter of the $175 \mathrm{LNs}$ was $1.6 \mathrm{~cm}$. $75 \mathrm{LNs}$ were oval $(S / L=0.5-0.7)$ and 86 were elongated $(S / L<0.5)$. Eight of them were round $(S / L$ $>0.7$ ) and six were conglomerated. LNs were mainly located in levels II and V. 17 LNs showed gross necrosis and 149 had an echogenic hilum. 76\%(133/175) of the LNs showed increased perinodal echogenicity which is a dominant finding of $K D$. Increased perinodal echogenicity was seen in $93.5 \%(102 / 109)$ of the necrotising type and $43.8 \%(25 / 57)$ of the proliferative type; difference between the two was statistically significant $(p=0.001)$. On power Doppler, $161 \mathrm{LNs}$ showed norma hilar vascularity, whereas $14 \mathrm{LNs}$ showed absent hilar vascularity and one demonstrated displaced hilar flow.

Conclusion: Understanding the US findings of KD can contribute in differentiating $\mathrm{KD}$ with other causes of cervical lymphadenopathy.
10:30 - 12:00

Room I/K

\section{Cardiac}

\section{SS 403a}

\section{Cardiac MR: myocardiopathies and more}

Moderators:

A. Carneiro; Porto/PT

L. Natale; Rome/lT

B-201 10:30

Does myocardial mass influence in the presence of myocardial fibrosis?

Does it have prognostic implications?

A.B. Alcolado Jaramillo, J. Gonzalez Mirelis, M.A. Cavero Guibanel,

M.A. Pastrana Ledesma, P. Garcia Pavia; Majadahonda/ES

(anaalcolado_8@hotmail.com)

Purpose: Our aim is to assess the distribution of left ventricular mass (LV) in patients with hypertrophic cardiomyopathy $(\mathrm{HCM})$ and its relation to the presence and location of myocardial fibrosis in left ventricule in sequences of delayed contrastenhancement cardiovascular magnetic resonance (CMR). We analyse the role of myocardial fibrosis in the prediction of sudden death and heart failure in HCM. Methods and Materials: In $50 \mathrm{HCM}$ patients, LV mass by CMR was measured and compared with healthy control subjectss. In all patients left ventricular function was analysed. We evaluated and identified localisation of myocardial fibrosis with delayed enhancement. We have assessed the presence and amount of myocardial fibrosis in HCM patients and analysed their risk factors for sudden death.

Results: The mean of left ventricular myocardial mass from which there exists delayed enhancement was $238.9 \pm 61$ gr. The most frequent distribution pattern for delayed enhancement was multifocal. The LV mass showed a modest relationship to the myocardial fibrosis. $75 \%$ of patients showing myocardial fibrosis had at least one risk factor for sudden death.

Conclusion: Myocardial fibrosis was not observed in all patients with HCM, but when they presented the delayed enhancement, there was a correlation between the presence of myocardial fibrosis and the degree of hypertrophy. The presence of delayed enhancement was more prevalent among patients with a higher risk profile. This can change the therapeutic approach in primary prevention, using automatic implantable desfibrillators in patients with risk factor for sudden death.

\section{B-202 10:39}

Late gadolinium enhanced-cardiac magnetic resonance (LGE-CMR) quantification of myocardial damage as prognostic indicator in hypertrophic cardiomyopathy (HCM)

G. Ligabue ${ }^{1}$, S. Bertugno' ${ }^{1}$, F. De Cobelli ${ }^{2}$, L. Natale ${ }^{3}$, C. De Cecco ${ }^{3}$ I. Carbone ${ }^{3}$, M. Luzietti ${ }^{3}$, P. Torricelli', R. Fattori ${ }^{4} ;{ }^{1}$ Modena/IT, ${ }^{2}$ Milan $/ I T,{ }^{3}$ Rome $/ I T$, ${ }^{4}$ Bologna/lT (gligabue@sirm.org)

Purpose: To investigate the prognostic value of LGE-CMR, throughout the association between LGE extension/evolution and major adverse cardiac events (MACE) in HCM patients.

Methods and Materials: 42 patients (mean age: $57 \pm 16.2 ; 73.8 \%$ males) with documented HCM underwent CMR. LGE-CMR images were obtained 15 min after injection of $0.2 \mathrm{mmol} / \mathrm{kg}$ of gadolinium-based contrast agent. Indexed end-diastolic left ventricular mass was (IEDLV mass) assessed and myocardial damage (expressed as \% of total LV myocardial mass) was quantified and defined as intensity $6 \mathrm{SD}$ above the mean signal of a non-enhanced myocardium area. Ooccurrence of clinical adverse events during follow-up period was recorded. Mean follow-up duration was $21.6 \pm 16.2$ months.

Results: The mean indexed LV mass was $87.31 \pm 22.7 \mathrm{~g} / \mathrm{m}^{2}$. On LGE-CMR, 36 (85.7\%) patients exhibited myocardial scar. The mean LGE was $7.05 \pm 6.4 \%$ o IEDLV mass. There was no association between IEDLV mass, LGE and ventricula tachycardia (VT), NYHA functional classes III or IV and LV outflow obstruction. During the follow-up 19 MACE occurred. There was no association between MACE occurrence and IEDLV mass. Both the presence and the size of the scar were significant predictors of the occurrence of MACE. Patients who presented MACE had significantly higher value of LGE percentage compared to those without: 1 MACE occurred with $2>$ LGE\%LGE\% $22 \%, P<0.013$.

Conclusion: Presence and extension of LGE seem to be important predictive factors for the occurrence of MACE in HCM patients. 


\section{B-203 10:48}

Interaction between myocardial fibrosis, outflow obstruction and early left ventricular dysfunction in hypertrophic cardiomyopathy: an MRI study G. Rinaldi, E. Biagini, L. Lovato, V. Russo, C. Rapezzi, R. Fattori; Bologna/lT (gio_rinaldi_81@yahoo.it)

Purpose: To evaluate the influence of myocardial fibrosis, detected by magnetic resonance imaging (MRI) as late gadolinium enhancement (LGE), on left ventricular outflow tract (LVOT) obstruction, left ventricular function during exercise in $\mathrm{HC}$ patients with normal ejection fraction (EF) at rest.

Methods and Materials: $52 \mathrm{HC}$ patients underwent cardiac MRI and performed bicycle exercise echocardiogram and tissue Doppler. LGE was considered to be relevant when $>3.93 \%$ of total LV mass $\left(66^{\text {th }}\right.$ percentile). Patients were divided into two groups: LGE - (LGE < 3.93\%, 34 patients) and LGE + (LGE > 3.93\%, 18 patients).

Results: At peak exercise, the increase in LVOT gradient was lower in the LGE + group ( $16 \pm 14 \mathrm{mmHg}$ vs $32 \pm 35 \mathrm{mmHg}$; $=0.02$ ) while on tissue-Doppler measurements, patients in the LGE + group showed a higher E' velocity at peak exercise $(11.5 \pm 5.2 \mathrm{~cm} / \mathrm{s}$ vs $8.8 \pm 3.4 \mathrm{~cm} / \mathrm{s} ; p=0.028)$, a greater increase in $E^{\prime}$ velocity from rest to peak exercise $(4.3 \pm 4.0 \mathrm{~cm} / \mathrm{s}$ vs $1.1 \pm 3.8 \mathrm{~cm} / \mathrm{s} ; \mathrm{p}=0.008)$ and a less impaired diastolic reserve index $(5.4 \pm 4.0$ vs $2.6 \pm 3.5 ; p=0.011)$.

Conclusion: In patients with $\mathrm{HC}$ and normal EF at rest, myocardial fibrosis is associated with a lower LVOT gradient and with a less impaired longitudinal diastolic function during exercise.

\section{B-204 10:57}

Cardiac magnetic resonance: correlation between left atrial area and late enhancement and myocardial mass in hypertrophic cardiomyopathy C. De Cecco, G. Muscogiuri, M. Di Girolamo, S. Fierro, F. Mancuso, V. David; Rome/IT (g.muscogiuri@hotmail.it)

Purpose: To correlate left atrial area with late enhancement and myocardial mass in patients with hypertrophic cardiomiopathy (HCM).

Methods and Materials: We retrospectively evaluated 28 patients with $\mathrm{HCM}$ diagnosis and late enhancement underwent cardiac MR using a 1.5 T system (Magnetom Sonata, Siemens). The MRI protocol consisted of a left ventricular functional study (4-chamber, vertical long axis, short axis) (TR: $3.09 \mathrm{~ms}, \mathrm{TE}: 1.3 \mathrm{~ms}$, FA: 80 , thick: $8 \mathrm{~mm}$ ) followed by late enhancement data set acquired $10-15$ minutes after iv administration of $0.2 \mathrm{mmol} / \mathrm{kg}$ of Gd contrast agent. Late enhancement was evaluated on Inversion recovery Turbo-Flash sequences (TR: $8 \mathrm{~ms}$, TE: $4 \mathrm{~ms}$, TI: 250-340 ms, thick: $8 \mathrm{~mm}$ ) as an area with SI > 6SD from normal tissue. We evaluated left atrial area on 4-chamber plane and myocardial mass was calculated with a dedicated software. LGE mass was measured by manual planimetry on short axis. For statistical analysis we used Spearman's correlation.

Results: The patients showed the following parameters: mean LE mass $9.1 \pm 8.8 \mathrm{~g}$, left atrial area $29.1 \pm 7.1 \mathrm{~cm}^{2}$ and myocardial mass $199.7 \pm 79.5 \mathrm{~g}$. A significant correlation between left atrial area and late gadolinium mass $(p=0.03)$ was observed; no evidence of significant correlation between myocardial mass and left atrial surface $(p=0.2)$ was observed.

Conclusion: Our results demonstrate a positive correlation between late enhancement mass and left atrial area. Late enhancement is not only a marker of myocardial disarray and fibrosis but also correlated with cardiac function. Our study confirms the importance of late enhancement quantification in $\mathrm{HCM}$ for patient stratification and prognosis.

\section{B-205 11:06}

Myocardial late enhancement is the stronger predictor of left ventricle systolic dysfunction in Becker muscular dystrophy: a cardiac magnetic resonance and ${ }^{31} \mathrm{P}$ MR spectroscopy study

A. Esposito ${ }^{1}$, F. De Cobelli', M. Cava ${ }^{1}$, G. Perseghin'1 , G. D'Angelo², T. Canu¹, C. Sallemi ${ }^{1}$, E. Clementi ${ }^{1}$, A. Del Maschio ${ }^{1} ;{ }^{1}$ Milan/IT, ${ }^{2}$ Bosisio Parini/IT (esposito.antonio@hsr.it)

Purpose: Becker muscular dystrophy (BMD) is a progressive myopathic disorder. Cardiac involvement represents the primary cause of death but the pathogenic events remain obscure. Aim was to establish the relative relevance of functional, structural and metabolic cardiac alterations characterising BMD.

Methods and Materials: $13 \mathrm{BMD}$ patients (age: $36 \pm 12$ years; range: $20-57$ ) underwent neurological evaluation with grading of skeletal muscle dysfunction (average muscle score, AMS, 10: normal to 0: global dysfunction), comprehensive cardiac magnetic resonance (CMR) study including late-enhancement (LE) assessment and cardiac phosphorous MR-spectroscopy ( ${ }^{31} \mathrm{P}$-MRS) to quantify PCr/ATP-ratio. 27 normal subjects (CS) matched for age, sex and BMI underwent CMR and ${ }^{31} \mathrm{P}-\mathrm{MRS}$ as control group.
Results: Patients showed lower LV ejection fraction (LVEF) (BMD: $54 \pm 10 \%$ vs CS: $61 \pm 4 \% ; p=0.021$ ), end-diastolic-wall-mass (EDWM) (BMD: $103 \pm 14 \mathrm{gr}$ vs CS: $136 \pm 25 \mathrm{gr} ; \mathrm{p}<0.001$ ), left ventricle mass index (BMD: $58 \pm 7$ vs CS: $71 \pm 14$; $p<0.001$ ) and higher end-diastolic-volume/EDWM ratio (BMD: $1.61 \pm 0.38$ vs CS: $1.04 \pm 0.14, p<0.001)$. In BMD patients PCr/ATP-ratio tended to be reduced (BMD: $1.73 \pm 0.45$ vs CS: $1.99 \pm 0.41 ; p=0.07$ ). All BMD patients had LE in the LV wall with highly repetitive distribution pattern (basal-medium lateral wall with subepicardial/mesocadial distribution). LE percentage (LE\%) was $18 \pm 14 \%$ (range: $3 \%-55 \%$ ). LE\% showed a trend to correlate with AMS and was higher in $\mathrm{BMD}$ patients with $\mathrm{EF}<50 \%$. In multivariate analysis including age, BMI, EDV EDWM-ratio, PCr/ATP-ratio, LE\% and AMS, LE\% resulted the stronger predictor of LVEF $\left(R^{2}=0.74 ; p<0.001\right)$.

Conclusion: BMD cardiac involvement is characterised by alterations of morphology, function, energy metabolism and structure, but the amount of myocardial LE explains alone the $76 \%$ of the LVEF variability. LE is an important biomarker of the degree of severity of cardiac involvement in BMD.

\section{B-206 11:15}

Cardiac iron distribution within myocardium measured by MRI-R2* in patients with thalassemia

J. Yamamura ${ }^{1}$, B. Schännagel ${ }^{1}$, R. Grosse ${ }^{1}$, J. Graessner ${ }^{1}$, P. Nielsen ${ }^{1}$, G.E. Janka ${ }^{1}$, G. Adam ${ }^{1}$, R. Fischer ${ }^{2} ;{ }^{1}$ Hamburg/DE, ${ }^{2}$ Oakland, CA/US

(j.yamamura@uke.uni-hamburg.de)

Purpose: To assess regional iron distribution by MRI-R2* within the heart of patients with $\beta$-thalassemia major $\left({ }^{\mathrm{TM}}\right)$ and other iron overload diseases.

Methods and Materials: Breathhold ECG gated MRI (1.5 T) of the heart was used for the measurement of transverse relaxation rates $\mathrm{R} 2^{*}$ in 32 patients (11-79 y). In a mid-papillary short-axis slice divided into septal, anterior, lateral, and posterior quadrants, $\mathrm{R} 2^{*}$ was analysed from ROI-based signal intensities from 12 echo times ( $T E=1.3-26 \mathrm{~ms}$ ). Typical boundary effects were evaluated in detail.

Results: The segmentation of the cardiac wall resulted in highly significant correlations of $\mathrm{R}^{*}$ between septal and all other quadrants. In the patient group with $\mathrm{R} 2{ }^{*}$ $<50$ s- 1 (normal), all quadrants show significantly higher median rates $(126 \pm 25 \%$, $150 \pm 24 \%, 174 \pm 51 \%)$ than the septum $(p<10-4)$. Typical boundary effects on segmental R2* from blood, lung tissue, epicardial fat and hepatic iron could not be easily separated from segmental iron distribution.

Conclusion: The measurement of MRI-R2* in the interventricular septum is representative for the cardiac wall and the least affected method by boundary effects to detect patients with iron overload at risk of developing heart failure.

\section{B-207 11:24}

Prognostic role of cardiac magnetic resonance in patients with premature ventricular complexes of left bundle branch block morphology

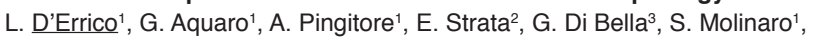

M. Lombardi', C. Bartolozzi ${ }^{1} ;{ }^{1}$ Pisa/IT, ${ }^{2}$ Florence $/ I T,{ }^{3}$ Messina/IT

(ginaderr@hotmail.com)

Purpose: CMR is a component of the diagnostic work up for the differential diagnosis between arrhythmogenic right ventricular cardiomyopathy/dysplasia (ARVC/D) and idiopathic RV tachycardia. RV abnormalities evaluated by CMR could have prognostic importance. We investigated whether the presence of right ventricular (RV) abnormalities detected by cardiovascular magnetic resonance (CMR) predict adverse outcome in patients presenting with frequent premature ventricular complexes (PVCs) of left bundle branch block (LBBB) morphology.

Methods and Materials: Four hundred and forty consecutive patients with $>1,000 \mathrm{PVCs}$ of LBBB morphology (minor diagnostic criterion of ARVC/D) and no other pre-existing criteria were prospectively enrolled. RV wall motion (WM), signal abnormalities, dilation, and reduced jection fraction evaluated by CMR were considered imaging criteria of ARVC/D. Follow-up was performed evaluating an index composite endpoint of 3 cardiac events: cardiac death, resuscitated cardiac arrest, and appropriate implantable cardiac-defibrillator shock.

Results: Subjects with multiple RV abnormalities (RVA-2 group) had worse outcome than the no-RVA group (hazard ratio [HR]: 48.6; 95\% confidence interval [Cl]: 6.1 to $384.8 ; p<0.001$ ). Of the 61 patients in the RVA-2 group, only 6 had a definite diagnosis of ARVC/D applying the Task Force Criteria. Also, subjects with a single imaging criterion (RVA-1 group) had worse outcome than the no-RVA group (HR: $18.2 ; 95 \% \mathrm{Cl}$ : 2.0 to $162.6 ; p=0.01)$. Patients with only WM abnormalities had higher prevalence of cardiac events thanno-RVA (HR: $27.2 ; 95 \% \mathrm{Cl}: 3.0$ to $244.0 ; p=0.03$ ). Conclusion: In subjects with frequent PVC of LBBB morphology, CMR allows risk stratification. RV abnormalities were associated with worse outcome. 


\author{
B-208 11:33 \\ Septal late gadolinium enhancement at the right ventricular insertions in \\ contrast-enhanced cardiac MRI: prevalence and underlying pathologies \\ P. Bischoff, P.W. Radke, R.L. Duschka, S. Kapsimalakou, \\ S. Hollmann, A. Kovacs, F.M. Vogt, J. Barkhausen, P. Hunold; \\ Lübeck/DE (peter.bischoff@uk-sh.de)
}

Purpose: Myocardial late Gd enhancement (LGE) in MRI is a reliable tool in the detection and differential diagnosis of myocardial disease. Aim of this study was to identify septal LGE at the right ventricular insertions and to define its prevalence and the underlying pathologies.

Methods and Materials: A total of 370 contrast-enhanced cardiac MRI studies were performed (1.5 T/3.0 T, Achieva, Philips). The MRI protocol consistently contained Cine SSFP and 3D T1w IR-LGE acquired 8-15 min after administration of $0.2 \mathrm{mmol} / \mathrm{kg}$ gadubotrol (Gadovist $₫$ ). Septal LGE at the right ventricular insertions was evaluated regarding its prevalence and the underlying pathology.

Results: Non-infarction-related LGE was found in 142/370 (38\%) patients. In 43 cases (30\%), typical LGE was detected at the right ventricular insertion, $21 \mathrm{pts}(49 \%)$ had LGE both of the anterior and inferior (ant./inf). insertion, $22(51 \%)$ pts only a the inferior (inf). insertion: The underlying pathology was dilated cardiomyopathy in $10 / 43(23 \%)$ pts (ant./inf.: 7 ; inf. 3), left ventricular hypertrophy due to aortic valve stenosis in 10/42 (23\%) pts (ant./inf.: 4; inf. 6), hypertrophic cardiomyopathy in $8 / 43$ (19\%) pts (ant./inf.: 5 ; inf. 3), pulmonary hypertension in 6/43 (14\%) pts (ant./inf.: 4 inf. 2), storage disease in $3 / 43(7 \%)$ pts (ant./inf.: 1 ; inf. 2), myocarditis in $3 / 43(7 \%)$ pts (inf.: 3 ). In 3 of 43 pts ( $7 \%$ ), the underlying pathology remained unclear (inf. 3) Conclusion: Septal LGE at the right ventricular insertions is a frequent finding This specific LGE pattern seems to be useful in the differential diagnosis of nonischaemic myocardial disease.

\section{B-209 11:42}

Feasibility of cardiac diffusion-weighted MR imaging in acute myocarditis: a preliminary bicentric study

J.-P. Laissy', V. Gaxotte', N. Pasi', A. Ribet'2, J.-M. Serfaty'; ${ }^{1}$ Paris/FR,

${ }^{2}$ Velizy/FR (jean-pierre.laissy@bch.ap-hop-paris.fr)

Purpose: To prospectively determine whether diffusion-weighted cardiac MR imaging (DWI) is feasible and to determine cardiac DWI patterns in patients with acute myocarditis, by comparison with delayed-enhancement (DE) cardiac magnetic resonance (CMR) sequences.

Methods and Materials: Twenty-nine consecutive patients referred for CMR with a clinical presentation suggestive of acute myocarditis were included. Three patients with inconclusive final diagnosis were excluded, resulting in a final cohort of 26 patients. Rest MR examinations included DWI, black-blood fat-suppressed T2-weighted, first-pass perfusion, and DE sequences. Abnormal DWI areas were matched to abnormal DE areas. The apparent diffusion coefficient (ADC) was measured in involved areas and compared to remote normal areas.

Results: DWI patterns were significantly correlated to DE patterns, with focal or multifocal nonsegmental nonsubendocardial hypersignals (DWI, $2.96 \pm 0.66$ segments vs $D E, 3.5 \pm 0.65$ segments, $p=n s$ ), predominantly in an inferolatera location. Segment-by-segment comparison showed a high level of correlation (y $=(1.15) \mathrm{x}-0.44, r=0.9337)$. The ADC of affected segments vs normal remote myocardium was $70.3 \% \pm 5.3 \%$

Conclusion: Cardiac DWI should provide useful additional findings in acute myocarditis. Moreover, ADC maps should help better understand inflammatory phenomena.

\section{B-210 11:51}

Differences in perfusion of malignant and benign cardiac tumours

K.U. Bauner, S. Sourbron, C. Schmitz, M.F. Reiser, A. Huber;

Munich/DE (Kerstin.Bauner@med.uni-muenchen.de)

Purpose: To determine the diagnostic value of MR perfusion imaging in differentiation of benign from malignant cardiac tumours.

Methods and Materials: 21 patients with cardiac tumours (myxoma $(n=7)$, rhabdomyoma $(n=2)$, angiolipoma $(n=1)$, angiosarcoma $(n=5)$, rhabdomyosarcoma $(n=1)$, leiomyosarcoma $(n=2)$, lymphoma $(n=1)$, metastasis $(n=2$, sarcoma and melanoma)) were examined (histopathologic correlation was present in all cases). A saturation recovery T1w GRE technique was implemented for tumour perfusion imaging. The following perfusion parameters were assessed for tumour tissue, normal myocardium and the arterial input function (AIF): 1. maximum-contrastenhancement-ratio $\left(\mathrm{CER}_{\text {mex }}[\%]\right.$ ) and 2. the maximum-slope-of-CER-curve (slopeCER[\%/sec]). Tumour perfusion relative to normal myocardium and to the AIF was calculated. Differences between benign and malignant tumours, sensitivities and specificities were calculated.
Results: CERmax and slopeCER of malignant cardiac tumours was significantly higher compared with benign lesions $(p<0.001)$. Calculation of CERmax and slopeCER of myocardium and AIF was not significantly different between malignant and benign lesions. $\mathrm{CER}_{\max }$ (tumour/myo) and $\mathrm{CER}_{\max }$ tumour/AIF) were significantly higher in malignant tumours compared with benign lesions $(\mathrm{p}=0.0015$ and $p=0.0016)$ as was the slopeCER (tumour/myo) $(p=0.037)$. The calculated sensitivity and specificity of $\mathrm{CER}_{\text {max }}$ tumour/myo), $\mathrm{CER}_{\text {max }}$ tumour/AIF) and the slopeCER (tumour/myo) for differentiating malignant from benign lesions resulted in $100 \%$ and $63.3 \%, 100 \%$ and $63.3 \%$ and $100 \%$ and $54.5 \%$, respectively, with cut-off values of $1.8,1.08$ and 0.20 .

Conclusion: MR pefusion imaging delineates significant differences between malignant and benign cardiac tumours. Higher CERmax of tumour tissue relative to normal myocardium and relative to the AIF indicates malignancy as well as a high-slopeCER of tumour in relation to normal myovardium.

10:30 - 12:00 Room L/M

Neuro

\section{SS 411}

\section{Brain tumours: detection and characterisation}

Moderators

P. Demaerel; Leuven/BE

P. Zamecnik; Heidelberg/DE

\section{B-211 10:30}

Correlation between apparent diffusion coefficient values and

histopathological subtype of meningiomas

B. Ozgen Mocan, E. Sanverdi, K.K. Oguz, M. Mut, A. Dolgun, F. Soylemezoglu; Ankara/TR (burce@hacettepe.edu.tr)

Purpose: Meningiomas are mostly benign tumours; however, atypical or malignant subtypes with more aggressive clinical course and higher recurrence rates are also seen. There is no reliable diagnostic feature for the preoperative differentiation of the high-grade subtypes. We sought to determine with a large series, whether DW imaging features could differentiate benign and malignant/atypical meningiomas or predict histopathological subgroup of the meningiomas.

Methods and Materials: Patients with pathologically proven meningiomas that had their preoperative study and surgery performed in our institution were included. MR imaging studies of 177 patients were retrospectively evaluated by a radiologist blinded to the pathology results. Mean ADC values of the lesions as well as of the normal appearing contralateral parietal white matter were measured. ADC ratios of meningioma/contralateral WM were calculated.

Results: The histopathological analysis revealed 135 benign, 37 atypical and 5 malignant lesions with 61 transitional, 38 meningothelial, 5 angiomatous, 15 fibroblastic, 11 psammomatous and 5 secretory subtypes. The mean ADC value and $A D C$ ratios were $0.99 \pm 0.4 \times 10^{-3} \mathrm{~mm}^{2} / \mathrm{s}$ and $1.22 \pm 0.6$ for benign lesions $0.84 \pm 0.1 \times 10^{-3} \mathrm{~mm}^{2} / \mathrm{s}$ and $1.05 \pm 0.1$ for atypical lesions; $0.84 \pm 0.1 \times 10^{-3} \mathrm{~mm}^{2} / \mathrm{s}$ and $0.96 \pm 0.2$ for malignant subtypes. There was no statistically significant difference between the mean ADC ratios of the different grades (ANOVA test; $p>0.001$ ) as well as among the ADC values of different histological subtypes $(p>0.3)$. Conclusion: The preoperative differentiation of the atypical/malignant meningiomas would make a significant impact on the patient counseling, treatment decision and surgical planning. However, our study failed to prove any additional value of DW imaging in this aspect.

\section{B-212 10:39}

Differentiation between benign and malignant meningiomas using diffusion and perfusion MR imaging

S. Chedia, M. Gagua, N. Kbilashvili; Kutaisi/GE (kaxabery@mail.ru)

Purpose: Our purpose was to investigate the utility of diffusion-weighted (DW) and perfusion-weighted (PW) MR imaging to differentiate benign and malignant meningiomas which were verified histologically.

Method and Materials: A total of 29 patients with meningiomas (22 benign and 7 malignant) underwent conventional, perfusion and diffusion MR imaging. Conventional MRI revealed the ordinary signs of tumour. The absolute apparent diffusion coefficient (ADC) and normalised ADC (NADC) ratio, maximal relative cerebral blood volume (rCBV) and the corresponding relative mean time to enhance (rMTE) in both tumour parenchyma and peritumoral oedema were measured.

Results: The mean ADC of malignant meningiomas $\left(0.64 \pm 0.12 \times 10^{-3} \mathrm{~mm}^{2} / \mathrm{s}\right)$ was significantly lower compared with benign meningiomas $\left(0.89 \pm 0.09 \times 10^{-3} \mathrm{~mm}^{2} / \mathrm{s}\right.$ 
$P<.0001)$. Mean NADC ratio in the malignant group $(0.93 \pm 0.18)$ was also significantly lower than the benign group $(1.29 \pm 0.12 ; \mathrm{P}<.0001)$. The mean maximal rCBV values of benign and malignant meningiomas were $7.18 \pm 4.12$ and $6.01 \pm 3.89$, in the parenchyma, and $1.07 \pm 0.95$ and $3.85 \pm 1.41$ in the peritumoral edema. The mean rMTE values were $1.14 \pm 0.23$ and $1.24 \pm 0.27$, in the parenchyma, and $0.88 \pm 0.24$ and 1.21 \pm 0.31 , in the peritumoral oedema. The defferences in rCBV and rMTE values between benign and malignant meningiomas were not significant $(P>0.05)$ in the parenchyma, but both were significant $(p<0.05)$ in the peritumoral oedema. Conclusion: Diffusion and perfusion MR imaging are useful in the preoperative differentiation between benign and malignant meningiomas.

\section{B-213 10:48}

Contribution of diffusion and perfusion weighted magnetic resonance imaging in the differentiation of meningiomas and other extra-axial tumours

A. Zimny, J. Bladowska, P. Szewczyk, J. Wagel, M. Sasiadek; Wroclaw/PL (abernac@wp.pl)

Purpose: Different extraaxial tumours often show very similar appearance in standard MRI; there are many tumours that can mimic meningiomas, e.g. dural metastases or schwannomas. The aim of our study was to evaluate usefulness of diffusion (DWI) and perfusion weighted MR imaging (PWI) in the differential diagnosis of extraaxial tumours.

Methods and Material: Forty-six extraaxial tumours (32 meningiomas including one located intraventricularly, 11 schwannomas, 2 dural metastases, and 1 choroid plexus papilloma) underwent standard MR imaging as well as DWI and PWI using 1.5 Tesla MR unit. In each tumour min. ADC and max. rCBV measurements were performed as well as analyses of perfusion curves with evaluation of the rate of their return to the baseline (return coefficient - $R C$ ).

Results: The values of min. ADC, max. rCBV and RC for different tumour types were as follows: meningiomas $(0.79 ; 8.61 ; 0.21)$, schwannomas $(1.2 ; 0.7 ; 1.8)$, dural metastases $(0.8 ; 1.2 ; 1.0)$, choroid plexus papilloma $(1.1 ; 2.0 ;-0.6)$, respectively. There were significant differences between meningiomas and all other tumour types in perfusion parameters and between meningiomas and schwannomas also in min. ADC values. There were no significant differences in min. ADC between meningiomas and dural metastases or between intraventricular meningioma (ADC $=0.9$ ) and choroid plexus papilloma.

Conclusion: Though several of the evaluated extraaxial tumours showed identical appearance in standard MRI, they differed significantly in PWI. PWI and less significantly DWI seem to be very useful in distinguishing different dural tumours, neoplasms located in the cerebello-pontine angle or intraventricularly.

\section{B-214 10:57}

Comparison of contrast-enhanced susceptibility-weighted imaging with contrast-enhanced T1-weighted imaging at 3 Tesla in patients with newly diagnosed Glioblastoma

A. Radbruch, P. Bäumer, B. Wiestler, B. Stieltjes, L. Kramp,

C. Hartmann, W. Wick, S. Heiland, M. Bendszus;

Heidelberg/DE (alexander-radbruch@gmx.de)

Purpose: Susceptibility-weighted imaging (SWI) is a promising tool for evaluating brain tumours by assessing microbleeds/intratumoural susceptibility signals (ITSS). The purpose of this study was to determine the added value of contrast-enhanced susceptibility-weighted imaging (CE-SWI) to visualise enhancement in CE-SWI in addition to ITSSs, and to compare both parameters with contrast-enhanced T1 Images (CE-T1).

Methods and Materials: CE-SWI (TR 26, TE 19.2; flip-angle $15^{\circ}$ ) and CE-T1 (MPRAGE TI 1100, TR 1710; TE 4.0 flip-angle $15^{\circ}$ ) images were acquired from 40 patients with newly diagnosed and histologically confirmed glioblastoma, before surgery at a 3-Tesla MRI. The images were overlaid on the slice demonstrating the largest tumour area. Two experienced neuroradiologists a) measured the area of the enhancement and calculated percentage-wise overlap of the enhancement in both sequences and b) quantified the number of ITSSs and assessed their overlap in both sequences. Results: All 40 patients presented a nearly identical enhancement in CE-SWI and CE-T1 sequences with a percentage-wise overlap of the enhancement of $92.2 \pm 4.6 \%$. ITSSs were detected in $36 / 40$ patients on CE-SWI and in 15/40 on CE-T1. There was a significant correlation between tumour area and number of ITSSs $(R 2=0.80)$. Only in $15.0 \pm 12.4 \%$ ITSS that were detected on CE-SWI could also be detected on CE-T1.

Conclusion: CE-SWI images provide additional information regarding the appearance of ITSSs that often cannot be visualised in CE-T1 images, whereas the enhancement in both sequences is nearly equivalent. Therefore, CE-SWI could be a useful sequence, e.g. for biopsy-planning, to avoid taking the biopsy from an ITSS.
B-215 11:06

Noninvasive tumour hot spot localisation of primary cerebral tumours: the role of MR spectroscopy and arterial spin labelling

J. Furtner, G. Kasprian, M. Krssak, M. Weber, G. Widhalm, J. Hainfellner,

D. Prayer; Vienna/AT (julia.furtner@meduniwien.ac.at)

Purpose: To determine whether there is an accordance between the region of the most malignant part of the tumour related to metabolic changes as demonstrated by multivoxel MR-spectroscopy and the region of the highest tumour perfusion using arterial spin labelling (ASL).

Methods and Materials: 45 patients with primary brain tumours, histologically classified as low-grade $(n=16)$ or high-grade $(n=29)$ gliomas according to the WHO brain tumour classification were included. A 3 Tesla MR scanner was used to perform multivoxel MR-spectroscopy and pulsed-ASL sequences at an inversion time of $614 \mathrm{~ms}$. The two different sequences were coregistrated and the cerebral lesions were assessed for location of the Cho/Naa ratio peak on MR-spectroscopy and highest signal intensity on the ASL sequence.

Results: The accordance of the Cho/Naa ratio peak in multivoxel MR-spectroscopy and the region of highest signal intensity in the ASL sequence was $85 \%$ in highgrade gliomas and $72.5 \%$ in low-grade gliomas.

Conclusion: Our findings may suggest that ASL allows to detect the region of the highest tumour vessel density, which shows a high correlation with the metabolically most malignant region of the brain tumour. The combination of these two non-invasive MR sequences is able to define the tumour hot spot without the use of any contrast media. In the clinical setting this would mainly be an advantage for patients with elevated serum creatinine values or known previous allergic reaction associated with contrast media, in which case contrast media application would be a contra indication.

\section{B-216 11:15}

Estimation of water diffusion and tumour micro-circulation in human glioblastomas: repeatability of the appliedtechniques, effect of the b-values, and relationship between the functional parameters

S. Bisdas, A. Dimostheni, R. Ritz, T. Nägele, U. Ernemann;

Tübingen/DE (sotirios.bisdas@med.uni-tuebingen.de)

Purpose: To assess the repeatability of dynamic contrast-enhanced (DCE)-MRIderived microcirculatory parameters and apparent diffusion coefficient (ADC) calculations in glioblastomas and to examine the association between them taking account the effect of b-value.

Methods and Materials: 25 patients with glioblastomas underwent multi-shot echoplanar DWI and DCE-MRI. ADC images sets were generated using 0, 150, 500, 1000, and $2000 \mathrm{~s} / \mathrm{mm}^{2}$ as b-values. Commercially available software using an arterial input function (AIF) was applied for the calculation of the transfer constant between the intravascular and extravascular space ( $\left.K^{\text {trans }}\right)$, extravascular blood volume $\left(v_{e}\right)$, transfer constant from the extracellular extravascular space to plasma $\left(\mathrm{k}_{\mathrm{ep}}\right)$, and initial area under the signal intensity-time curve (iAUC). The qualitative and quantitative DCE$\mathrm{MRI}$ and $\mathrm{ADC}$ assessments were analysed using parametrical and non-parametrical tests. Measurement repeatability was determined by the Bland-Altman method. Results: $K^{\text {trans }}, v_{e}$, and iAUC values in tumour tissue were higher than in peritumoral area $(P=0.02)$. In the tumour tissue, significant correlations were observed between $\mathrm{K}^{\text {trans }}$ and $v_{e}, K^{\text {trans }}$ and iAUC, iAUC and $v_{e}(r=0.64, P=0.0014)$. Further significant correlations were found in the tumour tissue between $v_{e}$ and $A D C_{0-500-1000}(r=0.56$, $P=0.007)$, in the peritumoral region between $K^{\text {trans }}$ and $A D C_{150-500-1000-2000}(r=0.55$, $P=0.05)$ as well as between $v_{e}$ and $A D C_{0-500-1000}(r=0.56, P=0.05)$. The most satisfactory repeatability was shown for $\mathrm{K}^{\text {trans }}, \mathrm{v}$ and for $\mathrm{ADC}$ calculated by $4 \mathrm{~b}$-values. Conclusion: DCE-MRI and ADC using a 4-point calculation showed satisfactory repeatability in glioblastomas. The demonstrated correlations imply that the choice of the b-values is crucial for the association of ADC and DCE-MRI parameters.

\section{B-217 11:24}

Orbital lymphoma: imaging findings at diagnosis and follow-up

G. Priego, C. Majos, F. Climent, A. Muntane, N. Romero, S. Mercadal; Barcelona/ES (gemika@gmail.com)

Purpose: Imaging findings in orbital lymphoma, at diagnosis and follow-up, allow a proper radiological assessment, and hence an appropriate staging.

Methods and Materials: We included 44 patients (mean age 61 years, 20 men and 24 women) with orbital lymphoma studied by imaging (CT or MRI) between 1996 and 2010. Twenty three cases were collected with radiological exams at diagnosis. Radiological data were analysed including orbital distribution, quadrants involved and infiltration of orbital structures. In the follow-up, 39 cases were collected and radiological findings were reviewed regarding treatment response. 
Results: Orbital relapses were detected in 9\% (4/44). Mortality occurred in 1 case. The distribution at diagnosis was 3 bilateral and 20 unilateral. The predominant involvement was one quadrant $(13 / 23,57 \%)$ and superiorexternal quadrant (14/22, $64 \%)$. The dominant quadrant was not defined in one case. The most commonly infiltrated orbital structures were the superior rectus muscle $(16 / 23,70 \%)$, latera rectus muscle $(13 / 23,57 \%)$, eyelid $(13 / 23,57 \%)$ and lacrimal gland $(12 / 23,52 \%)$. Analysing the first control post-treatment, the following findings were observed: complete response in 36\% (14/39), major response in 59\% (23/39) and minor response (including growth) in 5\% (2/39). Controls every 12 months in average showed stability of the images (or even decrease) for 2 years (range 1-5 years). Conclusion: Lymphoma can involve the orbit, being the most common the superiorexternal quadrant and the orbital structures described. In follow-up there may be residual images that remain stable over time. However, recurrence is a possibility and follow-up by imaging is required.

\section{B-218 11:33}

(EPOS)

Volumetric increase in Schwannoma on surveillance brain MRIs following stereotactic radiosurgery: a review of predictive factors

K.J. Opfermann, S.L. Cooper, J.M. Jenrette, III; Charleston, SC/US

(opferman@musc.edu)

Purpose: A percentage of patients receiving stereotactic radiosurgery (SRS)/ fractionated stereotactic radiosurgery (SRT) for Schwannomas initially respond with a transient volumetric enlargement. The objective of this study is to evaluate possible predictive factors of transient MRI volumetric changes.

Methods and Materials: Twenty-five Schwannoma cases were retrospectively reviewed following definitive radiotherapy at our institution (1990-2009). Patients had initial MRI, Radiation Oncologist/Neurosurgery consultation and follow-up brain MRIs. The median duration of imaging follow-up was 28 months (2.9mos-158mos). Volumetric changes were measured by a single reviewer on T1-contrasted images. Change was classified as: enlargement, transient enlargement, direct shrinkage, or alternating enlargement and regression based on a $13 \%$ increase in volumetric tumour size. Characteristics reviewed were: patient co-morbidities, previous interventions, tumor characteristics, radiotherapy, post-treatment symptoms and management.

Results: Nine of the cases (36\%) had transient enlargement or alternating enlargement and regression based on post-treatment imaging. The increase in tumour size ranged from $123 \%-653 \%$, although tumours undergoing regression decreased to as small as $17 \%$ of the original size. Patients with prior surgical intervention, radiation dose greater than $16 \mathrm{~Gy}$, and SRS were more likely to be represented in this population. Only one patient with transient tumor enlargement but all patients with alternating tumor volume had a history of administered steroids.

Conclusion: Results still mandate close post-treatment MRI surveillance for volumetric increase of Schwannomas following SRS/SRT. Of special concern for transient/alternating increase in volume may be those patients having had prior surgical intervention, higher radiation doses or a single fraction treatment.

\section{B-219 11:42}

Choline elevation in MR spectroscopy of brain tuberculomas: how often does it occur?

Y. $\underline{\text { Ragab }}^{1}$, F. Zienhom 1 , M.M.A. Awadalla', H.M.H. Hamza', N. Elsiofy²,

K. Kamal'; ${ }^{1}$ Cairo/EG, ${ }^{2}$ Jeddah/SA (yragab61@hotmail.com)

Purpose: To determine the frequency of choline elevation in cases of brain tuberculomas, based on MR spectroscopy.

Methods and Materials: 35 patients with intra-axial tuberculomas underwent single and multi voxel proton MR spectroscopy. MRI and ${ }^{1} \mathrm{H}$-MRS were performed on 1.5 Tesla systems. ${ }^{1} \mathrm{H}-\mathrm{MRS}$ indices [ $\mathrm{N}$-acetyl aspartate (NAA); creatine (Cr); choline (Cho); and lipid lactate (Lip/Lac)] were computed from the tuberculomas and surrounding perifocal oedema. Correlations were done with contralateral normal-appearing brain tissue. Cho/NAA ratios were obtained for both sides. Tuberculomas were confirmed by stereotactic biopsy in nine patients; other patients showed lesion regression on antituberculous drugs.

Results: $39 \%$ of patients showed elevation of choline compared with NAA (elevated Cho/NAA ratios). The mean Cho/NAA ratios for tuberculomas was $1.8 \pm 0.3$ compared with $0.6 \pm 0.24$ for normal brain tissue in the contralateral side. Lip/ Lac waveforms were elevated in $67 \%$ of patients. The perifocal hyperintensity (oedema) showed no elevation of Cho or Lip/Lac compared with the normal brain tissue, but reduction of NAA was observed in $86 \%$ of cases.

Conclusion: Choline is frequently elevated in relatively high proportion of patients with intracranial tuberculomas; knowledge of possible choline elevation can avoid the misinterpretation of neoplasms. Awareness of the fact that a significant proportion of tuberculomas show Choline (Cho) elevation on MRS is important in clinical imaging interpretation of brain masses and mass-like lesions.
B-220 11:51

CT perfusion imaging: does it correlate with CD105 in rat brain $\mathrm{C} 6$ glioma? J.W. Zhang, X.Y. Feng; Shanghai/CN (zhangjw2000@hotmail.com)

Purpose: To investigate CT perfusion paremeter changes in a rat brain C6 glioma model using CT perfusion imaging, and analyse correlations between CT perfusion paremeter and CD105.

Methods and Materials: A total 20 male SD rats were divided into glioma and control groups (normal rats) at random. Rats in glioma group were implanted C6 glioma cells at right caudate nucleus of the brain. After 3 weeks, perfusion CT was performed in glioma and control groups. Perfusion CT values (CBV, CBF, PS and MTT) were measured at the right nucleus caudatus in rat brain. The samples at nucleus caudatus in rat brain were examined histologically using $\mathrm{HE}$ and immunohistochemical staining for CD105. The data were analysed using SPSS11.5 statistical software. Spearman correlation coefficients were used to investigate relationships between perfusion CT parameters (CBV, CBF, and PS) and immunohistochemical staining for CD105. Results: Normal rat brain CBV, CBF, PS and MTT values (mean \pm SD) were $(10.26 \pm 3.58) \mathrm{mL} / 100 \mathrm{~g},(292.12 \pm 77.50) \mathrm{mL} / 100 \mathrm{~g} \cdot \mathrm{min},(0.44 \pm 0.43) \mathrm{mL} / 100 \mathrm{~g} \cdot \mathrm{min}$ $(1.49 \pm 0.05) \mathrm{s}$, respectively. Rat gliomas CBV, CBF, PS and MTT values (mean \pm SD) were $(17.35 \pm 6.73) \mathrm{mL} / 100 \mathrm{~g},(508.66 \pm 158.88) \mathrm{mL} / 100 \mathrm{~g} . \mathrm{min},(13.92 \pm 8.96)$ $\mathrm{mL} / 100$ g.min, $(1.79 \pm 0.44) \mathrm{s}$, respectively. CBV, CBF and PS values in glioma group were significant higher than those in control group $(\mathrm{t}=2.85, \mathrm{P}=0.011 ; \mathrm{t}=$ $3.54, P=0.003 ; t=4.82, P<0.001$, respectively). Significant correlations were observed between CD105 and CBV, PS and CBF in glioma group, while MTT was not in significant correlation with CD105 in glioma group.

Conclusion: Perfusion CT can reflect angiogenesis within rat C6 gliomas.

10:30 - 12:00

Room N/O

\section{Contrast Media}

\section{SS 406}

\section{Breaking news on MR contrast media: from cell specificity to clinical safety}

Moderators:

T. Allkemper; Münster/DE

A. Giovagnoni; Ancona/IT

\section{B-221 10:30}

Gadofosveset trisodium-enhanced MRI offers a radiation-free, stand-alone examination for venous thromboembolism

A. Hansch, A. Pfeil, S. Betge, W.A. Kaiser, H.-J. Mentzel; Jena/DE

Purpose: In this study, evaluation of leg veins and lung arteries for thrombosis was performed twice with a new intravascular contrast agent gadofosveset trisodium. Methods and Materials: Seventy patients with sonography-confirmed DVT but no clinical signs of PE were prospectively enrolled in the study. Complete MRI examination was performed with a single dose of Gadofosveset trisodium $(0.03 \mathrm{mmol} /$ $\mathrm{kg}$ body weight). Pulmonary arteries were imaged with the a fat-suppressed 3D gradient echo volume interpolated breath-hold examination (VIBE FS), 3D fast lowangle shot gradient recalled echo sequence and true fast imaging with steady-state precession 3D acquisitions using respiratory gating with navigator technique. Image quality of was evaluated by a 5-point scale. Magnetic resonance venography of the pelvic region and the leg was performed using the VIBE FS.

Results: The diagnostic quality of gadofosveset trisodium-enhanced 3D fast low-angle shot (FLASH) gradient recalled echo sequence showed best diagnostical quality at the lung arteries. PE was detected in 29 of the 70 DVT patients (41.4\%). Additionally, we detected significantly more thrombi at the leg than did the Doppler ultrasonography $(p<0.05)$. Conclusion: This study shows the feasibility of a combined protocol for the MRI diagnosis of DVT and PE using gadofosveset trisodium. We suggest performing imaging of pulmonary arteries with the 3D fast low-angle shot (FLASH) gradient recalled echo sequence and MRV of of the leg using the VIBE FS.

\section{B-222 10:39}

Ischaemic-type biliary lesions following liver transplantation: usefulness of Gd-EOB-DTPA-enhanced MR cholangiography

P. Boraschi, F. Donati, R. Gigoni, S. Salemi, F. Filipponi, C. Bartolozzi,

F. Falaschi; Pisa/IT (p.boraschi@do.med.unipi.it)

Purpose: To evaluate the usefulness of contrast-enhanced MR cholangiography (MRC) using Gd-EOB-DTPA for evaluating ischaemic-type biliary lesions (ITBLs) in liver transplant recipients. 
Methods and Materials: Twenty-eight liver transplant patients with ischaemic changes of the biliary tree underwent MRI at $1.5 \mathrm{~T}$-device. After acquisition of $\mathrm{T} 1 \mathrm{w} / \mathrm{T} 2 \mathrm{w}$ images, conventional MRC was performed through thin-slab 3D FRFSE T2w and thick-slab SSFSE T2w sequences. In each patient a 3D fat-suppressed breath-hold T1w sequence (TR/TE, $3.6 \mathrm{~ms} / 1.6 \mathrm{~ms} ; 2.4 \mathrm{~mm}$ thk/-1.8 mmsp; matrix, 224 x 192; one NEX) was performed before and 15, 20, 25, 30, 35, 40 minutes after intravenous administration of gadolinium-EOB-DTPA (Primovist $\circledast$ ), Bayer Schering Pharma; $10 \mathrm{ml}$ ) at a flow-rate of $2 \mathrm{ml} / \mathrm{s}$, followed by injection of isotonic saline $(20 \mathrm{ml})$. T1w sequence was also obtained after 90 minutes in 9/28 cases. Depiction of intra-/extra-hepatic bile ducts, dilation of biliary system, presence of biliary stricture and stone/sludge were evaluated on images by consensus reading of two observers using a 3-point-scale: 0 , absent; 1 , visible; 2 , excellent. Imaging results were correlated with direct cholangiography and/or follow-up.

Results: The grading of visualisation and depiction of dilation of the intra-hepatic bile ducts was significantly superior on T2w MRC compared with Gd-EOB-DTPAenhanced T1w MRC; on the contrary, this latter significantly tended to out-perform conventional T2W MRC in delineating strictures involving hepatic bifurcation and extrahepatic donor bile duct $(p<0.0001)$. Sludge/stone formation was correctly identified with both techniques. Biliary enhancement was recognised within 20 minutes post-Gd-EOB-DTPA injection in 6 subjects, between 25 and 40 minutes in 16, and at 90 minutes in 6 cases.

Conclusion: Gd-EOB-DTPA-enhanced MRC may provide both anatomical and functional information of ITBLs in liver transplants.

\section{B-223 10:48}

Multi-center, randomised comparison study to eVALUatE outcomes and resource needs of imaging and treatment following Primovist-enhanced MRI of the liver in comparison to extracellular contrast media-enhanced MRI and contrast-enhanced CT in patients with colorectal cancer and suspected metachronous liver metastases: an interim analysis

C.J. Zech 1 , S. Gschwend ${ }^{2}$, V. STUDY Group $2 ;{ }^{1}$ Munich/DE

${ }^{2}$ Berlin/DE (Christoph.Zech@med.uni-muenchen.de)

Purpose: To perform an economic evaluation of Gd-EOB-DTPA-enhanced MR (PV-MRI) compared to MRI with extracellular-contrast-media (ECCM-MRI) and contrast-enhanced MDCT (CE-MDCT) as initial diagnostic modalities in the workup of patients with colorectal liver metastases.

Methods and Materials: 35 study centres included 296 patients from 10/2008 to $07 / 2010$, which were referred for staging of the liver in case of suspected liver metastases. The initial imaging study was randomised. A consensus between the investigating surgeon and radiologist was reached whether further imaging was required to obtain a therapeutic decision. The primary efficacy variable was the proportion of patients for whom further imaging was required after initial imaging with PV-MRI as compared to ECCM-MRI and CE-MDCT. The results were compared using a one-sided approximate test for equality at a significance level of $2.5 \%$. The diagnostic confidence was rated by the investigator on a four-point-scale.

Results: 98 patients received PV-MRI, 95 ECCM-MRI and 91 CE-MDCT. Further imaging was required in $0(0 \%), 15(15.8 \%)$ and 33 cases $(36.3 \%)$, respectively. These results are statistically significant. Diagnostic confidence was high/very high in $96(98 \%), 84(88.4 \%)$ and 51 cases $(67.1 \%)$, respectively.

Conclusion: The results show superiority of PV-MRI over CE-MDCT and ECCMMRI with regard to the requirement for further imaging. With PV-MRI as the initial staging strategy, no further tests to assess operability were needed, which has implications for work-flow and costs. Further evaluation of the collected data with regard to diagnostic efficacy of each method compared to surgery and an cost calculation is planned.

\section{B-224 10:57}

(EPOS)

\section{Hepatic uptake and pharmacokinetics of the MRI contrast agent} gadoxetate in healthy subjects: role of OATP1B1, OATP1B3 and ABCC2 J.P. Kühn, A. Nassif, S. Oswald, W. Weitschies, W. Siegmund, R. Puls, N. Hosten; Greifswald/DE (kuehn@uni-greifswald.de)

Purpose: Liver-MRI with the specific contrast agent gadoxetate improves detection of focal lesions. There is evidence that gadoxetate is a substrate of hepatic organ ion transport proteins (OATPS) and ABCC2. To confirm their role in hepatic uptake and elimination of gadoxetate in humans, we measured hepatic enhancement and pharmacokinetics of the agent in volunteers with functional polymorphisms of OATP1B1, OATP1B3 and ABCC2.

Methods and Materials: The study was performed in 41 white subjects, among them 8 with wild-type of OATP 1 B 1,8 carriers of the ${ }^{*} 1 \mathrm{~b} /{ }^{*} 1 \mathrm{~b}$ haplotype and 12 of the ${ }^{*} 15 /{ }^{*} 15$ and ${ }^{*} 1 a /{ }^{*} 5$ haplotype. The remaining subjects were carriers of OATP1B3 G699 A or ABCC2 C-24T and G1249 A. Gadoxetate-induced hepatic enhancement was measured up to $480 \mathrm{~min}$ after bolus injection of $25 \mu \mathrm{M} / \mathrm{kg}$ at 1.5 Tesla using a T1-weighted GRE-flash-3D sequence. Pharmacokinetics in serum and renal and faecal excretion of gadoxetate were quantified using an LC-MS/MS-assay. Results: Peak signal intensity was reached significantly earlier $\left(T_{\max }\right)$ in subjects with variant OATP1B1 alleles $\left({ }^{*} 1 \mathrm{a} /{ }^{*} 1 \mathrm{a}, 66 \pm 30 \mathrm{~min} ;{ }^{*} 1 \mathrm{~b} /{ }^{*} 1 \mathrm{~b}, 43 \pm 21 \mathrm{~min} ;{ }^{*} 15 /{ }^{*} 15-{ }^{*} 1 \mathrm{a} /{ }^{*} 5\right.$ $35 \pm 9 \mathrm{~min}, \mathrm{p}<0.05)$. Hepatic enhancement $\left(\mathrm{AUC}_{3-*}\right.$ in arbitrary units) was significantly lower in carriers of ${ }^{*} 15$ or ${ }^{*} 5(28 \pm 8.8, p<0.05)$ compared with ${ }^{*} 1 a /{ }^{*} 1 a$ wild-type $(42 \pm 8.8)$ and ${ }^{*} 1 \mathrm{~b} /{ }^{*} 1 \mathrm{~b}(40 \pm 15)$. None of the genetic polymorphisms of OATP1B1, OATP1B3, or ABCC2 had any significant effect on systemic exposure to gadoxetate. Conclusion: OATP1B1 is a major factor influencing hepatic uptake of gadoxetate in humans. Hepatic enhancement is reduced in subjects with deficient OATP1B1 function. OATP1B3 and ABCC2 polymorphisms have no clinical relevance in gadoxetate-enhanced liver-MRI.

\section{B-225 11:06}

Gd-EOB-DTPA-enhanced MR imaging (MRI) in the early dynamic phase: is bolus timing necessary to obtain an optimal arterial phase for liver MRI? C. Schmid-Tannwald, K.A. Herrmann, M.F. Reiser, C.J. Zech;

Munich/DE (christine.schmid-tannwald@med.uni-muenchen.de)

Purpose: To evaluate the necessity to perform bolus timing for the early dynamic phase in Gd-EOB-DTPA-enhanced liver MRI.

Methods and Materials: Three groups of patients which were referred to a Gd-EOB-DTPA-enhanced liver MRI were retrospectively evaluated. In group 1 CareBolus technique $(n=20)$, in group 2 a test bolus technique $(n=18)$, in group 3 no bolus timing with a fixed delay of 20 seconds $(n=18)$ was used to determine the start of the arterial-dominant phase after bolus injection. Two readers independently evaluated the arterial phase with five criteria (proper enhancement in aorta; in liver arteries, slight enhancement in portal vein, missing enhancement in hepatic veins, cortical enhancement of kidney). If a criterion was fulfilled, one point was assigned. Sums per patient were calculated. Significance was tested with Wilcoxon-test. Results: The mean of the sum scores for the CareBolus technique and the test bolus technique were similar with 9.1 and 9.0 , respectively, and significantly higher than the mean for the fixed delay with $6.2(p=0.0013$ and 0.0028 , respectively). The most critical parameter was the enhancement in the portal vein, which was correctly hit in $12 / 20$ cases with CareBolus, $11 / 18$ cases with test bolus and only in $3 / 18$ cases for the fixed delay.

Conclusion: The results indicate that for Gd-EOB-DTPA-enhanced liver MRI bolus-timing technique is mandatory. The differences between CareBolus and test bolus technique are negligible.

\section{B-226 11:15}

Clinical and pathological effects of gadodiamide on a new, adeninesensitised, rat model of nephrogenic systemic fibrosis

N. Bouzian ${ }^{1}$, N. Fretellier ${ }^{1}$, P. Bruneval ${ }^{2}$, F. Daubiné ${ }^{3}$, J.-M. Idée ${ }^{1}$, C. Corot ${ }^{1}$; ${ }^{1}$ Roissy/FR, ${ }^{2}$ Paris/FR, ${ }^{3}$ Nantes/FR (ideej@guerbet-group.com)

Purpose: To set up a new rat model of nephrogenic systemic fibrosis (NSF) and investigate the role of baseline renal function.

Methods and Materials: Wistar rats received a $0.75 \%$ adenine-enriched diet for either 4 weeks (characterisation study vs. control rats), 16 days (Study 1 ) or 8 days (Study 2). Rats received daily injections of gadodiamide (Study 1: $5 \times 2.5 \mathrm{mmol} /$ $\mathrm{kg} /$ day, Day 14 to Day 18, Study 2: 5 x 1.0 or $2.5 \mathrm{mmol} / \mathrm{kg} /$ day, Day 7 to Day 11). The model was characterised from a clinical, pathological (femur and kidneys) and biochemical standpoint up to 3 weeks after completion of the adenine treatment. In studies 1 and 2, adenine-treated rats were sacrificed 3 weeks after the last injection of gadodiamide or saline. Skin biopsies were performed on $1^{\text {st }}$ and $9^{\text {th }}$ days of treatment and at sacrifice. All histopathological studies were blinded.

Results: Adenine induced major tubular lesions with lithiasis and interstitial fibrosis. Fibrous osteitis-like lesions occurred in both femoral epiphyses and diaphyses. Early decrease in creatinine clearance (Day 8: $0.12 \pm 0.03$ vs. $1.2 \pm 0.3 \mathrm{~mL} / \mathrm{mn} / 100 \mathrm{~g}$ $p<0.01$ ), hyperphosphoraemia (peak at Day $22: 4.1 \pm 1.0 \mathrm{vs.} 2.4 \pm 0.2 \mathrm{mmol} / \mathrm{L}$ ), hypocalcaemia $(2.0 \pm 0.2 \mathrm{vs} .2 .3 \pm 0.1 \mathrm{mmol} / \mathrm{L})$ and gradual mortality were found Gadodiamide-induced mortality (Study 1: 10/11, Study 2: 1 death at $2.5 \mathrm{mmol} / \mathrm{kg} /$ day) and skin lesions (dermal fibrosis, calcification, inflammation) were related to the baseline renal function.

Conclusion: Gadodiamide-induced cutaneous and systemic toxicity depends on baseline renal function in an adenine-sensitised model of NSF. 


\begin{abstract}
B-227 11:24
Is the subtotal-nephrectomy rat model of nephrogenic systemic nephropathy associated with osteodystrophy-like bone lesions? F. Daubiné1, N. Fretellier², N. Bouzian², R. Le Bot', J.-M. Idée ${ }^{2}$, C. Corot²; 'Nantes/FR, ${ }^{2}$ Roissy/FR (ideej@guerbet-group.com)
\end{abstract}

Purpose: To investigate the bone lesions associated with the subtotal nephrectomy rat model of nephrogenic systemic fibrosis.

Methods and Materials: Wistar rats were submitted to subtotal (5/6 $\left.6^{\text {th }}\right)$ nephrectomy $(\mathrm{SNx})$ and received a high-phosphate diet or were sham-operated and received a normal diet (6 rats/group, randomised). Rats were sacrificed 34 days after surgery and both tibia and femur were submitted to blinded histological analysis using Von Kossa (bone structure), Goldner's Trichrome (osteoid and bone marrow), Masson's trichrome (bone structure, osteoid and bone marrow) and tartrate-resistant acid phosphatase (activated osteoclasts) stainings. All diaphyses and epiphyses were investigated.

Results: Increase in serum creatinine $(66.1 \pm 39.2$ vs. $25.8 \pm 2.1 \mu \mathrm{mol} / \mathrm{L})$ and phosphorus $(3.2 \pm 1.4 \mathrm{mmol} / \mathrm{L}$ vs. $1.6 \pm 0.1 \mathrm{mmol} / \mathrm{L})$ levels were found in $\mathrm{SNx}$ rats. Serum calcium levels remained unchanged $(2.3 \pm 0.2 \mathrm{vs} .2 .4 \pm 0.1 \mathrm{mmol} / \mathrm{L})$. Important decrease in bone tissue mineralisation was found in all femoral epiphyses of SNx rats (Von Kossa's stain). These lesions were associated with a dramatic decrease in the density of bone marrow in both femur (Goldner's Trichrome) and tibia (Masson's Trichrome). Fibrous structures were found in the bone marrow of all $\mathrm{SNx}$ rats. No difference between SNx and sham-operated groups was evidenced in femur diaphyses as well as activated osteoclasts. Histopathological lesions were consistent with fibrous osteitis, the most common form of osteodystrophy. Conclusion: In hyperphosphoraemic rats submitted to subtotal nephrectomy, clinically relevant osteodystrophy-like lesions were found 34 days after surgery, thus allowing mechanistic studies involving storage of $\mathrm{Gd}$ chelates in bones.

\section{B-228 11:33}

Observational post-marketing study on the safety of Gd-DOTA: the secure study

F. Laurent; Pessac/FR (francois.laurent@chu-bordeaux.fr)

Purpose: To assess the general safety profile of meglumine gadoterate (Gd-DOTA) as well as the occurrence of nephrogenic systemic fibrosis (NSF).

Methods and Materials: This worldwide post marketing study (PMS) is being conducted to collect safety data in about 40000 patients with or without renal insufficiency undergoing routine contrast-enhanced magnetic resonance imaging (MRI) using Gd-DOTA, as well as risk factors at inclusion, MRI indications, and conditions of use/administration of the product. For any patient identified as renally impaired at the time of inclusion (i.e. estimated glomerular filtration rate or estimated creatinine clearance $<60 \mathrm{ml} / \mathrm{min} 1.73 \mathrm{~m}^{2}$ ), a 3-month follow-up has to be performed in order to detect any suspicion/occurrence of NSF. As of August 19, 2010, the cut-off date for the interim safety analysis, this ongoing PMS includes data from 9936 patients. Results: Among these included patients, $54.5 \%$ were females, $58.7 \%$ aged $18-59$ years. The main indications for MRI examinations were central nervous system $(48.1 \%)$ and whole body $(26.6 \%)$. The mean total volume of Gd-DOTA injected was $15.4 \mathrm{ml}$ (range: $1-65 \mathrm{ml}$ ). The main risk factors were hypertension $(15.3 \%)$ and allergies $(12.9 \%)$. Moderate to severe impaired renal function was reported in 315 patients $(3.2 \%)$. Among them, $199(63.2 \%)$ had no suspicion of NSF, and for $116(36.8 \%)$ follow-up data are not yet collected. A total of 15 patients $(0.15 \%)$ had at least one adverse event (AE) (mainly urticaria, vomiting, nausea) including 2 deaths (unrelated to Gd-DOTA).

Conclusion: This interim safety analysis already confirms a very good safety profile of Gd-DOTA.

\section{B-229 11:42}

\section{Pharmacovigilance based on spontaneous adverse event reporting of} meglumine gadoterate (Gd-DOTA) after 15 million administrations and 20 years of clinical use

E. de Kerviler' 1 , S. Gaillard 2 ; ${ }^{\text {Paris/FR, }}{ }^{2}$ Villepinte/FR

(eric.de-kerviler@sls.aphp.fr)

Purpose: To assess the safety of meglumine gadoterate (Gd-DOTA) from adverse drug reactions (ADRs) reporting over 20 years.

Methods and Materials: Gd-DOTA marketed since 1989 is currently available in more than 65 countries. All adverse drug reactions (ADRs) from different sources (clinical trials, health authorities, literature, spontaneous) were collected, coded with MedDRA (version 12.0) and analysed, using the manufacturer's continuous and cumulative pharmacovigilance database.
Results: ADRs were reported in 1020 patients $(<0.007 \%)$ out of $15,022,425$ exposures. Among all cases reported, $80.5 \%$ were spontaneous cases. The majority of ADRs reported were non-serious $(638 / 1020,62.5 \%)$ and already known effects of the product $(695 / 1020,68.1 \%)$. Most patients $(764 / 1020,74.9 \%)$ recovered promptly without sequels. The most frequently reported ADRs were urticaria $(0.0012 \%)$, nausea $(0.0011 \%)$, vomiting $(0.0009 \%)$, rash $(0.0008 \%)$, pruritus $(0.0007 \%)$ and erythema $(0.0007 \%)$. Serious ADRs occurred in 345 patients $(0.0022 \%)$ including 28 fatal cases (6 not related to ADR) and 11 cases of nephrogenic systemic fibrosis (NSF) (10 confounded and confirmed cases and 1 case of aggravation of a pre-existing NSF, causality of Gd-DOTA not established).

Conclusion: Based on the pharmacovigilance analysis, we reported a very low incidence of serious ADRs, thereby confirming the excellent safety profile of Gd-DOTA.

\section{B-230 11:51}

Prevalence of nephrogenic systemic fibrosis in dialysis patients: the ProFINEST study

S. Amet' ${ }^{1}$, G. Choukroun ${ }^{2}$, V. Launay-Vacher ${ }^{1}$, C. Francès' 1 A. Castot ${ }^{3}$

G. Deray', O. Clement' $;{ }^{1}$ Paris/FR, ${ }^{2}$ Amiens/FR, ${ }^{3}$ Saint-Denis/FR

(vincent.launay-vacher@psl.aphp.fr)

Purpose: NSF is a cutaneous and systemic disorder characterised by widespread tissue fibrosis. It has been suggested that gadolinium-based contrast agents (GBCA) may be responsible for NSF, especially in dialysis patients. The ProFINEST study is a national prospective study endorsed by the French Drug Agency (Afssaps), and the French Societies of Nephrology, Dermatology, and Radiology. It aims at determining the prevalence of NSF after a magnetic resonance imaging (MRI) examination, $\pm \mathrm{GBCA}$, in dialysis patients.

Methods and Materials: The study is based on a 3-section patient form. Section 1: demographics and dialysis; section 2: MRI examination; section 3: any dermatological event (DE). Further investigations are planned in case of DE. When a NSF diagnosis is confirmed, an ancillary study is to be performed, with random selection of 4 patients (same gender, dialysis technique, centre, and without any DE after MRI with the same GBCA, if injected).

Results: Since 01/2009, 368 patients have been included (247 centres): mean age 63.5 years, $58.4 \%$ men, $57 \%$ of the patients received GBCA among whom $87.3 \%$ gadoterate. 9 patients reported a DE: feet skin lesions linked to arteriopathy, haemodialysis-related prurit, localised skin reaction to a trinitrine patch, thoracic zona, panniculitis, psoriasis and lupus erythematosus. Dermatological diagnoses did not report any evidence of NSF.

Conclusion: So far thus, no case of NSF has been reported in 368 dialysis patients among whom the majority received a GBCA. Most patients received a macrocyclic gadolinium chelate for which no case of NSF has been observed worldwide (gadoterate).

10:30 - 12:00 Room P

Cardiac

\section{SS 403b}

\section{Coronary CT angiography}

Moderators:

H. Alkadhi; Zurich/CH

E. Konen; Tel Hashomer/lL

\section{B-231 10:30}

National multicenter multivendor, prospective study to evaluate in real life the diagnostic value of cardiac multidetector CT in a large cohort of stable patients with chest pain scheduled for coronary angiography: the EVASCAN study

J.-P. Laissy', P. Gueret' ${ }^{2}$ L. Bonello 3 , J.-F. Deux ${ }^{2}$, D. Bertrand ${ }^{4}$, L. Christiaens ${ }^{5}$, C. Caussin ${ }^{6}$, D. Crochet 1 , V. Chabbert $7{ }^{7}$ Paris/FR, ${ }^{2}$ Créteil/FR,

${ }^{3}$ Marseilles/FR, ${ }^{4}$ Rouen/FR, ${ }^{5}$ Poitiers/FR, ${ }^{6}$ Le Plessi-Robinson/FR, ${ }^{7}$ Toulouse/FR (jean-pierre.laissy@bch.ap-hop-paris.fr)

Purpose: EVASCAN is a national multicenter multivendor prospective study aimed at assessing the diagnostic performance of MDCT as compared with conventional coronary angiography $(\mathrm{CA})$ considered the reference. The study was fully supported by a grant of the French Health Ministry.

Methods and Materials: Between June 2006 and June 2008, 40 centers prospectively enrolled 1254 symptomatic patients who underwent MDCT angiography $<4$ days before elective CA, using a standardised optimised protocol for each system (16, 40, 64-slice or dual-source scanner). MDCT and CA examinations 
were blindly analysed in central core-laboratories. Significant CAD was defined as $\geq 50 \%$ stenosis. The assessment of MDCT accuracy was performed according to a per-patient, per-vessel and per-segment approach analysis.

Results: Two hundred and six patients were excluded from analysis (protocol deviations, MDCT or CA not conducted). The final study population comprised 1048 patients. On a per-patient analysis, sensitivity, specificity, positive and negative predictive value were $83 \%, 74 \%, 78 \%$ and $79 \%$, respectively. On a per-vessel analysis, they were $62 \%$, $90 \%, 66 \%$ and $88 \%$, respectively, and on a per-segment analysis, they were $38 \%, 94 \%$, $43 \%$ and $93 \%$, respectively. The best sensitivity and worst specificity were observed in heavily calcified segments (Agatston score $\geq 600$ ), as well as in the population at high risk of $C A D$ when compared to patients with low or intermediate probability of CAD. Conclusion: In this large cohort of stable patients in whom an elective CA was scheduled, diagnostic accuracy of MDCT may help establish clinical application guidelines in patients with suspected CAD.

\section{B-232 10:39}

Long-term predictive value of assessment of coronary atherosclerosis by contrast-enhanced coronary computed tomography angiography: metaanalysis and systematic review

F. Bamberg ${ }^{1}$, W. Sommer ${ }^{1}$, V. Hoffmann ${ }^{1}$, K. Nikolaou', M.F. Reiser ${ }^{1}$,

U. Hoffmann' ${ }^{2}$ C. Becker ${ }^{1} ;{ }^{1}$ Munich/DE, ${ }^{2}$ Boston, MA/US

(fabian.bamberg@med.uni-muenchen.de)

Purpose: We conducted a systematic review and meta-analysis to determine the predictive value of findings of coronary CT angiography for incident cardiovascular events.

Methods and Materials: We searched PubMed, EMBASE and the Cochrane Library through January 2010 for studies that followed $\geq 100$ individuals for $\geq 1$ year and reported at $\geq 1$ hazard ratio $(\mathrm{HR})$ of interest. Risk estimates for the presence of significant coronary stenosis (primary endpoint; $\geq 50 \%$ diameter stenosis), left main stenosis, each coronary stenosis, 3-vessel disease, any plaque, per coronary segment containing plaque, and non-calcified plaque were derived in random effect regression analysis and causes of heterogeneity were determined in meta-regression analysis. Results: We identified 11 eligible articles including 7,335 participants $(59.1 \pm 2.6$ years, $62.8 \%$ males) with suspected CAD. The presence of $\geq 1$ significant coronary stenosis (9 studies, 3,670 participants and 252 outcome events [6.8\%] with $62 \%$ revascularisations) was associated with a tenfold higher risk (HR: $10.74,95 \%-\mathrm{Cl}$ : $6.37-18.11$ ) with an $11.9 \%$ annualised event rate; $6.4 \%$ in studies excluding revascularisation (HR: 6.15 [95\%-Cl: 3.22-11.74]). Adjustment for coronary calcification did not attenuate the prognostic significance $(p=0.79)$. The estimated HR $(95 \%-C l)$ for left main stenosis, presence of plaque and each coronary segment containing plaque were 6.64 (2.6-17.3), 4.51 (2.2-9.4), and 1.23 (1.17-1.29), respectively. Conclusion: Presence and extent of CAD on coronary CT angiography are strong, independent predictors of cardiovascular events despite heterogeneity in endpoints, categorisation of CT findings and study population.

\section{B-233 10:48}

Study repetition of nonsatisfactory prospective high pitch acquisitions in cardiac imaging with 128 DSCT: frequency, radiation dose, and meaning G. Tardaguila de la Fuente, C. Delgado Sanchez-Gracián, C. Martínez, D. Castellón Plaza, F. Tardáguila Montero; Vigo/ES (gonzatar@gmail.com)

Purpose: Prospective high pitch acquisitions in cardiac imaging allow us to obtain the whole heart in one beat. Low dose studies are performed but when artefact appears we cannot edit the ECG; the solution is to repeat the study. The aim of this study is to determinate how often studies have to be repeated, why they have to be done again and radiation dose.

Methods and Materials: 229 patients underwent high pitch cardiac 128-DSCT. High pitch technique was used when heart rate $<65 \mathrm{bpm}$ spontaneously or after the administration of betablockers. Imaging protocol: rotation speed: $0.28 \mathrm{~s}$, table speed: $460 \mathrm{~mm} / \mathrm{s}$, pitch: 3.4 , collimation: $2 \times 128 \times 0.6 \mathrm{~mm}$ and tube current $320 \mathrm{mAs} /$ rotation. Tube potential was $100 \mathrm{kV}$ (patients $<80 \mathrm{~kg}$ ), $120 \mathrm{kV}$ (patients $>80 \mathrm{~kg}$ ) and $140 \mathrm{kV}$ (stent studies). We analysed dose of the study, number of cases that need to be repeated, cause of the repetition, type of acquisition of the repetition (high pitch, prospectively or retrospectively triggered) and total dose of repeated studies. Results: 40 studies needed to be repeated (17.5\%). Causes: 26 studies had to be repeated because of rhythm alterations, 9 due to isolated extrasystoles and 5 for unclear reasons. The second acquisition was another high pitch in 27 patients $(68 \%)$, in $4(10 \%)$ prospectively triggered scan was obtained and a retrospectively triggered CT was performed in $9(22 \%)$. Radiation dose of non-repeated studies was $1.23 \pm 0.49 \mathrm{mSv}$. Total dose of repeated studies (including the initial acquisition) was $3.98 \mathrm{mSv} \pm 2.62$. Conclusion: Although a relevant number of high pitch cardiac studies $(17.5 \%)$ had to be repeated, the low dose of these studies makes it worth to choose this type of acquisition when possible.

\section{B-234 10:57}

Heart rate and heart rate variability: what is the effect on motion and stairstep artefacts in step-and-shoot coronary CT angiography at a 256-slice CT scanner?

D. Muenzel, P.B. Noel, F. Dorn, M. Dobritz, E.J. Rummeny, A. Huber; Munich/DE (muenzel@roe.med.tum.de)

Purpose: To evaluate the effect of heart rate variability and heart rate on intra-scan and inter-scan "stairstep" artefacts in step-and-shoot coronary CT angiography (CCTA) with the use of a wide detector.

Methods and Materials: 70 patients underwent CCTA using a step-and-shoot technique with prospective ECG-gating at a 256-slice CT scanner (Brilliance iCT, Philips). Heart rate during the $C T$ scan was registered. Variability of heart rate was estimated by the difference of maximum and minimum heart rate during the scan. Motion artefacts were quantified using a 5-point scale with 1 point indicating no motion artefacts whereas coronary segments rated with 5 points were considered as nondiagnostic. Stairstep artefacts between single CT scan volumes per step were defined as misalignment of continuous cardiac structures quantified by measurement of the off-set on coronal and sagittal CT multiplanar reformations (MPR) of transverse single slices.

Results: Mean heart rate during the CT scans was $66 \mathrm{bpm}$ (standard deviation SD: 16.7). Mean variability of the heart rate was $10.7 \mathrm{bpm}$ (SD 17.5). A significant correlation between heart rate and stairstep artefacts $(r=0.43, p<0.001)$ and between heart rate and motion artefacts $(r=0.67, p<0.001)$ was found. There was no significant effect of heart rate variability on stairstep artefacts $(r=0.5, p<0.001)$ and motion artefacts $(r=0.18, p=0.154)$.

Conclusion: In contrast to CCTA using narrow CT detectors with low z-coverage heart rate variability has no significant effect on motion and stairstep artefacts using a wide CT detector with high z-coverage. However, a higher heart rate still increases stairstep and motion artefacts.

\section{B-235 11:06}

Dual-source 128-section CT coronary angiography at variable heart rates: comparison of image quality and radiation dose

L. Zhang, H.H. Pan, N. Xu, Y.S. Du; Shanghai/CN (zhanglei4302@hotmail.com)

Purpose: To determine the effect of heart rate frequency (HRF) and heart rate variability (HRV) on radiation exposure and image quality in patients undergoing DS CT coronary angiography with adaptive ECG pulsing.

Methods and Materials: 253 consecutive patients were categorised in three groups: group A: low HRF ( $\leq 65$ beats/min) with normal to minor HRV (mean interbeat difference (IBD), 0-3), coronary CTA proceeding with prospective ECG-triggered spiral model; group B: intermediate to high HRF ( $\geq 66$ beats $/ \mathrm{min}$ ) with normal to minor HRV (IBD, 0-3), with prospective ECG-triggered sequence scan; group C: intermediate to high HRF ( $\geq 66$ beats/min) with moderate to severe HRV (IBD $>4$ ), with retrospective ECG-gated spiral model. Optimal ECG pulsing windows for low ( $\leq 65$ beats/min), intermediate ( $66-79$ beats $/ \mathrm{min})$, and high HR ( $\geq 80$ beats $/ \mathrm{min}$ ) were at $60 \%-76 \%, 30 \%-77 \%$, and $31 \%-47 \%$ of the R-R interval, respectively. Outside the ECG-pulsing window, the tube current was reduced to $20 \%$ of the full current. Results: Coronary CTA yielded good image quality in $87.7 \%$ of patients $(222 / 253)$ No significant differences among three groups with different $\mathrm{HRF}$ and $\mathrm{HRV}$ in image quality and diagnostic performance were found. Significant difference $(P<0.001)$ was found among the effective doses of group A $(0.602 \pm 0.363 \mathrm{mSv})$, group B $(1.253 \pm 1.804 \mathrm{mSv})$ and group C (9.039 $\pm 5.657 \mathrm{mSv})$.

Conclusion: The higher temporal resolution of dual-source 128 sections CT coronary angiography performed with adaptive ECG pulsing results in preserved diagnostic image quality and performance independent of HRF or HRV at the cost of limited dose reduction in arrhythmic patients.

\section{B-236 $11: 15$}

Incremental value and safety of oral ivabradine for heart rate reduction in computed tomography coronary angiography

E. Maffei ${ }^{1}$, C. Martini ${ }^{1}$, T. Arcadi ${ }^{1}$, S. Seitun ${ }^{1}$, A. Clemente ${ }^{1}$,

N. Brunetti' ${ }^{2}$ F. Cademartiri ${ }^{1}$, A. Guaricci ${ }^{2} ;{ }^{1}$ Parma/IT, ${ }^{2}$ Foggia/IT

(filippocademartiri@hotmail.com)

Purpose: Heart rate (HR) reduction is essential to achieve optimal image quality and diagnostic accuracy with computed tomography coronary angiography (CTCA). Administration of ivabradine could be an attractive alternative to beta-blockade to reduce $\mathrm{HR}$.

Methods and Materials: One-hundred twenty-three patients referred for CTCA were prospectively enrolled. Patients were divided into two groups depending on the absence or presence of chronic beta-blockade treatment. Within the two groups 
patients were randomised to either no additional premedication or oral ivabradine for 5 days prior to CTCA. In the presence of chronic beta-blockade therapy it was shifted to atenolol $50 \mathrm{mg}$ twice a day for 5 days prior to CTCA. HR and blood pressure were assessed at admission (T0), immediately before CTCA (T1) and during CTCA (T2). The target HR was $<65$ bpm.

Results: Ivabradine significantly reduced HR during CTCA. Mean relative HR reduction was $15 \%$ for controls, $12 \%$ for chronic beta-blockade, $19 \%$ for ivabradine and $24 \%$ for both chronic beta-blockade and ivabradine at T2 ( $p$ for trend $<0.001$ ). The rate of patients who reached the target HR at T2 was $83 \%$ in controls, $71 \%$ with chronic beta-blockade, $97 \%$ with ivabradine and $97 \%$ with both ( $p$ for trend $<0.05$ ). The percentage of patients that needed additional IV beta-blockade at T1 decreased from $69 \%$ to $40 \%$ with ivabradine and $30 \%$ with both ( $p$ for trend $<0.05$ ). Conclusion: Ivabradine is safe and effective in increasing the rate of patients at target $\mathrm{HR}$ and in reducing the need for additional IV beta-blockade in patients referred for CTCA.

\section{B-237 11:24}

Individual optimisation of contrast enhancement in coronary CT angiography: a multivariate analysis

J.-F. Paul ${ }^{1}$, L. Grandin'², J.-P. Daurès ${ }^{2} ;{ }^{1}$ Le Plessis-Robinson/FR, ${ }^{2}$ Montpellier/FR (pauljf@ccml.fr)

Purpose: To optimise contrast enhancement in coronary CT angiography for each patient.

Methods and Materials: A first cohort of 58 patients underwent coronary CT angiography using a fixed protocol $(73 \mathrm{~mL}$ iobitridol $350 \mathrm{mgl} / \mathrm{mL}, 5.6 \mathrm{~mL} / \mathrm{s})$. Influence of various individual parameters (weight, height, body mass index (BMI), body surface area (BSA), cardiac frequency) were studied with regard to contrast enhancement in aortic root. The Pearson's correlation coefficient was used to assess the strengths of associations. Multivariate linear regression analysis with a backward selection process was performed to evaluate the impact of independent factors on aortic enhancement. Piecewise linear regression analysis was used for the factors where the relationship with enhancement was not linear. Then, a second cohort of 61 patients underwent an individually tailored injection protocol based on this model with various injection rates in order to achieve the range of target enhancement within 400-500 HU.

Results: From univariate analysis, a significant negative correlation was found with weight, height and BSA. No significant correlation was found with cardiac frequency, but enhancement tends to decrease for heart rate above $75 \mathrm{bpm}$. From multivariate analysis, the best model for explaining variability included height, weigh and cardiac frequency $\left(R^{2}=0.63\right)$. In the second cohort of patients using tailored protocol, $85 \%$ achieved the target range.

Conclusion: Simple physiologic/morphologic individual parameters may explain for more than $60 \%$ of aortic enhancement variability in coronary CT angiography. Individually tailored injection protocols may reduce variability of enhancement, and achieve a predefined target enhancement in most cases.

\section{B-238 11:33}

Computer-aided detection of coronary artery stenosis at computed tomography angiography: effect on performance of readers with different experience levels

E.M. Arnoldi ${ }^{1}$, U. Schoepf ${ }^{2}$, M. Rosenblum², P.L. Zwerner², R. Goldenberg ${ }^{3}$, M.F. Reiser ${ }^{1}$, C. Thilo ${ }^{4} ;{ }^{1}$ Munich/DE, ${ }^{2}$ Charleston, SC/US, ${ }^{3} \mathrm{Haifa} / \mathrm{IL}$,

${ }^{4}$ Augsburg/DE (elisabeth.arnoldi@med.uni-muenchen.de)

Purpose: To evaluate the effect of computer-aided detection on the performance of readers with different experience levels for diagnosing stenosis at coronary CT angiography (cCTA).

Methods and Materials: Five observers with different experience levels evaluated cCTA examinations of 50 patients for coronary artery stenosis ( $>=50 \%)$. After three months the same five observers reevaluated all studies, this time guided by a computer-aided detection system (CorAnalyzer, Rcadia) as a second reader. Without human interaction, this algorithm analyses the coronary arteries for the presence and location of significant stenosis. Quantitative catheter angiography served as the reference standard. Comparison of performance measures at baseline and at reevaluation was performed using likelihood-ratio Chi-square test. Results: QCA revealed 29 stenoses ( $>=50 \%$ ). For stenosis detection, the sensitivity of the computer-aided detection system alone averaged $67 \%$ per-vessel and $100 \%$ per-patient. Inexperienced readers' initial interpretations averaged $65 \%$ sensitivity per-vessel ( $86 \%$ per-patient) which improved to $82 \%(100 \%)$ with computer-aided detection. Intermediate reader sensitivity increased from $92 \%$ $(93 \%)$ to $96 \%(93 \%)$, whereas sensitivity of expert readers increased from $87 \%$ $(96 \%)$ to $89 \%$ (96\%). With computer-aided detection, the number of false negative patients was reduced from 2 to zero for the inexperienced readers but remained unchanged for experienced readers.

Conclusion: Computer-aided detection as a second reader has an overall beneficial impact on the performance of observers of all experience levels for diagnosing significant coronary artery stenosis during cCTA interpretation. In experienced readers benefit the most from the addition of computer-aided detection to their interpretation, while the added value is only modest for more experienced observers.

\section{B-239 11:42}

Correlation between myocardial bridge and myocardial ischemia by dual energy CT

S. Ou, Y. Qi, G. Peng; Guangzhou/CN (Ihq2008xxh@163.com)

Purpose: The aim of this investigation is to evaluate the detective rate, morphology of myocardial bridge-mural coronary artery (MB-MCA), and the relationship between MB-MCA and myocardial ischaemia by dual energy CT (DECT).

Methods and Materials: 252 patients suspected or confirmed ischaemic heart disease (IHD) underwent DECT were prospectively analysed. The length of MCA and thickness of MB were measured, the location of MB, type of MCA, and the distribution of atherosclerosis were observed. The relationship between myocardial bridge and the myocardial ischaemia was analysed by two experienced radiologists Results: Among 252 patients, 62 sites of MB-MCA were detected account for $21.4 \%$ (54). The average length of MCA was $23.61 \pm 6.40 \mathrm{~mm}$. The average thickness of MB was $2.21 \pm 0.38 \mathrm{~mm} .40(40 / 62,64.5 \%) \mathrm{MB}-\mathrm{MCA}$ were located at the LAD, 9 $(9 / 62.14 .5 \%)$ at the first diagonal branch and $13(13 / 62.21 .0 \%)$ on the CX. $66.2 \%$ (41/62) MCA was the superficial type and $33.8 \%$ (21/62) was the deep type. 32 $(32 / 41.78 \%)$ sites of myocardial perfusion defect were detected in superficial type and $18(18 / 21,85.75 \%)$ sites in deep type. The overall prevalence of coronary atherosclerosis at the proximal segment of MB-MCA was $53.2 \%(33 / 62)$, while at the distal segment was $11.2 \%(7 / 62)$, respectively.

Conclusion: The data of this study can demonstrate the anatomical structure of MB-MCA, location, the correlation between the types and MCA, and myocardial perfusion defect by DECT in IHD.

\section{B-240 11:51}

Bronchial artery evaluation in cardiac computed tomography (CCT) patients: is there an association between coronary artery disease (CAD) and bronchial arteries hypertrophy?

S. Tresoldi', F. Villa ${ }^{1}$, G. Di Leo ${ }^{2}$, N. Flor ${ }^{1}$, R. Bisogno ${ }^{1}$, G. Cornalba ${ }^{1} ;{ }^{1}$ Milan/IT ${ }^{2}$ San Donato Milanese/IT (silvia.3soldi@gmail.com)

Purpose: To estimate the correlation between the diameter of bronchial arteries at cardiac computed tomography (CCT) and the degree of coronary artery disease (CAD).

Methods and Materials: Two radiologists in consensus retrospectively evaluated CCT images of 100 patients ( 77 males; mean age $66 \pm 11$ years; range 34-88 years) with 4 degrees of CAD [gr1 = patent coronary arteries $(n=25)$, gr2 = significant disease $(n=25)$, gr3 = stent $(n=25)$, gr4 = by-pass $(n=25)]$ and measured the number and diameter of bronchial arteries. Examinations were performed either on a 64-MDCT or on a DSCT after administration of $85-100 \mathrm{ml}$ of contrast media $(370-400 \mathrm{mgl} / \mathrm{ml})$ at $5 \mathrm{ml} / \mathrm{s}$ followed by a $50-\mathrm{ml}$ saline flush at the same rate. Technical parameters were: $100-120 \mathrm{kVp}$; 200-700 mAs; retrospective ECG gating with mAs modulation; $64 \times 0.6 \mathrm{~mm}$ collimation. Lung-windowed images were reviewed to search for diseases possibly related with bronchial artery hypertrophy. KruskalWallis and Chi-square test were used.

Results: Patient demographics were similar in the 4 groups (mean age, respectively, $60 \pm 12,68 \pm 9,65 \pm 8,69 \pm 9$ years). Mean bronchial artery diameter in the 4 groups was, respectively, $1.5 \mathrm{~mm}, 1.7 \mathrm{~mm}, 1.6 \mathrm{~mm}, 1.9 \mathrm{~mm}$ with a statistically significant difference $(p=0.008)$. A significant difference $(p=0.023)$ was found also in the detection of hypertrophic $(>1.5 \mathrm{~mm})$ bronchial arteries, more represented in group $2(58 \%)$ and $4(63 \%)$ than in $1(35 \%)$ and $3(50 \%)$. There were no significant differences in pulmonary pathology between the four patients' groups.

Conclusion: Diameter of bronchial arteries significantly increases with CAD and particularly with surgical revascularisation, suggesting the existence of a physiological shunt between the two systems that can compensate each-other in case of need. 


\section{Vascular}

\section{SS 415}

\section{Neurovascular disorders}

\section{Moderators:}

G. Markose; London/UK

L.N. Tanenbaum; New York, NY/US

\section{B-241 10:30}

Do genetic effects or environmental factors influence the cerebrovascular atherosclerosis? Findings of the international twin study 2009

A.D. Tarnoki ${ }^{1}$, D.L. Tarnoki ${ }^{1}$, E. Medda ${ }^{2}$, M.F. Giannoni ${ }^{2}$, G. Meneghetti ${ }^{3}$, G. Cardaioli', L. Littvay', V. Berczi ${ }^{1}$, Z. Garami ${ }^{5} ;{ }^{1}$ Budapest/HU, ${ }^{2}$ Rome/IT,

${ }^{3}$ Padua/IT, ${ }^{4}$ Perugia/IT, ${ }^{5}$ Houston, TX/US (tarnoki2@gmail.com)

Purpose: To assess the heritability (A), shared (C) and unshared (E) environmental components of middle cerebral artery (MCA) mean flow velocities (MFV) and pulsatility indexes $(\mathrm{PI})$ and calculate the correlation with arterial stiffness (augmentation index on brachial artery, Aixbra, pulse wave velocity on aorta, PWVao).

Methods and Materials: 167 Italian and American (90 monozygotic /MZ/ and 77 dizygotic /DZ/) twin pairs (mean age $53 \pm 13$ years) underwent transcranial Doppler sonography (MFV, PI on left and right MCAs) and arterial stiffness test (Aixbra, PWVao, TensioMed Arteriograph).

Results: Heritability of right left and right MFV indicated $0.001(95 \% \mathrm{Cl}, 0.000$ to $0.287)$ and $0.107(95 \% \mathrm{Cl}, 0.000$ to 0.423$), \mathrm{C}$ was $0.724(95 \% \mathrm{Cl}, 0.616$ to 0.826$)$ and $0.577(95 \% \mathrm{Cl}, 0.320$ to 0.730$)$, and $\mathrm{E}$ was $0.275(95 \% \mathrm{Cl}, 0.201$ to 0.382$)$ and $0.317(95 \% \mathrm{Cl}, 0.219$ to 0.457$)$. Heritability of left and right $\mathrm{PI}$ values indicated 0.000 (95\% Cl, 0.000 to 0.094$)$ and 0.049 (95\% Cl, 0.000 to 0.332$)$, C was 0.293 $(95 \% \mathrm{Cl}, 0.105$ to 0.459$)$ and $0.411(95 \% \mathrm{Cl}, 0.210$ to 0.566$)$, and $\mathrm{E}$ was 0.707 (95\% Cl, 0.550 to 0.889$)$ and $0.540(95 \% \mathrm{Cl}, 0.415$ to 0.689$)$, respectively. MFV and PI had no significant relationship with Aixbra and PWVao.

Conclusion: No genetic but common environmental factors (familiar socialisation) seem to play a significant role on the onset of MCA MFVs. Environmenta factors related to individual experience (e.g., smoking, diet, diabetes and physical activity) influence the PIs. No relationship was found between MFV, PI and arterial stiffness (Aixbra, PWVao)

\section{B-242 10:39}

Association between carotid plaque type and cerebral microbleeds L. Saba' ${ }^{1}$, R. Sanfilippo ${ }^{1}$, M. Anzidei ${ }^{2}$, R. Bura ${ }^{1}$, R. Montisci ${ }^{1}$, G. Mallarini ${ }^{1}$; ${ }^{1}$ Cagliari/IT, ${ }^{2}$ Rome/IT (lucasaba@tiscali.it)

Purpose: Cerebral microbleeds (CMBs), are being increasingly recognised with the widespread use of MRI techniques that are sensitive to iron deposits The purpose of this work was to explore the association between carotid plaque type and CMBs. Methods and Materials: 43 consecutive (males 31; median age 64) patients were prospectively analysed. Carotid arteries were studied by using a 16-detector row CT scanner, whereas brain was explored with a 1.5 Tesla system. CMBs were studied using a T2*-weighted gradient-recalled echo (GRE) sequence. Microbleeds were classified as absent (grade 1), mild (grade 2; total number of microbleeds, 1-2), moderate (grade 3; total number of micro-bleeds, 3-10) and severe (grade 4; total number of microbleeds, $>10$ ). Chi square, multiple logistic regression analysis as well as ROC was calculated. Results: The prevalence of CMBs was $29.9 \%$. A statistically significant difference was observed between symptomatic $(43 \%)$ and asymptomatic $(15 \%)$ patients $(\mathrm{p}$ value $=$ 0.0425 ; $\mathrm{OR}=4.36$ ). Liner regression analysis demonstrated an association between the number of $C M B$ and the symptoms $(p=0.022)$. A statistically significant association was observed between the presence of fatty plaque and CMBs $(p=0.015)$. Conclusion: Results of this study confirm an increased number of CMBs in symptomatic patients and indicate an association between the presence of carotid artery fatty plaque and CMBs.

\section{B-243 10:48}

Prevalence of complicated carotid atherosclerotic plaques ispilateral to ischaemic cryptogenic stroke using high-resolution MRI

T. Saam ${ }^{1}$, T. Freilinger ${ }^{1}$, J. Grimm ${ }^{1}$, A. Schindler ${ }^{1}$, F. Bamberg', C. Yuan ${ }^{2}$,

M.F. Reiser ${ }^{1}$, M. Dichgans ${ }^{1}, \mathrm{~K}$. Nikolaou ${ }^{1} ;{ }^{1}$ Munich/DE, ${ }^{2}$ Seattle, WA/US

(Tobias.Saam@med.uni-muenchen.de)

Purpose: Although distinct pathogenetic mechanisms for ischaemic stroke have long been recognised, a definite or even probable aetiology cannot be established in about one-third of all patients ("cryptogenic strokes"). Recent studies have shown that high-resolution black-blood carotid MRI (hr-bb-cMRI) is able to identify complicated atherosclerotic AHA lesion type VI (AHA-LT6) with haemorrhage, thrombus or rupture of the fibrous cap with good correlation to histopathology. The purpose of our study was to evaluate the prevalence of AHA-LT6 in carotid arteries of subjects with cryptogenic stroke.

Methods and Materials: 30 consecutive patients (24 men, mean age $69.9 \pm 11.9$ years) with cryptogenic stroke and eccentric plaque by ultrasound were recruited from our stroke unit. All patients underwent extensive clinical workup (lab, brain MRI ultrasound, 24-hour ECG, transesophageal echocardiography) to exclude other causes of stroke. All patients received a hr-bb-cMRI at 3.0-Tesla with fat-saturated pre- and post-contrast T1w-, PDw-, T2w- and TOF images using surface coils and parallel imaging techniques. Prevalence of AHA-LT6 was determined in both carotid arteries based on previously published MRI criteria by two experienced reviewers who were blinded to the clinical information.

Results: AHA-LT6 with haemorrhage, rupture of the fibrous cap and / or thrombus was found in 14 out of 30 arteries (46.7\%) ipsilateral and in 1 out of 30 arteries $(3.3 \%)$ contralateral to the ischaemic, "cryptogenic" stroke $(P<0.001)$.

Conclusion: This study suggests that arterio-arterial embolism from non-stenotic carotid atherosclerotic plaques play a role in the pathogenesis in a subset of subjects previously diagnosed with a cryptogenic stroke.

\section{B-244 10:57}

Association between carotid artery plaque volume, composition and ulceration

L. Saba, S. Sannia, R. Montisci, R. Sanfilippo, R. Bura, G. Mallarini; Cagliari/IT (lucasaba@tiscali.it)

Purpose: Ulcerated carotid plaques are associated with a higher risk of embolism and occlusion, producing ischaemic neurological events. The purpose of this study was to evaluate the relationship between carotid artery plaque volume, composition and presence of ulceration.

Methods and Materials: 70 consecutive patients ( 46 males, 24 females; mean age 63 years; age range $42-81$ years), studied by using a multi-detector row CT were retrospectively analysed. Component types of the carotid plaque were defined according the following $\mathrm{HU}$ ranges: lipid $<60 \mathrm{HU}$; fibrous tissue from $60-130 \mathrm{HU}$; calcification $>130 \mathrm{HU}$ and plaque volumes of each component was calculated. Each carotid artery was analysed by 2 observers. Statistical analysis, by using ROC analysis, was performed to evaluate association between presence of ulceration and specific components of plaque (and its volume)

Results: 14 ulcerated plaque were detected. No significant association was observed between presence of ulceration and total carotid plaque volume $(A z=$ $0.641 ; p$ value $=0.39)$, calcified plaque volume $(A z=0.545 ; p$ value $=0.67)$ and fibrous plaque volume $(A z=0.612 ; p$ value $=0.18)$ whereas a significant statistica association was detected with lipid plaque $(A z=0.784 ; p$ value $=0.021)$.

Conclusion: Results of our study suggest that the amount of lipid plaque volume is associated with presence of ulceration; this finding suggests that the lipid component, identified by MDCTA, should be considered a significant determinant of carotid plaque vulnerability

\section{B-245 11:06}

Association between carotid plaque enhancement and micro-vessel density: preliminary results by using MDCTA and histological validation L. Saba, L. Lai, R. Sanfilippo, R. Montisci, G. Faa, G. Mallarini; Cagliari/IT (lucasaba@tiscali.it)

Purpose: Previous studies demonstrated that carotid plaques analysed by MDCTA can show contrast enhancement. The purpose of this preliminary work was to explore the association between carotid plaque enhancement (CPE) and micro-vessel density. Methods and Materials: 21 consecutive (males 15; median age 63) patients studied using a 16-detector row CT scanner were prospectively analysed. Exams were performed before and after contrast medium administration and analysis of plaque enhancement was performed. Patients underwent carotid endarterectomy "en bloc"; histological sections were prepared and the presence of micro-vessel was quantified. Logistic regression analysis as well as ROC curve and area under the curve was calculated.

Results: A statistically significant association between the degree of CPE and micro-vessel density $(p=0.009 ; R 2=0.32)$ was observed. The ROC curve analysis confirmed this association with an area under the curve of 0.735 (standard deviation $=0.112$ ) and a $p$ value of 0.0355

Conclusion: Results of this preliminary study indicated that the CPE is associated with the micro-vessel density. Histological analysis demonstrated that the degree of intra-plaque neo-vascularisation is statistically associated with the entity of CPE. 


\author{
B-246 11:15 \\ Characterisation of neovascularisation in carotid atherosclerotic plaques \\ with contrast-enhanced ultrasound \\ D.-A. Clevert, P. Zengel, S. Weckbach, T. Saam, M.F. Reiser; \\ Munich/DE (Dirk.Clevert@med.uni-muenchen.de)
}

Purpose: To evaluate the use of contrast-enhanced ultrasound in the neovascularisation within carotid atherosclerotic plaques.

Methods and Materials: 35 patients with known atherosclerotic plaques in the carotid artery were examined with contrast-enhanced ultrasound to role out the features of neovascularisation within this plaque. In addition, these plaques were analysed and correlated with plaque size and echogenicity. For contrast-enhanced ultrasound we injected $2.4 \mathrm{cc}$ of SonoVue (Bracco, Italy) i.v. The examinations were performed using the $\mathrm{S} 2000$ or Sequoia 512 (Siemens/Acuson, Mountain View) with an $9.15 \mathrm{MHz}$ or $17 \mathrm{MHz}$ probe using the CPS-software.

Results: There were 41 atherosclerotic plaques, 27 of which (19 soft and 8 mixed) enhanced after injection of SonoVue. The enhancement occurred from the carotid wall to the center of the plaque with a short-line pattern in 15 plaques, whereas 12 plaques enhanced from both the carotid wall and the carotid lumen, with just a little spot pattern. The arrival time of contrast was later in the plaques than in the carotid artery and the time to peak was longer in the plaques than in the carotid lumen. Among the 14 unenhanced plaques, 4 were hard, 3 were calcified, 2 were soft, and 5 were mixed. The unenhanced plaques had a thickness of $<2.7 \mathrm{~mm}$. Conclusion: In our small patient population, contrast-enhanced ultrasound allows the dynamic evaluation of neovascularisation within carotid plaques and neovascularisation may correlate with plaque morphology.

\section{B-247 11.24}

Distinct progression of atherosclerosis in carotid and femoral arteries of high-risk patients: 2-year follow-up by MRI vessel wall imaging

R. Wyttenbach ${ }^{1}$, N. Bianda ${ }^{1}$, M. Di Valentino ${ }^{1}$, D. Périat ${ }^{2}$, P. Santini ${ }^{1}$, J.-M. Segatto ${ }^{1}$, R. Corti ${ }^{2}$, A. Gallino ${ }^{1} ;{ }^{1}$ Bellinzona/CH, ${ }^{2} \mathrm{Zurich} / \mathrm{CH}$ (rolf.wyttenbach@bluewin.ch)

Purpose: To evaluate prospectively progression of atherosclerosis in high-risk patients with mild to moderate carotid and femoral artery stenosis by serial high resolution MRI.

Methods and Materials: Thirty-one asymptomatic patients (mean age, 69 years $\pm 7.5 \mathrm{sd}$ ) with high Framingham risk-score, mild to moderate carotid artery stenosis $(30-70 \%)$ and mild femoral artery stenosis $(<50 \%)$ underwent MRI plaque imaging (1.5 T) at baseline, 1 and 2 years at the level of carotid and femoral artery lesions. MRI studies $(n=186)$ were evaluated in consensus by two blinded readers for total vessel area (TVA), lumen area (LA) and vessel wall area (VWA). ANOVA test was used for repeated measures over time.

Results: For carotid artery, TVA remained unchanged during follow-up from 74.05 $(B L)$ to 74.56 (1y) and $75.35 \mathrm{~mm}^{2}(2 y)(p=0.45)$, LA decreased from 25.71 to 25.10 and $24.03 \mathrm{~mm}^{2}(p=0.015)$, whereas VWA tended to increase from 48.34 to 49.46 and $51.31 \mathrm{~mm}^{2}(\mathrm{p}=0.059)$. For femoral artery, TVA and VWA increased from 53.70 to 55.55 and $57.86 \mathrm{~mm}^{2}(\mathrm{p}=0.02)$, and 32.98 to 34.29 and $36.91 \mathrm{~mm}^{2}(\mathrm{p}=$ $0.007)$, respectively. LA remained unchanged $\left(20.71,21.26,20.95 \mathrm{~mm}^{2} ; p=0.67\right)$ Conclusion: High-resolution MRI demonstrated progression of atherosclerosis in the carotid and femoral arteries in patients with high Framingham risk-score. There appears to be a distinct pattern of progression of atherosclerotic burden in different vascular territories with progression of luminal narrowing in mild to moderately stenotic carotid arteries, whereas in mildly stenotic femoral arteries increase of plaque burden was compensated by positive (outward) remodelling of the vessel wall.

\section{B-248 11:33 \\ Nonenhanced ECG-gated time-resolved 4D steady-state free precession MR angiography (4D SSFP MRA) in assessment of intracranial collateral flow: comparison with digital subtraction angiography (DSA) \\ R.S. Lanzman ${ }^{1}$, P. Kröpil' ${ }^{1}$, P. Schmitt ${ }^{2}$, X. Bi ${ }^{3}$, F.R. Miese ${ }^{1}$, A. Scherer ${ }^{1}$ \\ D. Hänggi ${ }^{1}$, B. Turowski ${ }^{1}$, D. Blondin ${ }^{1} ;{ }^{1}$ Düsseldorf/DE, ${ }^{2}$ Erlangen/DE, \\ ${ }^{3}$ Chicago, IL/US (rotemshlomo@yahoo.de)}

Purpose: To evaluate a non-enhanced time-resolved 4D SSFP MRA for dynamic visualisation of intracranial collateral blood flow.

Methods and Materials: 22 patients $(59.0 \pm 11.8$ years $)$ with steno-occlusive disease of brain-supplying arteries were included in this study, 4D SSFP MRA of the intracranial arteries was acquired with 15 temporal phases and a temporal resolution of $115 \mathrm{~ms}$ on a 1.5 T MR scanner. ECG-gated image acquisition was performed with increasing trigger times following spatially selective and non-selective inversion (FAIR technique) to obtain time-resolved images. The presence of collateral flow on 4D SSFP MRA was analysed by two radiologists. Cerebral DSA served as the reference standard and was available in all patients.

Results: Non-enhanced 4D SSFP MRA was performed successfully in 21 of 22 $(95.5 \%)$ patients. Collateral flow was excluded with both 4D SSFP MRA and DSA in 4 patients. In 17 patients, DSA detected anterior collateral flow $(n=8)$, posterior collateral via the right $(n=8)$ and left $(n=7)$ posterior communicating artery as well as patent EC-IC bypasses $(n=8) .29$ of 31 collateral flow pathways were visualised by 4D SSFP MRA. As compared to DSA, 4D SSFP MRA showed a high sensitivity $(92.3 \%)$, specificity $(100 \%)$, positive predictive value $(100 \%)$ and negative predictive value $(95.2 \%)$ for dynamic visualisation of intracranial collateral flow.

Conclusion: 4D SSFP MRA is a promising non-invasive imaging technique for dynamic visualistion of intracranial collateral flow. As a non-enhanced imaging technique it can be applied safely in patients with contraindications for contrast material, as, for example, renal insufficiency.

\section{B-249 11:42}

Evaluation of anterior ethmoidal artery by 320-slice CT angiography with comparison to 3D spin digital subtraction angiography: initial experiences G. Sun, J. Ding, M. Li, Z.-H. Peng; Jinan/CN (cjr.sungang@vip.163.com)

Purpose: To explore the usage of 320-slice CT angiography (CTA) for evaluation of anterior ethmoidal artery (AEA) course and its relationship with adjacent structures, using 3D spin digital subtraction angiography (DSA) as standard reference. Methods and Materials: A total of 32 patients with cerebrovascular disease, who had both carotid 3D spin DSA and multiplanar volume rendering (MPVR) 320-slice cranium CT within 30 days interval from December 2008 to March 2010, were retrospectively reviewed. To detect the AEA course on 320-slice cranium CT, CTA and multiplanar reformations (MPR) were performed. In all cases, AEA course in ethmoid and bony anatomical landmarks (bony notch in medial orbital wall, anterio ethmoidal canal and anterior ethmoidal sulcus) for locating AEA were analysed. Results: AEA entire course in the ethmoid was seen in 100\% (32/32) and $90.1 \%$ (29/32) cases by 3D spine DSA and 320-slice CTA, respectively. In the three cases that AEA not visualized by 320 -slice CTA, 2/3 were due to dominant posterior ethmoidal artery and the other $1 / 3$ was due to diminutive AEA. In MPR of 320-slice CT, bony notch in bilateral orbital medial walls was detected in $100 \%(64 / 64)$, anterio bilateral ethmoidal canals in $43.75 \%(28 / 64)$ and anterior bilateral ethmoida sulcuses in $98.44 \%(63 / 64)$ cases.

Conclusion: 320 -slice CTA has high detection rate of AEA in the ethmoida comparable to $3 D$ spin DSA while not invasive and can be preferentially used for evalu ation of AEA with adjacent bony variations and pathologic changes of this region.

\section{B-250 11:51}

Reperfusion of cerebral aneurysms after endovascular coiling: CE and TOF MRA at 3 Tesla: a powerful alternative to DSA?

U. Wießpeiner, R. Vollmann, H. Deutschmann, M. Augustin, K. Leber, F. Ebner; Graz/AT (robert.vollmann@medunigraz.at)

Purpose: Rebleeding is a severe complication after incomplete endovascular coiling of cerebral aneurysms. Therefore, long-term follow-up imaging is required to detect remnants and reperfusion as well as de-novo aneurysms. For this purpose digital subtraction angiography (DSA) is considered as gold standard technique, but it is invasive and causes a high radiation exposure.

The aim of the present study was to evaluate the diagnostic accuracy of 3.0 T MRA versus conventional DSA.

Methods and Materials: Eighty-seven patients (58 females, 29 males) with previously coiled intracranial aneurysms underwent 3.0 T MRA and DSA within a time period of 3 to 12 months. The MR imaging protocol included time-of-flight (TOF)MRA (TR/TE/ $\alpha$ : 22 3.68/ 18 ${ }^{\circ}$, TA: 04:19 min) and contrast enhanced (CE)-MRA (FLASH 3D TR/TE/ $\alpha$ : 3.74/ 1.49/20 ; GRAPPA, Accel.Factor:2; TA: 00:22 min). Consecutively, complete occlusion, remnant/reperfusion or newly developed aneurysms were evaluated. Findings were assigned to 1 of 5 categories: exact match between MRA and DSA (1), minimal discrepancy (2), moderate discrepancy (3), considerable discrepancy (4), no match (5). All images were analysed retrospectively by 4 neuroradiologists.

Results: DSA has found complete occlusion in 44, neck remnant/reperfusion in 43 and de-novo aneurysms in 16 patients. 3.0.T MRA found occlusion in 44, remnant/ reperfusion in 47 and de novo aneurysms in 22 patients. Sensitivity, specificity and accuracy of TOF and CE-MRA together were $100 \%, 81 \%$ and $91 \%$, respectively. Conclusion: 3.0.T MRA has the capability to act as single follow-up exam after endovascular coiling and is an accurate tool to detect small de-novo aneurysms. 
$14: 00-15: 30$

Room A

\section{Abdominal Viscera}

\section{SS 501a}

\author{
CT perfusion and dual energy CT in abdominal \\ imaging \\ Moderators: \\ B. Marincek; Kilchberg/CH \\ G. Petralia; Milan/IT
}

\section{B-251 14:00}

CT perfusion: can be a useful tool for predicting early response after chemotherapy? Preliminary study

D. Kim, S. Kim, S.-A. Im, S. Han, J. Lee, J. Han, B. Choi; Seoul/KR

(mi4ri4@gmail.com)

Purpose: To evaluate the feasibility of CT-perfusion for the prediction of early response to chemotherapy in advanced gastric or colon cancer patients with liver metastasis using FDG-PET as a standard of reference.

Methods and Materials: Colonic $(n=18)$ or gastric $(n=2)$ cancers patients with liver metastasis underwent perfusion-CT and FDG-PET before and after 1 cycles of chemotherapy. CT-perfusion parameters (MIP), blood flow (BF), blood volume, permeability (PMB), arterial liver perfusion (ALP), portal liver perfusion, hepatic perfusion index for the largest hepatic metastasis were obtained. PET were analysed to determine maximum SUV (SUVmax) for the corresponding liver metastasis. Reduction rate was calculated for CT and PET. Patients who showed more than $25 \%$ reduction in PET-SUVmax after chemotherapy were designated as a responder group. Mann-Whitney-U test was used to determine the differences of CT-perfusion parameters between responders and non-responoders. Spearman correlation test was used to find correlation between perfusion parameters and SUVmax. Performance of CT-perfusion parameters in discriminating non-responders from responders was evaluated using $\mathrm{ROC}$ analysis.

Results: On PET, there were responder $(n=8)$ and non-responder groups $(n=$ 12). Among CT-perfusion parameters, BF, PMB, and ALP were significantly different between the groups. The reduction rate of $B F$ (correlation coefficient $=0.414$ ) and PMB (0.394) was significantly correlated with that of SUVmax. ROC analysis revealed area under the curve was highest in $\mathrm{BF}(0.781)$ for differentiating responders from non-responders. In the prediction of non-responders, BF showed $87.5 \%$ sensitivity and $75 \%$ specificity at a cut-off value of $15 \%$ reduction rate.

Conclusion: CT perfusion parameters, particularly BF, can be used as an alternative tool to PET in predicting early response after chemotherapy.

\section{B-252 14:09}

CT perfusion technique as an early predictor for tumour response to transarterial chemoembolisation in patients with HCC lesions: preliminary results

D. Ippolito, P. Bonaffini, L. Ratti, D. Leni, R. Corso, S. Sironi; Monza/IT (davide.atena@tiscalinet.it)

Purpose: To prospectively investigate the role of CT-perfusion (CT-p) technique in evaluation of perfusion changes in hepatocellular carcinomas (HCC) before and after transarterial chemoembolisation (TACE) therapy.

Methods and Materials: Twenty-seven patients with cirrhotic liver disease and histologically proven HCC were prospectively enrolled in our study. CT-p study was performed on 16 multidetectorCT (Brilliance 16,Philips,NL), dose exposure was 120 $\mathrm{Kv}, 80 \mathrm{mAs}$.In all cases bolus injection of $50 \mathrm{ml}$ of non-ionic contrast agent $(350 \mathrm{mgl} /$ $\mathrm{ml}$ ) at a flow rate of $6 \mathrm{ml} / \mathrm{sec}$ was performed and forty dynamic scans were acquired at a fixed table position.A dedicated perfusion software which generated a quantitative map of arterial and portal perfusion by means of colour scale was employed. The following perfusion parameters were assessed before and after TACE: Hepatic perfusion (HP),Arterial perfusion (AP), Blood Volume (BV) and Hepatic perfusion Index (HPI). Results:A complete HCC filling by lipiodol was found in 18 cases and partial filling in the 9 remaining cases. The following perfusion data were obtained in partially treated lesions: HP $32.7 \pm 15.1 \mathrm{ml} / \mathrm{sec} / 100 \mathrm{gr}$; AP:38, $4 \pm 8.8 \mathrm{ml} / \mathrm{min}$; BV $17.6 \pm 9.5 \mathrm{ml} / 100 \mathrm{mg}$; HPI $96.2 \pm 7.5 \%$. Corresponding value calculated in patients without residual tumor were: HP 13.6 \pm 6.3 ; AP 13.1 \pm 7 ; BV 6.8 \pm 4.8 and HPI 13.6 \pm 9.2 . While in normal liver the found parameters were HP 11.04 \pm 4 ; AP 10.3 \pm 3.4 ; BV $14.9 \pm 2.8$ and HPI 16.2 \pm 9.8 . A significant difference $(p<0.001)$ was found for all parameters between residual viable tumour tissue $(p<0.001)$ compared with successfully treated lesion due to the presence of residual arterial vascular structure in viable portion of treated $\mathrm{HCC}$
Conclusion: On the basis of the small patient population, this feasibility study shows that quantitative analysis of perfusion could provide an in vivo early biomarker for prediciting treatment response in patients with $\mathrm{HCC}$ lesions.

\section{B-253 14:18}

CT-perfusion in HCC patients: evaluation of quantitative map as early predictor of tumour response to radiofrequency ablation and transarterial chemoembolisation treatment

P.A. Bonaffini, D. Ippolito, F. Meloni, D. Leni, R. Corso, S. Sironi; Monza/IT (pa.bonaffini@gmail.com)

Purpose: To prospectively investigate the role of CT-perfusion (CT-p)technique in detection of blood flow changes related to therapeutic effects of transarterialchemoembolisation and radiofrequency ablation in patients with HCC.

Methods and Materials: Fifty-four patients with known cirrhotic liver disease and biopsy proven diagnosis of HCC lesion, that underwent to TACE or RFA treatment, were prospectively enrolled in our study. Perfusion study of hepatic parenchyma and of treated lesion was performed about 1 month after treatment on a multidetector 16-slice CT (Philips Brilliance,16p,NL). Dynamic-CT study was performed with static table position: 8 dynamic slice/scan; total of 40 scans; intravenous injection of $50 \mathrm{ml}$ of contrast medium. The treated lesion and surrounding parenchyma were evaluated using a dedicated perfusion software which generated a quantitative map of perfusion by means of colour scale. The following perfusion parameters for whole liver and $\mathrm{HCC}$ lesions were considered: hepatic perfusion (HP), arterial perfusion (AP), blood volume (BV), hepatic perfusion index (HPI) and time to peak (TTP). Results: Perfusion parameters of treated lesions could be quantitatively assessed using CTp analysis. 16/54 patients had a residual disease and values of perfusion parameters measured within tumour tissue were: $\mathrm{HP}=38.5 \pm 14.6 \mathrm{ml} / \mathrm{sec} / 100$ gr; $A P=41.5 \pm 8.9 \mathrm{ml} / \mathrm{min} ;$ TTP $=19.1 \pm 4.3 \mathrm{sec} ; \mathrm{BV}=18.5 \pm 6.3 \mathrm{ml} / 100 \mathrm{mg}$ $\mathrm{HPI}=57.8 \pm 32.3 \%$. The corresponding values obtained in remaining 26 cases in whom a complete necrosis were achieved were: $\mathrm{HP}=12.8 \pm 6.1 \mathrm{ml} / 100 \mathrm{gr} /$ sec; $\mathrm{AP}=13.1 \pm 7 \mathrm{ml} / \mathrm{min} ; \mathrm{BV}=7.4 \pm 9.8 \mathrm{ml} / 100 \mathrm{gr} ; \mathrm{HPI}=13.1 \pm 8.7 \%$ and TTP $=36.2 \pm 12.3 \mathrm{sec}$. A significant difference $(p<0.001)$ was observed in the mean value of all parameters calculated between treated lesions with residual tumou and those successfully treated. CT-p technique has demonstrated highly perfused areas related to the presence of residual arterial vessels within the viable portion of treated lesions.

Conclusion: This feasibility study shows that quantitative analysis of perfusion could provide an in vivo early biomarker for prediciting the treatment response in patients with $\mathrm{HCC}$ lesions.

\section{B-254 14:27}

Role of perfusion CT in evaluation of early acute pancreatitis and correlation with clinical outcome

M. Kang, A. Sagar, D. Bhasin, R. Gupta, A. Bhalla, S.S. Rana, N. Khandelwal; Chandigarh/IN (mandykang2005@yahoo.com)

Purpose: To evaluate efficacy of CT perfusion for detection of early ischaemic changes in patients with acute pancreatitis and to assess the correlation of CT perfusion measurements with the clinical outcome.

Methods and Materials: 39 consecutive patients with clinical diagnosis of acute pancreatitis presenting within 72 hours of symptom onset were enrolled in the study. A control group of 15 patients was also enrolled. Eleven perfusion parameters were evaluated for both the groups: EFP (extraction fraction product, FP (blood flow), VP (blood volume), PS (permeability surface product), KEP (outflow rate), VE (leakage rate into extravascular space), E (extraction measure), MTT (mean transit time), DT (delay time), PEI (peak enhancement intensity) and TTP (time to peak). The severity of disease was assessed using CTSI (Balthazar) and modified CTSI (Mortele) scores. The following parameters of clinical outcome were recorded: duration of hospital stay, need for intervention, development of infection, organ failure and mortality. Results: EFP, FP, VP, KEP and PEI were significantly lower in the study group as compared with the control group. VE, TTP and E were higher in the study group. EFP, FP, VP, KEP and PEI correlated well with regard to need for percutaneous intervention. FP, VP, KEP and PEI correlated well with duration of hospital stay. EFP correlated well with occurrence of infection. Between mild and severe pancreatitis groups the parameters EFP,FP,VP, KEP,VE,E,PEI and TTP were statistically significant.

Conclusion: Our data suggest that pancreatic perfusion measurement using MDCT could help in early assessment of severity and predict clinical outcome of acute pancreatitis. 


\author{
$B-255$ \\ $14: 36$ \\ Quantitative analysis of lodine concentration in hepatic tumours using \\ dual energy multiphasic CT as objective index for assessment of tumour \\ vascularity: initial experience \\ S. Kobayashi', O. Matsui', K. Otani², T. Gabata', W. Koda', \\ T. Minami', Y. Ryu', K. Kawai', K. Kozaka'; ' ${ }^{1}$ Kanazawa/JP, ${ }^{2}$ Tokyo/JP \\ (satoshik@staff.kanazawa-u.ac.jp)
}

Purpose: To investigate whether quantitative analysis of iodine concentration in hepatic tumours using multiphasic dual energy (DE) CT could be useful as objective index for assessment of tumour vascularity.

Methods and Materials: Eight patients (14 hepatic tumours) who underwent abdominal multiphasic dual source DE CT (non-enhanced single energy CT data followed by arterial, portal and equilibrium phase DE CT) and subsequent angiography-assisted CT for closer examination were included in this study of 176 consecutive abdominal multiphasic DE-CT cases. Virtual-non-contrast images and iodine maps of the livers were generated from the DE CT data using commercial software. lodine concentrations in the tumours were measured on the iodine maps. Average iodine concentration in hypervascular hepatocellular carcinoma $(\mathrm{HCC}),(\mathrm{n}=10)$ and hypovascular hepatocellular tumours $(n=4)$ were compared with t-test. Additionally, average iodine concentration in background liver parenchyma and abdominal aorta were measured. Results: Average iodine concentrations in each contrast phase (arterial, portal, and equilibrium phase) were as follows: hypervascular $\mathrm{HCC}=2.5 \pm 0.8,2.3 \pm 0.4$, $1.7 \pm 0.4(\mathrm{mg} / \mathrm{ml})$; hypovascular hepatocellular tumour $=1.1 \pm 0.8,1.8 \pm 1.2,1.4 \pm 0.8$ $(\mathrm{mg} / \mathrm{ml})$; background liver parenchyma $=1.0 \pm 0.5,2.3 \pm 0.5,1.4 \pm 0.3(\mathrm{mg} / \mathrm{ml}) ; \mathrm{ab}-$ dominal aorta $=12.5 \pm 2.8,5.3 \pm 1.0,4.0 \pm 0.6(\mathrm{mg} / \mathrm{ml})$. On arterial phase, average iodine concentration of hypervascular HCC was significantly higher than that of hypovascular hepatocellular tumour $(P=0.01)$.

Conclusion: Quantification of iodine concentration using dual energy CT might be useful as objective index to assess the degree of vascularity in hepatic tumours.

\section{B-256 14:45}

\section{Spectral CT with metal artefacts reduction software (MARS) for} improvement of tumour visibility in the vicinity of gold fiducial markers O.R. Brook', S. Gourtsoyianni', A. Brook², C. Wilcox', V. Raptopoulous'; ${ }^{1}$ Boston, MA/US, ${ }^{2} \mathrm{Haifa} / \mathrm{LL}$

Purpose: Metal artefacts reduction software (MARS) utilising spectral CT has been recently introduced for reduction of beam-hardening artefacts. We demonstrate its use for visualisation of lesions in the vicinity of implanted gold fiducials for imaging guided focused radiotherapy.

Methods and Materials: The study was approved by institutional IRB with a waiver of informed consent. Consecutive patients with implanted fiducial seeds for radiotherapy of various abdominal lesions scanned at one month post-treatment were included in the study. Spectral imaging was utilised for arterial phase CT performed on Discovery CT750 HD (GE Healthcare, Waukesha, WI). Images were reconstructed with and without MARS. Two observers independently evaluated images with and without MARS for single best image, visibility of the tumour and amount of artefacts in three planes. Mann Whitney test was used to test statistical significance.

Results: Seven patients (23 fiducials) were included in the study. Fiducials were placed in pancreas $(n=11)$, liver $(n=8)$ and periportal lymph nodes $(n=4)$. In $65 \%(95 \% \mathrm{Cl}, 51-77 \%)$ of the cases MARS images were graded better than regular reconstruction. Median score of visibility of the tumour for regular images was 2 and for MARS was 4 (possible scores: 1-worst to 5 -best). A significant reduction of blooming artefact was noted on MARS images $(p=0.01)$. Quadratic weighted kappa values showed moderate to perfect agreement between observers for the various measurements.

Conclusion: Metal artefacts reduction software utilising spectral CT improved visibility of the tumour in the vicinity of gold fiducial seeds used for focused radiotherapy planning

\section{B-257 14:54}

Dual-energy iodine subtraction in intravenous CT-cholangiography: virtual non-enhanced imaging or true non-enhanced imaging?

C.M. Sommer, C.B. Schwarzwaelder, W. Stiller, U. Stampfl,

M. Holzschuh, T. Heye, L. Grenacher, H.U. Kauczor, B.A. Radeleff;

Heidelberg/DE (cmsommer@gmx.com)

Purpose: To prospectively evaluate whether virtual non-enhanced imaging (VNI) is effective in replacing true non-enhanced imaging (TNI) applying dual-energy iodine subtraction in intravenous CT-cholangiography.

Methods and Materials: From April 2009 until February 2010, 15 consecutive potential donors for living-related liver transplantation (nine males and six females; mean age $37.6 \pm 10.8$ years) underwent a two-phase CT examination of the liver The first phase consisted of a single-energy non-enhanced CT scan providing TNI. A fter administration of intravenous cholangiographic contrast, the second phase was performed as a dual-energy cholangiographic CT scan. VNI was performed with a standard dual-energy iodine subtraction tool (Liver-VNC ${ }^{\oplus}$; Siemens Medical Solutions, Forchheim, Germany). TNI and VNI were compared with respect to objective image quality and subjective image quality.

Results: For TNI and VNI, attenuation values for the intrahepatic main ducts were $16.8 \pm 11.2 \mathrm{HU}$ and $5.5 \pm 17.0 \mathrm{HU}(p<0.05)$, for the gallbladder $14.3 \pm 7.2 \mathrm{HU}$ and $-1.8 \pm 13.1 \mathrm{HU}(p<0.001)$ and for the liver parenchyma $55.3 \pm 8.4 \mathrm{HU}$ and $58.1 \pm 10.6 \mathrm{HU}$ (n.s). For TNI and VNI, image noise for the liver parenchyma was $14.8 \pm 2.0 \mathrm{HU}$ and 7.6 $60.6 \mathrm{HU}(p<0.001)$, duct-to-liver signal-to-noise-ratio was $1.2 \pm 0.9 \mathrm{HU}$ and $0.7 \pm 2.2 \mathrm{HU}(\mathrm{n} . \mathrm{s})$. and duct-to-liver contrast-to-noise-ratio was $2.6 \pm 0.6 \mathrm{HU}$ and $6.9 \pm 2.1 \mathrm{HU}(\mathrm{p}<0.001)$. VNI was rated as adequate replacement for TNI in all subjects. Conclusion: For intravenous CT-cholangiography in potential donors for livingrelated liver transplantation, subjective image quality is comparable between VNI and TNI. However, objective image quality is significantly different when VNI is compared with TNI. Therefore, VNI is not effective enough to completely replace TNI.

\section{B-258 15:03}

Dynamic volume CT with iterative reconstruction in abdominal perfusion imaging: initial results

P. Rogalla, H. Meyer, S. Kandel; Toronto, ON/CA (Patrik.Rogalla@uhn.on.ca)

Purpose: To evaluate the effect of iterative reconstruction on perfusion values and perfusion pixel noise in dynamic volume CT.

Methods and Materials: 18 patients underwent dynamic volume 320-slice CT of the kidneys for pre-operative assessment of focal lesions. The study had ethics approval. Following intravenous injection of $60 \mathrm{ml}$ of iodined contrast material at a flow rate of $10 \mathrm{ml} / \mathrm{s}$, the imaging protocol consisted of 17 sequential low-dose scans at every 2-8 $\mathrm{s}$ (decreasing frequency) covering both kidneys. Scanning parameters were $100 \mathrm{kV}, 20-40 \mathrm{mAs}$ (depending on body habitus), $0.5 \mathrm{~s}$ gantry revolving time, $16 \mathrm{~cm}$ detector coverage, and $0.5 \mathrm{~mm}$ slice thickness for all dynamic scans. Raw data were reconstructed using conventional filtered back projection (FBP) with coneXact+ for full-field coverage and true iterative reconstruction (IR). ROIs were placed in the aorta as the reference and within 10 levels in each kidney cortex. Two postprocessing techniques were applied for calculation of perfusion maps and regional perfusion, and perfusion pixel noise values were statistically compared between both reconstruction methods.

Results: We found no difference in regional perfusion values between FBP and IR (2.61 and 2.79 in normal cortex, 1.92 and 1.71 in renal neoplasia (n.s)., respectively), independent of the postprocessing method used. Standard deviation of perfusion pixel noise was 43 and $21(p=0.01)$ in normal tissue, and 46 and 23 in renal tumours ( $p=0.001)$ for FBP and IR, respectively.

Conclusion: Iterative reconstruction leads to significant reduction in perfusion pixel noise while maintaining regional perfusion values, therefore potentially improving reproducibility and allowing for radiation dose reduction.

\section{B-259 15:12}

Iterative reconstruction algorithm for hepatic enhanced 256-slice CT: image quality of the liver tumours on routine and low-dose CT

S. Xu, Y. Hou, Q.Y. Guo; Shenyang/CN

Purpose: To evaluate 256-slice CT images of the liver tumours reconstructed using iterative reconstruction (IR) on routine-dose (RD) and low-dose (LD) CT. Methods and Materials: This prospective study was approved by the institutional review board. Informed patient consent was obtained. Forty eight patients ( 24 men, 24 women; average age: $58 \mathrm{yrs}$ ) with known or suspected liver tumours underwent hepatic enhanced 256-slice CT scans (Brilliance iCT, Philips). RD and LD CT scans were acquired sequentially during the hepatic portal venous phase of contrast enhancement. The CT dose index of $L D$ acquisition was $50 \%$ lower than RD acquisition. $\mathrm{RD}$ and $\mathrm{LD}$ data were reconstructed using IR algorithm (iDose,Philips) at eight different levels $(0 \%, 20 \%, 30 \%, 40 \%, 50 \%, 60 \%, 70 \%, 80 \%)$ separately. Quantitative noise measurements were performed. Two radiologists blinded to scanning technique evaluated the four iDose level $(0 \%, 20 \%, 50 \%, 80 \%)$ reconstructions. Sharpness of tumours, contrast between tumours and normal liver tissue, and image quality were graded [scale: 1 (worse) - 4 (best)]. Data were analysed using randomised block design analysis of variance and Friedman rank test.

Results: On RD and LD CT, the quantitative noise significantly improved with increasing level of IR $(P<0.05)$.As the level increased, however, sharpness of tumours tend to be obscure. The LD data reconstructed at $50 \%$ iDose level got identical observer image quality scores with RD data reconstructed at $0 \%$ iDose level. 
Conclusion: The iDose IR improves the image quality by decreasing image noise. When the radiation dose was reduced by $50 \%$, Images maintaining the diagnostic quality of RD CT ( $0 \%$ IR) could be acquired with $50 \%$ iDose level correspondingly. However, excessive IR may obscure the sharpness of tumours.

\section{B-260 15:21 \\ Is intravenous morphine comedication effective in improving bile duct visualisation in dual-energy CT-cholangiography? \\ C.M. Sommer, C.B. Schwarzwaelder, W. Stiller, U. Stampfl, \\ N. Bellemann, T. Heye, L. Grenacher, H.U. Kauczor, B.A. Radeleff; \\ Heidelberg/DE (cmsommer@gmx.com)}

Purpose: To prospectively evaluate whether intravenous morphine comedication is effective in improving bile duct visualisation in dual-energy CT-cholangiography in potential donors for living-related liver transplantation.

Methods and Materials: This prospective study was approved by our local ethics committee. Forty subjects underwent CT-cholangiography with infusion of a hepatobiliary contrast over $\mathbf{4 0}$ minutes. Twenty minutes after beginning of the contrast infusion, either normal saline ( $n=20$ patients; group 1$)$ or morphine sulphate $(n=$ 20 patients; group 2) was injected. Another 25 minutes later, a dual-energy CT scan of the liver was performed (tube currents of $140 \mathrm{kV}$ and $80 \mathrm{kV}$ ). Applying a dualenergy post-processing technique, pure iodine images were generated. Primary study goals were determination of bile duct visualisation scores (on a scale of 0 to 3: 0 - not visualized; 3 - excellent visualization) and diameters. The secondary study goal was determination of the bile duct volume.

Results: Bile duct visualisation scores for second- and third-order branch ducts were significantly higher in group 2 compared with group $1(2.9 \pm 0.1$ versus $2.6 \pm 0.2[p<0.001]$ and $2.7 \pm 0.3$ versus $2.1 \pm 0.6[p<0.01]$, respectively). Bile duct diameters for the common duct and main ducts were significantly higher in group 2 compared with group 1 $(5.9 \pm 1.3 \mathrm{~mm}$ versus $4.9 \pm 1.3 \mathrm{~mm}[\mathrm{p}<0.05]$ and $3.7 \pm 1.3 \mathrm{~mm}$ versus $2.6 \pm 0.5 \mathrm{~mm}[\mathrm{p}<0.01]$ respectively). The bile duct volume for the entire biliary system was significantly higher in group 2 compared with group $1(71.2 \pm 9.9 \mathrm{ml}$ versus $47.4 \pm 16.6 \mathrm{ml}[\mathrm{p}<0.001])$.

Conclusion: Intravenous morphine comedication is effective in improving bile duct visualisation in dual-energy CT-cholangiography in potential donors for living related liver transplantation.

\section{$14: 00-15: 30$} Room B

\section{Musculoskeletal}

\section{SS 510}

\section{Spine}

Moderators:

M.P. Aparisi Gomez; Valencia/ES

V.N. Cassar-Pullicino; Oswestry/UK

\section{B-261 14:00}

A careful evaluation of scout CT lateral radiograph may prevent unreported vertebral fractures

A. Bazzocchi' ${ }^{1}$ P. Spinnato ${ }^{1}$, P. Tchouante ${ }^{1}$, G. Battista ${ }^{1}$, G. Guglielmi ${ }^{2}$,

U. Albisinni', R. Canini ${ }^{1} ;{ }^{1}$ Bologna/IT, ${ }^{2}$ Foggia/lT (paolospinnato@email.it)

Purpose: Our aim was to review Scout CT Lateral Radiographs (SCT) to reveal vertebral fractures unreported by radiologists.

Methods and Materials: We retrospectively reviewed 234 CT examinations. Ou investigation was first focused on SCT data and subsequently confirmed by multiplanar sagittal reconstructions when a vertebral fracture was found. The target spine segment was T4-L4 for whole-body, T9-L4 for lumbar and T4-L2 for thoracic CT scans. A semiquantitative diagnostic approach (followed by a morphometric one) was used. When a fracture was detected a wide clinical anamnesis of the patient was sought to understand whether fractures or osteoporosis were already known. Time spent per single SCT reading session was recorded.

Results: We considered 121 whole-body, 54 thoracic and 59 abdominal CT scans In 25 patients (25/234 $-10.7 \%)$ the evaluation of the appropriate spine segment on SCT was incomplete or limited for patient/technical-based conditions. In 37 patients (37/234 - 15.8\%, 19 males - 18 females, age 63 $\$ 12$ years) 50 vertebral fractures were detected ( 27 mild, 21 moderate, 2 severe). Among 37 fractured patients only 10 were detected by radiologists. In $9 / 37$ patients $(24.3 \% ; 7 / 10-70.0 \%$ among "officially" detected patients) a condition of osteoporosis was previously settled or suspected. Mean time spent to evaluate a single SCT was 1 minute and 25 seconds. Conclusion: The perception and sensibility to vertebral fractures among radiologists are still poor when a vertebral fracture assessment is not the aim of the examination. Little time spent for the evaluation of SCT could improve our accuracy.

\section{B-262 14:09}

Vertebral fracture assessment: between experts and young physicians

A. Bazzocchi' ${ }^{1}$ P. Spinnato ${ }^{1}$, F. Fuzzi $^{1}$, C. Sassi ${ }^{1}$, E. Salizzoni ${ }^{1}$, G. Battista ${ }^{1}$

G. Guglielmi ${ }^{2}$, R. Canini1; ${ }^{1}$ Bologna/IT, ${ }^{2}$ Foggia/IT (paolospinnato@email.it)

Purpose: The aim of our study was to determine the diagnostic accuracy of new dual-energy $\mathrm{x}$-ray absorptiometry technologies in the detection of vertebral fractures and to analyse intra- and inter-observer variability and time consumption of densitometric methods (MXA) compared with radiographic ones (MRX) in two groups of readers: experts and residents.

Methods and Materials: Forty-five patients were submitted to both MRX and MXA (Lunar-iDXA ${ }^{\mathrm{TM}}$, GE-Healthcare, USA) on the same day. Lateral images of the spine were independently evaluated by 3 expert radiologists (group-1) and by 3 radiology residents (group-2) in two sessions with 7 days between evaluations of the same anonymous images. "True" fractures were defined by an independent expert radiologist on MRX evaluation. Cohen test was used to analyse interpretation agreement. Results: Forty-eight "true" fractures were detected (48/585 vertebrae, $8.2 \%$ - 28 mild, 16 moderate, 4 severe) in 45 analysed patients $(61.2 \pm 11.1$ years, $\left.\mathrm{BMI}=25.3 \pm 3.0 \mathrm{Kg} / \mathrm{m}^{2}\right)$. MXA accuracy was $97 \%$ and $80 \%$ on a lesion-based and patient-based analysis, respectively. Inter-observer agreement on presence/absence of vertebral fracture (lesion-based) was $67 \%$ for MXA versus $65 \%$ for MRX in group-1, $65 \%$ versus $58 \%$ in group- 2 . The average intra-observer agreement in the two groups was equally $98 \%$ for both methods. The mean time spent for a single examination was 1.35 (min.sec) for MXA versus 2.10 for MRX in group-1 and 2.11 for MXA versus 3.36 for MRX in group-2.

Conclusion: Nowadays technical improvements make MXA comparable with traditional radiographic gold standard, providing consistent advantages and attractions even for less-experienced physicians.

\section{B-263 14:18}

Can converted BMD values derived from sagittal reformations of contrastenhanced MDCT predict incidental osteoporotic vertebral fractures? T. Baum ${ }^{1}$, D. Müller ${ }^{1}$, M. Dobritz ${ }^{1}$, E.J. Rummeny', T.M. Link², J.S. Bauer ${ }^{1}$; ${ }^{1}$ Munich/DE, ${ }^{2}$ San Francisco, CA/US (thbaum@gmx.de)

Purpose: To obtain BMD values of the lumbar spine from sagittal reformations of routine abdominal contrast-enhanced MDCT and assess their performance in differentiating patients with and without prevalent and incidental osteoporotic fractures of the spine.

Methods and Materials: Eight postmenopausal women $(65 \pm 5 y e a r s)$ underwent standard lumbar QCT (L1-L3) and afterwards routine abdominal contrast-enhanced MDCT. The sagittal reformations were used for corresponding lumbar BMD measurements. The calculated MDCT-to-QCT conversion equation for BMD was applied to baseline and follow-up routine abdominal contrast-enhanced MDCT scans of 91 postmenopausal women $(68 \pm 8$ years). Their vertebral fracture status (no, prevalent or incidental osteoporotic fracture) was assessed in the sagittal reformations. Results: Acorrelation coefficient of $r=0.94(p<0.05)$ was calculated for the BMD values of MDCT and standard QCT with the conversion equation $\mathrm{BMD}_{\mathrm{OCT}}=0.69 \times \mathrm{BMD}_{\mathrm{MDCT}}-11 \mathrm{mg} /$ $\mathrm{ml}$. Mean follow-up time of the 91 patients was $10 \pm 3$ months. Ten patients $(11.0 \%)$ had a prevalent osteoporotic vertebral fracture at baseline. Incidental osteoporotic vertebral fractures were diagnosed in 12 patients (13.2\%). At baseline, patients with prevalent and incidental fractures showed significantly lower BMD values (averaged over L1-L3) than patients without fracture $(66.33 \mathrm{mg} / \mathrm{ml}$ vs. $73.46 \mathrm{mg} / \mathrm{ml}$ vs. $93.55 \mathrm{mg} / \mathrm{ml} ; \mathrm{p}<0.05)$ $B M D$ values of patients with incidental and prevalent fractures were not different at baseline ( $p>0.05)$. Short- and long-term reproducibility errors for BMD measurements in the sagittal reformations amounted $2.09 \%$ and $7.70 \%$, respectively.

Conclusion: In this longitudinal study, converted BMD values of the lumbar spine derived from sagittal reformations of routine abdominal contrast-enhanced MDCT could predict incidental osteoporotic vertebral fractures.

\section{B-264 14:27}

Cortical porosity identifies fragility fractures in type-2 diabetic postmenopausal women

S.P. Yap, T. Baum, A.J. Burghardt, T.M. Link; San Francisco, CA/US (thomas.baum@ucsf.edu)

Purpose: Subjects with type-2 diabetes mellitus (T2DM) have an increased risk of fragility fractures. Currently, fracture risk is assessed via BMD measurements from dual $x$-ray absorptiometry (DXA). However, patients with T2DM have normal to elevated BMD measurements with respect to normal controls. The purpose of this study is to determine whether T2DM patients with fragility fractures have higher cortical porosity using high-resolution peripheral quantitative computed tomography (HR-pQCT) 
Methods and Materials: 49 postmenopausal females (age $=60.5 \pm 5.3$ years) were recruited into four groups: controls $(n=19)$, controls with osteoporotic fractures ( $n$ $=10)$, T2DM $(n=17)$, T2DM with osteoporotic fractures $(n=6)$. Fractures were assessed from radiographs and QCT of hip and spine. DXA of the spine and hip was performed for BMD measurements. HR-pQCT of distal and ultra-distal (UD) regions of radius and tibia were performed and cortical porosity was assessed. Results: The HR-pQCT showed statistically significant and elevated cortical porosity values when T2DM with fractures were compared to controls, controls with fractures, and T2DM (distal radius $[p=0.0002,0.0005,0.0001]$, distal tibia $[p=$ $0.0178,0.0167,0.0015]$, and UD radius $[p=0.0004,0.0028,0.0008]$ respectively). Gold-standard DXA hip and spine were unable to differentiate T2DM with fractures from the other groups.

Conclusion: Cortical porosity assessed with HR-pQCT may provide a new useful tool to determine the risk for fragility fractures in T2DM postmenopausal women. In this study T2DM patients with fractures were differentiated from control subjects using cortical porosity, while DXA was unable to distinguish the different groups.

\section{B-265 14:36}

Craniometric measurements in the assessment of basilar invagination: are they still relevant in the age of cross-sectional imaging?

Y. Kwong, N. Rao, K. Latief; Nottingham/UK (dryune@hotmail.com)

Purpose: Basilar invagination is a serious complication of rheumatoid arthritis, and a number of plain radiographic measures at the craniocervical junction are in use to enable its diagnosis. However, these are hampered by the overlap of bony landmarks. We aim to establish accurate CT values for these measures to facilitate early diagnosis on cross-sectional imaging.

Methods and Materials: 100 male and 100 female patients who had their craniocervical junction imaged as part of their CT study were retrospectively identified. Patients aged between 18 and 49 were included. Two radiologists independently reformatted the images in the mid-sagittal plane and performed the following measurements: Wackenheim's line, McRae's line, Chamberlain's line, McGregor's line, Redlund-Johnell method and our modification of Ranawat's method.

Results: There were significant differences between CT values and accepted radiographic measurements for the Wackenheim, Chamberlain and McGregor lines. McRae's line was the easiest to measure, and the odontoid tip did not cross this line in any patient (distance from line ranged from 0.6 to $10.4 \mathrm{~mm}$ ). The measurements for the Redlund-Johnell method were similar to radiographic values (males $>31.6 \mathrm{~mm}$, females $>27.4 \mathrm{~mm}$ ), and we provide normal values for the modified Ranawat method (males $>23.7 \mathrm{~mm}$, females $>24.2 \mathrm{~mm}$ ).

Conclusion: We propose that McRae's line is used over other methods when assessing for basilar invagination on cross-sectional studies, as it is the easiest variable to understand and remember. If the odontoid tip is eroded, the RedlundJohnell and modified Ranawat methods are alternatives, and we have provided normal CT values for these.

\section{B-266 14:45}

Baastrup's disease - a 'disease' or a normal feature of the ageing spine? An MDCT population study

Y. Kwong, N. Rao, K. Latief; Nottingham/UK (dryune@hotmail.com)

Purpose: Baastrup's disease is characterised by the development of abnormal contact between adjacent spinous processes. The clinical significance remains unresolved, with a few studies reporting Baastrup's disease as a cause of back pain. The aim of this study was to perform a large population analysis of the prevalence of Baastrup's disease in an unselected population and to determine if it is part of the normal ageing spine.

Methods and Materials: The scans of 1,008 patients, divided equally between seven age groups, who had undergone abdominopelvic CT were retrospectively reviewed. Baastrup's disease was judged to be present, by two independent observers, if there was close contact between adjacent spinous processes, and if the apposing ends were sclerotic. The presence of degenerative changes, i.e. disk degeneration, spondylolisthesis and facet OA was also noted.

Results: 413 patients (41.0\%) had evidence of Baastrup's disease. A decade-ondecade increase in prevalence was seen, with a peak of $81.3 \%$ in the over 80 's. Up to 5 affected levels were seen in some patients ( $4.1 \%$ of positive cases), but most commonly, one level was affected (35.4\%). Baastrup's disease was most common at L4/5. There were associated degenerative changes at almost all affected levels (899/901). Conclusion: Baastrup's disease has a very high prevalence amongst the elderly. Our data demonstrates that it develops with increasing age, and is part of the normal ageing process in the spine. Further, given the near universal association with degenerative changes, we urge caution before diagnosing Baastrup's disease as the cause of a patient's back pain.

\section{B-267 14:54} Juxtafacet cysts of the lumbar spine: a positional MRI study P. Niggemann ${ }^{1}$, J. Kuchta ${ }^{1}$, J. Höffer ${ }^{2}$, D. Grosskurth ${ }^{2}$, H.-K. Beyer ${ }^{2}$, K.-S. Delank ${ }^{2} ;{ }^{1}$ Bonn/DE, ${ }^{2}$ Cologne/DE (p.niggemann@mrt-koeln.de)

Purpose: Juxtafacet cysts (JFC) are often found in degeneration of the facet joints. The method of choice to diagnose JFC is MRI. However, some JFC are missed and only found intraoperative. The present study addresses the incidence of JFC in a symptomatic population, positional MRI features and factors leading to variation in size of JFC.

Methods and Materials: 67 distinct intraspinal or intraneuroforaminal JFC in 50 patients were investigated using positional MRI. The signal intensity, size of the JFC, the pres ence of a slip, the variation of the slip and the angular movement of the affected segment were assessed in supine, neutral sitting, flexion (sitting) and extension (standing) positions. The overall movement of the spine and the lordosis angle were measured. Results: JFC varied in size in segments with an unstable slip and with an increased angular movement. JFC with bright signal tend to vary in size compared with JFC with intermediate or low signal. Joint effusion and displacement of the effusion leads to formation of the cysts and is responsible for variations in size. In our study, the sensitivity for JFC detection was $100 \%$ for extension (standing), $89 \%$ for supine and $78 \%$ for neutral sitting position. JFC were the most prominent in extension (standing) Conclusion: The present study proves that JFC are related to degenerative disease of the facet joints. Detection rate of JFC rises with increasing lordosis of the spine and under weight-bearing condition. Unstable slipping or increased angular movement affect the size of JFC.

\section{B-268 15:03}

Lateral lumbosacral radiological angular indices in standing and lateral lying positions and their relation with chronic nonspecific low back pain and body mass index

M. Tahmasebi, M. Shaterzadeh, E. Shokrollahi; Ahvaz/IR

(tahmasebilmn@yahoo.com)

Purpose: To investigate which lateral lumbosacral radiological indices are more related to chronic nonspecific low back pain and whether their relations are more significant in standing position or lateral lying position and how they are related to BMI. Methods and Materials: A total of 200 people were introduced to the research: 100 chronic low back pain patients and 100 people as control group, with the same age and sex range. Height and weight were measured, BMI calculated and quantitative Oswestry low back pain questionnaire completed. Lateral lumbosacral $\mathrm{x}$-ray was done in two positions: standing and lateral lying. Lumbar Stability Index was determined, Sacral Inclination Angle (SIA), Lumbo Sacral Angle (LSA), Sacral Horizontal Angle (SHA) and Segmental Lordotic Angle (SLA) - L3 to S1 - were measured in both positions. Relations between Oswestry number and BMI with each other and with different radiologic indices were calculated.

Results: Of all measured angles, LSA is significantly smaller in patients. Lumbar Stability Index is strongly changing to instability in both groups (patients and controls) with increasing BMl from 30 to 35 and also with changing position from lying to standing. The Oswestry number has a positive relation to BMI which is stronger in control group than in patients. Conclusion: In reporting lateral lumbosacral $x$-ray, jumbar stability index should be noticed in people with high BMI, especially measured in standing position, even if the person has no low back pain. Lumbosacral angle should be noticed especially in people with chronic nonspecific low back pain.

\section{B-269 15:12}

The diagnostic advancement of axial loaded lumbar spine MR in patients with clinically suspected central canal stenosis Y. $\mathrm{Kim}^{1}$, J. Lee ${ }^{2} ;{ }^{1}$ Seoul/KR, ${ }^{2}$ Sungnam/KR (selenenuevo@gmail.com)

Purpose: The purpose of this study was to define diagnostic advancement of L-spine MR with axial loading device in patients with clinically suspected central canal stenosis, and to show a relationship of facet joint instability with aggravated central canal stenosis in axial loaded studies.

Methods and Materials: From April 2006 to May 2010, lumbar MRI with axial loading device (DynaWell, DynaWell Inc., Las Vegas) was taken in 54 patients. Axial and sagittal T2 weighted images were obtained in preloaded phase and axia loaded phase. Axial images were evaluated with concerns of 1) gross change of central canal stenosis, 2) findings of facet joint change, including arthrosis, effusion, effacement of effusion, and 3) formation of ventral synovial cyst after axial loading. In addition, dural sac cross-sectional area (DSCSA) was measured in L3/4, L4/5 and $\mathrm{L} 5 / \mathrm{S} 1$ levels to quantify the change of stenosis. The significant change in DSCSA was defined as $10 \%$ decrease. Changes of neural foraminal stenosis, curvature and spondylolisthesis were evaluated with sagittal images. 
Results: With a use of axial loading device, the additional diagnosis of severe central canal stenosis was made in 13 patients $(25 \%)$ in both gross interpretation and quantitative study (DSCSA $<75 \mathrm{~mm}^{2}$ ). The significant decrease of DSCSA was demonstrated in 22 patients $(42 \%)$. The significant decrease was related to facet joint effusion and effacement of effusion.

Conclusion: Measurable advancement in diagnosis of severe central canal stenosis was possible with axial loaded MRI. Patients with facet joint instability had tendency to show significant changes in central canal area.

\section{B-270 15:21 \\ 3D-MRI imaging quantification of the rotation of the spinal cord in scoliosis preoperation \\ W.L.Q. Wang, Y.J.L. Yang, Y.Z.Y. Yang; Guangzhou/CN \\ (zwyzwy789@sohu.com)}

Purpose: To assess the rotation of the spinal cord and vertabra in axial planner using 3D-MRI in scoliosis patients.

Methods and Materials: Ten patients with a thoracic or thoracolumbar scoliosis underwent 3D-Space T2-weighted MRI. The rotation of the spinal cord and vertebra in axial section were measured for T4, the apical vertebra and L1 through multiplanar reconstruction. The rotational data were compared with Cobb angle; also their relationship was anlysed using non-parametric tests.

Results: There was different rotation of spinal cord at T4, the apical vertebra, $\mathrm{L} 1$ and rotation angle of spinal cord (RAc) was different from vertebra rotation angle (RAsag), especially in patients with large Cobb angle; most of them had smaller vertebra rotation than spinal cord. There were three rotation types : overrotation $(\mathrm{O})$ type, under-rotation $(\mathrm{U})$ type and reverse-rotation $(\mathrm{R})$ type. $80 \%(8$ of 10)patients were of $O$ type rotation. Statistical analysis showed a significant correlation between spinal cord rotation and Cobb angle at measured level. The position of apex was statistically correlated with the Cobb angle.

Conclusion: To quantify the degree and rotation type of the spinal cord using $3 \mathrm{D}-\mathrm{MRI}$ in scoliosis patients are important to make surgical planning. That may decrease the operation dangers and prevent the complication of nervous dysfunction caused by spinal cord injury due to the over derotation.

\section{4:00 - 15:30}

Room D1

\section{Chest}

\section{SS 504}

\section{Diffuse lung disease}

Moderators:

D. Hahn; Würzburg/DE

N. Sverzellati; Parma/IT

\section{B-271 14:00}

HRCT patterns that predict mortality in patients with chronic hypersensitivity pneumonitis

S.L.F. Walsh' ${ }^{1}$, N. Sverzellati ${ }^{2}$, A. Deveraj ${ }^{1}$, A.U. Wells ${ }^{1}$, D.M. Hansell';

${ }^{1}$ London/UK, ${ }^{2}$ Parma/IT (slfwalsh@gmail.com)

Purpose: The aim of this study was to identify HRCT features that predict mortality in patients with chronic fibrotic hypersensitivity pneumonitis (CHP).

Methods and Materials: A cohort of 95 patients with a clinico-radiologic diagnosis of $\mathrm{CHP}$ was identified (mean age $55.1 \pm 12.6$ years, 40 male:55 female, biopsy proven $=27$ ). HRCT scans at baseline were scored by two observers for the extent of abnormal lung and the proportional contribution of fine and coarse reticulation, microcystic (cysts $\leq 4 \mathrm{~mm}$ ) and macrocystic honeycombing. Main pulmonary artery and segmental pulmonary artery measurements were also assessed. Finally, a score for severity of traction bronchiectasis was assigned. Using death as the primary outcome measure, variables were analysed by Cox proportional hazards model. Results: On multivariate analysis, percentage of coarse reticulation $(\mathrm{HR}=1.02, \mathrm{Cl}$ $=1.01-1.04, \mathrm{p}=0.014)$, microcystic honeycombing $(\mathrm{HR}=1.09, \mathrm{Cl}=1.01-1.17, \mathrm{p}=$ $0.019)$ and macrocystic honeycombing $(\mathrm{HR}=1.06, \mathrm{Cl}=1.01-1.10, \mathrm{p}=0.005)$ were independently associated with an increased mortality. Macrocystic honeycombing was a stronger prognostic index than microcystic honeycombing. The degree of traction bronchiectasis was also a strong predictor of poor prognosis $(\mathrm{HR}=1.11, \mathrm{Cl}$ $=1.05-1.17, p<0.001)$. Pulmonary vasculature measurements were not predictive of mortality either on univariate or multivariate analysis.

Conclusion: Patterns of coarse fibrosis such as coarse reticulation and honeycombing are associated with increased mortality in patients with a diagnosis of CHP. In addition, degree of traction bronchiectasis is strongly predictive of mortality.

\section{B-272 14:09}

Prognostic HRCT patterns in fibrotic pulmonary sarcoidosis S.L.F. Walsh ${ }^{1}$, N. Sverzellati ${ }^{2}$, A. Devaraj ${ }^{1}$, A.U. Wells ${ }^{1}$, D.M. Hansell'; ${ }^{1}$ London/UK, ${ }^{2}$ Parma/IT (slfwalsh@gmail.com)

Purpose: The aim of this study was to identify prognostic HRCT patterns in a group of patients with a diagnosis of fibrotic pulmonary sarcoidosis.

Methods and Materials: The need for patient consent was waived by the institutional ethics committee. HRCTs of 96 patients (mean age $42.1 \pm 13.3$ years, 48 male:48 female) with a clinico-radiologic diagnosis fibrotic pulmonary sarcoidosis were studied. Visual estimates were made of the extent of abnormal lung and the proportional contribution of fine and coarse reticulation, microcystic (cysts $\leq 4 \mathrm{~mm}$ ) and macrocystic honeycombing. In addition, a score for severity of traction bronchiectasis was assigned. Using death as the primary outcome measure, variables were analysed by Cox proportional hazards model.

Results: CT features predictive of a worse outcome on bivariate analysis were proportion of coarse reticulation $(\mathrm{HR}=1.06, \mathrm{Cl}=1.01-1.08, \mathrm{p}<0.004)$ and main pulmonary artery size $(\mathrm{HR}=2.45, \mathrm{Cl}=1.18-5.08, \mathrm{p}<0.016)$. Patterns of honeycombing and traction bronchiectasis were not significantly associated with increased mortality.

Conclusion: In fibrotic sarcoidosis the HRCT pattern of coarse reticulation is significantly associated with increased mortality. Unlike, in the setting of fibrotic interstitial pneumonias, patterns of honeycombing and traction bronchiectasis have no significant predictive value. In addition, main pulmonary artery size is a strong predictor of mortality in patients with fibrotic pulmonary sarcoidosis.

\section{B-273 14:18}

Acute exacerbation of usual interstitial pneumonia after resection of lung cancer: CT manifestations of the preoperative lung

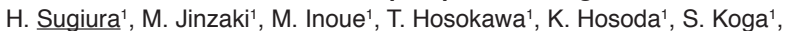

T. Hoshi ${ }^{2}$, Y. Kawabata ${ }^{2}$, S. Kuribayashi' ${ }^{1}$ Tokyo/JP, ${ }^{2}$ Saitama/JP (hsugiura@momo.so-net.ne.jp)

Purpose: To investigate whether graphical changes in preoperative chest $\mathrm{CT}$ were correlated with the occurrence of acute exacerbation (AE) of usual interstitial pneumonia (UIP) after resection of lung cancer.

Methods and Materials: This study included 205 cases (182 males and 23 females) of lung cancer with histologically proven UIP pattern in the specimen of the resected lungs. The preoperative chest CT scans were retrospectively evaluated, and assessed for the presence of interstitial pneumonia (IP) findings. The IP pattern was classified into three groups on the basis of IP findings as follows: group 1, with typical honeycombing; group 2, with atypical honeycombing; group 3 , neither typical nor atypical honeycombing (only ground glass opacities and/ or reticulation). Atypical honeycombing indicated the finding of cysts with some fibrosis whose appearance corresponds neither to the definition of honeycombing nor pulmonary emphysema.

Results: Acute exacerbation of UIP developed in 11 cases after thoracotomy. The incidence of AE occurred in the groups 1, 2, and 3 of IP pattern was $13.6 \%(6 / 44)$, $6.4 \%(5 / 78)$, and $0.0 \%(0 / 83)$, respectively. Comparison between the IP pattern and the incidence of $A E$ indicated significant difference $(P<0.01)$.

Conclusion: Postoperative AE of UIP developed in cases with atypical honeycombing as well as in cases with typical honeycombing, while AE was not observed in cases with no findings of typical nor atypical honeycombing. Therefore, atypical honeycombing may also be a risk factor of postoperative AE of UIP, and is important to be recognised before surgery for lung cancer.

\section{B-274 14:27}

Automatic detection and quantification of emphysema in healthy smokers: CT findings in correlation with pulmonary function tests (PFTs)

K. Yasunaga, N. Cherot-Kornobis, J.-L. Edme, A. Sobaszek, C. Boulenguez, A. Duhamel, J. Rémy, M. Rémy-Jardin; Lille/FR (martine.remy@chru-lille.fr)

Purpose: To provide morphological and quantitative CT information on emphysema in healthy smokers in correlation with pulmonary function tests (PFT).

Methods and Materials: The study population included 75 smokers (current smokers: $\mathrm{n}=39$; ex-smokers: $\mathrm{n}=36$ ) and 25 non-smokers who underwent volumetric high-resolution CT of the chest and PFTs with automated quantification of emphysema.

Results: The frequency $(29 / 75 ; 39 \%$ vs $1 / 25 ; 4 \% ; p=0.001)$ and extent $(0.13 \%$ vs $0.0004 \% ; p=0.01$ ) of emphysema was significantly higher in smokers compared to nonsmokers. Among smokers: (a) emphysema was an isolated finding in 8 subjects whereas it was found in association with CT features of airway disease in 21 smokers; (b) the severity of emphysema did not differ according to the GOLD 
stage $(p=0.77)$. Smokers with emphysema had significantly higher mean values of FRC ( $p=0.0012)$, RV (0.5). Correlations were found between the percentage of emphysema and: (a) tobacco consumption of current $(r=0.34215 ; p=0.0330)$ and exsmokers $(r=0.44104 ; p=0.0071)$; $(b)$ alterations of TLC, FRC, RV, FEV1) VC and DLCO of smokers. Although there was no statistically significant difference in the overall percentage of emphysema between current smokers and exsmokers, current smokers had a higher percentage of emphysema in the right lung ( $p$ $=0.041)$ and in the right upper lobe $(p=0.037)$.

Conclusion: Quantitative CT allows recognition of regional specificities and subclinical functional alterations in smokers with emphysema.

\section{B-275 14:36}

The value of quantitative lung parenchymal assessment in bronchiolitis obliterans following hemopoietic stem cell transplantation

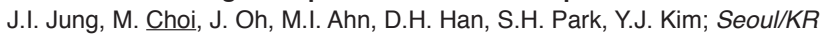
(asteria1208@catholic.ac.kr)

Purpose: To evaluate the usefulness of quantitative lung parenchymal assessment in bronchiolitis obliterans (BO) following hemopoietic stem cell transplantation (HSCT).

Methods and Materials: Ten patients with BO after HSCT and 20 volunteers were included. They underwent MDCT including inspiration and expiration scans. The patients performed pulmonary function tests (PFTs). The source images were reconstructed two ways for visual (VA) and qunatitative assessment (QA). In VA, radiologic abnormalities were reviewed. In $Q A$, mean lung density (MLD), relative areas of lung (RA) with attenuation coefficients below specific thresholds and nine percentiles of the distribution of attenuation coefficients were computed. The relationships between these parameters and PFT results of patients were correlated. Results: All patients showed abnormal CT findings including expiratory air trapping on VA. In QA, MLD of inspiration scan (MLDin), MLD of expiration scan (MLDex), difference between MLDin and MLDex ( $\triangle M L D$ ) were -862.6, -813.2 and 49.4 for patients with $\mathrm{BO}$, and $-815,-690.25$ and 124.75 for volunteers. There was significant difference between all these parameters for two groups $(p=0.001)$. Relative area with attenuation coefficient less than $-800 \mathrm{HU}\left(\mathrm{RA}_{800}\right)$ and $-750 \mathrm{HU}\left(\mathrm{RA}_{750}\right)$ and the $80^{\text {th }}$ percentile on expiration were most highly correlated with patients' residual FEV1 (rho $=-0.927, p<0.001$ ). MLDin had a slight tendency to be lower in patients whose disease was more severe. But this was not statistically significant $(r=0.46, p=0.07)$

Conclusion: $\mathrm{RA}_{800}, \mathrm{RA}_{750}$ and the $80^{\text {th }}$ percentile on expiratory scan are valid index to quantify BO on MDCT. Quantitative lung analysis is useful tool for the assessment of $\mathrm{BO}$ after HSCT.

\section{B-276 14:45}

\section{Pulmonary findings in ankylosing spondylitis by HRCT: correlation with} disease indexes

Z.M. Metafratzi', E. Alexiou', M. Fanariotis ${ }^{1}$, I. Saougou², K. Vlahos ${ }^{2}$, A. Drosos², K. Tsampoulas $2 ;{ }^{1}$ Larisa/GR, ${ }^{2}$ loannina/GR

Purpose: To identify the lung abnormalities in ankylosing spondylitis (AS) on HRCT and to correlate them with the disease severity indexes.

Methods and Materials: Thirty-two patients with AS (age 48 $\pm 8 y$ s, and disease duration $17.5 \pm 7 \mathrm{ys}$ ) free of respiratory symptoms, underwent rheumatological examination and lung HRCT. A control group of fifteen age- and sex-matched subjects were investigated with the same technique.

Results: HRCT revealed at least three different coexisting abnormalities in twentythree $(74 \%)$ patients, predominantly pleural tags $(19,59.4 \%)$, air-trapping (17, $53.1 \%)$, pleural thickening $(14,43.8 \%)$, parenchymal bands $(13,40.6 \%)$, irregular interfaces $(10,31.3 \%)$, interlobular thickened septa $(9,28.1 \%)$, GGOs $(8,25 \%)$, bronchiectasis $(7,21.9 \%)$, interstitial lung disease $(5,16.6 \%)$, bullae $(3,9.4 \%)$, honeycombing $(2,6.3 \%)$ and tracheal dilatation $(8,25 \%)$. None of the control group had more than two coexisting HRCT findings including mild air-trapping $(5,35.7 \%)$ and mild bronchiectasis (4, 26.7\%). No correlation was noted between HRCT findings and the smoking history or drug administration of the patient group. The only HRCT abnormality observed to be statistically significant in the patients compared with the controls was the parenchymal bands $(p=0.002)$. Furthermore, CRP was significantly correlated to the presence of parenchymal bands $(p<0.001)$. No other correlation was detected between HRCT findings and the disease severity indexes or duration of the disease.

Conclusion: A spectrum of HRCT lung abnormalities is often detected in asymptomatic patients with AS. The most common findings demonstrate evidence of mild airway and interstitial disease and tracheal dilatation.

\section{B-277 14:54}

Gemcitabine-induced lung toxicity: the role of HRCT

R. Argirò, E.A. d'Adamo, B. Sacconi, D. Conte, F. Sandolo, M. Bezzi, E. Cortesi,

R. Passariello; Rome/IT (r85ino@hotmail.com)

Purpose: To evaluate the incidence of gemcitabine-induced lung toxicity and the role of HRCT in detecting pulmonary drug-related events in asymptomatic patients. Methods and Materials: 140 patients with proven neoplasia (NSCLC, pancreas, breast and urotelial cancer) were treated with gemcitabine (as single or combined therapy) for 3-18 months. All of them underwent at least two HRCTs before and during treatment. Respiratory symptoms were assessed the same day of the exam, using the WHO toxicity grading. Three patients treated also with radiation therapy were not included in the study.

Results: During treatment, 15 patients (10.9\%) presented with HRCT findings compatible with drug-related toxicity. The HRCT showed four major radiological appearances: sub-lobar or segmental opacities $(n=6)(40 \%)$, interstitial pneumonitis with ground glass opacities $(n=6)(40 \%)$, bronchiolitis $(n=2)(13.3 \%)$ and diffuse alveolar damage (DAD) $(n=1)(6.7 \%)$. Respiratoty symptoms, however, were present in only 8 patients $(5.8 \%)$ : Grade $1(n=4)$; grade $2(n=5)$; grade 4 $(n=1)$. There was no correlation between incidence of lung disease and gemcitabine dosage. All symptomatic patients were successfully treated with steroids and gemcitabine dose reduction or suspension. Asymptomatic patients underwent steroid treatment with close clinical and HRCT follow-up.

Conclusion: The incidence of gemcitabine-induced lung toxicity in our series was similar to those reported in the literature. HRCT allowed for identification of lung alteration in an asymptomatic phase and can therefore be considered a valid instrument to support clinical management of oncologic patients treated with gemcitabine.

\section{B-278 15:03}

Assessment of the relationship between morphological emphysema phenotype and corresponding pulmonary perfusion pattern on a segmental level

M.G.H. Bryant, S. Ley, R. Eberhardt, F. Herth, C. Heussel, H.-U. Kauczor, J. Ley-Zaporozhan; Heidelberg/DE (mghbryant@gmail.com)

Purpose: The hypothesis of this study was that distinct morphological emphysema phenotypes assessed by CT show characteristic perfusion defect pattern.

Methods and Materials: Forty-one patients with severe (GOLD III or IV) emphysema underwent inspiratory 3D-HRCT and contrast-enhanced MR-perfusion (1.5 T spatial resolution $3.5 \mathrm{~mm} \times 1.9 \mathrm{~mm} \times 4 \mathrm{~mm}$ ). 3D-HRCT data were visually analysed in consensus by 3 experts in chest radiology, for phenotyping and quantification of emphysema. The predominant phenotype per segment was categorised as normal, centrilobular, panlobular or paraseptal. Segmental lung perfusion was visually analysed using 6 patterns of pulmonary perfusion (1 - normal, 2 - mild homogenous reduction in perfusion, 3 -inhomogenous perfusion without focal defects, 4 - inhomogenous perfusion with focal defects, 5 - inhomogenous absense of perfusion, 6 - homogenous absence of perfusion), the extent of the defect given as a percentage.

Results: A total of 730 segments were evaluated. CT phenotyping categorised 566 $(78 \%)$ as centrilobular, $159(22 \%)$ as panlobular and $5(<1 \%)$ as paraseptal with no normals. Scores with regard to MR perfusion patterns: 1 - 0; 2 - 0; 3 - 28 (4\%); 4 - 425 (58\%); 5 - 169 (23\%); 6 - 108 (15\%). The predominant perfusion pattern matched as follows: $70 \%$ centrilobular emphysema - inhomogenous perfusion with focal defects (score 4); $42 \%$ panlobular - homogenous absense of perfusion (score 6 ) and $43 \%$ panlobular - inhomogenous absense of perfusion (score 5). Conclusion: MR pulmonary perfusion pattern correlates with the CT phenotype at a segmental level in patients with severe emphysema.

\section{B-279 15:12}

Inspiratory and expiratory MDCT (multidetector computed tomography) scans: automatic airways analysis in patients with chronic obstructive pulmonary disease (COPD)

M. Amato, A.R. Larici, A. del Ciello, R. Silvestri, M. Occhipinti, P. Franchi, A. Contegiacomo, L. Bonomo; Rome/IT (michele.amato81@gmail.com)

Purpose: To assess the role of MDCT with automated measurement of airways in the quantification of airflow obstruction in inspiratory and expiratory scans in patients with COPD.

Methods and Materials: 20 patients (age range $48-85$ years) with clinical diagnosis of COPD prospectively underwent pulmonary functional tests (PFTs) and chest CT scan. All CT exams were performed with a 64-rows scanner (slice thickness/ interval $0.625 \mathrm{~mm}$ ). Two consecutive acquisitions were obtained, one standard dose full inspiration scan followed by one low-dose $(20 \mathrm{mAs})$ scan at the end of forced 
expiration. Datasets were analysed using an automated commercial software for airways analysis (Thoracic VCAR, GE Healthcare). One chest radiologist reported morphologic airway parameters, automatically calculated from lobar (second generation) to sub-subsegmental bronchi (fifth generation: diameter $<4 \mathrm{~mm}$ ) on both inspiratory and expiratory scans: lumen diameter (LD), lumen area (LA), wall thickness (WT), wall area (WA), wall area ratio (WA\%: wall area/total bronchial area\%). Ratio between mean values of all automatic measurements obtained in inspiratory and expiratory scans were correlated with PFTs for each patient and each anatomic level (Pearson correlation coefficient).

Results: The best correlation ( $p=0.04 ; p=0.08$ ) was observed between functional parameters of airflow obstruction $\left(\mathrm{FEV}_{1}, \mathrm{FEV} / \mathrm{FVC}\right)$ and the ratio between mean values of $L A$ in expiration and mean values of $L A$ in inspiration $\left(L A_{\text {exp }} / L A_{\text {insp }}\right)$, at the level of the fifth bronchial generation.

Conclusion: Dynamic modifications of distal airways lumen area correlate with functional parameters indicative of airflow obstruction. MDCT with automatic measurement of the airway parameters may have a role in quantification of airflow obstruction in COPD patients.

\section{B-280 15:21}

CT quantitative measurements correlated with pulmonary function tests in chronic obstructive pulmonary disease: a meta-analysis

X. Xie, R. Vliegenthart Proença, Y. Wang, G.H. de Bock, M. Oudkerk;

Groningen/NL (x.xueqian@rad.umcg.nl)

Purpose: To determine the relationship between CT quantitative emphysema or small airway measurements and pulmonary function test (PFT) in chronic obstructive pulmonary disease (COPD).

Methods and Materials: Web of Science, PUBMED, EMBASE and Cochrane Central were searched for studies before September 2010. Out of 1386 citations, 18 articles were included in the meta-analysis according to pre-defined inclusion criteria. Attenuation area percentage < -950 HU (\%LAA-950), mean lung density (MLD) and wall area percentage (WA\%) were considered as CT quantitative parameters. The predicted forced expiratory volume in the first second (FEV1, \%p), and FEV1 divided by the forced volume vital capacity (FEV1/FVC) were considered as reference PFT parameters. Data were independently evaluated and abstracted with a standardized protocol and abstraction form. Pooled correlation coefficients as well as $95 \%$ confidence intervals $(95 \% \mathrm{Cl})$ were calculated in a meta-analysis using a random effect model.

Results: The 18 studies included 2406 participants. The pooled correlation coefficient was -0.42 (95\%Cl: $-0.49,-0.35)$ for \%LAA-950 and FEV1,\%p; -0.68 (-0.77,$0.57)$ for \%LAA-950 and FEV1/FVC; $0.46(0.23 .0 .64)$ for MLD and FEV1,\%p; $0.66(0.52,0.77)$ for MLD and FEV1/FVC; and $-0.46(-0.58,-0.33)$ for WA\% and FEV1,\%p.

Conclusion: In this meta-analysis, CT quantitative emphysema and small airway measurements had moderate correlations with lung function tests in COPD patients. The strongest association was found between CT-derived lung density measurements and FEV1/FVC

$$
14: 00-15: 30
$$
Room D2

\section{Interventional Radiology}

\section{SS 509}

\section{US, MR and CT guided interventions}

Moderators:

G. Goh; London/UK

R.W. Günther; Aachen/DE

\section{B-281 14:00}

Effectiveness of transforaminal and interlaminar epidural steroid injection under CT guidance: a prospective randomised controlled study

A. Fontaine ${ }^{1}$, D. Hoa ${ }^{1}, \mathrm{~N}$. Amoretti ${ }^{2} ;{ }^{1}$ Montpellier/FR, ${ }^{2}$ Nice/FR (annaudrey2000@yahoo.fr)

Purpose: To prospectively assess short- and long-term effectiveness of both transforaminal and interlaminar steroïd injections under CT guidance for lumbosacral radiculopathy compared with a single transforaminal approach.

Methods and Materials: Institutional review board approval and written informed consent were obtained. From December 2009 to April 2010, 112 consecutive patients ( 41 males, 71 females; mean age 57 years; range $18-90$ years) who were scheduled to undergo lumbar steroïd injection were enrolled. The patients were randomly assigned to either group 1 (both transforaminal and interlaminar injec- tions in the same time) or goup 2 (single transforaminal injection). Follow-up was conducted within 7 days and one month (short-term) and one year (long-term). Short- and long-term outcomes were measured using a visual analog scale (VAS) and the Oswestry Disability Index. Univariate analysis (using Fisher exact test and $\lambda 2$ tests) and multiple logistic regression analysis were performed to evaluate the relationship between possible outcome predictors and the therapeutic effect. Results: Significant pain relief (> 50\%) was seen at one month in $49 \%$ of patients in group 1 and $43 \%$ in group 2. Significant differences were noted in pain relief characterictics at one month between groups 1 and 2 in patients presenting a disc extrusion or a posterolateral herniated disc. The only significant outcome predictor at short-term follow-up was previous history of lumbar surgery. No major complication was noted at long-term follow-up.

Conclusion: Association of transforaminal and interlaminar steroïd lumbar injection under CT guidance is more effective than single transforaminal injection.

\section{B-282 14:09}

May CT-guided bone biopsy be considered helpful in advanced breast cancer (ABC) management?

M. Padrenostro, P. Della Vigna, L. Monfardini, S. Rizzo, G. Aurilio, L. Preda,

M. Bellomi; Milan/IT (mpadrenostro@libero.it)

Purpose: Biopsies of metastatic sites are usually not recommended to evaluate changes in biological features in women with $A B C$. We assessed discordant expression of estrogen receptor $(E R)$, progesterone receptor $(\mathrm{PgR})$ and epidermal growth factor receptor 2 (HER2), between primary tumour and bone metastases and its clinical impact on patients' management.

Methods and Materials: ABC patients who underwent CT-guided bone biopsy from 1997 to 2009 were included. Data on receptors status of primary tumour and bone metastases were collected and analysed.

Results: 109 patients were enrolled. Median time from primary diagnosis to bone biopsy was 4.2 years. Overall discordance rate (ODR) in ER expression was $20.5 \%: 3 / 7$ pts $(42.9 \%)$ shifted from negative to positive; $19 / 100$ pts $(19 \%)$ from positive to negative $(p=0.0006)$. ODR in PgR expression was $43.9 \%: 4 / 21$ pts (19\%) changed from PgR negative to positive and $43 / 86$ pts $(50 \%)$ from positive to negative $(p<0.0001)$. ODR in HER2 status was $6.9 \%$ : $4 / 78$ pts $(5.1 \%)$ changed from HER2 negative to positive and $2 / 8$ ts $(25 \%)$ from positive to negative $(p=$ $0.41)$. According to the ER status, $13 / 21$ pts $(61.9 \%)$ changed endocrine therapy when discordant and $23 / 77$ pts $(29.7 \%)$ when concordant $(p=0.01)$. According to the HER2 status, $4 / 6$ pts $(66.6 \%)$ changed immunologic therapy in discordan status and $6 / 72$ pts $(8.3 \%)$ in concordant status $(p=0.002)$. All patients well tolerated the bone biopsy.

Conclusion: Receptors profile may significantly change between primary breast cancer and bone metastases, with relevant impact on therapy. CT-guided bone biopsy may therefore be considered a safe procedure to optimise therapeutic choices.

\section{B-283 14:18}

Complications of CT-guided transgluteal pelvic abscess drainage with catheter placement

L. Zheng, M. Kataoka, A. Roycjowdhury, J. Ferrucci; Worcester, MA/US (larry.zheng@umassmemorial.org)

Purpose: To assess complications of CT-guided percutaneous drainage cathete of deep pelvic abscess by transgluteal approach in a teaching medical centre. Methods and Materials: 101 cases of CT-guided pelvic abscess drainage with catheter placement by the transgluteal approach performed in our institution from 1, 2006 up to 3, 2010 were reviewed. All the records were reviewed for clinical diagnosis, catheter size and follow-up. The CT images were evaluated for signs of inflammation at the procedure site, angle/location of the approach, technique of catheter placement (Trocar vs Seldinger) and numbers of attempts of catheter placement. Approaches were classified as parasacral or non-parasacral route. Results: Patient age ranged from 4 to 94 years (average 50 years; 69 females and 32 males). The majority of our patients had inflammation or vascular congestion $(76 \%)$ in the pelvis. The catheter was placed using the parasacral in 87 cases and non-parasacral approach in 14 cases. Seldinger technique was used in 66 cases and Trocar technique in 35 cases. The size of the catheter used varied from 6 to $14 \mathrm{Fr}$. The number of the punctures varied from 1 to 5 (average 1.4). Complications were seen in 8 cases $(8 \%)$ including 3 pseudoaneurysm (all done with nonparasacral approach), 3 haematomas $(2<3 \mathrm{~cm}$ and $1>5 \mathrm{~cm}$ ) and 2 fistulas. All complications occurred with theTrocar technique.

Conclusion: Complications of CT-guided transgluteal approach for pelvic abscess drainage are more common with non-parasacral approach and Trocar technique in our experience. 


\section{B-284 14:27 \\ Feasibility of CT-guided percutaneous fiducial marker placement for stereotactic radio-surgery of inoperable pancreatic cancers G.J. Mallarajapatna, N. Ramanna, S. PS, K. Swamy, A. BS; Bangalore/IN (revathigovind@gmail.com)}

Purpose: To assess CT scan as a guiding tool for percutaneous placement of internal fiducial markers for stereotactic radio-surgery of inoperable pancreatic cancers. Methods and Materials: Consecutive 31 patients with inoperable pancreatic cancer were treated with stereotactic radio-surgery at our institute in the last 12 months. CT scan was used as a guiding tool for placement of internal gold fiducial markers to track the translational and rotational movements of the organ during treatment. A total of 148 fiducials were placed. At least 4 fiducials each (not more than 5) were placed in and around the tumour in all patients in non-co-linear manner with at least $2 \mathrm{~cm}$ distance between any two fiducials.

Results: Stomach, small bowel, colon and liver were traversed in 22, 1, 1 and 2 patients, respectively. No major complications such as pancreatitis, significant haemorrhage or sepsis occured in any patient. Small self limiting focal haematomas occurred in 4 patients. A minority of fiducials (17 i.e. $12 \%$ ) were not usable of tracking due to either migration or improper placement.

Conclusion: CT scan guidance is accurate and safe for percutaneous fiducial placement in and around the pancreatic tumours. At least 4 fiducials (three are necessary for tracking) are necessary for each lesion, as migration of fiducials is unpredictable, sometimes rendering them useless for tracking. Thorough knowledge of principles of fiducial placement is necessary. Access routes and methods are similar to CT-guided pancreatic biopsies but may need more punctures. Antibiotic coverage is recommended when traversing colon is inevitable.

\section{B-285 14:36 \\ Ultrasound-guided aspiration and ethanol sclerotherapy for the treatment of endometriotic cysts \\ G. Gatta, V. Parlato, G. Di Grezia, A. Porto, R. Grassi, A. Rotondo; Naples/lT (graziella.digrezia@yahoo.it)}

Purpose: To check the effectiveness of ultrasound-guided aspiration and ethanol sclerotheraphy in treating endometriotic cysts and adopt this procedure as an alternative to surgery.

Methods and Materials: 50 consecutive patients with an average age of 25.2 years (SD 6.5; range 16-14 years) diagnosed with endometriotic cyst through ultrasonography, who were pregnant or presenting with highly anaesthesiologic risk, adherences, refusal of surgery, underwent ultrasound-guided aspiration of 54 endometriotic cysts ( 40 cysts with a transabdominal approach and the remaining 14 with a transvaginal one) and ethanol sclerotherapy.

Results: The procedure was successful in all patients. Imaging check-ups were carried out after a span period of: $12 / 24$ hours, 15 days and $3 / 6 / 12$ months. After 12 months, 4 of our patients ( $8 \%$ ) showed recurrence. 3 of them opted for a second session of ultrasound-guided aspiration and ethanol sclerotherapy.

Conclusion: Ultrasound-guided aspiration and sclerotherapy with $95^{\circ}$ ethanol provide a valid alternative to surgery in treating endometriotic cysts.

\section{B-286 14:45}

Ultrasound-guided fine needle aspiration biopsy: does cytopreparatory

technique influence specimen adequacy?

A.M. Scaranelo, B. Lord, W. Geddie; Toronto, ON/CA

(anabel.scaranelo@gmail.com)

Purpose: To evaluate the specimen adequacy rate of ultrasound-guided fine needle aspiration (FNA) biopsies performed by the same operator, with and without the use of liquid-based cytology preparations (LBP), in two cohorts of consecutive patients. Methods and Materials: During a four-year period, 2,517 consecutive FNA biopsies were performed in a tertiary hospital and evaluated without liquid-based cytology preparations using direct smears only. In a separate institution, over a three-year period 283 consecutive FNA biopsies were evaluated with LBP only. Ultrasoundguided FNA biopsies performed by the same operator at the two institutions were compared. McNemar's test was used to determine whether the adequacy rate obtained with the two techniques was significantly different.

Results: Adequate cytopathologic specimens were obtained for $98 \%$ (355 of 369) of the nodules biopsied under sonographic guidance and assessed using smears, and for $79 \%$ (221 of 279 ) of the nodules sampled under sonographic guidance and assessed using LBP $(p<0.01)$. The overall rate of inadequate cellularity for USguided FNAs was $1.6 \%$ using smears and $20.7 \%$ for US-guided FNAs using LBP. Conclusion: In two series of fine needle biopsies performed by the same operator with the same technique there was a higher inadequacy rate when liquid-based cytopreparatory technique was used. The use of direct smears for ultrasoundguided biopsies may impact the clinical utility of this interventional procedure. Further studies addressing optimisation and pre-analytic standardisation of fine needle biopsy are necessary.

\section{B-287 14:54}

Ultrasound-guided versus computed tomography-controlled pararadicular injections in the lumbar spine: a prospective randomised clinical trial A. Loizides, S. Peer, S. Ostermann, J. Obernauer, K. Galiano, H. Gruber; Innsbruck/AT (alexander.loizides@i-med.ac.at)

Purpose: Pararadicular injections are widely used for alleviation of back pain Injections are preferentially performed as fluoroscopy or computed tomography (CT)-controlled interventions. Ultrasound provides real-time monitoring, does not produce ionising radiation, and is broadly available. This study was performed to evaluate the overall effect of ultrasound-guided pararadicular injections.

Methods and Materials: We studied feasibility, accuracy, time-savings, radiation doses, and pain relief of ultrasound-guided pararadicular injections versus CT-controlled interventions in a prospective randomised clinical trial. 22 adult patients with chronic low back pain were consecutively enrolled and assigned to an ultrasound or a CT- group. Results: 12 subjects from the group randomised to ultrasound were judged (exclusion criteria: $\mathrm{BMl}>35$ + postsurgical state) to be feasible for this type of approach. In 12 patients of the ultrasound group the intertransverse ligament was clearly visible and all of the associated pararadicular injections were performed correctly. The mean duration of procedure and mean radiation dose was 4.82 minutes and 26.73 mGy.cm in the ultrasound group, and 6.9 minutes and 63.57 mGy.cm in the CT group. Both groups showed a benefit from pararadicular injections.

Conclusion: These initial results which will be evaluated in a larger study show that the ultrasound approach to the pararadicular compartment in the lumbar spine is feasible with minimal risks in a large majority of patients and results in a significant reduction of procedure duration and radiation dose.

\section{B-288 15:03}

Image-guided biopsy of pleuric and peripheral lung lesions: comparison between ultrasound (US) and computed tomography (CT) guidance

C. Murolo ${ }^{1}$, G. Serafini ${ }^{2}$, E. Savarino ${ }^{1}$, L.M. Sconfienza ${ }^{3} ;{ }^{1}$ Genoa/IT, ${ }^{2}$ Pietra Ligure/IT, ${ }^{3}$ Milan/IT (io@lucasconfienza.it)

Purpose: Image-guided biopsy represents the reference standard in the characterisation of pleuric and peripheral lung lesions that can be sampled under CT or US guidance. Our purpose was to compare the outcome of CT or US guidance when sampling peripheral lung or pleuric lesions.

Methods and Materials: From 1/ 2000 to 8/2010, 711 thoracic biopsies were performed at our institution. Among these, 284 lesions had pleuric origin or had a peripheral location in the lung with at least a small contact with the pleura. These lesions were biopsied either under CT (179 lesions; 170 patients, 71/99 males/ females, mean age $64 \pm 12.5$ years) or US guidance (105 lesions; 103 patients, $44 / 59,67 \pm 9.9$ years), according to location of the lesion and patients' general conditions, using a 23G modified-Menghini needle. For each biopsy, duration of the procedure, occurrence of post-procedural pneumothorax, and sample adequateness were recorded. Chi-square and U-Mann-Whitney statistics were used.

Results: No statistical difference was found for sex and age distribution $(p>0.544)$. CT-guided biopsies mean time was $588 \pm 175 \mathrm{~s}$, while US-guided biopsies mean time was $445 \pm 156 \mathrm{~s}(\mathrm{p}<0.001)$. Post-procedural pneumothorax was observed in $44 / 170$ patients (25.9\%) biopsied under CT guidance and in $25 / 103$ (24.3\%) biopsied under US guidance $(p=0.489)$. Adequate samples were obtained in 172/179 lesions (96.1\%) under CT guidance and in 101/105 (96.2\%) under US guidance. Conclusion: When dealing with pleuric or peripheral lung lesions, US guidance is comparable to CT in terms of sample accuracy and post-procedure pneumothorax, while allows for reducing significantly time of execution, being also free from ionising radiations.

\section{B-289 15:12}

Unresectable liver metastases of colorectal carcinoma: repeated transarterial chemoembolisation using different chemotherapeutic drug combinations followed by MR-guided laser-induced thermotherapy T.J. Vogl, N.N. Naguib, N.E. Nour Eldin, M.G. Mack, S. Zangos, A. Jost; Frankfurt a. Main/DE (t.vogl@em.uni-frankfurt.de)

Purpose: To evaluate a treatment protocol with repeated transarterial chemoembolisation (TACE) downstaging before MR-guided laser-induced interstitial thermotherapy (LITT) using different chemotherapeutic drug combinations in patients with primarily unresectable colorectal liver metastases. 
Methods and Materials: 224 patients were included in the current prospective study. TACE (mean 3.4 sessions/patient, SD 1.33, range 1-10) was performed as a downstaging treatment to meet the previously described LITT requirements (number of metastases $<5$, diameter of each metastasis $<5 \mathrm{~cm}$ ). The intraarterial protocol consisted of Irinotecan and Mitomycin (77 patients), Gemcitabine and Mitomycin (49 patients) or Mitomycin alone (98 patients) in addition to Lipiodol and Embocept in all patients. Post TACE, all patients included underwent MR-guided LITT (mean 2.2 sessions/patient).

Results: Overall, TACE resulted in a mean reduction in diameter of the target lesions of $21.4 \%$. The mean time to progression was 5 months, the mean local tumor control rate was 10.7 months, both calculated as of therapy completion. Calculated from the beginning of the TACE therapy, mean survival rate in the patients was 26.1 months (SD 16.01, range 4-110 months), in patients treated with Irinotecan and Mitomycin 24.17 months, in patients treated with Gemcitabine and Mitomycin 26.9 months and in patients treated with Mitomycin only 27.24 months with a statistically significant difference between the groups $(p<0.01)$.

Conclusion: Repeated TACE offers adequate downstaging of liver metastases of colorectal carcinoma to allow MR-guided LITT. The combined treatment illustrates substantial survival rates and high local tumour control with statistically significant differences between the three different chemotherapeutic drug combinations used.

\section{B-290 15:21}

Can real-time US-CT/MRI fusion imaging guidance enable ablation of liver malignancies that are undetectable with conventional US?

L. Solbiati ${ }^{1}$, T. Ierace ${ }^{1}$, L. Cova ${ }^{1}$, T. Tondolo ${ }^{2}$, N. Goldberg ${ }^{3} ;{ }^{1}$ Busto Arsizio/IT,

${ }^{2}$ Segrate/IT, ${ }^{3}$ Jerusalem/IL (Isolbiati@aobusto.it)

Purpose: To assess the ability of real-time US-CT/MRI fusion imaging for guiding percutaneous ablation of liver malignancies undetectable with US.

Methods and Materials: From 2003 to 2009, 334 patients with 884 focal liver tumours ( $544 \mathrm{HCCs}$ and 340 metastases) underwent percutaneous radiofrequency or microwave ablation guided by a image fusion system that combines real-time US with fusion to CT/MRI images based upon magnetic field tracking (Esaote, Genoa, Italy). Of these, $85 \mathrm{HCCs}$ and 68 metastases $(\mathrm{N}=153$ [17.3\%]) in 67 patients were detectable only with contrast-enhanced CT or MRI, but undetectable with US due to small size $(n=66)$, isoechogenicity with liver parenchyma $(n=46)$, obscuration due to location $(n=41) .138 / 153(90.2 \%)$ tumours were smaller than $2.0 \mathrm{~cm}$ and $58 / 153(37.9 \%)$ smaller than $1 \mathrm{~cm}$ (mean $1.3 \mathrm{~cm}$ ). CT or MRI was performed at 24 $\mathrm{hr}$ after ablation to assess the technical efficacy (completeness of ablation including a $5 \mathrm{~mm}$ ablative margin) and at 8 months to search for possible local tumour progression (clinical efficacy).

Results: At 24-hr follow-up imaging, 140/153 (91.5\%) malignancies were completely ablated; 4 (2.6\%) were partially ablated and 9 targets $(5.9 \%)$ were completely missed. The four partially ablated nodules were successfully re-treated at $28-40$ days. At 8-month follow-up, local tumour progression was detected in $27 / 144$ (18.7\%) tumours (11 HCCs and 16 metastases). Thus, overall efficacy was $91.5 \%$ per tumour and $86.6 \%$ per patient.

Conclusion: A real-time fusion imaging system enables sufficiently precise targeting of many tumours undetectable with US alone to achieve complete ablation in the majority of cases.

\section{4:00 - 15:30}

Room E1

\section{Neuro}

\section{SS 511a}

\section{Imaging in stroke}

\section{Moderators:}

J. Fiehler; Hamburg/DE

J. Frühwald-Pallamar; Vienna/AT

\section{B-291 14:00}

Order of CT stroke protocol (CTA before or after CTP): impact on image quality?

F. Dorn ${ }^{1}$, T. Liebig' ${ }^{2}$, D. Muenzel ${ }^{1}$, R. Meier ${ }^{1}$, H. Poppert ${ }^{1}$, E.J. Rummeny ${ }^{1}$

A. Huber ${ }^{1} ;{ }^{1}$ Munich/DE, ${ }^{2}$ Cologne/DE (dorn@roe.med.tum.de)

Purpose: To evaluate if the order of a CT stroke protocol (CT-perfusion before or after CT-angiography) has an impact on image quality.

Methods and Material: 40 consecutive patients with clinical suspicion of brain infarction underwent CT stroke protocol including non-enhanced CT, CT angiography (CTA) and CT perfusion (CTP). Twenty examinations were performed with CTP before CTA (group 1), 20 in reversed order (group 2). Mean density (Houndsfield Units, $\mathrm{HU}$ ) was determined at baseline and peak enhancement of CTP, as well as on source-images of CTA in distinct regions (basilar/ middle cerebral artery, transverse and superior sagittal sinus, grey/ white matter (GM/WM)). Contrast of extra-/intracranial arteries and veins was rated according to a 5-point-scale $1=$ excellent, 5 = poor). CTP maps were assessed by determining mean transit time (MTT), cerebral blood flow (CBF) and blood volume (CBV) in identical regions. Results: HU between groups 1 and 2 were not significantly different for CTA and CTP at peak enhancement. At CTP baseline, $\mathrm{HU}$ between group both groups were different for all points except for GM and WM. There was no significant difference between both groups for the mean delta (difference between baseline and peak enhancement), as well as for MTT, CBV and CBF. Quality of CTA was rated to be superior in group 2 concerning extracranial venous contrast; however, not for all other criteria. Conclusion: Reversal of CT stroke protocol had no significant influence on quantitative parameters of the CTP. Quality of CTA concerning extracranial venous contrast was superior when CTA was performed before CTP.

B-292 14:09

Whole brain CT perfusion maps with paradoxical low mean transit time to predict infarct core

S. Chakraborty, M.E. Ahmad, R. Glikstein, M. Hogan, D. Dowlatshahi, G. Stotts; Ottawa,ON/CA (santanoo@gmail.com)

Purpose: CT perfusion (CTP) is based upon central volume technique (CBF = CBV/MTT) and uses complex deconvulotional method to generate various perfusion maps. Traditionally, low CBV values are indicative of infarct core and low CBF with high MTT/ TTP is used to describe ischaemic penumbra. The paradoxical low MTT in the region of ischaemia has not described in the current CTP literature to best of our knowledge.

Methods and Materials: We retrospectively reviewed the clinical and whole brain perfusion data of 134 patients presenting with acute stroke in less than 6 hours from onset of symptoms over 18 months. 22 patients with low MTT in the region of ischaemia were further evaluated for clinical and imaging outcome.

Results: Out of 134 patients, 41 were excluded due to inadequate studies ( $\mathrm{n}=$ 14) and no follow-up scans $(n=27) .43$ of remaining 93 patients had obvious abnormality on MTT perfusion maps. 22 of these 43 patients showed low MTT (colour coded as blue on RGB scale) in the centre of otherwise high MTT (red on RGB scale) area. Overall, MTT map has lower sensitivity (71\%) for the detection of ischaemia and paradoxical low MTT sign has even lower sensitivity, seen only in $36 \%$ of cases. However, this has very high specificity and all 22 patients with low MTT developed infarct in the same region on follow-up imaging. However, the final volumes of infarct were approximately $20 \%$ bigger.

Conclusion: Low MTT in the region of ischaemia is an interesting finding that accurately predicts the infarct core.

\section{B-293 14:18}

Perfusion CT in stroke imaging: influence of post-processing

M.M. Lell' ${ }^{1}$, B. Abels ${ }^{1}$, E. Klotz ${ }^{2}$, B. Tomandl ${ }^{3}$, M. Uder ${ }^{1} ;{ }^{1}$ Erlangen/DE,

${ }^{2}$ Forchheim/DE, ${ }^{3}$ Bremen/DE (michael.lell@uk-erlangen.de)

Purpose: There is an ongoing discussion about the appropriateness slope (MS) or deconvolution (DC) approach for post-processing of perfusion CT data in stroke imaging. We performed a qualitative and quantitative comparison of DC and MS using identical source data sets and pre-processing parameters.

Methods and Materials: 50 data sets were used to calculate colour maps of cerebral blood flow (CBF), cerebral blood volume (CBV), and various temporal parameters with software implementing DC and MS algorithms. Colour maps were qualitatively rated/categorised. Quantitative region-of-interest-based measurements were made in non-ischaemic grey and white matter, suspected penumbra and suspected infarction core. Qualitative results, quantitative results as well as PCT lesion sizes from DC and MS were statistically compared.

Results: CBF and CBV colour maps based on DC and MS were of comparably high quality. Quantitative CBF and CBV values calculated by DC and MS were within the same range in non-ischaemic regions. In the suspected penumbra, average CBF (DC) values were lower than CBF (MS). In the suspected infarction core, average CBV (DC) was similar to CBV (MS). Using adapted tissue-at-risk/non-viable-tissue thresholds we found excellent correlation of DC and MS lesion sizes.

Conclusion: DC and MS yielded comparable qualitative and quantitative results. Lesion sizes indicated by DC and MS showed excellent agreement when using adapted thresholds. 


\section{B-294 14:27}

Evaluation of a novel post-processing filter of native CT images for detection of early ischemic stroke: improved differentiation of early infarct core

A. Kemmling, L. Feyen, J. Minnerup, V. Hesselmann, T. Niederstadt, W. Heindel, H. Seifarth; Münster/DE (akemmling@web.de)

Purpose: In non-enhanced CT identification of early infarct in acute ischaemic stroke is limited. This study evaluates the use of a novel post-processing filter for native $\mathrm{CT}$ images to detect early ischaemic infarct.

Methods and Materials: Non-enhanced CT (NE-CT) was performed in 25 patients with acute $(<6$ hours) middle cerebral arterial (MCA) stroke. Standard NE-CT images were processed using an novel edge-contrast enhancement filter (Neuro Best, Siemens). Standard and Neuro Best enhanced scans were evaluated for parenchymal hypoattenuation and loss of gray white-matter distinction (1-5 scale; definite absence to definite presence). Infarct lesions were segmented slice-byslice (fixed window, 10 to $80 \mathrm{HU}$ ). Infarct core in admission cerebral blood flow CT perfusion maps and follow-up infarct in NE-CT were segmented. All CT scans and lesion maps were co-registered (Analyze 9.0, AnalyzeDirect). Voxels in standard and Neuro Best CT images were defined as "true positive" (TP), "true negative" (TN), "false positive" (FP) or "false-negative" (FN) by comparison with reference lesion maps (follow-up CT and CTP). Sensitivity (TP/TP + FN) and specificity (TN/ $\mathrm{TN}+\mathrm{FP}$ ) for detection of infarct core was calculated.

Results: With standard NE-CT, sensitivity and specificity for stroke detection were $67 \%$ and $98 \%$. Sensitivity increased to $75 \%$ using Neuro Best CT images with slight loss of specificity $(96 \%)$. Receiver operating characteristic analysis of overall image rating revealed an improvement in detecting infarcted tissue using Neuro Best CT post-processing $(p=0.02)$.

Conclusion: In acute ischaemic stroke, detection of early infarct in NE-CT is facilitated using a novel edge-contrast enhancement filter (Neuro Best).

\section{B-295 14:36}

\section{Prediction of malignant middle cerebral artery infarction by a rater} independent model based on normalised stroke oedema and subarachnoid reserve volume

A. Kemmling, L. Feyen, T. Duning, T. Niederstadt, W. Heindel, J. Minnerup; Münster/DE (akemmling@web.de)

Purpose: Early identification of patients developing malignant ischaemic stroke is crucial. The purpose of this study was to evaluate rater-independent parameters based on precise anatomical volume measurements of infarct, cerebrospinal fluid reserve volume and midline-shift to quantify the risk of malignant infarction.

Methods and Materials: $\ln 25$ consecutive cases with acute proximal middle cerebral arterial occlusion, CT was used to determine cerebrospinal fluid (CSF) reserve volume in the healthy hemisphere normalised to intracranial volume (IV): after automated skull-stripping and thresholding ( $\mathrm{HU}$ range -10 to 20) of CT images, CSF volume was calculated and normalised to IV ( $\mathrm{nCSF}=$ volume of CSF/IV). Volume of infarct at admission (reduced CBV in CT perfusion) and stroke volumes (SV) at maximum infarction prior to decompression or death were segmented (Analyze 9.0) and normalised to IV (nSV = SV/IV). Herniation was quantified by volume of midline shift (MSV) normalised to IV (nMSV = MSV/IV). The ratio of $n S V / n C S F$ was correlated with nMSV. A voxel-wise probability map of midline-shift, strokevolume, and CSF in MNI-152 space was created. The cut-off value of nSV/nCSF for malignant infarction was determined by ROC curve analysis.

Results: The ratio of $n S V / n C S F$ significantly correlated with nMSV $(r=0.836$, $p<0.001$ ). The cut-off value for malignant infarction was 1.7 (sensitivity $92.3 \%$, specificity $90.9 \%$ ). The voxel-wise probability map in $\mathrm{MNI}-152$ space allows visual estimation of nMSV with a priori knowledge of nCSF and nSV.

Conclusion: The risk of malignant infarction highly correlates with rater independent parameters of volume of midline-shift and ratio of normalised infarct-volume and CSF reserve volume.

\section{B-296 14:45}

Dual energy computed tomography: the value of the virtual nonenhanced brain images in the assessment of acute stroke

J. Ferda, J. Baxa, H. Mírka, E. Ferdova, B. Kreuzberg; Plzen/CZ (ferda@fnplzen.cz)

Purpose: To compare virtual nonenhanced images created on the base of the dual energy CTA brain images with conventional nonenhanced images in patients with suspected stroke.

Methods and Materials: The nonslected group of 200 consecutive patients (113 males and 87 females) with the symptoms suspected from acute ischaemic stroke underwent dual energy $\mathrm{CT}$ angiography after intravenous application contrast mate- rial (iomeprol, $400 \mathrm{mgl} / \mathrm{ml}, 80 \mathrm{ml}, 4 \mathrm{ml} / \mathrm{s}$ ). The simultaneous exposition of $140 \mathrm{kV}$ and $80 \mathrm{kV}$ was used with the collimation of $2 \times 32 \times 0.6 \mathrm{~mm}$ of both system. The virtual nonenhanced images were compared with the "real" nonenhanced images previously acquired by conventional way. The radiologist blinded to the findings of the real images describe findings of the $\mathrm{CT}$ angiography including the evaluation of virtual nonenhanced images of $6 \mathrm{~mm}$ thickness were created with the threematerial decomposition algorithm called brain haemorrhage.

Results: The following findings were found on "real" noncontrast images: no hypodense area (112x) cases, territorial hypodense area (73x), intracranial bleeding (7x), space occupying masses (6x), extensively calcified tissue (2x). All findings were found on virtual nonenhanced images even if the image quality was decreased according higher noise level. The additional value of the vascular structures and masses enhancement was found in improved depiction of the hypodense area and the enhancement pattern of expansive processes.

Conclusion: In the nonselected population of the patients with clinically suspected stroke, the conventional nonenhanced CT images could be omitted without the risk of missed important finding. This approach could reasonably decrease the radiation dose burden to the patient

\section{B-297 14:54}

Comparison of susceptibility weighted imaging and dynamic susceptibility contrast MRI in patients with acute cerebral stroke

Y. Han, M. Wang, D. Shi, Y. Li; Zhengzhou/CN (hyh_yafeng@yahoo.cn)

Purpose: To compare susceptibility weighted imaging (SWI) with dynamic susceptibility contrast (DSC) MR imaging for evaluation of acute cerebral ischaemia at 3.0 T. Methods and Materials: A high-resolution SWI and DSC were both performed with a 3.0 T MRI on 34 patients with acute ischzemic stroke within 3 days of symptom onset. For qualitative analysis, MR findings of DSC were grouped into four categories (hyperperfusion, normal perfusion, delayed perfusion, or absent perfusion), while SWI was evaluated according to the changes of signal intensity. Three regions of interest (ROI) were defined by diffusion-weighted imaging abnormalities and mirror regions in the contralateral normal brain tissues, respectively. Signal intensity ratios (infracted region versus the normal contralateral region) were calculated and compared using a paired $t$ test.

Results: SWI showed vein dilatation in affected area in 26 of 34 patients, which were confirmed by DSC with hypoperfusion. In 8 cases, SWI showed norma whlie DSC showed delayed perfusion $(n=2)$, hyperfusion $(n=3)$ or perfusion deficit $(n=3)$. The signal intensity ratio on DSC was $0.82 \pm 0.23$, while SWI was $0.91 \pm 0.26$. Both qualitative and quantitative analyses demonstrated that there were no significant difference between the two techniques $(P>0.05)$. In addition, SWI revealed haemorrhage within ischaemia in 8 cases which were not showed by routine MRI and DSC.

Conclusion: The high-resolution SWI was sensitive in detecting cerebral ischaemic lesions and evaluating cerebral haemodynamics of decreased diffusion, and can be combined with plain MRI to provide meaningful information in the diagnosis of acute cerebral ischaemia.

\section{B-298 15:03}

Isolated cerebellar infarct: imaging findings, aetiopathogenesis and clinical outcome

A. Zourla, A. Vemmou, S. Papakostas, M. Tsalikis, P. Stavrakas, A. Chalazonitis; Athens/GR (mahizourla@yahoo.gr)

Purpose: To describe computed tomography (CT) and/or magnetic resonance imaging (MRI) findings in patients with isolated cerebellar infarct (ICl) correlated to aetiopathogenetic mechanisms and clinical outcome.

Methods and Materials: From a consecutive series of 568 patients with posterior circulation infarction evaluated prospectively we identified by imaging studies 140 (24.6\%) patients with cerebellum involvement. After extensive study, cases were (a) classified according to the aetiopathogenetic mechanisms: atherosclerosis cardioembolism, infarcts unknown cause (IUC) and other atiologies, (b) categorised based on vessel cerebellum territories and (c) followed up to 5 years regarding mortality and stroke recurrence.

Results: We identified 74/140 (53\%) cases with ICI. Infarcts distribution was: posterior inferior cerebellar artery (PICA) $23(31 \%)$, anterior inferior cerebellar artery (AICA) $8(11 \%)$, superior cerebellar artery (SCA) $37(50 \%)$ and multiple territories in $6(8 \%)$ cases. Haemorrhagic transformation was more common in SCA infarcts $(40.5 \%)$ compared to PICA $(8.7 \%)$ and AICA $(11.1 \%),(p=0.012)$, while brain oedema with mass effect was similar among vascular territories $(11.9 \%, 8.7 \%$, and $11.1 \%$, respectively, $p=0.923$ ). The most common causes were different $(p=$ 0.004 ) among vascular territories: cardioembolism in SCA (60\%), atherosclerosis in AICA (67\%) and in PICA equal cardioembolism and atherosclerosis (34\% and 
$31 \%)$. After a median follow-up of 30 months (interquartile range 20-60), mortality was $47 \%, 16 \%, 11 \%$ for SCA, PICA and AICA respectively, while for the same period recurrence rate was $65 \%, 29 \%$ and $62 \%$, respectively.

Conclusion: Cerebellar infarct vascular distribution is related to different stroke mechanism and radiological features and is associated with different long-term stroke outcome.

\section{B-299 15:12}

Longitudinal study on diffusion tensor imaging and clinical prognosis in brainstem infarction

M. Zhang, J. Lu, D. Rong, Y. Cao, K. Li; Beijing/CN

(zhangmiao_smile@163.com)

Purpose: To explore the evolving pattern of diffusion tensor characteristics in different stages of brainstem infarction and their relationships with clinical prognosis, and the relationship between corticospinal tract injury and motor function recovery. Methods and Materials: DTI examinations were performed on twenty patients at within 7 days, 15 days, 1 month, 3 months and 6 months after their brainstem infarction. The relative fractional anisotropy ( $\mathrm{rFA}$ ) values and relative apparent diffusion coefficient ( $\mathrm{rADC}$ ) values were measured. Motor function was assessed with Fugl-Meyer scores. Three-dimensional reconstructions of corticospinal tract were performed using DTT method. The changes of the rFA values, rADC values in the infarcted areas and their relations to motor function recovery were studied. The relationship between corticospinal tract injury and motor function outcome was also analysed.

Results: The rFA values significantly decreased with time, while the rADC values significantly increased $(p<0.05)$. However, there was no significant correlation between the rFA, rADC values and Fugl-Meyer scores. DTT maps showed that if the corticospinal tract was not compressed, motor function was almost intact; if the corticospinal tract was compressed, curved or slightly disrupted, motor function was impaired but improved in a short time; and if the corticospinal tract was mostly or completely disrupted, then motor function was severely impaired and recovered very slowly.

Conclusion: rFA and rADC values in brainstem infarction areas evolve dynamically and in regular patterns. There are relationships between the extent of corticospinal tract injury and clinical manifestations and motor function recovery, which can greatly help the evaluation of clinical prognosis.

\section{B-300 15:21}

Abciximab does not prevent ischaemic lesions related to cerebral angiography: a randomised placebo-controlled tria

C. Brockmann; Mannheim/DE

Purpose: To assess the efficiency of GB Ilb/Illa platelet receptor inhibition by abciximab in the prevention of silent embolism during DSA by diffusion weighted magnetic resonance imaging (DWI).

Methods and Materials: This randomised, double-blind, prospective study assessed 184 participants with a need for DSA. The participants were monitored by MRI before and after the angiography. DWI was evaluated by two independent neuroradiologists for the occurrence of silent embolism.

Results: Using DWI a total of 105 lesions consistent with silent embolism were counted in 31 patients. Fifteen of $90(16.7 \%)$ patients receiving abciximab and 16 of $94(17.0 \%)$ patients in the placebo group were affected by silent embolism. No significant relationship was found between the two groups and the post-angiographic appearance of silent emboli (Wilcoxon two-sample test: $p$-value 0.9 ).

Conclusion: The present study shows that IIb/llla receptor inhibition by abciximab does not diminish the occurrence of silent infarct during diagnostic catheter angiography. These results challenge the current concept of thrombembolism and air embolism equally contributing to post-angiographic ischaemic cerebral lesions. Our findings indicate that solid blood clots are not the origin of hyperintense lesions observed on DWI and enhance the role of alternative mechanisms such as air embolism. Silent embolism as a surrogate parameter for ischaemic stroke therefore must be questioned.
14:00 - 15:30

Room F1

\section{Genitourinary}

\section{SS 507}

\section{Prostate cancer}

Moderators:

U.G. Mueller-Lisse; Munich/DE

M. Secil; Izmir/TR

\section{B-301 14:00}

The relevance of diffusion-weighted MR-imaging at $3 \mathrm{~T}$ with different $\mathrm{b}$ value for the detection of prostate cancer $(500,1000,3000)$

V. Panebianco, D. Lisi, V. Buonocore, F. Galati, T. Biondi, R. Passariello; Rome/IT (valeria.panebianco@uniroma1.it)

Purpose: To prospectively determine the accuracy of diffusion-weighted (DW) magnetic resonance (MR) imaging for identifying cancer in the prostate peripheral zone (PZ) in association with T2-weighted and DCEMR imaging before biopsy. Methods and Materials: The institutional review board approved this study and all the patient subscribed an informed consent. Twenty-seven patients underwent endorectal MR at 3 T magnet (Discovery M750, GE Healthcare) equipped with surface phased array and endorectal coil. Scan protocol included morphologic imaging with TSE T2-weighted sequences on the axial, sagittal and coronal planes, DWI sequences at different b value $(500,1000,3000)$ and dynamic contrast enhanced imaging using GRE 3D T1-weigthed sequence. Image cluster analysis was performed on voxels within the suspected tumour regions. Two readers in consensus recorded the presence of prostate cancer at magnetic resonance imaging and rated the imaging quality of DWI.

Results: For all the patients, the DWI sequence images were suitable for the evaluation of the zonal anatomy of the prostate gland and the tumour localisation. In the prediction of prostate tumour foci, we noticed an improvement for tumour detection with a b value of 3000 in comparison with other b value (500 and 1000) with $89 \%$ and $87 \%$, respectively, for sensitivity and specificity, and was crucial especially in cases with negative or borderline pattern at DCEMR.

Conclusion: DWI is a feasible technique that can be used for the differentiation of malignant and benign tissues in the prostate gland tissue. We obtained a significant improvement using high value of b (1000-3000)

\section{B-302 14:09}

Diffusion-weighted MRI (DW-MRI) at 3 Tesla (3 T) for prostate cancer: evaluation of tumour detection and aggressiveness

H. Vargas, O. Akin, T. Franiel, Y. Mazaheri, K. Udo, J. Zheng, C. Moskowitz, H. Hricak; New York, NY/US (vargasah@mskcc.org)

Purpose: To evaluate the incremental value of DW-MRI over T2-weighted MR (T2WI) at $3 \mathrm{~T}$ for prostate cancer detection; and to investigate the use of quantitative diffusion parameters to characterise aggressiveness, using whole-mount step-section pathology as the reference standard.

Methods and Materials: 51 patients (median age $58 \mathrm{y}$ ) underwent prostate T2W and DW-MRI at $3 \mathrm{~T}$ for the assessment of prostate cancer; and were subsequently treated by prostatectomy. Two readers independently evaluated all the studies and provided a score for the qualitative analysis according to the level of suspicion for the presence of cancer on a 1-5 index scale, first using T2WI alone and then using T2WI and DW-MRI in conjunction. Areas under the curve (AUCs) were estimated to evaluate performance. Quantitative analysis was performed using generalised estimating equations to test the ADC difference between benign and malignant prostate regions, as well as the associations between the ADC and tumour Gleason scores.

Results: AUCs for readers 1 and 2 were 0.79 and 0.76 for T2WI and 0.79 and 0.78 for DW-MRI, respectively. There was a significant difference in the mean $A D C$ between malignant and benign prostate regions $\left[1.09\right.$ and $1.08 \times 10^{3} \mathrm{~mm}^{2} / \mathrm{s}$ for readers 1 and 2, respectively, vs $1.81 \times 10^{3} \mathrm{~mm}^{2} / \mathrm{s}$ for both readers]. A higher Gleason score was significantly associated with a lower mean ADC $(p=0.017)$ Conclusion: The addition of DW-MRI to T2WI did not significantly improve the readers' performance in detecting prostate cancer. However, quantitative analysis showed that ADC may be a useful tool to detect and establish the aggressiveness of prostate cancer. 
B-303 14:18

(also Prospective comparison of T2w-MRI and dynamic-contrast-enhanced MRI, 3D-MR spectroscopic imaging or diffusion-weighted MRI in repeat TRUSguided biopsies

D. Portalez, B. Malavaud; Toulouse/FR (dportalez@rx-infomed.com)

Purpose: To compare T2-weighted MRI and functional MRI techniques in repeat prostate biopsies.

Methods and Materials: Sixty-eight patients with a history of negative biopsies, negative digital rectal examination and elevated PSA were imaged on a 1.5 T MR system using an integrated endorectal-pelvic coil before repeat biopsies. T2-W MRI, diffusion-weighted imaging (DWI), 3D-MR spectroscopy imaging (MRSI) and dynamic-contrast-enhanced MRI (DCE-MRI) were successively acquired in all patients. Interpretation was based on robust literature-adapted dichotomous criteria with cutoffs for DWI and MRSI. Systematic sextant biopsies and additional biopsies were targeted towards areas whose features raised the suspicion of tumour. Results: Prostate cancer was found in 28/68 (41.2\%) patients corresponding to 45 segments. In 62 segments suspicious areas were evidenced in locations not sampled by sextant biopsies of which $20(32 \%)$ were found to be positive. The other 25 positive segments were found in the routine eight spots. The probability of a segment being malignant was calculated from logistic regression modelling from the results of T2w-MRI and of functional technique. T2-w-MRI + DWI exhibited the highest positive predictive value (0.52), compared with T2-w MRI alone (0.29), +DCE-MRI (0.33) and +3D-MRSI (0.25). The probability of cancer in a segment varied 12 -fold from $63.4 \%$ when T2-weighted and diffusion -weighted imaging MRI were both suspicious compared with $5.2 \%$ when both were non- suspicious. Conclusion: The proposed system of analysis and reporting was proven of clinical relevance in the decision process to repeat -or not to repeat -targeted biopsies.

\section{B-304 14:27}

\section{Biexponential apparent diffusion coefficients in different prostate tissue}

with echo planar imaging (EPI) sequences and high b value

X. Liu, L. Zhou, W. Peng; Shanghai/CN (liuxiang_1940@163.com)

Purpose: To investigate the feasibility of biexponential signal decay modelling for diffusion signal decays of different prostate tissues with EPI and high b value. Methods and Materials: Twenty-six patients ( 11 with prostate cancer, 15 benign) underwent EPI diffusion-weighted MR imaging in which b-factors from 0 to $3000 \mathrm{~s} / \mathrm{mm}^{2}$ in 10 steps were sampled. The acquired signal decay curves were fit with biexponential signal decay functions, the fast $A D C$ ( $A D C f$ ), fraction of fast component $(f)$ and the slow ADC (ADCs) were measured for the peripheral zone (PZ), central gland (CG), cancer and benign lesions. The data of different tissue were compared with a oneway bonferroni test. Results: The prostate cancer has the lowest ADCf $(1.67 \pm 0.18)$ and ADCs $(0.42 \pm 0.09)$ in all the tissue $(P<0.05$, Bonferroni), and smallest $f(43.3 \pm 3.7 \%)(P<0.05$, Bonferroni). The PZ tissue shows a higher $f(66.8 \pm 10.4 \%$ vs $52.7 \pm 4.4 \%)$ and $A D C s(1.40 \pm 0.32$ vs $0.75 \pm 0.18)$ than $C G(P<0.05)$; however, there is no significant difference between the ADCf of the two tissue $(3.87 \pm 0.42$ vs $4.01 \pm 0.70, P>0.05)$. The benign lesions exhit a lower $\operatorname{ADCf}(3.2 \pm 0.52)$ than both the normal tissue $(P<0.05)$, and a lower ADCs $(0.75 \pm 0.25)$ and $f(57.8 \pm 2.6 \%)$ than PZ; however, no significant different between the $C G$ and benign in the ADCs and $f$.

Conclusion: The biexponential apparent diffusion coefficients with EPI at high $b$ value provide additional, unique tissue characterisation parameters for prostate tissue and could be practical in clinical use.

\section{B-305 14:36}

Whole-torso MRI including DWI for patients with recurring prostate cancer: technical feasability and assessment of lesion conspicuity in DWI

M. Eiber, K. Holzapfel, E.J. Rummeny, A.J. Beer; Munich/DE

Purpose: In a technical development study the principal methodological aspects of whole-torso magnetic resonance imaging (MRI) including diffusion-weighted imaging (DWI) with background-suppression using a time optimised protocol for restaging of prostate cancer patients were evaluated.

Methods and Materials: Twenty-two patients underwent MRI at $1.5 \mathrm{~T}$ from the base of the skull to the proximal thigh using axial T1w, T2w STIR and DWI (b-values: 50 and $500 \mathrm{~s} / \mathrm{mm}^{2}$ ) and sagittal T1w and T2w STIR of the spine. ADC-values of liver, spleen, kidney, muscle and bone were measured. Image quality in DWI was assessed using a scale from 0 to 9 . Contrast-to-noise ratios (CNR) of lymph node and bone metastases were determined in T1w, T2w STIR and DWI. Bone metastases were further subclassified according to their Hounsfield units (HU) in CT. Results: Mean acquisition and mean room times were 66:52 min and 77:34 min, respectively. ADC values of normal organs showed good concordance with reported data. Good to excellent image quality was observed for DWI (mean scores
7.41-8.00) with exception of the neck (mean score 4.81). CNR of DWI (b-value $50 \mathrm{~s} / \mathrm{mm}^{2}$ ) for lymph node metastases was clearly superior compared to all other sequences. For bone metastases T1w performed significantly better for sclerotic lesions $(\mathrm{HU}>600)$, DWI (b-value $\left.50 \mathrm{~s} / \mathrm{mm}^{2}\right)$ for non-sclerotic lesion $(\mathrm{HU}<300)$. Conclusion: In patients with recurrent prostate cancer a whole-torso MR protocol including DWI is technically robust. Due to the high CNR of DWI compared to T1w and T2w STIR, detection of malignant lesions should be facilitated by DWI, except for sclerotic bone metastases.

\section{B-306 14:45}

Detection of localised prostate cancer using proton magnetic resonance spectroscopy and carbon-11-acetate PET/CT

I. Jambor, R.J. Borra, J. Kemppainen, V. Lepomäki, K. Dean, K. Alanen,

M. Nurmi, H.J. Aronen, H. Minn; Turku/FI (ivjamb@utu.fi)

Purpose: We assessed the ability of magnetic resonance imaging (MRI), proton magnetic resonance spectroscopy (1H MRS) and carbon-11-acetate PET/CT to image localised prostate cancer and detect its aggressiveness.

Methods and Materials: Twenty-one patients with untreated prostate cancer, diagnosed using transrectal ultrasound guided biopsy, underwent pelvic carbon-11-acetate PET/ CT and 1.5 TMRI using surface coils. Cancer laterality was based on the percentage of cancer and the highest Gleason score determined from biopsies. 3D 1 H MRS of the entire prostate volume was performed. Cramer-Rao minimum variance bound was automatically calculated for each individual metabolite. For each lobe choline + creatine + polyaminesto-citrate (CCP/C) ratios and standardised uptake values (SUV) were obtained from $1 \mathrm{H}$ MRS and carbon-11-acetate PET/CT, respectively. The visual and quantitative findings on MRI and PET/CT were compared with cancer laterality and aggressiveness based on the Gleason score, and with PSA velocity and international risk group classification Results: Using visual analysis, the sensitivity, specificity and accuracy of contrastenhanced MRI were $89 \%, 29 \%, 79 \%$ and using carbon-11-acetate PET/CT were $80 \%, 29 \%, 71 \%$, respectively, on a lobar level. The agreement of visual interpretation, with prostate cancer laterality based on biopsy findings, of contrast-enhanced MRI and carbon-11-acetate PET/CT and was $71 \%$. The dominant lesion CCP/C and SUV values were neither associated with prostate cancer aggressiveness nor did PSA velocity correlate with the SUV or CCP/C values from the entire gland. Conclusion: MRI, 1H MRS and carbon-11-acetate PET/CT enables detection of localised prostate cancer with comparable and limited accuracy but fail to provide information on cancer aggressiveness.

\section{B-307 14:54}

Comparison of qualitative and quantitative approach to prostate MR spectroscopy in peripheral zone cancer detection

G.M. Villeirs, S. Klijn, P.J. De Visschere, G.O. De Meerleer; Gent/BE (geert.villeirs@ugent.be)

Purpose: To compare the diagnostic performance of a qualitative (pattern recognition) and a quantitative (numerical assessment) approach to magnetic resonance spectroscopy (MRS) in the diagnosis of peripheral zone prostate cancer.

Methods and Materials: 185 patients (131 with histopathologically proven cancer, 54 normal/benign after at least 12 months of follow-up) were prospectively evaluated with qualitative MRS using a 4-point scale between March 2004 and January 2008 , and retrospectively reassessed using a prototype quantitative postprocessing software in April 2008. Based on pathology and follow-up data, diagnostic performance parameters were calculated.

Results: The qualitative and quantitative approaches were concordant in $78.9 \%$ (146/185) of cases. The difference between the areas under the ROC curve $(0.791$ versus 0.772 , respectively) were not statistically significant. The sensitivity, specificity and accuracy were $55.7 \%, 94.4 \%$ and $67.0 \%$ for the qualitative approach, and $55.0 \%, 83.3 \%$ and $63.2 \%$ for the quantitative approach. The sensitivity for high-grade tumours (Gleason $4+3$ or higher) was $85.2 \%$ (23/27) for both approaches. All cancers missed on either one approach separately (31/31) and $91 \%$ of cancers missed on both approaches together (23/27) were of lower grade (Gleason $3+4$ or lower). Conclusion: Qualitative and quantitative approaches to MRS yield similar diagnostic results. Discordances in tumour detection only occurred in lower grade cancers.

\section{B-308 15:03}

$3 \mathrm{~T} 1 \mathrm{H}-\mathrm{MRS}$ and DCEMR versus urinary PCA3 test in the detection of prostate cancer foci in patient with biochemical alteration V. Panebianco, G. Busetto, V. Buonocore, F. Galati, A. Sciarra, R. Passariello; Rome/IT (valeria.panebianco@uniroma1.it)

Purpose: We compared PCA3 test with MRSI-DCEMR combined examination in the detection of prostate tumour foci in patients with elevated PSA levels and prior negative random TRUS-guided biopsy. 
Methods and Materials: A total of 143 consecutive patients with a first negative random TRUS-guided prostate biopsy with elevated PSA levels (total PSA between $\geq 4 \mathrm{ng} / \mathrm{ml}$ and $<10 \mathrm{ng} / \mathrm{ml}$ ) underwent MR examination on $3 \mathrm{~T}$ magnet (Verio, Siemens, Germany) equipped with surface phased array and endorectal coil. Scan protocol included morphologic imaging with TSE T2-weighted sequences on three planes, spectroscopic imaging with $3 \mathrm{D}$ CSI sequences and dynamic contrast enhanced imaging using 3D FLASH T1-weigthed sequence. The second prostate biopsy was performed no later than 2 weeks from PCA3 test and MR examination. Results: For PCA3 test, the total number of urinary sediments that could be analysed successfully was $95.3 \%$ (141 on 143 specimens) as for MR, the examination and the following comparison with biopsy results were possible in all patients $(143 / 143,100 \%)$. The overall sensitivity and specificity of PCA3 in this cohort were $76.9 \%$ and $66.6 \%$, respectively, with a PPV of $80 \%$ and a NPV of $62.5 \%$. Sensitivity and specificity, for combining MRI, MRS and DCEMR, were respectively, 92.8\% and $86.6 \%$ with a positive predictive value (PPV) of $92.8 \%$ and a negative predictive value (NPV) of $86.6 \%$.

Conclusion: The association MRSI/DCEMR in comparison with PCA3 results shows a better detection of prostate cancer foci in patients with prior negative TRUS-guided biopsy and alterated PSA serum levels.

\section{B-310 15:12}

TRUS-MRI image fusion prostate biopsy in patients with no previous biopsy and a PSA level between 4 and $10 \mathrm{ng} / \mathrm{ml}$

F. Cornud, D. Eiss, N. Barry de Longchamps; Paris/FR

(francois.cornud@imagerie-tourville.com)

Purpose: To show the feasibility of TRUS-MRI image fusion prostate biopsy and to compare the positive biopsy rate and the prognosis value of TRUS-MRI fusion biopsy with those of a 12 cores sextant protocol.

Methods and Materials: 72 consecutive patients (mean age $64 \pm 6$ ) with a PSA level between 4 and 10 (mean $6.7 \pm 6$ ) and no palpable abnormality at digital rectal examination underwent an endorectal multiparametric MRI combining T2-weighted, diffusion-weighted and dynamic contrast enhanced MRI. During TRUS, a dedicated electromagnetic device (virtual navigator, ESAOTE, Italy) permitted a dynamic overlay of TRUS and MR images when a suspicious area, defined by a multiparametric MRI score, was detected, allowing for stereotaxic biopsies. Four TRUS-MRI fusion biopsies were performed in the target, followed by the sextant protocol.

Results: TRUS-MRI image fusion was possible in every case. Both targeted and sextant biopsies were positive in 28 patients $(28 / 72,39 \%)$. Fusion biopsies were solely positive in 12 patients $(12 / 72,17 \%)$, concerning tumours originating in the anterior part of the transition zone in $10 / 12$ cases $(83 \%)$. No patient had solely positive sextant biopsies. Increased cancer detection rate by fusion biopsies (40/72, $55.5 \%)$, approached significance $(p=0.057)$. No difference in Gleason score was observed between fusion and sextant biopsies, but the mean cancer length per core was significantly higher on fusion biopsies $(5.9 \mathrm{~mm} \pm 2.5)$ than that of sextant biopsies $(4.3 \mathrm{~mm} \pm 2.7, \mathrm{p}=0.02)$.

Conclusion: TRUS-MRI image fusion is a feasible technique which significantly increases the assessment of tumour burden. It questions the pertinence of the indication of MRI prior to biopsy.

\section{$14: 00-15: 30$} Room F2

\section{Breast}

\section{SS 502}

\section{Magnetic resonance imaging}

\section{Moderators:}

C. de Bazelaire; Paris/FR

A. Oktay; Izmir/TR

\section{B-311 14:00}

Role of breast MR in ruling out malignancy at surgical excision of B3 lesions diagnosed at core-needle-biopsy: a prospective observational study

A. Linda, V. Londero, A. Dal Col, L. Battigelli, C. Zuiani, M. Bazzocchi; Udine/IT (annalinda33@gmail.com)

Purpose: To determine whether breast MR can help exclude associated malignancy in case of B3 (borderline) lesions diagnosed at core-needle-biopsy (CNB). Methods and Materials: A total of 175 B3 lesions (69 papillomas, 51 radial sclerosing lesions-RSL, 18 atypical ductal hyperplasia-ADH and 37 lobular neoplasia-LN) were diagnosed at CNB in 168 women. All patients underwent breast MR, and two experienced breast radiologists reviewed the MR images according to the MR-BIRADS lexicon. Surgical excision was recommended regardless of the imaging findings. MR findings ("non suspicious lesions": BI-RADS 1 and 2 categories, versus "suspicious lesions": BI-RADS 3,4 and 5 categories) were correlated with histologic results on surgical specimen. Overall and by-lesion sensitivities, specificities, positive (PPV) and negative (NPV) predictive values of MR in predicting presence of malignancy were calculated.

Results: Lesions were categorized as follows: 63 BI-RADS 1.13 BI-RADS 2.42 BI-RADS 3.54 BI-RADS 4 and 3 BI-RADS 5. Surgical excision demonstrated $26 / 175$ malignancies, corresponding to an overall CNB underestimation rate of $14.8 \%$ ( $10 \%$ for papillomas; $4 \%$ for RSL, $28 \%$ for $A D H, 32 \%$ for $L N$ ). MR provided an overall sensitivity of $81 \%$, specificity of $48 \%$, PPV of $21 \%$ and NPV of $93 \%$ By-lesion analysis demonstrated the following specificities, sensitivities, PPV and NPV: $100 \%, 29 \%, 14 \%, 100 \%$, respectively, for papillomas; $50 \%, 55 \%, 4 \%, 97 \%$, respectively, for RSL; $60 \%, 46 \%, 30 \%, 75 \%$, respectively, for $\mathrm{ADH} ; 83 \%, 80 \%, 67 \%, 91 \%$, respectively, for $\mathrm{LN}$.

Conclusion: MR demonstrated high NPV for malignancy in case of B3 lesions associated with low underestimation rates at CNB (papillomas and RSL). In cases of non-suspicious MR findings, follow-up rather than surgical excision might be recommended for those lesions.

\section{B-312 14:09}

High-risk breast lesions (B3) diagnosed at imaging-guided needle biopsy: can MRI-BIRADS features predict likelihood of malignancy?

V. Londero, C. Zuiani, A. Linda, M. Lorenzon, L. Battigelli, M. Bazzocchi; Udine/IT (Iondero.viviana@aoud.sanita.fvg.it)

Purpose: To assess whether MRI-BIRADS lesions features can predict the probability of malignancy for high-risk breast lesions diagnosed at core-needle-biopsy (CNB). Methods and Materials: Retrospective review of MRI findings in 138 high-risk breast lesions - 71 benign papillomas (PAP), 30 radial sclerosing lesions (RSL), 23 lobular neoplasias (LN), 14 atypical ductal hyperplasias (ADH) - diagnosed at imaging-guided CNB was performed. MRI-BIRADS morphologic and kinetic features were compared with histology at surgical excision (SE) in $117(85 \%)$ and with imaging follow-up (>24 months) in 21 (15\%) lesions. Chi-square test was used for statistical analysis (statistically significant: $p$-value $<0.05$ )

Results: $23(17 \%)$ lesions were upgraded to malignancy at SE (seven papillomas, one RSL, eleven LN, four ADH): 9 (39\%) were invasive carcinomas and 14 (61\%) were ductal carcinomas in situ. Lesion-specific underestimation rates were: benign papillomas 10\% (7/71); RSL 3\% (1/30); LN 48\% (11/23); ADH 29\% (4/14). Lesions classified as nonmasslike enhancement (NMLE) were significantly more likely to be malignant $(36 \%)$ than those described as masses $(13 \%)$ [ $p=0.01]$. Lesions size was not a significant predictor of malignancy[ $p=0.19]$. For papillomas, $R S L$ and $A D H$ presenting as masses, BI-RADS margins, internal enhancement pattern and kinetic characteristics did not help to predict presence of malignancy. For LN, risk of underestimation was significantly higher for lesions classified as 'suspicious masses' according to Fischer score (7/8 [88\%]) rather than "non-suspicious" (1/7 $[14 \%])[p=0.01]$ and for lesions $>10 \mathrm{~mm}(5 / 5[100 \%])$ rather than $\leq 10 \mathrm{~mm}(3 / 10$ $[30 \%])[p=0.04]$.

Conclusion: Among MRI features, NMLE in any B3 lesion and 'suspicious mass' in the case of $L N$ are highly predictive of malignancy. MRI finding of focus or foci $(<5 \mathrm{~mm})$ does not exclude presence of malignancy.

\section{B-313 14:18}

Long-term follow-up of mammographically and sonographically occult likely benign (BI-RADS 3 ) lesions detected on screening MRI N.K. Singh, P. Crystal, S. Kulkarni; Toronto, ON/CA (drnitinsingh@hotmail.com)

Purpose: To evaluate outcome of otherwise occult likely benign breast lesions detected on screening MRI in asymptomatic high-risk women.

Methods and Materials: Retrospective review of breast MRI database performed to identify all nonpalpable, mammographically \& sonographically occult, MRIdetected likely benign lesions in asymptomatic women without prior history of breast carcinoma. These BI-RADS 3 "MRI-only" lesions were followed from 18 to 77 months (mean 28 months) and rate of malignancy was calculated. Characteristics of probably benign lesions on screening breast MRI were analyzed for predictors of malignancy.

Results: 131 lesions were reported as likely benign in 114 patients out of 695 screening breast MRI studies. These included $42(37 \%)$ foci, $48(42 \%)$ non-mass like enhancement and $41(36 \%)$ masses. Eighteen lesions were biopsied during follow-up period due to lesion progression on follow-up MRI or for other reasons. Malignancies were found in four women, constituting $22 \%$ of the lesions that underwent biopsy and $3 \%$ of probably benign lesions. Tumor histology was invasive 
ductal carcinoma in two patients and ductal carcinoma in situ in two patients. All four malignancies were diagnosed in enhancing masses ( $10 \%$ of enhancing masses). No cancers were diagnosed in subgroup of enhancement foci.

Conclusion: A probably benign interpretation was given to $16 \%$ of high-risk women at their breast MRI screening examination. In $3 \%$ of women with likely benign lesions, breast carcinoma was subsequently diagnosed in an area initially judged to be probably benign. Additional studies are required to refine criteria for probably benign lesions on breast MRI.

\section{B-314 14:27 \\ Management of incidental enhancing lesions detected by breast MR imaging \\ V. Girardi, G. Carbognin, L. Camera, I. Baglio, F. Bonetti, R. Pozzi Mucelli;} Verona/IT (giravero@yahoo.it)

Purpose: To evaluate the outcome of incidental enhancing lesions detected by breast MRI.

Methods and Materials: Among 1044 consecutive breast MRI, we analysed 154 lesions evident on MR images, non-palpable, first look ultrasound and mammographically occult. These cases were reviewed to assess the indication for MRI lesions size, MRI characteristics, method of biopsy (US, MRI, other) and association with pathologic results. The gold standard was pathological results and/or follow-up (>12 months). Statistical analysis was performed with Fisher exact test. Results: Of 154 incidental MRI lesions, targeted US identified 97 (63\%). The detection rate with targeted US was higher with mass-like MRI lesions $(68 \%)$ vs non-mass MRI lesions (38\%), with lesion size > $10 \mathrm{~mm}(71 \%)$ vs < $10 \mathrm{~mm}(58 \%)$ Of 154 incidental MRI lesions, 111 underwent biopsy ( 71 by US, 31 by MR, 9 by surgery) and 43 were followed-up for at least 12 months. Malignancy was found in $42 / 154$ cases (28\%: true positive rate). Targeted US-guided biopsy detected 20/42 $(47 \%)$ malignant lesions; the remaining $22 / 42(53 \%)$ cancer have no US correlate and were diagnosed with MRI-guided biopsy or surgery.

Conclusion: Given the relatively low rate of true positive (28\%), biopsy of incidental MR findings is recommended prior to therapeutic decision. Targeted US is a reliable, economical, and time sparing tool in the management of the majority $(63 \%)$ of incidental MRI findings. It contributes to accurately select the cases to submit to MRI-guided biopsy. The lack of US correlate has not to preclude biopsy by MR as $53 \%$ of cancers found were only MRI visible.

\section{B-315 14:36}

\section{Breast MR in patients showing suspicious microcalcifications on} mammography: is there any clinical value?

A. Linda, R. Zearo, V. Londero, C. Molinari, C. Zuiani, M. Bazzocchi; Udine/IT (annalinda33@gmail.com)

Purpose: To evaluate the clinical value of breast MR in patients with suspicious microcalcifications and negative ultrasound findings.

Methods and Materials: Between 2006 and 2008, 115 patients underwent breast MR before stereotactic vacuum-assisted-biopsy (SVAB) for microcalcifications (38 BI-RADS category 3, 65 BI-RADS 4 and 12 BI-RADS 5). Two experienced breast radiologists reviewed MR images according to the MR-BIRADS lexicon. Surgical pathology results and follow-up (> 24months) served as the reference standard for lesions diagnosed as malignant or high-risk and benign at SVAB, respectively. Overall and by-category sensitivities, specificities, positive- (PPV) and negative predictive values (NPV) for malignancy were calculated.

Results: Lesions were categorised as follows: 49 MR-BI-RADS 1, 27 MR-BI-RADS 2, 10 MR-BI-RADS 3, 9 MR-BI-RADS 4 and 20 MR-BI-RADS 5. Thirty-three of $115(29 \%)$ lesions proved to be malignant, corresponding to by-category malignancy rates of $8 \%, 29 \%$ and $92 \%$ for BI-RADS 3,4 and 5 microcalcifications, respectively. The remaining 82 lesions were diagnosed as benign at SVAB and remained unchanged at imaging follow-up (mean 31 months, range:24-43). MR provided an overall sensitivity of $79 \%$, specificity of $84 \%$, PPV of $67 \%$ and NPV of $91 \%$. By-category analysis demonstrated the following sensitivity, specificity, PPV and NPV for BI-RADS 3 microcalcifications: $67 \%, 89 \%, 33 \%$ and $97 \%$, respectively. Conclusion: Patients with highly suspicious microcalcifications (BI-RADS 4 and 5) should undergo SVAB, regardless MR findings. However, MR shows high NPV in case of BI-RADS 3 microcalcifications. Therefore, in case of non-suspicious MR findings, follow-up rather SVAB might be recommended in patients with probably benign microcalcifications.

\section{B-316 14:45}

Second-look ultrasound of incidental breast MRI lesions in patients with newly diagnosed breast cancer: correlating location with pathology V. Girardi, G. Carbognin, L. Camera, A. Brandalise, F. Bonetti, R. Pozzi Mucelli; Verona/IT (giravero@yahoo.it)

Purpose: To evaluate location and pathological outcome of breast MRI suspicious lesions in patients with newly diagnosed ipsilateral/controlateral breast cancer. Methods and Materials: Among 310 consecutive pre-operative breast MRI, 71 lesions (evident on MR images, non-palpable, first-look ultrasound and mammographically occult) were recommended for second-look ultrasound. The retrospective evaluation included second-look ultrasound identification according to lesion's location (ipsilateral - in the same/different quadrant - controlateral), distance from the primary lesions $(<4 \mathrm{~cm} />4 \mathrm{~cm})$. Results were correlated with pathological findings. Results: Of 50 lesions depicted at second-look ultrasound, 26 lesions were ipsilateral to the known cancer and 24 were controlateral. Overall, 16/26 (61\%) ipsilateral lesions were malignant. Of the 26 ipsilateral lesions, $12(24 \%)$ were in the same quadrant as the index cancer, of which $8(66 \%)$ were malignant (4-ILC, 3-IDC, 1-DCIS) and 4 were benign. The remaining 14/26 (28\%) ipsilateral lesions were in different quadrant from the known cancer; 8 (57\%) were malignant (5-ILC, 3-DCIS) and 6 were benign. Of the 24 controlateral lesions to known cancer, 4/24 (16\%) were malignant (2-ILC, 2-IDC). Overall, of the $20 / 50(40 \%)$ malignant lesions identified by second-look ultrasound, $13 / 20(65 \%)$ were within $4 \mathrm{~cm}$ distance from the primary $(p<0.05)$.

Conclusion: Second look ultrasound identified $71 \%$ of incidental breast MRI lesions in newly diagnosed breast cancer patients, of which $40 \%$ proved malignant. The majority $(65 \%)$ of additional tumour foci are in the same quadrant and within $4 \mathrm{~cm}$ of the index cancer. At a minimum, when performing ultrasound in a patient known to have cancer, thorough evaluation of at least that quadrant of the breast is recommended.

\section{B-317 14:54}

Breast MRI contribution to the study of papillary lesions

M. Vaño, J. Camps Herrero, M. Forment Navarro, V. Ricart Selma,

J. Cano Gimeno, C. Martínez Rubio; Alzira/ES (mavanyo@hospital-ribera.com)

Purpose: To evaluate the diagnostic performance of breast MRI in the evaluation of papillary lesions and to assess the influence of this technique on the therapeutic approach.

Methods and Materials: In a retrospective study of breast MRI exams performed from January 2003 to July 2010, three groups of patients were included: patients with nipple discharge $(n=40)$, patients with a radiological suspicion of a papillary lesion $(n=28)$ and patients with a retrospective pathological diagnosis of a papillary lesion $(n=16)$. Enhancing lesions associated with a dilated duct or cyst, lesions located in the nipple-areolar complex or with a segmental distribution were considered suspicious. Lesions were classified according to BI-RADS categories and the gold standard was pathological results after surgery.

Results: Of the 84 patients studied with MRI, 30 had a negative MRI study for a papillary lesion (with a mean follow-up of 20 months) and in 54 cases findings consistent with papillary lesions were diagnosed. They were pathologically confirmed: 17 intracystic papillomas (31'5\%), 15 papillary carcinomas $\left(27^{\prime} 7 \%\right)$ 7 papillary carcinomas in situ (13\%), 9 diffuse papillomatosis $\left(16{ }^{\prime} 6 \%\right)$ and six false positives. In 27 cases ( 26 '2\%), MRI provided new information that changed therapeutic approach. Sensitivity was $87.9 \%$, especificity $77 \%$, NPV was $90.9 \%$ and PPV was $70 \%$.

Conclusion: MRI helps to characterise not only papillary lesions but also allows us to identify other lesions not seen with conventional techniques and has a high NPV. New findings identified with MRI are important because they may change the therapeutic approach in 1 out of every 4 patients.

\section{B-318 15:03}

Kinetic analysis of non-mass-like enhancing lesions on breast MRI using manual and computer-assisted methods

T. $\mathrm{Vag}^{1}$, P.A.T. Baltzer ${ }^{2}$, M. Dietzel ${ }^{2}$, R. Zoubi ${ }^{2}$, W.A. Kaiser ${ }^{2} ;{ }^{1}$ Munich/DE, 2Jena/DE (Tibor.Vag@roe.med.tu-muenchen.de)

Purpose: To analyse the kinetic characteristics of non-mass-like enhancing lesions in dynamic breast MRI using manual and computer assisted methods.

Methods and Materials: The enhancement pattern of 82 histopathologically verified non-mass-like enhancing lesions ( 36 malignant, 46 benign) was evaluated on breas $\mathrm{MRI}$ using manual placement of a region of interest (ROI) and commercially available computer analysis software. The latter can automatically assess volume enhancement characteristics of a lesion voxelwise. Kinetic features evaluated included classification of the signal-intensity time curve as washout, plateau or persistent enhancement. A washout enhancement pattern is regarded as suggestive for malignancy. 
Results: Unlike manual ROI placement, computer-aided analysis demonstrated a significant difference in enhancement pattern between benign (washout: $32.6 \%$, plateau: $32.6 \%$, persistent: $34.8 \%)$ and malignant lesions $(77.1 \%, 8.6 \%, 14.3 \%$ respectively, $\mathrm{P}<0.01$, two-sided Chi-squared test) following initial rapid signal increase. Mean percentage of washout voxel volumes within a lesion was significantly higher in malignant lesions than in benign lesions (11.9\% \pm 12.7 (SD) vs. $6.9 \% \pm 11.3$ (SD), $\mathrm{P}<0.01$, Mann-Whitney $\mathrm{U}$ test). Conversely, the mean percentage of persistent voxel volumes was significantly lower in malignant lesions than in benign lesions $(60.1 \% \pm 21.1$ (SD) vs. $79 \% \pm 23$ (SD), $\mathrm{P}<0.01$, Mann-Whitney U test). Conclusion: Computer-assisted enhancement pattern analysis might have diagnostic benefit in the evaluation of non-mass-like enhancing lesions.

\section{B-319 15:12}

Assessment of perifocal oedema in MR-mammography: a systematic comparison of two pulse sequences using visual grading characteristics analysis

P.A.T. Baltzer, M. Dietzel, W.A. Kaiser

Jena/DE (pascal.baltzer@med.uni-jena.de)

Purpose: Perifocal oedema, defined as high T2w signal intensity around an enhancing lesion has been described as a specific feature of malignancy. In clinical MR-mammography (MRM), both fatsat and non-fatsat T2w sequences are available. However, there is no consensus on which technique should be used for oedema assessment. Consequently, this investigation was performed to compare two commonly used pulse sequences for oedema assessment in MRM.

Methods and Materials: Consecutive patients from a 22-month period with 197 malignant and 123 benign lesions verified by histopathology were included in this investigation. All underwent MRM according to European guidelines including a non-fatsat T2w-TSE sequence (TR/TE: $8900 / 207 \mathrm{~ms}$ ) and a short tau inversion recovery fatsat sequence (STIR, TR/TE: $8420 / 70 \mathrm{~ms}$ ). All images were acquired in the same orientation (axial) and slice thickness $(3 \mathrm{~mm})$. Two experienced radiologists in consensus rated presence of perifocal oedema according to an ordina scale: 0 = not present, $1=$ little, 2 = intermediate, 3 = distinct. Data analysis was performed using crosstabs and visual grading characteristics (VGC) analysis.

Results: Overall sensitivity/specificity was calculated with 64.8\%/92.7\% (T2w-TSE) and $62.3 \% / 93.5 \%$ (TIRM). VGC revealed an Area under the VGC Curve of 0.516 (standard error 0.021 ), $P=$ n.s.

Conclusion: Perifocal oedema is a specific feature of malignancy with moderate sensitivity. VGC analysis did not reveal significant differences between both pulse sequences analysed. Consequently, both T2-TSE and TIRM images are suitable for assessment of perifocal oedema.

\section{B-320 15:21}

The impact of pre-operative breast MRI and MRI-induced work-up on surgical delay

B. Lord, R. Zhu, L.M. Jacks, A.M. Scaranelo; Toronto, ON/CA

(bridgette.lord@uhn.on.ca)

Purpose: To assess the time delay due to MRI-induced work-up in women awaiting surgery for breast cancer and to examine MRI-induced work-up outcomes.

Methods and Materials: A prospectively collected database was used to select patients who visited a Rapid Diagnostic Breast Clinic (RDBC) from September 2006 to September 2008 were diagnosed with breast cancer, and had a pre-operative MRI with subsequent MRI-induced work-up.

Results: In 292 consecutive new RDBC patients, 199 (68\%) had surgery and 175 $(60 \%)$ were diagnosed with breast cancer. Of the 175 patients with breast cancer, $118(67 \%)$ had a pre-operative breast MRI and $32(18 \%)$ had an MRI call-back. Additional foci of ipsilateral cancer and contralateral cancer were found in $28 \%$ and $9.4 \%$ of the 32 patients, respectively. The false positive rate of MRI-induced work-up was $37.5 \%$. The mean time interval from RDBC to MRI was 15.35 days and from MRI to call-back 19.13 days. Patients with an MRI call-back had a mean time interval of 55.24 days from RDBC to surgery compared to 46.25 days for patients who had a MRI but no call-back.

Conclusion: The surgical delay caused by pre-operative breast MRI and MRIinduced work-up in breast cancer patients did not exceed 9 days. Additional foci of malignant disease were found in $28 \%$ of patients and contralateral breast cancer in $9.4 \%$ of RDBC patients who underwent a pre-operative MRI.
14:00 - 15:30

Room G/H

GI Tract

\section{SS 501b}

\section{CT colonography: 17 years on}

Moderators:

S. Gryspeerdt; Roeselare/BE

M.M. Morrin; Dublin/IE

\section{B-321 14:00}

Radiation risks for patients undergoing screening CT colonography with 256-slice CT scanners: how anxious should we be?

K. Perisinakis ${ }^{1}$, I. Seimenis ${ }^{2}$, A. Tzedakis ${ }^{1}$, A.E. Papadakis ${ }^{1}$, J. Damilakis ${ }^{1}$; ${ }^{1} /$ raklion/GR, ${ }^{2}$ Nicosia/CY (perisina@med.uoc.gr)

Purpose: To provide patient-specific estimates of doses and theoretical risks of radiation-induced cancer for patients undergoing 256-slice CT colonography (CTC) screening.

Methods and Materials: Patient-specific voxelized phantoms were generated using 256-slice CTC images of 8 males and 13 females, while corresponding dose-images were produced by employing a novel Monte Carlo CT dosimetry software. A low-dose screening, CTC $120 \mathrm{kVp}$ protocol was simulated with prone and supine imaging at 30 and $50 \mathrm{mAs}$, respectively. Tube load was adapted depending on patient body-size. Normalised (to free-in-air CTDI) organ dose data were derived for all primarily irradiated radiosensitive organs, i.e. 11 main organs and 11 remainder organs. Organ doses were determined for each patient for the specific exposure parameters. Effective dose was estimated for each patient using 2007 ICRP recommendations. Risks of radiation-induced cancer were estimated using appropriate sex-, age- and organ-specific cancer risk factors. Individualised total cancer risk was estimated by adding respective organ risks.

Results: The higher doses were received by colon, liver, stomach and bladder. Mean effective dose was estimated to be 1.9 and $2.1 \mathrm{mSv}$ for male and female patients, respectively. The mean total theoretical life-attributable risk factor for cancer induction following screening 256-slice CTC was estimated to be $20 \times 10^{-5}$ and $17 \times 10^{-5}$, for female and male individuals, respectively.

Conclusion: For a typical patient cohort undergoing low-dose screening CTC on a 256-slice CT scanner the total theoretical risk for radiation-induced cancer is as low as 1 in 5000 and 1 in 5900 for females and males, respectively.

B-322 14:09

(EPOS)

Low dose CT colonography: feasibility of using iterative reconstruction algorithms for improved 3D interpretation of polyps

D. Mehta, S. Virmani; Highland Heights, OH/US (dhruv.mehta@philips.com)

Purpose: $3 \mathrm{D}$ visualisation is used as primary interpretation method for CT colonography. Low-dose acquisition techniques are prone to significant artefacts particularly in the pelvis region. Previously published literature indicates high prevalence of polpys in the rectum. The purpose of this study was to assess the image quality impact of iterative reconstruction techniques on 3D interpretation of polpys.

Methods and Materials: Randomised selection of 30 low-dose CT colonography datasets was performed from the database. Selection criteria included scanne type (64-slice CT), acquisition protocol (120 kV, $15 \mathrm{mAs}$ ), and BMI $>25<30$. Reconstructions were performed using conventional filtered-backprojection (FBP) and an iterative reconstruction technique (iDose, Philips). Qualitative image quality assessments were performed relative to FBP [scale: 1 (worse than FBP) - 5 (superior to FBP)] by two blinded readers on a per-segment basis (rectum, sigmoid descending, transverse, ascending and caecum) using a 3D workstation (EBW, Philips). Quantitative noise measurements were performed in a ROI placed in the air-pockets of each segment. Statistical differences were tested using wilcoxon signed-rank test and t-test.

Results: Image quality scores were [segment, median-rank]; [rectum 5] [sigmoid 4] [descending 3] [transverse 3] [ascending $3 \mathrm{p}$ ] [cecum 3] and showed significant improvement in 3D visualisation for the rectum $(p<0.5)$ and sigmoid $(p<0.5)$. Quantitative noise measurements were [segment, FBP-noise, iDose-noise $p$-value]; [rectum 42.519 .1 ] [sigmoid 38 17.1] [descending 34 15.3] [transverse 25.311 .4 [ascending 3214.4 ] [caecum 3214.4 ]. Noise was significantly lower on the iDose reconstructions.

Conclusion: Iterative reconstruction techniques could help improve the $3 \mathrm{D}$ interpretation for low-dose CT colonography particularly in the rectum and sigmoid segments. 


\author{
B-323 14:18 \\ Diagnostic value of CT-colonography as compared to colonoscopy in an \\ asymptomatic screening population: meta-analysis \\ M.C. de Haan ${ }^{1}$, R.E. van Gelder', A. Graser ${ }^{2}$, S. Bipat ${ }^{1}$, J. Stoker ${ }^{1}$. \\ ${ }^{1}$ Amsterdam/NL, ${ }^{2}$ Munich/DE (m.c.dehaan@amc.uva.nl)
}

Purpose: Previous meta-analyses on computed tomography (CT)-colonography included both average risk and high risk individuals, which may overestimate the diagnostic value in screening. A meta-analysis was performed to obtain the diagnostic value of CT-colonography for screening.

Methods: A comprehensive search (until July 2010) was performed using the MEDLINE, EMBASE and Cochrane databases. Initial selection of articles and critical appraisal was done by two reviewers using QUADAS. Inclusion criteria were: prospective, randomised trials or cohort studies comparing CT-colonography with colonoscopy ( $\geq 50$ participants), $\geq 95 \%$ asymptomatic average risk participants $\geq 50$ years. Study-design, participant characteristics, CT-colonography techniques and $2 \times 2$ contingency tables were recorded. Sensitivity and specificity estimates were calculated on per patient basis and summary sensitivity on per polyp basis, using bivariate and univariate analyses.

Results: Of 1,021 studies identified, five studies were included, including 4,086 participants ( $<1 \%$ high risk). Based on QUADAS, all studies were of high quality. Pooled sensitivities for patients with polyps $\geq 6 \mathrm{~mm}$ and adenomas $\geq 6 \mathrm{~mm}$ were $75.9 \%$ and $82.9 \%$, respectively, while specificities were $94.6 \%$ and $91.4 \%$, respectively. Pooled sensitivities per patient regarding polyps $\geq 10 \mathrm{~mm}$ and adenomas $\geq 10 \mathrm{~mm}$, were $83.3 \%$ and $87.9 \%$, respectively, and specificities $99.0 \%$ and $97.6 \%$, respectively. Pooled sensitivities per polyp for advanced adenomas $\geq 6 \mathrm{~mm}$ and $\geq 10 \mathrm{~mm}$ were $84.4 \%$ and $86.9 \%$, respectively, while sensitivity for colorectal cancer was $100 \%$. Conclusion: Compared to colonoscopy, CT-colonography is highly sensitive for colorectal cancer. CT-colonography is less sensitive for clinically relevant (advanced) adenomas $\geq 6 \mathrm{~mm}$; however, its detection rate is acceptable for screening purposes especially when the attendance is higher than for colonoscopy.

\section{B-324 14.27}

Clinical results of a five-year $\mathrm{CT}$ colonography screening programme

E. Picano, E. Neri, L. Faggioni, P. Vagli, C. Bartolozzi; Pisa/IT

Purpose: To analyse the diagnostic results of the CT Colonography (CTC) screening programme at our Institution over a five-year period.

Methods and Materials: We retrospectively reviewed 1894 screening СTC examinations performed at our institution from January 2003 to March 2010 on asymptomatic patients. Out of them, 810 had been obtained on a 4-row CT scanner and the remaining 1084 on a 64-row CT scanner using a low-radiation dose protocol in the supine and prone positions and a same-day preparation with iodine faecal tagging ( $500 \mathrm{~mL}$ of water and $50 \mathrm{~mL}$ of diatrizoate dimeglumine). Lesions were classified according to the $\mathrm{C}$-RADS system ( $\mathrm{C} 0=$ not diagnostic, $\mathrm{C} 1=$ unremarkable, $\mathrm{C} 2=$ polyps sized 6-9 $\mathrm{mm}, \mathrm{C} 3=$ polyps over $10 \mathrm{~mm}, \mathrm{C} 4=$ colonic masses over $3 \mathrm{~cm}$ ). Results: Overall, 249/1894 examinations were classified as C0 (13.1\%), 1338 as C1 $(70.7 \%), 142$ as C2 $(7.5 \%), 95$ as C3 $(5.0 \%)$ and 70 as C4 (3.7\%). C0 cases were not diagnostic mainly due to the persistence of marked faecal residues. The greater axis of polyps was $7.6 \pm 1.1 \mathrm{~mm}$ in the $\mathrm{C} 2$ group and $16.3 \pm 3.2 \mathrm{~mm}$ in the C3 group, respectively. Among C4 lesions, 51/70 (72.9\%) were annular and the remaining 19/70 (27.1\%) vegetating cancers. Sensitivity of CTC in the C2 group was $95 \%$ (135/142), $98 \%$ in C3 (93/95) and 100\% in C4 (70/70).

Conclusion: CTC allows detection and classification of colonic polyps and masses based on C-RADS 2, 3 and 4 categories with very high sensitivity. Poor colonic cleansing can be responsible of CTC failure in a small, but still not negligible fraction of cases.

\section{B-325 14:36}

Patients' preferences about follow-up of medium-size polyps detected at screening CT colonography

L. Faggioni, E. Neri, P. Vagli, E. Picano, F. Cerri, C. Bartolozzi; Pisa/lT

Purpose: To evaluate patients' preferences about follow-up of medium-size polyps detected at screening CT Colonography (CTC).

Methods and Materials: 193 C-RADS2 asymptomatic patients were enrolled in the study. Patients were asked to fill in a written informed consent form explaining the indications, technique and potential complications of CTC, and they were also invited to choose their preferred examination technique (CTC or optical colonoscopy: OC) and their follow-up interval by repeated consultations at 3-month intervals. The follow-up interval for CTC and OC was recorded.

Results: $87 / 193$ C-RADS2 patients (45.1\%) accepted follow-up; of the latter, 42/87
(48.3\%) chose CTC and the remaining 45/87 (51.7\%) preferred OC

Average time interval for follow-up was comparable between CTC and OC $(9.00 \pm 4.24$ vs $9.00 \pm 4.39$ months, respectively; $p=0.7188)$. No patients chose to undergo a 3-year follow-up with either CTC or OC. Most patients elected to have follow-up with either CTC or OC before 18 months rather than later $\left(\mathrm{Chi}^{2}=\right.$ 12.66, $\mathrm{p}=0.0004$, and $\mathrm{Chi}^{2}=12.53, \mathrm{p}=0.0004$, respectively). Between the two techniques, no statistically significant difference was found between the ratios of patients undergoing follow-up before 18 months and from 18 months onwards $\left(\mathrm{Chi}^{2}=0.01, \mathrm{p}=0.9203\right)$

Conclusion: A substantial fraction of C-RADS2 patients prefer to undergo immediate $\mathrm{OC}$ and polyp removal rather than follow-up (either with CTC or OC), and the majority of those accepting follow-up are willing to wait for less than 18 months. Such findings may suggest a revision of the proposed C-RADS2 category.

B-326 14:45

(EPOS)

Digital colonic cleansing of CT colonography images: influence on quality of $2 \mathrm{D}$ and $3 \mathrm{D}$ reconstructions

P. Vagli, L. Faggioni, E. Picano, R. Scandiffio, A. Mantarro, P. Bemi, L. Cini,

E. Neri, C. Bartolozzi; Pisa/IT (paolavagli@yahoo.it)

Purpose: To evaluate the performance of digital colonic cleansing of CT Colonography (CTC) datasets with faecal tagging and its impact on quality of $2 \mathrm{D}$ and $3 \mathrm{D}$ post-processing images.

Methods and Materials: We reviewed 26 cases of CTC positive for small polyps confirmed by conventional colonoscopy, in which patients underwent a 3-day-noncathartic preparation and a same day faecal tagging protocol. Digital cleansing was performed using a dedicated plug-in embedded in the ColonVCAR software (GE Medical Systems, Milwaukee, WI). Two radiologists experienced in CTC, who were unaware of conventional colonoscopic findings, analysed CTC datasets in 2D mode, as well as in 3D mode using Virtual Endoscopy, with and without digital bowel cleansing. CTC image quality in terms of severity of digital subtraction artefacts in 2D mode before and after cleansing was estimated visually in consensus on a per-segment basis using a five-point scale (1=poor through $5=e x c e l l e n t)$, while 3D images with and without cleansing were classified as either diagnostic or not diagnostic.

Results: Quality of 2D images did not differ significantly with and without cleansing in the various colonic segments (ascending colon $4.31 \pm 0.48$ vs. $4.54 \pm 0.66$; transverse colon $4.38 \pm 0.65$ vs. $4.38 \pm 0.51$; descending colon $3.92 \pm 0.49$ vs $3.92 \pm 0.64$; sigmoid colon $3.38 \pm 0.77$ vs. $3.46 \pm 0.66$, respectively; Wilcoxon signed rank test, $\mathrm{p}>0.05$ ). A significantly higher diagnostic rate of $3 \mathrm{D}$ images occurred with cleansing $(22 / 26$ cases $=84.6 \%)$ than without it $(2 / 26$ cases $=7.7 \%$; Fisher's exact test, $\mathrm{p}<0.0001$ )

Conclusion: Digital colonic cleansing does not lead to a significant improvement of $2 \mathrm{D}$ images, but is a prerequisite for $3 \mathrm{D}$ post-processing of tagged CTC datasets.

\section{B-327 14:54}

Computer-aided detection in CT colonography (CTC): which CAD paradigm is best in a screening population?

G. Iussich, L. Correale, C. Senore, D. Campanella, G. Galatola, C. Laudi, P. Falco, N. Segnan, D. Regge; Turin/IT

Purpose: To prospectively compare the diagnostic performance and time efficiency of primary and second reader CAD paradigms.

Methods and Materials: Individuals participating to a CRC screening program and with a positive FOBT test were recruited for same day CTC and CC. Two experienced radiologists independently analysed the $C T C$ studies following randomisation, using CAD either as a first (CAD1) or as a second reader (CAD2); levels of confidence were assigned to positive findings. Reporting time, per-patient sensitivity for patients with adenomas or cancer of at least $6 \mathrm{~mm}$ in size, specificity, along with the relative $95 \%$ confidence intervals, and areas under ROC curves (AUC) were calculated for both reading paradigms. Colonoscopy (CC) and histology were the reference standard. Results: Seventeen of the 199 enrolled individuals (8.5\%) were excluded for: refusal to perform $\mathrm{CC}(3)$, unavailable histological data (4), protocol violation (3) and non-diagnostic image quality (7). The remaining 182 individuals included 93 patients $(51 \%)$ with at least one adenoma $6 \mathrm{~mm}$ or more. Sensitivity with CAD2 and CAD1 was $86 \%(80 / 93)(95 \% \mathrm{Cl}: 77-92 \%)$ and $89 \%(83 / 93)(95 \% \mathrm{Cl}: 81-95 \%)$, respectively $(P=0.5)$. There was no difference in specificity between CAD2 and CAD1 [90\% (80/89) (95\% Cl: 81-95\%) versus 91\% (81/89) $(95 \% \mathrm{Cl}: 83-96 \%)$ ]. The mean AUCs for CAD2 and CAD1 were similar $(P=0.09)$. CAD1 reading took 2 minutes less than CAD2 $(6 \mathrm{~m}: 2 \mathrm{~s} \pm 55 \mathrm{~s}$ versus $8 \mathrm{~m}: 7 \mathrm{~s} \pm 30 \mathrm{~s}, \mathrm{P}=0.008)$. Conclusion: CAD1 is more time efficient and has similar diagnostic performances to CAD2 and should be considered for future mass screening programs, where cost-effectiveness may represent a key issue. 


\section{B-328 15:03 \\ Comparative performance of novel context-sensitive CAD and conventional CAD in the detection of flat lesions in CT colonography J. Näppi', S.H. Park'2, D. Regge ${ }^{3}$, H. Yoshida'; ' 1 Boston, MA/US, ${ }^{2}$ Seoul/KR, ${ }_{3}^{3}$ Candiolo/IT (jnappi@partners.org)}

Purpose: To compare the detection performance of a context-sensitive computeraided detection (CS-CAD) scheme with that of conventional polyp-detection CAD in detecting flat lesions in CT colonography (CTC).

Methods and Materials: 105 colonoscopy-confirmed lesions $\geq 6 \mathrm{~mm}$ categorised as "flat" in cathartic or non-cathartic multi-center CTC studies were measured retrospectively for their maximum height in 2-D multi-planar views. The lesions with maximum height of $<3 \mathrm{~mm}$ were considered as flat lesions for this study. A fully automated CS-CAD scheme was developed to detect colorectal lesions based on context-sensitive algorithms and hyper-shape features. The detection performance of the CS-CAD and conventional polyp-detection CAD were compared with the flat-lesion patients and randomly sampled normal patients. Per-lesion sensitivity, false-positive (FP) rate, and normalised area under free-response operating characteristic curve (AUC) were assessed by the use of leave-one-patient-out evaluation and McNemar's test.

Results: There were 127 patients (male: $52 \%$; female: $48 \%$; mean age: 56 years) from 20 institutions: 109 were normal and 18 had 20 flat lesions ( $\geq 10 \mathrm{~mm}$ : 10 $6-9 \mathrm{~mm}: 10 ; 3$ cancers, 13 adenomas). The use of CS-CAD increased AUC from 0.28 to 0.92 . For flat adenomas/carcinomas, CS-CAD detected $100 \%(100 \%)$ of lesions $\geq 10 \mathrm{~mm}(6-9 \mathrm{~mm})$ with 0.8 (3.1) FPs per patient on average, whereas conventional polyp-detection CAD detected only $63 \%$ (13\%) of lesions $\geq 10 \mathrm{~mm}$ (6-9 mm) with 1.4 (4.8) FPs per patient. The improvement in detection sensitivity by CS-CAD was statistically significant $(p<0.001)$.

Conclusion: CS-CAD yields superior performance over conventional CAD in detecting flat lesions $<3 \mathrm{~mm}$ in maximum height.

\section{B-329 15:12}

CT colonography: development and validation of a novel registration algorithm to align prone and supine scans

D.J. Boone, H. Roth, T. Hampshire, J. McClelland, M. Hu, S.A. Taylor,

G. Slabaugh, D. Hawkes, S. Halligan; London/UK (darrenboone@hotmail.com)

Purpose: Despite colonic cleansing prior to CT colonography, prone and supine acquisitions remain necessary to differentiate pathology from luminal content Observers must therefore match endoluminal locations between prone and supine studies, a process that is complicated by considerable colorectal deformation. We developed and validated a novel algorithm that establishes endoluminal spatial correspondence between prone and supine CT acquisitions.

Methods and Materials: Ethical approval was obtained to use anonymised CT colonography data from 24 patients randomised into 12 development (13 polyps) and 12 validation sets (13 polyps). A radiologist (experience $>500$ validated datasets) indicated the polyp volumes in both prone and supine CTC scans using separate multiplanar reformats. These polyps were masked from the algorithm so as not to influence registration and used as reference points for validation. To perform registration, a triangulated mesh of the endoluminal colonic surface was first extracted from segmented colonic data. Surface parameterisation was then achieved using the Ricci flow method followed by non-rigid B-spline registration in the 2 dimensions of colon length and circumference to recover the deformations. Results: Using the development set, 8 of the 13 polyps were registered successfully $(62 \%)$ with a mean error of $6.6 \mathrm{~mm}(+-4.8)$. In the validation set, 10 of the 13 polyps were registered successfully $(77 \%)$ with mean error of $6.7 \mathrm{~mm}(+-4.6)$ Conclusion: This novel algorithm allows accurate registration of prone and supine datasets, and should considerably simplify the interpretative task in CT colonography. It additionally has the potential to improve computer-aided detection.

\section{B-330 15:21}

CT colonography: comparison of visual search patterns in experienced and novice readers

D.J. Boone ${ }^{1}$, S. Halligan ${ }^{1}$, P. Phillips ${ }^{2}$, S. Mallett ${ }^{3}$, D. Altman ${ }^{3}$, S.A. Taylor' A. Gale ${ }^{4}$, D. Manning $2 ;{ }^{1}$ London/UK, ${ }^{2}$ Carlisle/UK, ${ }^{3}$ Oxford/UK, ${ }^{4}$ Leics/UK (darrenboone@hotmail.com)

Purpose: Diagnostic accuracy can vary between observers because of differences in ability to detect and characterise lesions. Eye tracking technology has been used to explore observers' visual search during plain radiographic interpretation but has not been applied previously to complex 3D tasks. We compared visual search strategies of novice and experienced readers interpreting CT colonography.
Methods and Materials: Endoscopically validated CTC datasets, containing 8 lesions reported by experienced readers in a prior study were selected (mean polyp size $=10 \mathrm{~mm}$; range $5-25 \mathrm{~mm}$ ), including true positive and false positive lesions. Video clips (mean $=26 \mathrm{~s}$ ) incorporating these lesions were interpreted by faculty members $(n=10)$ or participants $(n=19)$ selected from 3 CT colonography workshops. Case order and polyp appearance time were randomised. Visual search was recorded using a Tobii X120 eye tracker and observers indicated potentia polyps with a mouse click.

Results: Visual search patterns were recorded satisfactorily. Of 5 true positive lesions, novice readers indicated a median of 3 lesions (range 1-5) and experienced readers indicated a median of 4 lesions (range 2-5), giving good accuracy calibration data for the development of new 3D vision measures. Both novices and experts identified true positive lesions at a median of 3.3 seconds after the lesion was first visible, although times varied by lesion.

Conclusion: Visual tracking during 3D interpretation is technically feasible. Experienced readers on average identified more lesions, although both novices and experts took on average similar times to indicate polyps. Further studies will assess the effect of training on visual search pattern.

14:00 - 15:30

Room I/K

Cardiac

\section{SS 503}

\section{Coronary CT: economic impact and dose issues}

Moderators

D. Fleischmann; Stanford, CA/US

D. Piotrowska-Kownacka; Warsaw/PL

\section{B-331 14:00}

CT coronary angiography: optimisation of image quality in patients with different body habitus by combined $\mathrm{kV}$ and $\mathrm{mA}$ adaptation

P. Rogalla ${ }^{1}$, N. Paul ${ }^{1}$, H. Meyer ${ }^{1}$, A. Lembcke ${ }^{2}$, J. Mews ${ }^{3}$, S. Kandel ${ }^{1}$;

${ }^{1}$ Toronto, ON/CA, ${ }^{2}$ Berlin/DE, ${ }^{3}$ Neuss/DE (Patrik.Rogalla@uhn.on.ca)

Purpose: We developed a dose adaptation strategy modulated to the patients AP chest diameter (APD) with the goal to minimise variation in image quality while ensuring the highest possible vascular attenuation.

Methods and Materials: 92 patients (mean age 56 year, BMI $27.7 \pm 4.4 \mathrm{~kg} / \mathrm{sqm}$ ) in two institutions underwent prospectively triggered 320 -slice single-beat cardiac CT. Tube voltage was $100 \mathrm{kV}$ in patients with APD up to $25 \mathrm{~cm}, 120 \mathrm{kV}$ between 26 and $30 \mathrm{~cm}$, and $135 \mathrm{kV}$ with APD greater than $30 \mathrm{~cm} ; \mathrm{mA}$ was gradually adjusted to the patient's APD within the kV settings. All other imaging parameters were kept constant including scan-field-of-view, slice thickness, reconstruction method and kernels, and contrast material injection volume and flow. Image noise was defined as standard deviation of attenuation and measured using a ROI in the descending aorta. Variation in image noise, aortic attenuation, signal-to-noise ratios were calculated and statistically compared between the three $\mathrm{kV}$ settings.

Results: Average pixel noise (SD) for 100, 120, $135 \mathrm{kV}$ scans were 29.35 (4.23), 27.7 (3.89), 27.9 (3.98) HU, respectively, without statistical difference. Vascular attenuation (SD) were 488.7 (99.5), 400.0 (67.8), 361.2 (45.9) HU, respectively, and signal-to-noise ratios (SD) were 16.73 (3.07), 14.70 (3.01), 13.24 (3.16) respectively, all significantly different.

Conclusion: Tube output modulation with stepwise increase of kV and gradual adaptation of $m A$ s based on patients' APD is an effective means to reduce variation in image quality in CT coronary angiography whilst assuring the highest possible vascular attenuation in patients with varying body habitus.

\section{B-332 14:09}

Economic analysis of the use of coronary calcium scoring as an alternative to stress ECG in the non-invasive diagnosis of coronary artery disease

V. Raman ${ }^{1}$, E.T.M. McWilliams ${ }^{2}$, S.R.M. Holmberg ${ }^{1}$, K. Miles ${ }^{1} ;{ }^{1}$ Brighton/UK, ${ }^{2}$ Hastings/UK (ramanvivek@doctors.org.uk)

Purpose: A publication by National Institute of Health and Clinical Excellence (NICE) recommends that in low pre-test probability population suspected of coronary artery disease (CAD), a zero coronary calcium score (CCS) excludes CAD. This study evaluates this recommendation by a) undertaking a meta-analysis of studies involving CCS and CAD and b) using the meta-analysis to inform an economic evaluation of a range of diagnostic pathways that use CCS as an alternative to stress-ECG (sECG) 
Methods and Materials: Literature review incorporates CCS involving both electron beam and multi-detector technologies in patients with suspected CAD who underwent invasive angiography (IA). Economic-analysis using decision tree modelling was based on a previous technology appraisal by NICE for Myocardial Perfusion Scintigraphy (MPS). Input data were taken from three sources: aforementioned document, national tariffs and our meta-analysis. Quality adjusted life years (QALY) and treatment costs for different heath states included in our analysis were derived from a Markov model found in NICE appraisal. Parameters for cost-effectiveness include incremental cost effective ratio (ICER) and Cost/QALY.

Results: Meta-analysis of 10760 patients shows CCS of $>0$ is highly sensitive, $96 \%$ (95\% Cl 95.4-96.4) but not specific, $40 \%(95 \% \mathrm{Cl} 38.7-41.4)$ for CAD. A definite cost benefit using CCS testing in non-invasive diagnostic algorithms for CAD is demonstrated. At all prevalence intervals CCS-based methods have ICER values considerably lower than the recommended $£ 30,000$ when compared with sECG strategies. At lower pre-test probabilities it also cost-effective to employ CCS as the first stratifying test prior to MPS or IA.

Conclusion: CCS is a cost-effective alternative to SECG in suspected CAD.

\section{B-333 14:18}

\section{Role of coronary angiography MDCT in the clinical setting: changes of}

diagnostic workup and economic implications

R. Malago, A. Pezzato, C. Barbiani, R. Pozzi Mucelli; Verona/IT

(robertomalag@yahoo.it)

Purpose: To assess the incremental value of MDCT- coronary angiography (MDCT$\mathrm{CA}$ ) in the diagnostic workup and cost management of patients with suspected coronary artery disease (CAD).

Methods and Materials: 550 consecutive patients underwent MDCT-CA between $04 / 2008$ and $07 / 2010$. For each patient pre-test probability of CAD using Morise score was related to exercise test, MDCT-CA and CA. We calculated thereafter the incremental diagnostic value of stress test to MDCT-CA for each category of cardiovascular risk. The traditional diagnostic workup (without MDCT-CA) to the modified workup (with MDCT-CA) in terms of pre-test CAD probabilityleffectiveness and costleffectiveness were compared.

Results: The diagnostic performance of stress test had a sensitivity and specificity of $46.2 \%$ and $73.4 \%$ with PPV and NPV of $48 \%$ and $72 \%$. MDCT-CA demonstrated a sensitivity of $100 \%$, a specificity of $94.7 \%$, PPV of $96.7 \%$ and NPV of $100 \%$. According to Morise Score, in low pre-test probability, stress test accuracy resulted $30 \%$, in moderate pre-test $38 \%$, in high pre-test $45 \%$ considering MDCT-CA as reference. The MDCT-CA modified diagnostic protocol offers an average increased diagnostic performance of $60 \%$ compared with the traditional protocol, and an average cost saving of $€ 1323$ per patient. Conclusion: MDCT-CA is the reference method for the non-invasive exclusion of critical coronary stenosis. Up to a low-medium CAD risk, the MDCT-CA diagnostic workup is the most cost-effective protocol, being superior to the traditional exercise ECG-based protocol.

\section{B-334 14:27}

Cost-effectiveness and lifetime benefits of substituting dual-energy CT for SPECT in the assessment of myocardial perfusion in patients with suspected or known coronary artery disease

M. Meyer ${ }^{1}$, M. Weininger ${ }^{2}$, U.J. Schoepf ${ }^{2}$, G. Rowe ${ }^{2}$, J.W. Nance ${ }^{2}$, D. Kang ${ }^{2}$, S.A. Chiaramida ${ }^{2}$, C. Fink ${ }^{1}$, T. Henzler ${ }^{1} ;{ }^{1}$ Mannheim/DE, ${ }^{2}$ Charleston, SC/US (M.Meyer@stud.uni-heidelberg.de)

Purpose:To compare the cost-effectiveness and potential lifetime benefits of replacing SPECT with dual-energy computed tomography (DECT) for myocardial perfusion assessment in coronary artery disease (CAD).

Methods and Materials: A decision and micro-simulation model was developed to estimate the health effects of using DECT myocardial perfusion imaging instead of SPECT for identifying patients in need of revascularisation. The model was based on the performance of stress/rest DECT and stress/rest SPECT for detecting myocardial perfusion deficits in 50 patients (13 female; $63 \pm 11$ years) with known or suspected CAD. Stress/rest perfusion cardiac MRI with delayed enhancement and decision to revascularise served as the reference standards. For DECT, a reimbursement of $\$ 1700$ was assumed. All other actual healthcare costs in these patients were derived from our hospital billing system using Cranware software. Results: Compared with MRI, DECT (versus SPECT) had 90\% (85\%) sensitivity, $71 \%(58 \%)$ specificity and $87 \%(79 \%)$ accuracy for identifying patients with obstructive CAD in need of revascularisation. Compared with no-imaging and no-treatment strategies, routine SPECT gained 13.49 quality-adjusted life-years (QALYs) with an incremental cost-effectiveness ratio (ICER) of US $\$ 3,557$ (in 2010) per QALY. DECT gained an additional 0.64 QALYs over the SPECT strategy and 14.13 QALYs if compared with no-imaging and no-treatment strategies, with an ICER of US\$3.191 per QALY $(p=0.0002)$.
Conclusion: Using DECT as the first-line imaging modality for myocardial perfusion assessment for known or suspected CAD has the potential to increase QALYs at lower cost compared with SPECT. Prospective studies are needed to verify these findings.

\section{B-335 14:36}

The impact of NICE guidelines for the investigation of chest pain on outpatient radiology services in the UK

C.M. Patterson, E.D. Nicol, L. Bryan, T. Woodcock, D. Bell, S.P. Padley; London/UK (cmpatterson@doctors.net.uk)

Purpose: The National Institute for Health and Clinical Excellence (NICE) have released guidelines for the investigation of chest pain of recent onset. There is concern that the guidelines will increase the burden on imaging services, requiring service reconfiguration and investment $(2,3)$. This study was performed to assess the impact of the guidelines on outpatient radiology services in the UK.

Methods and Materials: 595 consecutive patients attending chest pain clinics at two hospitals over six months preceding release of the NICE guidelines (51\% male; median age 55 yrs (range 22-94 yrs)) were risk stratified using NICE criteria. Preliminary cardiac investigations recommended by NICE were compared with existing clinical practice and the relative costs calculated.

Results: NICE would have recommended 443 patients $(74 \%)$ for discharge without cardiac investigation, 10 (2\%) for cardiac computed tomography (CCT), 69 (12\%) for functional cardiac imaging and $73(12 \%)$ for invasive coronary angiography (ICA). Relative to existing practice there would have been a trend towards reduced functional cardiac imaging $(-24 \% ; p=0.06)$ and increased CCT $(+43 \% ; p=0.436)$ but a significant increase in ICA $(+508 \%$; $p<0.001)$. The cost of investigations recommended by NICE would have been $£ 15,881$ greater than existing practice. Conclusion: This study suggests implementation of the NICE guidelines will require investment in imaging services, particularly ICA. It will be necessary to establish and maintain CCT for relatively few patients; also to establish and maintain functional cardiac imaging even though referrals are likely to decline. Individual hospitals should assess their local cardiac populations prior to service reconfiguration.

\section{B-336 14:45}

Image quality and dose performance of $80 \mathrm{kV}$ low-dose scan protocol in high-pitch spiral coronary CT angiography: feasibility study

D. Wang, X. Hu, Q. Zhang, S. Xie, S. Zhang; Hangzhou/CN

(wd.radiology@gmail.com)

Purpose: To investigate the image quality (IQ) and dose performance of low-kV high-pitch spiral (HPS) coronary CT angiography (CCTA)

Methods and Materials: 106 patients consecutively enrolled into HPS CCTA (pitch = 3.4) were scanned on Definition Flash using $80 \mathrm{kV} / 400$ ref. mAs, $100 \mathrm{kV} / 370$ ref. $\mathrm{mAs}$, and $120 \mathrm{kV} / 370$ ref. $\mathrm{mAs}$ when patient $\mathrm{BMl}$ was $\leq 22.5(\mathrm{n}=40)$, between 22.5 and $27.5(n=53)$ and $>27.5 \mathrm{~kg} / \mathrm{m}^{2}(n=13)$. IQ was assessed per-segment by two observers independently using a 4-point scale (1 - excellent, 4 - undiagnosable). Image noise and signal-to-noise ratio (SNR) at aorta root were measured. The effective dose was calculated using conversion factor of 0.017 .

Results: Diagnostic IQ was obtained in 503 of 507,687 of 668,164 of 167 coronary segments in $80,100,120 \mathrm{kV}$ groups without significant difference $(p=0.482)$. The proportions of segments with score 1 to 4 were not significantly different among three $\mathrm{kV}$ groups (all $\mathrm{p}>0.05$ ). Image noises were significantly higher in $80 \mathrm{kV}$ group than 100 and 120 groups $(P<0.001)$, while SNR was not $(p=0.097)$. The effective dose of $80 \mathrm{kV}$ group $(0.36 \pm 0.03 \mathrm{mSv})$ was significantly lower than that of $100 \mathrm{kV}$ group $(0.86 \pm 0.08 \mathrm{mSv})$ and $120 \mathrm{kV}$ group $(1.77 \pm 0.18 \mathrm{mSv})$.

Conclusion: $80 \mathrm{kV}$ HPS CCTA is feasible for patients with $\mathrm{BMI} \leq 22.5 \mathrm{~kg} / \mathrm{m}^{2}$ which can save $58 \%$ dose than $100 \mathrm{kV}$ group, while maintain diagnosable IQ.

\section{B-337 14:54}

Radiation dose threshold for coronary artery calcium score with MDCT: how low can you go?

T. Newton, K. Wong, H. Mehrez, R. Menezes, A. Crean, E. Nguyn, N.S. Paul; Toronto, ON/CA (narinder.paul@uhn.on.ca)

Purpose:To confirm coronary calcium scoring (CCS) can be performed using an effective dose of $<1 \mathrm{mSv}$. 2 , and to create a reference exposure chart for CCS according to body habitus.

Methods and Materials:1. Phantom validation: Initial validation of noise simulating software using a commercial plaque phantom. 2. In vivo validation: A prospective study including 50 patients with CCS performed. The image raw data of each patient wereprocessed using proprietary software to generate seven new complete CCS image datasets at simulated tube currents ranging from 20 to $300 \mathrm{~mA}$. The 
anonymised datasets were analysed by three readers. The lowest level of tube current at which CCS remained accurate was correlated with six parameters $A$ weight, B: body mass index, $\mathrm{C}$ and $\mathrm{D}$ : $\mathrm{AP}$ and lateral thoracic diameters, $\mathrm{E}$ : the arithmetic mean of (C) and (D) and F: Scout attenuation coefficient (SAC) measured from AP and lateral CT scout projections.

Results: SAC had the closest correlation with the lowest tube current for accurate CCS. The 95\% confidence intervals were established for SAC and lateral thoracic diameter. The lowest value in this range was used to predict the lowest possible tube current that could have been used. These data were used to create an algorithm for dose stratification. Using SAC or lateral diameter, mean effective radiation dose for CCS could be reduced from $1.83 \mathrm{mSv}$ to $0.80 \mathrm{mSv}$ or $0.87 \mathrm{mSv}$, respectively. Conclusion: CCS can be performed using at least $50 \%$ reduction in tube current Radiation dose can be easily tailored to the patient by utilising data from CT scout projections.

\section{B-338 15:03}

(EPOS)

Radiation dose reduction with 320-multidetector computed tomography coronary angiography

M.C. Williams, F.R. Millar, N. Weir, T. Smith, D. Brian, N. Uren, G. McKillop,

E.J.R. van Beek, D.E. Newby; Edinburgh/UK (michelle.williams@ed.ac.uk)

Purpose: Radiation exposure is a major healthcare concern. We aimed to optimise scanning protocols in order to reduce radiation dose, while maintaining image quality.

Methods and Materials: In a single centre cohort study, consecutive patients underwent contrast-enhanced prospective, electrocardiogram-gated, CTCA using a 320-multidetector scanner. We assessed patients before (group 1, $\mathrm{n}=31$ ) and after (group 2 , $\mathrm{n}=32$ ) the implementation of a dose reduction protocol. Dose reduction techniques included reducing detector range, tube current and voltage, limiting acquisition to one heart-beat with a $70-80 \%$ phase window, reducing heart rate to 60 rather than 65 beats per minute (bpm) and using delayed, intermittent and low dose bolus tracking. We present effective dose calculated with $\mathrm{k}$-factors of 0.014 (and 0.023$) \mathrm{mSv} /\left(\mathrm{mGy}{ }^{*} \mathrm{~cm}\right)$. Results: Patients in both groups were of similar age $(58 \pm 14,55 \pm 11), \mathrm{BMI}(28 \pm 6$, $\left.27 \pm 5 \mathrm{~kg} / \mathrm{m}^{2}\right)$ and heart rate $(59 \pm 13,56 \pm 8 \mathrm{bpm})$. The median DLP was lower in group 2 (171 (interquartile range 137-259) vs 308.60 (182-393), $p<0.001)$. The median DLP for bolus tracking was lower in group 2 (20 (10-23.4) vs 31.7 (19.5$51.4), p<0.001)$. Thus, the median effective dose fell from $4.3(7.1)$ to 2.1 (3.9) $\mathrm{mSv}$ for CTCA and $0.72(0.44)$ to $0.46(0.28) \mathrm{mSv}$ for bolus tracking

Conclusion: Radiation dose for 320-multidetector CTCA can be reduced by optimising protocols, without compromising image quality. It is important to consider which $\mathrm{k}$-factor is used when assessing CTCA radiation dose. The radiation dose from bolus tracking and scout images should not be ignored when considering patient exposure, and these can also be markedly reduced.

\section{B-339 15:12}

The influence of chest wall tissue composition in determining image noise during cardiac computed tomography

N.S. Paul, H. Kashani, D. Odedra, P. Rogalla; Toronto, ON/CA

(narinder.paul@uhn.on.ca)

Purpose: To determine the influence of chest wall composition on image quality during cardiac CT compared with other biometric parameters.

Methods and Materials: A prospective study recruiting 50 consecutive patients (35M), mean age 55.2 years, were referred for coronary artery calcium quantification. All scans used $120 \mathrm{kVp}$ and $150 \mathrm{mAs}$. Images were analysed on a 3d workstation (Aquarius Intuition, Terrarecon, CA). Patient weight and body mass index were recorded. Following parameters were measured:anteroposterior and lateral diameters, total (CS Total), fat (CS fat) and muscle and bone cross-sectional area (CSMB). Image noise was measured by prescribing an $\mathrm{ROI}$ in descending aorta. Correlation coefficient $(R)$ was calculated to assess the association between image noise and biometric parameters (MedCal, v11.2, Belgium). Data were stratified based on gender and BMI. For BMI groups, data were stratified based on fat to muscle ratio. Results: For total study population, image noise was highly correlated with weight $(R=0.72)$ and $C S$ total $(R=0.68)$. The strongest association with image noise in males is observed for weight and BMI $(R=0.68)$ followed by CS total $(R=0.59)$. For females it is seen for CS total $(R=0.76)$ with a CS fat $R$ value of 0.72 . For $\mathrm{BMI}$, in groups with a fat: muscle ratio $>1, \mathrm{CS}$ total and tat had the strongest correlations with image noise.

Conclusion: Image noise in cardiac CT correlates more strongly with chest wall component than other patient parameters including BMI and weight. Incorporating this parameter into dose reduction strategies will potentially result in improving protocols.
B-340 15:21

Steroid-induced left ventricular hypertrophy in strength athletes on cardiac MRI

T. Luijkx, B.K. Velthuis, N.H.J. Prakken, W.P.T.M. Mali, M.J.M. Cramer; Utrecht/NL (tluijkx@gmail.com)

Purpose: Cardiac MRI (CMR) is often used to distinguish physiologic cardiac adaptation in athletes from cardiomyopathies when screening remains inconclusive. Earlier research, both with echocardiography and CMR, is ambivalent on the nature of cardiac changes in strength athletes. In an attempt to clarify the extent of ventricular adaptations in strength athletes we investigated strength athletes either admitting or denying the use of anabolic androgenic steroids (AAS) and compared them with non-athletes and athletes practising combined strength-endurance sports. Methods and Materials: 150 men aged 18-62 years underwent CMR (mean age $28 \pm 8$ years): 52 non-athletes ( $0-3 \mathrm{hrs} / \mathrm{wk}$ training), 52 strength-endurance athletes and 46 strength athletes (> $6 \mathrm{hrs} / \mathrm{wk}$ training) of which 20 admitted and 26 denied the use of AAS. Blinded observers experienced in cardiac MRI used a reproducible contour tracing protocol for the assessment of volumes, function, and wall mass. Results: Non-using strength athletes show little difference in cardiac dimensions as compared to non-athletes (LV volume $103.5 \mathrm{vs} .100 .9 \mathrm{ml} / \mathrm{m}^{2}$, LV wall mass $48.2 \mathrm{vs} 47.0 \mathrm{~g} / \mathrm{m}^{2}$ ). Steroid-using strength athletes show an increased LV volume $\left(118.5 \mathrm{ml} / \mathrm{m}^{2}\right)$ and LV wall mass $\left(60.8 \mathrm{~g} / \mathrm{m}^{2}\right)$, not exceeding dimensions found in strength-endurance athletes $\left(127.5 \mathrm{ml} / \mathrm{m}^{2}\right.$ and $65.9 \mathrm{~g} / \mathrm{m}^{2}$, respectively). Ventricular and septal diameters show similar results, but maximum septal wall thickness is higher in steroid-using strength athletes than in strength-endurance athletes (4 chamber/short axis view $12.5 / 12.0$ vs. $11.0 / 11.1 \mathrm{~mm}$ ).

Conclusion: Left ventricular dimensions in steroid-using strength athletes are increased as compared to non-using strength athletes and non-athletes, but do not exceed those of strength-endurance athletes.

14:00 - 15:30

Room L/M

Vascular

\section{SS 515}

\section{Peripheral arteries}

Moderators

F. Fanelli; Rome/IT

V. Sarajlic; Sarajevo/BA

\section{B-341 14:00}

Feasibility of ECG-triggered non-enhanced MR angiography of peripheral arteries in patients with symtomatic PAOD in comparison with DSA as goldstandard

M. Rasmus, S. Partovi, A. Jacob, M. Aschwanden, G. Bongartz, D. Bilecen; Basle/CH (mrasmus@uhbs.ch)

Purpose: To evaluate non-enhanced-MRA (NE-MRA) acquired with a 3D Turbo Spin Echo sequence with ECG triggering versus DSA as reference standard in symptomatic PAOD patients.

Methods and Materials: 23 PAOD patients with clinical indication for DSA-guided intravascular intervention underwent NE-MRA 1day after intervention (native SPACE, Magnetom Espree 1.5 T, Siemens). Two blinded readers ( $r 1$, r2) scored 66 preselected stenoses-grades (StG) from $1(<10 \%)$ to $4(>90 \%)$. The intervetionalist scored the same preselected stenosis-grades from DSA as goldstandard (DSAStG). Segmentwise comparison consisted of comparison between MRA and DSA in 95 vessel segments from 1 (good quality) to 4 (inadequate).

Results: 3 datasets were excluded due to insufficient image data. Stenosis were graded higher in NE-MRA than in DSA (mean StG r1:3.0, r2:3.1, $\kappa$ 0.59; DSAStG 2.7; $p(r 1), p(r 2) 0.001)$. Segmentwise comparison revealed mean $r 1: 2.7$ and r2:2.9 (к 0.34)

Conclusion: This study can be seen in context of a NE-MRA renaissance due to NSF and in second line increasing cost pressure. The new NE-MRA technique revealed only limited visualisation quality in symptomatic patients with multiple- and high-grade stenosis. We assume that especially flow disturbance due to multiple stenosis may have hampered visualisation quality of the flow-dependent technique in this collective. Even though we assume restricted clinical potential as "fall back" after failed CE-MRA, anatomic pre-imaging for future MRI-guided interventions and maybe in subjects with less flow disturbance (i.e. screening). A further advance of NE-MRA techniques towards a reliable diagnostic tool for patients with renal impairment and PAOD remains desirable. 


\section{B-342 14:09}

MRA of the calf station at $3 \mathrm{~T}$ : intraindividual comparison between nonenhanced ECG-gated flow-dependent MRA, continuous table movement MRA and time-resolved MRA

S. Haneder, U.I. Attenberger, P. Riffel, T. Henzler, S.O. Schönberg,

H.J. Michaely; Mannheim/DE

Purpose: To compare 3D-non-enhanced ECG-gated inflow-dependent MRangiography (NATIVE-MRA) vs. continuous table movement MR-angiography (C ${ }^{\text {TM}}$-MRA) and time-resolved TWIST-MRA in the calf station at 3.0 T.

Methods and Materials: 36 consecutive patients $(27 \mathrm{~m} / 9 f, 66.1 \pm 14.4$ years) with PAOD (stages II-IV) were included in this IRB-approved prospective study. During a single MR-exam, patients underwent NATIVE-MRA (TR $=2 \mathrm{R}-\mathrm{R}$ intervals $/ \mathrm{TE}=$ $34 \mathrm{~ms} / 1.3 \mathrm{~mm}$ isotropic resolution-ir) of the calf station as well as contrast-enhanced $\mathrm{C}^{\text {TM}}$-MRA (TR $=2.4 \mathrm{~ms} / \mathrm{TE}=1.0 \mathrm{~ms} / 1.2 \mathrm{~mm}$ ir ) and TWIST-MRA (TR $=2.8 \mathrm{~ms} / \mathrm{TE}=$ $1.1 \mathrm{~ms}) / 1.1 \mathrm{~mm}$ ir). A total of $0.1 \mathrm{mmol} / \mathrm{kg} \mathrm{BW}$ gadobutrol were injected at $1.5 \mathrm{~mL} / \mathrm{s}$ (70\% C TM $^{\mathrm{TM}}$-MRA; $30 \%$ TWIST-MRA). The image quality (IQ) as well as the degree of tenosis were rated on a four-point scale ( $4=$ excellent- $1=$ non-diagnostic; $4=$ occlusion-1 $=$ insignificant wall changes). Positive (PPV) and negative predictive values (NPV), sensitivity (SS) and specificity (SP) for stenosis detection were calculated for NATIVE-MRA vs. C'M-MRA and NATIVE-MRA vs. TWIST-MRA. Values were obtained for overall graduation of vessel wall changes and for severe stenosis $(>70 \%)$. Results: With NATIVE-MRA 122/288 (42.4\%) segments were not assessable due to patient motion. Compared to $\mathrm{C}^{\mathrm{TM}}$-MRA and TWIST-MRA the IQ was significantly inferior $(p<0.0001-p=0.0426)$. C ${ }^{T M}$-MRA/TWIST-MRA detected stenoses in $44.9 \% / 46.1 \%$ of segments, whereof $85.0 \% / 82.9 \%$ were high-grade stenoses. NATIVE-MRA detected stenoses in $53.5 \%$ of segments thereof $94.0 \%$ high grade. SS/NPV of the NATIVE-MRA ranged from $97.8 \%$ to $100 \%$. The SP and PPV ranged from $72.7 \%$ to $85.5 \%$ and $66.7 \%$ to $78.2 \%$.

Conclusion: Contrast-enhanced MRA techniques are superior to the NATIVE-MRA in terms of IQ and detection of stenosis. If technically successful, NATIVE-MRA is characterised by high NPV and overestimation of the degree of stenosis.

\section{B-343 14:18}

MR angiography of the peripheral arteries with gadobenate dimeglumine: comparison of image quality and diagnostic accuracy of a standard dose at $1.5 \mathrm{~T}$ versus a low dose at $3 \mathrm{~T}$

M. Anzidei, A. Napoli, B. Cavallo Marincola, L. Di Mare, G. Cartocci,

C. Catalano, R. Passariello; Rome/IT (michele.anzidei@gmail.com)

Purpose: To compare a standard $(0.1 \mathrm{mmol} / \mathrm{kg} \mathrm{BW})$ and a low $(0.05 \mathrm{mmol} / \mathrm{kg} \mathrm{BW})$ gadobenate dimeglumine dose, respectively, at $1.5 \mathrm{~T}$ and $3 \mathrm{~T}$ in terms of image quality and diagnostic accuracy for contrast-enhanced MR-angiography (CE-MRA) of the peripheral vessels relative to CT-angiography (CTA).

Methods and Materials: 60 patients were randomly assigned to 2 groups of 30 subjects. Group 1 underwent CE-MRA at $1.5 \mathrm{~T}$ using a $0.1 \mathrm{mmol} / \mathrm{kg}$ dose while group 2 underwent CE-MRA at $3 \mathrm{~T}$ using a $0.05 \mathrm{mmol} / \mathrm{kg}$ dose of gadobenate dimeglumine. All patients underwent CTA. Three blinded observers reviewed MRA datasets while one independent observer evaluated CTA images for the assessment of stenosis degree (>50\%), extent and location. Signal-to-noise (SNR) and contrast-to-noise (CNR) ratios were calculated for each MRA dataset. Interobserver agreement for CE-MRA was analysed using Cohen-k statistics. Sensitivity, specificity, accuracy, positive predictive value (PPV) and negative predictive value (NPV) were calculated for each group. Differences between groups were assessed using McNemar's test. Results: Interobserver agreement was substantial for image quality and stenosis assessment. Similar values for SNR and CNR were obtained for both groups $(p>0.05)$. Sensitivity, specificity accuracy, PPV and NPV values of Group1/Group2 for stenosis assessment compared to CTA were 95/96\%, 95/95\% 97/97\%, 96/95\% and 97/97\%, respectively. The diagnostic performance of groups 1 and 2 was not significantly different $(p>0.05)$

Conclusion: Image quality and diagnostic performance of CE-MRA with a low $0.05 \mathrm{mmol} / \mathrm{kg}$ BW dose of gadobenate dimeglumine at $3 \mathrm{~T}$ is similar to that achieved with a standard $0.1 \mathrm{mmol} / \mathrm{kg} \mathrm{BW}$ dose at $1.5 \mathrm{~T}$.

\section{B-344 14:27}

Dynamic contrast-enhanced MRI with gadofosveset in peripheral arterial disease: initial findings of hyperemic fractional microvascular blood volume assessment

B. Versluis ${ }^{1}$, T. Leiner ${ }^{2}$, P.J. Nelemans ${ }^{1}$, G.-W.H. Schurink ${ }^{1}$, J.E. Wildberger ${ }^{1}$ W.H. Backes ${ }^{1} ;{ }^{1}$ Maastricht/NL, ${ }^{2}$ Utrecht/NL (bas.versluis@rad.unimaas.nl)

Purpose: The aim of the current study was to describe a method to assess the fractional hyperaemic microvascular blood volume of the calf musculature in patients with peripheral arterial disease (PAD) and healthy control subjects, using a blood pool contrast agent (Gadofosveset) in dynamic contrast-enhanced (DCE) MRI.
Methods and Materials: Ten patients with proven PAD (Fontaine II, TASC-II type-A lesion of the superficial femoral artery) and 10 healthy control subjects were included. Patients underwent DCE perfusion imaging of the calf musculature once, whereas controls were examined twice on different days to determine normative values and the reproducibility of the technique. Hyperaemic fractional microvascular blood volume $\left(\mathrm{V}_{\mathrm{p}}\right.$, unit: \%) of the anterior tibial, gastrocnemius and soleus muscle were determined during reactive hyperaemic conditions as provoked by a standardised cuff paradigm.

Results: Fractional microvascular blood volume $\mathrm{V}_{\mathrm{p}}$ was significantly lower for all muscle groups in PAD patients compared to controls (range differences 3.2-4.8\%, $p<0.01)$. Differences in $V_{p}$ between the muscle groups were not significant $(p=$ 0.49 and $p=0.15$ in patients and controls, respectively). Interscan and interreader coefficient of variation in controls ranged from 10 to $13 \%$ and 3 to $4 \%$, respectively, for the different muscle groups.

Conclusion: DCE MRI using a blood pool contrast agent is able to reproducibly determine the fractional hyperaemic microvascular blood volume of the calf musculature and to discriminate between PAD patients and healthy control subjects. Blood pool agents are therefore a valuable addition to DCE MRI in PAD.

\section{B-345 14:36}

Diagnostic efficacy and image quality of 64-slice CT angiography of the abdominal aorta and abdominal arteries at $100 \mathrm{kVp}$ versus $120 \mathrm{kVp}$ J.-N. Dacher ${ }^{1}$, C. Becker ${ }^{2}$, J. De Mey ${ }^{3}$, M. Favat ${ }^{4}$, S. Heye ${ }^{5}$, A. Larralde ${ }^{6}$, R. Marangoni ${ }^{7}$, P. Reimer ${ }^{8}$, C. Loewe ${ }^{9} ;{ }^{1}$ Rouen/FR, ${ }^{2}$ Munich/DE, ${ }^{3}$ Brussels/BE, ${ }^{4}$ Belluno/IT, ${ }^{5}$ Leuven/BE, ${ }^{6}$ Rennes/FR, ${ }^{7}$ Turin/IT, ${ }^{8}$ Karlsruhe/DE, ${ }^{9}$ Vienna/AT (Jean-Nicolas.Dacher@chu-rouen.fr)

Purpose: To assess the influence of $x$-ray tube voltage (100 vs $120 \mathrm{kVp}$ ) on various efficacy parameters and dose reduction in abdominal 64-slice MDCT angiography. Methods and Materials: The present research is an ancillary study of a previously published prospective multicentre multivendor trial. Patients $(n=310)$ were randomised to undergo MDCT angiography with administration of iobitridol (350 mgl/ $\mathrm{mL}$ ) or iomeprol (400 mgl/mL). Each centre applied its own $\mathrm{mAs} / \mathrm{kVp}$ for abdominal MDCT. Types of contrast medium (CM), body mass index (BMI) and presence or absence of endoprosthesis (EP) were studied as confounding factors. Dose estimates were retrospectively collected. The primary endpoint was to assess the influence of $x$-ray tube voltage on the diagnostic efficacy. Secondary objectives were to assess the image quality and dose savings obtained at $100 \mathrm{vs} 120 \mathrm{kVp}$. Results: Among the 307 analysed patients, $18 \%$ were explored at $100 \mathrm{kVp}$. Baseline characteristics including confounding factors were balanced between both $\mathrm{kV}$ groups. The diagnostic contribution was equivalent between both $\mathrm{kV}$ groups with no impact of BMI, EP or CM iodine concentration. Effective dose for MDCT angiography was $5.7 \pm 1.9 \mathrm{mSv}$ versus $15.5 \pm 8.4 \mathrm{mSv}$, respectively, in 100 - and $120 \mathrm{kVp}$ patients. Image quality, including arterial enhancement and vessel wall visualisation, was deemed significantly superior at $120 \mathrm{kVp}$.

Conclusion: Although setting the $\mathrm{x}$-ray tube at $100 \mathrm{kVp}$ does not impact the diagnostic efficacy of abdominal 64-slice MDCT angiography, it negatively impacts the image quality. Scanning patients at $100 \mathrm{kVp}$ is compatible with a two-third reduction of the radiation dose.

\section{B-346 14:45}

Role of dual energy CT angiography in the evaluation of peripheral arteries disease

A. Bottari, S. Caloggero, T. Mandolfino, R. Romeo, V. Cassone, S. Lamberto; Messina/IT (bottaria@unime.it)

Purpose: To evaluate advantages and limitations of DE CTA in comparison with DSA in the study of lower limbs arteries.

Methods and Materials: From April 2007 to September 2009, 150 patients underwent DE CTA of the aorta and lower limbs with the following study protocol: collimation 2 × 32 × 0.6 mm; pitch 0.65; kV 140 (A tube) and 80 (B tube). Reconstruction parameters were: thickness $1.0 \mathrm{~mm}$, gap $0.7 \mathrm{~mm}$ and DE composition 0.3 . In all the patients $80-110 \mathrm{ml}$ of iodinated contrast material was injected at a rate of $4 \mathrm{ml} / \mathrm{sec}$. After images evaluation 70 patients were collected as candidate for endovascular treatment and underwent DSA. Findings obtained by angiography were compared with pre-treatment DE CTA.

Results: In all patients a very close correspondence between the two technique were reported showing for the DE CTA, a sensibility of $99 \%$ and specificity of $97 \%$. Moreover, using thin slice $(1 \mathrm{~mm})$ for 3D reconstruction we reduce artefacts from bone-vessel closeness. Thus, diagnostic accuracy was improved and no significant difference in lesion detection was reported between above or below the knee. 
Conclusion: DE CTA has diagnostic accuracy higher than other non-invasive imaging technique and very similar to DSA that still represent the gold standard in vascular imaging. Moreover, automatic bone removal application allow fast post-processing time generating in few seconds 3D MIP images of vascular tree (luminogram) similar to DSA images. This improve confidence for non-vascula radiologist and other specialists and also allow a perfect therapeutic planning for endovascular treatment.

\section{B-347 14:54}

Dose reduction in lower extremity arterial imaging using a reduced tube voltage and automatic tube current modulation technique with 64-slice computed tomography

Y. $\mathrm{Ge}^{1}$, Y. Guo 1 , Z. Wen ${ }^{1}, \mathrm{~J} . \mathrm{Li}^{2}, \mathrm{X} . \mathrm{Su}^{1} ;{ }^{1}$ Zhengzhou/CN, ${ }^{2}$ Beijing/CN

(cjr.geyinghui@vip.163.com)

Purpose: To evaluate the use of automatic tube current modulation (ATCM) and $80 \mathrm{kVp}$ tube voltage for radiation dose reduction for lower extremity arterial imaging in multi-slice CT.

Methods and Materials: Eighty-two consecutive diabetic outpatients with lower extremity occlusive disease were randomly divided into three groups with different scanning techniques on a 64-slices CT scanner. The first group used standard $120 \mathrm{kVp}$ and fixed tube current of $180 \mathrm{mAs}$ (group1, $\mathrm{n}=26$ ), and the second (group2, $\mathrm{n}=26$ ) and third groups (group3, $\mathrm{n}=30$ ) used $120 \mathrm{kVp}$ and $80 \mathrm{kVp}$ with ATCM, respectively. The pelvic, knee, calf and foot were four observing levels for noise measurement and image quality assessment with a 3-point scale. Paired analyses were performed on radiation dose, image quality and image noise using t-test.

Results: The scan lengths for all three groups were statistically the same $(p>0.05)$. The CTDI $\mathrm{vol}_{\text {vol }}$ and DLP values for the three groups were $(14.48 \pm 3.60,5.56 \pm 2.16$ and $2.99 \pm 0.73 \mathrm{mGy})$ and $(1703.54 \pm 347.7,679.6 \pm 267.3$ and $360.95 \pm 91.47 \mathrm{mGy} \cdot \mathrm{cm})$. Radiation dose was significantly reduced by $60 \%$ and $79 \%$ for group2 and group3, respectively, compared with group $1(p<0.05)$. Group3 and group 1 had the highest and lowest image noises, respectively. However, there was no significant difference in image quality scores $(2.57 \pm 0.50,2.26 \pm 0.48$ and $2.56 \pm 0.56)$ between the three groups $(p>0.05)$

Conclusion: Using ATCM for lower extremity arterial scanning can reduce radiation dose. Radiation dose can further be reduced with equivalent image quality by using $80 \mathrm{kVp}$.

\section{B-348 15:03}

Evaluation of an iterative reconstruction algorithm (iDose) to reduce image noise in a preclinical model of low dose CT angiography

A.A. Fingerle, P.B. Noël, A. Huber, E.J. Rummeny, M. Dobritz; Munich/DE (alexander.fingerle@gmx.de)

Purpose: In this work we present the evaluation of a hybrid-iterative reconstructionalgorithm (iDose, PhilipsHealthcare) for the reduction of image noise and improvement of image-quality in a preclinical model of low-dose CTA.

Methods and Materials: A swine intestinal specimen consisting of the bowel and the mesentery was used as a model. After injection of a contrast solution in the SMA, the intestinal specimen was scanned in a water bath on a 256-slice multi-detector CT (Brilliance iCT, PhilipsHealthcare) using 15 low-dose combinations of tube-voltage and tube-current settings (80-120 kVp; 15-130 mAs). In additiona, a high-dose scan (140 kV/650 mAs) was performed as reference to the low-dose acquisitions. Low-dose images were reconstructed with a conventional reconstruction-algorithm and in three different iDose-levels. Altogether 60 different image sets were generated. Image-noise was measured in vessels and the intestinal wall. Subjective image-quality and delineation of vessels were addressed. Statistical analysis was performed.

Results: CTA performed with the low-dose protocol showed an average dose reduction by a factor of 2 compared to the standard-dose protocol. Image-noise was higher by a factor of 2 in low-dose images reconstructed with the standard reconstruction. Reconstructing low-dose data with the iDose reconstruction-algorithm, image-noise was lowered to a comparable level of standard-dose CT angiography $(p<0.01)$. On this note, all image information was retained in low-dose images reconstructed with iDose.

Conclusion: iDose allows dose reduction in MDCT angiography. Further iDose provides a significantly reduction of image-noise and thereby retains the imagequality equivalent of standard-dose CT. This may allow a routine dose reduction in the clinic with high impact on patient health.

\section{B-349 15:12}

"Fail to plan and you plan to fail". Patient management and therapy decision according to transatlantic inter-society consensus (TASC) guidelines for peripheral artery obstructive disease (PAOD): a prospective 64-CT angiography study using DSA as reference

A. Napoli, F. Zaccagna, M. Anzidei, G. Cartocci, L. Molisso, L. di Mare,

C. Catalano, R. Passariello; Rome/IT (alessandro.napoli@uniroma1.it)

Purpose: To evaluate the diagnostic accuracy of 64-slice CTA for the detection of peripheral artery stenosis and to compare provisional therapeutic approach suggested after 64-CTA using DSA as reference standard.

Methods and Materials: 212 consecutive patients with disabling intermittent claudication scheduled for DSA underwent CTA $(64 \times 0.6 \mathrm{~mm} ; 110 \mathrm{ml}$ of lomeprol-400 at $4 \mathrm{ml} / \mathrm{sec}$ ). Image evaluation was performed with the arterial vascular system divided into 35 segments, detected stenoses were graded using a 4-point scale (0-4: normal-occlusion). Lesions were classified according to TASC guidelines and a provisional indication was made as to whether treatment should most likely be conservative, endovascular, surgical or combined. Diagnostic performance (accuracy, sensitivity, specificity, PPV and NPV) was obtained considering also uninterpretable CTA results. Chi-square, McNemar and Wilcoxon's rank tests were used as appropriate.

Results: A total of 7420 arterial segments pooled into 1060 vascular regions were assessed. McNemar's test showed no significant difference between CTA and DSA $(0.62<\mathrm{p} 0.05)$. After DSA, 50/210 (28\%) patients were referred for conservative treatment, 94/210 (44\%) underwent endovascular procedures, 39/210 (19\%) underwent surgery and 18/210 (9\%) underwent an intra-operative hybrid (endovascular and surgical) treatment. Therapy recommendation based on CTA findings alone was identical to that of DSA for all but one patient who had a type $D$ infra-inguinal lesion. Conclusion: 64-CTA yields very high diagnostic accuracy without influence of plaque composition; these data translate into reliable therapy decision-making and patient management as compared to DSA.

\section{B-350 15:21}

Correlation between arterial peak flow and ankle-brachial index in peripheral arterial disease

B. Versluis ${ }^{1}$, T. Leiner ${ }^{2}$, P.J. Nelemans ${ }^{1}$, G.-W.H. Schurink ${ }^{1}$, J.E. Wildberger ${ }^{1}$, W.H. Backes ${ }^{1} ;{ }^{1}$ Maastricht/NL, ${ }^{2}$ Utrecht/NL (bas.versluis@rad.unimaas.nl)

Purpose: The ankle-brachial index (ABI) measurement is an important functional measure in the diagnosis and follow-up of peripheral arterial disease (PAD). $A B I$ measurements, however, are hampered by large interobserver variability and inconclusive results in patients with uncompressible arteries. Alternatively, MRI can be used to determine arterial peak flow (APF) as a substitute functional measure. In this study, APF of the popliteal artery and the ABI in PAD patients is compared. Methods and Materials: 124 patients with $P A D$, varying from intermittent claudication $(n=67)$ to critical ischaemia $(n=57)$, were included. All subjects underwent vector cardiography triggered quantitative $2 \mathrm{D}$ cine MR phase-contrast angiography of the popliteal artery to determine APF during a routine contrast-enhanced MR angiography. All APF measurements were analysed by one radiologist with 3 years of experience. $\mathrm{ABI}$ measurements were performed by a small team of Doppleroperators with $>10$ years of experience.

Results: APF was successfully measured in $87 \%$ of the patients, whereas the ABI could only be determined in $70 \%$ of the patients. Pearson correlation between APF and $A B I$ was 0.72 in intermittent claudication and 0.47 in critical ischaemia, both were significant at the 0.01 level. Both APF and ABI were significantly lower in critical ischaemia as compared to intermittent claudication $(p<0.01)$.

Conclusion: The APF is a functional vascular measure that can be determined in more patients than the $A B I$, is not influenced by uncompressible arteries, and correlates significantly with the $\mathrm{ABI}$ and is therefore a valuable measure in PAD patients, both for diagnostic and follow-up purposes. 


\section{Neuro}

\section{SS 511b}

\section{Neurovascular diseases}

Moderators:

I.Q. Grunwald; Oxford/UK

A. van der Lugt; Rotterdam/NL

\section{B-351 14:00}

Lenticulostriate arterial lumina are normal in CADASIL: a high field in vivo magnetic resonance imaging study

M.K. Liem ${ }^{1}$, J. van der Grond'1, M.J. Versluis', J. Haan², A.G. Webb',

M.D. Ferrari', M.A. van Buchem'1, S.A.J. Lesnik Oberstein'; ${ }^{1}$ Leiden/NL,

${ }^{2}$ Leiderdorp/NL (m.k.liem@lumc.nl)

Purpose: Cerebral autosomal dominant arteriopathy with subcortical infarcts and leukoencephalopathy (CADASIL) is a hereditary small vessel disease. Although postmortem studies have demonstrated mural thickening in leptomeningeal arteries and lenticulostriate perforating arteries, it is unclear whether this also leads to luminal narrowing. High-field MRI scanners enable in vivo imaging of the lumen of the lenticulostriate arteries. The aim of this study was to examine the luminal diameters of lenticulostriate arteries in living CADASIL patients and to investigate whether luminal narrowing is correlated with the number of lacunar infarcts in the basal ganglia.

Methods and Materials: $22 \mathrm{NOTCH} 3$ mutation carriers (MCs) and 11 healthy controls were examined using high-resolution 3D-time-of-flight magnetic resonance angiography (TOF MRA) imaging on a 7-Tesla MRI scanner. Scans were analysed for the presence of focal stenotic segments. The total number, length and total cross-sectional area of lenticulostriate arteries were measured and compared between mutation carriers and controls. These measurements were correlated with age, disease duration and number of lacunar infarcts in the basal ganglia. Results: No stenotic segments were observed. No differences between MCs and controls were found in total number of end branches (MCs: mean $=14.6$; controls: mean $=12.8$ ), length of the lenticulostriate system or total cross-sectional area of lenticulostriate artery lumina. Measurements of lenticulostriate artery lumina were not associated with lacunar infarct load in the basal ganglia area or with basal ganglia hyperintensities.

Conclusion: 3D TOF MRA on 7-Tesla showed no differences in luminal diameters of lenticulostriate arteries between CADASIL patients and controls.

\section{B-352 14:09}

$7 \mathrm{~T} \mathrm{MRI} \mathrm{reveals} \mathrm{diffuse} \mathrm{areas} \mathrm{of} \mathrm{T}^{*}$ hypointensity in the putamen and caudate nucleus in CADASIL

M.K. Liem ${ }^{1}$, S.A.J. Lesnik Oberstein', M.J. Versluis', J. Haan², A.G. Webb', M.D. Ferrari', M.A. van Buchem 1 , J. van der Grond'; ' 'Leiden/NL, ${ }^{2}$ Leiderdorp/NL (m.k.liem@lumc.nl)

Purpose: Cerebral autosomal dominant arteriopathy with subcortical infarcts and leukoencephalopathy (CADASIL) is a hereditary form of small vessel disease. Cerebral microbleeds can be visualised in up to $40 \%$ of patients with MRI scanners at standard field strengths. However, these scanners have a limited sensitivity in detecting more subtle cerebral damage associated with iron deposition. The aim of this study was to quantify focal and diffuse iron deposition in CADASIL, using 7 Tesla MRI.

Methods and Materials: Twenty-five NOTCH3 mutation carriers and 15 healthy controls were examined using high-resolution $\mathrm{T}^{*}$-weighted imaging on a 7 Tesla whole body MRI scanner. MRI scans were analysed for focal and diffuse areas of decreased signal intensity. Mean signal intensity measurements in affected brain regions were compared between mutation carriers and controls.

Results: Diffuse areas of decreased signal intensity were found in mutation carriers. Compared with healthy controls, mutation carriers had significantly lower signal intensity in the putamen $(p=0.005)$ and caudate nucleus $(p=0.0007)$. Focal areas of decreased signal intensity were found in $36 \%$ of mutation carriers and in none of the healthy controls. These areas were predominantly $(66 \%)$ located in the thalamus. Other locations included the subcortical and deep white matter. Conclusion: 7 Tesla MRI reveals increased areas of hypointensity in the putamen and caudate nucleus of CADASIL patients that is likely caused by increased diffuse iron accumulation. Focal areas of decreased signal intensity were also found in $36 \%$ of CADASIL patients, in a pattern consistent with microbleeds.
B-353 14:18

Cocaine-induced vasculitis in subarachnoid haemorrage (SAH): main diagnostic and angiographic patterns

E. Puglielli, M. Fuschi, V. Di Egidio, M. Monina; Teramo/IT (edopug@hotmail.com)

Purpose: Aim of the study is to describe the main angiographic patterns of the cocaine-induced vasculitis changes on cerebral angiograms in $\mathrm{SAH} /$ stroke patients. Methods and Materials: From April 2007 to September 2010, we retrospectively identified thirteen patients (mean age 43.7 years), referred for acute $\mathrm{SAH} / \mathrm{stroke}$, after use of cocaine, with positive urine toxicology or its metabolites. The subjects underwent computed tomography angiography (CTA), magnetic resonance angiography (MRA) and digital subtraction angiography (DSA) valuation shortly after. Results: The main symptoms were headache, hemiparesis, agitation, and aphasia. Seven patients showed on CT mild SAH and a large putaminal haemorrhage, while at DSA multiple mild- and small-vessel occlusions were found. In four patients, CT showed a large SAH in the basal cisternal, while MRA and DSA demonstrated segmental narrowing/dilatations of medium-sized intracranial arteries. In two patients, a wide basal cisternal and interemispherical SAH were found on $\mathrm{CT}$; in one of the above patients DSA revealed multiple infundibulomas of the main intracranial bifurcations, with a $23 \mathrm{~mm}$ diameter size, anterior communicating aneurism that were subsequently embolised. Data were analysed with a Fischer exact test $(p<0.05)$. Conclusion: The rate of strokes among cocaine abusers is broadly increasing in the last few years. The exact mechanism of cocaine-induced stroke remains still unclear, but is probably a consequence of alteration in the cerebral flow autoregulation, due to several factors including vasospasm, cerebral vasculitis, enhanced platelet aggregation, and hypertension. Although histopathologic examination is required for a definitive diagnosis, CTA, MRA, and DSA can support the neuroradiologic evaluations.

\section{B-354 14:27}

Manganese-induced toxic-metabolic encephalopathy: clinical and radiological findings

F.I. Todua, D. Miminoshvili, M. Okujava; Tbilisi/GE (dmiminoshvili@yahoo.com)

Purpose: To review neurological abnormalities (clinical and neuropsychological changes) and results of radiological (Multi-slice CT, MRI) examinations in methcathinone users. This psychoactive substance is synthesised from drugs which contain ephedrine and potassium permanganate.

Methods and Materials: We have investigated 32 patients (28 males and 4 females) with chronic intravenous use of methcathinone. Patient age ranged from 23 to 54 years. All patients underwent MRI and MSCT of the brain.

Results: On MRI examination the brain abnormalities were revealed in 28 cases: there was a hyperintense signal on T1-weighted images in globus pallidum $(n=24)$ substantia nigra $(n=22)$, nuclei dentatus of cerebellum $(n=18)$, anterior pituitary lobe $(n=11)$, and white matter of the great hemispheres $(n=3)$. The signal intensity was higher after recent and frequent use of the Methcathinone. The signal abnormalities on MR were caused by the accumulation of the manganese, which is byproduct of the synthesised street drug methcathinone, in the basal ganglia and other structures. On CT investigation leukoaraiosis was revealed in 15 cases, basal gangia calcifications in five and cerebellar atrophy in four cases. Most patients $(85 \%)$ had extrapyramidal disorders: bradykinesia, postural disturbances, ataxia, tremor, muscular dystonia (mostly of the face and lower extremities). Pseudobulbar syndrome (dysarthria, dysphonia) were revealed in $10 \%$ cases. Cognitive abnormalities (personality changes, stupor) were noted in $5 \%$ cases.

Conclusion: Thus, in cases of methcathinone abuse MRI and MSCT examinations are strongly recommended to find out the cerebral anomalies and determine treatment tactics.

B-355 14:36

Supratentorial superficial siderosis: localisation, clinical symptoms and differential diagnoses

J. Linn ${ }^{1}$, K. Bochmann', T. Pfefferkorn', P. Demaerel ${ }^{2} ;{ }^{1}$ Munich/DE, ${ }^{2}$ Leuven/NL (linn@nrad.de)

Purpose: Supratentorial superficial siderosis (SupS) has recently been shown to be a typical MRI sign of cerebral amyloid angiopathy (CAA). Case reports suggest that SupS is responsible for transitory ischaemic attack or aura-like symptoms in CAA. We investigated the typical localisation and clinical symptoms of SupS in a larger patient population and identified its most probable causes.

Methods and Materials: We searched the electronic databases of our institutions to identify all patients in whom a SupS was detectable on T2*-weighted gradient echo $\left(T 2^{*} \mathrm{~W}\right)$ sequences. Exclusion criteria were history of aneurysmal subarachnoid 
haemorrhage, head trauma or neurosurgical intervention. T2* $\mathrm{W}$ was analysed by two neuroradiologists in consensus regarding the localisation of SupS and with regard to the presence of microbleeds (MBs) or macrohaemorrhages (ICHs). Clinical records were assessed to determine the clinical symptoms. The most probable causes underlying the SupS were identified based on all clinical, laboratory, imaging and histological information.

Results: 52 patients ( $73 \pm 4$ years) matched the inclusion criteria. In most cases SupS involved the central sulcus. Additional ICHs were detected in 29 patients, 17 patients had MBs. Most common symptoms were headaches, focal seizures or transitory ischaemic attacks. The following causes were identified: probable CAA (39 cases), definite CAA ( 3 cases), reversible cerebral vasoconstriction ( 2 cases). In 7 cases the underlying diagnosis could not be determined.

Conclusion: SupS presents with typical clinical symptoms, which are most probably caused by the common involvement of the central sulcus. In older patients, CAA is the most important cause of SupS.

\section{B-357 14:45}

Carotid artery plaque histology and brain MR

L. Saba, L. Lai, R. Sanfilippo, R. Montisci, G. Faa, G. Mallarini; Cagliari/lT (lucasaba@tiscali.it)

Purpose: There are several evidences that carotid plaque structure could be an independent risk factor for ischaemic stroke. The purpose of this work was to explore the relationship between carotid plaque histological composition and brain MRI findings.

Methods and Materials: 18 consecutive (males 14; median age 64) patients were prospectively analysed. Brain MRI was performed using a 1.5 Tesla system and lesion's pertinence to the anterior circulation were recorded. Patients underwent carotid endarterectomy "en bloc"; histological sections were prepared and the presence and integrity of fibrous cap, haemorrhagelthrombus and plaque composition (calcification, lipid rich necrotic core) were analysed.

Results: The prevalence of haemorrhagelthrombus was 56\% (10118) and the prevalence of ruptured fibrous cap was $72 \%$ (13\18). Liner regression analysis demonstrated an association between the number of brain infarct (lacunar and non-lacunar) and the presence of haemorrhagelthrombus ( $p=0.003)$, whereas no statistically significant association was observed between the presence of fatty plaque, ruptured fibrous cap and the type of the plaque.

Conclusion: Results of this preliminary study indicate that haemorrhagelthrombus play a crucial role in the pathogenesis of stroke, and it supports the notion that cerebrovascular lesions are probably caused by emboli arising from thrombotic lesions.

\section{B-358 $\quad 14: 54$}

Vertebral artery variations at the C1-2 level diagnosed by MR angiography A. Uchino, N. Saito, T. Watadani, Y. Okada, E. Kozawa, N. Nishi, W. Mizukoshi, R. Nakajima, M. Takahashi; Hidaka/JP (auchino@saitama-med.ac.jp)

Purpose: The craniovertebral junction is clinically important as the location of many kinds of congenital or acquired lesions requires surgical interventions. The vertebra artery (VA) in its several variations runs within this area. We report the incidence of these VA variations on magnetic resonance angiography.

Methods and Materials: We retrospectively reviewed MRA images, obtained using two 1.5-tesla imagers, of 2,739 patients, and paid special attention to the course and branching of the VA at the level of the C1-2 vertebral bodies.

Results: There were 3 types of VA variation at the C1-2 level-(1) persistent first intersegmental artery (FIA), (2) VA fenestration, and (3) posterior inferior cerebellar artery (PICA) originating from the C1/2 level. The overall incidence of these 3 variations was $5.0 \%$. There was no laterality in frequency, but we found female predominance $(P<0.05)$. We most frequently observed the persistent $F I A(3.2 \%)$, which was sometimes bilateral. We found VA fenestration $(0.9 \%)$ and PICA of C1/2 origin (1.1\%) with almost equal frequency. Two PICAs of $\mathrm{C} 1 / 2$ origin had no normal VA branch.

Conclusion: We frequently observed VA variations at the C1-2 level and with female predominance. The persistent FIA was most prevalent and sometimes seen bilaterally. Preoperative identification of these variations in VA is necessary to avoid complications during craniovertebral junction surgery.

\section{B-359 15:03}

Diagnostic accuracy of thin slice angiographic reconstructions of volume perfusion CT: comparison with CT angiography

A.M.J. Frölich ${ }^{1}$, E. Klotz ${ }^{2}$, R. Schramm¹, M. Knauth ${ }^{1}$, P. Schramm ${ }^{1}$;

${ }^{1}$ Göttingen/DE, ${ }^{2}$ Forchheim/DE (andreas.froelich@med.uni-goettingen.de)

Purpose: Volume perfusion CT (VPCT) is increasingly used for diagnostic imaging in acute stroke. The goal of this study was to compare the diagnostic accuracy of thin slice angiographic reconstructions obtained from VPCT datasets (4D-CTA) with conventional CT angiography (CTA) data.

Methods and Materials: 59 consecutive patients were examined with a comprehensive CT protocol including non-enhanced CT (NECT), CTA and VPCT (Siemens Definition AS+). Contrast protocol for CTA was $60 \mathrm{ml}$ at $6 \mathrm{ml} / \mathrm{sec}$, for VPCT $45 \mathrm{ml}$ at $6 \mathrm{ml} / \mathrm{sec}$. VPCT used a periodic spiral technique: 30 spirals within $45 \mathrm{~s}$, z-coverage $96 \mathrm{~mm}$, 4D-CTA recons every $1 \mathrm{~mm}$ with a slice width of $1.5 \mathrm{~mm}$. Axia and coronal maximum-intensity projections $(10 \mathrm{~mm}$ every $3 \mathrm{~mm}$ ) were generated from CTA and 4D-CTA at peak arterial time. Two experienced neuroradiologists blinded to diagnosis and patient data evaluated all images for presence of vessel occlusion or stenosis.

Results: $34 \%$ of patients had an acute intracranial occlusion which was detected on both 4D-CTA and CTA. 54\% showed no acute vascular pathology on CTA or 4D-CTA. In $24 \%$ arterial stenosis was detected in agreement with CTA and 4D-CTA. In $12 \%$ of cases, a relevant pathology of the extracranial arteries was not detected because they were not included in the VPCT scan volume.

Conclusion: For the evaluation of acute stroke, obtaining NECT and VPCT alone (with 4D-CTA reconstructions instead of traditional CTA) may be a time- and radiation dose-efficient approach to detect acute intracranial vessel occlusion and intracranial stenosis. CTA remains mandatory in cases of extracranial vessel pathology outside of the VPCT scan range.

\section{B-360 15:12}

Evaluation of middle cerebral artery stenosis: comparison of highresolution MR imaging and digital subtraction angiography M. Wang', D. Shi', T. Li', Y. Han'1 Y. Dai'; ; Zhengzhou/CN, ${ }^{2}$ Shanghai/CN (meiyun9999@gmail.com)

Purpose: To explore the accuracy of high-resolution MR imaging (HR-MRI) in quantifying the severity of middle cerebral artery (MCA) stenosis by comparing with gold standard, conventional digital subtraction angiography (DSA).

Methods and Materials: A prospective study of 26 patients with symptomatic MCA stenosis was enrolled in this study. HR-MRI was performed on a 3.0 T MRI system (TrioTim, Siemens Medical Solutions) and compared to DSA. High-resolution T1T2- and PD-weighted images were acquired along MCA short axis, and T1- and PD-weighted postcontrast images were obtained 5 minutes after $15 \mathrm{ml}$ Gd-DTPA injection. A quantification of wall thickness area, lumen area, and degree of stenosis was performed on T2-weighted cross-sectional images at the level of maximal MCA stenosis by three independent blinded readers, the degree of stenosis was calculated and compared with that defined by DSA.

Results: Spearman correlation demonstrated that HR-MRI correlated well with DSA in evaluating the severity of MCA stenosis $(r=0.81, p<0.05)$. The inter-observer agreement was good (Kappa $=0.72)$. The sensitivity and specificity of HR-MRI in the detection of severe stenosis was $83 \%$ and $80 \%$, respectively.

Conclusion: HR-MRI could potentially be a useful tool in providing information about severity of MCA stenosis. 


\begin{tabular}{ll}
\hline $14: 00-15: 30$ & Room $P$
\end{tabular}

\section{Physics in Radiology}

\section{SS 513}

\section{New CT reconstruction and post processing techniques}

Moderators:

J. Damilakis; Iraklion/GR

C. Leidecker; Forchheim/DE

\section{B-361 14:00}

Iterative reconstruction in image space using a raw-data-based non-

isotropic noise model

H. Bruder, R. Raupach, J. Sunnegardh, T. Flohr, K. Stierstorfer;

Forchheim/DE (rainer.raupach@siemens.com)

Purpose: We present an iterative reconstruction scheme in image space based on a non-isotropic noise model for purpose of reducing radiation dose and image noise. Methods and Materials: Most recently, iterative reconstruction in image space was introduced providing a huge potential of noise and/or dose reduction, while maintaining spatial resolution. In all currently published methods, non-linear regularisation is based on an isotropic, local noise model. We propose an improvement of this noise model, which takes into account the higher noise of projection data in directions of large attenuation. This is done by applying non-isotropic attenuation maps derived from parallel forward projections of axial images, and can be efficiently implemented using standard Fourier techniques. The strength of non-linear regularisation becomes non-isotropic adjusted by the non-isotropic local noise values, derived from the attenuation maps.

Results: Based on simulation data and clinical data, respectively, it is shown that for non-symmetric objects non-isotropic image noise can be substantially reduced, revealing low contrast details otherwise obscured by noise. Clinical data of thoracicabdominal scans demonstrate a potential radiation dose reduction by $70 \%$, while maintaining image noise and visually perceived sharpness for high contrast objects. Conclusion: Iterative reconstruction in image space based on the presented nonisotropic noise model is an efficient iterative reconstruction scheme providing significant reduction of image noise and/or radiation dose by up to $70 \%$. For each image slice, a single-slice forward projection has to be computed prior to the iteration loop.

\section{B-362 14:09}

Translation of statistical iterative reconstruction into non-linear image processing

H. Bruder, R. Raupach, J. Sunnegardh, K. Stierstorfer, T. Flohr;

Forchheim/DE (rainer.raupach@siemens.com)

Purpose: We demonstrate that statistical iterative reconstruction can be translated to non-linear image processing in case of data-dependent Gaussian noise. Methods and Materials: Statistical iterative reconstruction is known to produce images with better signal-to-noise ratio compared to conventional FBP-type reconstruction. Originally based on the Poisson noise model, it can be simplified to a data-dependent Gaussian noise model for large numbers of quanta, manifesting as a signal weighting of sinogram data according to their statistical reliability. Based on the update equation of Iterative Filtered Backprojection reconstruction (IFBP), we introduce the signal weighting operator (method A) and show that, even in case of non-linear regularisation, under certain conditions the update equation can be formulated as an iterative reconstruction in image space. We also derive an equivalent approximation of iterative reconstruction in image space based on a non-isotropic noise model (method B).

Results: In case of non-linear regularisation it is demonstrated that methods A and $B$ are equivalent regarding noise characteristic and spatial resolution. For non-symmetric objects, non-isotropic image noise can be substantially reduced, revealing previously invisible low contrast details. Image sharpness of objects with contrast beyond the noise level is maintained.

Conclusion: We prove that statistical iterative reconstruction with data-dependent Gaussian noise and noise weighted IFBP is equivalent. Under certain conditions, it can be translated into a non-linear image processing. If iterative reconstruction is to be used for the reduction of artefacts due to non-exact reconstruction, a few IFBP iterations can be applied prior to image space processing.

\section{B-363 14:18}

A new method for scatter correction in cone-beam CT and its application to patient data and metal artefact reduction

E. Meyer ${ }^{1}$, M. Baer ${ }^{1}$, R. Raupach ${ }^{2}$, M. Kachelrie ${ }^{1} ;{ }^{1}$ Erlangen/DE

${ }^{2}$ Forchheim/DE (esther.meyer@imp.uni-erlangen.de)

Purpose: To remove scatter artefacts from cone-beam CT images.

Methods and Materials: Scatter artefacts show up as cupping artefacts, dark streaks between dense objects and blurred edges of objects. Algorithmically, scatter artefacts can be reduced by estimating the scatter content of a projection and reconstructing the original raw data minus the estimated scatter. All methods available today need to be well calibrated to the scanner specifics. We propose an empirical scatter correction (ESC) that does not need calibration. It rather uses the pep model [EuRad, Vol. 9: 563-569, 1999] and combines it with various scatter convolution functions to come up with scatter estimates. Using no calibration, ESC simply assumes that there is a linear combination of the uncorrected projection and the scatter estimates which is scatter-free. The coefficients are determined in image domain by maximising the flatness of the corrected volume. We used simulated data and patient data from a clinical dual source cone-beam spiral CT scanner. Some of them suffer from meta artefacts where scatter is expected to be a dominant contribution.

Results: Our ESC significantly reduces scatter artefacts. In case of metal implants, ESC provides an efficient method to reduce metal artefacts. For flat detector CT, cupping and other inhomogeneities due to scatter are reduced.

Conclusion: ESC is a convenient method which does not need prior knowledge for calibration. Scatter artefacts impair image quality and accuracy of CT values. Especially in cases with metal artefacts and in wide cone-angle flat-detector CT scans scatter artefact removal is of greatest value.

\section{B-364 14:27}

Model-based iterative reconstruction improves $C T$ image quality

K. Zeng ${ }^{1}$, B. De Man ${ }^{1}$, J.-B. Thibault ${ }^{2}$, A. Hagiwara ${ }^{3} ;{ }^{1}$ Niskayuna, NY/US,

${ }^{2}$ Waukesha, WI/US, ${ }^{3}$ Hino/JP (zengkai@ge.com)

Purpose: The application of MBIR to clinical CT applications is still a relatively new area and a number of challenges have to be overcome for clinical application of MBIR. One of them is the elimination of pinwheel artefacts, which are sometimes also observed in FBP reconstructions from helical scans, in the presence of strong longitudinal gradients. We proposed an improved MBIR method to reconstruction images with fewer artefacts. Methods and Materials: Pinwheel artefact is caused by high-z gradient in object and non-perfect sampling of detector along $z$ direction. Here, we present a method to prevent such kind of artefacts using MBIR by improving its forward model. Instead of a uniform voxel as used in conventional methods, we incorporate a longitudinal slope in each voxel to better model longitudinal gradients, which can better represent edges within a voxel. This eliminates possible model mismatches and therefore reduces artefacts.

Results: We validated the proposed method to a simple post-processing method to minimise pinwheel artefacts, which is commonly used for FBP reconstructions. Both methods have been implemented and tested using patient data sets acquired from GE Lightspeed CT systems. Both result in significant artefact reduction. The improved voxel model method outperformed the post-processing method, especially for the region close to bone structures.

Conclusion: The proposed method can efficiently suppress pinwheel artefacts without significant computational penalty.

\section{B-365 14:36}

Advanced interpolation for normalised metal artefact reduction (NMAR) in CT E. Meyer ${ }^{1}$, R. Raupach ${ }^{2}$, M. Lell ${ }^{1}$, B. Schmidt ${ }^{2}$, M. Kachelrie ${ }^{1} ;{ }^{1}$ Erlangen/DE, ${ }^{2}$ Forchheim/DE (esther.meyer@imp.uni-erlangen.de)

Purpose: To correct metal artefacts in clinical circular and spiral cone-beam CT. Methods and Materials: Metal artefact reduction (MAR) is typically performed by replacing corrupt projection data by interpolation. We have recently proposed NMAR that consists of a normalisation step prior to interpolation and that turned out to significantly outperform all other known MAR techniques and thereby appears to be the most promising MAR approach available today [MedPhys 37 (10):inpress]. Nevertheless, even with NMAR slight artefacts remain. We therefore enhanced NMAR and replaced its simple linear interpolation by better interpolation and inpainting methods: directional interpolation and anisotropic diffusion inpainting. Directional interpolation aims at properly connecting traces of high-contrast objects though the metal parts in the projections. Inpainting with diffusion type equations ensures a smooth transition between measured and surrogate projection data. For the evaluation, ten patient data sets with hip prostheses and dental fillings were processed with NMAR and the image quality was evaluated. 
Results: Subtle artefacts remaining with NMAR, such as in the presence of multiple dental fillings, can be significantly reduced when using the more advanced interpolation techniques. For example tiny dark regions close to the dental fillings are removed. For the cases studied, the directional interpolation is slightly more efficient than the anisotropic diffusion inpainting.

Conclusion: NMAR's image quality can be further enhanced using sophisticated interpolation methods. Considerable improvements are found in particular for structures in the utmost vicinity of implants and dental fillings.

\section{B-366 14:45}

\section{Intrinsic beam hardening correction (IBHC) for cone-beam CT}

R. Grimmer, M. Kachelrieß; Erlangen/DE (rainer.grimmer@imp.uni-erlangen.de)

Purpose: To remove first and higher order non-linear beam hardening and scatter artefacts from patient CT images.

Methods and Materials: X-ray polychromacy and scatter are non-linear effects appearing as cupping and banding artefacts in CT images. A first order water correction (e.g. [MedPhys 33 (5):1269-1274]) followed by higher order BHC (e.g. [MedPhys 37 (10):5179-5187]) are typically used to remove those artefacts. To avoid this two-step process and to avoid any calibration steps depending on the scanner or the spectrum, we propose IBHC which performs a simultaneous correction of the first and higher order beam hardening artefacts. It separates the initial image into different materials, and forward projects those to obtain various rawdata sets. A combination of these is reconstructed and used as basis images to correct the initial image such that the final image is nearly artefact-free. A wavelet decomposition is used to determine those frequency components that contain artefacts to find the best combination of the basis images. Patient data from clinical and flat panel CT scanners are used to evaluate IBHC. Results: The The IBHC can almost completely eliminate cupping and streak artifacts. Typically, more than $90 \%$ artefact reduction is observed. For example, dark streaks were reduced from $-246 \mathrm{HU}$ to $-8 \mathrm{HU}$ in one of our head scans.

Conclusion: IBHC is an efficient and stable new approach to reduce cupping and beam hardening. It is scanner-independent and therefore can be applied also offline to any CT image.

\section{B-367 14:54}

Dose-efficient deformation-constrained 4D cone-beam CT image reconstruction

M. Brehm, F. Bergner, M. Kachelriess;

Erlangen/DE (marcus.brehm@imp.uni-erlangen.de)

Purpose: To reconstruct high-quality respiratory-correlated 4D volumes from flat panel detector cone-beam CT scans and to obtain 100\% dose usage.

Methods and Materials: Respiratory-correlated cone-beam CT suffers from streak artefacts and from reduced dose usage because only a subset of the acquired projection data contributes to each time frame. We developed a new approach that iteratively reconstructs the volumes and simultaneously estimates the respiratory deformation fields. This estimate is used to generate a non-moving reference volume from which the other motion phases are derived by deformation. A spatiotemporal regularisation is used to suppress the effect of artefacts from the iterative 4D CBCT reconstruction. The method is verified using simulated rawdata obtained by deforming a clinical patient dataset using realistic deformation fields and by processing patient data acquired with the flat panel cone-beam CT scanner. Results: The streak artefacts in the reconstructions from both, simulated data and patient data, are highly reduced. Compared to the conventional phase-correlated method with 10 reconstructed phases, the noise level is reduced by more than $60 \%$ with our method in all cases. This corresponds to a dose reduction of more than $80 \%$. Streak artefacts can almost completely be suppressed.

Conclusion: Motion-compensated, spatiotemporal regularisation combined with iterative 4DCBCT reconstruction is capable of removing the streak artefacts to a large degree while maintain a good spatial and good temporal resolution. The dose usage is significantly increased because all data now contribute to each time frame.

\section{B-368 15:03}

Using a histogram constraint to enhance temporal resolution in CT

H. Schöndube, T. Allmendinger, K. Stierstorfer, H. Bruder, T. Flohr;

Forchheim/DE

Purpose: To present and evaluate a new algorithm to enhance temporal resolution in CT beyond the short-scan limit by making use of a histogram constraint. Methods and Materials: A minimum scan angle of $180^{\circ}$ plus fan-angle is needed to acquire complete data for reconstructing an image. Conventionally, this means that a temporal resolution of half the gantry rotation time is achievable and that an enhancement of temporal resolution can only be accomplished by a faster gantry rotation or using a dual-source system. In this work, we pursue a different approach, namely employing an iterative algorithm to reconstruct images from less than $180^{\circ}$ of projections and using a histogram constraint to prevent the occurrence of limited-angle artifacts. The method is fundamentally different from previously published approaches using prior images and TV minimisation. Furthermore, motion detection is used to enhance dose usage in those parts of the image where temporal resolution is not critical.

Results: The proposed method yields good results, both with simulated and with clinical data. Our evaluations also show that an enhancement of temporal resolution to a value equivalent to about $120^{\circ}-135^{\circ}$ of projections is viable. Furthermore, by employing motion detection, a substantial noise reduction can be achieved in those parts of the image where no motion occurs.

Conclusion: Using our proposed method, an enhancement of temporal resolution in CT images by about $30 \%$ is possible. Furthermore, an optimisation of dose usage can be achieved by use of a motion detection system.

\section{B-369 15:12}

CT image reconstruction from limited data

L. Ritschl ${ }^{1}$, C. Fleischmann ${ }^{2}$, M. Kachelrie ${ }^{1}{ }^{1} ;{ }^{1}$ Erlangen/DE,

${ }^{2}$ Nürnberg/DE (marc.kachelriess@imp.uni-erlangen.de)

Purpose: To obtain clinically relevant and artefact-free images from incomplete CT data (limited angle, metal implants, few projections, projection truncation).

Methods and Materials: Image reconstruction based on compressed sensing (CS) is a promising technique to reconstruct from incomplete raw data. Existing algorithms (TV-Min, PICCS, ASD-POCS), however, do not always yield clinical image quality and require to adjust several object-dependent parameters. We propose an improved total variation (iTV) reconstruction algorithm to overcome these difficulties. It controls the reduction of the TV while improving raw data agreement throughout each iteration step. Thereby, iTV does not need to be tuned for a specific patient. For example, the same set of parameters for metal artefact reduction, for sparse projection sampling, for limited angle artifact reduction and for projection detruncation is used for both clinical CT and flat detector CT. To evaluate the performance of ITV clinical patient cases (from a dual source clinical CT scanner and from a mobile C-arm CT scanner) with limited data were processed and compared with today's gold standard cone-beam filtered backprojection.

Results: In all examples, the algorithm was able to suppress most of the image artefacts. Especially in the case of metal implants and dental fillings and in the case of limited angle tomograhy $\left(160^{\circ}\right)$ the resulting images were of surprisingly high image quality.

Conclusion: With iTV a highly robust and highly versatile algorithm that guarantees high-fidelity images for actual patient data has become available.

\section{B-370 15:21}

Assessment of CT spectral imaging with metal artefact reduction sequence for metal artefact reduction: in vitro and clinical studies

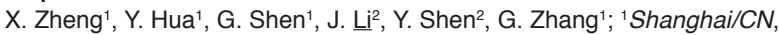
${ }^{2}$ Beijing/CN (jianying.li@med.ge.com)

Purpose: To assess CT spectral imaging with metal artefact reduction sequence (MARS) in reducing metal artefacts in vitro and in patients.

Methods and Materials: A phantom consisting of two rows of 16 needles (7-22G), two 10-mm diameter aluminum tubes and four tibial bone nails in a homogenous agar gel block within a water bath was scanned with the regular CT at $120 \mathrm{kVp}$ and the single-tube, instantaneous kV switching spectral imaging on GE HDCT for monochromatic images. Twenty orthopaedic patients with intramedullary nails received CT for evaluation. Multiple regions of interest were placed around implants for CT value and standard deviation measurement and comparison. In patient study, images were also scored for implant margin visualisation, surrounding tissue details, and artefact severity. The surface profile and profile plot through implants were also analysed. Results: The monochromatic images with MARS in phantom study reduced the maximal CT number variation in areas surrounding implants from $32 \mathrm{HU}$ in regular CT to $11 \mathrm{HU}$ $(p<0.05)$. In patient study, metal artefacts significantly compromised the delineation of adjacent structures and implant margins in the regular $\mathrm{kVp}$ images. These artefacts were dramatically reduced in the $2 \mathrm{D}$ and $3 \mathrm{D}$ monochromatic images with MARS, which made the implant margins, intramedullar content, trabecular integrity, periosteal reaction and soft tissue abnormalities readily visualised and evaluated (score of 4.2 vs. $2.8, p<0.01$ ). The plots from the HDCT monochromatic images also reflected the actual structural layers. Conclusion: HDCT spectral imaging with MARS reduced metal artefact from metal implants and improved the depiction of surrounding focal structures. 


\section{Saturday, March 5}




\section{Musculoskeletal}

\section{SS 810}

\section{Hip and ankle}

Moderators:

A. Feydy; Paris/FR

V. Zubler; Zurich/CH

\section{B-371 10:30}

Imaging displacement and strain in the medial gastrocnemius muscle during ankle-joint motion using 2D cine DENSE MRI

A. Lawson ${ }^{1}$, B. Spottiswoode ${ }^{1}$, A. Hess ${ }^{1}$, E. Meintjies ${ }^{1}$, S. Beningfield ${ }^{1}$

X. Zhong 2 ; ${ }^{1}$ Cape Town/ZA, ${ }^{2}$ Virginia, VA/US (drajlawson@gmail.com)

Purpose: The objective of this study was to apply magnetic resonance imaging (MRI) using displacement encoding with stimulated echoes (DENSE) to investigate the dynamic displacements and strain fields in the medial gastrocnemius muscle. Methods and Materials: Five volunteers were imaged using a $1.5 \mathrm{~T}$ Siemens MRI scanner (MAGNETOM Symphony, Siemens, Erlangen). Informed consent was obtained and all studies were approved by our institutional review board. The volunteers were positioned feet-first and right-side down into the MRI bore with their right ankle resting on a platform with a straight right knee and a flexi-coil around the right calf. The sequence was gated using a plantarflexion trigger mechanism and a cine series of echo-planar DENSE images was acquired over several successive plantar flexions. Displacement encoding was applied in three orthogonal directions. First and second principle strain measurements were derived from the displacement fields after spatio-temporal phase unwrapping.

Results: Regional displacements were largest in the medial portion of the medial gasctrocnemius adjacent to the interface with the soleus muscle. The directions of the first principle strain vectors agreed well with the pennation angle of the myofibres. The magnitude of the first principle strain was largest in the middle of the muscle belly and the mean first principle strain along this midline at maximum plantar flexion for all five volunteers was $74.5 \pm 38.4 \%$.

Conclusion: This study shows how DENSE MRI can be used to quantitatively determine regional displacement and strain in the gastrocnemius muscle. These strain measurements compare favourably with previous studies using ultrasound.

\section{B-372 10:39}

Sonoelastography of plantar fascia: comparison among healthy subjects and patients affected by plantar fasciitis

D. Orlandi ${ }^{1}$, L. Sconfienza ${ }^{2}$, C. Martini ${ }^{1}$, G. Ferrero ${ }^{1}$, E. Silvestri ${ }^{1} ;{ }^{1}$ Genoa/IT, ${ }^{2}$ San Donato Milanese/IT (theabo@libero.it)

Purpose: Plantar fasciitis is a common condition in athletes and general population. In early stages, the diagnosis of such affection can be challenging. Our aim was to evaluate real-time sonoelastography in the assessment of the plantar fascia in healthy volunteers and in patients with clinical suspicion of plantar fasciitis, comparing these findings with those obtained at conventional ultrasound.

Methods and Materials: IRB approval and patients' informed consent were obtained. We included in our study 42 feet of 21 healthy volunteers ( 13 males, mean age $28 \pm 8.1$ years) and 19 feet of 19 patients (11 males, mean age $44 \pm 12$. 2 years) referring for clinical symptoms of plantar fasciitis. All subjects underwent conventional ultrasound and sonoelastography assessment of the hindfoot (13$6 \mathrm{MHz}$ linear probe, MyLab $70 \mathrm{XvG}$, Esaote, Italy) and clinical evaluation of pain with a visual analogue scale.

Results: Sonoelastography demonstrated an elastic structure in $42 / 42$ healthy feet $(100 \%)$, while loss of elasticity was detected in $17 / 19$ patients $(89 \%)$. The difference between the two groups was statistically significant $(p<.001)$. Among patients who presented altered elasticity values $(n=15)$, conventional ultrasonography detected signs of plantar fasciitis in $15 / 19$ patients $(79 \%)$. Compared with clinical examination, sensitivity of sonoelastography was $89 \%$, specificity was $100 \%$ and accuracy was $94 \%$, while conventional ultrasound showed $79 \%, 100 \%$,and $90 \%$, respectively. Good correlation between sonoelastography and ultrasonography was observed $(r=.897)$.

Conclusion: Sonoelastography represents a valuable tool in differentiating healthy subjects from patients and also in those cases where ultrasound was not conclusive. Sonoelastography showed remarkable sensitivity, specificity and accuracy in detecting elasticity alterations of plantar fascia, with good correlation with conventional ultrasonography.

\section{B-373 10:48}

Five-year ultrasound survey of functional overload injuries of patellar and Achilles tendons in elite rugby players

C. Martini', G. Ferrero', G. Cicio', L. Scofienza², E. Silvestri'; ' 'Genoa/IT, ${ }^{2}$ San Donato Milanese/IT (chiarapio@libero.it)

Purpose: Tendon overload syndromes affecting patellar (PT)and Achilles tendons (AT) represent a frequently disabling painful condition in rugby athletes. We used US to re-evaluate AT and PT in elite rugby athletes five years after a previous report, comparing the results with a group of healthy non-athletes volunteers in order to highlight any morpho-structural differences related to functional overload. Methods and Materials: US evaluation of each player tendons was performed at baseline (July 2005) and five years later (July 2010) using two different US systems ( $\left.1^{\text {st: }}: H D I 5000, A T L ; 2^{\text {nd }}: M y L a b 70 X v G, E s a o t e\right)$ equipped with high-resolution probes (8-18 MHz). Weight bearing and at rest bilateral PT evaluation was performed and proximal (PPT), mean (MPT) and distal (DPT) longitudinal measures were recorded. Weight bearing and at rest bilateral AT evaluation was performed and latero-lateral (LL)/antero-posterior (AP) diameter was measured and noted.Tendons were also scanned to highlight fibrillar structure alterations, focal hipoechoic areas and calcifications. The examination was repeated on 26 healthy non-athletes volunteers matched for age and sex.

Results: Athletes' mean tendon thickness was significantly higher than that of controls (mean value PT atlete/controls:PPT $5.3 \mathrm{~mm} / 3.8 \mathrm{~mm}$;MPT $5.1 \mathrm{~mm} / 3.6 \mathrm{~mm}$;DPT $5.0 \mathrm{~mm} / 3.6 \mathrm{~mm}$; AT:AP $6.4 \mathrm{~mm} / 5.7 \mathrm{~mm}$;LL $16.1 \mathrm{~mm} / 12.8 \mathrm{~mm}$ ). Hypoechoic areas were found in $27 \%$ of athletes and $5 \%$ of controls, calcifications in $15 \%$ and $3 \%$, respectively. All athletes who presented focal hypoechoic areas and/or calcifications at baseline were found to have them increased in number and size at five years. Two athletes healthy at baseline were found to have one focal hypoechoic area and one calcification at five years.

Conclusion: AT and PT US evaluation in rugby players allows for detecting and highlighting overload degenerative alterations without clinical symptoms. US seems to have a great potential in prediction and prevention of damage progression in high-level athletes.

\section{B-374 10:57}

Tendon and ligament injuries of the ankle and foot: the role of diffusion weighted MR imaging in the diagnosis

H. Aydin; Ankara/TR (dr.hasanaydin@hotmail.com)

Purpose: Our aim was to determine the diagnostic performance and utility of diffusion weighted imaging (DWI) against the routine magnetic resonance imaging (MRI) in evaluation of the patients with ankle and foot injuries.

Methods and Materials: After institutional review board approval, ankle-foot MR imaging and DWI-apparent diffusion coefficient (ADC) mapping was performed to the 106 injured tendons and ligaments of 60 patients. All the injuries of tendons and ligaments were named as Rupture (R)- Partial tear (PT) and Tenosynovitis (T). Diagnostic interpretation was based on the MRI-DWI and ADC mapping, verified by either open surgery-diagnostic artroscopy or conservative procedures-splint application. Statistical analysis of this research was assessed by Fischer's exact test, variance analysis test between dependent groups, receiver operating characteristics (ROC) curve and Pearson chi square statistics.

Results: For the all the ligaments of ankle included in this research, DWI had $100 \%$ sensitivity in depicting the R and T, $67 \%$ sensitivity in the diagnosis of PT. For the injured tendons; DWI had $100 \%$ success rate in the diagnosis of R, $92-96 \%$ sensitivity corresponding to PT and T. DWI had significant statistical superiority over MRI in the visualisation of R of ligaments, PT and T of all tendons included in this research $(p<0.05)$.

Conclusion: DWI is a good imaging modality in the diagnosis and depiction of injured ankles, might further improve the sensitivity of classical ankle and foot MR imaging, influence patient treatment options especially in the ruptured ligaments and injured tendons, supply more beneficial and diagnostic information than the routine MRI.

\section{B-375 11:06}

Physiological changes of the acetabulum during maturation: MR assessed data in a cross sectional asymptomatic cohort from $\mathbf{9}$ to 16 years K.-P. Kienle ${ }^{1}$, J. Keck', K.A. Siebenrock ${ }^{1}$, S. Werlen ${ }^{1}$, Y.-J. Kim², T.C. Mamisch'; ${ }^{1}$ Berne/CH, ${ }^{2}$ Boston, MA/US (philipp.kienle@googlemail.com)

Purpose: As base for understanding and comparison to disease process we assessed children's acetabular morphology.

Methods and Materials: All 331 pupils from a primary- and high-school were asked to take part in this study. 64 pupils (127 asymptomatic hips) were willing to participate and obtained MRI on $3 \mathrm{~T}$ machine. We assessed on 7 radial positions around the 
acetabular roof, rotating from anterior to superior to posterior: (1) acetabular diameter (width of the cup), (2) acetabular-extension (depth of the cup), (3) acetabular depth as defined by Pfirrmann et al (depth correlated to the centre of the femoral head) and (4) the beta-angle, as distance to the head-neck offset. We related these parameters to age, status of the epiphyseal scar and location in the joint.

Results: Looking at the influence of age, we found that the acetabular diameter increases highly significant $(p<0.001)$. The acetabular extension, also growth by aging highly significant $(p=0.05)$. The beta angle decreases with growing older, but not significant $(p>0.05)$. Dividing the population into two groups, one with open epiphysis and the other with a closed one, we could not find any significan changes between these both groups $(p<0.05)$. Significant changes could mostly be found in the superior, ventral superior and dorsal superior parts.

Conclusion: Within our normal cohort no asymmetric changes could be observed in coverage during maturation using MRI. These data can be compared to hips with pathological coverage (hip dysplasia, pincer-type FAI) to better understand potential influences during growth, genetic preposition and to develop preserving possibilities.

\section{B-376 11:15}

The femoral epiphyseal torsion angle in MR arthrography of the hip: diagnostic utility in patients with femoroacetabular impingement syndrome

C. Schaeffeler, K. Holzapfel, M. Eiber, H. Gollwitzer, E.J. Rummeny, K. Woertler; Munich/DE (schaeffeler@roe.med.tum.de)

Purpose: To evaluate if the epiphyseal torsion angle (ET-angle) shows pathologic values in patients with clinically suspected femoroacetabular impingement syndrome (FAl) referred to MR arthrography (MR-A) of the hip and to analyse whether patients with pathologic values show higher incidence of hip pathology. Methods and Materials: 68 consecutive MR-A of the hip in patients with positive clinical impingement tests were included to the study group. The control group was composed by 30 hips of healthy volunteers without clinical signs of FAI, who underwent standard MRI. The angle between a line perpendicular to the proxima epiphyseal scar of the femur and the long axis of the femoral neck (ET-angle) was measured independently in both groups on oblique axial T1w images by three radiologists. Furthermore, all MR-A were retrospectively evaluated for internal derangement and clinical data were reviewed.

Results: Mean values of the ET-angle in the FAl-group were $12.1^{\circ}$ (range: $-3.1^{\circ}$ $\left.29.4^{\circ}\right)$ for reader $1,12.2^{\circ}\left(0.8^{\circ}-28.9^{\circ}\right)$ for reader 2 and $12.8^{\circ}\left(0.5^{\circ}-29.9^{\circ}\right)$ for reader 3 . In the control group the mean values were $7.5^{\circ}\left(2.4^{\circ}-17.8^{\circ}\right)$ for reader $1,6.9^{\circ}$ $\left(0.7^{\circ}-18.5^{\circ}\right)$ for reader 2 and $9.6^{\circ}\left(2.1^{\circ}-19.8^{\circ}\right)$ for reader 3 . T-test analysis showed highly significant lower ET-angles in healthy volunteers compared to the FAl-group for all three readers $(p<0.010)$. The calculated intraclass correlation coefficient of 0.75 represented good interrater agreement. Patients with labral lesions detected by MR-A did not show significantly increased ET-angles.

Conclusion: Patients with clinically suspected FAI show significant higher ETangles than healthy volunteers. Values $>20^{\circ}$ should be considered pathologic.

\section{B-377 11:24}

(EPOS)

Superior acetabular notch (pseudocartilage defect): prevalence in MRarthrography and correlation with surgery

T.J. Dietrich, A. Suter, C.W.A. Pfirrmann, C. Dora, M. Zanetti; Zurich/CH (tdiet@gmx.net)

Purpose: In literature, based on a few dried skeletal specimens, the supracetabular notch has been described as an anatomic variant. The purpose of this study was to evaluate the prevalence of such superior acetabular notches (SAN) (pseudocartilage defects) in MR-arthrographies of the hip and to correlate with surgery. Methods and Materials: 838 consecutive MR-arthrograms were reviewed for the presence of superior acetabular notches (SANs). SANs were classified into two types: SAN type-1 with an accessory fossa in the apex of the acetabulum and a distinct contrast media inflow into the fossa, and SAN type-2 with a fossa without contrast media inflow. MR-arthrographies with SANs were correlated with surgery if available. Results: Twenty-three (2.7\%) patients (10 females, 13 males; mean age 29.3 years) presented with SAN type-1: depth, $2.6 \mathrm{~mm}$; width $5.0 \times 4.5 \mathrm{~mm}$; localisation lateral to the acetabular notch, $7.7 \mathrm{~mm}$. Seventy-seven (9.2\%) patients had a SAN type-2 (37 females, 40 males; average age 38.6 years): depth $2.4 \mathrm{~mm}$, width 4.8 $\times 4.6 \mathrm{~mm}$, localisation lateral to the acetabular notch $8.1 \mathrm{~mm}$. Four hip joints with SAN type- 1 and 11 joints with SAN type-2 underwent surgery. No cartilage defect was found in the area of the SAN; however, in one case the surgeon described an accessory fossa in the dome of the acetabulum as an anatomic variant.

Conclusion: Superior acetabular notches (SANs) are commonly seen on MRarthrograms and should not be interpreted as a deep cartilage defect. Surgery confirms that SAN represents most likely an anatomic variant.
B-378 11:33

Delayed gadolinium-enhanced magnetic resonance imaging of cartilage (dGERMIC) in femoroacetabular impingement

S.R. Apprich, K. Siebenrock, B. Bittersohl, M. Beck, S. Werlen, T.C. Mamisch; Berne/CH (sebastian.apprich@meduniwien.ac.at)

Purpose: Femoroacetabular impingement is a pre-arthritic condition with two main types; cam and pincer. Each type has been described at surgery to lead to specific patterns of articular cartilage wear. We sought to evaluate if the dGEMRIC-index would produce any identifiable patterns of cartilage degeneration similar to these. Methods and Materials: We compared the dGEMRIC-indices in a group of cam ( $n=6$; mean age 33 years) and pincer patients $(n=7$; mean age 36.3 years) to a control group of asymptomatic controls ( $n=12$; mean age 25.2 years) with no MRI findings of osteoarthritis. T1maps and dGERMIC indices were obtained from a dual-flip angle 3D GRE-sequence at 1.5 Tesla. The superior portion of the hip joint was divided into seven regions from posterior anterior. These regions were then subdivided into peripheral and central regions and at each ROI, a dGERMIC index was recorded. Differences between study groups were determined using Student's t-test.

Results: The cam (488.1 ms) and pincer $(462.0 \mathrm{~ms})$ groups both had statistically lower dGEMRIC values compared to the control group (643.3 ms). The cam group demonstrated not only peripheral but also central involvement of the joint and this was concentrated in the anterior portion of the joint. The pincer group exhibited more global hip involvement with all areas of the hip averaging a dGEMRIC index $28 \%$ less than controls.

Conclusion: With the use of dGEMRIC more specific patterns of cartilage wear can be elicited in patients with impingement, which may improve patient selection and help better understand the progression of osteoarthritis throughout the hip joint.

\section{B-379 11:42}

Semiquantitative assessment of hip MRI in patients with hip pain (Hip OsteoArthritis MRI Score/HOAMS): reliability and association with radiographic scoring

F.W. Roemer ${ }^{1}$, A. Winterstein ${ }^{1}$, D.J. Hunter ${ }^{2}$, L. Li ${ }^{3}$, Y.-j. Kim³

T.C. Mamisch ${ }^{4}$, J. Cibere ${ }^{5}$, K. Bohndorf ${ }^{1}$, A. Guermazi $^{3}$;

${ }^{1}$ Augsburg/DE, ${ }^{2}$ Sydney/AU, ${ }^{3}$ Boston, MA/US, ${ }^{4}$ Berne/CH, ${ }^{5}$ Vancouver, BC/CA (frank.roemer@klinikum-augsburg.de)

Purpose: The aim of this study was to describe a novel semiquantitative scoring system for MRI of hip osteoarthritis, to test its reliability and analyse associations of intraarticular damage with radiographic OA severity.

Methods and Materials: 52 patients ( $>50$ years of age) with hip pain were examined with $1.5 \mathrm{~T} \mathrm{MRI}$. Of these, $44(85 \%)$ had weight-bearing x-rays and $37(71 \%)$ received i.v. gadolinium administration. All MRIs were read by one expert radiologist. Intra- and interobserver reliability exercise was performed on a subset of 15 examinations. Cartilage, bone marrow lesions (BMLs), cysts, osteophytes, labra damage, synovitis and effusion were assessed. X-rays were scored in consensus according to the Kellgren-Lawrence (KL) grading scheme. Reliability was calculated using weighted (w) kappa statistics. Associations between presence of MR features (large BMLs, severe cartilage damage, labral damage and synovitis) and radiographic OA severity were calculated using Cochran-Armitage test for trend. Results: Distribution of radiographic grading was: KL0 12 (27.3\%), KL1 11 (25\%), KL2 14 (31.8\%), KL3 5 (11.4\%) and KL4 2 (4.6\%). Intra-reader reliability for the different features ranged between 0.69 (synovitis) and 0.85 (cartilage). Inter-reader reliability ranged between 0.48 (labrum) and 0.85 (BMLs). Scoring of subchondra cysts was not reliable with a w-kappa of 0.18 and 0.15 . There was a strong association between presence of MRI-detected lesions and radiographic $\mathrm{KL}$ grade $(p=0.002)$ apart from labral damage $(p=0.26)$.

Conclusion: MRI-based semiquantitative assessment of the hip joint appears to be reliable apart form scoring of subchondral cysts. Presence of more severe MRI-detected intraarticular pathology shows a strong association with radiographic OA apart from labral damage. 


\section{Abdominal Viscera}

\section{SS 801a}

\section{Imaging evaluation of liver fat, iron and fibrosis}

\section{Moderators:}

J.M. Alústiza; San Sebastián/ES

M.A. Bali; Brussels/BE

\section{B-381 10:30}

Effect of iron burden on hepatic apparent diffusion coefficient (ADC) in patients with thalassemia major

G. Restaino ${ }^{1}$, M. Occhionero ${ }^{1}$, S. Roiati ${ }^{2}$, A. Caulo², S. Costanzo ${ }^{1}$, A. Pepe ${ }^{3}$, G. Sallustio ${ }^{1} ;{ }^{1}$ Campobasso/IT, ${ }^{2}$ Rome/IT, ${ }^{3}$ Pisa/IT (gennares@hotmail.com)

Purpose: Hepatic ADC is commonly used for characterisation of liver disease. DWI-EPI sequence is very sensitive to susceptivity effects. Aim of the present study was to assess the effect of hepatic iron burden on ADC values of liver measured with 3 different b-values $(200,600$ and 1000$)$

Methods and Materials: 53 patients with thalassemia major (35.2 y/o; $27 \mathrm{~F}$ ) underwent liver MRI with 1.5 T scanner. Liver T2*, as expression of iron overload, was measured with a multiecho GRE sequence and with a dedicated software (HyppoMIOT, ifc-CNR, Pisa-Italy). ADC values were measured in the same hepatic region with EPI-DWI sequence with 3 different b-values $(200,600$ and 1000$)$ and a dedicated software (Functool2, ADW 4.2, GEMS). The T2* and ADC values were compared with Pearson's test.

Results: Liver iron overload resulted absent in 9 patients, borderline in 24, sligh in 8 , moderate in 11 , and severe in 1 . ADC values measured with $b=200$ showed no significant correlation to $\mathrm{T}^{*}(p=0.44)$ and weak correlation to $\log 2^{*}(p=$ $0.0258 ; r=0.31$ ). ADC with $b=600$ showed significant linear correlation to $T 2$ and to $\log T 2^{*}(p<0.0001 ; r=0.60$ and, respectively, $p<0.0001 ; r=0.76)$. ADC values with $b=1000$ showed the most significant correlation to T2* $(p<0.0001 ; r$ $=0.83)$ and even stronger correlation to $\log 2^{*}(p<0.0001 ; r=0.91)$.

Conclusion: Iron overload has a strong influence on hepatic ADC values measured with $E P I-D W I$ with $b=600$ and $b=1000$, with a significant linear correlation between $\mathrm{T}^{*}$ and $\mathrm{ADC}$ values. This effect must be considered when measuring $\mathrm{ADC}$ in liver of patients with known or suspected iron overload.

\section{B-382 10:39}

Quantification of liver fat in the presence of iron and iodinated contrast media: an ex-vivo dual energy CT study

M.A.F. Fischer ${ }^{1}$, R. Gnannt ${ }^{1}$, D. Raptis ${ }^{1}$, C. Reiner ${ }^{1}$, P.-A. Clavien ${ }^{1}$, B. Schmidt ${ }^{2}$ S. Leschka ${ }^{3}$, H. Alkadhi', R. Götti'; ${ }^{1}$ Zurich/CH, ${ }^{2}$ Forchheim/DE, ${ }^{3}$ St. Gallen/CH (michaelalexander.fischer@usz.ch)

Purpose: To evaluate the feasibility and accuracy of dual energy CT (DECT) for ex-vivo quantification of liver fat content (LFC) in the presence of iron and contrast media (CM)

Methods and Materials: Sixteen phantoms with a defined LFC (0-50\%) and with varying liver iron content were scanned with a second-generation DECT-system. Phantoms were scanned unenhanced and contrast-enhanced using an iron-specific dual energy 3-material decomposition algorithm providing virtual non-iron images (VNI) to subtract iron and CM from the data. Single-energy (SECT) and DECT measurements (HU) of unenhanced and contrast-enhanced phantoms were compared to the known LFC using Pearson's analysis and Student's t-test for related samples. Results: For fat quantification in the absence of iron and CM, CT numbers were similar in SECT and DECT (all, $p>0.05$ ), showing a linear correlation with titrated LFC ( $r$ ranging from 0.981 to $0.999 ; p<0.01$ ). For fat quantification in the presence of iron but without CM, significant underestimation of LFC was observed for al measurements in SECT and DECT (all, $p<0.05$ ), except for VNI (all, $p>0.25$ ). For fat quantification in the presence of iron and $\mathrm{CM}$, further underestimation of LFC was seen for measurements in SECT and DECT (all, $p<0.015$ ), whereas VNI showed a high accuracy for estimating LFC (all, $p>0.2$ ).

Conclusion: In-vitro, DECT with use of VNI images allows for the accurate quantification of LFC even in the presence of iron and iodinated contrast media. No added value was seen for DECT as compared to SECT for LFC quantification in the absence of iron and iodine.
B-383 10:48

Noninvasive quantification of hepatic fat and iron content in an epidemiological study using $\mathrm{T}^{*}$-corrected three-point Dixon MR J.P. Kühn' ${ }^{1}$ N. Friedrich', K. Hegenscheid ${ }^{1}$, M. Evert ${ }^{1}$, A. Kickhefel ${ }^{2}$, N. Hosten ${ }^{1}$ H. Völzke', R. Puls'; ${ }^{1}$ Greifswald/DE, ${ }^{2}$ Erlangen/DE (kuehn@uni-greifswald.de)

Purpose: To investigate noninvasive MRI using a calibrated three-point Dixon technique for quantifying hepatic fat (HFC) and liver iron content (LIC) in a largescale epidemiological study.

Methods and Materials: Calibration and screening were performed on a 1.5 T MRI system (Magnetom Avanto, Siemens) using three-point Dixon MRI with correction for T2* effects (WIP_432_rev.1). The sequence was first tested in 87 patients who underwent a clinically indicated percutaneous liver biopsy ( 45 men, 42 women; mean age $55.8 \pm 14.2$ years). Voxel-based calibration was performed using the histological results for HFC and LIC. Second, in an ongoing population-based study, 1,518 volunteers (722 men, 796 women; mean age $52.7 \pm 14.3$ years) underwent liver MRI including the T2*-corrected three-point Dixon sequence. Mean calculated HFC was graded according to pathologic S2-guidelines. Mean LIC was determined from T2-values. Results: The correlation between signal intensity and histological HFC was $r=$ 0.95 . Functional association between $\mathrm{SI}$ and histological HFC was evaluated by a nonlinear saturation-growth regression model. In the population-based study, all MRIs were successfully performed. Mean HFC was $13.2 \pm 17.9 \% .52 .4 \%$ had no; $33.4 \%$ had low-grade, $12.2 \%$ moderate, and $2.0 \%$ high-grade hepatic steatosis Mean LIC was $1.7 \pm 1.4 \mathrm{mg} / \mathrm{g}$ (NR: $0.2-1.8 \mathrm{mg} / \mathrm{g}$ ) with $29.4 \%$ having abnormally high LIC. Results of HFC and LIC are available for further statistical analysis in the epidemiological study.

Conclusion: Three-point Dixon MRI with $\mathrm{T}^{*}$ correction is a cost-effective and noninvasive technique for quantifying hepatic fat and iron in a screening population.

\section{B-384 10:57}

Assessment of hepatic steatosis in morbidly obese patients undergoing gastric bypass surgery using open magnet 1H-MR spectroscopy J.R. van Werven ${ }^{1}$, T.C.M.A. Schreuder ${ }^{1}$, E. Aarts ${ }^{2}$, A.J. Nederveen ${ }^{1}$, J. Meijer ${ }^{2}$, F.J. Berends ${ }^{2}$, I.M.C. Janssen ${ }^{2}$, P.L.M. Jansen ${ }^{1}$, J. Stoker ${ }^{1} ;{ }^{1}$ Amsterdam/NL, ${ }^{2}$ Arnhem/NL (j.r.vanwerven@amc.uva.nl)

Purpose: The purpose of this study was to assess hepatic steatosis in morbidly obese patients undergoing gastric bypass surgery using open magnet $1.0 \mathrm{~T} 1 \mathrm{H}$ MRS with histopathological control.

Methods and Materials: Patients underwent 1H-MRS for the assessment of steatosis before and three months after surgery. Liver biopsy was performed during surgery. Steatosis was expressed as a ratio of the fat peak area over the cumulative water and fat peak areas. Histopathological percentages of steatosis were graded as follows: none (0-5\%), mild (5-33\%), moderate (33-66\%) and severe (>66\%). Spearman's correlation and accuracy was assessed. Differences between groups were assessed with the Wilcoxon signed rank and Mann-Whitney $U$ analysis. Results: We included 38 patients, with mean age and BMI of 45.6 years and $47.9 \mathrm{~kg} / \mathrm{m}^{2}$, respectively. Before surgery mean steatosis measured with $1 \mathrm{H}-\mathrm{MRS}$ was $10.3 \%$. $1 \mathrm{H}$-MRS showed an accuracy of $32 / 36(89 \%)$ and significantly correlated with histopathological assessment of steatosis $(r=0.85, p<0.001) .1 \mathrm{H}-\mathrm{MRS}$ was able to discriminate none from mild steatosis $(p=0.011)$, mild from moderate steatosis $(p<0.001)$ and moderate from severe steatosis $(p=0.037)$. Three months after surgery steatosis decreased to $5.2 \%(p<0.001)$. The prevalence of steatosis measured by $1 \mathrm{H}-\mathrm{MRS}$ decreased from $53 \%$ to $33 \%$.

Conclusion: $1 \mathrm{H}-\mathrm{MRS}$ for assessment of hepatic steatosis and changes in steatosis after gastric bypass surgery is feasible in morbidly obese patients using an open 1.0 T MR scanner. 1H-MRS measurements of hepatic fat are accurate and correlate with histopathology results. 1H-MRS using an open 1.0 T MR scanner should be considered when morbidly obese patients do not fit in cylindrical MR scanners.

\section{B-385 11:06}

Absolute liver fat quantification by dual-echo MRI: new evidence from a computer-based histopatholgic volume measurement M.A.F. Fischer ${ }^{1}$, D. Raptis ${ }^{1}$, M. Montani ${ }^{2}$, C. Reiner ${ }^{1}$, R. Graf ${ }^{1}$ P.-A. Clavien ${ }^{1}$, J. Hodler ${ }^{1}$, D. Nanz ${ }^{1}$, H. Scheffel ${ }^{1} ;{ }^{1}$ Zurich $/ \mathrm{CH},{ }^{2} \mathrm{Berne} / \mathrm{CH}$ (michaelalexander.fischer@usz.ch)

Purpose: To evaluate the accuracy of dual-echo gradient echo (DE-SPGR) magnetic resonance imaging (MRI) for the absolute quantification of liver fat conten (LFC) in vitro using a liver-fat phantom and in vivo with histopathology as the standard of reference. 
Methods and Materials: MRI was performed on a 1.5-Tesla scanner using a 3DDE-SPGR sequence with automatic chemical-shift selective reconstruction of in-/ out-of-phase (IP/OP) and fat-signal-only images. Absolute LFC was estimated by means of MR fat-fractions (FF-MRI) as derived from IP/OP (FF-IP/OP) and from fat-only images (FF-FAT). In vitro, seven phantoms containing homogenised liver/ fat concentrations from 0 to $50 \%$ (FF-Phantom) were examined. In vivo, 43 biopsies in 22 patients were retrospectively analysed for the percentage of hepatocytes containing lipid droplets (cell fraction; CF-HISTO) and for the absolute fat-fraction per field-of-view (FF-Phantom).

Results: In vitro significant linear correlation was observed between FF-MRI and FF-Phantom $(r=0.993 ; p<0.0001)$. Mean measurement bias of absolute values was significantly smaller for FF-IP/OP compared to FF-FAT $(+2.6 /+4.5)$. In vivo histopathological measurements ranged from 5 to $90 \%$ for CF-HISTO and from 0 to $20 \%$ for FF-HISTO. Correlation of FF-MRI with FF-HISTO was significantly better when compared to CF-HISTO ( $<<0.0001)$. Mean difference of FF-MRI to CF-HISTO was $21.1 \%$ and to FF-HISTO $4.8 \%$. Mean measurement bias was significantly smaller for FF-IP/OP compared to FF-FAT $(p<0.0001)$.

Conclusion: FF-MRI derived from 3D-DE-SPGR reflects the absolute LFC per area or volume and not the percentage of fatty hepatocytes as determined with traditional histopathological evaluation. FF-IP/OP is superior to FF-FAT for estimation of LFC showing significant less overestimation especially in a clinically relevant range from 0 to $5 \%$.

\section{B-386 11:15}

Liver focal fatty changes at ultrasound (LFFC-US) after percutaneous intraportal islet transplantation in islet-transplant-alone (ITA) and isletafter-kidney (IAK) transplanted patients: an early sign of altered graft function?

M. Cellina, M. Venturini, P. Maffi, C. Losio, C. Gremizzi, A. Palmisano, P. Fiorina, A. Secchi, A. Del Maschio; Milan/IT (cellina.michaela@hsr.it)

Purpose: Few longitudinal imaging studies of liver-engrafted-islets are available for ITA/IAK patients. The link between islet-function and the appearance of islet-induced-LFFC-US is controversial. The aims of this study were to assess prospectively LFFC-US and their relationship with islet-function.

Methods and Materials: LFFC-US timing of detection, prevalence and duration were assessed in 30 IAK, in 5 ITA and, retrospectively, in full-, partial-, and no-function-groups, according to islet-function evaluated one year after islet-transplantation. Patients with persistent LFFC-US underwent liver biopsy. LFFC-US-positive and LFFC-US-negative patients with functioning-islet were compared for islet-function and C-peptide-levels during the follow-up.

Results: LFFC-US were found in 12 patients (10/30 IAK, 2/5 ITA). First detection was at 6 months in 8 cases, at 12 months in 4 cases. LFFC-US last longer than 1 year in 8 cases. Steatosis was found histologically in $8 / 8$ patients. At 12 months, LFFC-US were detected at a higher extent in patients with partial-islet-function (10/12, 8 IAK, 2 ITA) compared with patients with full-islet-function. C-peptide-levels were significantly lower in LFFC-US-positive than in LFFC-US-negative patients. At 18 months, LFFC-US-positive were more prone to worsen their function (9/12) compared with LFFC-US-negative-patients (3/18).

Conclusion: US represents a sensitive tool to diagnose/monitor LFFC-US, resulting more related to partial- than full-islet-function, in both IAK and ITA patients. We could suppose that in case of full-function the local insulin secretion is not enough to induce steatosis. Differently, in some cases of partial function, steatosis could be the expression of remaining vital islets stressed in insulin overproduction due to lost function of other islets.

\section{B-387 11:24}

Quantification of liver fibrosis using diffusion-weighted MR imaging and proton MR spectroscopy in experimental rabbit models

Q. Wang, C. Liang, H. Liu; Guangzhou/CN (wangqscmu@gmail.com)

Purpose: To investigate whether the degree of liver fibrosis can be quantified non-invasively with diffusion-weighted MR imaging and proton MR spectroscopy using an experimental rabbit liver fibrosis model.

Methods and Materials: 5 male New Zealand White rabbits of control group and 40 rabbits of liver fibrosis group were examined with diffusion-weighted MR imaging and ${ }^{1} \mathrm{H}$ MR spectroscopy. Rabbit liver fibrosis models were induced by transperitoneal injection of $\mathrm{CCl}_{4}$ and were divided into no fibrosis (stage 0), mild/ moderate fibrosis (stages I and II) and severe fibrosis/cirrhosis (stages III and IV) groups based on histological examinations from resected liver. Diffusion-weighted SE EPI single shot sequence $\left(\mathrm{b} 1=0 \mathrm{sec} / \mathrm{mm}^{2}\right.$, b2 $=600 \mathrm{sec} / \mathrm{mm}^{2}$ ) and ${ }^{1} \mathrm{H}-\mathrm{MRS}$ PRESS technique (TR $=1500 \mathrm{msec}, \mathrm{TE}=35 \mathrm{msec}$ ) were used to measure ADC values and choline-to-lipid ratios. We compared the mean ADC value and choline/ lipid ratio among different groups of liver fibrosis.
Results: The mean ADC value decreased as the severity of liver fibrosis increased $(P<0.01)$, and was significantly different between all groups of fibrosis $(P<0.05)$ The mean choline/lipid ratio increased as the severity of liver fibrosis progressed $(P<0.05)$, and was statistically different between severe fibrosis/cirrhosis and the other groups of fibrosis $(P<0.05)$.

Conclusion: There is a decrease of ADC value and increase of choline/lipid ratio with increasing degrees of liver fibrosis. The preliminary results demonstrate the potential usefulness of diffusion-weighted imaging and proton MR spectroscopy for quantification of liver fibrosis.

\section{B-388 11:33}

Tissue echo patterns analysis with acoustic structure quantification (ASQ): preliminary experience of new non-invasive method for staging liver fibrosis in chronic hepatitis

V. Cantisani, P. Ricci, A. Marcantonio, C. Marigliano, V. Maldur, A. Porfiri, R. Passariello; Rome/lT

Purpose: The acoustic structure quantification technology is a novel ultrasound method that provides analysis of the fibrosis level in the liver using the statistical information in the raw data echo signals. The aim of this study was to evaluate ASQ in the assessment of liver fibrosis in patients with chronic autoimmune, criptogenetic and viral hepatitis $B$ and $C$.

Methods and Materials: 57 patients (26 females, 31 males, mean age 57 years) with chronic viral hepatitis underwent ASQ imaging and consecutively liver biopsy. The results were compared with the histological fibrosis degree $(F)$, which served as the reference. 20 healthy volunteers received $A S Q$ quantification of different segments of the liver.

Results: The best ASQ assessments with the lowest rate of invalid measurements were carried out by an intercostal approach to segment VII/VIII of the liver. The ASQ values of the healthy group had a mean of 108 (range 88-122), the means of the patient group ranged from 100 to 150 . ASQ quantification correlated significantly with the histological fibrosis stage: no fibrosis or mild fibrosis ( $<$ or = F2), severe fibrosis (F4 or F5) and cirrhosis (= F6), respectively.

Conclusion: This study shows the ASQ is a noninvasive, quick and suitable method for assessing liver fibrosis or cirrhosis in patients with HBV, HCV or autoimmune disorder. ASQ measurements of the liver should be performed via a subcostal access. Increasing ASQ values velocities correlate with higher degree of hepatic fibrosis.

\section{B-389 $\quad$ 11:42}

MR elastography and fibro-C index for quantification of liver fibrosis in chronic hepatitis $\mathrm{B}$ : preliminary results

S.K. Venkatesh, S. Xu, D.C.S. Tai, H.S. Alsaif, H. Yu, A. Wee; Singapore/SG (dnrskv@nus.edu.sg)

Purpose: Comparison of MR elastography (MRE) and fibro-C index for quantification of liver fibrosis in chronic hepatitis $B$.

Methods and Materials: A retrospective comparison of quantification of liver fibrosis with MRE and fibro-C index was performed in 30 patients with chronic hepatitis B. Stiffness of the liver was estimated by placing regions of interest on the stiffness maps MRE and a mean value in kilopascals $(\mathrm{kPa})$ obtained. The live biopsy samples were processed with standard histology technique and an experienced hepatopathologist graded for fibrosis using METAVIR score. On the same biopsy sample, quantification of the amount of collagen with fibro-C index was performed with Gaussian mixture model segmentation method and quantification index representing total aggregated collagen amount was obtained. The stiffness values, METAVIR scores and quantification index scores were correlated. Receive operator curve $(\mathrm{ROC})$ analysis was performed for accuracy of MRE and fibro-C index for detection of fibrosis.

Results: Liver stiffness values with MRE and quantification by fibro-C index correlated well $(R 2=0.64, p<0.01)$. There was no significant difference between the accuracies of MRE and fibro-C index for detection of fibrosis (0.94 vs. 0.90 $P=0.5)$, significant fibrosis ( 0.95 vs. $0.90, P=0.41)$ and cirrhosis $(0.99$ vs. 0.94 $P=0.2)$. However, MRE performed better than fibro-C index in differentiating the different grades of fibrosis from normal liver.

Conclusion: MRE derived stiffness of liver correlates well with fibrous content of liver and differentiates fibrotic liver and normal liver, therefore MRE may be suitable alternative for non-invasive assessment of liver fibrosis burden in chronic hepatitis $\mathrm{B}$ patients. 


\begin{abstract}
B-390 11:51
Intraobserver and interobserver variability (ie-iaOVar). in the estimation of liver fibrosis with shear wave elastography (SWE) in chronic liver disease: a proposition for standardisation through a modified protocol

P.S. Zoumpoulis, I. Theotokas, A. Plagou, K. Mpouhra, E. Vafeiadis; Athens/GR (p.zoumpoulis@echomed.gr)
\end{abstract}

Purpose: To estimate and correct ie-iaOVar. of measurements of liver fibrosis using SWE.

Methods and Materials: We studied 60 cirrhotic patients using SWE. All patients had liver biopsy performed in a period not exceeding \pm 4 months from US examination. During the first part of the study, all patients had a SWE examination of the liver performed by two independent examiners. Both examiners obtained two SWE measurements representing the mean tissue elasticity in $\mathrm{KPa}$. During the second part of the study, each examiner performed a third SWE examination which was processed by a prototype analysis software to quantify the degree of liver fibrosis. The findings were compared for fibrous liver staging ( $F$ stage) using liver biopsy. Results: We observed large ie-iaOVar. differences in the mean elasticity measurements during the first part of the study. The SWE images collected during the second part of the study were analysed by calculating statistical features (mean, standard deviation, kurtosis, skewness and entropy) of the elasticity values within multiple measurement ROls as well as relative ratios between different ROls. Multiple regression analysis was then performed to quantify the index of liver fibrosis. We found good correlation of the SWE-based quantification of liver fibrosis and the histologically derived liver fibrosis staging results.

Conclusion: SWE of the liver is an effective approach for estimating liver fibrosis, which exhibits good correlation with histologically proven $\mathrm{F}$ stage results. A modified analysis, adapted to the liver anatomic features and relying on multiple statistical features was found to reduce the ie-iaOVar. of measurements.

Room D1

Chest

\section{SS 804}

\section{Pulmonary embolism and beyond}

Moderators:

D. Musset; Clamart/FR

G. Staskiewicz; Lublin/PL

\section{B-391 10:30}

Severity assessment of pulmonary embolism using dual energy CT: correlation of a pulmonary perfusion defect score with clinical and morphological parameters of blood oxygenation and right ventricular failure S.F. Thieme, N. Ashoori, W.H. Sommer, T.R.C. Johnson, H. Leuchte, A. Becker, J. Behr, M.F. Reiser, K. Nikolaou; Munich/DE (sven.thieme@med.uni-muenchen.de)

Purpose: To establish a dual energy (DE)-based perfusion defect scoring system and correlate it with established pulmonary embolism (PE) severity scores in CT and clinical parameters of PE severity.

Methods and Materials: In 65 patients with acute PE in dual energy CT (DECT) angiography, DE perfusion maps were visually scored for perfusion defects using a novel scoring system (P-Score). Vascular obstruction score was determined using an established scoring system (Mastora) based on the CT pulmonary angiography (CTPA) data. Both scores were correlated with short-axis diameters of the left heart ventricle, right ventricular to left ventricular diameter ratio (RV/LV ratio), and width of the pulmonary trunk, as well as clinical data related to PE severity (oxygen saturation, plasma troponin I, arterial partial pressure of oxygen $\left(\mathrm{PaO}_{2}\right)$, heart rate, blood pressure, shock index, 30-days-survival). Reading times were measured. Results: P-Score correlated significantly $(\mathrm{p}<0.05)$ with vascular obstruction score (O-score) $(r=0.65)$, RV/LV ratio $(r=0.47)$, left ventricular diameter $(r=-0.36)$, width of the pulmonary trunk $(r=0.26)$, and with the clinical parameters troponin I $(r=0.43)$, and $\mathrm{PaO}_{2}(\mathrm{r}=-0.50)$. O-Score correlated significantly with $\mathrm{RV} / \mathrm{LV}$ ratio $(\mathrm{r}=0.36)$, width of the pulmonary trunk $(r=0.27), \mathrm{PaO}_{2}(r=-0.41)$, and troponin I $(r=0.37)$. Mean time for generation of P-score and O-score was $140 \pm 72$ and $252 \pm 108$ seconds. Conclusion: A newly introduced, DECT-based pulmonary perfusion defect scoring system correlates well with clinical and CT parameters of PE severity. It is an easy-to-perform method and represents a valuable clinical tool for diagnosis and prognosis of acute PE.

\section{B-392 10:39}

Impact on diagnostic performance and reading time of a computer aided detection algorithm for the detection of acute PE: second reading versus concurrent reading

R. Wittenberg ${ }^{1}$, J.F. Peters ${ }^{2}$, I.A.H. van den Berk' ${ }^{3}$, N.J.M. Freling ${ }^{3}$, R.J. Lely ${ }^{3}$, B. de Hoop 1 , K. Horsthuis ${ }^{3}$, C.J. Ravesloot ${ }^{1}$, C.M. Schaefer-Prokop ${ }^{4}{ }^{1}$ Utrecht/NL, ${ }^{2}$ Best/NL, ${ }^{3}$ Amsterdam/NL, ${ }^{4}$ Amersfoort/NL (rianne_wittenberg@hotmail.com)

Purpose: Evaluation of a CTPA study for the detection of pulmonary embolism (PE) represents a time consuming and tiring reading process. Computer-aided detection (CAD), when used as second reader, was found to improve reader performance especially for the detection of small emboli but inevitably at the expense of a substantial increase of reading time. We hypothesised that CAD may be used more advantageously when used as concurrent reading tool.

Methods and Materials: In this institutional review board-approved retrospective study, six observers of varying experience evaluated 157 negative and 39 positive 64-slice consecutively acquired CTPA. With a time interval of 6 weeks, all cases were read twice in different order using $C A D$ as second and as concurrent reader, respectively. Per patient, observers were asked to determine the presence of PE using a 5 point confidence scale and to document their reading time with and without CAD. Sensitivity and specificity were calculated by comparing the reader data with an independent consensus standard

Results: Baseline performance without CAD was high with a mean sensitivity of $91 \%$ and increased further to $95 \%$ with CAD as second reader and $94 \%$ with CAD as concurrent reader. Mean specificity was not adversely affected $(96 \%, 94 \%$ and $96 \%$, respectively). The mean reading time with CAD as concurrent reader significantly decreased (108 s, $136 \mathrm{~s}$ and $91 \mathrm{~s}$, respectively, $\mathrm{p}<0.001$ ).

Conclusion: CAD used as concurrent reader has the potential to achieve the same high sensitivities without loss of specificity at the advantage of a significantly shorter reading time.

\section{B-393 10:48}

Assessment of operability by means of CTPA and perfusion SPECT in patients with chronic thromboembolic pulmonary hypertension A. Grgic, F. Miodek, A. Buecker, D. Hellwig, M. Böhm, H.-J. Schäfers, R. Bals, H. Wilkens, C.-M. Kirsch; Homburg a.d. Saar/DE (aleksandar.grgic@gmx.de)

Purpose: Chronic thromboembolic pulmonary hypertension (CTEPH) is potentially curable with pulmonary endarterectomy (PEA). The criteria for identification of PEA-amenable patients need to be standardised. The aim of this study was to evaluate the value of rigidly registered CT pulmonary angiography (CTPA) and perfusion SPECT in differentiating between operable and non-operable patients. Methods and Materials: 45 patients with CTEPH ( 20 men, $59 \pm 12$ years) were evaluated for PEA by a local interdisciplinary board using all available diagnostic information and served as the gold standard. For the evaluation of SPECT a lobebased perfusion score was visually assessed using the score of 0 [lack of perfusion] to 1 [normal perfusion]), after which the percentage of vascular obstruction (PVO) was calculated: $P V O=[1$ - perfusion score $] \times 100$. By CTPA, the vascular obstruction index (OI) of central, peripheral, and global PA bed and diameters of large vessels (pulmonary artery (PA), aorta (Ao) and PA/Ao) was determined. In angiography, PA pressure (PAP), PA resistance (PVR) and wedge pressure $(\mathrm{PCm})$ were determined. Receiver operating characteristics (ROC) analysis was performed.

Results: Mean PAP, PVR and PCm was $48 \pm 12 \mathrm{mmHg}, 896 \pm 467$ dynes $^{*} \mathrm{sec}^{*} \mathrm{~cm}^{-5}$, and $11 \pm 5 \mathrm{mmHg}$. The PA diameter and PA/Ao correlated with mean PAP $(r=0.32$; $p=0.04 ;$ and $r=0.41 ; p=0.008)$ and PVR $(r=0.42 ; p=0.006 ;$ and $r=0.51 ; p=$ $0.001) .24$ patients were chosen as candidates for PEA. Haemodynamic values were not able to differentiate between operable and non-operable patients. PVO and central OI separated PEA-amenable patients (both $p<0.001$ ) resulting in the area under the curve of 0.847 (sensitivity of $83 \%$ and specificity of $80 \%$ ) and 0.805 (sensitivity and specificity of $79 \%$ and $80 \%$ ).

Conclusion: An accurate interpretation of rigidly registered CTPA and perfusion SPECT may contribute to stratification of operability in patients with CTEPH.

\section{B-394 10:57}

Thrombus load as a direct predictor for right ventricular failure in acute pulmonary embolism as demonstrated by computerised tomography pulmonary angiography

L.F. Wong ${ }^{1}$, A.R. Akram¹, J.T. Murchison'1, E.J.R. Van Beek ${ }^{1}$, J.H. Reid² ${ }^{1}$ Edinburgh/UK, ${ }^{2}$ Melrose/UK (Ifwong@doctors.org.uk)

Purpose: To determine the correlation between increasing thrombus load as a predictor of right ventricular failure as determined by computerised tomography pulmonary angiography (CTPA) findings to form a basis for correlation with clinical outcome. 
Methods and Materials: Retrospective cohort analysis of positive CTPA versus negative CTPA scans was performed over 40 months. Right ventricular:left ventricular ratio (RV:LV ratio), presence of septal shift, pulmonary artery and aortic size were analysed with respect to thrombus load as determined by the modified Miller score (MMS) using non-parametric analysis (two-tailed or Chi-squared test) and Pearsons rank correlation.

Results: 504 positive CTPA scans and a representative cohort of 100 negative CTPA scans were included. Increasing MMS was found to correlate with higher $\mathrm{RV}: \mathrm{LV}$ ratio with statistically significant difference between the RV:LV ratio between the negative cohort versus the positive cohort with MMS 12 or more (mean RV:LV ratio 1.323 vs $0.930, p<0.0001$ ) with no significant difference with $M M S<12$. Septal shift was also more likely with a MMS 12 or more compared to MMS $<12(63.8 \%$ vs $18.6 \%, p<0.001$ ) with an increase in pulmonary artery size with increasing MMS (Pearson rank coefficient $r=0.221, p<0.001$ )

Conclusion: This is the first study to accurately describe the relationship between thrombus load and an increase in RV:LV ratio beyond a MMS score of 12 using CT pulmonary angiography. This suggests a 'tipping point' beyond which right ventricular decompensation occurs and may add to further risk stratification in patients with acute pulmonary embolism on CTPA.

\section{B-395 11:06}

MRI for acute pulmonary embolism diagnosis: prospective evaluation in 300 patients with comparison to 64-detector multislice CT

M.-P. Revel, S. Couchon, A. Hernigou, R. Niarra, G. Chatellier, G. Meyer,

C. Lefort, G. Frija; Paris/FR (marie-pierre.revel@egp.aphp.fr)

Purpose: To evaluate current MRI performance for PE diagnosis, by reference to 64-detector multislice CT angiography (CTA) findings.

Methods and Materials: IRB approval and informed consent were obtained. Three hundred patients out of 1796 patients referred for PE suspicion in our institution between June 2007 and June 2009 were included. MRI was performed within 24 hours from CTA on a 1.5 Tesla unit and included 3 different sequences: 1- Unenhanced steady state free precession (SSFP) (2D FIESTA) axial transverse sequences, with ECG gating, with and without breath-holding, 2- fast SPGR coronal perfusion sequence (TR: 2, TE: 0.8 ) after injection of $0.1 \mathrm{mmol} / \mathrm{kg}$ DOTA-Gd at $5 \mathrm{ml} / \mathrm{s}$, 3- fast SPGR axial transverse angiographic sequence (TR: 3.4, TE:1.2) after injection of $0.1 \mathrm{mmol} / \mathrm{kg}$ DOTA-Gd at $3 \mathrm{ml} / \mathrm{s}$. Two independent readers analysed all sequences together (global reading) and each sequence separately in a random order.

Results: MRI protocol was completed in 274 patients who had a conclusive CTA. The rate of indeterminate result was $28 \%$ for reader 1 and $30 \%$ for reader 2 . Excluding indeterminate results, MRI sensitivity and specificity on global readings were 84 and $99 \%$ for reader 1 and 79 and $100 \%$ for reader 2 . The kappa value for interobserver agreement was 0.59 (95\% Cl: 0.50 - 0.67). Unenhanced breath-holded SSFP sequence sensitivity and specificity were 76 and $96 \%$ for reader 1 and 69 and $99 \%$ for reader 2 . Conclusion: MRI shows high specificity, even on unenhanced sequences and acceptable sensitivity for PE diagnosis at the cost of a high rate of indeterminate results.

\section{B-397 11:15}

CT angiography on the management of emergency department patients suspected of having pulmonary embolism: the impact of nonthromboembolic findings in $\mathbf{4 9 6}$ patients

J.-O.F. Johnson, E. Cento, S. Gupta, R.A. Novelline; Boston, MA/US (jamlikomari@gmail.com)

Purpose: Pulmonary embolism (PE) is potentially life-threatening. Its clinical findings can mimic other conditions. CT angiography (CTA) is the cornerstone of evaluating patients with suspected PE presenting to the Emergency Department (ED). The purpose of this study was to 1. determine the incidence acute PE among adult $E D$ patients undergoing CTA and 2. determine the frequency of alternate diagnoses which affect patient management and its concordance with chest radiography. Methods and Materials: Two board-certified radiologists retrospectively reviewed the clinical information and radiologic findings of 496 patients suspected of $\mathrm{PE}$ who presented to the ED in 2009. We documented the presence of PE, alternate diagnoses and correlation to radiography. Non-PE CT findings were categorized into three groups: $A=$ acute change in clinical management; $B=$ follow-up, but no change in clinical management; $\mathrm{C}=$ normal or no clinical significance.

Results: CTA was nondiagnostic in nine patients. The frequency of $P E$ among 487 patients was $10.3 \%$. The prevalence of the categorized groups was $A=17 \%, B=$ $24 \%$, and $C=69 \%$. The predominate category $A$ finding was pneumonia $(88 \%)$. Fifty-two per cent of these pneumonia patients had concordant findings on chest radiograph. The most common group $B$ finding was an indeterminate pulmonary nodule $(70 \%)$ requiring follow-up in $17 \%$ of total patients.
Conclusion: CTA identified PE in $10.3 \%$ of ED patients. In addition, CTA provided an alternate diagnosis which impacted acute treatment in $17 \%$ of patients. Pneumonia was the most common non-PE diagnosis and was radiographically occult in nearly $50 \%$ of patients. Seventeen per cent of patients had indeterminate nodule (s)

\section{B-398 $11 \cdot 24$}

Do incidentally discovered pulmonary emboli on contrast-enhanced abdominal CT scan warrant further evaluation?

Y. Yagil, A. Engel, L. Guralnik; Haifa/IL (y_yagil@rambam.health.gov.il)

Purpose: Unsuspected pulmonary embolism (PE) is a well-recognised entity however, there are no publications regarding incidentally discovered PE on contrastenhanced (CE) abdominal CT scans. The aim was to determine the added value of a CE-chest CT scan to abdominal CT scan for the confirmation of PE.

Methods and Materials: CE-chest CT and abdominal CT examinations of 35 patients in whom $\mathrm{PE}$ was detected on the abdominal CT examination were retrospectively studied. Data pertaining to patient characteristics and CT examinations were obtained from computerised medical records. PE was evaluated by the observation of occlusive pulmonary arterial filling defects on contiguous images. Verification of PE and quantification of arterial clot load using the Miller scoring system was assessed on both examinations.

Results: The study population included 15 (43\%) men and 20 (57\%) women, with a mean age of 63.5 years (range $22-89$ years). Seventeen $(49 \%)$ patients had a history of active malignancy and $7(19 \%)$ of previous thromboembolic episode. All patients diagnosed with PE by the abdominal CT scan had positive PE findings on the chest CT scan as well. The average difference in arterial clot load score between the two scans was $22 \%$. In $46 \%$ of the cases no difference in score was observed. Conclusion: Positive PE findings on CE-abdominal CT scan can provide the necessary information required for decision on patient management. Therefore, incidental discovery of PE on CE-abdominal CT scan does not warrant further evaluation by a CE-chest CT scan.

\section{B-399 11:33}

Clinicians' response to a report of an incidental pulmonary embolism detected on contrast-enhanced CT

J.M. Franklin, F.V. Gleeson; Oxford/UK (jamiemfranklin@hotmail.com)

Purpose: Incidental pulmonary emboli (iPE) may be detected on contrast-enhanced CT (ceCT) of the chest performed for other reasons; the prevalence of this finding is approximately $2.5 \%$. The purpose of this study was to report on both clinical response related to the finding of an $\mathrm{PPE}$ on $\mathrm{CeCT}$ in the UK, and the reasons for the responses.

Methods and Materials: Patients with an iPE detected on a ceCT incorporating the chest were retrospectively identified for a 12-month period. Case note review was performed to assess clinical response to this finding. Patient and iPE characteristics, subsequent treatment, and outcome related to treatment and nontreatment were recorded.

Results: There were 68 patients with iPE. 37\% of iPE were in the pulmonary trunk or main pulmonary arteries, $35 \%$ were lobar and $28 \%$ were in the segmental or subsegmental arteries. 62 of $68(91.2 \%)$ patients with iPE were treated with therapeutic anticoagulation. There were $2(3.2 \%)$ serious adverse events related to treatment. Six (8.8\%) patients were not treated. Two (33\%) developed recurrent venous thromboembolism (VTE); the remainder suffered no adverse sequelae. Clinicians' decisions to treat iPE were not significantly associated with patient age, $\mathrm{PE}$ risk factors or PE size. A smaller proportion of single iPE were treated than multiple iPE (19/24 vs 43/44; $p=0.033)$.

Conclusion: There are adverse outcomes associated with both treatment and non-treatment of iPE. However, despite the uncertainty about the natural history and clinical significance of iPE, the majority of patients at our institution with iPE receive prompt anticoagulation.

\section{B-400 11:42}

Pulmonary arterial hypertension associated with systemic sclerosis: prevalence in the absence of interstitial lung disease

V. Wilkinson, K. Karunasaagarar, J. Hurdman, C. Elliot, D. Kiely, C. Hill; Sheffield/UK (Victoria.Wilkinson@sth.nhs.uk)

Purpose: Pulmonary arterial hypertension (PAH) may occur independenly of interstitial lung disease (ILD) in systemic sclerosis (SSc). We aimed to assess the proportion of patients in our SSc population with $\mathrm{PAH}$, in the absence of ILD. Methods and Materials: Retrospective analysis of 153 SSc patients investigated for possible PAH. 102 patients included who had high resolution computed tomography (HRCT) and right heart catheterisation (RHC) within 3 months. PAH diagnosed by 
$\mathrm{RHC}$ based on a mean systolic pulmonary arterial pressure of $\geq 25 \mathrm{mmHg}$ at rest or $\geq 30 \mathrm{mmHg}$ on exercise (EPAH). HRCT consensus read by 2 blinded chest radiologists. Presence/severity of ILD evaluated at 3 lung levels based on a scoring system adapted from Desai et al. (2004). Degree of ILD categorised as absent or $\mathrm{mild} /$ moderate/severe accordingly. 29 patients with evidence of other lung disease associated with PAH or any lung levels not assessable were excluded.

Results: 45 patients had PAH at rest, 14 had EPAH and 14 did not have PAH in the study group. $49 \%$ had no evidence of ILD, $44 \%$ had mild ILD, $7 \%$ had moderate ILD, and no patients had severe ILD in the PAH at rest group. 50\% had no HRCT evidence of ILD and $50 \%$ had mild ILD in the EPAH group.

Conclusion: This study provides further confirmation that PAH occurs independently of ILD in SSc. The fact that PAH was also seen frequently in patients with only mild ILD suggests disproportionate pulmonary vascular involvement.

\section{0:30 - 12:00}

Room D2

\section{Interventional Radiology}

\section{SS 809}

\section{Peripheral vascular intervention}

Moderators:

M. Cerna; Olomouc/CZ

D.K. Tsetis; Iraklion/GR

\section{B-401 10:30}

Analysing clinical and cost effectiveness of endovascular treatment in patients with critical ischaemia of crural arteries in a tertiary university hospital from 2005 to 2010

T.A. Sandner, C. Degenhart, J. Becker-Lienau, M.F. Reiser, M. Treitl;

Munich/DE (torleif.sandner@med.uni-muenchen.de)

Purpose: Studies reporting efficacy of endovascular therapy are characterised by heterogeneous definition of endpoints. As a result, in particular, for crural arteries only poor information regarding functional patient outcome is available. Also, data analysing treatment costs in those patients are rare. We report our results from a tertiary university hospital in Munich/Germany.

Methods and Materials: A retrospective analysis of all patients who underwent infrapopliteal angioplasty for critical limb ischaemia (CLI) from 2005 to 2010 was performed. Clinical, procedural and haemodynamic outcome was analysed. The primary end point was limb salvage. Secondary end points were technical success, improvement according to Rutherford, ulcer healing, overall survival, recurrence and complications. In addition, material consumption and costs were reviewed. Results: In 191 patients (mean age 64.7 yrs; 70.7\% male) 486 percutaneous endovascular interventions (PEI) were performed. Single vessel run-off to the foot was present in 120 (62.8\%) patients and complete occlusion of all crural vessels in $42(22.0 \%)$ at initial presentation. The mean follow-up was 6.5 months, 17 patients were lost during follow-up. Technical success rate was $81.1 \%$ (394/486 interventions) and limb salvage could be achieved in $71.7 \%$ (123/174 patients). A high recurrence rate was especially found in diabetic patients. The mortality rate was $5.2 \%$ and major complications occurred in $7.3 \%$. Average total cost/patient/ intervention was 1118.02 \pm 309.42 Euro and $2381.76 \pm 518.41$ Euro for the total length-of-stay in hospital.

Conclusion: PEI is a valuable option particularly in multimorbid patients in which the use of bypass surgery is limited and who otherwise might face amputation. Taking into account a longer hospitalisation and higher periprocedural complication rates in patients undergoing surgery, PEI is economically advantageous.

\section{B-402 10:39}

Long-term results and cost analysis of an interdisciplinary approach for the treatment of critical limb ischaemia (CLI)

M. Cejna, N. Durakovic, R. Mathies, W. Hofmann; Feldkirch/AT

(manfred.cejna@lkhf.at)

Purpose: TASC guidelines for CLI require an interdisciplinary approach. What are the long-term success rates at what costs?

Methods and Materials: Retrospective analysis of all invasive treatments for CLI performed 2005 in the Vascular Centre of the LKH Feldkirch. All treatment- and follow-up treatment data up to 2009 (imaging, endovascular interventions and surgical treatment, complications, major and minor amputations, survival) were recorded from patient records. In addition, cost-calculations were performed. Results: 90 limbs were treated in 78 patients $(73.8 \pm 9.6$ years mean age) 18 limbs had Fontaine stage III and 72 limbs had Fontaine stage IV disease. Of 90 limbs 40 were primarily treated by endovascular-, 45 by surgical- and 5 by combined techniques. Endovascular treatment was applied to significantly lower TASC stages $(p=0.001)$. Limb salvage rates were 95\% (1 year), 95\% (2 years), 95\% (3 years) and $87 \%$ (4 years), respectively. Survival rates were $86 \%$ (1 year), $81 \%$ (2 years), $70 \%$ (3 years), and $66 \%$ (4 years), respectively. There were no significant differences between the treatment groups for limb salvage $(n=0.20)$ and survival $(n=0.58)$, respectively. Costs for initial surgical treatment were 6.298 Euro, and 3.869 Euro (for endovascular treatment) per patient. Total treatment costs (including 5-year follow-up) were 27.429 Euro for the surgery first approach and 17.443 Euro for the endovascular first approach per patient

Conclusion: Interdisciplinary co-operation achieves good long-term results for limb salvage and survival. An endovascular first approach has lower costs than a surgery first approach and should be preferred when applicable.

\section{B-403 10:48}

Randomised clinical study of the Zilver ${ }^{\circledast}$ PTX ${ }^{\circledR}$ self-expanding Nitinol stent with polymer-free paclitaxel coating shows improved 12-month effectiveness over angioplasty and bare metal stents for the superficial femoral artery

F. Fanelli; Rome/IT (fabrizio.fanelli@uniroma1.it)

Purpose: Zilver ${ }^{\circledR}$ PTX ${ }^{\circledR}$ is the first drug-eluting stent with CE approval for the superficial femoral artery (SFA). This multicenter, randomised study compared the safety and effectiveness of the Zilver PTX to balloon angioplasty (PTA) and bare metal stenting (BMS).

Methods and Materials: Patients with de novo or restenotic SFA lesions were randomised to PTA or Zilver PTX stent placement. PTA patients experiencing procedural failure ( $>30 \%$ residual stenosis) underwent secondary randomisation to provisiona stenting with Zilver BMS or Zilver PTX. Endpoints included event-free survival (EFS) and primary patency by Duplex ultrasound (peak systolic velocity ratio $<2.0$ ). Results: 479 patients were enrolled, with 241 randomised to the Zilver PTXgroup and 238 to the PTA group. Demographics and lesion characteristics were similar for the groups. Approximately half the PTA group experienced procedural failure and underwent secondary randomisation, assigning 59 and 61 patients to provisional stenting with Zilver BMS and Zilver PTX, respectively.

12-month results included non-inferiority of EFS rate and superiority of patency rate for the Zilver PTX groups compared to the PTA group. The stent fracture rate was less than $1 \%$. There was significant clinical improvement with Zilver PTX over both PTA and BMS. Furthermore, provisional Zilver PTX showed significantly improved primary patency over provisional BMS, demonstrating the benefit of the polymer-free paclitaxel coating.

Conclusion: The Zilver PTX results support safety and show significantly improved effectiveness over PTA and BMS for treatment of the SFA.

\section{B-404 10:57}

Subintimal angioplasty for superficial femoral artery tasc II d lesions in critical limb ischaemia: outcomes with and without stenting and value of stent position for secondary patency after subintimal channel occlusion R. Gandini, E. Pampana, L. Boi, G. Loreni, M. Stefanini, G. Simonetti; Rome/IT (boiluca.md@gmail.com)

Purpose: In a prospective, double arm, non-randomised, single center consecutive series of subintimal angioplasty (SIA) with selective stent placement for superficial femoral and popliteal artery TASC II D lesions, critical limb ischaemia patients' data were analysed with regard to immediate outcome and follow-up success.

Methods and Materials: SIA was successful in 398 patients of 406 critically limb ischaemia (98\%). Stents were released in the subintimal channel in 83 patients (21\%) after suboptimal SIA.

Results: Primary patency at one-year follow-up was 79.5 and $66.7 \%$, respectively, in patients treated with and without stent $(p=0.02)$. One-year follow-up secondary patency was 87.5 and $92.0 \%$ in patients treated with and without stent $(p=$ NS). Re-occlusions occurred in 147 patients treated by SIA alone (47\%) and 27 patients treated by SIA and stent $(32 \%)(p=0.02)$. Successful recanalisation rate was higher for patients treated by SIA alone (95\%) compared to patients in who a stent was released $(81 \%)(p=0.04)$. Feasibility of stent recanalisation was higher in patients treated by a stent released in contact with the true lumen $(p=0.02)$.

Conclusion: SIA is a feasible and effective primary treatment for patient with critical limb ischaemia due to TASC II D superficial femoral and popliteal artery lesions. Use of stent in subintimal angioplasty improved primary patency compared to SIA alone treatment. Stent position affects the technical approach for possible future recanalisation after stent occlusion 


\section{B-405 11:06}

Transfemoral and transtibial combined approach in subintimal recanalisation of SFA obstructions extending on popliteal and distal vessels origin

R. Gandini, E. Pampana, V. Da Ros, L. Boi, M. Stefanini, G. Simonetti; Rome/IT (boiluca.md@gmail.com)

Purpose: To propose our technique, to obtain the optimal recanalisation to the foot in case of long SFA occlusions involving the popliteal trifurcation, using a combined antegrade and retrograde subintimal approach in patients presenting critical limb ischemia (CLI).

Methods and Materials: On 664 diabetic patients treated for limb salvage, in 75 with SFA long occlusions involving the leg vessel tree, subintimal antegrade and retrograde approaches were performed (posterior tibial artery in 43 and anterior tibial artery in 32 cases). The patent portion of the runoff vessel was previously assessed by magnetic resonance angiography and directly punctured under ultrasound Doppler guidance. A subintimal channel rendezvous was performed to allow snaring of the guidewires. Subsequent balloon dilatation was performed.

Results: We achieved $98.2 \%$ technical success. At Doppler-US mean follow-up of 24.5 months, the patent vessels were $71.2 \%$, but we had a $92.3 \%$ in limb salvage with complete healing of limb lesions and rest pain resolution. The oximetry value showed an increase from mean original value of $17.8 \mathrm{mmHg}$ to $45.1 \mathrm{mmHg}$ at 6 months follow-up.

Conclusion: In patients with SFA occlusion involving the popliteal trifurcation, secure candidate in amputation, combined antegrade and retrograde subintima recanalisation approaches, is probably the most suitable and efficacy endovascular option to obtain a direct flow to the foot and so, an high percentage of limb salvage.

\section{B-406 11:15}

Directional atherectomy of heavily calcified stenotic lesions of the lower limb within joint segments

P. Minko, S. Jaeger, A. Buecker, M. Katoh;

Homburg a.d. Saar/DE (peterminko@yahoo.com)

Purpose: To evaluate the mid-term outcomes (2 year) of patients with peripheral occlusive disease (POD) and heavily calcified stenotic lesions in segments with high biomechanical stress after atherectomy.

Method and Materials: Patients suffering from POD (Rutherford 3 to 6) were treated with the Silverhawk atherectomy device (ev3 Endovascular, MN, USA) if heavily calcified lesions in the most proximal or distal $3 \mathrm{~cm}$ of the superficial femoral artery and/or popliteal artery were present. Overall 42 patients with 46 lesions (15 females, 27 males; mean age: $69 \pm 8.8$ ) were included into this prospective study. Patients were followed up after 6, 12, 18 and 24 months for clinical re-evaluation including the measurement of the maximum walking distance and the ankle brachial index $(\mathrm{ABI})$ and to perform duplex sonography.

Results: The primary success rate of the procedure was $89 \%$. In five cases additional PTA and/or stenting was necessary. Procedure-related complications namely peripheral embolism occurred in 3 cases $(6 \%)$. The mean Rutherford score decreased significantly from 4 to $0.8(p<0.001)$, while the mean $A B I$ increased from 0.62 to $0.94(p=0.088)$ after 24 months. The primary and assisted primary patency rate was $66 \%$ and $79 \%$, respectively.

Conclusion: Directional atherectomy should be considered as an alternative for treatment of heavily calcified stenotic lesions in segments with high biomechanical stress as in most cases stenting can be avoided. Results after two year demonstrated a significant decrease of the Rutherford score and an increase of the $A B$ and a reasonable patency rate.

\section{B-407 11:24}

Haemodynamic endpoint definition of directional atherectomy in the superficial femoral and popliteal artery by direct intraarterial pressurewire measurments

J. Becker-Lienau, M.F. Reiser, M. Treitl;

Munich/DE (mtreitl@med.uni-muenchen.de)

Purpose: A major drawback of directional atherectomy is potential over-excision with the risk of vessel rupture or aneurysm formation. Since there is no study concerning the endpoint of atherectomy, the interventionalist decides by angiogram and experience when to stop atherectomy. The typically irregularly vessel silhouette induces the interventionalist to do more passes then maybe necessary. Our study compares the least necessary count of passes of directional atherectomy for sufficient stenosis treatment based on (1) intra-arterial pressure measurements and (2) satisfactory angiographic results as considered by the interventionalist.
Methods and Materials: In a prospective trial consecutive patients with stenosis/ occlusion of the femoro-popliteal vasculature with a length $\leq 90 \mathrm{~mm}$ are treated by atherectomy with the SilverHawk/TurboHawk catheter. The intra-arterial pressure gradient over the target lesion, measured by Radi PressureWire, is controlled before and after each pass with the atherectomy device. Haemodynamically sufficien treatment is defined as a complete reduction of the pressure gradient to 1.0. The endpoint of atherectomy is defined angiographically by the interventionalist who is blinded to the results of pressure measurements.

Results: 20 patients were enrolled. Mean lesion length was $5 \mathrm{~cm}$, mean pressure gradient 0.72 . Complete reduction of the pressure gradient was achieved with $4 \pm 2$ passes in mean. For a satisfactory angiographic result in mean $11 \pm 4$ passes were necessary $(p<0.001)$.

Conclusion: Our results demonstrate that directional atherectomy can achieve haemodynamically sufficient stenosis treatment with significantly less catheter passes than angiography indicates, allowing for a shortening of the procedure and reduced complication rate.

\section{B-408 11:33}

Alternative techniques for below-the-knee recanalisation: procedural and clinical results from a single center experience

R. Gandini, L. Boi, C. Del Giudice, A. Spinelli, C. Reale, G. Simonetti; Rome/IT (boiluca.md@gmail.com)

Purpose: Percutaneous arterial recanalisation with PTA is becoming cornerstone of treatment of below-the-knee vascular peripheral disease. Nonetheless, successful recanalisation is not always obtained with traditional antegrade approach. Recently, new alternatives techniques have been introduced to recanalise these cases not treatable with antegrade approach. We report our results focusing on anatomical indications technical aspects and rationale of these new approaches. Methods and Materials: In a single-center retrospective clinical analysis, from January 2005 to July 2010, we collected 1602 patients with critical limb ischaemia for complex lesions of the popliteal and infrapopliteal vascular territory. In 224 patients (14\%), traditional antegrade revascularisation failed and alternative techniques were adopted. Of these subgroups, 85 limbs were treated with transpedal retrograde approach, in 46 patients a pedal to plantar and 36 patients a plantar to pedal retrograde revascularisation was performed. A transcollateral angioplasty and retrograde revascularisation was suitable in 57 patients.

Results and Conclusion: Successful recanalisation was obtained in 219 patients $(98 \%)$ with alternative techniques. Adjunctive popliteal stenting was performed only in 13 patients.

\section{B-409 11:42}

Use of a percutaneous suture-mediated closure system to achieve haemostasis after 6-8 $\mathrm{F}$ transfemoral approaches

P. Kamusella ${ }^{1}$, C. Wissgott ${ }^{1}$, H.J. Steinkamp ${ }^{2}$, R. Andresen ${ }^{1} ;{ }^{1}$ Heide/DE, ${ }^{2}$ Berlin/DE (pkamusella@wkk-hei.de)

Purpose: The safety and effectiveness of a percutaneous suture-mediated closure system for achieving haemostasis after a transarterial femoral approach were investigated.

Methods and Materials: A percutaneous suture closure system (Perclose/ PercloseProglide, Abbott Vascular) was used in 2200 patients (average age $67.5 \pm 12.5$ years) who had undergone angiographic intervention. Vascular access sites with a sheath size of between $6 \mathrm{~F}$ and $8 \mathrm{~F}$ were closed. Platelet aggregation was inhibited with $100 \mathrm{mg} / \mathrm{d}$ ASA and all of the patients additionally received $5000 \mathrm{IU}$ heparin periinterventionally. After application of the suture-mediated closure system and achievement of sufficient haemostasis, a light compression bandage was applied in all patients and 6 hours' bed rest was recommended. Post-interventionally (following day and after 6 weeks), the puncture site of all patients was checked using colour-coded duplex sonography.

Results: Immediate haemostasis was achieved in 2103/2200 patients (95.6\%). In the remaining $97 / 2200$ cases, a correct development of the suture was not possible, because of calcifications. In such cases, haemostasis was achieved by manual compression. Major complications involved three infections that required vascular surgical debridement with the use of an interposition graft. In addition, there were five cases of secondary bleeding requiring transfusion. Minor complications involved $3(0.14 \%)$ pseudoaneurysms, $256(11.6 \%)$ groin haematomas (up to $\max .3 \mathrm{~cm}$ ) and $85(3.9 \%)$ palpable suture granulomas.

Conclusion: At puncture sizes of 6-8 F, safe and effective haemostasis is possible with the percutaneous suture-mediated closure system. Insufficient suture closure is to be expected in the case of strongly calcified vessels. 


\section{B-410 11:51}

The length of right basilic vein from elbow crease to carina measured during peripherally inserted central catheter insertion under fluoroscopy J.-Y. Yoon, E.-Y. Jeon, S. Choi, H. Kim, J.-M. Park, B.-S. Cho; Anyang/KR (yoonji@hallym.or.kr)

Purpose: For central placement of tip of peripherally inserted central catheter (PICC), individual length of upper extremity vein in relation to the body size of the patient is important, because blind bedside procedure of PICC is increasing. Methods and Materials: From April 2008 to August 2010, 100 (49 males, 51 females) out of 156 patients were selected in whom PICC was inserted through right basilic vein under fluoroscopy and measurement data were available. Measurement of right basilica vein from elbow crease to the carina was done during the procedure (catheter length, length of elbow crease to puncture site) and post-procedural chest radiography (length of carina to catheter tip). These data were compared with the height of the patient and formula of right upper extremity vein from elbow crease to carina in relation to the height was acquired by linear regression analysis.

Results: The mean length of elbow crease to carina was $42.5 \mathrm{~cm}$ with standard deviation (SD) of 4.03 , and height was 160.6 with SD of $10.0 \mathrm{~cm}$. The formula of length of right upper extremity vein through basilic vein from elbow crease to carina was 0.2178 by height plus $7.47 \mathrm{~cm}$. Although the height and right upper extremity vein length were longer in male than in female, the data of height minus length of right upper extremity vein were not statistically different.

Conclusion: For blind bedside procedure of PICC, the formula of our study would be greatly helpful for the correct central location of the tip of PICC.

\section{Cardiac}

\section{SS 803}

\section{Cardiac stress imaging and functional analysis}

\section{Moderators:}

S. Katsilouli; Athens/GR

F. Pugliese; London/UK

\section{B-411 10:30}

Diagnostic accuracy of CT-based dynamic adenosine-mediated myocardial perfusion imaging for the detection of hemodynamically relevant coronary artery stenosis

F. Bamberg, A. Becker, K. Bauner, F. Schwarz, R. Marcus, T. Johnson, M.F. Reiser, W. Sommer, K. Nikolaou; Munich/DE (fabian.bamberg@med.uni-muenchen.de)

Purpose: To determine the diagnostic accuracy of CT-based dynamic myocardial perfusion imaging for the detection of hemodynamic relevant coronary artery stenosis as defined by invasive measurement of fractional-flow reserve (FFR).

Methods and Materials: Subjects scheduled for invasive angiography underwent dynamic adenosin-stress myocardial perfusion imaging (100 kV, 320 mAs/rot, ECG-triggered) every second for 30 s using a fast Dual Source CT. At invasive angiography, FFR measurement of all coronay lesions was performed. Myocardial blood flow and volume (MBF and MBV) were derived from CT using a model-based parametric deconvolution method for each myocardial segment pertaining to each coronary artery. A FFR $\leq 0.75$ was defined as hemodynamic relevant.

Results: Of 31 enrolled subjects ( $81 \%$ male, mean age: $64 \pm 3$ years), 26 completed the study protocol ( $10.3 \pm 2 \mathrm{mSv})$. Among the remaining 22 subjects, 416 myocardial segments were analyzed of which $59(14.2 \%)$ were classified as hemodynamically impaired (FFR $\leq 0.75$ ). On average, MBF and MBV were $86.4 \pm 29 \mathrm{ml} / 100 \mathrm{ml} / \mathrm{min}$ and $17.2 \pm 6 \mathrm{ml} / 100 \mathrm{ml}$; respectively and significantly lower in territories pertaining to a hemodynamic relevant coronary stenosis $(56.2 \pm 15 \mathrm{vs} .91 .4 \pm 28 \mathrm{ml} / 100 \mathrm{ml} / \mathrm{min}$ and $11.5 \pm 4$ vs. $18.1 \pm 5 \mathrm{ml} / 100 \mathrm{ml}$, both $\mathrm{p}<0.001)$. Using $70 \mathrm{ml} / 100 \mathrm{ml} / \mathrm{min}$ as a positivity criterion (optimization of the AUC), sensitivity and specificity of MBF for the detection of a significant stenosis was $88 \%[95 \%-\mathrm{Cl}$ : $0.73-0.97]$ and $85 \%[95 \%-\mathrm{Cl}$ : 0.80-0.90], respectively [NPV and PPV: 97 and 50\%, respectively).

Conclusion: Dynamic CT-based stress myocardial perfusion imaging permits detection of hemodynamic relevant coronary artery stenosis and may thus allow for simultaneous assessment of both coronary morphology and hemodynamic relevance.

\section{B-412 10:39}

Dynamic myocardial stress perfusion imaging using $2^{\text {nd }}$ generation dualsource computed tomography

M. Weininger ${ }^{1}$, U. Schoepf ${ }^{1}$, T. Henzler ${ }^{2}$, G.W. Rowe', P. Costello', G. Bastarrika ${ }^{3} ;{ }^{\prime}$ Charleston, SC/US, ${ }^{2}$ Mannheim/DE, ${ }^{3}$ Pamplona/ES (weininge@musc.edu)

Purpose: Most recent advances in computed tomography (CT) suggest CT as standalone modality for an integrative assessment of all aspects of coronary artery disease. We evaluated the feasibility of first-pass myocardial stress perfusion imaging using $2^{\text {nd }}$ generation dual-source $C T$.

Methods and Materials: Dynamic myocardial adenosine-stress CT perfusion imaging (Somatom Definition Flash, Siemens) was performed on 30 consecutive patients (18 males, 12 females; mean age $62 \pm 12$ years) using a dedicated "shuttle" mode. Additionally, all patients underwent rest/stress MRI and SPECT perfusion, and delayed-enhancement MRI. Two blinded observers visually assessed for myocardial perfusion defects on CT compared to MRI and SPECT. For semi-quantitative evaluation, CT- and MRI-derived myocardial-to-left ventricular upslope indices were compared. In addition, absolute MBF was quantified based on dynamic perfusion $\mathrm{CT}$ and correlated with semi-quantitative $\mathrm{CT}$ measurements. Results: A total of 459 segments (90\%) were suitable for evaluation. Sensitivity, specificity, positive and negative predictive values for detecting myocardial perfusion defects at CT (versus SPECT) compared to MRI were as follows: $86 \%$ ( $84 \%$ ), $98 \%$ $(92 \%), 93 \%(88 \%), 94 \%(92 \%)$. Semi-quantitative analysis of CT data displayed differences between ischaemic and non-ischaemic myocardium with a signal intensity upslope comparable to MRI-derived values (CT: $5.2 \pm 2 \mathrm{SI} / \mathrm{s}$; MRI: $4.7 \pm 2.3$ $\mathrm{SI} / \mathrm{s}$ ). Moderate correlation was observed between absolute $\mathrm{CT}$ quantification of myocardial blood flow and semi-quantitative CT measurements.

Conclusion: Adenosine-stress first pass myocardial CT perfusion imaging is capable to evaluate qualitative and semi-quantitative parameters of myocardial perfusion in a comparable fashion as MRI and SPECT. In addition, it may enable absolute quantification of the myocardial blood flow.

\section{B-413 10:48}

Adenosine stress dual energy $\mathrm{CT}$ for the diagnosis of coronary heart disease: do we need rest images?

T. Henzler' ${ }^{1}$, J.W.J. Nance'2, U. Schoepf ${ }^{2}$, C. Fink', S.O. Schönberg',

M. Weininger ${ }^{2} ;{ }^{1}$ Mannheim/DE, ${ }^{2}$ Charleston, SC/US

(thomas.henzler@medma.uni-heidelberg.de)

Purpose: To prospectively compare the diagnostic accuracy of stress-only dual energy-CT (DECT) combined with delayed enhancement imaging with a stressrest DECT protocol for detection of myocardial perfusion defects seen on SPECT. Methods and Materials: 57 patients underwent three-phase contrast (Isovue, Bracco)enhanced ECG-gated DECT (Definition, Siemens) with adenosine-stress, rest, and delayed enhancement imaging. DECT datasets were used to map the myocardial iodine distribution within the left ventricular myocardium. Myocardial segments were classified, in a blinded fashion, as normal, reversible ischaemia, or fixed perfusion defects. Segmental analysis was done based on a combination of stress-, rest-, and delayed enhancement DECT series as well as using a combination of stress-only and delayed-enhancement-DECT. Results were compared with stress-rest SPECT. Results: 214 of all analysed 969 myocardial segments showed perfusion defects on rest DECT and 227/969 on adenosine stress DECT studies. Delayed enhancement was positive in 175/969 myocardial segments. Rest DECT confirmed rest SPECT findings with 92.1\% sensitivity, $96.4 \%$ specificity, and $96.5 \%$ accuracy. Stress-DECT confirmed stressSPECT findings with $94.6 \%$ sensitivity, $97.5 \%$ specificity, and $96.8 \%$ accuracy. The overall sensitivity, specificity, and accuracy of combined stress-, rest-, and delayed-enhancement DECT for diagnosing normal, ischaemic, and infarcted myocardial segments were $93 \%$, $97.1 \%$, and $96.0 \%$, respectively. Stress-only combined with delayed enhancement DECT confirmed SPECT findings with $89 \%$ sensitivity, $97.5 \%$ specificity, and $95.5 \%$ accuracy. Conclusion: Stress-, rest-, and delayed enhancement DECT shows excellent agreement with SPECT for characterising myocardial perfusion patterns. However, similar to recent findings in nuclear myocardial perfusion imaging, almost identical accuracies can be achieved without rest imaging. 


\author{
B-414 10:57 \\ Patient-specific left ventricular function and biomechanical parameters \\ from cardiac $C T$ imaging \\ A. Baali', R. Moreno', M. Chau², H. Rousseau', F. Nicoud ${ }^{3} ;{ }^{1}$ Toulouse/FR, \\ ${ }^{2}$ Castelnau le Lez/FR, ${ }^{3}$ Montpellier/FR (baali.adil@gmail.com)
}

Purpose: Oriented towards a more complete diagnosis for clinicians, we propose a new Computational Fluid Dynamics (CFD) that uses cardiac computed tomography (CT) explorations to automatically generate functional results including computational haemodynamics and segmental contractile parameters. This is done within the same numerical domain all over the cardiac cycle. This new method provides us relevant patient-specific functional and biomechanical parameters since they are known to be related to pathological processes.

Methods and Materials: We performed a dynamic multi-slice computed tomography coronary angiography on fifteen patients. Left ventricle was extracted by segmentation and non-structured numerical domain was generated in order to represent the cavity as a grid-based on tetrahedral finite volumes with an inlet (mitral valve) and an outlet (aortic valve). The grid volume, qualified as the native mesh, is then transformed over the cardiac cycle through realistic morphological. Results: Ejection fraction, time-resolved spherical index and segmental strain rate indexes were performed for each study from deformation process. Functional imaging with typical biomechanical fields: velocity, pressure, wall shear stress and vorticity were obtained from CFD by means of arbitrary lagrangian eulerian (ALE) formulation into the AVBP code (Cerfacs-Toulouse). All results were available after $3 \mathrm{~h}$, including geometrical extraction (semi-automatic), deformation process and CFD results. Conclusion: This new non-invasive method for assessment of both the myocardial deformation and the global left ventricular function opens a very wide spectrum of clinical applications since it is realised for a short time, easily, reproducibly and is much less operator-dependant than echocardiographic imaging.

\section{B-415 11:06}

Assessment of left ventricular diastolic function by multislice spiral computed tomography

A. Belgour, F. Vallet, S. Ragot, J.-P. Tasu; Poitiers/FR (alibelgour@gmail.com)

Purpose: Diastolic dysfunction is defined as an impaired left ventricular filling. The aim of this study was to establish the ability of multislice spiral computed tomography (MSCT) in the assessment of left ventricular diastolic function.

Methods and Materials: Left ventricular filling was assessed in 38 subjects (12 without any cardiac abnormality, 26 with various cardiac diseases) by transthoracic echocardiography (TTE) and MSCT. The time separating the two modalities was less than ten days. The early left ventricular filling percentage (ELVFP) defined as the sum of LV volumes during the two first diastolic phases on the ejection volume and left atrial volume indexed to body surface area (LAVi) were measured by MSCT. Mitral inflow velocity and myocardial tissue velocities were measured by TTE. Adysfunction was diagnosed from the echocardiography abnormalities. The MSCT parameters were evaluated to classify the patients into normal or abnormal diastolic function. Results: Diastolic dysfunction was found in seventeen patients by TTE. There was a significant difference of ELVFR measured by MSCT between normal diastolic function group and abnormal diastolic function group $(p<0.0001)$. ELVFR correlated well with $\mathrm{E} / \mathrm{Ea}$ used in the estimation of the left ventricular filling pressures (Rhô $=-0.52 ; p=0.002$ ). Enlarged LAVi was found in $36 \%$ of patients with diastolic dysfunction, whereas it was enlarged in only $20 \%$ with normal diastolic function. Conclusion: MSCT is a feasible method to assess left ventricular diastolic function. It can separate patients with diastolic dysfunction from patients with normal diastolic function.

\section{B-416 11:15}

Evaluation of global left ventricular function with DS-CT: comparison of three workstations with magnetic resonance as gold standard M. Rengo' ${ }^{1}$, N.R. Mollet', L.A. Neefjes ${ }^{2}$, A. Rossi'2, G.P. Krestin', P.J. de Feyter², A. Laghi'; ' 'Latina/IT, ${ }^{2}$ Rotterdam/NL (marco.rengo@gmail.com)

Purpose: To compare the accuracy and reliability of left ventricular (LV) volumes assessment of three different workstations (WS) and to compare results to cine MRI as standard reference.

Methods and Materials: Two expert radiologists evaluated DS-CTCA 30 datase on three different WS: Leonardo (Siemens), Aquarius (Terarecon) and Vitrea (Vital). LV end-diastolic (EDV) and end-systolic volumes (ESV) and ejection fraction (EF) were evaluated for each WS using an automatic and semiautomatic analysis. Mean time, inter-reader and intra-reader agreement were calculated. Results were compared with those obtained by cine MRI.
Results: A low correlation between the automatic method and MRI results was found for all workstations. Correlation and Bland and Altman plots between DS-CT and MRI, obtained with the semiautomatic analysis on all WS were good $(\mathrm{r} 2=0.81$, 0.86 and 0.83 , respectively). A constant error was found for all WS $(+5 \%,-3 \%$ and $+4 \%)$ for the automatic evaluation of EF. Mean time was significantly shorter for Leonardo WS (8'35") compared to Vitrea WS (10'20") and not significantly longer than for Aquarius WS ( $\left.7^{\prime} 32^{\prime \prime}\right)$. Intra-reader agreement was good for all WS (wK = $0.83,0.79$ and 0.81 ). Inter-reader agreement was significantly better for Leonardo and Aquarius than for Vitrea (wK $=0.85$ and 0.91 vs wK $=0.75$ ).

Conclusion: A good correlation with MRI was obtained for all workstations using a semiautomatic analysis. The LV function is a predictive marker for morbidity and mortality in patients with coronary artery disease. This study investigates the variability of LV function assessment on three commercially available WS.

\section{B-417 11:24}

Gender differences in response to cold pressor test assessed with velocity-encoded cine-MR imaging of the coronary sinus

A. Jacquier, A. Flavian, F. Kober, P. Cozzone, M. Bernard; Marseille/FR (alexis.jacquier@ap-hm.fr)

Purpose: Gender-specific differences in cardiovascular risk are well known, and current evidence supports an existing role of endothelium in these differences. The purpose of this study was to assess non-invasively coronary endothelial function in male and female young volunteers by myocardial blood flow (MBF) measurement using coronary sinus (CS) flow quantification by velocity encoded cine MRI at rest and during cold pressor test (CPT).

Methods and Materials: Twenty-four healthy volunteers (12 men, 12 women) underwent MRI in a 3 tesla scanner. Coronary sinus flow was measured at rest and during CPT using non-breath-hold velocity-encoded phase contrast cine-MRI. Myocardial function and morphology were acquired using a cine steady-state free precession sequence.

Results: At baseline, MBF was $0.63 \pm 0.23 \mathrm{~mL} \cdot \mathrm{g}^{-1} \cdot \mathrm{min}^{-1}$ in men and $0.79 \pm 0.21 \mathrm{~mL}$ $\cdot \mathrm{g}^{-1} \cdot \mathrm{min}^{-1}$ in women. During CPT, the rate pressure product in men significantly increased by $49 \pm 36 \%(p<0.0001)$ and in women by $52 \pm 22 \%(p<0.0001)$. MBF increased significantly in both men and women by $0.22 \pm 0.19 \mathrm{~mL} \cdot \mathrm{g}^{-1} \cdot \mathrm{min}^{-1}(\mathrm{p}=$ $0.0022)$ and by $0.73 \pm 0.43 \mathrm{~mL} \cdot g^{-1} \cdot \mathrm{min}^{-1}(p=0.0001)$, respectively. The increase in MBF was significantly higher in women than in men $(p=0.0012)$.

Conclusion: MRI coronary sinus flow quantification for measuring myocardial blood flow revealed a higher response of MBF to CPT in women than in men. This finding may reflect gender differences in endothelial-dependent vasodilatation. This non-invasive rest/stress protocol may become helpful to study endothelial function in normal physiology and in physiopathology.

\section{B-418 11:33}

\section{Chemoradiotherapy for thoracic oesophageal cancer impairs left} ventricular function: MRI evaluation

M. Hatakenaka, M. Nagao, Y. Shioyama, Y. Matsuo, S. Sunami,

T. Kamitani, M. Jinnouchi, H. Yabuuchi, H. Honda; Fukuoka/JP (mhatake@radiol.med.kyushu-u.ac.jp)

Purpose: To determine whether chemoradiotherapy for oesophageal cancer affects left ventricular function (LVF).

Methods and Materials: The LVF of 27 patients (21 males, 47-85 years) with oesophageal cancer (CE 6, UT 3, MT 13, LT 5, Stage I-IVa) who received concurrent chemoradiotherapy (total dose to primary lesion of 40-71 Gy, 5-FU and CDDP) was evaluated with 1.5-T MRI before $(n=27)$, at about 40Gy $(n=23)$, and after chemoradiotherapy ( $\geq 61.4 G y, n=18$ ). Several parameters related to LVF (end diastolic volume index (EDVI), end systolic volume index (ESVI), stroke volume index (STVI), ejection fraction (EF), cardiac output index (COI), heart rate (HR), wall motion (WM) and wall thickening (WT)) were compared among three time points using Wilcoxon single rank test. The dose of the left ventricular segments was also evaluated.

Results: In thoracic oesophageal cancer, significant change $(p<0.05)$ was observed between before and after chemoradiotherapy in STVI $\left(38.7 \pm 1.8 \mathrm{~mL} / \mathrm{m}^{2}\right.$ vs. $33.5 \pm 2.8)$, EF $(59.4 \pm 2.1 \%$ vs. $56.3 \pm 2.0)$, HR $\left(67.8 \pm 3.0 \mathrm{~min}^{-1}\right.$ vs. $\left.82.3 \pm 4.6\right)$, and WM of segment $9(7.6 \pm 0.49 \mathrm{~mm}$ vs. $6.3 \pm 0.88)$ and segment $10(9.8 \pm 0.54 \mathrm{vs}$ $8.9 \pm 0.7)$, and between before and at about 40 Gy in $\mathrm{HR}\left(67.8 \pm 3.0 \mathrm{~min}^{-1} \mathrm{vs} .72 .2 \pm 3.8\right)$ and WM of segment $8(7.3 \pm 0.43 \mathrm{~mm}$ vs. $6.1 \pm 0.58)$ and segment $9(7.6 \pm 0.49$ vs. $6.2 \pm 0.63)$. No significant difference was observed in cervical oesophageal cancer except EF between before and at about $40 \mathrm{~Gy}(57.6 \pm 3.8 \%$ vs. $57.4 \pm 5.0, p<0.05)$. Irradiated dose of segments 8, 9 and 10 was more than 40Gy in the patients with thoracic oesophageal cancer.

Conclusion: These results indicate that chemoradiotherapy for thoracic oesophageal cancer impairs LVF. 


\section{B-419 $11: 42$}

Three-dimensional magnetic resonance imaging using single breathhold kt-BLAST for assessment of global leftventricular functional parameters A.M. Huber, J. Jaroni, K. Herrmann, M. Settles, A. Beer, E. Rummeny; Munich/DE (armin.huber@roe.med.tu-muenchen.de)

Purpose: To determine the accuracy of three-dimensional (3D) kt-broad-use linear acquisition speed-up technique (BLAST) accelerated MRI (kt-BLAST) for the assessment of LV volumes and mass standard compared to segmented multiple breath-hold cine imaging.

Methods and Materials: 55 patients were included. In each patient a standard multislice cine steady-state free precession (SSFP) sequence was performed with complete ventricular coverage during multiple breath-holds (temporal resolution $47 \mathrm{msec}$, voxel size $\left.1.25 \times 1.25 \times 8 \mathrm{~mm}^{3}\right)$. In addition, two kt-BLAST-accelerated 3D sequences with complete ventricular coverage were acquired kT1 (TR $3.2 \mathrm{msec}$ TE $1.6 \mathrm{msec}$, temporal resolution $57 \mathrm{msec}$, voxel size $1.25 \times 1.25 \times 4 \mathrm{~mm}^{3}$ ) and kT2 (TR $3.2 \mathrm{msec}$, TE $1.6 \mathrm{msec}$, temporal resolution $57 \mathrm{msec}$, voxel size $1.25 \mathrm{x}$ $1.25 \times 8 \mathrm{~mm}^{3}$ ) during a single breath-hold after a separate breath-hold for a training scan. For comparison of SSFP and kt-BLAST, the following LV parameters were determined: EF, EDV, ESV and LV mass.

Results: The EF was slightly underestimated by the kT1 (47\%) and kT2 (48\%) compared to the reference sequence (53\%). All parameters showed high correlation of the kT-BLAST sequences and the reference sequence $(r=88$ to $r=0.98$ $r<0.001)$. The relative mean difference for KT1/KT2 compared to the reference sequence was $0.11 / 0.09$ for the EDV, $-0.073 /-0.086$ for the EDV, $0.044 /-0.051$ for the ESV and $0.085 / 0.12$ for the LV mass.

Conclusion: 3D kt-BLAST-accelerated MRI enabled to determine the LV parameters with high correlation compared to the reference sequence. EF was slightly underestimated, LV mass slighly overestimated.

\section{B-420 11:51}

Right ventricular fractional area change reflects right ventricular ejection fraction in pulmonary hypertension: a CMR study

N. Creuze, S. Hoette, D. Chemla, D. Montani, F. Parent, O. Sitbon, M. Humbert, G. Simonneau, D. Musset; Clamart/FR (nicos1977@hotmail.com)

Purpose: Right ventricular (RV) function is an important prognostic factor in pulmonary arterial hypertension $(\mathrm{PH})$. The RV fractional area change (RVFAC) is a widely used echocardiographic index of RV function but has been poorly documented in cardiac magnetic resonance imaging (CMR) studies, and this was the aim of our prospective study.

Methods and Materials: 43 patients $(22 \mathrm{~F} / 21 \mathrm{M}$; aged $60 \pm 12 \mathrm{yrs}$ ) with precapillary $\mathrm{PH}$ underwent right heart catheterisation and CMR on a 1.5 T scanner (Siemens) with eletrocardiographic gating (delay $\pm 48 \mathrm{~h}$ ). In the four chamber cine, the tricuspid annular plane systolic excursion (TAPSE) was measured and RV end-diastolic and end-systolic areas (RVEDA, RVESA) were manually delineated. RVFAC was calculated (100 x (RVEDA-RVESA)/RVEDA). RVEF was calculated using contiguous $6 \mathrm{~mm}$ RV short axis cines (balanced SSFP).

Results: Patients had increased mean pulmonary arterial pressure $(45 \pm 10 \mathrm{mmHg})$ and pulmonary vascular resistance $\left(16 \pm 7\right.$ wu. $\left.\mathrm{m}^{2}\right)$. CMR results were as follows: RVEF $=31 \pm 17 \%$ (range 3-75), RVFAC $=26 \pm 13 \%$ (4-65) and TAPSE $=$ $13.6 \pm 8 \mathrm{~mm}(6-26)$. The RVEF was related to RVFAC $\left(r^{2}=0.78\right)$ and TAPSE $\left(r^{2}\right.$ $=0.56$ ) (each $p<0.001)$. Twenty-seven patients $(63 \%)$ had severe RV dysfunction (RVEF <35\%). TAPSE $<15 \mathrm{~mm}$ predicted severe RV dysfunction with $92 \%$ sensitivity, $81 \%$ specificity, $89 \%$ positive predictive value and $86 \%$ negative predictive value. RVFAC $<30 \%$ predicted severe RV dysfunction with $96 \%$ sensitivity, $94 \%$ specificity, $96 \%$ positive predictive value and $94 \%$ negative predictive value. Conclusion: The RVFAC is strongly related to RVEF, and RVFAC $<30 \%$ is a simple, sensitive and specific marker of severe RV dysfunction in $\mathrm{PH}$ patients. The prognostic implications of RVFAC still need confirmation.
$10: 30-12: 00$

Room F1

\section{Abdominal Viscera}

\section{SS $801 b$ \\ Imaging evaluation of abdominal tumours response to therapy}

Moderators:

D.J. Breen; Southampton/UK

A. Siemianowicz; Piekary Slaskie/PL

\section{B-421 10:30}

Prediction of chemotherapeutic response of liver metastases from colorectal cancer by diffusion-weighted and dynamic contrast-enhanced MR imaging: value of pretreatment apparent diffusion coefficient and $\mathbf{K}^{\text {trans }}$ values

K. Holzapfel ${ }^{1}$, A.J. Beer ${ }^{1}$, C. Ganter ${ }^{1}$, R. Strecker ${ }^{2}$, E.J. Rummeny ${ }^{1}$, S. Metz ${ }^{1}$; ${ }^{1}$ Munich/DE, ${ }^{2}$ Erlangen/DE (holzapfel@roe.med.tum.de)

Purpose: To evaluate apparent diffusion coefficient (ADC) and $\mathrm{K}^{\text {trans }}$ as functional magnetic resonance (MR) biomarkers for pretreatment prediction of chemotherapeutic response of liver metastases in patients with colorectal cancer.

Methods and Materials: Twenty-three patients (15 males, 8 females) with 54 liver metastases from colorectal cancer underwent MR imaging (1.5 T) before starting of chemotherapy. All patients received standard $1^{\text {st }}$-line chemotherapy for metastatic disease. The protocol comprised diffusion-weighted imaging (DWI) using an echoplanar single-shot sequence (b values: 50/300/600 s/mm ${ }^{2}$ ) and dynamic contrast-enhanced MR imaging (DCE-MRI) using a TWIST (time-resolved angiography with interleaved stochastic trajectories) sequence with application of Gd-DTPA ( $0.1 \mathrm{mmol} / \mathrm{kg} \mathrm{BW})$. Quantitative ADC and $\mathrm{K}^{\text {trans }}$ data were calculated (Tissue 4D software, Siemens). In addition, lesions were classified either as responding or non-responding, according to changes in size based on response evaluation criteria in solid tumors (RECIST) after two cycles of chemotherapy.

Results: Thirty-three responding and 21 non-responding metastases were evaluated The pretherapeutic mean ADC value $\left(\times 10^{-3} \mathrm{~mm}^{2} / \mathrm{s}\right)$ in responding lesions $(0.97 \pm 0.23)$ was significantly lower than in non-responding lesions $(1.12 \pm 0.16 ; p<0.01$; MannWhitney $\mathrm{U}$ test). For dynamic analyses, the responding lesions had higher pretreatment mean $\mathrm{K}^{\text {trans }}$ values $\left(\mathrm{min}^{-1}\right)$ than non-responding lesions, however, without reaching statistical significance $(0.17 \pm 0.04$ vs. $0.15 \pm 0.03$; $p=0.12$; Mann-Whitney U test).

Conclusion: Prediction of tumour response before onset of chemotherapeutic treatment using MR imaging biomarkers could have considerable clinical benefit. Our results indicate that responding lesions have significantly lower ADC values and tend to have higher $\mathrm{K}^{\text {trans }}$ values compared with non-responding lesions in patients with liver metastases from colorectal cancer.

\section{B-422 10:39}

Prognostic value of different CT measurements in therapy response evaluation in patients with colorectal carcinoma and synchronous hepatic metastases at initial staging

M.W. Huellner, T. Zander, R. Winterhalder, T. Treumann, K. Strobel,

P. Veit-Haibach; Lucerne/CH (martin.huellner@ksl.ch)

Purpose: To evaluate the prognostic value of different CT-measurement for response assessment after initiation of chemotherapy in patients with synchronous colorectal cancer liver metastases at initial staging

Methods and Materials: 35 patients with colorectal carcinoma and synchronous hepatic metastases at initial staging were retrospectively evaluated. The prognostic value of different CT measurements concerning overall survival were evaluated after three cycles of capecitabine (Xeloda)-based chemotherapy. Response parameters were: the sum of longest diameters (SLD) (RECIST 1.1 criteria), product of the two longest diameters in one plane, product of the three longest diameters in two planes, tumour-to-liver ratio, tumour shrinkage ratio and Houndsfield units (HUmax. HUmean and HUmin). The prognostic value of CT data was evaluated using Cox regression. Results: 35 patients (25 males, 10 females, mean age 66.1 years, range $41-84$ years) were included. The overall mean survival time was 858 days (range 1891888 days). Eleven patients died during follow-up. Follow-up CT using RECIST 1.1 criteria identified 11 patients with PR (mean decrease: $-51.0 \%$ vs. baseline), 14 with SD (mean decrease: $-13.0 \%$ vs. baseline) and 2 with PD (increase vs. baseline: $+32.5 \%$ ). Overall, CT measurement using the SLD (RECIST 1.1) did not show a statistically significant correlation with overall survival $(p>0.05)$. In addition, none of the other above-mentioned parameters showed any statistically significant values $(p>0.05)$. 
Conclusion: Morphological response assessment using different CT-measurements has no prognostic value concerning overall survival in patients with synchronous colorectal liver metastases. Larger studies need to investigate possible differences in patients with k-ras mutation.

\section{B-423 10:48 \\ (EPOS) Detectability of liver metastatic breast lesions with both the C-arm CT and MRI: assessment of lesion vascularisation at the first and last TACE E.C. Mbalisike, J. Gurung, A. Azizi, P. Farshid, B. Al hazaimeh, V. Jacobi, T.J. Vogl; Frankfurt a. Main/DE (embalisike@yahoo.com)}

Purpose: To assess the detectability of liver metastatic breast lesions with both $\mathrm{C}$-arm CT and MRI, and determining TACE effects on lesion vascularisation after treatment.

Methods and Materials: Both C-arm CT and CE-MRI were done on 10 female patients. Detectability was assessed using the contrast-to-noise ratio (CNR) of the $\mathrm{C}$-arm CT at the first and fourth sessions. Lesion vascularisation was assessed quantitatively using a 4-tier grading system. Signal-to-noise ratio (SNR) of CE-MRI was assessed and tested with the $\mathrm{C}$-arm CT (CNR). Calculations of the $\mathrm{C}$-arm CT (CNR) and CE-MRI (SNR) were done as CNR= (ROI Lesion-ROI Liver)/SDnoise, and $\mathrm{SNR}=\mathrm{S} / \mathrm{N}$ (Noise $(\mathrm{n})=\mathrm{SD}$ liver/0.66), respectively. Statistical tests used here were the Wilcoxon's matched pair test and the Spearman-Rang correlation test. Results: Using the CE-MRI, 29 lesions were assessed. 28 of these lesions were found by the C-arm CT. One hypovascular lesion missed by the C-arm CT showed similar signal with its surrounding normal liver tissues. Detectability of 26 lesions reduced significantly by their fourth $\mathrm{C}$-arm $\mathrm{CT}$ session, though only 12 lesions los their vascularization. C-arm CT contrast-to-noise ratio showed a $\mathrm{P}$ value of 0.005 , while CE-MRI signal-to-noise ratio and contrast-to-noise ratio of C-arm CT showed $P$ value of 0.036 , and a Spearman-Rang coefficient of 0.391 .

Conclusion: $96.6 \%$ of metastatic liver lesions were detected by the C-arm CT using the MRI as the gold standard. This lesion detectability was significantly reduced by the fourth/last TACE session, signifying loss of lesion vascularisation.

\section{B-424 10:57}

C-arm CT versus MRI: degree of vascularisation with influence on the response of liver metastatic colorectal carcinoma during TACE

E.C. Mbalisike, A. Azizi, A. Darvishi Nakhl Ebrahimi, P. Farshid, M. Elsabaie, T.J. Vogl; Frankfurt a. Main/DE (embalisike@yahoo.com)

Purpose: To determine the influence of lesion vascularisation assessed by both intra-arterial C-arm CT (IACCT) and contrast-enhanced MRI (CE-MRI) on lesion response to TACE for liver metastatic colorectal carcinoma (CRC).

Methods and Materials: 37 patients (mean age 58.1, SD 11.7) with 120 focal metastasis were assessed. 151 TACE sessions (mean session of $4.1 /$ patient) were done. Assessment on vascularisation was done using four grades from hypovascular, heterogeneously hypervascular, homogeneously hypervascular and mixed vascular. Responses to therapy were assessed using MRI at 3 months and 6 months. Statistical tests used in this study were Spearman-Rang's test, and Wilcoxon's-matched-pairs-test.

Results: Using both CE-MRI and intra-arterial C-arm CT, 64.7\% $(n=24)$ were grouped as hypovascular, $27 \%(n=10)$ heterogeneously hypervascular, $5.4 \%(n$ $=2$ ) mixed vascular and $2.7 \%(n=1)$ homogeneously hypervascular. C-arm CT confirmed CE-MRI findings on lesion vascularisation in $33 / 37$ patients. SpearmanRang's correlation coefficient used to compare pre-treatment volumes of intraarterial C-arm CT and MRI, obtained statistical correlation of $0.736(P=0.0001)$. Lesion response was assessed using Wilcoxon-matched-pairs-tests. $P$ value of 0.003 was obtained at 3 months, and $P$ value of 0.002 at 6 months.

Conclusion: C-arm CT was able to confirm CE-MRI findings on vascularisation of lesion. Significant responses were seen in patients with CRC after therapy with TACE.

\section{$B-425 \quad 11 \cdot 06$}

French multicentric prospective study of dynamic contrast-enhanced ultrasound (DCE-US) for the evaluation of antiangiogenic treatments: final results

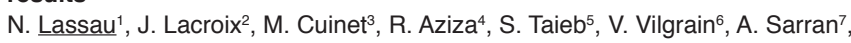
J. Bonastre ${ }^{1}$, S. Koscielny $1 ;{ }^{1}$ Villejuif/FR, ${ }^{2}$ Caen/FR, ${ }^{3}$ Lyon/FR, ${ }^{4}$ Toulouse/FR, ${ }^{5}$ Lille/FR, ${ }^{6}$ Clichy/FR, ${ }^{7}$ Marseille/FR (lassau@igr.fr)

Purpose: A prospective study of dynamic contrast-enhanced ultrasound (DCE-US) with quantification for the evaluation of antiangiogenic treatments was launched in 2007 (19 centers), supported by the French National Cancer Institute. The objectives were the diffusion of the standardised method, a cost evaluation and the identification of perfusion parameters predicting tumour response.
Methods and Materials: All patients had DCE-US at baseline, D7, D14, D30, D60 and every 2 months. Each examination included a bolus injection of sonovue (Bracco $\left.{ }^{\circledR}\right)$ and registration of 3 minutes of raw linear data with an Aplio (Toshiba). Raw data were analysed with a mathematical model (patent PCT/IB2006/003742) to evaluate 7 parameters characterising the tumour perfusion curve. Response to treatment was evaluated every 2 months with RECIST criteria. In order to have sufficient follow-up data, the statistical analysis has to be performed more than 6 months after the inclusion of the last analysed patient. Inclusions were closed in March 2010.

Results: A total of 539 patients have been included (mainly RCC (157) and HCC (107)); more than 2000 DCE-US examinations were performed. Preliminary results concerning 400 patients followed more than 6 months show that 5 parameters have a significant difference $(P<0.003)$ according to the response at 6 months A cut-off between responders and non-responders was determined (decrease of $80 \%$ and $40 \%$ for AUC, respectively).

Conclusion: Preliminary results (400 patients) confirm the usefulness of this too to monitor anti-angiogenic treatments. The final result will allow us to present a complete analysis of 539 patients followed of 12 months.

\section{B-426 11:15}

Detection of colorectal metastases in patients being treated with chemotherapy utilising SPIO-contrast MRI

J.T. Smith, J. Ward, D. Wilson, J. Wyatt, D. Treanor, M. Sheridan, S. Boyes, A. Guthrie; Leeds/UK (drjontsmith@aol.com)

Purpose: Chemotherapy commonly causes liver injury through sinusoidal obstructive syndrome and steatosis. Chemotherapy-induced liver injury may make it more difficult to detect metastases secondary to reduced contrast between the injured liver and metastases. The aim of this study was to determine the accuracy of SPIO-contrast enhanced imaging in patients who have undergone chemotherapy prior to liver surgery.

Methods and Materials: Local ethics committee approval was obtained. Thirty-one patients with hepatic metastases completing pre-operative chemotherapy were recruited. Images were reviewed independently by two blinded observers who identified and localised lesions with a four-point confidence scale. The alternative free-response receiver operator characteristic method was used to analyse the results, which were correlated with findings from surgery, intra-operative ultrasonography and histopathology.

Results: The sensitivity in detecting colorectal metastases following chemotherapy was $78 \%$ and $76 \%$ for observers 1 and $2(95 \% \mathrm{Cl}, 71 \%-85 \%$ and $68 \%-82 \%$, respectively). The area under the alternative free-response receiver operator curves were 0.73 and 0.80 for observers 1 and 2, respectively. The inter-observer Kappa value $=0.41$

Conclusion: Compared to previous published work, on chemotherapy naive patients, it is clear that the accuracy of SPIO-enhanced MR imaging in detecting colorectal metastases following chemotherapy is substantially reduced. It is therefore critical that all imaging, both pre- and during chemotherapy, is reviewed when reporting liver MRI prior to surgery. It is important that authors clarify whether patients are chemotherapy naive or not in further studies.

\section{B-427 11:24}

Functional MRI evaluation of liver tumour response after radiofrequency: short- and mid-term evolution of diffusion parameters

T.-L.C. Lu, F. Becce, P. Frascarolo, A. Denys, P. Bize, S. Schmidt; Lausanne/CH

Purpose: To evaluate the short- and mid-term evolutions of the apparent diffusion coefficient of lesions treated with RF, in order to determine if the ADC can be used as a marker of tumour response.

Methods and Materials: Twenty patients were treated for a liver malignancy with $\mathrm{RF}$ and were examined on a 1.5 T/3.0 T machine with T2, gadolinium-enhanced $T 1$ and diffusion sequences: before treatment ( $<1$ month), just after treatment $(<1$ month) and midterm (3-6 months). The ADC was measured in the whole lesion and in the area with the most restricted diffusion (MRDA). The ROI size was also measured on the diffusion map. The Pearson/ANOVA tests were used.

Results: All patients were successfully treated with complete disappearance of CE. The lesional size on T2 showed a negative evolution in time $(p<0.002)$. The $A D C$ in the whole lesion showed a bell-shaped evolution (increasing just after RF, then decreasing, $p=0.02$ ). The ROI size on the diffusion map followed a similar course $(p=0.01)$. For the MRDA, such evolutions were also found, but they were not significant. There was a negative correlation between CE and the ADC $(p<0.02)$ and between the lesional size on T2 and ADC $(p=0.03)$ in the whole lesion. There 
were also positive correlations between the ROI size and ADC $(p=0.0008)$ and between CE and the size on T2 $(p=0.0002)$. The ADC in MRDA showed some non-significant correlations with other variables.

Conclusion: The lesions successfully treated with RF have a clear and predictable evolution in terms of $\mathrm{T} 2$ size, CE and ADC

\section{B-428 11:33}

Diffusion-weighted MRI in metastatic gastrointestinal tumours (GIST): a pilot study on the assessment of treatment response in comparison with 18 F-FDG PET/CT

M. Koehli, V. Dunet, M. Montemurro, S. Leyvraz, R. Meuli, J. Prior, S. Schmidt; Lausanne/CH (sabine.schmidt@chuv.ch)

Purpose: To evaluate the clinical potential of diffusion-weighted MR imaging with apparent diffusion coefficient (ADC) mapping for the assessment of gastrointestinal stromal tumour (GIST) response to targeted therapy in comparison with ${ }^{18} \mathrm{~F}-\mathrm{FDG}$ PET/CT Methods and Materials: Five patients ( $3 \mathrm{~W} / 2 \mathrm{M}$, aged $56 \pm 13 \mathrm{y}$ ) with metastatic GIST underwent both a ${ }^{18} \mathrm{~F}-\mathrm{FDG}$ PET/CT (Discovery LS, GE Healthcare) and a MRI (VIBE T1 Gd, DWI [b = 50,300,600] and ADC mapping) before and after change in therapy. Exams were first analysed blindly and then PET/CT images were coregistered to T1 Gd MR images for lesion detection. SUV and ADC were measured for the six largest lesions on MRI. The relationship between $\mathrm{SUV}_{\max }$ and ADC was analysed using Spearman's correlation.

Results: Altogether, 24 lesions (15 hepatic and 9 non-hepatic) were analysed on both modalities. Three PET/CT lesions (12.5\%) were initially not considered on ADC and 4 lesions on the second PET/CT were excluded because of hepatic vascular activity spillover. SUV ${ }_{\max }$ decreased from $7.2 \pm 7.7 \mathrm{~g} / \mathrm{mL}$ to $5.9 \pm 5.9 \mathrm{~g} / \mathrm{mL}(P=0.53)$ and ADC increased from $1.2 \times 10^{-3} \mathrm{~mm}^{2} / \mathrm{s} \pm 0.4$ to $1.4 \times 10^{-3} \mathrm{~mm}^{2} / \mathrm{s} \pm 0.4(P=0.07)$

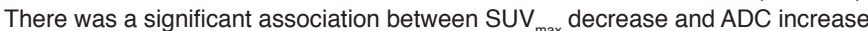
(rho $=-0.64, \mathrm{P}=0.004$ )

Conclusion: Changes in ADC from diffusion-weighted MRI reflect response of ${ }^{18} \mathrm{~F}-\mathrm{FDG}$-avid GIST to therapy. The exact diagnostic value of DWI needs to be investigated further, as well as the effect of lesion size and time under therapy before imaging. Furthermore, the proven association between SUV $_{\max }$ and ADC may be useful for the assessment of treatment response in ${ }^{18} \mathrm{~F}-\mathrm{FDG}$ non-avid GIST.

\section{B-429 11:42}

(EPOS)

Therapeutic evaluation after transcatheter arterial chemoembolisation of hepatocellular carcinoma using diffusion-weighted and dynamic contrastenhanced magnetic resonance imaging

H. Wang, Q. Zou, P.-F. Liu, Z.-X. Ye; Tianjin/CN (hua0718@sina.com)

Purpose: To evaluate the efficacy of treatment of hepatocellular carcinoma (HCC) after transcatheter arterial chemoembolisation (TACE) with diffusion-weighted (DW) and dynamic contrast-enhanced (DCE) MR imaging.

Methods and Materials: DW $\left(b=500,0 \mathrm{~s} / \mathrm{mm}^{2}\right)$ and DCE MR imaging was performed before and 4-6 weeks after TACE in 20 patients with 39 HCCs. Apparent diffusion coefficient $(A D C)$ values and relative enhancement rate $(r E R)$ of arterial and portal venous phase were compared before and after treatment using Wilcoxon signed ranks test. The agreement of decrease/increase of ADC value and increase/ decrease of rER of arterial or portal venous phase was analysed using McNemar test. Results: Tumour ADC value increased significantly from $1.17 \pm 0.30 \mathrm{~mm}^{2} / \mathrm{s}$ to $1.27 \pm 0.29 \mathrm{~mm}^{2} / \mathrm{s}(\mathrm{p}=0.003)$. There was no significant difference between $\mathrm{rER}$ of arterial (from $0.73 \pm 0.48$ to $0.69 \pm 0.38$ ) or portal venous phase (from $1.27 \pm 0.37$ to $1.21 \pm 0.36$ ) before and after TACE. The decrease/increase of ADC value was not coincident with increase/decrease of $r E R$ of arterial phase $(p=0.001)$, but coincident with that of portal venous phase. For lesions with increased $r E R$ of portal venous phase, the difference between ADC value before TACE $\left(1.24 \pm 0.21 \mathrm{~mm}^{2} / \mathrm{s}\right)$ and after TACE $\left(1.29 \pm 0.28 \mathrm{~mm}^{2} / \mathrm{s}\right)$ was not significant; for lesions with decreased $\mathrm{rER}$ of portal venous phase, the ADC value increased significantly from $1.12 \pm 0.34 \mathrm{~mm}^{2} / \mathrm{s}$ to $1.26 \pm 0.30 \mathrm{~mm}^{2} / \mathrm{s}$ after TACE $(p=0.004)$.

Conclusion: ADC value may be a useful tool for assessing HCC response to TACE. It is related to perfusion effect of portal venous phase to some extent, and may not be sensitive to tumour recurrence as compared with DCE MRI.

\section{B-430 11:51}

MRI-monitoring of angiogenesis inhibition in an orthotopic pancreatic cancer model

H.-J. Raatschen, B. Hotz, S. Fischer, B. Zsivcsec, M. Arndt, P. Mall, K.-J. Wolf, H.G. Hotz; Berlin/DE

Purpose: To quantify effects of different angiogenesis-inhibiting drugs on microvascular parameters in a pancreatic cancer model using dynamic MRI.
Methods and Materials: DSL-6 A/C1 pancreatic tumour fragments were orthotopically implanted in 46 male Lewis rats. Three weeks after implantation, angiogenesis inhibition was initiated by intraperitoneal administration of bevacizumab $(n=13)$ or suramin $(n=19)$. The control group $(n=13)$ remained untreated. In 23 rats, dynamic albumin-(Gd-DTPA)-enhanced MRI at $1.5 \mathrm{~T}$ was performed 24 hours after the first treatment, the remaining animals were imaged after 4 weeks of therapy. Fractional plasma volumes ( $\mathrm{fPV}$ ) and vascular permeabilities $\left(\mathrm{K}^{\mathrm{PS}}\right)$ were calculated based on the dynamic MRI data using a pharmacokinetic model.

Results: fPV at 24 hours was similar among the bevacizumab, suramin and control group $(7.3 \pm 5.9 \%, 6.1 \pm 3.3 \%$ and $6.1 \pm 4.3 \%$; $p=0.86)$. After 4 weeks, PV in suramin-treated tumours $(2.7 \pm 1.0 \%)$ declined significantly compared to the 24 hours measurements $(p<0.05)$ and to the control tumours $(6.3 \pm 4.0 \% ;<0.05)$, whereas changes in bevacizumab-treated tumours were not significant $(4.0 \pm 1.2 \%$, $\mathrm{p}=0.22)$. $\mathrm{K}^{\mathrm{PS}}$ at 24 hours was zero in bevacizumab-treated tumours and as such differed significantly from the suramin and control group $\left(K^{P S}=0 \pm 0,0.062 \pm 0.066\right.$ and $0.082 \pm 0.039 \mathrm{~mL} / \mathrm{min} / 100 \mathrm{cc} ; \mathrm{p}<0.05)$. After 4 weeks no significant differences in $\mathrm{K}^{\mathrm{PS}}$ were detected among the 3 groups $(0.035 \pm 0.059,0.049 \pm 0.026$ and $0.086 \pm 0.088 \mathrm{~mL} / \mathrm{min} / 100 \mathrm{cc} ; \mathrm{p}=0.28)$ nor between the two time points.

Conclusion: Dynamic albumin-(Gd-DTPA)-enhanced MRI can be used to detect and quantify effects of different angiogenesis inhibitors on microvascular parameters in an orthotopic pancreatic cancer model and as such may help to identify new drugs for cancer treatment and to test their efficacy.

10:30 - 12:00

Room F2

GI Tract

\section{SS 801C}

\section{Small bowel: assessment of function and inflammation}

Moderators

D.D.T. Maglinte; Indianapolis, IN/US

G. Masselli; Rome/IT

\section{B-431 10:30}

Replacing VoLumen ${ }^{\mathrm{R}}$ with a better, cost effective, neutral oral contrast; methylcellulose plus sorbitol

S. Raza, M. Batool, M.O. Aslam, N.A. Al-Nakshabandi, U. Bhopal; Riyadh/SA (sibtainr@hotmail.com)

Purpose: To assess the performance of a new combination for neutral oral contrast for CT abdomen and CT Enterography. We wanted to compare the performance of a combination of methyl cellulose and sorbitol in water to that of commercially available $0.1 \%$ barium suspension, VoLumen.

Methods and Materials: A total of 120 patients were included in the study, randomly divided into two groups. Group 1 received 1000-1500 ml of VoLumen and group 2 was given equal amounts of combination of $0.4 \%$ methyl cellulose plus $1.5 \%$ sorbitol solution. Contrast-enhanced CT scan was performed on 64 detector row CT. Random, blinded evaluation of the studies was done by two of the four authors. Data for luminal distension in duodenum, jejunum and ileum were recorded along with assessment of mural details. Patient acceptance was also recorded along with cost effectiveness of each type of contrast. Student $t$ test and Chi square test were applied to compare luminal distension between groups and within each group. Results: Our new combination contrast gave better luminal distension than VoLumen ( $p$ less than 0.05 ) but no significant difference for mural details. Moreover, it was much better tolerated and had a very significantly low cost.

Conclusion: The new combination of methyl cellulose and sorbitol as neutral contrast agent provides better luminal bowel distension than VoLumen. The significantly low cost and better patient acceptance makes this our recommendation, for use as a routine in all abdominal CT scans.

\section{B-432 10:39}

Can we estimate oro-caecal time using MRI? A comparison with hydrogen breath test (HBT) in healthy volunteers: preliminary results L.M. Sconfienza ${ }^{1}$, E. Savarino ${ }^{2}$, A. Parodi ${ }^{3}$, C. Murolo ${ }^{2}$, V. Savarino ${ }^{2}$, F. Sardanelli'; ${ }^{1}$ San Donato Milanese/IT, ${ }^{2}$ Genoa/IT, ${ }^{3}$ Sanremo/IT (io@lucasconfienza.it)

Purpose: MRI is a reliable imaging modality in the evaluation of small bowel, both in basal condition and with MR-enterography technique. HBT is one of the methods used to assess oro-caecal transit time. No data exist about the use of MRI in the assessment of oro-caecal transit time. We determined the role of MRI in the assessment of oro-caecal transit time of a bolus of lactulose, having HBT as reference standard. 
Methods and Materials: Twenty-one asymptomatic volunteers (10 males, 11 females, mean age $32 \pm 8.4$ years) were asked to eat a light dinner on the previous evening and to fast on the day of examinations. They underwent abdominal $1.5 \mathrm{~T}$ MRI using axial and coronal single-shot fast-spin-echo T2-weighted sequences before and after oral administration of $10 \mathrm{~g}$ of lactulose diluted in $125 \mathrm{~mL}$ of water. $\mathrm{MRI}$ acquisition was repeated every $15 \mathrm{~min}$ up to $180 \mathrm{~min}$. Before each MR acquisition, breath gas-cromatography was performed (Microlizer DP Plus, Quintron, Milwaukee, IL). Correlation between presence of fluid bolus in the caecum at MR and the detection of a hydrogen concentration peak was estimated.

Results: Median oro-cecal transit time estimated by MRI was 135 min (range 113-136 $\mathrm{min}$ ) while it was $128 \mathrm{~min}$ (120-146 $\mathrm{min}$ ) by HBT. Excellent correlation between the two techniques was found $(r=0.910)$

Conclusion: MRI can be effectively used in the estimation of oro-caecal transit time. If confirmed on patients affected with gut dismotility and similar conditions, MRI could play a role in the diagnosis of a number of gastrointestinal diseases.

\section{B-433 10:48}

Test-retest reliability of dynamic physiological small bowel MR assessment of peristalsis

A.S. Farghal, A.P. Toms, B. Kasmai, R. Greenwood, P. Malcolm; Norwich/UK (afarghal@gmail.com)

Purpose: A novel technique for quantifying small bowel peristalsis using measurements derived from dynamic small bowel MR has been developed in our institution in which peristalsis is defined using descriptive statistics summarising the profile of mean signal amplitude per voxel. The aim of this study was to assess the testretest reliability of this technique.

Methods and Materials: Dynamic coronal MR sequences covering the whole abdomen (breath hold True FISP 21 frames) were acquired from 5 volunteers who fasted for six hours and drunk $1 \mathrm{~L}$ of water. MR data were analysed with an in-house software package, which calculated the mean absolute change in signal per voxel for each dynamic sequence. The total voxel activity (TVA) per slice was then calculated as a sum of all mean absolute changes in voxel signal. All studies were repeated with the same parameters and analysis with at least a two-week gap between studies. Descriptive statistics were performed on each study. Reliability was measured using a Pearson Product-Moment Correlation Coefficien comparing each slice in the two studies as well as total abdominal voxel activity. Results: The correlation coefficient demonstrated "almost perfect agreement" between first and second studies. The slice by slice correlation was $r=0.849$ $(95 \% \mathrm{Cl} 0.765$ to 0.904$)$, the total abdominal correlation was $r=0.9898(95 \% \mathrm{Cl}$ 0.8483 to 0.9994$)$.

Conclusion: The test-retest reliability of the technique described for quantifying small bowel peristalsis with dynamic MR is extremely good suggesting that this may be a robust technique for serial studies.

\section{B-434 10:57}

\section{Global assessment of small bowel activity using dynamic MR with} automated computerised method: protocol optimisation A.S. Farghal, A.P. Toms, B. Kasmai, A. Kerner-Bignell, P. Malcolm; Norwich/UK (afarghal@gmail.com)

Purpose: The aim of this study was to optimise the MR protocol for evaluating global small bowel peristalsis in vivo using a simple automated method from dynamic MR. The minimal acquisition time, number of slices and study repetitions required to produce reproducible data were assessed.

Methods and Materials: Twenty-five dynamic MR studies (True FISP, a single breath hold, ECG triggering) were obtained from three fasting healthy volunteers. The abdomen was covered by 10 dynamic coronal acquisitions. Parametric maps were automatically generated using in-house built software representing the mean absolute signal change in each pixel. Thresholds selected from histograms were used to decrease background noise. An index of peristalsis was generated by dividing the sum of the mean pixel values of the resulting parametric images divided by the number of pixels. ANOVA tests were performed to determine the shortest acquisition time, minimum number of slices and minimum number of study repetition required for a reproducible index of peristalsis.

Results: There was no statistical significance between the 30, 25, 20 and 15 frames acquisition protocols. For acquisitions of 15 frames or less, there was significant variability in the data. Using half or less of the dataset by selecting alternate coronal slices results in significant differences in variance. There was no significant difference between a single acquisition and the combined data from up to five acquisitions.

Conclusion: For optimal physiological small bowel dynamic MR studies, a single acquisition, with dynamic MR consisting of a minimum of 15 frames, covering the whole abdomen is satisfactory.

\section{B-435 11:06}

Dynamic contrast-enhanced MRI (DCE-MRI) as an indicator of disease activity in patients with luminal Crohn's disease

M.L.W. Ziech, C. Lavini, A.J. Nederveen, C.Y. Ponsioen, P.C.F. Stokkers, J. Stoker; Amsterdam/NL (m.l.ziech@amc.uva.nl)

Purpose: To assess if DCE-MRI parameters are correlated with disease activity in patients with luminal Crohn's disease (CD).

Methods and Materials: Ethical permission and informed consent were obtained. Patients with luminal CD undergoing MRI and ileocolonoscopy were recruited. Patients drank $1600 \mathrm{ml}$ of mannitol $2.5 \%$. Coronal DCE-MRI was performed at $3 \mathrm{~T}$ during intravenous contrast injection of Gadolinium (TE/TR=1.8/2.9 ms, FA $6^{\circ}, 14$ slices, dynamic scan duration 6.11 min, 450 dynamics, temporal resolution 0.81 $\mathrm{sec})$. ME images were calculated. A region of interest (ROI) was drawn around the most abnormal region assessed on MRI and, if not present, a normal bowel loop and ME were averaged over these ROI. C-reactive protein and Crohn's disease activity index (CDAI) were determined. Also Crohn's disease endoscopic index of severity (CDEIS) was determined which evaluates bowel involvement. CDEIS evaluation included also a partial CDEIS per segment to assess different bowe segments. Correlations were measured with Spearman's test.

Results: Sixteen patients were included (mean age 39 years range 24-72). Mean time between MRI and ileocolonoscopy was 15 days (range 2-32). Median CDAI was 329 (range 112-735). Median CDEIS was 5.5 (range 0.4 - 39.0). Median Creactive protein was 22 (range $<1.1-95$ ). $\mathrm{ME}$ had a significant positive correlation with CDEIS per-segment $(r=0.601, p=0.014)$. All other per-patient indices (CDAI, $\mathrm{CRP}$ and CDEIS) had no significant correlation with ME, although for CDEIS there was a trend towards a positive correlation $(r=0.487, p=0.056)$.

Conclusion: ME measured with DCE-MRI is an objective non-invasive parameter to assess segmental disease activity in CD patients.

\section{B-436 11:15}

\section{Non perforating small bowel Crohn's disease assessed by MR} enterography: derivation and histopathological validation of an MR-based activity index

S.A. Taylor, M. Steward, S. Punwani, I. Proctor, Y. Adjei-Gyamfi, F. Chaterjee,

M. Rodriguez-Justo, S. Halligan; London/UK (csytaylor@yahoo.co.uk)

Purpose: To develop and validate a qualitative scoring system for enteric Crohn's disease activity using MR enterography (MRE).

Methods and Materials: MRE was performed in 16 patients (mean age 33, 8 male) undergoing small bowel resection. Mural thickness, T2 signal, contrast enhancement, and perimural oedema were scored qualitatively (0-3) at 44 locations. Transmural histopathological scoring of acute inflammation (AIS) was performed at all locations (score 0-13). MRI parameters best predicting AIS were derived using multivariate analysis. The MRI activity index was prospectively applied to 26 Crohn's patients (mean age 32, range 13-69 years, 15 males) and correlated to terminal ileal biopsy scores of acute inflammation ("eAIS" score 1-6). Receiver operator characteristic curves were calculated.

Results: Mural thickness (coefficient $1.65[95 \% \mathrm{Cl} 1.35-2.69], \mathrm{p}=0.01$ ) and T2 signal (coefficient 0.90 [-0.24-2.04], $p=0.1$ ) best predicted AIS (AIS $=2.16+1.65$ $\mathrm{x}$ mural thickness $+0.90 \times \mathrm{T} 2$ signal [R-squared 0.53]). There was a significant correlation between the MRI index and eAIS (Kendall's tau $=0.40,95 \% \mathrm{Cl} 0.11-0.64$, $\mathrm{p}=0.02)$. The model achieved a sensitivity of $0.81(95 \% \mathrm{Cl} 0.54-0.96)$, specificity of 0.70 (0.35-0.93) and AUC 0.77 for predicting acute inflammation (eAIS $\geq 2$ ). Conclusion: A simple qualitative MRI Crohn's disease activity score appears predictive against a histopathological standard of reference.

\section{B-437 11:24}

Using CT enterography to monitor Crohn's disease activity: a follow-up study

Y. Wu, N. Hao, Y. Tang, F. Miao; Shanghai/CN (wuyw0103@hotmail.com)

Purpose: To evaluate CTE values in CD patient follow-up through quantitative measures of CD-associated parameters.

Methods and Materials: We prospectively included 40 known CD patients from 2008-2010.Endoscopy, CTE and laboratory tests (Hs-CRP, ESR) were performed in two weeks and CDAl index, Rutgeers score was calculated by experienced gastroenterologists. When CDAI>150, active disease was considered. Endoscopic active disease was identified when Rutgeerts Score $\geq 1$. CTE parameters, including bowel wall attenuation, bowel wall thickness, comb sign, perienteric fat density and luminal stenosis were evaluated by quantitative or semiquantitative methods. CTE.Hs-CRP,ESR ,Endoscopy and CDAI index were repeated 12 months later. 
Results: Rutgeerts score and CDAI were correlated with CTE findings at baseline $(P<0.05)$. Follow-up results showed $18(45 \%)$ patients get progression with Rutgeerts score, whereas $6(15 \%)$ patients were found regression and $16(40 \%)$ patients were stable. Similarly, using CDAl index progression was found in $14(35 \%)$ patients whereas regression in $8(20 \%)$ patients and $18(45 \%)$ patients were found to be stable. Variations between two times Rutgeerts score was shown positively correlated with the variations of bowel wall attenuation, bowel wall thickness, comb sign, perienteric fat density and luminal stenosis.(Spearmen index 0.362-0.614, $\mathrm{P}<0.05$ ).CDAl variations were detected correlated with variations of bowel wall attenuation, bowel wall thickness, comb sign and perienteric fat density. (Spearmen Index 0.334-0.624, $P<0.05$ ). Comb sign highest correlated with either CDAI (Spearmen Index $=0.624$, $P<0.01$ ) or Rutgeers score (Spearmen Index $=0.614, P=1.7 \times 10^{-5}$ ).

Conclusion: CTE is a valuable tool in Crohn's disease patients follow-up.Comb sign did best in monitoring disease activity.

\section{B-438 11:33}

Quantitative measures of comb sign-correlation with disease activity and laboratory indications

Y. Wu, N. Hao, M. Fei, Y. Tang; Shanghai/CN (wuyw0103@hotmail.com)

Purpose: To determine if quantitative measures of the comb sign at CT enterography correlate with laboratory indications in Crohn's disease patients.

Methods and Materials: We retrospectively included 120 known CD patients (65 male and 55 female patients) who had undergone CT enterography from 2008.92010.9 and had high sensitive $C$ reaction protein (HS- CRP) and erythrocyte sedimentation rate (ESR) results. The interval time between CTE and laboratory tests is less than one week (1-7days). We divided 120 patients into two groups based on disease activity. 78 patients were characterised as active disease while 42 were inactive. For each patient, coronal maximum intensity projection (MIP) image in which comb sign can be well displayed was selected and 5 region of interest $(\mathrm{ROI})\left(1 \mathrm{~cm}^{2}\right)$ were placed over the area where comb sign exists. Comb sign amount was counted in each ROI to assess the comb sign intensity. Quantitative comb sign results were compared with CRP and ESR level.

Results: Quantitative measures of comb sign is significantly higher in active group than in inactive group ( 86 vs.47, $p<0.01$ ). HS-CRP level is also obviously higher in active group than in inactive group (5.96 vs.3.33 mg/L, $P<0.05$ ), whereas ESR has no significant difference between two groups. Quantitative comb sign results well correlated with CRP level s with the coefficient of $0.49(P<0.05)$. Quantitative comb sign results did well in predicting CD activity with the sensitivity of $0.81(95 \%$ $\mathrm{Cl}, 0.70-0.89)$ and specificity of $0.78(95 \% \mathrm{Cl}, 0.66-0.84)$ based on ROC curve. Conclusion: Quantitative measures of comb sign is highly correlated with CRP levels and becomes a good parameter in predicting CD activity.

\section{B-439 11:42}

Contrast-enhanced ultrasound in ileal Crohn's disease: correlation between quantitative parameters and simple endoscopic score for Crohn's disease activity

E. Bock, A. Di Veronica, L. Guidi, A. Armuzzi, F.M. Danza, L. Bonomo,

A. De Franco; Rome/IT (antonio.defranco3@tin.it)

Purpose: Evaluate biological activity in patients with ileal Crohn's disease (CD) using CEUS with blood pool contrast agent (sulphur hexafluoride, SonoVue ${ }^{\circledR}$ SV-Bracco Italy) and HDI-QLab software elaboration and correlate quantitative data obtained with the simple endoscopic score for Crohn's disease (SES-CD).

Methods and Materials: Thirty-six patients (M25, F11, m.a.31) affected by ileal Crohn's disease (CD) were enrolled after informed consent and studied by CEUS with second generation contrast agent (SonoVue ${ }^{\circledR}$ i.v. $4.8 \mathrm{ml}$ ). All patients have had ileoscopy and scored from 0 to 3 in conformity to SES-CD as suggested by Daperno-Rutgeerts as gold standard. By HDI-QLab software elaboration of timeintensity curves maximum peak intensity (MPI) and coefficient of the wash-in slope $(\beta)$ were selected as quantitative parameters.

Results: MPI (Spearman's $\rho=0.47, p<0.0052)$ and slope $(\beta)$ (Spearman's $\rho=$ $0.55, p<0.0011)$ significantly correlated with SES-CD. MPI values $(45.85-85.04)$ are significantly higher in patients with SES-CD $\neq 0$ (median 60.47, $\mathrm{Cl} 95 \%$ ) in confront to patients with SES-CD $=0$ (median $0.00, \mathrm{Cl} 95 \% 0-18.48$ ): Mann Whitney test $\mathrm{p}<0.0001$. $\beta$ values $(6.11-10.13)$ are significantly higher in patients with SES-CD $\neq 0$ (median 7.24, $\mathrm{Cl} 95 \%$ ) in confront to patients with SES-CD $=0$ (median 0.00 , Cl 95\%, 0-4.04): Mann Whitney test $\mathrm{p}<0.0001$.

Conclusion: Significant correlation in predicting active CD were found between MPI and slope evaluated by CEUS and SES-CD.
B-440 11:51

Contrast-enhanced ultrasound in intestinal GvHD: microbubble penetration as a new diagnostic feature

A.G. Schreyer, C. Friedrich, M. Jung;

Regensburg/DE (andreas.schreyer@klinik.uni-r.de)

Purpose: Graft-versus-host-disease (GVHD) is an important complication in patients after allogeneic stem cell transplantation. Early detection is of paramount interest, because early therapy can improve the outcome. We evaluated contrast-enhanced ultrasound (CEUS) in patients with GVHD to assess typical imaging features.

Methods and Materials: CEUS was performed in 9 patients with histologically proven GVHD. As a control 4 healthy volunteers and 6 patients with Crohn's disease (CD) were examined. We employed a high-resolution multi-frequency transducer (6-9 MHz) with contrast harmonic imaging and low mechanical index technique to preserve microbubble integrity. After the injection of $2.4 \mathrm{ml}$ SonoVue ${ }^{\circledR}$ intravenously data were acquired and stored digitally. ROls (region of interest) were manually placed over the surrounding mesenteric fat, bowel wall and bowel lumen. Movement compensation of the ROls during the scan was performed. Time intensity curves were calculated based on the ultrasound signal and the maximum signal increase of each compartment was calculated.

Results: Patients with CD as well as those suffering from GVHD showed a significant contrast uptake of the bowel wall. Only patients with GVHD showed a transmural penetration of microbubbles into the bowel lumen (average maximum signal increase - GVHD: $7.88 \mathrm{~dB}$; CD: $1.13 \mathrm{~dB}$, healthy volunteer: $0.28 \mathrm{~dB}$ ).

Conclusion: We assume that the damaged gut mucosal barrier in GVHD enables the microbubbles to penetrate through the bowel wall into the bowel lumen. We consider the penetration of microbubbles into the bowel lumen as a novel diagnostic feature for GVHD.

10:30 - 12:00
Radiographers
SS 814
Improving productivity and quality: the
radiographer's area of responsibility

Moderators:

B. Bjorkman; Huskvarna/SE

D. Tscholakoff; Vienna/AT

\section{B-441 10:30}

Interobserver variability in pre-endovascular aneurism repair assessment by CT-scan angiography

M.M.C.P. Ribeiro, G.E.I. Vaz, C. Silva-Fortes, L.J.O.C. Lança, J.C. Maurício,

J.E.G. O' Neill; Lisbon/PT (margarida.ribeiro@estesl.ipl.pt)

Purpose: The vascular measurements for abdominal aortic stent graph endoprosthesis are often replicated by different health professionals groups. This study aimed to calculate the interobserver variability between three professional groups - 4 radiographers $(R D) ; 4$ radiologists (RG); and 4 vascular surgeons (VS) - using angio-CT technique, according the pre-EVAR (endocvascular aneurism repair) measurement protocol.

Methods and Materials: 480 observations from patients with abdominal aortic aneurism were analysed by 12 observers from 5 abdominal CT studies. Eight specific measures were applied ( 6 diameters and 2 distances) following the same pre-EVAR protocol. For statistical analysis Bland-Altman and analysis of variance test were used by Excel 2007 (Microsoft ${ }^{\circledR}$ ) and analyze-it ${ }^{\circledR}$ applications to evaluate the mean difference between observers.

Results: The mean difference between observers was $1.55 \pm 3.39 \mathrm{~mm}$ and $3.38 \pm 5.39 \mathrm{~mm}$ for vessel diameters and distances, respectively. Pairs of observations differences showed that $75.1 \%$ were $\leq 2 \mathrm{~mm}$ and $4.2 \% \geq 5 \mathrm{~mm}$. Among professional groups was found a variation between RG and VS with $-1.15 \mathrm{~mm}$ (95\% Cl: $-1.597 \mathrm{~mm},-0.702 \mathrm{~mm})$ and between RD and CV with $-2.7 \mathrm{~mm}(95 \% \mathrm{Cl}$ : $-3.848 \mathrm{~mm},-1.557 \mathrm{~mm})$. There were no significant differences $(p>0.05)$ in results obtained between professional groups. The mean difference for the aneurysm diameter location was a $1.7 \mathrm{~mm}$, less than the gold-standard considered $(2.04 \mathrm{~mm})$. Conclusion: There was agreement between the three different professional groups. The difference values we found could be considered as acceptable. The pre-EVAR measurements may be performed by radiographers and the replication is not justified unless when applied the multi-observational method for reliability purpose. 


\section{B-442 10:39 \\ Implementing evidence-based protocols for i.v. contrast media; the process, outcome and follow-up}

B. Vee, A. Taule, N. Kleven-Madsen; Bergen/NO (bente.vee@helse-bergen.no)

Purpose: The department of radiology at $\mathrm{HUH}$ aims to provide patients with services based on best available evidence in all aspects of imaging. Based on risk assessment and ESUR guidelines for administering i.v. contrast media (IVCM), a need was recognised for systematic review of current practice. The objective was to 1) survey and 2) improve all processes involving the use of IVCM. The overall aim is to utilise and increase the competence of all professions involved in the various steps involved to provide the best achievable quality in the use of IVCM; evidence-based practice.

Methods and Materials: All diagnostic use of IVCM; referral, radiology report, follow-up of sideeffects was reviewed. Main focuses were identifying precise manageable measurement of kidney function (eGFR), patients at risk for CIN, NSF, thyroid patients, use of, i.e. metformin, classification and handling of sideeffects, responsibility (clinician, radiologist and radiographer) and patient information.

Results: By reviewing routines and implementing new procedures, the process has created awareness on the use of IVCM so that patient care and safety are met. It has been challenging to clarify the responsibility for each professional participant. Continued staff information and education throughout the process was important. Conclusion: Evidence-based routines and procedures have been established, and are clearly communicated to all involved participants to achieve and maintain good practice. A successful implementation requires continuous focus (dynamic process) from dedicated staff to follow-up; implement new knowledge and adjust the daily routines. Quality indicators for evaluating the effect of implementation must be developed.

\section{B-443 10:48}

Effect of informed consent for administration of intravenous contrast material in the anxiety level of patients submitted to computed tomography exams

K.B. Azevedo, A.F.C.L. Abrantes, L.P.V. Ribeiro, R.P.P. Almeida, C.A. Silva; Faro/PT (kbazevedo@ualg.pt)

Purpose: Evaluation and relation of the state and trait anxiety level in patients before the delivery of informed consent with patients after that, and the evaluation of eventual relations between psychometric tests and sociodemographic characteristics. Methods and Materials: The present study is quasi-experimental. The total sample of 161 patients was divided in a control group and an experimental group, and then state-trait anxiety inventory was applied to both, after the sociodemographic interview and always after the consent of participation of the patient.

Results: At a $95 \%$ confidence interval, the intergroup difference between means of state anxiety ranged from -0.929 and -0.810 . The value of $t$ student test was -28.701 ( $\mathrm{df}=159$ and 2-tail sig $=0.000$ ). On the other hand, the intergroup difference between means of trait anxiety ranged from -0.141 and -0.022 . The value of t student test was $-2.722(\mathrm{df}=159$ e 2-tail sig $=0.007$ ). Application of Spearman's Ro revealed the correlation between values of state and trait anxiety based in a non-normal approximation: for control group $0.409(\mathrm{sig}=0.000)$ and for experimental group 0.321 ( $\mathrm{sig}=0.004)$, whereas it did not reveal any statistically significant correlation between sociodemographic characteristics and psychometric tests. Conclusion: There exists a significant intergroup difference between the anxiety state and the anxiety trait. A significant correlation is verified between state and trait anxiety within each group, what is not verified between psychometric tests and sociodemographic characteristics.

\section{B-444 10:57}

Can collaboration between radiographers and non-consultant hospital doctors during image interpretation improve the accuracy of diagnostic decisions?

M.F. Mc Entee, B. Kelly, L. Rainford; Dublin/IE (mark.mcentee@ucd.ie)

Purpose: In emergency departments (ED) junior doctors regularly make diagnostic decisions based on radiographic images [1]. This study investigates whether collaboration between juniour doctors and RTs impacts on diagnostic accuracy.

Methods and Materials: Research was carried out in the ED of a university teaching hospital and included 10 pairs of participants. RTs and juniour doctors were shown 42 wrist radiographs and $40 \mathrm{CT}$ brains and were asked for their level of confidence of the presence or absence of distal radius fractures or fresh intracranial bleeds, respectively, using ViewDEX software, first working alone and then in pairs. Receiver operating characteristic was used to analyse performance. Results were compared using one-way analysis of variance.
Results: The results showed statistically significant improvements in the area under the curve values (AUC) of the juniour doctors when working with the RTs for both sets of images (wrist and CT) treated as random readers and cases $(p<0.008$ and $p<0.0026$, respectively). While the RTs' results saw no significant changes, their mean $A z$ values did show an increasing trend when working in collaboration. Conclusion: Improvement in performance of juniour doctors following collaboration strongly suggests changes in the potential to improve accuracy of patient diagnosis and therefore patient care. Further training for juniour doctors in the interpretation of diagnostic images should also be considered. Decision making of juniour doctors was positively impacted on after introducing the opinion of an RT. Collaboration exceeds the sum of the parts; the two professions are better together.

\section{B-445 11:06}

Novel, portable, cassette-sized, and wireless flat-panel digital radiography (DR) system: initial workflow results versus computed radiography (CR)

T. Lehnert, M.J. Kerl, R.W. Bauer, N. Naguib, M. Nour-Eldin, T.J. Vogl;

Frankfurt a. Main/DE (thomas.lehnert@kgu.de)

Purpose: To compare workflow efficiency between a conventional computed radiography (CR) system and a novel, portable, cassette-sized, and wireless flat-pane digital radiography (DR) system.

Methods and Materials: Observational time-motion analyses were performed at one site at which CR, and the new portable DR system are used concurrently. The workflow steps of both systems were identified and categorised to facilitate comparison. The time required for examination preparation, patient positioning, exposure, post-acquisition processing, and for the examination as a whole, was recorded by a neutral observer. Timing differences between the $\mathrm{CR}$ and portable DR systems were compared, and all data were analysed using commercially available statistical software. Nine general radiographic exam types were selected with approximately 50 patients per exam type.

Results: A total of 941 examinations ( $C R, n=474$; portable $D R, n=467$ ) were timed in this study. Total examination time differences between $\mathrm{CR}$ and portable DR system (mean 26.44s; median 26.99s) were found to be statistically significant $(\mathrm{P}<.001)$, with DR proving faster than $\mathrm{CR}$. The single largest contributor to the time difference between $\mathrm{CR}$ and portable DR was post-acquisition processing (mean 26.58s, median 25.91s), a composite of multiple individual steps, including cassette transport (CR only, mean 13.22s, median 12.74s), cassette readout (mean 10.15s, median 10.4s) and post-processing (mean 3.21s, median 3.11s).

Conclusion: Overall radiographer time was significantly shorter when performing exam-related tasks with the novel, portable DR system than when performing comparable tasks with $\mathrm{CR}$, a difference that appears to result largely from technology configuration.

\section{B-446 $\quad$ 11:15}

Interobserver agreement among PGMI radiographer in quality classification of mammograms: a pilot study

R. Gullien, J. Andersen, A. Haakull, E. Rostad, R. Hammond; Oslo/NO (raul@uus.no)

Purpose: To evaluate the inter-observer agreement between internal and external radiographers with and without experience in PGMI classification of mammograms using the classifications recommended in the quality assurance manual for the Norwegian Breast Cancer Screening Program (NBCSP).

Methods and Materials: Three PGMI radiographers, $A=$ internal experienced radiographer, $\mathrm{B}=$ external experienced radiographer, $\mathrm{C}=$ internal inexperienced radiographer, reviewed and classified 480 screening mammograms from 120 women. Using the PGMI classifications were $\mathrm{P}=$ perfect, $\mathrm{G}=$ good, $\mathrm{M}=$ moderate and $\mathrm{I}=$ inadequate mammograms, each radiographer classified the mammograms independently, by scoring them as: $P=1, G=2, M=3$, and $I=4$. Inter-observer agreement was assessed (Kappa analysis).

Results: The agreement between the PGMI radiographers was: $\kappa=0.40,0.40$, and 0.44 , respectively, for $A+B, B+C, A+C$. This level of agreement is classified as fair to moderate according to Kappa classification. The exact agreement between $A+B, B+C, A+C$ was, respectively, $57 \%, 56 \%$ and $61 \%$. The best agreement appeared between the experienced and inexperienced internal radiographer $(A+$ $C)$, while there is less agreement between the two experienced $(A+B)$. The lowest agreement was between the external experienced and the internal inexperienced radiographer $(B+C)$. This indicate that there are a different competency levels between the three radiographers.

Conclusion: Interobserver agreement with the PGMI quality classification in our pilot study was fair to moderate. This indicates that PGMI classification is a challenge in mammography screening, since there is room for individual assessments - despite guidelines. 


\section{B-447 11:24}

Radiographer led real time review of CT colonography: initial experiences R.J. Moore, D.P.G. Mullan, P. Healey, M. Hughes; Liverpool/UK (rachelmoore01@btinternet.com)

Purpose: To evaluate and report our experiences of role extension for radiographic advanced practitioners (GI) in CT colonography (CTC) techniques.

Methods and Materials: Barium oenemas have been superceded by CTC in our institution. This reduced fluroscopic workload could lead to 'de-skilling' of Gl radiographic advanced practitioners who are treained to masters level, with several years of specific experience. Dedicated CTC lists are performed with a seperate CT and GI radiographer in attendance. The GI radiographer assesses the adequacy of bowel prep, performs PR examinations, and continually analyses the quality of pneumocolon. There is focused real-time review of the first aquisition of images whilst the patient is prepared for the subsequent aquisition. If a tumour is discovered on real-time review, the $\mathrm{G}$ radiographer will justify and perform a concurrent $\mathrm{CT}$ thorax for completion imaging. Results: 401 studies were performed with real-time review between 1/6/10 and 1/9/10. This lead to same day CT thorax studies in 23 cases of previously undiagnosed colorectal tumours. Diagnostic quality pneumocolon was achieved in $97 \%$ of cases. Conclusion: Real-time review of CTC by a GI advanced practitioner leads to concurrent performing of $\mathrm{CT}$ thorax in appropriate cases. This leads to timely full body staging and reduces delays in appropriate cases. Understanding of colon anatomy and pathology allows the GI radiographer to safely achieve diagnostic quality pneumocolon in the majority of cases.

\section{B-448 11:33}

The effect of utilising SMS technology on patient care in radiology N.M. Mishah; Jeddah/SA (nabeel747@hotmail.com)

Purpose: To present the effect of utilising SMS (short message service) on patient care in radiology department since SMS technology was recently utilised to remind and/or confirm patient's appointment and to send patient preparations. The efficiency and effectiveness of this tool will be compared to other conventional telecommunication methods.

Methods and Materials: Statistical data for the first half of year 2010 (6 months period) were retrieved from the system then, the electronic data were analysed in order to measure the percentage of SMS message delivery rate and its relation to better patient care and the list of no show patients (or) badly prepared patients for rebooking. Results: A tremendous improvement in patient care, attandence and communication if compared to the situation before implementing SMS. All the patients received the SMS ( $100 \%$ delivery rate) and no show list was decreased by $70 \%$ and bad patient preparation was decreased by $35 \%$.

Conclusion: SMS technology is available, easy to use, cost-effective, and efficient way to communicate with patients for better patient care and better resource utilisation.

\section{B-449 11:42}

Radiologists' responses to inadequate referrals

K.B. Lysdahl', B.M. Hofmann 2 , A. Espeland ${ }^{3} ;{ }^{1} O$ Slo/NO, ${ }^{2}$ Gjøvik/NO, ${ }^{3}$ Bergen/NO (kristin.bakke-lysdahl@hf.hio.no)

Purpose: To investigate radiologists' responses to inadequate imaging referrals. Methods and Materials: A survey was mailed to Norwegian radiologists; $69 \%$ responded. They graded the frequencies of actions related to referrals with ambiguous indications or inappropriate examination choices and the contribution of factors preventing and not preventing an examination of doubtful usefulness from being performed as requested.

Results: Ninety-five percent (344/361) reported daily or weekly actions related to inadequate referrals. Actions differed among subspecialties. The most frequent were contacting the referrer to clarify the clinical problem and checking test results/ information in the medical records. Both actions were more frequent among registrars than specialists and among hospital radiologists than institute radiologists. Institute radiologists were more likely to ask the patient for additional information and to examine the patient clinically. Factors rated as contributing most to prevent doubtful examinations were high risk of serious complications/side effects, high radiation dose and low patient age. Factors facilitating doubtful examinations included respect for the referrer's judgment, patient/next-of-kin wants the examination, patient has arrived, unreachable referrer, and time pressure.

Conclusion: Radiologists frequently take action in response to inadequate referrals, mainly by searching for complementary information. Their actions seem to be mainly motivated by patient safety considerations. Vetting of referrals at arrival, easier access to referring clinicians, and sufficient time for radiologists to handle inadequate referrals, may contribute to improved use of imaging.
B-450 11:51

Which ethics for radiographers?

A. Santos; Coimbra/PT (adelinosantos@estescoimbra.pt)

Purpose: Radiographers citizenship behaviours are spontaneous actions that go beyond the requirements of organisations. This research focuses on ethical fields because ethically correct behaviour has been gradually moving away from the daily practice.

Methods and Materials: An original electronic survey was used. The representative sample consists of 472 radiographers and sought to discover the (in)congruence between the perceived ethical climate and the preferred ethical climates relates to actions taken at the same time and if training in ethics, bioethics and deontology could guide these same behaviours.

Results: Respondents (73\%) believe that what was desired to happen in compliance with laws/rules did not fit in practice. Radiographers feel that, within the organisation, colleagues break the rules and institutional norms, in most cases. Technicians (82\%) believe that the climate of benevolence that exists in their institutions falls short of expectations. It highlights a weakened sense of collegiality that, in turn, translates into a weak spirit of labor solidarity.

Conclusion: Technicians' training does not interfere in how they perceive the climate of benevolence. The most consistent result is that which occurs in self-interest climate. Within a context where the latter feeling prevails people are reluctant to practice good ethical behaviour but instead defend their own interests. Education can and should influence the exercise of citizenship. It becomes a cornerstone in personal and professional development. Training in ethics, bioethics and deontology should be assumed as vital in the consolidation of professional identity of radiographers and strategies for success in the departments of radiology.

10:30 - 12:00 Room N/O

Neuro

\section{SS 811}

\section{Neurovascular intervention planning and follow-up}

Moderators:

S.J. Bakke; Oslo/NO

S. Puchner; Vienna/AT

\section{B-451 10:30}

Non-invasive imaging after stent-assisted coiling of intracranial aneurysms: comparison of 3 T-MRI and 64-MDCT

A. Kovács'1, M. Möhlenbruch'², D.R. Hadizadeh², M. Seifert'², W.A. Willinek², S. Greschus ${ }^{2}$, S. Flacke ${ }^{2}$, H. Clusmann', H. Urbach ${ }^{2} ;{ }^{1}$ Lübeck/DE,

2Bonn/DE (attila.kovacs@uk-sh.de)

Purpose: Follow-up imaging after stent-assisted coiling (SAC) of intracranial aneurysms is limited by signal loss in the stented vessel segment in MRI and by streak artefacts caused by aneurysm coils in MDCT. Searching for a non-invasive surveillance in this condition we propose a technique to minimise streak artefacts in MDCT by gated data acquisition and shifting the reconstruction window (gCTA). Methods and Materials: The effect of gCTA on artefact reduction was evaluated in a preliminary phantom study and compared with non-gated-CTA, TOF-MRA and DSA. To show translational feasibility gCTA and TOF-MRA were also performed on five patients treated with SAC as part of a follow-up protocol. The length of impaired vessel segments (LIVS) were compared and correlated with the stent length, the number of coils and the packing density. The assessment of treatment outcome (ATO) was compared with DSA as the standard of reference.

Results: The phantom study revealed a distinct reduction of streak artefacts using gCTA. In MRI all stented vessel segments showed signal loss in both, in the phantom and in patients. The LIVS was $78 \%$ shorter in $\mathrm{GCTA}(4.86 \pm 6.93 \mathrm{~mm})$ as compared to TOF-MRA $(21.82 \pm 7.47 \mathrm{~mm}, \mathrm{p}<.01)$. LIVS correlated in TOF-MRA the with the stent length, in gCTA with the number of coils. With regard to ATO gCTA and TOF-MRA correlated with DSA in $3 / 5$ and $0 / 5$ patients, respectively. Conclusion: Gated CTA is a promising technique to reduce the amount of artefacts induced by stent-assisted intracranial coils. Image quality and assessment of treatment outcome in patients with stent-assisted coiling is superior compared to TOF-MRA. 


\section{B-452 10:39 \\ Circle of Willis single-source dual energy CTA: "metal artefact reduction software" in evaluation of vasospasm and residual aneurysm after endovascular approach for intracranial aneurysms \\ S. Comelli, F. Fusaro, D. Savio, G. Vaudano; Turin/lT (fedefusaro@libero.it)}

Purpose: Single-source dual energy CT (Discovery 750HD, GE Med. Systems, WI) with a particular post-processing software allows to significantly decrease, even though not completely avoid, metal artefacts. We experienced it in patients previously treated for intracranial aneurysm with endovascular approach, either after coiling or flow-diverting device. Aim of the study is to evaluate vasospasm during ten days following treatment, and post-operative recurrent or residual aneurysms at mid-term (3 to 6 months) follow-up controls.

Methods and Materials: From January to July 2010, 25 post-operative aneurysms ( 6 up to $28 \mathrm{~mm}$ in maximum diameter, mean $9.2 \mathrm{~mm}$ ) in 24 patients (age 42-79, mean 58.3) have been studied with dual energy CT angiography (DECTA). 23 aneurysms were treated with detachable coils, 2 with pipeline flow-diverting stent (ev3, Irvine, CA). DECTA raw-data were post-processed with metal artefacts reduction software (MARS), using levels $64 \mathrm{KeV}$ up to $72 \mathrm{KeV}$.

Results: DECTA with MARS has proven to be diagnostic in vasospasm grading evaluation for $25 / 25$ patients $(100 \%)$. Compared with digital subtraction angiography (DSA, GE Innova 4100) performed in all patients after CT, MARS demonstrates 100\% sensitivity and specificity in vasospasm evaluation. On mid-term follow-up controls, MARS significantly reduced metal artefacts, but does not seem to be enough accurate to detect $2 \mathrm{~mm}$ or smaller aneurysm residual (sensitivity $54 \%$, specificity $68 \%$ ). Conclusion: Our initial results suggest DECTA with MARS protocol shows high diagnostic accuracy compared with DSA for vasospasm evaluation in post-coiled aneurysms. More data are required in mid- and long-term follow-up post-operative aneurysms evaluation with DECTA.

\section{B-453 10:48}

\section{A comparison study with contrast-enhanced MRA and time-of-flight MRA}

at 3.0 $\mathrm{T}$ for evaluation of intracranial aneurysms

Q. Wu, M. Li, J. Zhang, Y. Li; Shanghai/CN (wuqianlily@163.com)

Purpose: To compare the diagnostic accuracy and reliability of TOF-MRA and CEMRA at $3 \mathrm{~T}$ for detection and characterisation of untreated intracranial aneurysms Methods and Materials: 3D-TOF-MRA and 3D-CE-MRA at $3 \mathrm{~T}$ were performed in 52 patients with suspected or known aneurysms sequently, prior to rotational subtraction angiography (RDSA). Accuracy, sensitivities, specificities, positive predictive value (PPV) and negative predictive value (NPV) on aneurysm-, vessel-, and size-based evaluation were calculated to detect or rule out intracranial aneurysms The diagnostic relativity of VR 3D-TOF-MRA at 3 T and VR-DSA, 3D-CE-MRA at 3 T and VR-DSA was conducted, respectively, to compare the relativity of both two modalities to assess the intracranial aneurysms.

Results: A total of 58 intracranial aneurysms were visualised on VR 3D-TOF-MRA. For aneurysm $<3 \mathrm{~mm}$, one aneurysm was missed on VR 3D-TOF-MRA, while on VR 3D-CE-MRA, there were eight false-negative cases. Accuracy, sensitivity, specificity, PPV and NPV of VR 3D-TOF-MRA at $3 \mathrm{~T}$ for the detection of intracranial aneurysm $<3 \mathrm{~mm}$ were $97.7 \%, 94.7 \%, 100 \%, 100 \%, 95.7 \%$, respectively, while on VR 3D-CE-MRA, $80.5 \%, 57.9 \%, 100 \%, 100 \%, 73.3 \%$, respectively. Fo the detection of intracranial aneurysm $\geq 3 \mathrm{~mm}$, there was no statistical difference between two modalities.

Conclusion: 3D-TOF-MRA at $3 \mathrm{~T}$ is revealed to have overwhelming advantages over 3D-CE-MRA for the detection and characterisation of untreated intracrania aneurysms. 3D-CE-MRA is only superior to 3D-TOF-MRA for depicting the morphology of giant aneurysms with partial thrombosis or turbulent flow and in uncooperative patients with SAH. Thus, 3D-TOF-MRA at $3 \mathrm{~T}$ is recommended for detection and follow-up of untreated intracranial aneurysms.

\section{B-454 10:57}

Digital subtraction CT angiography for the diagnosis of intracranial aneurysms in $\mathbf{5 0 6}$ patients: correlation of three-dimensional digital subtraction angiography

L. Lu, L. Zhang, C. Zhou, M. Wang, G. Lu; Nanjing/CN (xuzhoululi@163.com)

Purpose: To further evaluate the clinical value of digital subtraction computed tomography angiography (DS-CTA) in the diagnosis of intracranial aneurysms in a large cohort with three-dimensional rotational digital subtraction angiography (3D-DSA) as rference standard.

Methods and Materials: Five-hundred and six patients clinically suspected of intracranial aneurysms underwent both DS-CTA in a dual source CT scanner and 3D- DSA with median time interval of 1 day. Accuracy of aneurysm detection with
DS-CTA was analysed on a per-patient and a per-aneurysm basis with 3D-DSA as reference standard.

Results: Of 506 patients, 107 patients (21.1\%) had no aneurysms, while 399 patients (78.9\%) had 452 aneurysms on 3D-DSA.DS-CTA correctly detected 450 aneurysms in 402 patients. Using 3D-DSA as the standard of reference, sensitivity and specificity of DS-CTA to detect intracranial aneurysms were $97.7 \%$ and $88.8 \%$ on a per-patient basis, $96.5 \%$ and $87.2 \%$ on a per-aneurysm basis. DS-CTA had sensitivities of $90.9 \%, 93.8 \%, 98.3 \%, 100 \%$ for the detection of aneurysms with the diameter of less than $3 \mathrm{~mm}$, larger than $3 \mathrm{~mm}$ but less than $5 \mathrm{~mm}$, larger than $5 \mathrm{~mm}$ but less than $10 \mathrm{~mm}$, and larger than $10 \mathrm{~mm}$. Excellent correlation was found between 3D-DSA and DS-CTA in the diagnosis of intracranial aneurysms (Kappa $=0.830, P<0.001)$ Conclusion: On the basis of this large cohort, DS-CTA has a high sensitivity and specificity for the detection of intracranial aneurysms compared with 3D-DSA, even for the aneurysms with the diameter less than $3 \mathrm{~mm}$.

\section{B-455 11:06}

Intravenous flat detector $\mathrm{CT}$ to visualise patency of intracranial stents: first results with an optimised protocol

T. Struffert' ${ }^{1}$, S. Kloska', T. Engelhorn', Y. Deuerling-Zheng' ${ }^{2}$, S. Ott', M. Doelken ${ }^{1}$ M. Saake ${ }^{1}$, A. Doerfler ${ }^{1} ;{ }^{1}$ Erlangen/DE, ${ }^{2}$ Forchheim/DE (tobias.struffert@uk-erlangen.de)

Purpose: As stents for treating intracranial atherosclerotic stenosis may develop in-stent re-stenosis (ISR) in up to $30 \%$, follow-up imaging is mandatory. Residual stenosis (RS) is not rare. We evaluated an minimal invasive optimised flat detector CT protocol with intravenous contrast application (i.v. FD-CTA) to conventional angiography to evaluate the stent lumen.

Methods and Materials: In 12 patients treated with a self expandable stent follow-up imaging was performed using i.v. FD-CTA and intra-arterial angiography (DSA). Location of intracranial stenosis was M1 in 6, V4/BA in 5 and C5 segment in 1 patient. MPR, subtracted MIP and VRT reconstructions were used to correlate to DSA. Two neuroradiologists evaluated the images in anonymous consensus reading and calculated ISR or RS, respectively. Correlation coefficients and a Wilcoxon test were used for statistical analysis.

Results: In 4 patients, no stenosis was detected. In 6 patients RS and in two cases ISR perfectly visible on MPR reconstructions of i.v. FD-CTA were detected. Wilcoxon's test showed no significant differences between the methods $(p>0.05)$. We found a high correlation with coefficients of the pairs DSA/ FD-CT MIP $r=0.91$, DSA/ FD-CT MPR $r=0.82$ and FD-CT MIP/ FD-CT MPR $r=0.8$.

Conclusion: Intravenous FD-CTA could clearly visualise the stent and the lumen, allowing ISR or RS to be recognised. FD-CTA provides a non-invasive imaging modality of intracranial stents and might replace DSA for non-invasive follow-up imaging.

\section{B-456 11:15}

MRI-defined recent silent ischaemia before thrombolysis: prevalence and impact on bleeding risk

M. Tisserand ${ }^{1}$, L. Le Guennec ${ }^{1}$, E. Touzé1, A. Hess' ${ }^{1}$, A.-D. Devauchelle ${ }^{1}$ S. Bracard ${ }^{2}$, J.-L. Mas', J.-F. Méder', C. Oppenheim ${ }^{1} ;{ }^{1}$ Paris/FR, ${ }^{2}$ Nancy/FR (marietisserand@hotmail.com)

Purpose: Uncertainties about the frequency and the associated bleeding risk of recent silent ischaemia (RSI), incidentally found on pretreatment MRI, in candidates for thrombolysis require clarification because exclusion from therapy is a serious consequence for patients with such MRI findings.

Methods and Materials: We retrospectively analysed the fluid-attenuated inversion recovery (FLAIR)/diffusion-weighted imaging (DWI) before intravenous (IV) thrombolysis in 115 patients to search for MRI-defined RSI; these corresponded to well-developed FLAIR/DWI brain hyperintensities (RSI+), as distinct from the acute index ischaemia, which typically lacked FLAIR changes. Patients without such findings were assigned to the RSI- group. Groups were compared for baseline characteristics and for rates of symptomatic and asymptomatic haemorrhagic transformation (HT) using odds ratio (OR) and their $95 \%$ confidence interval $(\mathrm{Cl})$. Results: We observed RSI in 21 patients (18.3\%). The mean (standard deviation) volume of RSI was $6.5(12) \mathrm{mL}$ (interquartile range $0.6-9$ ). None of the baseline parameters differed between groups. There was no significant difference in rates of any type of $\mathrm{HT}$ between groups. Parenchymal haemorrhage type $1(\mathrm{PH}-1)$ or type $2(\mathrm{PH}-2)$ according to ECASS criteria occurred in $10(11 \%)$ RSI- patients and in two $(10 \%) \mathrm{RSI}$ + patients $(\mathrm{OR}=0.88 ; 95 \% \mathrm{Cl}=0.18-4.37)$. Symptomatic $\mathrm{HT}$, defined according to NINDS criteria, occurred in $10(11 \%)$ RSI- patients and in one $(5 \%)$ $\mathrm{RSI}+$ patient $(\mathrm{OR}=0.42 ; 95 \% \mathrm{Cl}=0.05-3.47)$.

Conclusion: We found that $18.3 \%$ of acute stroke patients treated by IV thrombolysis in a stroke unit had RSI on pretreatment MRI. However, the presence of RSI was not associated with an increased risk of asymptomatic or symptomatic $\mathrm{HT}$. 


\author{
B-457 11:24 \\ The penumbra system - a mechanical device for the treatment of acute \\ ischaemic stroke: a single center experience \\ T. Kim, S. Kim, E. Kim, J. Lee, Y. Lim, M. Han; Suwon/KR \\ (piglett82@hanmail.net)
}

Purpose: To analyse the feasibility, the safety and performance of the Penumbra System.

Methods and Materials: A prospective, single arm, independently monitored and core laboratory adjudicated trial enrolled subjects with acute stroke, within 8 hrs of symptom onset and an angiographically verified occlusion (TIMI grade 0 or 1). The primary end point was revascularisation of the target vessel to TIMI grade 2 or 3 . Secondary end points were achievement of $\mathrm{mRs}$ core $\leq 2$, or $\geq 4$-point NIHSS improvement score at 30-day follow-up, as well as mortality rate.

Results: 20 target arteries of 18 patients were treated by the penumbra system. 14 cases of anterior circulation and 4 basilar artery occlusions. At baseline, mean mRS score was 4.5, and mean NIHSS score was 20. After procedure, 17 arteries were successfully revascularised ( $81 \%$ ) to TIMI 2 or 3 . At 30 day follow-up, 9 subjects $(45 \%)$ had mRs core $\leq 2$ or $\geq 4$-point NIHSS. None of the patients developed sICH. The all-cause mortality rate was $22 \%$ ( 4 of 18), which is lower than expected in this severe stroke cohort, where $65 \%$ of the subjects at baseline had either an NIHSS score of more than 20 or a basilar occlusion.

Conclusion: Penumbra system showed a high potential for recanalisation of acute thromboembolic occlusions of the large cerebral arteries. Clinical improvement can be observed in patients with ischaemic stroke using low-dose thrombolytic agents with adjunctive mechanical disruption of clot. Moreover, this strategy may reduce the risk of intracerebral haemorrhage observed with thrombolytics.

\section{B-458 11:33}

Endovascular treatment of very small $(2 \mathrm{~mm})$ intracranial aneurysms

M.H.J. Voormolen ${ }^{1}$, T. van der Zijden ${ }^{1}$, T. Menovsky ${ }^{1}$, O. d'Archambeau ${ }^{1}$

H. Fransen', P.M. Parizel'; ' ${ }^{2}$ Edegem/BE, ${ }^{2}$ Gent/BE (maurits.voormolen@uza.be)

Purpose: Evaluation of endovascular occlusion of very small intracranial aneurysms Methods and Materials: From January 2006 until July 2010, 18 patients (6 males/12 females, age 17 - 75 years) with 20 intracranial aneurysms of $2 \mathrm{~mm}$ were treated in our hospital by endovascular occlusion. Aneurysms were located in the internal carotid artery (siphon $(n=3)$, supraclinoid $(n=4)$ ), anterior communicating artery $(n=4)$, middle cerebral artery $(n=3)$, distal PICA, distal posterior cerebral artery and basilar tip. Seven patients presented with subarachnoid haemorrhage (SAH) and one with neurological deficit (mass effect). Six aneurysms were additional to ruptured aneurysms and four aneurysms were incidentally detected. Patients underwent endovascular treatment with coils $(n=5)$, balloon-assisted coiling $(n=$ $10)$, stent-assisted coiling $(n=2)$ and parent artery occlusion $(n=1)$. Clinical and angiographic follow-up was done at regular intervals.

Results: All but one aneurysm were technically successfully occluded. Two aneurysms had neck remnants that disappeared upon follow-up. Five procedural complications happened $(28 \%)$. In two patients with SAH coil perforation occurred; one patient recovered and the other died. One patient died of SAH from another untreated aneurysm. Two patients with unruptured aneurysms had local thrombus formation that resolved without sequel. Upon follow-up 17 of 18 aneurysms (94\%) remained occluded. One aneurysm with gradual re-opening was surgically clipped. Mean follow-up 11 months (range 0-24 months). Two patients (11\%) had permanent neurological deficit. Two patients died of SAH complications.

Conclusion: Endovascular occlusion of very small intracranial aneurysms poses a considerable treatment challenge with a significant morbidity/mortality rate, but can be performed in selected cases.

\section{B-459 11:42}

Intracranial endovascular stent implantation of middle cerebral artery atherosclerotic stenoses: results of 33 lesions

D. Hwang, K. Kim, Y. Ko, I. Kim, C. Hur; Seoul/KR

Purpose: The purpose of this study is to evaluate the initial success rate and follow-up result of the stent-assisted angioplasty for atherosclerotic stenoses of MCA. Methods and Materials: Thirty three lesions of twenty-nine patients were included in this study from March 2004 to September 2009. All patients had cerebal infarction averagely 54.75 days before the procedure. The locations of the stent implantation were all in M1 segment in MCA. Average follow-up period was 36 months.

Results: The stent implantation was successful in 26 lesions (78.8\%). We had 7 cases of failure $(n=7,21.2 \%)$. We had two cases $(6.06 \%)$ of microselection failure due to the tortuosity of the ICA. There was one case $(3.03 \%)$ of selection failure due to tortuousity of aortic arch. In one case (3.03\%), advance at the cavnernous siphon was not achieved. In one case (3.03\%), the patient did not cooperate nor was sedated enough for the procedure. There were two severe procedure-related complications $(6.06 \%)$. In one case $(3.03 \%)$, MCA rupture occurred after the ballooning of the stent. Immediate coiling was done, but the patient became comatose. In one case, $\mathrm{ICH}$ developed 2 weeks after the procedure. Mild restenoses occured in 2 patients without significant symptom changes during the follow-up period. Conclusion: Stent implantation in MCA stenosis is technically feasible and has relatively low rate of periprocedural complication. Still there is risk of serious complications such as rupture, haemorrhage, and restenosis. Further long-term follow-up study is necessary.

\section{B-460 11:51}

Mechanical thrombolysis for acute carotid occlusion: single center experience

R. Gandini, E. Pampana, F. Massari, A. Chiaravallotti, L. Boi, G. Simonetti; Rome/IT (boiluca.md@gmail.com)

Purpose: Acute stroke due to internal carotid artery occlusion has a poor prognosis. Clinical outcome is related to efficacy and timing of vessel recanalisation. We report our single center experience focusing on the adequate pre-procedural and post-procedural management.

Methods and Materials: We retrospectively reviewed 15 consecutive endovascular cases of acute internal carotid artery occlusions treated at our center between July 2009 and November 2009. Preprocedural symptoms were evaluated through NIHSS. Mechanical thrombolysis was perfomed through Penumbra system. Adjunctive intra-arterial pharmacological therapy (urokinase) was performed only if time to stroke was inferior to 6 hours. If thrombosis was associated with a significant vessel stenosis, a stent was deployed. All patients underwent post-procedural neurologic examinations and angio-MR performed at 3 and 6 months after the procedure.

Results: Penumbra mechanical thrombolysis was performed in all cases. $86.6 \%$ of the treated vessels were revascularised to TIMI 2 of 3 . Intra-arterial thrombolysis was associated with mechanical approach in 13 patients $(86.6 \%)$. One patient died after the procedure (7\%). In 5 patients $(33.3 \%$ ) a carotid stenting procedure was necessary. In 4 patients $(26.6 \%)$ stenting of the middle cerebral artery was perfomed. In the other patients we observed a significant improvement of symptoms (mean baseline NIHSS $19.5 \pm 0.3$; mean postprocedural NIHSS $3.5 \pm 0.2, p<0.0001$ ). Conclusion: Mechanical thrombolysis associated with intra-arterial pharmacological therapy and stenting is an efficient treatment for acute stroke.

10:30 - 12:00 Room P

Vascular

\section{SS 815}

\section{Vessel wall, plaque, diabetes}

Moderators:

I.E. Chemelli-Steingruber; Innsbruck/AT

A. Cieszanowski; Warsaw/PL

\section{B-461 10:30}

Dual source CT angiographic evaluation of coronary disease in symptomatic type 2 diabetic patients

Z.-G. Chu; Chengdu/CN (chuzg0815@yahoo.cn)

Purpose: Coronary artery disease (CAD) is a common and severe complication of type 2 diabetes mellitus (DM). We aim to identify the features of CAD in diabetic patients using dual source CT angiography (DSCTA).

Methods and Materials: During the period from July. $1^{\text {st }}, 2009$ to Mar. $20^{\text {th }}, 2010$ 113 consecutive patients (70 males, 43 females; mean age, $68 \pm 10$ years) with type $2 \mathrm{DM}$ were found with coronary plaques by performing coronary DSCTA and enrolled into this study. Their CT data were reviewed, and the extent, distribution and types of plaque as well as luminal narrowing were evaluated.

Results: In total, $287(2.5 \pm 1.1$ per patient) coronary vessels and $470(4.2 \pm 2.8$ per patient) segments were found with plaques, respectively. Multivessel disease was more common than single vessel disease $(p<0.001)$, and the left anterior descending (LAD) (35.8\%) and the sixth segment (19.1\%) was the most frequently involved vessel and segment (each $p<0.001$ ), respectively. The calcified plaque $(48.8 \%)$ was the most common type of plaque $(p<0.001)$, followed by mixed plaque $(38.1 \%)$. To different degrees of luminal narrowing, mild narrowing $(36.9 \%)$ was more common than others $(p<0.001)$, but the significant difference between nonobstructive stenosis and obstructive stenosis was not observed $(50.4 \%$ vs. $49.6 \%, p=0.855$ ) 
Conclusion: Coronary DSCTA detected a high plaque burden in patients with type $2 \mathrm{DM}$. Plaques were mainly detected in the proximal segments of LAD. More calcified and less non-calcified plaques were observed. More attention should be paid to the significant prevalence of obstructive stenosis.

\section{B-462 10:39}

(EPOS) Improved function of circulating angiogenic cells (CACs) is evident at US in islet-transplanted patients (ITA) affected by type 1 diabetes (T1D) M. Cellina, M. Venturini, P. Maffi, A. Petrelli, P. Fiorina, C. Soldati, A. Palmisano, A. Secchi, A. Del Maschio; Milan/IT (corrado.soldati@hsr.it)

Purpose: A reversal of endothelial dysfunction in T1D was previously described after pancreas transplant. CACs are vascular-committed bone-marrow-derived-cells expressing endothelial- and stem-cell markers, dysfunctional in T1D. We studied if restoration of endogenous-beta-cell-function with transplantation is associated with better CACs-function.

Methods and Materials: We studied with US the right brachial arteries of 14 insulin-independent- ITA, 18 T1D untransplanted patients and 14 controls. Endothelial-dependent-dilation (EED) was ultrasonically assessed by antero-posterior diameter increase of the right brachial artery during a post ischaemic hyperaemia, while endothelial-independent-dilation was studied by nitrate-induced vasodilation (NDD) as control. An occluding forearm cuff placed $5 \mathrm{~cm}$ below the antecubital fossa was inflated to $50 \mathrm{mmHg}$ above systolic pressure for 5 minutes, then released to induce reactive local hyperaemia. Recordings were made 60 seconds after onset of hyperaemia and 3 minutes after sublingual administration of nitro-glycerine spray. The parameters of endothelial-function were correlated with $\mathrm{Hba1c}$.

Results: T1D showed a lower EED compared with healthy controls (T1D = $4.1 \pm 1.4 \%$ vs. $C=8.6 \pm 0.6 \%, p=0.0001)$. Interestingly, ITA showed a normal EED being statistically higher compared with T1D group (ITA $=16.4 \pm 2.3 \%, p=0.0007$ vs. T1D). EED negatively correlated with $\mathrm{HbA1c}(R=-0.40, \mathrm{p}=0.01)$. No differences in NDD were found in the three groups studied ( $T 1 D=17.3 \pm 3.0 \%, C=23.6 \pm 4.5 \%$ and ITA $=19.2 \pm 3.5 \%$; $n$ s)

Conclusion: Diabetic patients showed a lower percentage of EDD compared with healty controls, while after islet-transplantation a fully recovery of endothelial function is evident. Instead, no differences in NDD were found in three groups studied, confirming the hypotesis that the defect of CACs may reflect into a macroscopic alteration of endothelial function in patients.

\section{B-464 10:48}

Comparison of symptomatic and asymptomatic atherosclerotic carotid plaques using parallel imaging and $3 \mathrm{~T}$ high-resolution in vivo MR imaging J.M. Grimm ${ }^{1}$, A. Schindler ${ }^{1}$, C. Schmidt ${ }^{1}$, M. Dichgans ${ }^{1}$, C. Yuan², C.C. Cyran ${ }^{1}$, M.F. Reiser ${ }^{1}$, K. Nikolaou 1 , T. Saam $1 ;{ }^{1}$ Munich/DE, ${ }^{2}$ Seattle, WA/US (jochen.grimm@med.uni-muenchen.de)

Purpose: To determine if high-resolution 3 T magnetic resonance imaging (hr-MRI) can depict differences between symptomatic and asymptomatic atherosclerotic carotid plaques.

Methods and Materials: In this prospective monocentric observational study, 31 patients ( 7 females; $73 \pm 8.3$ years) with symptomatic carotid disease defined as ischaemic brain lesions in one carotid artery territory on diffusion weighted images underwent a carotid black-blood hr-MRI at 3.0-Tesla with fat-saturated pre- and post-contrast T1w-, PDw-, T2w- and TOF images using surface coils and parallel Imaging techniques (PAT factor $=2$ ) within 7 days after symptom onset. All patients underwent extensive clinical workup (lab, brain MRI, duplex sonography, 24-hour ECG, transoesophageal echocardiography) to exclude other causes of ischaemic stroke. Prevalence of American Heart Association lesion type VI (AHA-LT6) and thrombus, status of the fibrous cap, presence and type of haemorrhage were determined in both carotid arteries based on previously published MRI criteria by one reviewer who was blinded to the clinical information. The Mc Nemar Test was used for statistical comparison. A p-value $<0.05$ was considered statistically significant. Results: Symptomatic plaques had, compared to asymptomatic plaques, a higher prevalence of AHA-LT6 (65.5\% vs. $10.3 \%$; P < 0.001$)$, ruptured/ulcerated fibrous caps $(34.5 \%$ vs. $3.5 \%$; $P=0.012)$, plaque haemorrhage $(58.6 \%$ vs. $10.3 \% ; P=$ $0.001)$, early subacute haemorrhage $(27.6 \%$ vs. $0 \% ; P=0.008)$ and juxtaluminal thrombus ( $10.3 \%$ vs. $0 \%$; $=$ n.s)

Conclusion: $3 \mathrm{~T} \mathrm{hr-MRI}$ is able to differentiate between symptomatic and asymptomatic carotid plaques, demonstrating the high potential of hr-MRI to differentiate between stable and vulnerable lesions and ultimately identify patients with low versus high risk for cardiovascular complications.

\author{
B-466 10:57 \\ The effect of cervical spondylosis on vertebral arterial flow and its \\ association with hearing impairment \\ O. Kiritsi ${ }^{1}$, G. Mlkroulis ${ }^{2}$, G. Noussios ${ }^{3}$, K. Tsitas ${ }^{4} ;{ }^{1}$ Kozani/GR, ${ }^{2}$ Thessaloniki/GR \\ ${ }^{3}$ Serres/GR, ${ }^{4}$ Katerini/GR (kirolga@hotmail.com)
}

Purpose: The aim of the present study was to evaluate the effect of cervical spondylosis on vertebral arterial flow and its association with hearing impairment. Methods and Materials: From April 2004 till February 2006 a prospective horizontal trial was performed. 56 individuals [mean age $53 \pm 14$ years] with a clinical diagnosis of cervical spondylosis were enrolled in the trial. A clinical investigation, including history, physical examination, $x$-rays and colour Doppler ultrasound, was carried out initially. 56 healthy individuals [mean age $29.34 \pm 6.98$ years] were used as control. Results: Sixteen controls and 3 group B participants dropped out before the radiological investigation was completed. Of the 53 participants 28 [52\%] had hearing impairment and consisted group A. The rest 25/53 [48\%] subjects scored 0 to the aforementioned test and formed group B. Group A demonstrated a total degeneration score of 227 , while group $B$ and controls showed a total score of 128 and 3 , respectively. In group A, mean peak systolic velocity, mean end diastolic velocity and mean resistance index on the right was significantly lower compared to mean values in subjects without hearing disorders and healthy individuals. Pearson product-moment correlation demonstrated strong correlation between cervical spine spondylosis and vertebral artery flow parameters. Both the Mann-Whitney $\mathrm{U}$ test and the Pearson product-moment correlation coefficient depicted strong correlation between total degeneration score and hearing impairment in group $A$. Conclusion: In conclusion, a strong relationship was depicted between cervival spine spondylosis and vertebral artery flow parameters or hearing impairment.

\section{B-467 11:06}

Evaluation and comparison of vascular ${ }^{18} \mathrm{~F}$-sodium fluoride uptake and arterial wall calcifications using PET/CT

P. Bannas, T. Derlin, S. Klutmann, J. Mester, G. Adam, M. Clausen, C. Weber; Hamburg/DE

Purpose: Calcification is a prominent feature of atherosclerotic plaques. Currently, F-18-sodium fluoride is extensively used for imaging of bone remodelling. There are no data concerning its potential for characterisation of atherosclerotic plaques. In this study the prevalence and distribution of vascular F-18-sodium fluoride uptake and arterial calcification in major arteries were examined with combined PET/CT. In addition, the topographic relationship between increased radiotracer accumulation and calcification was correlated.

Methods and Materials: Image data of 75 patients (48 women, 27 men, age $65.2 \pm 12.4$ y) undergoing whole-body F-18-sodium fluoride PET/CT for assessment of bone metastases were evaluated retrospectively. F-18-sodium fluoride uptake and arterial wall calcifications in major arteries (left/right common carotid arteries, thoracic aorta, abdominal aorta, left/right common iliac arteries, and left/righ femoral arteries) were analysed qualitatively and semiquantitatively, and compared. Results: F-18-Sodium fluoride uptake was observed at 254 sites in 57 (76\%) of the 75 study patients, calcification at 1930 sites in 63 (84\%) patients throughout all major arteries. The localisation was consistent with established atherosclerotic topography, e.g. increased uptake predominantly in the thoracic and abdominal aorta, and in the femoral arteries. The mean maximum standardised uptake value (SUVmax) of lesions showing regional uptake was 1.9 (range 0.8-3.5). Colocalisation of radiotracer accumulation and calcification could be observed in $223(88 \%)$ uptake areas. However, only these $223(12 \%)$ arterial calcification sites showed increased F-18-sodium fluoride uptake.

Conclusion: Our data indicate for the first time the feasibility of F-18-sodium fluoride for imaging of calcified atherosclerotic lesions in humans.

\section{B-468 11:15}

Relationship between progression of arterial stiffness and atherosclerotic burden over time

S. Oberoi, J.W. Nance, M. Meyer, J.M. Barraza, U.J. Schoepf;

Charleston, SC/US (nancej@musc.edu)

Purpose: Both arterial stiffness and atherosclerosis are independent predictors of adverse cardiovascular events. They have also been shown to be independently associated with each other in cross-sectional studies, but the nature of the relationship is vague. The purpose of this study was to examine changes in arterial stiffness and atherosclerosis over time to determine if there is parallel progression of disease. Methods and Materials: 164 patients with suspected coronary artery disease underwent 2 coronary computed tomography (CCTA) scans an average of $12 \pm 10$ months apart. Arterial stiffness was represented by the aortic distensibility index 
(ADI) and atherosclerosis by segment involvement score (SIS, defined as number of coronary artery segments per patient displaying atherosclerotic plaque). Changes in ADI and SIS between the scans were compared using linear and logistic regression accounting for differences in clinical and demographic baseline characteristics. Results: Age $(p=0.004)$, time between scans $(p=0.02)$, and an increase in SIS $(p<0.001)$ were associated with a decrease in ADI on univariate analysis. Increases in SIS remained a significant independent predictor of decreased ADI on both multivariate logistic regression (with change in ADI represented categorically) and multivariate linear regression (both $p<0.001$ ).

Conclusion: Progression of aortic stiffness and progression of atherosclerosis have a temporal relationship. While outcome studies are needed, a comprehensive assessment of vascular health with CCTA, including both indices of arterial stiffness and atherosclerotic burden, may improve risk stratification and therapy monitoring.

\section{B-469 11:24}

\section{Use of 3D rather than 2D ultrasound results in more accurate} determination of both endothelial and vascular function L. Kavanagh, C. Brown, P. Fitzgerald, M. Lee, A. Stanton; Dublin/IE (liamkavanagh@rcsi.ie)

Purpose: Brachial artery flow-mediated dilatation (FMD) and nitrate-induced dilatation (NID), using 2D ultrasound, are non-invasive methods for assessing endothelial and vascular function. However, operator-dependence and poor reproducibility have limited the use of these assessments to research rather than clinical practice. We tested whether the use of 3D ultrasound could diminish operator dependence, and improve validity and reproducibility.

Methods and Materials: 10 fasting males (18-80 years) attended the clinic twice. On each occasion the brachial artery ( $5 \mathrm{~cm}$ above the elbow) was imaged pre- and post-ischaemia (FMD), and pre- and post-sublingual glyceryl-tri-nitrate (NID), using a Phillips iU22 ultrasound system with L17-5 (2D) and VL13-5 (3D) probes. 2D cineloops and 3D volume sets were ECG-gated. Diastolic diameters (median of 3 R-wave images) were measured offline using AMS semi-automated edge-detection software. FMD and NID were calculated as the \% changes in diastolic diameter with ischaemia and nitrates, respectively.

Results: The use of 3D ultrasonic imaging resulted in improved reproducibility by comparison with $2 \mathrm{D}$ imaging - mean difference \pm SD of differences between repeated measures for each of the assessments were as follows; 3D-FMD $0 \% \pm 2.3 \%$ versus $2 \mathrm{D}-\mathrm{FMD} 1.5 \% \pm 5.9 \%$; 3D-NID $0.7 \% \pm 6.9 \%$ versus $2 \mathrm{D}-\mathrm{NID} 2.7 \% \pm 10.6 \%$. Conclusion: Most likely due to avoidance of probe mal-alignment errors, use of $3 \mathrm{D}$ ultrasonic imaging rather than $2 \mathrm{D}$ results in more accurate determination of both endothelial and vascular function. This improved accuracy and operator independence is likely to facilitate more widespread clinical usage in patients at risk of cardiovascular events.

\section{B-470 11:33}

Study of total aorta and coronary arteries with prospective ECG-triggered 320-MDCT angiography

Y. Li., Z. Fan, Z. Zhang; Beijing/CN (athen06@hotmail.com)

Purpose: To investigate the feasibility of prospective ECG-gating wide-volume protocol in CT angiography of whole aorta and coronary arteries

Methods and Materials: Consecutively 55 patients suspected with acute aortic syndrome (AAS) underwent total aorta angiography with prospective ECG-gating wide-volume without heart rate control. The exposure window was set at $40-50 \%$ $\mathrm{R}-\mathrm{R}$ interval (heart $\geq 70 \mathrm{bpm}$ ) or $70-80 \% \mathrm{R}-\mathrm{R}$ interval (heart $<70 \mathrm{bpm}$ ) within one forced heart beat. The parameters for the acquisition were $0.35 \mathrm{~s} / \mathrm{r}, 120 \mathrm{kV}$, $450 \mathrm{mAs}$. The imaging quality of ascending aorta, aortic valve, and coronary artery was evaluated for motion artifact. The mean attenuation was measured in the ascending aorta, middle descending aorta and distal descending aorta. The radiation dose and contrast medium were recorded.

Results: All of the examinations were performed successfully. The stair artefact could be found at thoracic part in patients with arrhythmia (16.4\%). The imaging qualities were acceptable for evaluating the ascending aorta $(100 \%)$, aortic valve $(94.5 \%)$ and coronary arteries segments $(97 \%)$. The mean attenuations were higher than $315 \mathrm{Hu}$ at every segment of the aorta. The mean radiation dose was $20 \pm 4.7 \mathrm{mSv}$. The average agent was $88.2 \pm 5 \mathrm{ml}$. Of the 55 patients ( 48 patients with aortic diseases, 3 paitents with aortic valve calcification), $21.8 \%$ patients had coronary artery diseases. In 2 patients with type A aortic dissection, right coronary artery came from false lumen.

Conclusion: For patients suspected with AAS, prospective ECG-gating widevolume CTA of whole aorta has the potential to provide additional information about the coronary artery and aortic valve with low radiation exposure.
10:30 - 12:00

Room Q

Paediatric

\section{SS 812}

\section{Neuroradiology: pre- and postnatal studies}

Moderators:

A. Borthne; Lorenskog/NO

L. Tzarouchi; loannina/GR

B-471 10:30

Posterior fossa measurements in open and closed neural tube defects on foetal MRI

R. Woitek, G. Kasprian, M. Weber, P.C. Brugger, T. Czech, D. Bettelheim,

D. Prayer; Vienna/AT (ramona.woitek@meduniwien.ac.at)

Purpose: Differentiation between open and closed neural tube defects (ONTDs, CNTDs) on foetal MRI is crucial due to differences in prognosis. This study aims to quantify posterior fossa abnormalities in dependency of the presence of ONTD or CNTD, and to provide further diagnostic and potentially prognostic parameters. Methods and Materials: On orthogonal T2-weighted sequences, acquired during clinically indicated MR examinations (1.5 Tesla) of foetuses without spinal dysraphism $(n=81)$, with ONTDs ( $n=18 ; 21$ examinations) and CNTDs ( $n=6 ; 9$ examinations), the clivus-occiput angle $(\alpha)$ and the maximum transverse diameter of the posterior fossa $\left(\mathrm{DM}_{\mathrm{PF}}\right)$ were measured. For statistical camparison of foetuses with normal brain development with foetuses with spinal dysraphisms foetuses were age and sex matched.

Results: In foetuses without NTDs a significant positive correlation between gestational age and $\alpha(r=.952 ; p<.001)$ as well as $\mathrm{DM}_{\mathrm{PF}}(r=.601 ; p<001)$ was found. In foetuses without spinal dysraphism $\alpha$ was significantly different from foetuses with ONTDs $(p<.001)$ but not with CNTDs. $\alpha$ was also significantly different between fetuses with ONTDs and CNTDs $(p=.029)$. DM $M_{P F}$ of foetuses without spinal dysraphism was significantly different from $D_{\mathrm{PF}}$ of foetuses with ONTDs or CNTDs (both $\mathrm{p}<.001$ ). Between foetuses with ONTDs and CNTDs no significant difference in $\mathrm{DM}_{\mathrm{PF}}$ was found $(\mathrm{p}=.618)$.

Conclusion: The morphology and size of the posterior fossa and especially the clivus-occiput angle $(\alpha)$ depend on the absence or presence of an ONTD or CNTD. In contrast to a published ultrasound study $\alpha$ does not remain constant during pregnancy but in our study correlates positively with gestational age.

\section{B-472 10:39}

\section{Early foetal MRI diagnosis of lissencephaly}

G. Kasprian, C. Mitter, P.C. Brugger, L. Perju-Dumbrava, M. Schmid, D. Prayer; Vienna/AT (gregor.kasprian@meduniwien.ac.at)

Purpose: So far the detection of abnormalities in ventricular size and shape are the most important sonographic clues in the early detection of developmental brain pathology. This study aims to analyse early foetal MR imaging signs in cases of lissencephaly.

Methods and Materials: 26 foetal MRI examinations ( 1.5 Tesla) of 19 cases (mean age at initial MRI: $25.5 \pm 4.3$ gestational weeks - GW) with clinical, neuroimaging or neuropathological evidence for lissencephaly were retrospectively analysed. In 7 cases foetal MR and in 3 cases postnatal MR follow-up examinations including $\mathrm{T} 1$, multiplanar T2-weighted and diffusion weighted sequences were available. Overall image quality was rated excellent $(54 \% ; 14 / 26)$, moderate $(38 \% ; 10 / 26)$ and poor $(8 \% ; 2 / 26)$.

Results: An average mismatch between the morphologically estimated and actual gestational age of $5.1 \pm 4.0 \mathrm{GW}$ could be detected. 9/10 cases imaged before 24 GW showed abnormal opercularisation. Overall the characteristic pattern of foetal brain asymmetry was present in one third of cases. Signs of abnormal gyration were detected in $7 / 19$ cases with 2/19 cases of Type 2 Lissencephaly showing an irregular cortical surface. The characteristic laminar organisation could not be discriminated in $60 \%$ of examinations on T2-w and in $40 \%$ of examinations on diffusion weighted sequences. Abnormally enlarged ganglionic eminence (6/19) and hippocampal malrotation (16/19) were commonly encountered. Infratentorial abnormalities included a wide posterior fossa (9/19), kinking of the brainstem (3/19) and an abnormally flat pons (9/19).

Conclusion: Foetal MR detection of a variety of structural brain abnormalities can strengthen the confidence in the early in utero MR diagnosis of lissencephaly. 


\author{
B-473 10:48 \\ Perinatal brain injury after hypoxia and resuscitation in piglets assessed \\ by dynamic FDG PET \\ C.E. de Lange, E. Malinen, H. Qu, K. Johnsrud, A. Skretting, O. Saugstad, \\ B. Munkeby; Oslo/NO (charlotte.de.lange@rr-research.no)
}

Purpose: Changes in cerebral glucose metabolism may be an early prognostic indicator of hypoxic ischaemic injury. The aim of this study was to use dynamic FDG-PET for evaluating changes in cerebral glucose metabolism immediately after global hypoxia and the influence of the oxygen content in the resuscitation air. Methods and Materials: 16 anaesthesized piglets were subjected to global hypoxia using $\mathrm{FiO}_{2} 0.08$ for 40 min followed by resuscitation with $\mathrm{FiO}_{2} 0.21$ or 1.0 for 30 minutes and reoxygenation. Dynamic PET was performed by injection of $18 \mathrm{~F}$ Fluoro-2deoxy-glucose before and after hypoxia+resuscitation. T2-weighted MR images were acquired for anatomic imaging and coregistered with the FDG-PET images. ROls were traced around the basal ganglia, cerebrum, cerebellum, cortex and white matter. Global and regional cerebral metabolic rates of glucose (CMRgl) before and after hypoxia+resuscitation was assessed by Patlak analysis for the two resuscitation groups. Statistical analysis was performed with paired $t$ test with a significance level of $p=.05$. Results: CMRgl was significantly reduced from a mean baseline level $( \pm 1 \mathrm{SD})$ of $21.3 \pm 1.9$ to $12.3 \pm 0.9 \mu \mathrm{mol} / \mathrm{min} / 100 \mathrm{~g}$ after hypoxia+resuscitation, for all piglets $(p<0.01)$. There was no significant difference between the different regions or between the 21 and $100 \%$ group, but a trend of less decrease in CMRgl in white matter was observed.

Conclusion: Global hypoxia causes immediate decrease of cerebral glucose metabolism in newborn pigs independent of resuscitation with 21 or $100 \%$ of $\mathrm{O}_{2}$ Early regional and global cell death or a downregulation of the glucose metabolism are likely mechanisms.

\section{B-475 10:57}

Brainstem dysgenesis: MR imaging findings in 24 patients

E. Vázquez, I. Delgado, A. Sanchez-Montañez, M. Flotats, G. Enriquez, M. Roig; Barcelona/ES (evazquez@vhebron.net)

Purpose: Brainstem dysgenesis is a generic, umbrella, term that designates all patients with congenital dysfunction of cranial nerves and decreased muscle tone due to prenatal lesions or malformations of the brainstem. Cause is atributted to a vascular event occurring during early foetal life involving the developing brainstem tegmentum and provoking different clinical syndromes. A retrospective analysis of radiological-clinical findings in 24 children with brainstem dysgenesis is presented. Methods and Materials: A total of 24 patients with clinical brainstem dysgenesis were retrospectively reviewed. All patients were imaged at birth or shortly after and some of them were followed by MR imaging at older ages, including at that time DTI and tractography. Studies were performed using either a 1.5 or a 3.0-Tesla MRI scanner (Magnetom Avanto and Trio; Siemens, Erlangen, Germany). Images were reviewed independently by two paediatric neuroradiologists and findings were agreed on by consensus.

Results: Brainstem tegmental hypoplasia was found in 11 of our 24 cases, being classified as symmetrical $(n=10)$, and asymmetrical $(n=1)$. One patient showed a tiny calcification within the pons on cranial CT. Supratentorial anomalies were found in 4 patients, mainly polymicrogyria (3) and ventriculomegaly (1). Miscellaneous imaging findings were agenesis/hypoplasia of cranial nerves, better depicted on CISS images. DTIFT demonstrated additional findings beyond those seen with conventional MR imaging. Conclusion: Traditional MRI may be insufficient to evaluate brainstem hypoplasia or cranial nerves absence-hypoplasia, particularly during neonatal period. Modern sequences such as CISS or DTI-FT may depict greater detail providing important information in children with braistem dysgenesis.

\section{B-476 11:06}

\section{Spatial dependency of phase shift in the cerebral venous system of} neonates using susceptibility-weighted magnetic resonance imaging T. Niwa ${ }^{1}$, N. Aida' ${ }^{1}$ H. Kawaguchi ${ }^{2}$, T. Obata ${ }^{2}$, Y. Tachibana ${ }^{1}$, R. Watanabe ${ }^{1}$, J. Shibasaki'; ${ }^{1}$ Yokohama/JP, ${ }^{2}$ Chiba/JP (tniwa@kcmc.jp)

Purpose: Phase analysis on susceptibility-weighted imaging (SWI) has been introduced to be related to blood oxygenation. The purpose of this study was to assess the spatial distribution of phase shift (PS) in the neonatal cerebral venous system using phase image on SWI.

Methods and Materials: Thirty-five neonates (gestational age, range 24-40 weeks; corrected age, range 36-44 weeks), who had undergone SWI without intracranial abnormal findings or systemic abnormality, were retrospectively assessed. Veins were masked out on the phase image using custom software. PS in the vein at the deep gray matter as well as cortical veins at the rolandic area, frontal and parietal lobe was measured. The differences in PS in each area were assessed on each neonate. Ratios of the PS in the deep grey matter to PS in the cortical veins were compared with the corrected age, gestational age and age after birth.

Results: There was a significant difference in PS among the regions $(p<0.01)$ pairwise comparison revealed that PS at the deep grey matter was significantly larger than that in the cortical veins $(p<0.01)$; PS in the rolandic area was significantly larger than that in the frontal or parietal area $(p<0.01)$. There was a negative correlation between the phase ratio and the corrected age $(p<0.01)$, but not correlated with gestational age and age after birth.

Conclusion: A characteristic distribution of PS was identified in the neonatal venous system. The reduction of difference in PS in the vein at deep grey matter and cortex may reflect brain development.

\section{B-477 11:15}

Spontaneous modifications of contrast enhancement in childhood pilocytic astrocytomas: a new MRI sign?

R. Calandrelli, S. Gaudino, G.M. Di Lella, F.R. Quaglio, A. Costantini, L. Bonomo, C. Colosimo; Rome/lT (rocalan1@virgilio.it)

Purpose: MRI is the gold standard in the diagnosis and follow-up of pilocytic astrocytoma (PA). PA may appear as a cystic lesion with mural nodule, solid with intrinsic cysts or purely solid lesion; the solid part may enhance in a homogeneous or a heterogeneous fashion. Assessment of contrast enhancement (CE) pattern is essential for the diagnosis and follow-up. Sometime PA may mimic high grade tumour, and biopsy may not be always possible. Our purpose was to evaluate CE changes of PA with serial MR, and to assess these CE "fluctuations" as a possible new MRI sign for PA. Methods and Materials: Retrospective evaluation of 912 MR exams of 140 pts (with histologically proven PA) followed for an average of $4.5 \mathrm{ys}$ with serial MRI. We selected 39 pts: off therapy, without NF1, without dimensional changes of tumour/ residual tumour. In pts with CE changes, tumour size and areas of CE were calculated with a cross-product. Descriptive statistics were calculated for continuous variables, effects of possible factors influencing changes of $C E$ areas were tested. Results: Of the 39 pts without dimensional changes in tumour size, 12/39 showed CE changes in terms of increase/appear and/or decrease/disappear of the CE. 3/12 PA were infratentorial, $8 / 12$ supratentorial and $1 / 12$ endoventricular. There were no significant correlation between age, gender, tumour localisation, tumour size and CE areas. Conclusion: In our experience PA may show a variable CE during the years, in the absence of tumour/residual tumour dimensions change. CE fluctuation can be considered a MRI sign of PA, but not considered as an indicator of tumou progression or tumour response.

\section{B-478 11:24}

Functional significance of the left arcuate fasciculus with respect to language development in paediatric epilepsy patients with malformations of cortical development

M.J. Paldino, K.M. Rodrigues, A. Poduri, R. Pienaar, P.E. Grant; Boston, MA/US (michael.paldino@gmail.com)

Purpose: To evaluate the significance of absence of the left arcuate fasciculus $(\mathrm{AF})$ with respect to language development in a cohort of paediatric patients with malformations of cortical development (MCD)

Methods and Materials: Patients were retrospectively identified with 1. MCD. 2 30-direction diffusion tensor imaging (DTI) at 3 Tesla. 3. Language characterized by paediatric neurologist. DTI was used for deterministic tract reconstruction. AF was categorized as left-only, right-only or bilateral. Language was categorized as intact (age-appropriate), mild-to-moderate impairment (some development, but delayed compared to piers) or profound impairment (non-verbal). Proportions were compared using Fisher exact test.

Results: 30 patients met the criteria: 10 with polymicrogyria, 14 with focal cortical dysplasia (pathologically-proven), 1 lissencephaly, 4 schizencephaly, and 1 grey-matter heterotopia. 15 patients had intact language, 11 mild-to-moderate impairment and 4 profound impairment. 100\% (10/10) of patients without a left AF had some degree of language impairment ( 6 mild-to-moderate, 4 profound); $25 \%$ $(5 / 20)$ of patients with a left AF were impaired ( 5 mild-to-moderate). This difference was significant $(p<0.0001) .100 \%(15 / 15)$ of patients with intact language had an identifiable left AF. The frequency of absence of the left AF in patients with intact language was significantly less than that in patients with some degree of impairment $(p<0.0001)$. Absence of the left AF had $100 \%$ specificity $[95 \% \mathrm{Cl}(75 \%, 100 \%)]$, $67 \%$ sensitivity and $100 \%$ positive predictive value $[95 \% \mathrm{Cl}(68 \%, 100 \%)]$ for some degree of language impairment.

Conclusion: These preliminary results suggest that absence of the left AF may be a specific biomarker of language impairment in patients with MCDs. 
B-479 11:33

Ultrasound of brachial plexus birth injury in infants: experience in $\mathbf{8 0}$ patients

M. Valle, F. Senes, N. Catena, F. Maiuri, C. Martinoli; Genoa/IT

(maura.valle@libero.it)

Purpose: To describe the diagnostic performance of ultrasound (US) in infants with a brachial plexus birth injury.

Methods and Materials: From January 2002 to August 2010, a series of consecutive $n=80$ children (mean age 2.3 months; 54 males) with obstetrical palsy were examined with high-frequency $17-5 \mathrm{MHz}$ US. All patients underwent cervical and brachial plexus MR imaging and MR myelography within one week of the US study. Then, 53/80 (66\%) patients had surgery and underwent intraoperative electrophysiological studies. Analysis of US findings included exiting nerve root injuries with or without pseudomeningoceles and posttraumatic neuromas.

Results: In patients who underwent surgery, the sensitivity and specificity of the US findings of posttraumatic neuromas in the interscalenic area were $89 \%$ and $95 \%$ respectively, using surgical findings and somatosensory-evoked potentials as the standard of reference. Dividing the brachial plexus in an upper and lower part, US was able to establish the level of injury in $70 \%$ of cases. Paraforaminal nerve root injuries and pseudomeningoceles were identified with US in only $12 \%$ and $8 \%$ of surgically-proven root avulsions. In the group of infants who were managed conservatively, US was $92 \%$ sensitive and $100 \%$ specific to detect neuromas using MR imaging as the standard of reference. In this group, posttraumatic neuromas were smaller in size $(p<.0001)$ or absent ( 9 cases).

Conclusion: US is promising for imaging brachial plexus birth injuries. The information provided has a prognostic value and is useful for the surgeon in a preoperative setting.

\section{B-480 11:42}

Why does this happen? The use of post-mortem radiological imaging in unclear child death

K. Glutig, U. Schmidt, C. Erfurt, G. Hahn; Dresden/DE

(katja.glutig@uniklinikum-dresden.de)

Purpose: The aim of our study consisted in describing the need for a standardised imaging before autopsy in the case of an unclear child death. Can combination with a traditional radiographic skeletal overview imaging, multislice computertomography (MSCT) and magnetic resonance imaging (MRI) receive additional important information of the death circumstances of the child to the legal doctor?

Methods and Materials: In the period between March 2005 until September 201038 dead children were examined. $37 \mathrm{x}$-ray skeleton overview admissions, 34 MS-CT and in five cases MRI of brain and spine were carried out. Retrospectively all investigations were evaluated by two experienced child radiologists regardless of each other. Results: In the skeletal overview admissions show pathological findings in 6 cases (15.8\%): for example, fractures of rib and skull and two children had no air in the lung and in the stomach. In the MSCT 18 cases showed a pathology (33.3\%): signs for infection, pneumonia, otitis media, pulmonary interstitial emphysema (PIE), fracture, bleeding. Remarkable intracranial and intraspinal bleeding findings could be proved in the MRT (7.9\%).

Conclusion: In case of an unclear child death skeletal overview x-ray imaging in combination with MSCT and MRI before the legal-medical autopsy can give enhanced diagnostic information. MSCT can illustrate whether the child was liveborn. The detection of pulmonary interstitial emphysema (PIE) proving suffocation as a cause of neonate death. 


\section{Sunday, March 6}




\section{Abdominal Viscera}

\section{SS 1201a}

\section{Diffusion-weighted MRI: advances and applications}

Moderators:

S. Gourtsoyianni; Iraklion/GR

B.J. Op de Beeck; Antwerp/BE

\section{B-481 10:30}

Which b-values should I use for ADC calculation in the liver?

P. Summers ${ }^{1}$, G. Petralia' ${ }^{1}$ L. Bonello ${ }^{1}$, M. Pasin ${ }^{1}$, R. Di Filippi ${ }^{1}$, L. Antiga ${ }^{2}$,

A. Caroli ${ }^{2}$, M. Bellomi'; ${ }^{1}$ Milan/IT, ${ }^{2}$ Ranica/IT (paul.summers@ieo.it)

Purpose: To systematically investigate the impact of b-values on inter- and intrasubject repeatability of ADC measurement in healthy liver.

Methods and Materials: Ten healthy volunteers (age: $33 \pm 11$ years, $2 \mathrm{~F}$ ) underwent repeated DW-MRI during gentle free breathing at $1.5 \mathrm{~T}$, with b-values: $0,50,100$, $200,400,500,700,900,1000 \mathrm{~s} / \mathrm{mm}^{2}$ (30 slices, 340 × $280 \mathrm{~mm}, 256$ x 192 matrix, thickness/gap 7/1 mm, TE/TR, 71/3200 ms, 4 averages and fatsat) for three diffusion encoding directions. ADC maps were obtained by fitting a monoexponential model to the individual voxel decay data including all nine b-values, and repeating the calculation while progressively leaving out the lowest remaining $b$-value until only the $b=900$ and $b=1000$ images remained. Whole liver ROls drawn to avoid major vessel and bile ducts on the $b=0$ image were thresholded to eliminate remaining visible bile ducts and vessels. Mean and variance in the ADC values for each ROI were examined across calculated maps.

Results: With minimum b-values of 400 or 500 , liver ADC values were lowest $(0.884$ and $0.885 \mathrm{~mm}^{2} / \mathrm{s}$ respectively), suggestive that flow contamination was minimised. Including b-values 0 or 50 , or using only b-values 900 and 1000 led to significantly higher ADC values $(p<0.02)$ and greater absolute variance. Intra-subject standard deviation averaged about one-third the observed inter-subject variation.

Conclusion: Our results indicate that $b=0$ and 50 images should be excluded from calculation of ADC. Eliminating visible vessels and bile ducts by thresholding the $b$ $=0$ image likely explains the low ADCs we observed relative to prior publications.

\section{B-482 10:39}

MR diffusion-weighted imaging (DWI) of the healthy liver parenchyma: repeatability and reproducibility of apparent diffusion coefficient (ADC) measurements by intraclass correlation coefficient (ICC)

F. Pasquinelli, G. Virgili, L.N. Mazzoni, G. Belli, F. Regini, C. Nardi,

S. Colagrande; Florence/IT (filippopasquinelli@yahoo.it)

Purpose: To evaluate repeatability and reproducibility of 4 different ADC sampling methods in the healthy liver parenchyma.

Methods and Materials: DWI breath-old echo-planar acquisitions $(b=0-300-1000$ $\mathrm{s} / \mathrm{mm}^{2}$ ) were executed on a $1.5 \mathrm{~T}$ MR scanner. Two observers evaluated 30 healthy subjects (age range 18-> 65 years) DWI images, with 4 different sampling methods: one region of interest (ROI) per section (OR), one $\mathrm{ROI}$ per segment (OS), partial liver volume (PV), total volume (TV), considering, respectively $4 \%, 4 \%, 30 \%$ and $70 \%$ of the entire liver volume parenchyma. Data were reported per group (mean $\pm S D$ ). Statistical evaluation was done by ANOVA test.

Results: ADC $\left(10^{-6} \mathrm{~mm}^{2} / \mathrm{s}\right)$ mean increases, enlarging ROI size: 1343 $\pm 201(\mathrm{OR})$, $1373 \pm 221$ (OS), $1463 \pm 253$ (PV), 1560 \pm 211 (TV). Left liver lobe was difficult to be evaluated, given the presence of artefacts.

Repeatability was greater than reproducibility (ICC 0.80 vs $<0.45)$; both increase averaging 3 measurements of the lower-right liver lobe (ICC $\geq 0.87$ and 0.82 , respectively). Conclusion: Inter-observer variability was greater than intra-observer one. Reproducibility can be increased averaging measurements of the lower-right liver lobe, using a large-size ROI. ADC increment, observed using a large ROI, can be explained with the more pronounced contribution of perfusion and vessels.

\section{B-483 10:48}

Variation with age of the apparent diffusion coefficient (ADC) of the liver parenchyma: an in vivo versus in vitro study

L.N. Mazzoni, F. Pasquinelli, C. Nardi, G. Belli, F. Regini, S. Colagrande; Florence/lT (mazzoni@fi.infn.it)

Purpose: To evaluate mean and dispersion of liver ADC measurements varying the size of the region of interest $(\mathrm{ROI})$ and the age of the subjects at two different $\mathrm{b}$ values, comparing results with phantom measurements
Methods and Materials: Two observers evaluated 40 healthy subjects, divided in 4 age classes $(18-30,31-45,46-65,>65)$, comparing the results with phantom measurements. DWI breath-old echo-planar acquisitions $\left(b=0-300-1000 \mathrm{~s} / \mathrm{mm}^{2}\right)$ were executed on a 1.5 T MR scanner. Liver sampling methods: total volume (TV) one ROI per slice (OR), considering, respectively, $70 \%$ and $4 \%$ of the entire liver volume. The same sampling methods were applied on phantom measurements. Data were reported as mean $\pm S D$.

Results: Absence of significant ADC $\left(10^{-6} \mathrm{~mm}^{2} / \mathrm{s}\right)$ correlation with age. Wide data dispersion, more pronounced at $b=300$. In vivo the larger the ROI, the higher the ADC mean: $2563 \pm 572$ versus $1558 \pm 247$ (TV), $2412 \pm 512$ versus $1375 \pm 261(\mathrm{OR})$ respectively, $b=300$ versus $b=1000$. In vitro, no $A D C$ variation was osberved at different $b$ values.

Conclusion: Absence of ADC correlation with age. ADC increment with ROI size can be explained by the greater perfusion contribution. This hypothesis is confirmed by the absence of ADC increment, varying the ROI size, in phantom measurements.

\section{B-484 10:57}

Detection and characterisation of focal liver lesions: value of diffusionweighted MRI, gadoxetic acid-enhanced MRI and combination of both methods

K. Holzapfel, M. Eiber, A.A. Fingerle, E.J. Rummeny, J. Gaa; Munich/DE (holzapfel@roe.med.tum.de)

Purpose: To evaluate diffusion-weighted MRI (DWI) and gadoxetic acid (GdEOB-DTPA)-enhanced MRI in the detection and characterisation of focal liver lesions (FLL).

Methods and Materials: Forty-five patients with FLL underwent MRI (1.5 T). First respiratory-triggered DWI was performed (b: $50 / 300 / 600 \mathrm{~s} / \mathrm{mm}^{2}$ ) followed by Gd-EOB DTPA-enhanced MRI using a 3D T1-weighted volumetric interpolated breath-hold sequence including image acquisition during hepatocyte-selective phases 10 and $20 \mathrm{~min}$ after injection. Imaging results were correlated with histopathology and imaging follow-up. Two readers reviewed three image sets in consensus (DWI, Gd-EOBDTPA, and combined DWI/Gd-EOB-DTPA) to detect and characterise FLL. Readers' confidence for both detecting and characterising FLL was scored on an ordinal five-point scale. Accuracies of the three image sets for detecting and characterising FLL were evaluated using free-response receiver operating characteristic analysis. Results: A total of 146 FLL (39 metastases, 16 HCCs, 40 haemangiomas, 31 cysts, $15 \mathrm{FNHs}, 5$ adenomas) were analysed. For detection of FLL, there was no significant difference in accuracy between the image sets (reader-averaged figures of merit: 0.920 for DWI, 0.909 for Gd-EOB-DTPA, 0.928 for combined set). However, for lesions $\leq 10 \mathrm{~mm}$, the combined set (92.8\%) showed a significantly higher sensitivity than DWI $(83.2 \%, \mathrm{p}<0.05)$ and Gd-EOB-DTPA sets $(82.4 \%$, $\mathrm{p}<0.01)$. For characterising FLL, Gd-EOB-DTPA $(0.914, \mathrm{p}<0.05)$ and combined set $(0.923, p<0.01)$ were significantly superior to DWI $(0.852)$.

Conclusion: Combination of DWI and Gd-EOB-DTPA-enhanced MRI allows detecting FLL with high accuracy. Adding DWI to Gd-EOB-DTPA-enhanced MRI improves the sensitivity in detecting small lesions. For characterising FLL, GdEOB-DTPA-enhanced MRI is superior to DWI.

\section{B-485 11:06}

Diffusion weighted imaging (DWI): evaluation of apparent diffusion coefficient (ADC), diffusion coefficient (D) and perfusion fraction (f) in progressive diffuse liver disease

F. Pasquinelli, G. Belli, L.N. Mazzoni, F. Regini, C. Nardi, S. Colagrande; Florence/IT (filippopasquinelli@yahoo.it)

Purpose: To estimate the ADC, D and f variations in progressive chronic liver disease, in different evolution grading, in comparison with the healthy liver parenchyma. Methods and Materials: 27 subjects, divided into 4 groups (A-D), were evaluated with 1.5 T MR scanner: 7 healthy volounteers $(A), 6$ with fibrosis histologically determinated F0-F1-F2 (B), 6 F3-F4 Child A (C), 8 F4 Child B- (D). ADWI free-breath, EP multi-b acquisition (0-150-300-450-600-800-1000 s/mm²) was used. Two observers evaluated the liver parenchyma with large region of interest (right lobe). Data were reported per group (mean $\pm S D$ ). Statistical evaluation was done by ANOVA test. Results: ADC $\left(10^{-6} \mathrm{~mm}^{2} / \mathrm{s}\right)$ value decreases increasing the liver fibrosis $(p<0.059)$ : $1319 \pm 68$ vs $1205 \pm 162$ for group $A$ and $D$ respectively. $F$ presents the same behav iour $(p<0.01)$ : $0.181 \pm 0.048$ vs $0.098 \pm 0.027$ for group $A$ and $D$, respectivelly. $D(10$ ${ }^{-6} \mathrm{~mm}^{2} / \mathrm{s}$ ) measurements shows no significant variation: $1201 \pm 84$ versus $1199 \pm 92$, for group $A$ and $D$, respectivelly.

Conclusion: The ADC value decrease correlates to the f-reducing, during the progressive liver fibrosis, but not to $D$. This is probably due to the diminishing of perfusion in the cirrhotic liver parenchyma: the f parameter could be an important tool to evaluate the fibrosis grading 


\section{B-486 11:15}

Diffusion-weighted MR imaging after administration of different gadolinium contrast agents: is it still reliable?

S. Kinner, L. Umutlu, G. Antoch, T.C. Lauenstein; Essen/DE

(Sonja.Kinner@uni-due.de)

Purpose: Diffusion weighted imaging (DWI) is an emerging technique for abdominal MRI. DWI is usually performed before intravenous contrast injection. However, it may be necessary or time-saving to acquire DWI data after gadolinium administration. We aimed to compare DWI before and after the administration of different gadolinium compounds.

Methods and Materials: 15 patients with known $\mathrm{HCC}$ underwent liver MRI at $1.5 \mathrm{~T}$ (Magnetom Avanto, Siemens) including DWI on day 1 before and after administration of gadobutrol (Gadovist $\left.{ }^{\circledR}\right)$ and on day 2 after administration of EOB-gadoliniumDTPA (Primovist $\AA$ ). Signal-to-noise ratios (SNR) and contrast-to-noise ratios (CNR) of $\mathrm{HCC}$ lesions and liver parenchyma were determined for all acquired DWI data sets. Furthermore, ADC values were calculated and compared using a Wilcoxon test. A p-value $<0.05$ indicated statistical significance.

Results: There were no statistically significant differences regarding SNR precontrast (mean: 43.3) after gadobutrol (43.2) or after EOB-gadolinium-DTPA (46.5). Similarly, no significant differences were found for CNR (average values: 34.7 vs. 32.1 vs. 35.7) nor for ADC-values (1.5 vs. 1.4 vs. $1.5 \times 10^{-3}$ ).

Conclusion: There is no significant difference regarding DWI of HCC before and after intravenous injection of different gadolinium compounds. Hence, it is feasible to run DWI after gadolinium either as an alternative for unsuccessful pre-contrast DWI or as a gap filler to spare time in EOB-gadolinium-DTPA imaging.

\section{B-487 11.24}

Diffusion weighted imaging of liver lesions: is there an influence of Gd-EOBDTPA on lesion contrast and apparent diffusion coefficient (ADC) values?

P.A.T. Baltzer ${ }^{1}$, M. Benndorf ${ }^{1}$, J. Schelhorn ${ }^{2}$, M. Dietzel ${ }^{1}$, W.A. Kaiser ${ }^{1} ;{ }^{1}$ Jena/DE, ${ }^{2}$ Weimar/DE (pascal.baltzer@med.uni-jena.de)

Purpose: Gd-EOB-DTPA-enhanced liver MRI is widely used for detection and differentiation of focal liver lesions. Diffusion weighted imaging (DWI) including apparent diffusion coefficient (ADC) measurements is increasingly utilised as a fast and, with limitations, quantitative method for liver lesion characterisation. DW can be acquired before and after contrast agent injection. However, knowledge on Gd-EOB-DTPA-related influences on DWI of the liver is limited. Consequently, this single center prospective intraindividual comparison study was performed in order to systematically investigate this matter.

Methods and Materials: Consecutive patients referred to standardised liver MR (1.5 T, Gd-EOB-DTPA, $0.025 \mathrm{mmol} / \mathrm{kg}$ ) for screening of metastases over 13 months were investigated. All underwent a breathold DWI sequence before and after contrast agent application (EPI-DWI, TR/TE (eff): 2100/62 ms, b-values: 0/800 s/ $\mathrm{mm}^{2}$ ). Patients with treated liver lesions were excluded. Signal intensity of lesion, parenchyma and noise on DWI images as well as the ADC value was measured after identification by two observers in consensus using manually placed regions of interest. Reference standard was either histopathology or imaging follow-up determined separately by two radiologists. Data analysis included contrast-to-noise ratio (CNR) calculation and Wilcoxon signed-rank test.

Results: 50 malignant and 39 benign lesions were identified. Neither CNR nor $A D C$ values showed significant differences between pre- and postcontrast DWI. Both pre- and postcontrast $A D C$ values differed highly significant between benign and malignant lesions $(P<0.001)$

Conclusion: The present study did not identify a significant influence of GdEOB-DTPA on DWI of liver lesions. This allows for individual tailoring of imaging protocols to clinical needs.

\section{B-488 $11: 33$}

Value of respiratory-triggered echo-planar diffusion-weighted MR imaging in the differentation between benign and malignant focal liver lesions

M. Di Martino, M. Anzidei, R. Di Miscio, F. Zaccagna, C. Catalano,

R. Passariello; Rome/IT (dimase81_6@hotmail.com)

Purpose: To evaluate the usefullness of diffusion-weighted MR imaging (DWI) using a respiratory-triggered SSEPI sequence in the differentation between benign and malignant focal liver lesions.

Methods and Materials: 154 patients with 154 malignant and 120 benign focal liver lesions underwent MR imaging at 1.5T using a body-phased array coil. DWI was perfomed by a respiratory-triggered SSEPI sequence $(T R=2028, T E=76$, matrix $=192 \times 125$, slice thickness $5.00 \mathrm{~mm}$, GRAPPA, b-values 0, 50, 500, 800 $\left.\mathrm{sec} / \mathrm{mm}^{2}\right)$. ADC values were obtained. Imaging results were correlated with histo- pathologic findings and imaging follow-up. Mann-Whitney test and a ROC curve were used for statistical analysis.

Results: The mean ADC values $\left(x 10 \exp ^{-3} \mathrm{~mm}^{2} / \mathrm{sec}\right) \pm$ standard deviation were $1.77 \pm 0.57$ for haemangiomas, $1.25 \pm 0.28$ for adenomas; $1,257 \pm 21$ for CCC $0.98 \pm 0.21$ for HCCs, $1,276 \pm 0.299$ for shunts, $1.6 \pm 0.278$ for regenerative nodules, and 2,676 \pm 0.37 for cysts, $1,066 \pm 0.26$ for metastases. A good positive correlation was found between ADC value of liver lesions and reference standard (Spearman's rho $=.67$ ) with a significantly lower ADC value of malignant lesions compared to that of benign $(p<.0001) .89 \%$ of lesions were correctly classified as malignant and benign at a threshold ADC value of 1.18.It is not possible to establish a cut-off point due to the wide variability into the benign lesion group.

Conclusion: DWI using the respiratory-triggered SSEPI sequence could be usefu to differentiate benign from malignant focal liver lesions. ADC value of malignan lesions is significantly lower than that of benign; however, it is not possible to establish a cut-off point.

\section{B-489 11:42}

Diffusion-weighted MRI for assessment of colorectal metastases: could it substitute Gd-EOB-DTPA-enhanced MRI?

R. Cianci, A. Filippone, F. Sabatino, E. Pace, V. Bianco, A. Tartaro,

A.R. Cotroneo; Chieti/IT (r.cianci@rad.unich.it)

Purpose: To evaluate the role of diffusion-weighted imaging (DWI) as a substitute for Gd-EOB-DTPA-enhanced MRI in the assessment of colorectal hepatic metastases

Methods and Materials: We included 54 patients with $\leq 5$ surgically proven colorectal hepatic metastases, who underwent Gd-EOB-DTPA-enhanced MR examination including DWI $\left(b=0-500 \mathrm{~s} / \mathrm{mm}^{2}\right)$. All patients underwent liver resection after intraoperative ultrasound (IOUS) within two weeks from MRI. Two independent readers, with different experience in abdominal imaging, reviewed three image sets (DWI, Gd-EOBDTPA, DWI and Gd-EOB-DTPA) to detect hepatic metastases using a four-grade scale. ROC analysis was performed and sensitivity was calculated for each reader and each reading session, using IOUS and histologic findings as reference standard. Results: There were a total of 115 liver metastases (mean diameter $2.3 \mathrm{~cm}$, range 0.4-6.3 cm). The accuracy in detecting hepatic metastases was lowest for DWI alone (reader 1, 0.823; reader 2, 0.872), preceded by Gd-EOB-DTPA set (reader 1, 0.923; reader $2,0.895$ ) and combined set (reader 1, 0.985; reader 2, 0.994). The differences resulted statistically significant only between combined and DWI set $(p=0.034)$. For the less experienced reader, combined set showed a significantly higher sensitivity than DWI and Gd-EOB-DTPA set for lesions smaller than $1 \mathrm{~cm}$, whereas no significant differences were observed for lesions bigger than $1 \mathrm{~cm}$ and for the experienced reader. Conclusion: DWI can not substitute Gd-EOB-DTPA-enhanced MRI in the assessment of hepatic metastases. Nevertheless, it seems to improve overall lesion detection and diagnostic confidence when combined with Gd-EOB-DTPA-enhanced $\mathrm{MRI}$, especially for the less experienced radiologist.

\section{B-490 11:51}

\section{Diffusion-weighted MR imaging for differentiation of benign from}

malignant lesions in the periampullary region

S. Kim, H. Kwon, N. Lee, G. Kim, D. Kim, H. Seo; Pusan/KR

(kh8310@naver.com)

Purpose: The purpose of our study was investigating the value of DWI for distinguishing benign from malignant lesions of the periampullary region.

Methods and Materials: Eighty-three patients underwent respiratory-triggered breath-hold DWI at 1.5 or 3.0 TMR. DWI was performed with three $b$ factors $(0,500$, and $800 \mathrm{~s} / \mathrm{mm}^{2}$ ), and apparent diffusion coefficients (ADCs) were also calculated in the periampullary lesions. On DWI, signal intensity (SI) of the periampullary lesions was compared with SI of the back muscle and pancreas. In addition, we compared ADCs of malignant from benign lesions at MR by the Mann-Whitney $U$ test.

Results: Seventy-eight patients were evaluated excluding 5 patients due to previous interventions or technical errors. Nine patients with malignant lesions and 23 patients with benign lesions were evaluated at $1.5 \mathrm{~T}$. Thirteen patients with malignant lesions and 33 patients with benign lesions were evaluated at $3.0 \mathrm{~T}$. On DWI with a b factor $800 \mathrm{sec} / \mathrm{mm}^{2}, 20$ of 22 malignant lesions demonstrated slightly high SI (1/22, 4.5\%) and significantly high SI (19/22, 86.4\%). Fourteen of 56 benign lesions demonstrated slightly high SI (10/56, 17.9\%) and significantly high SI $(4 / 56,7.1 \%)$. The mean ADC of malignant and benign periampullary lesions were $1.25 \pm 0.26 \times 10^{-3} \mathrm{~mm}^{2} / \mathrm{s}$ and $1.89 \pm 0.33 \times 10^{-3} \mathrm{~mm}^{2} / \mathrm{s}$, respectively at $1.5 \mathrm{~T}$ MR $(p=.00)$. The mean ADC of malignant and benign periampullary lesions were $1.39 \pm 0.35 \times 10^{-3} \mathrm{~mm}^{2} / \mathrm{s}$ and $1.96 \pm 0.35 \times 10^{-3} \mathrm{~mm}^{2} / \mathrm{s}$, respectively at $3.0 \mathrm{TMR}(\mathrm{p}=.00)$ Conclusion: DWI can be reliable in the differentiation of benign from malignant lesions in the periampullary region. 
10:30 - 12:00

Room D1

\section{Cardiac}

\section{SS 1203}

\section{Atherosclerosis imaging}

\section{Moderators:}

F. Knollmann; Pittsburgh, PA/US

E.A. Mershina; Moscow/RU

\section{B-491 10:30}

In vivo quantification of total atherosclerotic burden: prognostic accuracy of whole body CTA in relation to traditional cardiovascular risk index and 5-year follow-up

A. Napoli, F. Zaccagna, M. Anzidei, G. Cartocci, L. Molisso, L. di Mare

C. Catalano, R. Passariello; Rome/IT (fulvio.zaccagna@libero.it)

Purpose: To investigate if mid-term prognostic value of WB-CTA to predict cardiovascular (CV) events in asymptomatic patients with CV risk factors can be superior to traditional method of risk stratification and can more accurately guide primary preventive strategies in asymptomatic patients.

Methods and Materials: 341 patients with CV risk factors (age 63.39 \pm 10.4 [3489]) underwent WB-CTA $(64 \times 0.6 \mathrm{~mm}$; lomeprol- $40070+50 \mathrm{ml}$ at $4 \mathrm{ml} / \mathrm{s})$. Coronary arteries were divided into 15 segments, extra-coronary arteries into 32 segments and detected stenoses were graded using a 5-point scale (0-4 normal-occlusion; 5 aneurysm). An atherosclerosis burden score (ABS) was generated for each individual and correlated to traditional $\mathrm{CV}$ risk (Framingham risk index; FRI). ABS and FRI were compared using Kaplan-Meier survival analysis, ROC analysis and stepwise multivariable Cox proportional hazards regression models.

Results: At baseline, ABS was $19.5 \pm 20.1$ and FRI $12 \pm 10.7 ; 48.4 \pm 11.2$ months after WB-CTA, all patients received an interview to determine health status during this period. According to Kaplan-Meier curves, mean event-free time was of $54.2 \pm 2.1 \mathrm{~m}$ for $A B S<10,48.2 \pm 1 \mathrm{~m}$ for $10<A B S 20(p=0.018)$. Mean event-free time was $50.1 \pm 0.5 \mathrm{~m}$ for FRI mild-risk group and $48.3 \pm 0.7 \mathrm{~m}$ for high-risk group without significant differences between the two groups $(p=0.054)$. For the prediction of CV events, in the high-risk group, ABS showed an accuracy of 0.88 , meanwhile FRI accuracy was of $0.67(p=0.004)$. Multivariable analysis showed that only age $(p=0.011), A B S(p=0.000)$ and therapy $(p=0.001)$ were independent predictors of hard CV events.

Conclusion: WBCTA-derived ABS reflects real atherosclerotic burden and provides superior risk stratification and event prediction with respect to $\mathrm{FRI}$; hard event prediction was significantly associated to age, ABS and therapy but not to FRI.

\section{B-492 10:39}

CT characterisation of coronary atherosclerosis: prognostic value in patients with acute chest pain

J.W. Nance ${ }^{1}$, U.J. Schoep ${ }^{1}$, S. Oberoi ${ }^{1}$, J.M. Barraza' ${ }^{1}$, G.F. Headden ${ }^{1}$,

F. Bamberg ${ }^{2} ;{ }^{1}$ Charleston, SC/US, ${ }^{2}$ Munich/DE (nancej@musc.edu)

Purpose: Coronary CT angiography (CCTA) enables comprehensive evaluation of the coronary atherosclerotic plaque burden; however, the prognostic value of this test is not well documented. Our purpose was to correlate findings at CCTA with clinical outcomes in patients with acute chest pain.

Methods and Materials: 460 consecutive patients (35\% male, mean age $55 \pm 11$ years) with acute chest pain underwent cCTA. The presence, extent (number of affected segments), severity ( $\geq 50 \%$ or $<50 \%$ stenosis), and type (calcified, noncalcified, or mixed) of atherosclerotic lesions were evaluated. Patients were clinically followed (mean follow-up $16.7 \pm 10.8$ months) for major adverse cardiac events (MACE: MI, unstable angina, revascularisation, cardiac death). Cox regression was used to identify CT-based predictors of MACE independent of clinical risk factors. Results: 70 (15\%) patients had a MACE during follow-up. Clinical characteristics associated with MACE were male gender, age, hypertension, hypercholesterolemia, and smoking status. CT parameters associated with MACE included presence and extent of plaque, obstructive plaque, calcified plaque, non-calcified plaque, and mixed plaque. All parameters except presence of plaque remained significant after adjustment for clinical risk factors; the most significant predictors were extent of non-calcified plaque (HR: $1.7, \mathrm{p}<0.001)$ and obstructive plaque (HR: 1.4, $p<0.001$ ). Absence of coronary atherosclerosis on cCTA had a $100 \%$ negative predictive value for MACE during follow-up.

Conclusion: CCTA characterisation of the atherosclerotic plaque burden predicts MACE independent of traditional risk factors. Non-calcified plaque bestows the highest relative risk. Understanding the relationship between plaque type and prognosis may establish CCTA as a robust tool for patient-specific risk stratification.

\section{B-493 10:48}

Prognostic value of prospective ECG-gated dual-source CT coronary angiography in patients with suspected coronary artery disease

R. Donato, M. Micchìa, S. Racchiusa, A. Sergi, G. Cicciò, A. Bottari, C. Anfuso, F. Zimbaro, G. Ascenti; Messina/IT (roccodonato81@libero.it)

Purpose: To evaluate the prognostic value of prospective ECG-gated dual source coronary CT angiography (DSCT-CA) for major adverse cardiac events (MACE) in patients with suspected coronary artery disease (CAD).

Methods and Materials: A total of 102 patients (58 males and 44 females, age $62 \pm 11.7$ years) with suspected coronary disease underwent prospective ECGgated DSCT-CA to assess the presence, severity and positive remodelling of coronary atherosclerotic plaques. The occurrence of MACE (cardiac death, myocardial infarction and unstable angina with hospitalisation) was evaluated in each patient during an average 28-month follow-up (24-32 months). CAD was defined significant when stenosis was $>=50 \%$ in any coronary artery.

Results: DSCT-CA noted the absence of CAD in 42 (41.2\%) patients, no significant CAD in $28(27.4 \%)$ and significant CAD in $32(31.4 \%)$. The presence of positive remodelling plaques was found in $22(21.6 \%)$ patients. MACE occurred in 14 patients ( 8 myocardial infarctions, 4 cardiac deaths, 8 unstable angina), of which 10 with significant $C A D$ and 4 with no significant CAD. 10 out of 14 patients with MACE $(71.4 \%)$ showed presence of positive remodeling plaque. Patients with normal coronary arteries at DSCT-CA showed an event rate of $0 \%$ at follow-up. Conclusion: The prospective ECG-gated DSCT-CA proved to be an excellent method to predict the prognosis in patients with suspected CAD. DSCT-CA identifies an increased risk of cardiac events related to the presence of positive remodelling plaque and the severity of CAD.

\section{B-494 10:57}

Prognostic value of stenosis severity and atherosclerotic plaque burden at coronary CT angiography for major adverse cardiac events (MACE): a population-based outcome study

J.A. Abro, R.E. Sweeney, U. Schoepf, M. Gebregziabher, P. Costello; Charleston, SC/US (abro@musc.edu)

Purpose: Recent studies have established the prognostic value of CCTA for allcause mortality. However, the predictive value of cCTA specifically for cardiac events is less well documented. We hypothesize that extent and severity of coronary artery disease $(C A D)$ as defined at CCTA are predictive of major adverse cardiac events (MACE).

Methods and Materials: We examined a consecutive cohort of 454 patients (233 women, mean age 57 5 years) who had undergone cCTA using either 64-slice or dual-source CT for atypical chest pain. Stenosis at CCTA was scored as minimal $(<30 \%)$, mild $(30 \%-49 \%)$, moderate $(50 \%-69 \%)$, or severe $(\geq 70 \%)$ for each coronary artery. A > 12-month follow-up of MACE was assessed using Cox proportional hazards models adjusted for pretest CAD likelihood and risk factors. MACE were determined using the Social Security Death Index, medical record review and phone calls to patients and referring physicians.

Results: Of the 452 patients, 198 patients did not show any atherosclerosis at cCTA. Of these, none had a MACE during follow-up (100\% NPV). 254 patients had atherosclerosis at CCTA ( 28 non-calcified, 254 calcified, 180 mixed). $87 / 254$ patients had stenosis $>50 \%$. 26 patients had a MACE during follow-up. The presence of stenosis $>50 \%$ was significantly $(p=0.003)$ associated with MACE during follow-up. MACE rates were not significantly $(p>0.05)$ associated with plaque type or calcium score. Conclusion: Our results suggest that in individuals presenting with atypical chest pain, a normal cCTA has $100 \%$ NPV for ruling out MACE during follow-up. Stenosis $>50 \%$ determined at CCTA significantly predicts the risk of MACE.

\section{$B-495 \quad 11: 06$}

Coronary artery plaque imaging: comparison of black-blood MRI and 64MDCT

Y. $\underline{\mathrm{He}}^{1}$, Z. Zhang ${ }^{1}$, Q. Dai ${ }^{1}, \mathrm{~J} . \mathrm{An}^{2}$, L. Jin ${ }^{3}$, J. Renate ${ }^{3}$, D. Li ${ }^{4} ;{ }^{1}$ Beijing/CN,

${ }^{2}$ Shenzhen/CN, ${ }^{3}$ Shanghai/CN, ${ }^{4}$ Los Angeles, CA/US (heyi139@sina.com)

Purpose: To evaluate the ability of black-blood coronary arterial wall MRI to identify and classify coronary artery plaques by comparing with 64-MDCT.

Methods and Materials: 15 patients with confirmed coronary artery plaques in the proximal or middle segments of coronary arteries by 64-MDCT underwent black-blood coronary wall MRI. All scans were performed on a $1.5 \mathrm{~T}$ scanner. Crosssectional coronary wall images were acquired on the lesion coronary artery from the ostium to the middle segment continuously without gap. The vessel cross-sectional area (CSA), luminal CSA, maximal wall thickness, plaque burden, CNR and SNR were measured in each slice, which were then compared with the CTA images. 
Results: 3 patients were excluded from the analysis. In 12 patients, coronary plaques were found in 46 slices on both CTA and MRI. These plaques were classified to 3 groups based on CTA: calcified plaques $(n=11)$, soft plaques $(n=23)$, and mixed plaques $(n=12)$. In MRI, the plaque burden, maximal wall thickness, SNR, CNR in the coronary walls containing plaques were greater than those of the normal coronary walls $(0.83 \pm 0.08$ vs $0.73 \pm 0.08,1.88 \pm 0.51$ vs $1.51 \pm 0.26 \mathrm{~mm}$, $12.95 \pm 2.78$ vs $9.93 \pm 2.31,6.76 \pm 2.52$ vs $3.89 \pm 1.54$, respectively, $p<0.05$ ). The luminal CSA at the plaque was smaller than that at normal coronary walls $\left(2.50 \pm 1.50\right.$ vs $\left.4.72 \pm 2.28 \mathrm{~mm}^{2}, p<0.05\right)$. The SNR in the soft plaque was significantly greater than those in calcified and mixed plaques $(p<0.05)$.

Conclusion: Coronary wall MRI can identify coronary plaques in the proximal and middle segments, and has the potential to differentiate plaque types based on signal intensity.

\section{B-496 11:15}

Prognostic value of subclinic artery disease: correlation between MDCTCA and carotid artery MRI

R. Malago, C. Barbiani, S. Rainer, G. Caliari, A. Pezzato, P. Vallerio,

A. Fratta Pasini, R. Pozzi Mucelli; Verona/IT (robertomalag@yahoo.it)

Purpose: Acute cardiovascular events are related to type of coronary plaque rather than coronary artery stenosis degree. The aim of our study is to evaluate the prevalence of sub-clinic coronary artery disease (CAD) and relate plaque composition to carotid US and MRI.

Methods and Materials: 30 consecutive patients $(22 \mathrm{M}, 8 \mathrm{~F})$ mean age $67 \pm 8 \mathrm{YO}$ between 03 and 06/2010 underwent MDCT-CA and carotid artery US and MRI. All patients were on high level pre-test probability of CAD according to AHA risk score. For carotid arteries and coronaries type of plaque (calcific, mixed and fibrolipidic) and degree of stenosis was distinguished into significant (> $50 \%$ lumen narrowing) or not significant $(<50 \%$ lumen narrowing). Correlation of plaque composition and significance of stenosis was obtained by means of Spearman correlation.

Results: 38 plaques (1.26/patient) were detected with US and MRI (27/38 fibroli$\mathrm{pidic} / \mathrm{mixed}, 11 / 38$ calcific). Mean Ca score obtained was 2205.5 (0-2893.8). 105 plaques were detected in MDCT-CA fibrolipidic (20/105) 19\% mixed 29/105 (28\%) and calcific 56/105 (53\%). Correlation between US and MRI in plaque morphology was high ( $r=0.95$ for mixed, $r=1$ for calcified and $r=0.8$ for soft plaques). Correlation between the two MRI and MDCT-CA was $r=0.311, P=0.101$ for fibrolipidic plaque, $r=0.579, P=0.001$ for mixed plaque, $r=0.471, P=0.010$ for calcific plaque. Correlation for stenosis degree and between MRI and US was $r=0.8$ and between the MDCT. CA and MRI technique was $r=0.518 \mathrm{P}=0.004$

Conclusion: Plaque morphology and pattern of stenosis on carotid arteries can help to predict the presence of significative CAD.

\section{B-497 11:24}

Coronary atherosclerosis and coronary artery disease in patients with obstructive sleep apnoea: assessment with coronary CT angiography G.W. Rowe, R.B. Wilson, A.M. Armstrong, J.A. Abro, A.T. Parker, U. Schoepf, S. Sharma; Charleston, SC/US (wilsonro@musc.edu)

Purpose: There is increasing evidence linking OSA with coronary artery disease (CAD). We aimed at using coronary CT angiography for studying the association between OSA and coronary atherosclerosis and CAD.

Methods and Materials: 49 patients having documented OSA by polysomnogram and Berlin questionnaire underwent dual-source cCTA. They were matched with 46 patients with similar cardiovascular risk, but free of OSA. All patients underwent cCTA for atypical chest pain and/or prior equivocal physiological testing. In all 95 patients, two experienced observers in consensus analysed the cCTA data for the presence and degree of coronary artery stenosis. The association between OSA status, stenosis, and plaque type was tested using $X^{2}$ and Fisher's exact statistics. Results: There was no significant $(p>0.05)$ difference in demographics between patients with and without OSA. Global coronary artery calcium scores were not significantly different between the two groups (mean Agatston score $272 \pm 422$ with OSA versus $241 \pm 415$ without OSA, $p=0.5$ ). However, in patients with OSA there was a significantly $(p=0.0013)$ higher prevalence of stenotic CAD and more extensive vessel involvement (6\% one-vessel, $27 \%$ two-vessel, $22 \%$ three-vessel, 33\% four-vessel CAD in OSA versus $15 \% / 7 \% / 13 \% / 24 \%$ without OSA, $p=0.0017$ ). Composition of stenotic lesions was notably different between the two groups, with a significantly $(p=0.0043)$ higher prevalence of non-calcified and mixed plaque in the OSA group.

Conclusion: Our initial results suggest that patients with OSA have more active coronary atherosclerotic disease, with a greater degree of vessel involvement with stenosis and more stenotic non-calcified and mixed plaque than patients without OSA.

\author{
B-498 11:33 \\ Ex-vivo classification of non-calcified coronary atherosclerotic plaque \\ components on CT coronary angiography: impact of neighbouring \\ attenuation and density thresholds \\ E. Maffei, C. Martini, T. Arcadi, S. Seitun, E. Capuano, I. Romano, I. Romano, \\ C. Lario, F. Cademartiri; Parma/IT (filippocademartiri@hotmail.com)
}

Purpose: To assess the effect of neighbouring attenuation and density thresholds on attenuation values of non-calcified plaque by computed tomography coronary angiography (CTCA) in an ex-vivo model.

Methods and Materials: We studied 7 ex-vivo left coronary artery specimens surrounded by olive-oil and filled with saline and 4 solutions with decreasing dilutions of contrast material: control (saline), 1/200, 1/80, 1/50 and 1/20. The CTCA protocol was: slice/collimation $32 \times 2 \times 0.6 \mathrm{~mm}$, and rotation time $330 \mathrm{~ms}$. The attenuation $(\mathrm{HU})$ value of atherosclerotic plaques was measured for each dilution in: lumen, plaque (non-calcified coronary wall thickening), calcium and surrounding oil. Based on the measured CT attenuation values, plaques were classified as lipid-rich or fibrous using $30 \mathrm{HU}, 50 \mathrm{HU}$ and $70 \mathrm{HU}$ density thresholds. The results were compared with one-way ANOVA-test and correlated with Pearson's test. Results: 500 cross sections with plaques were detected and assessed. Mean lumen $(45 \pm 38 \mathrm{HU}$ to $669 \pm 151 \mathrm{HU})$ and plaque $(11 \pm 35 \mathrm{HU}$ to $101 \pm 72 \mathrm{HU})$ attenuation differed significantly $(p<0.001)$ among the different dilutions. The attenuation of lumen and plaque of coronary plaques showed moderate correlation $(r=0.54$ $\mathrm{p}<0.001$ ) while the correlation between plaque attenuations in neighbouring dilutions was good $(r>0.70)$. Using a $50 \mathrm{HU}$ threshold, the prevalence of lipid-rich plaques would progressively shift from $441(88 \%)$ to $136(27 \%)$ with increasing lumen attenuation $(\mathrm{p}<0.05)$. The same was observed using a threshold of $30 \mathrm{HU}$ (from $75 \%$ to $19 \% ; p<0.05$ ) and $70 \mathrm{HU}$ (from $88 \%$ to $27 \%$; $p<0.05$ ).

Conclusion: Classification of coronary plaques as lipid-rich or fibrous based on absolute CT attenuation values is significantly affected by the neighbouring attenuation and density thresholds.

\section{B-499 11:42}

Impact of metabolic syndrome on coronary artery stenosis and plaque characteristics assessed by 64 -slice multidetector-row cardiac computed tomography

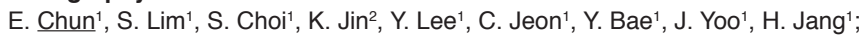
${ }^{1}$ Seongnam-si/KR, ${ }^{2}$ Seoul/KR (drejchun@hanmail.net)

Purpose: We investigated plaque characteristics as well as coronary artery stenosis and plaque burden according to the presence of metabolic syndrome (MS) by 64-slice multidetector-row cardiac computed tomography (MDCT).

Methods and Materials: Coronary artery stenosis (>50\% of diameter), plaque burden and plaque characteristics as well as coronary artery calcium score (CACS) were assessed by MDCT in 3,000 age and sex-matched asymptomatic individuals. Anthropometric parameters and metabolic profiles were also measured. Results: Subjects with MS had more coronary stenosis, multivessel involvement, greater plaque burden and higher CACS than subjects without MS (all, $\mathrm{P}<0.01$ ). Mixed or non-calcified plaques were also more prominent in subjects with MS than those without MS. After adjustment for confounding factors, MS was strongly associated with significant coronary stenosis, greater plaque burden and multivesse involvement. In plaque types, mixed plaque was associated with MS and correlated significantly with the Framingham risk scores.

Conclusion: In asymptomatic subjects with MS, presence of mixed plaque, plaque burden and coronary stenosis were significantly greater than subjects without MS as assessed by MDCT. MDCT is an effective imaging modality for detecting coronary atherosclerosis and plaque characteristic in asymptomatic patients but future study on its clinical effect is warranted to further confirm its role.

\section{B-500 11:51}

Reproducibility of assessment of non-calcified coronary artery plaque burden across different image analysis platforms

S. Oberoi, J.M. Barraza, C. Thilo, U.J. Schoepf; Charleston, SC/US (barraza@musc.edu)

Purpose: Coronary CT angiography (CCTA) assessment of non-calcified coronary artery plaque (NCP) burden is gaining use for risk stratification and monitoring therapy. Sufficient reproducibility is essential for serial assessment. We investigated reproducibility of non-calcified plaque burden quantification across multiple commercial image-analysis platforms.

Methods and Materials: For 47 patients with documented NCP on CCTA, automated quantification of NCP burden was performed on identical data sets using 3 different image analysis platforms (Aquarius, TeraRecon; Circulation, Siemens; Vitrea, Vital). 
NCP burden in the left anterior descending (LAD), left circumflex (LCX), and right coronary artery (RCA) was quantified with each program. Identical tissue attenuation ranges of $0-50 \mathrm{HU}$ for low and $50-130 \mathrm{HU}$ for medium attenuation plaques were applied. Duplicate measurements were performed to ensure reproducibility. Results: Mean values for low attenuation plaques for LAD, RCA, and LCX were 75.14, 47.67, and 51.88 HU, respectively, for Circulation, 75.89, 79.36, and $79.85 \mathrm{HU}$ for Aquarius, and 185.1, 200.3, and 139.4 HU for Vitrea. Similarly, for medium attenuation plaque: $174.9,109.9$, and $136.1 \mathrm{HU}$ for Circulation, 129.4 134.3, and 150.2 HU for Aquarius, and 424.9, 493.4, and 391.9 HU for Vitrea. For low attenuation plaque, the log (volumes) overall and for each coronary artery were significantly different $(p<0.05)$ among platforms. Medium attenuation plaque results were similar, but Circulation and Aquarius were not significantly different in the LAD and LCX. Duplicate measurement results were not significantly different ( $p>0.05$ ), indicating reproducibility within each platform.

Conclusion: Current software platforms do not allow reproducible quantification of NCP burden across different workstations. Serial assessment requires use of the identical system.

\begin{tabular}{ll}
\hline $10: 30-12: 00$ & Room D2 \\
\hline
\end{tabular}

\section{Interventional Radiology}

\section{SS 1209 \\ Musculoskeletal intervention}

Moderators:

X. Buy; Strasbourg/FR

T. Sikdar; Harlow/UK

\section{B-501 10:30}

Does saline solution temperature influence patient short-term outcome of a double-needle ultrasound-guided treatment of calcifying tendinitis of the rotator cuff (CTRC)? The discovery of hot water

L.M. Sconfienza 1 , G. Serafini ${ }^{2}$, M. Bandirali ${ }^{1}$, F. Lacelli², E. Silvestri ${ }^{3}$,

F. Sardanelli'; ${ }^{1}$ San Donato Milanese/IT, ${ }^{2}$ Pietra Ligure/IT, ${ }^{3}$ Genoa/IT (io@lucasconfienza.it)

Purpose: We determined whether the temperature of saline influences procedure performance and patient short-term outcome of an ultrasound-guided lavage technique for treating CTRC.

Methods and Materials: 88 patients ( 49 females, mean age $47 \pm 11.1$ years) with painful shoulder and ultrasound diagnosis of CTRC were prospectically randomised. Group A $(n=44)$ underwent ultrasound-guided percutaneous treatment of CTRC (local anaesthesia, double-needle saline lavage and calcium aspiration, intrabursal steroid injection) using saline at room temperature, group $B(n=44)$ underwent the same treatment with saline at about $42^{\circ} \mathrm{C}$. Duration of procedure was recorded and ease of calcium dissolution was subjectively scored 1 (easy), 2 (intermediate), or 3 (difficult). Ultrasound appearance of the calcium deposit was judged as hard, soft, fluid. Visual analogue scale (VAS) was used for patients' discomfort.

Results: Procedure duration was significantly lower $(P=.030)$ in group $B(544 \pm 241$ s) compared to group $A(791 \pm 311 \mathrm{~s})$. Calcium dissolution was significantly improved $(P=.034)$ in group $B$ (median score 1) compared to group $A$ (median score 2 ); in the subgroup with hard deposits (group $A, n=14$; group $B=16$ ), ease of calcium dissolution was larger and more significant $(P<.001)$. VAS was significantly lower after treatments in both groups compared to baseline (group A, VAS before = $9.0 \pm 0.6 ;$ VAS 1 month $=4.7 \pm 0.5 ;$ VAS 3 months $=3.4 \pm 0.4$; group B, $9.1 \pm 0.4$ $4.5 \pm 0.4,3.3 \pm 0.5$, respectively, $P<.001$ for all). While four post-procedural bursitis were observed in group $A$, none of them was observed in group $B$.

Conclusion: When treating CTRC, using warm saline solution could reduce procedure duration, improve calcium deposit dissolution, and reduce the occurrence of post-procedural bursitis.

\section{B-502 10:39}

Cement-directed kyphoplasty reduces cement leakage as compared with vertebroplasty: results of a controlled, randomised trial

T.J. Vogl ${ }^{1}$, R. Pflugmacher ${ }^{2}$, J. Hierholzer ${ }^{3}$, G. Stender ${ }^{4}$, M. Gounis ${ }^{5}$, A. Wakhloo; ${ }^{1}$ Frankfurt a. Main/DE, ${ }^{2}$ Berlin/DE, ${ }^{3}$ Potsdam/DE, ${ }^{4}$ Marl/DE, ${ }^{5}$ Worcester, MA/US (t.vogl@em.uni-frankfurt.de)

Purpose: To prospectively compare cement leakage-rates, leak-locations, painrelief and patient-disability for cement-directing kyphoplasty system (CDKS) and vertebroplasty in painful vertebral compression-fractures.
Methods and Materials: Seventy-seven patients $\geq 50$ years ( $1-3$ osteoporotic vertebral compression-fractures between T4 and L5) were enrolled in an open-label, prospective, randomised study (randomization 2:1 for CDKS (49patients/65 levels) or vertebroplasty (28patients/39 levels) treatment. Polymethylmethacrylate (PMMA)cement was used in both treatments. Cement-leakage and leak-locations were evaluated by CT and post-operative radiographs. Pain-levels were assessed pre-/ post-operatively, at 3 and 12 months using ten-point visual-analogue-scale (VAS). Disability was assessed pre-operatively, at 3 and 12 months using the OswestryDisability-Index (ODI). Radiographs and CTs were obtained at 3 months and final radiographs at 12 months. Anterior vertebral body height was determined from CT and heights were compared between post-op and 3 months. Radiographs and CTs were evaluated for refractures or new adjacent level-fractures at each follow-up. Results: CT provided increased resolution and additional axial-view for detection of more cement-leaks versus radiography. Treatment with CDKS significantly reduced number of levels with leaks compared with vertebroplasty $(47.7 \%$ vs.71.8\%,p = 0.0132 ). When leaks were classified by location, significantly fewer cortical and basivertebral vein-leaks occurred in the CDKS-group $(p=0.0158 / p=0.0217$, respectively). Immediate pain-relief sustained throughout the study for both treatmentgroups. ODI-scores were similarly improved for CDKS and vertebroplasty at 3 and 12 months, compared with pre-operative levels. Examining height maintenance over time revealed that mean change in anterior vertebral body height was $5.90 \%$ $( \pm 11.36)$ for CDKS and $9.49 \%( \pm 8.28)$ for vertebroplasty: CDKS may provide improved long-term height-maintenance $(p=0.2148)$.

Conclusion: Cement-directed kyphoplasty effectively relieves pain and reduces cement-leakage, particularly in the posterior direction, by guiding cement-flow in anterior, superior and inferior directions.

B-503 10:48

Vertebroplasty and baropodometry: a comparison of load distribution among normal population and patients with vertebral fractures treated with vertebroplasty

D.K. Filippiadis, G. Velonakis, A. Mazioti, E. Brountzos, N. Kelekis, A. Kelekis; Athens/GR (dfilippiadis@yahoo.gr)

Purpose: Through comparison of patients with vertebral fractures and normal population we illustrate percutaneous vertebroplasty (PV) effect upon load distribution changes (among the two feet - among rear and front of the same foot) during standing and walking.

Methods and Materials: Last year, we prospectively compared 2 groups. Group A (36 patients, $75 \pm 15$ years) with vertebral fractures were evaluated on electronic baropodometer. Load distribution between right and left foot during standing and walking was recorded prior (group A1) and the day after (group A2) PV. Group B (30 patients, $42 \pm 13$ years) with no back pain or surgery record (normal population) was evaluated on the same electronic baropodometer. The two groups were compared by means of related samples Wilkoxon Signed Rank test.

Results: Mean value of load distribution variation between rear and front of the same foot for the normal population was $9.45 \pm 6.79 \%$ (standing) and $14.76 \pm 7.09 \%$ (walking). Mean value of load distribution variation between rear and front of the same foot prior to PV was $16.52 \pm 11.23 \%$ (standing) and $30.91 \pm 19.26 \%$ (walking). Load distribution variation post-PV was $10.08 \pm 6.26 \%$ (standing) and $14.25 \pm 7.68 \%$ (walking). Comparison of load distribution variation among groups $\mathrm{A} 1$ and $\mathrm{A} 2$ or groups $A 1$ and $B$ is statistically significant ( $p=0.001$ and $p=0.011$, respectively). Comparison of load distribution variation among groups $A 2$ and group $B$ is not statistically significant $(p=0.486)$.

Conclusion: Comparative groups enable us to consider that PV apart from the already known pain reduction effect is efficient on equilibrium and load distribution improvement as well.

\section{B-504 10:57}

Efficacy of percutaneous vertebroplasty with a bone substitute: a preliminary experience

S. Marcia, C. Boi, V. Erbì, S. Marini, M. Marras, G. Mallarini; Cagliari/IT (stemarcia@gmail.com)

Purpose: To prove the effectiveness of a bone substitute in the treatment of osteoporotic or traumatic vertebral fractures with percutaneous vertebroplasty. Methods and Materials: We performed PVP on twenty-three patients treating 47 vertebral bodies by means of injection of calcium sulphate $60 \%+$ hydroxyapatite $40 \%$. About $3 \mathrm{cc}$ of bone cement was injected for each vertebra. Patients had been previously evaluated with clinical examination and with $\mathrm{x}$-ray, CTms and MRI T2w STIR. All the procedures were executed with local anaesthesia under digital fluoroscopic guidance. Clinical evaluation, assessment of pain by mean of a visual analogue scale (VAS, $0-10$ ) and of function by means of the Oswestry 
disability scale (ODI 0-50) was performed baseline, at one and six months after the procedure. We also carried out $\mathrm{x}$-ray, CTms and MRI checks at one and six months. The use of analgesic drugs before and after the procedure was also evaluated. Results: we have obtained a progressive reduction of the pain in all the patients (VAS baseline: 8.36, at $1 \mathrm{M} 2.18$, at $6 \mathrm{M} 2.17$ ) and a good improvment of the quality of life (ODI baseline 24, at $1 \mathrm{M}: 11.7$, at $6 \mathrm{M}: 9.3$ ), a progressive reduction of bone oedema and a progressive inclusion of the bone cement. $100 \%$ of patients were used to take analgesic drugs before the implantation, while only $18 \%$ continued to do. No complications arose.

Conclusion: Our preliminary studies prove that calcium sulphate could result in being an actual substitute of PMMA in the treatment of osteoporotic and traumatic vertebral fractures, especially in young patients.

\section{B-505 $11: 06$}

\section{Percutaneous vertebroplasty for pain management in spinal extradural metastasis}

G. Sun, P. Jin, M. Li, L. Li, Z.-H. Peng; Jinan/CN (cjr.sungang@vip.163.com)

Purpose: To evaluate safety and effectiveness of performance of percutaneous vertebroplasty $(\mathrm{PV})$ in patients with spinal extradural metastasis, with or without definite signs or symptoms of spinal cord or cauda equina.

Methods and Materials: We performed PV procedures in 32 patients with metastatic disease, who presented at least one vertebral lesion with destruction of the posterio vertebral body wall or epidural extension of tumour. Amongst this patients, nine had signs of spinal cord or cauda equina compression. Procedural safety was evaluated by any post-procedure adverse events. The PV analgesic efficacy was evaluated by means of follow-up clinic reexamination or phone calls to assess change in patients' pain level prior and post procedure. The effectiveness was defined as at least a $50 \%$ improvement in pain as compared with baseline of prior procedure. Follow-up for assessments were performed after the procedure at 1 day; 1 week; 1,3 , and 6 months. Results: Fifty-three levels in 32 patients were treated in this group. Analgesic efficacy of PV was obtained in $97 \%$ patients (31/32) at 1 day and 1 week, $86 \%$ at 1 month, $89 \%$ at 3 months and $87 \%$ at 6 months after procedure. Leakage of PMMA was detected in $64 \%(33 / 53)$ treated vertebrae. No complications with clinical sequelae were encountered.

Conclusion: Epidural involvement with or without neurological signs of spinal cord or cauda equina should not be a contraindication to performance of $\mathrm{PV}$ in patients with few other treatment options.

\section{B-506 11:15}

A prospective study comparing percutaneous decompression, combination of minimally invasive techniques and conservative therapy for the treatment of intervertebral disc herniation

D.K. Filippiadis, G. Velonakis, A. Mazioti, A. Malagari, N.L. Kelekis, A. Kelekis; Athens/GR (dfilippiadis@yahoo.gr)

Purpose: To compare efficacy (pain reduction and mobility improvement) among percutaneous disc decompression (PDD), combination of minimally invasive techniques and conservative therapy for intervertebral disc herniation treatment. Methods and Materials: During the last 4 years, we prospectively compared 3 groups of 15 patients, each suffering from sciatica due to intervertebral disc herniation. Group Aunderwent fluoroscopically guided percutaneous intervertebral disc decompression (PDD). Group B underwent a session where PDD was combined either to intradiscal discogel infusion, or to percutaneous nucleoplasty (plasma energy coblation). Group $\mathrm{C}$ underwent conservative therapy (analgesics, anti-inflammatory drugs, muscle relaxants, physiotherapy) for 6 weeks. Pain reduction and mobility improvement were recorded [clinical evaluation, 0-10 units Numeric Visual Scale (NVS)].

Results: Within a 24 months follow-up period, Group A patients presented a mean pain reduction of $6.60 \pm 3.31$ NVS units $(80.38 \%$ success rate), Group B patients a mean pain reduction of $5.73 \pm 2.60$ NVS units $(76.82 \%$ success rate) and Group C patients presented a mean pain reduction of $2.33 \pm 2.94$ NVS units $(34.30 \%$ success rate). No complications were noted. Comparison of pain reduction among Group $A$ and Group C or among Group B and Group $C$ is statistically significant ( $p=0.005$ and $p=0.004$, respectively). Comparison of pain reduction among Group A and Group B is not statistically significant $(p=0.43)$.

Conclusion: Comparative groups enable us to consider minimally invasive techniques (solely performed) rather than conservative treatment as the treatment of choice for symptomatic small to medium-sized intervertebral disc hernias. There is no proof that combining minimally invasive techniques will improve the success rate.

\section{B-507 11:24}

Pain palliation of bone metastasis: initial clinical experience using high intensity focused ultrasound therapy with magnetic resonance guidance

A. Napoli, M. Anzidei, C. Catalano, M. Mastantuono, F. Zaccagna,

L. Molisso, G. Cartocci, L. di Mare, R. Passariello; Rome/IT

(alessandro.napoli@uniroma1.it)

Purpose: To determine the efficacy of non-invasive high intensity MR-guided focused ultrasound (MRgFUS) treatment for palliation of bone metastasis pain in patients not candidated for External Beam Radiotherapy (EBRT).

Methods and Materials: Under the IRB approval, 18 patients with 21 lesions underwent MRgFUS treatment using the ExAblate 2000 system (InSightec). Treatments were done in a single session, in an ambulatory setting. 12 patients underwent prior EBRT with a mean 6 months recurrent pain. In 6 patients, MRgFUS treatment was performed as first treatment modality. Effectiveness of pain palliation was evaluated at follow-up using the visual analog pain score (VAS) and measurable changes in analgesics intake. For tumour control perfusion, T1w images were obtained preand post-treatment in order to determine the non-perfused sonication-related area. Results: All patients and all lesions were treated. Mean follow-up time was 4 months. At baseline median, VAS was 7; it was 5 at 3 days, 3 at two weeks and 2 at one and four months. Wilcoxon's rank test showed a statistically significant difference between baseline and follow-up VAS values $(0.01<p<0.038)$. No heating-related adverse event was recorded during this clinical application; patient medication intake was considerably reduced. Variable degree on non-perfused volume was observed after treatment, mainly within the pericortical region. Deeper penetration of the acoustic energy is at present desirable even if technically difficult to achieve with the current system.

Conclusion: MRgFUS is a promising non-invasive treatment modality for successful palliation of bone metastasis pain in patients who are not candidate for EBRT.

B-508 11:33

(EPOS)

Clinical application of CT-guided radiofrequency ablation for the treatment of metastatic bone neoplasms

Z. Wang, L. Zhang, Y. Zheng, J. Gong, Z. Chen; Shanghai/CN (wzm0722@hotmail.com)

Purpose: To investigate the clinical efficacy of CT-guided radiofrequency ablation (RFA) for the treatment of metastatic bone neoplasms.

Methods and Materials: Under intravenous aneaesthesia. CT-guided RFA was performed in 20 patients with metastatic bone tumour. The degree of pain was evaluated at 24 hours, 3 and 6 months after the operation by brief pain inventory (BPI). Results: All patients were followed up for 6 months and survived so far. The average peak pain score before the operation was 8.1 (6-10), which decreased significantly to $6.1,4.6,3.3$ and 3.0 at 24 hours, 1,3 and 6 months after the operation, respectively $(P<0.001)$. The mean pain score before the operation was 63 , which decreased significantly to $4.0,2.3,2.1$ and 1.9 at 24 hours, 1,3 and 6 months after operation, respectively $(\mathrm{P}<0.001)$. After RFA treatment. The KPS scores of all patients increased while the CT values of the bones lesions decreased. No major complications occurred both during and after the operation. One patient with vertebral lamina destruction suffered from lower limb hypoesthesia after RFA procedure, and the lower limb sensation was restored within 48 hours after the injection of prednisone was employed. Conclusion: CT-guided radiofrequency ablation is a safe, effective, minimallyinvasive and up-to-date technique for the treatment of metastatic bone neoplasms with excellent anti-pain effect; its short-term response is sure and reliable.

\section{B-509 11:42}

MR-guided discography in an open high-field MRI scanner

F. Streitparth ${ }^{1}$, T. Hartwig ${ }^{1}$, B. Schnackenburg ${ }^{2}$, P. Strube ${ }^{1}$, M. De Bucourt ${ }^{1}$

B. Hamm ${ }^{1}$, U. Teichgräber ${ }^{1} ;{ }^{1}$ Berlin/DE, ${ }^{2}$ Hamburg/DE

(florian.streitparth@charite.de)

Purpose: To evaluate the feasibility of MR-guided discography in an open highfield MRI.

Methods and Materials: 48 disc segments of 41 patients scheduled for intradiscal thermal treatment, total disc replacement or spondylodesis were examined. An 1.0 T open MRI was used for instrument guidance and imaging. After primary disc puncture under guidance of fast interactive PDW TSE imaging, 1-2 ml of gadolinium contrast saline mixture was injected into the disc. The occurrence of memory pain during injection was recorded. Axial and sagittal T1w TSE images with and without fat-saturation were obtained. All MRI discograms were analysed by two readers, who were blinded to the clinical findings.

Results: Overall, the placement of the puncture needle in the targeted disc was accurate under real-time MR guidance. Injections were technically successful in all cases. No major complications occurred. The mean procedure time was $17 \mathrm{~min}$ 
(range 13-34 min). Image quality of contrast-enhanced MR discograms was excellent. Memory pain was detected in 16 of 48 affected segments.

Conclusion: MR-guided discography is accurte and safe. Our results suggest that this method may substitute fluoroscopy or CT-discography. Multiplanar dynamic imaging facilitates the puncture of discs and provides high-quality MR-discograms.

\section{B-510 11:51 \\ Guidance and thermometry of laser diskectomies in open high-field MRI \\ F. Streitparth ${ }^{1}$, T. Walter ${ }^{1}$, B. Schnackenburg ${ }^{2}$, U. Wonneberger ${ }^{1}$, J. Rump ${ }^{1}$, \\ B. Hamm ${ }^{1}$, U. Teichgräber ${ }^{1} ;{ }^{1}$ Berlin/DE, ${ }^{2}$ Hamburg/DE \\ (florian.streitparth@charite.de)}

Purpose: To evaluate MR guidance and thermometry during percutaneous laser disc decompression (PLDD) in a high-field open 1.0 T MRI.

Methods and Materials: In 30 discs of human cadaveric lumbar spines, a fluoroscopic PD-w turbo spin echo (TSE) sequence was used for positioning of a laser fiber and a reference temperature probe within the targeted disc. Three non-spoiled gradient echo (GRE) sequences were compared to monitor laser effects at 15 Watt using a $1064 \mathrm{~nm}$ Nd:YAG laser. Temperature distribution was visualised on the basis of T1- and proton resonance frequency method (PRF). Image quality, temperature accuracy and correlation to macroscopic lesion sizes were analysed. Subsequently, MR-guided PLDD was performed in 15 patients.

Results: MR-guided placement of the laser fiber in the targeted disk was precise. Laser effects were monitored online in all cases. The best image quality and temperature accuracy $(r 2=0.94)$ was achieved with the GRE sequence with an echo time (TE) of $7 \mathrm{~ms}$. The correlation between the macroscopic and MR-lesions was sufficient for the GRE with a TE of 2 and $7 \mathrm{~ms}(\mathrm{r} 2=0.76,0.63)$. In vivo, PLDD was technically successful in all cases. No major complications occurred.

Conclusion: Instrument guidance and PRF-based thermometry of PLDD in the lumbar spine are feasible and accurate. Using the open high-field MRI with fast TSE and GRE sequence designs may render laser diskectomies more effective and controllable.

$$
\text { 10:30 - 12:00 }
$$

Room E1

\section{Neuro}

\section{SS 1211}

\section{Added value of DTI and fMRI in brain and spine}

\section{Moderators:}

A. Bizzi; Milan/IT

D. Seixas; Vila Nova de Gaia/PT

\section{B-511 10:30}

Morphological subgroups in Alzheimer's disease based on the precuneus atrophy

A. Krainik, O. Moreaud, S. Cantin, A. Memin, S. Keignart, O. Eker, S. Grand, J.-F. Le Bas; Grenoble/FR (akrainik@chu-grenoble.fr)

Purpose: To determine the relationships between the precuneus atrophy (PCA) and common imaging parameters (hippocampal atrophy and microangiopathy) in patients with Alzheimer's disease (AD) and prodromal AD.

Methods and Materials: 105 patients suspected of $A D$ and prodromal $A D$ according to the NINCDS-ADRDA and Petersen's criteria had a brain MRI, including T2-WI, T2*-WI, FLAIR, DWI, and 3D-GRE T1. Hippocampal atrophy was rated by two radiologists using the Scheltens's score (0-4), the microangiopahty with the Fazekas's score (0-3). The precuneus atrophy (PCA) was rated using an original visual score based on the antero-posterior enlargement of the cingular sulcus, ranging from -2 (anterior $>$ posterior enlargement) to +3 (anterior $<$ posterior enlargement). Results: The precuneus atrophy was defined by a PCA score $\geq 2$. Thus, 2 groups were identified: $P C A+$ for patients with atrophy $(n=53)$, and PCA- for patients without $(\mathrm{n}=52)$. No difference for gender and age was detected with: male/female $(\mathrm{PCA}+)$ $=24 / 29 ; \mathrm{M} / \mathrm{F}(\mathrm{PCA}-)$ 23/29; age $(\mathrm{PCA}+)=74.2 \pm 8.0$, age $(P C A-)=75.3 \pm 10.3$. Significant differences were detected for hippocampal atrophy with Scheltens's (PCA+) $=2.5 \pm 0.9$, Scheltens's $(P C A-)=2.9 \pm 0.8(p=0.04)$, and for microangiopathy with Fazekas's $(\mathrm{PCA}+)=0.6 \pm 0.8$, Fazekas's $(\mathrm{PCA}-)=1.5 \pm 0.9(\mathrm{p}<0.001)$. The PCA score was negatively correlated with the severity of both the hippocampal atrophy ( $R$ Pearson $=-0.26 ; p=0.01$ ), and the microangiopathy ( $R$ Pearson $=-0.44 ; p<0.001)$. Conclusion: In patients with $A D$ and prodromal $A D$, at least two distinct patterns can be identified based on the occurrence of the precuneus atrophy. When present, the microangiopathy is less severe, suggesting different pathophysiological mechanisms. This result may help to better distinguish mixed dementia from AD, and to constitute homogeneous populations in future therapeutics trials.

\author{
B-512 10:39 \\ Impaired cerebral vasoreactivity to $\mathrm{CO}_{2}$ in Alzheimer disease using BOLD \\ fMRI
}

S. Cantin, M. Villien, O. Moreaud, I. Tropres, S. Keignart, E. Chipon,

J.-F. Le Bas, J. Warnking, A. Krainik; Grenoble/FR (scantin@chu-grenoble.fr)

Purpose: To evaluate the cerebral vasoreactivity (CVR) using BOLD fMRI during carbogen inhalation with $7 \% \mathrm{CO}_{2}$ in Alzheimer disease (AD) and mild cognitive impairment $(\mathrm{MCl})$.

Methods and Materials: Thirty nine subjects were included to be investigated using BOLD fMRI (1.5 T) during a block-design carbogen inhalation paradigm, with a high concentration face-mask under physiological monitoring. Basal cerebra perfusion was measured using pulsed arterial spin labeling (PASL) with a Q2TIPS sequence. Data were analyzed using Matlab® and SPM5 with the group-average capnia responses as regressors and corrected for gray-matter partial volume. Results: Among participants, 12 subjects were excluded because of incomplete protocol, leaving 27 subjects without significant microangiopathy diagnosed for AD $(n=9), M C l(n=7)$, and matched controls $(n=11)$ to be analyzed. No adverse reaction related to the $\mathrm{CO}_{2}$ challenge was reported. Carbogen inhalation induced a whole-brain BOLD signal increase, predominant in the gray matter. In patients, BOLD responses were decreased $(0.36 \pm 0.13 \% B O L D / m m H g$ in $A D, 0.36 \pm 0.12$ in $\mathrm{MCl}, 0.62 \pm 0.20$ in controls). CVR impairments were diffuse but seemed predominant in posterior areas. The basal hypoperfusion in AD was not significantly different from $\mathrm{MCl}$ and controls. Among clinical and biological parameters, the effect of the apoE4 genotype was not significant. CVR values were correlated with cognitive performances and hippocampal volumes.

Conclusion: This BOLD fMRI study on $\mathrm{CO}_{2}$ challenge shows impaired CVR in $A D$ and $\mathrm{MCl}$. This new fMRI approach may help to better characterize patients with cognitive disorders and further investigate vaso-protective therapeutics.

\section{B-513 10:48}

Diffusion tensor tractography indicates cervical spinal cord alterations in patients with amyotrophic lateral sclerosis

W. Van Hecke ${ }^{1}$, C. Sage ${ }^{2}$, L. Emsell ${ }^{3}$, P.M. Parizel ${ }^{1}$, S. Sunaert ${ }^{1} ;{ }^{1}$ Antwerp/BE, ${ }^{2}$ Leuven/BE, ${ }^{3}$ Galway/IE (wim.vanhecke@ua.ac.be)

Purpose: To study the cervical spinal cord involvement in patients with amyotrophic lateral sclerosis (ALS) using diffusion tensor imaging (DTI) and tractography. Methods and Materials: 28 ALS patients (14 M, $14 \mathrm{~F})$ with age range between 35 and 78 years, average time from onset of 14 years, and a mean score on the revised ALS functional rating scale of 39.7 were examined. Data sets of 26 healthy age- and sex-matched controls (11 M, $15 \mathrm{~F}$ ) were also acquired. DTI data sets were acquired on a $3 \mathrm{~T}$ scanner, with 16 diffusion-weighted images, resolution of $0.98 \times 0.98 \times 1.2 \mathrm{~mm}^{3}$ and a b-value of $800 \mathrm{~s} / \mathrm{mm}^{2}$. Regions of interest (ROIs) were used to delineate the cervical spinal cord on the non-diffusion weighted images. Tractography was then performed on these ROls and fractional anisotropy (FA), mean diffusivity (MD), longitudinal (E1), and transverse eigenvalues (E23) were calculated along the tracts.

Results: FA, L23, and MD values were significantly different in the cervical spinal cord between the ALS subjects and the controls $(p<0.05)$. The FA was significantly higher and the L23 and MD significantly lower in the control group compared to the ALS patients. No differences were found between L1 of both groups. All diffusion measures were correlated with the ALS functional rating scale and the disease duration. Although some trends were observed, no significant correlations were found. Conclusion: This DTI study demonstrated the spinal cord involvement on patients with ALS, in which a lower FA and a higher L23 and MS were observed compared to control subjects.

\section{B-514 10:57}

A quantitive analysis of neurovascular compression in trigeminal nerve: 3-T MR imaging using DTI sequence

C. You, W.M. Chai, L. Tan, K.M. Chen, Z.Y. Wu, H. Wang; Shanghai/CN (youchao8888@yahoo.com.cn)

Purpose: As neurovascular compression (NVC) is not only observed in trigeminal neuralgia (TN) patients, but also in some asymptomatic individiduals, the aim of this study was to identify, using diffusion tensor imaging (DTI), functional characteristics of NVC in patients with and without TN.

Methods and Materials: By using 3D-TOF and 3D-FIESTA sequence in 3.0 T MR 40 patients with unilateral TN, 40 asymptommatic subjects with unilateral NVC and 40 asymptomatic subjects without NVC were enrolled in this study. Meanwhile, all the patients also underwent coronal DTI scanning for quantitive analysis of bilateral trigeminal nerve. The values of fractional anisotropy (FA) and mean diffusivity (MD) 
were measured on coronal images of four continuous slices in which the trigeminal nerves from the root exit point to the distal cisternal segment were clearly shown. Results: A significant reduction in $\mathrm{FA}(\mathrm{t}=-0.987, \mathrm{p}<0.001)$, as well as a significant increase in MD ( $t=3.266, p=0.002)$ were observed in the affected nerve of $T N$, while either health volunteers with NVC or normal subjects without NVC did not show any difference of FA and MD in both sides.

Conclusion: The preliminary study of DTI demonstrates that trigeminal NVC may occur in asymptomatic patients, but functional abnormality is only detected in affected nerve with both TN and NVC. The application of DTI could be useful for diagnostic and therapeutic decisions in TN, and it could also help to understand its pathogenesis.

\section{B-515 11:06}

Diffusion tensor weighted MRI of optic nerve in MS patients

Y. Anik, O. Temel, A. Demirci, H. Efendi, Z. Sakci, S. Gumustas; Kocaeli/TR (yoncaanik@yahoo.com)

Purpose: The aim of this study is to evaluate the optic nerve in multiple sclerosis patients with diffusion tensor imaging and to correlate fractional anisotropy (FA) and apparent diffusion coefficient (ADC) values with visual acuity.

Methods and Materials: Forty MS patients; 20 that had optic neuritis attack previously and 20 that had no optic neuritis attack before are included. As a control group 20 healthy volunteered patients without eye pathology were integrated. Al patients were examined with $3 \mathrm{~T}$ MR equipment with 8-channel head coil with conventional sequences and DTI sequence FA and ADC values of both optic nerves were calculated in all patients. Findings were correlated with visual acuity. Results: In MS patients with optic neuritis attack previously, FA values of the affected optic nerves were significantly decreased compared with that of the contro group $(p<0.001)$ and with clinically unaffected contralateral nerves $(p<0.01)$. Also significant correlation was found among FA reduction and visual acuity $(p<0.01)$. Conclusion: Axonal disruption of the affected nerve in MS patients can be demonstrated via DTI FA measurements.

\section{B-517 11:15}

Diffusion tensor imaging differences between prosocial and individualistic-oriented healthy subjects

W. Van Hecke, G. Emonds, C.H. Declerck, C. Boone, P.M. Parizel; Antwerp/BE (wim.vanhecke@ua.ac.be)

Purpose: To investigate white matter differences in subjects with a "prosocial" and an "individualistic" character orientation. These differences were examined using a voxel-based diffusion tensor imaging (DTI) approach.

Methods and Materials: 28 healthy subjects, aged between 19 and 33 years, were recruited. Social value orientation was assessed by means of the "decomposed games measure". As a result, two groups were determined: 14 subjects with strong prosocial orientation, and 14 subjects with strong individualistic orientation. DTI data sets were acquired on a 3 T scanner with 64 diffusion directions, resolution of $2 \times 2 \times 2 \mathrm{~mm}^{3}$ and a b-value of $900 \mathrm{~s} / \mathrm{mm}^{2}$. All data sets were aligned to a DTI atlas with a non-rigid coregistration algorithm. After anisotropic smoothing with a kernel of $3 \mathrm{~mm}$, FA values were compared between both subject groups in every voxel. Clusters of at least 10 voxels with a $p<0.005$ were considered as significant. Results: FA differences between both groups were found in the inferior longitudinal fasciculus, thalamus, forceps minor, forceps major, fornix, superior longitudinal fasciculus, and corona radiata.

Conclusion: Based on diffusion tensor measurements, there are differences in structural organisation of white matter fiber tracts between healthy subjects with a prosocial and individualistic character orientation.

\section{B-518 11:24}

Altered amplitude of low-frequency fluctuation in adult developmental stuttering

C. Yu; Tianjin/CN (chunshuiyu@yahoo.cn)

Purpose: Although developmental stuttering (DS) has been extensively studied using structural and task-based functional magnetic resonance imaging (fMRI), there is no study focused on the resting-state brain activity in this disorder or the effect of family history of stuttering on brain imaging alterations. Using the amplitude of low-frequency fluctuation (ALFF) as a measure of resting-state brain activity, we aim to investigate changes of the ALFF in adults with DS and the effect of family history of stuttering on this measure.

Methods and Materials: Forty-nine adults with DS and 51 age- and gendermatched fluent controls were scanned using fMRI. In a voxel-wise manner, the ALFF was compared among stuttering subjects with and without family histories, and fluent controls.
Results: Stuttering subjects showed increased ALFF in the left inferior frontal gyrus, superior, middle and inferior temporal gyri, ventral sensorimotor and Rolandic operculum cortices, and right superior and middle frontal gyri, and decreased ALFF in the bilateral paracentral lobules and supplementary motor areas, and the right occipital lobe. Stuttering subjects with and without family histories had differential distributions and patterns of abnormal ALFF relative to control subjects. Moreover, when directly compared with those without family histories, stuttering subjects with family histories showed significantly increased ALFF in the right auditory- and speech-related areas and decreased ALFF in the semantic-related brain areas. Conclusion: We suggest stuttering subjects with and without genetic propensity have differential alterations in brain spontaneous neuronal activity.

\section{B-519 11:33}

Functional MRI of regional brain responses to pleasant and unpleasant IAPS pictures: different networks

F. Aldhafeeri, J. Alghamdi, V. Sluming; Liverpool/UK

(F.M.Aldhafeeri@liverpool.ac.uk)

Purpose: The main purpose of this study was to evaluate the efficiency of IAPS pictures and to investigate the neural substrates of positive and negative emotions Methods and Materials: We have scanned 14 healthy volunteers (8 males and 6 females) using Trio MRI scanner (Siemens, Germany). High-arousing pleasant pictures $(N=100)$ and high-arousing unpleasant pictures $(N=100)$ have been selected from IAPS. The experimental paradigm consisted of 10 blocks of emotionally-evocative visual stimuli in addition to neutral conditions in which blank screen with cross hair is presented. The stimulus duration was $21 \mathrm{~s}$, during which seven pleasant/unpleasant visual stimuli presented for 3s. Images were analysed using Brain Voyager QX software package (Brain Innovation, Maatricht the Netherlands). We compared BOLD activation patterns to complex emotional pictures to determine if these stimuli would activate similar or distinct brain regions Results: Pleasant visual stimuli showed significant BOLD signal reduction in the visual cortex $\mathrm{P}$ ( $<0.05$ with increased BOLD signal in the: PFC and posterior cingulated cortex. The unpleasnt visual stimuli demonstrated statistically significant, $\mathrm{P}$ ( gyrus and cuneus. However, extensive increase in BOLD signal, $\mathrm{P}$ (

$<0.05$, was observed in: visual cortex, parietal lobe, hippocampus, amygdale, temporal lobe and frontal lobe.

Conclusion: Findings from our study prove the efficiency of IAPS pictures in promoting emotional centres in the brain. Although BOLD signal reduction is poorly understood, our findings demonstrate that positive and negative emotions can be distinguished using fMRI.

\section{B-520 11:42}

Functional connectivity MRI (FC-MRI) predicts linguistic performance after surgical treatment for drug-resistant epilepsy

E. Pravata'1, M. Caulo², C. Sestieri², D. Mantini², C. Briganti²,

G. Colicchio ${ }^{1}$, A. Tartaro ${ }^{2}$, G. Romani ${ }^{2}$, C. Colosimo $1{ }^{1}$ Rome $/ I T,{ }^{2}$ Chieti/lT (emanuele.pravata@gmail.com)

Purpose: Functional connectivity (FC) of the language network (LN) is known to be reduced in the brain of epileptic patients and to positively correlate with linguistic performance. We investigated, in a group of patients with drug-resistant epilepsy, the existence of correlations between the pre-surgical LN FC and the post-operative linguistic outcome.

Methods and Materials: We retrospectively evaluated the FC of the LN of twelve right-handed patients with non-tumoral intractable epilepsy. FC was estimated between six common cortical regions for language in the frontal and temporal lobes of both hemispheres. Correlation between FC z-scores and post-operative (6+ months) neuropsychological follow-up vIQ scores (WAIS-R) was estimated using Pearson's test

Results: All patients experienced no seizures during the follow-up period. A negative correlation was found between the post-operative verbal-IQ scores and the preoperative mean inter-hemispheric FC $(r=-0.56 ; p=0.04)$ whereas no significant correlation was found with the mean intra-hemispheric FC $(p>0.05)$.

Conclusion: A reduced FC of the $\mathrm{LN}$ in the dominant hemisphere of epileptic patients is correlated with a low verbal scores, as opposite a pre-operative high inter-hemispheric FC seems to predict a poorer linguistic outcome after surgery. Including an MRI assessment of the FC of the LN may help to better stratify the post-operative risk of language deficit. 


\section{0:30 - 12:00}

\section{GI Tract}

\section{SS 1201b}

\section{Colon and rectum: a multimodality perspective}

Moderators:

E. Fraile Moreno; Madrid/ES

F. lafrate; Rome/lT

\section{B-521 10:30}

Time-intensity curve parameters in colorectal tumour measured by double contrast-enhanced ultrasonography: correlations with tumour angiogenesis

H. Zhuang, Z.-G. Yang; Chengdu/CN (zhuanghua120@yahoo.cn)

Purpose: To investigate the value of double contrast-enhanced ultrasonography (DCEUS) in evaluation of perfusion status of the colorectal tumours, in which a kind of luminal contrast agent was used combined with SonoVue, and to assess the correlation between time-intensity (TIC) parameters and tumour angiogenesis. Methods and Materials: Thirty-nine patients with colorectal tumours were examined preoperatively using DCEUS. Of the 39 tumours examined, 32 were adenocarcinoma and 7 were adenoma. The arrival time (AT), time to peak (TTP), peak intensity (PI) and area under the curve (AUC) in colorectal tumours were measured. The post-surgical specimens were assessed for microvessel density (MVD) and vessel endothelial growth factor (VEGF). Correlation between TIC parameters and the expression of VEGF or MVD were studied.

Results: The mean values of AT, TTP, PI and AUC of the colorectal tumours were $14.32 \pm 11.36 \mathrm{~s}, 30.61 \pm 18.65 \mathrm{~s}, 20.38 \pm 17.45 \mathrm{~dB}$ and $221.10 \pm 156.09$, respectively. Both AUC and MVD were significantly higher in colorectal adenocarcinomas than in adenomas (all $p<0.05$ ). There was a positive linear correlation between AUC and MVD in colorectal tumours and adenocarcinomas $(r=0.686, p=0.0019$; and $r=0.736, p=0.0235$, respectively). No correlation was found between VEGF and any ultrasound perfusion parameter.

Conclusion: DCEUS is a valuable method for evaluation of the tumour angiogenisis in colorectal tumours in vivo. AUC has a positive linear correlation with MVD and could form a new index for assessing angiogenesis and the biological behaviour of colorectal tumours.

\section{B-522 10:39}

Intersegment variation in MRI-derived perfusion in normal colon S.A. Taylor, R. Hafeez, S. Punwani, M. Steward, P. Boulos, S. Halligan; London/UK (csytaylor@yahoo.co.uk)

Purpose: Abnormal contrast enhancement on MRI is advocated as a biomarker for inflammation in colitis, although perfusion kinetics of normal colon are poorly described. The purpose was to assess enhancement patterns in normal colon and test for inter segmental differences.

Methods and Materials: 11 patients (mean age 61) without prior history of inflammatory bowel disease underwent standard MR colonography followed by norma same day colonoscopy. Acquired sequences included a volumetric interpolated breath hold examination (VIBE) to encompass the whole colonic volume, performed three times prior to and every 30 seconds following intravenous Dotarem for 210 seconds. A region of interest was placed in each of 6 colonic segments and perfusion curves (averaged across all patients) were generated by plotting signal intensity [SI] (normalised to the maximum value in each segment) agains time. Curve morphology was analysed and SI compared across segments using Mann Whitney statistics.

Results: There was a distinct difference between the right colon (caecum and ascending) which showed typical type 2 curve morphology (peaking at $150 \mathrm{sec}$ onds followed by a plateau phase) and the left colon (rectum, sigmoid, descending and transverse) which demonstrated type 1 morphology (peak enhancement at 210 seconds, no plateau). There was a significant difference in normalised SI at 180 and 210 seconds between the right colon (mean 0.81 ) and left colon (mean $0.92), p=0.02$

Conclusion: Normal colon shows intersegmental differences in perfusion kinetics which should be considered when used as a biomarker for colonic inflammation.

\section{B-523 10:48}

Referral rate of CT colonography versus double-contrast barium enema in a university hospital over a 7-year period

E. Picano, E. Neri, L. Faggioni, P. Vagli, C. Bartolozzi; Pisa/IT

Purpose: To assess the variation of referral rate of CT Colonography (CTC) examinations at our university hospital over a 7-year period and its economical impact on the local national healthcare system (NHS), compared with double-contrast barium enema (DCBE)

Methods and Materials: We retrospectively searched annual activity registries of the diagnostic and interventional radiology unit of our university hospital from January 2003 to March 2010 for CTC and DCBE examinations. Until April 2008 CTC had been obtained on a 4-row CT scanner (LightSpeed CT: GE Healthcare, Milwaukee, WI), and subsequently on a 64-row CT scanner (LightSpeed VCT: GE Healthcare) using a low-radiation dose protocol, while all DCBE studies had been carried out on a MTX20E system (Italray, Florence, Italy). The number of CTC and DCBE examinations per year was calculated and their cost to the NHS was estimated based on current local prices.

Results: A total of 2504 CTC examinations were performed. The number of CTC studies rose from 78 in 2003 to 600 between January and March 2010, with a mean annual growth rate of 74.6 new CTC. In parallel, referral to DCBE increased at a mean growth rate of 32.4 new examinations per year, i.e. less than half that of CTC. The mean cost of each CTC and DCBE examination is 186 Euro and 103 Euro, respectively.

Conclusion: CTC is being increasingly used as the imaging technique of choice for detection of colonic lesions, although it is still more expensive than conventional DCBE.

\section{B-524 10:57}

Chronic diverticulitis versus colorectal cancer: findings on CT colonography

S.E.H. Cremers ${ }^{1}$, S. Gryspeerdt ${ }^{2}$, P. Lefere ${ }^{2}$, S. Houterman ${ }^{3}$, G. Slooter ${ }^{3}$, M.L. Verhulst ${ }^{3}$, P.T.J. Cremers 3 ; ${ }^{1}$ Dordrecht/NL, ${ }^{2}$ Hooglede/BE, ${ }^{3}$ Veldhoven/NL (simonecremers@hotmail.com)

Purpose: In CT colonography chronic diverticulitis (CD) can mimic colorectal cancer (CRC) masses. In order to distinguish these two entities several findings were analysed. Methods and Materials: 318 symptomatic patients consecutively underwent CT colonography between June 2008 and August 2010. We included all patients with pathologic confirmation of CD (9) and CRC (26) on surgical specimens. The images were analysed by a consensus of two reviewers. The following findings were evaluated: length of the mass, diverticula included in the mass, growth pattern, lumina narrowing, presence of perilesional lymph nodes and mesenteric fat stranding Results: There was a significant difference in median length of the mass between $C D$ and $C R C, 95$ and $49 \mathrm{~mm}$, respectively $(p=0.001)$. Diverticula included in the mass were visible in all patients with $C D$, but in none of the patients with $C R C$ $(p<0.001)$. Furthermore, a curved growth pattern of the mass was noted in al but one patient with $C D$ versus two out of 26 patients with CRC $(p<0.001)$. There were no significant differences between the groups with respect to median lumina narrowing $(85 \mathrm{vs} 77 \%, p=0.5)$, presence of perilesional lymph nodes $(p=0.6)$ and presence of perilesional mesenteric fat stranding $(p=0.4)$

Conclusion: To differentiate $\mathrm{CD}$ and $\mathrm{CRC}$ masses the length of the mass, inclusion of diverticula and growth pattern are important discriminating factors.

\section{B-525 11:06}

Contrast-enhanced multidetector computed tomography (CE-MDCT) and virtual colonoscopy $(\mathrm{VC})$ in preoperative $\mathrm{T}$ staging of colorectal cancer N. Flor, M. Mezzanzanica, A. Ceretti Pisani, G. Di Leo, F. Sardanelli, G. Cornalba, S. Bosari, F. Villa, P. Rigamonti; Milan/IT (flornic@hotmail.com)

Purpose: To predict the T stage of colorectal cancer (CRC) using CE-MDCT with VC

Methods and Materials: Forty-four patients with CRC underwent 64-MDCT using faecal tagging. Apple-core deformity (ACD) involving $\geq 50 \%$ of the lumen on double contrast enema-like VC and inhomogeneous peritumoral fat (IPF) on multiplanar reconstructions were considered suggestive of $\mathrm{T}$ stage $\geq 3$ by two independent readers. One of them repeated the evaluation after two weeks. Pathological $T$ stage was the standard of reference. McNemar and Cohen $\mathrm{k}$ statistics were used. Results: Pathology revealed T1 $(n=1)$, T2 $(n=9)$, T3 $(n=28)$, and T4 $(n=6)$. The more experienced reader found: 8 cases without ACD or IPF, with a predictive value for $\mathrm{T} \leq 2$ of $7 / 8(0.88,95 \%$ confidence interval $[\mathrm{Cl}] 0.47-1.00) ; 19$ cases of ACD without IPF with a predictive value for T3 of $16 / 19(0.84 \% 95 \% \mathrm{Cl} 0.60-0.97)$, the remaining 3 cases being T2; 17 cases with both $A C D$ and IPF with a predictive 
value for $\mathrm{T} \geq 3$ of $17 / 17(1.00,95 \% \mathrm{Cl}, 0.81 \%-1.00), 6$ of them (35\%) being $\mathrm{T} 4$; no cases of IPF without ACD. Accuracy of ACD for T $\geq 3$ was $40 / 44(0.91,95 \%$ $\mathrm{Cl} 0.78-0.98)$, that of IPF $27 / 44(0.61,95 \% \mathrm{Cl} 0.46-0.76)(\mathrm{p}<0.001)$. Intra- and interobserver reproducibilities were excellent for $A C D(k=1 ; k=0.93$, respectively) and good for IPF ( $\mathrm{k}=0.69 ; \mathrm{k}=0.53$ ).

Conclusion: Both variables are highly reproducible. The presence of ACD outperformed that of IPF in predicting a T stage $\geq 3$. The co-existence of ACD and IPF is highly suggestive of $\mathrm{T} \geq 3$, with about one-third of $\mathrm{T} 4$.

\section{B-526 11:15}

The impact of $18 \mathrm{~F}$ FDG-PET/CT in high and low risk primary rectal cancer C.J. Hunter ${ }^{1}$, A. Garant ${ }^{2}$, T. Vuong ${ }^{3}$, G. Artho ${ }^{3}$, R. Lisbona ${ }^{3}$, M. Abulafi ${ }^{1}$, G. Brown ${ }^{4} ;{ }^{1}$ Croydon/UK, ${ }^{2}$ Quebec, QC/CA, ${ }^{3}$ Montreal, QC/CA, ${ }^{4}$ Sutton/UK (chris_j_hunter@hotmail.com)

Purpose: FDG-PET/CT is not currently recommended for routine staging of rectal cancer. We evaluated the diagnostic yield of FDG-PET/CT in MRI staged high and low risk rectal cancers.

Methods and Materials: In a multicentre prospective observational trial, 236 patients with rectal cancer were recruited to a study evaluating FDG-PET/CT. All patients underwent MRI staging and were stratified into high and low risk (high risk: extramural venous invasion, extramural spread $>5 \mathrm{~mm}$ or T4, pelvic sidewall lymph nodes, N2 or involved circumferential resection margin). The primary aim was to evaluate detection rates of metastatic disease using FDG-PET/CT. The secondary aim was to determine the diagnostic yield of FDG-PET/CT according to MRI risk features.

Results: Imaging data were available for 230/236 (97.5\%) patients. Overall, FDG$\mathrm{PET} / \mathrm{CT}$ did not significantly increase detection of metastatic disease compared with computed tomography for the whole group 33/230 (14.4\%, 95\% $\mathrm{Cl} 10.4-19.5 \%)$ versus $22 / 230(9.6 \%, 95 \% \mathrm{Cl} 6.4-14.1 \%)$. FDG-PET/CT had a significantly higher diagnostic yield for distant metastases in MRI high risk rectal cancers, $(28 / 136$, $20.6 \%, 95 \% \mathrm{Cl} 14.7-28.2)$ versus $(5 / 94,5.3 \%, 95 \% \mathrm{Cl} 2.3-11.9, \mathrm{OR} 6.4, \mathrm{p}=0.001)$ for low risk patients and also identified significantly more metastases not visible on conventional CT (OR 4.6, 95\% $\mathrm{Cl} 1.3-16.2, \mathrm{p}=0.001$ ). In patients with high risk feature on MRI the rate of distant metastases uniquely identified on FDG-PET/ CT was $13.2 \%(95 \% \mathrm{Cl}$ 8.5-20.0).

Conclusion: It is possible to identify a high risk group using rectal MRI features with a high diagnostic yield on PET/CT. Patients in this group have significantly more metastases identified on PET/CT than CT alone.

\section{B-527 11:24}

Influence of FDG PET-CT on management decisions in patients with potentially operable metastatic colorectal cancer

A.F. Scarsbrook, F.U. Chowdhury, R.H. Briggs; Leeds/UK

(andrew.scarsbrook@leedsth.nhs.uk)

Purpose: To assess the impact of FDG PET-CT in patients with potentially resectable metastatic colorectal carcinoma.

Methods and Materials: All patients with potentially resectable metastatic colorectal undergoing FDG PET-CT between November 2007 and March 2009 were retrospectively analysed. Findings on PET-CT were compared to conventional imaging and impact on subsequent patient management was evaluated. Clinical impact was divided into: Major: Detection of occult disease or further characterisation of indeterminate lesion (s); Minor: Confirmation of suspected metastases; No impact. Outcome data was obtained from clinical and radiological databases. Results: 102 patients underwent PET-CT during the study period (mean age 67 years, range 27-85) including 94 patients with liver metastases, 5 patients with isolated lung metastases and 3 patients with limited peritoneal disease. In 31 patients (30\%) PET-CT had a major impact on subsequent management. 16 patients had inoperable disease confirmed, previously unsuspected metastatic disease was detected in 9 patients, 3 patients had occult second primary tumours and 3 patients were down-staged. PET-CT had a minor impact in 12 patients $(12 \%)$ and no impact in 59 cases (58\%). The introduction of PET-CT into the pre-operative imaging algorithm had a statistically significant impact on patient management $(p<0.05$, Chi squared test). At 18 month follow-up 17 patients remained radiologically disease free after liver (15/34) or lung (2/3) surgery.

Conclusion: FDG PET-CT has a valuable role in selected patients with metastatic colorectal carcinoma by improving staging accuracy and characterising indeterminate lesions and can help triage patients to the most appropriate treatment.

\author{
B-528 11:33 \\ Whole-body imaging for the detection and evaluation of metastatic and \\ recurrent disease in the follow-up for colorectal cancer \\ M. Maas, D.M.J. Lambregts, I.J.G. Rutten, P.J. Nelemans, V.C. Cappendijk, \\ G.L. Beets, R.G.H. Beets-Tan; Maastricht/NL (moniquemaas@live.nl)
}

Purpose: Up to $40 \%$ of patients with colorectal cancer (CRC) develop metastases/ recurrence. Early and accurate identification is important because survival is improved when metastases/recurrences are curatively resected. The aim of this study is to determine which is the most accurate whole-body imaging (WBI) modality for detection of recurrent/metastatic disease during follow-up of patients with CRC. Methods and Materials: Medline and Embase were searched. Studies evaluating the accuracy of WBI with CT, PET-CT, MRI and/or PET for metastasis/recurrence detection during follow-up for $\mathrm{CRC}$ were included. Studies evaluating liver only or response to therapy were excluded. $2 \times 2$ contingency tables were extracted and summary receiver operator characteristics (sROC)-curves with area-under-thecurves $(A \cup C)$ were constructed by a regression model. Subgroup analyses were performed to correct for heterogeneity.

Results: 14 studies (published between 1997 and 2009) were included, some of which investigated multiple modalities. Grouping the articles according to modality, 12 investigated PET, 7 CT, 5 PET/CT and one MRI. Study populations comprised of patients with high suspicion for metastasis/recurrence. All studies used histopathology and clinical and radiological follow-up as reference method. PET-CT and PET both had an AUC of 0.94. CT had significantly lower performance: AUC 0.79 , $p<0.05$. After correction for sources of heterogeneity CT remained the modality with lowest diagnostic performance.

Conclusion: PET-CT and PET have the highest diagnostic performance for the detection of metastasis/recurrence during follow-up of CRC patients with high suspicion for metastasis/recurrence. Particularly in these high-risk populations, CT has significantly lower diagnostic performance, partly due to its limitations in the detection of extrahepatic metastases.

\section{B-529 11:42}

Evaluation of MR diffusion-weighted imaging in differentiating endometriosis infiltrating the bowel from colorectal carcinoma M.P.H. Busard, V. Mijatovic, C. van Kuijk, M.C.G. Bleeker, P.G.A. Hompes, J.H.T.M. van Waesberghe; Amsterdam/NL (m.busard@vumc.nl)

Purpose: Endometriosis infiltrating the bowel may simulate colorectal carcinoma in specific cases. The aim of this study was to evaluate MR DWI in differentiating endometriosis infiltrating the bowel from colorectal carcinoma.

Methods and Materials: In 66 patients, MR DWI was added to the standard imaging protocol in patients visiting our outdoor MR clinic for the analysis of suspected or known deep infiltrating endometriosis (DIE). A total of 15 patients were diagnosed with DIE infiltrating the bowel. High b-value diffusion-weighted images were qualitatively assessed by two readers in consensus and compared with high b-value diffusion-weighted images in 15 patients evaluated for colorectal carcinoma. In addition, ADC values of lesions were calculated (b-values of $50,400,800 \mathrm{~s} / \mathrm{mm}^{2}$ ). Results: Endometriosis infiltrating the bowel showed low signal intensity on high b-value diffusion-weighted images, whereas colorectal carcinoma showed high signal intensity on high b-value diffusion-weighted images. Mean ADC value in endometriosis infiltrating the bowel $\left(0.80 \pm 0.06 \times 10^{-3} \mathrm{~mm}^{2} / \mathrm{s}\right)$ was significantly lower compared with mean ADC value in colorectal carcinoma $\left(0.86 \pm 0.06 \times 10^{-3} \mathrm{~mm}^{2} / \mathrm{s}\right)$ but with considerable overlap between ADC values.

Conclusion: Only qualitative assessment of MR DWI may be valuable to differentiate between endometriosis infiltrating the bowel and colorectal carcinoma.

\section{B-530 11:51}

Rectal cancer: mucinous carcinoma on MR imaging indicates poor response to neoadjuvant chemoradiation

K. Oberholzer, M. Menig, A. Hoetker, A. Kreft, T. Junginger, A. Heintz, H. Schmidberger; Mainz/DE (Andreas.Hoetker@uni-mainz.de)

Purpose: To assess response of locally advanced rectal carcinoma to chemoradiation with regard to mucinous status and local tumour invasion found at pretherapeutic magnetic resonance imaging (MRI).

Methods and Materials: Eighty-eight patients were included in this prospective study (advanced mrT3 and mrT4 carcinomas). Carcinomas were categorised by MRI as mucinous (mucin proportion $>50 \%$ within the tumour volume), and as nonmucinous Patients received neoadjuvant chemoradiation consisting of 50.4 Gy (1.8 Gy/fraction) and 5-fluorouracil. Therapy response was assessed by comparing pretherapeutic MRI with histopathology of surgical specimens (minimum distance between outer tumour edge and circumferential resection margin $=\mathrm{CRM}, \mathrm{T}$ and $\mathrm{N}$ category). 
Results: A mucinous carcinoma was found in 21/88 patients. Pretherapeutic mrCRM was $0 \mathrm{~mm}$ (median) in the mucinous and nonmucinous group. 83/88 patients underwent surgery with tumour resection. The ypCRM $(\mathrm{mm})$ at histopathology was significantly lower in mucinous carcinomas than in nonmucinous carcinomas $(p<=0.001)$. Positive resection margins ( $y p C R M<=1 \mathrm{~mm}$ ) were found more frequently in mucinous carcinomas than in nonmucinous ones $(p<=0.001)$. Treatment had less effect on local tumour stage in mucinous carcinomas than in nonmucinous carcinomas (for $\mathrm{T}$ downsizing $\mathrm{p}=0.012$, for $\mathrm{N}$ downstaging $\mathrm{p}=0.007$ ). Disease progression was only observed in patients with mucinous carcinomas $(n=5)$. Conclusion: Mucinous status at pretherapeutic MRI was associated with a noticeably worse response to chemoradiation and should be assessed by MRI in addition to local tumour staging to predict estimate of treatment before it is initiated.

\section{0:30 - 12:00} Room F1

\section{Genitourinary}

\section{SS 1207 \\ Renal MRI}

Moderators:

O. Buckley; Dublin/IE

S. Moussa; Edinburgh/UK

\section{B-531 10:30}

Arterial spin labelling (ASL) perfusion MRI of renal masses: correlation with histopathological findings

R.S. Lanzman 1 , P. Robson², K. Mentore ${ }^{2}$, A.A. Wagner², D.C. Alsop², I. Pedrosa²; ${ }^{1}$ Düsseldorf/DE, ${ }^{2}$ Boston, MA/US (rotemshlomo@yahoo.de)

Purpose: To assess the value of arterial spin labelling (ASL) perfusion magnetic resonance imaging (MRI) for characterisation of renal masses using histopathological findings as the reference standard.

Methods and Materials: Forty-two patients (34 males, 8 females, mean age $60.1 \pm 11.3$ years) with suspected renal masses were prospectively included in this study. Pseudocontinous ASL images were acquired though the centre of the mass on a 1.5 T clinical MR scanner. Region-of-interest $(\mathrm{ROI})$ analyses were performed to quantify the perfusion of the entire tumour $\left(\mathrm{P}_{\text {whole }}\right)$, the solid portion of the tumour $\left(P_{\text {solid }}\right)$, as well as the maximum tumour perfusion $\left(P_{\text {max }}\right)$.

Results: Histopathologic analysis was available in thirty-four patients that were included in the final analysis. $P_{\text {whole }}$ and $P_{\text {solid }}$ of papillary RCC (32.5 \pm 13.7 and $41.9 \pm 18.4 \mathrm{ml} / \mathrm{min} / 100 \mathrm{~g}$, respectively) were significantly lower and $P_{\text {whole }}$ and $P_{\text {solid }}$ of oncocytomas $(307.9 \pm 74.3 \mathrm{ml} / \mathrm{min} / 100 \mathrm{~g})$ were significantly higher as compared with clear cell $(167.5 \pm 57.8$ and $189.1 \pm 47.7$, respectively), chromophobe $(161.5 \pm 71.4)$ and unclassified $(211.1 \pm 36.4 \mathrm{ml} / \mathrm{min} / 100 \mathrm{~g}) \mathrm{RCC}(\mathrm{p}<0.01) . \mathrm{P}_{\max }$ of oncocytoma $(488.8 \pm 119.7 \mathrm{ml} / \mathrm{min} / 100 \mathrm{~g})$ was significantly higher than $\mathrm{P}_{\max }$ of papillary $(111.3 \pm 60.4 \mathrm{ml} / \mathrm{min} / 100 \mathrm{~g})$, unclassified $(277.8 \pm 78.3 \mathrm{ml} / \mathrm{min} / 100 \mathrm{~g})$ and chromophobe $(280.3 \pm 41.8 \mathrm{ml} / \mathrm{min} / 100 \mathrm{~g}) \mathrm{RCC}$, but did not differ significantly from $P_{\text {max }}$ of clear cell RCC $(332.2 \pm 106.9 \mathrm{ml} / \mathrm{min} / 100 \mathrm{~g})$.

Conclusion: ASL MRI allows for distinction among different histopathological subtypes based on their perfusion level. Oncocytomas demonstrate higher perfusion levels whereas papillary RCC exhibit lower perfusion levels than that of other RCC subtypes.

\section{B-532 10:39}

Static renal BOLD-MRI does not reflect renal function: a prospective study in 246 patients

L. Metzger, U.I. Attenberger, S. Haneder, S.O. Schönberg, H.J. Michaely; Mannheim/DE (henrik.michaely@umm.de)

Purpose: To assess whether static, single measurement renal blood oxygen leveldependent (BOLD)-MRI allows characterisation of the renal function.

Methods and Materials: In this IRB-approved study 246 consecutive patients (164 at $1.5 \mathrm{~T}, 82$ at $3 \mathrm{~T}$ ) underwent transversal BOLD-measurements with a GRE with 12 echo times (TR $=106 \mathrm{~ms}, \mathrm{TE}=5-33 \mathrm{~ms}, 1.4 \times 1.4 \times 4 \mathrm{~mm}^{3}$ spatial resolution). Using OsiriX 3.7 T2*-maps were generated in which ROls were placed in the medulla and cortex of the kidneys. $R 2^{*}$ was calculated as $R 2^{*}=1 / T 2^{*}$. Individual $\mathrm{R} 2^{*}$-values were correlated to the patients' MDRD-eGFR, age and gender for 1.5 $\mathrm{T}$ and 3.0 T seperately. Patients were classified according to the National Kidney Foundation system of chronic kidney disease (CKD).

Results: Measured R2* values at $3.0 \mathrm{~T}$ were on average $50 \%$ higher than at $1.5 \mathrm{~T}$ $(p<0.0001)$. At both field strengths no differences in $\mathrm{R} 2^{*}$ of cortex and medulla were found between male (R2* medulla $22.6 \mathrm{~s}^{-1}$ at $\left.1.5 \mathrm{~T}\right)$ and female $\left(22.3 \mathrm{~s}^{-1}\right)$ patients $(p>0.05)$. At both field strengths, there was no correlation of the $R 2^{*}$-values of cortex and medulla with patients age $(r=0.02)$ or with the eGFR $(r=0.007)$. No differences in $\mathrm{R}^{*}$ * were found between the different CKD-stages (medullary $\mathrm{R}^{*}$ * range $27-33 \mathrm{~s}^{-1}$ at $3 \mathrm{~T}$ and $21-22 \mathrm{~s}^{-1}$ at $1.5 \mathrm{~T}, \mathrm{p}>0.05$ ).

Conclusion: Static BOLD-MRI measurements fail to discriminate between patients with various CKD-stages most likely due to superimposed external factors such as degree of hydration, medications and renal perfusion. For valuable clinical application of renal BOLD-MRI, dynamic pharmacodynamic studies with intraindividual assessment of signal variation seem mandatory

\section{B-534 10:48}

Comparison of Gd-DTPA and Gd-BOPTA for studying renal perfusion and filtration with MR-renography

M. Notohamiprodjo ${ }^{1}$, M. Pedersen ${ }^{2}$, C. Glaser ${ }^{1}$, A.D. Helck ${ }^{1}$

M.F. Reiser ${ }^{1}$, S.P. Sourbron ${ }^{3} ;{ }^{1}$ Munich/DE, ${ }^{2}$ Aarhus/DK, ${ }^{3}$ Leeds/UK (mike.notohamiprodjo@med.uni-muenchen.de)

Purpose: Gadobenate-dimeglumine (Gd-BOPTA) exhibits an increased relaxivity in the presence of serum-proteins. The purpose of this study was to compare GdBOPTA to the unbound contrast agent gadopentate-dimeglumine (Gd-DTPA) for studying renal perfusion and filtration with MR-renography.

Methods and Materials: Eight Danish-Landrace-pigs were examined at $1.5 \mathrm{~T}$ with a saturation-recovery TurboFLASH-sequence (TR $194 \mathrm{~ms}$; TE $0.96 \mathrm{~ms}$; temporalresolution $1 \mathrm{~s}$; acquisition-time $10 \mathrm{~min}$ ) using a double-bolus protocol with Gd-DTPA as first bolus (Magnevist, Bayer Schering) followed by Gd-BOPTA (Multihance, Bracco Spa) as second bolus or vice versa $(n=4 / 4)$. Time between injections was $20 \mathrm{~min}$. Data were fitted to a 2-compartment-filtration model, comprising an individual arterial-input-function (AIF) and taking into account tubular-outflow. Cortical regions-of-interest were semi-automatically defined by threshold-setting on a pixel-based plasma-flow-map. Calculation of split renal plasma-flow $(\mathrm{ml} / 100 \mathrm{ml} / \mathrm{min})$ and -volume $(\mathrm{ml} / 100 \mathrm{ml})$ and glomerular-filtration-rate (GFR, $\mathrm{ml} / 100 \mathrm{ml} / \mathrm{min}$ ) were performed. Statistical analysis was performed with the Wilcoxon-signed-rank-test. Results: No significant differences were found between Gd-DTPA and Gd-BOPTA for plasma-flow (Gd-DTPA: $226.3 \pm 70.0 / G d-B O P T A: 222.9 \pm 44.9)$ and -volume (Gd-DTPA $13.3 \pm 3.3 /$ Gd-BOPTA $13.5 \pm 1.3$ ). Compared to Gd-DTPA, GFR calculated with GdBOPTA was significantly lower by $29.2 \%$ (Gd-DTPA: $26.6 \pm 5.3 /$ Gd-BOPTA: $18.8 \pm 3.5$ ) $(p<0.01)$. There were no significant effects related to the sequence of injection. Conclusion: Perfusion-parameters calculated with Gd-DTPA and Gd-BOPTA are comparable, whereas GFR is systemically underestimated with Gd-BOPTA. The potential explanation lies in the decreased relaxivity of Gd-BOPTA in the absence of serum proteins in the renal ultrafiltrate, so that a lower tubular gadolinium concentration and thus lower filtration is presumed by mistake. This has to be taken into account when comparing MR-renography-studies with different contrast agents.

\section{B-535 10:57}

A new look at kidneys: contrast-enhanced kidney MRI in humans at 7 Tesla L. Umutlu ${ }^{1}$, O. Kraff ${ }^{1}$, S. Orzada ${ }^{1}$, S. Kinner ${ }^{1}$, S. Maderwald ${ }^{1}$, G. Antoch ${ }^{1}$ H.H. Quick ${ }^{2}$, M. Forsting ${ }^{1}$, M.E. Ladd ${ }^{1}$, T.C. Lauenstein ${ }^{1} ;{ }^{1}$ Essen/DE ${ }^{2}$ Nürnberg/DE (Lale.Umutlu@uk-essen.de)

Purpose: The aim of this study was to assess the feasibility of dynamic contrastenhanced renal MRI at 7 Tesla with optimisation and implementation of a dedicated examination protocol.

Methods and Materials: 12 healthy volunteers were examined on a 7 Tesla whole-body MR-system (Magnetom 7T, Siemens) utilising a custom-built 8-channe transmit/receive radiofrequency body coil suitable for RF-shimming. The following non-enhanced sequences were obtained: 1) T1w fs 2D FLASH, 2) T1w 2D in-andopposed phase, 3) True-FISP, 4) T2w TSE. For dynamic imaging, gadobutrol was injected intravenously and T1w 3D FLASH images were obtained pre-contrast and at 20,70 , and $120 \mathrm{sec}$ delay. Qualitative image analysis was performed using a three-point scale ( 1 = poor, 2 = moderate, $3=$ good quality). SNR and CNR of the renal cortex/medulla were measured for all sequences.

Results: 2D FLASH imaging showed best overall image quality with an average score of 2.57 for overall image quality and being least prone to artefacts. Nonenhanced $2 \mathrm{D}$ and $3 \mathrm{D}$ T1w imaging revealed high signal intensity of renal vasculature (arteries: 2.7). Quantitative analysis showed highest CNR between cortex and medulla for arterial phase 3D FLASH imaging $(C N R=12.2)$, providing a statistically significant difference to all other sequences except for the in-and-opposed-phase and the fat-saturated 2D FLASH sequence. Conversely, equilibrium phase FLASH imaging yielded the weakest CNR score of 3.6.

Conclusion: Our results demonstrate the feasibility and diagnostic potential of 7 Tesla kidney imaging, yielding an excellent delineation of anatomical details and non-enhanced vasculature with the potential of a non-enhanced angiographic application. 


\section{B-536 11.06}

Diffusion tensor imaging of the kidneys: influence of b-value and number of encoding directions

N.C. Chuck ${ }^{1}$, O. Donati ${ }^{1}$, G. Steidle ${ }^{2}$, J. Hodler ${ }^{1}$, D. Nanz ${ }^{1}$, A. Boss ${ }^{1} ;{ }^{1}$ Zurich/CH, ${ }^{2}$ Tübingen/DE (natalie.chuck@usz.ch)

Purpose: The purpose of this study was to assess the dependence of image quality and diffusion tensor imaging (DTI) parameters (fractional anisotropy FA mean diffusivity $\mathrm{MD}$ ) on b-value selection and number of encoding directions in DTI of the kidney.

Methods and Materials: Eight healthy volunteers consented to an examination on a 1.5 T whole-body MR scanner (Philips Achieva). After localisation, oblique coronal DTI data sets of the kidneys were acquired with systematic variation of b-value $(300,500$ and $700 \mathrm{~s} / \mathrm{mm} 2)$ and number of diffusion-encoding directions $(6,15$ and 32). A respiratory triggered (breathing pad) single-shot echo planar sequence (TR/ TE $267 \mathrm{~ms} / 67 \mathrm{~ms}$, parallel imaging SENSE factor 4, matrix size $124 \times 128$, scan time 2-6 minutes) was used for DTI data acquisition. Quality of FA and MD maps was assessed on a 4-point score by 2 readers.

Results: In the visual read, the parametrical DTI maps obtained with all evaluated diffusion encoding schemes were attested good quality. Best scores were reached for encoding schemes with 32 encoding directions and b-values of either 500 or 700 $\mathrm{s} / \mathrm{mm} 2$. FA values ranged between 0.42 and 0.46 (MD $1.5-2.3 \times 10(-3) \mathrm{mm}(2) / \mathrm{s})$ for renal medulla and between 0.21 and 0.28 (MD 1.9 - 2.9) for renal cortex. Conclusion: DTI of the kidneys with high $b$-values is feasible using parallel imaging techniques. Best image quality was achieved with 32 encoding directions, which, however, also required longest acquisition times.

\section{B-537 $11: 15$}

Diffusion tensor imaging, tractography and arterial spin labelling in transplanted kidneys

K. Hueper, M. Gutberlet, T. Rodt, W. Gwinner, F. Lehner, D. Hartung,

M. Galanski; Hannover/DE (hueper.katja@mh-hannover.de)

Purpose: Monitoring of kidney transplanted patients is important to early detect allograft damage and finally to ensure long-term allograft survival. In this study, we evaluated MR diffusion tensor imaging (DTI) and arterial spin labelling (ASL) for the detection of acute and chronic changes of allograft microstructure and perfusion. Methods and Materials: 10 kidney transplanted patients with differing degrees of allograft dysfunction and 8 healthy volunteers were examined using a fat-saturated echo-planar DTI-sequence (1.5 T MAGNETOM Avanto, 6 diffusion directions, $b=$ $0.600 \mathrm{~s} / \mathrm{mm}^{2}$ ) and an ASL-sequence (FAIR trueFISP, $\mathrm{TI}=1,200 \mathrm{~ms}$ ). Mean ADC and fractional anisotropy (FA) as well as specific perfusion rate were calculated and compared between healthy and transplanted kidneys. Tractography was performed for visualisation of diffusion properties.

Results: The ADC in the cortex and in the medulla was lower in transplanted than in healthy kidneys $(p<0.01)$. FA in the cortex and in the medulla of healthy kidneys were $0.169 \pm 0.009$ and $0.417 \pm 0.007$. In allografts these values were significantly reduced to $0.122 \pm 0.008$ and $0.264 \pm 0.014(p<0.001)$ and the perfusion was significantly decreased as well $(p<0.01)$. Tractography was able to visualise renal microstructure and functional units and thus allowed for demonstration of pathological changes in the allografts.

Conclusion: The feasibility of DTI and ASL for assessment of renal transplants was demonstrated and differences in diffusion and perfusion properties were shown. Further investigations, e.g. comparison to kidney biopsy results and differentiation of subgroups are intended to evaluate DTI and ASL for routine clinical application.

\section{B-538 11:24 \\ Can diffusion-weighted MRI (DW-MRI) improve the detection of pathologies in transplanted kidneys? \\ M. Triantafyllou, M. Ith, J.M. Froehlich, S. Eigenheer, D. Ott, H.C. Thoeny; Berne/CH (maria_tr2@yahoo.de)}

Purpose: To retrospectively evaluate whether DW-MRI of transplanted kidneys helps for the detection of renal pathologies.

Methods and Materials: A total of 52 transplanted kidneys (46 patients, $12 \mathrm{w} ; 34 \mathrm{~m}$ ) presenting with various pathologies were included. Unenhanced T1-/T2-weighted sequences and coronal EPI-DW-MRI (10 b-values) with automatic calculation of ADC-maps were acquired on a 1.5 T MR-scanner. Qualitative criteria such as homogeneity of renal parenchyma were rated besides detection of focal lesions on both morphological and/or DW-MRI. Mean ADC-values of renal parenchyma and focal lesions were measured. Results were correlated to clinical follow-up (n = 11) and/or histology $(n=41)$.
Results: DW-MRI rated 23/52 kidneys as normal, 29/52 abnormal. Histology/ follow-up attributed pathologies in 41 kidneys. Among abnormal kidneys on DWMRI $(n=29)$ only 5 showed also reduced/absent corticomedullary differentiation on morphology. Pathologies missed on morphological images were rejection (n $=5)$, vascular abnormalities $(n=5)$, reflux $(n=4)$, cyclosporine-A-toxicity $(n=3)$, chronic nephritis $(n=4)$, glomerulonephritis $(n=1)$, pyelonephritis $(n=1)$. In 12 kidneys DW-MRI/morphological imaging missed the pathology. This corresponds to a sensitivity of $72.5 \%$, specificity of $100 \%$, positive-predictive-value of $100 \%$, negative-predictive-value of $47.8 \%$ and diagnostic-accuracy of $79 \%$ for DW-MRI. Pathologies missed on DW-MRI/morphology were cyclosporine-A-toxicity $(n=6)$, vascular abnormalities $(n=4)$, glomerulonephritis $(n=2)$ and rejection $(n=4)$. In 25 kidneys a total of 78 focal pathological lesions were only visible on DW-MR with mean ADC-values beyond $1.88 \times 10^{-3} \mathrm{~mm} / \mathrm{s}^{2}$. Renal parenchyma of abnormal kidneys without focal lesions on DW-MRI $(n=7)$ had mean ADC-values beyond a threshold-value of $2.00 \times 10^{-3} \mathrm{~mm} / \mathrm{s} 2$ (mean: $1.83+/-0.13 \times 10^{-3} \mathrm{~mm} / \mathrm{s}^{2}$ ) indicating diffuse parenchymal injury whereas normal kidneys had a value of $2.03+/-0.13$ $\times 10^{-3} \mathrm{~mm} / \mathrm{s}^{2}$

Conclusion: DW-MRI improves the diagnosis of focal and parenchymal pathologies in transplanted kidneys. Measurement of ADC-values may be a helpful diagnostic tool.

\section{B-539 11:33}

Comparison of three different in-phase and out-of-phase gradient-echo imaging techniques for the characterisation of adrenal lesions at $1.5 \mathrm{~T}$ : preliminary observations

M. Ramalho ${ }^{1}$, R. de Campos$^{2}$, V. Heredia², R. Azevedo², R. Semelka²;

${ }^{1}$ Almada/PT, ${ }^{2}$ Chapel Hill, NC/US (jmpmramalho@gmail.com)

Purpose: To compare the performance of three-dimensional (3D) gradient recalledecho (GRE) and magnetization-prepared (MP) GRE in-phase (IP) and out-of-phase (OP) imaging with standard two-dimensional (2D) GRE, for the characterisation of adrenal lesions, using previously reported quantitative evaluation methods. Methods and Materials: Forty-three consecutive subjects ( 21 males and 22 females; mean age, $59.5 \pm 11.4$ years) with 49 adrenal lesions constituted our study population. Adrenal signal intensity (SI) index, and adrenal-to-spleen, adrenal-toliver, and adrenal-to-muscle SI ratios were calculated for each lesion and compared between the three techniques by using independent samples student- $t$ test. The area under the receiver operating characteristic (ROC) curve (AUC) for each evaluation method was determined and comparisons of independent ROC curves were performed between all sequences.

Results: For all sequences, the mean adrenal SI index and SI ratios were significantly different between adenomas and nonadenomas $(p<0.002)$. For the 3D-GRE and MP-GRE techniques, the adrenal SI index and the modified adrenal-to-spleen ratio showed the higher AUCs, respectively, although not statistically significant. Different thresholds were recommended for discriminating adenomas from nonadenomas among the three techniques.

Conclusion: 3D-GRE and MPGRE IP/OP sequences can be employed for adrenal lesion characterisation with comparable results with the reference standard of 2D-GRE. Different recommended thresholds should be selected accordingly to the used IP/OP technique and for the various evaluation methods.

\section{B-540 11:42}

Quantification of viscoelastic properties of the kidney using supersonic shear wave imaging in an experimental rat model of fibrosis induced by L-NAME

M. Derieppe ${ }^{1}$, Y. Delmas ${ }^{2}$, C. Deminière ${ }^{2}$, S. Placier ${ }^{1}$, J.-L. Genisson ${ }^{1}$,

M. Tanter ${ }^{1}$, C. Combe ${ }^{2}$, N. Grenier ${ }^{2} ;{ }^{1}$ Paris/FR, ${ }^{2}$ Bordeaux/FR (nicolas.grenier@chu-bordeaux.fr)

Purpose: Chronic renal disease is characterised by progressive development of glomerulosclerosis and/or interstitial fibrosis. Renal fibrosis can be assessed by kidney biopsy only. The aim of our study was to validate the use of ultrasonic shearwave elastography in an experimental rat model of glomerulosclerosis induced by chronic administration of L-NAME (inhibitor of nitric oxyde synthesis).

Methods and Materials: Four groups of rats were studied: a control group ( $n=$ $8)$, a group before and after 4 weeks of L-NAME administration $(20 \mathrm{mg} / \mathrm{kg} / \mathrm{day})$ $(\mathrm{H} 4, \mathrm{n}=8)$, a group before and after 6 weeks of L-NAME $(\mathrm{H} 6, \mathrm{n}=15)$, and a group studied longitudinally $(n=9)$ before, after 4 weeks $(L 4)$ and after 7 weeks (L7) of administration. Elasticity of renal cortex was quantified through the shear modulus $(\mu)$ using a $8 \mathrm{MHz}$ probe and ultrafast image sampling. After killing, urines were analysed for dosage of protein/creatinine ratio. Kidneys were removed for histological quantification of fibrosis. 
Results: Diseased rats showed an increased urinary protein/creatinine ratio ( mean $=6.7 \mathrm{mg} / \mathrm{mg}$ ) but moderate glomerulosclerosis at histological examination Cortical elasticity was $4.55 \pm 1.54 \mathrm{kPa}$ in control kidneys. Compared to controls, it was increased in all pathological groups, except for $\mathrm{H} 6: \mathrm{H} 4=6.87 \pm 2.42 \mathrm{kPa}$ $(\mathrm{p}=0.046) ; \mathrm{H} 6=4.79 \pm 1.08 \mathrm{kPa}(\mathrm{p}=0.70) ; \mathrm{L} 4=7.18 \pm 1.51 \mathrm{kPa}(\mathrm{p}=0.012) ; \mathrm{L} 7$ $=7.04 \pm 1.55 \mathrm{kPa}(p=0.012)$. However, considering all animals, no correlation was observed between biological data, degree of glomerulosclerosis and level of cortical elasticity.

Conclusion: Quantification of level of fibrosis by ultrasonic shearwave elastography is feasible. However, more experience is necessary to understand all the factors responsible for the increase of cortical elasticity.

\section{$10: 30-12: 00$} Room F2

\section{Breast}

\section{SS 1202}

\section{Magnetic resonance imaging}

Moderators:

U. Bick; Berlin/DE

E. Taheri; Tehran/IR

\section{B-541 10:30}

Vascular map asymmetry at breast contrast-enhanced MRI: a ROC analysis N. Verardi', R.M. Trimboli' ${ }^{1}$ L.A. Carbonaro ${ }^{2}$, G. Di Leo ${ }^{2}$, F. Sardanelli2; ${ }^{1}$ Milan/IT ${ }^{2}$ San Donato Milanese/IT

Purpose: To introduce a new breast vascular map asymmetry index (VMAI) as a marker of malignancy.

Methods and Materials: 173 consecutive breast MR examinations were reviewed. Using MIP reconstructions (FLASH sequences with $0.1 \mathrm{mmol} / \mathrm{kg}$ of gadobenate dimeglumine), two residents independently assigned a 5-point score at number, length, and diameter of vessels. In the presence of a mass-like lesion, -2 and -1 indicated marked and mild contralateral asymmetry; 0 symmetry; +1 and +2 mild and marked ipsilateral asymmetry. Asymmetry in negative examinations was assigned +1 or +2 . Image analysis was repeated for axial, sagittal, and coronal MIP reconstructions. The score sum over number, length, and diameter of vessels and over the 3 planes was considered as VMAI. Histology/1-year follow-up served as reference standard. Spearman, ROC, Kruskal-Wallis, and reproducibility statistics. Results: 87 (50\%) examinations showed malignant lesions (19 DCIS) and 25 (14\%) benign lesions; the remaining $61(36 \%)$ were negative. For the more experienced resident, ROC-AUC was 0.745 : the VMAI threshold corresponding to a fixed specificity of $93 \%$ was 7.5 (sensitivity $38 \%$; PPV $85 \%$; NPV 60\%). For invasive cancers, the median VMAI was 1 for G1 lesions, 5 for G2, and 12 for $G 3(p<0.001)$. The VMAI correlated with the diameter of malignant lesions $(r=0.507, p<0.001)$. In only $6(3 \%)$ cases the VMAI difference between the two residents was $>3$. Conclusion: Our index is reproducible and correlates with both the diameter of malignant lesions and the histological grade of invasive cancers. Lesions with VMAI $>7.5$ have a high probability $(85 \%)$ of being malignant.

\section{B-542 10:39}

Split dynamic magnetic resonance imaging: a novel method for improved morphologic and functional assessment of breast tumours

K.-I. Gjesdal' ${ }^{1}$ E. Grøvik ${ }^{2} ;{ }^{1}$ Ålesund/NO, ${ }^{2}$ Oslo/NO (k.i.gjesdal@medisin.uio.no)

Purpose: One of the major issues concerning dynamic contrast-enhanced MRI of the breast is the application of either high-spatial resolution for the assessment of tumour morphology or high-temporal resolution for an adequate evaluation of contrast kinetics. These features can only be obtained separately using conventional MRI-techniques, with two injections of contrast agent (CA) causing higher cost for health care and inconvenience for the patients. There is a universal agreement that an optimal combination of both features may improve the diagnostic accuracy in the assessment of breast tumours. This work presents a novel method in which a high-spatial and a high-temporal resolution sequence are applied in an alternating fashion during a single CA injection, allowing a comprehensive assessment of tumour morphology and contrast kinetics, simultaneously.

Methods and Materials: The high-temporal resolution 3D T1 doubleecho EPI-sequence had the following key parameters: TR/TE1/TE2/FA = $42 \mathrm{~ms} / 5.5 \mathrm{~ms} / 23 \mathrm{~ms} / 28^{\circ}$, temporal resolution $=2.8 \mathrm{~s}$. The high-spatial resolution 3D T1-THRIVE sequence used the following parameters: TR/TE/FA = $5.4 \mathrm{~ms} / 2.7 \mathrm{~ms} / 10^{\circ}$, voxel size $=0.85^{\star} 0.85^{\star} 1.25 \mathrm{~mm}^{3}$, number of slices $=125$, temporal resolution $=63 \mathrm{~s}$. Both sequences were performed with fat suppression.
Results: Examination of the high-temporal signal-intensity-time-curves allows the extraction of 4 different pharmacokinetic biomarkers $\left(\mathrm{K}^{\text {trans }}, \mathrm{K}_{\mathrm{ep}}, \mathrm{V}_{\mathrm{e}}, \mathrm{V}_{\mathrm{p}}\right), 5$ different descriptive biomarkers (Wash-in, Wash-out, AUC, TTP, Peak ${ }_{\text {enh }}$ ) and an absolute measurement of the dynamic change in the transverse relaxation rate $\left(R_{2}{ }^{*}\right)$. In addition, the high-spatial resolution images yield an adequate assessment of tumour morphology.

Conclusion: The novel split-dynamic sequence generates high-spatial resolution and high-temporal resolution images during a single CA-injection, providing both morphological features and contrast kinetics simultaneously.

\section{B-543 10:48}

Potential of diffusion MRI as a biomarker of low-risk DCIS

M. $\varliminf^{1}{ }^{1}$, D. Le Bihan'1, R. Okumura², T. Okada'1, K. Fujimoto'1, S. Kanao',

S. Tanaka1', K. Togashi'; ' Kyoto/JP, ${ }^{2}$ Osaka/JP (mamiiima1@gmail.com)

Purpose: To evaluate the potential of breast diffusion MRI as a biomarker of lowgrade ductal carcinoma in situ (DCIS)

Methods and Materials: This study was a retrospective analysis of 19 patients (mean age 55 years) who underwent breast MRI examinations at $1.5 \mathrm{~T}$, and informed consent was waived. MRI scans were performed with a four-channel breast array coil and included contrast-enhanced (Gadoteridol) T1-weighted and diffusion-weighted images (DWI) with b values of 0 and $1,000 \mathrm{~s} / \mathrm{mm}^{2}$. All patients underwent surgical resection and were diagnosed with DCIS based on histopathology (6 low-grade; 7 intermediate-grade; 5 high-grade).

Results: The mean apparent diffusion coefficient (ADC) values (and their 95\% confidence intervals) were $1.43(1.32-1.55) \times 10^{-3} \mathrm{~mm}^{2} / \mathrm{s}, 1.28(1.16-1.41) \times 10$ ${ }^{3} \mathrm{~mm}^{2} / \mathrm{s}, 1.20(1.07-1.32) \times 10^{-3} \mathrm{~mm}^{2} / \mathrm{s}$ and $2.10(1.96-2.22) \times 10^{-3} \mathrm{~mm}^{2} / \mathrm{s}$, for low-, intermediate-, high-grade DCIS and normal breast tissues, respectively. The ADC value of high-grade DCIS was significantly lower than that of low-grade DCIS $(p=0.01)$, and there was a significant negative trend between ADC and tumour grade $(p<0.01)$. A receiver operating characteristic $(R O C)$ analysis gave an ADC threshold of $1.3 \times 10^{-3} \mathrm{~mm}^{2} / \mathrm{s}$ for the diagnosis of high-grade DCIS $(81 \%$ sensitivity, $62 \%$ specificity). Patients whose ADC values were above this threshold in all ROIs were identified as low grade with $100 \%$ specificity.

Conclusion: These results suggest that quantitative DWI could be used to identify patients with low-grade, low-risk DCIS with very high specificity. Such patients could be spared from unnecessary invasive approaches, such as lumpectomy or mastectomy.

\section{B-544 10:57}

Breast MRI at $3 \mathrm{~T}$ : a pilot study to compare unenhanced MRI (DWI combined with a T2 IDEAL sequence) with contrast-enhanced MRI for the assessment of response to neo-adjuvant chemotherapy

F. Pediconi, M. Telesca, V. Casali, F. Vasselli, E. Miglio, L. Ballesio, C. Catalano, R. Passariello; Rome/IT (federica.pediconi@uniroma1.it)

Purpose: To determine sensitivity and specificity of contrast agent free approach at 3 T using only diffusion weighted imaging (DWI) combined with T2w IDEAL images (ueMRI) and to compare findings with contrast-enhanced MRI (CE-MRI) for assessment of response to neoadjuvant chemotherapy.

Methods and Materials: Twelve consecutive patients undergoing neoadjuvant chemotherapy for biopsy-proven breast cancer ( $>2 \mathrm{~cm}$ lesions) underwent MR at $3 \mathrm{~T}$ prior to chemotherapy (every two cycles) and before surgery. MRI protocol comprised FSE-T2w-IDEAL and DWI sequences, and a Vibrant-3D-T1w sequence acquired before and after administration of gadobenate dimeglumine (MultiHance). Tumour response was classified using RECIST criteria based on tumour size at MR imaging. Tumor response at MR imaging after chemotherapy was compared with histological specimens obtained after surgery. Two blinded observers rated images from ueMRI first and then images from CE-MRI for the evaluation of tumou response. Lesion size, ADC values and FSE IDEAL descriptors were assessed. Results: Observers 1 and 2 determined sensitivities of $92 \%$ and $84 \%$, respectively, for ueMRI and $100 \%$ (both observers) for CE-MRI. Similar specificity was noted by the two observers ( $82 \%$ for ueMRI; $86 \%$ for CE-MRI). Differences between methods and between observers were not statistically significant $(P \geq 0.09)$. Similarly, no significant differences in lesion size measurements were noted between sequences. Visibility of malignant lesions was considerably worse using ueMRI. The mean tumour ADC values for responders and non-responders were significantly different after the first II cycles of treatment.

Conclusion: Combined DWI (for tumour density) and T2w IDEAL (for lesion morphology and size) sequences permit high sensitivity and specificity in evaluating tumour response to neoadjuvant chemotherapy although better performance is achieved with CE-MRI.ADC values may be useful for differentiating responders from nonresponders earlier during treatment. 


\author{
B-545 11:06 \\ Value of MRI and diffusion-weighted imaging in evaluating early response \\ of patients with locally advanced breast cancer undergoing recombinant \\ human endostatin chemotherapy \\ J.Q. Xu, Q.X. Jia, Y. Huan; Xi'an/CN (junqingxu@126.com)
}

Purpose: To evaluate the role and the performance of MR and diffusion weighted imaging (DWI) for assessing the response of patients to recombinant human endostatin (endostar, China) chemotherapy in local advanced breast cancer (LABC). Methods and Materials: 96 women with LABC (99 lesions) underwent routine MRI and DWI was carried out at 3.0 T before treatment and after three cycles of endostar chemotherapy. For each patient, analysis of the percentage change in $\mathrm{ADC}$, volume and diameter after each cycle of chemotherapy were compared between the baseline and post-chemotherapy to predict the response to endostar. The residual tumour volumes were obtained using $3 \mathrm{D}$ maximum intensity projections (MIP) of dynamic enhanced scan and DWI map, respectively, and were compared with pathological findings to assess the accuracy of DWI in detecting and measuring residual tumour. All results were proved by histopathological findings.

Results: There were 83 lesions responding to endostar, while 16 non-responders. After the third cycle, the sensitivity for differentiating responders from non-responders was $78 \%$ for volume and $62 \%$ for $A D C$. In the response group, there was a significant difference in ADC value between prior to and post-endostar $(P<0.005)$, while no significant differences were found in non-responders. A cut-off value for $\mathrm{ADC}$, volume and diameter was obtained after the third cycles of endostar.

Conclusion: DWI appears to provide functional information regarding changes in $A D C$ value of tumours due to endostar chemotherapy. DWI may be useful in monitoring the pathological response and evaluating the residual tumour after endostar chemotherapy.

\section{B-546 11:15}

Is oedema reduction a predictive factor for evaluating the response of advanced breast cancer to neoadjuvant chemotherapy?

M. Telesca, F. Pediconi, M. Luciani, V. Casali, E. Miglio, F. Vasselli, C. Catalano, R. Passariello; Rome/IT (mariannatelesca@yahoo.it)

Purpose: To retrospectively evaluate changes in oedema in patients with advanced breast cancer undergoing neoadjuvant chemotherapy (NAC) and to determine whether reduction or absence of oedema can be used to predict final clinic-pathologic response.

Methods and Materials: From January 2005 to 2010, 146 patients with advanced breast cancer associated with oedema were identified. Dynamic MR imaging (MRI) was performed in all patients before and during neoadjuvant chemotherapy. Results were compared with findings from pathologic assessment of surgical samples obtained after NAC. The breast MRI protocol comprised a precontrast T2W STIR sequence and a dynamic 3D T1w FLASH sequence acquired before and at 2-minute intervals after administration of gadobenate dimeglumine (MultiHance) at $0.1 \mathrm{mmol} /$ $\mathrm{kg}$ bodyweight. Patients were divided into 2 groups according to the oedema changes observed during NAC: patients with oedema that appeared reduced or absent and patients with no apparent variation of the oedema.

Results: Of the 146 patients evaluated, 120 had invasive ductal cancer, 18 had lobular cancer, and 8 had mixed ductal/lobular cancer. 114/146 (78.1\%) patients were deemed responders to NAC; in these patients, oedema was reduced or absent in 79/114 (69.3\%) cases. The remaining $32(21.9 \%)$ patients were considered nonresponders to NAC; in these patients, oedema was reduced or absent in $8 / 32(25 \%)$ cases. There was a good correlation between the reduction of oedema on MR imaging during NAC and response to NAC $(p<0.003)$. How was correlation determined. Conclusion: Evaluation of oedema detected on MR imaging is a useful adjunct to findings from conventional $\mathrm{T} 1 \mathrm{w}$ dynamic imaging to accurately assess the pathologic response of breast cancer after NAC.

\section{B-547 11:24 \\ Comparison of MRI and PET-CT scan for evaluation of response to} neoadjuvant chemotherapy in patients with locally advanced breast cancer K.R. Cho, K.W. Hwang, B.K. Seo, O.H. Woo, H.J. Lim, Y.W. Oh, K.H. Park, E.S. Lee, E.K. Park; Seoul/KR (krcho@korea.ac.kr)

Purpose: To compare the accuracy of breast MRI and PET-CT scan in predicting tumour response to neoadjuvant chemotherapy (NAC) and to investigate which modality is more helpful to predict tumour response according to biological status. Methods and Materials: Between January 2008 and March 2010, 20 patients with pathologically confirmed breast cancer who underwent both breast MRI and PET-CT scan before and after receiving NAC were enrolled prospectively in this study. All had undergone surgical treatment after completion of NAC. The tumour response to NAC was assessed with change of tumour size on breast MRI using RECIST. The tumour response to NAC was defined as a $>50 \%$ decline of peak standard uptake value (SUV) on PET-CT based on EORTC. MRI and PET-CT findings were correlated with pathologic results, and their association with biologic markers (ER, PR, and Her2) was evaluated.

Results: Complete, partial and no response was seen in $9(45 \%), 8(40 \%)$ and $3(15 \%)$ patients on breast MRI, and $9(45 \%), 10(50 \%)$ and $1(5 \%)$ patients on PET-CT scan, respectively. MRI was more accurate in predicting tumour response to neoadjuvant chemotherapy than PET-CT (55\%). MRI was more accurate than PET-CT scan in cases of HER2-positive (100\% vs $80 \%$ ) and ER-positive $(57 \%$ vs. $45 \%)$ breast cancer, but identical in cases of triple negative cases $(60 \%$ predictive on both imaging modalities). However, these differences were not significant statistically $(p>0.05)$.

Conclusion: Although there was no statistical significance, breast MRI for predicting tumour response to NAC could be more accurate than PET-CT scan, especially in cases of Her2- and ER-positive groups.

\section{B-548 11:33}

Diagnostic performance of breast MR imaging according to patients' characteristics and radiologists: before and after using computer-aided evaluation system for breast MR imaging

B. Seo', E. Pisano ${ }^{2}$, E. Cole ${ }^{2}$, D. Zeng ${ }^{2}$, K. Cho ${ }^{3}$, C. Kuzmiak2; ${ }^{1}$ Ansan/KR, ${ }^{2}$ Chapel Hill, NC/US, ${ }^{3}$ Seoul/KR (seoboky@korea.ac.kr)

Purpose: To compare diagnostic performance of breast MR imaging before and after the introduction of a breast MRI computer-aided evaluation (CAD) system in an academic diagnostic breast imaging clinic.

Methods and Materials: A total of 445 breast lesions (158 malignant, 287 benign) in 390 patients who underwent contrast-enhanced breast MR imaging at ou institution between 2005 and 2008 were included. 258 of the 445 breast lesions $(58 \%)$ were assessed using a breast MRI CAD system. One of four radiologists assessed morphologic findings and kinetics. In lesions that were evaluated with the CAD system, the lesion was considered positive for malignancy if a lesion had significant enhancement using the $100 \%$ threshold or washout enhancement pattern. We compared diagnostic performance of radiologists before and after the introduction of the CAD system according to patient characteristics and interpreting radiologist. Statistically, univariate and multivariate analyses were performed to compare diagnostic performance before and after introduction of CAD system. Results: The specificity increased after the introduction of the CAD system but not significantly $(p=0.798)$. There was no statistical difference in specificity before and after introduction of CAD controlling for each variable $(p=0.618)$. There was a decrease in sensitivity that approached significance after the introduction of the breast MRI CAD system but only for heterogeneously dense breasts $(p=0.0528)$. The difference in the sensitivity before and after introduction of the CAD system was not significant $(p=0.947)$.

Conclusion: Based on our results, the use of a CAD system for breast MRI does not significantly improve diagnostic performance.

\section{B-549 11:42}

Potential of CAD to predict occurrence of distant metastasis in primary breast cancer

M. Dietzel, P.A.T. Baltzer, R. Zoubi, C. Jerowski, M. Gajda, I.B. Runnebaum, W.A. Kaiser; Jena/DE

Purpose: Breast-MRI is highly sensitive for the detection of breast cancer. Furthermore, it provides functional information on tissue vascularisation. Computerassisted diagnosis (CAD) is feasible to semi-automatically and to quantitatively assess imaging data. This breast-MRI investigation was performed to identify the potential of CAD to predict occurrence of distant metastasis in primary breast cancer. Methods and Materials: We enrolled 59 consecutive patients with primary breas cancer (data acquisition: 01.01.2004 to 31.12.2004). All received one staging breast-MRI examination at 1.5 Tesla according to international guidelines before therapy was initiated. Follow-up-interval and occurrence of distant metastasis was documented. For breast-MRI analysis dedicated commercial software was used (Brevis, cooperation of Siemens \& Mevis, Erlangen/Bremen, Germany). It allows semiautomatic quantitative measurement of breast-MRI enhancement patterns (initial-enhancement, wash-out, peak-enhancement, time to peak enhancemen [TTP]). Results were evaluated to identify significant and independent parameters to predict occurrence of distant metastasis. Significant parameters were further analysed by Kaplan-Meier statistics.

Results: Median follow-up time was 1560 days. 6 patients were diagnosed with metachronous distant metastasis during follow-up. Wash-out above $40 \%$ could be identified as significant and independent predictor for occurrence of distant me- 
tastasis (negative predictive value 100\%, sensitivity 100\%). Using this parameter Kaplan-Meier statistics were performed successfully (Logrank-test: $P<0.001$ ).

Conclusion: Semi-automatic analysis of breast MRI using CAD provides quantitative data, which can be used to predict occurrence of distant metastasis in primary breast cancer. Such data should be further investigated regarding risk stratification of patients and potential impact on the therapeutic approach.

\section{B-550 11:51}

Breast MRI for the prediction of lymph node metastases: systematic approach using individual descriptors and a dedicated decision tree M. Dietzel ${ }^{1}$, P.A.T. Baltzer ${ }^{1}$, T. Vag ${ }^{2}$, T. Gröschel ${ }^{1}$, H. Habrecht ${ }^{1}$, R. Zoubi ${ }^{1}$, M. Gajda ${ }^{1}$, O. Camara ${ }^{1}$, W.A. Kaiser ${ }^{1} ;{ }^{1}$ Jena/DE, ${ }^{2}$ Munich/DE

Purpose: To correlate a detailed catalogue of 17 descriptors in breast MRI (bMRI) with the presence of lymph node metastases (LNM) and to identify useful combination of such descriptors for the prediction of LNM using a dedicated decision-tree. Methods and Materials: Standardised protocol and study design were applied in this IRB-approved study (T1w-FLASH; $0.1 \mathrm{mmol} / \mathrm{kg}$ BW Gd-DTPA; T2w-TSE; histological verification after bMRI). Two experienced (>500 MR-mammographies) radiologists performed prospective evaluation of the previously acquired examination in consensus. In every lesion 17 previously published descriptors were assessed. Subgroups of primary breast cancers with $(\mathrm{N}+:$ 97) and w/o LNM were created (N+: 253). Prevalence and diagnostic accuracy of each descriptor was correlated with presence of LNM (Chi-square-test; diagnostic-odd's-ratio/DOR). To identify useful combinations of descriptors for the prediction of LNM a CHAID decision-tree was applied.

Results: 7 of 17 descriptors were significantly associated with LNM. The most accurate were "skin thickening" $(P<0.001$; DOR 5.9) and "internal enhancement" $(\mathrm{P}<0.001$; DOR $\leq 13.7)$. The CHAID decision-tree identified useful combinations of descriptors: skin thickening" plus "destruction of nipple line" raised the probability of $\mathrm{N}+$ by $40 \%(\mathrm{P}<0.001)$. In case of absence of "skin thickening", "oedema" and "irregular margins" likelihood of $\mathrm{N}+$ was $0 \%(\mathrm{P}<0.05)$.

Conclusion: Our data demonstrate close association of breast MRI descriptors with nodal status. If present, such descriptors can be used - as standalone or in combination - to accurately predict lymph node metastasis and such to stratify prognosis of the patient.

\section{Molecular Imaging}

\section{SS 1206}

\section{MRI as a tool for molecular imaging}

Moderators:

A. Rahmouni; Creteil/FR

W. Semmler; Heidelberg/DE

\section{B-551 10:30}

\section{Diffusion-weighted MR imaging (DWI) allows monitoring the effect of} combretastatin A4 phosphate on the rabbit VX2 tumour models: 12-day dynamic results

H. Shao ${ }^{1}$, K. Xu' ${ }^{1}$ J. Zhang ${ }^{2}$, F. Chen ${ }^{3}$, Z. Sun ${ }^{4}, \mathrm{Y} \mathrm{Ni}^{3} ;{ }^{1}$ Shenyang/CN,

${ }^{2}$ Nanjing/CN, ${ }^{3}$ Leuven/BE, ${ }^{4}$ Jinan/CN (haiboshao@yahoo.com.cn)

Purpose: To investigate the 12-day dynamic characteristics of tumour response to a vascular disrupting agent combretastatin A4 phosphate (CA4P) in rabbit VX2 tumour models.

Methods and Materials: The study protocol was approved by local ethical committee for animal care and use. Thirty-two VX2 tumours implanted in the bilateral leg muscles of 16 rabbits were employed. Conventional and DWI images were acquired with a 3.0-T MR unit before and 24h, 4days, 8days and 12days after intravenous administration of CA4P at $20 \mathrm{mg} / \mathrm{kg}$. The apparent diffusion coefficient (ADC) of tumour was compared between consecutive two time points by using paired Student $t$ tests.

Results: The mean ADC acquired at baseline was $[1.33 \pm 0.16] \times 10^{-3} \mathrm{~mm}^{2} / \mathrm{sec}$ with an obvious contrast enhancement on T1-weighted images. Twenty-four hours after CA4P administration, the ADC of entire tumour slightly decreased $\left([1.29 \pm 0.12] \times 10^{-3} \mathrm{~mm}^{2} / \mathrm{sec}, \mathrm{p}>0.05\right)$ although the tumour enhancement disappeared almost completely on enhanced T1WI corresponding histologically to viable but oedematous tumour cells. At 4days, without change on enhanced T1WI, the ADC increased significantly $\left([1.45 \pm 0.19] \times 10^{-3} \mathrm{~mm}^{2} / \mathrm{sec}, \mathrm{p}<0.01\right)$ corresponding to massive central necrosis and peripheral tumour re-growth at histology. The entire
ADC had no change at 8 days $\left([1.42 \pm 0.21] \times 10^{-3} \mathrm{~mm}^{2} / \mathrm{sec}, \mathrm{p}>0.05\right)$ but significantly decreased at 12 days $\left([1.28 \pm 0.21] \times 10^{-3} \mathrm{~mm}^{2} / \mathrm{sec}, \mathrm{p}<0.05\right)$ because of progressive tumour proliferation.

Conclusion: The dynamic histological changes of tumour caused by CA4P as reflected exactly by DWI indicate a non-invasive measure for monitoring tumour vascular targeting treatment.

\section{B-552 10:39}

Magnetic resonance imaging of murine myocardial infarction with an elastin-binding contrast agent

M. Wildgruber ${ }^{1}$, M. Settles ${ }^{1}$, R. Braren ${ }^{1}$, V. Ntziachristos ${ }^{2}$, E. Rummeny ${ }^{1}$,

R. Botnar ${ }^{3}$, A. Huber ${ }^{1} ;{ }^{1}$ Munich/DE, ${ }^{2}$ Neuherberg/DE, ${ }^{3}$ London/UK (moritz.wildgruber@roe.med.tum.de)

Purpose: To improve non-invasive magnetic resonance imaging (MRI) of myocardial infarction in order to investigate subsequent myocardial remodelling by targeting Elastin in the injured myocardium.

Methods and Materials: Myocardial infarction (MI) was induced in $11 \mathrm{C} 57 \mathrm{BL} / 6 \mathrm{~J}$ mice by permanent ligation of the left anterior descending artery (LAD). Cardiac MRI was performed on day 7 after MI. Each mouse was subsequently studied with Gd-DTPA (reference method) and the Elastin-binding contrast agent CP-1052 both injected at $0.6 \mathrm{mmol} / \mathrm{Kg}$. Dynamic MRI was performed over a period of 90 minutes using a phase-sensitive inversion recovery gradient echo sequence. Contrast-tonoise ratios (Slinfarct-SImyocardium)/SDnoise after injection of Gd-DTPA and CP-1052 were calculated and compared at 5, 10, 15, 30, 45, 60 and 90 minutes. Results: CNR after Gd-DPTA injection peaked at 10 minutes and returned to pre-injection values at 60 minutes. After injection of CP-1052 the CNR peaked at 45 minutes and was significantly higher than Gd-DTPA at 30 minutes $(p=0.04)$, 45 minutes $(p=0.02)$ and 60 minutes $(p=0.02)$. Specific CNR for Gd-DTPA/CP1052 were as follows: at 5 minutes 9.9/14.0, at 10 minutes $11.9 / 14.3$, at 15 minutes $10.7 / 14.6$, at 30 minutes $7.1 / 24.1$, at 45 minutes $3.3 / 31$, at 60 minutes $3.0 / 28$, at 90 minutes $1.6 / 20$

Conclusion: Elastin targeted gadolinium enables visualisation of injured myocardium in an experimental mouse model of myocardial infarction with a higher CNR and prolonged binding characteristics. Improved dynamic MRI with CP-1052 may be attributed to its higher relaxivity and its specific affinity for Elastin.

\section{B-553 10:48}

Dynamic contrast-enhanced (DCE)-MRI with gadobutrol for monitoring sorafenib effect on experimental prostate carcinomas with immunohistochemical validation

C.C. Cyran ${ }^{1}$, P.M. Paprottka', J. Von Einem¹, B. Schwarz'1, O. Dietrich', M.F. Reiser ${ }^{1}$, B.J. Wintersperger ${ }^{2}$, K. Nikolaou'; ${ }^{1}$ Munich/DE, ${ }^{2}$ Toronto, ON/CA (clemens.cyran@med.uni-muenchen.de)

Purpose: To investigate the effects of the multikinase inhibitor sorafenib on experimental prostate carcinomas in rats with dynamic contrast-enhanced (DCE-) MRI quantifications of tumour perfusion and endothelial permeability. Non-invasive MRI results were validated by immunohistochemistry.

Methods and Materials: Copenhagen rats $(n=20)$ implanted with subcutaneous prostate carcinoma allografts (MLLB-2) were imaged on days 0 and 7 using DCE$\mathrm{MRI}$ at 3 T enhanced with gadobutrol. The treatment group $(n=10)$ received daily applications of $10 \mathrm{mg} / \mathrm{kg} / \mathrm{bodyweight} \mathrm{Sorafenib} \mathrm{(Nexavar}{ }^{\circledR}$, Bayer, Leverkusen, Germany) via gavage. Quantitative measurements of tumour perfusion ( $\mathrm{ml} / 100 \mathrm{ml} /$ $\mathrm{min}$ ) and endothelial permeability $(\mathrm{ml} / 100 \mathrm{ml} / \mathrm{min}$ ) were calculated based on a two-compartment kinetic model. Tumours were excised on day 7 to undergo immunohistochemical staining for tumour vascularity (RECA-1), cell proliferation (TUNEL) and apoptosis (Ki-67).

Results: Tumour perfusion in sorafenib-treated prostate carcinoma allografts declined significantly from days 0 to $7(47.9 \pm 36.9 \mathrm{vs} .24 .4 \pm 18.5 \mathrm{ml} / 100 \mathrm{ml} /$ $\mathrm{min}, \mathrm{p}<0.05)$. In the control group, tumour perfusion increased significantly from days 0 to $7(37.6 \pm 12.3$ vs. $49.8 \pm 15.0 \mathrm{ml} / 100 \mathrm{ml} / \mathrm{min}, \mathrm{p}<0.05)$. No significant effect on endothelial permeability was observed either in the therapy or in the control group ( $p>0.05$ ). Immunohistochemical measurements revealed significantly lower tumour vascularity in the therapy than in the control group (RECA-1 $5.1 \pm 1.9$ vs. $23.1 \pm 7.7, p<0.05)$. In the sorafenib-treated tumours, significantly more apoptotic cells (TUNEL $427 \pm 283$ vs. $218 \pm 312, p<0.05$ ) and significantly less proliferating cells (Ki-67 $847 \pm 307$ vs. $1692 \pm 469, p<0.05)$ were observed than in the control group.

Conclusion: Tumour perfusion quantified by gadobutrol-enhanced DCE-MRI can be applied as non-invasive surrogate parameter for monitoring the anti-angiogenic anti-proliferative and pro-apoptotic effects of sorafenib on prostate carcinoma allografts as validated by immunohistochemistry. 


\section{B-554 10:57}

A novel model demonstrating MRI molecular imaging in vivo E.A. Wallnöfer, G.C. Thurner, C. Kremser, H. Talasz, A. Abdelmoez, N. Klammsteiner, W. Jaschke, P. Debbage; Innsbruck/AT (Paul.Debbage@i-med.ac.at)

Purpose: Tissue-specific MR imaging in living organisms requires accumulation of high gadolinium concentrations at specific target sites of the tissue. In this work nanoparticles, each bearing hundreds of gadolinium ions and targeted to oligosaccharides present in the glycocalyx at the endothelial luminal surface, are presented and vascular molecular imaging demonstrated in an animal model.

Methods and Materials: Nanoparticles, bearing covalently attached gadoliniumDTPA chelates and covalently attached targeting groups, were prepared from human serum albumin. As targeting molecule the tomato lectin (LEA) was used. After intravenous injection of these nanoparticles into Sprague-Dawley rats MR images were acquired, from the first 30 seconds after injection onwards and then during a period up to 4 hours.

Results: Nanoparticles with appropriate internal cross-linking produced strong MR signals from the vascular walls. The initial MR images after nanoparticle injection were blood-volume images. However, within 2-3 minutes the larger blood vessels appeared as ring like structures, indicating that the contrast agent was accumulating in the blood vessel walls at concentrations significantly higher than those in the blood. These results were confirmed by light microscopy showing accumulation of nanoparticles in the endothelium of microvessels, and in the endothelium, the media and the adventitia of arteries.

Conclusion: All materials used to synthesise the presented nanoparticles are inexpensive and can be obtained or prepared in high purity. These inexpensive, high-quality nanoparticles, delivering strong MR signals from the vascular walls of living laboratory animals, offer the potential for improved vascular imaging, especially for detection of diseases affecting the endothelium.

\section{B-555 11:06}

Cellular imaging of inflammation in obesity: 4.7 T MR imaging of adipose tissue macrophages after USPIO injection

S. Dechoux ${ }^{1}$, V. Deveaux ${ }^{1}$, M. Poirier-Quinot ${ }^{2}$, M. Levy ${ }^{3}$, S. Manin ${ }^{1}$, C. Wilhelm ${ }^{3}$, S. Lotersztajn ${ }^{1}$, F. Gazeau ${ }^{3}$, A. Luciani ${ }^{1},{ }^{1}$ Creteil/FR, ${ }^{2}$ Orsay/FR, ${ }^{3}$ Paris/FR (sdechoux@yahoo.fr)

Purpose: To assess the sensitivity of 4.7T MR imaging for adipose tissue macrophage $\left(\mathrm{A}^{\mathrm{TM}}\right)$ detection and quantification in an animal model of obesity.

Methods and Materials: This study complied with national recommendations on animal experiment. Systemic injection of $1000 \mathrm{umol} / \mathrm{kg}$ of a macrophage-targetted contrast media (USPIO - P904, Guerbet, Aulnay, France) was performed in leptin deficient obese mice $(n=17)$, and their lean littermates $(n=17)$, at different ages $(7,10,14$ weeks). The effect of USPIO injection on adipose tissue signal variance observed at 4.7T MRI on GE T2 sequences was determined. MRI results were correlated to $\mathrm{A}^{\mathrm{TM}}$ quantification by $\mathrm{F} 4 / 80$ immunostaining, and pro-inflammatory markers quantification by RT-PCR (MCP1, TNF, F4/80). The amount of iron in adipose tissue was quantified by ferromagnetic resonance.

Results: A significant increase in signal variance was seen in obese mice compared to lean mice $(p<0.0001)$ and non-injected obese mice $(p=0.0002)$. This was correlated to an increase of pro-inflammatory markers in AT (Pearson $r=0.5952$ ), an increase of $A^{T M}$ quantification (Pearson $r=0.5749$ ). Iron amount was increased in obese mice AT compared to lean animals $(p=0.0288)$. Progressive increase in signal variance was also demonstrated in aging animals, paralleling increased inflammation $(p=0.005)$.

Conclusion: $\mathrm{A}^{\mathrm{TM}}$ inflammation detection and quantification is feasible on 4.7T MRI in an animal model of obesity.

\section{B-556 11:15}

Heterogeneity in DCE-MRI maps: a biomarker for tumour response? L. Alic, M. van Vliet, C.F. van Dijke, A. Eggermont, W. Niessen, J.F. Veenland; Rotterdam/NL

Purpose: The heterogeneity in DCE-MRI maps provides essential information for the assessment of tumour response to treatment. We evaluated the ability of DCE-MRI heterogeneity to differentiate between responders and non-responders. Methods and Materials: 18 patients (males 10, females 8 median age: $56.5 \mathrm{y}$ ) with irresectable extremity soft tissue sarcoma were treated with isolated limb perfusion (ILP). DCE-MR images were acquired before and after ILP with a 1.5 T MRI scanner (Vision, Siemens, Erlangen, Germany) using a bolus injection of $0.1 \mathrm{mmol} / \mathrm{kg}$ gadopentetate dimeglumine. Perfusion parametric maps were calculated using a two-compartment pharmacokinetic model and a model free method. Heterogeneity measures based on the co-occurrence method were calculated for the various parametric maps: Ktrans, slope, area under the curve (AUC) and maximum enhancement. The response categories were based on histopathological findings: responders (more than $50 \%$ necrosis) and non-responders (less than $50 \%$ necrosis). The differences in heterogeneity measures before and after treatment were compared for both response categories using a Wilcoxon signed rank test. Results: There is a clear correlation between pharmacokinetic and model free parametric maps. The heterogeneity measures computed from both type of maps were able to measure significant changes due to treatment in the response group (Coherence feature: for the Ktrans map $p<0.002$; for the slope map $p<0.003$; for the maximum enhancement map $p<0.004$ for the AUC map $p<0.005$ ), and concurrently measured no significant changes in the non-responder group. Conclusion: Heterogeneity features, computed from pharmacokinetic and heuristic parametric maps, show potential as a biomarker for monitoring tumour response in sarcomas.

\section{B-557 11:24}

Targeting human colon adenocarcioma with novel SPIO-loaded single unilamellar nanovesicles in xenograft mouse MR model

S.-T. Feng ${ }^{1}$, H. Li' ${ }^{1}$ A.-C. Ching ${ }^{2}$, C.-H. Sun ${ }^{1}$, P.-X. Qiu' ${ }^{1}$, X.-T. Shuai ${ }^{1}$,

Q.-F. Meng', Z.-P. Li ${ }^{1}$, J.-Y. Yang ${ }^{1} ;{ }^{1}$ Guangzhou/CN, ${ }^{2}$ Paris/FR (fst1977@163.com)

Purpose: We present in vitro and in vivo MR studies of the human colon adenocarcioma with novel single unilamellar nanovesicles encapsulating supraparamagnetic iron oxides (SPIOSUV).

Methods and Materials: In vitro: experimental designs of new forms of PEGylated SPIOSUV were performed with size control. LoVo-6-luc-1 human colon adenocarcinoma cells and L-O2 human hepatic cells were cultured in media containing different concentrations of SPIOSUV. MR imaging was performed and T2 relaxivity was assessed using T2-weighted mapping software package. In vivo: conventional SPIO, hydrophobic and hydrophilic forms of SPIOSUV were injected into BALB/C nude mice with LoVo xenograft $(n=3 \times 6)$. Dynamic MRI was done and T2 relaxivity was measured in the tumour, liver and muscles.

Results: In vitro: SPIOSUV was more superparamagnetic than conventional SPIO $(p<0.01)$. Higher T2 relaxivity of SPIOSUV with LoVo cells than L-O2 cells was shown $(p<0.01)$. Hydrophilic SPIOPN showed higher T2 relaxivity rate than hydrophobic form in vitro $(p<0.01)$. In vivo: T2-weighted MR signal of the human colon adenocarcinoma LoVo xenografts was significantly decreased with SPIOSUV than conventional SPIO in rodent model $(p<0.01)$. TEM confirmed SPIOSUV in tumour cell cytoplasm.

Conclusion: We successfully manufactured two (hydrophobic and hydrophilic) forms of SPIOSUV. The feasibility of in vitro and in vivo MRI of the LoVo human colon adenocarcinoma had been shown. These new types of SPIOSUV may open the door to diagnosing early phenotype of human colon adenocarcinoma due to their unique structures, kinetics and biodistribution.

B-558 11:33

(EPOS)

Imaging labelled tumour cell growth and lymphatic micrometastasis in mice xenograft model with MR

Z.-H. Dong, T. Liu, F. Gao; Chengdu/CN

Purpose: To investigate primary tumour formation and mechanism of tumour lymphatic micrometastasis in nude mouse using MRI with histology.

Methods and Materials: Human colorectal cancer LOVO cells were labelled with USPIO. The labelling efficiency was evaluated by Prussian blue staining. Then the labelled and unlabelled LOVO cells $\left(10^{8}\right.$ cells $\left./ 0.05 \mathrm{ml}\right)$ were inoculated in claw pad and groin area of nude mice. A 7T MR with T2, T2* of the tumours and surrounding lymph nodes was performed in the following 4 weeks. After imaging, tumour tissues and lymph nodes were collected and subjected to immunohistologic analysis, which include haematoxylin and eosin (H\&E) staining, Prussian blue (PB) staining, CD68 staining, lymphatic vessel endothelial hyaluronan receptor (LYVE-1) staining and vascular endothelial growth factor C (VEGF-C) staining.

Results: The labelling efficiency was $90 \%$. MRI demonstrated hypointense regions where USPIO labelled tumour cells located; then the low signal increased gradually. The increase in signal of the periphery was more rapid than central parts of the tumour. MR T2* weighted image detected lymphatic micrometastasis at 8 days P.I. and lymphatic metastasis of tumour cells happened prior to exponential growth. Lymph node metastasis was verified by H\&E staining and Prussian blue staining Immunohistochemical analysis of primary tumour sections collected at 8,28 days P.I. revealed existence of macrophages, lymphangiogenesis, Prussian blue positive tumour cells and overexpression of VEGF-C.

Conclusion: The growth of tumour cells and lymphatic micrometastasis can be observed by MRI. There was a close relationship between tumour lymphatic metastasis and lymphangiogenesis. 


\section{B-559 $11: 42$}

Effect of size criteria on the diagnosis of malignant lymph nodes in Iymphomas: comparing whole-body MRI including DWI with FDG-PET/CT G. AbdulQadhr, D. Molin, G. Åström, M. Suurküla, L. Johansson, H. Ahlström; Uppsala/SE (goran.abdul-qadhr@radiol.uu.se)

Purpose: In lymphoma patients, to compare conventional whole-body MRI and whole-body DWI with FDG-PET/CT, and to measure the effect of changing the size definition of positive nodes on the sensitivity and specificity of whole-body MRI using FDG-PET/CT as a reference.

Methods and Materials: Thirty patients, 8 with Hodgkin lymphoma and 22 with non-Hodgkin's lymphoma underwent whole-body MRI including DWI and FDG-PET/ CT. The body was divided into 15 lymph node regions; largest node on each region was measured. Two size definitions, a longitudinal diameter of $\geq 1.5 \mathrm{~cm}$ with longest transverse diameter of $<1$ and $\geq 1 \mathrm{~cm}$ for positive lymph nodes were used. Three groups, DWI alone, conventional MRI alone and combined DWI and conventional MRI (combined group) were compared to PET/CT.

Results: The combined group had the highest sensitivity and specificity in 30 patients using both definitions $(77.2 \%$ and $96.6 \%)$ for $\geq 1.5 x<1 \mathrm{~cm}$, and $(72.1 \%$ and $97.9 \%)$ for $\geq 1.5 x \geq 1 \mathrm{~cm}$, respectively, $\mathrm{k}=0.74(0.65-0.83)$ and $\mathrm{k}=0.76(0.69-0.84)$, respectively. The highest number of false negative regions were in mediastinum and neck and the lowest were in paraaortic regions.

Conclusion: The combination of conventional whole-body MRI and DWI with size criteria of $\geq 1.5 \mathrm{x}<1 \mathrm{~cm}$ gives the best results compared to separate sequences and lower size criteria.

\section{B-560 11:51}

Validation of DCE-MRI parametric maps: developing a tool using 3D-histology

K. Bol, J.C. Haeck, L. Alic, M. Bernsen, M. de Jong, W.J. Niessen,

J.F. Veenland; Rotterdam/NL (k.bol@erasmusmc.nl)

Purpose: To develop a validation tool for DCE-MRI parametric maps of tumour tissue, based on exact co-registration of MRI with 3D-histology.

Methods and Materials: In literature, many different DCE-analysis methods are used to characterise tumour tissue. To validate these methods histology is the gold standard, exact co-localisation between histology and MR images is therefore a prerequisite. This co-localisation is complicated by deformation and shrinking of the tissue during histological processing. To meet this problem, 3D-histology stacks were created and registered to DCE-MRI images using non-rigid B-splines with a mutual information metric. Semi-quantitative and quantitative parameters (using a two-compartment model) were computed from the DCE data. For the evaluation, regions of interest (ROIs) consisting of vital and non-vital tumour tissue were drawn in histology and transformed to DCE-parametric maps.

Results: In quantitative DCE-MRI parametric maps, the average values in vita and non-vital ROls were significantly different for the standard Tofts Model. For the semi-quantitative parameters, a significant difference between vital and non-vital tissue was observed for the wash-in, wash-out, maximum enhancement and area under the curve (AUC). Whereas the Tofs Model and the wash-in parameters can discriminate between vital and non-vital tissue, the wash-out, maximum enhancement and the AUC showed large overlap between vital and non-vital tissue.

Conclusion: Non-rigid registration is necessary for exact co-localisation of DCEMRI data with histology. The pharmacokinetic DCE-parameters, and the heuristic parameter wash-in, discriminate between vital and non-vital tumour regions. In future, this method will be expanded to evaluate several quantitative DCE-MRI analysis methods.
$10: 30-12: 00$

Room I/K

\section{Musculoskeletal}

SS 1210

Shoulder and wrist

Moderators:

I.W. McCall; Oswestry/UK

S.P. Morozov; Moscow/RU

\section{B-561 10:30}

Diagnostic value of direct MR arthrography of the shoulder in superior

labrum anterior: posterior lesions

S. Khedr ${ }^{1}$, A. Shabana ${ }^{2}$, A. Gaballa ${ }^{1} ;{ }^{1}$ Jiddah/SA, ${ }^{2}$ Cairo/EG

Purpose: To determine the accuracy of direct magnetic resonance (MR) arthrography in the diagnosis of superior labrum anterior-posterior (SLAP) lesions of the shoulder.

Methods and Materials: From January 2007 to June 2009, MR arthrography of the shoulder was performed in 49 patients who underwent arthroscopy or open surgery 12 days to 5 months after MR arthrography. Diagnostic criteria for SLAP lesion included marked fraying of the articular aspect of the labrum, biceps anchor avulsion, inferiorly displaced bucket handle fragment, and extension of the tear into the biceps tendon fibres. Surgical findings were correlated with those from MR arthrography. Type I SLAP was considered negative because it has no significant clinical relevance.

Results: SLAP injuries were diagnosed at surgery in 34 of the 49 patients $(69.3 \%)$. 12 of the 34 lesions (35.2\%) were classified as type II, $8(23.5 \%)$ as type III, 6 $(17.6 \%)$ as type IV, and $4(11.7 \%)$ as type IV, 2 as type V (5.8\%), 1 as type VI $(2.9 \%)$ and 1 as type VII (2.9\%). MR arthrography had a sensitivity of $83.3 \%$, a specificity of $88 \%$, positive predective value of $86.9 \%$ and negative predective value of $84.6 \%$. The MR arthrographic findings showed correlation with the arthroscopic or surgical classification in $69 \%$.

Conclusion: MR arthrography is a useful and accurate technique in the diagnosis of SLAP lesions of the shoulder. MR arthrography provides pertinent preoperative information with regard to the exact location of tears and grade of involvement of the biceps tendon.

\section{B-562 10:39}

Diagnostic value of direct MR arthrography in evaluation of rotator interval lesions

S. Khedr; Jiddah/SA

Purpose: To evaluate the diagnostic role of direct MR arthrography in evaluation of rotator interval lesions.

Methods and Materials: 36 patients with clinical history of anterior and inferior glenohumeral joint instability (GHI), recurrent shoulder pain or clicking were referred from orthopaedic department in the period from February 2008 to March 2010. Direct MR arthrography with intra-articular injection of diluted gadolinium (1:200) was done in all patients. 3D-T1Wl fat-suppressed images in axial, coronal and sagittal planes in addition to the standard protocols for shoulder MR examinations were done for all patients. The oblique sagittal plane, taken parallel to the glenoid fossa and orthogonal to the long axis of the rotator interval and cuff, is the single most valuable sequence to evaluate the interval and its contents. MR arthrograms were analysed for the presence of rotator interval lesions. MR arthrographic findings were correlated with arthroscopic and surgical findings.

Results: Arthroscopic and surgical results confirmed the presence of rotator interval lesions in 23 patients including supraspinatous tendon tear in four patients, subscapularis tendon tear in 7 patients, SGL tear in 3 patients, coracohumeral ligament injury in 2 patients, biceps tendon tear in 6 patients, rotator interval defect in 2 patients, and adhesive capsulitis in 3 patients. The sensitivity, specificity and accuracy of direct MR arthrography was $95.6 \%, 100 \%$ and $97.2 \%$, respectively. Conclusion: Direct MRA is an accurate method in detection of rotator interval lesions. 


\begin{abstract}
B-563 10:48
Detection of hyaline cartilage lesions of the glenohumeral joint: a comparative study of multiple detector CT-arthrography and MRarthrography at $1.5 \mathrm{~T}$, with arthroscopic correlation

P. Omoumi, A. Rubini, B.C. Vande Berg, F.E. Lecouvet; Brussels/BE (patrick.omoumi@uclouvain.be)
\end{abstract}

Purpose: To compare the diagnostic performance of MDCT arthrography (CTA) and MR arthrography (MRA) at $1.5 \mathrm{~T}$ in detecting hyaline cartilage lesions of the shoulder joint, with correlation to arthroscopy.

Methods and Materials: CTA and MRA examinations prospectively obtained in 57 consecutive patients (mean age 47 [16-72]; 23 females, 34 males) during the same arthrographic procedure were evaluated for glenohumeral cartilage lesions. Two radiologists independently analysed the cartilage surfaces of the humeral head and the glenoid fossa in 9 anatomical surface areas. Arthroscopy, performed within one month of the imaging, was taken as the gold-standard. We determined the sensitivity and specificity of CTA and MRA for detecting cartilage lesions with substance loss, the Spearman correlation coefficient between CTA/MRA and arthroscopic gradings, as well as $\mathrm{K}$ statistics for assessing interobserver reproducibility.

Results: CTA showed significantly higher sensitivity than MRA for the detection of cartilage lesions with substance loss ( 81 vs. $66 \%$ for grade $\geq 2$ lesions, respectively $[p<0.001]$ ) ( 91 vs. $64 \%$ for grade 4 lesions, respectively [ $<<0.001]$. The specificity for the detection of grade $\geq 2$ lesions was not statistically different $(96 \%$ for both techniques). Spearman correlation coefficients between CTA/MRA and arthroscopic grading of cartilage lesions were $0.773 / 0.689$, respectively. Interobserver agreement with CTA and MRA was moderate to substantial for grading all articular surfaces and for detecting lesions with substance loss ( $\kappa=0.656$ and 0.588 , respectively). Conclusion: CTA and MRA show similar diagnostic performance for detecting cartilage lesions with substance loss in the entire glenohumeral joint at $1.5 \mathrm{~T}$, although CTA has higher sensitivity than MRA.

\section{B-564 10:57}

Evaluation of rotator cuff lesions: a comparative study of multi-detector CT-arthrography and MR-arthrography at $1.5 \mathrm{~T}$, with arthroscopic correlation

P. Omoumi, B.C. Vande Berg, F.E. Lecouvet; Brussels/BE (patrick.omoumi@uclouvain.be)

Purpose: To compare the diagnostic performance of MDCT arthrography (CTA) and MR arthrography (MRA) at $1.5 \mathrm{~T}$ in detecting rotator cuff tears, with correlation to arthroscopy.

Methods and Materials: CTA and MRA examinations prospectively obtained in 58 consecutive patients (mean age, 45 [16-72]; 26 females, 32 males) during the same arthrographic procedure were independently evaluated for rotator cuff tears by two radiologists. Arthroscopy, performed within one month of the imaging, was taken as the gold-standard. We determined the sensitivity and specificity of CTA and MRA for detecting rotator cuff tears (supraspinatus (SS), infraspinatus (IS), subscapularis (SC), long tendon of the biceps brachii (Bc)), the Spearman correlation coefficient between CTA/MRA and arthroscopic gradings, as well as the interobserver reproducibility using $\mathrm{K}$ statistics.

Results: For partial and complete tears considered together, there was no statistical difference in sensitivity or specificity between CTA and MRA $(p<0.05)$ (sensitivity: SS: 89/93, IS: 89/100, SC: 100/100, Bc: 70/80\%, respectively; specificity: SS: 97/87, IS: $76 / 80$, SC: $90 / 90$, Bc: $100 / 100$, respectively). For complete tears of the SS tendon, both CTA and MRA showed a sensitivity and specificity of $100 \%$. Spearman correlation coefficients between CTA/MRA and arthroscopic detection of SS tears were 0.951 and 0.928 , respectively. Interobserver agreement for the detection of rotator cuff tears was almost excellent with CTA $(\kappa, 0.862-1.000)$ and substantial to almost excellent with MRA $(\kappa, 0.741-0.914)$.

Conclusion: CTA and MRA have similar diagnostic performance for detecting rotator cuff tears at 1.5 T. CTA has slightly better reproducibility.

\section{B-565 11:06}

Preoperative evaluation of anterior shoulder instability: comparison of MR arthrography and CT arthrography with arthroscopic correlation

S. Acid, T. Le Corroller, R. Aswad, B. Maurel, V. Pauly, P. Champsaur; Marseille/FR (acidsouad@hotmail.fr)

Purpose: To compare MR arthrography (MRA) and CT arthrography (CTA) for the preoperative planning of shoulder anterior instability.

Methods and Materials: Forty consecutive patients (10 women and 30 men range 15-48) who underwent on the same day shoulder MRA and CTA before instability repair were prospectively included in the study. A 1:1 mixture of diluted gadoteridol
(4 mmol/L) and iopamidol (300 $\mathrm{mg}$ iodine $/ \mathrm{mL}$ ) was injected. MR and CT images were analysed in a randomised fashion by two musculoskeletal radiologists. The arthroscopic findings represented the reference standard.

Results: In detecting glenoid fractures, sensitivity and specificity were $100 \%$ and $96.4 \%$ for CTA, and $66.6 \%$ and $100 \%$ for MRA. In demonstrating ALPSA lesions, sensitivity and specificity were $92.9 \%$ and $100 \%$ for CTA, $100 \%$ and $91.7 \%$ for MRA. For HAGL lesions diagnosis, sensitivity and specificity were $100 \%$ and $100 \%$ for CTA, $100 \%$ and $97.4 \%$ for MRA. In detecting middle and inferior glenohumeral ligamentous tears, sensitivity and specificity were $57.9 \%$ and $85.7 \%$ for CTA, $57.9 \%$ and $95.2 \%$ for MRA. In depicting glenoid cartilage lesions, sensitivity and specificity were $77.3 \%$ and $88.8 \%$ for CTA, $72.7 \%$ and $94.4 \%$ for MRA. No difference was observed for detection of rotator cuff tendons tears.

Conclusion: CTA and MRA were equivalent in demonstrating labro-ligamentous and cartilaginous lesions associated with shoulder instability. CTA was superior in detecting glenoid rim osseous lesions that are known to be a decisional element in the surgical strategy. Hence, CTA may be considered a method of choice in the preoperative evaluation of shoulder anterior instability.

\section{B-566 11:15}

The clinical relevance of lateral acromial sclerosis, the 'sourcil' sign and other radiographic acromial features in rotator cuff disease

T.D. Turmezei, W.A. Wallace, K.J. Fairbairn; Nottingham/UK

(tom@diagnosticradiology.eu)

Purpose: The purpose of this study was to investigate which radiographic acromial features could be used to reliably identify a full-thickness (FT) rotator cuff tear and what combination of these features might represent the 'sourcil' sign in a symptomatic population.

Methods and Materials: A retrospective study was performed on 158 patients undergoing first-time surgery for rotator cuff disease taken from a single surgeon's series over 3-and-a-half years. Dedicated antero-posterior gleno-humeral joint radiographs of 83 patients with impingement, 21 with a partial thickness (PT) tear and 54 with a FT tear were randomised and blindly assessed for seven individual acromial features, acromio-humeral distance (AHD) and acromial coverage index (ACl). PT tear and impingement subjects were combined (group 1) for comparison with FT tear subjects (group 2).

Results: Lateral acromial inferior surface sclerosis was seen more commonly in group $2(61.4 \%)$ than group $1(51.4 \%)$ (odds ratio $=1.809)$ but not significantly $(p$ $=0.162$ ). Whole acromial inferior surface sclerosis and erosion was observed with a frequency of $0 \%$ in group 1 and $7 \%$ in group 2, giving a positive predictive value (PPV) of $100 \%$ for a FT tear. Age, AHD and ACl-adjusted odds ratios showed no change in significance of results.

Conclusion: Lateral acromial inferior surface sclerosis is common in rotator cuft disease but is not a discriminator for a FT tear. For useful identification of a FT tear with plain radiography, the 'sourcil' sign could be described as a combination of whole acromial inferior surface sclerosis and erosion.

\section{B-567 11:24}

BLADE versus conventional TSE sequences in shoulder-MRI: benefit or not?

A. Horng ${ }^{1}$, R. Vieira ${ }^{2}$, M. Pietschmann', M. Notohamiprodjo', P. Müller',

M.F. Reiser', C. Glaser'; ' $M$ Munich/DE, ${ }^{2}$ New York, NY/US (annie.horng@med.uni-muenchen.de)

Purpose: TSE sequences with Cartesian read-out of $k$-space are prone to artefacts caused by involuntary patient movements, which might result in non-diagnostic images. The recently developed T2-weighted sequence with rotating rectangular read-out of $k$-space (BLADE) is expected to reduce motion artefacts and improve image quality. This study compares BLADE to a fat-saturated TSE-sequence (CTSE) for image quality, anatomical detail depiction and confidence in detection of pathologies in shoulder-MRI.

Methods and Materials: Moderately T2-w coronal and axial BLADE and cTSEsequences in identical positioning were acquired in 60 consecutive patients (TR $3000 \mathrm{~ms} / \mathrm{TE} 45 \mathrm{~ms} / \mathrm{FOV}$ 160/BW $260 \mathrm{~Hz}$ ) at $3 \mathrm{~T}$. Images were rated using a 5-pointLikert-Scale (1-bad to 5-good) for image quality, artefacts and clinically important anatomical structures (muscles/tendons of musculi supraspinatus/infraspinatus/ subscapularis, long biceps tendon, labrum, glenoid/humeral cartilage, inferior/ middle glenohumeral ligaments). Differences were calculated by a Wilcoxon test. Results: Average rating for image quality and artefacts was 4.6/4.8 for BLADE and 3.6/3.7 for CTSE. BLADE demonstrated significantly better image quality ( $85 \%$ better than cTSE, $p<0.01$ ), less artefacts $(78 \%$ less than cTSE, $p<0.01$ ) and better depiction of anatomical structures (4.8 vs $4.7, p<0.01$ ) as compared to cTSE. Also, confidence of diagnosis for pathologies was higher for BLADE (4.8 vs 4.6) than for CTSE $(p<0.01)$. 
Conclusion: Rotating read-out of k-space (BLADE) yields significant reduction of motion artefacts and better depiction of anatomical details in comparison to cTSE sequences used in musculoskeletal radiology. Thus, BLADE provides a promising alternative with possible benefits in image quality for the examination of children, critically ill or claustrophobic patients, who express a higher probability for motion artefacts.

\section{B-568 11:33}

Athletic injuries of the extensor carpi ulnaris subsheath: MRI findings and utility of gadolinium-enhanced fat-saturated T1-weighted sequences with wrist pronation and supination

F. Becce ${ }^{1}$, J. Jeantroux ${ }^{2}$, H. Guerini² ${ }^{2}$ B. Montalvan² ${ }^{2}$ D. Le Viet ${ }^{2}$, J.-L. Drapé2;

1'Lausanne/CH, ${ }^{2}$ Paris/FR (fabio.becce@chuv.ch)

Purpose: To report the magnetic resonance imaging (MRI) findings in athletic injuries of the extensor carpi ulnaris (ECU) subsheath, assessing the utility of gadolinium-enhanced (Gd) fat-saturated (FS) T1-weighted sequences with wrist pronation and supination.

Methods and Materials: Sixteen patients (13 males, 3 females; mean age 30.3 years) with athletic injuries of the ECU subsheath sustained between January 2003 and June 2009 were included in this retrospective study. Initial and follow-up 1.5-T wrist MRIs were performed with transverse T1-weighted and STIR sequences in pronation, and Gd FS T1-weighted sequences with wrist pronation and supination. Two radiologists assessed the type of injury (A to $\mathrm{C}$ ), ECU tendon stability, associated lesions and rated pulse sequences using a three-point scale: $1=$ poor 2 = good and 3 = excellent

Results: Gd-enhanced FS T1-weighted transverse sequences in supination (2.63) and pronation (2.56) were most valuable, compared with STIR (2.19) and T1 weighted (1.94). Nine type $A$, one type $B$ and six type $C$ injuries were found. There were trends towards diminution in size, signal intensity and enhancement of associated pouches on follow-up MRI and tendon stabilisation within the ulnar groove. Conclusion: Gd-enhanced FS T1-weighted sequences with wrist pronation and supination are most valuable in assessing and follow-up athletic injuries of the ECU subsheath on 1.5-T MRI

\section{B-569 11:42}

The usefulness of a new technique, dynamic cine-arthrography of wrist, compared with MR arthrography

T. $\mathrm{Kim}^{1}$, G. Lee ${ }^{1}$, B. Kim${ }^{1}$, C. Kang${ }^{2}$, S. Hong ${ }^{2}$, Y. Kim ${ }^{2} ;{ }^{1}$ Kyoungki-do/KR ${ }^{2}$ Seoul/KR

Purpose: We aimed to introduce a new technique of wrist arthrography, dynamic cine-arthrography (DCA) and compare its results with that of wrist MR arthrography (MRA) using in diagnosis of scapholunate (SL) and lunotriquetral (LT) ligament tears and triangular fibrocartilage complex (TFCC) injuries, based on arthroscopic operations.

Methods and Materials: Between Jan 2002 and May 2010, 38 wrists of 38 patients which underwent both DCA and MRA were enrolled in this study. DCA was performed as follows; after puncture of radiocarpal joint by needle, contrast solution was injected on continuous fluoroscopic guidance during passive wrist exercise. We reviewed DCA and MRA findings about SL or LT ligament tear and TFCC injury according to Palmer classification system. Based on arthroscopic findings, we measured and compared the diagnostic values between DCA and MRA using kappa value.

Results: For SL ligament tear, sensitivity, specificity, positive predictive value, negative predictive value and accuracy were $66.7,100,100,82.0$ and $86.8 \%$ in DCA, while $80,95.7,92.3,88$ and $89.5 \%$ in MRA. For LT ligament tear, sensitivity, specificity, positive predictive value, negative predictive value and accuracy were 75.0, 94.1, 60.0, 97.0 and 92.1 in DCA, while 75.0, 91.2, 50.0, 96.9 and $89.5 \%$ in MRA. ForTFCCtear, sensitivity, specificity, positive predictive value, negative predictive value and accuracy were $96.4,100,100,90.9$ and $97.4 \%$ in both DCA and MRA. Inter-examination agreement was almost perfect (kappa value $=0.814$ ) for SL ligament tear, substantial (kappa value $=0.682$ ) for LT ligament tear and perfect (kappa value $=1.000$ ) for TFCC tear between DCA and MRA.

Conclusion: DCA is cheap and simple examination and valuable in diagnosis of intrinsic ligament tear or TFCC tear, as well as MRA.
B-570 11:51

Optimisation of b-value for diffusion tensor imaging of the median nerve at 3.0 $\mathrm{T}$ and of reconstruction parameter for fiber tractography R. Guggenberger ${ }^{1}$, D. Markovic ${ }^{1}$, P. Eppenberger ${ }^{1}$, D. Nanz ${ }^{1}$, M. Wyss ${ }^{1}$

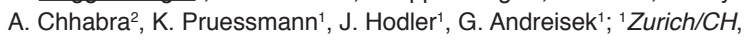
2Baltimore, MD/US (roman.guggenberger@usz.ch)

Purpose: Recently, several studies on diffusion tensor imaging (DTI) of the median nerve have been published. However, various imaging and reconstruction parameters were used. Thus, the purpose of this study was to systematically assess the optimal b-value for DTI at 3.0 T and reconstruction parameter for fiber tractography. Methods and Materials: Local ethical board approved study with written informed consent from 45 healthy volunteers ( 30 women, 15 men; mean age, $41 \pm 3.4$ ys) who underwent DTI of the right median nerve at 3.0 T (Achieva, Phillips, Best, the Netherlands) using a dedicated 8-channel wrist coil. A single-shot echo-planarimaging sequence (TR/TE $10123 / 40 \mathrm{~ms}$ ) was acquired at four different b-values $\left(800,1000,1200\right.$, and $\left.1400 \mathrm{~s} / \mathrm{mm}^{2}\right)$. Fiber tractography was performed by two independent readers using dedicated software (Phillips) and four different tracking algorithms which included different FA and reconstruction angle thresholds. Fiber tractography was then evaluated quantitatively and qualitatively.

Results: DTI acquisitions at higher b-values (1200 and $1400 \mathrm{~s} / \mathrm{mm}^{2}$ ) resulted in significantly longer fibers at tractography when compared to tractography at b-values of 800 and $1000 \mathrm{~s} / \mathrm{mm}^{2}(p<0.001)$. Overall fiber quality (fiber length, homogeneity, density and accordance with anatomy) was best at a b-value of 1200 $\mathrm{s} / \mathrm{mm}^{2}(\mathrm{p}<0.001)$. Tracking algorithms using a FA and angle threshold of 0.2 and $10^{\circ}$, respectively, were significantly better than other reconstruction algorithms. Conclusion: At 3.0 T, the optimal b-value for DTI is $1200 \mathrm{~s} / \mathrm{mm}^{2}$ and the optimal reconstruction parameter for fiber tractography includes a minimum FA and angle threshold of 0.2 and $10^{\circ}$, respectively.

10:30 - 12:00

Room L/M

\section{Radiographers}

\section{SS 1214}

\section{The radiographer's role in optimising radiation} exposure

Moderators:

M. Maas; Amsterdam/NL

G. Paulo; Coimbra/PT

\section{B-571 10:30}

Establishing the dose optimisation threshold for CT using psychophysical methods

F. Zarb' 1 L. Rainford ${ }^{2}$ M.F. McEntee ${ }^{2:}{ }^{1}$ Msida/MT, ${ }^{2}$ Dublin/IE (francis.zarb@um.edu.mt)

Purpose: The increasing frequency of $\mathrm{CT}$ leads to concerns about radiation risks. However, the consequences of not performing the CT examination thus missing injuries and disease have an impact upon patient management. For justified examinations, dose optimisation is needed. CTDI and DLP are used to indicate dose and quantify improvements achieved through optimisation. The aim of this study was to optimise $\mathrm{mA} ; \mathrm{kV}$ and pitch, while maintaining image quality and reducing dose Methods and Materials: Current CT scan parameters for head, abdomen and chest examinations were collected from four CT suites. These protocols were used to image a Catphan $\circledast 600$ CT QA phantom whereby image quality was assessed. Each of $\mathrm{mA} ; \mathrm{kV}$ and pitch was then systematically reduced until the contrast resolution (CR), spatial resolution (SR) and noise were significantly lowered. The Catphan ${ }^{\circledR} 600$ images were evaluated by two expert observers. The protocol considered as the optimisation threshold was the one just above the setting that resulted in a significant reduction in CR and noise but not affecting SR at the $95 \%$ confidence interval. Results: Using optimised parameters, CTDI and DLP were significantly reduced ( $p<0.001$ ) by $0-49 \%$ and $0-51 \%$, respectively. Below the optimisation threshold, $\mathrm{CR}$ and noise were significantly affected ( $p$ 0.083)

Conclusion: To affect an optimisation strategy we have established the limits at which image quality is detrimentally reduced by dose reduction. This work established the $\mathrm{mA}, \mathrm{kV}$ and pitch changes to achieve the optimisation threshold, reducing dose to the limit at which image quality is significantly decreased. 


\section{B-572 10:39}

Optimisation and dose reduction in CT of the skull

M. Monteiro, A. Catarino, E. Fernandes; Coimbra/PT

(mmonteiro@estescoimbra.pt)

Purpose: This study aims to assess the CT scan parameters: noise, spatial resolution and low contrast resolution by reducing only the $\mathrm{mA} 10 \%$ to $40 \%$ in order to find a compromise between quality image and the dose administered.

Methods and Materials: To collect data we used CT equipment Light Speed - GE 4-slices and a Light Speed VCT-GE 64-slices, and a LightSpeed ${ }^{\mathrm{TM}}$ 7.X phantom that simulates the density of the skull using the usual protocol for examinations of skull images obtained from both CT equipment in Sections 1 and 2 of the phantom. Subsequently reductions were made in the value of $\mathrm{mA}$ from $10 \%$ to $40 \%$ keeping constant the time of scan and kV.

Results: Based on a QA phantom using a FOV similar to the skull, we conclude that for both $\mathrm{CT}$ equipment in the images of $2.5 \mathrm{~mm}$ for the study of the posterior fossa, we can reduce to $20 \%$ of the value of $\mathrm{mA}$, for images related to the study of supra-tentorial region the $\mathrm{mA}$ can be reduced by $30 \%$ without loss of image quality. In both $\mathrm{CT}$ equipment the limiting factor reduction $\mathrm{mA}$ was the noise parameter. Conclusion: We find that for the equipment 4 slices $\mathrm{CT}$ we can reduce the value of DLP by $20 \%$, in 64 slices CT equipment we can reduce the value of DLP in about $27 \%$, compared with the dose protocol commonly used for examining the skull on both CT.

\section{B-573 10:48}

CT diagnostic reference levels (DRLs) in Malta

F. Zarb ${ }^{1}$, L. Rainford ${ }^{2}$, M.F. McEntee ${ }^{2} ;{ }^{1}$ Msida/MT, ${ }^{2}$ Dublin/IE

(francis.zarb@um.edu.mt)

Purpose: DRLs are dose levels that should not normally be exceeded, where good practice is applied. EU directive (97/43/EURATOM) makes the implementation of DRLs mandatory for member states. The current work establishes DRLs for three common CT examinations as the first step towards $\mathrm{CT}$ dose optimisation nationwide. Methods and Materials: The study included $57 \%$ of CT units in Malta, including three CT manufacturers. CT head, chest and abdomen were included being the most frequent CT examinations identified through a survey based on NRPB W67 guidelines. CTDI and DLP were obtained from the units' display console for 276 CT examinations, while effective dose (E) was estimated using ImPACT CT dose calculator.

Results: Maltese $3^{\text {rd }}$ quartile CTDIw values for abdomen: $11.7 \mathrm{mGy}$; chest: 12.5 mGy; head: $36.1 \mathrm{mGy}$ and DLP values for head: $736 \mathrm{mGycm}$ were well below EU recommendations. However, $3^{\text {rd }}$ quartile DLP values for abdomen: $539.4 \mathrm{mGycm}$ and chest: $492 \mathrm{mGycm}$ were fairly higher indicating the need for dose optimisation in these areas. Displayed CTDI and DLP correlate very well to calculated values using the ImPact method ( $(>0.7)$, indicating the use of both as appropriate CT dose descriptors. Variations in choice of scan parameters, scanner type and design are main reasons for significant differences in mean CTDI, DLP and $E$ between hospitals.

Conclusion: $3^{\text {rd }}$ quartile levels have been established for three $\mathrm{CT}$ examinations in Malta. Further work to minimise dose without affecting image quality and bring all DRLs below EU levels is also needed. DRLs should also be established for other CT examinations.

\section{B-574 10:57}

\section{Optimisation of compression, image quality and radiation dose in} mammography in Ireland

D. O'Leary, L. Rainford; Dublin/IE (desiree.oleary@ucd.ie)

Purpose: The study objective was to find achievable, objective and standardised compression depths to assist radiographers to achieve the lowest possible radiation dose with highest diagnostic image quality in mammography within the symptomatic breast service in Ireland.

Methods and Materials: This large quantitative and qualitative study of symptomatic breast units within the Republic of Ireland, collected image quality, compression and radiation dose data ( $n=4790$ images). The data were analysed using mathematical modelling and SPSS.

Results: Objective compression levels could not be proposed due to higher than expected inadequate image quality rates $(6.2 \%)$ and inconsistent compression forces; other factors may also impact on the compression levels attained which cannot be teased out by mathematical modelling of the current data set. Compression forces are low affecting image quality; a significant finding is that greater compression force by $11-15 \mathrm{~N}$ is needed to achieve perfect images. Mean glandular doses (MGDs) received by perfect images are significantly lower than inadequate images.
Conclusion: More explicit guidelines regarding radiographer training and bi-annual individual mammographer technique and image quality review must be enforced including greater training of radiographers performing mammography to standardise undertaking of mammographic projections with regard to achievable compression depth, application of compression force and MGDs delivered to the breasts of Irish women attending symptomatic breast services. MGD is proposed at the $95^{\text {th }}$ percentile for $55-65 \mathrm{~mm}$ breast compression for full field digital mammography units at $2.4 \mathrm{mGy}$ and for film-screen units at the mean MGD in line with other European publications as 2.64 mGy.

\section{B-575 11:06}

Impact of an optimization training program for radiological institutes on the reduction of $\mathrm{CT}$ radiation dose

S.T. Schindera, G. von Allmen, R. Treier, P.R. Trueb, C. Nauer, P. Vock,

Z. Szucs-Farkas; Berne/CH (sschindera@aol.com)

Purpose: To assess the efficacy of an optimisation training program for radiologists and technologists on the reduction of CT radiation dose.

Methods and Materials: Ten radiological institutes (seven public and three private institutes) were counselled by a radiation consulting team, including a radiologist and technologist with extensive experience in optimisation of CT protocols. The optimisation training program contained a small group teaching on protocol optimisation and a lecture on radiation dose reduction strategies. The radiation dose was assessed before and after training by the dose-length product (DLP) of five adult CT protocols (sinus, brain, chest, pulmonary angiography and abdomen). Only the DLP values of average-sized patients were included in the study. The mean DLP values were compared with the dagnostic reference levels (DRLs) of Switzerland. Results: During the pre- and post-optimisation period the DLP values of $180 \mathrm{CT}$ examinations each were collected. The average reduction of DLP in the post-optimisation period was $34 \%$ for sinus ( 170 vs. $112 \mathrm{mGy}{ }^{\bullet} \mathrm{cm}, \mathrm{P}<0.001$ ), $13 \%$ for brain (1005 vs. $874 \mathrm{mGy} \cdot \mathrm{cm}, \mathrm{P}<0.05), 30 \%$ for chest ( 443 vs. $311 \mathrm{mGy} \cdot \mathrm{cm}, \mathrm{P}<0.05), 40 \%$ for pulmonary arteries ( 364 vs. $217 \mathrm{mGy} \cdot \mathrm{cm}, P<0.001$ ) and $4 \%$ for abdomen $(575 \mathrm{vs}$. $555 \mathrm{mGy} \cdot \mathrm{cm}, \mathrm{P}=0.60$ ). The post-optimisation DLP values of the sinus, brain, chest, pulmonary arteries and abdomen were $68 \%, 13 \%, 22 \%, 52 \%$ and $15 \%$, respectively, below the DRL which is $350,1000,400,450$ and $650 \mathrm{mGy} \cdot \mathrm{cm}$, respectively. Conclusion: The optimization training program is a very effective method to achieve substantial radiation dose reduction in $\mathrm{CT}$ independent of the scanner type.

\section{B-576 11:15}

Reducing dose to the patient through PA techniques for the clavicle M.F. Mc Entee, C. Kinsella; Dublin/IE (mark.mcentee@ucd.ie)

Purpose: This study compares dose and image quality during PA and AP radiography of the clavicle to determine the optimum technique and quantify and difference. Methods and Materials: The methodology involved a cadaver-based dose and image quality study recording dose to the thyroid, eye, breast, entrance and exit surfaces. An image quality VGA study analysing AP and PA projections was also carried out. Results: A statistically significant $56.1 \%(p<0.001)$ and $56.5 \%(p<0.001)$ reductions to breast tissue and $62.3 \%(p<0.001)$ and $78 \%(p<0.001)$ reductions in the thyroid dose with employment of PA and PA15 caudal projections. Reductions of $28.5 \%(p<0.08)$ and $11.8 \%(p<0.12)$ in the doses to the eye were demonstrated for the PA. Differences in entrance-surface and exit doses were deemed nonsignificant. A 5.9\% $(p<0.39)$ reduction in overall image quality was seen with PA positioning. Reductions in image quality were evaluated to be nonsignificant at $95 \%$ (AP vs PA $(p<0.06)$, AP15 vs PA15 $(p<0.28)$.

Conclusion: The current study demonstrated consistent significant dose reductions to the thyroid and breast when PA projection is chosen over the AP projection. The authors recommend the implementation of PA positioning for clavicle radiography.

\section{B-577 11:24}

Patient's dose optimisation in projection lumbar spine examination with CR

A. Henner ${ }^{1}$, A.-R. Pietilä2 ${ }^{2}$ H. Pöyskö2 ${ }^{2}$ J. Sergejeff', H. Paso'; ${ }^{1}$ Oulu/FI,

${ }^{2}$ Haukipudas/FI (anja.henner@oamk.fi)

Purpose: The purpose of this study was to find out methods to evaluate the adult patient's dose in projection lumbar spine examination with computed radiography (CR) in health center after the change of procedure.

Methods and Materials: Data from lumbar spine ap on Bucky table were collected regularly as said in state 423/2000. In spring 2009 the procedure was changed due to the order of the central hospital. The radiographers find out increase in patient dose with AEC (automatic exposure control) when changing the procedure from Bucky table (patient lying on the table) to wall-stand (patient standing). The 
patient's dose was evaluated with ESD and after that the manual settings were taken in use. The next step was to adjust the sensitivity of the AEC by the vendor. Results: The doses increased significantly when changing to the stand wall. The radiographers decided to change to the manual settings and reached the earlier or lower dose level. Later the sensitivity of the AEC was increased and after that the use of AEC was possible without any dose increase.

Conclusion: The radiographers must be aware of the dose levels. Doses must be followed up regularly, especially when changing the procedures. The decrease in dose can be made by easy and cheap ways. The key person in dose optimisation is the radiographer. Working in this way demonstrates very high commitment to the radiation safety culture.

\section{B-578 11:33}

Mobile chest radiography on special care baby units: can we improve our practice?

K. Matthews, M. McEntee; Dublin/IE (kate.matthews@ucd.ie)

Purpose: To analyse potential for improvement in mobile chest radiography on neonates and infants by investigating: (1) does referral information provide evidence for justification; (2) what national diagnostic reference levels (DRLs) are appropriate (3) could practice be improved by modifications drawn from survey data analysis? Methods and Materials: Data were collected on 64 examinations in six hospitals: two tertiary referral, two neonatal and two general, representing $29 \%$ of hospitals nationwide providing mobile radiography to a Special Care Baby Unit. Radiation dose was recorded with calibrated DAP meters in centiGray ${ }^{*} \mathrm{~cm}^{2}\left(\mathrm{CGy}^{*} \mathrm{Cm}^{2}\right)$. Radiographers recorded the research data.

Results: Referral information was generally complete, although some requests had sparse or no clinical details. DAP dose correlated poorly with age, but reasonably with weight $(r=0.464)$. At specific weights, DAP dose varied within and between hospitals by factors up to 17 and 24 , respectively. Discounting aberrant dose outliers, there was never greater than five times variation between hospitals. Smal samples at particular weights impacted onto regression analysis of influences on DAP. However, trends indicate that technically, field size, $\mathrm{kVp}$ and $\mathrm{mAs}$ have the greatest influence.

Conclusion: The majority of mobile chest examinations have reasonable clinical justification. Weight-specific, Irish DRLs in the range 0.53 to $1.35 \mathrm{cGy}{ }^{*} \mathrm{~cm}^{2}$ are proposed for babies under one year and up to $6 \mathrm{kgs}$. Survey doses are comparable to published data. Unnecessary variations in DAP are apparent. Analysis of DAP variations suggests that more objective matching of baby weight to $k \mathrm{p}$ and mAs could considerably reduce the DAP dose without detriment to image quality.

\section{B-579 11:42 \\ Radiographer's role in the optimisation of MR-guided HDR prostate brachytherapy \\ C. Vandulek, J. Pall, F. Lakosi, G. Antal, A. Kovacs, J. Hadjiev, I. Repa;}

Kaposvár/HU (cvandulek@gmail.com)

Purpose: MRI provides excellent visualisation of the prostate and surrounding tissues, making it the choice for guiding and monitoring brachytherapy. The purpose of this study was to optimise the initial protocol of transperineal MR-guided highdose-rate prostate brachytherapy. The radiographers played an important role in the fine-tuning of the methodology

Methods and Materials: The procedures were performed using an open, 0.35T MRI scanner (GE Healthcare). Study volunteers were scanned comparing signalto-noise ratio (SNR) of the 9-inch GP coil and abdomen coil. Volunteers were scanned feet first in lateral decubitus and supine position to study patient comfort and ergonomics. Standard and modified T1 and T2 MRI sequences were compared in respect of artefacts and SNR

Results: The tested and modified imaging protocol allows clear definition of the prostate, coaxial needles and catheters. The results of the study justified the transition in methodology from GP surface coil to abdomen coil, change in positioning from lateral decubitus to supine position. Patients confirmed improvements in comfort and short-term side-effects. Modified pulse sequences resulted in improved SNR and reduction of artefacts.

Conclusion: The initial methodology implemented for MR-guided prostate brachytherapy has been further developed leading to improvements in patient comfort, ergonomics, patient access and SNR. The radiographers played an important role in the optimisation of the protocol contributing to the success of the study. Our results facilitate us to implement the optimised techniques in further MR-guided brachytherapy protocols other than the prostate.

\section{B-580 11:51}

The consequence of the referral to patient's dose

A. Henner ${ }^{1}$, A.-R. Pietilä ${ }^{2}$, H. Pöyskö ${ }^{2}$, M. Pohjola ${ }^{1} ;{ }^{1}$ Oulu/FI, ${ }^{2}$ Haukipudas/FI

(anja.henner@oamk.fi)

Purpose: The purpose of this study was to find out the consequence of referral to the procedure in orthopantomogramic imaging of the teeth in order to decrease the patient's dose.

Methods and Materials: The dose area product (DAP) of different programs was measured without patient. The variation in different program is very large, e.g. when using the partial program instead of whole mandible to a seven years old child, the dose is only one third (from $6.13 \mathrm{cGycm}^{2}$ to $2.31 \mathrm{cGycm}^{2}$ ). The radiographers and dentists evaluated critically the present procedures: very poor referrals without any focused indication and due to this ortopantomogram was always taken by the same program (whole mandible). The doses of the different programs were presented to the dentists.

Results: After the meeting the dentists started to write better referrals with clear indications and detailed information telling what they want to see from the x-ray. On the basis of this information the radiographer can take whole or partial mandible and set the $\mathrm{kV}$ and $\mathrm{mA}$ according the required image quality.

Conclusion: The examinations of teeth are very common in Finland. About 2.1 million intraoral and about 300000 ortopantomograms are taken yearly and a lot of them to children. That is why the image quality and dose optimisation is important. It is very easy to decrease the patient dose in co-operation the referral dentists. The multiprofessional co-operation, open discussions and awareness of the dose levels are the key points.

$10: 30-12: 00$ Room P

\section{Physics in Radiology}

\section{SS 1213}

\section{Novel techniques and new technologies}

Moderators:

L. Struelens; Mol/BE

A. Todd-Pokropek; London/UK

\section{B-581 10:30}

Human medical imaging with a reformed x-ray interferometry using a practical $x$-ray tube: a preliminary cadaveric study

J. Tanaka ${ }^{1}$, A. Momose ${ }^{2}$, M. Nagashima1, K. Kido ${ }^{3} ;{ }^{1}$ Iruma-gun/JP, ${ }^{2}$ Kashiwa/JP ${ }^{3}$ Hachiouji/JP (jtanaka@saitama-med.ac.jp)

Purpose: To evaluate the image characteristics from human cadavers obtained with a reformed $\mathrm{x}$-ray interferometry in which a practical $\mathrm{x}$-ray tube is used. Methods and Materials: A reformed x-ray Talbot interferometry following the theory of Lau in which a practical incoherent $x$-ray source is used, i.e. Talbot-Lau interferometry was created on the basis of design with wave-optic simulation. In this system, a source grating which consists of multiple slits of $10 \mu \mathrm{m}$ or less in width, arranged periodically, is placed immediately after an incoherent $x$-ray source. Each slit emits coherent $x$-ray and if the pitch of the grating is set appropriately, an $x$-ray source with coherent light and enough power can be achieved. Because this system requires neither synchrotron nor microfocus $\mathrm{x}$-ray tube, medical application of x-ray interferometry has become realistic. After confirming the performance of the system using samples such as fruits, chicken wings and fish, we have started to image parts of human cadaveric body such as a hand and a knee under approval of university ethics committee. The images obtained have been correlated with macroscopic anatomical findings.

Results: Certain components of a hand and knee such as ligaments, tendons and cartilages, which are hardly seen by conventional x-ray imaging, have been clearly depicted by this new system.

Conclusion: This new imaging technology is assumed to be clinically applicable. Our trial should be advanced to the next step with normal volunteers.

\section{B-582 $\quad 10: 39$}

Will x-ray phase contrast imaging revolutionise diagnostic imaging?

R. Raupach, M. Grasruck, B. Schmidt, T.G. Flohr;

Forchheim/DE (rainer.raupach@siemens.com)

Purpose: To quantify the inherent properties, the potential, and the limitations of $\mathrm{x}$-ray phase contrast imaging $(\mathrm{PCl})$

Methods and Materials: The refractive index of materials can be characterised by its real part (phase shift) and imaginary part (absorption). $\mathrm{PCl}$ visualises the rea part of the refractive index by exploiting the differential phase shift. It is believed 
to provide additional information on tissue properties compared with conventional absorption-based imaging (Al) techniques. We have developed a mathematical formalism to assess the contrast-to-noise ratio (CNR) per dose analytically. This allows for a direct performance comparison of $\mathrm{PCl}$ versus $\mathrm{Al}$. The mathematical model was validated by numerical simulations and compared to experimental data, particularly, with regard to CT.

Results: 1. PCl shows a fundamentally different noise power spectrum (NPS). $\mathrm{PCl}$ images show strong statistical fluctuations on growing length scales. The predicted characteristics are confirmed by simulations as well as experimental data. Consequently, the performance of $\mathrm{PCl}$ deteriorates with decreasing spatial resolution. 2 . Due to the measurement of periodic quantities, information propagation collapses at low dose. Simulations and experimental findings are in agreement with this result. This implies a minimally required radiation dose. In contrast, $\mathrm{Al}$ does not collapse at low dose.

Conclusion: To achieve a benefit of $\mathrm{PCl}$ versus $\mathrm{Al}$ a minimum spatial resolution is necessary. This implies increased radiation dose if the resolution to break even is higher than that of a specific diagnostic application today. On the other hand, $\mathrm{PCl}$ cannot be performed at arbitrarily low dose as a matter of principle.

\section{B-583 10:48}

\section{Can intravenous contrast be used for PET-CT attenuation correction in} head and neck cancer?

R.C. Flamini, L. Yamaga, J. Wagner, M.L. Cunha, A. Osawa, G.C. Campos, R. Gomes, M.B.G. Funari; SãoPaulo/BR (rcflamini@yahoo.com.br)

Purpose: Evaluate the influence of iodine intravenous contrast in calculating standard uptake value (SUV) in PET-CT studies with ${ }^{18} \mathrm{~F}$-fluordeoxyglucose (FDG). Methods and Materials: We retrospectively analyzed 106 PET-CT studies of 63 consecutive patients diagnosed with head and neck cancer referred to our institution from 01/01/2006 to 07/31/2009. The protocol included PET-CT from the top of the head to the mid-thigh without intravenous contrast, followed by a head and neck PET-CT after intravenous contrast. The SUV values were calculated on the lesions $\left(S_{U V} V_{\text {LESION }}\right)$ and on the great vessels $\left(S_{\text {VESSEL }}\right)$ before and after contrast administration.

Results: Patients' average age were 56.5 years and 53 were male. From the 106 studies analysed, 59 showed areas of abnormal FDG uptake on the head and neck, suggestive of neoplastic disease. Analysing the 59 positive studies, there was an

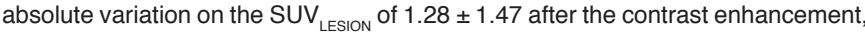
with a percentage change in the SUV LESION $_{\text {of }} 16.5 \pm 16.8 \%$. The average value of the SUV between the studies performed before and after the contrast raised from 6.64 to $7.65(p=0.191)$, not achieving statistical significance. Including all the studies performed, there was an absolute variation on the value of the SUV $0.05 \pm 0.22$ and a percentage change of $3.6 \pm 12.0 \%$ after contrast enhancement. The average value of the SUV VESSEL ranged from 1.82 before the contrast to 1.86 after the contrast $(p=0.389)$, also without statistical significance.

Conclusion: Intravenous contrast does not alter significantly the value of SUV, thus it can be used in the PET-CT studies for attenuation correction.

\section{B-584 10:57}

Dose reduction by on-line tube voltage modulation (TVM) in clinical conebeam $\mathrm{CT}$

M. Baer, M. Kachelrieß; Erlangen/DE (matthias.baer@imp.uni-erlangen.de)

Purpose: To evaluate the potential benefits of anatomy-dependent tube current and tube voltage modulation.

Methods and Materials: All CT manufacturers recognised the dose reduction potential of patient-dependent tube current modulation (TCM) and provide manufacturer-specific implementations. There are two drawbacks: the dose is estimated to be proportional to the tube current and the tube voltage always remains at a constant level. We derived a new approach that both accounts for the actual patient dose and that modulates the tube current as a function of the patient anatomy. The potential of combining TCM with TVM was evaluated in a simulation study using clinical patient data. Patient dose was estimated for each projection by analysing the rawdata using a Monte Carlo-calibrated polychromatic deterministic model. Rawdata were water precorrected using the approach of reference [Phys Med Biol 55:4107-4117]. The dose required to obtain a specified image quality (spatial resolution and noise) was recorded to quantify the value of combined TCM and TVM. Results: With TVM noise variance, respectively, dose can be lowered by up to $18 \%$ for both the hip and the shoulder region compared to TCM at a constant tube voltage of $120 \mathrm{kV}$. CT image quality is not impaired by TVM

Conclusion: Combining TVM with TCM reduces dose by $10 \%$ to $20 \%$ compared to TCM alone. It can therefore be an important contribution to the world-wide efforts to reduce patient dose.

\author{
B-585 11:06 \\ Assessment of a tube voltage of $70 \mathrm{kV}$ for paediatric CTA examinations
}

B. Schmidt, O. Saba, R. Banckwitz, H. Wolf, T. Flohr; Forchheim/DE

Purpose: Published studies have shown that in patients with small cross-section, using lower kVs ( 80 or $100 \mathrm{kV}$ ) allows acquiring data with a similar image quality but lower dose. In our study we assessed the impact of $70 \mathrm{kV}$ on image quality and dose. Methods and Materials: Measurements were performed on a prototype system (Definition AS+, Siemens), which was modified to allow scanning at $70 \mathrm{kV}$. To assess image quality, measurements with different sized phantoms simulating paediatric patients were performed. Noise was determined with ROI measurements. To assess contrast, probes with diluted iodinated contrast agent were placed in centra and peripheral positions of the phantoms and the enhancement measured. Dose was determined by CTDIvol measurements on CTDI phantoms (diameter 8 and $16 \mathrm{~cm}$ ). In addition, patient dose for a CT of the abdomen was estimated using a paediatric anthropomorphic phantom and TLD measurements.

Results: In case of the $8 \mathrm{~cm}$ phantom, for the same CTDIvol, noise does not change significantly for lower voltages. However, iodine contrast increases from $427 \mathrm{HU}$ at $120 \mathrm{kV}$ to $998 \mathrm{HU}$ at $80 \mathrm{kV}$ and $1125 \mathrm{HU}$ at $70 \mathrm{kV}$. Differences in effective dose between 70 and $80 \mathrm{kV}$ for the abdominal scan were strongly correlated with respective the difference in CTDIvol $(<10 \%)$.

Conclusion: For small patients a further reduction in tube voltage from $80 \mathrm{kV}$ down to $70 \mathrm{kV}$ allows either to acquire images with an increased iodine contract $(+13 \%)$ or a dose reduction of $28 \%$ for the image quality in terms of iodine contrast to noise.

\section{B-586 11:15}

Dual-energy spectral CT (DESCT) allows selection of the optimal monochromatic energy for imaging the instrumented spine and improves diagnostic quality over traditional imaging

J.M. Kessler, M. Ellestad, J. Rios, E.G. Stein, L. Tanenbaum; New York, NY/US (lawrence.tanenbaum@mountsinai.org)

Purpose: Leveraging unique atomic densities of different elements, and the ability of modern CT systems to image at multiple energies, dual-energy-spectral CT (DESCT) provides novel imaging information for CT diagnosis of spine disease. Analysis of DESCT scan spectral Hounsfield signal behaviour in instrumented spines allows selection of appropriate energy levels that minimise artefact and optimise image quality. We sought to determine energy levels that maximise signal-to-noise ratios (SNRs) for instrumented spines and compared these optimal images with those from traditional polychromatic $140 \mathrm{kVp}$ and routine monochromatic $70 \mathrm{keV}$ images. Methods and Materials: Ten instrumented spine patients were imaged on a GE CT750HD using DESCT. Data were processed and spectral Hounsfield unit curves (SHUCs) were generated from region of interests placed in spinal canals adjacent to hardware. An optimal SNR keV level was identified and a set of multiplanar images were generated at that level. These images were compared with standard $70 \mathrm{keV}$ monochromatic and $140 \mathrm{kVp}$ polychromatic sets and evaluated for artifact obscuring the spinal canal, and overall diagnostic quality.

Results: Evaluation of SHUCs demonstrates that optimal SNRs were obtained at energy levels 90 - 95keV. Comparing monochromatic images produced at optimal energy levels to standard monochromatic $70 \mathrm{keV}$ and $140 \mathrm{kVp}$ polychromatic images significantly reduces spinal canal noise and artefact. Furthermore, these images remained adequate for assessing other findings (e.g. degenerative disease). Conclusion: Monochromatic images of instrumented spines obtained at $93 \mathrm{keV}$ (range 90-95 keV) produced images with fewer artefacts and better hardware visualisation, with superior overall quality.

\section{B-587 11:24}

Performance of static MDCT beam-shaping filtration for elliptical subject geometries in dependence of projection angle

L.-S. Veloza, H.-U. Kauczor, W. Stiller; Heidelberg/DE

(stella.veloza@med.uni-heidelberg.de)

Purpose: MDCT beam-shaping filters modulate $x$-ray beam intensity across the fan compensating for decreasing absorption due to shorter $\mathrm{x}$-ray path lengths through patient body periphery compared to its center. To investigate projection-angle dependence of detector statistics for elliptical subject geometries, performance of experimentally measured filter geometry and theoretical filter geometry adapted to elliptical specimen has been compared.

Methods and Materials: Using a Compton spectrometer, primary MDCT $\mathrm{x}$-ray spectra $\left(120 \mathrm{kV}_{\mathrm{p}}\right)$ have been measured at different angular distance $\left(0^{\circ}-21^{\circ}\right)$ from the central ray of the $x$-ray fan. These have been used to calculate the geometrical shape of the aluminum bowtie filter. A theoretical filter model was determined for elliptical subject geometries. For both shape models, detector statistics after x-ray 
passage through a homogeneous elliptical phantom $(16.0 \mathrm{~cm}$ semimajor axis, $12.5 \mathrm{~cm}$ semiminor axis, $8.0 \mathrm{~cm}$ thickness) of polypropylene, PMMA and water was Monte-Carlo simulated with Geant4. The number of surviving quanta after xray passage through beam-shaping filter plus phantom was counted by a detector array positioned for projections a.p. to lateral in $15^{\circ}$-steps.

Results: At the fan edge, measured filter shape is systematically too thin: for lateral projections maximum difference is $28 \%$ compared to the theoretical filter model for elliptical subject geometries. Performance of the latter varies with projection angle and thus requires adaption of geometry to view angle.

Conclusion: Static beam-shaping filter geometry and its material composition should be reevaluated to reduce signal and thus patients' skin dose for lateral projections. Dynamic beam-shaping concepts could allow for radiation exposure reduction in MDCT.

\section{B-588 11:33}

Three-dimensional fluid-attenuated inversion recovery imaging: reduced cerebrospinal fluid artefacts and enhanced sensitivity and specificity for subarachnoid haemorrhages

N. Lummel ${ }^{1}$, V. Schoepf ${ }^{2}$, M. Burke ${ }^{3}$, H. Brückmann ${ }^{1}$, J. Linn ${ }^{1} ;{ }^{1}$ Munich/DE

${ }^{2}$ Vienna/AT, ${ }^{3}$ Solingen/DE

Purpose: Fluid-attenuated inversion recovery (FLAIR) images are highly sensitive to subarachnoid haemorrhages (SAH). Yet, cerebrospinal fluid (CSF) flow artefacts on conventional FLAIR can result in false-positive results. Here, we compared two-dimensional (2D) and three-dimensional (3D) FLAIR sequences concerning their proneness to these artefacts, and their sensitivity and specificity for $\mathrm{SAHs}$.

Methods and Materials: The following four FLAIR sequences were evaluated: (1) 2D FLAIR at 1.5 T, (2) 2D FLAIR, (3) 2D PROPELLER (= periodically rotated overlapping parallel lines with enhanced reconstruction) FLAIR, and (4) 3D FLAIR (Cube-FLAIR) at $3 \mathrm{~T}$. All sequences were applied in five healthy volunteers; sequences 2 and 4 were also applied under routine conditions in ten patients with focal epilepsy, and in five patients with $\mathrm{SAH}$. Analysis was done independently by two neuroradiologists. For volunteers and epilepsy patients the presence of flow artefacts in ventricles and cisterns was evaluated and scored on a four-point scale. Mean values were calculated and compared using paired t-tests. Sensitivity and specificity of sequences 2 and 4 for SAH detection were determined.

Results: Cube-FLAIR showed almost no CSF artefacts at all, either in the volunteers or in the epilepsy patients, and therefore was significantly superior to any other FLAIR $(p<0.001)$. Sensitivity and specificity of the 3 T 2D FLAIR for SAHs were $68.8 \%$ and $90.3 \%$, respectively, while the Cube-FLAIR showed a sensitivity of $96.9 \%$ and a specificity of $100 \%$.

Conclusion: The Cube-FLAIR allows FLAIR imaging with almost no CSF artefacts, and thus seems to be very helpful to detect SAHs.

\section{B-589 11:42}

Effective detective quantum efficiency of digital radiography systems in chest examinations: comparison between a wireless direct radiography detector and other widely used digital radiography system

A. Nitrosi ${ }^{1}$, M. Bertolini ${ }^{1}$, S. Rivetti ${ }^{2}$, N. Lanconelli ${ }^{3}$, P. Pattacini ${ }^{4}$, V. Ginocchi ${ }^{5}$,

M. Iori ${ }^{1} ;{ }^{1}$ Reggio Emilia/IT, ${ }^{2}$ Modena/IT, ${ }^{3}$ Bologna/IT, ${ }^{4}$ Guastalla/IT,

${ }^{5}$ Castelnovo ne'Monti/IT (andrea.nitrosi@asmn.re.it)

Purpose: To compare digital radiography systems in terms of effective detective quantum efficiency (eDQE) in chest examinations.

Methods and Materials: A wireless direct radiography (DR) Gadolinium-oxysulphide phosphor (GOS) based system was compared in terms of eDQE with respect to widely used CsI-DR based systems and a computed radiography (CR) system. Four systems were evaluated in a total of seven configurations. The PA chest phantom adopted in AAPM Report \#60 was used. The eDQE was calculated by the modulation transfer function (MTF), the normalized noise power spectrum (NNPS), the phantom attenuation and scatter, and the x-ray flux. Focal spot size effect and the use of air-gap instead of grid were investigated, as well. The image quality was measured with contrast-to-noise ratio (CNR) and a figure of merit (FOM) defined as the ratio between $\mathrm{CNR}^{2}$ and the air kerma at detector level.

Results: The MTF showed an important degradation. The NNPS was influenced by the grid (in some systems as structured noise). eDQE (0) doubled with a 20-cm-air-gap configuration. FOM was consistent with the eDQE results. In terms of eDQE (0) GOS-DR and CsI-DR systems showed better performance in respect to $C R$ system respectively of a factor of 3 and 4 times). FOMs confirmed eDQE (0) differences among different systems although DQE (0) differences were smaller. Conclusion: The eDQE comparison method showed DR system's wide margins of optimisation: among these, scatter reduction technique plays a fundamental rule.
B-590 11:51

MRgFUS: methodology and physical aspects

A. Torresin, P. Colombo, F. Zucconi, S. Pasetto, C. Ticca, A. Rampoldi,

A. Lascialfari; Milan/IT (alberto.torresin@unimi.it)

Purpose: The MR-guided High Intensity Focused Ultrasounds (MRgFUS) is a rapidly developing methodology for lesion treatments. The purpose of this work is to describe the methodology and characterise the device from physical point of view. Methods and Materials: The ExAblalte 2000v.4.2 (Insightec (IL)) is integrated with MRI-GE-Signa-HDxt-Advantage-1.5 T. The system controls the position of the US beam and the real time temperature of the tissue using the MRI system. The thermometry is based on PRF shift. We studied geometrical characteristics of a single sonification beam (3-4 cm lengths, half centimeters diameter) using specific HiFU gels. We investigated the relationship between temperature and the phase shift with an independent heating source and high accuracy fibre optics temperature sensors by means the $\alpha$ value evaluation. Also, T1 method for temperature evaluation was analysed. Before every treatment, phantom quality control protocol (DQA) was applied: the constancy of size focus, energy, temperature was tested. Results and Conclusion: The results show good correlation between chemical shift and temperature. We estimate the $\alpha$ value as $-0.0109 \pm 0.0002 \mathrm{ppm} /{ }^{\circ} \mathrm{C}$. Our data are in agreements with literature. Using PRF method, our precision of the single pixel readout temperature is $4.2^{\circ} \mathrm{C}$; the accuracy is $0.26^{\circ} \mathrm{C}\left(\Delta \mathrm{T}=10^{\circ} \mathrm{C}\right)$ and $0.65^{\circ} \mathrm{C}\left(\Delta \mathrm{T}=20^{\circ} \mathrm{C}\right)$. Using $\mathrm{T} 1$ method, our precision of the single pixel readout temperature is $9.1^{\circ} \mathrm{C}$; the accuracy is $1.7^{\circ} \mathrm{C}\left(\Delta \mathrm{T}=10^{\circ} \mathrm{C}\right)$ and $7{ }^{\circ} \mathrm{C}\left(\Delta \mathrm{T}=20^{\circ} \mathrm{C}\right)$. This lack of precision and accuracy of the T1 method should be explained with a choice of TR in the MRI protocol. DQA analysis: the stability of the physical parameters for the treatment is inside the experimental errors.

10:30 - 12:00 Room Q

Paediatric

\section{SS 1212}

\section{Cardiothoracic imaging}

Moderators

A.D. Calder; London/UK

M.A. Lucic; Sremska Kamenica/RS

\section{B-591 10:30}

Low dose computer tomography (LDCT) imaging of the paediatric chest: a phantom study on the use of iterative reconstruction (IR) as a new advanced reconstruction technique

C.-P. Wallner, M. Körner, U. Linsenmaier, K. Schneider, M.F. Reiser;

Munich/DE (cwallner@med.uni-muenchen.de)

Purpose: To evaluate the use of iterative reconstruction technique for dose reduction and image quality improvement in computer tomography (CT) of the paediatric chest.

Methods and Materials: Low-dose paediatric chest CT scans using the adaptive statistical iterative reconstruction (ASIR) method and the standard paediatric LDCT protocol were performed on a GE Discovery CT 750HD. Natural sponges as lung equivalent saturated with iodinated contrast media inserted within a chicken specimen simulating a neonatal patient and within a turkey specimen as an infant phantom were used. For the neonatal studies tube current was $80 \mathrm{kVp}$, for the infant studies $100 \mathrm{kVp}$. The ASIR reconstruction level was $40 \%$ for both the neonatal and the infant examinations. The noise index was identical for all protocols with a value of 45 . Results: The radiation dose delivered during the LDCT scan without IR was $1.64 \mathrm{mGy}$ CTDI (25.25 mGy ${ }^{*} \mathrm{~cm}$ DLP) for the neonatal phantom and $4.06 \mathrm{mGy}$ CTDI (100.79 $\mathrm{mGy}{ }^{*} \mathrm{~cm}$ DLP) for the infant phantom. For the neonatal phantom the dose delivered using LDCT with IR was $0.93 \mathrm{mGy}$ CTDI (14.30 $\left.\mathrm{mGy}^{*} \mathrm{~cm} \mathrm{DLP}\right)$ and for the infant phantom $2.29 \mathrm{mGy}$ CTDI $\left(57.92 \mathrm{mGy}^{*} \mathrm{~cm}\right.$ DLP). Dose reduction was $43.3 \%$ for the $80 \mathrm{kVp}$ neonatal protocol and $43.6 \%$ for the $100 \mathrm{kVp}$ infant protocol. Conclusion: With iterative reconstruction technique compared to a standard LDCT paediatric chest protocol we found the patient radiation dose being significantly reduced up to $44 \%$ when using the low-dose IR method. The resulting diagnostic image quality was similar for both methods. 


\author{
B-592 10:39 \\ Dual source CT: diagnostic, motion artefact free chest CT images in non \\ cooperative, free breathing children avoiding anaesthesia \\ K. Gonzalez Graniel, M.L. Dijkshoorn, A.M. Koch, I.J.C. Hartmann, \\ M. van Straten, H.A.W.M. Tiddens; Rotterdam/NL (kgraniel@gmail.com)
}

Purpose: To obtain a motion free and low dose chest CT scan in non-cooperative children, normally a controlled volume ventilation procedure under general anaesthesia is required, we use a high speed scanner to perform chest CT in uncooperative children to avoid the use of anaesthesia.

Methods and Materials: 31 Children (mean age 2.8 years, range 0 to 7.1 years) were scanned while freely breathing using a dual source CT scanner (Siemens, Somatom Definition Flash). Acquisition parameters: collimation $2 \times 64 \times 0.6 \mathrm{~mm}$; tube voltage $80 \mathrm{kV}$; pitch 3; rotation time $0.28 \mathrm{~s}$; Quality of images was scored independently by 2 radiologists in random order and blinded for identifiers as follows: motion artefact: $0=$ none to minimal, $1=$ moderate, $2=$ severe. diagnostic quality: $0=$ poor, $1=$ suboptimal, but satisfactory, $2=$ good, $3=$ excellent.

Results: Motion artefact scores were: 0 in 24, 1 in 6 , and 2 in 1 child. Diagnostic quality scores were 1 in 5,2 in 22, and 3 in 4 children. Mean dose-length-product was $16.84 \mathrm{mGycm}$ (range $3-72$ ). Mean scan length was $13.98 \mathrm{~cm}$ (range 8.70 19.80). Scan time for acquisition was less than 1 second

Conclusion: High speed CT allows obtaining high quality chest CTs in noncooperative patients avoiding the use of general anesthesia

\section{B-593 10:48}

High-pitch spiral CT: image quality and radiation dose in paediatric chest CT M.M. Lell, M. May, P. Deak, S. Alibek, T. Radkow, W. Kalender, M. Uder;

Erlangen/DE (michael.lell@uk-erlangen.de)

Purpose: We evaluated a new, subsecond high-pitch scan mode (HPM), which obviates the need of sedation and to hold the breath.

Methods and Materials: 60 patients were included in this study. 30 patients (mean age 14 months) were examined with a dual source CT system in an HPM $(P=3.0)$. None of the children was sedated. Image quality was assessed focusing on motion artefacts and delineation of the vascular structures and lung parenchyma. 30 patients (mean age 15 months) were examined under sedation in conventional pitch mode (CPM) on 10/64-slice CT systems. Dose values were calculated and Monte Carlo simulations were performed to assess dose distribution for CPM and HPM. Results: Image quality was superior with HPM, because of a significant reduction in motion artefacts, as compared to CPM with 10- and 64-slice CT. In the control group, artefacts were encountered at the level of the diaphragm (100\%), the borders of the heart $(100 \%)$, and the ribs $(67 \%)$ and spine $(20 \%)$, whereas motion artefacts were detected in the HPM-group only in 6 patients in the lung parenchyma next to the diaphragm or the heart $(P<0.001)$. Dose values were within the same range in the patient examinations (CPM, $1.9 \pm 0.6 \mathrm{mSv}$; HPM, $1.9 \pm 0.5 \mathrm{mSv}$; $\mathrm{P}=0.95)$. Conclusion: High-pitch chest CT is a robust method to provide highest image quality making sedation or controlled ventilation for the examination of infants, small or uncooperative children unnecessary, whereas maintaining low radiation dose values.

\section{B-594 10:57}

PoMoCT (postmortem computed tomography) investigations on deceased neonates: comparative analysis on chest findings of perinatal postmortem CT examinations

K. Karlinger, D. Ibolyka, A. Fintha, T. Glasz; Budapest/HU

(kinga.karlking@gmail.com)

Purpose: The aim of this study was to compare the postmortem neonatal chest-CTs with autopsy findings as a gold standard, and clinical data to determine the validity of our new supplementary postmortem examination method in the evaluation of the chest pathology.

Methods and Materials: Between July 2007 and January 2010 PoMo whole-body CTs were performed on 20 human neonatal corpses, ten of them also had whole-body CT-angiography with water-soluble contrast medium: eleven females, nine males, mean gestation week was 32.9 (23-41). From times of death till to imaging passed 2.5 days ( 14 hours to 5 days). Evaluation: MPR, MIP, volume rendering reconstructions. Subsequently conventional autopsy and histological examinations were performed. The independently established final diagnoses were compared by both investigating staff. Results: Chest/skeletal: three fractures, fifteen developmental rib-alteratons, one osseal metastasis, one hemivertebra. None of those were mentioned in the final report. Angiography: three double superior v. cava, three widely open Botallo ducts, one ventricular septumdefect, one heart of two chambers, one common arterial trunk, one pulmonal vein variation, one thrombosis of SVC. Lungs: seven atelectases, two haemorrhages, three hypoplasias, one metastasis, three bullae, two ptx/interstitial ptx, one hydroptx. Airways: one trachea/bronchus malformation. Pleura: five fluid collections, five diaphragm herniations.

Conclusion: Because of concordant data of conventional and virtual autopsies in demonstrating morphological alterations, one feels encouraged to regard the PoMoCT data to be relevant when deciding the final diagnosis. It draws the pathologist's attention to morphological situations, which are difficult to assess by conventional dissecting methods alone.

\section{B-595 11:06}

Prediction of bronchopulmonary dysplasia using lung ultrasonography within the first 24 hours of life

J. Lovrenski' ${ }^{1}$ E. Sorantin' ${ }^{2}$ S. Stojanović'; ${ }^{1}$ Novi Sad/RS, ${ }^{2}$ Graz/AT (jolo@neobee.net)

Purpose: To present a new grading system for lung ultrasonography (US) findings and its application in early prediction of bronchopulmonary dysplasia (BPD). Methods and Materials: Lung US was performed within the first 24 hours of life in 120 preterm infants with clinically diagnosed respiratory distress syndrome (RDS). Thirty of them developed BPD, all below 31 gestational week, weighing less than $1500 \mathrm{~g}$. Based on these criteria the control group was formed. US findings at each examined lung area were graded from 1 (normal) to 6 (subpleural consolidation). Grades 2 to 5 represented different amounts of $B$ lines. Prediction possibilities of BPD development and its mild and severe forms (types 1 and 2), both clinical and radiographic, were evaluated. MANOVA and discriminant analysis defined the difference between the groups, their characteristics and homogeneity.

Results: Statistically significant difference was verified between US findings of examined and control group at each lung area. Homogeneity of examined and control group was $83.33 \%$ and $94.12 \%$, respectively. Groups of BPD clinical types 1 and 2 had a homogeneity of $91.7 \%$ and $88.9 \%$, while for radiographic types 1 and 2 homogeneity was $93.75 \%$ and $92.86 \%$, respectively. Border equations for the each data set were determined. Pleural thickness also showed statistically significant difference between examined (confidence interval (Cl) $1.74-2.02 \mathrm{~mm}$ ) and control (Cl $1.40-1.63 \mathrm{~mm})$ group.

Conclusion: Lung ultrasonography findings might enable an early prediction of BPD. Their association with clinical parameters should be considered in studies to come.

\section{B-596 11:15}

Diagnostic performance of low-dose prospective ECG-triggering dualsource $\mathrm{CT}$ angiography compared with conventional $\mathrm{x}$-ray angiography in Kawasaki disease

Z. Cheng, X. Wang, L. Wu; Ji Nan/CN (chengzhaoping110@sina.com)

Purpose: This study was designed to compare the results of low-dose prospective ECG-triggering dual-source CT angiography (DSCTA) with $\mathrm{x}$-ray coronary angiography $(X C A)$ in infants and children with Kawasaki disease (KD).

Methods and Materials: This study was local ethics board approved; written informed consent was obtained from all patients. Sixteen children (9 male; mean age: 15 months, range: 6 months to 5 years; mean heart rate: $106 \mathrm{bmp}$, range: 88-121 bmp) underwent low-dose prospective ECG-triggering DSCTA with free breathing. All patients underwent XCA within a week. The maximal diameter and length of the aneurysm were recorded. Radiation dose values were calculated. Results: In eleven patients, aneurysms of the coronary arteries were identified, while coronary ectasia alone was present in the remaining five patients. DSCTA and XCA diagnosis of coronary artery aneurysm agreed completely. Maximal aneurysm diameter and length and ectasia diameter by DSCTA and XCA were similar. No stenotic lesion was identified by either technique. The mean effective dose of the protocol was $0.34 \pm 0.06 \mathrm{mSv}$.

Conclusion: Prospective ECG-triggering DSCTA with very low effective radiation dose allows the accurate diagnosis of coronary artery lesions in infants and children with KD compared with XCA.

\section{B-597 11:24}

Radiation dose and diagnostic accuracy of ECG-gated coronary CT angiography in paediatric patients

T. Henzler ${ }^{1}$, U. Schoept ${ }^{2}$, W. Huda ${ }^{2}$, C. Fink', S.O. Schönberg ${ }^{1}$,

A. Hlavacek ${ }^{2} ;{ }^{1}$ Mannheim/DE, ${ }^{2}$ Charleston, SC/US

(thomas.henzler@medma.uni-heidelberg.de)

Purpose: Over the past years paediatric ECG-gated coronary-CT-angiography (CCTA) has become feasible. We aimed to assess diagnostic accuracy of paediatric ECG-gated CCTA, to calculate the effective radiation dose (ED) and to identify the main determinants of radiation exposure. 
Methods and Materials: Between 01/2006 and 02/2010, 73 paediatric cCTA were performed to evaluate patients with known or suspected coronary abnormalities (48 males, 24 females, mean age 10.3 \pm 5.8$)$. Weight, height, CT dose index (CDTI), dose-length product (DLP), tube voltage (kV) and effective tube current (eff mAs) were recorded and the ED was calculated. To determine the diagnostic accuracy of cCTA, findings were compared with other diagnostic studies or with surgery. Results: CCTA findings were confirmed by other diagnostic studies or by surgery in all patients. The overall ED was $9.3 \mathrm{mSv} \pm 6.0 \mathrm{mSv}$. Patients scanned with $80 \mathrm{kV}$ had lower ED than patients scanned with $100-120 \mathrm{kV}(5.6 \mathrm{mSv} \pm 2.7 \mathrm{mSv}$ vs. 14.9 $\mathrm{mSv} \pm 5.4 \mathrm{mSv}(\mathrm{p}<0.0001))$. Patients between 0 and 5 years were exposed to lower ED compared to patients of age $5-10$ years $(4.0 \mathrm{mSv} \pm 1.9 \mathrm{mSv}$ vs. $7.4 \mathrm{mSv} \pm 2.9$ $\mathrm{mSv}(\mathrm{p} \leq 0.005))$ and age $10-18$ years $(4.0 \mathrm{mSv} \pm 2.9 \mathrm{mSv}$ vs. $11.9 \mathrm{mSv} \pm 6.4 \mathrm{mSv}$ $(p \leq 0.0002)$ ). Patients who underwent cCTA later than 12/2007 were exposed to lower ED than those who underwent cCTA before 12/2007 ( $8.0 \pm 5.2$ vs. $13.7 \pm 6.6$ $(p \leq 0.002))$. KV and eff mAS independently predict a higher ED, whereas age, weight and $\mathrm{BMI}$ are not predictive.

Conclusion: CCTA is highly accurate for the evaluation of coronary arteries in paediatric patients. Using dedicated paediatric cCTA protocols the ED can be significantly reduced.

\section{B-598 11:33}

Whole heart flow-sensitive 4D MRI in patients after repair of tetralogy of Fallot

J. Geiger, M. Markl, R. Arnold, U. Saueressig, B. Stiller, J. Hennig, M. Langer; Freiburg/DE (julia.geiger@uniklinik-freiburg.de)

Purpose: To evaluate flow-sensitive 4D MRI for the analysis of haemodynamics in patients after repair of tetralogy of Fallot (TOF), particularly with regard to altered flow patterns in the pulmonary system and their correlation with pulmonary vascular geometry. Methods and Materials: ECG and respiration synchronised flow-sensitive 4D MR with $3 \mathrm{D}$ velocity encoding $(\mathrm{TE}=2.4 \mathrm{~ms}, \mathrm{TR}=4.8 \mathrm{~ms}$, venc $=200 \mathrm{~cm} / \mathrm{s}$, spatial resolution $2.5 \mathrm{~mm}^{3}$, temporal resolution $=38.4 \mathrm{~ms}$ ) was acquired in ten patients after repair of TOF (mean age 12 years) and in four healthy controls (mean age 26 years). Analysis included the assessment of vascular geometry (vessel diameters and branching angles) in MR angiography data, evaluation of haemodynamics by 3D blood flow visualisation (particle traces) and quantification in the aorta, in the pulmonary trunk (TP) and left (IPA) and right (rPA) pulmonary arteries.

Results: Systolic peak velocity in the TP was significantly higher in patients $(1.9 \mathrm{~m} /$ $\mathrm{S} \pm 0.7 \mathrm{~m} / \mathrm{s})$ than in controls $(0.9 \mathrm{~m} / \mathrm{s} \pm 0.1 \mathrm{~m} / \mathrm{s})$. Regurgitation fraction in patients was $29 \% \pm 11 \%$ and no regurgitation was measured in controls. A higher blood flow in the rPA of the TOF patients was measured: rPA/IPA flow ratio of $2.6 \pm 2.5$ versus $1.1 \pm 0.1$ in controls. Correlation analysis detected relationships between the severity of vortices in the pulmonary system and vessel size $(r=0.79)$ and between the regurgitation fraction in the rPA and the pulmonary arteries branching angles $(r=0.73)$. Conclusion: 4D MR flow analysis showed abnormal flow patterns in the entire group of TOF patients which correlated significantly with vascular geometry. 4D MRI has the potential to demonstrate the complex nature of pulmonary morphology and haemodynamics in corrected TOF patients.

\section{B-599 11:42}

Haemodynamics in patients with transposition of the great arteries (D-TGA) after switch procedure visualised by flow-sensitive 4D MRI J. Geiger, M. Markl, R. Arnold, P. Franke, J. Mauch, B. Stiller, M. Langer; Freiburg/DE (julia.geiger@uniklinik-freiburg.de)

Purpose: To evaluate postoperative vessel morphology and haemodynamics by flow-sensitive 4D MRI in children after arterial switch procedure for transposition of the great arteries (D-TGA).

Methods and Materials: 4D MRI with $3 \mathrm{D}$ velocity encoding (TE $=2.4 \mathrm{~ms}$, TR $=$ $4.8 \mathrm{~ms}$, venc $200 \mathrm{~cm} / \mathrm{s}$, spatial resolution $\sim 2.5 \mathrm{~mm}^{3}$, temporal resolution $=38.4 \mathrm{~ms}$ ) was performed in nine patients after repair of TGA and in four healthy controls (mean age 12.2 years vs. 26 years). Blood flow was visualised by time-resolved $3 \mathrm{D}$ particle traces emitted from planes in the ascending aorta and the pulmonary trunk (TP). Visual grading was performed using a 3 grade ranking scale (0-2) with regard to the extent of flow vortices, helices, retrograde flow and presence of high peak velocities in the aorta and pulmonary system $(v>1.5 \mathrm{~m} / \mathrm{s})$.

Results: Six patients had vortices in the TP, mean vortex severity was graded as 0.78 . Vortex formation was less pronounced in the right (rPA) and left pulmonary artery (IPA) (0.33 and 0) and aorta (0.22). Helices were observed in the IPA and aorta with an average grading of 0.78 . Retrograde flow was rare in patients: 0.33 in TP and 0.22 in IPA. No reflux was detected in the rPA and aorta. Peak velocities $>1.5 \mathrm{~m} / \mathrm{s}$ were seen in TP, rPA and IPA in all nine patients, in the aorta in 7/9. No vortices, helices, reflux or accelerated flow were detected in volunteers.
Conclusion: 4D MRI enables visualisation of postsurgical haemodynamics in TGA-patients after switch repair showing elevated flow velocity in the pulmonary outflow tract and increased vortical and helical flow patterns.

\section{B-600 11:51}

Cardiac MRI of the foetal heart using a MR compatible Doppler ultrasound (CTG) for cardiac triggering

J. Yamamura, M. Frisch, I. Kopp, J. Graessner, K. Hecher, G. Adam,

U. Wedegärtner; Hamburg/DE (wedegaertner@uke.de)

Purpose: The aim of this study was to perform foetal cardiac MR imaging with triggering of the foetal heart beat in utero with a novel MR compatible cadiotocogramm (CTG) in a sheep model.

Methods and Materials: Images of the foetal sheep heart were performed on 5 pregnant ewes at a $1.5 \mathrm{~T}$ scanner (Siemens, Erlangen, Germany). All ferromagnetic materials were removed in a commercial CTG and it was made MR compatible. Using this CTG, a cardiac triggered, breath-hold cine MRI with steady-state free precession (SSFP) of the foetal heart were achieved in short axis view, two, four and three chamber view. From the short-axis, the left ventricular volume and thus the function was measured. Results: The foetal heart frequencies were between 130 and 160 beats per minute. The novel MR compatible CTG allowed a stable signal during the whole MRI measurement. It was possible to perform the CTG triggered foetal cardiac MRI in all foetuses. In cine sequences the contraction was shown. The average blood volumes at end systole was $2.9 \mathrm{ml}(\mathrm{SD} \pm 0.2)$, at end diastole $4.6 \mathrm{ml}( \pm 0.2)$; with ejection fractions between $38.4 \%$ and $40 \%$, respectively. The mitral, the tricuspid, aortic and the pulmonary valves as well as the foramen ovale were clearly depicted. Conclusion: The novel MR compatible CTG allowed an excellent trigger of the foetal heart rate. An evaluation of anatomical structures and functional information could be obtained from the cMRI. 
Monday, March 7 


\section{Musculoskeletal}

\section{SS 1610}

\section{Inflammation and infection}

\section{Moderators:}

G. Mantzikopoulos; Athens/GR

M.C. Wick; Innsbruck/AT

\author{
B-601 10:30 \\ Impact of motion correction on image quality in dGEMRIC of finger joints \\ in arthritis \\ C. Buchbender ${ }^{1}$, H.-J. Wittsack ${ }^{1}$, A. Scherer ${ }^{1}$, M. Quentin ${ }^{1}$, B. Ostendorf ${ }^{1}$, \\ T.C. Mamisch ${ }^{2}$, C. Zilkens ${ }^{1}$, B. Bittersohl ${ }^{1}$, F.R. Miese ${ }^{1} ;{ }^{1}$ Düsseldorf/DE, \\ ${ }^{2}$ Berne/CH (Christian.Buchbender@med.uni-duesseldorf.de)
}

Purpose: To assess misregistration artefacts in dGEMRIC of finger joints and to evaluate the effectiveness of motion correction.

Methods and Materials: In 40 cases dGEMRIC of metacarpophalangeal joint II was performed. Imaging used a dual flip angle approach (TE $3.72 \mathrm{~ms}$, TR $15 \mathrm{~ms}$, flip angles $5^{\circ}$ and $26^{\circ}$ ). Two sets of T1 maps for were calculated for dGEMRIC analysis from the imaging data for each patient: one with and one without motion correction. To compare image quality, visual grading analysis and precision of dGEMRIC measurement of both dGEMRIC maps for each case were evaluated. Results: Motion artefacts were present in $82 \%$ (33/40) of uncorrected dGEMRIC maps. Motion artefacts were graded as severe or as rendering evaluation impossible in $43 \%$ (17/40) of uncorrected dGEMRIC maps. Motion-corrected maps showed significantly less motion artefacts $(P<0.001)$ and were graded as evaluable in $97 \%(39 / 40)$ of cases. CV was significantly lower in motion-corrected images $(0.176 \pm 0.077)$, compared with uncorrected images $(0.445 \pm 0.347)(P<0.001)$. Motion-corrected dGERMIC was different in volunteers and patients $(P=0.044)$, whereas uncorrected dGEMRIC was not $(P=0.234)$. Conclusion: Motion correction improves image quality, dGEMRIC measurement precision and diagnostic performance in dGEMRIC of finger joints.

\section{B-602 10:39 \\ Repair of joint erosions in rheumatoid arthritis patients after one year treatment with anti-TNF therapy: a magnetic resonance imaging study M. Navallas, M.P. Lisbona, J. Ares, J. Maymo, A. Esteban, A. Solano; Barcelona/ES (mnavallas@gmail.com)}

Purpose: To determine if regression or repair of erosions occur after one year of treatment with etanercept in patients with rheumatoid arthritis (RA) using hand-MRI Methods and Materials: 29 patients with active RA, defined by a DAS28 highe than 3.2 and naïve for biologic therapy, were treated with etanercept added to previous DMARD therapy during a year. Clinical evaluation, laboratory tests, and $M R I$ of the dominant hand were performed before and after one year of treatment Paired sets of $h-M R I$ were read blinded for time-sequence by 2 experienced readers following RAMRIS method, tenosynovitis was also scored. Regression or repair was studied in three different ways: 1 . The observed yearly progression rate was lowe than the estimated rate, 2. RAMRIS score for erosion after one year was lower than the baseline score, or 3. Change of RAMRIS score for erosion exceeding the smallest detectable difference (SDD) and minimal detectable change (MDC $<20 \%$ ). Results: 29 patients ( 25 women) started therapy with etanercept combined with DMARDs. At baseline, the mean DAS28 was $4.7 \pm 1.1$ decreasing to $2.9 \pm 1.4$ $(p<0.001)$ after one year. All the other clinical parameters also showed significant decreases. Mean RAMRIS score for erosions did not change, but all the other MR parameters showed a significant decrease.

Conclusion: Etanercept added to DMARDs stopped progression of erosions measured by RAMRIS hand-MRI in $82.7 \%$ (24 patients) with active RA after one year of treatment. Five patients $(17.2 \%)$ showed regression or repair of erosions predominantly in the carpal bones.

\section{B-603 10:48}

Detection of acute inflammation in patients with arthritic finger joints with optical imaging in comparison to MRI

R. Meier, K. Thürmel, P. Moog, P. Noel, C. Ahari, M. Sievert, F. Dorn,

C. Schäffeler, S. Waldt, K. Wörtler, E.J. Rummeny; Munich/DE

(reinhardt.meier@gmail.com)

Purpose: To evaluate an Indocyanine Green (ICG)-enhanced Optical Imaging (OI) system for detection of active inflammation in patients with arthritic finger joints in comparison to MRI.
Methods and Materials: 45 Patients $(67 \%$ female, mean age $=53.7)$ with clinica suspicion of an arthritic joint disease were examined with an ICG-enhanced optical imaging system (Mivenion $\mathrm{GmbH}$, Berlin, Germany). The degree of inflammation in the carpal, metacarpophalangeal, proximal and distal interphalangeal joints of both hands was graded objectively by four independent radiologists on a 4-pointordinate scale (0: no inflammation, 1: mild, 2: moderate, 3: severe). Results were correlated with MRI (3 T Verio, Siemens, Germany) as standard of reference. Results: Of the 1350 joints of the evaluated 45 patients, in MRI a total of 608 $(45.0 \%)$ joints showed mild $(37.8 \%)$, moderate $(6.4 \%)$ or severe $(0.8 \%)$ inflammation. In OI an average of $414(30.7 \%)$ joints showed mild $(61.2 \%)$, moderate $(24.6 \%)$ or severe $(14.3 \%)$ inflammation. Using MRI as standard of reference, OI had a sensitivity of $43.5 \%(35.3 \%-52.1 \%)$, a specificity of $80.3 \%(74.8 \%-84.8 \%)$, and accuracy of $65.7 \%$ for detection of active inflammation in patients with RA. Conclusion: The evaluated ICG enhanced OI system showed substantial limitations in the detection of active inflammation in patients with arthritic finger joints in comparison to $\mathrm{MRI}$ as standard of reference. Despite its advantages of its minimal invasiveness, lower costs and easy operability the OI system appears to be of limited value for the clinical routine.

\section{B-604 10:57}

Do we really need the full dose of multihance for contrast-enhanced MR imaging for the assessment of synovial enhancement in patients with rheumatoid arthritis?

C. Schueller-Weidekamm ${ }^{1}$, H. Platzgummer ${ }^{1}$, M. Weber ${ }^{1}$, K.-P. Lodemann²,

F. Kainberger ${ }^{1} ;{ }^{1}$ Vienna/AT, ${ }^{2}$ Konstanz/DE

(claudia.schueller-weidekamm@meduniwien.ac.at)

Purpose: To investigate the diagnostic accuracy of a half-dose of multihance (Bracco, Italy) in the assessment of synovitis in hand and finger joints in RA.

Methods and Materials: Thirty patients with early rheumatoid arthritis underwent 3 T high-resolution MRI. Coronal T1-weighted TSE and STIR sequences and transversal T2-weighted SE sequences were acquired before contrast media. Coronal T1-weighted TSE sequences with fat saturation were obtained after 0.05 $\mathrm{mM} / \mathrm{kgKG}$ multihance (half-dose $=\mathrm{HD}$ ) with a delay of five minutes, and, five minutes later, additional coronal T1-weighted TSE sequences were obtained after a second application of $0.05 \mathrm{mM} / \mathrm{kgKG}$ multihance (full dose $=F D$ ) with a delay of five minutes. The contrast enhancement in the region of interest was measured in inflamed synovial tissue in exactly the same slice on half- and full-dose contrastenhanced sequences. Differences and the correlation of signal intensities on HD and FD contrast-enhanced sequences were compared using the paired t-test for statistical analysis as well as the Bland-Altman plot.

Results: A statistically significant difference was found for half- and full-dose multihance in contrast-enhanced synovial tissue (mean: 1184.527 vs. 1363.137 $\mathrm{p}<0.001)$. As the signal intensities showed a high correlation between HD and FD $(r=0.779)$, the formula FD $=375.858+0.833^{*} \mathrm{HD}$ can be used to convert HD to FD. Conclusion: In inflamed synovial tissue, the post-contrast signal intensities showed a high correlation between HD and FD. Therefore, HD multihance is sufficient for the assessment of synovial enhancement in patients with early rheumatoid arthritis with 3 T MRI. No additional relevant information for clinical purposes can be obtained by giving the FD multihance.

\section{B-605 $11 \cdot 06$}

Enthesitis and related changes in the knees in seronegative spondyloarthropathies: MRI control study

Y. Ragab, Y. Emad, H. Hamza; Cairo/EG (yragab61@hotmail.com)

Purpose: To evaluate the role of magnetic resonance imaging (MRI) in the detection of knee changes in patients with seronegative spondyloarthropathy (SpA)

Methods and Materials: 56 patients including 30 with psoriatic arthritis, 5 with ankylosing spondylitis, 5 with reactive arthritis, 5 with ulcerative colitis (UC), 5 with Crohn's disease, and another 6 with skin psoriasis. Controls were 20 healthy subjects without knee complaints.

MRI was performed in all participants including Gd-DTPA enhancement.

Results: Both knees were studied in $45(80.3 \%)$ patients and one knee in 11 (19.6\%). MRI showed evidence of bone marrow oedema in $13(23.2 \%)$ patients, cartilaginous erosions in $18(32.1 \%)$, and bone erosions in $9(16.1 \%)$. Enthesitis was found in medial collateral ligaments in $18(32.1 \%)$, lateral collateral ligaments in $8(14.3 \%)$, posterior cruciate ligaments in $3(5.35 \%)$, patellar tendon in $18(32.1 \%)$, biceps femoris insertion in (5.35\%), medial patellofemoral ligaments (MPFL) in 5 $(8.9 \%)$ and lateral patellofemoral ligament in 1 patient (1.8\%). In the UC and Crohn's patients $(n=10), 2$ had bone erosions and 5 had enthesitis. In the skin psoriasis group $(n=6)$, one had bone marrow oedema; enthesitis was detected in 5 at the patellar tendon insertion and in one in the MPFL. 
Conclusion: Entheseal-related changes can be found in patients with skin psoriasis or inflammatory bowel disease with no clinical arthritis. Enthesitis of the knee joint may be an early sign on MRI and a characteristic finding in the SpA group of diseases.

\section{B-606 $11: 15$}

In vivo monitoring of septic arthritis using macrophage MR imaging

G. Bierry, S. Lefevre, D. Ruimy, F. Jehl, A. Neuville, J.-L. Dietemann;

Strasbourg/FR (guillaume.bierry@chru-strasbourg.fr)

Purpose: To prospectively evaluate macrophage imaging using ultrasmall superparamagnetic iron oxide (USPIO)-enhanced MR imaging for the in vivo non-invasive monitoring of experimental infectious arthritis under antibiotic therapy.

Methods and Materials: Unilateral knee infection was induced by intra-articular injection of Staphylococcus aureus in 12 rabbits randomly assigned in 2 groups. All animals were explored by MRI at the acute phase before and after USPIO administration. Animals of group $1(n=6)$ were then killed. Animals of group $2(n=6)$ received successful antibiotic treatment. Two MRI sessions were then performed, before and after UPSIO administration $(n=6)$ and animals were then killed. MRI protocol included unenhanced and USPIO-enhanced T2*GRE, unenhanced and gadolinium-enhanced FS T1 SE sequences. MR data were qualitatively and quantitatively analysed and compared with bacteriological and histopathologic findings. Results: At acute phase: an intense synovitis with marked signal increase of the synovium on Gd-enhanced FS T1 image was observed in all animals, associated with areas of signal loss within infected synovium on USPIO-enhanced T2*GRE images reflecting an intense infiltration of USPIO-loaded macrophages. At healing phase: significant reduction of areas of the synovium that presented with signal loss on T2* GRE images as compared to acute phase, due to a significant reduction of macrophage infiltration. At the opposite, signal increase on Gd-enhanced FS T1 image remained unchanged.

Conclusion: In contrast to gadolinium-enhanced sequences, macrophage imaging using USPIO-enhanced MRI can accurately demonstrate resolution of experimental bacterial joint infection.

\section{B-607 11:24}

Assessment of fever of unknown origin: whole body diffusion weighted imaging and continuous table movement MRI in comparison to leucocyte scintigraphy/FDG-PET CT

S. Weckbach ${ }^{1}$, D. Buchheidt², H.J. Michaely², S.O. Schönberg², D. Dinter²,

S. Haneder ${ }^{2} ;{ }^{1}$ Munich/DE, ${ }^{2}$ Mannheim/DE (stefan.haneder@umm.de)

Purpose: To evaluate WB-MRI using DWI as a new specific modality for the aetiologic workup of fever of unknown origin (FUO) without radiation exposure and less technical effort in comparison to scintigraphy/PET-CT.

Methods and Materials: 20 patients with FUO were examined at $3 / 1.5 \mathrm{~T}$ (Siemens Magnetom Trio/Avanto) using a combined protocol with coronal WB diffusionweighted sequences (EPI-SE, $2.0 \times 2.0 \times 4.0 \mathrm{~mm}$ ), axial WB TimCT HASTE STIR sequences $\left(2.0 \times 1.0 \times 6.0 \mathrm{~mm}^{3}\right)$ and ce T1-weighted GRE -sequences as reference. In 8 patients ${ }^{67}$ Gallium-scintigraphy (Ecam, Siemens) was performed as standard of reference, in 5 patients 18 F-FDG-PET-CT (Biograph mCT, Siemens). In 3 adolescents ultrasound correlation was available. Scintigraphy/PET-CT and WB MRI were read by two blinded readers. Sensitivities/specificities were assessed. Results were compared, findings were clinically assured and modalities were reevaluated after diagnosis

Results: In 14 patients 17 fever foci/fever causing entities found were found by WBMRI. In 6 patients no focus was found by WB-MRI and scintigraphy /PET-CT and clinical follow-up was negative. In 4 patients (20\%) with positive MRI scintigraphy was negative, in these patients clinical assessment confirmed the MR diagnosis. Sensitivity of DWI was $94 \%$, specificity $100 \%$, PPV $100 \%$, NPV $80 \%$. In 3 patients negative DWI ruled out suspected infection from STIR and ce GRE images. Conclusion: WB-MRI excellently depicts fever foci and shows higher detection rates than the current standard of reference. DWI has a high sensitivity, specificity and PPV and offers additional functional information to standard morphological imaging.

\section{B-608 11:33}

An indigenous MRI scoring system for differentiating advanced femoral osteonecrosis from tubercular arthritis: myth or mystique?

V. Venugopal, I. Afshan, E. Ullah, I. Ahmad, S. Wahab; Aligarh/IN

Purpose: A MRI-based scoring system for differentiating advanced osteonecrosis of femoral head from tubercular arthritis is introduced.

Methods and Materials: MRI findings in 18 hips with tubercular arthritis and 36 hips with advanced osteonecrosis of femur were reviewed retrospectively. Confirmation of tuberculous arthritis was based on ELISA and/ or synovial biopsy. Osteonecrosis was confirmed by histopathology and only Arlet and Durroux types 3 and 4 were included in the study. The findings were analysed with emphasis on the changes in femoral head marrow, joint cavity, synovium, acetabulum and contrast-enhancement patterns.A score of two was assigned for presence of each of T2 hyperintensity of femoral head, synovial hypertrophy, articular cartilage erosion, unilateral involvement of femoral head, acetabular oedema/sclerosis and enhancement of involved head. A score of one was assigned for each of joint effusion, oedema of adjacent marrow and enhancement of adjacent soft tissue. A cut-off value of 10 out 15 points was considered to be positive for TB arthritis.

Results: 16 out of 18 cases of TB arthritis were correctly identifiable on the basis of this scoring system. The two remaining cases had a score of 9 . None of the AVN cases scored more than 10. A score of 10 for had a sensitivity $88.89 \%$ and specificity $100 \%$ the positive diagnosis of tuberculous arthritis. Positive and negative predictive values were 1 and 0.94 , respectively.

Conclusion: This indigenous scoring system is very effective in solving the imaging dilemma pertinent to endemic regions of differentiating advanced femora osteonecrosis from avascular necrosis.

\section{B-609 11:42}

Dual energy "virtual non-calcium" technique: detecting posttraumatic

bone bruises of the knee with computed tomography

G. Pache ${ }^{1}$, B. Krauss ${ }^{2}$, P. Blanke1, S. Bulla1, M. Langer ${ }^{1}$, T. Baumann ${ }^{1}$

${ }^{1}$ Freiburg/DE, ${ }^{2}$ Forchheim/DE (gregor.pache@uniklinik-freiburg.de)

Purpose: To evaluate bone marrow assessment with a dual energy CT "virtual non-calcium" technique utilising MRI proven bone bruises as a model for typical marrow pathology so far indiscernible via CT.

Methods and Materials: In this prospective, institutional review board approved the study; 21 patients with an acute knee trauma underwent dual energy CT (Somatom Definiton, Siemens) and MRI scans. The application LiverVNC (Syngo Dual-Energy, Siemens) was used to virtually subtract calcium from the images. Presence of bone bruise was rated by 2 radiologists on a 4-point scale for 6 femoral and tibial regions each. HU measurements were performed in the same regions. Consensus reading of independently randomised MRI scans served as the standard of reference. Image ratings and $\mathrm{HU}$ values were subjected to receiver-operator-characteristic (ROC) analysis.

Results: MRI revealed 59 bone bruises in a total of 236 regions (19 of 114 femoral, 40 of 122 tibial, 16 excluded due to artifacts). Visual rating revealed area-under-thecurve (AUC) values of $0.886(0.897)$ in the femur and $0.974(0.953)$ in the tibia for observer 1 and 2, respectively. For $\mathrm{HU}$ measurements the respective AUC values were 0.922 and 0.974 . If scores of 1 and 2 (strong/mild bone bruise) were counted as positive, sensitivity and specificity were $86.4 \%(86.4 \%)$ and $94.4 \%(95.5 \%)$ for observers 1 and 2, respectively. Kappa analysis demonstrated good to excellent agreement ( $\kappa$-value: 0.78/0.87 femoral/tibial).

Conclusion: Dual energy CT "virtual non-calcium" technique can subtract calcium from cancellous bone, allowing bone marrow assessment and making posttraumatic bone bruises of the knee detectable with computed tomography.

\section{B-610 11:51}

The initial assessment of clinical value of gouty arthritis by DSCT dualenergy technique

D. Han, L. Wu, B. He; Kunming/CN (kmhandan@sina.com)

Purpose: To explore the diagnostic value of gouty arthritis from crystal deposition of uric acid inside joints and related soft tissues by dual-source CT dual-energy mode (DECT) technique.

Methods and Materials: Plain DECT scans under gout program had been done on 132 patients that include 50 cases secured diagnosed, 37 suspected and 45 as control, respectively. The output images were sent to DE gout software, which can display the crystal deposit of uric acid on target positions by colour marked MPR and VRT images. The diagnosis of gouty arthritis was made based on DECT findings and the clinical manifestation.In the control group, colour signs were believed to be false positive. 
Results: Uric acid crystal deposits were detected in 71 patients by DECT. Combing with the clinical manifestation, 50 cases were secured diagnosed and 21 suspected. The detection rate was $81.6 \%$. 69 patients treated as gouty arthritis have showed positive response; the remaining two patients were confirmed by detecting uric acid in the joint effusion from surgery. The sensitivity was $100 \%$. The false-positive rate was $6.7 \%$ in control group. The detection rate was higher in patients with increased blood uric acid than those with normal blood uric acid $(P<0.01)$. However, uric acid crystals could be detected in joints and related soft tissues with normal blood uric acid (23/35.66\%).

Conclusion: DECT technique has relatively higher sensitivity and specificity in detecting uric acid crystal in joints and related areas. It is quite useful in the diagnosis and differentiation of gouty arthritis.

\section{Abdominal Viscera}

\section{SS 1601a}

\section{Advances in MRI and US in abdominal imaging}

Moderators:

S. Bohata: Brno/CZ

E. Danse; Brussels/BE

\section{B-611 10:30}

Acoustic radiation force impulse (ARFI) elastography for evaluation of diffuse liver disease: phantom and clinical studies

S. Moon, S. Kim, K.-S. Suh, N.-J. Yi, J. Lee, J. Han, B. Choi; Seoul/KR

(radmsk@hotmail.com)

Purpose: To validate the accuracy of ARFI elastography using phantom models and to evaluate its usefulness in diagnosing diffuse liver disease using histopathology as reference.

Methods and Materials: Shear wave velocities (SWVs) of thee plastic-based phantoms with different Young's moduli (soft $5 \mathrm{kPa}$, medium $10.6 \mathrm{kPa}$, and hard $25.7 \mathrm{kPa}$ ) were measured using ARFI elastography and correlated with their Young's moduli. Then, 26 prospectively recruited patients underwent preoperative ARFI elastography (Siemens) and were grouped into normal, fatty liver (FL), chronic liver disease (CLD), and liver cirrhosis (LC) by their histopathology. SWVs and liver function test (LFT) results of each group were compared using one-way ANOVA test. Then, their performance in diagnosing $\mathrm{LC}$ was evaluated using $\mathrm{ROC}$ analysis. Results: Mean SWVs of phantoms were significantly different; soft $1.86 \mathrm{~m} / \mathrm{sec}$, medium $3.20 \mathrm{~m} / \mathrm{sec}$, and hard $4.27 \mathrm{~m} / \mathrm{sec}$, and showed a perfect correlation with Youngs moduli $(r=1.000, p<0.01)$. Twenty-six patients were composed of 8 normal, $2 \mathrm{FL}, 8$ CLD, and 8 LC. Mean SWVs were significantly different among the groups; normal $1.70 \mathrm{~m} / \mathrm{sec}$, FL $1.72 \mathrm{~m} / \mathrm{sec}$, CLD $1.83 \mathrm{~m} / \mathrm{sec}$, and LC $3.64 \mathrm{~m} / \mathrm{sec}(\mathrm{p}<0.001)$. Post-hoc study revealed the mean SWV of LC was significantly different from non-LC group. Although albumin level $(p=0.049)$ and platelet count $(p=0.02)$ were significantly different among the groups, ROC analysis revealed AUC was much higher in SWV $(0.972)$ than these LFT parameters for differentiating LC from non-LC. In diagnosing LC, SWV showed $100 \%$ sensitivity and $77.8 \%$ specificity at a cut-off value of 1.99 $\mathrm{m} / \mathrm{sec}$ and $87.5 \%$ sensitivity and $100 \%$ specificity at a cut-off value of $3.00 \mathrm{~m} / \mathrm{sec}$. Conclusion: The accuracy of ARFI elastography is well validated with plastic-based phantoms. In diagnosing LC, ARFI elastography outperforms laboratory tests and can be an accurate diagnostic tool.

\section{B-612 10:39}

\section{Acoustic radiation force impulse (ARFI) imaging: repeatability of} measurements in selected liver segments and influence of age, body mass index (BMI) and liver capsule-to-box (CB) distance

O.S. Jaffer, P. Lung, D. Bosanac, V. Patel, S. Ryan, P. Sidhu; London/UK (ounali.jaffer@googlemail.com)

Purpose: To assess the validity of quantifying liver tissue stiffness using ARF imaging with influence of age, BMI and CB distance.

Methods and Materials: Ten healthy volunteers with normal B-mode liver ultrasound appearances were enrolled in the study. Three experienced radiologists measured 10 shear velocities on each of the following liver regions: segment 3 , $5 / 6$ and $7 / 8$. Similar measurements were taken one week later. The radiologists were blinded to each other and their previous week's measurements.

Analysis of variance and Student's t test were used to determine inter-observer and intra-observer variability, respectively. Multiple linear regression was used to determine correlation of age, BMI and $\mathrm{CB}$ distance.
Results: The mean age was 31 years (range 27-40), mean BMI was 23.6 (range 18.4-30.3) and mean CB distance was $2.0 \mathrm{~cm}$ (range 0.6-4.2 cm).

Out of 600 shear velocity measurements, mean values from segment 3 [1.31 $\mathrm{m} / \mathrm{s}(0.99-1.83 \mathrm{~m} / \mathrm{s})]$ were significantly higher than segment $5 / 6[1.22 \mathrm{~m} / \mathrm{s}(0.75$ $2.36 \mathrm{~m} / \mathrm{s})]$, and segment $7 / 8[1.12 \mathrm{~m} / \mathrm{s}(0.79-1.46 \mathrm{~m} / \mathrm{s})](\mathrm{p}=<0.0001)$. There was no significant difference between the mean values of segment $5 / 6$ and $7 / 8$. There was no inter- and intra-observer variability $(p=n s)$. Shear velocity weakly correlated between $B M I(r=+0.05, p=0.04)$, and age $(r=-0.09, p=0.002)$. A stronger correlation was observed with $C B$ distance $(r=-0.21, p=<0.0001)$.

Conclusion: With experienced radiologists, ARFI imaging is a robust and reproducible method for quantifying liver stiffness. It is reliable when taking shear velocity measurements from segments $5 / 6$ or $7 / 8$ but segment 3 should not be used. The influence of $\mathrm{CB}$ distance should be considered in future study design.

\section{B-613 10:48}

Haemodynamic changes occurring in liver cirrhosis: evaluation by a contrast-enhanced ultrasound dedicated software and potentially clinical applications

T. Abbattista, F. Ridolfi, E. Brunelli, P. Busilacchi; Senigallia/IT

(t.abbattista@tin.it)

Purpose: The early enhancement of hepatic veins (HVAT) distinguishes cirrhosis from chronic liver diseases. The aim of this study was to determine if a contrastenhanced ultrasound dedicated software (Quontrast, Bracco, Italy) could be used to give a map of haemodynamic changes of hepatic parenchyma and to differentiate liver cirrhosis from chronic hepatitis.

Methods and Materials: CEUS (Sonovue, Bracco S.p.a., Milan, Italy) was performed on 17 patients with liver cirrhosis, 20 patients with chronic hepatitis B/C and 10 healthy controls. Qontrast was used to obtain maps of the enhancement of hepatic parenchyma, including a main hepatic vein. Post-processing enhancement intensity was analysed to determine TTP (time to reach peak of enhancement) and peak (enhancement peak quantity).

Results: TTP was significantly reduced in patients affected by liver cirrhosis both in respect of patients with chronic hepatitis $(69.2 \pm 12 \mathrm{vs}$. $81.3 \pm 15.3$ seconds, $p=0.01)$ and to control patients $(86.3 \pm 20.3$ seconds, $p=0.007)$. Peak was higher in patients with chronic hepatitis $(25.5 \pm 8.7$ with respect to $22.4 \pm 6.4$ in patients with liver cirrhosis and $18.9 \pm 7.1$ in control patients): this difference approched but did not reach the statistical significance $(p=0.055)$. Quontrast revealed a high reproducibility: inter and intraobserver kappa coefficients for TTPs were 0.77 and 0.85 , respectively. Conclusion: Early TTPs seem to be predictive of liver cirrhosis and confirm the analysis of HVAT. This should be due to intra-hepatic shunts developed during chronic injury. The trend in reaching the highest peak of enhancement in chronic hepatitis should be due to wider vascular bed present in chronic liver diseases of viral origin.

\section{B-614 10:57}

Effects of contrast agent and outer volume saturation bands on water suppression and shimming of hepatic single-volume proton MR spectroscopy at $3.0 \mathrm{~T}$

L. Xu; Guangzhou/CN

Purpose: To determine whether administration of gadolinium diethylenetriamine pentaacetic acid (Gd-DTPA) and placement of the outer volume saturation bands significantly affect shimming and water

suppression on hepatic MRS prescan adjustments on a 3.0 T system.

Methods and Materials: Region of interest (ROI) of $2 \mathrm{~cm} \times 2 \mathrm{~cm} \times 2 \mathrm{~cm}$ were carefully positioned in the region of the middle portion of the right hepatic lobe. 32 patients were examined before and after administration of Gd-DTPA with and without outer-volume saturation bands. Linewidths (FWHM) and water suppression were obtained. A paired t-test for comparison of means was used.

Results: (1) The group with the outer volume saturation bands has slightly better water suppression effect than the group without outer volume saturation bands before administration $(94.0 \pm 2.4 \%, 93.2 \pm 2.8 \%, t=3.763, P=0.001)$. (2) The group with the outer volume saturation bands has better water suppression effect than the group without outer volume saturation bands after administration $(91.2 \pm 2.2 \%$, $88.9 \pm 3.0 \%, t=10.811, P<0.000)$. (3) Both shimming $(20.6 \pm 5.7 \mathrm{~Hz}, 19.5 \pm 5.8 \mathrm{~Hz}$ $\mathrm{t}=-2.137, \mathrm{P}=0.041)$ and water suppression effects $(91.2 \pm 2.2 \%, 94.0 \pm 2.4 \%, \mathrm{t}=8.649$, $\mathrm{P}<0.000$ ) were decreased on enhanced MRS prescan adjustments.

Conclusion: Placement of the outer volume saturation bands is helpful to improve water suppression both before and after contrast agent administration. Gd-DTPA exerts an adverse effect on water suppression and shimming of hepatic singlevolume proton MR spectroscopy at 3.0 T. 


\begin{abstract}
B-615 11:06
Multiecho MR sequences and high-resolution magic angle spinning (HRMAS) ex-vivo spectroscopy in the qualitative analysis and differentiation between steatohepatitis and steatosis

G. Besutti, G. Ligabue, L. Nocetti, F. Fiocchi, C. Stentarelli, A. Mucci, P. Loria,

G. Guaraldi, P. Torricelli; Modena/IT (gligabue@sirm.org)
\end{abstract}

Purpose: To compare multiecho gradient-echo MR (magnetic resonance) sequences in the differentiation between steatohepatitis and steatosis and to describe HR-MAS spectra of liver biopsy showing steatohepatitis or steatosis.

Methods and Materials: Fourteen patients with indication for biopsy assessment of steatosis underwent liver biopsy (reference standard) and MR imaging. Liver biopsy of both viral and metabolic steatosis were classified using NAFLD activity score (NAS) which depicts the degree of necro-inflammatrory activity allowing to differentiate between steatohepatitis and steatosis. Besides liver fat content (LFC), multiecho sequences were also used to calculate water and fat relaxation times $\left(\mathrm{T} 2^{\star}\right)$, which are influenced by microenvironmental characteristics, so potentially associated with necro-inflammatory activity. Relation between each multiecho parameter (LFC/T2* water/T2* fat) and NAS was estimated using univariate linear regression and Pearson coefficient. A fragment of biopsy specimen was analysed through HR-MAS to obtain metabolic tissue characterisation.

Results: Association was found between: NAS and LFCmulti $(r=0.7 ; p=0.006)$, NAS and T2*fat $\left(r=-0.73, p=0.063, n s, T 2^{*}\right.$ fat was available for 7 patients only). No correlation was found between NAS and T2*water. HR-MAS spectra showed tissue metabolic heterogeneity, with particular regard to the contents of free glucose, alanine, glutamine/glutamate and phospholipids.

Conclusion: This pilot study describes multiecho parameters associated with histological necro-inflammatory activity, allowing to study the potential capability of MR to differentiate between steatohepatitis and steatosis. Description of HR-MAS spectral heterogeneity in NAFLD and NASH may allow to find biochemical indicators of steatosis progression to be used in differentiating between steatohepatitis and steatosis in spectra acquired with in vivo MR Spectroscopy too.

\section{B-616 11:15}

\section{Accurate quantification of liver fat by H-MR spectroscopy and its} correlation with risk of coronary heart disease and diabetes mellitus A.C. Dhapare, A. Kohli, D. Rajput, M. Singh; Mumbai/IN (amoldhapare@yahoo.com)

Purpose: To evaluate the effectiveness of MR spectroscopy as a non-invasive tool for accurate detection of fat in liver and also to evaluate the risk of coronary heart disease and diabetes mellitus in individuals with fatty liver.

Methods and Materials: Spin echo sngle voxel proton MR spectroscopy with TR $2000 \mathrm{~ms}$ and TE $30 \mathrm{~ms}$ with a voxel size of $20 \times 15 \times 15 \mathrm{~mm}$ without breath holding is performed. Water peak is seen at $4.7 \mathrm{ppm}$ and fat peaks between 0.9 to $2.1 \mathrm{ppm}$. Percentage of fat is calculated from the area under the peaks. Around 80 healthy subjects in age group 25-50 years were scanned on 3 T Verio Siemens machine. Subjects with liver fat more than $5 \%$ were advised complete lipid profile along with fasting sugar and fasting insulin blood levels to calculate insulin resistance. Presence of obesity was also evaluated by measuring the waist circumference and BMI. Results: MR spectroscopy was accurate in measuring liver fat content. The startling finding was high incidence of hyperlipidaemia and insulin resistance in non obese subjects with fatty liver as compared with obese individuals without fatty liver with high incidence even in young individuals.

Conclusion: There is high incidence of fatty liver in general population with rising incidence in younger age group. It is emerging as a single most important risk factor for development of coronary heart disease and diabetes mellitus. Thus, MR spectroscopy proves to be an effective non invasive modality for early detection of fatty liver and preventing its complications.

\section{B-617 11:24}

Comparison of different in-vivo MR techniques and extracorporeal ex-vivo spectroscopy (HR-MAS) in the quantitative assessment of liver steatosis G. Besutti, G. Ligabue, L. Nocetti, C. Stentarelli, V. Righi, P. Loria, G. Guaraldi, P. Torricelli, S. Zona; Modena/IT (gligabue@sirm.org)

Purpose: To evaluate different in-vivo MR techniques and high-resolution magic angle spinnig (HR-MAS) spectroscopy in the quantitative assessment of liver steatosis, using liver biopsy as the reference standard.

Methods and Materials: Fourteen patients with indication for biopsy assessment of steatosis underwent liver biopsy and MR. MR liver fat content (LFC) was estimated using different techniques: T2-weighted and fat-suppressed T2-weighted Spin-Echo; dual phase T1-weighted gradient-echo; multiecho gradient-echo;
1H MR spectroscopy; HR-MAS spectroscopy on biopsy specimen. Measures of association between LFC and histological steatosis percentage were calculated using univariate linear regressions and Pearson $r$ coefficient. Respective ROC curves were used to compare specificity and sensibility of in-vivo MR methods in diagnosis of steatosis (cut-off value 5\%) and in quantitative evaluation of steatosis severity (cut-off value $33 \%$ ).

Results: High association was found between LFC and histological steatosis percentage (fat-suppressed $r=0.80, p=0.0005$; dual phase $r=0.89, p<0.0001$ multiecho $r=0.94, p<0.0001 ;$ spectroscopy $r=0.79, p=0.002$; HR-MAS $r=0.71$, $p=0.006$ ). According to the ROC curves, in-vivo MR techniques had high sensitivity and specificity in diagnosis and in quantitative evaluation of steatosis severity (area under the curves from 0.81 to 0.97 ), but no statistically significant difference was found between the four techniques $(p=0.39$ and 0.62$)$.

Conclusion: MR imaging can be considered a valid non-invasive alternative to biopsy for the diagnosis of steatosis and quantification of liver fat content. In addition, the small sample size may be responsible for the lack of significant difference between MRI techniques, but Multiecho seems to be the most promising.

\section{B-618 11:33}

In vivo ${ }^{1} \mathrm{H}$ MR spectroscopy in the early diagnosis and staging of liver fibrosis induced by viral hepatitis $B$

W. Li, N. Zhu, X. Zhao, K. Chen, W. Chai; Shanghai/CN (Iwxyjck@126.com)

Purpose: To explore the value of ${ }^{1} \mathrm{H}$ MRS for the early diagnosis and staging of liver fibrosis induced by viral hepatitis B.

Methods and Materials: 24 subjects underwent ${ }^{1} \mathrm{H}$-MRS of liver. Among them, 5 subjects were volunteers without hepatic disease and the other 19 subjects were patients with liver fibrosis who were further divided into two groups according to the Ishak system and whether it should be treated: 12 cases were slight liver fibrosis (F0 2) and the other 7 cases were advanced liver fibrosis (F3 6), the area under the peak of choline (Cho), lipid (Lip) and the relative Cho-to-Lip ratio (Cho/Lip) were calculated by GE spectra analysis software.

Results: The areas under the peaks of Cho, Lip and the ratio of Cho/Lip tended to increase gradually with the increasing degree of liver fibrosis (Cho: $4.99 \times 10^{-3}$ $29.86 \times 10^{-3}, 233.52 \times 10^{-3} ;$ Lip: $45.05 \times 10^{-3}, 328.26 \times 10^{-3}, 406.62 \times 10^{-3} ; \mathrm{Cho} /$ Lip: $0.10,0.23,0.51)$. There were significant correlations between Cho, Lip, Cho/ Lip and the degree of liver fibrosis $(P<0.05)$. The AUC of Cho, Lip and Cho/Lip for diagnosis of slight liver fibrosis were $0.95,0.83$ and 0.75 , their specificities were $80 \%, 100 \%$ and $80 \%$, sensitivities were $100 \%, 75 \%$ and $83.3 \%$ respectively. The AUC of Cho, Lip and Cho/Lip for diagnosis of advanced liver fibrosis were 0.87 , 0.61 and 0.80 , their specificities were $100 \%, 33.3 \%$ and $66.7 \%$, sensitivities were $71.4 \%, 100 \%$ and $85.7 \%$, respectively.

Conclusion: The metabolite (the area under the peak of Cho, Lip and the ratio of Cho/Lip) analysed with ${ }^{~} \mathrm{H}$-MRS is feasible, non-invasive makers in the early diagnosis and staging of liver fibrosis.

\section{B-619 11:42}

In-phase and out-of-phase gradient-echo imaging in abdominal studies: intra-individual comparison of three different techniques

M. Ramalho', V. Heredia', R.O.P. de Campos ${ }^{1}$, R.M. Azevedo',

B.M. Dale ${ }^{2}$, R.C. Semelka'; ${ }^{1}$ Chapel Hill, NC/US, ${ }^{2}$ Morrisville, NC/US (miguel-ramalho@netcabo.pt)

Purpose: To compare three T1-weighted in-phase and out-of-phase (IP/OP) gradient-echo imaging techniques in an intra-individual fashion, and to determine whether advantages exist for each of these sequences for various patient types. Methods and Materials: Institutional review board approval was obtained for this retrospective HIPAA-compliant study with waived informed consent. 118 consecutive subjects ( 74 males, 44 females; mean age $53.9 \pm 13.8$ ) who underwent a standard abdominal MRI protocol at $1.5 \mathrm{~T}$, between January and February 2010, including all three different IP/OP sequences [two-dimensional spoiled gradient-echo (2D-SGE), three-dimensional gradient-echo (3D-GRE) and magnetisation-prepared gradient-recall echo (MP-GRE)] were included. Two different reviewers independently and blindly evaluated IP/OP sequences to determine image quality, extent of artifacts, lesion detectability and conspicuity, and subjective grading of liver steatosis for the various sequences. Qualitative and quantitative data were subjected to statistical analysis. Results: Respiratory ghosting, parallel imaging and truncation artefacts as well as shading and blurring were more pronounced with 3D-GRE IP/OP imaging. Overall image quality was higher with 2D-SGE $(p<0.05)$. Detectability of low-fluid content lesions was lower with IP/OP MP-GRE sequences. MP-GRE sequences had the lowest SNRs $(p<0.001)$. Liver-to-spleen and liver-to-lesion CNRs were significantly lower with 3D-GRE and MP-GR, respectively $(p<0.001)$. Fat liver indexes showed strongly positive correlation between all sequences. 
Conclusion: All sequences resulted in adequate IP and OP information and image quality. 2D-SGE remains the best approach for IP/OP imaging. The good image quality of MP-GRE sequences acquired in a free breathing manner should recommend its use in patients unable to suspend breathing

\section{B-620 11:51 \\ Diagnostic accuracy of post-mortem whole-body magnetic resonance imaging in comparison with conventional autopsy \\ F. Henes, K. Püschel, G. Adam, M. Lorenzen; Hamburg/DE \\ (f.henes@uke.uni-hamburg.de)}

Purpose: Evaluation of diagnostic performance of whole-body magnetic resonance imaging (MRI) for detection of pathologic findings and prediction of causes of death as an alternative to conventional autopsy (CA).

Methods and Materials: In a prospective cohort study, thirty consecutive deceased patients underwent whole-body MRI (Philips Achieva 1.5-T, Best, Netherlands) prior to $C A$. In each cadaver coronal images of head and neck, thorax, abdomen, pelvis and lower extremities were acquired using a 3D-GRE- and a T2-STIR-sequence (TE: $3.3 \mathrm{~ms}$, TR: $93 \mathrm{~ms}$, FOV 400 and TE: $70 \mathrm{~ms}$, TR: $12.6 \mathrm{~s}$, IR: $190 \mathrm{~ms}$; FOV 400 , respectively). Subsequently, conventional autopsy was performed within 24 h. MR images were evaluated by two radiologist in consensus. Pathologic findings were documented and causes of death were predicted for each patient. Finally, results were correlated with autopsy findings.

Results: In 25 patients (83\%) MRI and CA were in agreement on the cause of death. In particular, in the detection of cardiac causes of death, post-mortem MRI achieved a high diagnostic accuracy (sensitiviy $84 \%$, specificity $100 \%$ ). MRI failed to demonstrate causes of death in 5 patients (1 pneumonia, 1 gastric ulcera, 1 alcohol intoxication, 2 myocardial infarction). With regard to the secondary findings a high finding agreement, $>95 \%$ was observed for pleural and pericardial effusion, ascites and rip fractures. Finding agreement for pulmonary emphysema, pulmonary oedema and renal infarction emerged to be poor (23\%)

Conclusion: Post-mortem whole-body magnetic resonance imaging achieves a high percentage of major finding agreement in comparison to conventional autopsy.

\section{Chest}

\section{SS 1604}

\section{Pulmonary infection and cystic fibrosis}

\section{Moderators:}

1. Hartmann; Rotterdam/NL

G.H. Mostbeck; Vienna/AT

\section{B-621 10:30}

Chest radiographic findings in H1N1 infection: comparison with seasonal influenza infection

M. Marti, I. Pinilla, M. Quintana, H. Rodriguez, J.C. Figueira, C. Prados;

Madrid/ES (mmarti.hulp@salud.madrid.org)

Purpose: To assess the initial radiographic findings in patients with $\mathrm{H} 1 \mathrm{~N} 1$ infection and compare them with those in patients with seasonal influenza.

Methods and Materials: 95 patients (50 males, 45 females) with microbiologically confirmed H1N1 infection seen from July to November 2009 who underwent chest radiographs at the emergency department formed the study group. The control group was formed by 98 patients ( 65 males, 33 females) with suspected seasonal flu seen from January to February 2009 at the same department. The initial radiographs were evaluated for the pattern (alveolar, interstitial, and alveolointerstitial), distribution and extent (focal, multifocal, and diffuse as the volumen of one lung). Radiographs were reviewed independently by 2 observers who reached a consensus decision.

Results: The study group showed a lower mean age ( 40.2 vs 50.9 years; $p<0.001)$ and more underlying comorbidities (48 vs 35 patients; $p<0.001$ ). The initial radiograph was abnormal in $52(54.7 \%)$ patients in the study group and $41(41.8 \%)$ in the control group. The most common finding in the study group was multifocal patchy consolidation $(41.2 \%, \mathrm{p}<0.001)$ whereas in the control group was lobar consolidation (43.9\%). Fourteen patients had involvement of more than 2 lobes and 5 patients showed diffuse involvement in the study group and none of the patients in the control group had more than 2 lobes affected.

Conclusion: Radiographs reveal abnormalities in more than half of the patients with $\mathrm{H} 1 \mathrm{~N} 1$ infection requiring admission. Multifocal patchy alveolar consolidation is the most frequent pattern in contrast with seasonal influenza.
B-622 10:39

Radiological prognostic factors in patients with $\mathrm{H} 1 \mathrm{~N} 1$ infection requiring hospital admission

M. Martí, I. Pinilla, M. Quintana, H. Rodriguez, J.C. Figueira, C. Prados; Madrid/ES (mmarti.hulp@salud.madrid.org)

Purpose: To determine the radiologic evolution in patients with $\mathrm{H} 1 \mathrm{~N} 1$ infection and findings associated with admission on the intensive care unit (ICU) and development of acute respiratory distress syndrome (ARDS).

Methods and Materials: 104 patients (53 males, 51 females; median age 40 years, range 15-96 years) with microbiologically confirmed $\mathrm{H} 1 \mathrm{~N} 1$ infection seen from July to December 2009 who underwent chest radiographs at the emergency department were studied. Radiographs were evaluated for the pattern (alveolar, interstitial, alveolointerstitial), distribution and extent (focal, multifocal, and diffuse), and findings of ARDS. Radiographs were reviewed by 2 observers who reached a consensus decision.

Results: 42 patients had abnormal initial chest radiograph and at least one more radiograph was obtained. Abnormalities improved and resolved in most patients in the first 3 weeks. Progression of findings was observed within the first 48 hours in 15 cases (14.4\%). 17 patients (16.3\%) required admission in ICU and mechanical ventilation. All of them showed an abnormal initial radiograph (16 alveolar o alveolo-intersticial and 1 interstitial pattern). Evolution to ARDS was found in 8 cases $(6.7 \%)$. All of them had on the initial radiograph alveolar multifocal involvement ( $p$ $=0.001$ ) with bilateral lesions in 4 cases.

In the multivariant analysis, a higher number of lung zones involved, the alveolar pattern and quickly progression were identified in patients requiring ICU admission and development ARDS.

Conclusion: The risk is higher for those showing alveolar pattern, a higher number of lung zones involved on the initial radiograph and quickly initial progression.

\section{B-623 10:48}

Initial CT and thoracic radiographic findings of H1N1 influenza virus infection in immunocompromised patients

A. Laqmani, G. Adam, M. Regier; Hamburg/DE (azien.laqmani@gmx.de)

Purpose: To retrospectively describe initial thoracic $\mathrm{CT}$ and radiographic findings of $\mathrm{H} 1 \mathrm{~N} 1$ influenza virus in immunocompromised patients and to determine whether initial radiologic findings can help triage patients who are at risk for an adverse clinical outcome.

Methods and Materials: The study population consisted of 33 patients with confirmed $\mathrm{H} 1 \mathrm{~N} 1$ infection suffering from an underlying immunocompromising disease who underwent initial chest radiographs. Three radiologists evaluated al initial radiographs $(n=33)$ and CT scans $(n=14)$ for type and pattern of opacities, distribution and extent of the observed abnormalities. Adverse outcome measures were defined as the need for mechanical ventilation, extracorporeal membrane oxygenation or death.

Results: The initial radiograph was abnormal in 19 of 33 and the corresponding CT in 13 of 14 of the patients. 10 patients showed an adverse outcome resulting in a death rate of $50 \%(n=5) .14$ patients revealed no abnormal radiographic findings. None of them had an adverse outcome. The most common radiographic findings included the following: consolidation (74\%), ground-glass opacity $(63 \%)$, reticular pattern $(32 \%)$, tree-in-bud pattern $(31 \%)$. The distribution was bilateral in $58 \%$ and affected predominantely the lower lung zones. A bilateral combination of consolidation and peribronchovascular ground-glass opacities affecting more than three lung zones was most predictive for a severe clinical course.

Conclusion: The majority of immunocompromised patients with pulmonary manifestation of $\mathrm{H} 1 \mathrm{~N} 1$ infection reveal abnormal radiologic findings. Early presumption of an adverse clinical outcome can be made by initial radiography and MDCT scans depicting multizonal bilateral consolidations and ground-glass opacities.

\section{B-624 10:57}

Diffusion weighted imaging (DWI): a new method to detect active lung inflammation in cystic fibrosis (CF)?

G. Serra ${ }^{1}$, P. Ciet $^{2}$, M. Mennini' ${ }^{1}$ S. Bertolo ${ }^{3}$, M. Ros ${ }^{4}$, S. Quattrucci'

H.A.W.M. Tiddens ${ }^{2}$, C. Catalano 1 , G. Morana ${ }^{3} ;{ }^{1}$ Rome $/ I T,{ }^{2}$ Rotterdam $/ N L$,

${ }^{3}$ Treviso/IT, ${ }^{4}$ Verona/IT (goffredo_serra@hotmail.it)

Purpose: To investigate DWI as tool to detect and quantify inflammation in CF lung disease.

Methods and Materials: 33 patients had a chest CT and MRI, including a morphological (T2w BLADE: TR/TE/alpha/TA: $\infty / 28 \mathrm{~ms} / 180 \% / 18 \mathrm{~s}$; slice thickness: $4 \mathrm{~mm}$ ) and DWI (TR/TE/FA: $5632,12 / 83 \mathrm{~ms} / 90^{\circ}$; slice thickness $5 \mathrm{~mm}$ ) study on the same day. CT and morphological MRI scored using a validated CF-CT score and equiva- 
lent CF-MRI scoring system; DWI scored using a newly developed score. Overlap between DWI and morphological MRI assessed with overlay techniques. DWI score was correlated to clinical and radiological parameters of disease severity: FEV1, body mass index (BMI), CT and MR scores and CT bronchiectasis score (CT-BE). Patients with DWI hot spots were compared to those without. Results expressed as mean (range). Scores expressed as \% of the maximal score.

Results: Mean age 24.6 years (6 to51); FEV1 78.64 (38 to 113); DWI score 9.89 (0 to 37); CT scores 22.88 (3 to 49); MRI scores 20.65 (7 to 40). Total DWI score correlated to CT score $(r=0.66, p=0.0001)$, MRI score $(r=0.599, p=0.001)$ and CT-BE score $(r=0.615, p=0.001)$. FEV1 of patients without DWI hotspots was higher $(p<0.0001)$ compared to patients with hot spots and DWI score correlated negatively with FEV1 $(r=-0.641 ; p<0.0001)$. No correlation was found between $\mathrm{BMI}$ and DWI score. Diffusion pattern only in part overlapped structural abnormalities on morphological MRI or CT.

Conclusion: DWI could be used to localise and quantify lung inflammation in CF. DWI is able to distinguish between hot and cold spots, that only partly overlap abnormalities on MRI and CT.

\section{B-625 11:06}

Comparison of chest-MRI to chest-CT to monitor cystic fibrosis (CF) lung disease

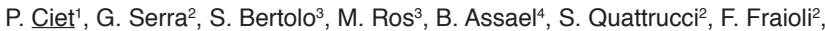

G. Morana ${ }^{3}$, H.A.W.M. Tiddens ${ }^{1} ;{ }^{1}$ Rotterdam $/ N L,{ }^{2}$ Rome $/ I T,{ }^{3}$ Treviso/IT,

${ }^{4}$ Verona/IT (pierluigi.ciet@libero.it)

Purpose: The most important components of CF lung disease are bronchiectasis (BE) and trapped air (TA). CT is the current gold standard to diagnose and monitor $\mathrm{BE}$ and TA. MRI has been suggested as a radiation free alternative for $\mathrm{CT}$, but its spatial resolution is inferior to CT. The purpose of our study was to compare the sensitivity of CT vs. MRI to monitor BE and TA in CF.

Methods and Materials: 39 stable CF patients (20 female; mean age 16.7 years) had a chest-CT and -MRI performed on the same day. MRI (Siemens Avanto): BLADE proton density (PD) transversal (TR/TE/alpha $\left.\infty / 28 \mathrm{~ms} / 180^{\circ}\right)$. CT (Siemens Somatom): low dose; volumetric; end inspiration and end expiration. CTs and MRIs were anonymised and scored in random order by 2 independent observers using the CF-CT score and an equivalent CF-MRI score. Scores are expressed as $\%$ of the maximal score. Statistics: Pearson; intra-class coefficient (ICC); Bland-Altman plots; results mean (range)

Results: Inter-observer variability (ICC): CT-BE (0.931); MRI-BE (0.892); CT-TA (0.917); MRI-TA (0.677). Correlation between CT-BE and MRI-BE score $(R=0.940$, $\mathrm{p}=0.0001)$; between CT-TA and MRI-TA $(R=0.511, p=0.005)$. Intra-observer variability (ICC): CT-BE (0.925), CT-TA (0.966); MRI-BE (0.838), MRI-TA (0.819). Bland-Altman plots showed that for mild CF-lung alterations MRI overestimates $\mathrm{BE}$ and TA compared to CT. For severe-CF MRI underestimates BE and TA compared to CT.

Conclusion: MRI shows less accuracy in the evaluation of CF-lung disease compared to CT, especially for TA. Further studies are needed to define the role of lung-MRI in CF follow-up.

\section{B-626 11:15}

Quantitative airway analysis on thin-section MD-CT in cystic fibrosis M.O. Wielpütz ${ }^{1}$, M. Puderbach ${ }^{1}$, M. Eichinger ${ }^{1}$, O. Weinheimer ${ }^{2}$, S. Ley ${ }^{1}$, H.-U. Kauczor ${ }^{1}$, C.-P. Heußel' ${ }^{1}{ }^{1}$ Heidelberg/DE, ${ }^{2}$ Mainz/DE (mark.wielpuetz@web.de)

Purpose: Major findings in multi-detector computed tomograms (MD-CT) of the chest of patients suffering from cystic fibrosis (CF) are bronchial wall thickening, mucous obstruction, and bronchiectasis. Aim of this study was to evaluate fully automatic airway detection and quantitative analysis of bronchial wall thickness on thin-section MD-CT in patients with CF compared to a control group without airway changes scanned for other reasons (NORMAL).

Methods and Materials: MD-CT ( $4 \times 1.25 \mathrm{~mm}, 70 \mathrm{mAs}, 120 \mathrm{kV})$ acquired from 22 CF patients and 33 NORMAL were subjected to a self-written airway analysis tool (YACTA). Automatic segmentation of the whole bronchial tree was performed and total bronchial diameter (TD), wall thickness (WT), and percentage of wall thickness of TD (WP) were calculated for each bronchial generation separately. Results: In CF patients, a mean of 768 measurements of the segmented bronchial tree compared to 373 in NORMAL group could be performed $\left(1^{\text {st }}-14^{\text {th }}\right.$ generation, $p<0.0001)$. WT was significantly increased in CF from $2^{\text {nd }}-9^{\text {th }}$ generation bronchi (mean 2.1-1.1 mm in CF vs. 1.8-0.7 mm in NORMAL, $p<0.0001$ ). TD was larger in $5^{\text {th }}-9^{\text {th }}$ generation bronchi in CF (mean 8.8-7.0 mm vs. 7.3-6.4 mm, p $<0.05$ 0.0001 ). Consecutively, WP was increased in CF from $2^{\text {nd }}-9^{\text {th }}$ generation (mean ranges $41-61 \%$ vs. $37-47 \%, p<0.05-0.0001)$.
Conclusion: Automatic airway detection and segmentation succeeded to measure bronchial wall thickening and wider bronchial diameters in CF as compared to NORMAL. Moreover, a lack of bronchial tapering could be detected for distal bronchi in CF. Automatic computational results might be used for quantitative monitoring of the disease instead of manual scoring.

\section{B-627 11:24}

Radiologic manifestations of tuberculosis in HIV-positive patients B. Saidi, M. Bakhshayesh-Karam, P. Tabarsi, S. Zahirifard, M. Ghofrani; Tehran/IR (bahare_saidi@yahoo.com)

Purpose: The chest presentation of tuberculosis in HIV positive differs from that of immuno-competent patients. The association of CD4 count and radiologic pattern has been previously investigated. The aim of this study was to review the pulmonary manifestations of tuberculosis in HIV-positive patients at our centre.

Methods and Materials: The radiographs of 52 male HIV-seropositive patients (mean age $39.9 \pm 9.5$ ) with sputum or bronchoalveolar lavage, culture or PCR proven tuberculosis who were referred to our center from April 2005 to January 2010 were studied. The CD4 count and ADA levels were available for 43 patients. The radiographs were reviewed regarding pattern 1. postprimary 2 . the patterns not characteristic of postprimary: pleural effusion, hilar or mediastinal lymphadenopathy, middle lobe or basal lower lobe involvement (atypical pattern) 3.miliary. The patterns were correlated with CD4 level.

Results: Seven (13.5\%) patients died. Thirty-six (70.6\%) patients had CD4 count less than 200. $19(36.5 \%)$ had post-primary pattern tuberculosis on x-ray, 23 $(44.2 \%)$ had atypical and $10(19.2 \%)$ had miliary pattern. $10(19.2 \%)$ had cavity, $15(28.8 \%)$ had bronchiectasis, $5(9.6 \%)$ had atelectasis, one had pneumothorax and one hydropneumothorax.12 (23.1\%) had pleural effusion, $14(26.9 \%)$ had hilar or mediastinal adenopathy. The mean CD4 count in the post-primary pattern was $176.8 \pm 193$; in the atypical pattern $137.05 \pm 133$ and miliary pattern $46.56 \pm 55$, the difference was statistically significant between the groups $(p<0.05)$.

Conclusion: The atypical pattern and miliary tuberculosis are common observations in HIV-positive patients. The CD4 counts were generally low in our patients; however, the mean CD4 count was significantly lower in the miliary pattern.

B-629 11:33

(EPOS)

Comparison of the spectrum of the clinical and radiological manifestations of pulmonary disease caused by Mycobacterium avium complex (MAC) and Mycobacterium xenopi (M. xenopi)

M.C. Carrillo, D. Patsios, U. Wagnetz, F. Jamieson, T. Marras; Toronto, ON/CA (maclacarrillo@gmail.com)

Purpose: To retrospectively describe and compare computed tomography (CT) chest manifestations of pulmonary disease caused by MAC and M. xenopi

Methods and Materials: We reviewed records and imaging of all patients that had either MAC or M. xenopi lung disease, and chest CT scans, between January 2002 and December 2009. Data regarding the clinical presentation, relevant treatment history, and other past medical history were recorded. Patterns of measurable disease were depicted on chest CT.

Results: There were 74 eligible patients; 54 with MAC and 20 with M. xenopi infection. Patients with MAC infection were older. M. xenopi patients had a higher rate of any malignancy (58\% versus $11 \%, \mathrm{p}=0.002)$ and pulmonary malignancy (25\% versus $5 \%, p=0.08$ ). Patients with $M$. xenopi more commonly had cavities ( $46 \%$ versus $16 \%, p=0.02), \leq 5 \mathrm{~mm}$ nodules ( $88 \%$ versus $58 \%, p=0.02)$, and $>5 \mathrm{~mm}$ nodules $(67 \%$ versus $46 \%, p=0.16)$. M. xenopi disease also more often demonstrated the so-called "classic fibrocavitary type" of radiological pattern ( $88 \%$ versus $21 \%, p=0.0002)$. MAC was more often associated with bronchiectasis $(60 \%$ MAC vs $33 \% \mathrm{M}$. xenopi, $\mathrm{p}=0.057)$ and a nodular-bronchiectatic pattern $(79 \%$ MAC vs $11 \% \mathrm{M}$. xenopi, $\mathrm{p}=0.0002$ ). Emphysema was more prevalent in patients with M. xenopi (50\% versus $20 \%, p=0.02)$.

Conclusion: Compared with MAC, patients with M. xenopi tend to be younger and more often have malignancies and emphysema.m. xenopi is more often associated with cavities, a "fibro-cavitary" CT pattern and nodules. MAC is more often associated with bronchiectasis and a "nodular-bronchiectatic" CT pattern. 


\author{
B-630 $11: 42$ \\ Pleuropulmonary paragonimiasis: why is this disease delayed or \\ misdiagnosed on CT? \\ S.K. Kim, G.Y. Jin; Jeonju-si/KP (inacien7777@paran.com)
}

Purpose: We investigated $\mathrm{CT}$ findings of pleuropulmonary paragonimiasis and evaluated the cause of delay or misdiagnosis of paragonimiasis.

Methods and Materials: This study was performed prospectively with informed questionnaire from January 2009 to June 2009 in four tertiary hospital. Electronical survey with several topics (personal information, method of diagnosis, kinds of CT machine, CT findings, extrapulmonary diagnosis, first impression) was done in each hospital for the patients who were diagnosed as pleuropulmonary paragonimiasis from 2003 to 2008. The data were collected and analysed at one hospital.

Results: 103 patients ( 58 men and 45 women; age $46.1 \pm 14.6$ ) from four hospitals were enrolled. The main lesion was at upper lobe in $58 \%$, lower lobe in $35 \%$ and right middle lobe in $7 \%$, with peripheral distribution in $78 \%$, central distribution in $14 \%$, and both in $8 \%$. CT findings were non-cavitary nodule in $32.9 \%$, cavitary nodule in $23.4 \%$, pneumonic consolidation in $26.5 \%$, lung mass in $8.5 \%$, linear density in $5.3 \%$ and others in $3.2 \%$. Other superimposed findings were bronchiectasis in $53 \%$, cyst in $29 \%$, bronchial wall thickening in $15 \%$ and mucoid impaction in $3 \%$. Worm tract sign, which is known as typical finding of pleuropulmonary paragonimiasis, was only present in $18.5 \%$. First impression on CT was paragonimiasis in $46 \%$, pneumonia in $18 \%$, tuberculosis in $14 \%$, lung cancer in $7 \%$, and others in $15 \%$. The diagnostic accuracy of CT was $43.7 \%$.

Conclusion: Diagnosis of pleuropulmonary paragonimiasis is often delayed or missed especially when the lesion is at upper lobe, which occasionally mimics tuberculosis or malignancy.

\section{$10: 30-12: 00$} Room D2

\section{Interventional Radiology}

\section{SS 1609}

\section{Aorta, vein and lymphatic interventions}

Moderators:

S. Müller-Hülsbeck; Flensburg/DE

J. Raupach; Hradec Kralove/CZ

\section{B-631 10:30}

Endovascular treatment for ruptured abdominal aortic aneurysms

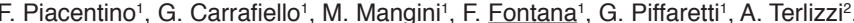
N. Sorrentino ${ }^{2}$, C. Fugazzola'; ' Varese/lT, ${ }^{2}$ Napoli/IT (f.piacentino@live.it)

Purpose: We report the results of our retrospective study comparing the outcome for the different endograft configuration.

Methods and Materials: Only patients with a ruptured abdominal aortic aneurysm confirmed by contrast-enhanced computed tomography angiography were eligible for the analysis. Out of a group of 67 patients, 42 patients $(62.7 \%)$ were treated with endograft. Patients were divided for comparative analysis according to the configuration of the endograft implanted. Twenty-five patients $(59.5 \%)$ were operated on general anaesthesia and oro-tracheal intubation. Thirteen patients (30.9\%) received an aorto-uni-iliac endograft (group A), and 29 a bifurcated endograft (group B). Database included 29 variables (18 preoperative, 8 intraoperative, 3 postoperative) Results: Primary technical success rate was 95\% (40/42). No patient required conversion to open repair. Overall, a total of 12 patients $(28.5 \%)$ died within 30 days: there were 2 intra-operative deaths (4.7\%), 9 patients died within 48 hours of operation, 3 further patients within two weeks. Hospitalisation death rate was $30.9 \%(13 / 42)$. At univariate and multivariate analyses, the subgroups were well-matched for gender, age, aneurysm size and morphology, type of diagnosis, delay, and values of the parameters at admission; shock was statistically more frequent in the group A. Hospital mortality was statistically higher in group A; type of endograft and intensive care unit admission were the only independent predictors of hospital mortality.

Conclusion: In our experience, an higher mortality rate for the aorto-uni-iliac configuration was observed; shock at admission was confirmed the most important factor for postoperative survival.

\section{B-632 10:39}

Stent collapse after uncovered abdominal aortic stenting in sub-/acute complicated Stanford type B dissection

A. Massmann, M. Katoh, D. Tscholl, R. Seidel, P. Fries, G.K. Schneider, H.-J. Schaefers, A. Buecker; Homburg/DE (Alexander.Massmann@uks.eu)

Purpose: To evaluate technical feasibility and outcome of stent placement in sub-/ acute complicated Stanford type B dissection.

Methods and Materials: 12 patients ( 1 female, range 44-71 years) with aortic dissection suffering from severe gastrointestinal malperfusion and claudication underwent uncovered stent placement (diameter 7-25 mm, length 40-100 mm) into the aorta to achieve sufficient perfusion. Additional stents were placed into the visceral arteries in 3 patients.

Results: Thoracoabdominal stent placement yielded to considerable clinical improvement in 11 of 12 patients. Additional stents (up to four) were placed in seven patients (1 celiac, 1 mesenteric, 2 renal, 6 iliac). Follow-up CTA showed near complete collapse of four stents (diameter $9-25 \mathrm{~mm}$, length $100 \mathrm{~mm}$ ) after one week. Catheterisation and balloon dilatation of all four stents were possible. But all cases showed re-collapse on follow-up CTA. However, duplex-ultrasound and CTA showed perfusion of the superior mesenteric artery in all patients. Follow-up ranged from 3 months up to 3 years (average 1.7 years). During this time, one patient required an iliaco-mesenteric bypass for treatment of chronic mesenteric ischaemia, one month after stent placement. All other patients did well without further interventional or surgical therapy.

Conclusion: Thoracoabdominal stent placement in sub-/acute complicated Stanford type B dissection is technically feasible and can be considered as a minimal-invasive therapy with similar clinical results compared to the placement of an endoprosthesis. Stent size should be adapted to the diameter of the true aortic lumen to avoid stent collapse, although even collapsed stents maintained sufficient perfusion.

\section{B-633 10:48}

Reliability and interobserver variability of preoperative measurements for thoracic endovascular aortic repair: semiautomatic centerline analysis versus manual measurement techniques

F. Rengier, S. Partovi, T.F. Weber, M. Müller-Eschner, Y. Hoo, F.L. Giesel, D. Böckler, H.-U. Kauczor, H. von Tengg-Kobligk; Heidelberg/DE

Purpose: Reliable preoperative measurements are crucial for successful thoracic endovascular aortic repair (TEVAR). The purpose of this study was to test whether reliability and interobserver variability of preoperative measurements for TEVAR are improved by means of semiautomatic centerline analysis compared to manual assessment on axial slices and double oblique multiplanar reformations (MPR). Methods and Materials: Preoperative CT angiographies of 30 patients with thoracic aortic disease (mean age $66.8 \pm 11.6$ years, 23 men) were analysed by one blinded vascular expert (reference standard) and three blinded non-experts. Maximum aortic diameters were measured at four positions relevant to TEVAR using three measurement techniques (manual axial slices, manual MPR, semiautomatic centerline analysis). Reliability was calculated as the absolute measurement deviation (AMD) from the reference standard and interobserver variability as the coefficient of variance $(\mathrm{CV})$. Results: Mean AMD was $7.3 \pm 7.7 \%$ (axial), $6.7 \pm 4.5 \%$ (MPR), and $4.7 \pm 4.8 \%$ (centerline). Mean CV was $5.2 \pm 4.2 \%$ (axial), $5.8 \pm 4.8 \%$ (MPR), and $3.9 \pm 5.4 \%$ (centerline). Both AMD and CV were significantly lower for centerline analysis compared to axial technique $(p=0.001 / 0.042)$ and MPR $(p=0.009 / 0.003)$. Deviations from reference standard were above $1 \mathrm{~mm}$ in $66 \%$ (axial), 64\% (MPR), and $42 \%$ (centerline). A stent graft diameter with $\geq 4 \mathrm{~mm}$ difference from the reference standard was chosen in $20 \%$ based on axial technique or MPR, and only in $8 \%$ based on centerline analysis.

Conclusion: Semiautomatic centerline analysis provides the most reliable and least variable diameter measurements in TEVAR patients. MPR should be performed by vascular experts due to significantly lower reliability and significantly higher interobserver variability among non-experts.

\section{B-634 10:57}

Predictive factors for endoleaks after thoracic aortic aneurysm endograft repair

A. Di Massa, M. Colosimo, E. Cotta, M. Mangini, G. Piffaretti, G. Carrafiello,

C. Fugazzola; Varese/IT

Purpose: Our prospective investigation aimed to determine and analyse the incidence and the determinants of endoleaks after thoracic stent graft.

Methods and Materials: Sixty-one patients affected by thoracic aortic aneurysms were treated. The study cohort contained 54 men, with a mean age of $63.6 \pm 17.9$ 
years. The follow-up imaging protocol included chest radiographs and triple-phase computed tomographic angiography performed at 1,4 and 12 postoperative months and annually thereafter.

Results: Median follow-up was 32.4 months (range: 1-96 months). Endoleaks were detected in $9(14.7 \%)$ patients, of which 7 were type 1 . Five endoleaks were detected at 30 postoperative days, and the other 4 developed with a mean delay of 12 months. Endovascular or hybrid interventions were used to treat the endoleaks. Secondary technical success rate was $100 \%$. Multivariate analysis demonstrated that the diameter of the aneurysmal aorta (odds ratio 1.75.95\%confidence interval 1.07-2.86) and the coverage of the left subclavian artery (odds ratio $12.05,95 \%$ confidence interval 1.28-113.30) were independently associated with endoleak development. The percentages of patients in whom reinterventions were unnecessary were $94.6 \% \pm 3.0 \%, 88.3 \% \pm 4.5 \%$, and $85.4 \% \pm 5.2 \%$, at 1,2 , and 5 years, respectively. The actuarial survival estimates at 1, 2, and 5 years were $85.2 \% \pm 4.6 \%, 78.1 \% \pm 5.4 \%$, and $70.6 \% \pm 6.4 \%$, respectively.

Conclusion: The diameter of the aneurysmal aorta and the position of the landing zone are independent predictors of endoleak occurrence after thoracic stent-graft procedures. A careful follow-up program should be considered in patients in whom these indices are unfavourable, because most of the endoleaks may be successfully and promptly treated by additional endovascular procedures.

\section{B-635 11:06}

\section{Stenting as an effective endovascular treatment of superior vena} cava syndrome: review of hundred and fifty-four cases. Single-centre experience

S. Kudrnova, B. Nemes, E. Rimely, K. Hüttl; Budapest/HU

(skudrnova@gmail.com)

Purpose: VCSS constitutes a life-threatening clinical manifestation of central vein obstruction mainly due to external compression by malignancies. Prompt restoration of VCS patency is required to enable diagnostic and therapeutical procedures ameliorating and prolonging patients' lives. VCS stenting represents an effective alternative to insufficient or high-risk best medical treatment, irradiation or opensurgery. We evaluate its technical success, clinical effectiveness, patency rate and patients' overall survival period in a large group of patients.

Methods and Materials: 154 patients (age range 23-87 years) underwent endovascular treatment for SVCS in our centre between November 2002 and May 2009 (malignant etiology in 143 cases, benign in 11). SVC was primarily stented in 145 , balloon dilated in 1 and interventional attempt was unsuccessful in 8 cases. Patients were invited back upon recurrence of symptoms.

Results: All successful interventions led to immediate relief of symptoms. No periprocedural mortality occurred, but 1 SVC rupture, 1 stent fracture and 1 haemopericardium. Reocclusion occurred in 11 cases (including 5 and 3 times in a single patient), within a time range of 2 days- 2.5 months (thrombolysis reintervention). Restenosis was reported in 7 cases (including 5 and 2 times in a single patient), within a time range of 2 weeks- 9 months (stent-in-stent reintervention). Survival time follow-up could be obtained in $94(64 \%)$ patients so far (1 week Survival Rate 93\%, 1 month SR 83\%, 6 months SR 45\%, 1 year SR $10 \%, 3$ year SR 3\%). Conclusion: Stenting has in our centre proved to be a highly technically successful, clinically effective, low-morbidity, low-mortality, low-restenosis treatment option for VCSS.

\section{B-636 11:15}

Catheter-directed thrombolysis in caval thrombosis with the EKOS catheter: preliminary results

A.H. Mahnken ${ }^{1}$, J. Grommes ${ }^{1}$, C. Plumhans ${ }^{1}$, S. Langer ${ }^{1}$, C. Wittens ${ }^{2}$;

${ }^{1}$ Aachen/DE, ${ }^{2}$ Maastricht/NL (mahnken@rad.rwth-aachen.de)

Purpose: Currently, one in four patients with primary iliofemoral deep vein thrombosis (DVT) develops a post thrombotic syndrome within one year. Pharmacomechanical thrombolysis is a viable option in the treatment of DVT. The aim of this study was to access the feasibility of new pharmaco-mechanical thrombolysis system in DVT with involvement in inferior vena cava.

Methods and Materials: 12 patients ( 7 males, mean age 44 [5-79] years) with severe ilio-femoral and caval thrombosis were treated using the EKOS ${ }^{\circledR}$ EndoWave $^{\mathrm{TM}}$ Peripheral Infusion System. The system combines a proprietary multiple-side-hole drug infusion catheter with a guidewire-exchangeable ultrasound core for simultaneously infusing therapeutic agents and delivering high- frequency, low-power ultrasound energy. Lysis was initiated with a bolus of $2.5 \mathrm{mg}$ rtPA followed a continuous infusion of $1 \mathrm{mg} \mathrm{trPA} / \mathrm{h}$. All patients were treated with an additional continuous intravenous infusion of $1000 \mathrm{U}$ Heparin/h.

Results: Complete recanalisation was achieved in 10/13 patients. In one patien partial recanalisation and in two patients no recanalisation was achieved. Median time for lysis was 41 hours. Median total dose of thrombolytic drug was $44 \mathrm{mg}$ rtPA (26-79). In three patients an iliac vein was stented to remove a flow obstructing lesion and to avoid recurrent thrombosis. During 7 months of follow-up early rethrombosis occurred in 4 patients. One patient developed a haematoma in the calf which was treated conservatively.

Conclusion: Pharmaco-mechanical lysis using the EKOS ${ }^{\circledR}$ EndoWave $^{\mathrm{TM}}$ system appears to be a safe and viable alternative option for treating caval and ileo-femoral DVT, warranting further studies.

\section{B-637 11:24}

Experience with superior vena cava filters

F. Pollice ${ }^{1}$, P. Pollice ${ }^{1}$, R. Muller ${ }^{2} ;{ }^{1}$ Andria/IT, ${ }^{2}$ Paris/IT

(francesco.pollice@tiscali.it)

Purpose: Therapy to prevent pulmonary embolism (PE) resulting from upper extremity deep venous thrombosis (UEDVT) remains controversial despite an increasing incidence of DVT of upper extremity origin. The purpose of this study was to evaluate the results of 72 superior vena cava Greenfield filters (SVC-GFs) placed at risk for PE arising from UEDVT.

Methods and Materials: During the past 78 months, we placed SVC-GFs in 72 patients with UEDVT in whom anticoagulation was either deemed contraindicated $(n=$ 67)or proved ineffective in preventing recurrent $\mathrm{PE}(4=)$ or extension of the thrombus $(n=1)$. There were 25 male $(35 \%)$ and $47(65 \%)$ female patients whose ages ranged from 25 to 99 years. Follow-up ranged from 10 days to 78 months. Sequential chest radiographs revealed no filter migration or displacement in 26 patients.

Results: Thirty-four patients died in the hospital of causes unrelated to the SVC filter or recurrent thromboembolism (mean time to death, 20 days). Follow-up of the surviving 38 patients ranged from 1 month to 78 months (mean 22 months); none of these patients were seen with any evidence of PE. One SVC-GF was incorrectly discharged into the innominate vein and left in place. This vein remains patent 2 months after insertion without evidence of filter migration.

Conclusion: We think that insertion of SVC-GFs is a safe, efficacious, and feasible therapy and may prevent recurrent thromboembolism in patients with UEDVT who are resistant to anticoagulation or have contraindications to anticoagulation.

B-638 11:33

Concomitant use of endovenous laser and foamed sclerosant for

treatment of lower limb varicosities

M. Bhalla, N. Bhalla; Ahmedabad/IN (namratamanav@hotmail.com)

Purpose: To report mid-term results (upto 3 years) of concomitant use of two modes of treating varicose veins - endovenous laser treatment for Great Saphenous Vein (GSV) reflux and sclerotherapy for incompetent perforators and accessory venous channels.

Methods and Materials: 253 lower limbs (76 Left,43 Right,134 billateral) of 186 patients ( 66 women, 120 men;mean age 45.5 years) were treated with concomitant use of endovenous laser and sclerotherapy. 5 patients (18 legs;7\%) had ulcers. GSV was mapped using $10 \mathrm{MHZ}$ linear Ultrasound (US) probe. Following local anaesthesia, 0.035 inch J-Guidewire was introduced in GSV from lowest point of reflux. A $5 \mathrm{~F}$ sheath and $610 \mu \mathrm{m}$ laser fibre was used. Laser energy was delivered intraluminally at $14 \mathrm{~W}$ continous mode. Fibre was uniformly withdrawn. Sclerosant, mixed with air to produce foam, was introduced through needle in perforators and additional venous channels under US guidance. All patients were advised ibuprofen and class II stockings. Follow-up was done at 1 week, 3.6,12,24 and 36 months. Results: $98 \%$ laser-treated GSV remained ablated at 3-year follow-up. $7 \%$ limbs in first year and additional $3 \%$ limbs in next 2 years (i.e. $10 \%$ limbs in 3 years) required one more sclerotherapy session for new incompetent perforators. No complications were noted. $90 \%$ patients complained of tightness along the course of treated GSV. Patients resumed routine activities immediately.

Conclusion: Laser has already proven its use in treating GSV. Sclerotherapy is effective in dealing with perforators and provides rapid healing of chronic venous ulcers. Concomitant use of both modes limits the need of subsequent procedure.

\section{B-639 11:42}

A comparison of standard dual-tip and split lumen heamodialysis catheters

H. Lee, S. Park, Y.-I. Jo, I. Chang, I. Yun; Seoul/KR (20080136@kuh.ac.kr)

Purpose: To compare a newer split lumen haemodialysis catheter with the standard dual-tip haemodialysis catheter.

Methods and Materials: From August 2005 to December 2009, among patients who were referred for tunneled haemodialysis catheters, the patients who underwent standard dual-tip haemodialysis catheter (DTC) insertion or split lumen 
haemodialysis catheter (SLC) insertion were enrolled. During haemodialysis with those haemodialysis catheters, initial catheter dwell times and catheter-related complications were compared between the two groups.

Results: A total of 164 patients were enrolled with 80 SLC insertions and 133 DTC insertions. Initial catheter dwell time was 71.94 days (range 4-277 days) in SLC and 68.55 days (range $1-292$ days) in DTC $(p=0.76)$. In terms of catheterrelated complications, catheter migration was detected in 8 patients $(10.5 \%)$ and 11 catheters $(12.4 \%)$ in the SLC group and in 2 patients $(1.7 \%)$ and 3 catheters $(2.0 \%)$ in the DTC group $(p=0.0026)$.

Conclusion: SLC did not extend the initial catheter dwell time compared to DTC. Furthermore, SLC was more prone to catheter-related complications, particularly catheter migration, than DTC.

\section{$B-640 \quad 11: 51$}

Effectiveness of therapeutic lymphography on lymphatic leakage

E. Alejandre Lafont, C. Krompiec, W.S. Rau, G. Krombach; Giessen/DE

(enrique.alejandre-lafont@radiol.med.uni-giessen.de)

Purpose: To investigate if therapeutic lymphography is a reliable method to treat lymphatic leakage when conservative treatment fails and to investigate which parameters influence the success rate.

Methods and Materials: Between 08/1995 and 1/2008, 50 patients with lymphatic leakage in the form of chylothorax, chylous ascites, lymphocele, and lymphatic fistula had undergone conventional therapeutic lymphography after conservative therapy had failed. Of these 50 lymphographies, 7 could not be statistically evaluated in our retrospective study: 1 patient had died of cancer a day after lymphography; 6 were excluded due to various technical problems. The remaining 43 patients were evaluated. Therapeutic success was evaluated and correlated to the volume of lymphatic leakage (more or less than $500 \mathrm{ml} /$ day), as assessed by drainage. Results: In nearly $78.8 \%$ of patients, the location of the leak could be detected, and surgical intervention could be planned when therapeutic lymphography failed. The lymphatic leak could be completely occluded in $70 \%$ of patients when the lymphatic drainage volume was less than $500 \mathrm{ml} /$ day. Even when lymphatic drainage was higher than $500 \mathrm{ml} / \mathrm{d}$, therapeutic lymphography was still successful in $35 \%$ of patients. The overall success rate in patients with failed conservative treatment was $51.2 \%$. Success did not depend on other factors such as age, sex of the patient, or time elapsed between lymphatic injury and intervention.

Conclusion: Therapeutic lymphography is an effective method in the treatment of lymphatic leakage when conservative therapy fails. The volume of lymphatic drainage per day is a significant predictor of the therapeutical success rate.

\section{$10: 30-12: 00$}

Room E1

\section{Computer Applications}

\section{SS 1605}

\section{Image analysis in oncology}

Moderators:

M. Onu; Bucharest/RO

J. Reponen; Raahe/FI

\section{B-641 10:30}

Creation of a database of 3D models of microcalcification clusters for future observer studies

E. Shaheen, C. Van Ongeval, L. Cockmartin, H. Bosmans; Leuven/BE (eman.shaheen@uzleuven.be)

Purpose: Breast cancer is a major health concern for women. Its early diagnosis relies on the detection and characterization of microcalcifications and/or masses. Observer studies related to the detectability of lesions are an important topic of research. Therefore, the availability of a database representing different types of lesions while preserving the morphological shape is crucial. We report on creating a database of 3D models of microcalcification clusters to be further simulated into projection images of 2D, breast-tomosynthesis and breast-CT.

Methods and Materials: Biopsy specimens containing microcalcification clusters were collected and imaged by a micro-CT scanner. A segmentation procedure was applied to every reconstructed image to build the 3D model. Several algorithms implemented by the computer-aided diagnosis (CADx) that preserve the morphology of microcalcifications have been investigated, but failed to segment perfectly due to the different anatomical background of the micro-CT images compared with mammograms. Therefore, an adapted segmentation procedure was developed resulting in a good morphological segmentation. However, it suffered from false positives that were enhanced by applying the top-hat routine.
Results: The 3D models were rotated into different orientations, re-simulated into 2D mammograms. An experienced radiologist judged their realistic appearance and classified them into Le Gal types. From 26 biopsy specimens, 100 models were generated and 81 models were accepted by the radiologist.

Conclusion: The proposed method proved its robustness for preparing a realistic database of microcalcifications clusters to be used in observer studies related to the clinical performance evaluation of digital mammographic modalities in terms of lesion detectability.

\section{B-642 10:39}

Combination of multiparametric data from computer-assisted analysis of dynamic enhancement features in MR-mammography: a comparison of artificial neural networks, logistic regression, classification and regression trees and k-nearest neighbour analysis for differential diagnosis of breast lesions

P.A.T. Baltzer, M. Dietzel, W.A. Kaiser; Jena/DE

(pascal.baltzer@med.uni-jena.de)

Purpose: In MR-mammography (MRM), computer-assisted methods are frequently used for dynamic enhancement data (DED) analysis. These software tools provide multiparametric information of DED. Combining these data into a diagnosis represents a dilemma for the radiologists. This investigation was performed to investigate and compare four different methods for combination of multiparametric DED into a diagnosis

Methods and Materials: 51 consecutive patients (mean age 58y) with 88 lesions (42 benign, 46 malignant) were investigated. All underwent MRM according to European guidelines at $1.5 \mathrm{~T}$. Reference standard was histopathology or imaging follow-up > 24 months. DED was analysed using a commercial software tool (iCAD former CADsciences). Analysis provided most suspect curve characteristics, worst Washin, worst Washout, fraction of different curve types (i.e. Persistent, Plateau, Washout) and distribution of pharmacokinetic parameters (ktrans, ve) inside the analysed lesion. Combination of these multiparametric data for the task of lesion classification (i.e. benign vs. malignant) was performed using four methods: artificial neural network (ANN), logistic regression (LR), classification and regression trees (CART) and k-nearest neighbour (KNN) analysis. Receiver operating characteristics $(R O C)$ analysis was used to compare diagnostic accuracy of these methods. Results: ROC analysis revealed superior classification accuracy for ANN (93.0\%) and LR (93.4\%) compared to CART (87.0\%) and especially KNN (80.1\%). As a comparison, most suspect curve washin/washout showed an accuracy of $73.4 / 64.7 \%$. Conclusion: Multiparametric combination of DED is possible, increasing classification accuracy. Significant differences were observed between the multivariate methods applied. Further study on larger patient collectives is needed to validate these findings.

\section{B-643 10:48}

Potential of MR-mammography to predict survival in breast cancer patients: systematic approach using survival time analysis and computerassisted diagnosis for quantitative assessment of tumour vasculature P.A.T. Baltzer ${ }^{1}$, M. Dietzel' ${ }^{1}$, R. Zoubi ${ }^{1}$, M. Gajda ${ }^{1}$, I.B. Runnebaum¹, W.A. Kaiser ${ }^{2}$ ${ }^{1}$ Jena/DE, ${ }^{2}$ Boston, MA/US

Purpose: In breast cancer accurate and non-invasive assessment of prognosis might potentially impact both patient management and therapeutic approach. This observational-unicenter study was conducted to identify the potential of magnetic-resonance-mammography (MRM) to predict disease-related death in primary breast cancer.

Methods and Materials: In this IRB-approved investigation, standardised protocols and study design were applied according to international guidelines (T1w-FLASH $0.1 \mathrm{mmol} / \mathrm{kg}$ BW Gd-DTPA; T2w-TSE; 1.5 -Tesla). All patients with invasive breast cancer and subsequent surgicopathological verification (no secondary/recurrent breast-cancer; study-duration: 24 consecutive months) were clinically followed up. Interval between initial staging-MRM and last follow-up, as well as occurrence of "disease-related death" were documented. For quantitative analysis of tumour-vasculature dedicated computer-assisted-diagnosis software was used. Pharmacokinetic parameters were automatically calculated for every tumour on a voxel by voxel basis and correlated with occurrence and time interval to "diseaserelated death" (Cox proportional-hazards-regression: $\mathrm{CR}$ )

Results: 839 patients were enrolled for MRM imaging. 122 were eligible for CR analysis. Median follow-up time was 52 months. In 15/122 patients "disease-related death" occurred. Survival time analysis by CR was possible with 4 CAD-parameters on a high level of significance $(P<0.001)$, demonstrating appropriate overall model fit (2 Log-Likelihood: 138.2; Chi-square: 37.7 ). 
Conclusion: Our data demonstrate the potential of MRM to significantly predict disease-related death in primary breast-cancer. As results were extracted from a routine staging examination, MRM non-invasively provides not only diagnostic information but also quantitative outcome data at one step. Future studies should address the impact of these findings on patient management and therapeutic approach.

\section{B-644 10:57}

Development and testing of an artificial neural network for the noninvasive differentiation of papillomas and fibroadenomas based on breast MRI characteristics

P.A. Baltzer ${ }^{1}$, M. Dietzel ${ }^{1}$, A. Dietzel ${ }^{2}$, T. Gröschel ${ }^{1}$, R. Zoubi ${ }^{1}$, M. Bogdan ${ }^{2}$, W.A. Kaiser ${ }^{3} ;{ }^{1}$ Jena/DE, ${ }^{2}$ Tübingen/DE, ${ }^{3}$ Boston, MA/US

Purpose: Non-invasive differential diagnosis of papillomas and fibroadenomas is of potential clinical importance, as it might aid in the indication for surgical resection of benign breast masses. The aim of this study was to design an artificial neural network (ANN) to predict presence of papillomas based on magnetic-resonancemammography (MRM) findings.

Methods and Materials: 103 fibroadenomas and 83 papillomas were included. All were imaged by MRM applying standardised protocols and study design (T1wFLASH; $0.1 \mathrm{mmol} / \mathrm{kg}$ BW Gd-DTPA; T2w-TSE; histological verification after MRM; IRB-approval). Expierenced radiologists without knowledge of final histological diagnosis evaluated this dataset using predefined MRM-descriptors. Based on such an ANN was developed to differentiate papillomas and fibroadenomas (The-MathWorks/ Inc., Feed-Forward-Architecture/Resilient Back-propagation-Algorithm). Performance of this ANN was evaluated 19 times independently and quantified on an unknown testing sample applying five-fold cross-validation (AUC: the area under the curve). Results: The ANN was able to significantly differentiate fibroadenomas and papillomas $(P<0.001)$. One hidden layer including 10 neurons at a learning rate of 1 , 2 and scaling-factor of 0.85 was identified as the optimal network-topology. This ANN reached a maximum AUC of 0.726 for the differentiation of papillomas and fibroadenomas (standard error: $0.0367 ; 95 \%$ confidence-interval 0.656 to 0.789 ). Conclusion: On unknown data, the optimised neural network showed significant performance and moderate diagnostic accuracy. Accordingly, multivariate differentiation of papillomas and fibroadenomas is possible based on MRM-criteria and might be used to non-invasively aid clinical decisions for surgical resection of benign breast masses.

\section{B-645 11:06}

Automated quantitative computer-assisted analysis of elastography to discriminate breast lesions

A. Malich, A. Kott, R. Gorna; Nordhausen/DE (ansgar.malich@shk-ndh.de)

Purpose: This study aimed to verify the diagnostic value of strain ratio as a quantitative elastographic parameter in ultrasound of breast lesion and related influencing factors.

Methods and Materials: 180 breast lesions were analysed sonographically $(14 \mathrm{MHz}$ probe) by two experiences radiologists in consensus including elastography analysis (Ueno-scale) and computer-based calculation of strain ratio. Values were related to size and pathological outcome. The following size-related groups were used (largest available diameter): $\mathrm{S} 1:<5 \mathrm{~mm} ; \mathrm{S} 2<10 \mathrm{~mm}$; $\mathrm{S} 3<15 \mathrm{~mm} ; \mathrm{S} 4<20 \mathrm{~mm} ; \mathrm{S} 5>30 \mathrm{~mm}$. Mean overall size was $10.4 \mathrm{~mm}$. ROC-analysis for cut-off values was performed.

Results: Strain ratio of malignant versus benign lesions was 6.36 versus 2.27 Mean strain ratios of benign lesions reflecting pathology were 0.98 (fibrolipoma); 1.05 (adenosis); 1.31 (fibrosis); 1.58 (cysts) 1.53 (intraglandular lymph nodes) 2.40 (fibroadenoma); 3.31 (calcifications-liponecrosis); 2.49 (papillomata); 4.44 (scars); and 2.06 (remaining benign entities). Size-related analysis of strain ratio was calculated for malignancies/fibroadenoma/cysts/other benign lesions as S1: 1.66/1.74/1.44/0.91; S2: 2.50/2.08/1.22/2.95; S3: 6.72/2.52/2.20/3.03; S4: 10.25/3.76/0.05/3.09; S5: 4.75/8.00/2.09/1.45. Best performing cut off values are (according to ROC-analysis) S2: 2.4 ; S3: 2.8 ; S4: 3.9

Conclusion: Strain ratio is influenced by size and histopathology. In the diagnostically most relevant group of 5-20 mm lesions, malignant lesions are characterised by a higher strain ratio versus all other entitites. Large malignant lesions are typically characterized by a lowered SR versus fibroadenomata (probably due to necrotic liquid components in cancer versus macrocalcifications in fibroadenomata). Typically fibroadenomas, scars and papillomas are characterised by increased SR-values as well. Elastography is of diagnostic use in the differential diagnosis of breast lesions.
B-646 11:15

Machine learning for the multivariate diagnosis of malignant tumours: development and testing of a dedicated software for the automatic selection and optimisation of classification algorithms

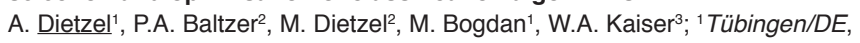
${ }^{2}$ Jena/DE, ${ }^{3}$ Boston, MA/US

Purpose: Machine-Learning is a promising field to solve the challenging task of multivariate diagnosis of malignant tumors. However, there is a multitude of algorithms usually requiring adjustment of a significant range of parameters to the specific radiological setting. Usually, such adjustments are done empirically by computer-scientists. In order to decrease operator related-bias and to simplify clinical application we developed a software to automatically apply and adjust Machine-learning algorithms on quantitative imaging data. Finally, the software was tested on a large clinical dataset.

Methods and Materials: A software tool was implemented to automatically test different Machine-learning algorithms and adjustments for the diagnosis of malignancy. Evaluation methods were implemented as default (e.g. multiple k-fold Cross-Validation). This software was tested on a clinical dataset. It included 329 benign and malignant breast lesions imaged by MRI (imaging-protocols according to EUSOBI-guidelines; standard-of-reference: histological verification). All lesions were characterized by a commercially CAD-software using selected standard enhancement-parameters. Based on this dataset (input variables: enhancement-parameters; classification variable: histology) different Machine-learning algorithms were tested and fine-tuned applying > 100 network parameters using the newly developed software.

Results: The software identified a medium sized network as most appropriate (one hidden-layer containing 5 neurons; learning-epochs: 50; learning-rate: 1.1). Test results demonstrated significant potential for the multivariate diagnosis of malignant tumors $(p<0.001)$ at moderated accuracy (median AUC[Area-under-the-Curve] 0.7249; Confidence-Interval: 0.70122-0.73923)

Conclusion: A computer tool for testing different Machine-learning settings was successfully implemented and tested on quantitative radiological data. Optimal parameter settings could be identified and appropriate performance was reached.

\section{$B-647 \quad 11: 24$} MSCT follow-up in patients with malignant lymphoma: improved therapy response classification using semi-automated volumetry compared to unidimensional measurements

M. Puesken ${ }^{1}$, B. Buerke ${ }^{1}$, N. Kohlhase ${ }^{1}$, V. Dicken ${ }^{2}$, C. Schuelke ${ }^{1}$, W. Heindel ${ }^{1}$, J. Wessling $1 ;{ }^{1}$ Münster/DE, ${ }^{2}$ Bremen/DE

Purpose: Impact of semi-automated volumetry compared to unidimensional measurements on therapy response classification in CT follow-up of malignant lymphoma.

Methods and Materials: MSCT scans of 63 patients with malignant lymphoma prior to therapy (baseline) and after 2 cycles of chemotherapy (follow-up) were included. A total of 313 target lymph nodes (56 cervical, 131 thoracic and 126 abdominal) were evaluated by two radiologists independently. Long axis diameter (LAD), short axis diameter (SAD) and volume were determined manually and using semi-automated segmentation software. Relative interobserver difference (RID) and time for manual and semi-automated segmentation were evaluated. Therapy response was calculated for each parameter based on "IWC" lymphoma-guidelines and "RECIST"-adapted guidelines. Mean of metric and volumetric measurements served as the reference standard. Statistical analysis encompassed intraclass correlation coefficients (ICC), t- and McNemar-test.

Results:Over all regions mean lymph node size in baseline/follow-up was $23.8 \pm 10.3 \mathrm{~mm} / 17.0 \pm 9.2 \mathrm{~mm}$ for $L A D$ and $7.2 \pm 13.5 \mathrm{ml} / 3.4 \pm 9.9 \mathrm{ml}$ for volume RID was consistently low in baseline and follow-up with high ICC $>0.96$ for semiautomated measurements. Mean evaluation time for semi-automated segmentation without need for correction was shorter $(16.6 \pm 11.7 \mathrm{sec})$ than for manual measurements $(29.0 \pm 14.5 \mathrm{sec})$. In $65 \%$ of all lymph nodes correction was necessary and evaluation time increased to $39.5 \pm 25.9 \mathrm{sec}$. Regarding therapy response, semi-automated volumetry obtained significantly more accurate classifications than semi-automated and manual LAD and SAD (e.g. volume $87.8 \%$ vs. semi-automated LAD 83.8\%, manual SAD $78.9 \%$, all $p<0.05$ )

Conclusion: Semi-automated lymph node volumetry is more accurate for therapy response classification in patients with malignant lymphoma as compared to established LAD. 


\author{
B-648 11:33 \\ A new algorithm for automatic segmentation of the left ventricular cavity \\ in cardiac MRI \\ M. Mazonakis, K. Pagonidis, E. Grinias, J. Damilakis; Iraklion/GR \\ (mazonak@med.uoc.gr)
}

Purpose: To present an algorithm for automatic segmentation of the left ventricular (LV) cavity in MR images.

Methods and Materials: Short-axis cine cardiac MR images were acquired from sixteen consecutive patients with known or suspected coronary artery disease. The automatic segmentation involved the classification of image pixels in three classes according to their grey value. The initial regions of high confidence were determined per class based on a histogram analysis and, then, the regions were grown using a flooding process. The algorithm could automatically detect and correct segmentation errors. The corrected segmentation map was presented to the user to select the LV endocardial region. The endocardial contours extracted from MR images were used to calculate the end-diastolic volume (EDV), end-systolic volume (ESV) and ejection fraction (EF). The automatically determined LV parameters were compared with the reference values obtained by manually tracing the endocardial borders. Results: Bland-Altman analysis showed a small underestimation of LV volumes (EDV: mean difference $=7.8 \pm 9.5 \mathrm{ml}$; ESV: mean difference $=2.3 \pm 4.6 \mathrm{ml}$ ) and a slight overestimation of $\mathrm{EF}$ (mean difference $=0.6 \pm 4.8 \%$ ) with the automatic segmentation algorithm compared to manual tracing. The differences between the two segmentation methods were not statistically significant (EDV: $p=0.06$; ESV: $p=0.08 ; E F: p=0.45)$. The parameters determined by automatic segmentation correlated well with those obtained by manual tracing (EDV: $r=0.86$; ESV: $r=0.92$ $E F: r=0.81$ ). The automatic image analysis time was less than 1.5 min per patient. Conclusion: The new algorithm enables the accurate assessment of LV volumes and $E F$ from MR images with great saving in labour.

\section{B-649 11:42}

Automatic lesion tracking for CT-based chemotherapy monitoring

J.H. Moltz', M. Schwier ${ }^{1}$, G. Soza ${ }^{2}$, C. Tietjen'2, H.-O. Peitgen ${ }^{1} ;{ }^{1}$ Bremen/DE,

${ }^{2}$ Forchheim/DE (jan.moltz@mevis.fraunhofer.de)

Purpose: In oncological therapy monitoring, an estimation of the change in size of a tumour is an important criterion for the assessment of treatment success. This requires finding correspondences between lesions in the baseline and follow-up CT scans, which can be a tedious task. We present an automatic tracking algorithm that identifies the positions of the corresponding follow-up lesions for a given set of segmented baseline lesions.

Methods and Materials: The two CT scans are coarsely aligned by a rigid registration which defines a search area in the follow-up image. Here, lesion candidates are detected by grey value and shape features. Finally, the candidate with the highest cross-correlation to the baseline lesion is chosen. We evaluated our method by applying it to 711 lesions (220 lung nodules, 263 liver metastases, and 228 lymph nodes) in CT scans of 172 patients from four different sites. It was considered successful when the point was inside an expert segmentation that was available for the follow-up lesions. Results: The tracking was successful for $95 \%$ of the lung nodules, $88.6 \%$ of the liver metastases, and $83.8 \%$ of the lymph nodes. In cases of failure, the mean distance to the correct lesion was $4.7 \mathrm{~mm}$. The average computation time was $3.5 \mathrm{~s}$. Conclusion: We developed a robust and fast method for automatic lesion tracking In combination with a segmentation algorithm, it can accelerate the radiological workflow by allowing fully automatic precomputations of follow-up examinations which only have to be confirmed or corrected by the radiologist.

\section{B-650 11:51}

New strategies for assessment Hodgkin lymphoma during and after therapy: preliminary results in comparison CT perfusion, whole body DWI and FDG-PET

A. Fausto, M.A. Mazzei, F.G. Mazzei, A. Fabbri, F. Lauria, L. Volterrani; Siena/IT (afausto@sirm.org)

Purpose: To assess the advantage of new techniques as CT perfusion and WB DWI versus FDG-PET in evaluating treatment response for Hodgkin disease (HD) during and after therapy.

Methods and Materials: 21 patients with $\mathrm{HD}$ underwent to CT perfusion $(100 \mathrm{~m}$ contrast media, $4 \mathrm{ml} / \mathrm{s}, 64$-row VCT, GE Healthcare), WB DWI ( $\mathrm{b}=600,1.5 \mathrm{~T}$ Signa HDx scanner, GE Healthcare), and FDG-PET at early assessment and restaging time during and after induction therapy $(n=9)$, only at restaging time after induction therapy $(n=9)$ and at staging, early assessment and restaging time prior, during and after induction therapy $(n=3)$, for a total of 36 studies. All CT perfusion and DWI studies were analysed using commercially available software (CT Perfusion
3 - Functool; GE Healthcare Technologies); blood volume (BV), blood flow (BF), mean transit time (MTT) and permeability surface (PS) and ADC values for selected region of interest were measured. Signed rank test was used.

Results: Residual masses were observed in all patients (12/12) and in $48 \%$ of subjects at restaging time (10/21). There were significant differences in PS $(p<0.01)$ between vital tumour and tissue fibrosis. Six CT perfusion and 5 WB DWI studies resulted negative even before FDG-PET studies.

Conclusion: CT perfusion and WB DWI offer functional tissue characterisation as well as FDG-PET; WB DWI can spares radiation to young subjects and CT perfusion can show better morphologic appearance. They can represent new alternative strategies in evaluating response to treatment in early assessment of HD.

10:30 - 12:00

Room E2

GI Tract

\section{SS $1601 b$}

Imaging of non-neoplastic conditions of the GI tract

Moderators:

M.M. Maher; Cork/IE

D.J.M. Tolan; Leeds/UK

\section{B-651 10:30}

High variability of the appearance of the appendix in adult cystic fibrosis: a computed tomography study

C. Pham, D. Grenet, M. Stern, A. Scherrer; Suresnes/FR

(a.scherrer@hopital-foch.org)

Purpose: Appendiceal changes in cystic fibrosis (CF) are well known but there is no previous study with systematic description on computed tomography (CT). Our objective was to describe the appearance of the appendix of adult CF and to see if it can be linked to clinical symptoms.

Methods and Materials: In a retrospective study of abdominal and pelvic CT scans of adult patients with CF: 55 CT scans from 55 patients (18 to 52 years old), cause of examination and diagnosis were noted. There was one case of colonic perforation with appendiceal micro-abscesses; for the other cases, there was no proven acute appendicitis. Findings studied: maximum appendiceal outer diameter and intraluminal fluid diameter, mucocele, appendicolith, peritoneal fat infiltration and effusion.

Results: Appendiceal diameter was enlarged $(10.9 \pm 2.6 \mathrm{~mm})$. There was a mucocele in 23/55 and appendicolith in 2 cases. Peritoneal fat infiltration was present in $9 / 55$ and effusion in 11/55 cases. Abdominal pain was mostly due to distal intestinal obstruction syndrome (DIOS) $\left(\chi^{2}=17.6 ; p<0.05\right)$. Appendiceal diameter did not correlated with age $\left(R^{2}<0.001 ; p=0.88\right)$ but did strongly with wall thickness $\left(R^{2}=0.23 ; p<0.05\right)$. No difference of diameter between the DIOS and the non-DIOS groups $(p>0.05)$ was observed. Conclusion: There is a high variability of the $\mathrm{CT}$ appearance of the appendix in adult CF patients. Appendiceal diameter is routinely enlarged but is age and symptomindependent. The CT diagnosis of acute appendicitis in this population is therefore not reliable. Clinical examination and comparison with previous CTs are essential.

\section{B-652 10:39}

Training MRI readers in detecting appendicitis

M.M.N. Leeuwenburgh', M.E. Thieme'2 , B.M. Wiarda², S. Bipat ${ }^{1}$

P.M.M. Bossuyt ${ }^{1}$, M.A. Boermeester ${ }^{1}$, J. Stoker ${ }^{1} ;{ }^{1}$ Amsterdam/NL, ${ }^{2}$ Alkmaar/NL (m.m.leeuwenburgh@amc.uva.nl)

Purpose: As MRI is introduced for diagnosing appendicitis, we evaluated the effect of direct feedback on the sensitivity and specificity of MRI for appendicitis in inexperienced radiologists and residents.

Methods and Materials: Twelve inexperienced readers ( 6 radiologists, 6 residents) evaluated 102 abdominal MRI's for appendicitis receiving direct feedback (final diagnosis) after each examination. The readers recorded the diagnosis and reading time per case. Prevalence of appendicitis was $44 \%$; cases were presented in random order. Mean sensitivity, specificity and time were calculated for the first and the last set of 25 scans. Results: In radiologists, sensitivity for appendicitis improved from $73 \%$ in the first set of 25 cases to $88 \%(95 \% \mathrm{Cl}: 71 \%$ to $95 \%)$ in the last set of cases. In residents, the sensitivity increased from $73 \%$ to $87 \%(95 \% \mathrm{Cl}: 67 \%$ to $96 \%)$. The specificity in radiologists was $88 \%$ in the first and $91 \%$ (95\% Cl: 83 to $96 \%$ ) in the last set, versus $87 \%$ and $93 \%(95 \% \mathrm{Cl}: 84 \%$ to $97 \%$ ) in residents. None of these differences reached statistical significance. The mean reading time decreased significantly from 231 seconds per scan in the first set to 140 seconds in the last set for radiologists $(p<0.001)$ and from 480 seconds per scan to 339 seconds for residents $(p<0.001)$. 
Conclusion: While reading time for inexperienced $M R I$ readers decreased significantly with systematic feedback in a series of 102 suspected appendicitis cases, sensitivity and specificity did not improve significantly, and diagnostic accuracy did not reach the level of experts.

\section{B-653 10:48 \\ In vivo visualisation of SPIO-loaded surgical mesh implants by MRI \\ N. Kühnert' ${ }^{1}$, N.A. Krämer ${ }^{1}$, H.C.W. Donker ${ }^{1}$, J. Otto ${ }^{1}$, I. Slabu ${ }^{1}$, \\ M. Baumann', G.A. Krombach ${ }^{2}$, C. Kuhl ${ }^{1}$, U. Klinge ${ }^{1} ;{ }^{1}$ Aachen/DE \\ ${ }^{2}$ Giessen/DE (nicolas.kuehnert@rwth-aachen.de)}

Purpose: Prosthetic mesh implants are widely used in hernia surgery. To revea long-term mesh-related complications such as shrinkage, dislocation or deformation, a precise visualisation of meshes in vivo is important. Modification of mesh MRI properties can help to delineate them. The aim of this study was to demonstrate time-dependent mesh shrinkage in vivo by MRI.

Methods and Materials: PVDF-meshes with incorporated super-paramagnetic iron oxides (SPIO) were implanted as an abdominal wall replacement in 30 rats. MRI was performed at day $1,7,14$, or 21 using gradient-echo sequences TR/TE $=50 / 4.6$, flip-angle $20^{\circ}$. Length and width were measured on axial, coronal and sagittal images and geometrical deformation was assessed. These data were compared to the post-mortem explanted meshes.

Results: In all orientations, MRI visualised all meshes and showed a mean shrinkage of length and width (day $21,13.1 \%, 3.1 \%$; day $14,9.2 \%, 4.1 \%$; day $7,5.9 \%$, $0.5 \%$ ). Post-mortem measurements validated these results.

Conclusion: SPIO loading of surgical meshes allows their visualisation in MRI and an accurate in vivo assessment of their shrinkage as well. MR imaging of such meshes could help reveal mesh-related complications, obviating the need for open surgical revision.

\section{B-654 10:57}

Application of $7 \mathrm{~T}$ micro-MR imaging to the detection of intestinal infarction due to venous occlusion: a rat model

F. Somma, D. Berritto, C. Cavaliere, N. Landi, R. Grassi; Naples/IT (fra1585@hotmail.it)

Purpose: The poor prognosis of gut ischaemia is due to the lack of specific findings, either clinical or radiological, that leads to delayed diagnosis and ineffective treatment. Early diagnosis assures a remarkable reduction of mortality rate. Therefore, the aim of this study is to value the experimental use of $7 \mathrm{~T}$ microMR in the detection of acute intestinal ischaemia (AMI) due to venous occlusion, relating the MR imaging patterns to the evolution of intestinal morphodynamism and histological analysis. Methods and Materials: The study was conducted on 16 Sprague Dawley rats. After anaesthesia, animals in the 'control group' ( $n=8)$ underwent the superior mesenteric vein (SMV) occlusion by tight ligation and, after macroscopical monitoring, were killed at different timing and the bowel removed for histological analysis; in reverse, rats in the 'experimental group' $(n=8)$ had a loop tied around SMV without occluding it. 3 days after surgery, basal MR scans were collected using a Bruker-Biospec-70/16-US; then SMV was occluded squeezing the loop and MR sessions were repeated after $5 \mathrm{~min}, 4$ and $8 \mathrm{hrs}$.

Results: Rats scanned using rare-T2 sequences showed no pathological pattern at the first time-point, while significant bowel wall thickening $(>1.5 \mathrm{~mm})$ and mesenteric hyperintensity were detected at following time-points ( 4 and $8 \mathrm{hrs}$ ).

Conclusion: Compared to histological analysis and macroscopical evidences, MR imaging correctly detected morpho-functional alterations of ischaemic gut and early identify ( $4 \mathrm{hrs}$ ) the specific pattern of lesions caused by SMV occlusion. Its future application in the early diagnosis of mesenteric venous ischaemia is highly reasonable.

\section{B-655 11:06}

A specific rat model of acute gut ischaemia due to arterial occlusion: a $7 \mathrm{~T}$ micro-MR imaging study

F. Somma, D. Berritto, N. Landi, C. Cavaliere, S. Cappabianca, A. Rotondo,

R. Grassi; Naples/IT (daniela.berritto@libero.it)

Purpose: Mesenteric ischaemia is an uncommon but often underestimated cause of nontraumatic acute abdomen and its global prevalence is around $0.1 \%$ of all hospital recovers. Despite the great improvement in its diagnostic techniques, bowel infarction is still a fatal disease, with high mortality rate. Thanks to a simple animal model, intestinal ischaemia was induced occluding superior mesenteric artery (SMA) without laparotomy. The aim of the study was to identify MR imaging patterns of lesions due to SMA acute occlusion, avoiding pneumoperitoneum and ileus post surgery.
Methods and Materials: 13 Sprague Dawley rats were anesthetised and a loop (3-0 gut) was tied loosely around the SMA, without occluding the artery. 3 days later, each rat underwent micro-MR (Bruker Biospin) abdominal scans before squeezing the loop and after that at different timepoints ( 4 and $8 \mathrm{hrs}$ ). Then the entire bowe was removed and processed for histological analysis on HE-stained sections. Results: One rat was excluded from the analysis because it showed an incomplete occlusion in angio-MR sequences $8 \mathrm{hrs}$ after SMA ligation. Basal micro-MR scans showed no gas in the abdomen and no mesentery irritation. Rare-T2 micro-MR sequences identified several signs of vascular occlusion such as loops dilation, bowel wall thinning and peritoneal fluid. These changes were confirmed by ex-vivo histological examination.

Conclusion: This animal model could represent a reproducible tool to evaluate by imaging the evolution of ischaemic gut due to acute arterial occlusion and to test the effectiveness of new therapeutic modalities.

\section{B-656 11:15}

Conventional defecography versus MR defecography for diagnosing obstructive defecation syndrome: is patient exposure to radiation still justified?

H. Neubauer, B. Hartung, F. Wendel, T. Pabst, W. Kenn, D. Hahn; Würzburg/DE (gwendolinhartung@yahoo.de)

Purpose: High-resolution MR defecography (MRD) is established for imaging obstructive defecation syndrome. Referral to conventional defecography (CD) is still common, as radiographic images appear more easily interpretable to clinicians. We compared both modalities for feasibility and diagnostic value in clinical rountine. Methods and Materials: Seventy-two patients (68 females, mean age $53 \pm 13$ ) referred to conventional defecography (oral, vesical, vaginal and high rectosigmoidal contrast, temporal resolution $0.5 \mathrm{~s}$ ) also underwent MR defecography (1.5 T, highres TRUFI, three sagittal $8 \mathrm{~mm}$ slices, rectal contrast $200 \mathrm{ml}$ ultrasonography gel, temporal resolution 1.2s) with imaging at rest, retention, during evacuation and post-evacuation. All examinations were read by two radiologists in consensus. Results: Periprocedural problems include failure to evacuate (MRD $n=11, C D$ $n=2$ ), spontaneous loss of rectal contrast (MRD $n=7, C D n=14$ ) and insufficient opacification of the rectosigmoid junction $(C D n=22)$. Diagnostic accuracy for intussusception, enterocele, rectocele, cystocele and sigmoidocele was $85 \%, 97 \%$, $100 \%, 85 \%$ and $98 \%$ (MRD) and 97\%, 89\%, 92\%, 97\% and $88 \%$ (CD). Kappa values ranged $0.42 \sim 0.65$. Of 39 cases with intussusception, 9 were seen on CD and 2 on MRD only. Of 9 cases with sigmoidocele and 22 cases with enterocele, $56 \%$ and $22 \%$ were visible on MRD only. Mean dose area product for CD was $11.359 \pm 8292 \mathrm{cGy} / \mathrm{cm} 2$.

Conclusion: Conventional and MR defecography both show high diagnostic accuracy and are complementary to some extent. Conventional imaging allows better evaluation of intussusception. MR defecography is superior for diagnosis of sigmoidocele and enterocele and should be modality of choice in all eligible patients.

\section{B-657 11:24}

MR-defecography of pelvic floor disorders: static and dynamic evaluation with an open low-field tilting MR system

E. Squillaci, V. Fiaschetti, V. Funel, M. Rascioni, D. Pastorelli, G. Simonetti; Rome/IT (ettoresquillaci@tiscali.it)

Purpose: To assess the feasibility of MR-Defecography (MR-D) in pelvic floor disorders using an open tilting magnet with a $0.25 \mathrm{~T}$ static field and to compare results obtained in the same subject both in supine and orthostatic position.

Methods and Materials: 14 symptomatic female subjects with pelvic floor disorders underwent MR-D with a permanent open magnet with changeable position and static $0.25 \mathrm{~T}$ field (G-SCAN, Esaote, Italy). The magnet table was provided with a tilting mechanism going from $0^{\circ}$ to $90^{\circ}$ with two steps and allowed the evaluation both in supine and orthostatic position. Images acquired on the sagittal plane were used for quantitative analysis and were evaluated separately by two radiologists to establish inter-observer concordance. Conventional Defecography (CD) was performed in all patients.

Results: The comparison between $\mathrm{CD}$ and MR-D showed statistically significant differences in the evaluation of anterior rectocele during evacuation in both positions (inaccuracy of $0.43 \mathrm{~cm}$ for overestimation by MR-D in orthostatism and inaccuracy of $0.81 \mathrm{~cm}$ for underestimation by MR-D in the supine position) and of ano-rectal junction descent from pubo-coccygeal line (PCL) during evacuation, only in the supine position (with inaccuracy of $1.66 \mathrm{~cm}$ of underestimation by MR-D). The value of intra-observer intra-class correlation coefficient (ICC) ranged from good to excellent; the inter-observer ICC ranged from moderate to excellent.

Conclusion: MR-D is more accurate in orthostatic than in supine position for the evaluation of pelvic floor disorders. 


\author{
B-658 $11: 33$ \\ A statistical analysis of appropriateness of US and XR exams in \\ outpatients referred for acute care at our emergency department: database \\ review \\ F. Pinto, A. Pinto, A. Rizzo, L. Romano, E. Sarno; Naples/IT \\ (fpinto1966@libero.it)
}

Purpose: To study appropriateness of ultrasound (US) and x-ray (XR) exams performed in a group of patients referred at our emergency dept. for acute care in order to identify best predictors for inappropriateness.

Methods and Materials: The dataset consists of medical records from 461 consecutive outpatients obtained since an intranet-based outpatient system was introduced in August 2009. Each record has information on (1) the clinical history of patients at the time of the radiological exam; (2) the clinic notes provided by the ordering physician and the previous lab results; (3) Findings at US and XR exams and the subsequent clinic visit reports. Evidence-based appropriateness criteria were used to determine if the exams would have met criteria for approval. Statistical analysis was finally carried out.

Results: Of the 461 US exams reviewed, 174 (37.7\%) were considered as inappropriate and $287(62.3 \%)$ were considered appropriate. Of the 461 XR exams reviewed, 203 (44\%) were considered as inappropriate and $258(56 \%)$ were considered appropriate. More frequent examples of inappropriate exams included XR plain film for diarrhoea, abdominal US exam for hypertension, and renal US for haematuria during a urinary tract infection. Fifty-eight percent of the appropriate US studies had positive results and affected subsequent management, whereas only $13 \%$ of inappropriate studies had positive results and affected management. Best predictors of imaging inappropriateness were: self-referred patients and patients referred by general practitioners. Conclusion: There is a low utilisation of the appropriateness criteria by clinicians when ordering XR and US imaging studies in acute patients.

\section{B-659 11:42}

Body packages in low dose $\mathrm{CT}$ : identification of packages containing illicit drugs using low dose (LD) computed tomography in an experimental study using a porcine body packer model

M.K. Scherr, S. Wirth, E. Ziegeler, J. Grimm, M. Uhl, M.F. Reiser;

Munich/DE (michael.scherr@med.uni-muenchen.de)

Purpose: To evaluate, using a porcine model, LD CT strategies to examine suspected body packers.

Methods and Materials: Nine samples of typical illicit drug compounds packed in standardised ovoid plastic containers were rectally inserted in a pig cadaver. Using a 64-row CT (CT750HD, GE Healthcare), images were obtained at $120 \mathrm{kVp}$ and fixed tube currents of $80 \mathrm{~mA}$ (LD80), $30 \mathrm{~mA}$ (LD30), and $10 \mathrm{~mA}$ (LD10). Images were compared to standard dose abdominal CT (STD) and rated, first, for general diagnostic image quality in abdominal indications (general assessment score: 0 $=$ impossible, 1 = limited, $2=$ relevant diagnostics, $3=$ excellent). Second, with respect to body pack detection, visibility of three main characteristics (wrapping, content and shape) was rated (visibility: $0=$ definitely not, $1=$ presumably not, 2 $=$ presumably, $3=$ definitely) and summarised to a detection score (DS) ranging from 0 to 9 with a score $\geq 6$ representing sufficient detectability.

Results: Mean detection scores (DS) for the different LD-protocols were $8.2 \pm 0.7$ (LD80), $6.8 \pm 1.3$ (LD30), and $4.6 \pm 1.9$ (LD10), compared to $8.8 \pm 0.5$ (STD), with $\mathrm{p}<0.05$, respectively. Effective doses were calculated at $2.3 \mathrm{mSv}$ (LD80), $0.9 \mathrm{mSv}$ (LD30), $0.3 \mathrm{mSv}$ (LD10), and $9.4 \mathrm{mSv}$ (STD). For all LD-protocols, mean general assessment scores were inferior to STD: $1.4 \pm 0.7$ (LD80), $1.1 \pm 0.7$ (LD30), and $0.3 \pm 0.6$ (LD10) compared to $2.3 \pm 1.0$ (STD), with $p<0.001$, respectively. Conclusion: While general diagnostic image quality was limited for all LD protocols, LD80 provided an excellent level of body pack detectability at a tolerable effective dose while LD30 reached a still sufficient level of detectability at a dose comparable to $\mathrm{x}$-ray.

\section{B-660 11:51}

Cumulated radiation dose and risk of developing radiation-induced cancer in IBD patients treated with biological therapy

C. Larsen', A. Reitan'2, A. Rydning'2, H. Olerud ${ }^{3}$, A. Negård2; ; Oslo/NO,

${ }^{2}$ Lørenskog/NO, ${ }^{3}$ Østerås/NO (charlotte.kile.larsen@gmail.com)

Purpose: To estimate the cumulative radiation dose and the risk of developing radiation-induced cancer in patients with IBD (inflammatory bowel diseases) and biological medication.

Methods and Materials: From April 2008 to August 2009 patients with IBD and biological medication at our gastrointestinal outpatient clinic were included. Based on data from the patients' clinical records, the cumulative radiation doses (CRD) were calculated retrospectively. The effective doses for each exam were calculated according to the NRPB - W4 report "Radiation Exposure of the UK Population from Medical and Dental x-ray Examinations". The risk of developing cancer was estimated as described in the BEIR VII report. Student's T-test was used with a p-value $<0.05$ considered significant.

Results: 60 consecutive patients were included (male/female 27/33, Crohns disease/ulcerative colitis 42/18). Mean age was 28 years (youngest/oldest 11/61). The median CRD was $23.5 \mathrm{mSv}$ (highest CRD: $290 \mathrm{mSv}$, lowest was $0 \mathrm{mSv}$ ). Radiation dose from CT represented $74 \%$ of the total CRD. Patients with Crohns disease did not receive a significantly higher CRD than patients with ulcerative colitis $(p=$ 0.07). Female patients received a higher CRD compared to male patients (28.8 $\mathrm{mSv} / 13.4 \mathrm{mSv}, \mathrm{p}=0.025)$. However, the increased risk of developing cancer was relatively low $0.21 \%$.

Conclusion: Patients with IBD and biological treatment have already at an early age, received a high CRD after several radiological exams. Especially patients with Crohns disease and female patients receive a high CRD. Considering the chronic aspects of IBD, non-ionising radiology exams should be used if possible.

\begin{tabular}{ll}
\hline $10: 30-12: 00$ & Room F1 \\
\hline
\end{tabular}

\section{Genitourinary}

SS 1607

\section{Male lower urinary tract}

Moderators:

D. Beyersdorff; Berlin/DE

O. Nikolic; Novi Sad/RS

\section{B-661 10:30}

Diffusion-weighted MR imaging of normal and abnormal scrotum: preliminary results

A.C. Isili, M.I. Argyropoulou, D. Giannakis, S. Tsampalas, A. Silakos, N. Sofikitis, K. Tsampoulas; loannina/GR (a_tsili@yahoo.gr)

Purpose: To evaluate the diagnostic performance of DW MR imaging in the characterisation of scrotal lesions.

Methods and Materials: We retrospectively evaluated 31 scrotal lesions (23 intratesticular and 8 extratesticular) in 26 men. For comparison, 13 men with normal scrotal sonograms were also examined. All MR examinations were performed on a 1.5 T unit. DW sequences were obtained using a single shot, multi-slice spinecho planar diffusion pulse sequence and $a b$ factor of $900 \mathrm{~s} / \mathrm{mm}^{2}$. The DW MR characteristics and the $\mathrm{ADC}$ values of normal scrotal contents and scrotal diseases were evaluated. We compared the ADC values between normal scrotum, benign lesions and scrotal malignancies using one-way ANOVA analysis and independentsamples T test. The accuracy of conventional sequences and DW imaging in differentiating benign from malignant scrotal lesions was calculated, using the clinical or histopathologic diagnosis as the standard of reference.

Results: The mean and SD of ADC values $\left(\times 10^{-3} \mathrm{~mm}^{2} / \mathrm{sec}\right)$ were as follows: norma testis $1.11+0.18$, benign intratesticular lesions $1.56+0.85$, testicular carcinoma $0.85+0.62$, normal epididymis $1.39+0.14$, and benign paratesticular lesions $1.72+0.60$. The ADC values of testicular malignancies differed significantly from those of normal testis and benign intratesticular lesions, and the ADC calculations of benign extratesticular lesions from those of normal epididymis $(P<0.05)$. The overall accuracy of conventional imaging, DW imaging alone and DW MR combined with conventional sequences in the characterisation of intratesticular lesions was $91 \%, 87 \%$ and $100 \%$, respectively.

Conclusion: DW MR imaging and ADC values may provide valuable information for characterisation of scrotal diseases.

\section{B-662 10:39}

Realtime-sonoelastography for evaluation of testicular masses: a retrospective analysis

F. Aigner, T. De Zordo, D. Junker, G. Mikuz, G. Pinggera, H. Steiner, W. Jaschke, L. Pallwein, F. Frauscher; Innsbruck/AT (friedrich.aigner@uki.at)

Purpose: To assess the performance of realtime-sonoelastography (RTE), a method able to calculate tissue stiffness by ultrasound, for evaluation of testicular masses.

Methods and Materials: Twenty-seven testicular masses were investigated by RTE on a EUB-8500 Hitachi ultrasound unit (Hitachi-Medical-Systems, Tokyo) with a $8 \mathrm{MHz}$ linear probe to assess tissue elasticity of the testis. Elastograms were obtained by slight compression and decompression of the testis, which was 
done with the linear probe. Hard lesions (encoloured in blue) were considered suspicious for testicular cancer and histopathologic findings were used as gold standard. Lesions with normal or decreased tissue stiffness (encoloured in red to green) have been thought to be benign changes, i.e. focal orchitis or ischaemia, which can mimic testicular cancer on gray scale ultrasound. When benign lesions were suspected, follow-ups were performed to exclude progression of the mass. Results: Overall, 19 (70\%) hard lesions and $8(30 \%)$ soft lesions were detected. Seventeen hard lesions (89.5\%) histopathologically revealed testicular cancer. The 2 other hard lesions were thought to be scars and had no progression at follow-up. No soft lesion was found to be cancer (100\%). Because of clinical findings and findings on B-mode ultrasound soft tissue changes were thought to be cysts or inflammation and all showed no growth at follow-up.

Conclusion: Our preliminary results suggest the capability of RTE to distinguish between benign and malign testicular masses and may therefore refrain men scheduled for unnecessary surgery of the testis in future.

\section{B-663 10:48}

Voiding MR-cystourethrography: a new diagnostic imaging technique for the evaluation of male lower urinary tract

M. Di Girolamo, C. De Cecco, E. Pandolfi, P. Venneri, S. Galassi, V. David Rome/IT (digirolamomarco@hotmail.com)

Purpose: To evaluate the diagnostic accuracy of a new diagnostic imaging technique called voiding MR-cystourethrography that allows the visualisation of the male urethra

Methods and Materials: 10 normal volunteers and 86 male patients with bladder outlet obstruction (evaluated with urine-flow velocity recording) underwent voiding MR-cystourethrography performed with an $1.5 \mathrm{~T}$ magnet with the patient placed in supine position. The filling of the urinary bladder with paramagnetic contrast agent was obtainded by the i.v. administration $20 \mathrm{mg}$ of furosemide followed by the i.v. administration of $3 / 4$ of the normal dose of a paramagnetic contrast agent (Magnevist, Schering, Germany). When the bladder was filled with contrast-material-enhanced urine, the patient was asked to urinate. During the micturition T1-weighted spoiled $3 \mathrm{D}$ gradient-echo acquisitions on sagittal plane were performed. 25 patients performed conventional cystourethrography in the month preceding MRI.

Results: Homogeneous opacification of the bladder lumen was always obtained. 10 patients were unable to perform the MR examination. In all the volunteers and in all the patients studied (76 pts) we obtained a perfect evaluation of the male urethra with voiding MR-cystourethrography. The visualisation of the urethra with MIP reconstructed images was considered comparable to that obtained with conventional cystourethrography. We detected 24 cases of bladder neck obstruction, 36 cases of urethral stricture and 2 urethral papillomatosis. The site, length and the number of urethral strictures were accurately determined by MRI.

Conclusion: Voiding MR-cystourethrography demonstrates the morphology of the bladder neck and urethra during the micturition and can substitute standard retrograde and micturating cystourethrogram.

\section{B-664 10:57}

Radiological reporting errors identified in the prostate, bladder and penile cancer multidisciplinary team meeting - a prospective review to identify the patterns and severity of errors at second reporting: beware the common pitfalls

S.J. Kennish, J.T. Smith; Leeds/UK (s_kennish@yahoo.co.uk)

Purpose: The urology multidisciplinary meeting (MDT) offers an opportunity for second reporting of urological malignancy imaging. Errors can be detected, categorised and graded as to potential severity. The frequency, category and pattern of common errors are presented with case illustrations to help radiologists avoid common pitfalls and improve personal reporting quality.

Methods and Materials: Continuous prospective collection and analysis of errors identified during independent second review of urological malignancy imaging at multi-disciplinary meetings over a 2-year period. Cases identified by a single radiologist and confirmed by consensus. Errors were categorised into: false negative, satisfaction of search, interpretation error, typographical error and false positive findings. Severity of error was graded from 1 to 4 with 4 representing the highest potential clinical significance.

Results: Of 3347 MDT patients, 41 radiological errors were identified (1.2\%). The average delay to identification was 47 days. The majority of errors were false negative $(37 \%)$, interpretation $(29 \%)$ or satisfaction of search $(22 \%)$. Failing to identify significant lymph nodes (false negative) and missing significant foci of disease in the presence of disseminated malignancy (satisfaction of search) were amongst the commonest patterns. Mean severity of error was $3(2-4)$, a high potential for significant consequences.
Conclusion: The incidence of errors was low. Nevertheless common patterns of error were detected with potentially significant consequences. The MDT offers an opportunity for double reporting and increasing accuracy. Education to illustrate common pitfalls may help to reduce the number of future reporting errors for both urological and non-urological malignant disease.

\section{B-665 11:06}

The incremental value of contrast-enhanced MR imaging in biopsy-proven local recurrence after radical prostatectomy

C. Wassberg, O. Akin, A. Shukla-Dave, H. Vargas, J. Zhang, H. Hricak;

New York, NY/US (cecilia.wassberg@gmail.com)

Purpose: To assess the impact of contrast-enhanced magnetic resonance imaging (CE-MRI) on the accuracy of readers of different experience levels in the detection of post-surgical local recurrence of prostate cancer by MRI, using biopsy as a standard of reference.

Methods and Materials: The Institutional Review Board approved and waived the informed consent requirement for this HIPAA-compliant study of 52 patients who, between March 2005 and December 2008, underwent 1.5-Tesla-MRI with multiphase post-contrast endorectal MRI following post-surgical biochemical recurrence of prostate cancer and had biopsy within 3 months of MRI. Two radiologists (reader 1 , with one year and reader 2 , with six years of experience) retrospectively and independently reviewed each MRI study and recorded their level of suspicion for recurrent cancer on a five-point scale. Areas under receiver operating characteristic curves $(A Z)$ were calculated to assess diagnostic performance of T2W imaging with and without CE-MRI. Inter-reader agreement was assessed using kappa statistics. Results: Thirty-three patients (63\%) had prostate cancer recurrence on biopsy. With the addition of CE-MRI, the AZ for cancer detection increased significantly for reader 1 ( 0.77 vs. $0.85 ; P=0.04$ ) but not for reader 2 (0.86 vs. $0.88 ; P=0.72$ ). Use of CE-MRI increased inter-reader agreement from fair $(\mathrm{K}=0.39)$ to moderate $(K=0.58)$; it also allowed correct reclassification and greater certainty in image interpretation in $13 / 52$ patients $(25 \%)$ for reader 1 and 6 patients $(12 \%)$ for reader 2 . Conclusion: Adding CE-MRI to T2W endorectal MRI can improve post-operative detection of locally recurrent prostate cancer, especially by relatively inexperienced readers, while reducing interobserver variability.

\section{B-666 11:15}

Role of 1H-MRS and DCE-MR in identification of pattern changes after neoadjuvant hormonal therapy

F. Galati, V. Panebianco, A. Sciarra, V. Buonocore, D. Lisi, R. Passariello; Rome/IT

Purpose: To determine the time-dependent metabolic and angiogenic changes that occur in PC during neoadjuvant HT using multiparametric MR.

Methods and Materials: Seventy-five patients were submitted combined endorectal MRI with MRSI and DCE-MRI using 3-T magnet (Verio, Siemens, Erlangen, Germany). Scan protocol included morphologic imaging with TSE T2-weighted sequences on three planes, spectroscopic imaging with 3D CSI and 3D FLASH $\mathrm{T} 1$-weigthed sequence. All patients underwent MRI examination before therapy, at 4,12 and 24 weeks from therapy. The time course of loss of prostate metabolites was delayed in PCa tissue as compared to healthy tissue.

Results: We noticed that at 4 weeks from therapy there was a citrate reduction with an apparent increase in choline peak in $90 \%$ of patients; during the last 24 weeks there was a significant time-dependent loss of the prostatic metabolites (citrate level decreased faster than creatine and choline levels, resulting in an increase in ratio value) till the complete loss of all metabolites (metabolic atrophy) in $30 \%$ of patients on long-term therapy. In patients with metabolic atrophy we observed neoangiogenic pattern reduction at 6 months.

Conclusion: Metabolic and angiogenic changes during a neoadjuvant HT may provide important prognostic informations and may help to define the optimal time to start the definitive therapy in each patient.

\section{B-667 11:24}

Assessment of early response to external beam radiation therapy for prostate cancer: a prospective study with $3 \mathrm{~T}$ diffusion-weighted imaging J. Seo, C. Kim, S. Park, B. Park, W. Park; Seoul/KR (noir1130.seo@samsung.com)

Purpose: To investigate the changes of apparent diffusion coefficient (ADC) in prostate cancers before, during and after external beam radiation therapy (EBRT), and to assess the reproducibility of ADC measurement in the prostate.

Methods and Materials: Eight consecutive patients with biopsy-proven prostate cancer were examined by T2-weighted, dynamic contrast-enhanced and diffusionweighted imaging (DWI) at $3 \mathrm{~T}\left[\mathrm{~b}\right.$-values, 0 and $1000 \mathrm{~s} / \mathrm{mm}^{2}$. All patients treated 
with EBRT underwent four serial MR examinations [i.e. prior to therapy (preTx), at the first week of therapy (postT1), at the third week of therapy (postT2) and 1 month after the completion of therapy (postT3)]. At each time, ADC was calculated in both tumours and normal tissues. For the reproducibility of the ADC measurement, five patients also underwent two separate pre-treatment DWI examinations with less than 2 weeks' interval.

Results: 13 tumours [peripheral zone $(P Z)=10$; central gland $(C G)=3$ ] were found in 8 patients. For the tumour, mean ADC was 0.86 at preTx, 1.03 at postT1, 1.15 at postT2 and $1.26 \times 10^{-3} / \mathrm{mm}^{2} / \mathrm{s}$ at postT3, respectively $(\mathrm{P}=0.001)$. However, mean $\mathrm{ADC}$ of benign $\mathrm{PZ}$ and $\mathrm{CG}$ had decreased consecutively before, during and after therapy. Between two separate pre-treatment DWI examinations, mean difference in ADC value of normal prostate tissues to reflect the reproducibility was $1.3 \%$. Conclusion: Our preliminary results suggest that DWI in prostate cancer may detect tumour response as early as one week following the start of therapy and moreover, the reproducibility of ADC measurements was demonstrated.

\section{B-669 11:33 \\ Prognostic implications of transrectal ultrasonographic appearance of prostate cancer \\ V.C.N. Junqueira' ${ }^{1}$, O. Zogbi-Neto ${ }^{1}$, J. Elias-Jr' ${ }^{1}$, A.J. Cologna' ${ }^{1}$, S. Tucci Jr ${ }^{1}$, R.B. Reis ${ }^{1}$, A.C.A. Westphalen ${ }^{2}$, V.F. Muglia ${ }^{1} ;{ }^{1}$ Ribeirão Prêto/BR, \\ ${ }^{2}$ San Francisco, CA/US (fmuglia@fmrp.usp.br)}

Purpose: The aim of this study was to evaluate the prognostic implications of transrectal ultrasonographic appearance of prostate cancers.

Methods and Materials: We selected, retrospectively, all patients with biopsy proven prostate cancer between January 2003 and July $2004(n=152)$. Patients who had, at least, 5 years of follow-up were enrolled in this study. After exclusions 101 constitute our study population. Patients were divided into two groups, according to sonographic appearance of the lesion at the time of biopsy, in isoechoic or non-visible and hypoechoic or visible lesions and the clinical outcome of these two groups was compared.

Results: Forty-one (40.6\%) patients had non-visible and $60(59.4 \%)$ had visible lesions. There was significant difference for the percentage of positive fragments in biopsy $(p<0.01)$, mean PSA value $(p=0.02)$, clinical staging and for Gleason Score, $(p=0.05)$. The outcomes for the two groups were significant different $(p<0.01)$. For non-visible lesions, 37 of 41 patients $(90.2 \%)$ remained free of disease, 2 had biochemical failure $(4.9 \%)$ and 2 died of non-related causes $(4.9 \%)$ For the visible lesions, 37 of $60(61.6 \%)$ patients were disease-free, $7(11.7 \%)$ had systemic metastases, $10(16.7 \%)$ died of complications related to prostate cancer and $6(10 \%)$ had non-related death. Kaplan-Meyer analysis showed significant difference for the risk of developing systemic metastases $(p=0.01)$ and death $(p$ $=0.01)$, but not for biochemical failure $(p=0.83)$.

Conclusion: Our data show that patients with non-visible prostate cancer have significant better outcome than patients with visible lesions on 5-year period of evaluation.

\section{B-670 11:42}

Contrast-enhanced US-guided biopsies following MRI in men with elevated PSA and previous negative biopsy

G. Rigou, Y. Le Bras, R. Hubrecht, X. Coutouly, G. Pasticier, N. Grenier;

Bordeaux/FR (geraldrigou@hotmail.fr)

Purpose: A first set of US-guided biopsy does not exclude prostate cancer in men with elevated PSA. Repeated biopsy detection rate is only 10 to $30 \%$ and increases risks of complications. Localisation of suspected targets on MRI with subsequent sonography may be difficult. Therefore, we set out to investigate how contrast-enhanced US-guided biopsies increase prostate cancer detection rate after MRI localisation.

Methods and Materials: 59 patients (mean age $63 \mathrm{y}$ ) with elevated PSA value (mean $=12.9 \mathrm{ng} / \mathrm{ml})$ and prior negative biopsy (1-5, mean 2.3) were included in a prospective study. They underwent prostate MRI examination: T1w, T2w, diffusionweighted and dynamic contrast-enhanced sequences. One examiner performed a first set of targeted biopsies on suspected lesion on MRI and identified on contrastenhanced US and, subsequently, a set of random biopsies. Diagnostic efficiency of each biopsy strategy was evaluated based on pathological results.

Results: $51 / 724$ non-targeted biopsies $(6.6 \%)$ and $39 / 83$ targeted biopsies (32.9\%) were positive for cancer. Mean cancer detection rate was $35.6 \%$ (21/59 patients), reaching $47.4 \%$ for the second set of biopsy (9/19 patients). 9/21 patients with cancer were identified by targeted biopsy only $(42.9 \%)$ versus $4 / 21$ identified by systematic biopsy only (19\%) and $8 / 21$ by both $(38.1 \%)$

Conclusion: In men with elevated PSA and prior prostate negative biopsy, detection rate of contrast-enhanced US-targeted biopsy is superior to repeated systematic biopsy after MRI, with a decreased number of samples.
$10: 30-12: 00$

Room F2

Breast

\section{SS 1602}

\section{Biomarkers in breast MRI}

Moderators:

S.H. Heywang-Köbrunner; Munich/DE

C.C. Riedl; Vienna/AT

\section{B-671 10:30}

Multicenter, double-blind, randomised, intraindividual crossover comparison of gadobenate dimeglumine and gadopentetate dimeglumine for MR imaging of the breast (DETECT)

L. Martincich ${ }^{1}$, M. Faivre-Pierret ${ }^{2}$, C.M. Zechmann ${ }^{3}$, S. Corcione ${ }^{4}$,

H.C. van den Bosch ${ }^{5}$, W.-J. Peng ${ }^{6}$, A. Petrillo7 , K. Siegmann ${ }^{8}$, J.T. Heverhagen ${ }^{9}$;

${ }^{1}$ Candiolo/IT, ${ }^{2}$ Lille/FR, ${ }^{3}$ Heidelberg/DE, ${ }^{4}$ Ferrara/IT, ${ }^{5}$ Eindhoven/NL,

${ }^{6}$ Shangai/CN, ${ }^{7}$ Napoli/IT, ${ }^{8}$ Tübingen/DE, ${ }^{9}$ Marburg/DE (laura.martincich@ircc.it)

Purpose: To intra-individually compare $0.1 \mathrm{mmol} / \mathrm{kg}$ doses of gadobenate dimeglumine and gadopentetate dimeglumine for contrast-enhanced breast MRI using a prospective, multicenter double-blinded, randomised protocol.

Methods and Materials: Institutional review board approval and patient informed consent was obtained. One hundred sixty-two women ( $52.8 \pm 12.3$ years) enrolled at 17 sites in Europe and China between 07/07 and 05/09 and underwent at least one breast MRI exam at 1.5 T using 3D spoiled GRE sequences. Of these, 151 women received both agents in randomised order in otherwise identical exams separated by $>2$ but $<7$ days. Images, acquired at $\leq 2$ min intervals after contrast injection, were evaluated independently by three blinded radiologists unaffiliated with enrolment centers. Histopathological confirmation was available for all malignant lesions ( $n=144)$, while benign lesions were confirmed either by histopathology $(n=52)$ or by 12 -month diagnostic follow-up $(n=20)$ with mammography and/or ultrasound. Determinations of malignant lesion detection rates and diagnostic performance (sensitivity, specificity, accuracy, positive and negative predictive values [PPV and NPV]) were performed and compared (McNemar and Wald tests). A full safety assessment was performed.

Results: Significant superiority for gadobenate dimeglumine was noted by each reader for malignant lesion detection $(91.7-94.4 \%$ vs. $79.9-83.3 \%$; $\leq \leq 0.0003)$. Each reader reported significantly superior sensitivity, specificity and accuracy for breast cancer detection with gadobenate dimeglumine $(91.1-95.2 \%$ vs. $81.2-84.6 \%$; 96.9 $99.0 \%$ vs. $93.8-97.8 \%$; $96.7-98.2 \%$ vs. $92.8-96.1 \% ; p \leq 0.0094)$ and significantly superior PPV (77.2-91.1\% vs. $60.9-80.7 \% ; p \leq 0.0002)$ and NPV $(99.0-99.4 \%$ vs. $97.8-98.1 \% ; p \leq 0.0003)$. No safety concerns were noted with either agent. Conclusion: Gadobenate dimeglumine is superior to gadopentetate dimeglumine for breast cancer diagnosis.

\section{B-672 10:39}

Advances in breast cancer detection and diagnosis using diffusion tensor imaging (DTI)

S. Feinberg Shapiro' ${ }^{1}$ E. Eyal ${ }^{2}$, E. Furman-Haran², D. Grobgeld², T. Golan ${ }^{3}$, Y. Itzchak ${ }^{3}$, R. Catane ${ }^{3}$, M. Papa ${ }^{3}$, H. Degani ${ }^{2} ;{ }^{1} \mathrm{Kvar}$ Saba/IL, ${ }^{2}$ Rehovot/IL,

${ }^{3} \mathrm{Tel}$ Hashomer/IL (afeinber@netvision.net.il)

Purpose: To determine the water diffusion tensor and track the mammary ductal trees in order to identify breast malignancy without the need to inject a contrast agent. Methods and Materials: Recruitment: 21 Healthy volunteers, 40 patients (33 cancers, 20 benign lesions) were scanned at $3 \mathrm{~T}$ using a PGSE-EPI-based diffusion protocol, TE $/ \mathrm{TR}=0.12 / 10.4 \mathrm{~s} ; \mathrm{b}$-values $=0$ and $700 \mathrm{~mm}^{2} / \mathrm{s} ; 30$ or 64 gradient directions; resolution $1.9 \times 1.9 \times 2 \mathrm{~mm}^{3}$ followed by DCE-MRI. Pixel-by-pixel analysis yielded parametric diffusion tensor maps and parametric DCE maps. Diagnosis was based on histopathology findings.

Results: The diffusion coefficients (L1, L2, L3) in three orthogonal directions were high in the normal fibroglandular tissue, close to free water values. The high fractional anisotropy and alignment of the prime diffusion direction enabled tracking the ductal trees and separating them from fibrous tissue. Cancers exhibited significantly lower L1 diffusion coefficients than normal tissue, yielding a $96 \%$ sensitivity $/ 96 \%$ specificity for differentiating cancers from normal tissue. L1 values in cancers were also significantly different from those in benign lesions yielding $92 \%$ sensitivity $/ 92 \%$ specificity for differentiating malignancy from benignancy. The average diffusion coefficient $[(\mathrm{L} 1+\mathrm{L} 2+\mathrm{L} 3) / 3]$ provided a similar performance as L1 but with a slightly lower specificity for differentiating cancers from normal tissue. Cancers also exhibited significantly lower values of anisotropy (L1-L3) than norma tissue with a $91 \%$ sensitivity $/ 72 \%$ specificity. 
Conclusion: Breast DTI is a completely non-invasive, fast (approx. 5 minutes) method that demonstrates a high potential for cancer detection and diagnosis as a stand alone method or in conjunction with DCE-MRI.

\section{B-673 10:48}

Diffusion-weighted magnetic resonance imaging (DWI) of the breast: high-resolution sagittally-oriented versus conventional axial echo-planar sequences

C. Losio, P. Panizza, A. Salerno, G. Cristel, I. Fedele, V. De lorgi, F. De Cobelli, A. Del Maschio; Milan/IT (losio.claudio@hsr.it)

Purpose: DWI is known to improve the specificity of dynamic breast MRI. However echo-planar sequences, usually acquired in the axial plane, suffer from low spatia resolution and artefacts that may significantly degrade image quality. Our purpose was to set-up a high-resolution sagittally-oriented DWI sequence and to test its performance during clinical breast MRI compared to conventional axial imaging. Methods and Materials: 84 women underwent breast DWI (b-value: 900) in the axial plane (both breasts acquired together) and sagittal plane (each breast acquired separately), followed by dynamic breast MRI. Standard post-processing of dynamic study was completed with qualitative and quantitative assessment of water diffusivity (ADC maps). ADC values were calculated for all enhancing lesions $>5 \mathrm{~mm}$ and compared for the two sequences. DW Images were also analysed in terms of lesion conspicuity and image quality. Diagnostic performance was assessed for both sequences, using cyto-histology or follow-up as reference standard. Results: DWI identified 44/52 (axial) and 48/52 (sagittal) enhancing lesions (range: $5-38 \mathrm{~mm}$ ). ADC values were significantly lower in malignant than benign lesions $(0.98 \pm 0.28$ vs $1.76 \pm 0.29 ; p<0.001)$, with no differences between the two sequences. High-resolution sagittal sequences better depicted small enhancing foci, with less artefacts and more accurate determination of ADC; however, the conspicuity of benign lesions was inferior compared to axial DWI.

Conclusion: DWI with high-resolution sagittally-oriented sequences is superior to axial one in terms of image quality, reducing distorsions and artefacts particularly in the right breast, with benefits in both qualitative and quantitative assessment of malignant lesions, and without significant increase of examination time.

\section{B-674 10:57}

Correlation of apparent diffusion coefficient (ADC) value of invasive ductal carcinoma on diffusion-weighted MR imaging with pathologic prognostic factors

S. Park, H. Choi, S. Hahn, H. Kim, H. Jung; Incheon/KR

(shpark@gilhospital.com)

Purpose: To measure the apparent diffusion coefficient (ADC) values of invasive ductal carcinoma (IDC) of the breast and to analyse correlations between ADC values and prognostic factors including tumour size, axillary lymph node status, histologic grade, estrogen receptor (ER), progesterone receptor (PR), and c-erb-2 (HER2) using diffusion-weighted MR imaging.

Methods and Materials: A total of 110 patients (mean \pm SD: $48.1 \pm 9.2 \mathrm{yrs}$ ) with 110 pathologically confirmed IDC were examined on 3.0 T MRI with diffusion weighted MR imaging. The ADC values of breast cancer were calculated using two $\mathrm{b}$ factors $\left(0\right.$ and $\left.1000 \mathrm{~s} / \mathrm{mm}^{2}\right)$. The mean and standard deviation (SD) of the $A D C$ value for each prognostic factor (size, grade, lymph node metastasis) and hormonal receptor (ER, PR, HER2) was calculated using independent sampled Student-t test. The correlations between ADC values and prognostic factors were analysed and assessed by one-way ANOVA test and Spearman's correlation test. Results: The mean ADC value of IDC $(n=110)$ was $0.88 \pm 0.15 \times 10^{-3} \mathrm{~mm}^{2} / \mathrm{s}$. The mean ADC of HER2-positive IDC $(n=36)$ was significantly higher than that of HER2-negative IDC $(n=74)\left(0.92 \pm 0.13 \mathrm{~mm}^{2} / \mathrm{s}\right.$ vs. $\left.0.86 \pm 0.16 \mathrm{~mm}^{2} / \mathrm{s}\right)(\mathrm{p}=0.02)$. On the other hand, ER status $(p=0.16)$, PR status $(p=0.57)$, histologic grade $(p=0.12)$, axillary lymph node status ( $p=0.27$ ) showed no statistically significant correlation with ADC values. Conclusion: HER2-positive IDC showed higher ADC than HER2-negative IDC, respectively, ER status, PR status, histologic grade and axillary lymph node status showed no statistically significant correlation with ADC.

\section{B-675 11:06}

Correlation between apparent diffusion coefficient and molecular and histological prognostic factors in breast cancer: initial observations in 53 patients

G. Petralia, L. Bonello, P. Summers, S. Raimondi, M. Pasin, R. Di Filippi,

G. Curigliano, L. Preda, M. Bellomi; Milan/IT (giuseppe.petralia@ieo.it)

Purpose: To correlate apparent diffusion coefficient (ADC) with molecular and histological prognostic factors for breast cancer (BCa).
Methods and Materials: Fifty-three female patients (mean age 48.1, range 28-81 years) with histologically proven $\mathrm{BCa}$ underwent conventional MRI (including T2weighted and T1 dynamic imaging post-contrast) and diffusion-weighted (DW) MRI (b-values 0, 250, 500 and $1000 \mathrm{~s} / \mathrm{mm}^{2}$ ). The ADC for the BCa tumour volume, as defined manually on the highest b-value images, was correlated with molecular and histological prognostic factors. ADC values were assessed for difference between gene profiling subtypes (Luminal A, Luminal B, HER-2, triple receptor negative), vascular invasion (present/absent), grading (G1/G2/G3), ER/PgR/HER-2 expression (positive/negative), Ki67 ( $<14 \%$ or $>14 \%$ ) and TNM staging as factors using analysis of variance (ANOVA). Spearman coefficient was used to assess correlation between $A D C$ values and continuous variables (age, percentage of ER, PgR, HER-2, Ki-67). Results: The mean $A D C$ of $B C$ a lesions visible on the high b-value image was $1.14 \pm 0.20 \times 10^{-3} \mathrm{~mm}^{2} / \mathrm{sec}$. The ADC of HER-2 was lower than other subtypes, reaching significance when compared with triple receptor negative $(p=0.055)$. The ADC for the small T3 subgroup $(n=8)$ was lower than other T grades, but only significantly so relative to $T 1(p=0.03)$. There was a marginally significant correlation (Spearman $r=-0.35, P<0.05$ ) between ADC and HER-2 expression. Conclusion: Our results showed little support for a correlation between ADC and the molecular and histological prognostic factors examined. A possible exception is HER-2 expression. The T3 subgroup may also bear a distinct ADC behaviour.

\section{B-676 11:15}

Diffusion-weighted imaging of breast cancer at $3 \mathrm{~T}$ : does the apparent diffusion coefficient value correlate with prognostic factors

M. Telesca, F. Pediconi, V. Casali, M. Luciani, F. Vasselli, E. Miglio, C. Catalano, R. Passariello; Rome/IT (mariannatelesca@yahoo.it)

Purpose: To evaluate the correlation of the apparent diffusion coefficient (ADC) value with prognostic factors in patients with biopsy-diagnosed breast cancer. Methods and Materials: A prospective study was conducted in 45 patients (mean age 46 years) with biopsy-proven malignant breast lesions measuring $\geq 6 \mathrm{~mm}$. Al patients were examined at 3 Tesla using dedicated bilateral breast coil. Diffusion weighted imaging (DWI) of the breast was performed using a single shot echo planar imaging with $a b$-factor of 0 and $800 \mathrm{sec} / \mathrm{mm}^{2}$. ADC maps were reconstructed and findings were compared with findings from histology. Lesion size, histotype, receptor status (ER, PgR) and Ki67 expression were determined.

Results: Breast cancer was detected in 41 patients $(91.1 \%)$. The mean ADC value was $1.41 \pm 0.23 \times 10^{-5} \mathrm{~mm}^{2} / \mathrm{s}$. There were no correlations between the ADC value and prognostic factors. In particular, there was no correlation between ADC and ER $(p=0.5)$ or between ADC and PRG $(p=0.6)$. A small correlation was noted with Ki67 expression, but it was not statistically significant.

Conclusion: ADC values are potentially an additional tool to predict tumour malignancy, although no statistically significant correlation with prognosis factors was found in this study.

\section{B-677 11:24}

3.0 Tesla diffusion tensor magnetic resonance imaging of the breast: preliminary results

A. Tagliafico, G. Rescinito, F. Monetti, C. Faedda, M. Calabrese, F. Chiesa, A. Villa; Genoa/IT (chiesa.fabio@gmail.com)

Purpose: To evaluate whether 3.0-T diffusion tensor imaging (DTI) allows differentiation between breast tumours and normal breast tissue.

Methods and Materials: The study was IRB approved and written informed consent was obtained. The study included 54 women with 65 breast lesions (56 malignant, 9 benign). DTI was performed during 3.0-T breast MRI examinations, and fractiona anisotropy (FA) and apparent diffusion coefficient (ADC) values were measured for breast lesions and normal tissue in each subject. FA and ADC were compared between cancers, benign lesions, and normal tissue by univariate and multivariate analyses. Results: FA of carcinomas (mean \pm SD: $0.32 \pm 0.09$ ) was significantly lower than normal breast tissue in the same subjects $(0.42 \pm 0.12 ; \mathrm{P}<0.001)$. Multiple logistic regression showed that FA and ADC were each independent discriminators of malignancy $(P<0.001)$. FA improved discrimination between cancer and normal tissue over ADC alone. There was no difference in FA between malignan and benign lesions.

Conclusion: Diffusion anisotropy is significantly lower in breast cancers than normal tissue, which may reflect alterations in tissue organisation. Our preliminary results suggest that FA adds incremental value over ADC alone for discriminating malignant from normal tissue but does not help with distinguishing benign from malignant lesions. 


\author{
B-678 11:33 \\ To compare MR spectroscopy at $3 \mathrm{~T}$ with tumour type and grading of \\ breast cancers \\ F. Pediconi, M. Telesca, M. Luciani, F. Vasselli, V. Casali, E. Miglio, C. Catalano, \\ R. Passariello; Rome/lT (federica.pediconi@uniroma1.it)
}

Purpose: To evaluate the diagnostic performance of magnetic resonance (MR) spectroscopy at $3 \mathrm{~T}$ to detect different cancer types and prognostic factors in patients with biopsy-proven breast cancer.

Methods and Materials: Breast MR spectroscopy was performed at $3 \mathrm{~T}$ in patients with biopsy-proven malignant lesions measuring $6 \mathrm{~mm}$ or larger at MR imaging. Single-voxel MR spectroscopy data were collected from a single rectangular volume of interest that encompassed the lesion. MR spectroscopy findings were defined as positive if the signal-to-noise ratio of the choline resonance peak was greate than or equal to 2 and as negative in all other cases. MR spectroscopy findings were then compared with histologic findings. Lesion size, histotype, nuclear grade, receptor status (ER, PgR), and Ki67 and HER2 expression were evaluated. Results: A total of 17 patients with 21 lesions were evaluated. Histologic analysis confirmed 15 IDC, 5 ILC and 1 DCIS. The median lesion size at MR imaging was $1.3 \mathrm{~cm}$ (range $0.6-7 \mathrm{~cm}$ ). A choline peak was presented in 13 of 21 lesions (12 IDC and 1 ILC). Three IDC with no choline peak presented with large central area of necrosis. Thus, a choline peak was more frequently associated with IDC than with ILC, DCIS and necrosis. No statistically significant association of choline peak with receptor status (ER, PgR), Ki67 and HER2 was found, although the choline peak was always present in triple negative patients.

Conclusion: Proton MR spectroscopy at $3 \mathrm{~T}$ was successfully incorporated into breast MR imaging studies for lesions measuring $6 \mathrm{~mm}$ or larger. MR spectroscopy could be an additional tool to predict tumour aggressiveness. Results need to be validated in larger-scale studies.

\section{B-679 11:42}

Noninvasive risk stratification of breast cancer: can magnetic resonance spectroscopy of index lesions predict lymph node status of primary breast cancer?

P.A.T. Baltzer ${ }^{1}$, J. Schelhorn ${ }^{2}$, M. Benndorf ${ }^{1}$, M. Dietzel ${ }^{1}$, W.A. Kaiser ${ }^{1} ;{ }^{1}$ Jena/DE, ${ }^{2}$ Weimar/DE (pascal.baltzer@med.uni-jena.de)

Purpose: In vivo proton magnetic resonance spectroscopy (MRS) enables the radiologist to noninvasively assess tumour metabolism in vivo. Based on the assumption that breast cancer is a heterogeneous disease with different levels of biological aggressiveness, such insights in molecular information may be used for clinical risk stratification. Spectroscopy of fine needle aspirates of breast cancer has been shown to predict lymph node status with high accuracy. We wondered, whether this result is reproducible by in vivo MRS of breast cancer.

Methods and Materials: 67 consecutive patients (median age $59 \mathrm{y}$ ) with breast cancer were investigated using single voxel MRS (1.5 T, PRESS, TR 2000 ms, TE $270 \mathrm{~ms}$ ). Standard of reference for nodal status was sentinel lymph node biopsy or lymph node dissection. The AMARES algorithm implemented in the MRUI software package v4 (http://www.mrui.uab.es/) was used for spectra quantification. Choline concentration was calculated using the water resonance of unsuppressed spectra as internal reference. Choline concentration tested against lymph node status using receiver operating characteristics $(\mathrm{ROC})$ analysis.

Results: 20 out of $67(29.9 \%)$ patients presented with lymph node metastases. $\mathrm{ROC}$ analysis revealed significant potential (area under the curve: $0.667, \mathrm{P}<0.031$ ) to discriminate positive from negative lymph node status. At a cut-off value of $5.32 \mathrm{mmol}$ choline $/ \mathrm{kg}$, high sensitivity $(95.0 \%)$ and negative predictive value (94.1\%) were achieved.

Conclusion: According to the presented results, quantitative MRS allows for noninvasive prediction of lymph node status with high sensitivity and negative predictive value. Future investigations should focus on the prognostic value and clinical applicability of this finding.

\section{B-680 11:51}

Diffusion weighted imaging of the breast: apparent diffusion coefficient (ADC) reflects grading of invasive breast cancer

P.A.T. Baltzer, J. Halder, M. Dietzel, W.A. Kaiser; Jena/DE

(pascal.baltzer@med.uni-jena.de)

Purpose: Diffusion weighted imaging (DWI) is increasingly recognised as a powerful quantitative tool for breast lesion differentiation. Changes of the extracellular space, i.e. increased cellularity in malignant lesions, leads to characteristic restriction of water diffusion. Beyond differential diagnosis, this method may also be applied to the assessment of breast cancer aggressiveness. Higher grading is associated with increased proliferation rate and, thus, cellularity. This investigation was performed to clarify whether there is an association between breast cancer grading and apparent diffusion coefficient (ADC) values obtained by DWI.

Methods and Materials: 120 consecutive patients (median age $61 \mathrm{y}$ ) with breast cancer were investigated using DWI (1.5 T, EPI, TR/TE 3500/80 ms, b-values: $1000 / 750 / 0 \mathrm{~s} / \mathrm{mm}^{2}$ ). ADC values were obtained by two blinded observers in consensus and compared with tumour grading ( $1=$ low, 2 = intermediate, $3=$ high grading) Data analysis included summary statistics and analysis of variance (ANOVA). Results: Of 120 cancers, 9 were pathologically classified as G1, 36 as G2 and 75 as G3. ADC showed a negative correlation with tumour grading (mean ADC G1: $1.28, G 2: 1.20$, G3: $1.06 \mathrm{~mm}^{2} / \mathrm{s}, \mathrm{R}=-0.222, \mathrm{P}=0.015$, ANOVA: $\mathrm{P}=0.049$ ). Conclusion: Microstructural changes associated with different degrees of breast cancer aggressiveness seem to be reflected by ADC values obtained by $\mathrm{DWI}$. Further study should clarify, whether noninvasive risk classification of breast cancer is possible by a combination of quantitative MRI methods.

10:30 - 12:00 Room G/H

Vascular

\section{SS 1615}

\section{Special applications, functional imaging}

Moderators:

W.P.T.M. Mali; Utrecht/NL

M. Sadick; Mannheim/DE

\section{B-681 10:30}

MR-lymphangiography for visualisation of dermal backflow in patients with lymphoedema of the lower extremity

M. Notohamiprodjo, K.A. Herrmann, M. Weiss, R.G. Baumeister;

Munich/DE (mike.notohamiprodjo@med.uni-muenchen.de)

Purpose: Dermal backflow is associated with obstruction of the lymph collecting vessels. The aim of this study was to evaluate MR-lymphangiography for visualisation of dermal backflow in patients with lymphoedema of the lower extremity. Methods and Materials: 30 consecutive patients with lymphoedema of the lower extremity underwent MR-lymphangiography on a 3.0 T-scanner (Magnetom VERIO, Siemens) with a highly-resolved isotropic T1-weighted FLASH3D-sequence after intracutaneous injection of Gd-DTPA. Four levels were examined: lower leg, knee, upper leg and pelvis. One radiologist and one nuclear-physician evaluated depiction of lymph-collectors, diffusity of drainage and number of visualised levels in consensus. All examinations were correlated with corresponding lymphoscintigraphyexaminations performed with colloid-bound $99 \mathrm{Tcm}$. Correlation of the two methods was assessed with weighted-kappa-coefficients.

Results: All examinations were diagnostic and MR-lymphangiography and lymphoscintigraphy showed excellent correlation $(r=0.84)$. Normal and instant lymphatic drainage in unaffected extremities was observed in both modalities shortly after contrast application. Depiction of delayed drainage was concordan in both modalities. In 12 patients dermal backflow in terms of complete or partial diffuse lymphatic drainage was detected. Five of these patients showed localised dermal backflow with directed lymphatic drainage of the levels below. Compared to lymphoscintigraphy contrast media leakage was more clearly depicted in MRlymphangiography. The three-dimensional reconstruction capabilities proved beneficial for exact anatomical localisation of the lymph vessel obstruction.

Conclusion: MR-lymphangiography and lymphoscintigraphy show excellent correlation. MR-lymphangiography allows for a more accurate anatomical localisation of dermal backflow in patients with lymphoedema of the lower extremity and is a valuable tool for planning of microsurgical therapy.

\section{B-682 10:39}

Ultrasonographic appearance of varices within groin lymph nodes in patients with and without surgically treated varicose disease: the relation to lower limb oedema

S.M. Dudea, C. Ciuce, D. Dumitriu, C. Botar-Jid, A. Chiorean, A. Ciurea; Cluj-Napoca/RO (dudea@clicknet.ro)

Purpose: To assess the incidence of lymph node varices and their relation to lower limb oedema in patients with and without surgically treated varicose disease. Methods and Materials: $8-12 \mathrm{MHz}$ ultrasound of the groin was performed, over 48 months, in 152 patients with former operation of lower limb varices and in 112 patients with untreated lower limb varices. Intranodal varices when present were graded as: grade 1 - node sinus still visible; grade 2 - no node sinus visible. Pres- 
ence and grade of nodal varices was plotted against the presence of limb edema. Results: incidence of oedema was $35.5 \%$ in the operated group and $30.4 \%$ in the untreated one $(p=n s)$. The incidence of lymph node varices, oedema and no oedema, was $64.8 \%$ and $12.2 \%$ in the operated group $(p<0.01)$ and, respectively, $35.3 \%$ and $11.5 \%(p<0.05)$ in the untreated group. In the treated group, two patients without oedema presented grade 2 dilatation; this was encountered in 10 patients $(28.5 \%)$ in the oedema group. For the untreated group, there was no grade 2 dilatation in the nonoedema subgroup and three patients $(25 \%)$ showed grade 2 nodal varices in the oedema group. Transnodal veins were only observed in the operated group.

Conclusion: The results infer a high prevalence of groin intranodal venous varices in patients with lower limb venous varices and oedema. Transnodal veins have, to the best of our knowledge, not been previously described. This finding may represent a compensatory mechanism or an indirect cause of oedema.

\section{B-683 10:48}

Renal artery stenosis: inflow inversion recovery MR angiography versus three-dimensional contrast-enhanced MR angiography

C.-L. Wu, R.-C. Lee, S.-H. Shen, C.-Y. Chang; Taipei/TW (clwu423@gmail.com)

Purpose: To compare inflow inversion recovery (IFIR) MR angiography (MRA) and three-dimensional contrast-enhanced (3DCE) MRA in the evaluation of renal artery stenosis.

Methods and Materials: From April 2009 to March 2010, 87 patients (55 males, 32 females; mean age, 70.9 years) were referred for MRA at 1.5 T MR (GE signa HD excite) under the clinical suspicion of renal artery stenosis and had both IFIR and 3DCE MRA. The images were reviewed by two radiologists independently. The image quality was graded using a five-point scale: grade 5, good demonstration of interlobar artery; grade 4, well demonstration of segmental artery; grade 3, good demonstration of anterior or posterior renal artery; grade 2, fair demonstration of main renal artery only; grade 1, poor demonstration of main renal artery. The diagnostic confidence was graded by a four-point scale: excellent, good, fair and incapable to make diagnosis. The Wilcoxon signed rank test was used for comparison between the two techniques. A two-tailed $P$ value of 0.05 or less was considered statistically significant.

Results: IFIR MRA showed comparable imaging quality as compared with 3DCE MRA in 40 cases (46\%), inferior in 33 cases (37.9\%) and superior in 14 cases $(16.1 \%)$ $(P=0.001)$. IFIR MRA showed equal diagnostic confidence as compared with 3DCE MRA in the majority of the cases ( $N=65,74.7 \%)$ and even superior in 6 cases $(6.9 \%)$ There was no statistical significance between the two techniques $(P=0.059)$. Conclusion: IFIR MRA is a non-contrast-enhanced MRA technique which has high diagnostic confidence for evaluation of renal artery stenosis.

\section{B-684 10:57}

Simultaneous acquisition of renal morphology and function using a CT perfusion protocol

A. Helck, M. Notohamiprodjo, U. Schönermarck, M. Wessely, R. Morello,

K. Nikolaou, D.-A. Clevert, M.F. Reiser, C. Becker; Munich/DE

(andreas.helck@med.uni-muenchen.de)

Purpose: To verify the accuracy of a renal CT-perfusion protocol for assessing renal function.

Methods and Materials: Eleven patients with renal graft dysfunction received a CTperfusion protocol using a 128-slice CT-scanner with continuous bidirectional tablemovement, allowing to cover a scan range of $14 \mathrm{~cm}$ within $1.5 \mathrm{sec}$. Twelve scans of the entire kidney were acquired every 3.5 seconds to obtain renal perfusion data. Tube potential and current were lowered to $80 \mathrm{kV} / 120 \mathrm{mAs}(\mathrm{BMl}<25)$ and $100 \mathrm{kV} / 120 \mathrm{mAs}$ $(\mathrm{BMI} \geq 25)$, respectively. Perfusion data were corrected for haematocrit and CT-derived glomerular filtration rate (GFR) was calculated by the modified Patlak method. GFR obtained from a two-point plasma disappearance curve of lomeprol served as the standard of reference (blood samples $2 \mathrm{~h}$ and $4 \mathrm{~h}$ after $\mathrm{CT} /$ slope-intercept method). Maximal HU-values in the iliac, renal artery, and renal vein were quantified.

Results: GFR obtained from CT-perfusion correlates well with the GFR derived by lomeprol clearance with a correlation coefficient of $r=0.85(p<0.005)$. Using the optimal of the 12 dynamic phases, maximum mean attenuation in the iliac artery was $357 \pm 129 \mathrm{HU}$, in the renal artery $341 \pm 118 \mathrm{HU}$, and the renal vein $176 \pm 28 \mathrm{HU}$. The mean amount of contrast medium required was $35 \mathrm{ml}$.

Conclusion: A renal CT-perfusion protocol offers the possibility to determine the GFR and thus facilitates simultaneous assessment of morphology and function. In addition, the CT-perfusion protocol helps to reduce the amount of contrast medium, which is beneficial for patients with impaired renal function.

\section{B-685 11:06}

Foot perfusion CT in patients with peripheral arterial occlusive disease (PAOD): a novel chance for treatment planning?

R. lezzi, R. Dattesi, F. Pirro, M. Santoro, G. Tinelli, F. Snider, L. Bonomo; Rome/IT (r.iezzi@rad.unich.it)

Purpose: To prospectively assess the technical feasibility and reproducibility of a quantitative foot perfusion multidetector-row computed tomography (MDCT) technique in patients with PAOD.

Methods and Materials: After institutional review board approval and informed patient consent was obtained, 10 patients with PAOD referred to our department to undergo a single-limb endovascular treatment were prospectively enrolled. All patients underwent dynamic foot 64-row-CT examinations before and after (within 72 hours) endovascular treatment, acquiring eight contiguous 5-mm reconstructed sections, with 60 -second acquisition time, during injection of $50 \mathrm{~mL}$ of contrast medium (lomeprol $400 \mathrm{mgl} / \mathrm{mL}$, at $5 \mathrm{~mL} / \mathrm{sec}$ ). Data were analysed by two experienced blinded readers using a dedicated software to calculate perfusion parameters, such as blood flow (BF), blood volume (BV), mean transit time (MTT), and permeabilitysurface area product (PS). Interobserver and intraobserver agreement of perfusion CT analysis of untreated foot were statistically assessed using intraclass correlation coefficient (ICC) and Blant-Altman analyses.

Results: A good interobserver and intraobserver agreement of perfusion CT analysis was obtained in all patients. All perfusion parameters obtained for untreated foot showed good agreement between the two repeated studies.

Conclusion: Foot perfusion CT is a feasible and reproducible technique. By providing the functional foot microvasculature, it could be a promising method to select the best treatment available for patients with PAOD; furthermore, in case of endovascular treatment, it could be useful to determine which and how many arteries should be recanalised in order to obtain the best result.

\section{B-686 11:15}

Abdominal wall vascularisation mapping in microsurgeons preoperative planning: a retrospective study on $174 \mathrm{CT}$ angiographies. DIEA, SIEA and SICA prevalence, caliber and branching pattern

T. Stocca, M. Bertolotto, M. Belgrano, F. Pozzi Mucelli, Y. Zimolo, M.A. Cova; Trieste/IT (tizianostocca@virgilio.it)

Purpose: To describe the different branching patterns and mean caliber of DIEA (deep inferior epigastric artery), a costant vessel is used by microvascular surgeons in autologus breast recostruction. To assess the incidence and anatomy of SIEA (superficial inferior epigastric artery) which can be used by surgeons as an alternative to DIEP flaps. To evaluate the incidence of SICA (superficial iliac circumflex rtery), a vessel can mimic SIEA.

Methods and Materials: The volume datasets of 174 consecutive abdominal CT angiography studies (CTA) in female patients performed using a 64-detector-row scanner (rotation time $400 \mathrm{~ms}$, beam collimation $64 \times 0.5,120 \mathrm{kVp}$, reconstruction interval $0.5 \mathrm{~mm}$ ) were reviewed (mean age 72 years old). Branching pattern was classified following the Taylor and Moon classification which describe the DIEA arterial branches entity.

Results: DIEAs were identified bilaterally in all but one patient with previous appendicectomy, in whom right DIEA had been ligated during the operation ( $\mathrm{n}=$ 347). The Taylor branching pattern was: single branch (type I) $61 \%(n=211)$, two branches (type II) $34 \%(n=119)$, three branches (type III) $5 \%(n=17)$. DIEA median calibre was $3.1 \mathrm{~mm} \pm 0.5 \mathrm{DS}$. SIEA was present in $49 / 174$ patients $(28.1 \%)$, bilateral in 8 cases $(16 \%)$ and monolateral in $40(84 \%)$. Superficial iliac circumflex artery was present in $47 / 174$ patients $(27 \%)$, bilateral in 19 cases $(40 \%)$ and monolateral in $28(60 \%)$

Conclusion: Vascularisation of the inferior abdominal wall is variable, and detailed preoperative knowledge of the anatomical variations is necessary in patients undergoing autologous breast reconstruction. CTA allows detailed evaluation of all surgically relevant arteries.

\section{B-687 11:24}

Planning deep inferior epigastric artery flaps for breast reconstructions: a comparison between CT, MR and colour-Doppler US

A. Cina, L. Barone-Adesi, P. Rinaldi, A. Cipriani, S. Santoro, M. Salgarello,

L. Bonomo; Rome/IT (acina@sirm.org)

Purpose: Deep inferior epigastric artery perforators free flaps (DIEP) surgery has become the state of the art for autologous breast reconstructions borrowing skin and fat tissue from the abdominal wall. The role of imaging is crucial during the DIEP planning to select a perforator pedicle adequate to supply the flap. Caliber of perforator and its intra-muscular course (MC) are important data for the surgeon. 
We compared CT, MR and CDS to assess accuracy in selecting and measure the "dominant" perforator, employed to supply the flap, and to estimate its MC.

Methods and Materials: Ten women (mean age 49) underwent before surgery: 64-slices CT (90 cc iodine contrast $400 \mathrm{mgl} / \mathrm{d}$ )l; $1.5 \mathrm{~T}$ MR (LAVA sequences, $12 \mathrm{cc}$ Gadobutrol) and CDS (12 MHz probe). Surgical findings were the standard of reference.

Results: The dominant perforators was correctly identified in 9/10 cases (90\%) by CT, $9 / 10(90 \%)$ by MR and $10 / 10$ by CDS (100\%). Accuracy for evaluation of perforator's caliber (<1 mm, 1-1.2 mm, 1.3-1.5 mm, >1.5 mm) was $90 \%$ for CDS, $70 \%$ for CT and $60 \%$ for MR, with a systematic overestimation for CT (mean error $0.8 \mathrm{~mm})$ and MR $(1 \mathrm{~mm})$. Accuracy for IMC evaluation $(<1 \mathrm{~cm}, 1.1-2 \mathrm{~cm}, 2.1-3 \mathrm{~cm}$, $>3 \mathrm{~cm}$ ) was $100 \%$ for CT, $80 \%$ for MR and $90 \%$ for CDS.

Conclusion: MR, CT and CDS allow an high accuracy to identify the dominant perforator for DIEP reconstruction. In a strategy to optimise the DIEP planning by employing panoramic imaging MR plus CDS may be proposed alternatively to CT for DIEP planning.

\section{B-688 11:33}

Corticosteroid treatment effects on MRI and ultrasonography findings in giant cell (temporal) arteritis

T.A. Bley ${ }^{1}$, C. Hauenstein ${ }^{2}$, M. Reinhard², J. Geiger ${ }^{2}$, M. Uhl², M. Markl²;

${ }^{1}$ Hamburg/DE, ${ }^{2}$ Freiburg/DE (t.bley@uke.de)

Purpose: To compare the impact of initial corticosteroid treatment on high-resolution magnetic resonance imaging (MRI) and colour-coded duplex sonography (CCDS) of the superficial temporal arteries in patients with giant cell (temporal) arteritis (GCA). Methods and Materials: Results of high-resolution MRI and CCDS of the superficial temporal arteries in 59 patients with suspected GCA were compared with the final clinical diagnosis (based on the American College of Rheumatology GCA criteria and a 6-month follow-up study). Patients were grouped into three groups according to the length of steroid treatment prior to CCDS or MRI: 0 to 1 day, of 0 to 4 days and $<4$ days. In 41 patients imaging results were also compared with findings of a temporal artery biopsy (TAB).

Results: $61 \%$ (39/59) of the patients were ultimately diagnosed as having GCA The sensitivity of CCDS and MRI under steroid treatment 4 days $50 \%$ and $56 \%$. TAB findings were positive in $59 \%(24 / 41)$ of patients with TAB. Compared with TAB the sensitivity of CCDS and MRI under steroid treatment $<1$ day was $92 \%$ and $90 \%, 0$ to 4 days was $89 \%$ and $84 \%$, and $>4$ days $50 \%$ and $80 \%$.

Conclusion: Inflammatory changes of the temporal artery depicted by CCDS and MRI in patients with GCA decrease significantly under corticosteroid treatment within days. Therefore, the examination with CCDS or MRI of patients with suspected GCA should be performed before onset of treatment or within the first few days.

\section{B-690 11:42}

MRI and contrast-enhanced MRA for the initial detection of an involvement of Osler-Rendu-Weber disease/hereditary haemorrhagic telangiectasia and follow-up after embolisation therapy of pulmonary AVMs

A. Massmann ${ }^{1}$, M. Wirth ${ }^{1}$, M. Schreiber ${ }^{1}$, P. Fries ${ }^{1}$, R. Seidel ${ }^{1}$, M. Katoh ${ }^{1}$,

U.W. Geisthoff ${ }^{2}$, A. Buecker ${ }^{1}$, G.K. Schneider ${ }^{1} ;{ }^{1}$ Homburg/DE $^{2}$

${ }^{2}$ Cologne/DE (Alexander.Massmann@uks.eu)

Purpose: Magnetic resonance imaging (MRI) and contrast-enhanced magnetic resonance angiography (CE-MRA) for initial detection of involvement of OslerRendu-Weber disease/hereditary haemorrhagic telangiectasia (HHT) and follow-up after embolisation therapy of pulmonary arterio-venous malformations (PAVMs). Methods and Materials: In 2000-2010, 262 patients (females 154, 3-86 years, mean 46 years) with definite $\mathrm{HHT}$ according to Curacao criteria and their first-degree relatives underwent screening and follow-up MRI/CE-MRA (Gadolinium-BOPTA $0.1 \mathrm{mmol} / \mathrm{kg}$ ) for the detection of cerebral, pulmonary and hepatic arterio-venous malformations (AVMs). Patients with PAVM underwent catheter angiography (DSA) and embolotherapy with platinum coils.

Results: Initial MR screening revealed cerebral AVM (CAVM) in 12/224 (5.4\%), pulmonary AVM (PAVM) in 88/256 (34.4\%) and hepatic AVM (HAVM) in 47/215 (21.9\%) patients. All CAVMs were clinically insignificant. 61/88 patients with 195 PAVMs (including 61 complex PAVMs) underwent embolisation. CE-MRA was superior to global DSA $(76.4 \%)$ for the detection of PAVM. Follow-up CE-MRA showed 57 new PAVMs in 14 (interval 1-5 years), and 11 reperfused PAVMs (due to recanalisation, opening of collaterals, insufficient packing) in 7 patients (interval 3 months- 5 years). Interestingly, HAVM occurred predominantly in females (78.7\%). Arterio-venous shunts lead to right-heart-insufficiency and biliary necrosis due to a left-to-right shunt. Arterio-portal sinusoidal shunts lead to pseudo-cirrhosis and hyperplastic nodular liver changes similar to $\mathrm{FNH}$; arterio-portal pre-sinusoidal shunts lead to portal hypertension. 3 patients with symptomatic HAVM were treated symptomatically.
Conclusion: MRI and CE-MRA are suitable methods for screening and follow-up of patients with $\mathrm{HHT}$, especially for the detection of PAVM. Embolisation therapy is safe and efficient. Regular follow-up of PAVM is mandatory. HAVM and rare CAVM are mostly asymptomatically.

$10: 30-12: 00$ Room I/K

\section{Cardiac}

\section{SS 1603}

\section{Emerging techniques and applications}

Moderators:

G. Hadjidekov; Sofia/BG

P.K. Vanhoenacker; Aalst/BE

\section{B-691 10:30}

Effects of autologous bone marrow mononuclear cell transplantation through coronary artery bypass grafting in patients with old myocardial infarction assessed by MRI

M. Lu, S. Zhao, S. Liu, S. Jiang, Q. Liu; Beijing/CN (lumjcn@hotmail.com)

Purpose: Recent studies have shown that autologous bone marrow mononuclea cell (aBM-MNC) transplantation can be effectively performed in human beings either by the coronary route or by endoventricular injections. However, scanty data are available for patients undergoing coronary artery bypass grafting (CABG) Accordingly, the aim of this study was to use an "one-stop" non-invasive imaging examination-MRI to evaluate the feasibility and safety of aBM-MNC transplantation in patients with old myocardial infarction (OMI) undergoing CABG.

Methods and Materials: We did a randomised, double-blind, placebo-controlled study in 50 patients (male $=47$, female $=3$, age $57.48 \pm 7.98 \mathrm{y}$ ) with OMI. The patients were randomly divided into two groups (group $A: C A B G+a B M-M N C$, group $B: C A B G$ only). Preoperative global left ventricular functions and scar tissue were measured by MRI. The therapeutic effects were assessed by MRI one year after aBM-MNC transplantation. Results: All the patients were treated without major complications. There is no evidence of new ventricular arrhythmia or neoplasia. The LVEF was improved $13.5 \%$ in group A, while $8.04 \%$ in group $B(P=0.04), L V E D V / m 2$ and $L V E S V / m 2$ were decreased by $24.21 \pm 5.86 \mathrm{ml} / \mathrm{m} 2$ and $29.22 \pm 5.05$, respectively, which were significantly different from that in group $B[3.13 \pm 7.23 \mathrm{ml}(\mathrm{P}=0.028)$ and $7.71 \pm 5.93(\mathrm{P}=0.008)]$. The cardiac output $(\mathrm{CO})$,cardiac index $(\mathrm{Cl})$ and cardiac mass $(\mathrm{CM})$ did not show significant difference between the two groups. Compared with group $\mathrm{B}$, aBM-MNC group was associated with a significant reduction in myocardial infarct size $(25.4 \%$ vs. $3.5 \%, p=0.016)$ Conclusion: Comprehensive in vivo CMR reveals reversed remodelling and improved systolic function and scar characteristics after aBM-MNC transplantation.

\section{B-692 10:39}

MRI of the foetal heart: comparison of the obstetric sonography and foetal magnetic resonance imaging findings

F.P. Junqueira, T.D. Gasparetto, G.M. da Cunha, H. Werner, M.M. Cardoso, P.A. Daltro, R.C. Domingues; Rio de Janeiro/BR (mouracunha@hotmail.com)

Purpose: This study aimed to evaluate the feasibility of foetal magnetic resonance imaging (MRI) with steady-state free precession (SSFP) sequence to visualise the normal and pathological appearances of the foetal cardiovascular system, comparing with the ultrasound (US) images.

Methods and Materials: All the patients were evaluated with MRI and US at the same day. The study included two groups of patients: 05 with US suspicion of congenital heart disease (CHD) including 1 case of conjoined twins; and 55 control patients with normal US. All the patients had at least 26 weeks of gestation. Foetal cardiac MRI consisted of a static phase with multiplanar SSFP sequences and realtime cine SSFP sequence. An experienced obstetric sonographer performed the US examinations of the foetal heart. The statistical analysis assessed the relation between foetal cardiac findings on both MRI and US

Results: MRI of the foetal heart showed good anatomical correlation with the US images in the normal fetuses. MRI also showed good correlation in the pathologic cases, being superior to US in the conjoined twins case, as the US failed to demonstrate the correct anatomy of the two hearts.

Conclusion: Foetal MRI with SSFP sequences in static and cine-resonance acquisition may help in the evaluation of the normal foetal heart and identification of the main morphological alterations related to $\mathrm{CHD}$, when the US examination is limited by the foetal position, maternal obesity, and oligohydramnios. Additionally, both obstetric US and foetal MRI presented good correlation of findings and can be used as adjunct imaging techniques. 


\begin{abstract}
B-693 10:48
Measurements of the great vessels for the detection of aortic coarctation with fetal cardiovascular MRI: preliminary experience

L. Manganaro, V. Vinci, A. Tomei, F. Fierro, M.E. Sergi, P. Sollazzo, S. Bernardo, F. Ventriglia, M. Marini; Rome/IT (lucia.manganaro@uniroma1.it)
\end{abstract}

Purpose: Aortic coarctation $(\mathrm{CoA})$ is the most common duct-dependent cardiac defect missed at routine physical screening of the neonate; indeed, despite new development in foetal echocardiography, prenatal detection rate for congenital outflow tract anomalies is relatively low. This study aimed to evaluate the ability of foetal cardiovascular MRI to characterise and measure anatomical details in order to offer new diagnostic possibilities in the detection of CoA.

Methods and Materials: From November 2009 to July 2010, we enrolled 10 foetuses with the US suspect of CoA. Foetal cardiovascular MRI was performed with a 1.5-T Magnet System, in standard condition and without mother sedation. Retrospectively, we evaluated the mediastinal pulmonary artery to ascending aorta diameter ratio, according to the preliminary experiences in echocardiography which considered 1.60 as limit for normality.

Results: We diagnosed CoA in 7/10 foetuses; we disconfirmed the US suspect of CoA in $2 / 10$ cases identifying a ratio under 1.60 ; in one foetus the ratio was exactly 1.60 , therefore classified as diagnostically inconclusive. The $7 / 10$ pathological cases were confirmed either by after birth follow-up ( 5 cases) or autoptical findings ( 2 cases). After birth follow-up confirmed, in 2/10 cases, the normal aortic diameter findings of MRI. At last, the unclassified case at US and MRI find out to be normal in the after birth follow-up. Conclusion: Our preliminary experience demonstrated the potential role of foeta cardiovascular MRI in the detection of CoA and in the accurate delineation of the vascular anatomy, in case of US diagnostically inconclusive.

\section{B-694 10:57}

Towards real-time MR-guided transarterial aortic valve implantation (TAVI): in vivo evaluation in swine

H.H. Quick ${ }^{1}$, P. Kahlert ${ }^{2}$, H. Eggebrecht ${ }^{2}$, G.M. Kaiser ${ }^{2}$, N. Parohl' ,

J. Albert ${ }^{2}$, I. McDougall ${ }^{3}$, R. Erbel ${ }^{2}$, M.E. Ladd ${ }^{2} ;{ }^{1}$ Erlangen/DE, ${ }^{2}$ Essen/DE,

${ }^{3}$ Vancouver, BC/CA (Harald.Quick@imp.uni-erlangen.de)

Purpose: MR-guided transcatheter, transarterial aortic valve implantation (TAVI) has been performed in swine following device modifications towards MR compatibility of the comercially available stent valve delivery device.

Methods and Materials: The self-expandable Medtronic CoreValve ${ }^{\circledR}$ aortic bioprosthesis (Medtronic Inc). is composed of a nitinol stent frame with integrated trileaflet valve and is either implanted via femoral or subclavian artery. Its $18 \mathrm{Fr}$ delivery catheter comprises the crimped prosthesis which can be released stepwise maintaining transaortic blood flow. The original catheter shaft revealed ferromagnetic braiding, considerably compromising MR imaging and safety. Device modifications obviating any metal braiding resulted in full MR compatibility. MR-guided TAV was performed on 8 swine $(75-85 \mathrm{~kg})$ via subclavian access on a 1.5 TAvanto MR system (Siemens) equipped with in-room monitor. Catheter placement and stent release were performed under TrueFISP real-time MR guidance providing $5 \mathrm{fps}$. Results: Device modifications resulted in artefact elimination and excellent real-time visualisation of catheter movement and valve deployment using rt-TrueFISP imaging. MR-guided TAVI was successful in 6/8 swine. Post-interventional therapeutic success could be confirmed using ECG-triggered cine-TrueFISP sequences and flow-sensitive phase contrast sequences revealing or excluding regurgitation. Final stent valve position was confirmed by ex vivo histology.

Conclusion: The self-expandable CoreValve aortic stent-valve is potentially suited for real-time MRI-guided placement after suggested design modifications of the delivery-system. MR imaging provided excellent pre-interventional anatomic and functional evaluation of the native aortic valve, precise real-time instrument guidance allowing accurate placement of the stent-valve within the native aortic annulus, and finally, detailed post-interventional evaluation of therapeutic success.

\section{B-695 11:06}

Accuracy of dynamic CT-based adenosine stress myocardial perfusion imaging to estimate myocardial blood flow in a porcine animal model with various degrees of coronary artery stenosis

F. Bamberg, F. Schwarz, T. Sandner, B. Wintersperger, R. Hinkel, E. Baloch,

A. Becker, M.F. Reiser, K. Nikolaou; Munich/DE

(fabian.bamberg@med.uni-muenchen.de)

Purpose: To determine the accuracy of CT-based dynamic stress myocardial perfusion imaging to estimated myocardial blood flow (MBF) in a porcine anima model with variable degrees of coronary artery stenosis in comparison with microsphere-derived MBF.
Methods and Materials: Six country pigs $(46 \pm 4 \mathrm{~kg})$ received stent placement $(3.0 \mathrm{~mm})$ of the proximal LAD. and balloon catheter placement within the stent and inflated to obtain luminal narrowing of the vessel diameter $(50 \%$ and $75 \%$ diameter stenosis, respectively, by flow wire). All animal models underwent adenosine-mediated $(280 \mathrm{ukg} / \mathrm{min})$ dynamic stress and rest CT imaging using a fast Dual-Source CT system with acquisitions every second for 30 s. CT-based MBF was derived using a model-based parametric deconvolution method. Fluorescent microspheres were injected at each perfusion state.

Results: All study procedures were performed without complications. At rest and stress, the average MBF was $93 \pm 6$ and $125 \pm 11 \mathrm{ml} / 100 \mathrm{ml} / \mathrm{min}$ for rest and stress imaging, respectively. There was a strong positive association between CT-based and microsphere-based MBF $(r=0.86, p<0.001)$ without overestimation by CT (average difference: $0.005 \pm 0.9 \mathrm{ml} / \mathrm{g} / \mathrm{min}, \mathrm{p}=0.9$ ). In addition, CT-based MBF within myocardial segments pertaining to $50 \%$ and $75 \%$ coronary stenoses was strongly correlated with microsphere MBF $(r=0.67, p=0.005)$ with lowest MBF at $75 \%$ luminal narrowing (2.1 \pm 0.1 vs. $1.4 \pm 0.4$ vs. $0.8 \pm 0.3 \mathrm{ml} / \mathrm{g} / \mathrm{min}$ for $0 \%, 50 \%$, and $75 \%$ luminal narrowing under stress, respectively; $p<0.01$ ) similar to microsphere MBF. Conclusion: CT-derived MBF measurements correlate well with microsphere MBF. Perfusion CT may allow for assessment of haemodynamic significance of different degrees of coronary artery stenosis.

\section{B-696 11:15}

Quantification of regional myocardial perfusion at different levels of stenosis in a large animal model using dynamic perfusion $\mathrm{CT}$ A. Rossi, A. Uitterdijk, M. Dijkshoorn, M. van Straten, W.J. van der Giessen, G.P. Krestin, D.J. Duncker, D. Merkus, N.R. Mollet; Rotterdam/NL (alexia.rossi0@aliceposta.it)

Purpose: To quantify differences in regional myocardial perfusion at different levels of coronary stenosis using dynamic perfusion dual source CT (DSCT).

Methods and Materials: In five pigs, an adjustable, remote controlled hydraulic occluder was placed around the LAD to induce various degrees of coronary stenosis. In addition, catheters were placed into the aortic arch and the coronary artery distally to the occluder for pressure measurements and fractional flow reserve (FFR) determination. Myocardial blood flow (MBF) was assessed during infusion of contrast using a second generation DSCT. Images were acquired at baseline and under maximal adenosine vasodilatation at FFR $=1$ (no stenosis), $\mathrm{FFR}=0.7$ (moderate stenosis) and FFR $=0.5$ (severe stenosis). MBF measured in the downstream LAD perfusion area was compared with the remote posterior myocardium. In addition, correlation between MBF and FFR was investigated.

Results: Adenosine resulted in an increase in MBF from $110 \pm 13$ to $262 \pm 12 \mathrm{ml} / 100 \mathrm{ml} / \mathrm{min}(\mathrm{P}<0.05)$. In the presence of adenosine, both moderate and severe stenosis reduced MBF in the LAD perfusion territory (204 \pm 45 and $107 \pm 29 \mathrm{ml} / 100 \mathrm{ml} / \mathrm{min}$ both $\mathrm{P}<0.05$ vs no stenosis) as compared to MBF in the remote myocardium ( $317 \pm 29$ and $254 \pm 26 \mathrm{ml} / 100 \mathrm{ml} / \mathrm{min}$ ). Moreover, percent MBF reduction correlated very well with the FFR measurements $\left(R^{2}=0.90, P<0.05\right)$. Conclusion: Dynamic perfusion DSCT is suitable for the assessment of myocardia hypoperfusion and can be used to discriminate between several degrees of clinically relevant coronary stenoses in a large animal model.

\section{B-697 11:24}

Dual energy CT for the detection of delayed enhancement in reperfused chronic infarction: a comparison with magnetic resonance imaging and histopathology in a porcine model

J.M. Kerl, S. Deseive, R.W. Bauer, T. Lehnert, B. Schell, T.J. Vogl;

Frankfurt a. Main/DE (matthias.kerl@kgu.de)

Purpose: To evaluate the performance of delayed enhancement dual energy $\mathrm{C} T$ (LE-DECT) for the detection of irreversibly damaged myocardium compared with delayed-enhancement magnetic MRI and histology in a porcine model of reperfused chronic myocardial infarction.

Methods and Materials: MI were induced by 30-min occlusion of the proximal left anterior descending coronary artery in 8 healthy minipigs. 61 days after LAD occlusion LE-DECT was performed 5, 10 and 15 minutes subsequent to contrast material injection using a DSCT-scanner in dual energy mode. 50 minutes after LE-DECT imaging, LE-MRI and histopathology were acquired. Two experienced radiologists interpreted in consensus all imaging series in a random fashion and blinded to the results of the other techniques for the presence of LE using the AHA 17-segment model. Results were compared with histopathology.

Results: Of totally 136 myocardial segments in 8 minipigs, histopathology found scar tissue in 27 segments. The best results were observed in CT 10 minutes after contrast material injection. Here, LE-DECT was able to determine segments containing scar tissue with $76 \%$ sensitivity, $93 \%$ specificity, $70 \%$ PPV, $94 \%$ NPV and $89 \%$ 
accuracy. Using $100 \mathrm{kV}$ grey-scale images $62 \%$ sensitivity, $97 \%$ specificity, $84 \%$ PPV, $0.91 \%$ NPV and $90 \%$ accuracy were observed. LE-MRI detected those segments with $78 \%$ sensitivity, $93 \%$ specificity, $72 \%$ PPV, $94 \%$ NPV and $90 \%$ accuracy. Conclusion: LE- DECT gets the best results 10 minutes after contrast material injection for the detection of reperfused chronic myocardial infarction. In addition, LE-DECT improves diagnostic accuracy of CT-LE in a combined approach.

\section{B-698 11:33}

Investigation of myocardial metabolite concentrations: evaluation by localized $1 \mathrm{H}$ magnetic resonance spectroscopy in vivo

J. Wu, L. Yang; Beijing/CN (wujian621@sohu.com)

Purpose: To investigate the myocardial metabolite concentrations evaluated by ${ }^{1} \mathrm{H}$ magnetic resonance spectroscopy in vivo.

Methods and Materials: We recruited 100 healthy volunteers for the present study. Cardiac magnetic resonance imaging used to localise voxel and localised ${ }^{1} \mathrm{H}$ MRS quantifying myocardial metabolite concentrations were scanned on 1.5 T MR scanner. Metabolite and triglyceride signal strength in relation to water signal strength from the same voxel in spectra were estimated using General Electric's SAGE package. Results: The 100 participants completed all study procedures and 86 participants (46males and 40 females, age $29 \pm 11$ years; BMI $22.1 \pm 2.6 \mathrm{~kg} / \mathrm{m}^{2}$ ) were included in the final analysis. 14 participants (14\%) were excluded because of poor spectra. The spectral peaks were identified with following chemical shifts: water, choline, creatine and triglyeride. No statistical significant inter-gender difference of creatine and choline concentrations were observed $(p>0.05)$, while myocardial triglyceride concentration was $88 \%$ higher in myocardial tissue in females than in myocardia tissue in males $(p<0.05)$, despite the former having a lower mean body-mass index. Conclusion: The concentration of myocardial triglyceride is related to gender and higher in females, while the concentrations of myocardial creatin and choline are not related to gender.

\section{B-699 11:42}

Evaluation of aortic distensibility and cardiac function in normal wild type and diabetic mice with MRI at $9.4 \mathrm{~T}$

P. Fries, J. Reil, A. Müller, R. Seidel, K. Altmeyer, A. Massmann, A. Bücker,

G. Schneider; Homburg/DE (drpeterfries@googlemail.com)

Purpose: The aim of this study was to compare aortic distensibility (AD) and cardiac function in wild type (C57BL/6) and type II diabetic (db-/db-) mice by means of cine MRI.

Methods and Materials: C57BL/ 6 and db-/db- mice ( $n=5$ each) were evaluated using a horizontal bore 9.4 T MRI animal scanner (Bruker, Karlsruhe, Germany) with a dedicated $2 \times 2$ phased-array surface coil. A double-gated cine sequence (Intragate Flash, TR/TE $=8.9 / 2.1 \mathrm{~ms}, \mathrm{FA}=10^{\circ}, \mathrm{FOV}=2 \times 2 \mathrm{~cm}$, matrix $=384 \times$ 384) located perpendicular to the ascending aorta was acquired for imaging of $A D$. Cardiac function was assessed acquiring five consecutive short axis cine sequences covering the whole ventricles. $A D$ was defined as the difference of the subintimal vessel area at systoly and diastoly. Enddiastolic (ED), endsystolic (ES), stroke volume (SV) and ejection fraction (EF) were analysed using an image processing software (Osirix). Student's T-Test was used for statistical analysis $(P<0.05)$.

Results: Diabetic animals showed a significant lower AD (mean \pm SD: $\left.0.467 \pm 0.05 \mathrm{~mm}^{2}\right)$ compared to C57BL/6 $\left(0.668 \pm 0.1 \mathrm{~mm}^{2}, \mathrm{p}=0.004\right)$. ED volumes of diabetic animals were significantly smaller (db-/db-: $41.9 \pm 7.1 \mathrm{ml}$, C57BL/6: $55.5 \pm 11.8 \mathrm{ml}, \mathrm{P}=0.03$ ) while there were no significant differences in SV between the two groups (db-/db-: $32.7 \pm 8.1 \mathrm{ml} / \mathrm{C} 57 \mathrm{BL} / 6: 30.4 \pm 7.0 \mathrm{ml})$. EF was significantly larger in diabetic animals $(78.1 \pm 0.1 \%)$ compared to the C57BL/6-group $(54.9 \pm 0.2 \%, P<0.001)$.

Conclusion: Diabetic mice demonstrate a significantly lower AD. As there is no significant difference in SV these characteristics are most likely based on vascular changes. In addition, db-/db- animals show signs of restrictive cardiomyopathy with decreased LVEDV and increased EF as compared to the reference.

\section{B-700 11:51}

Rotating-C-arm-tomography for intraprocedural anatomic evaluation of the aortic root in percutaneous aortic valve replacement (PAVR): comparison with multidetector $\mathrm{CT}$

F. Schwarz, P. Lange, F. Bamberg, V. Renz, K. Nikolaou, M.F. Reiser,

P. Boekstegers, C. Becker; Munich/DE (florian.schwarz@med.Imu.de)

Purpose: Percutaneous aortic valve replacement (PAVR) has been established to treat patients with severe aortic stenosis who are poor surgical candidates. While multi-detector CT (MDCT) plays the central role in pre-procedural workup, clinicians typically rely on fluoroscopy during PAVR. Modern flat-panel-detector fluoroscopy units offer a tomography mode, using a half-rotation to reconstruct tomographic images. Here, we analysed the correlation of aortic valve diameters obtained from pre-procedural MDCT and intra-procedural C-arm-tomography acquisitions. Methods and Materials: 15 patients underwent ECG-triggered, high-pitch CTangiography of the thorax and abdomen 3-30 days prior to PAVR. During valve replacement, all patients underwent $\mathrm{C}$-arm-tomography while contrast material was administered intraarterially at the level of the aortic valve. Rapid ventricular pacing was used to reduce motion artefacts. For this study, two readers independently determined valve diameters on a coronal and double-oblique plane. All datasets were anonymised, presented in random order and readers had to adjust planes manually. Results: Aortic valve diameters could successfully be measured in all datasets. Although there was a tendency towards better interobserver agreement for MDCT, it was excellent in both modalities (ICC $=0.82$ vs. 0.89). Overall, agreement between both modalities was high $(\mathrm{ICC}=0.80)$. There was a small but significant difference in means of valve diameters in the coronal plane $(23.9 \mathrm{~mm}$ vs. $22.9 \mathrm{~mm}$, $\mathrm{p}=0.008$ ). Mean values of valve diameters in a double-oblique plane were not different ( $21.5 \mathrm{~mm}$ vs. $21.7 \mathrm{~mm}, \mathrm{p}=0.55)$.

Conclusion: This study shows good agreement between aortic valve diameters measured by MDCT prior to PAVR and intra-procedural C-arm-tomography. Minor differences in values might result from rapid ventricular pacing during $\mathrm{C}$-armtomography.

10:30 - 12:00

Room L/M

\section{Contrast Media}

\section{SS 1606}

\section{Contrast media: clinical evidence, news and facts}

Moderators

T. Leiner; Utrecht/NL

B. Tombach; Osnabrück/DE

\section{B-701 10:30}

Exploration of immediate hypersensitivity reactions to contrast media: the French multicenter CIRTACI study - final results

O. Clément ${ }^{1}$, P. Dewachter ${ }^{1}$, C. Mouton ${ }^{2}$, F. Gillaizeau ${ }^{1}$, D. Laroche ${ }^{3} ;{ }^{1}$ Paris/FR, ${ }^{2}$ Nancy/FR, ${ }^{3}$ Caen/FR (olivier.clement@inserm.fr)

Purpose: The CIRTACI is a prospective multicenter study exploring immediate reactions after contrast media. The goal was to differentiate between allergic and non-allergic hypersensitivity to iodinated and gadolinium contrasts. Final analysis of the 330 cases in 31 centers will be reported.

Methods and Materials: The following data were obtained: clinical severity (Ring and Messmer classification), plasmatic tryptase and histamin levels, allergologica skin tests performed 6 weeks to 6 months after the reaction. $5 \mathrm{Gd}$ chelates or 10 iodinated agents were tested. Positivity of the intradermal test was assessed by the increase in diameter of the injection papula together with inflammatory signs. The final diagnosis concerning the culprit contrast agent had to be made between allergic and non-allergic hypersensitivity reaction based on these clinical, biological and allergological criteria.

Results: Among 319 available cases, there were 179 grade 1, 91 grade 2, 39, grade 3,5 grade 4 and 5 undetermined. 275 patients could follow the entire protocol, among them 233 reactions to iodinated and 42 to gadolinium agents. All contrast agents could be responsible for a reaction. Allergic hypersensitivity reactions were almost always found in grade 3 and 4 reactions. In grade 1 (diffuse urticaria) allergy was found in about $30 \%$ of the cases. Detailed results of the study will be presented at the meeting.

Conclusion: Patients presenting an immediate reaction to a contrast media (either iodinated or gadolinated) should be explored with proper biological and allergological. A true allergic hypersensitivity mechanism contra-indicate the contrast for life.

\section{B-702 10:39}

Safety and tolerability of iobitridol in general and in patients with risk factors: results in more than 160,000 patients

M.H. Maurer ${ }^{1}$, O. Heine ${ }^{2}$, M. Wolf ${ }^{3}$, B. Hamm ${ }^{1} ;{ }^{1}$ Berlin/DE, ${ }^{2}$ Sulzbach/DE, ${ }^{3}$ Püttlingen/DE (martin.maurer@charite.de)

Purpose: To review the safety, the tolerability and the diagnostic effectiveness of iobitridol under daily practice conditions in the general population and at-risk patients. Methods and Materials: A total of 160,639 patients (55.1\% males, $43.6 \%$ females, mean age 58.6 years) were analysed in 555 German centres. Patients underwent x-ray examinations using iobitridol (Xenetix ${ }^{\circledR}$, Guerbet, Roissy CdG, France) as IV 
contrast medium (mean volume $85.6 \mathrm{ml}$ ). $21.8 \%$ of all patients had at least one risk factor (i. a., renal impairment), 7.3\% were at-risk patients with allergies or who had previously reacted to contrast medium. Antiallergic pretreatment before contrast medium administration was given in 1144 patients $(0.7 \%)$. Adverse events were documented and the image quality was assessed.

Results: A diagnosis was possible in $99.5 \%$ of all cases. The image quality was rated good or excellent in $92.2 \%$. The adverse event rate (e.g., nausea, urticaria) observed was $0.6 \%$ in all patients, $1.6 \%$ in patients with allergies and $6.0 \%$ in patients with a previous reaction to contrast medium. Adverse events occurred more often in women than in men $(p<0.001)$. The rate of adverse events was not increased in higher doses of lobitridol, even if administered to high-risk patients. Conclusion: lobitridol was shown to be a well-tolerated contrast medium that allowed a diagnosis with very good or excellent imaging quality to be made in more than $90 \%$ of all cases. The incidence of adverse events was low even in patients with risk factors.

\section{B-703 10:48}

Contrast-enhanced ultrasound (CEUS) to assess the perfusion of patients with peripheral arterial disease (PAD) treated with endothelial progenitor cells (EPCs)

F. Alessandrino, S. Alessi, C. Dellabianca, V. Arici, C. Perotti, F. Calliada Pavia/IT (pragia@hotmail.com)

Purpose: Since contrast-enhanced ultrasound (CEUS) has been recognised as a valid method for the assessment of muscle perfusion deficits, the aim of this study is to evaluate CEUS as a diagnostic tool to assess the perfusion of the muscle of patients with PAD treated with endothelial progenitor cells (EPCs) and its correlation with other diagnostic tools as ankle brachial index $(\mathrm{ABI})$ and clinical data. Methods and Materials: 12 Patients underwent intramuscular injection of EPCs from September 2008 to April 2010. CEUS was performed before therapy and scheduled at 3, 6, 9, 12 months after the therapy. Patient performed baseline and CEUS examination with Philips IU22 (Philips Bothell WA) and GE Logiq e9 (GE Milwaukee, WI). The perfusion was assessed evaluating contrast agent timeintensity curves with QLab (Philips Bothell WA) and Qontraxt (Amid and R\&D, Bracco, Milan, Italy) software. Region of interest (ROI) were placed on the calf muscle and on posterior tibial artery (PTA)

Results: All the enrolled patients showed relevant clinical improvement, except one, who, later, underwent leg amputation. Mean AUC before treatment was 688.43 +- SD $172.34 \mathrm{~dB}$ s on PTA and 545.18 +- SD $188.39 \mathrm{~dB}$ s on calf muscle. Mean AUC at six months after treatment was 1054.62 +- SD $360.74 \mathrm{~dB}$ s on PTA and $1372.9+-$ SD $478.21 \mathrm{~dB}$ s on calf muscle.

Conclusion: CEUS is a valuable tool to display variation of the perfusion of the calf muscle and tibial artery in patients treated with EPCs with good correlation with clinical data.

\section{B-704 10:57}

Changes of water diffusion coefficient in the kidney after application of iodinated contrast media

G. Jost, D. Lenhard, M.A. Sieber, P. Lengsfeld, J. Huetter, H. Pietsch; Berlin/DE (gregor.jost@bayerhealthcare.com)

Purpose: The viscosity of x-ray contrast media (CM) may have an influence on the diffusion of water molecules within the kidney. The aim of our preclinical study was to investigate the changes in the apparent diffusion coefficient (ADC) of the kidney after application of monomeric and dimeric CM using diffusion weighted MRI. Methods and Materials: Han-Wistar rats (8 per group) received an intravenous injection of dimeric CM (lodixanol) and monomeric CM (lopromide) at a dosage of $4 \mathrm{~g} \mathrm{l} / \mathrm{kg} \mathrm{b}$.w. The control group received saline $(0.9 \% \mathrm{NaCl})$ at the same volume. The renal $A D C$ was monitored up to 40 min p.i. by diffusion weighted MRI using a 1.5 T clinical MR-scanner (Avanto, Siemens). The correlation between CM viscosity and the ADC of water was assed by phantom measurements.

Results: A slight ADC increase was observed immediately after application of the monomeric agent $\left(\triangle \mathrm{ADC}=80 \mu \mathrm{m}^{2} / \mathrm{s}\right)$ and isotonic water $\left(\triangle \mathrm{ADC}=89 \mu \mathrm{m}^{2} / \mathrm{s}\right)$, which normalised nearly completely to the baseline level at 40 min p.i. In contrast, after application of the dimeric agent a strong decrease of the ADC was determined. This was most prominent 6-12 min p.i. and did not recover to baseline level during the investigation $\left(\triangle \mathrm{ADC}=-418 \mathrm{um}^{2} / \mathrm{s}\right)$. The in vitro measurements yield an agentspecific correlation between increasing viscosity and decreasing water diffusion. Conclusion: A clear difference in the renal ADC was observed between monomeric $\mathrm{CM}$ / water (saline) and dimeric $\mathrm{CM}$. The strong ADC decrease observed for the dimeric agent might be caused by the high viscosity of dimeric CMA

\section{B-705 11:06}

Simultaneous introduction of a new CT request form for ceCT and outpatient CIN clinic: evaluation at 6 months

H.M. Dekker, J. Wetzels; Nijmegen/NL (h.dekker@rad.umcn.nl)

Purpose: To evaluate the application of a new CT request form and the simultaneous introduction of a special outpatient clinic for patients at high risk for contrastinduced nephropathy $(\mathrm{CIN})$.

Methods and Materials: During six months, request forms were collected from outpatients of the department of Urology, who were $\geq 18$ years and undergoing elective ceCT examinations. Information to be indicated on the request form was: MDRD-GFR in $\mathrm{ml} / \mathrm{min} / 1.73 \mathrm{~m}^{2}$ and the risk factors: diabetes, heart failure, periphera vascular disease, age $>75$, anaemia, symptomatic hypotension, large doses of contrast medium (>150 ml), dehydration, and nephrotoxic drugs.

Based on the risk factors a risk score was calculated to define patients at high risk for CIN, who were referred to the "outpatient CIN clinic".

Results: Of the 402 patients included, $24 \%$ had a pre-CT MDRD-GFR $<60 \mathrm{ml} \mathrm{min} / 1.73$ $\mathrm{m}^{2}$. Of these, 50 patients were defined as high risk and referred to the "outpatient CIN clinic". Thusfar, 32 patients have completed the protocol. Five patients did not need hydration, 27 patients received pre- and post-hydration in combination with the ceCT. Five patient were hydrated overnight to avoid overhydration. In nearly all patients, data given on the request form were reliable, and the MDRD recently determined. In the patient group with a MDRD $<60 \mathrm{ml} / \mathrm{min} / 1.73 \mathrm{~m}^{2}$ pre-CT, there was one patient with $\mathrm{CIN}$. Conclusion: The introduction of a new request form allows good identification of patients at risk for nephropathy, while the "outpatient CIN clinic" resulted in closer patient monitoring and optimal preparation, thereby improving patient safety.

\section{B-706 11:15}

\section{Comparison of lesion detection and characterisation in patients with} neuroendocrine tumours using DOTATOC-PET in correlation with contrastenhanced MRI and contrast-enhanced CT

F.L. Giesel, K. Clemens, S. Wulfert, J. Weller, N. Schimpfke, U. Haberkorn, H.-U. Kauczor, S. Ley; Heidelberg/DE (f.giesel@dkfz.de)

Purpose: Most of the gastroenteropancreatic neuroendocrine tumours (GEP-NETs) present an increased somatostatin receptor expression. Therefore, PET with $68 \mathrm{Ga}$ DOTATOC has both high sensitivity and specificy in tumour staging. However, additional morphologic characterisation of tumour lesions is often essential, e.g the relation to adjacent structures to determine weather the lesion is resectable. We evaluated the rate of successful lesion characterisation comparing contrast enhanced CT with MRI each in correlation with DOTATOC-PET.

Methods and Materials: 8 patients with GEP-NET were investigated using late enhanced MRI (1.5-Tesla, T2w-BLADE and T1w-VIBE, before and 15 min after injection of Primovist ${ }^{\circledR}$, BayerSchering/GER), contrast enhanced (ce) CT and DOTATOC-PET imaging. DOTATOC-PET and ce-CT were performed in a BIOGRAPH-6 PET/CT (Siemens/GER).

Results: Using DOTATOC-PET as standard reference for specific detection of metastases, 28 liver metastases were diagnosed. All 28 liver metastases were also detected by ce-MRI and extension could be adequately assessed using the late phase Primovist ${ }^{\circledR}-\mathrm{MRI}$ in all lesions $(100 \%)$. In contrast, not all liver metastases were detectable by ce-CT (79\%) and neither relation to adjacent structures nor exact lesion extension was assessable in the occult (21\%) liver metastases. Conclusion: Late ce-MRI was superior to ce-CT in providing additional morphologic characterisation and exact lesion extension of hepatic metastases from neuroendocrine tumour detected with DOTATOC-PET. Therefore, late enhanced Primovist ${ }^{\circledR}-\mathrm{MRI}$ seems to be the adequate imaging modality for combination with DOTATOC-PET to provide complementary (macroscopic and molecular) tumour characterisation in hepatic metastasised GEP-NETs.

\section{B-707 11.24}

\section{lodine contrast media iso-attenuating with gadolinium doses proven} diagnostic in CTA and angiography: a way to void both CIN and NSF in azotemia?

U. Nyman' ${ }^{1}$, B. Elmståhl' ${ }^{2}$ H. Geijer ${ }^{3}$, P. Leander², T. Almén², M. Nilsson²; ${ }^{1}$ Trelleborg/SE, ${ }^{2} \mathrm{Malmö/SE},{ }^{3}$ Örebro/SE (ulf.nyman@bredband.net)

Purpose: To establish iodine (I) contrast medium (CM) doses iso-attenuating with gadolinium (Gd) CM doses regarded as diagnostic in computed tomography angiography (CTA) and percutaneous catheter-angiography/vascular interventions (PCA/PVI) in azotemic patients.

Methods and Materials: CT Hounsfield units (HU) were measured in 20-mL syringes containing $0.01 / 0.02, / 0.05 / 0.1 \mathrm{mmol} / \mathrm{mL}$ of iodine or gadolinium atoms and placed in phantoms. Relative contrast was measured in $20-\mathrm{mL}$ syringes filled 
with iohexol at $35 / 50 / 70 / 90 / 110 / 140 \mathrm{mg} \mathrm{l} / \mathrm{mL}$ and $0.5 \mathrm{M}$ gadodiamide using radiofluoroscopy (RF), digital radiography (DX) and x-ray angiography (XA) systems. Clinical doses of Gd-CM at CTA/PCA/PVI were reviewed.

Results: At CT $91-116,104-125 \mathrm{mg} \mathrm{l} / \mathrm{mL}$ in the chest and abdominal phantoms, respectively, were iso-attenuating with $0.5 \mathrm{M} \mathrm{Gd}$ at 80-140 kVp. At RF/DX/XA systems $35-90 \mathrm{mg} \mathrm{I} / \mathrm{mL}$ were iso-attenuating with $0.5 \mathrm{M}$ gadodiamide at $60-115 \mathrm{kVp}$. Clinically, $60 \mathrm{~mL}$ of $91-125 \mathrm{mg} \mathrm{I} / \mathrm{mL}$ (5.5-7.5 gram-iodine) at 80-140 kVp CTA or $60 \mathrm{~mL}$ of $35-90 \mathrm{mg} \mathrm{I} / \mathrm{mL}$ (2.1-5.4 gram-iodine) at 60-115 kVp PCA/PVI would be iso-attenuating with $60 \mathrm{~mL} 0.5 \mathrm{M} \mathrm{Gd}-\mathrm{CM}$ (=0.4 mmol Gd/kg in a $75-\mathrm{kg}$ person). Conclusion: Meticulous examination technique and judicious use of ultra-low $\mathrm{I}-\mathrm{CM}$ doses iso-attenuating with diagnostic Gd-CM doses in CTA and PCA/PVI may minimise the risk of nephrotoxicity in azotemic patients, while there should be no risk of NSF.

\section{B-708 11:33}

\section{Is a new routine for contrast medium injection rate and volume} compromising the quality of enhancement on CT scans?

C.A. McCann, T. Lima, M. Jayawardena, C. Farrell, C. Monaghan, J. Holemans, H. Fewins, S. Binukrishnan, K. Irion; Liverpool/UK (cmcca@btinternet.com)

Purpose: To investigate in computed tomography (CT) whether an exponentially decelerated contrast medium injection, as compared with a standard constant-rate injection, can facilitate uniform vascular contrast enhancement with a reduced contrast material volume.

Methods and Materials: A retrospective review of 182 patients undergoing a CT scan between 22/01/10 to 17/02/10 was undertaken. Some scans were performed using the new software (Optibolus) with $75 \mathrm{ml}$ of intravenous contrast medium, whilst the previous software was maintained for other patients, who received $100 \mathrm{ml}$ of intravenous contrast medium using a fixed injection rate. Examinations included chest (including mesothelioma) staging, chest with contrast and CT angiogram. The radiologist was blinded to the injection technique used. Coefficient attenuation values were measured manually at multiple cardiac and vascular structures in the thorax. For staging scans, attenuation values at multiple hepatic and portal vein sites were also recorded. Patients were separated into two groups depending on the technique used. The average mean attenuation value for both groups was compared, as was the mean attenuation values for all the individual structures. Results: There was no significant difference in the objective measurement of attenuation value between either technique of injection, both in the thorax and abdomen. Average thoracic mean attenuation for the low/high volume injection was $228 / 230 \mathrm{HU}$, respectively. Average hepatic mean attenuation for the low/high volume injection was $101 / 102 \mathrm{HU}$, respectively.

Conclusion: Uniform vascular enhancement, and therefore the quality of the test, is maintained with a reduced contrast material volume. This has beneficial implications for patient renal function and cost.

\section{B-709 $11: 42$}

Contrast-induced nephropathy at computed tomograhy: does it matter for high-risk patients? A systematic meta-analysis

M. Weininger ${ }^{1}$, K.H. Zou' ${ }^{2}$, P. Costello ${ }^{1}$, U. Schoepf ${ }^{1} ;{ }^{1}$ Charleston, SC/US,

${ }^{2}$ New York, NY/US (weininge@musc.edu)

Purpose: Various trials compare the effect of iodinated intravenous contrast media (CM) at CT on renal function, using contrast-induced nephropathy $(\mathrm{CIN})$ as primary endpoint. We systematically reviewed adverse outcomes in high-risk patients following intravenous CM at CT beyond laboratory markers.

Methods and Materials: A systematic literature search was performed. Inclusion criteria were 1) peer-reviewed article, 2) prospective trial, 3) intravenous non-ionic CM at CT; 4) high-risk patients with decreased renal function; 5) SCr measurements before and after $\mathrm{CM} ; 6$ ) evaluation for $\mathrm{CM}$-related adverse outcomes. Definition of $\mathrm{CIN}$, type of $\mathrm{CM}$, mechanisms to control for bias, incidence of CIN and adverse outcomes were abstracted. Results: Eight studies were identified, comprising 1625 patients with decreased renal function (baseline SCr $1.41 \pm 0.38$ to $2.1 \pm 0.6 \mathrm{mg} / \mathrm{dl}$; GFR $37 \pm 12$ to $52.98 \pm 26.02 \mathrm{ml} /$ $\mathrm{min}$ ). All studies defined CIN as absolute ( $\geq 0.5 \mathrm{mg} / \mathrm{dl}$ ) or relative $(\geq 25 \%)$ increase in baseline SCr. 292 patients received iopamidol, 794 iodixanol, 76 iomeprol, 59 iohexol and 56 iopromide. For 348 patients $\mathrm{CM}$ was not specified. Pooled proportions of patients of at risk patients with increases in SCr fulfilling definitions of absolute and relative CIN were $5.31 \%$ (95\% Cl: 3.83\%-6.79\%; range 0\%-17.86\%) and 7.07\% (95\% Cl: 5.50\%-8.63\%; range $3.90 \%-26.79 \%)$. No study observed clinically manifest CM-related adverse outcomes, corrected for natural $\mathrm{SCr}$ fluctuations or assessed long-term effects on renal function. Conclusion: Existing literature shows varied incidence of $\mathrm{SCr}$ increases after intravenous $\mathrm{CM}$, meeting the definition of CIN. However, no clinically manifest hard adverse outcomes are reported, limiting the usefulness of $\mathrm{CIN}$ as meaningful endpoint to compare the safety of different $\mathrm{CM}$.

\section{B-710 11:51}

The potential role of residual gadolinium in the induction of nephrogenic systemic fibrosis in rats

D. Lenhard, M.A. Sieber, G. Jost, P. Lengsfeld, J. Huetter, H. Pietsch; Berlin/DE

Purpose: Nephrogenic systemic fibrosis (NSF) is a rare, but serious, acquired, idiopathic disorder. To date, the exact pathogenesis of NSF has not been identified but an association has been postulated between the administration of gadoliniumbased contrast agents (GBCA) and the occurrence of NSF. The purpose of this study was to test the hypothesis that long-term gadolinium (Gd) deposits play a role in the induction of NSF-like lesions in an animal model.

Methods and Materials: Han-Wister rats were administered a triple intravenous injection of Gd-DTPA-BMA formulated without excess ligand at a dose of $2.5 \mathrm{mmol} /$ $\mathrm{kg}$ body weight at different time intervals (24h or 14-, 28-, and 56-days). Gd concentrations were measured by inductively coupled plasma mass spectrometry and occurring skin lesions were determined macroscopically and histologically. Results: The occurrence of NSF-like macroscopic skin lesions differed between the injection groups. Triple injections of Gd-DTPA-BMA with shorter time intervals showed more severe skin reactions compared to longer intervals, despite similar gadolinium concentrations in the skin at the end of the experiment. The injection interval did not influence the long-term presence or level of accumulation of $\mathrm{Gd}$ concentration in tissue.

Conclusion: The presence and severity of NSF-like skin lesions in rats was correlated with the injection interval and not the amount of Gd in tissue. Shorter injection intervals resulted in more severe skin reactions. Room N/O

\section{Neuro}

\section{SS 1611}

\section{Imaging applications, software and post-processing}

Moderators:

N. Bargalló; Barcelona/ES

A. Löve; Lund/SE

\section{B-711 10:30}

Radiation exposure and image quality of $\mathrm{CT}$ brain images acquired with organ based tube current modulation and iterative reconstruction F. Bamberg, D. Augart, L. Eftimov, D. Morhard, K. Neumaier, T. Johnson, A. Graser, M.F. Reiser, H.-C. Becker; Munich/DE (anno.graser@med.uni-muenchen.de)

Purpose: We sought to determine the impact of automated exposure contro (AEC), organ-based tube current modulation (TCM) and iterative reconstruction (IR) technique on effective radiation dose and image quality in an experimenta set-up and a consecutive cohort of patient undergoing CT scanning of the brain. Methods and Materials: An Alderson-Rando-phantom equipped with thermoluminescent dosimeters was used to estimate the radiation exposure of organs within the brain and neck scanned. We also measured the grey and white matter signal-to-noise ratios from CT brain images of 100 consecutive patients and images were subjectively evaluated for quality criteria in different territories of the brain. Moreover, measures of effective radiation exposure were collected.

Results: In the phantom, AEC and TCM significantly reduced the radiation exposure of the lenses, cerebrum, cerebellum and thyroid gland by $41.9 \%, 34.5 \%, 30.5 \%$ and $34.9 \%$, respectively. Head CT scan from patients investigated with AEC and TCM and IR had significantly higher image quality $(p<0.05)$ and identifiability of intracerebral structures $(<0.05)$. The CT dose index and dose length product was $24.1 \%$ and $20.2 \%$ lower, respectively.

Conclusion: The combination of AEC, TCM and IR improves image quality while radiation exposure can be reduced, particularly in dose-sensitive organs, such as the lenses and thyroid gland.

\section{B-712 10:39}

Feasibility of cerebral CT angiography using lower tube voltage ( $80 \mathrm{kVp})$ and moderate-concentration of iodine contrast material: quantitative and qualitative comparison with conventional $\mathrm{CT}$ angiography E.-S. Cho; Seoul/KR (jjondol@yuhs.ac)

Purpose: To investigate the feasibility of $80-\mathrm{kVp}$ with moderate-concentration of contrast material (MC-CM) in cerebral CT angiography compared to conventional $120 \mathrm{kVp}$ with high-concentration of contrast material (HC-CM). 
Methods and Materials: Eighty-two patients were referred for cerebral CT angiography because of screening for intracranial aneurysm. Forty-one patients were scanned with $120 \mathrm{kVp}$ and $150 \mathrm{mAs}_{\text {eff }}$ after $70 \mathrm{~mL}$ of $\mathrm{HC}-\mathrm{CM}(370 \mathrm{mgl} / \mathrm{mL})$ administration and the other 41 patients were scanned with $80 \mathrm{kVp}$ and $390 \mathrm{mAs}$ eff $_{\text {after }}$ $70 \mathrm{~mL} \mathrm{MC-CM}(300 \mathrm{mgl} / \mathrm{mL})$ administration. Two groups of patients were compared with each other in terms of Hounsfield number of cerebral arteries, signal-to-noise ratio (SNR), contrast-to-noise ratio (CNR), subjective degree of arterial enhancement, image noise, sharpness of arteries, and overall diagnostic image quality of both maximum-intensity projection and volume-rendered images.

Results: Mean attenuations of cerebral arteries, SNR, and CNR were significantly higher in $80 \mathrm{kVp}$ with MC-CM $(390.0 \pm 31.5 \mathrm{HU}, 28.4 \pm 3.7,26.4 \pm 3.7$, respectively) than $120 \mathrm{kVp}$ with $\mathrm{HC}-\mathrm{CM}(280.9 \pm 43.0 \mathrm{HU}, 26.8 \pm 4.5,24.0 \pm 4.4$, respectively $)$. $80-k V p$ protocol had significantly higher quality scores in arterial enhancement, sharpness of the artery, image noise, and overall diagnostic image quality compared to $120-\mathrm{kVp}$ protocol. Effective dose of $80-\mathrm{kVp}$ protocol $(0.7 \pm 0.0 \mathrm{mSv})$ was $26.5 \%$ lower than that of $120-\mathrm{kVp}$ protocol $(0.9 \pm 0.1 \mathrm{mSv})$.

Conclusion: The use of $80 \mathrm{kVp}$ with MC-CM could improve arterial enhancement, $\mathrm{SNR}$, and CNR, and provide superior image quality than $120 \mathrm{kVp}$ with $\mathrm{HC}-\mathrm{CM}$, despite using smaller amount of iodine administration and decreased radiation dose.

\section{B-713 10:48}

Virtual non-contrast images of head $\mathrm{CT}$ angiography by dual-source $\mathrm{CT}$ dual-energy mode: initial experience

D. Han, L. Wen, Y. Xu; Kunming/CN (kmhandan@sina.com)

Purpose: To investigate the clinical value of virtual non-contrast (VNC) images of head CT angiograpgy (CTA) by dual-source dual-energy mode (DECT).

Methods and Materials: For 100 cases including 46 normal and 54 abnormal cases of which 15 were with haemorrhage, 13 infarction, 22 tumour and 4 calcification head CTA by DECT was performed. VNC images were generated from contrast images by DECT and were imported into DE VNC software after real non-contrast scan and DE-CTA. Mean CT values of brain parenchyma, signal-to-noise ratio, image quality, sensitivity of lesion detection and radiation dose were compared between real non-contrast scan and VNC. Results: Image quality was measured by a five-score grading criterion. That the score was greater than or equal to 3 was believed to meet the diagnosing requirement. The score of image quality was statistically significant difference between non-contrast images and VNC images $(P<0.05)$. The image quality in non-contrast scan was better than VNC. However, the scores of all VNC images were beyond 3. (2) Radiation dose of DE head CTA was lower about $9.2 \%, 47.6 \%$ than non-contrast scan and Neruo-DSA (radiation dose of Neuro-DSA for sum on non-contrast and enhanced dose), respectively. (3) Sensitivity of lesion detection showed no significant difference between non-contrast scan and DE-CTA VNC. But tiny calcified lesions might not be demonstrated by VNC. Conclusion: DE head CTA could get head CTA images and cerebral VNC images in one scan and VNC images could meet the diagnosing requirement and significantly decrease the radiation dose.

\section{B-714 10:57}

\section{Detection of aneurysms by 320 -slice volume CT angiography in patients} acutely suspected of having an intracranial aneurysm: feasibility of reducing tube voltage

G. Sun, J. Ding, M. Li, Z.-H. Peng; Jinan/CN (cjr.sungang@vip.163.com)

Purpose: To prospectively assess the feasibility of lower tube voltage ( $80 \mathrm{KV}$ )for detection of intracranial aneurysms by 320 -slice volume CT angiogyaphy.

Methods and Materials: Forty-eight patients with suspected subarachnoid hemorrhages underwent both CTA with 320-slice volume CT and DSA.Patients were randomly assiged to two groups (GA:80 KV, GB:120 KV). Contrast medium was injected automatically with bolus tracking. We compared vascular enhancement,image quality,sensitivity and specificity for detection of aneurysms, and radiation dose in two groups of CTA images. Results: The $80 \mathrm{kV}$ protocol had a higher mean vessel attenuation (42\% to $58 \%$ increase), correlated with a higher image noise and SNR than $120 \mathrm{kV}$, There were no significant differences in subjective image quality between patient group $A$ and $B$ regarding the scores for arterial enhancement, depiction of small arterial detail, interference of venous structures, and overall scores. Mean effective dose for the $80 \mathrm{kV}$ was significantly lower than for the $120 \mathrm{kV}(0.56 \mathrm{mSv} \pm 0.25 \mathrm{vs} 1.84 \mathrm{mSv} \pm 0.002$; $69.73 \%$; $<0.001$ ), With DSA as the standard of reference ,the sensitivity and specificity of group A and group B were $94.12 \%, 100 \%$ and $100 \%, 100 \%$ respectively. There was excellent correlation between the maximum size of each aneurysm on CTA of both groups and 3D DSA.No statistical difference was found between mean maximum diameter measured on CTA of two groups and those measured on DSA images. Conclusion: Using 320-slice MDCT,dignostic image quality can be chieved with $80 \mathrm{kV}$ for detection of intracranial aneurysms, which resulted in significant reduction of effective dose without significant loss of dignostic image quality and accuracy of aneurysm.
B-715 11:06

Radiation dose delivered during one-stop scanning of the nervous system performed on 320-detector row dynamic volume CT

Z. Luo ${ }^{1}$, B. Shen 1 , D. Wang ${ }^{1}$, X. Sun ${ }^{1}$, N. Chan' ${ }^{2}$, H. Zhang ${ }^{3} ;{ }^{1}$ Harbin/CN,

${ }^{2}$ Stanford, CA/US, ${ }^{3}$ Beijing/CN (luozhuren1984@163.com)

Purpose: To determine the radiation dose delivered during a one-stop scanning programme of the nervous system performed on a 320-detector row dynamic volume CT.

Methods and Materials: Patients who were suspected to have suffered acute ischaemic stroke from September 2009 to January 2010 were evaluated using a one-stop scanning programme on a 320-detector CT. Scan plans included NCCT covering the whole brain as a mask image, CT arterial angiography, CT venous angiography and whole-brain perfusion imaging with 19 total exposures. DLP measurements were displayed after each scan on the scanner's console. Radiation exposure was quantified with a DLP conversion factor of $0.0021 \mathrm{mSv}$ - $\mathrm{mGy}-1 \cdot \mathrm{cm}-1$

Results: A total of 45 cases of suspected acute ischaemic stroke were successfully identified using the scanning procedure and used in the analysis of radiation dose. An average DLP covering the entire brain resulted in a dose of $2695.3 \mathrm{mGy} \cdot \mathrm{cm}$ for an average effective radiation dose of $5.66 \mathrm{mSv}$. The effective dose of the NCCT was $0.45 \mathrm{mSv}$. The greatest contribution to the overall dose was the CTA of 3.27 $\mathrm{mSv}$. The average dose generated from CTV was $1.96 \mathrm{mSv}$.

Conclusion: Although the radiation dose was higher than that of brain NCCT scanning, the one-stop scan mode provided significantly more anatomical detail. As it is possible to carry out subtraction in any phase, pure arterial and venous phase images can be obtained. Furthermore, cerebral ischaemia in any part of the brain or functional changes in any region of the brain can be detected.

\section{B-716 11:15}

Evaluation of dose reduction in 64-row CT of the cervical spine with adaptive statistical iterative reconstruction (ASIR) using standard clinical protocols

M. Körner, R. Hempel, S. Wirth, M.F. Reiser, U. Linsenmaier;

Munich/DE (markus.koerner@med.uni-muenchen.de)

Purpose: To evaluate radiation exposure from 64-row CT examinations of the cervical spine using adaptive statistical iterative reconstruction (ASIR) compared to standard filtered back projection (FBP) raw data processing.

Methods and Materials: 64 -row C-spine CT with a standard clinical protocol of 67 studies (mean age $55 \pm 20$ y, 30 males, 37 females) with FBP (LightSpeed VCT XT, GE Healthcare, Waukesha, WI) was retrospectively compared to 80 studies ( $50 \pm 20$ y, 49 males, 31 females) with ASIR (Discovery 750HD, GE Healthcare). The scan parameters for both examinations were identical $(120 \mathrm{kV}$, dose modulation, $0.625 \mathrm{~mm}$ collimation, pitch $0.531: 1$ ) but the noise index ( $\mathrm{NI}$ ) was increased from 5 to 25 with an ASIR level of $30 \%$. From the dose reports generated automatically, the scan length, CTDI, and DLP were recorded. Statistical tests were performed with the Mann-Whitney $U$ test.

Results: In the FBP group, mean CTDI was 21.43 ( \pm 1.43 standard deviation), scan length was $186.30 \mathrm{~mm}( \pm 23.60)$, and DLP was $441.15 \mathrm{mGy}^{*} \mathrm{~cm}( \pm 51.73)$. In the ASIR group, CTDI was $9.57( \pm 3.36)$, scan length was $195.21 \mathrm{~mm}( \pm 26.82)$, and DLP was $204.23 \mathrm{mGy}^{*} \mathrm{~cm}( \pm 68.01)$. The differences were significant for CTDI and DLP $(p<0.001)$ and scan length $(p=0.013)$. Estimation of mean effective dose resulted in $2.38 \mathrm{mSv}$ in the FBP group and $1.10 \mathrm{mSv}$ in the ASIR group. All images were of diagnostic quality as routine clinical acquisition protocols were used. Conclusion: Dose reduction of about $50 \%$ can be achieved with the use of ASIR for 64-row CT of the cervical spine in standard clinical protocols.

\section{B-717 11:24}

Ultrasound-guided pain control in the cervical spine: a prospective randomised clinical trial

A. Loizides, H. Gruber, S. Peer, K. Galiano, J. Obernauer, S. Ostermann; Innsbruck/AT (stefanie.ostermann@i-med.ac.at)

Purpose: Pararadicular and facet-joint injections are widely used for alleviation of cervical pain. Injections are preferentially performed as fluoroscopy or computed tomography (CT)-controlled interventions. Ultrasound (US) provides real-time monitoring, does not produce ionising radiation, and is broadly available.

Methods and Materials: We studied feasibility, accuracy, time-savings, radiation doses, and pain relief of pararadicular ultrasound-guided and CT-controlled facetjoint injections versus CT-controlled interventions in a prospective randomised clinical trial. Sixteen adult patients with chronic cervical pain were consecutively enrolled and evenly assigned to the ultrasound or a CT- group. 
Results: Out of the 16 subjects 6 met after assignment the criteria for the randomised US procedure of the present study. All (US and CT) combined pararadicular and facet-joint injections were performed correctly. The duration of procedure and radiation dose was mean $4.6(1.3-8.4)$ minutes and $22.7(16-40) \mathrm{mGy}^{*} \mathrm{~cm}$, respectively, for the ultrasound-guided CT-controlled group, and 10.4 (5.3-15.5) minutes and 144.8 (56-312) $\mathrm{mGy}^{*} \mathrm{~cm}$ for the CT group. Both groups showed a comparable overall benefit from pararadicular and facet-joint injections.

Conclusion: The US-guided approach to the pararadicular compartment and the facet-joints in the cervical spine is feasible with minimal risks and the results indicate to date comparable pain reduction in the proposed study setting. However, zero radiation by US-guided performance in daily routine must be taken into account additionally.

\section{B-718 $11: 33$}

Apple iPad and emergency CT brain interpretation: a phantom and clinical imaging study

P. Mc Laughlin, S. O Neill, A. Mc Garrigle, J. Coyle, C. Brennan, M.M. Maher; Cork/IE (mclaughlin.paddy@gmail.com)

Purpose: The specifications of the Apple iPad meet ACR criteria for CT image interpretation; namely its display has a spatial resolution of $1024 \times 768$ pixels and it can display 16-bit DICOM images allowing radiologists adjust window width and level. To better quantify the clinical utility of the recently introduced iPad we compared its performance with a 3-megapixel diagnostic-monochrome display using CT-brain datasets and a contrast-detail phantom.

Methods and Materials: 100 consecutive emergency CT-brain examinations were reported in consensus by two general radiologists using the Ipad. The datasets were also reported by one of two neuroradiologists using a PACs workstation. Reporting discrepancies were scored using the ACR Radpeer criteria. The Artinis CDRAD2.0 contrast-detail phantom was used to objectively evaluate the iPad and diagnostic display.

Results: $75 \%$ of the CT datasets were reported in complete agreement. There were 3 clinically significant but understandable misses (Radpeer2b) with the iPad including a cervical fusion anomaly and two cases of early basal ganglia stroke. It is noteworthy that there were 4 clinically significant pickups when reporting with the iPad including parietal and supraorbital fractures (Radpeer3b). No significant difference in CDRAD score was demonstrated between the diagnostic monochrome (169points) and Ipad displays (163points).

Conclusion: There were 3 clinically significant but understandable misses according to ACR criteria. The Ipad radiologists were blinded to previous imaging and clinical information which likely contributed to these discrepancies. No significant difference was demonstrated between displays using a contrast-detail phantom. iPad would appear to represent a very promising tool to facilitate remote review of imaging studies.

\section{B-719 $11: 42$}

Automated slice positioning in MRI: application and reproducibility in brain examinations

L. Gerigk ${ }^{1}$, M. Harder ${ }^{2}$, H.-P. Schlemmer ${ }^{1}$, M. Essig ${ }^{1} ;{ }^{1}$ Heidelberg/DE, 2Erlangen/DE (l.gerigk@dkfz.de)

Purpose: Different slice angulations often lead to limited comparability and inaccurate measurements of brain lesions in follow-up examinations. We evaluated a tool for automated slice positioning in brain MRI.

Methods and Materials: In 51 consecutive patients, a 3D localiser was acquired and retrospectively processed using the algorithm intended for automated positioning. Slices were reconstructed in predefined orientations and visually rated for accuracy, which was classified into optimal (2), acceptable (1) and need for manual intervention (0). For reproducibility, the categories optimal congruence (2), visible deviation but usable (1) and inacceptable (0) were used.

Results: The accuracy of the alignment to the corpus callosum (CC) was optimal or acceptable in all but one patient (medium score 1.4), the resulting axial slice was rated with a medium score of 1.8 . The best reproducibility was achieved parallel to the CC with a score of 1.7 , followed by alignment to the temporal lobe with a score of 1.5 (right) to 1.6 (left). The reproducibility of slices through the internal auditory canal and the optic nerves was acceptable with scores of 1.0 to 1.1. Identified disturbances were temporal lobe lesions, metal artefacts and distortions near the skull base.

Conclusion: Automated slice positioning performs very well in the alignment of slices to the CC, speeding up the examination and reducing variability in follow-up measurements. The good performance in the alignment to the temporal lobe can be useful in the follow-up of MS lesions. A visual check is recommended, especially in patients with larger temporal lobe lesions.
$10: 30-12: 00$ Room P

\section{Physics in Radiology}

\section{SS 1613}

\section{X-ray technologies: assessment, QA and dosimetry}

Moderators:

R. Grimmer; Erlangen/DE

U. Zdesar; Ljubljana/SI

\section{B-721 10:30}

Correlation of contrast-detail analysis and clinical image quality for chest radiography based on a human cadaver study

A. De Crop, K. Bacher, T. Van Hoof, P. Smeets, K. D'Herde, K. Verstraete, H. Thierens; Gent/BE (an.decrop@ugent.be)

Purpose: Contrast-detail studies are often used to evaluate image quality. However, as these phantom models are not related to patient anatomy, it is unclear whether this methodology is appropriate to evaluate clinical image quality and to optimise radiography systems. The aim of this study was to find the correlation between clinical image quality and contrast-detail analysis in chest radiography.

Methods and Materials: Clinical image quality was assessed by means of three Thiel embalmed human cadavers. This technique results in excellent preservation of the flexibility and plasticity of organs and tissues. As a result, lungs could be inflated during image acquisition to simulate the anatomy of a chest $x$-ray. Both CDRAD 2.0 contrast-detail images as chest images of the Thiel bodies were acquired on an amorphous silicon flat-panel detector. Tube voltage, copper filtration and exposure settings were altered in order to simulate different quality levels. Four experienced radiologists assessed the image quality, using a visual grading analysis (VGA) technique based on the European Quality Criteria for Chest Radiology. The CDRAD images were scored manually and automatically using dedicated software (CDRAD analyser 2.1), both resulting in an IQFinv value.

Results: A statistically significant correlation $(r=0.74, p<0.01)$ was observed between the VGA scores and the manually obtained IQFinv values. Comparison of the VGA scores and the automated evaluated CDRAD images showed an even better correlation $(r=0.96, p<0.001)$.

Conclusion: Our results support the value of contrast-detail phantom analysis to evaluate clinical image quality in chest radiography.

\section{B-722 10:39}

Comparison of new digital mammography systems: physical characterisation and image quality evaluation

N. Oberhofer; Bozen/IT (nadia.oberhofer@asbz.it)

Purpose: Recently, several new digital mammography equipments with different innovative features have been proposed on the market. This work compares 7 different systems with respect to physical characteristics, phantom image quality and dose.

Methods and Materials: The studied units are: Fujifilm Amulet (innovative photoconductive switching read-out), GE Senographe DS, Hologic Selenia (old and new target/filter materials), Hologic Dimensions (new filter, new tomosynthesis modality), Sectra Microdose, Siemens Mammomat Inspiration (new active matrix flat panel imager). All system detectors have been characterised in terms of MTF, NNPS and DQE according to IEC 62220-1-2. Image quality has been assessed by means of contrast detail evaluation with the phantom CDMAM ver. 3.4 and the software CDMAM Analyser for automated image analysis using the Inverse Im-

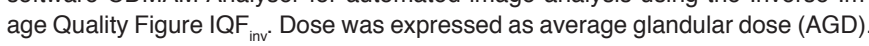
Performances have been evaluated in clinical setup using automatic exposure control (AEC) modality and in optimized setup. Optimization was based on contrast to noise ratio (CNR) measurements.

Results: DQE of all systems ranked between $55 \%$ and $80 \%$, being higher in systems applying direct conversion technology. Generally, a higher DQE reflected in

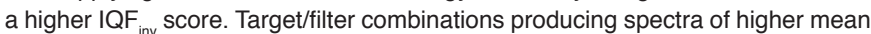
energy confirmed better performances than conventional Mo/Mo ones. Photoconductive switching readout exhibited a potential average $20 \%$ dose saving; also scanning acquisition showed improved image quality at equal dose compared to standard flat panel detectors.

Conclusion: The technological innovations allow better image quality with lower $A G D$, even though the AEC of some system could be further improved. 


\section{B-723 10:48}

Proposed European protocol for the estimation of average glandular dose for digital breast tomosynthesis

D.R. Dance ${ }^{1}$, K.C. Young', R.E. van Engen'; ; Guildford/UK, ${ }^{2}$ Nijmegen/NL (daviddance@nhs.net)

Purpose: There is a need for a European protocol for the estimation of average glandular dose for digital breast tomosynthesis. A protocol is proposed which is an extension of the current European methodology for dosimetry in conventional mammography.

Methods and Materials: The formalism introduces t-factors for single projections and T-factors for a complete examination which are used in conjunction with the current methodology for estimating dose for conventional mammography. The factors were calculated for a wide range of $x$-ray spectra, breast thicknesses and breast glandularities using a Monte Carlo model. Two geometries were considered: one using a fixed detector and the other a scanning narrow beam.

Results: $t$ and T-factors show little dependence on breast glandularity (less than $2 \%)$. For the fixed geometry, tables of t-factors are provided as a function of breast thickness and projection angle, which may be used to calculate $T$ for any selection of projection angles in the range $-/+30$ degrees. Dependence of T on X-ray spectrum and breast thickness is less than $+/ 4 \%$. Typical values of $T$ are in the range 0.94-0.99. For the geometry using a scanned beam, only tables of $\mathrm{T}$ are provided. Conclusion: The protocol is easy to implement and is a natural extension of the methodology currently used for dosimetry in conventional mammography. T-factors for the geometry with a fixed detector are close to 1 . For the same tube loading, the doses for conventional mammography and for breast tomosynthesis will be similar.

\section{B-724 10:57}

Contribution of compression paddle flexibility to estimation of breast glandularity

N. Geeraert, R. Klausz, M. Lemuhot, D. Sundermann, S. Muller; Buc/FR (nausikaa.geeraert@ge.com)

Purpose: Flexible compression paddles are used to improve patient comfort in mammography, but may reduce the accuracy on the compressed breast thickness (CBT). This study investigates if it is acceptable when CBT is used to determine breast glandularity for the automatic optimisation of parameters (AOP) at acquisition. Methods and Materials: The operation over one week of 1016 Senographe DS and essential mammography equipment (GE Healthcare, Chalfont, UK) was sampled using remote access. The parameters retrieved were paddle type, anode/filter, $\mathrm{kV}, \mathrm{mAs}$, detector dose and CBT. The detector dose is determined for the lowest level in the image compatible with a glandular area detected by the AOP from a pre-exposure. For each image the glandularity corresponding to these parameters (peak glandularity) was computed with the same algorithm as the AOP. Two populations were separated depending on the paddle: rigid or flexible, and the distribution of glandularities compared.

Results: Data from 58974 images were collected, with 30227 rigid paddles and 28747 flexible paddles. The difference in the mean peak glandularity between rigid and flexible paddles was found statistically significant: respectively, 38.3\% and $45.7 \%$. For the rigid paddle the $25^{\text {th }}, 50^{\text {th }}$ and $75^{\text {th }}$ percentile of the peak glandularity were $14 \%, 33 \%$ and $57 \%$, respectively, and $23 \%, 42 \%$ and $63 \%$ for the flexible paddle.

Conclusion: Depending on the compression paddle a significant difference was observed for the peak glandularity distribution over a priori equivalent populations. When using the flexible paddle the bias on the peak glandularity is small enough to justify its full usage.

\section{B-725 11:06}

Assessment of dose ratio for digital breast tomosynthesis vs FFDM mammograms for different BI-RADS density categorised breasts: a dose survey using the European dosimetry protocol

B. Østerås, R. Gullien, A. Haakull, P. Skaane; Oslo/NO (sbjo@uus.no)

Purpose: Assess the glandular dose ratio and mean glandular dose for $3 \mathrm{D}$ vs. 2D screening mammograms using the European breast dosimetry protocol, for different BI-RADS density categorised breasts.

Methods and Materials: 126 volunteers underwent 2D and 3D (combo) mammograms (468 combos in total), CC and MLO views. Protocol data were obtained from images. HVL and air KERMA was measured. Dose calculations were done using the European protocol including new proposed T-factors for tomosynthesis. Breast densities were categorised by an expert radiologist. $3 D$ vs. $2 D$ dose ratio was analysed separately for different BI-RADS categories.
Results: $\mathrm{BI}-\mathrm{RADS} 1$ and 2 ( $\mathrm{N}=287$ combo mammograms): Thickness: range 31 to $80 \mathrm{~mm}$, average $58 \mathrm{~mm}$, standard deviation $11.2 \mathrm{~mm}$. Dose ratio: range 0.81 to 2.92, average 1.69 (average mean glandular dose values for 2D and 3D was 1.60 and $2.68 \mathrm{mGy})$, standard deviation 0.40. BI-RADS 3 and $4(\mathrm{~N}=181)$ : thickness: range 17 to $79 \mathrm{~mm}$, average $47 \mathrm{~mm}$, standard deviation $13.4 \mathrm{~mm}$. Dose ratio: 0.68 to 2.11 , average 1.13 (average mean glandular dose values for $2 \mathrm{D}$ and $3 \mathrm{D}$ was 1.77 and $1.93 \mathrm{mGy}$ ), standard deviation 0.27.

Conclusion: Extra dose of doing a 3D compared to a 2D mammogram on this system is lower for dense breasts (BI-RADS 3 and 4) than for less dense breasts (BI-RADS 1 and 2). This should be taken into account when assessing the potential gain of using tomosynthesis in a screening setting

\section{B-726 11:15}

The determination of dose-area-product trigger levels to predict the exceeding of thresholds for deterministic effect at the skin for interventional radiology: TRIR project

L. Struelens ${ }^{1}$, K. Bacher ${ }^{2}$, F. Bleeser ${ }^{3}$, H. Bosmans ${ }^{4}$, E. D'Agostino ${ }^{1}$

M.-T. Hoornaert ${ }^{3}$, F. Malchair ${ }^{5} ;{ }^{1} M o l / B E,{ }^{2}$ Gent/BE, ${ }^{3}$ La Louvière/BE, ${ }^{4}$ Leuven/BE, ${ }^{5}$ Liège/BE (lara.struelens@sckcen.be)

Purpose: The dose to the skin of the patient in interventional radiology/cardiology can sometimes be high, and even get to the dose limits for deterministic effects. In practice, the radiologist/cardiologist has no direct means to know the doses that are given to the skin of the patients during the procedures. The goal of the TRIR project is to verify the correlation between maximum skin dose and DAP values for different interventional procedures. The final goal is to determine specific trigger levels for reaching threshold values of deterministic effects where follow-up of the patient is advisable.

Methods and Materials: Seven different interventional procedures are considered (cerebral embolisation (30 patients), chemo-embolisation (10 patients), radio-frequency ablations ( 30 patients), TIPSS ( 20 patients), embolisation of vena spermatica (40 patients), ERCP (30 patients) and biliary drainages ( 25 patients)) in 11 Belgium hospitals. For each procedure, the DAP value is registered. A sheet filled with up to 50 thermoluminescent dosemeters is wrapped around each patient to measure the procedure-specific maximum skin dose distribution.

Results: Especially for TIPSS, chemo-embolisations of the liver and cerebra embolisations the dose value for deterministic skin damage (2Gy) was attained, with maxima up to 7.5 Gy for TIPSS (trigger value: $35500 \mu \mathrm{Gy} . \mathrm{m}^{2}$ ). For the different procedures, a significant correlation was found between the DAP values and the measured maximum skin doses. However, this correlation is different for different procedures

Conclusion: DAP values are indicative for high skin doses. Procedure-specific trigger levels could be determined, separately for mono-plane and bi-plane systems.

\section{B-727 11:24}

Cumulative ionising radiation exposure in renal replacement therapy patients: a six-year retrospective analysis

J. Coyle, S. Kinsella, S. McCarthy, S. McWilliams, J. Eustace, M.M. Maher; Cork/IE (joecoyle1@gmail.com)

Purpose: To quantify cumulative exposure to ionising radiation in patients with end stage kidney disease (ESKD) and to investigate factors which may be independently associated with risk of high cumulative effective dose (CED) exposure.

Methods and Materials: The study had local institutional review board ethical approval. We conducted a retrospective study of 394 period prevalent ESKD patients attending a single tertiary referral centre between 2004 and 2009. Patien demographics were obtained from case records. Details of radiological investigations were obtained from a central radiology database. CED was calculated using standard procedure specific radiation levels. High exposure was defined as CED>50mSv, an exposure which has been reported to increase cancer mortality by $5 \%$. Data were compared using Pearson Chi-squared and Mann Whitney U test or Kruskal-Wallis tests.

Results: 394 patients were followed for a median of 4 years, (1518 patient years follow-up). Of these $63 \%$ were males. Seventeen per cent of patients had a cumulative effective dose (CED) of $>50 \mathrm{mSv}$. Computed Tomography (CT) accounted for $9 \%$ of total study numbers while contributing $61.4 \%$ of total study dose. Median cumulative dose and median dose per patient year were significantly higher in the Haemodialysis (HD) group (15.13mSv and $5.79 \mathrm{mSv}$ respectively) compared with the transplant group $(2.9 \mathrm{mSv}$ and $0.52 \mathrm{mSv}$ respectively), $\mathrm{p}<0.001$.

Conclusion: Patients requiring renal replacement therapy are at risk of cumulative exposure to significant levels of diagnostic radiation at young age. The majority of this exposure is imparted via CT examinations to patients in the Haemodialysis group. 


\section{B-728 $11: 33$}

Assessment of scatter radiation and over-scanning effect contribution to total effective dose in paediatric cardiac CT examinations

Y. Smal; Erlangen/DE (yulia.smal@imp.uni-erlangen.de)

Purpose: Patient-specific dose calculated by Monte Carlo (MC) techniques and based on available DICOM data is limited by scanned volume of interest. The aim of this study is to evaluate the contribution of dose (DC) received due to the scatter radiation and over-scanning effect to the total effective dose (E) during cardiac examinations in paediatrics.

Methods and Materials: MC simulations were performed for a SOMATOM Definition Flash (Siemens Healthcare, Forchheim, Germany) using a validated dose calculation tool (ImpactMC, CT Imaging GmbH, Erlangen, Germany). Differen cardiac scan protocols were considered by varying pitch $(0.17,0.22,0.3,0.38$ $0.44,0.52$, and 3.4$)$ and tube voltage $(80,100,120,140 \mathrm{kV})$. Organ dose values for a family of anthropomorphic paediatric phantoms mimicking 0-, 1-, 5-, 10-, and 15 -year-old patients were determined. Then, DC in organs of not-prime interest for examination with respect to $E$ was evaluated.

Results: The impact of scatter radiation and over-scanning effect to the overal dose increases with the decrease of age, contributing by 13 and $19 \%$ to $E$ for 15 years old and newborn, respectively. Regarding the gender for each age group the higher DC for male patients, with mean difference of $4 \%$ of $E$ for the same protocol, is to be expected. The results showed that DC slightly increases with the increase of tube voltage.

Conclusion: The scatter radiation and over-scanning should be taken into consideration evaluating effective dose for a particular patient, particularly for younge children. The radiation is used more efficiently at lower tube voltage.

\section{B-729 11:42}

Model and acquisition time-dependent differences in tumour perfusion CT E. Klotz ${ }^{1}$, M. Jürgens ${ }^{1}$, V.J. Goh ${ }^{2} ;{ }^{1}$ Forchheim/DE, ${ }^{2}$ Northwood/UK (ernst.klotz@siemens.com)

Purpose: It is known that the output of commercially available tumour perfusion CT packages is not necessarily directly comparable and acquisition protocol recommendations differ. Our goal was to better understand model-dependent differences between two frequently used commercial implementations.

Methods and Materials: We compared one implementation (MP4) that uses an impulse residue function with 4 independent parameters (scan time recommendation 60s to 120s) with another (MP3) which assumes no backflow from interstitial space ( 3 independent parameters, scan time recommendation $45 \mathrm{~s}$ ). We used measured arterial input functions from an ongoing breast tumour perfusion study and generated a large number of enhancement curves from them by convolution with MP4. The independent parameters were varied such that blood flow (BF), blood volume (BV), flow-extraction product $\left(E^{\star} F\right)$ and permeability-surface area product (PS) all covered the full range typically found for tumours. We then determined these parameters again by deconvolution with MP3 and analysed the differences for $45 \mathrm{~s}$ and 60 s scan time.

Results: 45 s data showed excellent correlation for BF and BV $\left(r^{2}>0.99\right)$, deviation from unity slope was less than $3 \%$. Permeability measures $E^{*} F$ and PS correlated equally well, but absolute differences increased for higher values to $20 \%$. These differences could be completely corrected for by a simple quadratic function. Absolute agreement for $45 \mathrm{~s}$ data was better than for $60 \mathrm{~s}$.

Conclusion: Using the model-specific scan time reommendations BF and BV are directly comparable. Permeability measures can be fully harmonised by a simple conversion function. This should improve comparison of existing studies.

\section{B-730 11:51}

Daily quality controls and cross-calibrations for PET/CT imaging in clinical setting and for clinical trial

C. Jégouic, D.O. Slosman; Geneva/CH (claude.jegouic@qualim.ch)

Purpose: PET/CT metabolic imaging using F-18 FDG is becoming increasingly used in oncology. Standardised uptake value (SUV) that reflects the metabolic activity measured within specific region of interest represents the most frequently used semi-quantitative parameter. Beside the biological variability of this measurement, intrinsic variability is related to performances of the PET/CT scanner and technical apparatus settings. Our aim was to validate a PET/CT cross-calibration protoco and to assess intra- and inter-apparatus reproducibility of SUV measurement. Methods and Materials: Dedicated phantom filled with a standardised FDG solution was used. Reference base-line was calculated. During 7 months, consecutive measurements were performed to monitor and evaluate the long-term intra-apparatus variability $(C V)$ and longitudinal daily quality control (QC). Statistical CUSUM and
Shewhart methods were applied. Automatic calibration correction factors (CCF) were calculated. Cross-calibration protocol was performed with 5 different PET/ CT scanners and validation was performed using SUV of standardised normal tissue of references.

Results: Intra-phantom CV was $0.17 \%(n=30)$ while inter-phantom CV achieved $0.73 \%$. Over a total of 145 measurements, the statistical CUSUM detected 2 significant abnormalities. The Shewhart did not detect significant shift/drift. The cross-calibration protocol demonstrated that even on a homogeneous phantom, in-vitro SUV variability was up to $19 \%$ while in-vivo variability reached $20 \%$. After cross-calibration corrections using CCF, in-vivo variability was reduced to $4 \%$.

Conclusion: These results consolidate the hypothesis that PET/CT scanners require daily quality control as other medical devices do. It also suggests that cross-calibration process is mandatory before comparing SUV measurements between PET/CT scanners or within clinical trials.

10:30 - 12:00 Room Q

Paediatric

\section{SS 1612}

\section{Abdominal and malignant diseases}

Moderators:

C. Balassy; Vienna/AT

L.-S. Ording-Müller; Tromsø/NO

\section{B-731 10:30}

Liver MRI with DWI in children with liver disease

S. Savelli, M. Esposito, A. Ciccarone, M. Di Maurizio, M. Mortilla, C. Fonda; Florence/IT (sarasavelli@hotmail.it)

Purpose: To evaluate the hepatic diffusion coefficient (D), perfusion coefficient $(F)$, fraction of perfusion (f) and ADC in paediatric patients with liver diseases. Methods and Materials: We enrolled 25 paediatric patients with liver diseases at possible fibrotic evolution scheduled for liver biopsy. All patients underwen clinical examination and laboratory tests for assessment of hepatic function and liver MRI. Liver MRI consisted in standard T1- and T2-weighted sequences and diffusion weighted sequences obtained with a total of six global gradients in three directions. $\mathrm{D}, \mathrm{F}$ and $\mathrm{f}$ were obtained, $\mathrm{ADC}$ maps were derived and mean liver ADC value for $b=500$ was calculated. All the results were correlated with histological findings and threshold values were calculated with ROC curve analysis. Statistical analysis was performed.

Results: Liver biopsy demonstrated liver fibrosis in 9 children. MRI with standard sequences showed an abnormal signal intensity in 2 patients. The measured threshold values for D, F, f and ADC value were $765 \mathrm{~mm}^{2} / \mathrm{s}, 70500 \mathrm{~mm}^{2} / \mathrm{s}, 0.2474$ and $1483 \mathrm{~mm}^{2} / \mathrm{s}$, respectively. D was reduced in $8 / 9$ patients with liver fibrosis (Se $88.9 \%$, Sp 92.9\%, p = 0.002), F in 6 (Se 66.7\%, Sp 57.1\%, p = 0.87), f in 7 (Se $77.8, \mathrm{Sp} 50 \%, \mathrm{p}=0.22$ ) and ADC in 4 (Se $44.4 \%$, Sp 57.1\%, p = 0.47).

Conclusion: Our results highlight the possibility to detect hepatic fibrotic changes in patients with liver diseases by means of DWI and, in particular, with D values, earlier than with standard sequences.

\section{B-732 10:39}

Imaging of complications in paediatric liver transplantation: findings in "split liver" transplantation

L. Monti, J. De Ville de Goyet, C. Lo Zupone, F. Gennari, M. Candusso,

F. D’Argento, S. Illuminati, R. Russo, P. Tomà; Rome/lT (cristinalz@yahoo.com)

Purpose: Illustrate the diagnostic role and the clinical contribution of imaging studies for following-up children after liver transplantation (LT).

Methods and Materials: Between 2008 and 2010, 89 LT recipients (age range: 4 months-18 years) (59 patients transplanted in another centre) have been followed at our institution and investigated by either colour Doppler ultrasound (CDU), MDCT, $\mathrm{MRI}$ and/or conventional angiography. Of these patients, 30 received whole liver (W), 57 a segmental (left lateral segment) cadaveric organ graft [using a supra-hilar technique $(S A): n=26$, or a trans-hilar technique $(S B): n=31,2$ received a reduced size graft $(R)$ and 1 a right split lobe $(S R)]$. All patients underwent routine screening using CDU. All patients with abnormal CDU or protocol biopsies, or recurrent abnormal laboratory tests underwent MRI, MDCT, or conventional angiography. Results: CDU was extensively used: 2136 CDU assessments (12/pat/year) out of 2253 studies. CDU was able to confirm either the absence or the type of problem in most cases. Vascular or biliary problems were diagnosed in 16 patients: 11 in SB group (30\%), 1 in SA group (4\%), and 4 in W group (13\%). Portal vein complica- 
tions were the most frequent findings and 5 patients underwent balloon dilatation and/or percutaneous stent placement. Two patients benefited from a meso-Rex bypass to restore portal flow.

Conclusion: 1- CDU plays the leading role for post-transplant assessment and MRI, MDCT and angiography should be reserved to confirm diagnosis before treatment. 2-In this series, a higher incidence of complications was observed after using trans-hilar split technique (SB).

\section{B-733 10:48}

Imaging the Rex recessus vein pre-operatively using wedged hepatic venous portography (WHVP) and Doppler ultrasonography and the role of post-operative Rex shunt surveillance

A. Lawson ${ }^{1}$, P. Rischbieter ${ }^{2}$, A. Numanoglu ${ }^{1} ;{ }^{1}$ Cape Town/ZA, ${ }^{2}$ Nelspruit/ZA (drajlawson@gmail.com)

Purpose: The objective of this study was to assess the efficacy of wedged hepatic venous portography (WHVP) at detecting a patent umbilical segment of the left portal vein (Rex vein) pre-operatively in paediatric patients suffering from extrahepatic portal vein obstruction (EHPVO) who were considered for mesentericoportal bypass. Methods and Materials: A retrospective chart review was done in patients with EHPVO who had been considered for a mesoportal bypass between January 2001 and January 2010.

Results: Sixteen patients (13 boys, 3 girls, mean age 5, range 1-13 years) were considered for mesoportal bypass including four post reduced-size liver transplant patients. Ten patients $(62 \%)$ underwent WHVP. The Rex vein was clearly identified in eight $(80 \%)$ patients. One Rex vein was seen at surgery despite not being demonstrated at WHVP. Six mesoportal bypasses were performed without WHVP of which three $(50 \%)$ were successful. The average shunt sizes on the first postoperative day as documented using Doppler ultrasonography was $7.8 \mathrm{~mm}$ with an average flow of $20.8 \mathrm{~cm} / \mathrm{s}$. The average flow rate increased to $27.1 \mathrm{~cm} / \mathrm{s}$ after one month. All shunt occlusions $(n=1)$ or partial thromboses $(n=2)$ were detected by post-operative Doppler ultrasound.

Conclusion: Our series demonstrates the use of WHVP as an effective tool with a sensitivity of $80 \%$ and specificity of $100 \%$ in the preoperative patency assessment of the Rex vein. Doppler ultrasonography is useful for evaluating the size and flow rates in the post-operative shunt and is sensitive (100\%) to the detection of early shunt thrombosis.

\section{B-734 10:57}

Colour-Doppler ultrasonography (CDU) assessment in children benefiting from the meso-Rex bypass (MRB) for extra-hepatic portal vein thrombosis (EPVT)

C. Lo Zupone, L. Monti, J. De Ville de Goyet, F. Gennari, A. Bertocchini,

F. D’Argento, S. Illuminati, R. Russo, P. Tomà; Rome/lT (cristinalz@yahoo.com)

Purpose: EPVT is a condition in which the obstruction of the portal vein trunk results in non-cirrhotic portal hypertension (pre-hepatic type) with complications ranging from variceal haemorrhage, hypersplenism, biliopathy to growth/development deficiency or subclinical encephalopathy. MRB (a direct bypass from mesenteric to left portal vein) has been shown to restore the physiologic portal flow to the liver. This study intends to evaluate the role of CDU for assessing of patency of the shunt and intra-hepatic portal flow at the long-term.

Methods and Materials: Between 2007 and 2010, 28 children affected by EPTV with recurrent bleeding from oesophageal varices and/or severe hypersplenism (age range: 9 months-18 years) underwent MRB. Intra- and extra-hepatic vascular anatomy was assessed preoperatively using $\mathrm{CDU}, \mathrm{CT}, \mathrm{MRI}$ and retrograde portography. CDU only was used for intra-operative checks, in the early (1 week) (EPO) and in the late post-operative period (LPO). Current follow-up ranges from 1 to 36 months.

Results: Peri-operative flow problems diagnosed at CDU led to successful immediate revision of the shunt in two cases and conversion into a meso-caval shunt in another one. Mean flow velocity in the MRB was $0.40 \mathrm{~m} / \mathrm{s}$ at EPO and $0.35 \mathrm{~m} / \mathrm{s}$ at LPO. At long-term patency of the MRB was $100 \%$ with $26 / 27$ MRB patients showed resolution of CDU signs of portal hypertension. MRB restores normal portal flow through the liver and cures portal hypertension.

Conclusion: CDU alone is adequate to efficiently check bypass patency and portal flow at long-term. Intra- and immediate post-operative CDU monitoring is essential for achieving best clinical results.

\section{B-735 11:06}

Manifestations of small bowel disease in paediatric Crohn's disease on magnetic resonance enterography

M.R. Torkzad ${ }^{1}$, U. Ullberg' ${ }^{2}$, N. Nyström¹, L. Blomqvist², P. Hellström¹,

U.L. Fagerberg'; ${ }^{1}$ Uppsala/SE, ${ }^{2}$ Stockholm/SE (michael.torkzad@radiol.uu.se)

Purpose: Magnetic resonance enterography (MRE) has rapidly gained popularity for imaging in patients with Crohn's disease (CD). We report the manifestations of $C D$ as observed at MRE in a paediatric population at the time of diagnosis of CD. Methods and Materials: MRE of 95 consecutive paediatric patients with suspected or confirmed inflammatory bowel disease (IBD) examined between 2006 and 2009 were retrospectively analysed. The findings were documented based on location of the small bowel disease and additional findings.

Results: 51 were boys and 44 girls (median age 13.7 years). 54 had CD, 31 other forms of IBD, and 10 no IBD. The most common site of small bowel involvement was the terminal ileum in $29(53.7 \%)$ patients, ileum in 10 (18.5\%) and jejunum in 9 $(16.7 \%)$. Solitary jejunal inflammation was seen in two patients $(3.7 \%)$. Two cases with small bowel stenoses leading to surgery were found. Perienteric lymphadenopathy could be seen in $30(55.6 \%)$ patients and fatty proliferation in $9(16.7 \%)$. Fistula formation and abscess/phlegmonous tissue were seen only in one patient each. The most common manifestation of small bowel inflammation was increased contrast enhancement of bowel wall (93.5\%), thickening of the bowel wall (90.3\%) and derangement of bowel shape with saccular formations $(25.8 \%)$.

Conclusion: MRE in the paediatric population often demonstrates acute inflammatory changes of the small bowel such as increased contrast uptake, bowel wall thickening and perienteral lymphadenopathy. More chronic small bowel changes, stenoses leading to surgery and jejunal involvements, are less commonly seen.

\section{B-736 11:15}

Safety of intravesical use of a second generation US contrast agent in children for vesicoureteric reflux detection

F. Papadopoulou' 1 , A. Ntoulia ${ }^{1}$, E. Siomou' ${ }^{1}$, K. Sarantidou' 1 , C. Tsampoulas ${ }^{1}$, K. Darge ${ }^{2} ;{ }^{1}$ loannina/GR, ${ }^{2}$ Philadelphia, PA/US (katerinantoulia@yahoo.com)

Purpose: To evaluate the safety of intravesical administration of a second generation US contrast agent (CA) for the diagnosis of vesicoureteric reflux (VUR) in children.

Methods and Materials: After institutional board review approval and written informed consent of parents 360 children (140 boys, 220 girls, mean age 33.8 months, range $1 \mathrm{~m}-17 \mathrm{y}$ ) underwent voiding urosonography (VUS) to rule out VUR. A second generation CA (SonoVue $\AA$, Bracco, Milan) was administered intravesically through a transurethral bladder catheter. Adverse events were recorded during the examination and also reported by phone call within 7 days thereafter. Urine analysis and culture were performed 3-5 days before and 24-48 hours after the VUS. Results: Minor adverse events were reported in $11(3 \%)$ patients. These included transient abdominal $(n=1)$, urethral pain $(n=6)$, transient macroscopic haematuria $(n=2)$, frequency of micturition $(n=1)$ and one episode of vomiting after 3 hours $(n=1)$. No severe or directly to the CA SonoVue ${ }^{\circledR}$ related adverse event was recorded. Symptoms subsided in the next 24-48 hours. The adverse events did not differ from those reported in VCUG studies and are most likely to be attributed to the catheter placement rather than CA. VUR was detected in 133 patients $(37 \%)$ Conclusion: Only a small percentage of patients experienced minor adverse events during VUS, most likely due to the catheter. The intravesical use of SonoVue ${ }^{\circledR}$ for possible VUR detection in children is a safe procedure.

\section{B-737 11:24}

MRI features and volumetric changes of suprarenal neuroblastoma following (wait and see) protocol of therapy

N.-E.A. Nour-Eldin, A. Ola, N.N. Naguib, T.J. Vogl; Frankfurt a. Main/DE (nour410@hotmail.com)

Purpose: To retrospectively evaluate the MRI features and volumetric changes of neuroblastoma following conservative therapy (wait and see protocol).

Methods and Materials: This retrospective study was approved by the institutional review board; informed consent was waived. The study included 72 patients of suprarenal neuroblastoma of which 10 patients undergone conservative therapy (wait and see protocol) at age of less than 6 months ( 6 females and 4 males) in the time period between January 1997 and January 2010. Initial tumour stage were stage 1: in $40 \%$ (4 out of 10 ), Stage 2 in $30 \%$ (3 out of 10 ) and stage $4 \mathrm{~S}$ in $30 \%$ (3 out of 10). CE-MRI was performed at 3 months interval. Mixed linear modelling and logistic regression were performed including correlation to tumour markers. Results: Mean tumour size at diagnosis was $5.2 \mathrm{~cm}$ in diameter (range 2.5-7.5). Mean tumour volume $55 \mathrm{cc}$ (range: $4.5-220 \mathrm{cc}, \mathrm{SD}$ : 15.5). The median follow-up 
time was 16 months (range 7-30 months). Seventy percent of cases showed spontaneous regression with complete remission after a median time of 6.3 months (range 5-9 months). Those cases with complete remission characterised by well-defined margin and homogenous MRI enhancement and absence of tumour necrosis $(p<0.05)$. Thirty percent showed residual tumour (incomplete remission) after 2 years follow-up in which surgery was indicated. MRI features tumours with incomplete remission were: ill defined margin and heterogenous contrast enhancement. Neither of the cases were associated with deletion of chromosome 1 (p36) nor amplificatrion of MYCN. Tumour markers were normal for all cases with complete remission.

Conclusion: MRI margin definition and tumour enhancement pattern are important imaging parameters to predict low risk suprarenal neuroblastoma response to conservative therapy.

\section{B-738 11:33}

The role of MR DWIBS examination in the imaging of childhood lymphomas

G. Rudas, M. Zsbán, Z. Tóth, L.R. Kozák, T. Györke, M. Csóka; Budapest/HU (grudas@mrkk.sote.hu)

Purpose: Ultrasound, CT, and conventional MRI provide information about tumour morphology, but not about viability. PET/CT has a good sensitivity and specificity to find viable tumours, but it has its limitations. We aimed to investigate whether diffusion-weighted whole-body imaging with background body-signal suppression (DWIBS) is a reliable method to differentiate between tumour and inflammation, and between scar and viable tumour. Moreover, we investigated correlations between CT and MR morphology.

Methods and Materials: 32 pairs of MR DWIBS (65 regions) and PET/CT examinations of 19 children with lymphoma (8 Hodgkin's and 11 non- Hodgkin's) were investigated. Average time between MRI and PET-CT was 4.8 days. MRI was performed at $3 \mathrm{~T}$; the protocol included axial and coronal T2 and 3D DWIBS of the chest and abdomen, and coronal T2 STIR and 3D DWIBS of the neck. The $b$ values for DWIBS acquisition were 0 and 800 .

Results: The correspondence between DWIBS and PET/CT was complete in $21 / 32$, incomplete in $7 / 32$, and conflicting in $4 / 32$ cases regarding viability assessment. Morphological correspondence was complete in $27 / 32$, incomplete in $3 / 32$ and conflicting in $2 / 32$ cases.

Conclusion: DWIBS has good correlation with PET/CT regarding viability assessment. However, the latter tends to result in higher rate of false positives, especially in the axillary and neck lymph nodes. DWIBS can help sorting out such false positives. MRI is also superior to PET/CT regarding morphology. MRI and DWIBS can be the first choice of examination in case of lymphomas while PET/CT can be reserved only for special cases.

\section{B-739 11:42}

Paediatric and adolescent lymphoma: can MR apparent diffusion coefficient (ADC) predict treatment response?

S. Punwani, S. Bandula, P. Humphries, S. Taylor; London/UK

(shonit.punwani@gmail.com)

Purpose: To assess pre-treatment apparent diffusion coefficient (ADC) for predicting treatment response in lymphoma.

Methods and Materials: Thirty-two patients ( $<18$ years) with histological confirmed lymphoma underwent whole-body anatomical MRI and diffusion weighted imaging (DWI) prior to and following treatment between August 2007 and July 2010. For the initial seventeen patients (group 1), the anatomical site of greatest nodal disease was selected at pre-treatment staging for localised DWI $(9.2 \mathrm{~cm}$ tissue block), and reassessed using DWI following 2 cycles of chemotherapy. DWI measurements from group 1 patients were used to derive a relationship between ADC (volume and single-slice) and percentage residual tissue volume following treatment. For the subsequent fifteen patients (group 2) whole-body DWI was performed as part of routine clinical care. The anatomical site post-treatment containing the maximum volume of residual tissue was identified for the assessment of ADC (volume and single-slice) and total volume of disease at pre-treatment, and residual volume of tissue at after treatment. The ability of pre-treatment ADC to predict disease response (based on the correlation established in group 1) was assessed for patients in group 2.

Results: A strong positive linear correlation was present between pre-treatment $A D C$ and percentage residual tissue volume following 2 cycles of chemotherapy (group 1: single-slice R2 $=0.84$, volume R2 $=0.70$ ). Sensitivity and specificity of pre-treatment ADC for predicting treatment response was $100 \%, 100 \%$ and $100 \%$, $92 \%$ for volumetric and single-slice measurements, respectively (group 2).

Conclusion: Pre-treatment ADC correlates positively with residual tissue volume and can predict response to chemotherapy.

\section{B-740 11:51}

Gonad protection in paediatric pelvic x-rays

M.J. Frantzen, L.A. Jacobi, S.G.F. Robben, G.J. Kemerink; Maastricht/NL (m.frantzen.wilmes@mumc.nl)

Purpose: To evaluate the benefit of gonad shielding in the light of attainable dose reduction and image quality.

Methods and Materials: Our study is retrospective and includes 712 examinations of children aged 0 - 10 years, covering the years 2007 and 2008. Positioning of gonad shields was visually evaluated in terms of protection obtained and unwanted coverage of radiologically relevant anatomy. The number of examinations not meeting the criteria was determined. Gonadal and effective doses were calculated using the dose area product.

Results: For girls it is impossible to fully protect the ovaries without eliminating essential image information, because of the varying position of ovaries in the pelvis. Added to this, shielding of the allowed area was poor, often paired to the coverage of relevant anatomy ( $10 \%$ correct). For boys shields were placed correctly in $33 \%$ of all cases. The average unshielded dose to the ovaries was $0.019 \pm 0.009 \mathrm{mGy}$, to the testes $0.086 \pm 0.040 \mathrm{mGy}$, corresponding to a detriment-adjusted hereditary risk of $0.1 \mathrm{E}-6$ and $0.5 \mathrm{E}-6$, respectively. The unshielded effective dose ranged from $0.006 \mathrm{mSv}$ to $0.011 \mathrm{mSv}$, corresponding to 1 - 2 days of natural background. Conclusion: Gonad shielding was poor, as consistently reported in an extensive body of literature, and the associated coverage of relevant anatomy caused suboptimal image quality. After weighting loss of image quality against the very low radiation dose reduction that can be achieved, we decided to discontinue the use of gonadal shields in children.

14:00 - 15:30 Room A

\section{Musculoskeletal}

\section{SS 1710a}

\section{Musculoskeletal intervention}

Moderators

J. Martel; Alcorcón/ES

C. van Rijswijk; Leiden/NL

\section{B-741 14:00}

Ultrasound-guided local anaesthetic hip joint injection as a diagnostic tool for osteoarthritis

P. Yoong ${ }^{1}$, M. Wijeratna ${ }^{2}$, R. Guirguis ${ }^{2}$, M. Porteous ${ }^{2} ;{ }^{1}$ Norwich/UK,

${ }^{2}$ Bury St Edmunds/UK (philipyoong@gmail.com)

Purpose: The diagnosis of hip osteoarthritis is often complicated by co-existing pathology in the knee and spine, and mismatch between clinical and radiologica signs. Temporary pain relief from a local anaesthetic injection into the hip joint has been reported to help localise symptoms, reducing the risk of unnecessary surgery being performed. We hypothesise that good surgical outcome is predicted by significant analgesia following diagnostic injection, and that alternative pathology can be demonstrated when there is no response to injection.

Methods and Materials: Data were analysed from a prospective database of 164 consecutive patients who underwent diagnostic hip injection for possible osteoarthritis. We recorded demographics, symptomatology, result of injection, and whether hip replacement was performed. A good outcome to surgery was defined as subsequent pain relief without significant residual symptoms.

Results: 56 patients had a total hip replacement following a good response to diagnostic injection. Of these 52 had a good outcome following surgery (93\%) $44 / 47$ patients had no surgery following no response to injection. A clear alternative diagnosis to hip osteoarthritis was made in 40 of these patients (91\%): spinal MR showed significant degenerative change in $18 / 19$ patients. There were two postinjection complications: a transient femoral nerve block and an infective arthritis requiring surgical washout.

Conclusion: Diagnostic ultrasound-guided local anaesthetic injection of the hip joint is a useful test in delineating hip pathology. Complete relief of hip pain following intracapsular injection of local anaesthetic predicts a good surgical outcome following joint replacement. 


\begin{abstract}
B-742 14:09
(also

One-year survey of two different ultrasound (US)-guided percutaneous treatments of lateral epicondylitis: results of a randomised controlled trial G. Ferrero', E. Fabbro', A. Muda', L. Sconfienza², E. Silvestri'; ${ }^{1}$ Genoa/lT, 2San Donato Milanese/IT (giulio.fer@libero.it)
\end{abstract}

Purpose: Lateral epicondylitis is a common cause of elbow pain in general population. We show the efficacy of a percutaneous treatment under US guidance in treating this condition.

Methods and Materials: 32 patients ( 18 males, 14 females, mean age $45 \pm 8.6$ ) suffering from lateral epicondylitis underwent an US-guided percutaneous treatment. They were randomly subdivided into two groups. In the first group (16 patients), under local anaesthesia and US guidance, a needle was advanced into the enthesis of the common extensor tendon. There, we performed multiple punctures to obtain a scarification of the enthesis and of the preinsertional portion of the tendon. In a second group (16 patients), an US-guided steroid injection was performed. A visua analogue scale was used to evaluate the degree of pain pre-and post-treatment at 2, 12, 24 and 48 weeks.

Results: In the first group, no significant improvement compared with baseline was found at 2 weeks but was present at 12,24 and 48 weeks ( $<.001$ for all). In the second group, significant improvement compared with baseline was found at 2 weeks $(p<.001)$ but not at 12,24 and 48 weeks. Comparison between the groups showed significantly different outcome in favour of the second group at two weeks $(p<.001)$ and in favour of the first group at 12,24 and 48 weeks $(p<.001)$. Conclusion: US-guided percutaneous dry needling alone is more effective than steroid injection. The efficacy of this treatment seems to be long-lasting. It can be considered as an effective and minimally invasive treatment for lateral epicondylitis.

\section{B-743 14:18}

Efficacy of dry needling for supraspinatus calcific tendonitis

B. Ogunwale ${ }^{1}$, S. Al-Islam¹, D. Young ${ }^{2}$, R. Carter ${ }^{1}$, N.R. Philips ${ }^{1}$, M. Mubashar ${ }^{1}$, W.W.Y. Chiu ${ }^{3}$, H. Benjamin-Laing ${ }^{4}$, W. Bhatti'; ${ }^{1}$ Manchester/UK, ${ }^{2}$ Glasgow/UK, ${ }^{3}$ London/UK, ${ }^{4}$ Liverpool/UK (bamikole_ogunwale@hotmail.com)

Purpose: In chronic supraspinatus clacific tendonitis refractory to conservative treatment, a number of non-surgical options are available. Most of these interventions incorporate needling of the calcific deposit as part of the procedure. However, an evaluation of needling without further intervention, e.g. attempting to aspitrate the deposits as in barbotage, has not been undertaken. This was recently highlighted by the National Institute of Clinical Excellence in England and Wales in its assessment of autologous blood use where dry needling often forms part of the procedure, and as such calls into question what is yielding the improvement in these procedures. We undertook a study to determine whether dry needling on its own would be of benefit to these patients.

Methods and Materials: Patients who had ultrasound guided dry needling for supraspinatus calcific tendonitis between April 2008 and February 2010 at South Manchester University Hospital were assessed using the Oxford shoulder score. Results: 31 patients were identified: 6 non-contactable, 1 unable to understand questionnaire and excluded. There was a $79 \%$ improvement demonstrated, which compares with an average of $60 \%$ with other interventional procedures utilised for this condition, including those that incorporate dry needling. There was an average increase in Oxford shoulder score of $17 \mathrm{p}<0.001,95 \% \mathrm{Cl}(12.0,23.0)$. The average pre-procedure (16) score is regarded as requiring surgical intervention, whilst the average post procedure score (33) is regarded as requiring lifestyle changes such as exercise.

Conclusion: The procedure was effective at relieving shoulder symptomatology from surpraspinatus calcific tendonitis and avoiding surgery.

\section{B-744 14:27}

Optimal injection site in shoulder MR arthrography: anterior or posterior approach?

M.G. Yilmabasar, B. Pekar, K. Akan, A. Hayirlioglu; Istanbul/TR

(yilmabasar@gmail.com)

Purpose: The purpose of our study was to optimise shoulder MR arthrography by comparing the anterior with the posterior ultrasound-guided arthrographic injection approach.

Methods and Materials: Thirty-nine patients were divided into two groups, according to the injection site selected: seventeen were injected using the anterior approach (just superior to subscapularis tendon, in external rotation), twenty-two were injected using the posterior approach (just superior to infraspinatus tendon, in internal rotation). The presence of enough intra-articular contrast material for interpretation, extra-articular leakage of contrast material and duration of procedure were recorded and the intensity of the patient's pain was measured using a visual analogue scale.

Results: Intra-articular contrast material was satisfactory for interpretation in $94.8 \%$ of all applications, without any significant difference between anterior and posterior application. Mean duration of procedure was $444 \pm 128$ (A490 $\pm 190, P 372 \pm 77)$ seconds and mean pain intensity was $4.6 \pm 0.7$ (A4.9 \pm 1.5 , P3.3 \pm 0.4$)$, contrast material leakage to extra-articular space was detected in seventeen $(A: 11, P: 6)$ cases; differences between the two applications were statistically significant $(p<0.005)$ Conclusion: MRI arthrography of the shoulder is known to increase diagnostic accuracy in comparison with conventional MRI. Especially in patients with sus pected anterior instability, the contrast injection using posterior approach has the advantage to avoid interpretation difficulties due to contrast extravasation which is more possible in anterior approach. In our clinic, the posterior approach is assumed as optimal injection site for MR arthrography, because it is less likely to cause interpretation difficulties due to contrast leakage. Moreover, the posterior approach is more tolerable to patients, easier to perform for the operator.

\section{B-745 14:36}

MR-guided biopsy of soft tissue tumours at 3 Tesla-MRI: site selection by the use of dynamic contrast enhanced MR imaging (DCE), multivoxel ${ }^{1} \mathrm{H}$ MR spectroscopy ( $\left.{ }^{1} \mathrm{H}-\mathrm{MRS}\right)$, and diffusion weighted imaging (DWI) I.-M. Noebauer-Huhmann, M. Krssak, G. Amann, J. Panotopoulos, P. Funovics, F. Kainberger, S. Trattnig; Vienna/AT (iris.noebauer@meduniwien.ac.at)

Purpose: To optimise MR-guided biopsy of soft tissue tumours, by selecting the biopsy region with the use of Multivoxel ${ }^{1} \mathrm{H}-\mathrm{MR}$ spectroscopy ( $\left.{ }^{1} \mathrm{H}-\mathrm{MRS}\right)$, diffusionweighted imaging (DWI) and a dynamic contrast enhanced sequence (DCE).

Methods and Materials: In 20 patients with suspected soft tissue tumours, preoperative MR-guided biopsy by the use of functional sequences was prospectively performed at 3 Tesla with a flexible surface coil. In addition to the routine sequences, in 18 patients, a DCE sequence was conducted over $3 \mathrm{~min}$, with a temporal resolution of $7.3 \mathrm{sec}$ during the first $72 \mathrm{sec}$, followed by $15 \mathrm{sec}$ image intervals. In 17 patients, DWI was applied with the use of DWI-MSh FH (b-value: 0-800), and the apparent diffusion coefficient (ADC) was calculated. Multivoxel ${ }^{1} \mathrm{H}$-spectroscopy could be conducted in 13 patients with sufficient tumour size.

Results: In 12/17 patients with heterogeneous tumours, DCE was available. The biopsy area was chosen at the site of the highest slope in the contrast enhancement curve, which corresponded with the maximal $\mathrm{Cho} / \mathrm{Cr}$ ratio in $8 / 11$ patients. The diffusion weighted sequence was of limited value for the selection of the biopsy area. In all patients, the biopsy was successful, in 19/20 patients, the highest mitotic rate was hit. The final histopathological results of the surgical specimen confirmed the diagnosis of 10 malignant tumours of different grades, 7 benign entities and 3 tumours of intermediate dignity.

Conclusion: Dynamic contrast-enhanced MR imaging and multivoxel ${ }^{1} \mathrm{H}-\mathrm{MR}$-spectroscopy are valuable tools for the selection of the biopsy site in MR-guided biopsy.

\section{B-746 $\quad$ 14:45}

Which is the more effective for the treatment of low back pain: intra-facet or peri-facet joint injection? A blinded randomised controlled trial F.W. Smith, A. Nandakumar, D. Wardlaw; Aberdeen/UK (franciswsmith@hotmail.com)

Purpose: There is controversy regarding the efficacy of facet joint steroid injections for the treatment of low back pain. This study is designed to identify any difference in clinical outcome between intra-facet (IF) and peri-facet (PF) injections in patients with low back pain (LBP). The difference in screening time and radiation dose, between the two techniques, are also recorded.

Methods and Materials: 88 patients were randomised into IF and PF groups. For the IF group bilateral L4/5 and L5/S1 levels were injected with $40 \mathrm{mg}$ of DepoMedrone with Lignocaine (total $2 \mathrm{ml}$ ). The PF group received $80 \mathrm{mg}$ of DepoMedrone with $1 \mathrm{ml}$ of $0.5 \%$ Marcaine (total $3 \mathrm{ml}$ ) at the same sites. Pain visual analogue score (VAS) and analgesic chart were completed weekly for six months. Results: 88 patients were entered into the study and 14 withdrew leaving 69 Sixty-three $(91 \%)$ had significant pain relief a week following injection, $86 \%$ and $94 \%$ in IF \& PF groups. The mean duration of pain relief was 12.4 weeks (IF-12.2, $\mathrm{PF}-12.5)$. The mean change in VAS before and after injection was 4 (IF-4, PF-4). Mean change in analgesic use before and after injection was two tablets. (IF-2.9, PF-2.3). The radiation dose area product (DAP) for IF injections was $603 \mathrm{mGy}$. $\mathrm{cm} 2$. compared with $45 \mathrm{mGy} . \mathrm{cm} 2$ for the PF group.

Conclusion: Both techniques afford significant pain relief (91\%), (PF $94 \%$ vs. IF $86 \%)$. PF is easier to perform at a lower radiation dose and is the preferred technique. 


\section{B-747 14:54}

Facet joint injections as a means of reducing the need for vertebroplasty in insufficiency fractures of the spine

D.J. Wilson, S.E. Owen, R.A. Corkill; Oxford/UK (djwilson@herald.ox.ac.uk)

Purpose: Two papers in the New England Journal (August 2009) compared treatment of vertebral fractures in randomised controlled trials reporting improvement in the majority of patients but with no significant difference between the local anaesthetic and vertebroplasty groups. Possible explanations include placebo response or therapeutic response to the "control procedure". Pain associated with osteoporotic compression fractures may be due to factors other than the fracture. Methods and Materials: Suitable patients referred for treatment by vertebroplasty were first offered a local anaesthetic and steroid facet joint injection at the most painful level; a two-week pain diary was employed. Those who failed to respond were offered a vertebroplasty.

Results: Of 91 patients referred, 16 went straight to vertebroplasty (13 successful). 61 out of the remaining 75 were offered facet joint injection as initial treatment. 21 were successful, 2 relapsed and had further facet injections with good results, 3 elected not to have treatment, 5 had temporary benefit and were offered rhizolysis and 1 died from unrelated causes. Of the 29 who failed to respond to facet injections, 24 underwent vertebroplasty (23 successful).

Conclusion: Around a third of patients referred for treatment of osteoporotic fractures by vertebroplasty responded beneficially to facet joint injection. It is possible that in this group the pain mediator is one of instability and overload on the face joints produced by adjacent wedge fracture. This protocol allows a reduction in the number of vertebroplasties undertaken with enhanced success rate from those vertebroplasties that are performed.

\section{B-748 15:03}

\section{Are we using discography appropriately?}

C.L. Gan, D. Lloyd; Cardiff/UK (cheelingan@yahoo.com)

Purpose: Discography is often used in conjunction of MRI findings to aid diagnosis of symptomatic lumbar disc degeneration. Positive discography with degenerate discs on MRI can help to differentiate between symptomatic and asymptomatic lumbar disc degeneration. Although discography is relatively safe, it is invasive and painful. We aim to investigate the appropriate use of discography in a U.K. tertiary centre.

Methods and Materials: All the discography requests within Cardiff \& Vale Healthcare NHS Trust from 01/11/2008 to 30/04/2009 were studied retrospectively. Electronic letters and test results from Clinical Workstation were reviewed to ascertain the indication for request and management plan following the procedure. Results: 73 requests were received in total. 42 patients had positive discography finding, whereas 21 were negative, 1 was inconclusive and the remaining 9 were abandoned either because of technical difficulty or patients' intolerance to procedure. All patients in the positive discography group had positive MRI correlation. However, only $55 \%$ of these patients either had operation or on surgical waiting list. $36 \%$ either were not offered operations or declined operations. Patients' decision towards operation was still awaited in the remaining $9 \%$.

Conclusion: Not all patients with positive discography had surgery. Possible implications following either positive or negative discography finding should be discussed with patients prior to referring for discography. This can help to prevent the patients with no intention for operation from going through this invasive and potentially painful procedure. The findings and recommendations can also apply to other hospitals in order to improve practice.

\section{B-749 $\quad 15: 12$}

Biomedical assessment of different bone biopsy needles

A.H. Mahnken, A. Keulers, V.C. Vargas da Silveira Cunha Cruz, P. Bruners,

C. Kuhl, T. Schmitz-Rode; Aachen/DE (mahnken@rad.rwth-aachen.de)

Purpose: To quantitatively analyse if bone biopsy needles differ in mechanical properties, subjective handling, signs of wear and tear and specimen quality. Methods and Materials: 19 different bone biopsy systems (total 38; 2/type) were examined. With each biopsy needle five consecutive samples were obtained from vertebral bodies of swine. The overall evaluation considered mechanical properties, subjective handling and sample quality. During puncture a force-torques sensor measured the mechanical properties at different stages of the procedure $(1=$ passage of cortical bone, 2 = bone sampling, $3=$ extraction of the needle). Before and after each biopsy each needle was investigated using a profile projector and signs of wear and tear were recorded. The samples were semi-quantitatively examined regarding sample quality.
Results: Measured forces showed remarkable variations in between the different biopsy systems (phase 1: - $55.99 \mathrm{~N} /-94.72 \mathrm{~N}$; phase 2: $-96.67 \mathrm{~N} /-145.64 \mathrm{~N}$; phase 3: $55.01 \mathrm{~N} / 81.55 \mathrm{~N}$ ). Increasing needle diameter correlates with expanding forces (Spearman correlation: $p=0.464$ ). In general, trokar cut required less force than mandrin design. Besides, there was a notable distinction in needle quality with length reduction as a surrogate marker of wear and tear varying from $0.025 \mathrm{~mm}$ to $0.556 \mathrm{~mm}$ depending on the type of needle.

Conclusion: Bone biopsy needles vary significantly in performance. Needle design has an important influence on mechanical properties, handling and specimen quality. Detailed knowledge of those parameters will help in selecting the appropriate bone biopsy needle.

\section{B-750 15:21}

Cervical infiltrations under CT guidance: potential risks (a 10-year experience)

D. Krausé, R. Loffroy, J.-M. Lerais, J.-P. Cercueil, B. Guiu; Dijon/FR (denis.krause@chu-dijon.fr)

Purpose: To demonstrate through a rigorous technique, the total safety of foraminal cervical infiltrations, for the patient in the case of a cervicobrachial neuralgia, due to a disk fragment.

Methods and Materials: January 2000-June 2010: To underline a 10-year experience in 450 patients treated by in situ corticoids infiltration under CT guidance, in the medial part of the foramen, just behind the ganglion. Systematic analysis with contrast $(0.5 \mathrm{ml})$ of the presumed diffusion space under $\mathrm{CT}$ control, before corticoids injection, and identification of an eventual vascular leak, either within the subarachnoid spaces, or within the synovial articular capsule.

Results: The venous vascular contamination represents the most important risk: contrast passage within the foraminal, intracanalar epidural vein, or also through the muscular deep veins, distant from the cervical canal (14\%, 64/450): CT depiction of abnormal contrast stagnation in a homo- or contra-lateral side. The total absence of any visible contrast during $\mathrm{CT}$ control must evoke a radiculo-medullary artery contamination, a very rare condition $(1.3 \%, 6 / 450)$. In all cases, the needle must absolutely be repositioned, with a new contrast control. The contrast injection of the articular capsule is very frequent, without any danger $(18-20 \%, 81-90 / 450)$. The reach of a radicular meningeal sheath is exceptional. No neurological complications were observed in this 10-year series.

Conclusion: The good knowledge of the presumed diffusion space (contrast) is absolutely necessary, routinely, in case of cervical infiltration with corticoids. The vascular contamination risks are the most dangerous and have to be immediately recognised before the corticoids injection.

\begin{tabular}{ll}
\hline 14:00 - 15:30 & Room D1 \\
\hline
\end{tabular}

Chest

SS 1704

CT angiography of the chest: dual energy and beyond

Moderators

N. Tacelli; Lille/FR

E.J.R. van Beek; Edinburgh/UK

\section{B-751 14:00}

Sensitivity of CT pulmonary angiography: intraindividual comparison of normal- and low-dose protocols in an experimental study Z. Szucs-Farkas ${ }^{1}$, F. Schibler', J. Cullmann' ${ }^{1}$, J. Charimo Torrente', M.A. Patak', S. Raible ${ }^{2}$, P. Vock 1 , S.T. Schindera'; ${ }^{1}$ Berne/CH, ${ }^{2}$ Biel/CH (zsolt.szuecs@insel.ch)

Purpose: To analyse the diagnostic accuracy of CT pulmonary angiography (CTPA) for the detection of pulmonary emboli (PE) with standard and low-dose protocol using reduced radiation and contrast material (CM) dose.

Methods and Materials: Normal-dose CTPAs using $120 \mathrm{kVp}$ and $100 \mathrm{~mL} \mathrm{CM}$ in 10 consecutive patients with no PE were retrospectively selected. The image characteristics of a $80 \mathrm{kVp}$ low-dose CTPA protocol (patient exposure reduction, $57 \%$ ) with $75 \mathrm{~mL} \mathrm{CM}$ were simulated. Four different sets of filling defects were visually simulated in identical locations in each normal-dose and corresponding low-dose series, equaling $783 \mathrm{PE}$ in 40 normal- and 40 low-dose datasets. Twenty series contained no emboli and were used as controls. The 100 CTPA studies were randomly assessed by 3 blinded readers for PE location and image quality. Results were assessed by non-parametrical and t-tests. 
Results: No difference was found between the CT protocols in sensitivity, specificity, positive and negative diagnostic likelihood ratios at all ramification levels of the pulmonary arteries ( $\mathrm{P}$ between.343 and 1 ). The overall sensitivity and specificity with the normal and simulated low-dose protocol were $79.9 \%$ vs $81.3 \%$ and $98.0 \%$ vs $98.2 \%(P=.444$ and .702$)$, respectively. The diagnostic confidence $(2.81 \pm .39$ vs $2.77 \pm .47 ; P=.297)$ and overall image quality (3.92 \pm .52 vs $3.83 \pm 0.54 ; P=.216)$ were similar at $120 \mathrm{kV}$ and $80 \mathrm{kV}$.

Conclusion: The intraindividual comparison of the diagnostic accuracy with normal-dose and simulated low-dose CTPA protocols revealed no difference under experimental conditions.

\section{B-752 14:09}

High-pitch dual-source CT angiography of the chest: is coronary artery imaging accessible from routine nonelectrocardiographically gated examinations?

M. De Malherbe, M. Rémy-Jardin, A.-L. Hachulla, F. Pontana, J.-B. Faivre, J. Rémy; Lille/FR (martine.remy@chru-lille.fr)

Purpose: To evaluate the assessibility of coronary arteries from standard chest CT angiographic examinations acquired with a high-pitch mode.

Methods and Materials: 242 patients underwent a nongated CT angiographic examination of the chest with dual-source, single-energy CT obtained with a pitch of 3 and a temporal resolution of $83 \mathrm{~ms}$ (prototype version; group $1 ; n=123$ ) or $75 \mathrm{~ms}$ (commercial version; group $2 ; \mathrm{n}=126$ ).

Results: The mean duration of data acquisition and mean dose-length-product were $1.49 \pm 0.42 \mathrm{~s}$ and $136.9 \pm 49.8 \mathrm{mGy} . \mathrm{cm}$, respectively. The median value of the heart rate was $81.7 \pm 15 \mathrm{bpm}$. The percentage of assessable segments was $88 \%$ at the proximal level (i.e., 4 segments), $75 \%$ at the proximal and mid-segment level (i.e., 7 segments), $61 \%$ and $48 \%$ when considering 10 and 15 segments, respectively. The mean $( \pm S D)$ number of assessable segments per patient was $3.5 \pm 0.78$ and $5.2 \pm 1.50$ when considering 4 and 7 segments per patient, respectively. The percentage of patients with 4 segments assessable was $67 \%(126 / 242)$, decreasing to $23 \%$ (55/242) with 7 segments assessable and $3 \%(7 / 242)$ with 10 segments assessable while no patient's entire coronary artery tree was assessable. No statistically significant difference was observed when comparing group 1 and group 2 patients.

Conclusion: Diagnostic image quality is attainable at the level of proximal and mid-coronary segments in $23 \%$ of patients with a dual-source, high-pitch scanning mode despite the absence of ECG gating and elevated heart rates.

\section{B-753 14:18}

Triphasic contrast injection improves evaluation of dual energy lung perfusion in pulmonary CT angiography

J.M. Kerl, R.W. Bauer, M. Renker, P. Weisser, B. Schell, T.J. Vogl;

Frankfurt a. Main/DE (matthias.kerl@kgu.de)

Purpose: Lung perfusion analysis in DECT is sensitive to beam hardening artefacts from dense contrast material. We compared two scan and four CM injection protocols in terms of severity of artefacts and attenuation levels in the thoracic vessels. Methods and Materials: Data of 120 patients who had undergone DECT pulmonary angiography were evaluated. Group $1(n=30)$ was scanned in a craniocaudal scan direction versus groups $2-4(n=30$ each) were scanned in a caudocranial scan direction. In group 1 - 3 biphasic injection protocols with different amounts of CM and $\mathrm{NaCl}$ were investigated. In group 4 a triphasic protocol with an initial $\mathrm{CM}$ bolus followed by a $\mathrm{NaCl} / \mathrm{CM}$ mixture and $\mathrm{NaCl}$ chaser bolus was used. Attenuation in the subclavian vein, superior vena cava and pulmonary artery tree was acquired. Artefacts on DE iodine distribution map were rated on a scale from 1 to 5 by two blinded readers in consensus.

Results: In protocol 4 mean attenuation in the SV $(645 \pm 158 \mathrm{HU})$ and SVC $(389 \pm 114 \mathrm{HU})$ was significantly lower compared with groups $1-3(p<0.002)$. Artefacts in group $4(1.1 \pm 0.4$ and $1.5 \pm 0.7)$ were rated significantly less severe compared with all other groups for the subclavian vein and SVC $(p<0.01)$. Attenuation in the PA was maintained on a diagnostic level in all 4 groups.

Conclusion: Split-bolus injection provides sufficient attenuation for pulmonary DECT angiography while beam hardening artefacts arising from high-density contrast material in the thoracic vessels can generally be avoided
B-754 14:27

Dual-energy CT pulmonary angiography of pulmonary embolism comparison of image quality and clot visualisation at $80 \mathrm{kvp}, 140 \mathrm{kvp}$ and weighted average images

L. Lu, L. Zhang, G. Lu, C. Zhou, Y. Zhao, S. Luo; Nanjing/CN (xuzhoululi@163.com)

Purpose: To quantitatively evaluate enhancement of pulmonary arteries (PA) and visualise pulmonary clots at different tube voltage settings at dual-energy CT (DECT). Methods and Materials: Sixty-six patients underwent DECT pulmonary angiography (CTPA), which was reconstructed into $80 \mathrm{kVp}, 140 \mathrm{kVp}$ and weighted average $120 \mathrm{kVp}$ images, respectively. CT value of $1^{\text {st }} 4^{\text {th }}$ branch of PA, pulmonary clots and back muscles were measured, and corresponding signal-to-noise ratio (SNR) and contrast-to-noise ratio (CNR) were calculated. Image quality of CTPA from three groups rated as 1-5 score was visually assessed by two chest radiologists. Results: There are significant differences for CT value, noise, SNR and CNR of PA enhancement $(\mathrm{P}<0.001)$ among three groups; CT value of PA enhancement in $80 \mathrm{kVp}$ images ( $1^{\text {st }}-4^{\text {th }}: 446.22 \pm 140.48 \mathrm{HU}, 432.89 \pm 129.59 \mathrm{HU}, 410.57 \pm 138.28 \mathrm{HU}$ $391.78 \pm 127.45 \mathrm{HU})$ was higher than that of $140 \mathrm{kVp}\left(1^{\text {st }}-4^{\text {th }}: 243.72 \pm 69.55 \mathrm{HU}\right.$ $229.62 \pm 63.44 \mathrm{H}, 216.40 \pm 71.55 \mathrm{HU}, 204.78 \pm 67.88 \mathrm{HU})$ and $120 \mathrm{kVp}\left(1^{\text {st }}-4^{\text {th }}\right.$ : $302.91 \pm 91.18 \mathrm{HU}, 290.42 \pm 85.01 \mathrm{HU}, 275.59 \pm 85.64 \mathrm{HU}, 263.32 \pm 84.54 \mathrm{HU})$, but with lower CNR $\left(1^{\text {st }}-4^{\text {th }}: 7.46 \pm 2.22,6.47 \pm 1.87,6.45 \pm 2.33,5.57 \pm 2.64\right)$ and SNR $\left(1^{\text {st }}\right.$. $\left.4^{\text {th }}: 8.34 \pm 2.19,7.25 \pm 1.81,7.31 \pm 2.37,6.35 \pm 2.76\right)$ than $140 \mathrm{kVp}$ with lowest CNR $\left(1^{\text {st }}-4^{\text {th }}: 5.54 \pm 2.03,4.67 \pm 1.49,4.93 \pm 2.06,4.12 \pm 2.19\right)$ and SNR $\left(1^{\text {st }}-4^{\text {th }}: 6.88 \pm 1.99\right.$ $5.91 \pm 1.44,6.33 \pm 2.07,5.38 \pm 2.42)$. CNR of pulmonary clots in $80 \mathrm{kVp}(8.30 \pm 3.26)$ was higher than that in $140 \mathrm{kVp}(5.56 \pm 2.24)$ and $120 \mathrm{kVp}(7.98 \pm 2.90)(\mathrm{P}<0.001)$ Image quality of CTPA derived from $80 \mathrm{kVp}(\mathrm{Kappa}=0.789)$ was superior to that of $140 \mathrm{kVp}(\mathrm{Kappa}=0.509)$ and $120 \mathrm{kVp}(\mathrm{Kappa}=0.652)(\mathrm{P}<0.05)$.

Conclusion: Image quality of $80 \mathrm{kVp}$ is superior to that of weighted average 120 and $140 \mathrm{kVp}$ for visualisation of pulmonary clots.

\section{B-755 14:36}

Dose optimisation at dual energy CT pulmonary angiography: a comparative study

R.W. Bauer, J.M. Kerl, S. Kramer, M. Renker, T. Lehnert, B. Schell, V. Jacobi, T.J. Vogl; Frankfurt a. Main/DE (ralfwbauer@aol.com)

Purpose: To compare dose and image quality of 64-slice, first and second generation dual energy CT (DECT) for CT pulmonary angiography (CTPA)

Methods and Materials: Each group consisted of 30 patients. Group 1 (single energy, $64 \times 0.6 \mathrm{~mm}, 120 \mathrm{kV} / 145 \mathrm{mAs}$ ) and group 2 (DE, $14 \times 1.2 \mathrm{~mm}, 140 / 80 \mathrm{kV}$ with $70 / 350 \mathrm{mAs}$ ) underwent CTPA on dual source dual energy scanner of the first generation (Definition, Siemens), group 3 (DE, $64 \times 0.6 \mathrm{~mm}, 100 / 140+S n$ filter kV with 120/102 mAs) and 4 (DE, $64 \times 0.6 \mathrm{~mm}, 80 / 140+S n$ filter $\mathrm{kV}$ with 202/86 mAs) on a scanner of the second generation (Flash, Siemens). Automated tube current modulation was used in all groups. CTDIvol, DLP, background noise (BN), thorax diameter and attenuation in the pulmonary trunk were compared.

Results: Thorax diameter and attenuation in the pulmonary trunk did not differ significantly $(p>0.4$ and $>0.19)$ between the groups. Mean CTDIvol and DLP were $8.5 \pm 2.6 \mathrm{mGy} / 235 \pm 117 \mathrm{mGycm}, 9.2 \pm 3.3 \mathrm{mGy} / 224 \pm 122 \mathrm{mGycm}$ $8.7 \pm 2.8 \mathrm{mGy} / 246 \pm 86 \mathrm{mGycm}$ and $6.2 \pm 1.6 \mathrm{mGy} / 170 \pm 41 \mathrm{mGycm}$ for groups $1,2,3$ and 4, respectively. Dose parameters were significantly lower in group 4 compared to groups $1-3(p<0.02)$ with no difference between groups $1-3$. BN was significantly lower $(p<0.01)$ in group $4(12 \pm 3 \mathrm{HU})$ and group $1(13 \pm 6 \mathrm{HU})$ compared to groups 3 and $2(16 \pm 6 \mathrm{HU}$ and $23 \pm 9)$.

Conclusion: On CTPA with a DECT scanner of the second generation noise levels are comparable to a $120 \mathrm{kV}$ protocol, while dose can be significantly reduced with a $80 \mathrm{kV} / 140 \mathrm{kV}+\mathrm{Sn}$ filter configuration.

\section{B-756 14:45}

Chest computed tomography using iterative reconstruction vs filtered back projection: image quality of low-dose CT examinations in 80 patients J. Pagniez, F. Pontana, J.-B. Faivre, A.-L. Hachulla, M. Rémy-Jardin, J. Rémy; Lille/FR (martine.remy@chru-lille.fr)

Purpose: To evaluate the image quality of an iterative reconstruction algorithm (IRIS) in low-dose chest CT in comparison with standard-dose filtered back projection (FBP) CT.

Methods and Materials: 80 consecutive patients referred for a follow-up CT examination of the chest, underwent a low-dose CT examination (group 2) in similar technical conditions to those of the initial examination (group 1) except for the milliamperage selection and the replacement of regular FBP reconstruction by iterative reconstructions using 3 (group 2a) and 5 iterations (group 2b). 
Results: Despite a mean decrease of $35.5 \%$ in the dose-length-product, there was no statistically significant difference between group 2a and group 1 in the objective noise, signal-to-noise (SNR) and contrast-to-noise (CNR) ratios and distribution of the overall image quality scores. Compared to group 1 , objective image noise in group $2 b$ was significantly reduced with increased SNR and CNR and a trend towards improved image quality.

Conclusion: Iterative reconstructions using 3 iterations provide similar image quality compared with the conventionally used FBP reconstruction at $35 \%$ less dose, thus enabling dose reduction without loss of diagnostic information. According to our preliminary results, even higher dose reductions than $35 \%$ may be feasible when using more than 3 iterations.

\section{B-757 14:54}

Dual-source nongated CT angiography of the chest: impact of high temporal resolution and high pitch mode on cardiogenic motion artefacts C. Darchis, N. Tacelli, J.-B. Faivre, T. Santangelo, M. Rémy-Jardin, J. Rémy; Lille/FR (martine.remy@chru-lille.fr)

Purpose: To evaluate the frequency and severity of cardiogenic motion artefacts on dual-source computed tomography (CT) angiograms of the chest acquired with a high-pitch and high-temporal resolution scanning mode.

Methods and Materials: 100 consecutive patients underwent nongated chest CT angiography with dual-source, single-energy CT without administration of betablockers (temporal resolution: $75 \mathrm{msec}$ ). The presence, location and severity of cardiogenic motion artefacts were evaluated at the level of mediastinal vessels, central and peripheral pulmonary arteries, heart borders and lung parenchyma.

Results: The mean $( \pm \mathrm{SD})$ duration of data acquisition and heart rate were 0.9 $( \pm 0.07) \mathrm{s}$ and $78.9( \pm 15.2) \mathrm{bpm}$, respectively. Cardiogenic motion artefacts were depicted at the level of the ascending aorta in 4 patients (4\%) and pulmonary trunk in 2 patients (2\%). Among the 7000 pulmonary arteries analysed ( 5 central, 5 lobar, 20 segmental, 40 subsegmental arteries/patient), pseudofilling defects were depicted at the level of 3 segmental arteries $(0.042 \%)$. A "double contour" of the left ventricle was noted in 52 patients $(52 \%)$ and in 7 patients $(7 \%)$ at the level of the right atrium, with a mean $( \pm \mathrm{SD})$ transverse displacement of $1.35( \pm 1.46) \mathrm{mm}$ and $2.14( \pm 0.21) \mathrm{mm}$ of the cardiac margin, respectively. Cardiogenic motion artefacts within lung parenchyma were depicted in 36 patients $(36 \%)$, rated as minimal $(29 / 36 ; 80.5 \%)$, moderate $(6 / 36 ; 16.7 \%)$ or severe $(1 / 36 ; 2.7 \%)$, most commonly seen in the cortical zones of the lower lungs.

Conclusion: Routine scanning of patients with high temporal resolution and high pitch provides examinations devoid of detrimental cardiogenic motion artefacts.

\section{B-758 15:03}

Clinical value of a high-pitch comprehensive cardiothoracic CT protocol for the early triage of patients with undifferentiated acute chest pain

T.R.C. Johnson, R. Marcus, W. Sommer, F. Schwarz, K. Nikolaou,

H.-C.R. Becker, M.F. Reiser, F. Bamberg; Munich/DE

Purpose: To evaluate image quality and diagnostic yield of a high-pitch dual source comprehensive cardiothoracic CT protocol in patients presenting with acute chest pain.

Methods and Materials: Consecutive symptomatic subjects with undifferentiated acute chest pain underwent ECG-synchronised high-pitch dual-spiral CT angiography (Definition Flash, Siemens) of the thorax. Independent investigators determined image quality of each vascular territory, measured contrast-to-noise-ratio (CNR) and determined radiation exposure. In addition, the prevalence of CT findings (pulmonary embolism, (PE), aortic dissection (AD), and significant coronary stenosis $(\geq 50 \%)$ was determined. Univariate and multivariate analyses were performed to determine the subpopulation with highest diagnostic yield.

Results: Among 51 subjects ( $66 \%$ males, average age: $63 \pm 15.8)$, the prevalence of positive CT findings was moderate (overall: $11.7 \%$ ). Overall, image quality of the pulmonary, aortic and coronary vasculature was good $(1.26 \pm 0.43$ and CNR 2.52 ) with an average radiation dose of 3.82 and only $3.2 \%$ of segments rated nonevaluable. Among the different vascular territories, image quality was lowest in the coronary artery tree $(p=0.02)$ and strongly associated with heart rate $(r=0.52$, $p<0.001)$. In subjects with a heart rate of $\leq 65 \mathrm{bpm}$, subjective image quality and CNR of the coronary arteries were higher $(1.6 \pm 0.5$ vs. $2.1 \pm 0.5, p=0.03$ and $1.21 \pm 0.3$ vs. $1.02 \pm 0.3, p=0.05$ ) with only $1.5 \%$ segments classified as non-evaluable. Conclusion: High-pitch dual-spiral comprehensive cardiothoracic CT provides low radiation exposure with excellent image quality at heart rates $\leq 65 \mathrm{bpm}$. In subjects with higher heart rates, image quality of the aortic and pulmonary vasculature remains excellent, while the assessability of the coronary vasculature degrades substantially.

\section{B-759 15:12}

Dual energy CT lung perfusion analysis: correlation of perfusion defect size with laboratory markers, right heart strain and clinical outcome R.W. Bauer 1 , C. Frellesen ${ }^{1}$, J.M. Kerl', M. Renker ${ }^{1}$, U.J. Schoepf ${ }^{2}$, H. Ackermann' ${ }^{1}$, V. Jacobi', T.J. Vogl' $;{ }^{1}$ Frankfurt a. Main/DE, ${ }^{2}$ Charleston, SC/US (ralfwbauer@aol.com)

Purpose: Dual energy CT (DECT) allows for imaging of perfusion defects (PD) caused by pulmonary emboli (PE). The effect of PD size on laboratory markers, right heart strain and clinical outcome was investigated.

Methods and Materials: Data of 53 patients with acute PE who underwent dual source DECT pulmonary angiography were retrospectively analysed. PD size in the lung parenchyma caused by PE was measured on DE iodine distribution maps. PD size was quantified absolutely (VolPD in $\mathrm{ml}$ ) and relatively to the tota lung volume (ReIPD in \%). Signs of right heart strain (RHS) were determined on MPRs in cardiac 4-chamber view. Patient files were searched for d-dimer, pO $\mathrm{pCO}_{2}$ levels and information on readmission for recurrent onset of $\mathrm{PE}$ and death. Results: D-dimer, $\mathrm{pO}_{2}$ and $\mathrm{pCO}_{2}$ levels were not correlated with PD size. Patients with RHS $(n=17)$ had significantly higher VolPD ( $215 \mathrm{vs.} 73 \mathrm{ml})$ and ReIPD ( $9.9 \mathrm{vs.}$ $2.9 \%$ ) than patients without RHS ( $\mathrm{p} 5 \%$ RelPD, while no PE-related hard endpoints were found for patients with $2.5-5 \%(n=16)$ or $<2.5 \%$ ReIPD $(n=19)$.

Conclusion: The extent of perfusion defects of total lung volume on DECT is correlated to right heart strain. Right heart dysfunction is a known predictor for patient outcome in PE. Further, patients with a relative PD size of $>5 \%$ were at risk for death and readmission due to PE. However, prospective trials are mandatory.

\section{$B-760 \quad 15: 21$}

Detection of acute pulmonary embolism in rabbits: correlation of dualenergy $C T$, MR angiography and perfusion imaging at $3 \mathrm{~T}$ L. Lu, L. Zhang, B. Chen, J. Bi, G. Lu, C. Zhou; Nanjing/CN (xuzhoululi@163.com)

Purpose: To compare the ability of dual-energy CT pulmonary angiography imaging (CTPA), dual-energy CT perfusion imaging (DEPI),MR pulmonary angiography (MRPA) and MR pulmonary perfusion (MRPP) to detect pulmonary embolism (PE) in rabbit models.

Methods and Materials: PE were established by injecting four gelatin sponge emboli of different sizes into femoral vein of rabbits ( $n=18,90$ lobes). Dual-energy CT (DECT) followed by 3 T MRI was performed at 2 hours after embolisation. Images of dual-energy CTPA, DEPI, MRPA and MRPP were obtained. The location and number of the emboli in per lobe were recorded. Detailed histopathological analysis was also performed for each rabbit. With the pathology results as the reference standards, sensitivity, specificity and agreement of the aforementioned methods were calculated.

Results: Histopathological results showed 24 lobes with gelatin sponge emboli and 66 lobes without evidence of emboli. The sensitivity and specificity of CTPA and DEPI were $95.8 \%, 95.8 \%$ and $94.1 \%, 90.2 \%$, respectively. The sensitivity and specificity of MRPA and MRPP was $83.3 \%, 37.5 \%$ and $90.2 \%, 98.0 \%$, respectively. Excellent inter-modality agreement was obtained between CTPA and DEPI (Kappa value $=0.971, P<0.001)$. Good inter-modality agreement was obtained between DECT and MRPA (Kappa value $=0.796, P<0.001$ ). There was no statistical significance between DECT and MRPA $(P>0.05)$; however, DEPI had a higher accuracy $(92 \%)$ than MRPP $(78.7 \%)(P<0.05)$.

Conclusion: DECT has a slightly higher sensitivity than MRPA with no statistically significance; DEPI has higher accuracy than MRPP to visualise perfusion defect derived from pulmonary emboli in a rabbit model. 
$14: 00-15: 30$

Room D2

\section{Interventional Radiology}

\section{SS 1709}

\section{Genitourinary and gastrointestinal interventions}

\section{Moderators:}

S.A. Thurnher; Vienna/AT

M.A.A.J. van den Bosch; Utrecht/NL

\section{B-761 14:00}

Evaluation of the effect on the expression quantity of endometrial integrin

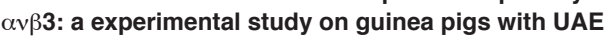

G. Tan, W. Guo, J. Yang; Guangzhou/CN (tgs1976@163.com)

Purpose: To explore the impact of uterine artery embolisation (UAE) upon the quantity of endometrial integrin $\alpha v \beta 3$ by experiment.

Methods and Materials: 40 female guinea pigs were randomly divided into control group $(n=10)$ and experimental group $(n=30$, further divided into A1 and A2 groups, 15/group). Bilateral UAE with Embosphere (trisacryl gelatin microspheres, $40-120 \mu \mathrm{m})$ was performed in the experimental group. Uterine specimens were obtained from all the animals 3 days after ovulation day and within 16 to 30 and 31 to 45 days, respectively, after UAE from the A1 and A2 experimental groups. The quantity of endometrial integrin $\alpha v \beta 3$ was evaluated by immunohistochemistry staining. The MOD (mean optical density) value of positive cells in endometrial glands was used as $\alpha v \beta 3$ content index.

Results: The average dosage of Embosphere was $0.040 \pm 0.008$ millilitre during the embolisation.Integrin $\alpha v \beta 3$ was expressed in endometrial glandular epithelial cells. The MOD values of endometrial integrin $\alpha v \beta 3$ for the control group and experimental group were $0.386 \pm 0.065$ (control group), $0.223 \pm 0.046$ (A1 subgroup) and $0.315 \pm 0.058$ (A2 subgroup), respectively. The statistical analysis indicated MOD values between control groups and two experimental subgroups were both significantly different $(P<0.05)$, as also between $A 1$ and $A 2$ subgroup $(P<0.05)$. Conclusion: The quantity of integrin $\alpha v \beta 3$ in endometrium would decrease after UAE and it might lead to negative influence on endometrial receptivity to some extent. The decrease of endometrial integrin $\alpha v \beta 3$ seems to be not persistent and irreversible. With time prolonged after embolization, the quantity of $\alpha v \beta 3$ might have the trend to recover gradually.

\section{B-762 14:09}

Recurrent bleeding within 24 hours after uterine artery embolisation for severe post-partum haemorrhage: are there predictive factors?

A. Kastler, S. Bros, P. Chabrot, L. Ouchchane, L. Cassagnes, E. Dumousset,

D. Gallot, L. Boyer; Clermont-Ferrand/FR (a_kastler@chu-clermontferrand.fr)

Purpose: To retrospectively identify predictive factors of recurrent bleeding within 24 hours after uterine artery embolisation (UAE) for post-partum haemorrhage (PPH). Methods and Materials: 194 patients were embolised for PPH between 08/1999 and 04/2009 in our institution. Twelve patients suffered from recurrent bleeding within the next 24 hours, making a second attempt at UAE necessary which was successful in ten cases. In two cases hemostatic hysterectomy was performed. We analysed epidemiological, obstetrical, anatomical and biological data.

Results: Complete data were available for $148 / 194$ women $(76 \%)$ included in the study among which mean age was 31.3 years, average pregnancy term was 37.7 weeks. Sixty-four patients $(43 \%)$ were primiparous, $18(12.2 \%)$ had a placenta accreta and $21(14 \%)$ suffered from a coagulopathy; $28(18.9 \%)$ patients presented with an anatomical variant of the uterine vasculature. Among the 12 patients with recurrent haemorrhage, the mean age (31.4 years) and pregnancy term ( 38 weeks and 5 days) were similar to those of the non-recurrent-bleeding series. However, after multivariate analysis, three criteria have emerged as risk factors of bleeding recurrence after initial UAE: primiparity (10 patients, $83 \% ; O R=18.84 ; \mathrm{p}=0.014)$ ), coagulation disorders ( 6 patients, $50 \% ; O R=12.08 ; p=0.006$ ) and anatomical variants of the uterine arterial vasculature ( 7 patients; ovarian collaterals: 3 cases, hypogastric: 3 cases, bladder: 1 case $)(O R=9.83 ; p=0.003)$

Conclusion: Search for uterine collaterals is mandatory prior to UAE for PPH. Primiparity and coagulation disorders increase the risk of recurrent bleeding after UAE for PPH.
B-763 14:18

Uterine artery chemoembolisation: its application in treating C-section scar pregnancy

G. Zhhang, S. Gu, T. Wang, Z. Han; Shanghai/CN (guofuzh@msn.com)

Purpose: To discuss the efficacy and safety of uterine artery chemoembolisation in treatment of Cesarean-section scar pregnancy.

Methods and Materials: During the period of Jun 2005-Jun 2010, uterine artery chemoembolisation was performed in 130 patients with C-section scar pregnancy in our hospital. Via bilateral uterine arteries, 100-150 mg MTX and $80000 \mathrm{U}$ gentamycin were infused separately, after that, the bilateral uterine arteries were embolised with gelfoam particles to slow down the blood flow and then bars of gelfoam to block blood flow. Among all the patients, 23 cases of them who were combined with leiomyoma were embolised with PVA instead of gelfoam. Curettage of uterus was carried out within $24 \mathrm{~h}$ to $72 \mathrm{~h}$ after the procedure.

Results: The success rate of bilateral uterine artery chemoembolisation was $100 \%$. And the success rate of curettage combined with the first time embolism operation is $96.92 \%$ (126/130), The blood loss was between 12 and 110 milliliters (mean $28.5 \mathrm{ml}$ ) during the procedure of curettage. The other 4 cases (4/130) whose embryonic month were large and combined with arteriovenous fistula were still rich in blood supply after the first embolism operation which were proved by ultrasound examination, need a second embolism 2 weeks later after the first time embolism operation. No serious complications occurred in the treatment of 130 cases and the uteruses of all cases were preserved in the treatment.

Conclusion: The uterine artery chemoembolisation is an effective and safe treatment for C-section scar pregnancy, which can greatly reduce the risk of hysterectomy and preserve fertility of the pregnant women.

\section{B-764 14:27}

Immediate and long-term follow-up after uterine artery embolisation in post-partum haemorrhages: monocentric study of 88 patients L. Mezzetta, R. Loffroy, B. Guiu, P. Sagot, J.-P. Cercueil, D. Krausé; Dijon/FR (denis.krause@chu-dijon.fr)

Purpose: To evaluate immediate efficacy and mid-long-term complications after uterine artery embolisations in post-partum haemorrhages (PPH).

Methods and Materials: 88 consecutives patients (mean age 31.3 years, (17-45) were treated by embolisation for PPH. The median follow-up was 55 months (8-117) The PPH risks factors were: uterine scars $(n=21)$; curettage $(n=15)$; multiparous women $(n=13)$; obesity $(n=8)$; previous PPH $(n=7)$; coagulopathies $(n=5)$; caesarean surgical interventions $(n=7)$.

Results: Immediate results: primary technical success (94.4\%, 83/88); 5 haemostasis uterine surgical ablations were necessary (in 3 cases, for abnormal placental insertion). The complications were: haematomas on the arterial puncture site ( $n$ $=16)$; pelvic pain during 72 hours $(n=22)$; endometrites $(n=3)$, with one uterine necrosis; endometrium atrophy $(n=7)$.

Mid- and long-term results: menses return $(86.4 \% ; 64 / 74)$, with a mean delay of 2.9 months without or with modifications of the regularity $(43.7 \%)$ and/or of the abundance $(56.3 \%)$. Oligo-amenorrheas $(20 \%, n=15)$, with uterine synechies $(4 \%, n=6)$ - sterilities $(19 \%, 17 / 88)$ : definitive menauposes $(n=5)$; voluntary tubal ligatures $(n=4)$. Pregnancy desires $(28 \%, 25 / 88) ; 17$ pregnancies for 14 patients, 14 at term, 4 with a new PPH; child birth deliveries at 38 amenorrheas weeks (mean weight of the foetus $3129 \mathrm{~g}$ ).

Conclusion: Embolisation of the uterine arteries for post-partum haemorrhages representa a very safe, efficient technique, which allows a secondary return of menses, and a satisfying fertility rate, especially in the long term.

\section{B-765 14:36}

Chorionic villus sampling and marked membrane separation

S. Akhlaghpoor, A. Aziz Ahari; Tehran/IR (alireza_ahdr@yahoo.com)

Purpose: The major concern about invasive prenatal diagnostic tests is the frequency of procedure induced pregnancy loss. Chorionic Villus Sampling (CVS) is the invasive test of choice in the first trimester after the $10^{\text {th }}$ gestational week. Our experience suggests after the $10^{\text {th }}$ gestational week the marked chorioamniotic separation is an uncommon finding. This study assesses the rate of marked membrane separation in 10- to 14-week gestational period and post CVS foetal loss in this group.

Methods and Materials: 41 (5.2\%) patients were selected among 782 patients as cases with marked membrane separation. The average maternal age was 26.85 years. With a 20-gauge Chiba needle attached to a $20-\mathrm{ml}$ syringe under ultrasound guidance CVS procedures were performed. Follow-up was by telephone and clinical visits until 24 weeks of gestation. Early foetal loss in first 2-week post-procedural 
period and late foetal loss from 2 weeks after procedure till $24^{\text {th }}$ gestational week were considered CVS complications.

Results: We detected $4.2 \%$ early foetal loss after the procedure. 14 cases voluntarily underwent therapeutic abortion, due to beta-thalassemia or haemophilia. One microcephal foetus spontaneously aborted in $21^{\text {th }}$ gestational week. 25 neonates were delivered alive at term and one prematurely at $32^{\text {nd }}$ week.

Conclusion: This study showed that only $5.2 \%$ of patients that underwent CVS have marked chorioamniotic separation. From loss rates, we can suggest that the procedure does not have a major impact on post CVS foetal loss in patients with marked membrane separation.

\section{B-766 14:45}

Non-invasive treatment of symptomatic leiomyomas: initial experience using magnetic resonance-guided focused ultrasound technology

A. Napoli, M. Anzidei, F. Ciolina, L. Di Mare, L. Molisso, G. Cartocci,

C. Catalano, R. Passariello; Rome/IT (alessandro.napoli@uniroma1.it)

Purpose: To establish the efficacy of sonication in leiomyomas volume reduction over time.

Methods and Materials: A total of 19 symptomatic leiomyomas in 16 women (average age 46 years, range 34-55 years) were treated with MRI-guided focused ultrasound (MRgFUS). The system (ExAblate 2000, InSightec), equipped with a phased-array transducer (0.95-1.3 MHz), computer-controlled positioning system, radiofrequency amplifier system, operates in conjunction with a 3 T MR unit (GE). Pre-treatment imaging set (T2-w and T1-w images, pre- and post-Gd - BOPTA) were obtained to measure leiomyoma volume. Immediately after treatment, T1-W contrast-enhanced fat-suppressed MR images in three planes were used to measure nonperfused volume ratio. The average volume of treated fibroids was $175 \pm 201$ (SD) $\mathrm{cm}^{3}$. Follow-up images were obtained 4 and 6 months after treatment and served to determine leiomyoma shrinkage. Qualitative and quantitative relations between fibroid volume, nonperfused volume ratio at treatment, and 6-month shrinkage were measured.

Results: The average nonperfused volume ratio was $65 \% \pm 25 \%$ immediately after treatment. At follow-up treated fibroids showed volume decrease to $112 \pm 141 \mathrm{~cm}^{3}$ with an average volume reduction of $36 \% \pm 25 \%$. A linear regression model showed highly significant correlation between post-treatment nonperfused volume ratio and shrinkage at 4 months.

Conclusion: MRgFUS therapy for leiomyoma results in substantial nonperfused volume ratio and shrinkage. Improved relief of symptoms can be achieved in a safe and noninvasive manner without affecting surrounding tissue.

\section{B-767 14:54}

Chronic mesenteric ischaemia: 28 year endovascular treatment experience U.C. Turba, S.S. Sabri, B. Arslan, J.R. Stone, A. Park, W.E. Saad, K.D. Hagspiel, J.F. Angle, A.H. Matsumoto; Charlottesville, VA/US (uct5d@virginia.edu)

Purpose: To report endovascular therapy and outcomes of the treatment for the patients who are suffering from chronic mesenteric ischaemia (CMI).

Methods and Materials: Institutional Review Board approval was obtained. This is a retrospective study with data collected from patients between April 1981 and September 2009. Two hundred and fifteen (215) blood vessels were treated in 166 patients. We are reporting mesenteric arterial procedural details. Procedural technical success, all mesenteric vessels that we attempted to treat in each patients and vessels. We are also reporting clinical success and failures.

Results: One hundred and sixty-six patients (54 men, 112 women) had CMI procedures. The mean age was 69 years (range $40-94$ years). Two hundred and fifteen mesenteric vessels were treated (average 1.3 vessels per patient). The specific vessels treated were: 118 superior mesenteric arteries (SMA; 55\%), 62 celiac arteries (CA; 29\%), and 35 inferior mesenteric arteries (IMA; 16\%). SMA between vessels that were treated using stents and vessels treated using PTA only $[\mathrm{F}(1,113)=22.4, \mathrm{p}=.00]$. For IMA, PTA only and stenting techniques [F (1, $34)=7.4, p=.01]$. 5-year primary patency for celiac artery, SMA and IMA was 81, $82,80 \%$, respectively.

Conclusion: Although PTA offers better primary patency for SMA and IMA, stents are increasingly utilised recently for the patients who have suboptimal PTA. There is no significant difference in mortality in each group of vessels or treatment methods.
B-768 15:03

Balloon dilatation for oesophageal strictures: interventional parameters and clinical outcomes in children

M. Cevener, K. Cira, S. Yilmaz, T. Sindel, G. Karaguzel, M. Melikoglu; Antalya/TR (metincevener@gmail.com)

Purpose: We retrospectively assessed the interventional parameters and outcomes of the oesophageal balloon dilatation (EBD) in children with benign oesophageal stricture due to corrosive material intake and surgical anastomosis.

Methods and Materials: Between January 2006 and August 2010, 58 patients (age range 3-249 months) who underwent EBD due to corrosive and surgical strictures were included the study. Associations between fallowing parameters were analysed by Fisher exact test and Mann Whitney test with using SPSS 16 software: period between initial and last EBD, number of EBD, complications, location and type of strictures, severity of lesions, balloon size and radiologic-clinic success.

Results: In all cases technical success was $100 \%$. Radiologic and clinic success rate was $92 \%$. There was no significant correlation between gender and procedure parameters. The most significant factors in success rate were severity of lesions and balloon size. There were no statistical significance between number of EBD, complications, location and type of stricture, period between initial and last EBD, but number of EBD and complications were higher in patients with severe-segmental/ diffuse stricture and anastomotic stricture, respectively.

Conclusion: EBD in children with surgical and corrosive oesophageal stricture is valuable and effective method. Especially severity of lesions and balloon size plays important role in radiologic and clinic success.

\section{B-769 15:12}

Predictive factors of spontaneous bacterial peritonitis among patients referred for paracentesis in the radiology department

J. Morin, J.-S. Billiard, D. Olivié, L. Lepanto, J. Murphy-Lavallée, A. Belblidia

G. Pomier Layrargues, A. Tang; Montréal, QC/CA (julie.morin.13@umontreal.ca)

Purpose: The objective of the present study was to use clinical and laboratory data to develop a model for predicting the presence of spontaneous bacterial peritonitis (SBP) in patients referred for paracentesis in the radiology department.

Methods and Materials: We calculated the prevalence of SBP from September through November 2007. We then performed a case-control study of 169 patients who were referred for paracentesis under ultrasound guidance, between January $1^{\text {rst }} 2008$ and March $7^{\text {th }}$ 2010. Variables analysed included demographic, antibiotics and laboratory findings. Ascites was evaluated for cell count and inoculation of aerobic and anaerobic blood culture bottles. We evaluated objective predictive factors of SBP; sensitivity and specificity by receiver operating characteristic $(\mathrm{ROC})$ curve. Factors associated with the presence of SBP were evaluated by univariate and multivariate logistic regression analysis.

Results: The prevalence of SBP was $7.3 \%$ based on positive ascites culture and $20.4 \%$ based on a polymorphonuclear count $>250 / \mathrm{mm}^{3}$. On multivariate analysis, SBP was associated with antibiotic prophylaxis before admission [odds ratio (OR) 8.4, 95\% confidence interval $(\mathrm{Cl}): 2.2-31.5, \mathrm{p}=0.002$ ], antibiotic prescription based on a clinical suspicion of SBP before paracentesis (OR 9.7, 95\% Cl 2.4-38.3, $\mathrm{p}=0.001$ ), and Child-Pugh score (OR 2.5, 95\% Cl 1.0-6.2, $\mathrm{p}=0.039$ ). Area under the curve (AUC) for this model was 0.82 . Sensitivity and specificity of the mode were 85.1 and $57.5 \%$, respectively.

Conclusion: Initiation of prophylactic antibiotic therapy prior to admission and underlying disease severity (i.e. Child-Pugh score) are predictors of SBP. validation of this predictive model for SBP warrants prospective examination. 
14:00 - 15:30

Room E1

\section{Molecular Imaging}

\section{SS 1706}

\section{Fancy new stuff in experimental and clinical molecular imaging}

Moderators:

C.D. Claussen; Tübingen/DE

M.G. Pomper; Baltimore, MD/US

\section{B-771 14:00}

Cerenkov optical imaging of radioisotope

A. Ruggiero ${ }^{1}$, J.P. Holland' ${ }^{2}$ J.S. Lewis ${ }^{2}$, J. Grimm ${ }^{2} ;{ }^{1}$ Rotterdam/NL,

${ }^{2}$ New York, NY/US (a.ruggiero@erasmusmc.nl)

Purpose: Harnessing the full potential of complementary imaging modalities is the driving force in the development of multimodal imaging probes. Here, we investigate the use of radiopharmaceuticals as a dual modality probe (PET-optical) by imaging the inherent optical emissions (Cerenkov radiation) from their radioactive decay. Methods and Materials: Cerenkov luminescence imaging (CLI) of a range of radionuclides $\left({ }^{18} \mathrm{~F},{ }^{89} \mathrm{Zr},{ }^{64} \mathrm{Cu},{ }^{124},{ }^{131} \mid,{ }^{225} \mathrm{Ac}\right.$ ) was investigated using in vitro phantom and a standard optical imaging device (Ivis 200 , Xenogen). In vivo, the novel radiolabelled monoclonal antibodies (mAb) ${ }^{89} \mathrm{Zr}$-DFO-J591 and ${ }^{89} \mathrm{Zr}$-DFO-trastuzumab were used for the simultaneous PET and optical imaging of PSMA positive prostate cancer and Her-2 positive breast cancer xenograft, respectively. Biodistribution studies combined with ex vivo $\mathrm{CLI}$ of radiotracer uptake in organs was performed. Results: Intensity of the Cerenkov radiation in phantoms correlated linearly $\left(\mathrm{R}^{2}=\right.$ 0.98 ) with both the activity concentration and the measured PET signal (\%ID/g). In vivo and ex vivo $\mathrm{CLI}$ provided accurate, quantitative information on $\mathrm{mAb}$ biodistribution and tumour uptake that increased over time from $24 \mathrm{~h}\left(3.4 \pm 0.35 \mathrm{p} / \mathrm{s} / \mathrm{cm}^{2} / \mathrm{sr}\right)$ to $96 \mathrm{~h}\left(6.0 \pm 0.45 \mathrm{p} / \mathrm{s} / \mathrm{cm}^{2} / \mathrm{sr}\right)$ correlating with the corresponding immuno-PET images. $\mathrm{CLI}$ was used to visualise tumour margins and successfully guide surgical resection Conclusion: CLI of medical isotopes shows excellent promises for the use of radiopharmaceuticals as a dual-modal imaging modality allowing both PET and direct optical imaging suitable for intraoperative surgical application.

\section{B-772 14:09}

Advantages and limitations of first whole-body hybrid PET-MR scanner in oncology

O. Ratib, C. Becker, M. Becker, M. Viallon, J.-P. Vallee, J.-P. Willi, M. Lord,

O. Rager, P. Loubeyre; Geneva/CH

Purpose: A whole-body hybrid PET-MR imaging unit was implemented and tested against PET-CT imaging for diagnostic and follow-up of oncology patients. Our goal was to evaluate the performance and clinical applicability of combined imaging protocols on hybrid PET-MR compared to PET-CT

Methods and Materials: A whole-body scanner combining a 3 T MR and a time-of-flight PET scanner sharing a single bed allowing sequential acquisition of co-registered MR and PET images was evaluated clinically in 35 patients referred for diagnostic PET-CT study. PET-MR images were acquired following standard clinical PET-MR studies. Images were interpreted by multidisciplinary teams of radiologists and nuclear physicians in the same way it is routinely done for hybrid PET-CT studies. Oncological studies included, lymphomas, head and neck tumours, prostate and breast tumours as well as lung and colon cancers.

Results: Optimised imaging protocols combining whole body MR attenuation correction data set with standard MR diagnostic protocols of both modalities while reducing the total time of the study were developed. Diagnostic quality of fused PET-MR images were comparable to corresponding PET-CT images and measured local SUV were comparable. None of the PET-MR studies had insufficient diagnostic quality and finding were comparable to PET-CT findings.

Conclusion: Optimised imaging protocols showed adequate clinical results of hybrid PET-MR imaging in oncology. Advantages and limitations of this new technique compared to results obtained from conventional PET-CT technique will be reported.

\section{B-773 14:18}

Novel conceptual PET design based on pixel CdTe detectors

M. $\underline{\text { Chmeissani }}{ }^{1}$, P. Arce ${ }^{2}$, M. Cañadas ${ }^{2} ;{ }^{1}$ Bellaterra/ES, ${ }^{2}$ Madrid/ES (mokhtar@ifae.es)

Purpose: Intrinsic limitations of the current PET scanners that are based on scintillating crystals, such as low detection efficiency, low energy resolution, low spatial resolution, image noise from scattered photons, and non-compatibility with MR can be overcome by novel design of PET scanner based on pixel solid-state detector.
Methods and Material: Pixel CdTe detector $1 \mathrm{~cm} \times 2 \mathrm{~cm} \times 2 \mathrm{~mm}$ bonded to dedicated ASIC that is thinned down to $50 \mu \mathrm{m}$. 4 of such assemblies mounted on $50 \mu \mathrm{m}$ thin kapton printed circuit board (PCB) form a unit detector with effective CdTe material of $4 \mathrm{~cm} \times 2 \mathrm{~cm} \times 2 \mathrm{~mm}$. A stack of such unit-detectors will form a module-detector of a can be arranges to form a PET scanner with effective $4 \mathrm{~cm}$ CdTe absorption and highly segmented in 3D (1 $\mathrm{mm} \times 1 \mathrm{~mm} \times 2 \mathrm{~mm})$ making it ideal for brain imaging. Results: Given the high detection efficiency of $4 \mathrm{~cm} \mathrm{CdTe}$ for $511 \mathrm{keV}(90 \%)$ and the excellent energy resolution ( $1 \% \mathrm{FWHM}$ ), simulation results followed by image reconstruction show, when compared with state-of-the-art PET scanner for head, the detection efficiency increases by a factor of 2.3 and the scattered photons are reduced from $98 \%$ to $3 \%$, thus allowing to resolve the $1.2 \mathrm{~mm}$ rod in Derenzo-like phantom with peak-to-valley ratio of 3 to 1 .

Conclusion: A PET scanner based on pixel CdTe detectors shows excellent results with a promise of big leap in PET image quality. A new funded project, by ERCAdG-2009 (FP7), voxel imaging PET pathfinder (VIP) has just started to construct the basic modules of this novel PET scanner.

\section{B-774 14:27}

Failure of annexin-based apoptosis imaging in the assessment of antiangiogenic therapy response

W. Lederle, S. Arns, A. Rix, F. Gremse, D. Doleschel, F. Kiessling,

M. Palmowski; Aachen/DE (wlederle@ukaachen.de)

Purpose: The accuracy of apoptosis imaging was analysed for assessing antiangiogenic therapy response. Treatment effects on tumour vascularisation were additionally addressed.

Methods and Materials: AnnexinVivo 750 (apoptosis) and AngioSense 680 (vascularisation) concentrations were determined by fluorescence molecular tomography (FMT2500) in nude mice with subcutaneous epidermoid carcinoma xenografts after 4 days of anti-angiogenic treatment with SU11248. Tumour localisation was facilitated by parallel morphological $\mu \mathrm{CT}$-scans. 3D FMT data were compared with corresponding 2D fluorescence intensities. Vascularisation was additionally analysed by contrast-enhanced ultrasound (2D MIOT). In vivo-data were validated on corresponding histological sections (CD31-staining: vascularisation; TUNELstaining: apoptosis). A Student's t test was applied for statistics.

Results: Strikingly lower AnnexinVivo concentrations were measured in treated tumours compared with controls (3D: control: $81.3 \mathrm{pmol} / \mathrm{cm}^{3}$, therapy: $27.5 \mathrm{pmol} /$ $\mathrm{cm}^{3}$; 2D: control: $13 \mathrm{Fl} / \mathrm{cm}^{2}$; therapy: $11 \mathrm{Fl} / \mathrm{cm}^{2}$ ). In contrast, immunohistology demonstrated a significantly increased apoptosis in treated tumours (TUNEL+ area: control: $0.011 \%$, therapy: $0.461 \% ; p<0.001)$. Lower AngioSense concentrations were detected in treated tumours (3D: control: $13.8 \mathrm{pmol} / \mathrm{cm}^{3}$, therapy: $6.3 \mathrm{pmol} /$ $\mathrm{cm}^{3}$; 2D: control: $38 \mathrm{Fl} / \mathrm{cm}^{2}$, therapy: $15 \mathrm{Fl} / \mathrm{cm}^{2}$ ). Ultrasound and immunohistology confirmed a significantly reduced vascularisation in treated tumours (ultrasound: control: 285.3 a.u. $/ \mathrm{mm}^{2}$, therapy: 87.3 a.u. $/ \mathrm{mm}^{2} ; \mathrm{p}<0.01$; histology: CD31+ area: control: $4.8 \%$, therapy: $1.7 \% ; p<0.001$ ).

Conclusion: In vivo apoptosis imaging fails in assessing increased apoptosis after anti-angiogenic therapy. This can be explained by the significant breakdown of the vasculature in response to treatment, thereby impairing the delivery of the annexin probe to the tumour. This favours apoptosis imaging only in therapies that do not severely interfere with the vasculature.

\section{B-775 14:36}

Combination of vascular endothelial growth factor antisense oligonucleotide therapy and radiotherapy increases the curative effects against maxillofacial VX2 tumours in rabbits

L.-F. Zheng, Y.-J. Li, H. Wang, J.-L. Zhao, X.-F. Wang, Y.-S. Hu, G.-X. Zhang; Shanghai/CN (zhenglinfeng04@yahoo.com.cn)

Purpose: To study effects of combination of vascular endothelial growth factor (VEGF) antisense oligonucleotide and radiotherapy on maxillofacial VX2 tumours in rabbits.

Methods and Materials: 24 New Zealand rabbits with maxillofacial VX2 tumour were randomly divided into the following 4 groups: group A, treated with 16 Gy of radiotherapy; group B, treated with an injection of $150 \mu \mathrm{g}$ of VEGF antisense oligonucleotide into tumour; group $C$, treated with an injection of $150 \mu \mathrm{g}$ of VEGF antisense oligonucleotide into tumour immediately after 16 Gy of radiotherapy; and group D, treated with an injection of $300 \mu \mathrm{l} 5 \%$ aqueous glucose solution into tumour. On days 3 and 14 after treatment, dynamic contrast-enhanced magnetic resonance imaging (DCE-MRI) was performed to calculate maximal enhancemen ratio (MER), slope of enhancement (SLE), and tumour volume change. Tumour samples were obtained for pathological examination and immunohistochemical staining for VEGF. 
Results: In group C, tumour volume was significantly reduced on day 14 after treatment, and the difference was statistically different as compared to that before treatment, on day 3 after treatment and other groups. Values of both MER and SLE after treatment were significantly lower than the values before treatment. Pathological specimen revealed decreased VEGF expression. The immunohistochemical score (IHS) of group $\mathrm{C}$ was significantly different from groups $\mathrm{A}$ and $\mathrm{D}$.

Conclusion: Injection of VEGF antisense oligonucleotide after radiotherapy can enhance the curative effect on rabbit maxillofacial VX2 tumour, and DCE-MRI can serve as a reliable technique for in vivo monitoring.

\section{B-776 14:45}

\section{The precise co-registration method of high-resolution SPECT and MR} images of in vivo mouse brains

K. Inoue ${ }^{1}$, M. Yamaguchi', I. Umeda' ${ }^{1}$ J. Hesterman², J. Hoppin ${ }^{3}$, C. Lackas ${ }^{3}$,

M. Fukushi ${ }^{4}$, N. Moriyama ${ }^{4}$, H. Fujii ; ${ }^{1}$ Kashiwa/JP, ${ }^{2}$ Washington, DC/US,

${ }^{3}$ Boston, MA/US, ${ }^{4}$ Tokyo/JP (kinoue@bidmc.harvard.edu)

Purpose: The precise co-registration of SPECT and MR images would be useful to evaluate detailed localisations of molecular probes in the small animal imaging. We investigated precise co-registration techniques of in vivo mouse brain images obtained from commercial small animal SPECT and MR scanners.

Methods and Materials: We developed a rodent holder with three external fiducial markers (FMs) to immobilize its head. To demonstrate the usefulness of these markers as guides, the resolution and the sensitivity at FMs on SPECT images were measured. A cylinder phantom and three mouse brains treated with I-123 iomazenil (IMZ) were scanned by both SPECT and MRI scanners. The obtained images were manually merged based on FMs. Co-registration errors were calculated using the mutual information and the rigid three-dimensional transformation algorithms.

Results: The resolution and the sensitivity at FMs on SPECT images were $713 \pm 42 \mu \mathrm{m} \mathrm{FWHM}$ and $425 \mathrm{cps} / \mathrm{kBq}$, respectively. These results indicated that FMs can be used as guides for the image co-registration. On the merged images, the maximum translational and rotational co-registration errors were $171 \pm 71 \mathrm{um}$ and $0.012 \pm 0.010^{\circ}$, respectively. In the animal studies, merged images clearly depicted the distribution of IMZ restricted to the cortex.

Conclusion: We proposed a precise co-registration technique of high-resolution SPECT and MR images of small animals. The images merged by this method were good enough to evaluate anatomical localization of molecular probes in mouse brains in vivo.

\section{B-778 14:54}

In vivo micro-CT and micro-PET imaging of liver lesions in a c-myc transgenic mouse model of hepatocellular carcinoma identifies tumour promoting agents

K. Hueper, M. Elalfy, T. Rodt, F. Länger, R. Halter, D. Hartung, M. Galanski, J. Borlak; Hannover/DE (hueper.katja@mh-hannover.de)

Purpose: To investigate whether in vivo micro-CT and micro-PET imagings enable an evaluation of different classes of tumour promoting agents in a c-myc transgenic mouse model of liver cancer.

Methods and Materials: Sixty c-myc transgenic mice were treated with different genotoxic or non-genotoxic chemicals or vehicle at the age of 2 months. In vivo contrast-enhanced CT (DHOG, Fenestra) and ${ }^{18} \mathrm{~F}$-FDG-PET of the abdomen were performed at 4 different time points between the age of 4 to 8.5 months. Liver volume, tumour percentage, tumour multiplicity and tumour-to-non-tumour ratio were determined and compared with histopathology results.

Results: MicroCT detected lesions of $\geq 1 \mathrm{~mm}$ with certainty. All animals that were positive for hepatocellular carcinoma in histopathology could be identified by in vivo imaging. There was a strong correlation between the determined tumour volume and the liver volume $(r=0.94)$. Significant differences $(p<0.01)$ in tumour percentage, tumour multiplicity and tumour-to-non-tumour ratio were observed between treated and control groups. Notably, in animals treated with the genotoxic carcinogen N-nitrodiethylamine hepatocellular carcinoma developed faster and tumour percentage was higher, therefore demonstrating its tumour promoting effect. Furthermore, metastatic spread to the lung as well as primary lung cancer could be observed in this group. Conclusion: Micro-CT and micro-PET imaging allowed in vivo detection and quantification of hepatocellular carcinoma; imaging also evidenced the tumour promoting effect of chemicals in the c-myc transgenic mouse model. The combination of in vivo imaging and the use of genetic mouse models of cancer may enable faster detection of hazardous drugs and chemicals.

\section{B-779 15:03}

Macrophage activity indicating $\mathbf{S} 100 \mathrm{~A} 9$ as novel imaging target for estimation of the malignant potential of tumour lesions in mice

M. Eisenblaetter, A. Becker, W. Heindel, T. Vogl, C. Bremer;

Münster/DE (eisenblaetter@uni-muenster.de)

Purpose: Tumours are not a homogenous compound of malignant cells but comprise a large variety of recruited host cells, inter alia a significant amount of macrophages. There is strong evidence that those tumour-associated macrophages (TAM) are promoters of tumour progression and metastasis, found, e.g. at sites of basement-membrane breakdown, enabling the tumour cells to locally egress into surrounding stroma. TAM density and activity correlates with poor prognosis in numerous malignant diseases, reflecting advanced tumour progression. This study should confirm the possibility to visualise TAM activity in tumour models using optical imaging (OI) and a macrophage-specific fluorescence contrast agent. Methods and Materials: An antibody addressing macrophage-specific S100 A9 was labelled with Cy5.5 for in vivo OI. IGG-Cy5.5 served as control for unspecific label distribution. Mice were inoculated with 4T1 murine breast cancer or MOS murine osteosarcoma, respectively. At the time, tumours reached a size of $4 \mathrm{~mm}$, OI was performed after injection of the fluorescence contrast agents. For correlation of imaging results, immunohistochemistry of tumour sections was performed. Tumour cells were analysed for $\mathrm{S} 100 \mathrm{~A} 9$ expression using ELISA.

Results: Neither 4T1 nor MOS cells showed significant S100 A9 production in ELISA. In fast-growing 4T1 tumours, anti-S100 A9-Cy5.5 injection resulted in fluorescence signal which was regularly about two-fold higher compared to IGG-Cy5.5 (2356.8 vs. $1283.2 ; p<0.005)$. At same size, slow-growing MOS accumulated less anti-S100 A9-Cy5.5 than 4T1 (1655.3 vs. 2356.8), reflecting lower macrophage activity. Histology confirmed in vivo imaging results.

Conclusion: Ol allows for in vivo visualisation of macrophage activity in primary tumour lesions and therefore for estimation of malignant potential.

\section{B-780 $15: 12$}

Dynamic contrast-enhanced computed tomography (DCE-CT) for monitoring anti-angiogenic effect of sorafenib on experimental prostate carcinomas with immunohistochemical validation

C.C. Cyran, P.M. Paprottka, B. Schwarz, J. Von Einem, R. Hinkel, O. Dietrich, M.F. Reiser, B.J. Wintersperger, K. Nikolaou; Munich/DE (clemens.cyran@med.uni-muenchen.de)

Purpose: To investigate dynamic, contrast-enhanced computed tomography (DCECT) for monitoring the effects of the multikinase inhibitor sorafenib on experimental prostate carcinomas in rats with immunohistochemical validation.

Methods and Materials: Copenhagen rats $(n=18)$ were implanted subcutaneously with prostate carcinoma allografts (MLLB-2) and underwent DCE-CT-enhanced with Iopromide (Ultravist $370^{\circledR}$, Bayer Schering, Berlin, Germany) on day 0 and after one week of therapy. The treatment group $(n=9)$ received daily applications of sorafenib (Nexavar ${ }^{\circledR}$, Bayer, Leverkusen, Germany, $10 \mathrm{mg} / \mathrm{kg}$ bodyweight) via gavage. Quantitative measurements of tumour perfusion (PF, $\mathrm{ml} / 100 \mathrm{ml} / \mathrm{min}$ ) and tumour vascularity (PV, \%) were calculated based on a two-compartment kinetic model. On day 7 tumors were excised for immunohistochemical staining including tumour vascularity (RECA-1), tumour cell proliferation (Ki-67) and apoptosis (TUNEL). Results: Under sorafenib therapy tumour perfusion declined significantly between days 0 and 7 (PF day $031.7 \pm 21.7 \mathrm{vs}$. PF day $79.3 \pm 5.7 \mathrm{ml} / 100 \mathrm{ml} / \mathrm{min}, \mathrm{p}<0.02$ ). Tumour vascularity decreased significantly under sorafenib therapy (PV day $0=14.3 \pm 8.0$ to $P V$ day $7=5.2 \pm 4.0 \% ; p<0.05)$. In the control group, no significant alteration $(p>0.05)$ of tumour perfusion or tumour vascularity was observed. Immunohistochemical measurements revealed significantly lower tumour vascularity in the therapy than in the contro group (RECA-1 $1.415 \pm 71$ vs. $2.391 \pm 120, p<0.01$ ). In Sorafenib-treated tumours, significantly more apoptotic cells (TUNEL $55.806 \pm 2.790$ vs. $22.332 \pm 1.116, p<0.01$ ) and significantly less proliferating cells (Ki-67 $77.026 \pm 3851$ vs. $140.457 \pm 7.022$, $p<0.01$ ) were observed than in the control group.

Conclusion: Tumour perfusion and tumour vascularity, quantified by DCE-CT, may be applicable as non-invasive surrogate parameters for monitoring the antiangiogenic, anti-proliferative and pro-apoptotic effects of sorafenib on experimental prostate carcinomas in rats as validated by immunohistochemistry. 
14:00 - 15:30

Room E2

\section{Musculoskeletal}

\section{SS $1710 b$}

\section{Imaging the knee}

Moderators:

A. Platkajis; Riga/LV

R.M. Rodrigo; Bilbao/ES

\section{B-781 14:00}

Diffusion tensor imaging and T2-maps in the assessment of muscle damage A. Esposito, F. De Cobelli, L. Campana, T. Canu, A. Manfredi, C. Sallemi,

G. Pezzetti, P. Rovere-Querini, A. Del Maschio; Milan/IT

(esposito.antonio@hsr.it)

Purpose: A non-invasive method to follow structural changes occurring during muscle damage/repair process was an essential need to investigate the role of immune system in muscle healing. Aim was to set up and validate a magnetic resonance (MR)-based follow-up of muscle repairing process.

Methods and Materials: The model of acute muscle damage was obtained through cardiotoxin injection (CTX) into tibialis-anterior and quadriceps muscles of C57BL/6N mice. MR imaging was performed before CTX injection and 1-3-5-7 and 10 days after injury on a 7T magnet (Bruker). T2w-MSME sequences for T2 relaxation-time (T2-rt) quantification and diffusion-tensor images (EPI-DTI) for fractional-anisotropy (FA) assessment were acquired. MR results were correlated with histological analysis performed by haematoxylin-eosin staining in the same animal model.

Results: In healthy muscles T2-rt resulted 16,723 $\pm 0.8149 \mathrm{~ms}$ and FA $0.404 \pm 0.0901$. T2-rt strongly increased after acute damage with maximum values at days 1 and 3 and a slow decrease in the later time points (day $1: 34.879 \mathrm{~ms}$, day 3: $44.84 \mathrm{~ms}$, day $5: 34.579 \mathrm{~ms}$, day $7: 36.875 \mathrm{~ms}$, day $10: 31.521 \mathrm{~ms})$. Modifications in T2-rt were apparently related to histological evidence of inflammatory infiltrate. Besides, we observed a marked reduction of $F A$ at days 1 and 3 after damage, with a complete recover of $F A$ values at day 7 , according to histological evidence of fibres regeneration (day $1: 0.161$, day $3: 0.113$, day $5: 0.3$, day $7: 0.336$, day $10: 0.377$ ). Conclusion: T2-rt and FA modifications seem sensitive and quantitative markers of two different phenomena occurring in the acute muscle damage, tissue oedema and muscular architecture disruption. MR imaging allows quantitative and non-invasive monitoring of muscle healing process with oedema reabsorption and fibres integrity recover.

\section{B-782 14:09}

Suprapatellar fat pad: MR imaging findings and clinical significance

N. Tsavalas, A. Ntailiani, E. Vassalou, A.H. Karantanas; Iraklion/GR

(nicktsavalas@yahoo.com)

Purpose: To evaluate the MRI characteristics of suprapatellar fat pad (SFP), correlate the findings with mass effect on the suprapatellar joint recess and determine its clinical significance.

Methods and Materials: We retrospectively reviewed 698 consecutive knee MRI examinations from 671 patients for the presence of SFP oedema with subsequent mass effect on the posterior joint recess. We measured the maximum anteroposterior (A-P), craniocaudal $(\mathrm{C}-\mathrm{C})$ and oblique $(\mathrm{OBL})$ diameter of the suprapatellar fat pad on sagittal fat-saturated intermediate-w TSE images. The ratio DI/SD was determined after assessing the difference of the signal intensities of the suprapatellar and prefemoral fat pad (DI) as well as the corresponding standard deviation of the background noise (SD). Findings of anterior knee pain according to history and physical examination were also recorded. Statistical analysis was performed with Mann-Whitney test and Spearman's correlation. Results: The presence of SFP oedema with mass effect on the posterior joint recess was detected in $12.89 \%(90 / 698)$ of MRI examinations. The A-P $(p<0.0001), C-C(p=$ $0.0158)$, and $O B L(p<0.0001)$ diameter of the pad as well the DI/SD ratio $(p<0.0001)$ were significantly larger in knees with mass effect. Significant correlation was found between the $\Delta \mathrm{l} / \mathrm{SD}$ ratio and the A-P (rho $=0.123, p=0.0011), C-C$ (rho $=0.105, p$ $=0.0057$ ) and $\mathrm{OBL}$ (rho $=0.140, p=0.0002$ ) diameters of the pad. Mass effect was significantly associated with anterior knee pain in 3 ball game players $(3 / 90=3.33 \%)$. Conclusion: SFP oedema with mass effect is a common finding in MRI examinations of the knee but very rarely associated with anterior knee pain.

\section{B-783 14:18}

3D-FIESTA MR imaging curved planar reconstruction for evaluating the anterior cruciate ligament of the knee joint

D.P. Hao, W.J. Xu, J.H. Liu; Qingdao/CN (haodp_2009@163.com)

Purpose: To determine the feasibility of the 3D-FIESTA MR curved planar reconstruction (CPR) for evaluating the anterior cruciate ligament of the knee joint. Methods and Materials: A magnetic resonance (MR) examination of the knee was performed on 30 subjects using a GE 3 T scanner and a phased-array extremity coil. MR 3D-FIESTA sequence with CPR and oblique sagittal proton density-weighted fast spin-echo (PD-FSE) sequence were performed. Signal-to-noise efficiency, and contrastto-noise ratio (CNR) measurements of the anterior cruciate ligament were calculated for all sequences and compared with the use of paired t-tests. The presence of the originating point, anteromedial $(\mathrm{AM})$ and posterolateral $(\mathrm{PL})$ bundle, inserting point of the anterior cruciate ligament on the two sequences were compared with the use of rank sum test. Results: The anterior cruciate ligament signal-to-noise efficiency of MR 3D-FIESTA sequence with CPR was not significantly different from that of the PD-FSE sequence $(P>0.05)$. MR 3D-FIESTA sequence with CPR produced CNR between anterior cruciate ligament and synovial fluid was not significantly different from that of the PD-FSE $(P>0.05)$. MR 3D-FIESTA sequence with CPR allowed excellent visualisation of the anterior cruciate ligament of the knee joint. The presence of the originating point, AM and PL bundle, inserting point of the anterior cruciate ligament on MR 3D-FIESTA sequence with CPR was significantly higher than oblique sagittal PD-FSE sequence $(P<0.05)$ Conclusion: MR 3D-FIESTA sequence with CPR significantly enhances our ability to identify the anterior cruciate ligament compared with oblique sagittal PD-FSE sequence.

\section{B-784 14:27}

Are all patients with anterior cruciate ligament tear able to undergo a double-bundle reconstruction? An MRI study

A. Ntailiani, G. Tzoanos, N. Tsavalas, E. Vassalou, A.H. Karantanas; Iraklion/GR (kdailiani@gmail.com)

Purpose: To estimate the incidence of anatomic restrictions which may prevent performing a double-bundle reconstruction of a torn anterior cruciate ligament (ACL). Methods and Materials: We retrospectively studied 658 knee MRI examinations, which corresponded to 635 patients. All MRI examinations were performed on a $1.5 \mathrm{~T}$ scanner using a synergy coil. Both the coronal and sagittal images were evaluated for the measurements. The femoral notch width (FNW) and the ACL tibial insertion site (TIS) length were digitally measured using a computer workstation. We measured FNW on coronal T1-w spin echo images at the level of decussation of ACL and posterior cruciate ligament and the ACL TIS length on sagittal fat suppressed proton density turbo-spin-echo images at the point of maximum length Exclusion criteria included patients with open physes, severe osteoarthritic changes (Grade III or greater) and multiligamentous injuries.

Results: We found $8.51 \%$ of patients to have open physes, $9.27 \%$ severe osteoarthritic changes and $0.759 \%$ multiligamentous injuries. The above were excluded from the analysis. $14.741 \%$ of subjects in our study group proved to be non-proper candidates for double-bundle reconstruction technique depending on the results of measurements. Of them, $13.677 \%$ had short TIS length, $1.367 \%$ had narrow femoral notch and $0.3 \%$ had both.

Conclusion: There is a significant rate of patients who do not meet the criteria for double-bundle ACL reconstruction technique. MRI is able to depict this subgroup of patients and thus is important to avoid failure of a demanding treatment.

\section{B-785 14:36}

Evaluation of external displacement of implanted menisci on MR imaging T. De Coninck ${ }^{1}$, W. Huysse ${ }^{1}$, P. Verdonk ${ }^{1}$, E.-L. Heinrichs ${ }^{2}$, K. Verstraete ${ }^{1}$; ${ }^{1}$ Gent/BE, ${ }^{2}$ London/UK (tineke.deconinck@ugent.be)

Purpose: To evaluate the external displacement of a polyurethane meniscal scaffold. The displacement is compared to that of 3 control populations: normal, torn, and transplanted menisci.

Methods and Materials: Fifty-two patients, implanted with a polyurethane scaffold (34 medial and 18 lateral scaffolds) received MR-scans at 1 week, 3, 12 and 24 months post-surgery. The findings of these patients were compared to 3 control populations: 100 normal, 100 torn and 16 allograft transplant menisci. Corona MR images were used to analyse the external displacement of the body of the meniscus, which was defined as the distance $(\mathrm{mm})$ between the edge of the tibial plateau and the peripheral edge of the meniscus.

Results: The external displacement of normal (mean $=1.36 \mathrm{~mm}$ ) and torn (mean $=1.37 \mathrm{~mm}$ ) menisci does not differ significantly. The lateral transplanted (mean = 4.04 ) and lateral scaffold (mean $=4.49 \mathrm{~mm}$ ) menisci have a similar displacement; however, both are significantly more displaced than normal and torn menisci. In the 
medial compartment there is also no difference between the external displacement of the scaffold (mean $=4.81 \mathrm{~mm}$ ) and transplanted allografts (mean $=4.71 \mathrm{~mm}$ ). The displacement of the medial scaffold is significantly less 1 week post-surgery compared to 3 and 12 months. The lateral displacement remains unchanged. Evaluation of the 24-month images is in progress.

Conclusion: The position of the medial polyurethane scaffold shifts between 1 week and 3 months postoperatively, but remains stable thereafter. Its final position is similar to that of a meniscal allograft.

\section{B-786 14:45}

Locoregional deformation patterns of knee cartilage: a 3D volumetric highresolution in-vivo study at $3 \mathrm{~T}$

A. Horng ${ }^{1}$, R. José ${ }^{2}$, M. Zscharn ${ }^{1}$, M. Notohamiprodjo' ${ }^{1}$ U. Hoehne-Hückstädt ${ }^{3}$,

U. Glitsch ${ }^{3}$, R. Ellegast ${ }^{3}$, M.F. Reiser ${ }^{1}$, C. Glaser ${ }^{2} ;{ }^{1}$ Munich/DE,

${ }^{2}$ New York, NY/US, ${ }^{3}$ St. Augustin/DE (annie.horng@med.uni-muenchen.de)

Purpose:To evaluate knee cartilage deformation as a surrogate parameter for joint contact areas under defined loading conditions.

Methods and Materials: Sagittal 3D-T1-w-FLASH-WE-sequences (TR14.2 ms/ TE7.2 $\mathrm{ms} / \mathrm{FA} 15^{\circ} /$ resolution $0.3^{2} \times 1.5 \mathrm{~mm}^{3} /$ matrix $512^{2}$ ) were acquired of 10 healthy knees before and after loading (10 min kneeling at $90 \%$ squatting/after 50 knee bends) and after further 90 min rest at $3 \mathrm{~T}$. After segmentation 3D-reconstruction of the cartilage was used to generate thickness- and $2 \sigma$-significance-thicknessdifference-maps. Volumetric parameters (volume, cartilage-bone-interface-area, mean thickness) were calculated. Voxel-based reproducibility for calculation of significant changes was assessed by RMSA, statistics by t-tests.

Results: Similar deformation pattern was found for patellar cartilage in the medial and caudolateral facet, most pronounced after squatting. Corresponding deformation was found in the posterior aspect of the femoral condyles (medial > lateral). Tibial cartilage revealed inner anterior and central deformation (lateral > medial), most pronounced after squatting. Dynamic revealed shallower changes than static loading. Voxel-based reproducibility depended on cartilage thickness (Th) ranging from 0.12 to $0.35 \mathrm{~mm}$. For Th $\geq 1 \mathrm{~mm}$ reproducibility was $<0.31 \mathrm{~mm}$ ( $<$ voxel size). Significant global changes for volume and mean thickness ranged from $1.1 \%$ to $4.9 \%$, while locoregional changes reached $14 \%$. Conclusion: These first data of direct in-vivo locoregional cartilage deformation patterns indicate potential contact and loading zones after kneeling, squatting and knee bends. They are anatomically and functionally plausible and may represent areas where stress-induced degeneration and subsequent osteoarthritis might originate. Voxel-based quantitative analysis of regional deformation combining information from all knee compartments complement common global analysis of cartilage volumetry. The approach may facilitate understanding of individual knee loading and kinematic properties and contribute to improving and validating biomechanical knee models.

\section{B-787 14:54}

Risk factors for cartilage loss of the knee joint over a 6-month period assessed at 3 T MR

F.W. Roemer ${ }^{1}$, C. Kwoh², M.J. Hannon², S.M. Green², J.M. Jakicic²,

R. Boudreauㄹ, C. Moore ${ }^{3}$, A. Guermazi4; ${ }^{1}$ Augsburg/DE, ${ }^{2}$ Pittsburgh, PA/US,

${ }^{3}$ Houston, TX/US, ${ }^{4}$ Boston, MA/US (frank.roemer@klinikum-augsburg.de)

Purpose: The aim of this study was to assess several MRI-features as baseline risk factors that may predict patello-femoral (PF) and tibio-femoral (TF) cartilage loss at 6-month follow-up.

Methods and Materials: For 177 subjects with chronic knee pain, 3 T MRI of both knees was performed at baseline and follow-up. Knees were semiquantitatively assessed using a modified WORMS score.

All MR joint morphologic features were dichotomised into present $(\geq 1)$ versus absent (0). Logistic regression models were applied to predict cartilage loss controlling for clustering by person and by knee. Models were adjusted for age, gender, treatment and $\mathrm{BMI}$. For the TF model, the interaction of BML and meniscal extrusion was included. Results: 304 knees (87.9\%) and 1153 subregions (23.8\%) exhibited prevalent cartilage damage at baseline. $79(1.6 \%)$ subregions showed incident or worsening cartilage damage at follow-up. Predictors for PF cartilage loss were effusion (adjusted odds ratio [aOR] $3.5 .95 \% \mathrm{Cl} 1.3-9.6$ ) and prevalent cartilage damage in same subregion (aOR 4.3.95\% Cl 1.3-13.8). Risk factors for TF cartilage loss were baseline ipsi-compartmental meniscal extrusion (aOR 3.6.95\% Cl 1.3-10.1), prevalent $\mathrm{BMLs}(\mathrm{aOR} 4.6 .95 \% \mathrm{Cl} 1.1-19.4$ ) and cartilage damage in same subregion (aOR 15.9.95\% $\mathrm{Cl}$ 5.1-49.8 The absence of BL mensical extrusion and concomitant ipsicompartmental BMLs were protective of cartilage loss (aOR 0.13.95\% $\mathrm{Cl} 0.02-0.67$ ). Conclusion: Cartilage loss over 6 months is rare, but may be detected semiquantitatively at $3 \mathrm{~T}$ MRI. Predictors of PF cartilage loss were effusion and prevalent cartilage damage in the same subregion. Predictors of TF cartilage loss was prevalent cartilage damage, BMLs and meniscal extrusion.
B-788 15:03

Evaluation of vascularisation of an implanted polyurethane scaffold with MR imaging

W. Huysse ${ }^{1}$, T. De Coninck ${ }^{1}$, P. Verdonk ${ }^{1}$, E.-L. Heinrichs², K. Verstraete ${ }^{1}$; ${ }^{1}$ Gent/BE, ${ }^{2}$ London/UK (wouter.huysse@gmail.com)

Purpose: To evaluate the presence of vascular tissue in a degradable polyurethane meniscal device implanted after partial meniscectomy. The vascularisation was analysed by dynamic contrast-enhanced magnetic resonance imaging (DCE-MRI). Methods and Materials: Fifty-two patients were assessed by DCE-MRI at 1 week and 3 months and a representative sample of subjects at 12 and 24 months postsurgery. The change in T1-relaxation time in a region-of-interest (ROI), as depicted on a time-intensity-curve (TIC), is used to measure gadolinium influx during the first 3 minutes after contrast administration. An increase of gadolinium can only be explained by the presence of blood vessels, hence this influx corresponds to vascularisation in this area. ROls were drawn over the inner and outer half of the scaffold. Results: In the first week after surgery, the outer half of the scaffold only shows enhancement in $6.8 \%(3 / 44)$ of the subjects. After 3 months signal enhancement, and thus evidence of tissue ingrowth into the peripheral half of the scaffold, is seen in $81.4 \%$ (35/43) subjects. After 12 months this decreases slightly to $72.7 \%(16 / 22)$, increasing again to $92.9 \%(13 / 14)$ at 24 months.

No enhancement is seen in the inner half in the first week. At 3, 12 and 24 months this is, respectively, $16.3 \%(7 / 43), 42.1 \%(8 / 19)$ and $72.7 \%(8 / 11)$.

Conclusion: DCE-MRI shows increasing enhancement over time in both the inner and outer half of the meniscal scaffold, demonstrating that there is capillary ingrowth into the device.

\section{B-789 15:12}

Radiological evaluation of cartilage after microfracture treatment: a longterm follow-up study

A.G. von Keudell, J. Atzwanger, R. Forstner, H. Resch, T. Hoffelner, M. Mayer; Salzburg/AT (mi.mayer@salk.at)

Purpose: The study purpose was a long- term evaluation of patients who received MFX through morphological pre- and postoperative MRI.

Methods and Materials: Between 2000 and 2007, 158 patients were treated with MFX for focal femoral or tibial defects. Patients with instabilities, secondary surgical intervention, patellofemoral lesions, plica mediopatellaris or more than one cartilage defect site and age $>55$ were excluded. 15 patients were included. Minimum postoperative follow-up (FU) was 18 months (18-78m). Mean age at surgery was 45 years (27-54) and average FU- interval 48 months (18-78m). Male to female ratio was 9:6. Radiological evaluation was performed with $\mathrm{x}$-ray and $3 \mathrm{~T}$-MRI

Results: $2 / 15$ patients received full knee replacement due to insufficient cartilage repair through MFX during FU period. Evaluation of pre- and postoperative MRI showed good cartilage repair tissue in one $(7.7 \%)$, moderate repair in two $(15.4 \%)$ and poor fill in ten patients $(76.9 \%)$. In these ten patients the defect size increased. Average defect size preoperatively was $187 \mathrm{~mm} 2(12-800 \mathrm{~mm} 2)$ and postoperatively $294 \mathrm{~mm} 2$ (40- $800 \mathrm{~mm} 2)$. Ten patients showed a varus leg axis deviation $\left(\varnothing 5.9^{\circ}\right)$ and three had a neutral alignment. The alignment correlated positively with declining knee function. Conclusion: Our study demonstrated that MFX as a treatment option for cartilage defect in the knee did not show the anticipated radiological long-term results. In 12/15 patients the cartilage defect size had increased after MFX, in 2/15 indicating full-knee- replacement.

\section{B-790 15:21}

Effect of gender in the follow-up after matrix-associated autologous chondrocyte transplantation (MACT) as assessed by morphological and biochemical magnetic resonance imaging

G.H. Welsch ${ }^{1}$, S. Werner ${ }^{1}$, S. Marlovits ${ }^{1}$, T.C. Mamisch², D. Stelzeneder ${ }^{1}$, A. Messner ${ }^{1}$, A. Kautzky-Willer ${ }^{1}$, S. Domayer ${ }^{1}$, S. Trattnig ${ }^{1} ;{ }^{1}$ Vienna/AT, ${ }^{2}$ Berne/CH (welsch@bwh.harvard.edu)

Purpose: The objective of this study was to determine gender-related differences in the repair tissue after MACT as assessed by advanced morphological and biochemical MRI.

Methods and Materials: 40 patients (20 females, 20 males) after MACT of one femoral condyle of the knee were included. Both groups were matched by age (females: $35.1 \pm 13.7$; males: $35.2 \pm 8.7$ years) and post-operative follow-up (females: $34.0 \pm 17.4$; males: $34.2 \pm 16.8$ months). At 3.0 Tesla MRI, morphological magnetic resonance observation of cartilage repair tissue (MOCART) scoring based on high-resolution PD-TSE, dual-FSE and TIRM sequences, was performed as well as biochemical T2 mapping, based on a multi-echo spin-echo sequence. A zonal (deep and superficial) region-of-interest analysis was performed to assess the quantitative T2-values in native control cartilage and cartilage repair tissue. 
Results: The MOCART score was comparable in female $(69.4 \pm 20.0)$ and male $(69.3 \pm 13.0)$ patients $(p=0.982)$. Seven of the nine variables did not differ significantly. Whereas the structure of the repair tissue was more homogeneous in female patients $(p=0.026)$, changes in the subchondral bone and bone marrow oedema were less often visible in male patients $(p=0.007)$.

Quantitative T2-values ( $\mathrm{ms}$ ) showed comparable results for the native control cartilage (female: deep $=47.7 \pm 9.6$, superficial $=53.1 \pm 9.2 ;$ male: deep $=49.8 \pm 12.6$, superficial $=53.7 \pm 10.5 ; p=0.445$ (deep), $p=0.826$ (superficial)); whereas the repair tissue showed significant shorter T2-values of the deep cartilage layer in female compared to male patients ( (female: deep $=43.5 \pm 9.8$, superficial $=48.5 \pm 9.8$; male: deep $=48.2 \pm 7.7$, superficial $=52.6 \pm 11.0 ; p=0.009$ (deep), $p=0.052$ (superficial)). Conclusion: Our initial results suggest that gender-related differences in the ultrastructure of the repair tissue, mainly in the subchondral bone and the adjacent deep cartilage layer, can be assessed using advanced morphological and biochemical MRI.

\begin{tabular}{ll}
\hline $14: 00-15: 30$ & Room F1 \\
\hline
\end{tabular}

\section{Genitourinary}

\section{SS 1707}

\section{Recent advances in GU}

Moderators:

G. Ivanac; Zagreb/HR

R.H. Oyen; Leuven/BE

\section{B-791 14:00}

Split-bolus CT-urography in dual-energy (DE) mode: there is a real dose reduction?

A. Mileto, S. Racchiusa, M. Gaeta, G. Ascenti, S. Mazziotti, F. Zimbaro,

E. Scribano, S. Caloggero, A. Blandino; Messina/IT (achille.mileto@gmail.com)

Purpose: To evaluate the potential dose reduction of a dual-energy (DE) singlephase split-bolus CT-urography protocol in comparison with standard single-energy (SE) dual-phase split-bolus CT-urography protocol. We also compared the quality of virtual nonenhanced (VNE) images derived from DE acquisition with that of true nonenhanced (TNE) data-set in the same patients.

Methods and Materials: 64 patients underwent CT urography with the following study protocol: TNE images acquisition in SE-mode, $50 \mathrm{~mL}$ bolus, 6-minute delay, $80 \mathrm{~mL}$ bolus, 100 seconds delay, urinary tract acquisition in DE-mode. On a secondary work-station VNE images were obtained. Two blinded and independent readers rated the quality of VNE respect to TNE data-set with a five-point scale: for 1-3 scores were judged comparable, not comparable for 4-5 scores. Effective radiation doses for $\mathrm{DE}$ single-phase protocol and standard SE dual-phase protocol (obtained by SE-mode scanning dose x2-multiplying) were calculated. Cohen's $\mathrm{k}$ was used to assess the interobserver agreement for VNE images evaluation. Results: VNE images were considered comparable (scores 1-3) to TNE data-set in the same 57 out of 64 patients $(k=.891)$. In the remaining 7 patients VNE images were judged not comparable (scores 4-5) for excessive noise, kidney exclusion or lower conspicuity of calcification. Mean effective dose of DE single-phase protocol was $6.37 \mathrm{mSv} \pm 1.54$ and that for standard SE dual-phase protocol was 8.92 $\mathrm{mSv} \pm 1.22$. Mean calculated dose reduction was $28.59 \%$.

Conclusion: Split-bolus CT-urography in DE-mode allows a radiation exposure lowering by $28 \%$, with VNE images that could replace TNE data-set.

\section{B-792 14:09}

Feasibility of CTA for renal arteries using lower tube voltage ( $80 \mathrm{kVp})$ and moderate-concentration of iodine contrast material: quantitative and qualitative comparison with conventional CTA

J.H. Lim, E.-S. Cho, J.-S. Yu; Seoul/KR (jihe@yuhs.ac)

Purpose: To investigate the feasibility of $80 \mathrm{kVp}$ with moderate-concentration of contrast material (MC-CM) in CT angiography of renal arteries compared to conventional $120 \mathrm{kVp}$ with high-concentration of contrast material (HC-CM).

Methods and Materials: Fifty patients with a clinical suspicion of renovascular hypertension were referred for CT angiography of renal arteries. Twenty-five patients were scanned with $120 \mathrm{kVp}$ and $200 \mathrm{mAs}$ after $110 \mathrm{~mL}$ of $\mathrm{HC}-\mathrm{CM}(370 \mathrm{mgl} / \mathrm{mL})$ administration and the other 25 patients were scanned with $80 \mathrm{kVp}$ and $585 \mathrm{mAs}_{\text {eff }}$ after $110 \mathrm{~mL}$ MC-CM $(300 \mathrm{mgl} / \mathrm{mL})$ administration. Two groups of patients were compared with each other in terms of Hounsfield number of renal arteries, signalto-noise ratio (SNR), contrast-to-noise ratio (CNR), subjective degree of arterial enhancement, image noise, sharpness of renal arteries, and overall diagnostic image quality of both maximum-intensity projection and volume-rendered images.
Results: Mean attenuation of main renal artery was significantly higher in $80 \mathrm{kVp}$ with MC-CM $(370.0 \pm 65.0 \mathrm{HU})$ than $120 \mathrm{kVp}$ with $\mathrm{HC}-\mathrm{CM}(269.9 \pm 27.8 \mathrm{HU}, \mathrm{p}<0.001)$ without significant differences in SNR and CNR. $80-\mathrm{kVp}$ protocol had significantly higher quality scores in arterial enhancement, sharpness of the artery, and overall diagnostic image quality compared to $120-\mathrm{kVp}$ protocol. Effective dose of $80-\mathrm{kVp}$ protocol $(4.5 \pm 0.3 \mathrm{mSv})$ was $8.2 \%$ lower than that of $120-\mathrm{kVp}$ protocol $(4.9 \pm 0.4 \mathrm{mSv}, \mathrm{p}<0.001)$. Conclusion: The use of $80 \mathrm{kVp}$ with MC-CM could improve arterial enhancement and provide superior image quality than $120 \mathrm{kVp}$ with $\mathrm{HC}-\mathrm{CM}$, despite using smaller amount of iodine administration and decreased radiation dose.

\section{B-793 14:18}

\section{The role of dual energy computed tomography in detection of}

intravascular invasion of renal cell carcinoma

J. Ferda, M. Hora, O. Hes, H. Mírka, J. Baxa, E. Ferdová, B. Kreuzberg;

Plzen/CZ (ferda@fnplzen.cz)

Purpose: To evaluate the accuracy of T3b-renal-cell-carcinoma (RCC) detection using dual energy-CT (DECT) and to evaluate the image guality.

Methods and Materials: 48 pt. (26 males, 22 females) who underwent surgery due to the suspected RCC were enrolled in the study. DECT in the arterial phase of contrast material distribution was performed using dual-source CT system with simultaneous exposition of 140 and $80 \mathrm{kV}$, respectively. The data were reconstructed in $0.6 \mathrm{~mm}$ thickness with one-third overlapping in the order of isotropic submilimeter resolution. The presence of vascular invasion or massive venous contrast return in the early arterial phase was signed as the suspected vascular invasion. The image analysis included the assessment of averaged, low-kV and virtual nonenhanced images. Following image quality was estimated: 1-excellent, 2-good, 3-evaluable, 4-insufficient. After resection or nephrectomy, all specimens were assessed, typed and staged by experienced pathologist according to $\mathrm{WHO}$ criteria and the pathologic results were compared with CT findings.

Results: The accuracy of the RCC detection reached 93/7\% (45/48) - two benign tumours and one inflammatory process were confirmed. The sensitivity according to the detection of T3b RCC stage was $87 \%(7 / 8)$, specificity reached $92 \%(37 / 40)$. The average image quality vas marked 1.3 , no one examination was marked as insufficient, the decreased quality of some patients were caused by obesity and expansion over the field of view of tube $B$.

Conclusion: The vascular invasion of RCC could be depicted with DECT with the high specificity and sensitivity using high quality imaging.

\section{B-794 14:27}

Dual energy CT in patients with polycystic kidney disease

A. Graser, N. Arndt, M. Staehler, M.F. Reiser, T.R.C. Johnson; Munich/DE (anno.graser@med.uni-muenchen.de)

Purpose: To evaluate the efficacy of dual source-dual energy CT (DECT) in the detection of neoplasia in patients with polycystic kidney disease.

Methods and Materials: 20 patients with polycystic kidney disease (ADPKD, $\mathrm{n}=11$; multicystic kidney disease, $\mathrm{n}=9$ ) underwent DECT on a dual source scanner (Somatom Definition Flash, Siemens Healthcare), using kVp settings of Sn140/100 kVp, $32 \times 0.6 \mathrm{~mm}$ collimation, and 232/310 mAs, online tube current modulation. A single phase examination 70 s after iv injection of $1.3 \mathrm{~mL} / \mathrm{kg}$ body weight of Imeron 400, Bracco Diagnostics was acquired. Colour-coded iodine maps and virtual nonenhanced images were used to determine enhancement within cysts and to differentiate haemorrhagic from simple cysts. A cut-off of $15 \mathrm{HU}$ was used as threshold for malignancy. The number of cysts on each kidney was recorded. In patients with malignancy, histopathology was the gold standard; otherwise, patients underwent follow-up imaging for 120-381 days. Average radiation exposure was calculated.

Results: Each patient had > 50 kidney cysts. Based on measured enhancement, malignant masses were seen in 8 patients, these were confirmed at histopathology as clear cell RCC $(n=5)$ and papillary RCC $(n=3)$; follow-up imaging showed no malignancy in 12 patients. Cysts did not enhance by more then $15 \mathrm{HU}$, while masses showed a mean enhancement of $45(25-123) \mathrm{HU}$. Mean radiation exposure was $5.5 \pm 1.3 \mathrm{mSv}$.

Conclusion: In patients with polycystic kidney disease DECT greatly facilitates the detection of malignancy, at the same time reducing radiation exposure by omission of a true nonenhanced phase. 


\author{
B-795 14:36 \\ MR imaging in endometriosis infiltrating the bowel wall \\ M.P.H. Busard, A.B. Luchinger, V. Mijatovic, I.C. Pieters-van den Bos, \\ M.A. Cuesta, M.C.G. Bleeker, C. van Kuijk, P.G.A. Hompes, \\ J.H.T.M. van Waesberghe; Amsterdam/NL (m.busard@vumc.nl)
}

Purpose: To describe MR imaging morphologic- and signal intensity abnormalities of endometriosis infiltrating the bowel and to assess the value of MR imaging in predicting depth of bowel wall infiltration.

Methods and Materials: This, single-centre, retrospective study was performed in a tertiary referral centre for endometriosis (2004-2010). A total of 473 consecutive patients were analysed for diagnosis of endometriosis infiltrating the bowel. All MR images were analysed by one experienced reader (number of lesions, location, size, $\mathrm{SI}$, and depth of bowel wall infiltration ("adhesions", serosal, muscular or suspicion of mucosal infiltration)). Inter-rater agreement for infiltration of the muscular layer of the bowel wall was calculated in a subset of patients. In 24 patients (27 lesions) MR imaging was correlated with histopathology.

Results: MR images revealed endometriosis infiltrating the bowel in $192(41 \%)$ patients (222 lesions). The inter-rater agreement for infiltration of the muscular layer of the bowel wall was 0.80 . All lesions showed isointense or slightly hyperintense signal compared with muscle with foci of high signal intensity on T2- and T1-weighted imaging in 42 and 33 cases, respectively. Depth of infiltration was assessed as serosal and muscular 29 and 178 lesions, respectively, and suspicion of mucosal infiltration was raised in 15 lesions. Compared with histopathology, bowel wall infiltration on MR imaging was correctly scored in 22 out of $27(81 \%)$ lesions. Conclusion: MR imaging is useful to predict serosal and muscular infiltration of the bowel wall, providing a roadmap to surgery.

\section{B-796 14:45}

Comparison of 3D and 2D FSE T2-weighted MR imaging in the diagnosis of pelvic endometriosis: preliminary results

M. Bazot' ${ }^{1}$, A. Stivalet', I. Thomassin-Naggara', E. Darai', C. Coudray²,

E. Poncelet ${ }^{3} ;{ }^{1}$ Paris/FR, ${ }^{2}$ Buc/FR, ${ }^{3}$ Lille/FR (marc.bazot@tnn.aphp.fr)

Purpose: To prospectively evaluate overall image quality and diagnostic accuracy of $3 \mathrm{D}$ and $2 \mathrm{D}$ T2-weighted MR imaging for the evaluation of pelvic endometriosis. Methods and Materials: One hundred and ten consecutive patients with clinical suspicion of endometriosis were recruited at two institutions. Two readers with different degrees of experience were independently asked to interpret images using both 3D and 2D MRI. Each dataset was independently evaluated by readers for the presence or absence of pelvic endometriosis (ovaries, uterosacral ligaments, vagina, rectosigmoid, Douglas pouch and bladder). Descriptive analysis, Mc Nemar test and Chi-square test were used. Intra- and interobserver agreement was calculated using $\mathrm{k}$ coefficients.

Results: Both readers found that $3 \mathrm{D}$ yielded significantly lower image quality than $2 \mathrm{D} \mathrm{MRI}(p<0.0001)$. Acquisition time for $3 \mathrm{D}$ was significantly less than that for $2 D$ MRI $(p<0.01)$. Out of twenty-three women undergoing surgery, 23 had endometriosis at histology. 3D offered a similar accuracy to diagnose ovarian and deep infiltrating endometriosis compared with 2D MRI. For all locations of endometriosis, a high or variable intraobserver agreement was observed for reader 1 and 2 , respectively.

Conclusion: Despite a lower overall image quality, 3D provides significant time saving and similar accuracy than multiplanar 2D MRI in the diagnosis of specific locations of endometriosis.

\section{B-797 14:54}

Occurrence of extrapelvic localisation of endometriosis in course of severe pelvic disease: role of MRI

G. Foti, N. Faccioli, M. Barillari, A. Zaccarella, R. Manfredi, R. Pozzi Mucelli; Verona/IT (gfoti81@yahoo.it)

Purpose: To evaluate the accuracy of magnetic resonance (MRI) in the assessment of extrapelvic endometriosis.

Methods and Materials: The MRI exams of 127 patients (mean age 29.8 years) suffering from severe pelvic endometriosis, performed within 1 week before laparoscopy, were retrospectively reviewed by two readers. Number, site, size of each implant were evaluated. At MRI, diagnosis was based on hyperintense lesions on T1-WI, plaque-like hypointense lesions on T1 and T2-WI, or indirect findings (tethering, thickening of peritoneal fat and visceral wall). Using laparoscopy as reference standard, MRI diagnostic accuracy was calculated. Pearson test was used to compare lesions size. Interobserver variability in determining the presence of extrapelvic disease was assessed with $k$ statistics. A value of $p<0.05$ was considered significant.
Results: At laparoscopy 97 lesions (bowel $=68$; peritoneum other than Douglas pouch $=15$; bladder $=7$; ureters $=7$ ) were identified in 75 patients, with 19 women presenting multiple implants. MR identified $77 / 97$ lesions, missing 14 bowel lesions, 3 of the peritoneum, 2 of the ureters, 1 of the bladder, with 10 false positive findings. MRI diagnosis was based on hyperintense lesions on T1-WI (36), plaque-like lesions (31), indirect findings (10). Sensitivity, specificity, PPV, NPV of MRI were $81 \%, 92 \%, 94 \%$ and $77 \%$, respectively. The correlation regarding lesion size at MRI (mean $33 \mathrm{~mm}$ ) and laparoscopy (mean $34 \mathrm{~mm}$ ) was good $(p<0.01)$. Interobserver variability was excellent $(k=0.92)$.

Conclusion: Identifying extrapelvic endometriosis preoperatively, MRI could be useful for treatment planning in case of severe pelvic disease.

\section{B-798 15:03}

Dose reduction for $\mathrm{CT}$ imaging of female pelvis with adaptive statistical iterative reconstruction on high-definition CT

Y. Cui, K. Cao, X.-Y. Zhang, J. Li, S.-Y. Gao, X.-P. Zhang; Beijing/CN (tjbjcui@yahoo.com.cn)

Purpose: To evaluate the dose reduction potential of an adaptive statistical iterative reconstruction (ASIR) on a high-definition CT (HDCT) for imaging female pelvis by comparing dose and image quality between HDCT and a 64-slice standarddefinition CT (SDCT).

Methods and Materials: Forty consecutive female patients for pelvic examination were included, with 23 scanned on SDCT and 17 on HDCT. Similar scan parameters were used on both scanners expect the tube currents of $310 \mathrm{~mA}$ and $200 \mathrm{~mA}$ with SDCT and HDCT, respectively. Images were reconstructed with a standard kernel on both scanners and ASIR (30\%) was applied on HDCT. Radiation dose was recorded and image noises for the background, uterine myometrium, internal obturator muscle and fat were measured and compared.

Results: All images were clinically acceptable. The image noises for SDCT group were $5.1 \pm 0.7 \mathrm{HU}, 11.5 \pm 2.6 \mathrm{HU}, 11.3 \pm 2.6 \mathrm{HU}$, and $9.8 \pm 1.6 \mathrm{HU}$ from the background, uterus myometrium, internal obturator muscle, and fat regions, respectively. The image noises for HDCT with ASIR were $5.5 \pm 0.9 \mathrm{HU}, 10.4 \pm 2.0 \mathrm{HU}$, $10.7 \pm 1.6 \mathrm{HU}$, and $10.2 \pm 1.7 \mathrm{HU}$ from the corresponding four regions, respectively. There was no statistical difference for the image noises between the two groups $(P>0.05)$. On the other hand, the average $x$-ray dose with HDCT was only $16.9 \pm 5.0 \mathrm{mGy}$, much lower than that of SDCT group $(26.1 \pm 2.0 \mathrm{mGy})(P<0.01)$. Conclusion: The use of $30 \%$ ASIR on HDCT maintained the same image quality for female pelvic scans as conventional SDCT while providing $35 \%$ dose reduction to patients.

\section{B-799 15:12}

Assessment of feto-placental unit oxygenation in an IUGR rat model using BOLD effect in MRI $4.7 \mathrm{~T}$

G.E. Chalouhi, M. Alison, L.J. Salomon, G. Autret, R. Thiam, D. Balvay, Y. Ville, O. Clément, N. Siauve; Paris/FR (gihad.chalouhi@gmail.com)

Purpose: This study aimed at investigating the use of the BOLD (blood oxygen level dependent) effect during an oxygen-challenge magnetic resonance imaging in an intra-uterine-growth-restriction (IUGR) rat model.

Methods and Materials: The IUGR rat model is based on a left uterine artery ligation. A small animal MRI 4.7 T unit was used, and a multiple gradient echo sequence (25 echo times [1.8 to $49.8 \mathrm{~ms}$ ]; TR $3500 \mathrm{~ms}$ ) was developed. T2* were measured in maternal livers, in the placentas, in foetal livers and brains, before and after the mother breathed oxygen $\left(6 / \mathrm{O}_{2} / \mathrm{min}\right)$. All foetuses and placentas were weighed afterwards to validate the IUGR model. Wilcoxon's signed rank sum tests were performed to compare the right and left foetoplacental units (FPU).

Results: Sixteen rats were studied, with a significant-induced growth restriction of $21.89 \%$ in the weight of the foetuses. A BOLD effect was detected in the maternal liver, as well as in all the placentas ( $n=61,34$ right, 27 left), the foetal livers $(n=$ 62,41 right, 21 left), and the foetal brains $(n=56,36$ right, 20 left $(p<0.002)$. The BOLD effect was significantly different $(p<0.0036)$ between the left ligated horn (IUGR) and the right horn for the placentas and the foetal brains. No significant difference was noted for the foetal livers.

Conclusion: BOLD MRI at $4.7 \mathrm{~T}$ is a good non-invasive tool to evaluate FPU oxygenation, and to detect IUGR. This technique may potentially be applied to the identification and understanding of placental dysfunction in IUGR in humans. 


\section{B-800 15:21 \\ Sexual development in utero: testicular descent on prenatal MRI U. Nemec, S.F. Nemec, M. Weber, P.C. Brugger, G. Kasprian, D. Prayer;} Vienna/AT

Purpose: Foetal gender determination including the evaluation of testicular descent is an important tool to assess normal male sexual development. In view of the increasing role of foetal magnetic resonance imaging (MRI) as an adjunct to US in prenatal diagnosis, we aim to demonstrate testicular descent in correlation to the gestational age on MRI in utero.

Methods and Materials: After chart review, this retrospective study included foetal MR scans of 202 male foetuses [17 to 39 gestational weeks (GW)] with normal anatomy or minor anomalies. On a 1.5 Tesla unit, multiplanar T2-weighted turbo-spin-echo sequences were applied to image the scrotal content. Relative frequencies of unilateral and bilateral testicular descent were calculated and correlated to the gestational age. Results: From 17 to $25 \mathrm{GW}$, no testicular descent was visualised. At $25+4 \mathrm{GW}$, unilateral descent was seen in $7.7 \%$ of cases. At $27 \mathrm{GW}, 62.5 \%$ of cases showed unilateral descent, and 50\% bilateral descent. At $30 \mathrm{GW}$, bilateral descent was seen in $100 \%$ of cases.

Conclusion: Our study charts the time course of testicular descent on prenatal MRI which may be useful in the identification of normal male sexual development and of congenital abnormalities, including cryptorchidism.

\section{4:00 - 15:30} Room F2

\section{Breast}

\section{SS 1702}

\section{Current problems in breast imaging}

\section{Moderators:}

E. Belloni; Piacenza/IT

A. Kanakas; Nicosia/CY

\section{B-801 14:00}

Male breast disease: optimising patient investigation

V. Ames ${ }^{1}$, J. Foreman ${ }^{2}$, M.G. Wallis ${ }^{2} ;{ }^{1}$ London/UK, ${ }^{2}$ Cambridge/UK

(v_ames_8@hotmail.com)

Purpose: Male patients represent approximately $1 \%$ of breast cancer diagnoses within the UK population. Our aim was to quantitatively assess the relative contribution of clinical examination and the standard imaging modalities, in the diagnosis of male breast disease.

Methods and Materials: All patients attending our Breast Unit have a prospective score (1-5) recorded for the clinical evaluation, mammography and ultrasound. We retrospectively evaluated the data on all male patients referred between January 2001 and December 2009. Findings were analysed to assess their contribution in making the diagnosis. For the purposes of this audit, scores 4 and 5 were classed as positive. Results: 1264 men were referred to the unit during the 9 years that we analysed. 130 men were excluded: 94 due to inadequate data; 20 referred for axillary node biopsy; 9 with nipple discharge as the sole symptom; 2 with known breast cancer; 5 with other malignancies. 23 of the remaining 1134 patients were diagnosed with breast cancer. The sensitivity, specificity, positive predictive value and negative predictive value for clinical examination $(n=1110)$ were: $71.4 \%, 99.4 \%, 71.4 \%$ and $99.4 \%$; for mammography $(n=671)$ were: $77.8 \%, 99.8 \%, 93.3 \%$ and $99.4 \%$; for ultrasound ( $n=361$ ) were: $90.1 \%, 99.1 \%, 87 \%$ and $99.4 \%$ respectively. Of note, two of the nine $(22 \%)$ patients excluded for nipple discharge only had biopsy-proven cancer. Conclusion: Nipple discharge is a very suspicious feature. Although all three modalities are highly specific, the lower sensitivity of clinical examination mandates the continuing use of imaging in the investigation of male breast disease.

\section{B-802 14:09}

Sonographically guided core cut biopsy: follow-up after 15 years

R. Schulz-Wendtland, B. Adamietz, M. Meier-Meitinger, E. Wenkel, M. Uder;

Erlangen/DE (ruediger.schulz-wendtland@uk-erlangen.de)

Purpose: From May 1992 to April 1993, we performed 307 ultrasonic guided core cut biopsies. In 119 of the 307 women, we dispensed with further surgical and histological procedures when the tentative diagnosis from complementary mammary diagnostic procedures revealed no pathological findings and concurred with the histological results of the core cut biopsy. The aim of our study was a follow-up after 15 years.
Methods and Materials: On 119 patients we performed the ultrasonic guided core cut biopsies $\left(7.5 \mathrm{mHz}\right.$-array, core cut biopsy systems BIP ${ }^{\text {TM }}$, today BARD Angiomed $^{\mathrm{TM}}$ and Pflugbeil ${ }^{\mathrm{TM}}$, coaxial- and core cut needle with a length of $10 \mathrm{~cm}$, diameter $2.1 \mathrm{~mm}$ (14G), core cut depth 1.6 resp. $2.2 \mathrm{~cm}, 1-3$ specimen) with a benign histology. Control intervals were 3.6,12 and 24 months sonographically, 12 and 24 months mammographically and a follow-up after 15 years.

Results: For 103 of the 117 patients it was possible to perform a follow-up. On one patient a histologically verified fibroadenoma with the tendency to grow was operated and the benign histology was confirmed. A second patient was therapied at a lobular carcinoma 43 months after core cut biopsy and the histology "simple mastopathy", and the third patient was operated at a breast cancer 56 months after intervention in that area, in which the result of the core cut biopsy was "to less material". Sensitivity, specificity $p p V$ and $n p V$ were in each case $98 \%$, at a $p<0.0001$. Conclusion: Precondition for the feasibility of ultrasonic guided core cut biopsies is the consequent use of the European guidelines.

\section{B-803 14:18}

The effect of experience and skill on volumetric helical perfusion CT quantification of primary breast cancer

A. Gogbashian, S. Li, V. Shah, I. Simcock, J. Stirling, A. Makris, V. Goh; Middlesex/UK (andrew@cardiacforum.com)

Purpose: To assess the effect of experience and skill on inter- and intra-observer reliability of quantitative volumetric helical perfusion CT (VPCT) in primary breast cancer.

Methods and Materials: Following ethical approval, 20 prospective patients with primary breast cancer underwent VPCT (4D adaptive spiral; Dual Source CT, Siemens) encompassing the whole tumour prior to surgery. Five observers participated (attending radiologist, oncology fellow, radiology fellow, radiology resident, CT technologist). Following dedicated training, each observer evaluated 20 datasets (deconvolution analysis; vPCT Body, Siemens). Maximum tumour size, volume, whole tumour blood flow (BF), blood volume (BV) and extraction fraction (ES) were recorded for the volume of interest. Each dataset was re-evaluated with $>4$ weeks between reads. Analysis of variance was performed to assess inter-observer differences in parameters. Bland-Altman statistics were applied to assess intra-observer reliability. Significance was at $5 \%$.

Results: Mean (SD) tumour size was $4.1(2.1) \mathrm{cm}$ and volume $42(90.9) \mathrm{cm}^{3}$. Within observers, the intra-class correlation coefficient ranged from 0.83 to 0.99 demonstrating excellent intra-observer agreement. There were no significant differences in quantitative parameters between observers; $B F(F=0.56 ; P=0.69)$, $\mathrm{BV}(\mathrm{F}=0.84 ; \mathrm{P}=0.50)$ and $E S(F=1.13 ; \mathrm{P}=0.34)$ indicating that quantification using a volumetric region of interest and $\mathrm{VPCT}$ is highly reproducible irrespective of experience and skill.

Conclusion: Quantitative vascular parameters are being applied increasingly to assess tumour biology and therapy response in clinical practice. With vPCT and volume of interest analysis, data can be reliably and reproducibly obtained by observers with differing experience.

\section{B-804 14:27}

Impact of real-time virtual sonography, a coordinated sonography and MRI system with magnetic navigation, on the preoperative staging of breast cancer

S. Nakano ${ }^{1}$, K. Yorozuya ${ }^{1}$, K. Fujiii', M. Yoshida' ${ }^{1}$, T. Fukutomi ${ }^{1}$, J. Kimura',

Y. Ohshima' ${ }^{1}$ T. Ishiguchi ${ }^{1}$, O. Arai ${ }^{2} ;{ }^{1}$ Aichi-gun/JP, ${ }^{2}$ Kashiwa/JP

(snakano1@aichi-med-u.ac.jp)

Purpose: We recently developed a real-time virtual sonography (RVS) that can simultaneously display images of the same obtained by sonography and magnetic resonance imaging (MRI) using magnetic navigation. The purpose of this study was to evaluate the role of RVS in the management of enhancing lesions visualised with MRI.

Methods and Materials: Between June 2006 and April 2007, 65 patients underwent MRI for staging of known breast cancer at our hospital. All patients were examined using mammography, sonography, MRI and RVS before surgical resection. Results were correlated with histopathologic findings. MRI was obtained on a 1.5 T imager, with the patient in the supine position using a flexible body surface coil. Detection rate was determined for main tumours and incidental enhancing lesions (IELs), with or without RVS.

Results: Overall sensitivity for detecting main tumours was 85\% (55/65) for mammography, $91 \%$ (59/65) for sonography, $97 \%$ (63/65) for MRI and 98\% (64/65) fo RVS. IELs were found in $26 \%(17 / 65)$ of the patients. Of 23 IELs that were detected by $\mathrm{MRI}, 30 \%(7 / 23)$ of IELs could be identified on repeated sonography alone, but $83 \%(19 / 23)$ of them were identified using the RVS system $(P=0.001)$. The 
RVS system was able to correctly project enhanced MRI information onto a body surface, as we checked sonography form images.

Conclusion: Our results suggest that RVS can sonographically identify enhancing breast lesions with excellent accuracy.

\section{B-805 14:36}

Medicolegal claims following screening mammography in the Netherlands W. Setz-Pels, V. van Breest Smallenburg, F.H. Jansen, L.E.M. Duijm;

Eindhoven/NL (w.setzpels@gmail.com)

Purpose: To determine the type and frequency of medicolegal claims at a Dutch breast cancer screening programme.

Methods and Materials: The study population consisted of all 80019 women who underwent screening mammography at a southern breast screening region of the Netherlands between January 1997 and July 2007 (301139 screens). We included all medicolegal claims that had been recorded at the central screening department within 3 years following screening mammography. During 2-year follow-up, we collected the biopsy results and surgery reports of all referred women. Two screening radiologists reviewed the screening mammograms of all screen-detected cancers (SDC) and interval cancers (IC) and determined whether the cancer had been missed at the previous screen (in case of SDC) or latest screen (in case of IC). The radiologists were blinded to each other's review; discrepant readings were followed by consensus reading.

Results: Just 3 medicolegal claims had been reported, all of them related to financial compensation following a diagnosis of IC. The verdicts of these cases stil have to be finalised. Excision biopsy had been performed in $10.7 \%(234 / 2183)$ of false-positive referrals. Review showed that $20.8 \%$ (261/1254) of SDCs had been missed at the previous screen and $23.6 \%(139 / 588)$ of ICs should have been detected at the latest screen.

Conclusion: Medicolegal claims were very rare, although a substantial proportion of referred women with benign follow-up had been confronted with excision biopsy and more than $20 \%$ of SDCs and ICs had been missed at the previous screen or latest screen, respectively.

\section{B-806 14:45}

Effect of previous benign breast surgery on cancer detection at screening mammography

J. Nederend ${ }^{1}$, V. van Breest Smallenburg'1, M.W.J. Louwman'1, A.C. Voogd ${ }^{2}$, J.H. Groenewoud ${ }^{3}$, F.H. Jansen ${ }^{1}$, M. van Beek ${ }^{1}$, L.E.M. Duijm ${ }^{1} ;{ }^{1}$ Eindhoven/NL, ${ }^{2}$ Maastricht/NL, ${ }^{3}$ Rotterdam/NL (joost.nederend@gmail.com)

Purpose: The influence of previous benign breast surgery on screening mammography accuracy is unknown. We determined whether the sensitivity of screening mammography and tumour characteristics are different for women with and without previous benign breast surgery.

Methods and Materials: We included a consecutive series of 317398 screening mammograms performed between 1997-2008. During 2-year follow-up, clinica data, breast imaging reports, biopsy results and breast surgery reports were collected of women with screen-detected or interval breast cancers. Screening sensitivity, tumour biology and tumour stages were compared between 168 women with breast cancer in the ipsilateral breast after prior benign surgery and 2039 women with breast cancer, but without previous ipsilateral, benign breast surgery. Results: The sensitivity of screening mammography was significantly lower for women with prior surgery $(64.3 \%$ (108/168) versus $73.4 \%(1496 / 2039), p=0.01)$. The concomitant increased interval cancer risk remained significant after logistic regression adjustment for age and breast density ( $\mathrm{OR}=1.5,95 \% \mathrm{Cl}: 1.1-2.1)$. Comparing screen-detected cancers in women with and without prior breast surgery, no significant differences in oestrogen-receptor status $(p=0.56)$, mitotic activity ( $p$ $=0.17)$, proportions of large $(\mathrm{T} 2+)$ tumors $(p=0.6)$ or lymph node positive tumors $(p=0.4)$ were found. For interval cancers, oestrogen-receptor status $(p=0.41)$ mitotic activity $(p=0.39)$ and proportions of large tumours $(p=0.9)$ and lymph node positive tumours $(p=0.5)$ were neither statistically significant.

Conclusion: Sensitivity of screening mammography is significantly lower in women with previous benign breast surgery than without, but tumour characteristics are comparable. At screening women should be informed about the decreased sensitivity of screening mammography in post-surgical breasts.
B-807 14:54

Is BI-RADS 3 a misinterpreted category in breast-diagnosis? Three years follow-up of probably benign lesions in a decentralised breast diagnosis project

S. Kapsimalakou, A. Bischof, T. Borberg, I. Grande-Nagel, P. Bischoff,

A. Kovacs, P. Hunold, J. Barkhausen, F.M. Vogt; Lübeck/DE

(Smaragda.Kapsimalakou@uk-sh.de)

Purpose: Our focus is the benefit of the 6-month surveillance in cases classified as BI-RADS 3.

Methods and Materials: 28,726 women (mean age $57 \pm 12$ years) were examined. Two independent radiologists read the mammogram as well as the supplemented ultrasound (in case of breast density ACR type 3 and 4). In case of discordance regarding the $\mathrm{BI}-\mathrm{RADS}$ classification, a third expert read the images and considered whether further examination was indicated or not.

Results: 3,081 diagnostic procedures ended with BI-RADS 3 results. Of those, $1,937(62.9 \%)$ women underwent a follow-up examination and for 1,930 cases the final BI-RADS classification was documented as follows: 36 (1.9\%) BI-RADS 1, 1086 (56.3\%) BI-RADS 2, 723 (37.5\%) BI-RADS 3, 60 (3.1\%) BI-RADS 4 and 25 (1.3\%) BI-RADS 6. This equals a tumour detection of $8.1 / 1,000$. The histologic tumour stadium and size were documented in 17 cases. The ratio in situ:invasive was $7: 10(1: 1.43)$ and the mean size was $10.6 \pm 4.3 \mathrm{~mm}$. In the total cohort of 28,726 diagnostic procedures, 533 carcinomas were detected with $17 \%$ in situ and $83 \%$ invasive breast cancer, ratio in situ:invasive $1: 4.9$ with and the mean size was $13.6 \pm 5.6 \mathrm{~mm}$.

Conlusions: The amount of detected tumours at follow-up of women with preceding BI-RADS 3 equates the associated potential of malignancy. Tumours that were diagnosed at follow-up examinations were on average smaller and had a higher proportion of early stage breast cancers compared to tumour findings of the total cohort.

B-808 15:03

Greater links to image quality should be applied to mean glandular dose diagnostic reference levels

D. O'Leary, L. Rainford, T. Grant; Dublin/IE (desiree.oleary@ucd.ie)

Purpose: This large study of symptomatic breast units geographically spread over the Republic of Ireland, collected image quality and radiation dose data from 18 mammography units. The effect of image quality on radiation dose (mean glandular dose; MGD) and vice versa was examined; the MGD diagnostic reference level has been to date proposed only with regard to compression level achieved not image quality required.

Methods and Materials: The quantitative and qualitative data were analysed using mathematical modelling and SPSS statistical tests including ANOVA. High image quality was matched to lowest achievable radiation doses; inadequate images were discarded from the dataset for recommendations of MGD.

Results: MGDs received by perfect images are significantly lower than the radiation doses received by inadequate images. This is seen in both digital images and film-screen images and for both mammographic projections: digital craniocaudal: $\mathrm{F}(3,978)=2.841 ; \mathrm{p}=0.37$; digital mediolateral oblique: $\mathrm{F}(3,977)=4.896 ; \mathrm{p}=$ 0.002; analogue craniocaudal: $F(3,785)=7.993 ; p<0.001$; analogue mediolatera oblique: $F(3,783)=7.961 ; p<0.001)$. The mean $M G D$ in $m G y$ required to produce a perfect image in each of the categories: analogue craniocaudal: $2.10 \mathrm{mGy}$ analogue mediolateral oblique: 2.25 mGy; digital craniocaudal: 1.23 mGy; digital mediolateral oblique: $1.28 \mathrm{mGy}$.

Conclusion: Greater training of radiographers performing mammography is required to standardise the undertaking of the mammographic projections with regard MGDs delivered to the breasts of Irish women attending the symptomatic breast services. MGD diagnostic reference levels should be linked to image quality achieved.

\section{B-809 15:12}

An objective method for classification of symptomatic mammography images in clinical audit

D. O'Leary, L. Rainford; Dublin/IE (desiree.oleary@ucd.ie)

Purpose: The ability to objectively critique and assess the image quality of mammograms obtained by each department is vital to assess the efficacy of the services provided by breast imaging units. As evidenced in the analysis of symptomatic breast images for a large national optimisation of mammographic examinations study, current methods of classification for symptomatic mammograms are largely subjective resulting in poor inter- and intra-departmental agreement on image quality. The subjectivity within current image quality criteria allows mammogra- 
phy departments to appear to achieve the high scoring requirements of only $3 \%$ inadequate images.

Methods and Materials: The European Quality Criteria for film-screen mammographic image quality, the proposed amended European criteria for digital mammography images and the breast screening quality criteria classification of images as inadequate, moderate, good and perfect were modified to remove all subjective criteria. These objective classifications of image quality were tested for inter- and intra-rater reliability by a panel of experts.

Results: Inter-rater reliability $(\mathrm{K}>0.701 ; \mathrm{p}<0.001)$ and agreement (Pearson's correlation $\rho>0.884 ; p<0.01$ ) by the evaluation panel was higher than using the original two methods. The intra-rater reliability was equally high $(K>0.7 ; p<0.001)$ and agreement via Pearson's correlation: $\rho>0.844 ; p<0.01$

Conclusion: A method of scoring images by combining the most objective components of two major European scoring systems for images is suggested as tested in a large national study within the symptomatic breast services of Ireland. The removal of subjectivity from the scoring systems will remove all doubt regarding the achievement of these image quality goals.

\section{B-810 15:21}

Artefacts in direct digital full-field mammography

Z.C. Milosevic, M. Nadrljanski; Belgrade/RS (dr.m.nadrljanski@gmail.com)

Purpose: The prospective analysis of artefacts in direct digital full-field mammography.

Methods and Materials: Between January 2009 and June 2010 screening or diagnostic mammography was performed in 4715 patients (Selenia, Hologic). The analysis of artefacts was based on following classification: patient-related artefacts (motion artefact, antiperspirant artefact, thin breast artifact), hardware-related artefacts (field inhomogeneity, detector-associated artefacts, collimator misalignment, underexposure, grid lines, grid misplacement, vibration artefact), and software processing artefacts ("breast-within-a-breast" artifact, vertical processing bars, loss of edge, high-density artifacts).

Results: The most common artefacts were patient-related artefacts (antiperspirant artefact) followed by intermittent field inhomogeneity artefact and high-density artefact (more than $10 \%$ of the mammograms). Motion artefact, thin breast artefact, underexposure artefact, breast-within a-breast artefact and loss of edge artefact were noted in less than $1 \%$ of the mammograms.

Conclusion: Artefacts can mask true abnormalities or create pseudolesions. Some artefacts in digital mammography are specific and, consecutively, should be identified and collected in order to avoid misinterpretation.

\section{4:00 - 15:30}

Room G/H

\section{Head and Neck}

\section{SS 1708}

\section{Insights into thyroid and parathyroid pathologies}

Moderators:

L. Grzycka-Kowalczyk; Lublin/PL

J. Olliff; Birmingham/UK

\section{B-811 14:00}

Hybrid imaging with Tc-99m Sestamibi SPECT-CT and ultrasonography performed together improves localisation of parathyroid adenomas

A. Parthipun; Carshalton/UK (parthipun@gmail.com)

Purpose: Pre-operative localisation of parathyroid adenomas is often performed with a combination of ${ }^{99 \mathrm{~m}} \mathrm{Tc}$-Sestamibi and ultrasonography (US) of the neck. In most institutions these examinations occur at different time points and are reported by different radiologists. The advent of hybrid imaging with single photon emission computed tomography with CT (SPECT-CT) improves localisation of parathyroid adenomas. The aim of this study is to evaluate whether SPECT-CT combined with US performed by a single radiologist improves localisation further.

Methods and Materials: 34 consecutive patients with biochemical evidence of primary hyperparathyroidism presenting to a university hospital were prospectively enrolled. Early and wash-out phase planar images and delayed SPECT-CT using $800 \mathrm{MBq}$ of ${ }^{99 \mathrm{~m} T \mathrm{C}-}$ Sestamibi were acquired on a Seimens Symbia Truepoint T6. US of the neck was performed on the same day by a single operator after reviewing the results of the SPECT-CT. Results: 28 parathyroid adenomas were identified in 34 patients. $20(71 \%)$ adenomas were visible on planar Sestamibi imaging, 27 (96\%) were visible on SPECT-CT and $23(82 \%)$ on US. US identified $1(3.5 \%)$ adenoma which was not visible on SPECT-CT and $6(21 \%)$ adenomas were visible on SPECT-CT but not on US
Conclusion: SPECT-CT improves localisation of parathyroid adenomas compared to planar imaging or US alone. Although US is not as sensitive as SPECT-CT, a combination of the two modalities reported by a single radiologist is the most accurate technique in the localisation of parathyroid adenomas.

B-812 14:09

Role of MDCTA in localization of parathyroid adenoma in primary hyperparathyroidism

R. Elias, C. van Eijck, W.W. de Herder, R. Valkema, A. van der Lugt; Rotterdam/NL (r.elias@erasmusmc.nl)

Purpose: To evaluate the role of multidetector computed tomography angiography (MDCTA) in preoperative localisation of hyperfunctioning parathyroid adenoma. Methods and Materials: Records of 77 patients with primary hyperparathyroidism between 2007 and 2009 were retrospectively reviewed. MDCTA was performed if there was discrepancy in the findings between ultrasound and Tc-99m-sestamibi scintigraphy (sestamibi). Patients with an MDCTA were selected for analysis. Ultrasound, sestamibi and MDCTA were reviewed and the results were compared with surgical and histopathologic findings. The operations were declared successful if the calcium and PTH levels were normalised after surgery.

Results: MDCTA was performed in 18 patients. Scan range included the neck and upper mediastinum. In 9 of 18 (50\%) patients ultrasound and sestamibi were positive, but there was discrepancy in accurate localising of adenoma $(n=7)$ or sestamibi revealed an adenoma in the upper mediastinum $(n=2)$. MDCTA identified and localised an adenoma in all cases. Surgical resection, which was performed in 7 patients, confirmed the imaging findings. In 7 of 18 patients (39\%) adenoma was revealed by either ultrasound or sestamibi. MDCTA confirmed the presence of an adenoma in all patients, which was surgically confirmed. In 2 patients (11\%) both ultrasound and sestamibi were negative. MDCTA revealed an adenoma in one patient, which was surgically confirmed.

Conclusion: MDCTA is a useful imaging technique in identifying and accurate localising parathyroid adenoma in patients with primary hyperparathyroidism in which ultrasound and sestamibi are discrepant or negative.

\section{B-813 14:18}

Thyroid cysts: their ultrasound worrisome appearance after evacuation

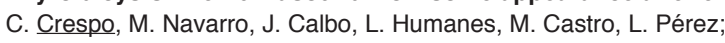
San Juan de Alicante/ES (crespo_conmar@gva.es)

Purpose: To show the worrisome ultrasound characteristics that thyroid cyst could became after their liquid content will be evacuated. The lesion wrinkled and appeared with characteristics suspicious of malignancy.

Methods and Materials: We reviewed the ultrasound examination of ten patients who had thyroid lesions with ultrasound criteria of malignancy. Only in two cases, there was medical information about previous puncture of a thyroid cyst. In five cases, it was the patient who told the radiologist about this.

Results: The studied thyroid nodules were solid and hypoechoic in ultrasound with irregular shape and ill defined margins. Three presented hyperechogenic foci and three showed a mixed pattern of vascularization. We performed follow-up ultrasound exam in all patients and biopsy in four of them. The pathologist found a benign lesion in all four cases. The review of the images of previous ultrasound exam (only disposable in six patients) showed characteristic features of thyroid cyst. Conclusion: All radiologists working in head and neck section knows the ultrasound appearance of a thyroid cyst. However, it is a habitual practice in clinical setting to evacuate a symptomatic thyroid cyst. In more instances the lesion is palpable and the procedure is done by the clinician or by the pathologist. The radiologist did not know this data and found a suspicious lesion in the gland. The knowledge of the lesion appearance at the initial ultrasound exam together with the information about the previous procedure could avoid an unnecessary biopsy and prevent the anxiety of the patient.

\section{B-814 14:27}

Correlations between shearwave elastography of thyroid nodules and cytological data: study about 157 patients

H. Monpeyssen, J.-M. Correas, J. Tramalloni, S. Poiree, N. Voillemot, J. Jais,

O. Hélénon; Paris/FR (hm-thyroide@wanadoo.fr)

Purpose: The correlation between a nodule stiffness and malignancy has been found by clinical palpation and demonstrated in in vitro studies. Strain elastography is able to provide stiffness information but the clinical routine use is suffering from limitations. Shear wave elastography (SWE) is becoming available and provides true quantitative measurement of stiffness with reduced variability between operators. The purpose of our study was to evaluate SWE in routine clinical practice. 
Methods and Materials: 161 thyroid nodules were studied (157 patients) using conventional US examination (MyLab Gold, Esaote. Italy) and SWE elastography (Aixplorer, Supersonic Imagine, France) with a high frequency linear transducer. Nodule stiffness and ratio were calculated. Cytological correlations were obtained in all cases using fine needle aspiration (FNAB). In addition, histological confirmation of cancer was obtained in case of suspicious cytological findings.

Results: SWE measurements from 32 nodules were finally excluded during the review process, corresponding to the learning curve. Statistical calculations were performed from the 129 nodules included. The stiffness of the embedding tissue was not significantly different between all the categories. Cancers exhibited a significant increased stiffness $(114 \pm 61 \mathrm{kPa})$ compared to normal parenchyma $(31 \pm 12 \mathrm{kPa})$ and other nodules $(34 \pm 17$ $\mathrm{kPa})$. The ratio between nodule and parenchyma was significantly higher $(\mathrm{p}<0.001)$. Conclusion: Shear wave elastography can provide additional information to the clinician to diagnose thyroid malignancy, and direct FNAB to the most suspicious lesions. It is an easy and promising imaging technique for characterisation of thyroid nodules. Multicentric study remains necessary to confirm these data.

\section{B-815 14:36 \\ Prospective evaluation of multiparametric ultrasound and quantitative elastosonography in the differential diagnosis of benign and malignant thyroid nodules \\ V. Cantisani, P. Ricci, O. Medvedeieva, M. Olive, M. Di Segni, E. Mancuso, \\ E. Maggini, R. Passariello; Rome/IT (vito.cantisani@uniroma1.it)}

Purpose: To assess the clinical value of quantitative sono-elastography in differentiating hystologically proven thyroid nodules as compared with multiparametric ultrasound evaluation.

Methods and Materials: 150 patients (range 21-83 years, mean age: 44 yrs) with thyroid nodules previously detected at colour-Doppler ultrasonound (CDUS), were prospectively examined with sono-elastography with dedicated quantitative software (Elasto-Q, Toshiba) before surgery (complete or partial thiroydectomy). Ultrasound examination and sono-elastography were evaluated by two readers in consensus. US features, colour-Doppler pattern, polychromatic map elastography and strain ratio value were evaluated. Sensitivity and specificity of CDUS and sono-elastography were compared using $\mathrm{X}^{2}$ test and ROC curves.

Results: Sensitivity and specificity considering hypoechogenicity, halo sign, colourDoppler pattern, polychromatic map elastography and strain ratio were $65,67,70$, 85 and $96 \%$ and $66,65,45,74,92 \%$, respectively. Sono-elastography was more sensitive and specific than all ultrasonographic in predicting malignancy of the thyroid nodules $(p<0.01)$. According to the different elastosonographic features the lesions characterised by score 3 or 4 as evidenced at polichromatic map or with a strain ratio $>2$ may be considered malignant.

Conclusion: Sonoelastography is highly sensitive in the characterisation of thyroid nodules, more accurate than colour-Doppler ultrasound; its application in the current daily clinical practice may limit unnecessary FNAB.

\section{B-816 14:45}

Spectral CT characterisation of thyroid solitary node: a pilot study X.-Y. Zhang, Y.-S. Sun, X.-T. Li, Y. Cui, J.-Y. Li, X.-P. Zhang; Beijing/CN (tjbjcui@yahoo.com.cn)

Purpose: To investigate the clinical utility of quantifying iodine concentration in spectral CT imaging for differentiating thyroid solitary nodes.

Methods and Materials: Thirty patients (ten solitary adenomas, ten solitary papillary carcinomas and ten normal) underwent thyroid gland CT scanning using spectral imaging mode with fast tube voltage switching. Material decomposition images were reconstructed. Regions of interest (ROIs) from unenhanced CT images of two thyroid gland lobes (including lesions) and carotid artery were chosen on iodine-basis images to measure the effective iodine concentrations (elC). Kruskal-Wallis test was used to analyse elC differences among the adenoma, carcinoma, and control groups. Results: There was no difference for elC in the thyroid of the control $(2.01 \pm 0.79 \mathrm{mg} /$ $\mathrm{cc})$, the uninjured lobes of the adenoma $(1.98 \pm 0.6 \mathrm{mg} / \mathrm{cc})$ and carcinoma $(2.05 \pm 0.72 \mathrm{mg} / \mathrm{cc})$ groups $(p=0.85)$. The elC for the carcinomas $(0.75 \pm 0.2 \mathrm{mg} /$ $c c)$ was significantly lower than that for the adenomas $(1.77 \pm 0.48 \mathrm{mg} / \mathrm{cc})(p<0.01)$, but no statistical difference was detected between the adenomas and the control groups $(p=0.51)$. There were statistical differences for the carotid arterial elC of the control, adenoma and carcinoma groups $(0.39 \pm 0.09 \mathrm{mg} / \mathrm{cc}, 0.83 \pm 0.12 \mathrm{mg} /$ $\mathrm{cc}, 1.89 \pm 0.26 \mathrm{mg} / \mathrm{cc}$, respectively, $\mathrm{p}<0.01)$. The carcinoma group had the highest carotid arterial elC

Conclusion: Spectral CT imaging allows for quantitative measurement of effective iodine concentration in solitary benign and malignant thyroid nodes. This may enable the identification of different thyroid diseases in the future.
B-817 14:54

PET/CT scanning in the management of differentiated thyroid carcinoma

F. Pomerri, F. Al Bunni, P. Muzzio; Padova/lT (fabio.pomerri@unipd.it)

Purpose: Differentiated thyroid carcinoma (DTC) has a good prognosis with a 10 -year survival rate higher than $90 \%$. However, $5-24 \%$ of patients experience persistent or recurrent disease. DTC management guidelines recommend PET for imaging patients with 131 I negative whole body scans and increasing serum thyroglobulin levels. The aim of this study was to assess the impact of PET on the therapeutic management of patients with recurrent DTC.

Methods and Materials: 100 DTC patients underwent surgery and postoperative thyroid remnant ablation with 131 and were studied using PET-CT because of rising basal or recombinant TSH-stimulated serum thyroglobulin levels. They were divided into 3 groups based on: diagnostic indications for 131l-negative scanning and increasing thyroglobulin level (group 1, 33 pts); surgical planning for recurrent disease (group 2, 45 pts); and effectiveness of systemic therapy (group 3, 22 pts). Results: PET-positive foci were found in 53/100 studies. PET located recurrent disease in $42 \%$ of group 1 patients, predicted curative resection in $58 \%$ of group 2 patients, and confirmed known metastatic disease in $59 \%$ of group 3 patients. PET results led to surgical treatment with radical intent in 19 patients (complete remission in 8 patients) and palliative in 6 cases. Moreover, $P E T$ results led to high-dose 131 I radio-metabolic treatment for 11 patients and external beam radiotherapy for 3 patients. The remaining 14 patients underwent only clinical follow-up.

Conclusion: The study emphasises how PET indications are changing from a purely diagnostic role in localising 131l-negative DTC recurrences to designing appropriate DTC surgical or combined therapeutic strategies.

\section{B-818 $\quad$ 15:03}

The role of proton-MR-spectroscopy and apparent diffusion coefficient values in the diagnosis of malignant thyroid nodules: preliminary results H. Aydin; Ankara/TR (dr.hasanaydin@hotmail.com)

Purpose: Our aim was to evaluate the role of Proton-MR-Spectroscopy (H-MRS) and apparent diffusion coefficient (ADC) values in the diagnosis of malignant thyroid nodules.

Methods and Materials: After institutional review board approval, a prospective study was conducted in 14 patients with malignant thyroid nodules, H-MRS, and Diffusion weighted imaging (DWI) with b factors $200-400 \mathrm{~s} / \mathrm{mm} 2$ were performed. $A D C$ mapping was constructed and $A D C$ values of the nodules were measured. Standard Cho peak, Cho /Cre ratio and ADC values of malignant nodules were correlated with the five benign nodules and four normal appearing thyroid lobe parenchyma. The gold standard reference was the fine needle aspiration biopsy (FNAB) and histopathology. Statistical analysis of this research was assessed by Fischer's exact t- test, Variance analysis test (ANOVA) and receiver operating characteristics (ROC) curve.

Results: Two acquisitions at TE: 40-144 msec.were applied in the H-MRS. Average Cho/Cre ratio for the malignant nodules was $2.95+\_1.54$ at short TE, $5.30+2.38$ at long TE, had significant statistical differences from the benign nodules, and normal thyroid parenchyma ( 0.805 at TE $40 \mathrm{msec}$. and > 1.225 at TE $144 \mathrm{msec}$ were assumed to be malignant. Average ADC values of malignant ones were $0.06+0.02$, had significantly lower than the benign nodules and the normal thyroid lobe ADC values $(\mathrm{p}<0.05)$

Conclusion: H-MRS acquisitions at short and long TEs, DWI and ADC mapping are new non-invasive imaging modalities, give diagnostic data about the nature of the nodules and can easily distinguish the malignant thyroid nodules from benign ones.

\section{B-819 15:12}

The efficacy of apparent diffusion coefficient value calculation in differentiation between malignant and benign thyroid nodules A. Dilli, U.Y. Ayaz, E. Cakir, E. Cakal, B. Hekimoglu; Çankaya/TR (umityasarayaz@yahoo.com)

Purpose: Differentiation of malignant and benign thyroid nodules is crucial for determination of therapeutic approach. Our purpose is to evaluate the efficacy of apparent diffusion coefficient (ADC) calculation in differentiation between malignant and benign thyroid nodules.

Methods and Materials: A prospective study was conducted in 52 consecutive patients ( 8 males, 44 females) with 59 nodules. Diffusion-weighted single-shot echo-planar magnetic resonance imaging was performed. The $b$ factors were 0 and $400 \mathrm{~s} / \mathrm{mm}^{2}$. ADC values of the thyroid nodules were calculated. Nineteen nodules were proved to be malignant by performing fine needle aspiration biopsy (FNAB) first and by histopathological examination during and after surgery. Forty nodules were accepted benign, as cytopathological examinations after FNAB were taken as 
reference. $A D C$ values were correlated with histopathological results in malignant nodules and FNAB results in benign nodules. Statistical analysis was performed. Results: The mean ADC value of malignant thyroid nodules was $0.829 \pm 0.1786 \mathrm{x}$ $10^{-3} \mathrm{~mm}^{2} / \mathrm{s}$ and mean ADC value of benign thyroid nodules was $1.984 \pm 0.4819 \mathrm{x}$ $10^{-3} \mathrm{~mm}^{2} / \mathrm{s}$. The mean ADC value of malignant nodules was significantly lower than the mean $A D C$ value of benign nodules $(P=0.0001,<0.05=\alpha)$. There was no significant difference between the mean ADC values of various malignant thyroid nodules. The $95 \%$ confidence interval for the mean ADC of malignant nodules was between 0.7429 and 0.9151 , whereas it was between 1.830 and 2.138 for the benign nodules. Conclusion: ADC value calculation is a noninvasive, effective method in differentiation of malignant thyroid nodules from benign ones.

\section{B-820 15:21}

(EPOS)

Vanishing thyroid in patients with renal cell carcinoma treated with tyrosine kinase inhibitor

S. Takahashi, K. Kitajima, T. Maeda, T. Yoshikawa, M. Fujii, Y. Ohno, H. Miyake, M. Fujisawa, K. Sugimura; Kobe/JP (kitajima@med.kobe-u.ac.jp)

Purpose: Hypothyroidism is a common complication in patients treated with tyrosine kinase inhibitors. We evaluated the relationship between the thyroid size shown on CT and thyroid function in patients with advanced RCC treated with tyrosine kinase inhibitors.

Methods and Materials: Thirty-two metastatic RCC patients treated with tyrosine kinase inhibitors (sorafenib; $n=22$, sunitinib; $n=10$ ), with follow-up period of $>12$ months were evaluated. The patients who ever showed elevated TSH of $>10$ $\mathrm{mU} / \mathrm{l}$ were defined as "hypothyroidism". CT scans were performed before and 3 , 6,9 , and 12 months after the initiation of the treatment. The area of the thyroid at the maximum section in each examination was measured and compared to those before the treatment. Differences in the thyroid size were also compared between the patients groups with or without "hypothyroidism", as well as the type of the drug. Results: 16 patients ( 8 sorafenib, 8 sunitinib) presented "hypothyroidism" $97.1 \pm 91.0$ days (range: $12-315$ days) after the beginning of the treatment. In patients with "hypothyroidism", the thyroid reduced in size of $87 \pm 18 \%$ after 3 months, $78 \pm 22 \%$ at 6 months, $67 \pm 22 \%$ at 9 months, and $64 \pm 23 \%$ at 12 months, whereas the patients without "hypothyroidism" maintained the size of $91 \pm 13 \%$ even 12 months after $(p=0.0027)$. The patients treated with sunitinib showed more prominent size reduction of the thyroid than sorafenib $(50 \pm 22 \%$ and $78 \pm 13 \%$ at 12 months; $p=0.0039$ ).

Conclusion: After tyrosine kinase inhibitor treatment, the thyroid showed apparent size reduction in the patients with hypothyroidism, especially treated with sunitinib.

\section{4:00 - 15:30}

Room I/K

\section{Cardiac}

\section{SS 1703}

\section{Valvular and congenital heart disease}

Moderators:

G. Bastarrika; Pamplona/ES

G.K. Schneider; Homburg a.d. Saar/DE

\section{B-821 14:00}

Cine imaging of the aortic valve with low radiation exposure using dynamic volume computed tomography: a feasibility study A. Lembcke ${ }^{1}$, T. Durmus ${ }^{1}$, P. Rogalla ${ }^{2}$, N. Paul ${ }^{2}$, P. Hein ${ }^{1} ;{ }^{1}$ Berlin/DE, ${ }^{2}$ Toronto, ON/CA (Alexander.Lembcke@gmx.de)

Purpose: To evaluate a low radiation scan protocol using dynamic volume computed tomography (DVCT) for aortic valve opening area (AVA) quantification. Methods and Materials: A total of 21 patients (body mass index $25.9 \pm 3.4 \mathrm{~kg} / \mathrm{m}^{2}$ ) with aortic valve stenosis underwent a contrast-enhanced electrocardiogram-gated scan using a DVCT equipped with a 320-row detector. A targeted dynamic data set of the aortic valve was acquired during a single heart beat with $160 \times 0.5 \mathrm{~mm}$ collimation, $350 \mathrm{~ms}$ gantry rotation time and fixed $\mathrm{x}$-ray tube settings of $100 \mathrm{kV}$ and $100 \mathrm{~mA}$. Aortic valve visibility was judged on a 5-point scale (from $0=$ insufficient to $4=$ excellent) and AVA was determined by planimetry. Transthoracic and transoesophageal echocardiography (TTE and TEE, respectively) served as references. The effective radiation dose was estimated from the displayed dose-length-product. Results: The aortic valve was visualised with invariably diagnostic quality rated as $2.1 \pm 0.8$ (range 1-4). The mean AVA on DVCT $\left(0.91 \pm 0.36 \mathrm{~cm}^{2}\right)$ was not significantly different from TEE $\left(0.94 \pm 0.39 \mathrm{~cm}^{2} ; p>0.05\right)$ but slightly larger compared to TTE $\left(0.83 \pm 0.28 \mathrm{~cm}^{2} ; p<0.01\right)$. A good correlation with acceptable limits of agreement was found between DVCT and TTE $\left(r=0.89\right.$, limits $\left.\pm 0.31 \mathrm{~cm}^{2}\right)$ and between DVCT and TEE $\left(r=0.84\right.$, limits $\left.\pm 0.39 \mathrm{~cm}^{2}\right)$. Absolute exposure time was $1.28 \pm 0.16 \mathrm{~s}$ ranging from 0.89 to $1.53 \mathrm{~s}$ depending on the heart rate $(71.9 \pm 10.2 \mathrm{bpm}$ ranging from 51 to $89 \mathrm{bpm})$. The resulting mean effective radiation dose was $0.61 \pm 0.07$ $\mathrm{mSv}$ ranging from 0.44 to $0.73 \mathrm{mSv}$.

Conclusion: Quantification of the AVA is feasible with a reasonable low radiation dose.

\section{B-822 14:09}

Evaluation of aortic valve morphology using multi-imaging modalities: comparison with intraoperative finding

J. Son, S. Ko, J. Choi, Y. Kim, M. Song, J. Shin, H. Hwang, S. Lee; Seoul/KR (20070035@kuh.ac.kr)

Purpose: To retrospectively compare the diagnostic accuracy of dual-source computed tomography (DSCT), cardiac magnetic resonance imaging (cMRI) and transthoracic echocardiography (TTE) for the assessments of the aortic valve (AV) morphology with intraoperative findings as reference standard

Methods and Materials: A total of 251 consecutive patients underwent DSCT, cMR and TTE before undergoing aortic valvuloplasty or replacement. Morphology of AVs evaluated visually by using short-axis DSCT, cine-cMRI and TTE images were compared with each other. Interobserver agreement was measured using Pearson's correlation analysis. The accuracy of diagnosis for multi-imaging modalities was obtained from the comparison with intraoperative findings using the chi-square test. Results: One hundred seventy-seven patients had tricuspid AV, 72 patients had bicuspid $\mathrm{AV}$, and three patients had quadricuspid AV. There was excellent correlation between cMRI and DSCT for AV morphology $(r=0.93, p<0.0001)$. There was good correlation between DSCT and TTE $(r=0.78, p<0.0001)$, MR and TTE $(r=$ $0.75, p<0.0001)$. DSCT, cine-cMRI and TTE had an excellent interobserver agreement $(r=0.93, r=0.92$ and $r=0.82, p<0.0001)$. DSCT $(n=248)$ had a sensitivity of $90.3 \%$ and a specificity of $92.3 \%$ for assessment of AV morphology (bicuspid vs tricuspid valve), and cardiac MRI ( $n=248)$ was $87.5 \%$ and $88.9 \%$, TTE $(n=243)$ was $90.9 \%$ and $73.1 \%$, respectively.

Conclusion: DSCT and cMRI enable accurate noninvasive assessment of the preoperative AV morphology.

\section{B-823 14:18}

Diagnostic accuracy of dual-source computed tomography coronary angiography in patients undergoing evaluation for transcatheter aortic valve implantation

P. Blanke, G. Pache, S. Bulla, C. Lang, M. Langer; Freiburg/DE (philipp.blanke@uniklinik-freiburg.de)

Purpose: To prospectively investigate diagnostic accuracy for dual-source computed tomography coronary angiography (CTCA) to diagnose significant coronary artery stenosis compared with catheter coronary angiography (CCA) in patients with severe aortic stenosis undergoing assessment of aortic root anatomy for transcatheter aortic valve implantation (TAVI).

Methods and Materials: One-hundred and twenty consecutive patients (64/56 female/male, mean age $79.0 \pm 10.9$ years $)$ with severe aortic stenosis $(0.7 \pm 0.1$ aortic valve area $\mathrm{cm}^{2}$ ) underwent a specific dual-source computed tomography protocol for evaluation of aortic root and coronay anatomy prior to TAVI. CCA was considered the standard of reference.

Results: Mean heart rate was $73.0 \pm 15.9$ beats/min. Thirty-four $(28 \%)$ patients had non-sinus rhythm. Prevalence of coronary calcifications was $98 \%$ (117 of 120). Mean Agatston score was 873 (median 475; range 0-7412). CCA identified 112 significantly stenosed segments in 46 patients. One-hundred and thirty-nine of 1699 segments $(8 \%)$ in 43 patients were considered non-diagnostic with computed tomography because of calcium $(n=116)$, motion artefacts $(n=17)$ or stents $(n=6)$. On a patientbased analysis, considering non-diagnostic segments as false positive, sensitivity, specificity and positive and negative predictive values for the detection of significan lesions ( $\geq 50 \%$ diameter stenosis) were $100 \%, 68 \%, 66 \%$ and $100 \%$, respectively. Conclusion: In patients undergoing DSCT for assessment of aortic root anatomy prior to TAVI, ruling out of significant coronary artery disease is impaired in a considerable portion of patients due to high calcium burden or heart rhythm irregularities. 


\section{B-824 14:27}

Imaging characteristics of PTFE felt pledgets used in prosthetic heart valve implantation on 256-slice CT: helpful in the detection of paravalvular leakage?

J. Habets, T.S. Meijer, R.C.A. Meijer, E.P.A. Vonken, W.P.T.M. Mali,

R.P.J. Budde; Utrecht/NL (J.Habets@umcutrecht.nl)

Purpose: Sutures with polytetrafluorethylene (PTFE) felt pledgets are commonly used in prosthetic heart valve (PHV) implantation. Paravalvular leakage is difficult to distinguish from PTFE felt pledgets on CT; both present as hyperdense structures. We assessed the ability to discriminate pledgets from contrast-enhanced solutions on MDCT images based on the difference in attenuation.

Methods and Materials: PTFE felt pledgets were scanned in 4 different contrastenhanced (Ultravist ${ }^{\circledR}$ - 300 mg jopromide/ml) saline concentrations (10.0, 12.0, 13.6 and $15.0 \mathrm{mg} / \mathrm{ml}$ ) in various experimental conditions; (1) pledget only, (2) sutured to the sewing ring of a mechanical PHV, and (3) sutured to the sewing ring of a mechanical PHV and porcine aortic annulus. Scanning was performed on a 256-slice scanner with 8 different scan protocols with various $\mathrm{kV}(100,120)$ and $\mathrm{mAs}(400,600,800,1000)$ settings. Attenuation of the pledgets and surrounding contrast-enhanced saline were measured. In addition, the attenuation of pledgets and contrast-enhanced blood was measured on scans of 20 patients with PHVs. Results: The attenuation of pledgets was consistently higher than that of the surrounding contrast-enhanced solution in all in vitro conditions. Attenuation values in the pledgets and solutions were significantly higher with $100 \mathrm{kV}$ tube voltage and increasing contrast concentration. No effect of tube current was present. Attenuation values of the pledgets and contrast-enhanced blood in patients were $420 \pm 26 \mathrm{HU}$ (range 383-494), and $288 \pm 41 \mathrm{HU}$ (range 202-367), respectively.

Conclusion: PTFE felt pledgets have an higher CT attenuation than surrounding contrast-enhanced blood. This helps to differentiate the pledgets from paravalvular leakage.

\section{B-825 14:36}

Percutaneous aortic valve replacement: predictive value of cusp calcium quantities for peri-procedural complications

F. Schwarz, P. Lange, F. Bamberg, K. Nikolaou, M.F. Reiser, P. Boekstegers,

C. Becker; Munich/DE (florian.schwarz@med.Imu.de)

Purpose: Percutaneous aortic valve replacement (PAVR) has been established to treat patients with severe aortic stenosis who are not amenable to surgical intervention. Although MDCT plays a central role in workup, little is known about CT-derivable parameters to predict procedural risk. Here, we analysed the predictive value of calcium on individual cusps for the occurrence of av-blocks and aortic regurgitation (AR)

Methods and Materials: After IRB-approval, 63 consecutive patients with severe aortic stenosis underwent ECG-synchronised high-pitch MDCT 3-30 days prior to intervention. Valve calcification was assessed quantitatively for each cusp using a semi-automated approach. Postinterventional aortography and diastolic aortic pressure were used to quantify AR. Patients were followed up clinically for 3 months. Results: PAVR was successfully performed in all patients. 30-day mortality was 1/63. 10 patients developed $A R>1^{\circ}$. Pacemaker implantation was required in 15 patients. Mean calcium-quantity was $572 \pm 454$, distributed evenly among the cusps. In the subgroup of patients with $A R>1^{\circ}$, overall calcium-quantity was significantly higher than in those with $A R<=1^{\circ}(919$ vs. $500, p<0.05)$. This relation was preserved on the cusp level. Patients requiring pacemaker implantation had lower amounts of overall calcium; however, this relation became significant for the non-coronary cusp only (145 vs. $266, p<0.05$ ). Calcium-quantity on the non-coronary cusp showed highest predictive value among all cusps and higher predictive value than overall calcium (ROC).

Conclusion: Analysis of the correlation between CT-derived parameters and clinical outcomes in PAVR can lead to more individual risk prediction models and thus to a further increase in safety of this emerging technique.

\section{B-826 14:45}

Low dose high-pitch 128-slice dual-source computed tomography for the evaluation of congenital heart disease in infants and children Z. Cheng, X. Wang; Ji Nan/CN (chengzhaoping110@sina.com)

Purpose: To assess ECG-triggered dual-source computed tomography (CT) in high-pitch spiral acquisition mode for the evaluation of congenital heart disease (CHD) in infants and children regarding image quality and radiation dose.

Methods and Materials: 26 consecutive patients ( 16 men, age 2.5 years) underwent clinically indicated 128-slice dual-source CT angiography of the entire thorax for the evaluation of $\mathrm{CHD}$ using a prospectively ECG-triggered high-pitch spiral acquisition mode (pitch $3.2 ; 80 \mathrm{kV}$ tube voltage, 0.28 seconds gantry rotation time). Overall image quality was evaluated using a 5-grade scoring system and was assessed by looking at cardiac and vascular structures. The image quality for the coronary arteries was also evaluated using a 5-grade scale. Quantitative image quality parameters (noise contrast-to-noise ratio) were measured. Effective radiation doses were calculated. Results: Images of diagnostic quality (grade $\geq 3$ ) were obtained in all 26 children with a mean image quality grade of $4.5 \pm 0.5$ (range 3-5). Interobserver agreement in grading image quality of graft segments was good (kappa $=0.75$ ). Mean coronary artery visibility grade was $4.1 \pm 0.6$ (range $3-5$ ) for the right coronary artery and $4.6 \pm 0.5$ (range $3-5$ ) for the left coronary artery. Image noise in the aorta was $28.9 \pm 7.5$, contrast-to-noise ratio was $11.9 \pm 5.2$. Effective radiation dose was $0.21 \pm 0.08 \mathrm{mSv}$.

Conclusion: Low dose high-pitch prospectively ECG-triggered DSCT angiography provides adequate thoracic and coronary artery image quality in children with $\mathrm{CHD}$.

\section{B-827 14:54}

Role of low-dose 64-slice multi detector CT in evaluation of children with cyanotic congenital heart disease, and its correlation with echocardiography and cardiac catheterization angiography

C. Guruprasadh, K.S. Sodhi, A.K. Saxena, R.M. Kumar, N. Khandelwal; Chandigarh/IN (cguruprasadh@gmail.com)

Purpose: To study the role of low-dose 64-slice multi detector CT (MDCT) in evaluation of children with cyanotic congenital heart disease, and its correlation with echocardiography and cardiac catheterization angiography (CCA).

Methods and Materials: This prospective study included 53 patients in the age group of 1 month to 24 years, with cyanotic congenital heart disease who underwent low-dose MDCT examination. The results were compared with echocardiography and CCA. 23 patients who did not undergo CCA were excluded from the study group. Results: Out of total study group of 30 patients, patent pulmonary arterial confluence was identified by low-dose MDCT in 23 out of the 29 patients, whereas echocardiography and cardiac catheterization angiography could identify it in only 19 patients. Low-dose MDCT was superior in detecting pulmonary artery and its stenosis, associated cardiovascular and extracardiac thoracic anomalies, whereas CCA was better in detection of major aortopulmonary collaterals. Echocardiography and cardiac catheterization angiography were also better in detection of intracardiac valvular and septal pathologies. The effective radiation dose to the patients less than 2 years of age was in the range of 1.7 to $2.5 \mathrm{mSv}$, while in patients more than 2 years of age was in the range of $2.1-4.2 \mathrm{mSv}$, which is much less than radiation dose entailed in CCA.

Conclusion: Low-dose MDCT is a useful tool and can replace invasive cardiac catheterization angiography for diagnosing cardiac and extracardiac thoracic anomalies with markedly reduced radiation dosage to the patient.

\section{B-828 15:03}

Diagnostic value of cardiac magnetic resonance (CMR) in estimation of pulmonary pressure gradient before and 4 months after transcatheter valve implantation

F. Secchi, A. Giardino, V. Nardella, G. Di Leo, M. Carminati, F. Sardanelli; Milan/IT (francescosecchimd@gmail.com)

Purpose: To evaluate the diagnostic value of CMR in estimation of pulmonary pressure gradient before and after transcatheter valve (Melody, Medtronic) implantation. Methods and Materials: After IRB approval and informed consent, patients with pulmonary valve disease underwent transcatheter valve implantation during which peak-to-peak gradient between right ventricle and pulmonary artery was measured (CAT). Before and 4-months after implantation, they were scheduled for echocardiography (US) and 1.5-T CMR. A turbo fast low-angle shot phase-velocity mapping sequence (TR/TE $=41 / 3.2 \mathrm{~ms}$, slice thickness $5 \mathrm{~mm}$; velocity of encoding $250 \mathrm{~ms}$ ) for pulmonary flow estimation was acquired. Pressure gradient $(\Delta P)$ was estimated with both US and CMR from peak flow velocity using Bernoulli's equation. Wilcoxon test were used for comparisons.

Results: we enrolled 19 patients, all of them studied within one week before valve implantation and 4 months later. All CMR examinations were diagnostic, with metallic artifacts limited to the inner space of the valve and not impairing the flow sequence. At CMR, $\Delta \mathrm{P}$ before valve implantation $(38 \mathrm{mmHg})$ was larger $(\mathrm{p}=$ 0.001 ) than that after valve implantation $(19 \mathrm{mmHg}$ ). Before valve implantation no significant differences were observed in terms of $\Delta P$ among the three techniques ( $p$ $>0.382$ ); after valve implantation no significant difference was observed between CMR and US ( $p=1.000)$.

Conclusion: the Melody valve implantation does not impair the pulmonary flow evaluation. It allows for a significant reduction of the pulmonary pressure gradient as confirmed at CMR. Finally, CMR is accurate and comparable with echocardiography. 


\author{
B-830 15:12 \\ Impact of revised task force criteria in distinguishing the athlete's heart \\ from ARVD/C using cardiac magnetic resonance imaging \\ T. Luijkx, B.K. Velthuis, N.H.J. Prakken, M.G.P.J. Cox, M.L. Bots, W.P.T.M. Mali, \\ R.N.W. Hauer, M.J. Cramer; Utrecht/NL (tluijkx@gmail.com)
}

Purpose:Arrhythmogenic right ventricular dysplasia/cardiomyopathy (ARVD/C) is an important cause of sudden cardiac death in young athletes. Cardiac magnetic resonance (CMR) evaluation of athletes to detect possible ARVD/C is complicated by overlapping features such as right ventricular (RV) volume increase.

The recently revised ARVD/C diagnostic Task Force Criteria (TFC) now incorporate cut-off values for RV ejection fraction (EF) and RV end-diastolic volume (EDV) on CMR. We compared CMR ventricular volume, function and cut-off values to distinguish ARVD/C patients from athletes.

Methods and Materials: CMR was performed in 33 ARVD/C patients diagnosed by the revised TFC ( $68 \%$ male), sex- and age-matched to 66 healthy non-athletes and 66 healthy athletes. Quantitative dimensions and volumes were compared and evaluated using existing (revised TFC) and new cut-off values.

Results: RV EDV in ARVD/C patients overlaps considerably with athletes. However, the ratio of left ventricular (LV)/RV EDV, end-systolic volume (ESV) and EF of athletes is similar to that of healthy non-athletes, but significantly different from ARVD/C patients. Areas under the ROC-curve are 0.683 (RV EDV index), 0.837 (LV/RV EDV ratio) and 0.932 (RV EF), demonstrating significantly $(p<0.001)$ better performance of RV EF and LV/RV EDV ratio.

Conclusion: If a wall motion abnormality is present, RV EF is suitable to distinguish ARVD/C from physiologic cardiac adaptation in athletes, whereas RV EDV index is not. The latter results in numerous false ARVD/C diagnoses in athletes. As an alternative we suggest using the LV/RV EDV ratio in athletes, which best represents the balance between left and right ventricles.

\section{4:00 - 15:30}

Room L/M

Neuro

\section{SS 1711}

\section{Paediatric neuroimaging: from foetus to child}

Moderators:

I.M. Björkman-Burtscher; Lund/SE

G. Morana; Genoa/IT

\section{B-831 14:00}

Asymmetric hippocampal development is common: a foetal magnetic resonance study

D. Bajic' ${ }^{1}$, N.C. Moreira' ${ }^{2}$ J. Wikström', R. Raininko ${ }^{1} ;{ }^{1}$ Uppsala/SE, ${ }^{2}$ Porto/PT (drabaj02@yahoo.se)

Purpose: To evaluate normal morphological development of the hippocampal region during the foetal period using magnetic resonance imaging (MRI)

Methods and Materials: MRIs of 63 foetuses without intracranial pathology were reviewed independently by two radiologists who did not know the gestation ages. Three MRls were performed post mortem and 60 in utero. Progress of the hippocampal inversion was analysed in the coronal slices and the left and right sides were compared in every case.

Results: The foetuses in the post mortem examinations were at gestation weeks (GW) 17-18 and in the in utero examinations at GW 19-36. The hippocampal sulcus was open, bi- or unilaterally, in 39 foetuses. The oldest of them was at GW 32. The sulcus was closed at GW 21 at the earliest, unilaterally, and always from GW 33 onwards bilaterally. In $26 / 63$ foetuses $(41 \%)$, the hippocampal development was asymmetric and in 22 foetuses, the right side had developed faster. The non-ovoid hippocampal shape was interpreted to be an earlier form than the ovoid shape. A shallow collateral sulcus was found at GW 17. A deep collateral sulcus was visible earliest at GW 26 unilaterally but in all foetuses from GW 31 onwards it was seen bilaterally. Orientation of the collateral sulcus was not related to the gestational age. Conclusion: There are wide individual temporal variations in the development of the hippocampus and collateral sulcus. Asymmetric development is common and in almost all asymmetric cases, the right hippocampus develops faster.

\section{B-832 14:09}

Measuring in-vivo foetal cortical surface development

G. Langs', G. Kasprian², E. Dittrich², M. Bittner', P.C. Brugger², D. Prayer²,

${ }^{1}$ Cambridge, MA/US, ${ }^{2}$ Vienna/AT (langs@csail.mit.edu)

Purpose: A comprehensive quantitative study of the three-dimensional foetal cortical development, its natural variability, and the correlates with specific pathologies, can aid early and accurate diagnosis of disease. It can foster the understanding of the development process, and can ultimately lead to effective treatment strategies. In this work, we evaluate a quantitative model of the development process derived from high resolution in utero magnetic resonance imaging.

Methods and Materials: We model the foetal cortical development from a data set containing 29 foetal brains with gestational ages from weeks 18 to 30 . After a coarse alignment and an expert annotation of the cortical surface, the temporal development is modeled by a group-wise spatio-temporal registration. We evaluated the accuracy of the model in a leave on out cross validation. We measured the reconstruction error of individual cases, and the automatic estimate of the gestational age derived from a cortical shape.

Results: The proposed spatio-temporal group-wise registration improves the erro to 1.48. The correlation coefficient between nominal gestational age, and automatically estimated age increases during the iterative spatio-temporal modelling process and reaches $\mathrm{r}=0.957$

Conclusion: Results on 29 foetal brains with gestational ages ranging from 18 to 30 gestational weeks indicate that the group-wise spatio-temporal registration has advantages over registration that does not take time into account. The accuracy of the gestational age estimate indicates that the time specificity of the resulting model allows for a precise comparison of individual cases to the population characteristics.

\section{B-833 14:18}

Functional resting-state measurements of the foetal brain

V. Schöpf, G. Kasprian, C. Mitter, P.C. Brugger, D. Prayer; Vienna/AT (veronika.schoepf@meduniwien.ac.at)

Purpose: FMRI has already been used to study foetal brain activity in utero using different kinds of stimulation methods. The underlying neuronal mechanisms strongly connected to gestational weeks of these activity networks have not been described yet. In this study, we used functional connectivity analyses allowing the characterisation of interregional neural interactions during spontaneous activity during rest to study those underlying mechanisms, aiming at specifying the differences of functionally connected cortical networks over foetal brain developmen stages. Furthermore, we aimed at describing subplate specific activity patterns and interactions with subcortical structures for different developmental stages.

Methods and Materials: Functional images of 10 foetuses with morphologically normal brain development, aged from gestational week 18-39, were acquired on a 1.5 T Philips Intera MR scanner using single-shot gradient-recalled echo-plana imaging (10-15 axial slices (5 mm thickness), matrix size 144 x 144, FOV $250 \times$ $250 \mathrm{~mm}$, TE/TR 50/1000 ms, flip angle $90^{\circ}$ ). Data sets were preprocessed including motion correction and brain extraction. Postprocessing was performed using functional connectivity analysis which was implemented in Matlab.

Results: Bilateral resting-state activity could be proved in cortical regions and in the subplate of the frontal, occipital, and temporal. Furthermore, functional correlations of thalamic structures to temporal and frontal subplate regions were obtained. Conclusion: Resting-state measurements in utero provide consistent results and can be analysed by the means of functional connectivity analysis. Our data reflec the high metabolic and functional activity of the subplate between 20 and $30 \mathrm{GW}$. Thus, the regional distribution of foetal brain function can be mapped in normal conditions and monitored in developmental abnormalities.

\section{B-834 14:27}

Prenatal diffusion tensor imaging in callosal agenesis

G. Kasprian, C. Mitter, P.C. Brugger, M. Schmid, V. Kulemann,

L. Perju-Dumbrava, D. Prayer; Vienna/AT (gregor.kasprian@meduniwien.ac.at)

Purpose: This in utero diffusion tensor imaging (DTI) study aims to visualise the 3D architecture of abnormal commissural connectivity in cases of complete (CCA) or partial (PCA) callosal agenesis.

Methods and Materials: 10 non-sedated foetuses with CCA and 3 foetuses with PCA (age: 20-37 gestational weeks-GW, mean: $26.9 \pm 5.4 \mathrm{GW}$ ) underwent in utero MRI at 1.5 T. An axial DTI sequence (16 diffusion encoding directions, reconstructed voxel size $0.94 \mathrm{~mm} \times 0.94 \mathrm{~mm} \times 3 \mathrm{~mm}$, b values of $0 \mathrm{~s} / \mathrm{mm}^{2}$ and $700 \mathrm{~s} / \mathrm{mm}^{2}$ ) was acquired and geometrically coregistered with multiplanar T2-weighted sequences of the foetal brain. After multiple regions of interest were defined in corresponding anatomical regions, the commissural tracts were visualised by a streamline algorithm. 
Results: In utero tractography initially allowed the consistent 3D visualisation of trajectories corresponding to the anatomical course of the bundles of Probst in 2/10 CCA cases uni- and in 8/10 CCA cases bilaterally from $20 \mathrm{GW}$ onwards. In PCA an atypical "sigmoid" bundle connecting the opposing frontal and occipital lobes, which could not be visualised by conventional (T2- and T1-weighted) foetal MR sequences, was depicted by DTI in $3 / 3$ cases.

Conclusion: Prenatal DTI provides further insights in the abnormal connectivity in cases of commissural abnormalities and thus helps to establish a more specific imaging diagnosis in a heterogeneous group of developmental brain pathologies.

\section{B-835 14:36}

\section{Correlations between pre- and postnatal cerebral MRI}

A. Dhouib Chargui, E. Blondiaux, M.-L. Moutard, T. Billette de Villemeur,

F. Chalard, J.-M. Jouannic, H. Ducou le Pointe, C. Garel; Paris/FR

(dhouibamira@yahoo.fr)

Purpose: To analyse the findings of a large series of foetal cerebral MRI and to compare pre-and postnatal MRI data.

Methods and Materials: A prospective study was performed between 2006 and 2009. The pre- and postnatal diagnoses established by MRI were compared and divided into five subgroups: Similar diagnosis (group 1) with possible different appearance related to the natural course of the disease (group 2), different diagnosis (group 3), similar diagnosis with additional findings discovered at postnatal MR (group 4), or with additional findings and different appearance related to the natural course of the disease (group 5). The prognostic impact of a possible disagreement between pre- and postnatal findings was evaluated.

Results: 100 foetuses were included. Foetal MRI was performed at a mean gestational age of 33 weeks (min: 24 weeks, max: 39 weeks) and postnatal MRI, at a mean age of 3 months 18 days. The results were subdivided as follows: group 1 ( $=52)$, group $2(n=32)$, group $3(n=4)$, group $4(n=11)$ and group $5(n=1)$. In 16 cases $(16 \%)$, the postnatal diagnosis was different from the prenatal one and this had a prognostic impact in 10/16 cases. Most discrepancies involved the corpus callosum anatomy (partial vs complete agenesis) and cortical and migration disorders. Conclusion: On a large cohort of 100 foetuses, pre- and postnatal MRI data showed good agreement in $84 \%$ of cases. Disagreement had a prognostic impact in $10 / 16$ cases.

\section{B-836 14:45}

Imaging characteristics of pilomyxoid astrocytomas in comparison with pilocytic astrocytomas

I. Lee' ${ }^{1}$, J. Kim², Y.-L. Suh², H. Eo², H. Shin², S.-Y. Yoo², K. Lee², H. Hwang1; ${ }^{1}$ Daejeon/KR, ${ }^{2}$ Seoul/KR (leeinho1974@hanmail.net)

Purpose: Pilomyxoid astrocytoma (PMA) is a recently described astrocytic tumour that has been previously diagnosed as pilocytic astrocytoma (PA). The purpose of this study was to describe the imaging features of PMAs in comparison with PAs. Methods and Materials: We retrospectively reviewed CT/MR images and medical records of 10 patients with PMA and 38 patients with PA. The mean ages of patients with PMA and PA were 10 and 15 years, respectively. Imaging features including location, composition, enhancement pattern, presence of calcification, haemorrhage and leptomeningeal dissemination were compared in patients with two tumoru types. Results: Six PMAs (60\%) occurred at the suprasellar area and the cerebellum was the most common (45\%) site of PA. Solid component was dominant in eight PMAs $(80 \%)$ and in 19 PAs $(50 \%)$. All of the PMAs containing solid mass $(n=8)$ included non-enhancing portion while $12 / 37$ (32\%) PAs included non-enhancing solid portion $(p<0.05)$. Leptomeningeal dissemination was noted in five PMAs $(50 \%)$ and one PA $(3 \%)(p<0.05)$. Other imaging findings were not significantly different. Conclusion: A younger age, more frequent occurrence at the suprasellar area mainly solid mass containing non-enhancing portion, and more frequent leptomeningeal dissemination are helpful differential features of PMAs as compared to PAs.

\section{B-837 14:54}

Tumour/normal brain apparent diffusion coefficient value ratios allow for distinguishing common paediatric cerebellar tumours

K. Koral ${ }^{1}$, L. Gargan 1 , K. Cederberg', D.C. Bowers ${ }^{1}$, B. Gimi'; ${ }^{1}$ Dallas, TX/US,

${ }^{2}$ Hanover, NH/US (korgun.koral@utsouthwestern.edu)

Purpose: To identify clinically useful tumour/normal brain ADC (apparent diffusion coefficient) ratio thresholds for distinguishing common paediatric cerebellar tumours.

Methods and Materials: 79 patients who had preoperative MR imaging and surgical pathology available between 01/2001 and 12/2009 were included. ADC values of solid tumour, normal cerebellum and right thalamus were measured on PACS by a paediatric neuroradiologist and a paediatric radiology fellow. Tumour/normal brain ratios were calculated and an ROC analysis was performed.

Results: There were 31 PAs (pilocytic astrocytoma) [ 15 males, mean age $=7.35$ years (1.44-18.73)], $27 \mathrm{MBs}$ (medulloblastoma) [19 males, mean age $=6.16$ years (1.15-14.18)], 14 ependymomas [13 males, mean age $=4.61$ years $(0.46-17.18)]$ and 7 AT/RTs (atypical teratoid/rhabdoid tumour) [ 4 males, mean age $=1.06$ years (0.25-2.29)]. The measurements performed by the paediatric neuroradiologist and fellow were concordant. The mean tumour/normal cerebellum brain ratios using measures obtained by the paediatric neuroradiologist were $2.36 \pm 0.44$ for PAs, $0.95 \pm 0.21$ for MBs, for $1.64 \pm 0.5$ ependymomas, and $0.83 \pm 0.11$ for AT/RTs; (mean $\pm \mathrm{std}$ ). Ratios to thalamus were consistently lower in all cases and again measurements performed by the paediatric neuroradiologist and fellow were concordant. ROC analysis distinguished between PAs and ependymomas (threshold $=1.8033$ : sensitivity $=93.55$, specificity $=78.57$ ) and also between ependymomas and MBs (threshold $=1.2461$ : sensitivity $=100$, specificity $=92.59$ ). MBs and AT/ RTs were not significantly distinguishable.

Conclusion: Tumour/normal brain ADC ratio is a useful clinical tool in distinguishing common paediatric cerebellar tumours.

\section{B-838 15:03}

Voxel-based morphometry in young patients with first-episode schizophrenia

A.O. Yakimov, A.N. Barkhatova, I.S. Lebedeva, V.G. Kaleda, A.V. Petraikin, T.A. Akhadov; Moscow/RU (iakimov@gmail.com)

Purpose: To characterise the brain volume abnormalities associated with the diagnosis of first-episode schizophrenia in young patients using voxel-based morphometry (VBM). This issue has never been investigated for the Russian population until now.

Methods and Materials: Group of 12 right-handed young patients with first-episode schizophrenia (F20.09, ICD-10; $19-25$ years) and group of matched mentally healthy subjects were compared utilising $3 \mathrm{~T} \mathrm{MRI}$. The data were acquired using isotropic T1WI based on 3D Turbo Field Echo. For VBM analysis we used Statistical Parametric Mapping program (SPM5) developed in the Wellcome Institute (London, UK). This technique allows the evaluation of grey matter volumes in subjects with schizophrenia compared with healthy controls in an automated fashion, across the whole brain. Original MR-images were imported into the SPM, preprocessed and statistically compared using two-sample t-test with false discovery rate (FDR) of 0.05 . Age and total intracranial volume were used as covariates.

Results: Patient group had significantly reduced grey matter in the left hemisphere Brodmann's area 9 and 10. Height threshold: $T=3.39, p=0.002$ FDR $p<0.05$ Extent threshold: $\mathrm{k}=15$ voxels, $\mathrm{p}=0.664$

Conclusion: Left hemisphere prefrontal cortex grey matter reduction in schizophrenia patients may be crucial to the pathogenesis of schizophrenia. This finding could become useful prognostic marker. Finally, we emphasise future research directions: study of the relationship between VBM findings of multifocal grey matter deficits and changes in the interconnecting white matter tracts; the need for populationbased VBM studies; and study for correlation between VBM measurements and other important schizophrenia markers.

\section{B-839 15:12}

Concentration changes of whole brain grey and white matter in child patients with Tourette's syndrome: evidence from voxel-based morphometry

Y. Liu, Y. Peng, P. Gao; Beijing/CN (liuyue20036@yahoo.com.cn)

Purpose: Pathophysiological evidence suggests involvement of fronto-striatal circuits in Tourette syndrome (TS). To identify TS-related abnormalities in grey and white matter,we used optimised voxel-based morphometry (VBM) which provides a quantitative measure of concentration.

Methods and Materials: Three-dimensional T1-weighted (3DT1) MRI were acquired in 31 TS children ( 28 boys, 3 girls, mean age 8.4 years, range 2-15 years) and 50 age- and sex-matched controls on a 1.5 Tesla Philips scanner. Images were pre-processed and analysed using a version of VBM2 in SPM2.

Results: Using VBM, TS patients showed significant increases in grey matter concentrations in prefrontal areas, sensorimotor areas, hippocampus, the left posterior cingulate gyrus, right caudate nucleus, right cerebellar hemisphere, left pons and parietal-occipital association cortex bilaterally. Decreases in grey matter concentrations were seen in medulla oblongata and left pons. Decreases in white matter concentrations were found in right thalamus, right caudate nucleus, right orbitofrontal structures, left anterior cingulate gyrus, left precentral and postcentral gyrus. Increases in white matter concentrations were detected in pons and anterior midbrain $\left(P<0.05, K_{E} \geq 10\right.$ voxels). Tic severity was not correlated with any con- 
centration changes of grey and white matter in brain. Tic course was negatively correlated with right caudate nucleus $(B e t a=-3.233, P=0.003$ ) and the left posterior cingulate gyrus $($ Beta $=-0.382, P=0.044)$.

Conclusion: Our MRI in vivo neuropathological findings support the hypothesis that alterations in frontostriatal circuitries underlie TS pathology. We also suggest that abnormalities of frontal- parietal-occipital regions, limbic system, cerebellar hemisphere and brain stem are associated with TS.

\section{$B-840$ \\ Preliminary fMRI study on the active dependence of visual cortex in children with anisometropic amblyopia}

L. Jiang, X.M. Wang, B.N. Luo; Guangzhou/CN (Ixjiang427@gmail.com)

Purpose: To investigate the active dependence of visual cortex in the visual cortex with blood oxygen level dependent functional magnetic resonance imaging (BOLD-fMRI) in children ametropic amblyopia and the relation to the spatial frequencies of visual signal.

Methods and Materials: Eight children with anisometropic amblyopia on single eye were performed BOLD-fMRI examination using Siemens Magnetom $3 \mathrm{~T}$ trio equipment. The brain cortex activated by amblyopic eye and contralateral norma eye were evoked and detected by visual stimuli of reversal black-and-white checkerboard pattern with different spatial frequencies of $0.2,0.4,0.7 \mathrm{cpd}$ individually. The data were processed with SPM software and the visual cortex activated by amblyopic eyes and contralateral normal eyes were compared.

Results: BA17, 18, 19 were activated by both eyes. Compared to the contralateral normal eyes, the size and the intensity of the visual cortex activated by amblyopic eyes decreased $(p<0.05)$ mainly in the areas of BA17, 18 . The spatial frequency of black-and-white checkerboard pattern would affect the activation of visual cortex on the active size and intensity. With higher spatial frequency, the size and intensity of the activation visual cortex activated by amblyopic eyes decreased in the area of BA18, $19(p<0.05)$, while there was no difference between the size and intensity of the activation visual cortex activated by contralateral normal eyes.

Conclusion: The active dependence of visual cortex activated by amblyopic eye decreases in size and intensity. And the higher spatial frequency will inhibit the excitability of visual cortex of amblyopic eye.

\section{4:00 - 15:30} Room N/O

\section{Abdominal Viscera}

\section{SS 1701}

\section{Liver MRI approaches function and structure}

\section{Moderators:}

V. Maniatis; Athens/GR

A. Mishra; Tripoli/LY

\section{B-841 14:00}

Dynamic contrast-enhanced MRI of the liver using Gd-EOB-DTPA for hepatic metastases from neuroendocrine tumours

W.H. Sommer ${ }^{1}$, S. Sourbron ${ }^{2}$, A. Haug ${ }^{1}$, K. Herrmann ${ }^{1}$, M.F. Reiser ${ }^{1}$, C. Zech ${ }^{1}$; ${ }^{1}$ Munich/DE, ${ }^{2}$ Leeds/UK (wieland.sommer@med.uni-muenchen.de)

Purpose: To correlate perfusion parameters from dynamic-contrast-enhanced MRI (DCE-MRI) with SUV derived from PET-CT imaging in liver metastases from neuroendocrine tumours (NET).

Methods and Materials: 24 patients with proven metastases of NET underwent DCE-MRI at 3 T (Siemens Verio) using a 3D gradient-echo sequence (TWIST; 2.1 sec temporal-resolution, 5 min acquisition-time) and liver-specific contrast-agen Gd-EOB-DTPA (Primovist, Bayer). All patients underwent PET-CT with the specific tracer Ga-DOTATATE within 1 week after DCE-MRI. ROls were placed in hepatic metastases $(>3 \mathrm{~cm}$ ) visible both on PET-CT and DCE-MRI-images. In addition, one ROI was placed in non-metastatic liver tissue. DCE-MRI parameters (arterial and venous plasma flow, extracellular MTT, extracellular volume and intracellula uptake of Gd-EOB-DTPA) and corrected SUVmax (SUVmax I SUVmax

) from PET-CT datasets were analysed for all ROls using the software PMI

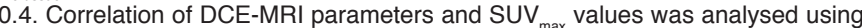
Pearson's correlation coefficient ( $r$ ).

Results: A total of 62 ROls were placed in hepatic metastases of NET. Arteria plasma flow showed a high correlation value with Ga-Dotatate uptake in PET-CT $(r=0.77 ; p<0.0001 ; 95 \% \mathrm{Cl}: 0.56$ to 0.89$)$ followed by extracellular volume $(r=$ $0.43 ; p=0.020 ; 95 \% \mathrm{Cl}: 0.08$ to 0.69$)$. Venous plasma flow $(r=-0.15 ; p=0.42$ $95 \% \mathrm{Cl}:-0.49$ to 0.22$)$, extracellular mean transit time $(r=-0.33 ; p=0.08 ; 95 \% \mathrm{Cl}$ :
-0.62 to 0.04$)$ and intracellular uptake of Gd-EOB-DTPA ( $r=-0.20 ; p=0.29 ; 95 \%$ $\mathrm{Cl}:-0.53$ to 0.17 ) were not significantly correlated to SUV

Conclusion: MRI-perfusion parameters from the DCE-MRI provide functional information for liver metastases of neuroendocrine tumours. Especially arterial plasma flow shows a good correlation with SUV-values derived from the specific PET tracer Ga-Dotatate.

\section{B-842 14:09}

Gadoxetic acid-enhanced MRI with MR cholangiography for the preoperative evaluation of bile duct cancer

H. Sun, J. Lee, H. Park, B. Choi, J. Han; Seoul/KR (hysunsun@hanmail.net)

Purpose: To assess the diagnostic performance of gadoxetic acid-enhanced MRI with MR cholangiography (MRC) in the preoperative evaluation of bile duct cancer staging and resectability.

Methods and Materials: 73 patients, with suspected of having bile duct cancer detected on CT, were included in this study. Two radiologists evaluated the MRI, including 3D-MRC and gadoxetic acid-enhanced dynamic images regarding the tumor respectability. Of the 73 patients, 69 patients underwent surgical exploration, otherwise 4 patients had palliative drainage because of unresectability. 69 patients were included in the assessment of tumour extent, vascular involvement, and lymph node metastasis. The results were compared with the surgical and pathology findings used as standard reference. Diagnostic performance was evaluated using receiver operating characteristics.

Results: The overall accuracy of each reviewer for determining the tumour respectability was 0.62 and 0.94 , respectively. The Az values were 0.802 for reviewer 1 and 0.892 for reviewer 2 for assessment of the both secondary biliary confluence tumour involvement, and 0.773 for reviewer 1 and 0.846 for reviewer 2 for assess ment of the intrapancreatic duct involvement. Reargding the vascular involvement, the $A z$ values were 0.718 and 0.906 , respectively, for the hepatic artery evaluation and 0.55 and 0.88 , respectively, for the portal vein evaluation. For assessment of lymph node metastasis, the overall accuracy was 0.69 and 0.79 , respectively. Conclusion: Gadoxetic acid-enhanced MRI with MRC is accurate and feasible for assessing the tumour extent of bile duct cancer; however, there was limitation in the evaluation of vascular and lymph node involvement, which influence diagnostic accuracy for assessing the tumour resectability.

\section{B-843 14:18}

Correlation between histologic diagnosis and MR signal intensity after GdEOB-DTPA administration of nodules detected within cirrhotic explanted livers: retrospective analysis

V. Battaglia, E. Bozzi, D. Campani, C. Bartolozzi; Pisa/IT (novetrequarti@yahoo.it)

Purpose: To retrospectively evaluate MR signal intensity on baseline, dynamic and hepatobiliary phases of nodules proven at histology on explanted cirrhotic livers. Methods and Materials: Histology detected 71 native nodules on 25 explanted livers (33 hepatocellular carcinomas-HCC, 20 high grade-HGDN, 18 low grade-LGDN dysplastic nodules). MR studies were retrospectively analysed to evaluate signal intensities of nodules. Nodules signal intensities were analysed on baseline T2w.i and T1w.i, dynamic arterial (AP) and late (DP) phases and hepatobiliary (HBP) phases (20 minutes after injection of Gd-EOB-DTPA). Dynamic enhancement patterns of nodules were classified as: A-isointense on AP and DP; B-iso/hypointense on AP and hypointense on DP; C-hyperintense on AP and hypointense on DP; $\mathrm{D}$-hyperintense on AP and isointense on DP. Nodules on HBP were classified as iso, hyper or hypointense.

Results: On baseline, a significant difference $(p<0.00001)$ among signal intensity of HGDN/LGDN and HCC was found. On dynamic study, pattern C showed a positive predictive value (PPV) in assessing the diagnosis of HCC of $100 \%$. Ten out of 33 HCC showed pattern B; no significant difference was observed between neoplasms' dynamic patterns and histological grade $(p<0.5)$. No diagnostic predictive dynamic pattern was found for HGDN, while a pattern A resulted to be typical of LGDN $(p<0.00001)$. On HBP, 48/71 lesions resulted to be hypointense, of whom 32 were HCC. The remnant 16 hypointense lesions on HBP were HGDN. LGDN were all iso or hyperintense.

Conclusion: Patterns B and C associated with nodular hypointensity on HBP are diagnostic for nodular premalignancy/malignancy. 


\section{B-844 14:27}

Diagnostic work-up of segmental cholangitisusing DWI and Gd-EOB-DTPenhanced MRI

S. Magnaldi, A. Wibmer, N. Bastati, D. Muin, S. Baroud, A. Ba-Ssalamah; Vienna/AT (silvia.magnaldi@meduniwien.ac.at)

Purpose: To describe the MRI features of segmental cholangitis using diffusionweighted imaging (DWI) and GD-EOB-DTPA dynamic enhanced MRI and distinguish it from THADs.

Methods and Materials: All patients underwent a standard Gd-EOB-DTPA enhanced MRI of the liver including axial DWI $(b=30,300$ and 600$)$. Patients with at least one wedge- or fan-shaped enhancing area in the arterial phase were selected for further analysis. THAD was defined as hyperenhancing area in the arterial phase and isointense in all remaining sequences. Segmental cholangitis was considered in the presence of hyperenhancing area in the arterial phase, hyperintensity in the DWI and hypointense in the hepatospecific phase. Blood laboratory parameters were compared with the Wilcoxon or the Kruskal-Wallis test.

Results: Of a total of 513 patients undergoing an enhanced MR examination of the liver, 42 showed at least one wedge- or fan-shaped enhancing area in the arterial phase. The typical appearance of a THAD was present in 12 patients $(28.6 \%)$ while 19 patients $(45.3 \%)$ had signs of segmental cholangitis. When compared to patients with THADs, patients with segemental cholangitis had significantly higher mean plasma levels of alkaline phosphatase (91.9 vs. $268.8 \mathrm{U} / \mathrm{l} ; \mathrm{p}=0.015)$ and C-reactive protein (0.05 vs. $3.27 \mathrm{mg} / \mathrm{dl} ; \mathrm{p}=0.006)$.

Conclusion: Segmental increased signal intensities on DWI, combined with segmental wash-out of a hepatospecific contrast agent after administration of Gd-EOB-DTPA, is an additional evidence of focal areas of parenchymal damage in the course of segmental cholangitis.

\section{B-845 14:36}

Tracer-kinetic analysis of Gd-EOB-DTPA in the liver with a dual-inlet twocompartment uptake model

W.H. Sommer ${ }^{1}$, S. Sourbron ${ }^{2}$, M.F. Reiser ${ }^{1}$, C. Zech ${ }^{1} ;{ }^{1}$ Munich/DE, ${ }^{2}$ Leeds/UK (wieland.sommer@med.uni-muenchen.de)

Purpose: Dynamic contrast-enhanced MRI (DCE-MRI) of the liver is able to quantify perfusion parameters. The aim of this study was to develop and validate a perfusion model for liver-specific contrast agent (Gd-EOB-DTPA) which is able to quantify the uptake into the hepatocytes.

Methods and Materials: In order to determine the intracellular uptake rate $\left(\mathrm{min}^{-1}\right)$, a dual-inlet two-compartment uptake model was designed, by modifying a singleinlet uptake model. DCE-MRI data were acquired at $3 \mathrm{~T}$ (Siemens Verio) in 25 patients using the 3D gradient-echo sequence TWIST (2.1sec temporal-resolution, $5 \mathrm{~min}$ acquisition). Gd-EOB-DTPA (Primovist, Bayer) was injected at $2 \mathrm{ml} / \mathrm{s}$. Data were postprocessed using the software PMI 0.4. ROls were drawn on parametric maps of descriptive indices in the abdominal aorta and the portal vein to measure arterial and venous input functions. In each patient, 5 circular ROIs were drawn in normal liver tissue.

Results: The one-compartment model did not provide a good fit to any of the data, and produced unphysical values for the total blood flow and the arterial flow fraction. The uptake model fitted all data accurately, and provided values in the expected range for all known parameters (bloodflow $110 \pm 35 \mathrm{ml} / \mathrm{min}$; arterial flow fraction: $29 \pm 14 \%$; MTT $12 \pm 3.8 \mathrm{sec}$ ). After correction for the difference in relaxivity between intra- and extracellular spaces, the average value for the uptake rate was $1.7 \pm 0.7 \times 10^{-2} \mathrm{~min}^{-1}$.

Conclusion: These data indicate that the proposed model is able to determine Gd-EOB-DTPA kinetics in the liver. The method may present a new and practical paradigm in functional liver MRI, producing quantitative measures of both perfusion and hepatobiliary function.

\section{B-846 14:45 \\ Gd-EOB-DTPA-enhanced liver MR imaging: correlation with liver function} tests

H.-K. Ryeom, S.-M. Lee, G.-C. Kim, Y.-J. Jang, J.-M. Lee; Taegu/KR

(amour7230@naver.com)

Purpose: To correlate commonly used liver function tests and Gd-EOB-DTPA enhanced MR imaging and to define laboratory tests that have potential to predict hepatobiliary phase imaging characteristics.

Methods and Materials: 121 patients who underwent liver MR imaging with Gd-EOB-DTPA were included in this study. Non-enhanced, dynamic phases, and two phases of hepatobiliary image sets (20 and 60 minutes) were obtained using a T1-weighed 3D gradient-echo sequence, The liver signal-to-noise ratio (SNR) and portal vein-to-liver contrast-to-noise ratio (CNR) were calculated from each image set. These calculated values and the results of clinical and laboratory tests were statistically analysed.

Results: Serum bilirubin level showed relatively good correlation with imaging results: liver SNR at 20 minutes $(r=-0.706, p<.01)$, portal vein-to-liver CNR at 20 minutes $(r=-0.7248, p<.01)$, portal vein-to-liver CNR at 60 minutes $(r=-0.7124$, $p<0.01)$, and the ratio of liver SNR at 20 minutes to non-enhanced phase $(r=$ $-0.784, p<.01)$. The liver SNR and portal vein-to-liver CNR were higher in patients with Child class $A$ than with Child class B or $C(p<.003)$, and patients with normal serum bilirubin level than those with elevated serum bilirubin level $(p<.003)$. In both groups, portal vein-to-liver CNR were higher in hepatobiliary phase images obtained at 60 minutes than those obtained at 20 minutes $(p<.001)$

Conclusion: Hepatocyte uptake (liver SNR) and shift of Gd-EOB-DTPA from the intravascular space to the hepatocytes (portal vein-to-liver $\mathrm{CNR}$ ) showed relatively good correlation with the serum bilirubin level.

\section{B-847 14:54}

Hepatobiliary phase of gadoxetic acid-enhanced MRI in the diagnosis of hepatocellular carcinoma in patients with impaired liver function J.-S. Yu, E.-S. Cho; Seoul/KR (yjsrad97@yuhs.ac)

Purpose: The degree of liver enhancement in the hepatobiliary $(\mathrm{HB})$ phase is known to be lower in patients with chronic liver dysfunction. The aim of our study was to evaluate the value of $\mathrm{HB}$ phase of gadoxetic acid-enhanced MRI in the diagnosis of hepatocellular carcinoma (HCC) in patients with impaired liver function. Methods and Materials: Fifty-eight patients with $75 \mathrm{HCCs}$ underwent gadoxetic acid-enhanced MR examination. Thirty-eight patients (group 1) had normal liver function, and the other 20 patients (group 2) had impaired liver function. Two radiologists reviewed two sets of MR images of two groups: set 1, unenhanced and gadoxetic acid-enhanced dynamic images; set 2, additional HB phase and unenhanced and gadoxetic acid-enhanced dynamic images. Diagnostic accuracy was compared using the area under the ROC curve (Az). Lesion-to-liver contrastto-noise ratio (CNR) was calculated in equilibrium phase (5-minute delayed) and HB phase.

Results: Az value increased with the addition of HB phase in both group 1 ( 0.962 to $0.998, p=0.365)$ and group $2(0.875$ to $0.929, p=0.202)$, but did not reach statistical significance. CNR on HB phase $(-126.8 \pm 66.1)$ was significantly lower than that on equilibrium phase $(-87.4 \pm 51.4, p<0.001)$ regardless of patients' liver function. However, CNR drop on HB phase of group $2(26.3 \pm 22.0)$ was significantly lesser than that of group $1(39.6 \pm 27.5, p=0.026)$.

Conclusion: Hepatobiliary phase images of gadoxetic acid-enhanced MR imaging may improve diagnosis of HCC although patient had impaired liver function.

\section{B-848 $\quad 15: 03$}

Optimisation of the dynamic, Gd-EOB-DTPA-enhanced MRI of the liver: the influence of the injection rate

C. Schmid-Tannwald, K.A. Herrmann, M.F. Reiser, C.J. Zech;

Munich/DE (christine.schmid-tannwald@med.uni-muenchen.de)

Purpose: To evaluate the influence of the injection rate on the enhancement of liver vasculature in Gd-EOB-DTPA-enhanced liver MRI.

Methods and Materials: Two groups of patients who were referred to a Gd-EOBDTPA-enhanced liver MRI (1.5 T) were retrospectively evaluated. In both groups a Care Bolus technique to determine the start of the arterial-dominant phase and a power injector were used. The injection rate of the contrast agent was $2 \mathrm{ml} / \mathrm{sec}$ (group 1: $\mathrm{n}=40$ ) and $1 \mathrm{ml} / \mathrm{sec}$ (group 2: $\mathrm{n}=40$ ), respectively. Signal intensitie (SI) in the unenhanced, early dynamic and hepato-biliary phase of T1w 3D GRE sequences were measured in normal liver parenchyma, in the aorta and in the intrahepatic portal vein (PV). Image noise was measured. Signal-to-noise-ratio (SNR), percentage enhancement (PE) and SNR increase were calculated. Statistical significance was tested with Student's t-test.

Results: The SNR of the aorta in the arterial phase was significantly higher in group 2 compared to group 1 (666.98 vs. 468.49; $p=0.007$ ). PE in the aorta was significantly higher in group 2 (593.74 vs. 427.67, $\mathrm{p}=0.0003)$. There was a significantly higher SNR increase of the aorta in group 2 (564.49 vs. $380.62 ; p=$ 0.004). The SNR, the PE and the SNR increase in the portal vein and the liver parenchyma were similar between both groups.

Conclusion: The lower injection rate of $1 \mathrm{ml} / \mathrm{sec}$ showed superior results compared to $2 \mathrm{ml} / \mathrm{sec}$ concerning the arterial enhancement. The reasons for these findings may be explained by saturation effects and a lower rate of protein binding in the dense contrast bolus of group 1. 


\author{
B-849 $\quad 15: 12$ \\ Free-breathing dynamic contrast-enhanced (DCE-MRI) of the abdomen at \\ 3.0 T using radial k-space sampling and k-space-weighted image contrast \\ (KWIC) reconstruction: preliminary study \\ K. Kim ', J. Lee', T. Block'², J. Han', B. Choi'; ' ${ }^{1}$ Seoul/KR, \\ 2Erlangen/DE (medimash@radiol.snu.ac.kr)
}

Purpose: To evaluate the feasibility of free-breathing DCE-MRI of the abdomen and thorax at 3.0 T using radial k-space sampling and KWIC reconstruction. Methods and Materials: Sixteen patients with malignant tumours (hepatocellular carcinoma, $n=5$; liver metastasis, $n=3$; pancreas cancer, $n=4$; lung cancer, $n$ = 4) underwent DCE-MRI at 3.0 T machine (Tim Trio, Siemens). Among them, 6 patients were scanned at two different time points. T1 map was generated from a triple flip-angle $\left(\alpha=2^{\circ}, 8^{\circ}, 15^{\circ}\right)$-based free-breathing radial T1 mapping procedure. Free-breathing dynamic scan was conducted using a modified 3D FLASH-VIBE sequence (TR/TE, 3.3/1.3 ms; flip angle, $11^{\circ}$; matrix size, 256 × 256) with Gd-DTPA bolus administration. The pixel-wise perfusion maps of the volume transfer coefficient (K-trans) and extraellular extravascular volme fraction (Ve) were generated using a software (4D Perfusion, Siemens). The overall image quality regarding the lesion conspicuity, artefact, and the sharpness of abdominal solid organ of the DCE-MRI were rated on a 4-point scale (1, poor, to 4, excellent quality) comparing the breath-hold T1-weighting gradient-echo sequence scanned immediately after the dynamic scan. Results: The proposed free-breathing DCE-MRI technique provided excellent motion-correction sum-up images with the reasonably high spatial resolution (1.48 $\times 1.48 \mathrm{~mm}$ ) and temporal resolution $(4.1 \mathrm{sec})$ that allowed for high frame-rate arterial input function sampling and excellent pixel-wise perfusion maps. Overall image quality score of DCE-MRI was $3.68 \pm 0.47$ with negligible radial artefact and motion artefact. Conclusion: Free-breathing DCE-MRI with radial k-space sampling and KWIC reconstruction is feasible for perfusion analysis of tumour in the abdomen and thorax. It can overcome respiratory motion while providing high spatial and temporal resolutions.

\section{B-850 15.21}

Value of MRI for the diagnosis of benign liver tumours: correlation of updated MRI criteria with histo-molecular analysis of resected specimens L. Baranes ${ }^{1}$, J. Tran-Van-Nhieu', J. Zucman-Rossi', A. Laurent', F. Pigneur ${ }^{1}$, M. Djabbari', A. Rahmouni', D. Cherqui', A. Luciani'; ' ${ }^{C}$ Creteil/FR, ${ }^{2}$ Paris/FR (laurencebaranes@yahoo.fr)

Purpose: To assess the sensitivity and specificity of updated MRI criteria for the diagnosis of benign liver tumour (BLT) with histology and molecular biology of resected liver specimens as reference standard.

Methods and Materials: 35 patients with 41 liver nodules referred between 2000 and 2008 for resection of BLT, and explored by a preoperative liver MRI were included. The resected specimens were analysed by microscopy, immunohistochemistry and molecular biology including CTNNB1 (coding for b-catenin), HNF1 A (coding for HNF1a), and IL6ST (coding for gp130) gene sequencing and RT-PCR quantification of LGR5, GLUL, FABP1, UGT2B7, CRP, SAA2, ANGPT1, ANGPT2. The sensitivity and specificity of updated MRI criteria and their combination were estimated, and the unconformities were analysed (Fisher exact test, Chi-square, Contingency table). Results: The histo-molecular diagnosis was focal nodular hyperplasia (FNH) (13 cases), adenoma (22 cases - 13 telangiectatic/inflammatory, 8 HNF1a-inactivated and 1 non-specific) and 6 non-hepatocellular tumors. The correlation between MR and histo-molecular diagnosis was $76.3 \%$. The specificity and sensitivity of MR for the diagnosis of $\mathrm{FNH}$ were $100 \%$ and $53.8 \%$. Marked hypersignal on T2W sequences associated with delayed persistent enhancement had a specificity of $96.4 \%$ and a sensitivity of $53.8 \%$ for the diagnosis of inflammatory/telangiectatic adenoma. The specificity and sensitivity of the homogeneous signal dropout on chemical shift images for the diagnosis of HNF1a-inactivated adenoma were 100\% and $75 \%$. MRI findings were correlated to molecular biology $(\mathrm{k}=0.85)$.

Conclusion: The specificity of MRI for the diagnosis of FNH is confirmed. MRI features of liver adenoma are correlated to molecular biology data.
$14: 00-15: 30$

Room P

Physics in Radiology

SS 1713

Technical validation of the new opportunities in CT imaging

Moderators:

J. Geleijns; Leiden/NL

W. Stiller; Heidelberg/DE

B-851 14:00

Accuracy estimation of spectral attenuation curves obtained by dual energy CT

G. Van Gompel, N. Buls, K. Nieboer, J. de Mey; Brussels/BE (gert.vangompel@uzbrussel.be)

Purpose: To quantify the accuracy of material attenuation data from synthesised monochromatic images generated by dual energy CT (DECT).

Methods and Materials: A DECT scan (Discovery HD, GE Healthcare) was performed on two patients and a technical phantom with relevant material inserts. From the monochromatic reconstruction images, attenuation curves were obtained for different materials of interest, i.e. plastics (polyethylene, nylon, polycarbonate, acry and polystyrene) and biological tissues (blood, adipose tissue and cortical bone). The attenuation curves were decomposed into a Compton (C) and photo-electric (PE) component by least squares fitting of the Klein-Nishina function and the inverse cubic function of the energy. Compton and PE decomposition coefficients and their ratio $(P E / C)$, reflecting the spectral material properties, were used to investigate the accuracy of the obtained attenuation curves.

Results: A sound congruence (variation $<1 \%$ ) was found between Compton coefficients computed from measured and tabulated attenuation curves (NIST) of the various plastics. For biological materials, the congruence of the Compton coefficients was observed to be lower ( $3 \%$ to $4 \%$ ). However, for these materials, the measured $\mathrm{PE} / \mathrm{C}$ ratios agree well $(<1.5 \%)$ with the $\mathrm{PE} / \mathrm{C}$ ratios obtained from the tabulated attenuation curves, indicating that the difference in Compton coefficients is due to a difference in density rather than a variation in spectral attenuation properties. Conclusion: Based on comparison of Compton and PE decomposition coefficients, we conclude that obtained attenuation data from DECT accurately match the tabulated attenuation data. Our results indicate that the decomposition coefficients are valuable metrics for tissue differentiation and characterisation based on DECT data.

\section{B-852 14:09}

Comparisons between two tube voltage combinations (80/Sn140 kV, $100 /$ $\mathrm{Sn} 140 \mathrm{kV}$ ) in dual energy CT imaging

Y. Ho', S. Yu', G. Lo', W. Lam¹, S. Liu', W. Wong', L. Johnson ${ }^{2} ;{ }^{1}$ Hong Kong/CN, 2Shanghai/CN (yickwingho@gmail.com)

Purpose: Two kV settings, 80/Sn140 kV (80/140) and 100/Sn140 kV (100/140), available for dual energy imaging in dual source CT (DSCT), were compared in the aspects of image quality and radiation dose.

Methods and Materials: A solid water phantom with 11 inserts of physical densities $\left(0.30-1.82 \mathrm{~g} / \mathrm{cm}^{3}\right)$ was scanned using a DSCT at $80 / 140$ and $100 / 140 \mathrm{kV}$ settings, utilising current modulation and keeping other scanning parameters the same and CTDI was recorded. Monochromatic images (40-190 keV) and basis-material iodine images were reconstructed. Signal-to-noise ratio (SNR) of inserts in monochromatic images at different energies were measured as the ratio of the mean $\mathrm{CT}$ number to standard deviation. Contrast ratio (CR) was measured as the ratio of the mean CT number in the insert to that of background. The measured iodine concentration was compared statistically between the two settings using paired t-test.

Results: Using 80/140 setting, the average SNR of inserts in monochromatic images were higher $(p<0.001)$ with CTDI being $18.9 \%$ lower than those of $100 / 140$. It demonstrated a higher CR in 40-90 keV (largest difference $=13 \%$ ), and lower CR in $100-190 \mathrm{keV}$ (largest difference $=6 \%$ ) than the $100 / 140$ setting in monochromatic images. No statistically significant difference was found in iodine concentration measurement $(p=0.126)$ between the two settings.

Conclusion: 80/140 was found to have better imaging quality in terms of better SNR and higher $C R$ in low keV range with lower radiation dose than 100/140, withou significant difference in quantitative measurements such as iodine concentration. 


\author{
B-853 14:18 \\ Iterative reconstruction technique for abdominal MDCT at low tube \\ voltages: assessment of diagnostic accuracy, image quality and radiation \\ dose in a phantom study \\ S.T. Schindera' ${ }^{1}$, L. Diedrichsen' ${ }^{1}$ H. Mueller', O. Rusch'1, R. Raupach², \\ B. Schmidt ${ }^{2}$, P. Vock' ${ }^{1}$ Z. Szucs-Farkas ${ }^{1} ;{ }^{1}$ Berne $/ C H,{ }^{2}$ Forchheim/DE \\ (sschindera@aol.com)
}

Purpose: To assess diagnostic accuracy, image quality and radiation dose of iterative reconstruction (IR) compared with the filtered back projection (FBP) technique for abdominal CT at low tube voltages.

Methods and Materials: A liver phantom with 45 hypovascular tumors (diameters 5-15 mm; tumor-to-liver contrast 10-50 HU) was placed into a water container that mimicked an intermediate-sized patient. The phantom was scanned with a 64-MDCT scanner at 120, 100 and $80 \mathrm{kVp}$. CT datasets were reconstructed with FBP and IR (IRIS, Siemens) algorithms. Image noise was measured and CNR of the tumours was calculated. Radiation dose was assessed by CTDI ${ }_{\text {vol }}$ Tumour detection was independently performed by three blinded radiologists.

Results: Compared with the FBP dataset at $120 \mathrm{kVp}$, the IR dataset at $100 \mathrm{kVp}$ demonstrated significantly lower noise $(20.9 \mathrm{HU}$ and $16.7 \mathrm{HU}$, respectively; $\mathrm{P}<.0001)$ and greater $\mathrm{CNR}$ values for the tumours $(\mathrm{P}<.05)$. The IR dataset collected at $120 \mathrm{kVp}$ yielded the highest sensitivity for tumour detection, while the FBP dataset at $80 \mathrm{kVp}$ yielded the lowest. The sensitivity for the IR dataset at $100 \mathrm{kVp}$ was greater than that for the FBP dataset at $120 \mathrm{kVp}(79.3 \%$ and $74.8 \%$, respectively; P>.05). The CTDI decreased by $39.8 \%$ between the $120-\mathrm{kVp}$ protocol and the $100-\mathrm{kVp}$ protocol and by $70.3 \%$ between the $120-k V p$ protocol and the $80-k V p$ protocol.

Conclusion: A 100-kVp abdominal CT protocol with an IR algorithm for intermediate-sized patients increased the image quality and improved the diagnostic accuracy at a reduced radiation dose when compared with a 120-kVp protocol with an FBP algorithm.

\section{B-854 14:27}

Significant dose reduction with preservation of diagnostic image quality: 128-row high pitch dual source CT

N.A. Amacker ${ }^{1}$, C. Mader', S. Leschka ${ }^{2}$, T. Frauenfelder'; ' ${ }^{1}$ urich/CH, ${ }^{2}$ St. Gallen/CH

Purpose: To investigate the radiation dose and image quality in high-pitch chest and abdominal CT compared to standard-pitch CT.

Methods and Materials: 131 patients (chest $n=100$, abdomen $n=85$ ) scanned on a DSCT with high-pitch mode (pitch 3; group 1) were retrospectively included and compared to 148 patients (chest $n=111$, abdomen $n=98$ ) scanned on a DSCT with standard-pitch mode (pitch 1; group 2). The groups matched for age, gender and BMI. The patients were subdivided into 4 subgroups (BMI $\leq 25$ and $>25$, chest and abdomen scan). Dose length product (DLP), image quality (image noise and scoring) were compared (Kruskal-Wallis test). DLP was correlated to BMI and noise (Pearson correlation). Interobserver agreement was analysed (Kappa-analysis). Results: DLP of group 1 was significant lower compared to group 2 (chest and abdomen: group 1 mean = $367 \pm 92$ mGy cm; group 2, $577 \pm 286 \mathrm{mGy} \mathrm{cm} ; \mathrm{p}<0.0001$ ). In group 1 image noise was significantly higher for abdomen scans when compared to group 2 (group 1: mean $=26.2 \pm 5.7 \mathrm{HU}$; group 2: $18.2 \pm 4.3 \mathrm{HU} ; \mathrm{p}=0.0001$ ), but not for chest scans (group 1: mean $=17.3 \pm 3 \mathrm{HU}$; group 2: $17.7 \pm 3.6 \mathrm{HU}$; $p=0.37)$. The highest correlation $(r=0.64)$ comparing noise to BMI was found for abdomen scans of group 1, reflecting stronger dependency of noise on BM in exams with reduced dose. The overall interobserver agreement was good for group $1(\mathrm{k}=0.75)$ and excellent for group $2(\mathrm{k}=0.92)$.

Conclusion: A significant dose reduction is possible using hp-DSCT. Diagnostic image quality is preserved with slightly higher image noise for abdominal CT scans.

\section{B-855 14:36}

Image quality in low-dose abdominal CT using a new model-based iterative reconstruction algorithm

D.B. Husarik, R.C. Nelson, D. Marin, M.S.R. Bashir, T.A. Jaffe, S. Richard,

J. Colsher, E. Samei; Durham, NC/US (danielahusarik@yahoo.com)

Purpose: To compare image quality of abdominal CT-scans at different radiationdose levels reconstructed with filtered back projection (FBP) and a new modelbased iterative reconstruction (MBiR).

Methods and Materials: An adult anthropomorphic phantom (CIRS, Norfolk, VA) with a custom-built liver insert simulating hepatic attenuation during the late arterial phase was used. The liver insert contained $15 \mathrm{~mm}$ spheres simulating hypervascular liver lesions. Imaging was performed using a 64-section multidetector-row CT scanner (Discovery CT 750HD; GE-Healthcare) with a tube-voltage of $100 \mathrm{kVp}$. Incrementally decreasing tube current-time products were used: $476 \mathrm{mAs}$ (100\%),
$357 \mathrm{mAs}(75 \%), 238 \mathrm{mAs}(50 \%), 119 \mathrm{mAs}(25 \%)$, and $48 \mathrm{mAs}(10 \%)$. All datasets were reconstructed with FBP and MBiR (GE-Healthcare). Lesions-to-liver contrastto-noise ratios (CNRs) were calculated. Objective lesion conspicuity and image quality were rated independently by three readers on a 5-point-scale ( $1=$ terrible to $5=$ excellent).

Results: When radiation-dose was decreased from $100 \%$ to $10 \%$, mean CNR decreased from 0.81 to 0.28 on FBP-images, and from 2.14 to 1.46 on MBiR-images. At the same time objective lesion conspicuity scores decreased from $4.0 \pm 0.9$ to $1.1 \pm 0.3(\mathrm{FBP})$, and from $4.4 \pm 0.9$ to $2.0 \pm 0.5$ (MBiR). Lesion conspicuity on $100 \%$ radiation-dose $\mathrm{FBP}$-images and $50 \%$ radiation-dose MBiR-images were rated equally. Decreasing radiation-dose from $100 \%$ to $10 \%$ increased mean image noise from $30.0 \mathrm{HU}$ to $87.7 \mathrm{HU}$ (FBP), and from $12.1 \mathrm{HU}$ to $17.2 \mathrm{HU}$ (MBiR). Objective image quality scores decreased from $3.3 \pm 0.9$ to $1.1 \pm 0.3$ (FBP), and from $4.3 \pm 0.7$ to $2.3 \pm 0.7$ (MBiR). Image quality scores on $100 \%$ radiation-dose FBP-images and on $25 \%$ radiation-dose MBiR-images were equal.

Conclusion: Image quality on $25 \%-50 \%$ radiation-dose MBiR-images is comparable to $100 \%$ radiation-dose FBP-images, therefore allowing for a $50-75 \%$ reduction in radiation-dose.

\section{B-856 14:45}

Image quality and dose saving aspects of adaptive statistical iterative reconstruction (ASIR) in 64-row abdominal CT imaging

S. Wirth, F. Mück, M. Körner, L. Brummund, M. Scherr, U. Linsenmaier,

J. Grimm, M. Treitl, M.F. Reiser; Munich/DE (swirth@med.uni-muenchen.de)

Purpose: To compare 64-row abdominal CT at different levels of ASIR to prior examinations with filtered back-projection (FBP) in regard to image quality and dose saving potential.

Methods and Materials: 24 patients with abdominal 64-row CT (LightSpeed VCT $\mathrm{XT}, \mathrm{GE})$ as reference examination and a follow-up study on a CT with ASIR option (Discovery $750 \mathrm{HD}, \mathrm{GE}$ ) were included. The noise index was increased from 29 (reference) to 43 (follow-up's) with other relevant scan parameters held constant. Images ( $5 \mathrm{~mm}$ ax, $3 \mathrm{~mm}$ sag and cor; standard kernel) were reconstructed using different ASIR levels $(0,30,50,70$, and $100 \%$, slice and volume mode). 2 experienced radiologists performed consensus reading with respect to image quality regarding soft tissue evaluation in comparison to the reference (-2:clearly inferior, -1 : inferior, 0 :equal, +1 :superior, +2 :clearly superior). The Mann-Whitney-U-test was used for statistical testing.

Results: ASIR 30 and 50 performed equal to the reference (mean $-0.1 ; p>0.05$ ), ASIR levels of 0 or $100 \%$ were rated inferior $(-0.3$ and $-0.7 ; p<0.01)$ without significant differences for slice and volume mode $(p>0.05)$. ASIR 70 volume mode performed equal to ASIR 30 and 50 slice mode $(0.0 ; p>0.05)$ while ASIR 70 slice mode was inferior $(-0.2 ; p<0.05)$. Mean CTDI of the follow-up studies decreased significantly from mean $19.8 \pm 5.3$ to $11.9 \pm 4.6 \mathrm{mGy}(\mathrm{p}<0.01)$. Mean effective dose was reduced to $9 \mathrm{mSv}$.

Conclusion: Image reconstruction with ASIR levels of 30 or 50 in slice mode allows for dose savings of $40 \%$ without significant loss of image quality. Time consuming volume ASIR showed no additional benefits.

\section{B-857 14:54}

Noise reduction and image quality improvement of low-dose and ultra-lowdose brain perfusion CT by HYPR-LR processing

R. Krissak ${ }^{1}$, C.A. Mistretta' ${ }^{2}$, T. Henzler', A. Chatzikonstantinou' ${ }^{1}$ J. Scharf ${ }^{1}$, S.O. Schönberg', C. Fink'; ${ }^{1}$ Mannheim/DE, ${ }^{2}$ Madison, WI/US (radkosk@yahoo.com)

Purpose: To evaluate image quality and signal characteristics of brain perfusion CT (BPCT) obtained by low dose (LD) and ultra low dose (ULD) protocol with and without post-processing using highly constrained back-projection (HYPR)-local reconstruction $(\mathrm{LR})$ technique.

Methods and Materials: Simultaneous BPCTs were acquired in 8 patients over 60 seconds on a dual-source CT system by applying LD ( $80 \mathrm{kV}, 200 \mathrm{mAs})$ on tube $A$ and ULD ( $80 \mathrm{kV}, 30 \mathrm{mAs}$ ) on tube B. Both datasets were post-processed using HYPR-LR. Correlation coefficients between mean attenuation values within corresponding ROIs, area under attenuation curve (AUC), maximum attenuation (MAX), and signal to noise ratio (SNR) of brain parenchyma were assessed. Subjective image quality was assessed on a 5-point scale by two blinded observers. Results: Radiation dose of ULD was more than six times lower compared to LD. SNR was improved by HYPR: ULD vs. ULD+HYPR: $1.9 \pm 0.3$ vs. $8.4 \pm 1.7$, LD vs. LD+HYPR: $5.0 \pm 0.7$ vs. $13.4 \pm 2.4$ (both $p<0.0001$ ). The subjective image quality was excellent (1.3) in LD+HYPR and non-diagnostic (5.0) in ULD. LD and ULD+HYPR images had moderate image quality (3.3 and 2.7). ULD+HYPR correlated better with the standard LD dataset $(r=0.672)$ than unprocessed ULD $(r=$ 0.542). There was no significant difference in AUC or MAX values. 
Conclusion: SNR and image quality of ULD BPCT can be improved to a level similar to LD when using HYPR-LR algorithm without distorting attenuation measurements. This can be used to substantially reduce radiation dose.

\section{B-858 15:03}

CT perfusion reproducibility assessment with a moving perfusion robot A. Hirschenbein 1 , S.Y. Mahgerefteh ${ }^{2}$, J. Sosna ${ }^{2} ;{ }^{1}$ Ra'anana/IL, ${ }^{2}$ Jerusalem/IL (avivhir@gmail.com)

Purpose: Dynamic contrast-enhanced CT is a potentially useful noninvasive technique for assessing tissue perfusion. Our aim was to assess the reproducibility of CT perfusion parameters derived with a perfusion robot.

Methods and Materials: We developed a unique moving CT perfusion robot containing 12 tubes placed 30 degrees apart, with varying concentrations of iodine (1.5 mgl/ml-60 mgl/ml corresponding to $15-1300 \mathrm{HU}$, respectively). The main robot components are motor, reduction gearbox, test-tube wheel, $\mathrm{x}$-ray sensor, position feedback, construction frame, and controller. To simulate repeated time density curves performed at different times, 30 experiments were performed on 5 different days on a 64-slice MDCT using parameters: $140 \mathrm{kVp}, 250 \mathrm{mAs}$, and $5 \mathrm{~mm}$ slice thickness. Cycle times ranged from 1.5 to 5 seconds for 3 minutes. ROI data were collected, plotted, and analysed for trends and reproducibility using a regression fit model.

Results: The CT perfusion robot produced constant movements for predetermined locations. Time density curves for different experiments had a perfect fit $(r 2=0.995)$. However, the changes in $\mathrm{HU}$ from the anticipated values ranged from $5 \%$ for the $60 \mathrm{mgl} / \mathrm{ml}$ iodine concentrations (1283 $\mathrm{HU}$ to $1343 \mathrm{HU}$ for anticipated $1300 \mathrm{HU}$ ) to $200 \%$ for the $1.5 \mathrm{mgl} / \mathrm{ml}$ iodine ( $16 \mathrm{HU}$ to $50.7 \mathrm{HU}$ for the anticipated $15 \mathrm{HU}$ ). Changes were more prominent for physiologic density ranges up to $400 \mathrm{HU}$ and decreased for the higher concentrations.

Conclusion: CT perfusion exam data simulated with a moving robot are not stable, with especially marked differences at the physiological range of iodine density in blood vessels.

\section{B-859 15:12}

Reducing abdominal CT radiation dose with iterative reconstruction in image space

X. uㅡ, W. Lin, G. Shen; Hangzhou/CN (lakewest@gmail.com)

Purpose: To assess radiation dose reduction and image quality for abdominal computed tomography (CT) examination with iterative reconstruction in image space (IRIS).

Methods and Materials: Low-dose abdominal CT examinations with IRIS were performed in 40 patients who had previously undergone routine-dose CT. Phantom also scanned with the same parameters. The image noise, high-contrast resolution and CT radiation dose were compared with and without IRIS. The images quality was graded on a scale of 0 (non-visualisation), 1 (poor), 2 (average), 3 (good), 4 (excellent). Signal-to-noise ratio (SNR) was compared with IRIS and without IRIS. Student t-test was used to determine differences of abdominal CT images with and without IRIS in numerical scores of images quality, SNR and radiation dose. Results: Radiation dose of low-dose abdominal CT examinations with IRIS was reduced by $33.33 \%$ compared with routine-dose CT.The image noise of phantom scanned with and without IRIS was no significantly difference (without IRIS, $4.38 \pm 0.44$; with IRIS, 4.48 \pm 0.38 . $t=0.66, p>0.01$ ). The high-contrast resolution shows 6 line pairs with IRIS as much as without IRIS. The numerical scores of details of abdominal CT images were no significantly difference with and without IRIS (without IRIS, $3.09 \pm 0.54$; with IRIS,3.18 $\pm 0.60 . t=0.32, p>0.01$ ). SNR with IRIS was higher than those without IRIS (without IRIS, 7.13 \pm 2.26 ; with IRIS, 9.15 $\pm 2.29 . t=2.93, p<0.01$ ).

Conclusion: IRIS allows radiation dose reduction sharply for abdominal CT examinations, whereas the overall image quality with IRIS has no significantly degradation, and even better than those without IRIS.

\section{B-860 15:21}

Temporal resolution in dual source CT: comparison between standard single energy scans and dual energy scans with a dedicated, mixed reconstruction technique

A. Rossi, M. van Straten, M.L. Dijkshoorn, R. Booij, G.P. Krestin, N.R. Mollet; Rotterdam/NL (a.rossi@erasmusmc.nl)

Purpose: To compare the motion artefacts and temporal resolution of single energy dual source (DS) cardiac CT with that of dual energy DS cardiac CT that uses a dedicated reconstruction technique to maintain the high temporal resolution. Methods and Materials: Both single energy and dual energy ECG-gated cardiac scans were performed in 15 pigs using a single contrast bolus injection. Single energy DSCT has a nominal temporal resolution of $75 \mathrm{~ms}$. The nominal temporal resolution of standard dual energy CT is $140 \mathrm{~ms}$. Therefore, additional images were generated using a dedicated, mixed single source/dual source reconstruction technique available at the scanner that should result in a resolution of $75 \mathrm{~ms}$. In addition, scans were reconstructed at 140 and $280 \mathrm{~ms}$. All coronary segments, the aortic valve and the epicardial border of the myocardium were evaluated with regard to motion artefacts on a four-point scale (1: non-diagnostic to 4: excellent). Results: Heart rate did not significantly differ between the single energy and dual energy acquisitions ( $49.8 \pm 9.6$ and $52.5 \pm 11.0$ beats/minute). Image quality was similar for single energy and dual energy CT at a temporal resolution of $75 \mathrm{~ms}$. However, moderate to severe artefacts were found more frequently in dual energy images. Significant lower image quality was scored at 140 and $280 \mathrm{~ms}$ temporal resolution. Conclusion: Temporal resolution of dual energy DSCT with a mixed reconstruction technique appears to be similar to the resolution of single energy DSCT. Only at high heart rates image quality of dual energy DSCT seems to be lower than single energy DSCT.

14:00 - 15:30 Room Q

\section{Computer Applications}

\section{SS 1705}

\section{Managing patient dose, quality assurance}

Moderators:

E. Bellon; Leuven/BE

J. Fernandez-Bayó; Sabadell/ES

\section{B-861 14:00}

Dose reduction in MSCT: results of the iterative reconstruction algorithm iDose to reduce image noise

M. Dobritz, A. Fingerle, P.B. Noel, A. Huber, E.J. Rummeny;

Munich/DE (dobritz@roe.med.tu-muenchen.de)

Purpose: To evaluate an intermediate hybrid iterative reconstruction algorithm (iDOSE, Philips Healthcare) for the reduction of image noise and improvement of image quality of low-dose computer tomography images.

Methods and Materials: 22 patients with malignant disease in tumour follow-up were included in the study. All examinations were performed on a 256-slice multidetector CT (iCT, Philips Healthcare) from chest to pelvis. All patients had a previous examination several months before, using a standard dose protocol (protocol A) adapted to body mass index (BMI). Patients were imaged with a low dose protocol (protocol B) and an iterative reconstruction algorithm (iDOSE, Philips Healthcare) was applied to the images. Image noise was measured in different body regions of standard dose, low dose and low dose images reconstructed with the iDOSE algorithm. Subjective image quality was addressed. Statistical analysis was performed. Results: CT examinations with low dose protocol (protocol B) showed an average dose reduction of approximately 50\% compared to the standard protocol (protocol A). Image noise was $21-55 \%$ higher in low dose images. By applying the iDOSE reconstruction algorithm image noise was lowered to the level of standard dose CT images $(p<0.01)$. Subjective image quality was equal in the low dose images reconstructed with iDOSE compared to the standard dose CT images.

Conclusion: The iDOSE reconstruction algorithm allows dose reduction of approximately $50 \%$ in multi-detector computer tomography examinations retaining an image quality equivalent of standard dose CT images without loss of information.

\section{B-863 14:09}

Dose reduction in 64-row whole-body trauma CT: initial experience with adaptive statistical iterative recontruction (ASIR)

M. Körner, A. Harrieder, S. Wirth, M.F. Reiser, U. Linsenmaier; Munich/DE

Purpose: To evaluate radiation exposure of 64-row whole-body CT in patients with major trauma using ASIR.

Methods and Materials: ASIR is a CT raw data processing method that compensates image noise more effectively than conventional filtered back projection (FBP), consequently allowing for reduction of exposure. Dose reports of 64-row whole-body trauma CT (head to symphysis) of 105 studies (72 males, 33 females) with FBP (LightSpeed VCT XT, GE Healthcare, Waukesha, WI) were retrospectively compared to 81 studies (52 males, 29 females) with ASIR (Discovery 750HD, GE Healthcare). The scan parameters for both examinations were identical but the noise index ( $\mathrm{NI}$ ) was increased from 5.2 to 6.0 (head) and 29.0 to 46.0 (chest, abdomen and pelvis) with an ASIR level of $30 \%$ (head) and $50 \%$ (chest, abdomen and pelvis). Scan length, CTDI, and DLP were tested for statistical difference with the Mann-Whitney-U test. 
Results: The mean CTDI for head, chest, and abdomen was significantly lower with ASIR (FBP: $53.8 \pm 2.1$ (standard deviation), $10.3 \pm 2.5,14.2 \pm 3.7$; ASIR $48.7 \pm 2.2,6.7 \pm 2.2,8.0 \pm 3.5, p<0.001)$. The mean DLP for head, chest, and abdomen was significantly lower with ASIR (FBP: $1308.55 \pm 202.0,505.4 \pm 135.4$ $836.0 \pm 250.1$; ASIR: $1189.3 \pm 162.8,342.9 \pm 124.4,572.4 \pm 234.6, p<0.001$ ) There was a significant increase in the mean scan length of the abdomen from $519.7 \pm 50.6$ to $681.8 \pm 263.9 \mathrm{~cm}$ with ASIR $(p<0.001)$. Total mean dose estimation was $24.1 \mathrm{mSv}$ (FBP) and 17.1 (ASIR), resulting in a reduction of $30 \%$.

Conclusion: 64 -row whole-body trauma CT with ASIR helps to significantly reduce exposure dose to the patients by $30 \%$ in comparison to CT with FBP.

\section{B-864 14:18}

Abdominal multidetector-row computed tomography (aMDCT): the unwanted screening?

L.M. Sconfienza, M. Bandirali, A. Esseridou, S. Tritella, A. Aliprandi,

F. Sardanelli; San Donato Milanese/IT (io@lucasconfienza.it)

Purpose: To evaluate the rate of relevant incidental findings (RIFs) at aMDCT Methods and Materials: We retrospectively evaluated the reports of two consecutive series of 200 inpatients and 200 outpatients aMDCT studies performed from February to April 2010. Structured reports included clinical suspect (CS). We considered additional findings which could be related to CS as CS-related additional findings (CSrAF). We considered non-CS-related additional findings requested to be actioned as RIFs: RIF1 (further investigations/follow-up); RIF2 (nonsurgical consultation); RIF3 (biopsy/surgical consultation). Incidental findings not requested to be actioned were excluded. Examinations were classified: R1 = confirmed-CS, no CSrAFs/RIFs; R2 = non-confirmed CS, no CSrAFs/RIFs; R3 = confirmed-CS, CSrAFs; R4 = non-confirmed-CS, CSrAFs; R5 = confirmed-CS, RIF; R6 = non-confirmed-CS, RIFs. Chi-square, 95\% confidence intervals $(95 \%$ IC) with binomial distribution were used.

Results: Rate of males and patients' age were not significantly different for inpatients and outpatients; $53.5 \%$ versus $54.0 \% ; 67.0 \pm 14.6 \mathrm{y}$ (mean \pm standard deviation) versus $67.0 \pm 12.3 \mathrm{y}$, respectively. Inpatients: $\mathrm{R} 1=61 \% ; \mathrm{R} 2=11 \%$; $\mathrm{R} 3$ $=10 \% ; R 4=3 \% ; R 5=12.5 \% ; R 6=2.5 \%$; outpatients: R1 = $66 \% ; R 2=13.5 \%$; $\mathrm{R} 3=5 \% ; \mathrm{R} 4=1.5 \% ; \mathrm{R} 5=10 \% ; \mathrm{R} 6=4 \%$, respectively $(\mathrm{p}=\mathrm{n} . \mathrm{s})$. RIFs rate was $58 / 400$ overall $(14.5 \%, 95 \%$ IC $11.2 \%, 18.3 \%) ; 15.0 \%$ for inpatients, $14.0 \%$ for outpatients $(p=n . s)$. Of 58 RIFs, 28 were RIF1 (48.3\%), 25 RIF2 $(43.1 \%), 5$ RIF3 (8.6\%); inpatients $33.7 \%, 60.0 \%, 6.7 \%$; outpatients $64.3 \%, 25.0 \%, 10.7 \%$, respectively $(\mathrm{P}=.001)$.

Conclusion: About 1/7 patients was diagnosed for a RIF prompting other medical actions. Request of further investigations/follow-up was more probable than medical consultation for outpatients. aMDCT exams work as an unwanted screening for undefined diseases. Consequences in terms of patient outcome and rate of overdiagnosis and overtreatment are not known.

\section{B-865 14:27}

\section{Results of a new quality assurance visual test for medical display} calibration

S. Busoni ${ }^{1}$, C. Fulcheri ${ }^{2}$, G. Belli' ${ }^{1}$, D. Deroo ${ }^{3}$, T. Kimpe ${ }^{3}$, C. Gori' ${ }^{1}{ }^{1}$ Florence/lT, ${ }^{2}$ Sesto Fiorentino/IT, ${ }^{3}$ Kortrijk/BE (busonis@aou-careggi.toscana.it)

Purpose: A new generation QA visual test, based on dynamically generated disks, was developed to test if displays are properly calibrated with respect to reading room illuminance and DICOM GSDF.

Methods and Materials: The visual test is based on detectability of discs, with variable size and luminance, displayed randomly over a uniform background. The user is asked to click on detected discs and the software registers the percentage of correct answers. Two types of visual tests were optimised to assess if the display is properly calibrated with respect to the ambient light settings and the GSDF. Optimal ranges for relevant parameters (disc size/luminance, background luminance) and the effect of a time constraint were investigated. A dataset, large enough to meet statistical significance, was assembled. Tests were performed on a BARCO Coronis medical display. Display calibration and illuminance were measured with a calibrated measurement device.

Results: Optimum disc size (between 8 and $20 \mathrm{~mm}$ ), background and disc contrast values ( $\pm 2, \pm 5, \pm 7$ JNDs) were defined to maximise test effectiveness. The $95 \%$ confidence level that the ambient illuminance is not correct, for $3 \mathrm{~lx}$ reading room calibrated display, requires at least 10 and 50 readings, if $75 \mathrm{~lx}$ and $25 \mathrm{Ix}$ are used, respectively. The $95 \%$ confidence level that the display is calibrated with a GSDF curve instead of a gamma 1.8 or 2.2 curve, requires at least 10 and 20 readings. Conclusion: The developed visual test provides a quick and reliable way to check if the ambient illuminance and the display calibration curve are set properly.
B-866 14:36

Error rates in breast imaging reports generated with automatic speech recognition technology versus traditional transcriptionist-generated reports

A.M. Scaranelo, S. Basma, B. Lord, L.M. Jacks, M. Rizki; Toronto, ON/CA (anabel.scaranelo@gmail.com)

Purpose: To compare the error rate of breast imaging reports generated with ASR versus conventional dictation transcriptionist service (CDT).

Methods and Materials: Breast imaging reports reviewed in multidisciplinary tumour board meetings from January 2009 to April 2010 were scrutinised for minor and significant errors in two separate hospitals.

Results: 615 reports were obtained. 308 reports were generated with ASR and 307 reports were generated with CDT. $23 \%$ of ASR reports contained at least one significant error versus $4 \%$ of CDT reports $(p<0.01)$. Significant errors were more common in breast MRI reports (35\% of ASR and $7 \%$ of CDT), with the lowest error rates occurring in interventional procedure reports (13\% of ASR and $4 \%$ of CDT) and mammography reports $(15 \%$ of ASR and $0 \%$ of CDT) $(p<0.01)$. There was no substantial difference in error rates between reports generated by staff radiologists and trainees or between reports generated from speakers with English as their first language and speakers with English not as their native language. After adjusting for degree of speaker training, native language and modality, reports generated with ASR were 8 times more likely to contain significant errors compared with reports generated with CDT $(p<0.01)$.

Conclusion: Reports generated using ASR are associated with a higher error rate than reports generated with CDT. Native language and training of the speaker do not have a significant impact on error rates. Conversely, the type of imaging of modality is a predictor of the occurrence of reporting errors.

\section{B-867 14:45}

Introduction of a radiological structured standardised reporting

application for abdominal aortic aneurysms

S. Karim ${ }^{1}$, C. Fegeler ${ }^{2}$, M. Mueller-Eschner ${ }^{1}$, D. Boeckler ${ }^{1}$,

H.-P. Schlemmer ${ }^{1}$, H.-U. Kauczor ${ }^{1}$, H. von Tengg-Kobligk ${ }^{1} ;{ }^{1}{ }^{H}$ Heidelberg/DE,

${ }^{2}$ Heilbronn/DE (s.karim@dkfz.de)

Purpose: Current radiological reports are individual and created by conventional free-text. Gathering clinical key parameters is therefore often time consuming and difficult to establish in clinical routine. To enhance diagnosis and therapy monitoring structured standardised reporting (SSR) might be beneficial.

Methods and Materials: To assess communication between the University departments of Vascular Surgery and Radiology, a technology acceptance model was adopted to evaluate the acceptance of current reports in the field of cardiovascular radiology. Based on a SWOT (strengths, weaknesses opportunities, threats) analysis and a detailed literature review of relevant clinical parameters, a new concept for SSR for abdominal aortic aneurysms (AAA) was developed. Qualitative data were analysed from field observations and interviews were conducted with experienced clinicians within the related departments.

Results: An innovative web-based reporting application standardised for AAA was implemented to enhance effectiveness of radiological reports. Clinical parameters of highest interest were subdivided into three main categories: characteristics of the pathology and adjacent anatomy, measurements, as well as additional findings. Integrated graphical widgets (e.g. drop-down-menus) provide the selection of predefined data entries with high usability. Figures for optional use to guide and standardise the reporting are embedded. The new system is open to communicate also with radiology and hospital information systems (RIS, HIS).

Conclusion: Structured standardised reporting has strong potential to improve clinical communication, to accelerate the reporting process and to enable a more comprehensive overview of the patient's diagnosis and monitoring. In summary, the presented SSR application may facilitate a faster extraction of relevant clinical information.

\section{B-868 14:54}

Development and long-term validation of web-based CAD server for synergic performance improvement of CAD software and radiologists Y. Nomura, N. Hayashi, Y. Masutani, T. Yoshikawa, M. Nemoto, S. Hanaoka, S. Miki, K. Ohtomo; Tokyo/JP (nomuray-tky@umin.ac.jp)

Purpose: In this study, we aimed at development and validation of our computerassisted diagnosis/detection (CAD) server with web interface for improving performance of both CAD software and radiologists.

Methods and Materials: We have been building a clinical CAD server system for processing DICOM data transferred from PACS systems, and for displaying the 
results as web pages. The data processing function is implemented as a "plug-in" of the server, for visualisation, automated lesion detection, measurement, etc Another important feature of the server is a function for radiologist's feedback. For example, each radiologist personally classifies lesion candidates of CAD result to four categories; true positive (TP) recognised before CAD reference, TP missed before CAD reference, false positive (FP), and pending. This feedback scheme is purposed not only for collecting additional learning samples for CAD software but also for explicit display of radiologist's interpretation characteristics by pointing out the number and the locations of lesions missed by the radiologist. That is, it realises synergic performance improvement of CAD software and radiologists. Results: In the clinical use for 20 months, feedback data of over 2,300 cases have been collected. Using additional learning samples, the sensitivity of the cerebral aneurysms detection in MRA data was improved from $83.6 \%$ to $90.9 \%$ at $6.0 \mathrm{FPs}$ per case. The display of the individual radiologist's interpretation characteristics has been useful for calling attention to the radiologist's weak points in lesion detection. Conclusion: We have developed a novel CAD server system, and validated its effectiveness through long-term clinical use.

\section{B-870 15:03}

\section{Cloud computing and radiology: applications, challenges and future} developments

C.J. McCarthy, D.P. Brophy, C.P. Cantwell; Dublin/IE (colin.mccarthy@iol.ie)

Purpose: We review the advent of cloud computing, its potential for use in radiology and outline how we used cloud computing to create an original web-based radiology portal.

Methods and Materials: The modern radiology department is heavily dependent on often expensive computing infrastructure. Cloud computing offers the possibility of online databases and customised application delivery over the internet, with the required infrastructure located off-site. Benefits such as savings on capital expenditure and scalability need to be balanced against issues such as data security and patient confidentiality. We developed a web-based resource using open source software and obtained seamless integration with a cloud-based application delivered securely from a remote server.

Results: The portal incorporates a teaching file, reporting tools, group calendar content-management system and a forum with private-messaging capabilities. In addition, dedicated modules allow tracking of discrepancy cases and monitoring of adverse patient events. The portal can be accessed at any radiology workstation on the campus or using a mobile device, and has lead to an exponential increase in data collection for quality assurance and teaching purposes.

Conclusion: Using open source software and cloud computing together has improved education, discrepancy reporting and quality assurance in our multi-site PACS environment. Ensuring compliance with data protection directives, such as HIPAA (US), the EU Data Protection Directive (95/46/EC) and local regulations poses an important challenge to the use of cloud computing in radiology. 
